\title{
Invited Lectures
}

\section{Overviews \\ Purinergic signalling: past, present and future}

\author{
Professor Geoffrey Burnstock PhD DSc FAA FRCS(Hon) FRCP(Hon) FMedSci FRS \\ President, Autonomic Neuroscience Centre
}

Following a brief account of the early history of the discovery of purinergic signalling, a personal view of some of the exciting cutting-edge directions being taken by research in the field will be considered. In particular, emphasis will be placed on the pathophysiology of purinergic signalling and its therapeutic potential.

\section{A Fuller Quiver Adenosine Receptors as Therapeutic Targets}

\author{
Bruce N. Cronstein, MD \\ NYU School of Medicine, 50 First Ave., New York, NY 10016 \\ Cronsb01@med.nyu.edu
}

Recent advances in understanding the role of adenosine and its receptors in physiology and pathophysiology as well as new developments in medicinal chemistry of these receptors have made it possible to begin to realize the therapeutic potential of adenosine and its receptors. Currently adenosine itself is targeted at the heart; a bolus of adenosine intravenously is commonly used to treat supraventricular tachycardia whereas an infusion of adenosine is used as a coronary vasodilator during pharmacologic stress testing. Non-selective adenosine receptor antagonists are used to maintain wakefulness (caffeine), as an analgesic (caffeine) and, less commonly at present, to treat bronchospasm (theophylline, aminophylline, enprofylline). Currently a number of new selective adenosine receptor agonists and antagonists are in testing for a variety of new indications and one adenosinetargeted indication. The older indication is pharmacologic stress testing and new selective $\mathrm{A}_{2 \mathrm{~A}}$ receptor agonists, ATL146e (Apadenoson, Adenosine Therapeutics) and Regadenoson (CV Therapeutics), are currently under study (Phase II-III) for this indication. In addition, a selective $\mathrm{A}_{1}$ receptor agonist is in trials for supraventricular tachycardia (tecadenoson, CV Therapeutics), Currently a number of companies are testing selective adenosine receptor agonists for newer indications including Rheumatoid Arthritis, cancer and wound healing, Adenosine $\mathrm{A}_{3}$ receptor agonists are in trials for the treatment of Rheumatoid Arthritis and Cancer (CF101, CF102, Can-Fite Biopharmaceuticals) and preliminary promising results in patients with RA have been reported. Topical application of an adenosine $\mathrm{A}_{2 \mathrm{~A}}$ receptor agonist to promote healing of diabetic foot ulcers is currently in Phase II trials after the successful completion of a Phase I safety/early efficacy trial (MRE94, King Pharmaceuticals). An allosteric enhancer at the adenosine $A_{1}$ receptor is in trials for neuropathic pain (T-62, King Pharmaceuticals). Because adenosine receptor stimulation may also be involved in disease pathogenesis selective adenosine receptor antagonists are being studied for select indications as well. Istradefylline (KW-6002, Kyowa Pharmaceuticals), a selective adenosine $A_{2 \mathrm{~A}}$ receptor antagonist, is now in late stage clinical trials for the treatment of Parkinson's Disease. Another approach to targeting adenosine receptors is to increase extracellular adenosine concentrations, mechanisms shown to mediate the anti-inflammatory effects of two drugs commonly used in the treatment of Rheumatoid Arthritis, methotrexate and sulfasalazine. Dipyridamole inhibits adenosine uptake and this clearly underlies its effect as a coronary vasodilator during pharmacologic cardiac stressing. New methotrexate analogues are in therapeutic trials in Rheumatoid Arthritis and recent pre-clinical studies of an adenosine uptake inhibitor for the treatment of inflammatory arthritis have been reported (KF24345, Kyowa Hakka Kogyo). In the future 
other therapeutic indications for adenosine receptor targeting may include fibrosis and scarring in the skin or liver, inflammation and inflammatory diseases, chronic pain syndromes and other yet to be described conditions.

\section{Activation of Adenosine $\mathrm{A}_{2 \mathrm{~A}}$ Receptors on $\mathrm{CD4}^{+} \mathrm{T}$ Cells Reduces Reperfusion Injury}

Joel Linden

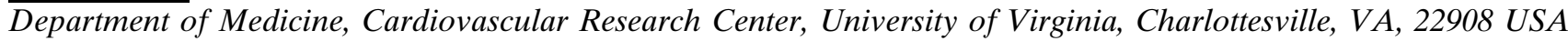

Agonists of adenosine $A_{2 A}$ receptors $\left(A_{2 A} R\right)$ such as ATL146e can substantially reduce necrosis of liver, heart and other tissues when added at the time of reperfusion following ischemia. Protection is attenuated by $\mathrm{A}_{2 \mathrm{~A}} \mathrm{R}$ antagonists and is absent in $\mathrm{A}_{2 \mathrm{~A}} \mathrm{R} \mathrm{KO}$ mice. We prepared bone marrow chimera mice by transplanting bone marrow from $A_{2 A} R$ KO mice to previously irradiated WT syngenic recipients (KO/WT chimera) or vice versa. Experiments with these mouse chimera revealed that $\mathrm{A}_{2 \mathrm{~A}} \mathrm{R}$-mediated protection from liver or heart reperfusion injury is entirely dependent on $\mathrm{A}_{2 \mathrm{~A}}$ Rs on bone marrow-derived cells. A mouse line with a floxed adora2a gene was constructed to enable specific deletion of receptors in tissues expressing Cre recombinase. The lysM promoter was used to express Cre selectively in macrophages and neutrophils. LysMCre- $\mathrm{A}_{2 \mathrm{~A}} \mathrm{R}^{\mathrm{f} / \mathrm{f}}$ mice effectively delete the $\mathrm{A}_{2 \mathrm{~A}} \mathrm{R}$ gene, adora2a, in macrophages and neutrophils but not $\mathrm{T}$ cells based both on functional assays and PCR based assays to quantify the floxed and recombined genes in purified cell populations. ATL146e was able to inhibit reperfusion injury in LysMCre- $\mathrm{A}_{2 \mathrm{~A}} \mathrm{R}^{\mathrm{f} / \mathrm{f}}$ mice, indicating that bone marrow derived cells other than neutrophils and macrophages contribute to protection. Macrophages participate in liver reperfusion injury because injury is reduced by depletion of macrophages with liposomal clodronate. Lymphocytes also appear to participate in reperfusion injury because Rag1 KO mice that lack lymphocytes and B cells are protected. Reperfusion injury is restored in Rag1 $\mathrm{KO}$ mice by adoptive transfer of $\mathrm{CD} 4^{+}$, but not $\mathrm{CD} 8^{+} \mathrm{T}$ cells, and injury is not restored by $\mathrm{CD}^{+}$cells derived from INF $\gamma \mathrm{KO}$ mice. Assays of $\mathrm{CD}^{+} \mathrm{T}$ cells in vitro indicates that activation of the TCR causes rapid induction of $A_{2 A} R$ mRNA, and that ATL146e binding to the $A_{2 A} R$ can reduce TCRstimulated INF $\gamma$ production by $98 \%$. ATL146e reduces reperfusion injury in Rag1 KO mice following adoptive transfer of WT, but not $\mathrm{A}_{2 \mathrm{~A}} \mathrm{R} \mathrm{KO} \mathrm{CD} 4^{+}$cells purified from donor spleens. As confirmation that $\mathrm{CD}^{+}$cells are important in reperfusion injury we found that depletion of these cells with anti-CD4 ${ }^{+}$antibodies also reduced liver or hear reperfusion injury but anti-CD8 ${ }^{+}$antibodies that effectively deplete $\mathrm{CD} 8^{+}$cells from blood are ineffective. Immunohistochemistry was used to measure the time course of accumulation of $\mathrm{T}$ cells into the heart and liver after initiating reperfusion following ischemia. In both tissues there is a small but significant accumulation of $\mathrm{T}$ cells within minutes that is largely attenuated by ATL146e. These data suggest that an important means by which $\mathrm{A}_{2 \mathrm{~A}} \mathrm{R}$ activation reduces reperfusion injury is by activating receptors on $\mathrm{CD} 4^{+} \mathrm{T}$ lymphocytes. This reduces macrophage-dependent $\mathrm{T}$ cell activation and $\mathrm{INF} \gamma$ release required for further rapid recruitment of additional lymphocytes and/or subsequent recruitment of neutrophils into the tissue. In ongoing work we are attempting to determine what factors are responsible for activating $\mathrm{CD}^{+} \mathrm{T}$ cells during reperfusion injury and if a particular subpopulation of $\mathrm{CD}^{+} \mathrm{T}$ cells are selectively activated. $\mathrm{A}_{2 \mathrm{~A}}$ agonists may be clinically useful for the treatment of ischemia-reperfusion injury, e.g. during coronary reperfusion following myocardial infarction, or tissue transplantation.

\section{Berne Award Lecture: Targeting Adenosine Receptors}

Bertil B. Fredholm

Department of Physiology and Pharmacology, Karolinska Institutet, S-171 77 Stockholm, Sweden

Bertil.fredholm@ki.se

My work in the adenosine area started, inspired by the professors Berne, Gerlach and Fain, with attempts to examine the physiological roles of adenosine in circulation and metabolism. It was realised early-especially by

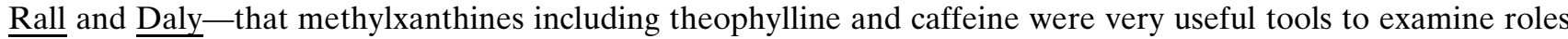


of adenosine, because they acted as receptor antagonists. The cardiovascular, central nervous and metabolic actions of adenosine also prompted several investigators, not least Westermann, to initiate development of adenosine analogues with some level of selectivity. Using these as tools we discovered that adenosine and ATP reduced neurotransmitter release, something that was independently discovered by Ribeiro.

The work of Van Calker, Hamprecht and Londos had clarified that there are at least two receptor subtypes, which we now call $\mathrm{A}_{1}$ and $\mathrm{A}_{2}$. With many collaborators, especially the late Tom Dunwiddie, we explored the roles of $A_{1}$ and $A_{2}$ receptors in brain tissue and related these actions to studies of adenosine formation. To the latter end, we used and modified techniques pioneered by Mcllwain and Daly, and found with Ungerstedt (in the first ever study using microdialysis) that there are resting levels of adenosine that are substantial (20-300 $\mathrm{nM})$. The effects of adenosine on transmitter release and neurotransmission were mediated by $\mathrm{A}_{1}$ receptors, whereas the marked effect on lymphocytes and blood vessels were mediated by $\mathrm{A}_{2}$ receptors. Radioligands were developed by several different groups and this enabled careful studies of receptor distribution. Autoradiographic techniques were especially important. When adenosine receptors were cloned this allowed even more precise localization studies and it was found that adenosine $\mathrm{A}_{2 \mathrm{~A}}$ receptors are co-localized with dopamine $\mathrm{D}_{2}$ receptors in one cell type of the basal ganglia and related structures. The $\mathrm{A}_{2 \mathrm{~A}}$ receptors were found to be of tremendous importance in the basic physiology of the basal ganglia and to be involved in many drug actions and physiological responses.

Cloning of the adenosine receptors also allowed the generation of knock-out mice to pinpoint the roles of the different receptors. These mice have shown that adenosine is not critically important for development or basic physiology, and that adenosine has, as suspected from the earlier investigations, a predominantly modulatory role. Therefore modulatory adaptations are limited and adenosine receptors are interesting drug targets. Using $\mathrm{A}_{2 \mathrm{~A}}$ receptor knock-out mice it has been shown that these receptors are of fundamental importance for the stimulatory actions of caffeine and for its effects on arousal. It is also shown that $\mathrm{A}_{2 \mathrm{~A}}$ receptors can be a target for drugs to combat Parkinsońs disease, as well as ischemia. Mice with targeted deletions of $\mathrm{A}_{1}$ receptors have shown that these receptors are critically important in kidney physiology. $\mathrm{A}_{1}$ receptors are important in limiting epileptic seizures and resulting neuronal death. They are very important in regulating transmitter release, but apparently play little role in ischemic cell death. This suggests that excitatory amino acid release may be overemphasized as a mechanism of neuronal cell death after ischemia. By contrast, $A_{1}$ receptors are very important in mediating protective effects in the heart, cause analgesia, and they play a role in metabolic adaptations.

Thus, many years of research targeting adenosine receptors have shown that they are important in some, but not many, physiological process. Adenosine receptors, do, however play several important pathophysiological roles. Therefore drugs that target adenosine receptors have a future.

\title{
$P 2 X$ and P2Y receptor-involvement in pain sensation; possible targets for new analgesics
}

\author{
Peter Illes $^{1}$, Wolfgang Schröder ${ }^{2}$ and Zoltan Gerevich ${ }^{1}$ \\ ${ }^{T}$ Rudolf-Boehm-Institute for Pharmacology and Toxicology, University of Leipzig, D-04107 Leipzig and \\ ${ }^{2}$ Department of Pharmacology, Grünenthal GmbH, D-52099 Aachen, Germany \\ illp@medizin.uni-leipzig.de
}

Cultured dorsal root ganglion (DRG) neurons of rats are known to be endowed at their cell bodies as well as at their peripheral and central processes with $\mathrm{P}_{2} \mathrm{X}_{3}$ receptors mediating rapidly desensitizing inward current responses to ATP or its structure analogue $\alpha, \beta$-methylene ATP $(\alpha, \beta$-meATP). In addition, we described the presence of $\mathrm{P}_{2} \mathrm{Y}_{1}$ receptors at these neurons, negatively coupled to $\mathrm{N}$-type voltage-sensitive $\mathrm{Ca}^{2+}$ channels. Whereas $\mathrm{P} 2 \mathrm{X}_{3}$ receptor-activation is supposed to induce propagated action potentials and thereby pain sensation, $\mathrm{P} 2 \mathrm{Y}$ receptor-activation may cause an opposite effect, by decreasing the $\mathrm{Ca}^{2+}$ dependent release of sensory transmitters from the central terminals of DRG neurons. According to the known transduction mechanisms of $\mathrm{P}_{2} \mathrm{X}_{3}$ and $\mathrm{P}_{2} \mathrm{Y}_{1}$ receptors an early and rapidly declining pronociceptive action of ATP is expected to be followed by a more protracted antinociceptive action of the same compound. In support of this assumption, we found a negative interaction between $\mathrm{P} 2 \mathrm{Y}_{1}$ and $\mathrm{P} 2 \mathrm{X}_{3}$ receptors at rat DRG neurons, which was due to $\mathrm{G}$ protein activation, but did not utilize any of the hitherto known second-messenger mechanisms including phospholipase $\mathrm{C}$, phosphatidylinositol 3 kinase, protein kinase $\mathrm{C}$, inositoltriphosphate or $\mathrm{Ca}^{2+} /$ calmodulin kinase II. In accordance with our in vitro data the intrathecal application of P2Y receptor agonists exhibited analgesic activity 
in an acute pain model (tail-flick test). In conclusion, the endogenous P2X/P2Y receptor agonist ATP either by itself or via its degradation product ADP may on the one hand induce pain and on the other hand may limit an overt pain reaction. These two opposing mechanisms may operate with a considerable time-lag between each other.

\title{
Structure and function in $\mathrm{P} 2 \mathrm{X}$ receptors
}

\author{
R. Alan North
}

Faculty of Life Sciences, University of Manchester, Manchester, M13 9PT, UK

P2X receptors belong to a superfamily of membrane proteins that are characterised by two membrane-spanning domains and a cysteine-rich ectodomain. Other members of this superfamily include (a) the nucleoside triphosphate diphosphohydrolase enzymes (NTPDase 1-8) which catalyse the extracellular degradation of ATP and ADP, and (b) the family of ion channels that includes the acid-sensing ion channels (ASIC 1-4), epithelial sodium channels (ENaC $\alpha-\delta$ ) and degenerins of $C$. elegans (e.g. MEC-4, MEC-10, DEG-1, DEL-1, UNC-8, UNC105). The three families of proteins are unrelated in primary structure. In terms of quaternary structure, $\mathrm{P} 2 \mathrm{X}$ receptors are considered to be trimers, whereas the body of evidence suggest that ENaC/ASIC channels are tetramers. This lecture will summarise and review experiments in which site-directed mutagenesis and functional expression have been used to deduce those parts of the P2X receptor involved in (a) ATP binding, (b) ion permeation, and (c) interactions with other proteins. The principal focus will be on $\mathrm{P} 2 \mathrm{X}_{1}, \mathrm{P} 2 \mathrm{X}_{2}, \mathrm{P} 2 \mathrm{X}_{2 / 3}$ and $\mathrm{P} 2 \mathrm{X}_{4}$ subunits, with comparisons from studies on other family members where appropriate.

This work was supported by Wellcome Trust.

North, RA Physiol. Rev 2002 82, 1013-1067

\section{The ecto-nucleotidase CD39/NTPDase1 is a key modulator of vascular inflammation and immunity}

\section{Simon C. Robson}

Liver and Transplant Centers, Beth Israel Deaconess Medical Center,Harvard Medical School, Boston. MA, USA srobson@bidmc.harvard.edu

Extracellular nucleotides (e.g. ATP, ADP, UTP) activate type-2 purinergic/pyrimidinergic (P2Y and P2X) receptors on platelets, endothelium and leukocytes. Ecto-nucleotidases hydrolyze these mediators, ultimately to the respective nucleosides, to regulate P2-signaling. Ecto-nucleotidases of the CD39/E-NTPDase family are expressed at high levels in the vasculature and immune systems. In addition to catalytic functions of the ectodomain of CD39, the palmitoylated intracytoplasmic N-terminus has been shown to structurally and functionally associate with a Ran binding protein, termed RanBPM. This multi-adaptor, scaffolding membrane protein regulates small GTPases and influences integrin signaling.

We have proposed that spatial and temporal expression of CD39/NTPDase1 within the vasculature, by immune cells and/or derived microparticles (viz. membrane vesicles) could regulate inflammatory processes, immune reactions and also impact development of cancers.

Expression of vascular CD39 appears crucial in regulating innate immunity, platelet thrombotic reactions, acute ischemic insults, altered vascular permeability, angiogenesis and tumor growth. For example, as visualized by in vivo video-microscopy, laser-induced arteriolar thrombus is characterized by rapid accumulation of platelets and microparticles. This process is then stabilized by platelet disaggregation with decreases in thrombus mass. The accumulation of NTPDase1 within thrombi blocks further ADP-mediated platelet activation. Mutant mice null for $C d 39$ and transgenic over-expressors of $C D 39$ show the predicted abnormalities with marked differences in clot formation in vivo.

Dendritic cell expression of CD39 influences antigen presentation and T cellular responses that are crucial in the evolution of adaptive immunity e.g. alloimmune reactions. CD39 is both an important surface marker of T 
regulatory cells (Treg) and an integral functional component of these cells. Co-ordinated expression of CD39 on Treg and the adenosine A2A receptor on activated effector $\mathrm{T}$ cells (Teff) generates a paracrine, immunosuppressive loop. Adoptive transfer of $C d 39$ null Treg fails to inhibit allograft rejection in vivo. Furthermore, $C d 39$ null mice develop autoimmune manifestations with deviated Th1 responses.

In addition to major recognized thromboregulatory roles, CD39 expression also has functional relevance for cellular immunoregulation, in both allo- and autoimmune reactions. These findings suggest integration of vascular inflammatory and immunologic purinergic mechanisms. Pharmacologic modalities to modulate or boost NTPDase1 expression may suppress unwanted, deleterious vascular or immune reactions, as seen in autoimmune disease and transplant graft rejection. In turn, related approaches could be employed to augment host protective responses promoting tissue regeneration and normal repair processes.

Grant support from the National Institutes of Health (HL57307, HL63972 and HL076540).

1. Mizumoto N et al. Nature Medicine 2002. 8: 358-365.

2. Robson SC et al. Semin Thromb Hemost. 2005;31(2):217-233.

3. Wu, Y et al. RanBPM associates with CD39. Biochem. J. 2006. In press.

4. Deaglio S and Dwyer K. et al. 2006. The ecto-nucleotidase CD39 is a surface marker of regulatory T cells and a catalyst for immunoregulation (submitted).

\title{
Giuliana Fassina Award: Therapeutic Potential of Partial A Agonists in Insulin Resistance and Diabetes.
}

\author{
Luiz Belardinelli, John Shryock, Arvinder Dhalla
}

Department of Pharmacological Sciences, CV Therapeutics Inc. Palo Alto, CA. USA 94304

luiz.belardinelli@cvt.com

$\mathrm{A}_{1}$ adenosine receptor $\left(\mathrm{A}_{1} \mathrm{AdoR}\right)$ agonists are potent anti-lipolytic agents that inhibit adipose tissue lipolysis and lower circulating free fatty acids (FFA) levels. A reduction of lipolysis in adipocytes is of potential benefit in treatments of dyslipidemia, type II diabetes, and metabolic syndrome. Therefore, an $\mathrm{A}_{1}$ AdoR agonist that reduces lipolysis in adipocytes may be useful in the therapy of insulin-resistant states. However, $\mathrm{A}_{1}$ AdoR agonists have potential unintended side effects as a result of the presence of $A_{1} A d o R$ in many tissues in addition to the adipose tissue. Functional selectivity of drug action (maximal or near-maximal anti-lipolytic effect with minimal or no cardiovascular effects) can be achieved by exploiting the differential receptor-effector coupling between adipose tissue and cardiac tissue. The undesired effects of $\mathrm{A}_{1} \mathrm{AdoR}$ in non-adipose tissues can be further minimized by use of low-efficacy agonists or partial agonists. We have discovered a novel partial agonist (CVT3619 ) of the $A_{1}$ AdoR that inhibits adipose tissue lipolysis and lowers circulating FFA levels. CVT-3619 is more than 10-30-fold selective for $\mathrm{A}_{1}$ vs other AdoRs as determined by binding assays. CVT-3619 reduces cyclic AMP content and release of FFA from rat epididymal adipocytes with $\mathrm{IC}_{50}$ values (potency) of 6 and $44 \mathrm{nM}$, respectively. CVT-3619 is a partial agonist relative to CPA (a full agonist) to reduce lipolysis in rat isolated adipocytes. CVT-3619 (at concentrations of $10 \mathrm{nM}-30 \mu \mathrm{M}$ ) does not change atrial rate in rat isolated heart and causes a small $(6-\mathrm{msec})$ prolongation of the AV nodal conduction time without causing atrioventricular block in guinea pig isolated heart. In in-vivo studies, CVT-3619 $(1-10 \mathrm{mg} / \mathrm{kg})$ decreases both FFA and triglyceride (TG) levels $(15-57 \%)$ in a dose-dependent manner in awake rats. CVT-3619 does not have any significant effect on heart rate and blood pressure at the doses that have maximal anti-lipolytic effects. At higher doses of CVT-3619 there is a significant but small decrease in heart rate. CVT-3619 also increases the potency of insulin to decrease lipolysis by 3-fold, suggesting that CVT-3619 increases insulin sensitivity. The potential anti-diabetic effect of CVT-3619 (10 mg/kg sc given twice daily for 5 consecutive days) was studied in ZDF rats, an animal model of type II diabetes. CVT-3619 treatment significantly lowers fasting plasma glucose, insulin, FFA and triglycerides as compared to vehicle treated rats. An oral glucose tolerance test performed on the 5th day of treatment with CVT3619 shows an improvement in insulin sensitivity as determined by the area under the curve of the timedependent changes in plasma glucose and insulin levels $\left(\mathrm{AUC}_{\mathrm{g}} \mathrm{x} \mathrm{AUC}_{\mathrm{i}}\right)$. In conclusion, CVT-3619 is a partial, orally bioavailable $\mathrm{A}_{1}$ AdoR agonist that lowers circulating FFA and TG levels resulting in improved insulin sensitivity, with minimal cardiovascular effects. 


\section{Burnstock Lecture: Versatile messengers in the brain: Nucleotide storage, signalling and hydrolysis}

\section{H. Zimmermann}

Institute of Cell Biology and Neuroscience, Biocenter, J.W.Goethe-University, 60439 Frankfurt am Main Germany h.zimmermann@cns.uni.frankfurt.de

ATP was first identified in muscle extracts in 1929. Proof for its role as an extracellular signaling molecule became apparent considerably later and was not without controversy in the beginning. Some of the strongest support for a potential extracellular function of ATP came from the demonstration of its storage inside granules of a variety of secretory cells such as in blood platelets and in chromaffin granules in the 1950ies, and later in adrenergic (1958/ 1963) and cholinergic (1974) synaptic vesicles. ATP was found to be costored with the noradrenaline or acetylcholine. On nerve stimulation, ATP is depleted from cholinergic synaptic vesicles in parallel with acetylcholine and is replenished together with acetylcholine during a subsequent period of rest. Adenosine taken up into cholinergic nerve terminals via a high affinity transporter becomes immediately phosphorylated, ends up in synaptic vesicles in the form of ATP and becomes coreleased with acetylcholine (Zimmermann, 1982) [1]. The costrorage of ATP with neurotransmitter substances as well as the coexistence of neuropeptides with classical neurotransmitters raised the question whether neurons may be able to employ more than one signaling substance, a new principle borne out by Geoffrey Burnstock in 1976 [2].

Also the notion of cell surface-located ATPases reaches far back into the 1950ies, based on both biochemical and enzyme histochemical evidence. Yet the functional role of extracellular ATP hydrolysis remained a matter of speculation. The evidence for an involvement of extracellular nucleotides in a variety of physiological functions increased dramatically form the 1970ies onwards. But the general acceptance of nucleotide signaling by the scientific community began only with the molecular cloning, heterologous expression and functional characterization of the molecular players involved. The first one was ecto-5'-nucleotidase (1990) followed by P2 receptors (1993), and NTPDase1 (CD39) (1996).

By now, nucleotides have been demonstrated to control major functions in the nervous system. These include synaptic transmission and its modulation, reciprocal signaling between neurons and glia, propagation of glial calcium waves, induction of gliosis or also activation, modulation and transmission in sensory systems. A major role of ecto-nucleotidases in these settings is in the modulation of ligand availability at nucleotide and nucleoside receptors. A novel and interesting function of nucleotides uncovered more recently concerns their role in adult and embryonic neurogenesis where ATP and UTP cooperate with growth factor receptors in activating progenitors (Mishra et al., 2006) [3]. This and other recent examples highlight the functional importance of interactive pathways between signaling via nucleotides and other cellular messengers.

(1) Zimmermann, H. 1982, In: Co-transmission, Cuello, A.C. (ed) London: The MacMillan Pess, p. $243-259$.

(2) Burnstock, G. Neuroscience 1976, 1, 239-248

(3) Mishra, S.K., Braun, N., Shukla, V., Füllgrabe, M., Schomerus, C., Korf, H.-W., Gachet, C., Ikehara, Y., Sévigny, J., Robson, S.C., and Zimmermann, H. Development 2006, 133, 675-684

\section{Basic Pharmacology}

\section{Molecular Physiology and Pharmacology of P2X 1 Receptors}

Jonathan Roberts, Catherine Vial, Helen Digby, Kelvin Agboh, Hairuo Wen \& Richard J. Evans Department of Cell Physiology and Pharmacology, Medical Sciences Building, University of Leicester, Leicester, U.K. LE1 $9 H N$

ATP binds to the large extracellular loop of P2X receptors and results in channel activation. It seems likely that residues important for ATP action at the receptor would be conserved in the family and in the extracellular loop 
of the P2X receptors $>90$ of the $\sim 280$ amino acids are identical in at least 5 of the seven P2X receptor subunits. Alanine replacement mutagenesis of the majority of conserved amino acids in the extracellular loop of the $\mathrm{P} 2 \mathrm{X}_{1}$ receptor has little or no effect on the response to ATP indicating that they do not play an essential role. The results from systematic alanine scanning of conserved residues in the extracellular loop give rise to a model of the site of ATP action at the $\mathrm{P}_{2} \mathrm{X}_{1}$ receptor with $\mathrm{K} 68$ and $\mathrm{K} 309$ binding to the phosphate tail and the motifs F185T186 and N290F291R292 co-ordinating the binding of the adenine ring. A similar binding environment has been described for the crystal structure of rat synapsin II. To test and refine the model we have used cysteine scanning mutagenesis of the region S286-I329 to investigate the environment adjacent to important conserved amino acids N290F291R292 and K309 and the residues close to the second transmembrane domain. Methanthiosulphonate (MTS) compounds can be used to modify any accessible free cysteine residues. For the majority of cysteine mutants there was little or no effect on ATP evoked responses or modification by either positively charged MTSEA or negatively charged MTSES. At mutants D316C, G321C, A323C and I328C responses were sensitive to MTS compounds, e.g. an $\sim 50 \%$ reduction in response to ATP on application of MTSEA for G321C. However at these mutants there was no shift in ATP potency of the point mutants from WT either before or after MTS reagent application. These results indicate that the modification of ATP response results from an effect on ionic permeation and not on agonist binding. At mutants N290C, F291C, R292C and K309C responses were sensitive to MTS reagent application, however in these cases ATP potency was significantly reduced compared to WT channels and MTS reagents resulted in a change in ATP potency, for example at $\mathrm{K} 309 \mathrm{C}$ the $\mathrm{EC}_{50}$ was decreased $\sim 40$ fold on addition of positively charged MTSEA showing that charge at this position is important. These support previous findings and suggest that these residues mediate part of the ATP binding pocket.

Supported by the Wellcome Trust.

\section{New insights into $\mathbf{P 2} X_{7}$ receptor signaling}

Annmarie Surprenant Institute of Molecular Physiology, Department of Biomedical Science, University of Sheffield, Sheffield, UK a.supprenant@sheffield.ac.uk

Abstract not received

\section{Novel Signal Transduction Pathways Regulated by P2Y $_{2}$ Nucleotide Receptors Mediate Inflammatory Responses in Mammalian Cells}

Weisman, G.A., Seye, C.I., Liao, Z., Yu, N., Wang, M., Liu, J., Chorna, N., ${ }^{1}$ Baker, O., Camden, J., Sun, G.Y., Gonzalez, F.A., ${ }^{1}$ and Erb, L.

University of Missouri-Columbia, Columbia, MO 65212 USA and *University of Puerto Rico, San Juan, PR 00931 USA

weismang@missouri.edu

The $\mathrm{G}_{\mathrm{q} / 11}$-coupled $\mathrm{P} 2 \mathrm{Y}_{2}$ nucleotide receptor $\left(\mathrm{P} 2 \mathrm{Y}_{2} \mathrm{R}\right)$ for ATP and UTP activates phospholipase $\mathrm{C}$ leading to an increase in $\mathrm{IP}_{3}$-dependent calcium mobilization and diacylglycerol-dependent activation of protein kinase $\mathrm{C}$, responses that regulate the activity of phospholipases $\mathrm{A}_{2}$ in primary murine astrocytes. Recent studies indicate that $\mathrm{P} 2 \mathrm{Y}_{2} \mathrm{Rs}$ possess novel molecular determinants that enable them to activate other signaling pathways independent of $\mathrm{G}_{\mathrm{q}}$ proteins. For example, data indicate that proline rich Src-homology-3 (SH3) binding domains in the intracellular C-terminus of the $\mathrm{P}_{2} \mathrm{Y}_{2} \mathrm{R}$ can mediate the transactivation of growth factor receptors (e.g., vascular endothelial growth factor receptor-2) to promote the up-regulation of cell adhesion molecules (e.g., vascular cell adhesion molecule-1) in endothelium that promote the binding and vascular infiltration of monocytes 
associated with inflammatory responses in cardiovascular diseases. In addition, an arginine-glycine-aspartic acid (RGD) motif in the extracellular domain of the P2 $\mathrm{Y}_{2} \mathrm{R}$ mediates interactions with $\alpha_{v} \beta_{3} / \beta_{5}$ integrins that are required for $G_{o}$ protein activation and nucleotide-induced increases in the motility of primary astrocytes, and $\mathrm{G}_{12-}$ mediated stress fiber formation that regulates cytoskeletal rearrangements required for cell chemotaxis. The $\mathrm{P}_{2} \mathrm{Y}_{2} \mathrm{R}$ is also capable of activating matrix metalloproteases including the adamalysins ADAM 10 and ADAM 17 that increase the degradation of amyloid precursor protein in astrocytoma cells to generate the nonamyloidogenic peptide s-APP $\alpha$, suggesting that $\mathrm{P} 2 \mathrm{Y}_{2} \mathrm{R}$ function may be neuroprotective in Alzheimer's disease. Other data indicate that $\mathrm{P} 2 \mathrm{Y}_{2} \mathrm{R}$-mediated activation of metalloproteases in salivary gland epithelial cells plays a role in the up-regulation of cell adhesion molecules that facilitate lymphocyte binding, a response associated with the auto-immune disease Sjögren's syndrome. Taken together, our results indicate that $\mathrm{P} 2 \mathrm{Y}_{2} \mathrm{R}$ expression in a variety of mammalian cell types can promote inflammatory responses due to the ability of the $\mathrm{P} 2 \mathrm{Y}_{2} \mathrm{R}$ to activate diverse signaling pathways in addition to the well-established activation of $\mathrm{G}_{\mathrm{q}}$ proteins. This study was supported by the National Institutes of Health Grants 1 P01-AG-018357 and 1 R01-DE-07389.

\title{
Nucleotide presynaptic ionotropic receptors are accurately tuned by other neurotransmitters
}

Miguel Díaz-Hernández, Rosa Gomez-Villafuertes, Javier Gualix, Maria Diez-Zaera, Miriam León-Otegui and M. Teresa Miras-Portugal

Presynaptic functional $\mathrm{P} 2 \mathrm{X}$ receptors and dinucleotide receptors are widely present in the CNS. Calcium responses induced by purinergic agonists evoke neurotramitter stores release of synaptic vesicles from Cholinergic, GABAergic and Glutamatergic nerve terminals. However, when a single cholinergic terminal was stimulated with nucleotide and nicotinic agonists altogether results in a significant decrease of the $\left[\mathrm{Ca}^{2+}\right]_{\mathrm{i}}$ signalling compared to responses of each independent agonist. Inhibitory interaction between both receptors is reverted when one of them is blocked by specific antagonists. The receptor's inhibitory cross-talk confirms the involvement of calcium/calmoduline-dependent protein kinase II, CaMKII, as the inhibitory effects are reverted in the presence of the specific inhibitors KN-62 and KN-93.

In GABAergic nerve endings it has been described that activation of GABA-B autoreceptors positively modulated the ATP and dinucleotide responses, inducing a significantly increase on the affinity of purinergic receptors.

Finally preliminary results obtained on Glutamatergic synaptic terminals appear indicate the existence of an inhibitory interaction between $\mathrm{P} 2 \mathrm{X}$ and glutamatergic autoreceptors.

All these results demonstrate the existence of an efficient interaction between purinergic and other autoreceptors populations, opening a new understanding on the functional regulation of terminals releasing the most abundant neurotransmitters in the CNS.

\section{Quantification and agonist-promoted regulation of the $\mathrm{P}_{2} \mathrm{Y}_{1}$ receptor}

\author{
T. K. Harden, D. Houston, D.M. Bourdon, G.L. Waldo, M. Ohno, and K.A. Jacobson \\ Dept. Pharmacology, University of North Carolina School of Medicine, Chapel Hill, NC, USA and NIDDKD, \\ NIH, Bethesda, MD, USA \\ tkh@med.unc.edu
}

The $\mathrm{P}_{2} \mathrm{Y}_{1}$ receptor $\left(\mathrm{P}_{2} \mathrm{Y}_{1}-\mathrm{R}\right)$ is expressed broadly in mammalian tissues, for example on the surface of platelets where it is essential for ADP-promoted aggregation. Our laboratories have focused on the development of $\mathrm{P}_{2} \mathrm{Y}_{1-}$ $\mathrm{R}$-selective antagonists, agonists, and radioligands as reagents for delineating the physiological and molecular properties of this important cell signaling protein. Synthesis of a series of selective, high affinity, non-nucleotide competitive antagonists for the $\mathrm{P}_{2} \mathrm{Y}_{1}-\mathrm{R}$ led to development of $\left[{ }^{3} \mathrm{H}\right] \mathrm{MRS} 2279$ as a radioligand for reliable 
screening of agonists and antagonists of the $\mathrm{P}_{2} \mathrm{Y}_{1}$ receptor. Moreover, the human $\mathrm{P} 2 \mathrm{Y}_{1}-\mathrm{R}$ was purified to homogeneity taking advantage of this radioligand and high level expression of recombinant receptor from a baculovirus in insect cells. We recently developed methodology for radiolabelling with ${ }^{32} \mathrm{P}$ the highest affinity $(\mathrm{Kd}$ $\sim 0.5 \mathrm{nM}) \quad \mathrm{P}_{2} \mathrm{Y}_{1}-\mathrm{R}$ antagonist, 2-iodo- $N^{6}$-methyl-(N)-methanocarba-2'-deoxyadenosine- $3^{\prime}, 5^{\prime}$-bisphosphate (MRS2500). This high specific radioactivity, high affinity radioligand has proved very useful for quantification of natively expressed $\mathrm{P} 2 \mathrm{Y}_{1}-\mathrm{R}$ across mammalian tissues. Moreover, we have applied this radioligand to quantify native $\mathrm{P}_{2} \mathrm{Y}_{1}-\mathrm{R}$ during agonist-induced trafficking in human platelets and in cell lines. Structure activity studies of $\mathrm{P}_{2} \mathrm{Y}_{1}-\mathrm{R}$ antagonists also were instrumental in development of (N)-methanocarba-2MeSADP (MRS2365), which is a high affinity full agonist at the ADP-activated P2 $\mathrm{Y}_{1}-\mathrm{R}$ but does not bind to the ADP-activated P2 $\mathrm{Y}_{12}-\mathrm{R}$ or $\mathrm{P}_{2} \mathrm{Y}_{13}-\mathrm{R}$. This molecule provides a reagent for specific activation of the $\mathrm{P}_{2} \mathrm{Y}_{1}$ receptor in tissues, e.g. platelets, where multiple ADP-activated receptors exist.

\section{Release of nucleotides and UDP-sugars from Airway Epithelia}

\section{Eduardo R. Lazarowski}

Cystic Fibrosis Center. University of North Carolina School of Medicine

Eduardo_Lazarowski@med.unc.edu

Extracellular nucleotides modulate multiple components of the innate lung defense by activating epithelial airway surface purinergic receptors. ATP, UTP, UDP, and adenosine control the production of airway surface liquid (ASL) by regulating ion transport. ASL nucleotides also promote cilia beating and mucin secretion, and thereby activate the mucociliary clearance (MCC) process that removes noxious materials from the airways. In addition, a recent study illustrated that the $\mathrm{P}_{2} \mathrm{Y}_{14}$ receptor (the cognate receptor for UDP-glucose, UDP-Glc) is expressed in alveolar epithelial type II and other lung epithelial cells and promotes the secretion of the potent neutrophil chemoattractant IL-8 (1). Thus, UDP-Glc may be an inflammatory mediator in the airways. Noteworthy, P2Y 14 receptor transcripts are most abundantly expressed in circulating neutrophils, relative to other peripheral tissues (2). Quantitative PCR analysis on human neutrophils illustrated the presence of $\mathrm{P}_{2} \mathrm{Y}_{14}$ receptor transcripts in copy number comparable to that of the abundantly expressed chemotactic peptide (fMLP) receptor FPR-1.

Despite the physiological and pathophysiological relevance of the responses triggered by extracellular nucleotides and UDP-sugars in the airways, the mechanisms and pathways for nucleotide release from airway epithelia are unknown. UDP-sugars are donor substrates for glycosylation reactions in the lumen of the secretory pathway. UDP is a byproduct of this process, which in turn is hydrolyzed to UMP by a Golgi resident UDPase. UDP-sugar/UMP antiporters translocate luminal UMP to the cytosol and cytosolic UDP-sugars to the lumen of the Golgi. We have discovered that in addition to ATP, airway epithelial cells release UDP-Glc constitutively and that release of UDP-Glc from resting epithelial cells is markedly affected by genetic manipulation of Golgi UDPsugar translocators. These observations suggest that UDP-Glc is released to the extracellular milieu from the secretory pathway, i.e., following translocation to the Golgi via UDP-sugar/UMP antiporters.

Impaired MCC, mucus hypersecretion, and neutrophil inflammation are characteristics of chronically diseased airways, e.g., cystic fibrosis (CF), chronic obstructive lung disease (COPD), and asthma. UDP-Glc levels in sputum samples from CF patients are markedly elevated, reaching concentrations ( $500 \mathrm{nM})$ capable of promoting robust activation of $\mathrm{P} 2 \mathrm{Y}_{14}$ receptors. Unlike ATP, UDP-Glc added either onto the mucosal surface of airway epithelial cells or to CF sputum was poorly hydrolyzed. In addition, enhanced ATP and UDP-Glc release occurs during $\mathrm{Ca}^{2+}$-promoted vesicle exocytosis and mucin granule secretion from airway epithelial goblet cells (3). Therefore, nucleotide release from goblet cells may be mechanistically associated with mucin secretion, e.g., as co-cargo molecules within mucin granules.

Together, our observations suggest that (i) airway epithelial cell nucleotide release has an exocytotic component, and (ii) sustained UDP-Glc accumulation in ASL during conditions associated with mucin hypersecretion e.g., CF, COPD, and asthma, provides long lasting pro-inflammatory signaling in the airways.

1. Muller T, Bayer H, Myrtek D et al. Am J Respir Cell Mol Biol 2005.

2. Moore DJ, Murdock PR, Watson JM et al. Brain Res Mol Brain Res 2003; 118(1-2): 10-23.

3. Kreda S, Okada S, van Heusden $\mathrm{C}$ et al. Abstract to the 8th International Symposium on Adenosine and Adenine Nucleotides (Ferrara, 2006). 


\section{Role of diadenosine polyphosphates in corneal wound healing}

Jesus Pintor $^{1}$, Aranzazu Mediero ${ }^{1}$ and Assumpta Peral ${ }^{2}$

${ }^{T}$ Dto. Bioquímica y Biología Molecular IV, ${ }^{2}$ Dep. Optica II, E. U. de Optica Universidad Complutense de Madrid, Spain.

jpintor@vet.ucm.es

As the epithelium is the most external layer of the cornea, it is often damaged by several factors causing corneal wounds. These wounds are repaired in a three-step process called corneal wound healing. Diadenosine polyphosphates, ApnA, are present in rabbit and human tears. This fact invites to think that these dinucleotides may participate in ocular surface processes such as corneal wound healing. Therefore, we have investigated the possible role of diadenosine polyphosphates on corneal wound healing by studying the changes in the rate of corneal epithelial cell migration after dinucleotide applications.

Primary corneal epithelial cell cultures were obtained from New Zealand white rabbits. Immunocytochemical experiments were carried out by fixing the cells with 4\% PFA and incubated with citokeratine 3 primary antibody which was subsequently incubated with a secondary Ig-G mouse labelled with FITC an cells were observed under confocal microscopy. Migration studies were carried out by taking confluent monolayers which were wounded with a pipette tip and challenged with different di- and mononucleotides $\left(\mathrm{Ap}_{3} \mathrm{~A}, \mathrm{Ap}_{4} \mathrm{~A}, \mathrm{Ap}_{5} \mathrm{~A}, \mathrm{Up} \mathrm{p}_{4} \mathrm{U}, \mathrm{ATP}, \mathrm{UTP}\right.$, ADP and UDP). For concentration-response analysis compounds were tested in doses ranging from $10^{-8}$ to $10^{-3}$ $\mathrm{M}$. When the P2 antagonists, PPADS, suramin and reactive blue 2, were used, they were assayed at $100 \mu \mathrm{M}$, and from $10^{-7}$ to $10^{-3} \mathrm{M}$ in concentration-response studies. In order to study the intracellular pathways involved in cell migration, several MAPK and citoskeleton inhibitors (U0126 $100 \mu \mathrm{M}$, Y27632 $100 \mu \mathrm{M}$, AG1478 $100 \mu \mathrm{M}$, PAO (phenylarsine oxide) $5 \mu \mathrm{M},(-)$-Blebblistatin $10 \mu \mathrm{M}$ and ML7 $25 \mu \mathrm{M}$ ) were assayed in the presence or absence of $\mathrm{Ap}_{4} \mathrm{~A}$ and $\mathrm{Ap}_{3} \mathrm{~A}$ both $100 \mu \mathrm{M}$. Stability of the dinucleotides was assayed by HPLC using an isocratic method.

Cells under study were identified as corneal epithelial cells due to this positive labelling to cytokeratine-3 in the immunocytochemical analysis. Cell migration experiments showed that $\mathrm{Ap}_{4} \mathrm{~A}$, UTP and ATP accelerate the rate of healing, while $\mathrm{Ap}_{3} \mathrm{~A}, \mathrm{Ap}_{5} \mathrm{~A}$ and UDP delay it. ADP and $\mathrm{Up}_{4} \mathrm{U}$ did not modify the rate of migration. For further experiments we took $\mathrm{Ap}_{4} \mathrm{~A}$ (which accelerates the rate of wound) and $\mathrm{Ap}_{3} \mathrm{~A}$ which delays it. $\mathrm{P} 2 \mathrm{Y}$ antagonists presented small differences between $\mathrm{Ap}_{4} \mathrm{~A}$ and $\mathrm{Ap}_{3} \mathrm{~A}$. The assays with MAPK and citoskeleton inhibitors, revealed that both, MAPK pathway and cdc-42/RAC/RhoA/ROCK pathways are involved in the epithelial migration. In this sense, $\mathrm{Ap}_{4} \mathrm{~A}$ activating cdc- 42 cascade increase the rate of corneal epithelial cell migration, while the delay caused by $\mathrm{Ap}_{3} \mathrm{~A}$ is mainly due to the MAPK pathway Finally, concerning the possible degradation of the dinucleotides it was almost impossible to detect any product as a consequence of their cleavage. Degradation of $1-3 \%$ of the dinucleotides in 2 min perfusion indicates that the active molecules are the diadenosine polyphosphates and not the generated mononucleotides.

In summary, analysing the pharmacological profile of all the compounds tested we can conclude that diadenosine polyphosphates activate two main P2Y receptors: a $\mathrm{P}_{2} \mathrm{Y}_{2}$ receptor accelerating the rate of healing and a $\mathrm{P}_{2} \mathrm{Y}_{6}$ receptor which delays this process.

\section{Medicinal Chemistry}

\section{Adenine nucleosides as reversible ${\mathrm{P} 2 Y_{12}}_{12}$ antagonists}

James G. Douglass, Adam Samuelson, J. Bryan deCamp, Emilee Glaub, Dima Smirnov, Sanjoy Mahanty, Anna Morgan, Chris Crean, Jose L. Boyer, Stephanie Anderson, and Paul S. Watson Inspire Pharmaceuticals Inc., 4222 Emperor Boulevard, Suite 200, Durham, North Carolina, USA 27703-8466 pwatson@inspirepharm.com

ADP serves as an endogenous agonist for both $\mathrm{P}_{2} \mathrm{Y}_{1}$ and $\mathrm{P} 2 \mathrm{Y}_{12}$ on platelets, and antagonism of its action at either receptor has been shown to inhibit platelet aggregation. Inhibition of platelet aggregation using irreversible $\mathrm{P}_{2} \mathrm{Y}_{12}$ antagonists has proven to be a useful therapy to treat Acute Coronary Syndrome (ACS). Previously we had 
identified several lipophilic modifications to adenine nucleotides that enable them to reversibly block aggregation mediated by $\mathrm{P}_{2} \mathrm{Y}_{12}$. This presentation will briefly discuss human clinical trial results of one of these reversible $\mathrm{P}_{2} \mathrm{Y}_{12}$ adenine nucleotide antagonists, INS50589, and the discovery of reversible $\mathrm{P}_{2} \mathrm{Y}_{12}$ adenine nucleoside antagonists stemming from observations gained from our original nucleotide research.

\title{
Development of pharmacological tools for studying P2Y receptors
}

\author{
C.E. Müller
}

Pharmaceutical Sciences Bonn (PSB), Pharmaceutical Chemistry, Pharmaceutical Institute Poppelsdorf, University of Bonn, Kreuzbergweg 26, 53115 Bonn, Germany

christa.mueller@uni-bonn.de

P2Y receptors are G protein-coupled receptors (GPCRs) activated by nucleotides, such as ATP, ADP, UTP, UDP, or UDPglucose, depending on the receptor subtype. Currently, 8 subtypes are known (physiological agonist

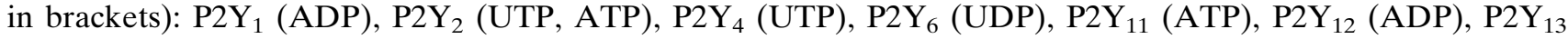
(ADP), and $\mathrm{P}_{2} \mathrm{Y}_{14}$ (UDPglucose) [1].

Our project focussed on the synthesis of base-modified uracil nucleotides and metabolically stable nucleotide mimetics as ligands for the uracil nucleotide-sensitive P2Y receptor subtypes. A series of nucleotides and analogs was synthesized, investigated at recombinant human $\mathrm{P}_{2} \mathrm{Y}_{2}, \mathrm{P}_{2} \mathrm{Y}_{4}$, and $\mathrm{P} 2 \mathrm{Y}_{6}$ receptors, and structure-activity relationships were analyzed. Alkyl substituents in the 6-position of UTP and UDP were not tolerated by the three receptor subtypes. Large, aromatic substituents at N3 of UDP were well tolerated by the $\mathrm{P}_{2} \mathrm{Y}_{6}$ receptor leading to potent, $\mathrm{P}_{2} \mathrm{Y}_{6}$-selective agonists. Modification of the triphosphate chain by introduction of a $\beta, \gamma$-dichloromethylene bridge in UTP derivatives, resulting in compounds with enhanced metabolic stability, was well tolerated by all three receptor subtypes.

Radioligands are valuable tools for studying GPCRs, however, selective radioligands have only been available for the $\mathrm{P}_{2} \mathrm{Y}_{1}$ receptor subtype so far. We have recently developed the first selective radioligand for the $\mathrm{P} 2 \mathrm{Y}_{12}$ receptor subtype [2], an ADP receptor predominantly expressed on platelets, and in lower density in the brain. The $\mathrm{P}_{2} \mathrm{Y}_{12}$ antagonist radioligand $\left[{ }^{3} \mathrm{H}\right] \mathrm{PSB}-0413$ (2-propylthioadenosine-5'-adenylic acid (1,1-dichloro-1-phosphonomethyl-1-phosphonyl) anhydride, AR-C67085MX) has been successfully used for characterizing P2 $\mathrm{Y}_{12}$ receptors on the protein level in various tissues and cells. The radioligand allowed to set up a fast and efficient screening assay for identifying novel lead structures for the development of $\mathrm{P}_{2} \mathrm{Y}_{12}$ antagonists, which are potent antithrombotic agents.

Extracellular nucleotides are quickly hydrolyzed by ectonucleotidases to limit their action. Inhibitors of such enzymes would act as indirect P2Y receptor agonists by prolonging the effects of endogenously released nucleotides. We have developed a fast and convenient nanoscale capillary electrophoresis assay to screen and characterize inhibitors of ectonucleotidases [3]. The enzymatic reaction is performed within the capillary followed by electrophoretic separation and quantification (by UV) of the reaction products. A novel class of ectonucleotidase inhibitors has been discovered by this approach.

1. Brunschweiger A, Müller CE. Curr Med Chem 2006; 12: 763-71.

2. El-Tayeb A, Griessmeier KJ, Müller CE. Bioorg Med Chem Lett 2005; 15: 5450-2

3. Iqbal J, Vollmayer P, Braun N, Zimmermann H, Müller CE. Purinergic Signalling 2005; 1: 349-58

\section{Molecular Recognition in P2Y Nucleotide Receptors}

Kenneth A. Jacobson

Molecular Recognition Section, Laboratory of Bioorganic Chemistry, National Institute of Diabetes and Digestive and Kidney Diseases, National Institutes of Health, Bethesda, MD 20892, USA

kajacobs@helix.nih.gov 
Agonists selective for $\mathrm{P}_{2} \mathrm{Y}_{1}, \mathrm{P}_{2} \mathrm{Y}_{2}$, and $\mathrm{P}_{2} \mathrm{Y}_{6}$ receptors and nucleotide antagonists selective for $\mathrm{P} 2 \mathrm{Y}_{1}$ and $\mathrm{P} 2 \mathrm{Y}_{12}$ receptors have been identified. Selective non-nucleotide antagonists have been reported for $\mathrm{P} 2 \mathrm{Y}_{1}, \mathrm{P}_{2} \mathrm{Y}_{2}, \mathrm{P}_{2} \mathrm{Y}_{6}$, $\mathrm{P}_{2} \mathrm{Y}_{11}, \mathrm{P}_{2} \mathrm{Y}_{12}$, and $\mathrm{P}_{2} \mathrm{Y}_{13}$ receptors. Three-dimensional structural models of $\mathrm{P} 2 \mathrm{Y}$ receptors, deduced mainly from rhodopsin-based homology modeling and mutagenesis studies, have aided in the development of selective ligands [1]. We have synthesized, as ligands for P2Y receptors, nucleotide analogues in which the ribose moiety is substituted by a variety of novel ring systems, including conformationally-locked moieties. The focus on conformational factors of the ribose-like moiety allows the inclusion of general modifications that lead to enhanced potency and selectivity. In solution, the ribose ring of an unbound nucleotide may exist in a dynamic equilibrium between (N) (North; 2'-exo/3'-endo) and (S) (South; 2'-endo/3'-exo) conformations, and X-ray crystallographic structures of diverse nucleotide complexes through nature indicate a clustering around these conformations. It is possible to stabilize each of these conformational clusters by chemical bridging within a ring. Replacement of the ribose moiety of ATP with a bicyclo[3.1.0]hexane ring (methanocarba, mc) ring system, i.e. fused cyclopropane and cyclopentane rings, locks the analogue in either a $(\mathrm{N})$ or $(\mathrm{S})$ conformation, depending on the position of the $\mathrm{CH}_{2}$-bridge. These two isomeric variants produce agonist analogues having widely differing activities at $\mathrm{P} 2$ receptors (collaboration w/T.K. Harden). At $\mathrm{P} 2 \mathrm{Y}_{1,2,4,11}$ receptors, there is a preference for the $(\mathrm{N})$ conformation as indicated with $(\mathrm{N})$-methanocarba analogues, such as the potent, competitive $\mathrm{P}_{2} \mathrm{Y}_{1}$ antagonist MRS2500. MRS2500 was sufficiently stable in vivo to inhibit ADP-induced platelet aggregation in mice [2]. $\left[{ }^{33} \mathrm{P}\right]$ MRS2500 was synthesized enzymatically and studied as a radioligand having a $\mathrm{K}_{\mathrm{d}}$ value of $0.3 \mathrm{nM}$ at the rat brain $\mathrm{P}_{2} \mathrm{Y}_{1}$ receptor [3]. MRS2365, an $(\mathrm{N})$-methanocarba analogue of 2-MeSADP, displayed potency $\left(\mathrm{EC}_{50}\right)$ of $0.4 \mathrm{nM}$ at the $\mathrm{P}_{2} \mathrm{Y}_{1}$ receptor with $>10,000$-fold selectivity in comparison to $\mathrm{P}_{2} \mathrm{Y}_{12}$ and $\mathrm{P}_{2} \mathrm{Y}_{13}$ receptors [3]. Basic structure activity relationships were explored at $\mathrm{P} 2 \mathrm{Y}_{2}$ and $\mathrm{P} 2 \mathrm{Y}_{4}$ receptors. However, there is a fundamental conformational difference between the binding sites of $\mathrm{P}_{2} \mathrm{Y}_{6}$ and various other $\mathrm{P} 2 \mathrm{Y}$ receptors. A uridine $5^{\prime}$ diphosphate analogue locked in the $(\mathrm{N})$ envelope conformation was inactive [4]. Based on a prediction from docking of nucleotide derivatives to a $\mathrm{P}_{2} \mathrm{Y}_{6}$ receptor model, (S)-mc-2'-deoxy-UDP was synthesized and found to be more potent than the corresponding riboside, dUDP, indicating a preference for the (S) conformation. At the recombinant human $\mathrm{P}_{13} \mathrm{Y}_{13}$ nucleotide receptor, MRS2211 (6-[(2-chloro-5-nitrophenyl)-azo]-pyridoxal-5'-phosphate) an analogue of the known P2 receptor antagonist PPADS was found to competitively and selectively inhibit functional activity with a $\mathrm{pIC}_{50}$ value of 5.97, being 45-fold more potent than PPADS [5].

1. Costanzi S et al. J Med Chem 2005; 48: 8108-11.

2. Hechler B et al. J Pharm Exp Therap 2006; 316; 556-63.

3. Houston $\mathrm{H}$ et al. Br J Pharmacol 2006; in press.

4. Chhatriwala M et al. J Pharm Exp Therap 2004; 311: 1038-43.

5. Kim YC et al. Bioorg Med Chem Lett 2006; 16: 596-601.

\title{
New Nucleotides and Nucleotides Analogs and their Activity on P2 Receptors
}

\author{
Gloria Cristalli and Rosaria Volpini \\ Dipartimento di Scienze Chimiche, Università di Camerino, via S. Agostino, 1, 62032 Camerino, Italy \\ Presenting author: gloria.cristalli@unicam.it
}

$\mathrm{P} 2$ receptors, activated by nucleotides like ATP, ADP, UTP, and UDP, belong to two families: the ionotropic $\mathrm{P} 2 \mathrm{X}$ receptors $\left(\mathrm{P} 2 \mathrm{X}_{1-7}\right)$, which represent a class of ligand-gated ion channels and the metabotropic $\mathrm{P} 2 \mathrm{Y}$ receptors ( $\left.\mathrm{P} 2 \mathrm{Y}_{1,2,4,6,11-14}\right)$, which are ubiquitously expressed in the human body. All these receptors play important pathophysiological roles, hence, the availability of new ligands can provide novel drugs for the treatment of a number of currently-incurable diseases including tumors and neurodegenerative disorders. However, a limited number of reports described the identification of potent and selective P2X/P2Y ligands. Hence, the present work will be focused on the design and characterization of novel P2 receptor ligands obtained through the phosphorylation of substituted purine and pyrimidine nucleosides [1]. Furthermore, the design of "mini nucleotides" based on the adenine skeleton, and their preparation using solid phase organic synthesis (SPOS) will be presented. 

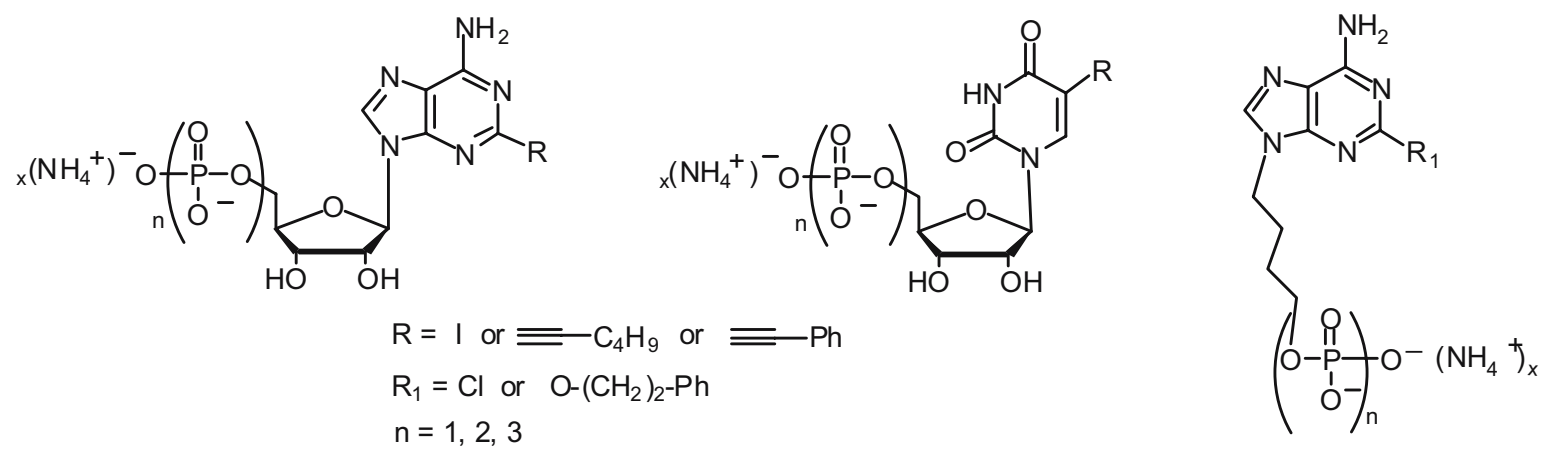

Studies on different pharmacological models such as: a) effects on the human platelet aggregation, b) neuronal differentiation and commitment to death in the human neuroblastoma SH-SY5Y cells, and c) activity on HEK 293 cells transfected by ionotropic P2X3 receptors, will be discussed. Preliminary results demonstrated that the above mentioned nucleotides interact with the $\mathrm{P} 2$ receptors; in particular, some 2-alkynyladenosine nucleotides behave as agonists or antagonists at platelet $\mathrm{P}_{2} \mathrm{Y}_{1}$ and $\mathrm{P} 2 \mathrm{Y}_{12}$ subtypes, depending on the nature of the substituent in 2position [2], while the 5-iodouridine triphosphate interacts with the metabotropic $\mathrm{P}_{2} \mathrm{Y}_{4}$ receptor subtype leading to neuroblastoma SH-SY5Y cell death. Furthermore, some mininucleotides demonstrated agonist activity on $\mathrm{P}_{2} \mathrm{X}_{3}$ receptors.

1. Cristalli G, Dal Ben D, Lambertucci C et al. Synthesis of new P2 receptor ligands. Collection Symposium Ser 2005; 7: 87-93.

2. Cristalli G, Podda GM, Costanzi S et al. Effects of 5'-phosphate derivatives of 2-hexynyl adenosine and 2-phenylethynyl adenosine on responses of human platelets mediated by P2Y receptors. J Med Chem 2005; 48: $2763-6$.

\section{Novel orthosteric and allosteric ligands for adenosine receptors.}

\section{Ad P. IJzerman}

Leiden/Amsterdam Center for Drug Research, PO Box 9502, 2300RA Leiden, The Netherlands (email: ijzerman@lacdr.leidenuniv.nl)

Adenosine receptor antagonists usually possess a bi- or tricyclic heteroaromatic structure at their core with varying substitution patterns to achieve selectivity and/or greater affinity. Taking into account molecular modelling results from a series of potent $\mathrm{A}_{1}$ adenosine receptor antagonists, we derived a pharmacophore suggesting that a monocyclic core can be equally effective. As a further design criterion we imposed a restriction on the polar surface area (PSA) of the molecules that would allow them to penetrate into the CNS. In consequence, we have synthesised two novel series of pyrimidines and a related series of purines with unanticipated substitution pattern, possessing good antagonistic potency at the $\mathrm{A}_{1}$ adenosine receptor and desirable PSA values. In particular, pyrimidine LUF5735 and purine LUF5962 display excellent, even subnanomolar affinity and selectivity at the human $\mathrm{A}_{1}$ receptor.

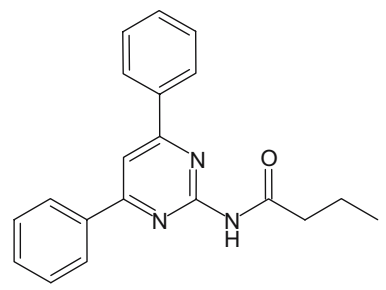

LUF5735

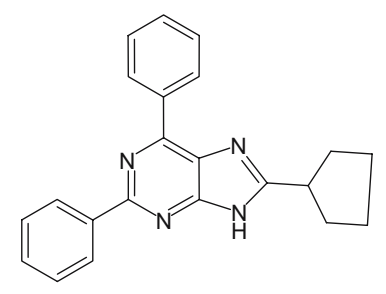

LUF5962<smiles>Clc1ccc(Nc2nc3ccccc3c3[nH]c(C4CCCCC4)nc23)cc1Cl</smiles>

LUF6000 
As a next challenge, a series of $1 \mathrm{H}$-imidazo-[4,5-c]quinolin-4-amine derivatives was synthesized as allosteric modulators of the human $\mathrm{A}_{3}$ adenosine receptor. Structural modifications were made at the 4-amino and 2positions. The compounds were tested in both binding and functional assays, and many were found to be allosteric enhancers of the action of $\mathrm{A}_{3} \mathrm{AR}$ agonists by several different criteria. First, a potentiation of the maximum efficacy of the agonist Cl-IB-MECA was observed for numerous derivatives, with the greatest increase of $45-50 \%$ observed for LUF6000. Also, a number of these compounds, including LUF6000, decreased the rate of dissociation of the agonist $\left[{ }^{125} \mathrm{I}\right] \mathrm{I}-\mathrm{AB}-\mathrm{MECA}$ from the $\mathrm{A}_{3}$ receptor. It was found that the capability of these compounds to increase agonist efficacy correlated with their ability to decrease the dissociation rate, but not their inhibition of equilibrium binding.

In conclusion we have prepared and evaluated novel series of compounds that display remarkable characteristics at either human adenosine $\mathrm{A}_{1}$ or $\mathrm{A}_{3}$ receptors.

\section{Recent developments in the field of $A_{2 B}$ and $A_{3}$ adenosine receptors antagonists}

Pier Giovanni Baraldi ${ }^{1}$, Delia Preti ${ }^{1}$, Mojgan Aghazadeh Tabrizi ${ }^{1}$, Francesca Fruttarolo ${ }^{1}$, Romeo Romagnoli $^{1}$, Naser Abdel Zaid ${ }^{3}$, Allan R. Moorman ${ }^{4}$, Stefania Merighi ${ }^{2}$, Katia Varani ${ }^{2}$, and Pier Andrea Borea ${ }^{2}$

${ }^{1}$ Dip. Scienze Farmaceutiche, ${ }^{2}$ Dip. di Medicina Clinica e Sperimentale-Sezione di Farmacologia, Università di Ferrara, ${ }^{3}$ College of Pharmacy, An-Najah National University, Nablus, ${ }^{4}$ King Pharmaceutical Research and Development, Inc., 4000 CentreGreen Way, Suite 300, Cary, North Carolina 27513

Adenosine, an endogenous modulator of a wide range of biological functions in the nervous, cardiovascular, renal, and immune systems, interacts with at least four cell surface receptor subtypes classified as $A_{1}, A_{2 A}, A_{2 B}$ and $A_{3}$ [1]. Clarification of the role of adenosine and its receptors in cancer development may hold great promise for the chemotherapeutic treatment of patients affected by malignancies [2].

Different classes of compounds with non-xanthine structures have been reported to be $\mathrm{A}_{3}$ adenosine receptor antagonists [3]. The pyrazolo[4,3-e]-1,2,4-triazolo[1,5-c]pyrimidine nucleus has been largely investigated by our group. Our interests were focused on the effects of substitution of the phenyl ring of the arylcarbamoyl moiety at $\mathrm{N}^{5}$ position and of substituents at $\mathrm{C}^{9}$ and/or at $\mathrm{N}^{7}-\mathrm{N}^{8}$ pyrazole nitrogens. These studies allowed us to obtain a large variety of compounds which showed affinities in the nanomolar range to human $\mathrm{A}_{2 \mathrm{~A}}$ or $\mathrm{A}_{3}$ adenosine receptors with high degree of selectivity [3].

Compounds presenting an additional fused ring on the xanthine nucleus have been reported to exhibit antagonistic activity with various levels of affinity and selectivity towards the four adenosine receptors subtypes [4]. We evaluated the effect of the introduction of a benzyl and a propyl at the 1 and 3 positions, respectively, in a new series of 7-aryl/alkyl-1H,6H-pyrrolo[2,1-f]purine-2,4-diones and 7-aryl/alkyl-1H,8H-imidazo[2,1-f]purine-2,4diones [5], among which, very potent and selective $\mathrm{A}_{3}$ receptors antagonists have been identified. In particular 1benzyl-7-methyl-3-propyl-1 $\mathrm{H}, 8 \mathrm{H}$-imidazo[2,1-f]purine-2,4-dione, shows a subnanomolar affinity with a noteworthy selectivity versus the other adenosine receptors subtypes $\left(\mathrm{K}_{\mathrm{i}}\left(\mathrm{hA}_{3}\right)=0.8 \mathrm{nM}, \mathrm{K}_{\mathrm{i}}\left(\mathrm{hA}_{1} / \mathrm{hA}_{3}\right)=3163, \mathrm{~K}_{\mathrm{i}}\left(\mathrm{hA}_{2 \mathrm{~A}} /\right.\right.$ $\left.\mathrm{hA} 3)>6250, \mathrm{IC}_{50}\left(\mathrm{hA}_{2 \mathrm{~B}}\right) / \mathrm{K}_{\mathrm{i}}\left(\mathrm{hA}_{3}\right)=2570\right)$.

Colotta et al. directed much effort toward the study of adenosine receptor antagonists investigating the 2arylpyrazolo[3,4-c]-quinoline nucleus [6]. In light of the reported activity profile, we decided to synthesize the structural isomers, 2-arylpyrazolo[4,3-c]quinolines [7], some of which showed high $\mathrm{A}_{3}$ receptor affinity and complete selectivity (2-p-tolyl-2,5-dihydro-pyrazolo[4,3-c]quinolin-4-one; $\mathrm{K}_{\mathrm{i}} \mathrm{hA}_{1}, \mathrm{~K}_{\mathrm{i}} \mathrm{hA} \mathrm{A}_{2 \mathrm{~A}}, \mathrm{EC}_{50} \mathrm{hA}_{2 \mathrm{~B}}>1000 \mathrm{nM}$, $\left.\mathrm{K}_{\mathrm{i}} \mathrm{hA}_{3}=9 \mathrm{nM}\right)$.

In the search for improved selective $\mathrm{A}_{2 \mathrm{~B}}$ antagonists for the treatment of asthma [8], we synthesized a variety of new 1,3-dipropyl-8-heterocyclic-substituted xanthines [9]. We introduced several heterocycles, such as pyrazole, isoxazole, pyridine and pyridazine at the 8-position of the xanthine nucleus. We have also investigated different spacers (substituted acetamide, oxyacetamide and urea moieties) on the heterocycle introduced. Some of the synthesized $\mathrm{C} 8$-substituted xanthines showed high affinity at $\mathrm{A}_{2 \mathrm{~B}}$ receptor subtype and very good selectivity $(\mathrm{N}$ benzo[1,3]dioxol-5-yl-2-[5-(2,6-dioxo-1,3-dipropyl-2,3,6,7-tetrahydro-1 $H$-purin-8-yl)-1-methyl- $1 H$-pyrazol-3yloxy]-acetamide; $\left.\mathrm{hA}_{2 \mathrm{~B}}=5.5 \mathrm{nM}, \mathrm{hA}_{1}, \mathrm{hA}_{2 \mathrm{~A}}, \mathrm{hA}_{3}>1000\right)$. 
(1). Fredholm et al. Pharmacol Rev 2001; 53: 527-52.

(2). Merighi et al. Pharmacol Ther 2003; 100(1): 31-48.

(3). Baraldi et al. Drug Dev Res 2003; 58: 315-29.

(4). Drabczyñska et al. Eur J Med Chem 2003; 38: 397-402.

(5). Baraldi et al. J Med Chem 2005; 48: 4697-701.

(6). Calotta et al. J Med Chem 2000; 43: 3118-24.

(7). Baraldi et al. J Med Chem 2005; 48: 5001-8.

(8). Auchampach et al. Mol Pharmacol 1997; 52: 846-60.

(9). Baraldi et al. J Med Chem 2004; 47: 1434-47.

\title{
Synthesis and molecular modeling studies of new pyrazolo[3,4-b]pyridines, potent and selective inhibitors of $A_{1}$ adenosine receptors
}

\author{
$\underline{\text { Maurizio Botta }}^{1}$, Fabrizio Manetti ${ }^{1}$, Cristina Tintori ${ }^{1}$, Adriano Martinelli ${ }^{2}$, Tiziano Tuccinardi $^{2}$, Letizia
} Trincavelli $^{2}$, Claudia Martini ${ }^{2}$, and Silvia Schenone ${ }^{3}$

${ }^{1}$ Dipartimento Farmaco Chimico Tecnologico, Università degli studi di Siena, Via Aldo Moro, 3, 53100 Siena, Italia;

${ }^{2}$ Dipartimento di Scienze Farmaceutiche, Università degli studi di Pisa, Via Bonanno Pisano, 6, 56126 Pisa, Italia;

${ }^{3}$ Dipartimento di Scienze Farmaceutiche, Università degli studi di Genova, Viale Benedetto 15, 16132 Genova, Italia

botta@unisi.it

Adenosine is an endogenous neuromodulator, which mediates its biological effects by interacting with four receptor subtypes named $\mathrm{A}_{1}, \mathrm{~A}_{2 \mathrm{~A}}, \mathrm{~A}_{2 \mathrm{~B}}$, and $\mathrm{A}_{3}$. The physiological significance and functions of adenosine have been extensively studied and in the last twenty years a large number of adenosine ligands, agonists and antagonists, have been developed. Particularly, many efforts have been invested in the synthesis of $\mathrm{A}_{1} \mathrm{ARs}$ antagonists, that can stimulate cerebral activity by blocking the adenosine central inhibitory activity. In this context, a number of new 4-amino-1-(2-chloro-2-phenylethyl)-1H-pyrazolo[3,4-b]pyridines 5-carboxylic acid esters have been synthesized by our research group and their binding affinity at the $A_{1}, A_{2 A}$ and $A_{3}$ adenosine receptors (AR) have been evaluated. The new compounds were characterized by a high affinity and selectivity toward the bovine $\mathrm{A} 1$ receptor subtype $\left(\mathrm{bA}_{1} \mathrm{AR}\right)$. In addition, to rationalize the relationships between structure and affinity of such compounds together with $\mathrm{A}_{1} \mathrm{AR}$ antagonists taken from the literature, molecular modeling studies were carried out. As a first approach, a pseudoreceptor model [1] was built to derive a hypothesis of the interaction pathway between the set of $\mathrm{A}_{1} \mathrm{AR}$ antagonists and a model of the putative $\mathrm{A}_{1}$ receptor. Nevertheless, biological evaluation of several compounds suggested by the model did not give the expected results, demonstrating that the model itself was scarcely able to predict the activity of inhibitors subjected to modifications at the position 5. As a consequence, a new and more accurate model was required to better predict the activity of $\mathrm{A}_{1} \mathrm{AR}$ inhibitors. To reach this goal, a highly predictive 3D QSAR model for antagonists of $\mathrm{A}_{1} \mathrm{AR}$ was generated [2]. Such model demonstrated to have the knowledge for estimating the affinity of a very large number of inhibitors belonging to different structural classes. The generated 3D QSAR has been also used to refine a model of the $\mathrm{A}_{1} \mathrm{AR}$ receptor built by homology and then molecular modeling study of the interaction of the compounds under investigation with the bovine $\mathrm{A}_{1}$ adenosine receptor was carried out. On the other hand, the ADME (absorption, distribution, metabolism, excretion) properties of the synthesized pyrazolo-[3,4$b$ ]pyridines were evaluated by means of the Volsurf software with the aim to have a prediction of the aqueous solubility and cell permeability of such molecules. All computational studies together with the synthesis of new pyrazolo-[3,4-b]pyridines will be reported during the oral presentation and the biological evaluation of the inhibitors on both the bovine and human $\mathrm{A}_{1}$ receptors $\left(\mathrm{bA}_{1} \mathrm{AR}\right.$ and $\mathrm{h} \mathrm{A}_{1} \mathrm{AR}$ respectively) will be also discussed.

(1). Bondavalli F, Botta M, Bruno O et al. J Med Chem 2002; 45: 4875-87.

(2). Manetti F, Schenone S, Bondavalli F et al. J Med Chem 2005; 48: 7172-85. 


\section{The Discovery of Selective, High Affinity $\mathbf{A}_{2 B}$ Adenosine Receptor Antagonists}

Jeff Zablocki $^{1}$, Rao Kalla ${ }^{1}$, Elfatih Elzein ${ }^{1}$, Thao Perry ${ }^{1}$, Xiaofen Li $^{1}$, Venkata Palle ${ }^{1}$, Vaibhav Varkhedkar $^{1}$, Art Gimbel ${ }^{2}$, David Lustig ${ }^{3}$, Kwan Leung ${ }^{3}$, Tim Glennon ${ }^{4}$, Hugh Genin ${ }^{4}$, and Dewan Zeng ${ }^{2}$

${ }^{1}$ Department of Bioorganic Chemistry, ${ }^{2}$ Department of Drug Research and Pharmacological Sciences, ${ }^{3}$ Department of Pre-Clinical Development CV Therapeutics Inc., 3172 Porter Drive, Palo Alto, CA 94304, USA Accelrys, Inc.

The $P_{1}$ family of adenosine receptors consists of two $G_{i}$ couple receptors, $A_{1}$ and $A_{3}$, and two Gs coupled receptors, $A_{2 A}$ and $A_{2 B}$. The $A_{2 B}$ adenosine receptor (AdoR) is found in multiple tissues including the lung where it is found in bronchial smooth muscle cells [1] and mast cells [2]. In vitro experiments, demonstrate the release of inflammatory cytokines from both bronchial smooth muscle cells and mast cells, effects that can be blocked by an $\mathrm{A}_{2 \mathrm{~B}}$ AdoR antagonist [1,2]. Thus, we hypothesize that the $\mathrm{A}_{2 \mathrm{~B}}$ AdoR may play a role in asthma. We will disclose an overview of our $A_{2 B}$ AdoR antagonist structure activity relationships starting with MRS-1754 [3] as a lead molecule to the discovery of 8-(1H-pyrazol-4-yl)xanthine $\mathbf{1}$, a compound with a $1 \mathrm{nM} \mathrm{K}_{\mathrm{i}}$ for the $\mathrm{A}_{2 \mathrm{~B}}$ AdoR and excellent selectivity (Figure 1). The complete SAR leading up to the discovery of 1 involved a systematic optimization of the heterocycle at the 8 position, followed by varying the chain length of the N-1 aralkyl (phenpropyl, phenethyl, and benzyl), optimizing substituents on the phenyl ring for affinity and selectivity for the $\mathrm{A}_{2 \mathrm{~B}}$ AdoR, and finally varying the $\mathrm{N}-1$ and $\mathrm{N}-3$ alkyl substituents. Unfortunately, $\mathbf{1}$ did not possess high oral bioavailability (rats), and this was attributed to low water solubility. Therefore, an effort to add charge to the molecule to enhance water solubility was explored. Initially, adding a carboxylate to the phenyl ring or benzylic methylene was explored, and the latter approach afforded a compound $\mathbf{2}$ of modest $\mathrm{A}_{2 \mathrm{~B}}$ AdoR affinity and selectivity (Figure 1). However, upon oral administration of $\mathbf{2}$ to rats it was rapidly converted to the unsubstituted pyrazole, a non-selective $A_{2 B}$ antagonist. Fortunately, replacement of the phenyl group with pyridyl as in 4 resulted in high affinity for the $\mathrm{A}_{2 \mathrm{~B}}$ AdoR and modest selectivity. Furthermore, 4 demonstrated improved oral bioavailability following dosing in rats. A homology model of the $\mathrm{A}_{2 \mathrm{~B}}$ AdoR receptor was built using MODELER based on the X-Ray of bovine rhodopsin. Various docking techniques (Ligand-Fit and Affinity), were used to develop a hypothetical binding pose for $\mathbf{1}$ and $\mathbf{4}$ consistent with the above SAR and published site-directed mutagenesis data, and the outcome of these efforts will be presented.

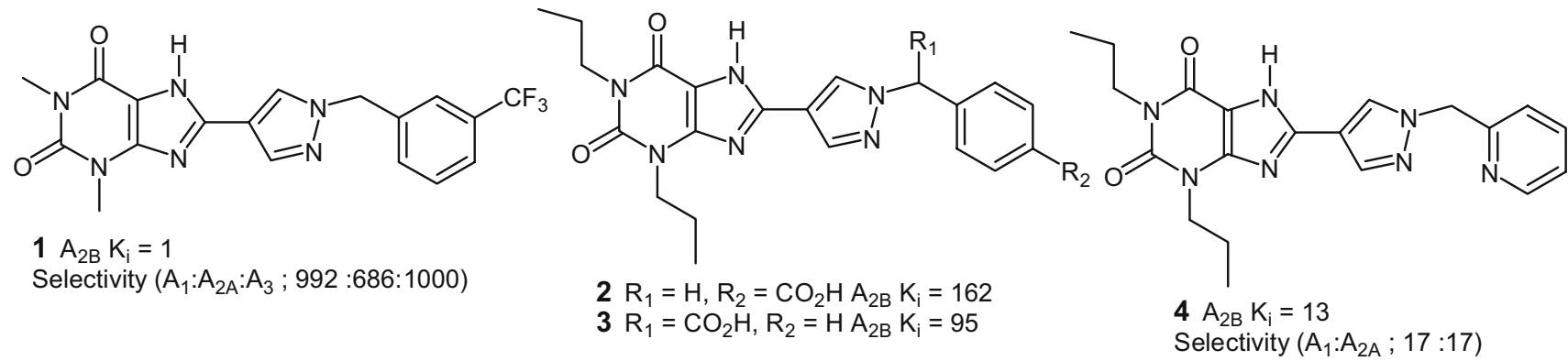

1. Zhong H, Belardinelli L, Maa T, Feoktistov I, Biaggioni I, Zeng D. Am J Respir Cell Mol Biol 2004; 30: 118-25.

2. Ryzhov S, Goldstein AE, Matafonov A et al. J Immunol 2004; 172: 7726-33.

3. Kim YC, Ji X, Melman N et al. J Med Chem 2000; 43: 1165-72. 


\title{
Cardiovascular System
}

\section{Adenosine and Vasomotor Regulation in the Coronary Microcirculation}

\author{
Lih Kuo $^{1}$ and Travis W. Hein ${ }^{2}$ \\ ${ }^{1}$ Department of Systems Biology and Translational Medicine, and ${ }^{2}$ Department of Surgery, Scott \& White Memorial \\ Hospital, College of Medicine, Texas A\&M University System Health Science Center, Temple, TX 76504, USA. \\ LKUO@tamu.edu
}

The nucleoside adenosine has been suggested to play a major role in the regulation of coronary blood flow during metabolic stress (i.e., hypoxia and ischemia) by the preferential dilation of small coronary arterioles primarily through the activation of adenosine $\mathrm{A}_{2 \mathrm{~A}}$ receptors. However, the signaling pathways leading to this dilation are incompletely understood. Since tissue acidosis and alteration in local hemodynamics are associated with metabolic stress, it is important to examine whether the adenosine-induced vasodilatory response can be influenced by these factors. Using an isolated vessel approach and videomicroscopic techniques, these issues were addressed recently in our laboratory. It was found that disruption of endothelium, blockade of endothelial ATP-sensitive potassium $\left(\mathrm{K}_{\mathrm{ATP}}\right)$ channels by glibenclamide, inhibition of nitric oxide $(\mathrm{NO})$ synthase by L-NAME and of soluble guanylyl cyclase by ODQ produced identical attenuation of vasodilation to adenosine. Combined administration of these inhibitors did not further attenuate the vasodilatory response. Pertussis toxin (PTX), but not cholera toxin, significantly inhibited vasodilation to adenosine, and this inhibitory effect was only evident in vessels with an intact endothelium. Both glibenclamide and a high concentration of extraluminal $\mathrm{KCl}$ abolished vasodilation of denuded vessels to adenosine; however, inhibition of calcium-activated potassium channels had no effect on this dilation. Rp-8-Br-cAMPS, a cAMP antagonist, inhibited vasodilation to cAMP analog 8-Br-cAMP but failed to block adenosine-induced dilation. These results suggest that adenosine activates both endothelial and smooth muscle pathways to exert its vasomotor activity. On one hand, adenosine opens endothelial $\mathrm{K}_{\mathrm{ATP}}$ channels through activation of PTX-sensitive G-proteins. This signaling leads to the influx of $\mathrm{Ca}^{2+}$ for NO synthesis, which subsequently increases smooth muscle cGMP for vasodilation. On the other hand, adenosine activates smooth muscle $\mathrm{K}_{\mathrm{ATP}}$ channels and leads to vasodilation through hyperpolarization. The latter pathway is independent of G-proteins and cAMP/cGMP signaling. In the presence of a threshold concentration of adenosine $(0.1 \mathrm{nM})$, the vasodilation in response to increased lumenal flow was significantly enhanced. Inhibiting endothelial $\mathrm{K}_{\mathrm{ATP}}$ channels (by lumenal glibenclamide) or depolarizing endothelial cells (by lumenal $\mathrm{KCl}, 40 \mathrm{mM}$ ) abolished the effect of adenosine. Reducing intraluminal pressure from 80 to $40 \mathrm{cmH}_{2} \mathrm{O}$ significantly enhanced vasodilations to adenosine and pinacidil ( $\mathrm{K}_{\mathrm{ATP}}$ channel activator). These enhanced responses were not affected by endothelial removal but were abolished by a subthreshold concentration of glibenclamide. In contrast to adenosine, sodium nitroprusside (SNP)-induced dilation was not affected by pressure changes. In the acidic solution $(\mathrm{pH}=7.3)$, the dilations to both adenosine and pinacidil, but not to SNP and $\mathrm{KCl}$ (10 to $20 \mathrm{mM}$ ), were enhanced in a glibenclamide-sensitive manner. In summary, these findings suggest that adenosine elicits coronary arteriolar dilation by activating $\mathrm{K}_{\mathrm{ATP}}$ channels for membrane hyperpolarization in the endothelial (i.e., NO release) and smooth muscle cells. Adenosine, by activating endothelial $\mathrm{K}_{\mathrm{ATP}}$ channels, can potentiate coronary arteriolar dilation in response to increased flow. In addition, activation of smooth muscle $\mathrm{K}_{\mathrm{ATP}}$ channels, by a mild acidosis or lowering intraluminal pressure, increases coronary arteriolar dilation to adenosine. It appears that adenosine can interact with local hemodynamics and chemical factors for integrative vasomotor regulation by selectively modulating resting $\mathrm{K}_{\mathrm{ATP}}$ channel activity in either endothelial or smooth muscle cells.

\section{An Emerging Role for Adenosine in Angiogenesis}

Thomas H. Adair

\author{
Department of Physiology \& Biophysics, University of Mississippi Medical Center, 2500 North State Street, \\ Jackson, MS 39216, USA \\ tadair@physiology.umsmed.edu
}

It is well-established that metabolic factors have a critical role in the regulation of angiogenesis [1]. An increase in metabolic activity leads to a decrease in tissue oxygenation causing tissues to become hypoxic. The hypoxia 
initiates a variety of signals that stimulate angiogenesis, and the increase in vascularity that follows promotes oxygen delivery to the tissues. When the tissues receive adequate amounts of oxygen even during peak levels of activity, the intermediate effectors return to normal levels, and angiogenesis ceases.

An emerging concept is that adenosine released from hypoxic tissues has an important role in driving the angiogenesis $[2,3]$. The following feedback control hypothesis is proposed: AMP is dephosphorylated by ecto-5'nucleotidase, producing adenosine under hypoxic conditions in the extracellular space adjacent to a parenchymal cell (eg, cardiomyocyte, skeletal muscle fiber, hepatocyte, etc.). Extracellular adenosine activates $\mathrm{A}_{2}$ receptors, which stimulates release of vascular endothelial growth factor (VEGF) from the parenchymal cells. VEGF binds to its receptor (VEGFR-2) on endothelial cells, stimulating their proliferation and migration. Adenosine can also stimulate endothelial cell proliferation independently of VEGF, which probably involves modulation of other proangiogenic and antiangiogenic growth factors, and perhaps an intracellular mechanism. In addition, hemodynamic factors associated with adenosine-induced vasodilation may have a role in development and remodelling of the vasculature. Once a new capillary network has been established, and the diffusion/perfusion capabilities of the vasculature are sufficient to supply the parenchymal cells with adequate amounts of oxygen, adenosine and VEGF as well as other proangiogenic and antiangiogenic growth factors return to near normal levels, thus closing the negative feedback loop. The available data indicate that adenosine might be an essential mediator for up to $50-70 \%$ of the hypoxia-induced angiogenesis in some situations; however, additional studies in intact animals will be required to understand fully the quantitative importance of adenosine.

Adenosine stimulation of angiogenesis is a natural phenomenon that occurs in the microenvironment of tissues in accordance with local metabolic needs. Amplification of this natural process through modulation of the enzymes of adenosine metabolism, or genetic or pharmacological induction of $\mathrm{A}_{2 \mathrm{~A}}$ or $\mathrm{A}_{2 \mathrm{~B}}$ receptors could, theoretically, lead to stimulation of angiogenesis in those areas of a tissue where hypoxia is most severe and adenosine levels are highest. This type of physiological therapy can be viewed as a departure from classical attempts to stimulate angiogenesis in a global manner, in which many or most cells of an organ or tissue are made to produce greater amounts of VEGF. In contrast to adenosine-based therapies, global induction of VEGF may not be an effective means to build capillary networks in the microenvironment of a tissue where hypoxia is most severe. (Supported by National Heart, Lung, and Blood Institute, HL51971.)

1. Adair TH, Gay WJ. Montani JP. Growth regulation of the vascular system: evidence for a metabolic hypothesis. Am J Physiol 1990; 259: R393-404.

2. Adair TH. Growth regulation of the vascular system: an emerging role for adenosine. Am J Physiol 2005; 289: R283-296.

3. Adair TH. An emerging role for adenosine in angiogenesis. Hypertension 2004; 44: 618-620.

\title{
Control of extracellular adenosine concentration by ecto-5' ${ }^{\prime}$-nucleotidase
}

\author{
A. Deussen, A. Pexa, J. Weichsel, S. Sternberger \\ Department for Physiology, Medical Faculty Carl Gustav Carus, TU Dresden
}

Adenosine is produced inside the cell by action of $5^{\prime}$-nucleotidase(s) and S-adenosylhomocysteine hydrolase. In addition, there is compelling evidence for extracellular adenosine production by ecto-5'-nucleotidase. A previous analysis of complex data sets of cardiac adenosine production has provided strong evidence that the prevailing path of adenosine production in the heart is intracellular (Deussen et al., Circulation 1999; 99: 2041-47). However, it was also found that intracellular removal of adenosine via adenosine kinase and adenosine deaminase was very rapid, and for physiological baseline conditions a transmembraneous adenosine concentration gradient was predicted, which was directed from extra- to intracellular. This scenario opens the possibility that extracellular adenosine production by ecto-5'-nucleotidase (CD73) is an important source of adenosine, which may significantly modulate the extracellular adenosine concentration.

This hypothesis was tested in isolated endothelial cells and isolated perfused mouse hearts with respect to an activation of CD73, pharmacological block of the enzyme and a genetic knock out approach. Baseline activity of CD73 was assessed by measurement of the conversion of $1, \mathrm{~N}^{6}$-etheno-AMP to $1, \mathrm{~N}^{6}$-etheno-adenosine in intact cells or hearts (HPLC assay with fluorescence detection). While conversion of the etheno-labeled AMP provided a read out of CD73 activity of intact cells or tissues, additional measurement of native adenosine release (HPLC- 
technique using UV-detection) gave information on the production of native (endogenous) adenosine. Application of lyso-phospholipids (lysophosphatidyl-choline, $10 \mu \mathrm{M}$ or platelet activation factor, $5 \mu \mathrm{M}$ ) enhanced catalytic activity of CD73 of micro- and macrovascular endothelial cells. Moreover, in presence of blockers of membrane transport inhibitors (nitrobenzylthioinosine $1 \mu \mathrm{M}$, dipyridamole $1 \mu \mathrm{M}$ ) the native extracellular adenosine concentration was doubled within 2 min after application of lyso-phospholipids. In contrast, block of CD73 by $\alpha, \beta$-methylene-ADP $(50 \mu \mathrm{M})$ significantly decreased the extracellular adenosine concentration and completely blocked the effect of lyso-phospholipids on CD73 activity. In isolated hearts from CD73 -/- mice the adenosine concentration in the venous effluent perfusate was only $1 / 3$ of the concentration measured in hearts of WT mice under identical conditions $\left(\mathrm{pO}_{2}>600 \mathrm{~mm} \mathrm{Hg}\right.$, perfusion pressure $\left.85 \mathrm{~mm} \mathrm{Hg}\right)$.

We conclude that ecto-5'-nucleotidase (CD73) represents a key enzyme for modulation of extracellular adenosine concentration in the cardiovascular system. This extracellular adenosine production may be of particular importance for conditions when total adenosine production is low. Lyso-phospholipids are of potential physiological or pathophysiological importance, because they can almost instantly enhance the activity of ecto-5'nucleotidase resulting in increased extracellular adenosine concentrations.

\section{The forearm model as a tool to study the clinical pharmacology of adenosine in humans}

Gerard A. Rongen, M.D., Ph.D.

Departments of Pharmacology-Toxicology and Internal Medicine, Radboud University Nijmegen Medical Center, Nijmegen, the Netherlands

G.Rongen@pharmtox.umcn.nl

Preclinical evidence identifies adenosine as a 'retaliatory metabolite', protecting cells against the deleterious consequences of ischemia and limited nutrient supply. Translation of this knowledge to humans in-vivo is needed because of inter-species differences in the pharmacology of adenosine. Various challenges are encountered in this translational effort including (1) extremely short plasma half-life of adenosine due to rapid cellular uptake and metabolism, preventing circulating adenosine from reaching its target cells, (2) adenosine-induced autonomic reflexes that mask the local actions of adenosine at autonomic nerve endings, vascular smooth muscle cells and cardiomyocytes [1] and (3) the lack of tools to study ischemic injury in an experimental setting in humans. Various strate-gies have successfully been applied to meet these challenges. The forearm model has proven its value in this regard. In this model, the brachial artery is cannulated for drug infusion and blood sampling. Intra-arterial delivery of drugs provides a concentration gradient between infused forearm and systemic circulation, allowing exploration of local effects of adenosine with minimal interference by autonomic reflexes or systemic actions [1, 2]. Using forearm strain gauge plethys-mography, the influence of adenosine on forearm blood flow can be studied. In this model, we have successfully demonstrated that the vasodilator property of adenosine is mediated by adenosine re-ceptors, and involves nitric oxide [3]. Using dipyridamole to inhibit cellular uptake of adenosine, we provided support in humans in-vivo for the hypothesis that $\mathrm{K}_{\mathrm{ATP}}$-channels are involved in adeno-sine-induced vasodilation [4] and that extracellular adenosine levels are reduced in patients with hyperhomocysteinemia but increased during oral treatment with methotrexate [5,6]. When the model is further refined by cannulation of an antecubital vein, effects on local and systemic release of endogenous substances such as norepinephrine can be explored. In this way, we have demonstrated that adenosine reduces norepinephrine release from sympathetic nerve endings [2,7]. Recently, we have developed forearm annexin A5 scintigraphy to quantify forearm ischemiareperfusion (I/R) injury. With this technique, we detected protection against $I / R$ injury by ischemic preconditioning, local infusion of adenosine and oral treatment with dipyridamole $[8,9]$. Future research will charac-terize environmental and genetic factors that determine protection against $\mathrm{I} / \mathrm{R}$ injury by adenosine.

1. Rongen GA, Smits P, Ver Donck K, Willemsen JJ, de Abreu RA, Van Belle H, Thien T. Hemodynamic and neurohumoral effects of various grades of selective adenosine transport inhibition in humans. Implications for its future role in cardioprotection. J Clin Invest 1995; 95: 658-68.

2. Rongen GA, Brooks SC, Ando S, Abramson BL, Floras JS. Angiotensin AT1 receptor blockade abolishes the reflex sympatho-excitatory response to adenosine. J Clin Invest 1998; 101: 769-76. 
3. Smits P, Williams SB, Lipson DE, Banitt P, Rongen GA, Creager MA. Endothelial release of nitric oxide contributes to the vasodilator effect of adenosine in humans. Circulation 1995; 92: 2135-41.

4. Bijlstra P, van Ginneken EEM, Huls M, van Dijk R, Smits P, Rongen GA. Glyburide inhibits dipyridamole-induced forearm vasodilation but not adenosine-induced forearm vasodilation. Clin Pharmacol Ther 2004; 75: 147-56.

5. Riksen NP, Rongen GA, Boers GH, Blom HJ, van den Broek PH, Smits P. Enhanced cellular adenosine uptake limits adenosine receptor stimulation in patients with hyperhomocysteinemia. Arterioscler. Thromb Vasc Biol 2005; $25(1)$ : $109-14$.

6. Riksen NP, Barrera P, van den Broek PH, van RP, Smits P, Ron-gen G. Methotrexate modulates the kinetics of adenosine in humans in vivo. Ann Rheum Dis 2006, in press.

7. Rongen GA, Lenders JWM, Lambrou G, Willemsen JJ, Van Belle H, Thien T, Smits P. Presynaptic inhibition of norepinephrine release from sympathetic nerve endings by endogenous adenosine. Hypertension 1996; 27 : $933-8$.

8. Riksen NP, Oyen WJ, Ramakers BP, van den Broek PH, Engbersen R, Boerman OC, Smits P, Rongen GA. Oral therapy with dipyridamole limits ischemia-reperfusion injury in humans. Clin Pharmacol Ther 2005; 78 (1): 52-9.

9. Rongen GA, Oyen WJG, Ramakers BP, Riksen NP, Boerman OC, Steinmetz N, Smits P. Annexin A5 scintigraphy of forearm as a novel in-vivo model of skeletal muscle preconditioning in man. Circulation 2005; 111: $182-7$.

\title{
Molecular mechanisms of coronary flow regulation by adenosine
}

\author{
S. Jamal Mustafa
}

Department of Physiology \& Pharmacology and Center for Interdisciplinary Research in Cardiovascular Sciences, West Virginia University, Morgantown, West Virginia, 26505, USA

E-mail address: smustafa@hsc.wvu.edu

Adenosine, a purine nucleoside acts through its cell surface receptors $\left(\mathrm{A}_{1}, \mathrm{~A}_{2 \mathrm{~A}}, \mathrm{~A}_{2 \mathrm{~B}}\right.$, and $\left.\mathrm{A}_{3}\right)$ via its coupling to G-proteins. Adenosine causes an increase in coronary flow mostly through $\mathrm{A}_{2 \mathrm{~A}}$ adenosine receptors. However, the involvement of other adenosine receptors in the modulation of coronary flow regulation is not well understood. Using adenosine receptor knockout mice, we have investigated the roles of each adenosine receptor subtype in the regulation of coronary flow. Recently, using the isolated mouse heart preparation from $\mathrm{A}_{3}$ adenosine receptor knockout mice, we reported an increased $\mathrm{A}_{2 \mathrm{~A}}$ receptor-mediated coronary flow [1]. Similarly, we found an increase in coronary flow in $\mathrm{A}_{1}$ adenosine receptor knockout mice [2]. These data support inhibitory roles for $A_{3}$ and $A_{1}$ adenosine receptors in the regulation of coronary flow. Additionally, in $A_{2 A}$ adenosine receptor knockout mice, the response to $\mathrm{A}_{2 \mathrm{~A}}$ selective agonist (CGS-21680) was totally abolished [3]. On the other hand, adenosine $\mathrm{A}_{2 \mathrm{~A}}$ receptor knockout mice showed a decrease in blood flow to NECA (non-selective agonist). Currently, we are investigating the coronary flow responses to both NECA and CGS-21680 in $\mathrm{A}_{2 \mathrm{~B}}$ adenosine receptor knockout mice. Further, real time-PCR for all four adenosine receptor knockout mice showed no significant change in all four adenosine receptors. These data support stimulatory roles for $\mathrm{A}_{2 \mathrm{~A}}$ and $\mathrm{A}_{2 \mathrm{~B}}$ adenosine receptors in the regulation of coronary flow. These observations provide new evidence for the presence and possible roles of all four adenosine receptor subtypes in coronary flow regulation. (Supported by National Heart Lung and Blood Institute grant \# HL-027339).

1. Morrison, RR, Talukder MAH, Ledent C, Mustafa SJ. Am J Physiol 2002; 282: H-437-H-444.

2. Tawfik HE., Teng B, Morrison RR, Schnermann J, Mustafa SJ. Am J Physiol Heart and Circulation-In Review.

3. Talukder MAH, Morrison RR, Jacobson MA, Jacobson KA, Ledent C, Mustafa SJ. Am J Physiol 2002; 282: H2183-H-2189.

\section{Subcellular Aspects of Adenosine Receptor-Mediated Signaling in Ventricular Myocardium}

\author{
Robert D. Lasley \\ Department of Surgery, University of Kentucky College of Medicine, Lexington, KY, USA \\ rlasley@uky.edu
}

For years the effects of adenosine in ventricular myocardium (intact hearts and myocytes) were attributed solely to $A_{1}$ receptor subtypes. However evidence generated by numerous laboratories over the past several years 
indicates that these effects are due to multiple adenosine receptor subtypes and interactions with various signaling pathways. Although the adenosine $A_{3}$ receptor subtype has received much interest in adenosine's effects in ischemic myocardium, there is additional evidence for the involvement of the $\mathrm{A}_{2 \mathrm{a}}$ receptor subtype. We have previously reported that: 1) intracoronary infusion of the $A_{2 a}$ agonist CGS21680 increased load-insensitive contractility in stunned, but not normal, in vivo porcine myocardium, independent of changes in coronary blood flow, [1] and 2) the preconditioning (PC) effect of the adenosine agonist AMP579 in in vivo rat myocardium is blocked by both the $\mathrm{A}_{1}$ antagonist DPCPX and the $\mathrm{A}_{2 \mathrm{a}}$ antagonist ZM241385 [2]. Studies from other investigators using isolated hearts and cardiomyocytes suggesting a role for $A_{2 a}$ receptor effects are consistent with our report that rat ventricular myocytes express $\mathrm{A}_{2 \mathrm{a}}$ receptors [3]. Additional observations from our laboratory indicate that although $\mathrm{A}_{2 \mathrm{a}}$ receptor activation does not induce PC, ZM241385 blocks the cardioprotective effects of the $\mathrm{A}_{1}$ agonist CCPA and the non-selective agonist NECA to a similar degree as DPCPX. Additional preliminary observations from our laboratory suggest that $\mathrm{A}_{2 \mathrm{~b}}$ receptors are expressed in ventricular myocardium and myocytes. However based on Western blot analysis and immunocytochemistry the majority of $\mathrm{A}_{2 \mathrm{a}}$ and $\mathrm{A}_{2 \mathrm{~b}}$ immunoreactivity is expressed in cytosolic, not membrane, fractions. In addition to adenosine receptor subtype interactions, adenosine receptors have been reported to interact with other G protein coupled receptors (GPCR), such as opioid receptors, in various tissues via signaling cross-talk and/or receptor hetero-dimerization. Although there have been few such studies in the heart, it has been reported that in vivo $\mathrm{A}_{1}$ agonist PC is blocked by a delta opioid receptor antagonist and morphine-induced PC is blocked by DPCPX [4]. We have observed similar findings that indicate that this interaction is selective for delta, but not kappa, opioid receptors. Adenosine receptors have been shown to couple to the activation of mitogen activated protein kinases (MAPKs) in various tissues. Studies in our laboratory indicate that adenosine receptors activate MAPKs in both intact myocardium and ventricular myocytes; however this activation occurs in distinct subcellular compartments. Our observations also indicate that the acute and delayed PC effects of adenosine receptor activation are dependent on MAPK signaling. Thus there is increasing evidence that the effects of adenosine in ventricular myocardium are mediated by multiple receptor subtypes and their interactions as well as via the modulation of cardiomyocyte subcellular signaling.

1. Lasley RD, Jahania MSA, Mentzer RM Jr. Am J Physiol (Heart Circ Physiol) 2001; 280: H1660-6.

2. Reid EA, Kristo G, Yoshimura Y, Ballard-Croft C, Keith BJ, Mentzer RM Jr, Lasley RD Am J Physiol (Heart Circ Physiol) 2005; 288: H2253-9.

3. Kilpatrick EL, Narayan P, Mentzer RM Jr, Lasley RD. Am J Physiol (Heart Circ Physiol) 2002; 282: H1035-40.

4. Peart JN, Gross GJ. Am J Physiol (Heart Circ Physiol) 2003; 285: H81-9.

\section{Use of Adenosine Compounds for Identifying Patients Prone to Suffer from Tachyarrhythmias}

Raphael Rosso, M.D.

Department of Cardiology, Tel Aviv - Sourasky Medical Center and Sackler School of Medicine, Tel Aviv University

In our presentation we review our experience in the use of adenosine-compounds for diagnostic purposes in cardiac arrhythmias. Adenosine-compounds are the treatment of choice for common supraventricular tachyarrhythmias involving the atrio-ventricular node. Moreover, adenosine-compounds can also be used for diagnostic purposes.

Injection of adenosine compounds during sinus rhythm leads to short lasting bradycardia (sinus arrest or block of conduction along the AV-node). This effect is short lasting and invariably resolves within seconds. The induced bradyarrhythmia is always followed by sinus tachycardia. This unique effects of adenosine compounds may be used for the following diagnostic purposes: 1) to reveal the presence of Wolff-Parkinson-White in patients with minor preexcitation due to a left sided location of the accessory pathway and fast AV nodal conduction, 2) to reveal the presence of dual AV node physiology in patients with AV-node reentry tachycardia, 3) to reveal the presence of concealed accessory pathways in patients with AV reentry tachycardia. The last two effects can be useful when evaluating patients who have a history of palpitations but no documented arrhythmias. Finally, since 
patients with long QT syndrome develop torsade de pointes during sudden deceleration (bradycardia-dependent) or sudden acceleration (tachycardia dependent torsade) of the heart rate, we have used adenosine-compounds for diagnosing long QT syndrome in patients with borderline QT intervals.

\title{
Bone and Cartilage
}

\section{Integrating Local and Systemic Regulation of Bone Turnover Through P2 Receptor Signalling}

\author{
J.A. Gallagher \\ Human Bone Cell Research Group, Department of Human Anatomy and Cell Biology, The University of \\ Liverpool, Liverpool, L69 3GE U.K. \\ jag1@liv.ac.uk
}

Bone is continually remodelled throughout life by bone-resorbing osteoclasts and bone-forming osteoblasts. This remodelling is regulated by a complex interaction of systemic hormones, including parathyroid hormone (PTH), and local factors, including mechanical loading, which together ensure that the skeleton adapts to physiological requirements. Extracellular nucleotides acting through multiple P2 receptors play a major role in integrating the local and systemic signalling. Since the early descriptions of P2 receptor expression in bone ${ }^{1}$, functional and molecular evidence has accumulated for the presence of multiple P2X and P2Y receptors on osteoblasts and osteoclasts. In addition to expressing P2 receptors, bone cells release ATP constitutively ${ }^{2}$ and this release is enhanced by mechanical stimulation. The exact mechanism for the constitutive release of ATP by osteoblasts remains to be identified, although exocytosis probably plays some part.

Some of the the functional consequences of ATP release in bone and subsequent activation of $\mathrm{P} 2$ receptors on bone cells have been identified. For example, activation of osteoblastic P2Y1 receptors leads to an up regulation of receptor activator of nuclear factor-kB ligand (RANKL), which stimulates the formation and activity of osteoclasts and thereby stimulates bone resorption ${ }^{3}$, and P2X7 receptors are expressed on osteoblasts and osteoclast and may play important roles in mechanotransduction and osteoclast formation ${ }^{4}$. One observation that we regard as particularly significant is the effect of activation of osteoblastic P2Y receptors on the induction of cfos, an immediate early gene that plays a key role in the proliferation and differentiation of bone cells. Activation of osteoblastic P2Y receptors alone results in a only a moderate induction of c-fos, whereas dual activation of P2 receptors and PTH receptors leads, through multiple levels of interaction in downstream signalling, to a massive synergy in c-fos expression ${ }^{5}$. This synergy provides a molecular mechanism whereby locally released extracellular nucleotides can sensitise cells to systemic PTH and thereby activate remodelling at discrete sites in bone. This may represent a common mechanism to integrate systemic and local signals in the regulation of turnover in other tissues.

1. Schöfl C, Cuthbertson KS, Walsh CA, Mayne C, Cobbold P, von zur Mühlen A, Hesch RD, Gallagher JA. Evidence for $\mathrm{P}_{2-}$ purinoceptors on human osteoblast-like cells. J Bone Miner Res 1992; 7: 485-91.

2. Buckley KA, Golding SL, Rice JM, Dillon JP, Gallagher JA. Release and interconversion of P2 receptor agonists by human osteoblast-like cells. FASEB J 2003; 17: 1401-10.

3. Buckley KA, Hipskind RA, Gartland A, Bowler WB, Gallagher JA. Adenosine triphosphate stimulates human osteoclast activity via upregulation of osteoblast-expressed receptor activator of nuclear factor-kB ligand. Bone 2002; 31: 582-90.

4. Gartland A, Buckley KA, Hipskind RA, Bowler WB, Gallagher JA. P2 receptors in bone-modulation of osteoclast formation and activity via P2X7 activation. Crit Rev Eukaryot Gene Expr 2003; 13(2-4): 237-42.

5. Buckley KA, Wagstaff SC, McKay G, Gaw A, Hipskind RA, Bilbe G, Gallagher JA, Bowler WB. Parathyroid hormone potentiates nucleotide-induced $\left[\mathrm{Ca}^{2+}\right]_{\mathrm{i}}$ release in rat osteoblasts independently of $\mathrm{Gq}$ activation or cyclic monophosphate accumulation: A mechanism for localizing systemic responses in bone. J Biol Chem 2001; 276: 9565-71. 


\section{P2X7 nucleotide receptor functions in bone cells}

Nattapon Panupinthu, Jasminka Korcok, Joseph T. Rogers, Stephen M. Sims and S. Jeffrey Dixon CIHR Group in Skeletal Development and Remodeling, Schulich School of Medicine \& Dentistry, The University of Western Ontario, London, Canada, N6A 5C1

E-mail: jeff.dixon@schulich.uwo.ca

Since ATP is released from cells in response to mechanical stimulation, it has been proposed that P2 nucleotide receptors mediate mechanotransduction in bone. ${ }^{1}$ Mice with targeted disruption of the P2X7 receptor gene exhibit diminished periosteal bone formation together with excessive trabecular bone resorption ${ }^{2}$ and impaired response to mechanical loading. ${ }^{3}$ Our goal was to understand the roles of $\mathrm{P} 2 \mathrm{X} 7$ receptors in osteoblasts (bone forming cells) and osteoclasts (bone resorbing cells). Whereas the expression of functional P2X7 receptors has been established in osteoclasts, ${ }^{4}$ their existence in osteoblasts has been controversial. Osteoblast-enriched cultures were obtained from calvariae of newborn rats, and wild-type (WT) and P2X7 receptor knockout (KO) mice. Osteoblast-enriched cultures were supplemented with ascorbic acid and $\beta$-glycerophosphate to induce formation of mineralized nodules. Cells expressing functional P2X7 receptors were associated with bone nodules. Benzoylbenzoyl-ATP (BzATP, P2X7 agonist), but not UTP, promoted mineralization in rat calvarial cell cultures. Moreover, mineralization in KO cultures was markedly reduced compared to WT, consistent with a role for P2X7 receptors. Expression of osteoblast marker genes (encoding Runx2, Osterix, osteocalcin and bone sialoprotein) was assessed by real-time PCR. Transcript levels were significantly enhanced by BzATP in rat calvarial cell cultures and suppressed in cultures from KO mice compared to WT (except Runx2). Cyclooxygenase inhibitors abolished the stimulatory effect of BzATP on bone formation without affecting mineralization in untreated cultures. Thus, P2X7 receptors stimulate osteoblast differentiation through a cell-autonomous, cyclooxygenase-dependent mechanism. The lifespan of osteoclasts is an important regulator of bone resorption. Osteoclasts were isolated from long bones of newborn WT and KO mice and cell survival was assessed. In the absence of exogenous nucleotides, higher numbers of WT than KO osteoclasts were apoptotic at $6 \mathrm{~h}$. Consistent with this observation, fewer WT osteoclasts survived at $12 \mathrm{~h}$. Interestingly, BzATP had no additional effect on either apoptosis or survival. Control experiments revealed that WT and KO osteoclasts were equally susceptible to apoptosis induced by the proapoptotic agent staurosporine. Brilliant blue G (P2X7 antagonist) decreased apoptosis and increased survival of WT osteoclasts, providing additional evidence for involvement of the P2X7 receptor. Treatment of WT osteoclasts with alkaline phosphatase (to hydrolyze endogenous nucleotides) increased their survival to levels comparable to $\mathrm{KO}$ osteoclasts, consistent with activation of $\mathrm{P} 2 \mathrm{X} 7$ receptors by constitutively released nucleotides. In summary, P2X7 receptors stimulate osteoblast differentiation and induce osteoclast apoptosis. Since the P2X7 receptor both enhances bone formation and suppresses bone resorption, it represents a potential target for the development of drugs with combined anabolic and antiresorptive effects. Supported by the Canadian Institutes of Health Research (CIHR).

(1) Dixon SJ, Sims SM. Drug Dev Res 2000; 49: 187-200.

(2) Ke HZ, Qi H, Weidema AF, Zhang Q, Panupinthu N, Crawford DT, Grasser WA, Paralkar VM, Li M, Audoly LP, Gabel CA, Jee WS, Dixon SJ, Sims SM, Thompson DD. Mol. Endocrinol. 2003; 17: 1356-67.

(3) Li J, Liu D, Ke HZ, Duncan RL, Turner CH. J Biol Chem 2005; 280: 42952-9.

(4) Naemsch LN, Dixon SJ, Sims SM. J Biol Chem 2001; 276: 39107-14.

\section{P2Y nucleotide receptor function in osteoblasts}

Isabel Orriss, Geoffrey Burnstock and Timothy R. Arnett

Department of Anatomy and Developmental Biology, University College London, London WC1E 6BT, UK. e-mail: t.arnett@ucl.ac.uk

Accumulating evidence suggests that extracellular nucleotides, signalling through P2 receptors, play a role in modulating bone cell function. ATP and ADP stimulate osteoclastic resorption, whilst ATP and UTP are 
powerful inhibitors of bone formation by osteoblasts ${ }^{1}$. We investigated changes in the expression of $\mathrm{P} 2$ receptors with cell differentiation in primary osteoblast cultures. Rat calvarial osteoblast, derived by trysin/collagenase digestion and cultured for up to 10 days, were loaded with the intracellular $\mathrm{Ca}^{2+}$-sensing fluorophore, Fluo-4 AM, and a fluorescence imaging plate reader (FLIPR) was used to measure responses to nucleotide agonists. Peak responses occurred within $20 \mathrm{sec}$, and were evoked by ATP or UTP at concentrations as low as $2 \mu \mathrm{M}$. Numbers of osteoblasts doubled between days 4 and 10 of culture but the peak intracellular $\mathrm{Ca}^{2+}$ response to ATP or UTP increased up to 6-fold over the same period, indicating that osteoblast responsiveness to nucleotides increased as cell differentiation proceeds. The approximate order of potency for the most active nucleotide agonists at day 8 of culture was ATP $>$ UTP \& ATP $\gamma \mathrm{S}>$ ADP $>$ UDP, consistent with the expression of functional $\mathrm{P}_{2} \mathrm{Y}_{2}, \mathrm{P}_{2} \mathrm{X}_{2}, \mathrm{P}_{2} \mathrm{Y}_{4}$, $\mathrm{P}_{2} \mathrm{Y}_{1}$ and $\mathrm{P}_{2} \mathrm{Y}_{6}$ receptors. Smaller responses were elicited by 2-MeSATP, Bz-ATP and $\alpha, \beta$-meATP, additionally suggesting the presence of functional $\mathrm{P} 2 \mathrm{X}_{1}, \mathrm{P} 2 \mathrm{X}_{3}, \mathrm{P} 2 \mathrm{X}_{5}$ and $\mathrm{P} 2 \mathrm{X}_{7}$ receptors. Expression of mRNA for the ATP and UTP-selective $\mathrm{P}_{2} \mathrm{Y}_{2}$ receptor increased strongly between days 6 and 15 in primary osteoblasts, whereas mRNAs for the $\mathrm{P}_{4} \mathrm{Y}_{4}$ (also ATP/UTP selective) and P2Y 6 (UDP/UTP selective) receptors were highly expressed at intermediate time points. In contrast, mRNA for the cell-proliferation-associated $\mathrm{P}_{2} \mathrm{X}_{5}$ receptor decreased to undetectable as osteoblasts matured, but mRNA for the cell death-associated $\mathrm{P} 2 \mathrm{X}_{7}$ receptor was detected at all time points. Similar trends were evident using immunostaining and Western blotting for P2 receptors. Exposure to 1-10 $\mu \mathrm{M}$ ATP or UTP during days 10-14 of culture was sufficient to cause near-total blockade of alizarin redstained bone nodules formed by osteoblasts; however, UDP and ADP were without effect. Microscopy revealed that ATP and UTP-treated osteoblasts deposited abundant collagenous material with the characteristic morphology of bone nodules, but that mineralisation failed to occur. In osteoblast cultures treated with $10 \mu \mathrm{M}$ ATP or UTP, the activity of alkaline phosphatase (thought to participate in bone mineralisation) was reduced by $>50 \%$. Our results show that there is a shift from $\mathrm{P} 2 \mathrm{X}$ to $\mathrm{P} 2 \mathrm{Y}$ expression during osteoblast differentiation in culture, with mature osteoblasts preferentially expressing the $\mathrm{P}_{2} \mathrm{Y}_{2}$ receptor and, to a lesser extent, $\mathrm{P}_{4} \mathrm{Y}_{4}$ and $\mathrm{P}_{2} \mathrm{Y}_{6}$ receptors. Taken together, these data suggest that the $\mathrm{P}_{2} \mathrm{Y}_{2}$ receptor and, possibly, the $\mathrm{P}_{2} \mathrm{Y}_{4}$ receptor could function as 'off-switches' for mineralised bone formation. It should be noted, however, that since several ectonucleotidases can generate pyrophosphate (PPi) from nucleotide triphosphates, and that PPi is a potent inhibitor of bone mineralisation (at $\geq 1 \mu \mathrm{M}$ in our in vitro system), the possibility remains that a component of the effect of extracellular nucleotides on mineralisation could also occur independently of $\mathrm{P} 2$ receptors. We are grateful for the support of the Arthritis Research Campaign.

1. Hoebertz A, Arnett TR, Burnstock G. Regulation of bone resorption and formation by purines and pyrimidines. Trends Pharmacol Sci 2003; 24: 290-7.

\title{
The role of nucleotides and $\mathrm{P} 2$ receptors in intercellular signaling in bone
}

\author{
Niklas Rye Jørgensen \\ Dep. of Clinical Biochemistry, Copenhagen University Hospital Hvidovre, Hvidovre, Denmark \\ Email:niklas@dadlnet.dk
}

The regulation of bone turnover is a complex and finely tuned process. Many factors regulate bone remodelling, including hormones, growth factors, cytokines etc. However, little is known about the signals coupling bone formation to bone resorption, and how mechanical forces are translated into biological effects in bone. Intercellular calcium waves are increases in intracellular calcium concentration in single cells, subsequently propagating to adjacent cells, and can be a possible mechanism for the coupling of bone formation to bone resorption and for the translation of mechanical forces on bone into biological signals.

Intercellular calcium waves have been demonstrated to occur not only among osteoblasts in vitro, but also between osteoblasts and osteoclasts, in response to mechanical stimuli. Calcium signaling among osteoblasts can be propagated by the secretion of a nucleotide, possibly ATP, acting in an autocrine action to purinergic P2Y2 receptors on the neighboring cells, leading to IP3 generation and subsequent release of calcium from intracellular stores. In the signaling from osteoblasts to osteoclasts, paracrine action of ATP seems to be responsible, with the involvement of purinergic $\mathrm{P} 2 \mathrm{X} 7$ pore forming receptor. 
Activation of $\mathrm{P} 2$ receptors in bone cells not only involves the propagation of intercellular calcium waves but also the proliferation and activity of the cells. Further, alterations in receptor expression and function have effects on bone development and turnover as well as on bone quality and fracture risk in vivo.

Thus, nucleotide-mediated intercellular calcium signalling is taking place in vitro among osteoblasts and between osteoblast and osteoclasts, and might be a mechanism by which mechanical forces regulate bone turnover in vivo. The mechanisms involved in the calcium signaling are definitely important in normal bone development and turnover, and might be future targets for pharmacological manipulation of bone metabolism.

\title{
Immunology and Inflammation
}

\section{Activation of P2X7 by ADP-Ribosylation}

Friedrich Koch-Nolte

Institute of Immunology, University Hospital, Martinistr. 52, Hamburg, 20246 Germany

nolte@uke.uni-hamburg.de

The cytolytic $\mathrm{P} 2 \mathrm{X} 7$ purinoceptor is widely expressed by inflammatory cells. $\mathrm{P} 2 \mathrm{X} 7$ has attracted attention because of its unique capacity to induce the formation of a large pore and cell death. Activation of $\mathrm{P} 2 \mathrm{X} 7$ requires much higher concentrations of ATP than activation of other P2X family members. P2X7 on murine T cells can also be activated by ADP-riboslyation of cell surface proteins catalyzed by the toxin like ecto-ADP-ribosyltransferase ART2 following release of the ART substrate, NAD, from cells [1]. Much lower concentrations of NAD than ATP suffice to activate P2X7. However, at saturating doses, ATP induces stronger activation signals than ADPribosylation. Moreover, activation of P2X7 is readily reversed after short exposures to ATP followed by washing away of the soluble ligand, wheras short exposures to NAD followed by washing chronically activate P2X7 by providing covalently bound ligands. Using site directed mutagenesis we have identified the sites of ADPribosylation on P2X7. The results shed light on the issue whether P2X7 is activated by ADP-ribose moieties attached to neighboring proteins or to P2X7 itself.

Supported by DFG grant No310/6-1 to FH and FKN

(1) Seman M, Adriouch S, Scheuplein F, Krebs C, Freese D, Glowacki G, Deterre P, Haag F, Koch-Nolte F. NAD-induced T cell death: ADP-ribosylation of cell surface proteins by ART2 activates the cytolytic P2X7 purinoceptor. Immunity 2003; 19 (4): 571-82.

\section{Adenosine 2A Receptor $\left(\mathrm{A}_{2 \mathrm{~A}} \mathrm{R}\right)$ Agonists Regulate Renal Nitric Oxide Production in Diabetic Nephropathy}

\author{
Alaa S. Awad, Elizabeth Duong, Liping Huang, Joel Linden and Mark D. Okusa \\ Departments of Medicine, University of Virginia, Charlottesville, VA 22908 \\ awad@virginia.edu
}

Background Nitric oxide (NO) regulates renal hemodynamics, endothelial function and inflammation. However, the role of $\mathrm{NO}$ in the pathophysiology of diabetic nephropathy (DN) remains controversial. We have shown previously that $\mathrm{A}_{2 \mathrm{~A}}$ receptor agonists prevent renal tissue injury and inflammation in DN [Am J Physiol Renal Physiol 2006, in press]. Whether the protective effect of $A_{2 A}$ receptor agonists is mediated by regulation of renal $\mathrm{NO}$ production is not known. We hypothesized that $\mathrm{A}_{2 \mathrm{~A}}$ agonists ameliorate renal endothelial dysfunction and inflammation associated with DN through an effect on NO production.

Methods Diabetes was induced with single iv injection of streptozotocin (STZ) in Sprague Dawley rats $(50 \mathrm{mg} /$ $\mathrm{kg}$ ). Urinary NO end products (NOX) and nitric oxide synthases (NOS) mRNA were evaluated in control, 
diabetes with vehicle and diabetes with ATL146e, a selective $\mathrm{A}_{2 \mathrm{~A}}$ agonist (10 $\left.\mathrm{ng} / \mathrm{kg} / \mathrm{min}\right)$ via osmotic pump over 6 weeks.

Results Blood glucose increased in the diabetes and treatment groups 48 hours after STZ-induction of diabetes. Renal medullary blood flow and urinary NOX excretion were reduced significantly in diabetes group $(67 \% ; \mathrm{p}<$ 0.001 and $60 \% ; \mathrm{p}<0.01$ from control) and restored to normal levels by ATL146e ( $86 \%$ and $100 \%$ from control), respectively. There was a significant correlation between urinary albumin excretion rate and urinary NOX $(\mathrm{r}=$ $0.84 ; \mathrm{p}<0.0001$ ). We next sought to determine renal NO synthase (NOS) isoforms altered in DN. By using realtime PCR, diabetes led to a decrease in renal eNOS mRNA expression (67\% from control) that was reversed by ATL146e (137\% from control; $\mathrm{p}<0.05$ from diabetes). iNOS mRNA expression was not detectable in control but was induced in diabetes, an effect that was reversed by ATL146e. Immunohistochemistry with iNOS antibody of the kidney tissue confirmed the result of mRNA expression and was corelated with increased macrophages infiltration, a major source of iNOS, in diabetic kidneys. In contrast, there was no significant change in nNOS gene expression between groups.

Summary These results demonstrate that DN is associated with decreased renal production of NO mainly through decreased expression of eNOS an effect reversed by $\mathrm{A}_{2 \mathrm{~A}}$ agonists. Furthermore an increase iNOS due in part to macrophage infiltration in DN is reversed with ATL146e.

Conclusion The effects of $\mathrm{A}_{2 \mathrm{~A}}$ agonists on ameliorating the previously described functional and morphological changes associated with DN may in part be due to effects on NO.

\title{
Adenosine in Dermal and Hepatic Fibrosis
}

\author{
Edwin SL Chan \\ New York University School of Medicine, New York, NY 10016, USA \\ Chane01@nyu.edu
}

Adenosine promotes the healing of wounds and matrix deposition in the skin, actions known to be mediated through the $\mathrm{A}_{2 \mathrm{~A}}$ receptor. The release of adenosine also accounts for the anti-inflammatory activity of methotrexate, one of the most widely used anti-rheumatic agents. We hypothesized that ligation of the $\mathrm{A}_{2 \mathrm{~A}}$ receptor is responsible for the development of hepatic fibrosis, one of the most serious complications of chronic methotrexate therapy, as well as cirrhosis arising from other causes such as ethanol consumption. Epidemiological studies have demonstrated a protective effect of caffeine in hepatic cirrhosis and we speculate that this protective effect is also related to the antagonistic activity of caffeine at the adenosine receptor. The capacity of adenosine for modulating fibrous tissue development in the skin is also relevant to the pathogenesis of another rheumatic disease, scleroderma, where skin fibrosis is a prominent feature. Using in vitro and in vivo models, we have demonstrated a role for the $\mathrm{A}_{2 \mathrm{~A}}$ receptor in the pathogenesis of these disorders. These findings provide a strong rationale for the exploitation of adenosine receptor antagonist therapies in future treatment of fibrotic diseases.

\section{Adenosine receptors and the regulation of chronic lung disease}

\author{
Michael R. Blackburn \\ Department of Biochemistry and Molecular Biology, University of Texas-Houston Medical School \\ michael.r.blackburn@uth.tmc.edu
}

Chronic lung diseases are associated with persistent lung inflammation and damage that lead to the progressive loss of lung function. In contrast to most injury and repair responses, the inflammation seen in these disorders is chronic and may last throughout the life of the afflicted individual. The mechanisms that are responsible for the intensity and chronicity of these disorders have not been elucidated. Adenosine is a signaling nucleoside that can elicit a variety of cellular functions by engaging cell surface adenosine receptors. Adenosine levels are elevated in the lungs of patients with chronic lung disease suggesting this signaling pathway may be involved in these disorders. My laboratory makes use of genetically modified mice to examine the importance of adenosine 
signaling pathways in aspects of chronic lung disease. We have generated mice deficient in the enzyme adenosine deaminase (ADA) that controls the levels of adenosine in tissues and cells. Adenosine levels accumulate in the lungs of these mice and are associated with increased lung inflammation and histopathologies seen in asthma and chronic obstructive pulmonary disease including fibrosis and alveolar airway enlargement. We have used both genetic and pharmacologic approaches to examine the contributions of the different adenosine receptors to the inflammation and damage seen in the lungs of ADA-deficient mice. In addition, we have examined the contribution of adenosine and its receptors in other models of chronic lung disease. My presentation will use data gathered in the above model systems to highlight the various pro-inflammatory/tissue destructive effects of adenosine receptors as well as anti-inflammatory/tissue protective effects, as they pertain to aspects of chronic lung disease.

\section{ADP-ribosylation by ARTs introduces diacritic modifications in the cytokine network}

Michel Seman

EA1556 University Denis Diderot-Paris7 and INSERM U519, Faculty of Medicine and Pharmacy, 22 Boulevard Gambetta, 76000, Rouen, France

michel.seman@univ-rouen.fr

Mammalian mono ADP-ribosyl transferases (ARTs) constitute a family of ectoenzymes catalyzing the transfer of ADP-ribose from $\mathrm{NAD}^{+}$onto arginine residues of target proteins. They are thus responsible for unusual posttranslational modifications of proteins in the extracellular compartment. $\mathrm{NAD}^{+}$is released during inflammation and can be detected in inflammatory liquids. This provides enough substrate to ARTs and renders possible ADPribosylation of neighboring membrane targets on the surface of ART expressing cells such as integrins or P2X7, and free soluble proteins present in surrounding extracellular fluids including cytokines and growth factors. Several cytokines can thus be ADP-ribosylated by ART1 but others are not although they all contain arginine residues in their primary sequence. This provides evidence for selective ADP-ribosylation sites. Cytokine ADPribosylation can either block their functions and/or modulate their bioavailability. ART-mediated ADPribosylation of cytokines represents a new mechanism of regulation in the immune system which may contribute to the control of inflammation and host-tumor interactions. It may also participate in the resistance of patients to cytokine-based therapies.

Supported by grants from the Ministère de la Recherche, Ligue Nationale contre le cancer, Association pour la recherche sur le cancer.

\section{Nicolò Copernico Award: From "Hellstrom Paradox" to Redox-Adenosinergic Rule of Immune Response}

Michail V. Sitkovsky

New England Inflammation and Tissue Protection Institute, Consortium at Northeastern University, Boston, $M A, U S A$

M.Sitkovsky@neu.edu

We will describe the redox-adenosinergic mechanism of protection of normal tissues from excessive immune damage. These studies were motivated by the long-standing "Hellstrom Paradox" of the peaceful co-existence of tumors and anti-tumor immune cells in the same cancer patient. We summarize the more than 25 years of research that was focused first on mechanisms of cell-cell interactions during T-cell mediated lethal hit delivery to tumor target cells, then on mechanisms that propagate or may inhibit the $\mathrm{T}$ cell receptor-triggered signaling. We provide evidence, which suggests that down-regulation of overactive immune cells in inflamed areas may be 
triggered by excessive collateral immune damage to endothelial cells and microcirculation. The ensuing interruption of normal blood and oxygen supply leads to low tissue oxygen tension. This is associated with an accumulation of extracellular adenosine and signaling through $\mathrm{A} 2 \mathrm{~A}$ and $\mathrm{A} 2 \mathrm{~B}$ adenosine receptors on the surface of surrounding cells, including activated myeloid and $\mathrm{T}$ cells. This chain of events culminates in inhibition of activated immune cells in a delayed, negative feedback manner due to the well-established immunosuppressive effects of cAMP-elevating A2 adenosine receptors. We also describe the rationale behind the model that the tissue protecting effects of hypoxia involve both hypoxia-stabilized Hypoxia Inducible Factor- $1 \alpha$ and A2 adenosine receptors. Similar mechanism mat explain Hellstrom paradox. An example of the predictive power of the redox-adenosinergic rule is provided by the confirmed exacerbation of acute lung injury by hypoxiaeliminating supplemental oxygen therapy.

\title{
Target genes of extracellular ATP in human dendritic cells
}

Didier Communi ${ }^{1}$, Frédéric Marteau ${ }^{1}$, Michael Horckmans ${ }^{1}$, Nathalie Bles ${ }^{1}$, Jean-Marie Boeynaems ${ }^{1,2}$ ${ }^{T}$ Institute of Interdisciplinary Research, IRIBHM, Université Libre de Bruxelles, Belgium; communid@ulb.ac.be

${ }^{2}$ Department of Medical Chemistry, Erasme Hospital, Université Libre de Bruxelles, Belgium

Extracellular ATP affects the maturation of human monocyte-derived dendritic cells (MoDCs), mainly by inhibiting Th1 cytokines, promoting Th2 cytokines, and modulating the expression of costimulatory molecules. Comparison of ATP and PGE2 expression profiles in human moDCs revealed an extensive number of target genes involved in immunosuppression, maturation and chemotaxis.

More particularly, ATP elicited a drastic up-regulation of two mediators involved in immunosuppression: thrombospondin-1 and indoleamine 2,3-dioxygenase. Extracellular ATP released from damaged cells and previously considered as danger signal is thus a potent regulator of mediators playing key roles in immune tolerance.

We also observed that ATP is able to regulate several chemokines and chemokine receptors. More particularly, adenine nucleotides induced down-regulation of major monocyte recruiters such as MCP-1 and MIP-1 $\alpha$ chemokines and reduced the capacity of MoDCs to attract monocytes and immature DCs.

Pharmacological data support the involvement of the $\mathrm{P}_{2} \mathrm{Y}_{11}$ receptor in the regulation of these ATP target genes in human MoDCs. Consequently nucleotides derivatives may be considered as useful tools for DC-based immunotherapies. Several other promising genes regulated by ATP have been identified in human DCs and their study is ongoing.

\section{P2 Receptors: Role in host defence against intracellular microorganisms}

\author{
$\underline{\text { Robson Coutinho-Silva }}^{1}$, Suzana Passos Chaves $^{1}$, Camila Marques da Silva ${ }^{1,2}$, Pedro M. Persechini ${ }^{1}$, \\ Bartira B Bergmann $^{1}$ and David Ojcius ${ }^{3}$ \\ ${ }^{1}$ Instituto de Biofísica Carlos Chagas Filho and ${ }^{2}$ Dept. Ciências Morfológicas - Universidade Federal do Rio de \\ Janeiro, Rio de Janeiro, Brasil; ${ }^{3}$ Division of Natural Sciences, University of California, Merced, CA \\ rcsilva@biof.ufrj.br
}

A growing number of studies have demonstrated the importance of $\mathrm{ATP}_{\mathrm{e}}$-signalling via $\mathrm{P} 2$ receptors as an important component of the inflammatory response to infection. More recent studies have shown that $\mathrm{ATP}_{\mathrm{e}} \mathrm{can}$ also have a direct effect on infection by intracellular pathogens, by modulating membrane trafficking in cells that contain vacuoles that harbor intracellular pathogens, such as mycobacteria and chlamydiae. A conserved mechanism appears to be involved in controlling infection by both of these pathogens, as a role for phospholipase $\mathrm{D}$ in inducing fusion between lysosomes and the vacuoles has been demonstrated. $\mathrm{P} 2 \mathrm{X}_{7}$ receptors is involved in clearance of intracellular bacteria, but in mycobacteria, infection seems to controlled by an additional $\mathrm{P} 2$ receptor. 
Other P2-dependent mechanisms are most likely operative in the cases of pathogens such as Leishmania, which survive in an acidic phagolysosomal-like compartment. We explored the possibility that P2 receptors could be used as a therapeutic drug against Leishmania infection. BALB/c mice infected with L. amazonensis were treated for three weeks, twice a week, with PBS, ATP $(50 \mathrm{mM})$, or UTP $(5 \mathrm{mM})$ in $20 \mu \mathrm{l}$ injections in foot lesions. We observed that ATP and UTP reduced the parasitic load by two-log units compared to control mice treated with PBS.. In vitro, peritoneal macrophages were infected for $48 \mathrm{~h}$ at $37^{\circ} \mathrm{C}$, and then treated with ATP $(500 \mu \mathrm{M})$, ADP $(100 \mu \mathrm{M})$, adenosine $(100 \mu \mathrm{M})$, UTP $(100 \mu \mathrm{M})$, UDP $(100 \mu \mathrm{M})$ or oATP $(300 \mu \mathrm{M})$ in PBS for 30 min. Only ATP showed significant anti-amastigotic activity ( $36 \%$ inhibition), with no significant cytotoxicity observed for any of the nucleotides, and UTP and UDP inducing production of nitric oxide. Measurements of calcium flux and apoptosis induced by the nucleotides data suggest possible modulation of at least two $\mathrm{P} 2$ receptor during in vitro infection with $L$. amazonensis, one being sensitive to ATP and the other, to UTP. These results suggest that P2 receptors are potential targets for anti-leishmanial therapy, that the immunological environment is probably important for this activity, and that there are several P2 receptors involved in the anti-microbial effect of the nucleotides.

Acknowledgment FAPERJ and CNPq.

\section{Polymorphisms in the $\mathbf{P 2 X} 7$ receptor and their clinical associations}

James S. Wiley, ${ }_{1}^{1}$ Suran Fernando, ${ }^{2}$ Kristy Skarratt, ${ }^{1}$ Bernadette Saunders, ${ }^{2}$ Ronald Sluyter, ${ }^{1}$ Ben Gu, ${ }^{1}$ Stephen Fuller ${ }^{1}$, Anne Shemon, ${ }^{1}$ Phuong Dao-Ung, ${ }^{1}$ Jenny Georgiou, ${ }^{1}$ and Warwick Britton ${ }^{2}$

${ }^{1}$ Department of Medicine, University of Sydney, Nepean Hospital, Penrith NSW 2750, and ${ }^{2}$ Centenary Institute of Cancer Medicine and Cell Biology, Camperdown, 2042, Australia

Email:wileyj@medicine.usyd.edu.au

The search for the function of the P2X7 receptor has drawn on results from two models; the gene-deleted mouse and rare genetic variants within the human population. Studies of the knock-out mouse by both the Pfizer and Glaxo groups have pointed to $\mathrm{P} 2 \mathrm{X} 7$ as a pro-inflammatory gene which may have a role in bone structure. Parallel clinical studies by our group and others have suggested that P2X7 may also have a role in innate immunity and resistance to certain infections.

The innate immune system is vital for the control of infection by intracellular pathogens such as mycobacteria, chlamydia and toxoplasma. These organisms evade the adaptive immune response by entering and replicating inside host cells and tissues. Recent studies have shown that activation of the P2X7 receptor on cells of the monocyte - macrophage series is needed for killing of intracellular mycobacteria and that the same activation signal leads to subsequent apoptotic death of the infected macrophage. Wide variations in P2X7 function have been observed between individuals and genetic factors play a major role in this variability. Much of the variability is due to the polymorphic structure of the gene with an average of one single nucleotide polymorphism (SNP) every 160 bp of coding DNA and we have described at least 5 SNPs causing loss of function and a further one has been reported giving gain of function.

In around $30 \%$ of the Caucasian population, a Glu-496 to Ala polymorphism in P2X7 receptors $(1513 \mathrm{~A} \rightarrow \mathrm{C})$, of the carboxyl terminus leads to loss of function. Homozygosity for this polymorphism abolishes ATP-induced channel dilatation (pore formation) while the heterozygous state gives cells with half the pore function of cells with wild type P2X7 protein. A second polymorphism, Ile-568 to Asn $(1729 \mathrm{~T} \rightarrow \mathrm{A})$ lies in a trafficking motif of the carboxyl terminus and prevents normal trafficking and surface expression of the receptor. A third polymorphism, Arg-307 to uncharged glutamine $(946 \mathrm{G} \rightarrow \mathrm{A})$ lies within the ATP binding pocket of the extracellular domain and abolishes the binding of ATP to the receptor with severe functional loss. A fourth polymorphism occurs as a splice site mutation at position +1 of the first intron of the gene $(151+1 \mathrm{~g} \rightarrow \mathrm{t})$ and gives rise to a null allele in $1-2 \%$ of the Caucasian population. While each of the latter three polymorphisms (1729A, 946A and 151+1t) occurs at an allele frequency of 1-2\% in Caucasians, none have been found in a large cohort of S.E. Asian immigrants to Australia.

The effect of loss-of-function SNPs appears to be additive such that macrophages from compound heterozygous subjects have total ablation of P2X7 function. Thus we have observed severe illness due to Toxoplasma gondii in two compound heterozygotes with total absence of $\mathrm{P} 2 \mathrm{X} 7$ function. In two large case control 
cohorts we have studied the reactivation of tuberculosis in immigrants of mainly S.E. Asian origin after arrival in Australia. The $1513 \mathrm{~A} \rightarrow \mathrm{C}$ polymorphism was strongly associated with extrapulmonary but not pulmonary tuberculosis in both the first $(\mathrm{OR} 3.99, \mathrm{P}<0.001)$ and second cohorts $(\mathrm{OR} 2.99, \mathrm{P}<0.01)$. These data confirm the major effects of $\mathrm{P} 2 \mathrm{X} 7$ polymorphisms on macrophage function and innate immunity of man.

\title{
Tissue-Derived Adenosine promotes Peripheral T cell Tolerance by Stimulating the $\mathbf{A}_{2 \mathrm{~A}}$ Receptor
}

Jonathan D. Powell* ${ }^{+}$Paul E. Zarek*, Charles Drake ${ }^{+}$, Drew M. Pardoll ${ }^{+}$

${ }^{*}$ Dept. of Pharmacology, ${ }^{+}$Dept of Oncology, The Johns Hopkins University, School of Medicine, Baltimore, MD, USA 21231

poweljo@jhmi.edu

A2a receptor $\left(A_{2 a} R\right)$ engagement by endogenous adenosine has been implicated as an important negative regulator of inflammation. Using microarray analysis, we determined that the $A_{2 \mathrm{a}} \mathrm{R}$ is differentially upregulated on $\mathrm{T}$ cells under conditions that induce $\mathrm{T}$ cell tolerance. In vitro, $\mathrm{A}_{2 \mathrm{a}} \mathrm{R} \mathrm{WT}$, but not $\mathrm{A}_{2 \mathrm{a}} \mathrm{R}$ null $\mathrm{T}$ cells that were initially activated in the presence of CGS-21680 (CGS) were hyporesponsive upon rechallenge; they were anergic. In vivo, the adoptive transfer of $\mathrm{A}_{2 \mathrm{a}} \mathrm{R}$ null $\mathrm{HA}$-specific $6.5 \mathrm{~T}$ cells into mice expressing $\mathrm{HA}$ as a self-antigen led to rapid autoimmune-induced death when compared with the transfer of $\mathrm{T}$ cells from WT mice. Furthermore, treating mice with just 4 days of CGS inhibited autoimmune-induced death and promoted the induction of $\mathrm{T}$ cell anergy. In this model, mice that survive the initial adoptive transfer develop inducible LAG-3+ regulatory T cells that protect the mice from a subsequent rechallenge with clonotypic T cells. LAG-3 expression levels and kinetics are enhanced in $\mathrm{A}_{2 \mathrm{a}} \mathrm{R}$ WT cells from CGS-treated mice, but decreased in $\mathrm{A}_{2 \mathrm{a}} \mathrm{R}$ null $\mathrm{T}$ cells, regardless of CGStreatement. CGS-treated mice that survived the initial adoptive transfer were protected against a subsequent rechallenge with lethal numbers of clonotypic cells. Conversely, CGS failed to protect mice from the adoptive transfer of LAG-3 null $\mathrm{T}$ cells. It is notable that the concentration of adenosine in the tumor mciroenvironment is in the $\mathrm{mM}$ range. This suggests that one mechanism by which tumors evade immune destruction is via $\mathrm{A}_{2 \mathrm{a}} \mathrm{R}$ induced tumor-antigen-induced tolerance. To test this hypothesis we examined the efficacy of tumor vaccines in $A_{2 a} R$ null mice or when given in conjunction with $A_{2 a} R$ antagonists. We found that in the $A_{2 a} R$ null mice or in mice which received $A_{2 a} R$ antagonists, the efficacy of immunotherapy was greatly enhanced. Overall, our data implicate tissue derived adenosine as a novel mechanism for promoting peripheral $\mathrm{T}$ cell tolerance.

\section{Round table on Caffeine and Health}

\section{Caffeine and Headache: An Update}

\author{
Astrid Nehlig \\ INSERM U 666, Strasbourg, France \\ e-mail: nehlig@neurochem.u-strasbg.fr
}

The use of caffeine as an adjunctive constituent of analgesic medications dates back to 1875 while the first clinical trials were performed after 1950. The combination of caffeine with current prescription and nonprescription drugs in alleviating migraine and tension-type headache-related symptoms and pain has been the subject of a number of studies.

Caffeine has intrinsic antinociceptive actions in animal models. These are linked to its antagonism at the level of adenosine receptors. Adenosine A1 receptor activation is antinociceptive by decreasing and A2 receptor activation is pronociceptive by increasing cAMP levels in the sensory nerve terminals. Adenosine A3 receptor activation produces pain behaviors due to the release of histamine and serotonin from mast cells and subsequent actions on nerve cell terminals. The intrinsic antinociceptive actions of caffeine have been proposed to result from 
actions at supraspinal sites and may involve inhibition of presynaptic adenosine receptors on cholinergic nerve terminals. In addition, during headaches, adenosine receptor-mediated vasodilation and/or irregular vascular tone contribute to pain.

Based on a pooled analysis of 30 clinical studies including about 10,000 patients suffering from various types of pain including headaches, the combination of caffeine with current analgesics was reported to increase the analgesic effect of aspirin or acetaminophen by $41 \%$. Caffeine is well known to alleviate withdrawal-induced headache which occurs after the abrupt cessation of caffeine intake. This effect is strongly linked to the psychological satisfaction related to the ingestion of caffeine; this is especially true for the first cup of coffee in the morning. In tension-type headaches, the efficacy of the combination of caffeine with acetaminophen, aspirin or ibuprofen has been repeatedly reported both for pain intensity and total pain relief. In this type of headache, caffeine displays also analgesic properties when given alone. In migraine, the combination of acetaminophen, aspirin and caffeine is more effective than the placebo in alleviating pain and associated symptoms even in severely disabled migraineurs. However, in the latter type of headache, the studies do not allow to outline the specific role of caffeine in the drug combination tested since no comparison was performed with combinations containing or not caffeine or with caffeine alone. A recent work on migraine suggested to use a combination of products that would act on a whole subset of physiological regulations that are impaired during migraine attacks.

The role of cerebral blood flow changes in headaches is unclear, except in caffeine withdrawal-induced headaches. In the latter ones, cerebral blood flow velocities are increased and they are significantly decreased within $30 \mathrm{~min}$ after caffeine intake, suggesting that increased blood volume may be involved in caffeine withdrawal headache. In tension-type headache, there does not seem to be vascular changes related to the attack and for migraine attacks, the literature is rather in favor of a decrease in cerebral blood flow during the attacks. Thus, in these two latter headache types, caffeine does not seem to exert analgesia via its vasoconstrictive properties.

\title{
Caffeine effects on blood pressure and stress responses in persons at risk for developing hypertension
}

\author{
William R. Lovallo \\ University of Oklahoma and Veterans Affairs Medical Center, Oklahoma City, Oklahoma, USA \\ bill@mindbody1.org
}

Caffeine is the worlds most widely consumed pharmacologically active substance. A constituent in coffee, tea, and soft drinks, caffeine acts to block adenosine receptors, and it is capable of interacting with all levels of the stress axis [1]. This action has implications for a range of systemic functions. Our work has concentrated on the effect of caffeine on blood pressure regulation and cortisol secretion, with an emphasis on these responses in hypertension. At the blood vessel wall, caffeine blocks A2a receptors on the prejunctional sympathetic nerve ending, reducing the ability of adenosine to regulate release of norepinephrine onto alpha adrenoreceptors on the smooth muscle cell. This increases contractile state of vascular smooth muscle and causes a rise in peripheral vascular resistance, providing a physiological basis for caffeine's ability to elevate blood pressure. This receptor blockade causes a greater and longer lasting rise in vascular resistance and blood pressures in persons with above-normal pressures [2,3]. Caffeine also increases the secretion of the stress hormone cortisol, and this effect is also greater in persons at high risk of developing hypertension. The effect of caffeine on blood pressure and cortisol secretion is not affected by the amount of caffeine the person normally consumes in their diet, raising the question of the extent of tolerance development in regular consumers. Our study of tolerance to caffeine shows that most persons do not develop a complete tolerance to caffeine's effect on blood pressure or cortisol output. Examination of individual differences in tolerance shows that half of the persons tested did not show tolerance to a morning dose of $250 \mathrm{mg}$ of caffeine given in even when given a structured diet of $600 \mathrm{mg}$ of caffeine per day (equivalent to 6 cups of brewed coffee) for the previous five days. These effects call into question the long-term effects of caffeine on blood pressure regulation in the development and progression of hypertension.

1. al'Absi M, Lovallo WR. Caffeine effects on the human stress axis. In Nehlig A (ed): Coffee, Tea, Chocolate and The Brain. Boca Raton, FL: CRC Press LLC 2004; 113-31. 
2. Hartley TR, Sung BH, Pincomb GA et al. Hypertension risk status and effect of caffeine on blood pressure. Hypertension 2000; 36(1): 137-41.

3. Shepard JD, al'Absi M, Whitsett TL et al. Additive pressor effects of caffeine and stress in male medical students at risk for hypertension. Am J Hypertens 2000; 13(5 Pt 1): 475-81.

\section{The effects of caffeine on anxiety- and mood-related behaviors in mice}

Malika El Yacoubi, Jean Costentin, Jean-Marie Vaugeois

FRE 2735 CNRS, IFRMP 23, U.F.R. de Medecine \& Pharmacie, 22 Boulevard Gambetta, 76183 Rouen Cedex, France

malika.el-yacoubi@univ-rouen.fr; jean-marie.vaugeois@univ-rouen.fr

It is now well established that the effects exerted in the brain by caffeine depend on its ability to act as an antagonist at $A_{1}$ and $A_{2 A}$ receptors of the ubiquitous neuromodulator adenosine. Caffeine can cause anxiety symptoms, especially in individuals with pre-existing anxiety disorders. It has been claimed too that caffeine use may be also associated with symptoms of depression, supporting a self-medication theory. However the precise mechanism of action of the drug is unclear. The elevated plus-maze (EPM) and the light/dark box are established behavioral tests useful to screen putative anxiogenic/anxiolytic effects of drugs. We have previously [1] investigated whether the anxiogenic effects of caffeine observed in mice depend on the blockade of $\mathrm{A}_{2 \mathrm{~A}}$ receptor. Caffeine acutely administered (50 or $100 \mathrm{mg} / \mathrm{kg}$ IP) induced anxiety-like effects in both procedures. Its chronic administration $(50 \mathrm{mg} / \mathrm{kg}$ IP twice daily) for 1 week or consumption in the drinking water $(0.3 \mathrm{~g} / \mathrm{l})$ for 8 days or 2 months were also anxiogenic in the EPM. The $A_{2 A}$ receptor antagonists ZM241385 (up to $60 \mathrm{mg} / \mathrm{kg}$ IP) and SCH58261 (up to $10 \mathrm{mg} / \mathrm{kg}$ IP) were devoid of acute effects in both tests. One week administration of ZM241385 (30 mg/kg IP) or SCH58261 (3 mg/kg IP) had no effects in the EPM. An antagonist (DPCPX) and an agonist (CPA) at $A_{1}$ receptors had no acute effects on anxiety-related indices in our hands but anxiolytic effects of adenosine acting through $A_{1}$ receptors or of other $A_{1}$ receptor agonists were found in other experimental conditions. An $\mathrm{A}_{2 \mathrm{~A}}$ receptor agonist (CGS 21680) displayed non-specific motor effects in the EPM. Acute administration of caffeine $(50 \mathrm{mg} / \mathrm{kg} \mathrm{IP})$ induced no clear-cut anxiety-like effects in the EPM in $\mathrm{A}_{2 \mathrm{~A}}$ receptor knockout mice that exhibited higher basal anxiety levels than wild-type mice. Chronic administration $(50 \mathrm{mg} / \mathrm{kg}$ IP twice daily) for 1 week elicited less anxiety-like behavior in $\mathrm{A}_{2 \mathrm{~A}}$ receptor knockout than in wild-type mice. Thus, adaptive mechanisms following mutation in $\mathrm{A}_{2 \mathrm{~A}}$ receptors or their long-term blockade after chronic ingestion of caffeine may be responsible for increased proneness to anxiety. However, the short-term anxiety-like effect of caffeine in mice may not be related solely to the blockade of adenosine $\mathrm{A}_{2 \mathrm{~A}}$ receptors, since $\mathrm{A} 2 \mathrm{~A}$ selective antagonists do not share it. It may likely be dependent on blockade of $\mathrm{A}_{1}$ receptors, in accordance with the increased anxiety-like behavior seen in $A_{1}$ receptor knockout mice [2]. Adenosine and its analogues have been shown to induce "behavioral despair" in animal models believed to be relevant to depression. Selective adenosine $\mathrm{A}_{2 \mathrm{~A}}$ receptor antagonists (e.g., SCH 58261, ZM241385, and KW6002) or genetic inactivation of the receptor were effective in reversing signs of behavioral despair in the tail suspension test (TST) and forced swim test (FST) [3]. $\mathrm{A}_{2 \mathrm{~A}}$ antagonists were active in the TST using either mice previously screened for having high immobility scores or mice that were selectively bred for their spontaneous "helplessness" in this test. We hypothesized that the antidepressant-like effect of selective $\mathrm{A}_{2 \mathrm{~A}}$ antagonists is linked to an interaction with dopaminergic transmission, possibly in the frontal cortex. However this view of antidepressant-like effects induced by an antagonism at $\mathrm{A}_{2 \mathrm{~A}}$ receptors has been recently challenged. Caffeine was effective in the FST at stimulant doses but a clear-cut antidepressant-like effect could not be ascribed to it. In conclusion, data from mice show that caffeine i) can induce anxiety at high doses through $\mathrm{A}_{1}$ and possibly $\mathrm{A}_{2 \mathrm{~A}}$ receptors and ii) cannot be viewed as a typical antidepressant.

1. El Yacoubi M, Ledent C, Parmentier M et al. Psychopharmacology (Berl) 2000; 148: 153-63.

2. Gimenez-Llort L, Fernandez-Teruel A, Escorihuela RM et al. Eur J Neurosci 2002; 16: 547-50.

3. El Yacoubi M, Costentin J, Vaugeois JM. Neurology 2003; 61(11 Suppl 6): S82-7. 


\title{
Respiratory System
}

\section{Adenosine: A promising clinical indicator of inflammation in asthma}

\author{
Riccardo Polosa \\ Dipartimento di Medicina Interna e Specialistica Ascoli-Tomaselli Hospitals, University of Catania, Italy \\ polosa@unict.it
}

Interest in the role of adenosine in asthma has escalated considerably since the early observation of its powerful bronchoconstrictor effects in asthmatic but not normal airways. A growing body of evidence has emerged in support of a pro-inflammatory and immunomodulatory role for the purine nucleoside adenosine in the pathogenic mechanisms of chronic inflammatory disorders of the airways such as asthma. The fact that adenosine enhances mast cell allergen-dependent activation, that elevated levels of adenosine are present in chronically inflamed airways, and that adenosine given by inhalation cause dose-dependent bronchoconstriction in subjects with asthmaemphasizes the importance of adenosine in the initiation, persistence and progression of these common inflammatory disorders of the airways. These distinctive features of adenosine have been recently exploited in the clinical and research setting to identify innovative diagnostic applications for asthma. In addition, because adenosine exerts its multiple biological activities by interacting with four adenosine receptor subtypes, selective activation or blockade of these receptors may lead to the development of novel therapies for asthma.

\section{Coordinated Nucleotide Metabolism and Vascular Leak}

\author{
Sean P. Colgan ${ }^{1}$, Linda F. Thompson ${ }^{2}$, and Holger K. Eltzschig ${ }^{3}$ \\ ${ }^{1}$ Center for Experimental Therapeutics, Brigham and Women's Hospital and Harvard Medical School, Boston, \\ Massachusetts, USA \\ ${ }^{2}$ Immunobiology and Cancer Research Program Oklahoma Medical Research Foundation, Oklahoma City, Oklahoma \\ ${ }^{3}$ Department of Anesthesiology and Intensive Care Medicine, Tübingen University Hospital, D-72076 Tübingen, \\ Germany \\ colgan@zeus.bwh.harvard.edu
}

At sites of ongoing inflammation, polymorphonuclear leukocytes (PMN, neutrophils) migrate across barrier cell types including vascular endothelia and mucosal epithelia. Such transmigration has the potential to disturb barrier properties and can result in organ dysfunction. It is recently appreciated that endogenous pathways exist to dampen barrier disruption during transmigration and may provide an important anti-inflammatory link. For example, during transmigration, PMN-derived adenine nucleotides activate endothelial and epithelial cells and induce a cyclic AMP-dependent re-sealing of barrier cell types. In recent work, we have sought to understand the link between extracellular nucleotide metabolism and vascular barrier function. In particular, these studies have revealed that activated PMN release ATP, which through the action of extracellular nucleotidases is enzymatically cleaved to adenosine. Once liberated, adenosine binds surface A2 receptors to coordinate barrier protection. As part of this analysis, we have recently addressed inflammatory microenvironmental influences, such as hypoxia, on adenine nucleotide metabolism. These studies have identified an hypoxia-dependent, transcriptionally-mediated program which coordinates the elevation of extracellular adenosine. Target molecules in this program include hypoxia-inducible factor (HIF)-dependent induction of ecto-5'-nucleotidase (CD73) and adenosine $\mathrm{A} 2 \mathrm{~b}$ receptor (A2bR), as well as HIF-dependent repression of the dipyridamole-sensitive equilibrative nucleoside transporters (ENT1 and ENT2)1-3. These studies provide new insight and potential therapeutic targets for diseases in which tissue permeability may be abnormal.

1. Eltzschig HK, Abdulla P, Hoffman E et al. HIF-1-dependent repression of equilibrative nucleoside transporter (ENT) in hypoxia. J Exp Med 2005; 202: 1493-505. 
2. Eltzschig HK, Ibla JC, Furuta GT et al. Coordinated adenine nucleotide phosphohydrolysis and nucleoside signaling in posthypoxic endothelium: role of ectonucleotidases and adenosine A2B receptors. J Exp Med 2003; 198: 783-96.

3. Synnestvedt K, Furuta GT, Comerford KM et al. Ecto-5'-nucleotidase (CD73) regulation by hypoxia-inducible factor-1 (HIF-1) mediates permeability changes in intestinal epithelia. J Clin Invest 2002; 110: 993-1002.

\title{
Inflammatory role of adenosine in human lung cells
}

\author{
Dewan Zeng, Hongyan Zhong, and Luiz Belardinelli \\ Department of Pharmacological Sciences, CV Therapeutics, Inc., Palo Alto, California 94304 \\ dewan.zeng@cvt.com
}

It has been suggested that adenosine might have both pro- and anti-inflammatory actions depending on the pathophysiology of the diseases. In the lungs of asthmatics, inhaled adenosine or adenosine monophosphate (AMP) increases the release of histamine, tryptase and leukotrienes, suggesting a pro-inflammatory role of adenosine presumably due to the activation of adenosine receptors present in mast cells. The objective of our study was to determine the potential role of adenosine and its receptors in the human lung cells such as bronchial epithelial cells, airway smooth muscle cells and lung fibroblasts. Among the four subtypes of adenosine receptors $\left(A_{1}, A_{2 A}, A_{2 B}\right.$ and $\left.A_{3}\right)$, the $A_{2 B}$ receptor is expressed at the highest level in all three types of lung cells. In airway smooth muscle cells, activation of $A_{2 B}$ receptors leads to the release of IL-6 and MCP-1. Similarly, in lung fibroblasts, activation of $\mathrm{A}_{2 \mathrm{~B}}$ receptors increases the release of IL-6, which in turn promotes the differentiations of fibroblasts into myofibroblasts. In bronchial epithelial cells, activation of $A_{2 B}$ receptors increases the release of IL19 , which leads to the release of TNF- $\alpha$ from a monocytic cell line. Altogether, these results suggest that activation of $A_{2 B}$ receptors in the human lung cells increases the release of several pro-inflammatory cytokines and chemokines, which could lead to deleterious inflammatory responses in the lung.

\section{Role of adenosine receptors in regulation of pro-inflammatory and pro- angiogenic cytokines in mast cells}

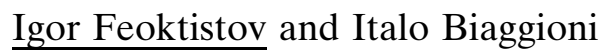

Vanderbilt University, Nashville, Tennessee 37232, USA

igor.feoktistov@vanderbilt.edu

Asthma is a chronic inflammatory disorder often accompanied by airway and vascular remodeling. Many of the pathologic features of asthma are attributed to the effects of mast cell-derived mediators. Adenosine is a powerful bronchoconstrictor of asthmatic, but not normal, airways. Growing lines of evidence, from early studies in isolated mast cells to more recent investigations in mice models, support a role of adenosine in Th2 and airway remodeling responses. Our own studies in this area suggested that the asthmatic response to adenosine is primarily mediated via the $\mathrm{A}_{2 \mathrm{~B}}$ receptors, which is selectively antagonized by enprofylline1. This work also provided evidence that adenosine is capable to regulate cytokine release from human mast cells. In recent studies, we showed that adenosine stimulates mast-dependent angiogenesis via cooperative interaction between $A_{2 B}$ and $A_{3}$ adenosine receptors. Activation of mast cell $\mathrm{A}_{2 \mathrm{~B}}$ adenosine receptors stimulates the synthesis of the pro-angiogenic factors VEGF and IL-8, whereas activation of $A_{3}$ receptor induces the expression of angiopoietin-2.2 Thus, adenosine can contribute to the vascular remodeling associated with asthma. In addition, adenosine can contribute to Th2 response through $\mathrm{A}_{2 \mathrm{~B}}$-mediated release of IL-4 and IL-13 from mast cells. The induction of IgE synthesis by the interaction between adenosine-stimulated mast cells and B-lymphocytes suggests that this mechanism is involved in the amplification of the allergic inflammatory responses associated with asthma3. The molecular mechanisms underlying this unique role of $\mathrm{A}_{2 \mathrm{~B}}$ adenosine receptors in promoting Th2 inflammation were explored in HMC-1 human mast cells. $\mathrm{A}_{2 \mathrm{~B}}$ receptors are coupled to Gs proteins linked to increase in cAMP, and are coupled to Gq to stimulate phospholipase $C \beta$. We found that $A_{2 B}$ receptors upregulate IL-4 through $\mathrm{Gq}$ signaling that is potentiated via crosstalk with Gs-coupled pathways. These data explain the presence and underscore the importance of dual coupling of $\mathrm{A}_{2 \mathrm{~B}}$ receptors to $\mathrm{Gs} / \mathrm{Gq}$ proteins with concurrent stimulation of diverse 
intracellular pathways for adenosine-dependent regulation of Th2 cytokines in human mast cells. These studies provide new insight and identify $A_{2 B}$ receptors as a potential therapeutic target for asthma.

1. Feoktistov I, Biaggioni I. Adenosine A2b receptors evoke interleukin-8 secretion in human mast cells. An enprofyllinesensitive mechanism with implications for asthma. J Clin Invest 1995; 96: 1979-86.

2. Feoktistov I, Ryzhov S, Goldstein AE, Biaggioni I. Mast cell-mediated stimulation of angiogenesis: cooperative interaction between A2B and A3 adenosine receptors. Circ Res 2003; 92: 485-92.

3. Ryzhov S, Goldstein AE, Matafonov A et al. Adenosine-activated mast cells induce IgE synthesis by B lymphocytes: an A2B-mediated process involving Th2 cytokines IL-4 and IL-13 with implications for asthma. J Immunol 2004; 172: 7726-33.

\section{Nervous System}

\section{Extracellular ATP mediates rapid retinal ganglion cell injury induced by intraocular pressure spikes}

Valentina Resta ${ }^{1}$, Elena Novelli ${ }^{1}$, Giovanni Vozzi $^{2}$, Cristiano Scarpa ${ }^{2}$, Matteo Caleo ${ }^{1}$, Arti Ahluwalia ${ }^{2}$, Anna Solini ${ }^{3}$, Eleonora Santini ${ }^{3}$, Vincenzo Parisi ${ }^{4}$, Francesco Di Virgilio ${ }^{5}$, Lucia Galli-Resta ${ }^{1}$

${ }^{1}$ Istituto di Neuroscienze CNR - Pisa, Italy,

${ }^{2}$ Centro E. Piaggio - Dept of. Electronic Engineering - University of Pisa, Italy,

${ }^{3}$ Dept. Internal Medicine, University of Pisa, Italy,

${ }^{4}$ G.B. Bietti Eye Foundation ONLUS and Eye Clinic, University of Rome Tor Vergata, Italy, ${ }^{5}$ Dept. Experimental and Diagnostic Medicine, Section General Pathology and Interdisciplinary Center for the Study of Inflammation (ICSI) - University of Ferrara, Italy

galli@in.cnr.it

Increased intraocular pressure (IOP) may lead to retinal ganglion cell (RGC) injury, and consequent visual deficits, as it is observed in glaucoma, a leading cause of blindness. Decades of studies have shown that chronic IOP alterations cause a number of detrimental effects, including mechanical damage of RGC axons, reduced blood supply, microglia activation and release of cytotoxic factors. Much less is known of the acute effects of pressure increments, mostly for the lack of adequate tools for this investigation.

Using a novel pressure incubator we have addressed this issue in isolated rat retinas, then extended our observations in vivo.

Imaging individual RGCs before and after pressure application we found that increasing pressure to $50 \mathrm{mmHg}$ for 1 min affects almost $30 \%$ of the RGCs within 1 hour. The percentage of damaged RGCs and the extent of cell injury increase when multiple pressure spikes are applied and pressure peaks are increased. However, even in the worst conditions, RGC damage is prevented by degrading extracellular ATP (eATP) with apyrase, or by blocking the P2X receptors with either oxidized ATP $(300 \mathrm{uM})$, or Brilliant Blue G $(0.5 \mathrm{uM})$.

In vivo, short IOP transients increase the levels of eATP in the eye fluids, damage RGCs within 1 hour, and impair RGC light-responses. Reducing eATP levels in the eye prevents RGC damage and shortens the impairment of light responses due to pressure.

These data show that pressure transients can injure RGCs within hours and demonstrates a causal link between eATP and such pressure-induced RGC damage. These findings may open the way to novel neuroprotective strategies in pathologies associated to high-pressure transients in the eye.

\section{$\mathbf{P 2}$ receptors in glial cells}

Maria P. Abbracchio

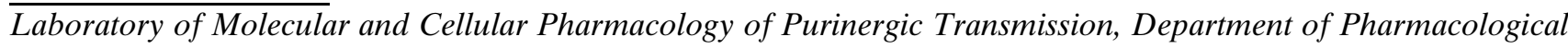
Sciences, University of Milan, Italy

mariapia.abbracchio@unimi.it 
All types of glial cells express P2 receptors which participate to nervous system function under both physiological and pathological conditions. In the CNS, ATP has been proposed as a main signaling molecule involved in shortterm, calcium-dependent cell-to-cell signaling [1,2]. ATP released from astrocytes activates P2 receptors on both astrocytes and other brain cells and generates a propagating wave of intracellular calcium increases allowing a homotypic and heterotypic signalling which also involves microglia, neurons and oligodendrocytes. Multiple P2X and P2Y receptors are expressed by both astrocytes and microglia [3-5]: however, these receptors are differentially recruited by nucleotides under specific patho-physiological conditions. $\mathrm{P} 2 \mathrm{Y}_{1,2,4}$ and, maybe, $\mathrm{P} 2 \mathrm{X}_{7}$ receptors, seem to be specifically recruited during calcium-dependent short-term signaling. Multiple P2 receptor subtypes (i.e., $\mathrm{P} 2 \mathrm{Y}_{1,2,6,12,13}$ and $\mathrm{P} 2 \mathrm{X}_{7}$ ) seem instead to cooperate to the long-term changes of astrocytes during inflammatory reactive gliosis. In these cells, P2 receptor-mediated gliosis occurs via activation of the ERK and protein kinase B/Akt pathways, and involves induction of both inflammatory and anti-inflammatory genes, cyclins, growth factors, adhesion and anti-apoptotic molecules. Conversely, genes involved in apoptotic cell death are down-regulated (for review, see: 6). Such a pattern of gene induction is consistent with the activation of neuroprotective mechanisms. Functional studies indeed suggest that, globally, the net effect of nucleotide-induced astrogliosis may be beneficial [6]. In microglial cells, exposure to inflammatory and immunological stimuli results in differential functional changes of distinct P2 receptor subtypes, suggesting that these receptors may play highly specific roles in the acquisition of the activated microglial phenotype [5,6]. On this basis, it is currently believed that nucleotide-induced activation of both astrocytes and microglia may originally start as an acute defense mechanism aimed at protecting neurons from cytotoxic and ischemic insults. Dysregulation of this process in chronic inflammatory diseases [7] eventually results in neuronal cell damage and loss. On this basis, the full elucidation of the specific roles of $\mathrm{P} 2$ receptors in these effects may help exploiting the beneficial neuroprotective features of activated glial cells while attenuating their harmful properties, and thus provide the basis for novel neuroprotective strategies which specifically target the purinergic system.

Partially supported by The Italian Ministry of University and Research, progetti FIRB2001 and FIRB2003, COFIN-MIUR2004 to MPA.

1. Haydon PG. Nat Rev Neurosci 2001; 2: 185-93.

2. Fields RD, Stevens B. Trends Neurosci 2000; 23: 625-33.

3. Fumagalli M, Brambilla R, D'Ambrosi N, Volonte C, Matteoli M, Verderio C, Abbracchio MP. Glia 2003; 43: $218-03$.

4. Fumagalli M, Trincavelli L, Lecca D, Martini C, Ciana P, Abbracchio MP. Biochem Pharmacol 2004; 68: 113-24.

5. Bianco F, Fumagalli M, Pravettoni E, D'Ambrosi N, Volonte C, Matteoli M, Abbracchio MP, Verderio C. Brain Res Brain Res Rev 2005; 48: 144-56.

6. Abbracchio MP, Ceruti S. Purinergic Signalling 2006, submitted for publication.

7. Marchetti B, Abbracchio MP. Trends Pharmacol Sci 2005; 26: 517-25.

\section{Presynaptic P2 receptors - interaction with other receptors and role in neuroprotection}

Lisiane O Porciúncula, Ricardo J Rodrigues, Paula M Canas, Teresa Almeida, Carla G. Silva, Jean P. Oses, A. Patrícia Simões, Catarina R Oliveira, Rodrigo A Cunha

Ctr. Neuroscience Coimbra, Fac.Medicine, Univ.Coimbra, Portugal racunha@ci.uc.pt

In the brain, ATP can either act as a neurotransmitter or neuromodulator or be involved in the communication with astrocytes or microglia. In hippocampal preparations, ATP is released in a calcium- and frequencydependent manner but its intensity-dependent release differs from that of classical transmitters (glutamate or acetylcholine, ACh). Also, ATP release was sensitive to L-type calcium channel blockers, in contrast to glutamate or ACh release. Thus, the pattern of ATP release seems to differ from that of classical neurotransmitters.

The investigation of the sub-synaptic localization of $\mathrm{P} 2$ receptors (P2Rs) revealed a robust immunoreactivity for $\mathrm{P} 2 \mathrm{X}_{1,2,3} \mathrm{Rs}$ and $\mathrm{P} 2 \mathrm{Y}_{1,2,4} \mathrm{Rs}$ in the presynaptic active zone of hippocampal terminals. Combined immunocytochemical and pharmacological characterization of glutamatergic terminals indicated a biphasic control of glutamate release by facilitatory $\mathrm{P} 2 \mathrm{X}_{1-3}$ Rs and inhibitory $\mathrm{P} 2 \mathrm{Y}_{1,2,4} \mathrm{Rs}$ in hippocampal terminals.

Not only P2Rs but also nicotinic ACh receptors (nAChRs), namely $\alpha 3^{*}$, but not $\alpha 4^{*}$, were enriched in hippocampal terminals. P2X ligands displaced binding to $\mathrm{AChRs}$ and $\mathrm{nAChR}$ ligands displaced binding to P2Rs. 
Glutamate or noradrenaline release from hippocampal synaptosomes was enhanced by epibatidine (10-300 nM, $\alpha$-conotonin MII-sensitive) or $\beta, \gamma$-imido ATP (3-60 $\mu \mathrm{M}$, prevented by NF-023 but not reactive blue-2) and, again, $\mathrm{P} 2 \mathrm{R}$ antagonists prevented $\mathrm{nAChR}$ responses and, conversely, nAChR antagonists prevented $\mathrm{P} 2 \mathrm{XR}$ responses. Also, $\mathrm{P} 2 \mathrm{X}_{2} \mathrm{Rs}$ co-immunoprecipitated with $\alpha 3$, but not $\alpha 4$ subunits, showing that $\mathrm{P} 2 \mathrm{X}_{1-3}$ and $\alpha 3 \beta 2 \mathrm{nAChRs}$ are physically linked and interact to control transmitter release.

Finally, we tested the role of P2Rs in the control of synaptotoxicity that is thought to predates neurotoxicity in neurodegenerative disorders. We tested if P2Rs controlled damage in cultured hippocampal neurons using two different noxious stimuli: 1) glutamate (100 $\mu \mathrm{M}$ for $25 \mathrm{~min})$, mimicking a general feature of excitotoxicity, causing $26 \pm 4 \%$ neuronal death $24 \mathrm{~h}$ after, and 2) $\mathrm{A} \beta_{1-42}(500 \mathrm{nM}, 48 \mathrm{~h})$, a causative factor of Alzheimer's disease, causing $22 \pm 3 \%$ neuronal death, which displayed apoptotic features (nuclear condensation, cytochrome c release, caspase 3 activation). This neurotoxicity was preceded by a synaptotoxicity (decreased synaptophysin immunoreactivity after $12 \mathrm{~h}$, when no neuronal death was observed) and dendritic atrophy (MAP-2 staining). Blockade of P2Rs (10 $\mu \mathrm{M}$ PPADS) or P2YRs (10 $\mu \mathrm{M}$ reactive blue 2$)$, but not P2X ${ }_{1-3}$ Rs $(10 \mu \mathrm{M}$ NF023 or TNP-ATP), prevented these neurotoxicity features. Notably, the selective $\mathrm{P} 2 \mathrm{Y}_{1} \mathrm{R}$ antagonist MRS2179 $(10 \mu \mathrm{M})$ was also neuroprotective. Furthermore, $\mathrm{P} 2 \mathrm{Y}_{1} \mathrm{R}$ immunoreactivity was enhanced in hippocampal terminals 15 days after $\mathrm{A} \beta_{1-42}(2 \mathrm{nmol}$, icv) administration, at a time where mice became amnesic (passive avoidance and Y maze tests) and displayed a loss of nerve terminals (loss of synaptophysin or SNAP-25 immunoreactivities) without neuronal loss (FluoroJade C staining). This indicates that extracellular ATP may be involved in the development of the synaptotoxicity and prompts the hypothesis that $\mathrm{P}_{2} \mathrm{Y}_{1} \mathrm{R}$ antagonists may be potential novel neuroprotective drugs. (Supported by Portuguese Society of Neuroscience, Pfizer and FCT).

\title{
Purinergic coordination of synaptic cross-talk mediated by astrocytes
}

\author{
Philip G. Haydon
}

Department of Neuroscience, Conte Center for Integration at the Tripartite Synapse, University of Pennsylvania School of Medicine, Philadelphia, PA 19104, USA

pghaydon@mail.med.upenn.edu

Though it is well known that there is a tonic accumulation of adenosine in the hippocampus which results in a suppression of synaptic transmission, there is little information regarding its source and control. Using inducible transgenic animals in which we used the tetracycline-regulated gene expression system to control the expression of transgenes selectively in astrocytes, we demonstrate that astrocytes control extracellular adenosine levels through the release of ATP(Pascual et al., 2005).

During the past decade it has become appreciated that astrocytes, the predominant glial cell type of the central nervous system, respond to neuronal activity with $\mathrm{Ca}^{2+}$ oscillations which induce the release of gliotransmitters that include glutamate, ATP and D-serine (Volterra and Meldolesi, 2005;Haydon, 2001). Our recent work has focused on the roles of these glial feedback pathways in the regulation of integration in the nervous system. Using a combination of imaging, electrophysiology and molecular genetics our studies indicate that astrocytes provide balanced excitation and inhibition to neurons through immediate actions of glutamate followed by delayed actions of accumulating adenosine. These gliotransmitters serve several functions that include the synchronization of neuronal activity, the suppression of synaptic transmission, as well as in mediating adenosine-dependent heterosynaptic depression (Pascual et al., 2005).

We generated inducible astrocyte specific transgenic animals which perturbed the release of ATP from these glial cells (Pascual et al., 2005). As a consequence we were able to identify critical roles for glial-derived purines in the regulation of synaptic transmission. We demonstrate that after releasing ATP this purine is hydrolysed to adenosine where it tonically suppresses excitatory synaptic transmission. As a consequence the dynamic range for synaptic plasticity (LTP) is enhanced. Additionally, in response to high frequency activity of presynaptic afferents, we have demonstrated that astrocytes releases ATP and cause a heterosynaptic suppression of neighboring unstimulated synapses that is also mediated through the actions of adenosine. Together these results allow us to conclude that astrocyte-derived purines coordinate synaptic networks by tonically regulate the strength of synaptic transmission, the range available for synaptic plasticity and heterosynaptic depression. 
1. Haydon PG. GLIA: listening and talking to the synapse. Nat Rev Neurosci 2001; Mar;2(3):185-93 2: $185-93$.

2. Pascual O, Casper KB, Kubera C, Zhang J, Revilla-Sanchez R, Sul JY, Takano H, Moss SJ, McCarthy K, Haydon PG. Astrocytic purinergic signaling coordinates synaptic networks. Science 2005; 310: 113-6.

3. Volterra A, Meldolesi J. Astrocytes, from brain glue to communication elements: the revolution continues. Nat Rev Neurosci 2005; 6: 626-40.

\title{
Purinergic signaling in regulating myelination by action potentials
}

\author{
R. Douglas Fields
}

Nervous System Development and Plasticity Section, National Institutes of Health, NICHD, Bethesda, MD, USA fieldsd@mail.nih.gov

Myelin is the membrane wrapping around axons that provides electrical insulation essential for rapid impulse conduction. Impulse activity can affect the formation of myelin, and this may be important in regulating nervous system structure and function according to environmental experience. Our studies performed on mouse dorsal root ganglion (DRG) neurons in cell culture show that impulse activity releases ATP from DRG axons, and that this is detected by different purinergic receptors on the two types of glia forming myelin in the PNS (Schwann cells) and CNS (oligodendrocytes). Both P1 and P2 receptors are involved in axon signaling to both types of myelinating glia, but ATP is of primary importance in regulating early development and myelination by Schwann cells, where it inhibits myelination; whereas adenosine is of primary importance in regulating early development of oligodendrocyte progenitor cells (OPCs), where it stimulates myelination. After OPCs mature, activitydependent release of ATP stimulates myelin formation through a different mechanism involving release of the cytokine LIF from astrocytes, which are non-myelin forming glia of the CNS. The activity-dependent release of ATP from axons provides a mechanism for regulating myelination in the central and peripheral nervous system, through both P1 and P2 signaling at different stages of development and in different types of glia.

\section{Cell Proliferation and Cancer}

\section{ATP: A new treatment modality in cancer?}

\author{
P.C. Dagnelie \\ Department of Epidemiology, Maastricht University, P.O. Box 616, 6200 MD Maastricht, The Netherlands \\ Email: Dagnelie@epid.unimaas.nl
}

Cancer is a devastating disease. Despite decades of research, the prognosis of many cancer types is still disappointingly poor. Adenosine 5'-triphosphate (ATP), given as intravenous infusions, may have several potential applications in cancer:

1. Inhibition of the cancer cachexia (weight loss) syndrome, improving fatigue and quality-of-life;

2. Attenuation of side effects of curative anti-cancer treatment;

3. Anti-tumour efficacy, either alone or in combination with current cancer treatments.

In this presentation, these three effects will be outlined and their prospects briefly discussed.

(1): So far, clinical experience with ATP treatment in cancer patients is limited. In 1995, following an uncontrolled phase I study in the US, we initiated a randomised clinical trial (RCT) with ATP infusions in cancer. ${ }^{1}$ A total of 58 patients with advanced non-small-cell lung cancer in the palliative treatment stage were randomised to ATP (continuous infusion for $30 \mathrm{~h}$, max. rate $75 \mathrm{ug} / \mathrm{kg} . \mathrm{min}$, frequency $1 \times / 2-4 \mathrm{wks}$ ) or control (palliative care only). Over a period of 6 months, ATP completely inhibited the loss in weight, fat mass, body cell mass, muscle strength and functional status (walking stairs, household activities, etc.) which were seen in the control group; also, ATP improved quality-of-life and fatigue.

The most intriguing finding was that ATP increased life expectancy from 3.5 to 9.2 months in a subgroup of cachectic lung cancer patients at a relatively early tumour stage (IIIB), Based on these results, further RCTs in 
cancer patients have been intiated. One such RCT (S. Beijer et al., presented at this meeting) is targeted at patients with mixed tumour types in the late palliative treatment stage cancer, and aims to improve weight, appetite, fatigue, physical disability and quality of life. ATP infusions in this protocol are applied in the home situation, which is feasible and safe provided a strict protocol is followed.

To (2/3) The strong favourable effect of ATP on life expectancy in lung cancer patients has triggered therapeutic studies, combining ATP with curative cancer treatment modalities such as radiotherapy and chemotherapy. At the same time, laboratory studies focus upon unravelling underlying mechanistic of the effects of ATP in cancer, especially the ATP-effects on therapy-induced inflammation and oxidative stress (Swennen et al., presented at this meeting).

We conclude that ATP holds promise for cancer treatment, not only for alleviating some of the devastating side effects of cancer, such as weight loss and fatigue, but also for increaing survival in selected patient groups. Moreover, ATP has potential to enhance the anti-tumour effects of current anti-tumour agents, while at the same time attenuating their side effects.

1. Dagnelie PC, Agteresch HJ. Drug Devel Res 2003; 59: 146-51 (7th Adenosine Meeting, Gold Coast, Australia).

2. Agteresch HJ, Burgers SA, van der Gaast A et al. Anti-Cancer Drugs 2003; 14: 639-44.

\section{Design, synthesis and antitumor activity of $4^{\prime}$-Thionucleosides as highly potent and selective $\mathbf{A}_{3}$ adenosine receptor agonists}

$\underline{\text { Lak Shin Jeong }}$

Laboratory of Medicinal Chemistry, College of Pharmacy, Ewha Womans University, Seoul 120-750, Korea

A number of ligands have been synthesized and tested for binding affinity at the rat, sheep, and human $\mathrm{A}_{3}$ versus $\mathrm{A}_{1}$ and $\mathrm{A}_{2 \mathrm{~A}}$ receptors. Among these ligands, IB-MECA (1) was found to be a highly potent human $\mathrm{A}_{3}$ agonist $\left(K_{\mathrm{i}}=1.8 \mathrm{nM}\right)$, which is 900 - and 1620 -fold selective for human $\mathrm{A}_{3}$ versus either human $\mathrm{A}_{1}$ or $\mathrm{A}_{2 \mathrm{~A}}$ receptors, respectively. Introduction of chlorine at the 2-position of IB-MECA, resulting in the formation of Cl-IB-MECA (2) also increased binding affinity and selectivity. Compound 2 has been reported to display a $K_{\mathrm{i}}$ value of $1.4 \mathrm{nM}$ and showed 900- and 3800-fold human $\mathrm{A}_{3}$ receptor selectivity versus human $\mathrm{A}_{1}$ and $\mathrm{A}_{2 \mathrm{~A}}$ receptors, respectively. Compounds $\mathbf{1}$ and $\mathbf{2}$ are in phase II clinical trials and preclinical trials as anticancer agents, respectively. Compound $\mathbf{2}$ is also used extensively as a pharmacological tool for studying $\mathrm{A}_{3}$ receptors.

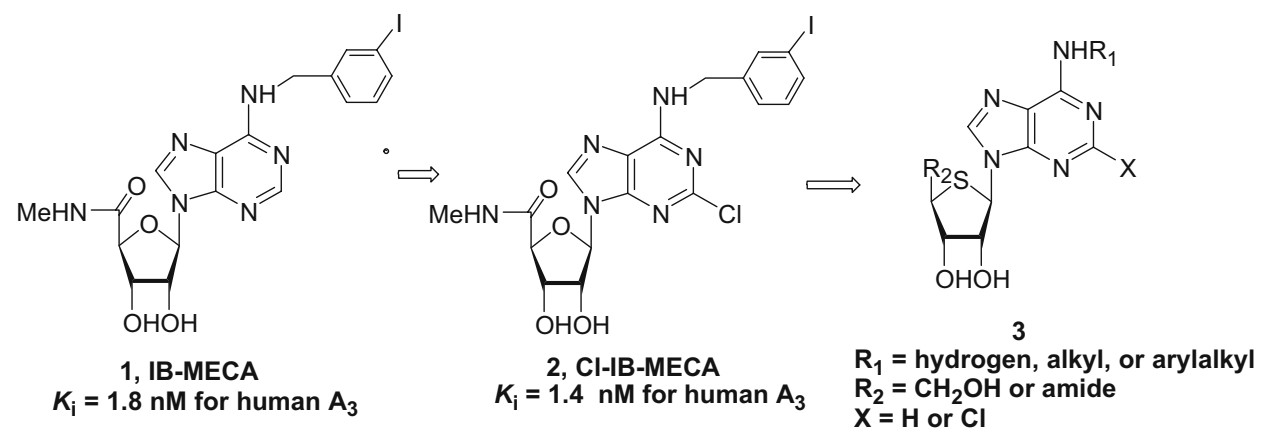

On the basis of high binding affinity and selectivity of $\mathbf{1}$ and $\mathbf{2}$ on human $\mathrm{A}_{3}$ adenosine receptors, we designed and synthesized the $4^{\prime}$-thio analogues $\mathbf{3}$, since a sulfur atom may serve as a bioisostere of an oxygen atom. The synthesized 4'-thionucleoside derivatives exhibited dramatic increases in affinity and selectivity for human $\mathrm{A}_{3}$ receptors versus human $\mathrm{A}_{1}$ and $\mathrm{A}_{2 \mathrm{~A}}$ receptors. Among compounds tested, 2-chloro- $N^{6}$-(3-iodobenzyl)- $4^{\prime}$ thioadenosine-5'-methyluronamide (Thio-Cl-IB-MECA) inhibited the growth of human promyelocytic leukemia HL-60 cells by arresting cell cycle and induction of apoptosis. The growth inhibitory activity of thio-Cl-IB-MECA 
was also found to be related with the modulation of Wnt signaling pathway by measuring the levels of $\beta$-catenin, phosphorylated forms of GSK- $\beta$ and Akt. Oral administration of Thio-Cl-IB-MECA exhibited highly potent antitumor activity in in vivo tumor xenograft model, indicating that it may be developed as a clinically useful anticancer agent.

Design, synthesis, and antitumor activity of the $4^{\prime}$-thionucleosides 3 as ultrapotent and selective agonists at the human $\mathrm{A}_{3}$ adenosine receptor will be presented in detail.

\title{
Growth regulation of tumor cells in hypoxia: Focus on $\mathbf{A}_{3}$ adenosine receptors
}

$\underline{\text { Stefania Merighi }}{ }^{1}$, Annalisa Benini ${ }^{1}$, Prisco Mirandola ${ }^{2}$, Stefania Gessi ${ }^{1}$, Katia Varani ${ }^{1}$, Edward Leung $^{3}$, Stephen Maclennan ${ }^{3}$, Pier Andrea Borea ${ }^{1,4}$

${ }^{1}$ Department of Clinical and Experimental Medicine, Pharmacology Unit; University of Ferrara, 44100, Ferrara, Italy

${ }^{2}$ Department of Anatomy, Pharmacology and Forensic Medicine, Human Anatomy Section, University of Parma, 43100, Parma, Italy

${ }^{3}$ King Pharmaceuticals Research \& Development, Cary, North Carolina 27513, USA

${ }^{4}$ Interdisciplinary Center for the Study of Inflammation; University of Ferrara, 44100, Ferrara, Italy. mhs@unife.it

Hypoxia appears to induce a program which shifts the cellular phenotype toward an increase in intracellular adenosine. Hypoxia-inducible factor-1 (HIF-1) is a key regulator of genes crucial to many aspects of cancer biology. Since the levels of both HIF-1 and adenosine are elevated within the hypoxic environment of solid tumors, we investigated whether adenosine may regulate HIF-1. In particular, we investigated the effect of $\mathrm{A}_{3}$ receptor antagonists on HIF-1, vascular endothelial growth factor and Angiopoietin-2 expression. Furthermore, this presentation will discuss new signaling pathways induced by $\mathrm{A}_{3}$ receptors in hypoxia and provide results of how human melanoma and glioblastoma tumor growth may be influenced through the adenosinergic system.

\section{Inhibition of hepatocellular carcinoma growth by an $\mathbf{A}_{3}$ adenosine receptor agonist: De-regulation of PKB/Akt-NF-kB signaling pathway}

\author{
Sara Bar-Yehuda, Avivit Ochaion, Shira Cohen, Faina Barer, Pnina Fishman
}

Can-Fite BioPharma, Petach Tikva, Israel

The $A_{3}$ adenosine receptor $\left(A_{3} A R\right)$ belongs to the family of the Gi-protein associated cell surface receptors and is highly expressed in various tumor cell types in comparison to the normal adjacent tissue. Lately it has been demonstrated that peripheral blood mononuclear cells (PBMNC) derived from colorectal patients also highly express $\mathrm{A}_{3} \mathrm{AR}$ in comparison to PBMNC derived from healthy volunteers. $\mathrm{A}_{3} \mathrm{AR}$ activation by the synthetic agonist suppress the development of melanoma, colon and prostate carcinoma in vitro and in vivo. The molecular mechanism is mediated via down-regulation of PKB/Akt which induces down-stream deregulation of the Wnt and the NF-kB signal transduction pathways.

In this study we followed $\mathrm{A}_{3} \mathrm{AR}$ expression in hepatocellular carcinoma (HCC) as well as in PBMNC both in human and in an animal model. The effect of an $\mathrm{A}_{3} \mathrm{AR}$ agonist administration on $\mathrm{A}_{3} \mathrm{AR}$ expression level, tumor development and signal transduction pathways involved were studied.

Utilizing paraffin embedded slides derived from human $\mathrm{HCC}$ samples, it was demonstrated that $\mathrm{A}_{3} \mathrm{AR}$ is highly expressed in HCC tumor tissues, in comparison to the normal adjacent tissue. The high expression of the $\mathrm{A}_{3} \mathrm{AR}$ in the tumor tissue was reflected in PBMNC derived from HCC patients. In addition, in tumor tissues as well as in PBMNC derived from N1S1 hepatoma bearing rats high $\mathrm{A}_{3} \mathrm{AR}$ expression level was noted in comparison to normal liver tissues. The expression level of the receptor was down-regulation upon oral treatment 
with the $\mathrm{A}_{3} \mathrm{AR}$ agonist Cl-IB-MECA (designated as CF102). These events were followed by a decreased levels of

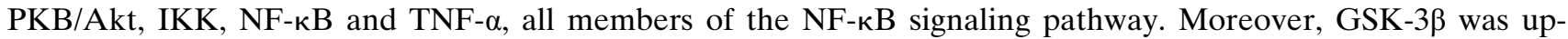
regulated while $\beta$-catenin, Lef/Tcf and c-Myc expression level was down-regulated, demonstrating that deregulation of the Wnt signaling pathway took place. As a result, a marked inhibiting in the growth of the N1S1 tumors was observed in the CF102 treated animals.

Taken together we conclude that the $\mathrm{A}_{3} \mathrm{AR}$ may be developed as a new surrogate marker. In addition the receptor may be suggested as target to be activated by synthetic small and orally bioavailable agonists which inhibits HCC growth.

\title{
P2X : A growth-promoting receptor
}

Francesco Di Virgilio

Department of Experimental and Diagnostic Medicine, Section of General Pathology, University of Ferrara fdv@unife.it

$\mathrm{P} 2 \mathrm{X}_{7}$ is a receptor for extracellular nucleotides expressed by different normal cell types. Activation of P2X7 may result in stimulation of cell proliferation or induction of apoptosis, depending on the level of activation. We have previously correlated the level of $\mathrm{P} 2 \mathrm{X}_{7}$ expression to disease severity in lymphocytes from patients affected by chronic lymphocytic leukemia (1). Furthermore, we have recently shown that expression of this receptor allows growth in the absence of serum (2). This growth-promoting effect appears to be mediated by three main factors: a) increased cytoplasmic $\mathrm{Ca}^{2+}$ levels; b) increased mitochondrial potential; c) enhanced ATP synthesis. P2 $\mathrm{X}_{7^{-}}$ expressing cells also release larger amounts of ATP into the pericellular space. We have found that tumours, among which human neuroblastoma, express a higher level of $\mathrm{P} 2 \mathrm{X}_{7}$ compared to non-tumour cells (3). Growthpromotion by $\mathrm{P} 2 \mathrm{X}_{7}$ may be mediated via additional mechanisms involving release of growth stimulatory factors among which substance $\mathrm{P}$ and ATP itself.

This novel findings suggest that overstressing the well known cytotoxic activity of this receptor may be limitative and in certain conditions even misleading.

1. Adinolfi E, Melchiorri M, Falzoni S et al. P2X7 receptor expression in evolutive and indolent forms of B lymphocytic leukemia. Blood 2002; 99: 706-8.

2. Adinolfi E, Callegari MG, Ferrari D et al. Basal activation of the P2X7 ATP receptor elevates mitochondrial calcium and potential, increases cellular ATP levels, and promotes serum-independent growth. Mol Biol Cell 2005; 16: 3260-72.

3. Raffaghello L, Chiozzi P, Falzoni S et al. The P2X7 receptor sustains the growth of human neuroblastoma cells through a substance P-dependent mechanism. Cancer Res 2006; 66; 907-14.

\section{Regulation of astrocyte proliferation by P2Y and P2X purinergic receptors}

\author{
Joseph T. Neary, Yuan Kang, and You-Fang Shi
}

Research Service, Miami VA Healthcare System, Departments of Pathology, Biochemistry \& Molecular Biology, and Neuroscience Program, University of Miami Miller School of Medicine, Miami, Florida 33125, USA

jneary@med.miami.edu

Extracellular ATP enhances the mitogenic activity of fibroblast growth factor-2 (FGF2) in astrocytes ${ }^{1}$, but the molecular mechanism(s) and the P2 purinergic receptor type(s) involved are not well understood. As one approach to determine whether the potentiating effect of extracellular ATP involves cell cycle control mechanisms, we measured the expression of cyclins that are induced in different phases of the cell cycle in primary cultures of rat cortical astrocytes ${ }^{2}$. ATP potentiated the ability of FGF2 to stimulate expression of cyclin D1, a regulator of cell cycle entry, as well as cyclin A, a regulator of DNA replication. The potentiating effect of ATP on cyclin expression was attenuated by inhibiting activation of extracellular signal regulated protein kinase 
(ERK) which is coupled to FGF2 and P2 receptors. P2 agonist studies revealed that UTP enhanced FGF2-induced cyclin expression and mitogenesis whereas 2-methylthio ADP was ineffective, thereby suggesting a role for P2Y2 or P2Y4 receptors in the potentiation of astrocyte proliferation induced by FGF2. In contrast to the effect of UTP, we found that an agonist of P2X receptors, $2^{\prime}, 3^{\prime}$-O-(4-benzoyl)benzoyl-ATP (BzATP), inhibited FGF2induced mitogenesis by $90 \%$. This effect was not explained by a cytotoxic response to BzATP because at least $95 \%$ of astrocytes treated with BzATP remained viable. BzATP is an agonist of P2X7 as well as P2X1 and P2X3 receptors, but $\alpha, \beta$-methylene ATP (in the media free of phenol red, an antagonist of P2X1 and P2X3 receptors ${ }^{3}$ ) did not inhibit FGF2-induced mitogenesis. In addition, dose-response studies demonstrated that 300 to 1000 micromolar ATP reduced the ability of FGF2 to stimulate DNA synthesis. These results indicate that P2X7 receptors mediate growth arrest in astrocytes. Consistent with opposing effects of $\mathrm{P} 2 \mathrm{Y}$ and $\mathrm{P} 2 \mathrm{X}$ receptors on mitogenesis, UTP stimulated a transient activation of ERK whereas BzATP stimulated a more sustained ERK signal. In addition, phospho-histone 3, a marker of mitosis during $\mathrm{M}$ phase of the cell cycle, was decreased by BzATP whereas UTP increased levels of phospho-histone 3 induced by FGF2. Collectively, these findings suggest that signaling by P2Y2/4 receptors enhance the ability of FGF2 to stimulate entry into a new cell cycle, DNA replication and mitosis by an ERK-dependent mechanism, whereas signaling by P2X7 receptors inhibits FGF2induced mitogenesis in astrocytes and leads to growth arrest. Interactions between P2Y, P2X and polypeptide growth factor signaling pathways may have important implications for CNS development as well as injury and repair. [This work was supported by the National Institutes of Health (NS046651) and the Department of Veterans Affairs.]

1. Neary JT, Whittemore SR, Zhu Q, Norenberg MD. Synergistic activation of DNA synthesis in astrocytes by fibroblast growth factors and extracellular ATP. J Neurochem 1994; 63: 490-4.

2. Neary JT, Kang Y, Shi YF. Cell cycle regulation of astrocytes by extracellular nucleotides and fibroblast growth factor-2. Purinergic Signaling 2005; 1: 329-36.

3. King BF, Liu M, Townsend-Nicholson A et al. Antagonism of ATP responses at P2X receptor subtypes by the pH indicator dye, Phenol red. Br J Pharmacol 145: 313-22, 2005.

\title{
Targeting the $\mathbf{A}_{3}$ adenosine receptor to combat cancer and autoimmune inflammatory diseases
}

\author{
Pnina Fishman, Avivit Ochaion, Shira Cohen, Faina Barer, and Sara Bar-Yehuda \\ Can-Fite BioPharma, Petach Tikva, Israel
}

CF101 is a small molecule adenosine derivative which binds specifically to the $A_{3}$ adenosine receptor $\left(A_{3} A R\right)$. The latter is abundantly expressed on the surface of cancer and inflammatory cells whereas only low expression was found in normal cells. Activation of this receptor de-regulates key intracellular signaling pathways and inhibits the proliferation of cancer and inflammatory cells. We have built a drug development platform around this novel target and utilized synthetic $\mathrm{A}_{3} \mathrm{AR}$ agonists, with high affinity to the receptor, to treat cancer and inflammatory diseases.

CF101 inhibited the growth of melanoma, colon prostate and pancreatic carcinoma tumors in experimental mice models when given daily orally. The molecular mechanism involves de-regulation of the Wnt and the NF-kB signal transduction pathway, i.e., downregulation of PKA and PKB/Akt and up-regulation of GSK-3 $\beta$ which subsequently phosphorylates $\beta$-catenin leading to its ubiquitination. As a result, a decrease in the level of the 2 cell cycle progression genes, cyclin D1 and c-Myc takes place.

CF101 inhibits the clinical and pathological manifestations of adjuvant induced arthritis in rats and collagen induced arthritis in DBA mice. On the molecular level, CF101 deregulates the PI3K-PKB/Akt-NF-kB pathway thus leading to the inhibition of TNF- $\alpha$ and the induction of apoptosis of inflammatory cells. CF101 also showed efficacy as a disease modifier in additional experimental inflammatory models including colitis and multiple sclerosis.

CF101 is an orally administered molecule with excellent safety profile and specific activity against targeted pathological cells. This molecule is being tested in several clinical trials, including an early PhII study in patients 
with active rheumatoid arthritis. A recent interim analysis of this trial demonstrated provocative evidence of therapeutic activity and an excellent safety profile.

\title{
Pain and Nerve Transmission
}

\section{Adenosine receptors in astrocytes, cytokines and neuroprotection}

\author{
$\underline{\text { Knut Biber }}$ \\ $\overline{\text { Department }}$ of Medical Physiology, University Medical Center Groningen, University of Groningen, The \\ Netherlands \\ K.Biber@med.umcg.nl
}

High concentrations of adenosine are released in the brain under neuropathological conditions. Recent evidence suggest that stimulation of adenosine $\left(\mathrm{A}_{1}, \mathrm{~A}_{2 \mathrm{~B}}\right.$ or $\left.\mathrm{A}_{3}\right)$ receptors in glial cells induces the release of neuroprotective substances, indicating that also adenosine receptors in glia cells contribute to adenosinedependent neuroprotection. The release of NGF in astrocytes for example is regulated by adenosine $\mathrm{A}_{1}$ receptor activation, whereas other adenosine receptors are involved in astrocytic release of neuroprotective cytokines. We have recently provided evidence that stimulation of adenosine $A_{3}$ receptors in cultured astrocytes stimulates the release of the neuroprotective chemokine CCL2 (formerly known as MCP-1). This is based on pharmacological evidence since the selective adenosine $\mathrm{A}_{3}$ receptor agonist 2-chloro-N6-(3-iodobenzyl)-N-methyl-5'-carbamoyladenosine (CL-IB-MECA) induced the release of CCL2, whereas specific ligands for adenosine $A_{1}$ or $A_{2}$ receptors did not affect CCL2 release. Furthermore, CL-IB-MECA-induced CCL2 synthesis was inhibited by adenosine $\mathrm{A}_{3}$ receptor antagonists [4]. Earlier studies of our group have shown that stimulation of astrocytic adenosine $\mathrm{A}_{2 \mathrm{~B}}$ receptor is instrumental for the release of IL-6, a cytokine with well-known neuroprotective properties [1,2]. Thus these data demonstrate that various adenosine receptor subtypes in astrocytes are responsible for the synthesis of different neuroprotective agents. Since these receptor subtypes display different affinities for adenosine it is suggested that depending on the concentration of extracellular adenosine specific patterns of neuroprotective substances are synthesized by astrocytes [3].

How cytokines like CCL2 or IL-6 protect neurons is not understood yet. We have recently obtained evidence that might explain the neuroprotective properties of IL-6. It is presented here that IL-6 enhances expression and function of neuronal adenosine $\mathrm{A}_{1}$ receptors in vitro and in vivo. It is therefore suggested that IL-6 increases neuronal survival in vitro and in vivo by enhancing the function of neuronal adenosine $\mathrm{A}_{1}$ receptors, the brains major protective system. The data presented here thus indicate a tight interplay between cytokines and the adenosinergic system in astrocytes and neurons with major impact in neuroprotection.

1. Fiebich BL, Biber K, Gyufko K et al. J Neurochem 1996; 66: 1426-31.

2. Fiebich BL, Akundi RS, Biber K et al. Neurochem 2005; 46: 501-12.

3. Van Calker D, Biber K. Neurochem Res 2005; 30: 1205-17.

4. Wittendorp MC, Boddeke HWGM, Biber K. GLIA 2004; 46: 410-8.

\section{Adenosine regulation of neurotrophin synaptic actions}

Ana M. Sebastião, M. José Diógenes, Paula I. Pousinha, Catarina C. Fernandes, António Pinto-Duarte, Catarina A. Gomes, Sandra H. Vaz and J. Alexandre Ribeiro

Institute of Pharmacology and Neurosciences, Faculty of Medicine and Institute of Molecular Medicine, University of Lisboa, Portugal

ATP and neurotrophins, in particular Brain Derived Neurotrophic factor (BDNF) have been implicated in generation and modulation of pain (e.g. Coull et al., 2005 - Nature 438: 1017-21). In contrast, adenosine has significant potential for alleviating various types of pain (see e.g. Hayashida et al., 2005 - J Anesth 19: 225-35). 
BDNF-induced long lasting changes in synaptic efficacy in the supraspinal descending pathway have been pointed out as one of the causes for BDNF-related development of persistent pain (Guo et al., 2006 - J Neurosci 26: 12637). BDNF-induced changes in synaptic activity and efficacy also occur in other areas of the central nervous system, including hippocampus (e.g. Braham and Messaoudi, 2005 - Prog Neurobiol 76: 99-125). Synaptic actions of BDNF are facilitated by enhanced neuronal activity (see Nagappan and Lu, 2005 - Trends Neurosci 28: 46471), depolarization (Boulanger and Poo, 1996 - Nature Neurosci 2: 346-51) and cyclic AMP (Boulanger and Poo, 1996 - Science 284: 1982-4). Depolarization induces the release of adenosine (e.g. Latini and Pedata, 2001 - J. Neurochem 79: 463-84) and enhanced neuronal activity facilitates adenosine $\mathrm{A}_{2 \mathrm{~A}}$ receptor mediated actions (Correia-de-Sá et al., 2000 - J. Neurochem 74: 2462-9). Since adenosine $A_{2 A}$ receptors are coupled to cyclic AMP and since activation of these receptors can induce transactivation of BDNF TrkB receptors (Lee and Chao, 2001 Proc Natl Acad Sci 98: 3555-60) we evaluated how activation of adenosine $\mathrm{A}_{2 \mathrm{~A}}$ receptors could influence synaptic actions of BDNF.

We observed that $A_{2 A}$ receptor activation by released adenosine is an essential pre-requisite to observe an effect of BDNF upon excitatory synaptic transmission in the CA1 area of the hippocampus (Diógenes et al. $2004-$ J Neurosci 24: 2905-13). The BDNF-induced enhancement of the release of acetylcholine at the hippocampus (Fernandes et al., Program No. 157.17. Abstract Viewer. SfN, 2005) and the BDNF-induced enhancement of neuromuscular transmission at the innervated phrenic-diaphragm (Pousinha et al., 2006 - Br. J. Pharmacol, proc sup, in the press) also require tonic activation of adenosine $A_{2 A}$ receptors. Triggering $B D N F$ actions by $A_{2 A}$ receptor activation involves cyclic AMP and protein kinase A (Diógenes et al., 2004). BDNF inhibits GABA uptake from hippocampal nerve terminals but this action is independent of $\mathrm{A}_{2 \mathrm{~A}}$ receptor activation ( $\mathrm{Vaz}$ et al., $\mathrm{Br}$ J. Pharmacol, Proc suppl, in the press). In the striatum, another neurotrophin, glial cell line-derived neurotrophic factor (GDNF) enhances the release of dopamine and this action is also modulated by $\mathrm{A}_{2 \mathrm{~A}}$ receptor activation (Gomes et al. 2005, J. Neurochem., 94, Supl 2, 264).

The above results clearly show that $\mathrm{A}_{2 \mathrm{~A}}$ receptors trigger synaptic actions of neurotrophins. Whether $\mathrm{A}_{2 \mathrm{~A}}$ receptors also facilitate $\mathrm{BDNF}$-induced enhancement of pain mechanisms and whether $\mathrm{A}_{2 \mathrm{~A}}$ receptor mediated inhibition of adenosine $\mathrm{A}_{1}$ receptor functioning (Cunha et al., 1994 - Brain Res 649: 208-16) and of $\mathrm{A}_{1}$ tonic inhibitory actions (Pinto-Duarte et al., 2005 - J. Neurochem 93: 595-604) may influence adenosine actions on pain, deserves further investigation.

Work supported by Fundação para a Ciência e Tecnologia, Portugal. BDNF was a gift by Regeneron.

\title{
Expression, function and modulation of $\mathrm{P}^{2} \mathrm{X}_{3}$ receptors on trigeminal ganglion nociceptors
}

\author{
A. Nistri, E. Fabbretti, A. Fabbro, M. D'Arco, M. Simonetti and R. Giniatullin \\ Neurobiology Sector, International School for Advanced Studies (SISSA), Trieste, Italy \\ nistri@sissa.it.
}

Painful stimuli from head tissues are transmitted to the brainstem via certain populations of trigeminal ganglion (TG) neuron. Such neurons can be activated by a wide range of signals which comprise locally released substances like ATP, or acid $\mathrm{pH}$, mechanical stimulation etc. In chronic pain states including migraine, it is suggested that there is a process of sensitization of trigeminal neurons which become hyper-responsive to stimuli. In the attempt to understand the molecular mechanisms responsible for chronic pain sensitization, we developed a primary culture of rat or mouse TG neurons to test the role of various pain mediators over an extended time period. Cultures were prepared from TGs of 2 week old animals and grown for 1-4 days in vitro without adding exogenous NGF. The first issue was to characterize the neuronal population in culture and to compare it with the one normally found in ganglia in situ. The focus of this study was on ATP-sensitive P2X receptors and capsaicinsensitive TRPV1 receptors: both classes are important transducers of nociception on sensory neurons.

Rat or mouse $\beta$-tubulinIII positive neurons demonstrated good viability in vitro with $<10 \%$ of them labeled against the early markers of apoptosis JNK or activated caspase3. Neurons were predominantly of small and medium diameter like those found in ganglia. Real time RT-PCR and Western blot analysis showed upregulation of $\mathrm{P} 2 \mathrm{X}_{3}$ and TRPV1 receptors in culture versus ganglia, while $\mathrm{P} 2 \mathrm{X}_{2}$ ones were unchanged. The number of neurons immunoreactive to $\mathrm{P}_{2} \mathrm{X}_{3}$ and TRPV1 receptors grew with more frequent co-localization. TRPV1 immunoreactivity was, however, comparatively low in mouse ganglia and cultures. $\mathrm{Ca}^{2+}$ imaging and whole-cell 
patch clamping showed functional P2X and TRPV1 receptors. The commonest effect was a fast desensitizing response mediated by $\mathrm{P} 2 \mathrm{X}_{3}$ receptor activity and antagonized by the selective blocker A-317491. More rat than mouse neurons generated mixed responses indicative of more efficient heteromerization of $\mathrm{P} 2 \mathrm{X}_{3}$ and $\mathrm{P} 2 \mathrm{X}_{2}$ subunits. We next tested if algogenic substances could modify P2X 3 or TRPV1 receptors in culture. Twentyfour $\mathrm{h}$ application of NGF $(50 \mathrm{ng} / \mathrm{ml})$ selectively upregulated $\mathrm{P}_{2} \mathrm{X}_{3}$ receptors without affecting TRPV1 receptors, while $10 \mu \mathrm{M}$ serotonin application $(24 \mathrm{~h})$ largely potentiated TRPV1 function without changing $\mathrm{P}_{2} \mathrm{X}_{3}$ receptor activity.

These data show that TG cultures allow studying early adaptive changes of nociception-transducing receptors including their selective modulation by algogenic substances. This work is supported by grants from Telethon Foundation (GGP 04037) and MIUR (FIRB).

\title{
Functional consequences of $\mathbf{P}^{2} X_{7}$ activation in astrocytes and microglial cells
}

\author{
F. Bianco, A. Colombo, R. Mele, M. Matteoli and C. Verderio \\ CNR-Institute of Neuroscience, University of Milano Department of Medical Pharmacology, Center of Excellence \\ on Neurodegenerative Diseases, Milano, Italy \\ c.verderio@in.cnr.it
}

Formation and shedding of vesicles from the plasmamembrane commonly occur in haematopoietic and immune cells and represents a process whereby signal molecules are released into the microenvironment. We have recently shown, in microglial cells, that ATP stimulation controls vesicle shedding through the activation of $\mathrm{P}_{2} \mathrm{X}_{7}$ receptors and that shed vesicles represent a pathway for the release of the pro-inflammatory cytokine IL1-beta. Isolation of shed vesicles, followed by IL-1 beta evaluation by a specific ELISA revealed the presence of the cytokine inside the vesicular organelles and its subsequent efflux into the extracellular medium. Western blot analysis revealed the presence of $\mathrm{P} 2 \mathrm{X}_{7}$ receptors, pro- IL-1 beta and pro-caspase- 1 in isolated vesicles, solubilized immediately after isolation. Processed bands of both IL-1 beta and caspase- 1 were detected in vesicles exposed 30 min to ATP before lysis, suggesting that shed vesicles are the site of IL1-beta processing. ATP-binding cassette (ABC) transporters, a large family of proteins whose role is to translocate various substances across biological membranes, mediate the efflux of IL1-beta from shed vesicles, as cytokine release was suppressed by the general $\mathrm{ABC}$ transporter inhibitor glibenclamide. Since shedding also occurs in resting microglia not expressing IL1-beta, it may represent a cargo system for the release of other signalling molecules from these cells. For these reasons, we are carrying out a biochemical characterization of isolated vesicles, to further characterize the protein composition of shed vesicles and investigate the role and function of these organelles.

\section{Intracellular signaling underlying ATP-induced chemotaxis of microglia}

\author{
K. Ohsawa ${ }^{1}$, Y. Irino ${ }^{1}$, Y. Nakamura ${ }^{1}$, C. Akazawa ${ }^{1}$, K. Inoue ${ }^{2}$, and S. Kohsaka ${ }^{1}$ \\ ${ }^{1}$ Department of Neurochem., Natl. Inst. Neurosci., Tokyo, Japan \\ ${ }^{2}$ Department of Pharmcol., Grad. Sch. Pharm. Sci., Kyushu Univ., Fukuoka, Japan \\ kohsaka@ncnp.go.jp
}

Resident microglia exhibit ramified shapes in the normal brain, however, in response to pathological stimuli, they transform rapidly themselves into more motile amoeboid form called activated microglia and migrate toward the lesioned sites, where the accumulating microglia secret a variety of substances [1] to repair the tissues. Thus, microglial cell migration is an important initial step of amelioration of the damaged nervous system.

Extracellular ATP is known to regulate physiological functions of microglia [2]. Microglia possesses both ionotropic P2X receptors (P2X4R and P2X7R) and G-protein-coupled P2Y receptors (P2Y2R, P2Y6R and $\mathrm{P} 2 \mathrm{Y} 12 \mathrm{R})$. We previously showed that ATP induced membrane ruffling and chemotaxis of microglia and suggested that the membrane ruffling is mediated by Gi/o-protein-coupled P2Y12R [3, 4]. We showed here that the ATPinduced chemotaxis of microglia is also regulated by the ionotropic receptor, P2X4R in addition to the P2Y12R.

Stimulation of G-protein-coupled receptor lead to activation of phospholipase C (PLC) and phosphoinositide 3-kinase (PI3K). We examined the effect of PLC and PI3K inhibitors on the formation of membrane ruffling and the chemotaxis of microglia following the stimulation by ATP. A PLC inhibitor inhibited both membrane ruffling 
and chemotaxis, while PI3K inhibitors suppressed only chemotaxis without inhibiting the membrane ruffling. These observations indicate that PLC activation is essential for both of the membrane ruffling and the chemotaxis, while the activation of PI3K is necessary only for the chemotaxis. Phosphorylation of Akt, which is known to be a downstream target of PI3K pathway, was enhanced by ATP stimulation. The increase in Akt phosphorylation was suppressed by chelating extracellular calcium. These results indicate that activation of PI3K pathway is modulated by the extracellular calcium influx suggesting a possibility that ionotropic P2XRs are involved in the PI3K activation. Therefore, we examined the effect of various P2XRs antagonists on the ATP-induced chemotaxis of microglia. TNP-ATP significantly inhibited the chemotaxis, but neither PPADS nor BBG were effective. Furthermore, we constructed the lentivirus vector expressing short hairpin RNAi against P2X4R and introduced the vector into microglia and showed that suppression of P2X4R reduced the ATP-induced chemotaxis of the cells. These results clearly indicate that P2X4R in addition to P2Y12R are involved in the ATP-induced chemotaxis of microglia.

1. Kreutzberg GW. Trends Neurosci 1996; 19: 312-8.

2. Inoue K. Glia 2002; 40; 156-63.

3. Honda S, Sasaki Y, Ohsawa K et al. J Neurosci 2001; 21: 1975-82.

4. Sasaki Y, Hoshi M, Akazawa C et al. Glia 2003; 44: 242-50.

\title{
P2X4-dependent neuropathic pain: A mechanism of the facilitation in pain transmission via microglia
}

Kazuhide Inoue and Makoto Tsuda

Department of Molecular and System Pharmacology, Graduate School of Pharmaceutical Sciences, Kyushu University, 3-1-1 Maidashi, Higashi, Fukuoka 812-8582, Japan

inoue@phar.kyushu-u.ac.jp

There is abundant evidence that extracellular ATP and other nucleotides have an important role in pain signaling at both the periphery and in the CNS. The focus of attention now is on the possibility that endogenous ATP and its receptor system might be involved in neuropathic pain. Neuropathic pain is often a consequence of nerve injury through surgery, bone compression, diabetes or infection. This type of pain can be so severe that even light touching can be intensely painful; unfortunately, this state is generally resistant to currently available treatments. We recently reported that the expression of P2X 4 receptors in the spinal cord is enhanced in spinal microglia after peripheral nerve injury, and blocking pharmacologically and suppressing molecularly P2X4 receptors produce a reduction of the neuropathic pain behaviour (Nature 2003; 424: 778-83). More recently, we have reported that brain-derived neurotrophic factor (BDNF) released from microglia by the stimulation of P2X4 causes the depolarizing shift in reversal potential of anion in LI neurons of rats with nerve injury (Nature 438, 1017-21, 2005), resulting in causing neuropathic pain. Understanding the key roles of these ATP receptors may lead to new strategies for the management of intractable chronic pain.

\section{The Role of P2X7 and P2X4 in pain processing; common or divergent pathways?}

\author{
$\underline{\text { I.P. Chessell }}^{1}$, J.P. Hatcher ${ }^{1}$, J.P. Hughes ${ }^{2}$, L Ulmann ${ }^{3}$, P. Green ${ }^{2}$, P.K. Mander ${ }^{2}$, A.J. Reeve ${ }^{1}$ and F.A. \\ Rassendren $^{3}$ \\ ${ }^{1}$ Pain Research, GlaxoSmithKline, Coldharbour Road, Harlow, Essex, UK \\ ${ }^{2}$ Neuro-Cell Sciences, GlaxoSmithKline, Coldharbour Road, Harlow, Essex, UK \\ ${ }^{3}$ Institut de Genomique Fonctionnelle, CNRS, Montpellier, France \\ IainP.Chessell@gsk.com
}

The $\mathrm{P} 2 \mathrm{X}_{7}$ receptor is unique in its ability to regulate the release of mature, biologically active interleukin-1 $\beta$ (IL$1 \beta$ ) [1]. We have demonstrated that in mice lacking this receptor, inflammatory and neuropathic hypersensitivity is completely absent to both mechanical and thermal stimuli, but that normal nociceptive responses are preserved [2]. 
The $\mathrm{P} 2 \mathrm{X}_{4}$ receptor has also been implicated in pain processing; the expression of $\mathrm{P}_{2} \mathrm{X}_{4}$ is upregulated in the spinal cord in microglial cells following nerve injury [3]. Whilst inhibition of $\mathrm{P}_{2} \mathrm{X}_{4}$ receptor expression by antisense oligonucleotides has been shown to inhibit nerve-injury pain behaviour [3], the role of this receptor in inflammatory pain, and its role in regulating inflammatory mediators has not been explored.

In this study, we describe the phenotypic characterization of $\mathrm{P} 2 \mathrm{X}_{4}$ knockout animals $(-/-)$ in inflammatory pain, and preliminary characterization of inflammatory mediator regulation in these animals, and compare these with phenotypic and cytokine characterization in $\mathrm{P} 2 \mathrm{X}_{7}-1-$ animals to aid elucidation of the role these $\mathrm{P} 2 \mathrm{X}$ receptor subtypes play in pain.

A fully backcrossed colony of $\mathrm{P} 2 \mathrm{X}_{4}-/-$ mice ( $\mathrm{n}=15$ per group) was examined in the Freund's complete adjuvant (FCA) model of inflammatory hypersensitivity and compared with responses in $\mathrm{P}_{2} \mathrm{X}_{4}+/+$ littermate controls $(\mathrm{n}=15$ per group). 24 hrs following intraplantar injection of FCA, significant mechanical hyperalgesia was observed in the $\mathrm{P} 2 \mathrm{X}_{4}+/+$ animals, but no significant hyperalgesia was observed in the $\mathrm{P}_{2} \mathrm{X}_{4}-/-$ cohort. These data are similar to those observed in the $\mathrm{P} 2 \mathrm{X}_{7}-1-$ animals [2], so a preliminary analysis of cytokine expression profile was performed in the $\mathrm{P}_{2} \mathrm{X}_{4}-/-$ animals to provide initial insight into commonality of mechanisms between the two transgenic lines.

Following intraplantar FCA injection, analysis of paw samples from the $\mathrm{P}_{2} \mathrm{X}_{7}-/-$ animals revealed significant attenuation of FCA-induced increases in IL-1 $\beta$ and IL-10, but a significant increase in IL-6 (see [2]). Conversely, in the $\mathrm{P}_{2} \mathrm{X}_{4}-/-$ animals, there was no reduction in FCA-induced increases in IL-1 $\beta$, and an increase in concentrations of IL-10.

Taken together, these data indicate the $\mathrm{P}_{2} \mathrm{X}_{4}-/-$ and $\mathrm{P} 2 \mathrm{X}_{7}-/-$ animals share a common pain phenotype, but this phenotype appears to be conferred via different mechanisms. Further studies are required to confirm and elucidate the mechanistic differences, and also to explore cross-talk between $\mathrm{P}_{2} \mathrm{X}_{4}$ and $\mathrm{P} 2 \mathrm{X}_{7}$ in both transgenic lines.

1. Perregaux DG, Gabel CA. J Biol Chem 1994; 269: 15195-203.

2. Chessell IP, Hatcher JP, Bountra C et al. Pain 2005; 114: 386-96.

3. Tsuda M, Shigemoto-Mogami Y, Koizumi S et al. Nature 2003; 424: 778-83

\title{
Round table on non-Adenine-Based Purines
}

\section{Evidence for a patho-physiological and pharmacological role of guanine-based purines as a new extracellular signalling system}

\author{
M.P. Rathbone ${ }^{1}$ and F. Caciagli ${ }^{2}$ \\ ${ }^{T}$ Department of Medicine, McMaster University, Hamilton, Ontario, Canada \\ ${ }^{2}$ Department of Biomedical Sciences, "G. d'Annunzio" University of Chieti-Pescara, Chieti, Italy \\ mrathbon@mcmaster.ca
}

In addition to the adenine-based purinergic intercellular signaling systems, involving adenine, adenosine and adenosine phosphates, over the last 15 years analogous guanine-based systems have been discovered. Most of these are involved in "trophic" effects, affecting the growth, differentiation and survival of various cells. Indeed, guanine-based purines act synergistically with certain growth factors such as NGF, and also stimulate the production and release from cells of several growth factors and cytokines. Guanine-based purinergic signaling has been particularly investigated in cells of the nervous system and muscle. However, in addition other tissues, including skin, respond to these compounds, indicating that, like adenine based purinergic signaling, guanine based signaling may be widespread throughout many cell types and organs.

Guanine-based purines are released from cells, and when cells are damaged the release increases substantially. Indeed, under conditions simulating ischemia, cells release more guanine-based purines than adenine based purines. Moreover, the extracellular concentration of the guanine-based purines is higher than that of the adenine-based counterparts.

There is evidence that in some cases guanosine produces its effects through entering cells and interacting with an NGF-inducible protein kinase. But there is also evidence that guanosine may interact with unique receptors on 
the surface of cells. Similarly, there is evidence that GTP may also have cell-surface receptors that mediate some of its effects. Moreover, guanosine is metabolized to guanine by the enzyme purine nucleoside phosphorylase (PNP). Experimental studies have indicated that exogenous extracellular guanosine is relatively persistent compared to adenosine, but a large proportion of guanosine is metabolized to guanine. Emerging evidence indicates that guanine may also have its own extracellular signaling system that is distinct from guanosine. Certainly, this would be of particular interest, since the enzyme that metabolizes guanine, guanine deaminase, shows 50 fold regional variations in brain. This degree of regional variation is characteristically associated with enzymes that degrade neurotransmitters.

It appears that the concept of intercellular signaling by guanine-based purines is now well substantiated. Since GTP, guanosine and guanine have different biological effects, different receptive mechanisms and likely different signal transduction mechanisms, it could be suggested the intriguing possibility that the extracellular interconversion of these guanine derivatives provides an extra layer of signal regulation by cells.

\title{
Guanosine stimulates remyelination in injured spinal cord through promotion of proliferation and differentiation of oligodendroglial precursor cells
}

\author{
S. Jiang ${ }^{1}$, M.P. Rathbone ${ }^{2}$, P. Giuliani ${ }^{3}$, F. Caciagli ${ }^{3}$ and P. Di Iorio ${ }^{3}$ \\ ${ }^{T}$ Department of Surgery, McMaster University, Hamilton, Ontario, Canada \\ ${ }^{2}$ Department of Medicine, McMaster University, Hamilton, Ontario, Canada \\ ${ }^{3}$ Department of Biomedical Sciences, "G. d'Annunzio" University of Chieti-Pescara, Chieti, Italy \\ jiangs@mcmaster.ca
}

The commonest type of spinal cord injury is a crush, which causes a central area of damage within the spinal cord, disrupting long tract nerve fibers passing up and down the spinal cord. Commonly, in the penumbra around the injury some nerve fibers survive, but the oligodendroglia that myelinate them die. Therefore these fibers do not conduct impulses, or do not conduct them well. However, the spinal cord contains endogenous precursors of the myelin-forming oligodendroglia, which, under certain circumstances, can differentiate into mature oligodendroglia and myelinate axons. This raises the possibility that after injury there is insufficient signal to trigger the differentiation of the oligodendroglial precursor cells into mature oligodendroglia that are capable of remyelinating axons. Guanosine (GUO) stimulates proliferation and differentiation of many types of cells in vitro and exerts neuroprotective effects in the central nervous system. Thirty five days after a standard moderate crush injury rats have a chronic, stable, permanent neurological motor deficit. We treated these rats with intraperitoneal (i.p.) GUO, $8 \mathrm{mg} / \mathrm{kg}$ body weight/day for up to 14 days. Their motor function began to improve after three days and continued to improve throughout the duration of the treatment. The improvement in motor function correlated with the increase in myelination in the penumbra of the injury. The myelin was characteristic of central nervous system myelin. The myelin arose from NG2-positive oligodendroglial precursor cells that matured into myelin-forming oligodendroglia. These may contribute to the myelin-forming cells. We hypothesize that the injury may 'prime' the intrinsic adult pregenitor cells in the penumbra of the lesion to respond to guanosine. Since GUO was administered systemically we questioned whether it acted directly on the nervous system as GUO, or as a metabolic product, or whether it did not even enter the central nervous system but instead exerted its effects by stimulating production of some peripheral hormone or cytokine. We used capillary electrophoresis combined with the radioactivity measurement to evaluate the distribution and metabolism of GUO $(8 \mathrm{mg} / \mathrm{Kg}$ body weight), containing an aliquot (about $0.005 \%$ of the total GUO) of $[3 \mathrm{H}]-\mathrm{GUO}(7.3 \mathrm{Ci} /$ mmol) administered by i.p. injection. GUO entered lesioned areas of spinal cord to the same extent as the remainder of the central nervous system. Over-basal exogenous GUO (eGUO) rapidly increased in plasma, reaching $80 \%$ maximum after 7.5 minutes and peaked after 30 minutes, thereafter remaining constant for the next hour. The amount of eGUO and its metabolite guanine (GUA) increased in the central nervous system and adipose tissue. Fifteen minutes after injection the amount of cerebral GUO was nearly doubled in comparison with basal value and the ratio of eGUO:eGUA was about 2:1, but after 60 minutes the eGUO:eGUA ratio was $<1$. Meanwhile the total eGUO + eGUA continued to increase in brain, spinal cord and adipose tissue, but not in other tissues, where it remained stable. These data indicate that systemically-administered GUO may certainly stimulate oligodendroglial precursor cells. But as well, it raises the possibility that GUA may also play a role. 


\section{Metabolism of extracellular nucleotides and nucleosides in the vasculature}

Gennady G. Yegutkin ${ }^{1}$, Tiina Henttinen ${ }^{2}$, Andrey Mikhailov ${ }^{2}$, Sergei Samburski ${ }^{1}$ and Sirpa Jalkanen ${ }^{1}$

${ }^{T}$ MediCity Laboratory and Department of Medical Microbiology, Turku University and National Public Health Institute, 20520 Turku, Finland

${ }^{2}$ Turku Centre for Biotechnology, University of Turku/Åbo Akademi University, POB 123, 20521 Turku, Finland gennady.yegutkin@utu.fi

Extracellular nucleotides and nucleosides trigger diverse immunomodulatory, prothrombotic and vasoactive signaling events in the vasculature. The duration and magnitude of purinergic signaling are thought to be mainly governed by ecto-nucleotidases expressed on endothelial and hematopoietic cells. In contrast to traditional paradigms that focus on cell-associated inactivating mechanisms, the existence of alternative pathways has received little attention. By using human vascular endothelial cells, normal and leukaemic lymphocytes, and cellfree serum as enzyme sources, we identified the following three groups of cell surface-associated and soluble activities: (1) nucleotide-inactivating enzymes, NTP diphosphohydrolase/CD39, nucleotide pyrophosphatase and ecto-5'-nucleotidase/CD73; (2) nucleotide-phosphorylating enzymes, adenylate kinase and nucleoside diphosphate kinase; (3) nucleoside-inactivating enzymes, adenosine deaminase and purine nucleoside phosphorylase. Evidence for this was obtained by using independent assays, including bioluminescent measurement of ATP metabolism, radio-TLC and reverse-phase HPLC analyses. The comparative measurements of enzymatic activities indicated the predominance of the nucleotide-inactivating pathway on the endothelial cells, whereas the lymphocytes are generally characterised by counteracting ATP-regenerating/nucleoside-eliminating phenotype. Next, given that ecto-adenylate kinase represents the only AMP-converting pathway on ecto-5-nucleotidase negative leukaemic T- and B-lymphocytes, we employed this ectoenzyme as an intrinsic probe for accurate "sensing" ATP in the pericellular space. This novel enzyme-coupled approach, together with confocal imaging of putative ATP stores, suggests that, along with predominant ATP accumulation within cytoplasmic granules, micromolar concentrations of ATP are constitutively retained on lymphoid cell surface without significant convection into bulk milieu. In conclusion, identification of the extensive network of enzymes directionally regulating active cycling between ATP and other nucleotides and nucleosides provides a novel insight into the purinergic homeostasis in the vasculature. Furthermore, the ability of lymphocytes to maintain micromolar ATP halo may represent an important route initiating signaling cascades within immunological synapses and facilitating leukocyte trafficking between the blood and tissues.

\section{The guanine-based purinergic system as a new target for neuroprotection against glutamatergic excitotoxicity}

\section{Diogo O. Souza}

Department of Biochemistry ICBS, Institute of Basic Sciences of Health, "Rio Grande do Sul" Federal University, Porto Alegre, RS, Brazil

diogo@ufrgs.br

Glutamate is the main excitatory neurotransmitter in mammalian CNS, essential for brain activities, as those involved in development, aging, memory, and adaptation to the environment. However, hyper-activation of the glutamatergic system may be potentially neurotoxic, involved in the pathogenesis of various acute and chronic brain injuries.

Our group has given strong evidence that the guanine-based purinergic system is effectively neuroprotective against glutamate toxicity, in acute and chronic animal models, both in vitro and in vivo studies. Although the administration of guanine derivatives (GD) exerts neuroprotection, our results strongly indicate that the active compound is the nucleoside guanosine (GUO).

In in vivo studies carried out in rat and mouse, GD protect against brain damage caused by hyper-activation of the glutamatergic system. Indeed: i) chronically, GMP administration in rat striatum protects cells against death 
caused by quinolinic acid (QA); ii) acutely, GMP or GUO i.c.v., i.p. or orally administered protect against seizures induced by QA (or $\alpha$-dendrotoxin). In in vitro studies carried out in brain slices, GUO protects cell against death caused by in vitro ischemia.

Searching for mechanisms implicated in this neuroprotection, we demonstrated that: i) GUO stimulates the astrocytic glutamate uptake (in astrocyte cultures and brain slices), the main process involved in endogenous neuronal protection; ii) QA induced-seizures decrease glutamate uptake by cortical brain slices and this decrease is reversed by GUO when it acts as anticonvulsant; iii) Brain ischemia decreases glutamate uptake by hippocampal slices and i.p. GUO administration prevents this decrease. Thus we propose that the stimulatory effect on glutamate uptake is involved in the neuroprotective actions of GUO.

These results encourage further studies aiming at the therapeutic use in humans of GUO in acute (hypoxia, ischemia, brain traumatism) and chronic (neurodegenerative diseases) brain injuries involving glutamate excitotoxicity.

\title{
The role of uridine kinase and uridine phosphorylase in the cross regulation of uracil and adenine nucleotides salvage synthesis in rat and human brain
}

\author{
Piero L. Ipata and Maria G. Tozzi
}

Department of Biology, University of Pisa, Via San Zeno 51, 56127 Pisa, Italy

ipata@dfb.unipi.it

A wealth of experimental data support the idea that uridine and its nucleotides (NT) have critical functions that help regulating a variety of biological systems $[1,2]$. In cultured cells the concentration of uridine $(\sim 3-8 \mu \mathrm{M})$ is sufficient to meet the pyrimidine requirement. At $12 \mu \mathrm{M}$ the UTP pool doubles, and the de novo pyrimidine synthesis is almost $100 \%$ inhibited, pointing for the importance of the nucleoside as a precursor in the pyrimidine salvage pathway. In contrast, purines are salvaged from their bases. Both in bacteria and in mammals the pathways for de novo pyrimidine and purine synthesis from simple precursors are cross regulated, i.e. accumulation of a purine NT (ATP) activates pyrimidine synthesis and inhibits that of purines, and vice versa. However, it is well established that several tissues and organs, including brain, rely more heavily in the salvage synthesis of NT from preformed purine and pyrimidine rings, rather than on de novo synthesis. This raises the following question: how do these districts maintain the right balance between their purine and pyrimidine pools? Recent in vitro evidence suggests that in rat and human brain cytosol the two processes of purine and pyrimidine NT salvage respond in an opposite manner to the fluctuation of intracellular [purine nucleoside triphosphates]/ [pyrimidine nucleoside triphosphates] ratio (PUR/PYR ratio). The metabolic sensor is a two enzyme system, composed of uridine kinase (Uk) and uridine phosphorylase (UPase), which contributes in maintaining uridine homeostasis. When the activity of Uk becomes inhibited by relatively high UTP and CTP concentrations (low PUR/PYR ratio), uridine accumulates and the equilibrium of the UPase reaction is shifted towards uridine phosphorolysis [3]. The ribose1-P produced forms PRPP [4], an obligate substrate of purine salvage. At a high ratio these effects are reversed. Uk is fully active, and pyrimidine salvage is favoured over purine salvage. Uridine might therefore be considered as a signal of pyrimidine abundance and a ribose carrier for purine nucleotides salvage.

An additional factor which might contribute in balancing the purine and pyrimidine salvage processes is the "oxypurine cycle," in which hypoxanthine (or guanine) is converted to IMP (or GMP) by HPRT, at the expense of PRPP. IMP (or GMP) is then dephosphorylated to inosine (or guanosine) by cN-II, and inosine (or guanosine) is converted back to hypoxanthine (or guanine) and Rib 1-P by PNP [5]. We notice that both PRPP, the "fuel" of the cycle, and Rib 1-P, its main product, are essential in NT salvage synthesis. PRPP is an obligate precursor of purine salvage, while the only known pathway for salvaging uracil is its Rib 1-P mediated ribosylation to give uridine, followed by multiple phosphorylation steps. The observation that in vitro uracil is readily converted into uridine in the presence of PRPP, provided hypoxanthine is present in the reaction mixture, led to the proposal that Rib 1-P needed to ribosylate uracil might well arise from PRPP breakdown occurring in the oxypurine cycle [5].

1. Löffler M, Fairbanks LD, Zameitat E, Marinaki AM, Simmonds HA. Trends Mol Med 2005; 11: 430-7.

2. Cao D, Leffert JJ, McCabe J, Kim B, Pizzorno G. J Biol Chem 2005; 280: 21169-75.

3. Tozzi MG, Camici M, Mascia L, Sgarrella F, Ipata PL. FEBS J 2006; in press.

4. Barsotti C, Tozzi MG, Ipata PL. J Biol Chem 2002; 277: 9865-9.

5. Barsotti C, Pesi R, Felice F, Ipata PL. Cell Mol Life Sci 2003; 60: 786-93. 


\section{Towards a patho-physiological role of non adenine purine nucleobases in the central nervous system}

F. Caciagli ${ }^{1}$, P. Ballerini ${ }^{1}$, R. Ciccarelli ${ }^{1}$, P. Di Iorio ${ }^{1}$, A. Poli ${ }^{2}$, F. Licastro ${ }^{2}$, S. Jiang ${ }^{3}$ and M.P. Rathbone ${ }^{4}$

${ }^{T}$ Department of Biomedical Sciences, "G. d'Annunzio" University of Chieti-Pescara, Chieti, Italy

${ }^{2}$ Department of Experimental Pathology and Biology, University of Bologna, Bologna, Italy

${ }^{3}$ Department of Surgery, McMaster University, Hamilton, Ontario, Canada and ${ }^{4}$ Department of Medicine, McMaster University, Hamilton, Ontario, Canada.

f.caciagli@dsb.unich.it

A growing evidence indicates that guanosine (GUO) exerts multiple neuroprotective activities. Indeed, it controls glutamate-induced excitotoxicity; causes astrogliosis and activates oligodendroglial cell precursors; modulates expression and function of some glial $\mathrm{K}^{+}, \mathrm{Cl}^{-}$and water channels; stimulates ApoE production and cholesterol efflux from astrocytes; enhances trophin production and exerts anti-apoptotic activity. These effects are not substantially affected by cell pre-treatment with nucleoside and nucleobase transporter inhibitors, thus indicating that they are due to the extracellular activity of GUO, likely mediated by specific plasma membrane receptor(s).

To correctly evaluate the effects caused by the extracellular GUO (eGUO) and to individuate receptor(s) mediating these effects, it is crucial to know whether and how eGUO is metabolized and affects the breakdown and the effects of the extracellular adenine-based purines.

We found that eGUO stimulates the efflux of purine and pyrimidine nucleotides from cultured glial cells and it is well known that the purine/pyrimidine nucleotide ratio as well as the concentrations of ATP and Pi control the activity of nucleotidases which play a pivotal role in the purine metabolism, especially in the nucleoside formation. On the other hand, the receptor-mediated activity of released nucleotides as well as that of the de novo formed nucleosides (especially adenosine) affect, in synergistic or, sometimes, in antagonistic manner, the effects caused by eGUO. Indeed, eGUO up-regulates the expression of $\mathrm{P}_{2} \mathrm{Y}_{2}$ receptors thus amplifying the ATP and UTP effects mediated by these receptors and extracellular adenosine, like eGUO, contributes, via $\mathrm{A}_{1}$ receptors, to the production of trophic factors whereas it affects, in opposite manner, the apoptotic death of glial cells via $\mathrm{A}_{1}$ and $\mathrm{A}_{3}$ receptors, respectively.

Moreover, we individuated the presence of a purine nucleoside phosphorylase (PNP) on the external surface of the plasma membranes of glial cells (ecto-PNP) which, in nature and in presence of $\mathrm{Pi}$, catalyzes the phosphorolysis of non adenine-based nucleosides (GUO and Inosine) thus producing the corresponding nucleobases (guanine and hypoxanthine). In this context, we recently addressed our attention to establish whether the ecto-PNP-mediated formation of purine nucleobases could also interfere with the effects caused by the respective nucleosides, by especially evaluating (in cells provided or not provided with PNP) the possible effects caused by extracellular guanine (eGUA) and by comparing them with those caused by eGUO, in the same cells. We found that eGUO and eGUA, by different molecular mechanisms, affect cell proliferation/death/ apoptosis and learning/memory processes. In particular, eGUA $(8 \mathrm{mg} / \mathrm{kg}$ orally administered $)$ almost completely counteracted the L-NAME-induced loss of memory in a passive avoidance task carried out in rats. Accordingly, an exonic polymorphism in PNP gene (transition G/A at +3052 position), compatible with changes in GUA formation, resulted to be coupled with a rapid and severe decay of cognitive activity (MMSE test) in patients with clinical diagnosis of Alzheimer's disease.

\section{Ectonucleotidases Structure and Function}

\section{Contibution of NPP-type ectophosphodiesterases to extracellular nucleotide metabolism}

Mathieu Bollen

Division of Biochemistry, Faculty of Medicine, Catholic University of Leuven, B-3000 Leuven, Belgium 
The extracellular level of nucleotides is dynamically controlled by the action of ecto-nucleoside diphosphokinases, ectoadenylate kinases and ectonucleotidases [1, 2]. There exist four structurally unrelated families of ectonucleotidases, namely E-NTPDases, alkaline phosphatases, NPP-type ectophosphodiesterases and 5nucleotidase. The family of NPPs belongs to the superfamily of phospho-/sulfo-coordinating metalloenzymes that all have a similar catalytic fold and action mechanism. The NPP-family consists of seven members but only three of these, NPP1-3, are known to hydrolyse nucleotides. They are expressed as secreted (NPP2) or transmembrane proteins (NPP1, NPP3). In addition to the catalytic domain, they contain one nuclease-like domain and two somatomedin-B like domains, which are essential for the expression of catalytic activity.

The enzymatic action of NPP1-3 (in)directly results in the termination of nucleotide signaling, the salvage of nucleotides and/or the generation of new messengers like ADP, adenosine or pyrophosphate [2]. NPP1 and NPP3 are only known to hydrolyze nucleotides but NPP2 also acts as an extracellylar lysophospholipase-D. The simultaneous hydrolysis of lysophospholipids and nucleotides generates products that have the potential to synergistically promote cell motility.

1. Stefan C, Jansen S, Bollen M. NPP-type ectophosphodiesterases: unity in diversity. Trends Biochem Sci 2005; 30: 542-50.

2. Stefan C, Jansen S, Bollen M. Modulation of purinergic signalling by NPP-type ectophosphodiesterases. PUSI 2006; in press.

\title{
Progress on the structures and functions of nucleoside triphosphate diphosphohydrolases and a calcium activated nucleotidase
}

\author{
Terence L. Kirley \\ Department of Pharmacology and Cell Biophysics, University of Cincinnati, P.O. Box 670575, Cincinnati, OH \\ 45267-0575, USA \\ terry.kirkley@uc.edu
}

My laboratory has studied aspects of several ecto-nucleotidases for more than a decade. Recently, we have focused on three such proteins-two members of the human Nucleoside TriPhosphate Diphosphohydrolase (NTPDase) family of nucleotidases, and one human Calcium Activated Nucleotidase (CAN) with homology to the soluble nucleotidases used by insects to enable blood feeding by hydrolyzing host ADP.

The two NTPDases are NTPDase3, a cell surface, integral membrane protein, whose likely function is modulation of purinergic signaling, and NTPDase6, which exists as a membrane-bound form in internal membranes, but can also be secreted as a soluble form by cleavage of its $\mathrm{N}$-terminal signal peptide. Studies on the soluble NTPDase6 have included methods of expression and refolding, as well as disulfide bond determination, and attempts to develop specific peptide inhibitors for the NTPDases using phage-display technology. Studies on NTPDase 3 have centered on mutagenic approaches to delineate residues and regions important for nucleotidase activities and for oligomer formation and stability. We developed computational models for the 3-D structure of the extracellular portions of the NTPDases, and have re-interpreted previous site-directed mutagenesis results using these models. We also propose a speculative cartoon model for how movements in the transmembrane domains of cell-surface NTPDases (demonstrated by Dr. Guidotti's group) might be linked to movements in the active site lobes of the 3-D model, mediated via several conserved proline residues near the juncture of the extracellular and transmembrane domains. The extracellular active site lobes predicted by the 3-D models are postulated to undergo hinge-like movements based on the homology of NTPDases with the actin superfamily of proteins, which exhibit butterfly-like domain movements.

Brain is one of the few tissues where NTPDase3 is relatively abundantly expressed. Therefore, we immunolocalized NTPDase 3 in adult rat brain, and found it to be associated with neurons and mostly limited to midline regions of the forebrain and midbrain. Based on co-localization with hypocretin-1/orexin-A cells and fibers in the hypothalamus, we hypothesize that NTPDase3 may modulate feeding and sleep-wake behaviors under purinergic control in this brain region.

A crystal structure of soluble human Calcium Activated Nucleotidase (CAN) has been published. However, we are investigating the possibility of multiple structural and functional roles for $\mathrm{Ca}^{2+}$ in this calcium-binding nucleotidase, as well as studying the newly discovered $\mathrm{Ca}^{2+}$-dependent dimerization that occurs in this human CAN.

This work was supported by NIH grants HL59915 and HL72882. 


\title{
The transmembrane domains of E-NTPDase 1, CD39, regulate its enzymatic activity
}

\author{
Alison Grinthal, Sari Paavilainen, Scott Jones, James Wu and Guido Guidotti \\ Department of Molecular and Cellular Biology, Harvard University, 7 Divinity Avenue, Cambridge, Massachusetts, \\ USA \\ guidotti@fas.harvard.edu
}

CD39 is anchored to the plasma membrane by two transmembrane domains, one at each end of the protein. Removal of either or both transmembrane domains or disruption of their native state by detergent solubilization reduces activity by $90 \%$, indicating that native function requires both transmembrane domains to be present and in the membrane. The transmembrane domains are also involved in dimerization of CD39. Here we report the results of experiments on the oligomeric state of CD39 in the plasma membrane of COS7 cells. We also investigate the interactions between the transmembrane domains of CD39 by mutation of selected residues analyzed by yeast selection, by disulfide crosslinking of cysteines introduced within the transmembrane helices and by manipulation of the lipid bilayer. The results indicate that the principal interactions between the transmembrane domains are intramolecular, that the helices are highly mobile, and that these dynamic motions are required for enzymatic activity and regulated by the status of the lipid bilayer.

\section{Ecto-ATPases and Metabolism}

\section{Co-ordinated regulation of P2-receptor signaling by membrane bound NTPDases}

J. Sévigny, F. Ben Yebdri, M. Fausther, G. Kauffenstein, F. Kukulski, E.G. Lavoie, J. Lecka, S.A. Lévesque, M.N. Munkonda and J. Pelletier

Centre de recherche en Rhumatologie et Immunologie, Université Laval, Ste-Foy, Québec, Canada

Jean.Sevigny@crchul.ulaval.ca

Through the activation of different and specific P2-receptors, extracellular nucleotides (ATP, ADP, UTP, UDP) regulate a variety of biological functions. The concentrations of these molecules are tightly regulated at the cell surface by ectonucleotidases. The most important family of enzymes that dephosphorylate ATP and other extracellular nucleotides are the members $1,2,3$ and 8 of the ecto-nucleoside triphosphate diphosphohydrolase (E-NTPDase) family. These enzymes sequentially hydrolyze the terminal phosphate residue of triphospho- and diphosphonucleosides, and, in conjunction with ecto-5'-nucleotidase, facilitates the formation of adenosine, another important biological messenger. NTPDases 1,2,3 and 8 differ in their catalytic abilities to dephosphorylate nucleotides, as for example ATP. Using this substrate, NTPDase1 gives a rapid accumulation of AMP with little formation of ADP. In contrast, NTPDase 2 hydrolyzes poorly the diphosphonucleoside derivative and accumulates it in its immediate environment. To give further options to the cells to tightly regulate nucleotide levels, NTPDases 3 and 8 generate a transient accumulation of ADP (a potent ecto-5'-nucleotidase inhibitor) with a late formation of AMP (the substrate of ecto-5'-nucleotidase).

These enzymatic properties are certainly of importance in the definition of functions of NTPDases. Another important parameter in the identification of functions is the cellular localization. With a set of antibodies that we have developed, we observed that NTPDase1 is mainly located on vascular endothelium, smooth muscles, as well as on macrophages. A number of experiments clearly demonstrated the function of NTPDase1 in platelet aggregation and thromboregulation. With mice deficient in NTPDase1 expression, preliminary data suggest a function of the enzyme in vascular smooth muscle cell contraction. Other recent data suggest that monocytic NTPDase1 may be involved in inflammation and cytokine secretion such as IL-8. NTPDase2 is localized to the adventitia of large blood vessels and on the external surface of some capillaries, in nerve structures, and in portal fibroblasts where a function in the control of cell proliferation has been proposed. NTPDase 3 have been recently localized to neurons by Dr. T.L. Kirley and co-workers. We have also observed high expression of the enzyme in 
few epithelia of the digestive system as well as in Langerhans islet cells of the pancreas. Finally, we have demonstrated that NTPDase8 is the long known liver canalicular ecto-ATPase.

Localization of these ectonucleotidases in different cells and tissue compartments suggests distinct roles of these enzymes. In this presentation, I will discuss about the characterization and function of plasma membrane bound NTPDases, namely NTPDase1,2,3 and 8 .

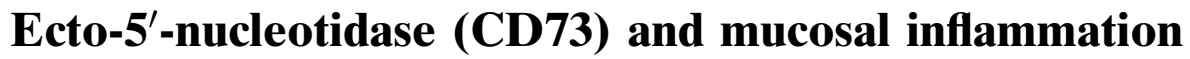

\author{
Sean P. Colgan ${ }^{1}$, Linda F. Thompson ${ }^{2}$, Andreas Robinson ${ }^{1}$ and Nancy A. Louis ${ }^{1,3}$
}

${ }^{1}$ Center for Experimental Therapeutics, Brigham and Women's Hospital and Harvard Medical School, Boston, Massachusetts USA

${ }^{2}$ Immunobiology and Cancer Research Program Oklahoma Medical Research Foundation, Oklahoma City, Oklahoma, USA

${ }^{3}$ Division of Newborn Medicine, Brigham and Women's Hospital and Harvard Medical School, Boston, Massachusetts USA

colgan@zeus.bwh.harvard.edu

Sites of inflammation are characterized by significant shifts in metabolic activity1. Shifts in energy supply and demand can result in diminished delivery and/or availability of oxygen, leading to inflammation-associated tissue hypoxia and metabolic acidosis. These shifts in tissue metabolism, as indicated by previous studies, are frequently associated with vasculitis and profound recruitment of inflammatory cell types, particularly myeloid cells such as neutrophils (PMN) and monocytes. Under such conditions, we and others have observed activation of the global hypoxia regulatory transcription factor hypoxia-inducible factor (HIF). A significant HIF target gene during mucosal inflammatory disease (e.g. inflammatory bowel disease, colitis) is CD732. Previous studies have determined that $\mathrm{CD} 73$ is regulated by HIF at the gene promoter level3. Since tissue profiling studies revealed highest expression of CD73 in mucosal tissues, particularly the intestine4, we have addressed the role of CD73 in model inflammatory disease in vitro and in vivo. Studies using intestinal epithelial cells have revealed that high expression of CD73 is protective for a number of epithelial properties (e.g. barrier function, ion transport), likely through generation of adenosine at the epithelial apical surface. Inflammatory studies in mice conditionally lacking the alpha subunit of HIF-1 in intestinal epithelial cells have shown that the loss of functional HIF-1 correlates with decreased expression of CD73 and enhanced inflammatory disease in these mice. More recent studies have addressed the role of CD73 colitic disease progression. These ongoing studies have revealed that the induction of colitis increases epithelial expression of CD73 mRNA and activity during the acute phase colitic disease. Studies utilizing Cd73-null mice4 have revealed that relative that the loss of CD73 correlated with more severe clinical symptoms of colitis (weight loss, colon length), and increased levels of inflammatory markers (neutrophil numbers). Mechanisms of increased susceptibility with decreased CD73 will be discussed. Taken together, these studies provide unique insight into tissue microenvironmental changes during model inflammatory disease and identify CD73 as a critical control point during mucosal insult.

1. Karhausen J, Haase VH, Colgan SP. Inflammatory Hypoxia: Role of Hypoxia-Inducible Factor. Cell Cycle 2005; 4: 256-8.

2. Karhausen JO, Furuta GT, Tomaszewski JE, Johnson RS, Colgan SP, Haase VH. Epithelial hypoxia-inducible factor-1 is protective in murine experimental colitis. J Clin Invest 2004; 114: 1098-106.

3. Synnestvedt K, Furuta GT, Comerford KM, Louis N, Karhausen J, Eltzschig HK, Hansen KR, Thompson LF, Colgan SP. Ecto-5'-nucleotidase (CD73) regulation by hypoxia-inducible factor-1 (HIF-1) mediates permeability changes in intestinal epithelia. J Clin Invest 2002; 110: 993-1002.

4. Thompson LF, Eltzschig HK, Ibla JC, Van De Wiele CJ, Resta R, Morote-Garcia JC, Colgan SP. Crucial role for ecto-5'nucleotidase (CD73) in vascular leak during hypoxia. J Exp Med 2004; 200: 1395-405. 


\section{Structure and function of ecto-5' ${ }^{\prime}$-nucleotidase based on structural studies of the bacterial 5 '-nucleotidases}

Norbert Sträter

Center for Biotechnology and Biomedicine, Faculty of Chemistry and Mineralogy, University of Leipzig, Deutscher

Platz 5, 04103 Leipzig

strater@bbz.uni-leipzig.de

On the basis of primary sequence alignments it is clear that the bacterial $5^{\prime}$-nucleotidases $\left(5^{\prime}\right.$-NT) are related to the vertebrate ecto- $5^{\prime}$-nucleotidases. The bacterial enzymes are present as monomers, whereas the ecto-enzymes form homodimers. A crystal structure is available for E. coli $5^{\prime}$-NT [1]. The enzyme consists of two domains: The $\mathrm{N}$-terminal domain (residues 25-342) binds two metal ions and contains an Asp-His dyad, which are important for the catalytic activity. This domain is related to other known enzyme structures of the calcineurin superfamily of dimetal phosphoesterases. The C-terminal domain (residues 362-550) has a unique structure, which has so far not been found in other protein structures. This domain provides the binding site for the adenosine moiety of the substrate. Thus, the active site is located between the two domains.

In the crystal structures, the protein has been characterised in two conformations, which differ in the relative orientation of the two domains [2]. The domain movement can be described as a rotation of the C-terminal domain around an axis, which passes through the center of the C-terminal domain. A rotation of up to $96^{\circ}$ is necessary for the change between the inactive open and the active closed conformation. The domain rotation is necessary for the catalytic action of the enzyme, presumably to allow for substrate binding and product release [3,4].

A sequence alignment of $E$. coli $5^{\prime}$-NT and the mammalian ecto-enzymes shows that both domains are conserved and a homology model can be built for ecto-5'-NT [5]. The ligands for the two catalytic metal ions are all conserved in ecto-5'-NT with the exception of M1-ligand Gln254 which corresponds to Asn245 in ecto-5'-NT. It appears unlikely that the shorter side chain supports metal coordination of Asn245, but the Asn245 carboxamide group may bind a water molecule which is coordinated to M1. Structures of E. coli $5^{\prime}$-NT in complex with the substrate analogue inhibitor $\alpha, \beta$-methylene-ADP revealed the characteristics of the substrate binding pocket of the C-terminal domain. A central feature is the hydrophobic stacking interaction of the adenine moiety, which is sandwiched between two phenylalanine side chains. The N1 nitrogen of the adenine ring is recognized by the carboxamide group of Asn431. The ribose group of the nucleotide is bound by Asp504, the backbone oxygen of Gly458 and Arg410.

Of these residues, the two phenylalanines are conserved in the ecto-enzymes or in electric ray $5^{\prime}$-NT are replaced by a tyrosine, which should also support the stacking interaction. Asn431 is replaced by a glycine residue. There is no other side chain nearby in the model which could replace the asparagine. Most likely, a water molecule interacts with $\mathrm{N} 1$ of the adenine ring. The aspartate residue bound to the hydroxyl groups of the ribose is conserved, as well as two of the three arginines. Arg375 of the E. coli enzyme is replaced by a serine. Thus, from the differences in the substrate binding site it is not obvious how the stronger substrate specificity of the mammalian enzymes towards AMP (vs. ADP and ATP) is achieved.

1. Knöfel T, Sträter N. Nat Struct Biol 1999; 6: 448-51.

2. Knöfel T, Sträter N. J Mol Biol 2001; 309: 255-66.

3. Schultz-Heienbrok R, Maier T, Sträter N. Biochemistry 2005; 44: 2244-52.

4. Schultz-Heienbrok R, Maier T, Sträter N. Protein Science 2004; 13: 1811-22.

5. Sträter N. Purinergic Signalling 2006, in press. 


\title{
The key regulators of skeletal mineralization: TNAP, NPP1 and ANK as therapeutic targets for the treatment of osteoarthritis, hypophosphatasia and arterial calcification
}

\author{
José Luis Millán \\ Burnham Institute for Medical Research, La Jolla, California, 92037 USA \\ millan@burnham.org
}

The mechanisms that regulate tissue calcification are of fundamental importance, as they ensure that ossification proceeds normally in the skeleton while pathological or ectopic calcification is prevented elsewhere in the body. Alterations in these regulatory mechanisms, either due to genetic defects or as a result of aging, lead to disease, such as the development of vascular calcification and osteoarthritis. Vascular calcification is a common occurrence in aging, atherosclerosis, diabetes, renal failure, aortic stenosis and prosthetic valve replacement. Osteoarthritis refers collectively to a number of disease states that affect the properties and functionality of the joints. Our work has shown that mice deficient in the nucleoside triphosphate pyrophosphohydrolase-1 (NPP1) isozyme (Enpp $1^{-1-}$ mice) or in the Ankylosis protein (ank/ank mutant mice) both have a deficit in inorganic pyrophosphate $\left(\mathrm{PP}_{\mathrm{i}}\right)$ production. $\mathrm{PP}_{\mathrm{i}}$ is a potent mineralization inhibitor and both these mutant mice share phenotypic features of softtissue ossification, corrected by simultaneous deletion of the tissue-nonspecific alkaline phosphatase (TNAP) gene $\left(A k p 2^{-1-}\right)^{1}$. Simultaneous inactivation of the $A k p 2$ gene in Enpp1 ${ }^{-1-}$ and ank/ank mutant mice led to normalization of inorganic pyrophosphate $\left(\mathrm{PP}_{\mathrm{i}}\right)$ levels, which in turn led to the normalization of depressed osteopontin (OPN) levels and importantly, correction of their calcification abnormalities [2, 3]. Enpp1 $1^{-/-}$and ank/ank mice also display arterial calcification similar to that seen in human patients with mutations in the NPP1 gene. Mice deficient in matrix Gla protein $\left(\mathrm{Mgp}^{-/-}\right)$mice are also characterized by ectopic calcification of all arteries in addition to bone abnormalities. When crossed to the Hyp "hypophosphatemic" mice, $\mathrm{Mgp}^{-1-}$ mice never developed arterial calcification and had a normal life span [4]. Similarly, [ank/ank; Hyp] double mutant mice had a normal life span, as opposed to ank/ank mice that usually die around 6 months of age. Also, injection of $\mathrm{PP}_{\mathrm{i}}$ into $\mathrm{Mgp}^{-1-}$ mice corrected their arterial calcification and normalized their life span. These results indicate that lowering the extracellular $\mathrm{P}_{\mathrm{i}} / \mathrm{PP}_{\mathrm{i}}$ ratio is sufficient to prevent the appearance of an osteoarthritis-like phenotype and of the arterial calcification in these different mouse models. Recent characterization of $\mathrm{Opn}^{-1-}$ and $\left[\mathrm{Akp2}^{-/-} ; \mathrm{Opn}^{-/-}\right]$mice has suggested that OPN may in fact be a more important mineralization inhibitor than $\mathrm{PP}_{\mathrm{i}}$, and that the effects of modulating $\mathrm{PP}_{\mathrm{i}}$ levels are carried out by the changes in OPN levels [5]. Our work currently focuses on developing therapeutic drugs to target the function of TNAP and NPP1 as a strategy to normalize the extracellular $\mathrm{P}_{\mathrm{i}} / \mathrm{PP}_{\mathrm{i}}$ levels and prevent/correct ectopic calcification. Funded by grants DE 12880 and AR 47908 from the National Institutes of Health, USA.

1. Hessle L, Johnsson KA, Anderson HC, Narisawa S, Sali A, Goding JW, Terkeltaub R, Millán JL. Proc Natl Acad Sci USA 2002; 99: 9445-9.

2. Johnson K, Goding J, Van Etten D, Sali A, Hu S-I, Farley D, Krug H, Hessle L, Millán JL, Terkeltaub R. J Bone Miner Res 2003; 18: 994-1004.

3. Harmey D, Hessle L, Narisawa S, Johnson K, Terkeltaub R, Millán JL. Am J Pathol 2004; 164: $1199-209$.

4. Murshed M, Harmey D, Millán JL, McKee MD, Karsenty G. Broadly expressed genes accounts for the special restriction of ECM mineralization to bone. Genes Dev 2005; 19: 1093-104.

5. Harmey D, Johnson K, Hoylaerts MF, Noda M, Terkeltaub R, Millán JL. Elevated osteopontin levels contribute to the hypophosphatasia phenotype in $A k p 2^{-1-}$ mice. J Bone Miner Res submitted 2005. 


\section{Adenosine and Neuroprotection}

\section{A2a adenosine receptors regulate CNS responses to ethanol and addicting substances: recent advances from cell biology to behavior}

Ivan Diamond

Vice_President, Neuroscience

CV Therapeutics

Palo Alto, California, USA

Very high levels of Gs(olf)-coupled A2a adenosine receptors (A2a) are found in the nucleus accumbens (NAcb), a mesolimbic brain reward region involved in alcoholism and addiction. Ethanol inhibits adenosine re-uptake and increases extracellular adenosine levels, thereby activating A2a receptors. Activation of A2a receptors promotes stimulation of adenylyl cyclase (AC) and cAMP/PKA signaling. In the NAcb, A2a receptor expression occurs postsynaptically on the same neurons expressing Gi-coupled D2 dopamine (D2), mu opioid (MOR) and cannabinoid (CB1) receptors. All of these receptors are involved in addiction. Activation Gi-coupled receptors for $30 \mathrm{~min}$. ordinarily inhibits cAMP production. By contrast, we show that a $10 \mathrm{~min}$. activation of Gi-receptors involved in addictive behaviors acts synergistically with A2a adenosine receptors to stimulate cAMP/PKA signaling and induction of CRE-mediated gene expression. In all instances, synergy is mediated by free Gi_betagamma subunit stimulation of AC II and IV. Synergistic stimulation of cAMP production requires A2a adenosine receptors. Importantly, knockdown or inhibition of the betagamma/cAMP signaling pathway prevents synergy in primary NAcb neurons and eliminates addictive behaviors. Relapse is the most serious complication of addiction that prevents effective medical treatment of human addicts. In rats, A2a receptor antagonists administered directly into the NAcb or indirectly by i.p. injection reduces voluntary ethanol drinking and eliminates reinstatement of heroin-seeking behavior in rats. These findings in a rodent model of human relapse suggest that A2a adenosine receptor antagonists may be useful therapeutic agents in the treatment of alcoholism and addiction. (Supported by grants from NIAAA and the Department of the Army).

\section{Adenosine $\mathbf{A}_{2 \mathrm{~A}}$ receptors and Parkinson's disease - Effects of Istradefylline on functional models of Parkinson's disease}

Tomoyuki Kanda, PhD.

Department of CNS Research, Pharmaceutical Research Center, Kyowa Hakko Kogyo Co., Ltd.

Currently, dopamine replacement therapy is most effective treatment for the motor symptoms of Parkinson's disease(PD) [1]. However, its association with the development of motor complications such as dyskinesia and wearing-off phenomenon, limits its usefulness in late stages of the PD [2]. Adenosine $\mathrm{A}_{2 \mathrm{~A}}$ receptors are abundantly localized to the indirect striato-pallidal output pathway, which is one of two major striatal output pathwayscontroling motor behavior via basalganglia network. Istradefylline is a novel selective adenosine $\mathrm{A}_{2 \mathrm{~A}}$ receptor antagonist currently in Phase III clinical trials in PD. The results from Phase II clinical trials has demonstrated that the coumpaund provided a clinically meaningful reduction in OFF time and an increased ON time with non-troublesome dyskinesia in the L-dopa treated patients with motor complications [3].

In PD, loss of dopaminergic neurons in substantia nigra causes imbalance in neuronal activity between the indirect strato-pallidal and direct striato-nigral pathways by loss of dopaminergic influences onto striatal medium spiny neurons gerenrating each pathway. This imbalance induces entire abnormality of basal ganglia network, resulting in abnormal movements (i.e., hypokinetic motor control). It has been demonstrated that the adenosine $\mathrm{A}_{2 \mathrm{~A}}$ receptor activation causesinduces excessive activation of the indirect striato-pallidal pathway via the dual modulation in the striatum and globus palidus [4]. Therefore, blockade of the $\mathrm{A}_{2 \mathrm{~A}}$ receptors should decrease such an excessive excitability of the indirect pathway and alleviated the motor symptoms of PD. The results from studies in animal models of PD have demonstrated that istradefylline increases locomotor activity and decreases motor disabilities without inducing involuntary movements in animals rendered dyskinetic by previous exposure 
to L-dopa [5]. In MPTP treated non-human primate(marmoset) models of PD, istradefylline was administered with a low dose of L-dopa for 21 days to animals primed to exhibit dyskinesia. The amplitude of involuntary movements observed was not greater than that produced by L-dopa alone in MPTP treated marmosets. In addtion, istradefylline shows significant reduction of L-dopa induced dyskinesias compared with L-dopa alone [6]. Results of studies on animal models of PD indicate that istradefylline did not exacerbate L-dopa-induced dyskinesia, and suggested that chronic coadministration of istradefylline with L-dopa may decrease dyskinesias caused by L-dopa. The results from functional models, providing relevance to clinical study outcomes in PD patients, described that adenosine $\mathrm{A}_{2 \mathrm{~A}}$ antagonists appear to be most promising as the first major nondopaminergic therapy for PD.

1. Bonuccelli U. Curr Opin Neurol 2003; 16(Suppl. 1): S13-9.

2. (a) Olanow CW Ann Rev Med 2004; 55: 41-60. (b) Rascul O, Payoux P, Ory F, Ferreira JJ, Brefel-Courbon C, Montastruc JL. Ann Neurol 2003; 53(Suppl. 3): S3-S12.

3. (a) Chase TN, Bibbiani F, Bara_Jimenez W, Dimitrova T, Oh-Lee JD. Neurology 2003; 61(Suppl. 6): S107-S111. (b) LeWitt PA, Parkinson Relat Disord 2005; 11(Supple. 2): 266.

4. (a) Mori A, Shindou T. Neurology 2003; 61 (Suppl. 6): S44-S48. (b) Agnati LF, Ferre S, Lluis C, Franco R, Fuxe K. Pharmacol Rev 2003; 55: 509-50.

5. Kase H, Neurology 2003; 61 (Suppl. 6): S97-S100.

6. Kanda T, Tashiro T, Shiozaki S. manuscript in preparation.

\section{Adenosine $\mathbf{A}_{2 \mathrm{~A}}$ Receptors Modulate Psychomotor Activity and Brain Injury by Distinct Cellular Mechanisms}

Liqun $\mathrm{Yu}^{1}$, Qing-yuan Huang ${ }^{1}$, Nelson Rebola ${ }^{4}$, Hai-Ying Shen ${ }^{1}$, Eric Kirsten Rapp ${ }^{1}$, Yuan-Ji Day ${ }^{3}$, Jarrod Ferrara ${ }^{1}$, Joana E. Coelho ${ }^{1}$, Paula M. Canas ${ }^{4}$, Zhi-Hong Huang ${ }^{2}$, Darcie Taylor ${ }^{1}$, Michael Moskowitz $^{2}$, Michael Schwarzschild ${ }^{2}$, Joel Linden ${ }^{3}$, Rodrigo A. Cunha ${ }^{4}$ and Jiang-Fan Chen ${ }^{1}$

${ }^{1}$ Department of Neurology, Boston University School of Medicine, Boston, MA; USA

${ }^{2}$ Neuroscience Center and Department of Neurology, Massachusetts General Hospital, Boston, MA; USA

${ }^{3}$ Department of Internal Medicine, University of Virginia, Charlottesville, VA; USA

${ }^{4}$ Center for Neuroscience, Institute of Biochemistry, Faculty of Medicine, University of Coimbra, Portugal chenjf@bu.edu

The adenosine $A_{2 A}$ receptor $\left(A_{2 A} R\right)$ has recently emerged as a leading non-dopaminergic therapeutic target for Parkinson's disease for its ability to regulate motor activity. Furthermore, $\mathrm{A}_{2 \mathrm{~A}}$ Rs influence brain injury outcome in variety of neurological disease models, presumably through modulation of glutamate release. Using forebrain neuronal-specific $A_{2 A} R$ knockout $(K O)$ mice, we here provide the first direct evidence that $A_{2 A} R$-mediated control of motor function and neuroprotection involve distinct cellular mechanisms. By crossing the floxed $\mathrm{A}_{2 \mathrm{~A}} \mathrm{R}$ mice with the CaMKII-Cre transgenic line, we selectively depleted $A_{2 A} R$ mRNA and protein in forebrain neurons to the background level of the global $\mathrm{A}_{2 \mathrm{~A}} \mathrm{R} \mathrm{KO}$ mice, as demonstrated by in situ hybridization, immunochemistry and receptor binding assays. This genetic deletion of $\mathrm{A}_{2 \mathrm{~A}} \mathrm{Rs}$ in forebrain neurons abolished the psychomotor effect of the $\mathrm{A}_{2 \mathrm{~A}} \mathrm{R}$ selective agonist CGS21680 and antagonist KW-6002 and of the non-selective antagonist caffeine, and largely attenuated the pyschostimulant effect of cocaine. This demonstrates the key role of forebrain neuronal $\mathrm{A}_{2 \mathrm{~A}} \mathrm{Rs}$ in the modulation of psychomotor activity. In contrast, genetic deletion of the $\mathrm{A}_{2 \mathrm{~A}} \mathrm{R}$ in forebrain neurons did not confer protection against ischemic brain injury by middle cerebral arterial occlusion or against MPTP-induced dopaminergic neurotoxicity, despite abolishing CGS21680-mediated presynaptic facilitation of glutamate release in forebrain $\mathrm{A}_{2 \mathrm{~A}} \mathrm{R} \mathrm{KO}$ mice. Furthermore, intracerebral ventricular administration of KW-6002 into forebrain $\mathrm{A}_{2 \mathrm{~A}} \mathrm{R}$ KO mice reinstated neuroprotection against MPTP neurotoxicity. These results provide the clearest data yet that $\mathrm{A}_{2 \mathrm{~A}} \mathrm{R}$ activity in forebrain neurons is critical to control psychomotor activity, but not for neuroprotection against brain injury, indicating that $\mathrm{A}_{2 \mathrm{~A}} \mathrm{Rs}$ modulate motor activity and brain damage by distinct cellular mechanisms. This opens up the new possibility of selectively manipulating $\mathrm{A}_{2 \mathrm{~A}} \mathrm{R}$ 's motor and neuroprotective effects by targeting different cellular elements. 


\title{
Adenosine and Huntington's Disease
}

Szu-Yi Chou ${ }^{1,2}$, Ming-Chang Chiang ${ }^{1,3}$, and Yijuang Chern ${ }^{1,2,3}$,

${ }^{1}$ Institute of Biomedical Sciences, Academia Sinica, Nankang, Taipei 115, Taiwan

${ }^{2}$ Institute of Life Sciences, National Defense Medical Center, Taipei 104, Taiwan

${ }^{3}$ Institute of Neuroscience, National Yang-Ming University, Taipei 112, Taiwan

bmychern@ibms.sinica.edu.tw

Huntington's disease (HD) is an autosomal dominant neurodegenerative disease characterized by chorea, dementia, and psychiatric symptoms. The causative mutation is a CAG trinucleotide expansion in exon 1 of the Huntingtin (Htt) gene. The normal Htt gene has 35 or fewer repeats in the N-terminal region, whereas the appearance of neurological symptoms is associated with 36 or more CAG repeats in the Htt gene. Among all brain areas, neurons within the striatum and cortex are most susceptible to HD. Besides the well-characterized neurological deficits, metabolic abnormalities have also been reported in HD. In addition to the brain, formation of $\mathrm{Htt}$ aggregates has also been documented in peripheral tissues including the liver. Several rodent models based on chemical lesions of the striatum or genetic manipulations of the Htt gene have been established and extensively used to develop beneficial treatments for $\mathrm{HD}$. Among the four adenosine receptors, the $\mathrm{A}_{1}$ and $\mathrm{A}_{2 \mathrm{~A}}$ adenosine receptors have been suggested as potential drug targets in HD. Using an HD transgenic mouse model (R6/2), we found that daily administration of an $\mathrm{A}_{2 \mathrm{~A}}$ adenosine receptor-selective agonist (CGS21680, CGS) delayed the progressive deterioration of motor performance and prevented a reduction in brain weight. 3D- $\mu$ MRI analysis revealed that CGS reversed the enlarged ventricle-to-brain ratio of $\mathrm{R} 6 / 2$ mice, with particular improvements in the left and right ventricles. ${ }^{1} \mathrm{H}-\mathrm{MRS}$ showed that CGS significantly reduced the increased choline levels in the striatum. Immunohistochemical analyses further demonstrated that CGS reduced the size of ubiquitin-positive neuronal intranuclear inclusions (NIIs) in the striatum of R6/2 mice and amended mutant Htt aggregation in a striatal progenitor cell line overexpressing mutant Htt with expanded polyQ. Moreover, chronic CGS treatment also ameliorated the formation of Htt aggregates and the decrease in two protein chaperones in the liver. Collectively, the above findings support the potential use of adenosine-related agents for treating HD.

\section{Adenosine receptor-receptor interactions: Relevance for ischemia and caffeine tolerance}

\author{
Rafael Franco, Sergi Ferré, Rodrigo Cunha, Vicent Casadó, Carmen Lluis, Francisco Ciruela \\ Molecular Neurobiology Unit. Institut d'Investigacions Biomèdiques August Pi i Sunyer (IDIBAPS). University of \\ Barcelona. Diagonal 645. 08028 Barcelona \\ rfranco@ub.edu
}

Of the four known adenosine receptors $\left(\mathrm{A}_{1}, \mathrm{~A}_{2 \mathrm{~A}}, \mathrm{~A}_{2 \mathrm{~B}}\right.$ and $\left.\mathrm{A}_{3}\right)$, adenosine $\mathrm{A}_{1}$ receptors $\left(\mathrm{A}_{1} \mathrm{Rs}\right)$ and adenosine $A_{2 A}$ receptors $\left(A_{2 A} R s\right)$ are primarily responsible for the central effects of adenosine. In addition to their postsynaptic location in different brain regions, $A_{1} R$ and $A_{2 A} R$ can be found presynaptically, where they modulate neurotransmitter release. $\mathrm{A}_{1} \mathrm{R}$ and $\mathrm{A}_{2 \mathrm{~A}} \mathrm{R}$ are coupled to $\mathrm{Gi} / \mathrm{o}$ and Gs/olf proteins, respectively. Presynaptic $A_{1} R s$ are the prototype of $G$ protein-coupled receptors whose stimulation decreases the probability of neurotransmitter release. On the other hand, presynaptic $\mathrm{A}_{2 \mathrm{~A}} \mathrm{Rs}$ are mostly excitatory and their stimulation induces neurotransmitter release. In this presentation evidence for heteromerization of $A_{1}$ and $A_{2 A}$ receptors is provided. This heteromerization have physiological consequences as described below.

Previous studies have provided evidence for colocalization and functional antagonistic interactions between $\mathrm{A}_{1}$ Rs and $\mathrm{A}_{2 \mathrm{~A}}$ Rs that modulate glutamate release in the striatum and hippocampus. A similar kind of interaction has been suggested to exist in the terminals of striatal cholinergic interneurons, although the localization and functional significance of $\mathrm{A}_{2 \mathrm{~A}} \mathrm{R}$ in striatal cholinergic neurons is controversial. The coexistence of both stimulatory $A_{2 A}$ Rs and inhibitory $A_{1} R s$ in the same terminal is intriguing particularly in view of their opposite functional effects. 
$\mathrm{A}_{1} \mathrm{R}$ and $\mathrm{A}_{2 \mathrm{~A}} \mathrm{R}$ form $\mathrm{A}_{1} \mathrm{R}-\mathrm{A}_{2 \mathrm{~A}} \mathrm{R}$ heteromers in transfected cells and in striatal glutamatergic nerve terminals. $A_{1} R-A_{2 A} R$ heteromerization plays a role in controlling the affinity of $A_{1} R$ and $A_{2 A} R$ for agonists and antagonists, like caffeine. Functional studies show that $A_{1} R-A_{2 A} R$ heteromers are responsible for a strong $A_{1} R-$ $\mathrm{A}_{2 \mathrm{~A}} \mathrm{R}$ antagonistic cross_talk. The strength of this cross-talk would depend on the concentration of extracellular adenosine, which is enhanced in ischemia. Tolerance to caffeine can be explained by the occurrence of $A_{1} R-A_{2 A} R$ heteromers. Heteromerization of receptors for a given neurotransmitter or neuromodulator constitutes a novel way to regulate presynaptic neurotransmission.

Part of the results in this presentation will be published in 2006: Ciruela et al., J Neurosc. In the press.

\title{
Ectonucleotidases Physiological Implication
}

\section{Biphasic regulation of airway E-NTPDases by Pseudomonas Aeruginosa lipopolysaccharide}

\author{
Maryse Picher $^{1}$ and Lauranell H. Burch ${ }^{2}$ \\ ${ }^{1}$ Cystic Fibrosis Center, University of North Carolina, North Carolina, USA \\ ${ }^{2}$ Laboratory of Respiratory Biology, NIEHS, Durham, North Carolina, USA \\ pichm@med.unc.edu
}

Chronic obstructive lung diseases are characterized by the inability to prevent bacterial infection causing recurrent inflammatory responses, epithelial damage and loss of lung function. In the past decade, numerous studies have demonstrated the importance of extracellular nucleotides for bacterial clearance. Their binding to P2 receptors on airway epithelia stimulates mucus secretion, cilia beating activity and hydration of the airway surface liquid layer. On the other hand, abnormally-high ATP levels resulting from damaged epithelia and bacterial lysis may cause lung edema and exacerbate inflammation-related epithelial damage. This study demonstrates the importance of ecto nucleoside triphosphate diphosphohydrolases (E-NTPDases; ATP $\rightarrow$ ADP $\rightarrow$ AMP) for the regulation of $\mathrm{P} 2$ receptor-mediated airway functions and identifies the mechanisms regulating their activities in lung diseases. All experiments were conducted on polarized primary cultures of human bronchial epithelial cells maintained at air-liquid interface. The E-NTPDase inhibitor, azide, reduced the metabolism of UTP and UDP by $45 \%$ and $55 \%$, respectively. Chronic lung diseases, including cystic fibrosis (CF) and primary ciliary dyskinesia (PCD), exhibited 4-6 fold higher rates of nucleotide elimination and azide-sensitive E-NTPDase activities. The mechanisms regulating these ectonucleotidases (NTPDase 1 and NTPDase 3) under pathological conditions were first studied using the common lung pathogen, Pseudomonas aeruginosa lipopolysaccharide (LPS). Bronchial cultures exposed to an optimum $(30 \mathrm{ng} / \mathrm{ml})$ endotoxin concentration displayed a biphasic response characterized by an acute reduction in total activity $(<8 \mathrm{~h})$ followed by chronic $(24 \mathrm{~h})$ increases in activity, NTPDase 1 and 3 expression. $P$. aeruginosa LPS induces several acute responses from airway epithelia, including oxidative stress, lipid peroxidation and inflammation. Bronchial cultures were exposed $(2 \mathrm{~h})$ on the mucosal surface to LPS ( $30 \mathrm{ng} /$ $\mathrm{ml})$ or $\mathrm{TNF} \alpha(10 \mathrm{ng} / \mathrm{ml})$ in the absence/presence of bilateral scavengers of peroxide (catalase) or superoxide radicals (superoxide dismutase; SOD). Although total azide-sensitive NTPDase activity was reduced by acute exposures to LPS or TNFa, ebselen-sensitive NTPDase 3 activity was increased 3-fold while NTPDase 1 activity was completely inhibited. Normal activity levels were partially restored by the scavengers (catalase $>$ SOD). The contribution of inflammatory pathways was demonstrated by the protective effect of the anti-inflammatory cytokine, IFN $\gamma$, and by SN50, cell permeable peptide preventing the translocation of the pro-inflammatory transcription factor, NFkB, to the nucleus. Altogether, these results suggest that NTPDase 1 and NTPDase 3 are co-expressed on human airway epithelia. Their opposite regulation by acute insults supports different roles in airway homeostasis. In contrast, elevated E-NTPDase activities in chronic lung diseases may represent an attempt to prevent $\mathrm{P} 2$ receptor desensitization and nucleotide-mediated lung damage.

This study was supported by the Cystic Fibrosis Foundation (Picher 05G0) 


\title{
CD38/CD157 gene family in health and disease
}

Fabio Malavasi

Laboratory of Immunogenetics, Department of Genetics, Biology and Biochemistry, University of Torino Medical School, Torino, Italy

fabio.malavasi@unito.it

The plasma membrane of human cells hosts a relatively large number $(\sim 5 \%)$ of molecules acting as enzymes apparently operating in an anti-economical manner. Nucleotide-metabolizing ectoenzymes constitute a family within this larger family and are represented by a set of molecules involved in the catabolism and scavenging of extracellular nucleotides. This process results in the synthesis of compounds that play a critical role in cell homeostasis and metabolism, suggesting that the physiological role of this complex family goes beyond the simple recycling of nucleotides.

In this context, the story behind the CD38 gene family (CD38 and CD157) is representative of a more general trend involving several nucleotide-metabolizing ectoenzymes, such as CD39 and CD26. Indeed, human CD38 is the mammalian prototype of a family of phylogenetically conserved proteins which share structural similarities and enzymatic activities involved in the production of an intracellular second messenger with calcium mobilizing effects. Engagement of CD38 by agonistic monoclonal antibodies and the CD31 ligand initiates a cytoplasmic signaling cascade involving tyrosine phosphorylation of the proto-oncogene c-cbl and of the extracellular regulated kinase 1/2 complex. Further requirements for signal transduction include a privileged localization within the cholesterol-rich areas of the plasma membrane and physical association with specialized surface receptors. CD38-mediated signals are crucial in heterotypic cell adhesion and migration as well as in the activation of proliferation / survival programs by normal and neoplastic cells. A direct role in the pathogenesis of diseases is also suggested by the involvement of CD38 in directing the prognosis of chronic lymphocytic leukemia. Further studies concerning the association between the CD38 gene family and human diseases will provide a better insight into the biological functions of the complex family of receptors and enzymes.

\section{Ectoenzymes of CD38 and CD39 gene families catalyze immunomodulation}

\author{
Silvia Deaglio ${ }^{1,2}$, Karen M. Dwyer ${ }^{2}$, David Friedman ${ }^{2}$, Terry B. Strom ${ }^{2,3}$ and Simon C. Robson ${ }^{2}$ \\ ${ }^{1}$ Department of Genetics, Biology and Biochemistry, University of Torino, Torino, Italy \\ ${ }^{2}$ Departments of Medicine and ${ }^{3}$ Surgery, Beth Israel Deaconess Medical Center, Harvard Medical School, Boston, \\ Massachusettes, USA \\ silvia.deaglio@unito.it
}

CD39 [ENTPD1 (ecto nucleoside triphosphate diphosphohydrolase-1), EC 3.6.1.5] is a member of a family of ecto-nucleotidases, surface molecules with extracellular catalytic sites that hydrolyze nucleotides. CD39 is the rate limiting enzyme that hydrolyses ATP/UTP and ADP/UDP to AMP; AMP is then rapidly degraded to adenosine by soluble or membrane-bound 5'-nucleotidase such as the ubiquitous CD73 (EC 3.1.3.5). This family of nucleotide-metabolizing ectoenzymes also includes molecules for which a clear-cut role in lymphocyte activation has been extensively proven, such as CD26 and CD38. Specifically, experience gathered with the CD38 gene family has clearly shown a non-redundant role in leukocyte trafficking and migration to inflammatory sites. Further, human disease models such as chronic lymphocytic leukemia, have highlighted a crucial receptor-like role for CD38 in controlling proliferation and survival signals to normal and neoplastic lymphocytes.

Little is known on the role of CD39 in lymphocyte activation. Preliminary data indicates that the enzymatic functions controlled by CD39 are involved in the recruitment, activation and polarization of naïve $\mathrm{T}$ cells by Langherhan's dendritic cells. We have now expanded these observations by exploring CD39 expression and the functional role within quiescent and activated T cell subsets. Our data indicate that CD39 efficiently distinguishes Treg from other resting or activated $\mathrm{T}$ cells in humans and mice. Furthermore, CD39 serves as an integral component of the suppressive machinery of Treg, acting, at least in part, through the modulation of extracellular levels of adenosine. We show that the coordinated regulation of CD39 expression and of the adenosine receptor A2A activates an immuno-inhibitory network that regulates Th1 responses. The in vivo relevance of this network 
is manifest in the phenotype of $C d 39$ null mice. These mice spontaneously develop autoimmune diseases associated with heightened Th1 responses. These data confirm the potential of CD39 as a phylogenetically conserved marker of Treg that has immunomodulatory functions.

Finally, these results may also be read in the wider context of a network of ectoenzymes acting in synergy to scavenge nucleotides and to generate powerful mediators of immune responses. Further work will be needed to determine the functional interactions between this complex family of cell surface enzymes.

\title{
Role of CD73-derived adenosine in acute and chronic inflammation
}

Schrader J. ${ }^{1}$, Zernecke A. ${ }^{2}$, Buchheiser A. ${ }^{1}$, Weber Ch. ${ }^{2}$, Özüyaman B. ${ }^{1}$

${ }^{1}$ Department of Cardiovascular Physiology, University of Duesseldorf, Germany

${ }^{2}$ Institute of Molecular Cardiovascular Research, RWTH Aachen, Germany

We have recently reported that mice with targeted deletion of ecto-5'-nucleotidase/CD73 are characterized by reduced coronary flow, enhanced platelet activation and increased adherence of monocytes to the endothelium. In the present study we have investigated the molecular mechanisms by which this proinflammatory response is mediated and explored whether CD73-derived adenosine modulates neointima formation in an acute injury model and inhibits development of arteriosclerosis in ApoE/CD73-/- double mutants.

$\mathrm{CD}^{2} 3^{-/}$mice exibit increased luminal staining for vascular adhesion molecule (VCAM)-1 in carotid arteries and increased expression of VCAM-1 transcripts and protein in whole carotid lysates. Endothelial cells cultured from $\mathrm{CD}^{-/-}$show an up-regulation of mRNA and protein expression of VCAM-1, which was associated with in creased nuclear factor (NF)-kB activity. Measurement of expression of the $A_{1}, A_{2 a}, A_{2 b}$ and $A_{3}$ receptor by RTPCR in the aorta and freshly harvested aortic endothelial cells revealed substantial downregulation the $A_{1}$ receptor (20\% of control) while the other adenosine receptors remained unchanged. Ex-vivo perfusion of carotid arteries show that the incresed monocyte arrest in carotid arteries of $\mathrm{CD} 73^{-/-}$is mediated by $\alpha 4 \beta 1$ integrin. After wire-induced injury of the carotid artery, CD73 expression was upregulated in WT mice, while neointima formation and macrophage content was increased in $\mathrm{CD}^{-/-}$mice, concomitant with elevated NF-kB activation, luminal VCAM-1 expression and soluble VCAM-1 concentration. Treatment of mice with the specific $\mathrm{A}_{2 \mathrm{a}}$ recetor agonist ATL-146e reversed the increased VCAM-1 transcript and protein expression in CD73 ${ }^{-/}$derived endothelial cells. Most importantly, ATL-145e fully prevented wire-induced neointima formation in CD73 ${ }^{-/-}$mice.

To explore whether CD73-derived adenosine also modulates chronic inflammation we have generated ApoE/ CD73-/- double mutants and found in 6 month old animals kept on normal diet, that the development of arteriosclerotic lesions in the aorta was 2.5 fold higher compared with ApoE controls. Measurement of various cytokines in plasma substantiate the increased inflammatory state in the double knockout.

Our data demonstrate that CD73-derived adenosine through activation of $\mathrm{A}_{2 \mathrm{a}}$ recetors protects against vascular inflammation, monocyte recruitment and neointima formation. This adenosine is also important in limiting the progression of arteriosclerosis.

\section{Platelets}

\section{Congenital and drug-induced $\mathrm{P}_{2} \mathrm{Y}_{12}$ defects in the clinical setting}

\author{
Marco Cattaneo \\ Unità di Ematologia e Trombosi, Ospedale San Paolo, Dipartimento di Medicina, Chirurgia e Odontoiatria, \\ Università di Milano, Milano, Italy \\ marco.cattaneo@unimi.it
}

$\mathrm{P}_{12} \mathrm{Y}_{12}$ plays an important role in platelet function. It mediates ADP-induced platelet aggregation and amplifies platelet secretion induced by release-inducing platelet agonists. Congenital defects of the platelet $\mathrm{P}_{2} \mathrm{Y}_{12}$ receptor is characterized by mild/moderate bleeding tendency. Six patients with congenital $\mathrm{P} 2 \mathrm{Y}_{12}$ defect, belonging to five 
kindred, have been described so far. Five patients had severe deficiency of the protein, while one expressed normal number of dysfunctional $\mathrm{P}_{2} \mathrm{Y}_{12}$ receptors on his platelets. $\mathrm{P} 2 \mathrm{Y}_{12}$ is important in the pathogenesis of arterial thrombosis, since substances inhibiting its function are potent antithrombotic drugs. The thienopyridines ticlopidone and clopidogrel, which irreversibly inactivate the receptor, dramatically decrease the incidence of arterial thrombosis in patients at risk. The extent of inhibition by clopidogrel (and ticlopidine) of ADP-mediated platelet responses varies widely among individuals, and insufficient inhibition has been called "clopidogrel resistance". Both ticlopidine and clopidogrel are a pro-drugs, which need to be metabolized by the liver to their active metabolites with anti-aggregating activity. Therefore, their pharmacological effects can be detected only some time after their first administration and, more importantly, the plasma levels of the active metabolites, and, consequently, the degree of inhibition of platelet aggregation induced by ADP, vary widely among subjects. In published studies, about $50 \%$ of the patients were either clopidogrel non responders or low responders. Interindividual variability in platelet inhibition by clopidogrel correlated well with the metabolic activity of the hepatic cytochrome P450, which activates the pro-drug to its active metabolite. Interference with clopidogrel metabolism by other drugs that are frequently given to patients with atherosclerosis, such as atorvastatin, can increase the number of patients who are resistant to clopidogrel, although this is still a controversial issue. A more recent thienopyridine, prasugrel, is currently under study. Preliminary data suggest that it may accomplish a greater inhibition of platelet aggregation than ticlopidine and clopidogrel and that the interindividual variability in response may be less marked. Cangrelor and AZD6140 are direct antagoinists of the platelet $\mathrm{P}_{2} \mathrm{Y}_{12}$ receptor. Both are currently under further clinical investigation.

\title{
$P 2 Y_{1}$ and $P_{2} X_{1}$ as targets for new antiplatelet agents
}

\author{
C. Gachet \\ INSERM U311, EFS-Alsace, University Louis Pasteur, Strasbourg, France \\ christian.gachet@efs-alsace.fr
}

ADP and ATP play a key role in normal haemostasis as well as in arterial thrombosis where platelet activation is pivotal. Upon activation, the nucleotides are released from the so-called dense granules, and stimulate three separate $\mathrm{P} 2$ receptors, the ATP-gated $\mathrm{P} 2 \mathrm{X}_{1}$ cation channel and the two ADP receptors $\mathrm{P}_{2} \mathrm{Y}_{1}$ and $\mathrm{P}_{2} \mathrm{Y}_{12}$. The $\mathrm{P} 2 \mathrm{X}_{1}$ receptor is involved in platelet shape change, activation by collagen and thrombus formation under high shear. The $\mathrm{P}_{2} \mathrm{Y}_{1}$ receptor initiates platelet aggregation through mobilisation of intracellular calcium stores while the $\mathrm{P}_{2} \mathrm{Y}_{12}$ receptor amplifies the responses to all agonists and stabilizes the aggregates. Among the platelet $\mathrm{P} 2$ receptors, the $\mathrm{P}_{2} \mathrm{Y}_{12}$ receptor is the molecular target of the efficient antiplatelet drug clopidogrel and several competitive antagonists which are under clinical evaluation. Its expression seems to be limited to the megakaryocytic lineage although several other cell types have been shown to express $\mathrm{P}_{2} \mathrm{Y}_{12}$ transcripts. It is thus a well established target for antiplatelet drugs. Less is known concerning the $\mathrm{P}_{2} \mathrm{X}_{1}$ and the $\mathrm{P} 2 \mathrm{Y}_{1}$ receptors which are broadly expressed in many tissues. However, studies in knock-out mice and experimental thrombosis models using selective antagonists have shown that both the $\mathrm{P} 2 \mathrm{X}_{1}$ and the $\mathrm{P} 2 \mathrm{Y}_{1}$ receptors are potential targets for new antithrombotic drugs.

$\mathrm{P}_{2} \mathrm{Y}_{1}$ deficient mice display increased resistance to thromboembolism induced either by a mixture of collagen and adrenaline or by tissue factor. Moreover, IV administration to mice of the P2 $\mathrm{Y}_{1}$ antagonists MRS2179 or MRS2500 similarly results in prolongation of the bleeding time, inhibition of ex vivo platelet aggregation to ADP and resistance to thromboembolism. Models of localized arterial thrombosis have also been used to further evaluate the $\mathrm{P}_{2} \mathrm{Y}_{1}$ receptor as a target for antiplatelet therapy. In a model of superfusion of ferric chloride to induce injury of mesenteric arterioles and intravital microscopy examination it could be shown that P2 $\mathrm{Y}_{1}$ inhibition was as efficient as $\mathrm{P}_{2} \mathrm{Y}_{12}$ inhibition. Interestingly, the treatment of $\mathrm{P}_{2} \mathrm{Y}_{1}$ deficient mice by clopidogrel resulted in additive inhibition of thrombosis, suggesting that a combination of drugs targeting both $\mathrm{P}_{2} \mathrm{Y}_{1}$ and $\mathrm{P} 2 \mathrm{Y}_{12}$ could be of benefit. A second model of localized arterial thrombosis consists in laser injury of mesenteric arterioles. In this case, $\mathrm{P}_{2} \mathrm{Y}_{1}$ deficient mice or MRS2500 treated mice again displayed reduced thrombosis as compared to the wild type but to a lesser extend as compared to mice treated with maximal doses of clopidogrel. Again, the combination of MRS2500 with clopidogrel resulted in additive inhibition of thrombosis. $\mathrm{P}_{2} \mathrm{X}_{1}$ deficient mice similarly display resistance to all these experimental thrombosis models, including systemic thromboembo- 
lism and localized thrombosis. Moreover, the suramin analog NF449 which displays some selectivity for $\mathrm{P}_{2} \mathrm{X}_{1}$ as been shown to reduce thrombosis in vivo.

Altogether, these results indicate that both $\mathrm{P} 2 \mathrm{Y}_{1}$ and $\mathrm{P} 2 \mathrm{X}_{1}$ are potential targets for new antiplatelet compounds, which should be evaluated now in experimental thrombosis in higher animals.

1. Gachet C. Annu Rev Pharmacol Toxicol 2006

2. Léon et al., J Clin Invest 1999

3. Léon et al., Circulation 2001

4. Hechler et al., J Exp Med 2003

5. Lenain et al., J Thromb Haemost 2003

6. Hechler et al., JPET 2005

7. Hechler et al., JPET 2006

\section{Purinoceptor-evoked signalling via $\mathbf{P 2} \mathrm{X}_{1}$ and other ion channels in the platelet}

Martyn Mahaut-Smith $^{1}$, Gwen Tolhurst ${ }^{1}$, C.Y. Eleanor Fung ${ }^{1}$, Richard N. Carter ${ }^{1}$, Catherine Vial ${ }^{2}$, Catherine Leon $^{3}$, Christian Gachet ${ }^{3}$, and Richard J. Evans ${ }^{2}$

${ }^{1}$ Department of Physiology, University of Cambridge, Cambridge, CB2 3EG, UK

${ }^{2}$ Department of Cell Physiology \& Pharmacology, University of Leicester, Leicester, LE1 9HN, UK

${ }^{3}$ Institut National de la Sante et de la Recherche Medicale (INSERM) U.311, EFS-Alsace 10, Strasbourg Cedex, 67065, France

mpm11@cam.ac.uk

The role of ion channels in platelet function has proven difficult to investigate due to the challenge of conducting direct electrophysiological recordings from these small and fragile cell fragments. As the megakaryocyte must generate most, if not all proteins of the anuclear platelet, the giant precursor cell provides a useful means to study the regulation and identity of platelet ion channels. Indeed, using confocal fluorescence imaging of fibrinogen binding to murine megakaryocytes, we show that ADP-evoked activation of $\alpha_{\mathrm{IIb}} \beta_{3}$ requires co-stimulation of P2Y and $\mathrm{P}_{2} \mathrm{Y}_{12}$ receptors as is well established in platelets. Under whole-cell patch clamp, hexokinase-purified ADP stimulated multiple phases of inward currents, which conduct both $\mathrm{Na}^{+}$and $\mathrm{Ca}^{2+}$ into the cell. All currents were dependent upon the presence of $\mathrm{P}_{2} \mathrm{Y}_{1}$ receptors, although $\mathrm{P}_{2} \mathrm{Y}_{12}$ receptors played a synergistic role through a $\mathrm{PI}$ 3-kinase-dependent pathway. The main conductance showed both early transient and delayed sustained phases, which mirrored the intracellular $\mathrm{Ca}^{2+}$ increase. In addition, $\mathrm{P} 2 \mathrm{X}_{1}$ receptor currents appeared as multiple transient currents, indicating that ATP secretion stimulates this non-selective cation channel in a repetitive, focal manner. Transient receptor potential (TRP) ion channels represent candidates for the direct P2Y receptor-dependent current and screening by RT-PCR from individually selected megakaryocytes indicates that TRPC6 is the dominant message amongst the TRPs known to be stimulated by phospholipase-C activity. To examine the relative role of $\mathrm{P} 2 \mathrm{X}_{1}$ receptors as an autocrine pathway for $\mathrm{Ca}^{2+}$ influx in human platelets, we established pharmacological conditions that selectively block this pathway (NF449 or $\alpha, \beta$ meATP predesensitisation). Multiple platelet agonists utilised the $\mathrm{P} 2 \mathrm{X}_{1}$ receptor as a major pathway by which they increase $\left[\mathrm{Ca}^{2+}\right]_{\mathrm{i}}$. The maximal reduction of peak $\left[\mathrm{Ca}^{2+}\right]_{\mathrm{i}}$ increases following $\mathrm{P} 2 \mathrm{X}_{1}$ inhibition was $48.3 \pm 1.3 \%, 75.5 \pm 8.8 \%, 65.3 \pm 3.1 \%$ and $30.9 \pm$ $6.6 \%$ for thrombin $\left(0.03 \mathrm{Uml}^{-1}\right)$, collagen $\left(0.5 \mu \mathrm{g} \mathrm{ml}^{-1}\right)$, U46619 (thromboxane $\mathrm{A}_{2}$ analogue, $\left.1.0 \mu \mathrm{M}\right)$ and ADP $(30 \mu \mathrm{M})$, respectively. We conclude that $\mathrm{P}_{2} \mathrm{X}_{1}$ receptors represent an important and widespread means of elevating $\mathrm{Ca}^{2+}$ in the platelet but that $\mathrm{P}_{2} \mathrm{Y}_{1 / 12}$ receptors stimulate another non-selective cation channel, most likely TRPC6, with a role in ADP-evoked $\mathrm{Ca}^{2+}$ influx.

Supported by the British Heart Foundation, Wellcome Trust and Medical Research Council 


\title{
Nucleotide Receptors and Cellular Functions
}

\section{Cardiovascular ATP release: Focus on the red blood cell}

\author{
David Erlinge
}

Department of Cardiology, Lund University, Sweden

Previous studies have demonstrated that nucleotides can be released from various sources in the cardiovascular system such as platelets, sympathetic nerves, endothelial cells and cardiomyocytes. We have focused on the regulation of nucleotide release in man, and the possible clinical implications.

ATP is released from red blood cells (RBC) when intracellular cAMP levels are increased in response to reductions in oxygen tension and $\mathrm{pH}$. The released ATP then binds to P2Y receptors on the endothelium and stimulates vasodilatation. Because blood consists of approximately $40 \%$ RBCs, containing a 1000-fold higher ATP concentration than plasma ( $\mathrm{mM}$ vs. $\mu \mathrm{M})$, even a minor release of ATP from the high intracellular concentrations could have major circulatory effects and may need to be regulated. We found that the ATP degradation product ADP inhibits ATP release by acting on $\mathrm{P}^{2} \mathrm{Y}_{13}$ receptors that inhibit cAMP in the RBC [1]. This negative feedback system could be important in the control of plasma ATP levels and tissue circulation. The first selective P2Y 13 antagonist MRS2211, blocked the ADP induced inhibition of ATP release in micromolar concentrations, giving us a tool to evaluate the importance of $\mathrm{P}_{2} \mathrm{Y}_{13}$ negative feedback pathway for circulatory control.

Nucleotide release in the heart comes from both cardiac myocytes and endothelial cells, but the RBC may also be an important source. In patients with acute myocardial infarction, we found that both ATP and UTP are released and may have effect on cardiac function and coronary flow [2]. The ATP receptor P2Y $\mathrm{Y}_{11}$, the UTP receptor $\mathrm{P}_{2} \mathrm{Y}_{2}$ and the UDP receptor $\mathrm{P}_{2} \mathrm{Y}_{6}$ are expressed in human myocardium and mediate inotropic effects [2, 3]. The prominent increase in blood flow following ischemia (reactive hyperemia) is to a major part mediated by ADP acting on endothelial $\mathrm{P}_{2} \mathrm{Y}_{1}$ receptors [4].

In the peripheral vasculature we found that purines and pyrimidines may provide a link between glucose and diabetic microvascular disease [5]. High glucose induced NFAT activation in cerebral microvessels by a mechanism totally blocked by apyrase and by $50 \%$ by the $\mathrm{P}_{2} \mathrm{Y}_{6}$ receptor blocker MRS2578, indicating that the vascular growth factors ATP, UTP and UDP are released in response to high glucose and may stimulate intracellular reactions associated with microvascular disease.Inflammation in the atherosclerotic plaque is important for destabilisation and plaque rupture that is the major cause of myocardial infarction. We have indirect evidence that ATP is released in the plaque. The common Ala-87-Thr polymorphism of the ATP receptor $\mathrm{P}_{2} \mathrm{Y}_{11}$ that is present in one fifth of the population was examined in 1200 patients with myocardial infarction and 2400 controls and markedly increased the risk of myocardial infarction, especially for early myocardial infarction and patients with family history. Interestingly, the inflammatory marker C-reactive protein was significantly increased in carriers of the Ala-87-Thr polymorphism, indicating an inflammatory mechanism.

In conclusion, purines and pyrimidines seem to be released and involved in most aspects of human cardiovascular pathophysiology.

1. Wang et al. Circ Res 2005.

2. Wihlborg et al. Circ Res 2006.

3. Balogh et al. J Mol Cell Card 2005.

4. Olivecrona et al. Purinergic Sign 2005.

5. Nilsson et al. ATVB 2005. 


\title{
Joachim Jankowski; Charite, Berlin "Uridine adenosine tetraphosphate: a novel endothelium- derived vasoconstrictive factor"
}

\author{
J. Jankowski \\ Charitè, Berlin
}

Beyond serving as a mechanical barrier, the endothelium shows important regulatory functions. The discovery of nitric oxide (NO) revolutionized our understanding of vasoregulation. In contrast, the identity of endothelialderived vasoconstrictive factors (EDCFs) remains unclear. The supernatant obtained from mechanically stimulated human endothelial cells obtained from dermal vessels elicited a vasoconstrictive response in an isolated perfused rat kidney. Endothelial-derived vasoconstriction in the isolated perfused rat kidney was decreased by a purinoceptor blocker more than by an endothelin receptor blocker. The nucleotide, uridine adenosine tetraphosphate $\left(\mathrm{Up}_{4} \mathrm{~A}\right)$ was isolated from the supernatant of stimulated human endothelium and identified by mass spectrometry. $\mathrm{Up}_{4} \mathrm{~A}$ most likely exerts vasoconstriction predominantly via $\mathrm{P} 2 \mathrm{X} 1$ receptors, and probably also via $\mathrm{P} 2 \mathrm{Y} 2$ and $\mathrm{P} 2 \mathrm{Y} 4$ receptors. In healthy subjects $\mathrm{Up}_{4} \mathrm{~A}$ plasma concentrations are found which cause vasoconstriction. Stimulation with adenosine $5^{\prime}$-triphosphate (ATP), uridine $5^{\prime}$-triphosphate (UTP), acetylcholine, endothelin, A23187 and mechanical stress release $\mathrm{Up}_{4} \mathrm{~A}$ from endothelium, suggesting that $\mathrm{Up}_{4} \mathrm{~A}$ contributes to vascular autoregulation. $\mathrm{Up}_{4} \mathrm{~A}$ is the first dinucleotide containing both purine and pyrimidine moieties isolated from living organisms. We conclude that $\mathrm{Up}_{4} \mathrm{~A}$ is a novel potent non-peptidic EDCF. Its vasoactive effects, plasma concentrations and its release upon endothelial stimulation strongly suggest that $\mathrm{Up}_{4} \mathrm{~A}$ has a functional vasoregulatory role.

\section{$\mathrm{P} 2 \mathrm{Y}$ and $\mathrm{P} 2 \mathrm{X}$ receptors and their role in exocrine pancreas}

Ivana Novak

August Krogh Building, Institute of Molecular Biology and Physiology, University of Copenhagen, Copenhagen, Denmark

inovak@aki.ku.ak

The exocrine pancreas secretes digestive enzymes and fluid rich in $\mathrm{HCO}_{3}{ }^{-}$and poor in $\mathrm{Ca}^{2+}$. These secretions originate from acini and ducts in response to classical secretagogues cholecystokinin and secretin. The function of these epithelia needs to be coordinated, such that digestive enzymes are not prematurely activated or that calcium stones do not form. We propose that along the acini-duct axis ATP and other components of the purinergic cascade mediate short-term regulation of secretion. The following findings lay the basis for these proposals. Acini release ATP into the interstitium and most importantly into the lumen leading to pancreatic ducts [1]. The initial ATP concentrations are in the high miromolar range, but since secretion also contains specific nucleotidases, CD39 and CD73, these would regulate concentration of nucleotides/nucleosides along the ductal tree [2].

Acini themselves are relatively poor in functional P2 receptors. In contrast, pancreatic ducts, which secrete $\mathrm{HCO}_{3}{ }^{-}$rich fluid, express several types of functional $\mathrm{P} 2$ receptors on the luminal and basolateral membranes [3]. On cellular level, $\mathrm{P} 2 \mathrm{X}_{4}$ and $\mathrm{P} 2 \mathrm{X}_{7}$ receptors stimulate $\mathrm{Na}^{+}$and $\mathrm{Ca}^{2+}$ influx, but seem to be without prominent effects on $\mathrm{Cl}^{-}$and $\mathrm{H}^{+} / \mathrm{HCO}_{3}{ }^{-}$transport usually associated with fluid secretion [3,4]. However, possibly via ERK1/ERK2 signalling pathways, they could be modulators of secretion. Initial studies on mice stimulated with pilocarpine in vivo indicate that exocrine gland function is affected in $\mathrm{P}_{2} \mathrm{X}_{7}$ knockout animals.

Pancreatic ducts also express metabotrophic $\mathrm{P}_{2} \mathrm{Y}_{2}$ and $\mathrm{P}_{2} \mathrm{Y}_{4}$ receptors, which in many epithelia, including human pancreatic duct cell lines, can stimulate $\mathrm{Ca}^{2+}$ activated $\mathrm{Cl}^{-}$channels [5]. In native rat pancreatic ducts, patch-clamp experiments indicated inhibition of $\mathrm{K}^{+}$conductance [3]. In a recent study we found that pancreatic ducts expresses $\mathrm{Ca}^{2+}$ activated $\mathrm{K}^{+}$channels of a big conductance (BK, maxi- $\mathrm{K}^{+}$channels) and intermediate conductance (IK). We used the oocytes expression and electophysiological recordings to study the interaction between individual $\mathrm{P} 2 \mathrm{Y}$ receptors and $\mathrm{Ca}^{2+}$-activated $\mathrm{K}^{+}$channels. The most interesting result was that stimulation of $\mathrm{P}_{2} \mathrm{Y}_{2}$ receptors inhibited BK [6]. Thus, as in the native epithelium, $\mathrm{P}_{2} \mathrm{Y}_{2}$ receptors inhibit $\mathrm{BK}$, which would down-regulate secretion, possibly preventing over-distension of the duct. In summary, elucidation of 
$\mathrm{P} 2 \mathrm{X}$ and $\mathrm{P} 2 \mathrm{Y}$ receptor regulated processes in pancreatic ducts will provide a better understanding of epithelial secretion in health and disease, such as in cystic fibrosis.

The projects were supported by the Danish Medical and Science Research Councils and the Lundbeck Foundation.

1. Sørensen CE, Novak I. J Biol Chem 2001; 276: 329525-32.

2. Yegutkin GG, Jalkanen S, Novak I. this volume

3. Hede SE, Amstrup J, Christoffersen BC, Novak I, J Biol Chem 1999; 274: 31784-91.

4. Henriksen, K. L. and I. Novak. Cell.Physiol.Biochem. 2003;13: 93-102.

5. Lazarowski ER, Boucher RC, Harden TK, Mol Pharmacol 2003; 64: 785-95.

6. Hede SE, Amstrup J, Klaerke DA, Novak I. Pflügers Arch Eur J Physiol 2005; 450: 429-36.

\section{Purinergic signalling triggers development of the eye}

Nicholas Dale

Department of Biological Sciences, University of Warwick, Coventry, UK

N.E.Dale@warwick.ac.uk

Abstract not received

\section{Round Table on Purinome-based drugs}

\section{Allosteric activators of glucokinase: The development of cyclopropane-derived agents and their potential for use in the treatment of type two diabetes}

Jochen Ammenn ${ }^{1}$, David G. Barrett ${ }^{1}$, Martina Berg ${ }^{1}$, Martin B. Brenner ${ }^{1}$, Stephen L. Briggs ${ }^{2}$, Annie Delaunois ${ }^{3}$, Jim D. Durbin ${ }^{2}$, Alexander M. Efanov ${ }^{1}$, Ulrich Giese ${ }^{1}$, Gema Sanz Gil ${ }^{1}$, Jesper Gromada ${ }^{1}$, Haihong Guo ${ }^{2}$, Ulrike Hary ${ }^{1}$, Stefan Heuser ${ }^{1}$, Astrid Kahl ${ }^{1}$, Carsten Ott $^{1}$, Mark Radloff ${ }^{1}$, Rainer Riedl $^{1}$, Ulrike Roettig ${ }^{1}$, Horst Schwetzler ${ }^{1}$, Erich Seger ${ }^{1}$, Sabine Sewing ${ }^{1}$, Birgit Sommer ${ }^{1}$, Yong Wang ${ }^{1}$, Jutta Wanner ${ }^{1}$, Andreas Weichert ${ }^{1}$, Andrea Zaliani ${ }^{1}$, and Christian Zechel ${ }_{-}^{1}$

${ }^{1}$ Lilly Research Laboratories, 22419 Hamburg, Germany

${ }^{2}$ Lilly Research Laboratories, Indianapolis, Indiana 46285

${ }^{3}$ Lilly Development Centre, 1348 Mont-Saint-Guibert, Belgium

barrett_david_g@lilly.com

The glucose-sensing enzyme glucokinase (GK) plays a key role in glucose metabolism. A member of the hexokinase family, it catalyzes the first step in glycolysis, phosphorylation of glucose to glucose 6-phosphate. It is a unique hexokinase in that it has a low affinity for glucose, demonstrates non-Michaelis-Menten kinetics and displays no inhibition by the product of the reaction [1]. These particular features make GK an ideal sensor of physiological changes in blood glucose levels, translating them into changes in the metabolic status of the cell.We report here the effects of a novel small molecule glucokinase activator, LY2121260, which enhances GK activity via binding to an allosteric site located in the hinge region of the enzyme [2]. We discuss the affect of LY2121260 on the glucose affinity of the enzyme and the velocity of the reaction, and present crystallographic data that support its mechanism of action. We show that LY2121260 stimulates insulin secretion in pancreatic â-cells and increases glucose use in rat hepatocytes in a glucose-dependent manner. Furthermore, animals treated with LY2121260 show an improved glucose tolerance following an oral glucose challenge. We also describe some structure-activity studies within this class of GK activators. Our results support the concept that GK activators increase both insulin secretion and hepatic glucose use and in doing so may prove to be effective agents for the control of blood glucose levels in patients with type 2 diabetes. 


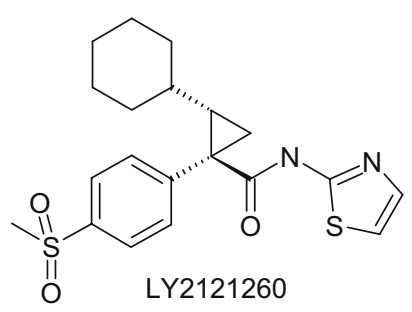

1. Matschinsky FM. Diabetes 1996; 45(2): 223-41.

2. Efanov AM et al. Endocrinology 2005; 146(9): 3696-701.

\section{Beyond purinergic receptors: Drugging the rest of the purine-binding proteome}

S. Hall, A. Barabasz, T. Barta, J. Daw, L. Dubois, J. Eaves, P. Fadden, B. Foley, T. Freed, L. Geng, G. Hanson, K. Hardemann, L. Hinkley, M. Jenks, M. Hu, K. Huang, M. Lewis, L. Liu, W. Ma, J. Otto, J. Partridge, B. Pronk, J. Rice, A. Scott, M. Silinski, P. Steed, J. Veal, K. Verleysen, R. Ware, and T. Wadkins

Serenex, Inc. 323 Foster Street, Durham, NC, 27701 USA

shall@serenex.com

Purinergic receptors represent an attractive class of druggable targets within the purine-binding proteome, yet there are many other enzyme families of interest as therapeutic targets within this large superfamily. We estimate that the purine-binding proteome contains at least 2000 members, with kinases composing the largest fraction( $25 \%)$ of this group. We report here a novel approach to screen hundreds of these targets in parallel, thus providing an efficient way to probe compound libraries as ligands to a broad variety of enzyme classes. We constructed and screened a targeted library of 8000 compounds using a novel affinity displacement assay (referred to as proteome mining). Multiple tissues were used as the source of native purine-binding proteins to profile this library. Hits were obtained for nearly 100 targets, including well validated targets such as dihydrofolate reductase and multiple kinases (e.g. CDK2, Her2, PDK1, AurA), and less well-validated targets such as the kinases Fer and Nek9. Importantly, the method also provided affinity information on potential toxicity targets (e.g. phosphorylase kinase, pyruvate carboxylase, glucose-6-phosphate dehydrogenase). Thus, this approach provides primary target affinity information as well as selectivity data across the entire purine-binding proteome. In addition, affinities of compounds for their protein targets could be determined directly from the same assay and these measurements were sufficiently quantitative and reproducible to drive the development of structure-activity relationships. Unexpectedly, selectivity within a gene family was not predictive of selectivity between gene families across the purine-binding proteome. The application of this screening approach to four diverse targets, dihydrofolate reductase, Hsp90, quinone reductase 2, and PI3kinase will be described. In each case, the use of the affinity displacement assay enabled the identification of novel, selective, orally active compounds. Taken together, these results support the use of proteome mining as both an alternative to single target biochemical screens and as a tool to drive SAR development. 


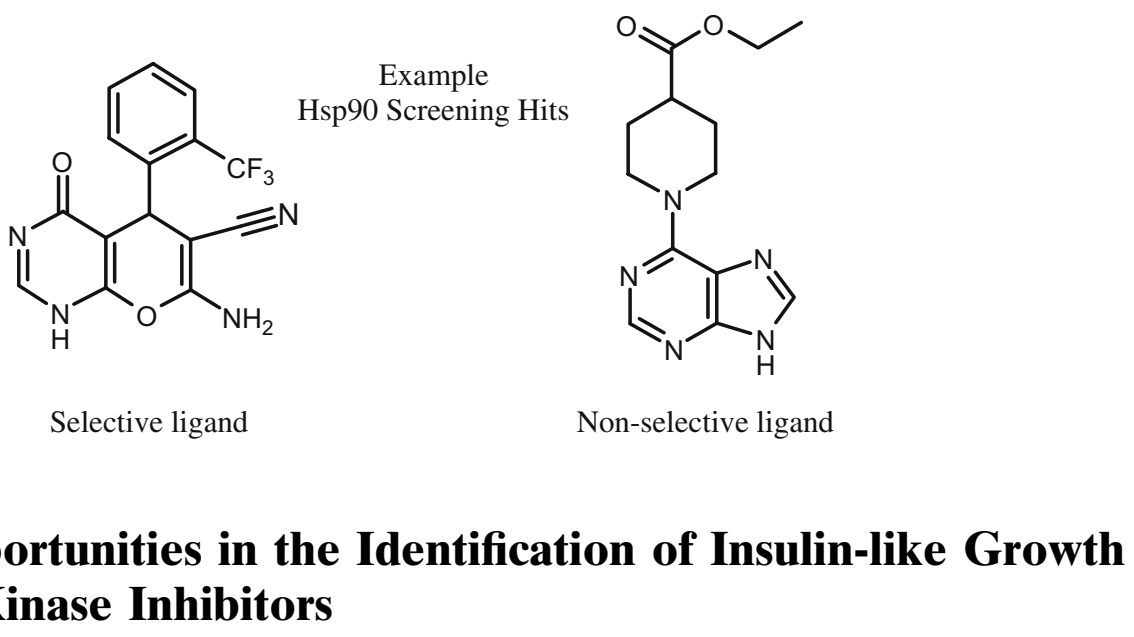

\section{Challenges and Opportunities in the Identification of Insulin-like Growth Factor-I Receptor Kinase Inhibitors}

C. García-Echeverría, M.A. Pearson, T. Meyer, J. Mestan, J. Zimmermann, J. Brueggen, H.-G. Capraro, R. Cozens, D.B. Evans, D. Fabbro, P. Furet, D. Graus Porta, J. Liebetanz, G. Martiny-Baron, S. Ruetz, S. Jacob, and F. Hofmann

Novartis Institutes for BioMedical Research, CH-4002 Basel, Switzerland

carlos.garcia-echeverria@novartis.com

The Insulin-like Growth Factor-I Receptor (IGF-IR) is a member of the insulin receptor family of tyrosine kinases. This transmembrane-spanning protein is composed of two $\alpha$ - and two $\beta$-subunits linked by disulfide bonds. While the $\alpha$-subunits are extracellular, the $\beta$-subunits span the plasma membrane and encompass an intracellular tyrosine kinase domain devoted to the initiation of several signal transduction cascades. Signaling through IGF-IR is initiated upon binding of the cognate ligand-Insulin-like growth factor-I (IGF-I) or II (IGF-II) - to the extracellular domain of the receptor. It is thought that this peptide-protein interaction induces a conformational change that results in auto-transphosphorylation of each $\beta$-subunit at specific tyrosine residues within the intracellular kinase domain and outside the catalytic domain. Activation of the receptor triggers, through docking and/or phosphorylation of several transduction molecules (e.g., IRS-1, IRS-2 or Shc), results in activation of downstream signaling pathways, of which the Ras/Raf/MAPK pathway is primarily responsible for mitogenesis, and the survival PI3K/PKB pathway appears to play a major role in mediating the IGF-IR biological functions.

A broad range of experimental studies have revealed that IGF-IR function is implicated in most of the hallmarks of cancer, but it is probably the anti-apoptotic activity of this receptor that makes its kinase activity an attractive therapeutic target in anti-cancer drug discovery. In this context, the identification of specific lowmolecular mass inhibitors of IGF-IR has proven to be a major challenge for medicinal chemistry due to the high sequence identity at the kinase domains of IGF-IR and InsR (around 84\%) and, in particular, at the ATP-binding pocket $(100 \%$ sequence identity). Selectivity over the InsR is a critical requirement for any IGF-IR kinase inhibitor to avoid disturbing glucose homeostasis. This presentation will cover the identification and characterization of a new series of IGF-IR kinase inhibitors. When used alone or in combination with cytotoxic agents, the synthetic IGF-IR kinase inhibitors suppress tumor growth and prolongs survival of mice with diffuse bone lesions of multiple myeloma. These preclinical findings and additional studies support the potential application of targeted therapeutic strategies directed at IGF-IR in combination with established antitumor modalities or for the treatment of IGFs-responsive neoplasias. Moreover, the selectivity achieved at the cellular level with these IGF-IR inhibitors suggest conformation differences between the native forms of IGF-IR and InsR -from the unactivated to the fully activated form - that can effectively be exploited for drug discovery. 


\section{Kinases: Important drug targets for novel anti-inflammatory therapies}

Alan J. Lewis

Novacell, 31 Technology Drive, Suite 100, Irvine, CA 92618sides " "

alan.lewis@hotmail.com

Protein kinases contribute to a diverse number of cellular processes and are the second most important group of drug targets after G-protein-coupled receptors. Moreover, aberrant kinase activity is implicated in many human diseases in particular those cancer and inflammatory diseases. Of the more than 500 kinases in the human kinome very few are targeted by currently marked drugs, notably the anticancer agents Gleevec (BCR Abl kinase, c-Kit), Tarceva (EGFR) Sorafenib (Raf, VEGFR) and Sutent (EGFR) Oriology remains the most fertile area for kinase inhibitors followed by inflammatory diseases. One reason is that many kinase inhibitors are multi-targeted ("dirty drugs") and the lack of selectivity may be beneficial in cancer to prevent resistance from occurring.

Several kinases including p38MAPK, JNK and IkB kinase-2 (IKK2) have been identified as key targets for a plethora of inflammatory diseases and clinical trials are underway. A major advantage in directing antiinflammatory drug discovery programs against such targets is the ability to modulate multiple inflammatory gene products including cytokines such as TNF and IL-1, cell adhesion molecules as well as enzymes such as Cox-2 and MMPs that are pivotal in the pathology of inflammatory disease.

Many of the current kinase inhibitors were developed from "focused" small molecule kinase libraries that target the ATP-binding site and the availability of crystal structures for several key kinases has greatly facilitated lead optimization. Allosteric strategies have also been used successfully to identify kinase inhibitors notably p38. The family of human protein kinases accounts for most cellular signal transduction and can provides an enormous opportunity to develop new treatments for anti-inflammatory therapies. 


\section{Communications}

\section{$\left[{ }^{3}\right.$ H]Adenine's High Filter Binding Precludes its Use as a Radioligand for Adenine Receptors}

Kai Ye, Thea Mulder-Krieger, Margot W. Beukers and Ad P. Ijzerman

Division of Medicinal Chemistry, Leiden/Amsterdam Center for Drug Research, Leiden University, PO Box 9502, 2300 RA Leiden, The Netherlands

k.ye@lacdr.leidenuniv.nl

An orphan G protein-coupled receptor, rat MrgA10, one of the MAS-related G protein-coupled receptors, has recently been characterized as an adenine receptor [1]. Among the methods used in the de-orphanization process were binding studies with $\left[{ }^{3} \mathrm{H}\right]$ adenine as a radioligand [1]. In subsequent studies from other research groups saturation analysis, kinetic studies and displacement experiments were performed on native tissues such as rat brain cortex with $\left[{ }^{3} \mathrm{H}\right]$ adenine as a radioligand, demonstrating the presence of adenine binding sites in these tissues $[2,3]$.

We initiated a program to address the medicinal chemistry aspects of the adenine receptor, with radioligand binding studies as a primary screen. We prepared membranes from $\mathrm{CHO}$ cells stably transfected with the rat adenine receptor (rAR) kindly donated by IriDM (Scherpenheuvel, Belgium), next to membranes of rat brain cortex. Subsequently, radioligand binding assays with $\left[{ }^{3} \mathrm{H}\right]$ adenine as the radioligand were developed.

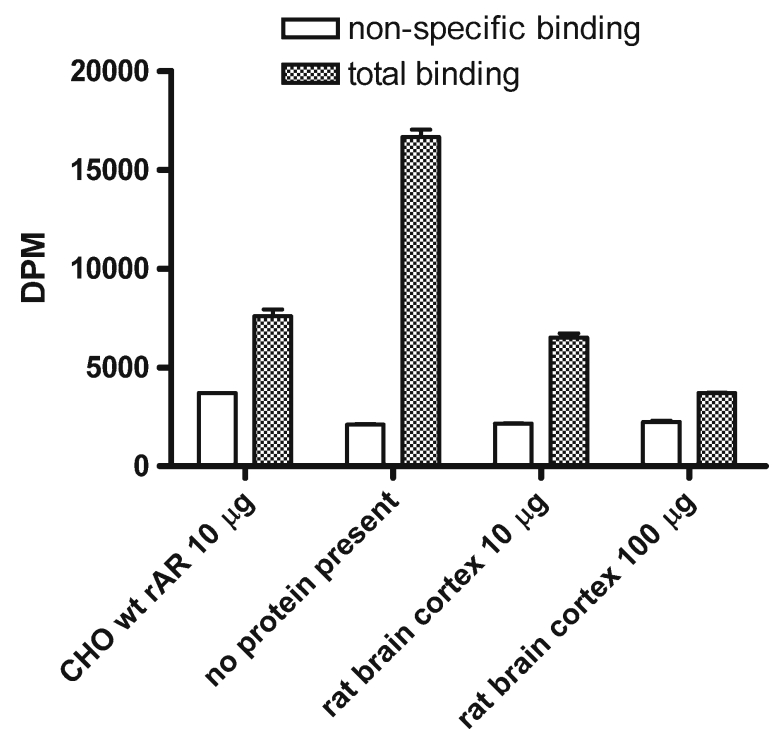

In that process we observed a number of worrying phenomena. Firstly, we observed an unusually rapid association of the radioligand in our kinetic studies, consistent with literature [2]. Secondly, in the displacement assays we learned that decreasing the membrane protein concentration (see Figure for rat brain cortex membranes) led to an increase in "specific" $\left[{ }^{3} \mathrm{H}\right]$ adenine binding, such that in the absence of protein the highest binding was observed. Thirdly, the affinities of adenine and a number of adenine derivatives to compete for $\left[{ }^{3} \mathrm{H}\right]$ adenine filter binding were in good agreement with the values in literature for the adenine receptor [1-3].

In conclusion, radioligand binding studies aimed at the adenine receptor with $\left[{ }^{3} \mathrm{H}\right]$ adenine as the radioligand should be performed with great care, if at all. 


\title{
$\left[{ }^{3}\right.$ H]HEMADO-A New Tritiated Adenosine Receptor Agonist Radioligand
}

\author{
Karl-Norbert Klotz ${ }^{1}$, Nico Falgner ${ }^{1}$, Sonja Kachler ${ }^{1}$, Rosaria Volpini ${ }^{2}$, Diego Dal Ben ${ }^{2}$, Catia Lambe- \\ rtucci $^{2}$, Ram Chandra Mishra ${ }^{2}$, Sauro Vittori ${ }^{2}$ and Gloria Cristalli ${ }^{2}$ \\ ${ }^{1}$ Universität Würzburg, Institut für Pharmakologie und Toxikologie, Versbacher Str. 9, D-97078 Würzburg, Ger- \\ many and ${ }^{2}$ Università di Camerino, Dipartimento di Scienze Chimiche, Via S. Agostino, 1, I-62032 Camerino, Italy \\ klotz@toxi.uni-wuerzbvurg.de
}

$\mathrm{A}_{3}$ adenosine receptors are promising drug targets for a number of conditions like inflammatory diseases including asthma, ischemic injury or certain types of cancer. Consequently, intense efforts in many laboratories are dedicated to the development of $\mathrm{A}_{3}$ selective agonists adn antagonists. A number of radioligands are in use for screening of new compounds in binding assays. In agonist development it is advantageous to use an agonist as the radioligand in order to avoid biphasic competition curves as a high affinity radiolabeled agonist will bind to high affinity binding sites only. In the case of $\mathrm{A}_{3}$ adenosine receptors the two radioligands ${ }^{125}$ I-AB-MECA and $\left[{ }^{3} \mathrm{H}\right]-$ NECA are widely used. A tritiated ligand offers a number of advantages in routine applications. However, $\left[{ }^{3} \mathrm{H}\right] \mathrm{NECA}$ is of limited value owing to the relatively low affinity for human $\mathrm{A}_{3}$ receptors $\left(\mathrm{K}_{\mathrm{i}}\right.$-value $\left.6.2 \mathrm{nM}\right)$, lack of subtype selectivity and its tendency to bind to numerous proteins other than adenosine receptors.

Based on a recently characterized series of potent 2-substituted adenosine receptor agonists we developed a tritiated $\mathrm{A}_{3}$ selective radioligand. The ligand of choice was HEMADO (2-hexyn-1-yl- $N^{6}$-methyladenosine) which exhibits an $\mathrm{K}_{\mathrm{i}}$-value at the human $\mathrm{A}_{3}$ subtype of $1 \mathrm{nM}$. HEMADO is 300 fold selective versus the $\mathrm{A}_{1}$ subtype and 1,100fold selective compared to the $\mathrm{A}_{2 \mathrm{~A}}$ receptor ${ }^{[1]}$. Starting from the precursor 2-hexyn-1-yl-6-iodopurine-9riboside $\left[{ }^{3} \mathrm{H}\right]$ HEMADO was generated by substitution of the 6-iodo with tritiated methylamine. The resulting radioligand has an specific radioactivity of $890 \mathrm{MBq} / \mathrm{mmol}(24 \mathrm{Ci} / \mathrm{mmol})$.

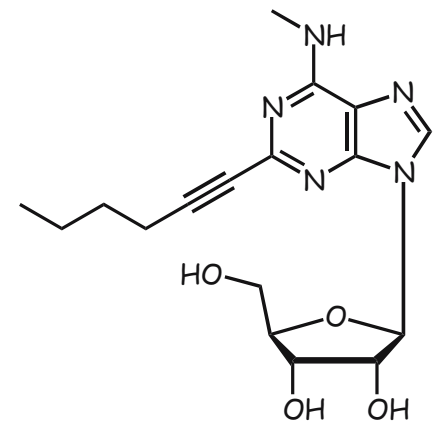

Characterization of $\left[{ }^{3} \mathrm{H}\right]$ HEMADO in radioligand binding studies revealed reversible binding to the human $\mathrm{A}_{3}$ adenosine receptor. In saturation binding studies for the $A_{3}$ subtype a $K_{D}$ value of $1.3 \mathrm{nM}$ was determined. The nonspecific binding at a radioligand concentration of $0.5 \mathrm{nM}$ amounted to $1-2 \%$ of total binding. Competition binding with a panel of agonists and antagonists clearly confirmed the correct $\mathrm{A}_{3}$ pharmacology of the binding site labeled by $\left[{ }^{3} \mathrm{H}\right] \mathrm{HEMADO}$. In concentrations up to $10 \mathrm{nM}$ no specific binding was detectable at any of the other adenosine receptor subtypes.

With $\left[{ }^{3} \mathrm{H}\right]$ HEMADO we present a tritiated high affinity agonist with $\geq 300$ fold $\mathrm{A}_{3}$ selectivity and very low nonspecific binding. $\left[{ }^{3} \mathrm{H}\right] \mathrm{HEMADO}$ is a useful tool for specific screening for $\mathrm{A}_{3}$ receptor agonists and antagonists in radioligand binding assays.

${ }^{1}$ Volpini R, Costanzi S, Lambertucci C, Taffi S, Vittori S, Klotz K-N et al. J Med Chem 2002; 45: 3271-9. 


\title{
$\left[{ }^{3}\right.$ H]-MRE 2029-F20, A Novel Selective Antagonist Radiolig and, for the Pharmacological and Biochemical Characterization of $\mathbf{A}_{2 \mathrm{~B}}$ Receptors
}

\author{
$\underline{\text { Stefania Gessi }}{ }^{1}$, Katia Varani ${ }^{1}$, Stefania Merighi ${ }^{1}$, Elena Cattabriga ${ }^{1}$, Youri Szabadkai ${ }^{2}$, \\ Rosario Rizzuto $^{2}$, Karl Norbert Klotz ${ }^{3}$, Edward Leung ${ }^{4}$, Stephen Mac Lennan ${ }^{4}$, Pier Giovanni Baraldi ${ }^{5}$ \\ and Pier Andrea Borea ${ }^{1}$ \\ ${ }^{1}$ Dpt of Clin. and Exp. Med., Pharmacology Unit and "Interdisciplinary Center for the Study of Inflammation \\ (ICSI)," ${ }^{2}$ Gen. Pat. Unit and ${ }^{3}$ Universitat Würzburg, Germany; ${ }^{4}$ King Pharmaceuticals Research \& Development, \\ Cary, North Carolina and ${ }^{5}$ Dpt of Pharm. Sci., University of Ferrara, 44100 Italy \\ gss@unife.it
}

This work investigates the pharmacological and biochemical properties of $\mathrm{A}_{2 \mathrm{~B}}$ adenosine receptors in $\mathrm{hA} \mathrm{A}_{2 \mathrm{~B}} \mathrm{HE}-$ K293 cells and in peripheral blood cells by using a new potent 8-pyrazole xanthine derivative, $\left[{ }^{3} \mathrm{H}\right]-\mathrm{N}$-benzo[1,3]dioxol-5-yl-2-[5-(1,3-dipropyl-2,6-dioxo-2,3,6,7-tetrahydro-1H-purin-8-yl)-1-methyl-1H-pyrazol-3-yl-oxy]-acetamide] $\left(\left[{ }^{3} \mathrm{H}\right]-\mathrm{MRE} 2029-\mathrm{F} 20\right)$, that shows high affinity and selectivity for $h A_{2 B}$ versus $h A_{1}, h_{2 A}$ and $h A_{3}$ subtypes. In particular MRE 2029-F20 displays low affinity for the human $\mathrm{A}_{1}$ receptor $(\mathrm{Ki}=245 \pm 31 \mathrm{nM})$ and no significant affinity for the human $A_{2 A}$ and $A_{3}$ subtypes $(\mathrm{Ki}>1,000 \mathrm{nM}) .\left[{ }^{3} \mathrm{H}\right]-\mathrm{MRE} 2029-\mathrm{F} 20$ bound specifically to the $\mathrm{hA}_{2 \mathrm{~B}}$ receptor stably transfected in HEK293 cells with $\mathrm{K}_{\mathrm{D}}$ of $2.8 \pm 0.2 \mathrm{nM}$ and $\mathrm{B}_{\max }$ of $450 \pm 42 \mathrm{fmol} / \mathrm{mg}$ protein. Saturation experiments of $\left[{ }^{3} \mathrm{H}\right]-\mathrm{MRE} 2029-\mathrm{F} 20$ binding in human neutrophils and lymphocytes detected a single high affinity binding site with $\mathrm{K}_{\mathrm{D}}$ of $2.4 \pm 0.5,2.7 \pm 0.7 \mathrm{nM}$ and $\mathrm{Bmax}$ of $79 \pm 10,54 \pm 8 \mathrm{fmol} / \mathrm{mg}$ of protein, respectively, in agreement with real-time RT-PCR studies showing the presence of $A_{2 B}$ mRNA. The rank order of potency of typical adenosine ligands with recombinant $\mathrm{h} \mathrm{A}_{2 \mathrm{~B}}$ receptors was consistent with that typically found for interactions with the $\mathrm{A}_{2 \mathrm{~B}}$ subtype and was also similar in peripheral blood cells. NECA stimulated cAMP accumulation in both $\mathrm{hA}_{2 \mathrm{~B}} \mathrm{HEK} 293$ and native cells whereas phospholipase $\mathrm{C}$ activation was observed in recombinant receptors and endogenous subtypes expressed in neutrophils but not in lymphocytes. MRE 2029-F20 revealed to be a potent antagonist in counteracting the agonist effect in both signal transduction pathways. In conclusion $\left[{ }^{3} \mathrm{H}\right]-$ MRE 2029-F20 is a selective and high affinity radioligand for the $h \mathrm{~A}_{2 \mathrm{~B}}$ adenosine subtype and in this work it has been used to compare the presence and functional coupling of $\mathrm{A}_{2 \mathrm{~B}}$ receptor in a recombinant system and in peripheral blood cells.

\section{2,6,8-Trisubstituted-1-Deazapurines as Adenosine $A_{1}$ Receptor Antagonists}

Jacobien K. von Frijtag Drabbe Künzel, Lisa C. W. Chang, Thea Mulder-Krieger, Joost Westerhout, Thomas Spangenberg, Johannes Brussee and Adriaan P. IJzerman

Leiden/Amsterdam Center for Drug Research, Division of Medicinal Chemistry, P.O. Box 9502, 2300 RA Leiden, The Netherlands

ijzerman@lacdr.leidenuniv.nl

Exploration of two particular pyrimidine and purine series in previous publications $[1,2]$ led to a refinement of a pharmacophore defined for antagonists of the adenosine $A_{1}$ receptor. This contribution details the adoption of these new criteria to produce a series of 1-deazapurines with consistently high affinity for the adenosine $\mathrm{A}_{1}$ receptor. 1-Deazapurines (otherwise known as $3 H$-imidazo[4,5-b]pyridines) are structurally very similar to the purines, however, in a synthetic sense they posed an array of challenges, mainly as a result of the reduced reactivity about the 6-membered ring. The desired double aromatic substituents at the 2- and 6-positions were amongst the most troublesome features to incorporate. An eventual adaptation of a known route resulted in a series with five of the derivatives displaying $\mathrm{K}_{\mathrm{i}}$ values in the sub-nanomolar range. Thus LUF5983 displayed an affinity of $0.87 \mathrm{nM}$ at the human adenosine $\mathrm{A}_{1}$ receptor with $>200$-fold selectivity vs. human $\mathrm{A}_{2 \mathrm{~A}}$ and $\mathrm{A}_{3}$ receptors. The compound was shown to behave as a potent antagonist/inverse agonist in cAMP second messenger studies in cells expressing the human adenosine $\mathrm{A}_{1}$ receptor. 
<smiles>[R2]c1cc([R1])c2nc([R3])[nH]c2n1</smiles>

target compounds<smiles>CCC(CC)c1nc2c(-c3ccccc3)cc(-c3ccccc3)nc2[nH]1</smiles>

LUF5983

1. Chang LCW et al. J Med Chem 2004; 47: 6529-40

2. Chang LCW et al. J Med Chem (in press)

\title{
2-Arylpyrazolo[3,4-c]quinolin-4-Heteroaroylamines as Potent and Selective Human $A_{3}$ Adenosine Receptor Antagonists. Synthesis and Biological Evaluation
}

\author{
Daniela Catarzi $^{\mathrm{a}}$, Vittoria Colotta ${ }^{\mathrm{a}}$, Flavia Varano ${ }^{\mathrm{a}}$, Francesca Capelli ${ }^{\mathrm{a}}$, Ombretta Lenzi $^{\mathrm{a}}$ and Katia \\ Varani $^{\mathrm{b}}$ \\ ${ }^{a}$ Dipartimento di Scienze Farmaceutiche, Università di Firenze, via U. Schiff 6, 50019, Sesto Fiorentino (FI). ${ }^{b}$ Di- \\ partimento di Medicina Clinica e Sperimentale, Sezione di Farmacologia, Università di Ferrara, via Fossato di \\ Mortara 17-19, 44100 Ferrara \\ daniela.catarzi@unifi.it
}

Adenosine mediates a wide variety of physiological effects by interacting with four receptor subtypes: $A_{1}, A_{2 A}$, $A_{2 B}$ and $A_{3}$. All four adenosine receptor (AR) subtypes are coupled via G-protein to the adenylate cyclase in either an inhibitory $\left(A_{1}\right.$ and $\left.A_{3}\right)$ or stimolatory manner $\left(A_{2 A}\right.$ and $\left.A_{2 B}\right)$ [1]. AR antagonists have attracted great attention for their potential therapeutic use, and the $A_{3} A R$ antagonists are sought as potential anti-inflammatory and anti-asthmatic agents [2]. In the last few years in our laboratory, much effort has been directed toward the synthesis of tricyclic heteroaromatic systems rationally designed as antagonists of the $\mathrm{hA}_{3} \mathrm{AR}$ subtype. In a recent paper we reported the study on 2-arylpyrazolo[3,4-c]quinolin-4-amines which were endowed with nanomolar $\mathrm{h} \mathrm{A}_{3}$ affinity [3]. On this class of derivatives, the presence of acyl residues on the 4-amino group produces profitable effects both in terms of $\mathrm{hA}_{3}$ affinity and selectivity [3,4]. On this basis, we decided to synthesize a new set of 2-arylpyrazolo[3,4-c]quinolin-4-amines in which diverse heteroaroyl moieties were introduced on the 4-amino group.

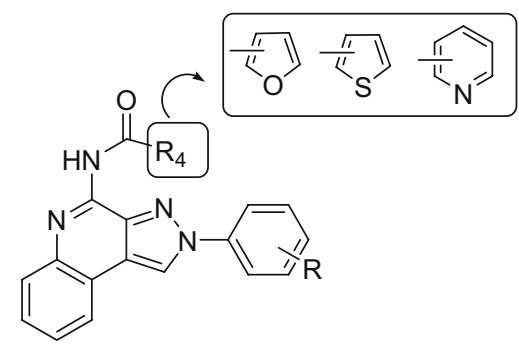

Affinities of the new derivatives at $\mathrm{hA}_{1}, \mathrm{hA}_{2 \mathrm{~A}}$ and $\mathrm{hA}_{3} \mathrm{AR}$ and their inhibitory effects on NECA-stimulated cAMP production in $\mathrm{hA}_{2 \mathrm{~B}} \mathrm{CHO}$ cells were determined. The obtained results show that the 2-arylpyrazolo[3,4c]quinoline-4-heteroaroylamines are potent and selective $\mathrm{hA}_{3}$ antagonists, indeed they possess $\mathrm{hA}_{3}$ nanomolar affinities while they are completely inactive at the other three AR subtypes.

1. Fredholm, B.B.; Ijzerman, A.P.; Jacobson, K.A.; Klotz, K.N.; Linden, J. Pharmacol. Rev. 2001, 53, 527-552.

2. Poulsen, S.A.; Quinn, R.J. Bioorg. Med. Chem. 1998, 6, 619-641.

3. Calotta, V.; Catarzi, D.; Varano, F.; Cecchi, L.; Filacchioni, G.; et al. J Med Chem, 2000, 43, 3118-3124.

4. Unpublished results reported in a Poster Presentation, 8th International Symposium on Adenosine and Adenine Nucleotides, May 24-28, 2006, Ferrara. 


\title{
2-Arylpyrazolo[3,4-c]quinolin-4-Acylamines as Potent and Selective Human $\mathbf{A}_{3}$ Adenosine Receptor Antagonists. Synthesis, Biological Evaluation and Molecular Modeling Studies
}

\author{
$\underline{\text { Vittoria Colotta }}^{1}$, Daniela Catarzi ${ }^{1}$, Flavia Varano ${ }^{1}$, Francesca Capelli ${ }^{1}$, Ombretta Lenzi $^{1}$, Letizia Trin- \\ cavelli $^{2}$ and Stefano Moro ${ }^{3}$ \\ ${ }^{1}$ Dipartimento di Scienze Farmaceutiche, Università di Firenze, via U. Schiff 6, 50019, Sesto Fiorentino (FI). \\ ${ }^{2}$ Dipartimento di Psichiatria, Neurobiologia, Farmacologia e Biotecnologie, Università di Pisa, via Bonanno 6, \\ 50126 Pisa. Molecular Modeling Section, Dipartimento di Scienze Farmaceutiche, Università di Padova, via \\ Marzolo 5, 35131 Padova. \\ vittoria.colotta@unifi.it
}

Adenosine is a ubiquitous neuromodulator eliciting many biological effects by activation of four different receptors subdivided into $\mathrm{A}_{1}, \mathrm{~A}_{2 \mathrm{~A}}, \mathrm{~A}_{2 \mathrm{~B}}$ and $\mathrm{A}_{3}$ subtypes and belonging to the $\mathrm{G}$ protein-coupled receptor family [1]. In the last decade much research has been directed towards the study of adenosine receptor (AR) antagonists which have proved to be attractive therapeutics in many pathophysiological conditions [2]. In particular, the $\mathrm{A}_{3}$ AR subtype antagonists are putative anti-inflammatory, anti-asthmatic or anti-ischemic agents [2]. A part of our recent studies were aimed at finding new tricyclic $\mathrm{A}_{3}$ AR antagonists and among them the 2-arylpyrazolo[3,4c]quinolin-4-amino series was investigated [3]. In the preliminary study a single modification at a time was apported at the $\mathrm{R}_{1}$ or $\mathrm{R}_{4}$ position of 2-phenylpyrazolo[3,4-c]quinolin-4-amino scaffold and some of the mono-substituted derivatives resulted to be potent human (h) $A_{3} A R$ antagonists. SAR studies pointed out that methyl and methoxy groups at meta or para positions were the most profitable $R_{1}$ substituents for enhancing $h A_{3} A R$ affinity. Advantageous effects were also elicited by introducing an acetyl or a benzoyl group at the $\mathrm{R}_{4}$ position. These interesting results prompted us to continue investigating this class of $h A_{3} A R$ antagonists with the purpose of evaluating whether the contemporary functionalization of the key positions $R_{1}$ and $R_{4}$ with the above cited groups could increase $\mathrm{hA}_{3} \mathrm{AR}$ affinity and selectivity. Also the bulkier diphenylacetyl substituent or two benzoyl residues were probed on the 4-amino group.

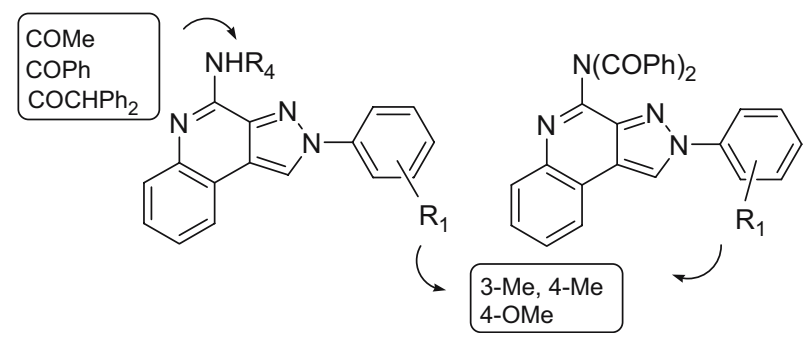

Preliminary binding data at the $\mathrm{hA}_{3} \mathrm{AR}$ show that the simultaneous presence of the suitable substituents on the 2phenyl ring $\left(\mathrm{R}_{1}\right)$ and the 4-amino group $\left(\mathrm{R}_{4}\right)$ maintained the $\mathrm{hA}$ AR affinity in the nanomolar range. Molecular modeling studies are in progress in order to elucidate the binding mode of this series of derivatives to the $\mathrm{hA}_{3}$ receptor site and to rationalize the affinity data.

1. Fredholm BB, Ijzerman AP, Jacobson KA, Klotz K-N, Linden J. Pharmacol Rev 2001; 53: 527-52.

2. Poulsen SA, Quinn RJ. Bioorg Med Chem 1998; 6: 619-41.

3. Colotta V, Catarzi D, Varano F, Cecchi L, Filacchioni G et al. J Med Chem 2000; 43: 3118-24. 


\title{
2-Chloro-2'-Deoxyadenosine-Induced Apoptosis of Human Cancer Cells Bearing a Mutated p53 Isoform: Role of MAP Kinases
}

\author{
Stefania Ceruti, Alessia Mazzola and Maria P. Abbracchio \\ Laboratory of Molecular and Cellular Pharmacology of Purinergic Transmission - Department of Pharmacological \\ Sciences, University of Milan - via Balzaretti 9 - 20133 - Milan, Italy \\ stefania.ceruti@unimi.it
}

The biochemical pathways controlling cell death are often mutated and/or defective in cancer cells, contributing to the development of resistance to chemotherapy. Thus, for any given anti-cancer agent, it is important to verify whether it can effectively induce cell death in cancerous cells carrying mutations of various apoptotic pathways. We have previously demonstrated that human astrocytoma ADF cells are resistant to agents activating the intrinsic pathway of apoptosis due the presence of a mutated caspase-9 isoform (1). Nevertheless, the anti-cancer agent 2-chloro-2'-deoxyadenosine (Cladribine, 2CdA) induces cell cycle block and is still able to effectively kill these cells by recruiting an atypical pathway of cell death, with caspase-2 acting as an "initiator" caspase, followed by caspase-3 (2). To better characterize 2CdA-activated apoptotic pathway in ADF cells, in this study we have focussed our attention on the tumor suppressor protein p53 which is mutated in more than $50 \%$ of human cancers. By cloning and sequencing the p53 isoform expressed by ADF cells, we detected a single nucleotide mutation in position 797 which translated into a G-to-E substitution at position 266 which belongs to the DNA-binding domain of the p53 protein. Since phosphorylation of the Ser15 residue of the p53 protein represents one of the most frequent post-translational modification controlling its proapoptotic activity, we have analyzed the importance of this phosphorylation reaction in cells exposed for various time periods to $2 \mathrm{CdA}$ by means of a specific antibody directed against phosphorylated p53. A very rapid apperance of the protein band corresponding to phospho-Ser15 p53 was observed, starting from a 30 min and declining after 3 hours of incubation with the adenosine analog. P53 phosphorylation was completely prevented by 2-deoxy-cytidine, which competes with 2CdA for its intracellular phosphorylation to the corresponding chloro-deoxy nucleotides. Indeed, co-incubation with SP600125, a selective inhibitor of c-jun-terminal kinases 1 and 2 (JNK1/2), completely blocked p53 phosphorylation, whereas inhibitors of other members of the MAP kinase family of enzymes (e.g., ERK1/2 and p38) had no effect. SP600125 was also able to significantly reduce the percentage of apoptosis induced by 2CdA. However, p53 phosphorylation and 2CdA-induced apoptosis are not correlated to each other, since $\alpha$-pifithrin, which selectively inhibits p53 transcriptional activity downstream of post-translational modifications, was completely ineffective in preventing cell death. Taken together, these results suggests that a JNK-dependent but p53-independent pathway of death is recruited by 2 CdA. JNK1/2 were also involved in the 2CdA-induced cell cycle block at G0/G1 phases and enhanced expression of genes controlling cell cycle progression (e.g., p21, GADD45A) as demonstrated by microarray analysis. Despite the previously mentioned inability of the selective inhibitor PD098059 to prevent p53phosphorylation, we have also detected a pro-apoptotic role for ERK1/2, since PD098059 significantly reduced 2CdA-mediated cell death. These results suggest that 2CdA treatment might represent an effective pharmacological approach for cancer cells bearing mutations of different apoptotic pathways of death, by recruting various members of the MAP kinases family.

(1) Ceruti S, Mazzola A, Abbracchio MP. J Pharmacol Exp Ther 2005; 314: 825-37

(2) Ceruti S, Beltrami E, Matarrese P et al. Mol Pharmacol 2003; 63: 1437-47

\section{2'-C-Methyl Derivatives of Tecadenoson and 2-Chloro-Tecadenoson with In- creased Selectivity for Human $A_{1}$ Adenosine Receptor}

Cappellacci L, ${ }^{1}$ Franchetti P, ${ }^{1}$ Vita $\mathrm{P},{ }^{1}$ Petrelli R, ${ }^{1}$ Pasqualini M, ${ }^{1}$ Costa $\mathrm{B},{ }^{2}$ Spinetti F, ${ }^{2}$ Martini C ${ }^{2}$, Klotz K-N, ${ }^{3}$ Lavecchia A, ${ }^{4}$ and Grifantini $\mathrm{M}^{1}$

${ }^{1}$ Department of Chemical Sciences, University of Camerino, 62032 Camerino, Italy; ${ }^{2}$ Department of Psychiatry, Neurobiology, Pharmacology and Biotechnology, University of Pisa, 56126 Pisa, Italy; ${ }^{3}$ Institut für Pharmakologie, 
Universitat Würzburg, Würzburg, Germany, ${ }^{4}$ Department of Medicinal Chemistry and Toxicology, University of Naple "Federico II," 80131 Naple, Italy

loredana.cappellacci@unicam.it

Adenosine mediates its physiological effects through four $G$ protein-coupled receptors $\left(A_{1}, A_{2 A}, A_{2 B}\right.$ and $\left.A_{3}\right)$. $A$ large number of agonists with high affinity at $A_{1}, A_{2 A}, A_{3}$ adenosine receptors and moderate affinity at $A_{2 B}$ receptor have been developed over the years. Many compounds originally thought to be selective for the $A_{1}$ or $\mathrm{A}_{2 \mathrm{~A}}$ subtypes later turned out to be also potent agonists at the more recently discovered $\mathrm{A}_{3}$ receptor. Owing to the great interest in $\mathrm{A}_{1}$ agonists as neuroprotective, antilipolytic, anti-arrhythmic, and antinociceptive agents, there is a need for novel agonists with high potency and selectivity at this receptor subtype to avoid side effects due to the stimulation of the other subtypes. We discovered that the substitution of the hydrogen in $2^{\prime}$-position of the ribose moiety of the $\mathrm{A}_{1}$ selective agonist CCPA with a methyl group (2'-Me-CCPA) reduces the affinity at human $\mathrm{A}_{2 \mathrm{~A}}$ and $\mathrm{A}_{3}$ receptors, thus increasing the selectivity for $\mathrm{A}_{1}$ subtype [1]. In this communication we report on the affinity at human adenosine receptors of $2^{\prime}-C$-methyl derivatives of Tecadenoson $\left(C V T 510, \mathrm{~N}^{6}-3(R)\right.$ tetrahydrofuranyl-adenosine), a potent and selective $\mathrm{A}_{1}$ agonist in clinical trials for treatment of supraventricular tachyarrhythmias, and of its 2-chloro derivative (2-Cl-Tecadenoson). Tecadenoson, 2-Cl-Tecadenoson and their $2^{\prime}$ - $C$-methyl derivatives were evaluated for the affinity at human $\mathrm{A}_{1}, \mathrm{~A}_{2 \mathrm{~A}}, \mathrm{~A}_{2 \mathrm{~B}}$, and $\mathrm{A}_{3} \mathrm{ARs}$ expressed in $\mathrm{CHO}$ cells in comparison with CPA, CCPA, 2'-Me-CPA and 2'-Me-CCPA. With the exception of Tecadenoson that proved to be equipotent to CPA, $\mathrm{N}^{6}-3(R)$-tetrahydrofuranyl substituted derivatives showed only 2.5- to 5-fold less affinity at human $\mathrm{A}_{1} \mathrm{AR}$ in comparison with the corresponding $\mathrm{N}^{6}$-cyclopentyl analogues. It was further confirmed that the introduction of a methyl group in the $2^{\prime}$-position of the ribose moiety of adenosine analogues induces a relevant decrease of affinity at $\mathrm{A}_{2 \mathrm{~A}}$ and $\mathrm{A}_{3}$ receptors. Thus, 2'-Me-Tecadenoson and 2'-Me-2-Cl-Tecadenoson turned out to be very selective compounds for human $\mathrm{A}_{1} \mathrm{AR}$ (>4,762- to $>6,452$-fold vs human $\mathrm{A}_{2 \mathrm{~A}}$, and 211- to 288 -fold vs $\mathrm{A}_{3}$ receptor, respectively).

[1] Cappellacci L, Franchetti P, Pasqualini M, Petrelli R, Vita P, Lavecchia A, Novellino E, Costa B, Martini C, Klotz K-N and Grifantini M. J Med Chem 2005; 48: 1550-62.

\title{
2'-C-Methyl-2-Chloro-N ${ }^{6}$-Cyclopentyladenosine, A Potent and Highly Selective $\mathbf{A}_{1}$ Adenosine Receptor Agonist, Has Antinociceptive Activity and Modulates RVM On- and Off-Cell Activities
}

\author{
de Novellis V., ${ }^{1}$ Mariani L., ${ }^{1}$ Vita D., ${ }^{1}$ Giordano C., ${ }^{1}$ Cappellacci L., ${ }^{2}$ Franchetti P., ${ }^{2}$ Grifantini M., ${ }^{2}$ \\ Rossi F., ${ }^{1}$ and Maione S. ${ }^{1}$ \\ ${ }^{1}$ Department of Experimental Medicine, Second University of Napoli, 80138 Napoli, Italy \\ ${ }^{2}$ Department of Chemical Sciences, University of Camerino, 62032 Camerino, Italy
}

This study was undertaken to investigate the effect of $2^{\prime}-C$-methyl-2-chloro- $\mathrm{N}^{6}$-cyclopentyladenosine $\left(2^{\prime}\right.$-Me-CCPA), a potent and highly selective $\mathrm{A}_{1}$ adenosine receptor agonist [1], on nociceptive responses and on spontaneous activity of ON- and OFF- neurons of rostral ventromedial medulla (RVM). Moreover, we investigated whether the intra-periaqueductal grey (PAG) microinjection of 2'-Me-CCPA induced changes in the tail flick latencies, as well as tail flick-related changes in RVM cell activities.

Systemic administrations of $2^{\prime}$-Me-CCPA (2-5 mg/kg, i.p.), $10 \mathrm{~min}$ before formalin, reduced the late hyperalgesic phase; in formalin test, these effects were prevented by DPCPX (3 mg/kg, i.p.), a $\mathrm{A}_{1}$ receptor antagonist, but not by DPMX (3 $\mathrm{mg} / \mathrm{kg}$, i.p.), a $\mathrm{A}_{2}$ receptor antagonist.

Intra-PAG microinjections of $2^{\prime}$-Me-CCPA $(0.5-1 \mathrm{nmol} / \mathrm{rat})$ increased the tail flick latencies, delayed the tail flickrelated onset to ON-cell burst and decreased the duration of OFF-cell pause in a dose dependent manner. Furthermore, 2'-Me-CCPA decreased RVM ON-cell and increased OFF-cell ongoing activities, in a dose dependent manner. These electrophysiological effects were prevented by DPCPX $(1 \mathrm{nmol} / \mathrm{rat}$.).

In conclusion, this study confirms the role of $\mathrm{A}_{1}$ receptors in the modulation of inflammatory pain and suggests a critical role of PAG purinergic system for the control of acute and chronic pain. 
[1] Cappellacci, L.; Franchetti, P.; Pasqualini. M.; Petrelli, R.; Vita P, Lavecchia, A.; Novellino, E.; Castor, B.; Martini, C.; Klotz, K.-N.; Grifantini, M. "Synthesis, Biological Evaluation and Molecular Modeling of Ribose-Modified Adenosine Analogues as Adenosine Receptor Agonists.” J. Med. Chem. 48, 1550-1562, 2005.

\title{
3-Modified 2-Aminothiophenes as $\mathbf{A}_{1} \mathbf{A} R$ Allosteric Enhancers
}

\author{
Peter J. Scammells ${ }^{1}$, George Nikolakopoulos ${ }^{1}$ and Joel Linden ${ }^{2}$ \\ ${ }^{T}$ Department of Medicinal Chemistry, Victorian College of Pharmacy, Monash University, 381 Royal Parade, Parkville \\ VIC 3052 Australia and ${ }^{2}$ Departments of Medicine (Cardiology) and Pharmacology, University of Virginia, \\ Charlottesville, VA 22908, USA \\ peter.scammells@vcp.monash.edu.au
}

The first allosteric enhancers (AEs) acting at the $A_{1}$ adenosine receptor $\left(A_{1} A R\right)$ were reported by Bruns et al. in $1990[1,2]$. PD81,723 (1) was one of the more potent and effective of the initial series of enhancers and has subsequently been commonly used for benchmarking new AEs. Since this initial discovery, other researchers have directed significant effort to refining the structure-activity relationships of the 3-, 4- and 5-positions of the 2aminothiophene core.

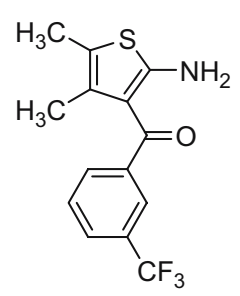

$1 \mathrm{AE}$ Score $=28 \%$ $\mathrm{ED}_{50}=13.6 \mu \mathrm{M}$<smiles>Cc1ccc(-c2c(-c3ccccc3)sc(N)c2C(=O)OCc2cccc(C(F)(F)F)c2)cc1</smiles>

2

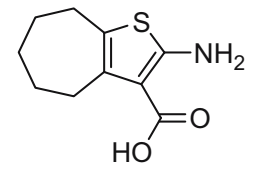

3 AE Score $=80 \%$ $\mathrm{ED}_{50}=1.4 \mu \mathrm{M}$

However, relatively few 2-aminothiophenes with esters or amides in the 3-position have been tested as allosteric enhancers. The initial study by Bruns and co-workers included two 2-amino-3-ethoxycarbonyl-4,5,6,7-tetrahydrothieno[2,3-c]pyridines [1,2]. Whilst these compounds maintained some enhancing ability, they proved to be less potent than the corresponding 3-benzoyl substituted 2-amino-4,5,6,7-tetrahydrothieno[2,3-c]pyridines. We recently synthesised a series of substituted 2-amino-3-benzoyl-4,5-diphenylthiophene allosteric enhancers. En route to these target compounds we also prepared some novel 2-aminothiophene-3-carboxylates and carboxamides to explore the effect of these substituents on AE activity [3]. Three of the five alkoxycarbonyl compounds proved to be clearly more effective than PD81,723 (1), with 3-trifluoromethylbenzyl 2-amino-4-(4-methylphenyl)-5-phenylthiophene-3-carboxylate (2) being the most effective compound in this group. We now report a more complete structure-activity study of this class of compound as $\mathrm{A}_{1} \mathrm{AR}$ allosteric enhancers. A number of compounds, notably 3, proved to be more potent and efficacious than PD81,723 (1). Conformationally restricted analogs have also been prepared and evaluated.

1. Bruns RF, Fergus JH Allosteric enhancement of adenosine A1 receptor binding and function by 2-amino-3-benzoylthiophenes. Mol. Pharmacol. 1990, 38, 939-949.

2. Bruns RF, Fergus JH, Coughenour LL, Courtland GE, Pugsley TA, Dodd, JH, et al. Structure-activity relationships for enhancement of adenosine A1 receptor binding by 2-amino-3-benzoylthiophenes. Mol. Pharmacol. 1990, 38, 950-958.

3. Lütjens H, Zickgraf A, Figler H, Linden J, Olsson RA, Scammells PJ. 2-amino-3-benzoylthiophene allosteric enhancers of A1 adenosine agonist binding: new 3, 4- and 5-modifications. J. Med. Chem. 2003, 46, 1870-1877. 


\title{
A C-terminal Lysine that Controls Human P2X $\mathbf{X}_{4}$ Receptor Desensitization
}

\author{
Samuel J. Fountain \& R. Alan North \\ Faculty of Life Sciences, Michael Smith Building, University of Manchester, Manchester, M13 9PT, UK. \\ Samuel.fountain@manchester.ac.uk
}

Receptor desensitization can determine the time course of transmitter action, and profoundly alter sensitivity to drugs. Among P2X receptors, ion currents through homomeric $\mathrm{P}_{2} \mathrm{X}_{4}$ receptors exhibit intermediate desensitization when compared to $\mathrm{P} 2 \mathrm{X}_{1}$ and $\mathrm{P} 2 \mathrm{X}_{3}$ (much faster) and $\mathrm{P} 2 \mathrm{X}_{2}$ and $\mathrm{P} 2 \mathrm{X}_{7}$ (slower) [1]. We recorded membrane currents in HEK293 cells transfected to express the human $\mathrm{P}_{2} \mathrm{X}_{4}$ receptor. The decline in current during a $4 \mathrm{~s}$ application of ATP $(100 \mu \mathrm{M})$ was about $30 \%$; this was not different during whole-cell or perforated patch recording. Alanine-scanning mutagenesis of the intracellular C-terminal identified two positions with much accelerated desensitization kinetics (Lys ${ }^{373}: 92 \pm 3.2 \%$ and $\mathrm{Tyr}^{374}: 74 \pm 4.2 \%$ ). At position 373, substitution of Arg or Cys also strongly accelerated desensitization: however, in the case of K373C the wild-type phenotype was fully restored by adding ethylammonium methanethiosulfonate. At position 374, phenylalanine could replace tyrosine. These results indicate that wild type desensitization properties requires an aromatic moiety at position 374 , and an amino rather than a guanidino group at position 373. These residues lie between previously identified motifs involved in membrane trafficking (YXXXK and YXXGL) $[2,3]$, and implicates the $\mathrm{C}$ terminal also in rearrangements leading to channel closing during the presence of agonist.

1. North RA (2002). Molecular physiology of P2X receptors. Physiol Rev. 82(4):1013-67.

2. Royle SJ et al. (2002). Identification of a non-canonical tyrosine-based endocytic motif in an ionotropic receptor. J Biol Chem 2002; 277(38):35378-85.

3. Chaumont $\mathrm{S}$ et al. (2004). Identification of a trafficking motif involved in the stabilization and polarization of $2 \mathrm{X}$ receptors. $\mathrm{J}$ Biol Chem 2004; 279(28): 29628-38.

\section{5-Heteroarylcarbamoylamino- Pyrazolo-TriazoloPyrimidines as $\mathbf{h} \mathbf{A}_{3}$ Adenosine Receptor Antagonists}

\author{
G. Pastorin ${ }^{1}$, T. Da Ros ${ }^{1}$, C. Bolcato ${ }^{1}$, C. Montopoli ${ }^{2}$, S. Moro ${ }^{2}$, B. Cacciari ${ }^{3}$, P. G. Baraldi ${ }^{3}$, K. Varani ${ }^{4}$, \\ P. A. Borea ${ }^{4}$ and G. Spalluto ${ }^{1}$ \\ ${ }^{1}$ Dipartimento di Scienze Farmaceutiche, Università degli Studi di Trieste; ${ }^{2}$ Molecular Modeling Section, Diparti- \\ mento di Scienze Farmaceutiche, Università degli Studi di Padova; ${ }^{3}$ Dipartimento di Scienze Farmaceutiche, Uni- \\ versità degli Studi di Ferrara ${ }^{4}$ Dipartimento di Medicina Clinica e Sperimentale-Sezione di Farmacologia, \\ Università degli Studi di Ferrara; Italy \\ Spalluto@units.it
}

A new series of pyrazolotriazolopyrimidines bearing at the N5-position different heteroarylcarbamoylamino moieties are described as an enlarged series of previously reported highly potent, selective and water soluble human $\mathrm{A}_{3}$ adenosine receptor antagonists (1) [1]. 


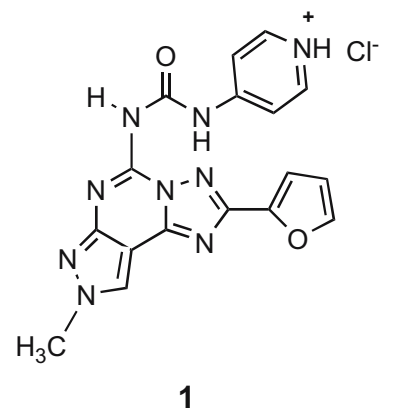

$\mathrm{hA}_{1} \mathrm{~K}_{\mathrm{i}}=350 \mathrm{nM} ; \mathrm{hA}_{2 \mathrm{~A}} \mathrm{~K}_{\mathrm{i}}=100 \mathrm{nM}$

$h A_{2 B} K_{i}=250 n M ; h A_{3} K_{i}=0.01 n M$

$\mathrm{hA}_{1} / \mathrm{hA}_{3}=35,000 ; \mathrm{hA}_{2 \mathrm{~A}} / \mathrm{hA}_{3}=10,000$

$\mathrm{hA}_{2 \mathrm{~B}} / \mathrm{hA}_{3}=25,000$

The synthesized compounds showed $\mathrm{A}_{3}$ adenosine receptor affinity in the nanomolar range and different levels of selectivity evaluated in radioligand binding assays at human $\mathrm{A}_{1}, \mathrm{~A}_{2 \mathrm{~A}}$, and $\mathrm{A}_{3}$ adenosine receptors. In particular, the effect of the heteroaryl substituents at the N5 position has been analyzed and this study allows recognizing that the presence of a pyridinium moiety in this position not only increases water solubility but also improves or retains potency and selectivity at the human $\mathrm{A}_{3}$ adenosine receptors. In contrast, replacement of pyridine with different heterocycles produces loss of affinity and selectivity at the human $\mathrm{A}_{3}$ adenosine receptors. A molecular modeling study has been carried out with the aim to explain these various binding profiles [2].

\title{
References
}

1. Maconi, A.; Pastorin G.; Da Ros T.; Spalluto G.; Gao, Z.G.; Jacobson, K.A. Baraldi, P.G.; Cacciari, B.; Varani, K.; Borea, P.A. J. Med. Chem. 2002, 45, 3579-3582.

2. Pastorin, G.; Da Ros, T.; Bolcato, C.; Montopoli, C.; Moro, S.; Caccairi, B.; Baraldi, P.G.; Varani, K.; Borea, P.A; Spalluto, G. J. Med. Chem. 2006, 49, 0000-0000.

\section{A Cell-based Assay Suitable for HTS of Adenosine A $A_{1}$ Receptor Agonists}

\author{
Giuliana Piazza, Lia Scarabottolo
}

Axxam S.r.l., San Raffaele Biomedical Science Park, Via Olgettina, 58, 20132 Milan, Italy giuliana.piazza.gp@axxam.it

Purinergic receptors are a family of seven ubiquitous transmembrane receptors comprising two classes, P1 and P2, which are activated by adenosine and extracellular nucleotides, respectively. The P1 receptors signal through multiple intracellular effectors in response to nucleoside activation. There are four subtypes of P1 receptors: $\mathrm{A}_{1}$, $\mathrm{A} 2_{\mathrm{A}}, \mathrm{A} 2_{\mathrm{B}}$ and $\mathrm{A}_{3}$.

In this study, we have focused our attention on the development of a cell-based system suitable for the identification of agonists of the adenosine $A_{1}$ receptor, suitable for High Throughput Screening applications (HTS).

The $A_{1}$ receptor is coupled to $G \alpha_{i}$, which inhibits adenylyl cyclase. Additionally, it mediates the activation of several types of $\mathrm{K}^{+}$-channels, the inactivation of $\mathrm{N}$-, $\mathrm{P}$ - and Q-type $\mathrm{Ca}^{2+}$ channels and the activation of phospholipase $C \beta$ (via $\beta \gamma$ subunits).

Mainly through the $\mathrm{A}_{1}$ receptor, adenosine mediates potent effects on myocardium and conduction tissue, endothelium and vascular smooth muscle, inducing delayed tolerance to ischemia-reperfusion injury.

In particular, $\mathrm{A}_{1}$-receptor activation produces preconditioning to protect the heart and other tissues from subsequent ischemic injury. Therefore, the pharmaceutical industry is interested in producing selective $\mathrm{A}_{1}$-receptor agonists as pharmacological treatment for cardiovascular diseases.

We have created a $\mathrm{CHO}$ cell line with stable expression of the reporter luciferase under the control of cAMP response elements and a chimera of the human $A_{1}$ receptor and $G \alpha_{s}$, which allows the switching of the receptormediated signal towards adenylil cyclase stimulation. We have demonstrated for the first time that activation of the $\mathrm{A}_{1}$ receptor can be detected by the direct increase of luminescence.

This generated $A_{1}-G \alpha_{s}$-expressing cell line is a sensitive and reliable functional cell-based assay suitable for the HTS of specific receptor agonists. 


\title{
A Negative Inotropic Effect of $\mathrm{Up}_{\mathbf{4}} \mathrm{A}$ in the Human Heart
}

Gergs U, ${ }^{1}$ Pönicke K, ${ }^{1}$ Simm A, ${ }^{2}$ Silber RE, ${ }^{2}$ Jankowski J, ${ }^{2}$ Jankowski V, ${ }^{2}$ Tepel M, ${ }^{2}$ Zidek W, Neumann J,

Institute for Pharmacology and Toxicology, Halle, ${ }^{1}$ Department of Cardio-Thoracic Surgery, Halle, ${ }^{2}$ Medizinische Klinik IV, Charité Campus Benjamin Franklin, Berlin

Joachim.neumann@medizin.uni-halle.de

Adenosine has been reported to exert negative inotropic effects in isolated cardiac preparations of numerous mammalian species, including man. The effects are assumed to be mediated via an $\mathrm{A}_{1}$ adenosine receptor. Interestingly, derivatives of adenosine like diadenosine polyphosphates e.g. $\mathrm{Ap}_{4} \mathrm{~A}$ exert similar effects via the $\mathrm{A}_{1}$ adenosine receptor in the human heart. Recently, uridine adenosine tetraphosphate $\left(\mathrm{Up}_{4} \mathrm{~A}\right)$ was described as a novel endothelian derived vasoconstrictive factor (Nat Med 11:223, 2005). $\mathrm{Up}_{4} \mathrm{~A}$ was released under certain

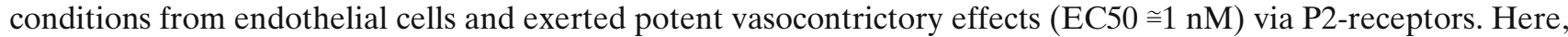
we studied the effect of $\mathrm{Up}_{4} \mathrm{~A}$ on force of contraction in isolated electrically driven atrial preparations from human hearts. The preparations were obtained from patients undergoing cardiac bypass surgery due to coronary heart disease. Preparations were studied under isometrical conditions driven at $0.5 \mathrm{~Hz}$.

Concentration response curves were obtained for the $\beta$-adrenoceptor agonist isoproterenol $(1 \mathrm{nM}-10 \mu \mathrm{M})$ and $\mathrm{Up}_{4} \mathrm{~A}(1 \mathrm{nM}-0.3 \mu \mathrm{M})$. In the very same preparations, isoproterenol exerted a positive inotropic effect while $\mathrm{Up}_{4} \mathrm{~A}$ exerted a concentration dependent negative inotropic effect. The effect started at about $0.03 \mu \mathrm{M}$ and amounted to $35 \%$ at $0.3 \mu \mathrm{M}$, the highest concentration of $\mathrm{Up}_{4} \mathrm{~A}$ tested $(\mathrm{n}=3)$. We assume that $\mathrm{Up}_{4} \mathrm{~A}$ exerts the effect probably via $A_{1}$ adenosine receptors. In summary, the new endogenous vasoconstrictor $\mathrm{Up}_{4} \mathrm{~A}$ can reduce force of contraction in the human heart.

Hence, it may play an autoregulatory function in the cardiovascular system.

\section{A Putative Dictyostelium Discoideum P2X Receptor}

\author{
Melanie Ludlow \& Steve Ennion \\ Cell Physiology and Pharmacology, University of Leicester, England, LE1 9HN \\ ml119@le.ac.uk
}

Completion of the Dictyostelium discoideum genome sequencing project has allowed a family of five putative P2X receptor genes to be provisionally identified in this species of cellular slime mould. Functional characterisation of a P2X or P2X-like receptor in this simple eukaryotic model organism could potentially contribute to our understanding of this class of ligand-gated ion channel. Extracellular ATP release from Dictyostelium and ATP induced calcium influx has previously been reported [1] supporting the presence of purinergic signalling pathways in this species.

The five putative Dictyostelium P2X subunits possess $35-75.8 \%$ sequence identity between each other and 11.4$16.1 \%$ sequence identity to human $\mathrm{P} 2 \mathrm{X}_{1-7}$ subunits with conservation of key $\mathrm{P} 2 \mathrm{X}$ receptor elements, such as an $\mathrm{N}$ terminal PKC motif, a C-terminal membrane stabilisation motif, two potential hydrophobic transmembrane domains and a similar overall size (368-407 amino acids in length). The Dictyostelium gene showing most homology to vertebrate P2X channels ( $\mathrm{DP} 2 \mathrm{XE}$ ) was selected for further investigation.

Initially we attempted to express $\mathrm{dP} 2 \mathrm{XE}$ protein in Xenopus laevis oocytes as this expression system has been used successfully for the characterisation of vertebrate and Schistosoma mansoni [2] P2X receptors. Expression of $\mathrm{dP} 2 \mathrm{XE}$ protein in oocytes was achieved following introduction of a vertebrate Kozak sequence to the N-terminus. However, cell surface biotinylation revealed that the protein was not trafficked to the plasma membrane in these cells and no ion channel currents could be detected by two-electrode voltage-clamp experiments.

In the presence of a food source Dictyostelium exist in a unicellular state. Upon starvation, cells aggregate and develop into a spore containing fruiting body. Northern blot analysis of dP2XE expression demonstrated that the $\mathrm{dP} 2 \mathrm{XE}$ gene is transcribed at a constant level through all stages of development, potentially indicating a housekeeping role. In order to determine the subcellular localisation of dP2XE in Dictyostelium, a C-terminal eGFP fusion protein (dP2XE-eGFP) was expressed. This protein displayed a diffuse cytoplasmic distribution with distinct localisation to the membranes of an intracellular organelle but absence from the plasma membrane. Conf- 
ocal imaging of cells stained with the styryl dye FM2-10 [3] demonstrated localisation of dP2XE-eGFP to the membranes of contractile vacuoles, intracellular organelles involved in water expulsion/osmoregulation and potentially calcium regulation. This intracellular localisation of $\mathrm{dP} 2 \mathrm{XE}$ indicates a novel function compared to that of the conventional cell surface membrane localised P2X receptors identified in vertebrates.

1. Parish, R.W.; Weibel, M., Extracellular ATP, ecto-ATPase and calcium influx in Dictyostelium discoideum cells. FEBS Lett 1980, 118, (2), 263-6.

2. Agboh, K.C.; Webb, T.E.; Evans, R.J.; Ennion, S.J., Functional characterisation of a P2X receptor from schistosoma mansoni. J Biol Chem 2004, 279, (40), 41650-41657.

3. Betapudi, V.; Mason, C.; Licate, L.; Egelhoff, T.T., Identification and characterization of a novel alpha-kinase with a von Willebrand factor A-like motif localized to the contractile vacuole and Golgi complex in Dictyostelium discoideum. Mol Biol Cell 2005, 16, (5), 2248-62.

\title{
A Role for the P2X7 Receptor in Control of Toxoplasma gondii Infection
}

\author{
$\underline{\text { S.J. Fuller }}{ }^{1}$, M. Lees ${ }^{2}$, H. Murray ${ }^{1}$, R. Sluyter ${ }^{1}$, B. Gu${ }^{1}$, N. Boulter ${ }^{2}$, K. Skarratt ${ }^{1}$, N.C. Smith ${ }^{2}$, J.S. \\ Wiley $^{1}$ \\ ${ }^{1}$ The University of Sydney, Department of Medicine, Nepean Hospital, Penrith 2750 and ${ }^{2}$ Institute for the Biotec- \\ hnology of Inectious Diseases, University of Technology, Sydney \\ sfuller@med.usyd.edu.au
}

The P2X7 receptor has a role in the host immune response to infection with the obligate intracellular pathogens, Mycoplasma and Chlamydia. We describe three immunocompetent subjects with absent P2X7 function due to one or a combination of inherited loss-of-function single nucleotide polymorphisms all of whom had a prolonged, severe illness due to infection with the obligate intracellular protozoan parasite, Toxoplasma gondii. $T$ gondii usually causes an asymptomatic infection in immunocompetent humans but infection in immunocompromised individuals can cause serious disease, including encephalitis while intrauterine infection can cause congenital blindness, mental retardation and death. Our first subject was a 20 -year-old female who presented with submandibular lymphadenopathy. Serology was positive for recent Toxoplasma infection and a lymph node biopsy showed features consistent with toxoplasmosis. The second subject was a 24 -year-old female who had a foetal ultrasound scan at 22 weeks gestation that showed gross cerebral ventriculomegaly consistent with congenital Toxoplasmosis and confirmed by PCR on amniotic fluid. The third subject, a 14-year-old male, presented with a 2year history of tiredness, lethargy and generalized painless lymphadenopathy. Serology for $T$ gondii was positive for acute infection and an excision biopsy of an enlarged left axillary lymph node showed lymphadenitis consistent with toxoplasmosis. Function of the P2X7 receptor, as measured by ethidium uptake using time resolved flow cytometry, was absent in two subjects and low in the third. Sequencing of the P2X7 gene confirmed the presence of one or a combination of loss-of-function single nucleotide polymorphisms previously described by our group. All subjects were HIV negative and had normal T-cell subsets and normal serum immunoglobulins Therefore, normal P2X7 function may be of importance in controlling Toxoplasma infection. Possible P2X7 dependent mechanisms of the host immune response to Toxoplasma infection include P2X7 dependent phagosome-lysosome fusion via phospholipase D activation and P2X7 triggered apoptosis of infected host cells, both of which have been shown to play a role in the host response to Chlamydia and Mycobacteria infection.

\section{A Role for Nucleotide Signaling Pathways in Adult Neurogenesis}

S.K. Mishra ${ }^{1}$, V. Shukla ${ }^{1}$, N. Braun ${ }^{1}$, C. Schomerus ${ }^{2}$, H.-W. Korf ${ }^{2}$, H. Kettenmann ${ }^{3}$, C. Gachet ${ }^{4}$, J. Sévigny $^{5}$, and S.C. Robson ${ }^{6}$, Y. Ikahara ${ }^{7}$ and H. Zimmermann ${ }^{1}$

${ }^{1}$ Frankfurt University, Biocenter, ${ }^{2}$ Frankfurt University, Medical School, Germany, ${ }^{3}$ Max Delbrück Center for Molecular Medicine Berlin-Buch, Germany, ${ }^{4}$ INSERM U.311, EFS-Alsace, Strasbourg, France, ${ }^{5}$ Sainte-Foy, Québec, Canada, ${ }^{6}$ Harvard Medical School, Boston, USA, ${ }^{7}$ Fukuoka University, School of Medicine, Japan

h.zimmermann@cns.uni-frankfurt.de 
In the adult rodent brain, active neurogenesis takes place in the subventricular zone of the lateral ventricles (SVZ) and in the dentate gyrus of the hippocampus. In both neurogenic regions, astrocyte-like cells function as stem cells/ precursor cells. To date the cellular and molecular events driving a subpopulation of precursor cells into neurogenesis as well as the cellular transition states leading to mature neurons are poorly understood. We showed that stem cells of the adult SVZ and progenitor cells in the dentate gyrus (residual radial glia) highly express the extracellular nucleoside triphosphate-hydrolyzing enzyme NTPDase2 [1]. Patch-lamp analysis of cells in hippocampal slices derived from mice transgenic for GFP under the control of the nestin promotor suggest that progenitor cells express P2X nucleotide receptors [2]. Progenitor cell-containing neurospheres cultured from the adult mouse SVZ in the presence of epidermal growth factor (EGF) and basic fibroblast growth factor (bFGF) express the ecto-nucleotidases NTPDase 2 and the tissue non-specific isoform of alkaline phosphatase, hydrolyzing extracellular ATP to adenosine. ATP, ADP and to a smaller extent UTP evoke rapid $\mathrm{Ca}^{2+}$ transients in neurospheres that are exclusively mediated by the metabotropic $\mathrm{P} 2 \mathrm{Y}_{1}$ and $\mathrm{P} 2 \mathrm{Y}_{2}$ nucleotide receptors. In addition, agonists of $\mathrm{P}_{2} \mathrm{Y}_{1}$ and $\mathrm{P} 2 \mathrm{Y}_{2}$ receptors and low concentrations of adenosine augment cell proliferation in the presence of the mitogenic growth factors EGF and bFGF. Neurosphere cell proliferation in the presence of growth factors is attenuated after application of the $\mathrm{P}_{2} \mathrm{Y}_{1}$ receptor antagonist MRS2179 and in neurospheres from $\mathrm{P}_{2} \mathrm{Y}_{1}$ receptor knockout mice. This suggests that constitutive P2 receptor activation via endogenously released nucleotide is necessary for optimal cell proliferation [3]. Our results infer nucleotide receptor-mediated synergism that augments growth factor-mediated proliferation of adult neural progenitor cells. They support the notion that extracellular nucleotides contribute to the control of adult neurogenesis in both, the SVZ and the dentate gyrus.

1. Braun, N., Sévigny, J., Mishra, S., Robson, S.C., Barth, S.W., Gerstberger, R., Hammer, K., and Zimmermann, H. 2003 Eur. J. Neurosci. 17, 1355-1364.

2. Shukla, V., Zimmermann, H., Wang, L., Kettenmann, H., Raab, S., Hammer, K., Sévigny, J., Robson, S.C., and Braun, N. J. Neurosci. Res 2005, 80, 600-610.

3. Mishra, S.K., Braun, N., Shukla, V., Füllgrabe, M., Schomerus, C., Korf, H.-W., Gachet, C., Ikehara, Y., Sévigny, J., Robson, S.C., and Zimmermann, H. Development 2006, 133, 675-684.

\title{
A SNP in the Adenosine A1 Receptor Associated with Fibromyalgia
}

\author{
Hailing Liu ${ }^{1}$, Bruce Solitar ${ }^{1}$, Jessica Stein ${ }^{1}$, Roland Staud ${ }^{2}$, Daniel Clauw ${ }^{3}$, Bruce Cronstein ${ }^{1}$ \\ ${ }^{T}$ New York University School of Medicine, New York, NY. ${ }^{2}$ University of Florida School of Medicine, Gainesville, \\ FL. ${ }^{3}$ University of Michigan, Ann Arbor, MI.
}

Background: Fibromyalgia (FM) is a chronic pain syndrome of unknown etiology characterized by widespread chronic pain and tender points. Although the exact pathophysiology of FM is unknown, individuals with FM are hyperalgesic to painful stimuli. Mice with targeted deletion of the adenosine A1 receptor (A1AR), like FM patients, demonstrate increased sensitivity to painful stimuli, and are characteristically anxious and aggressive. We therefore hypothesized that FM may be due to genetically-determined changes in A1AR.

Methods and Results: DNA was isolated and purified from peripheral blood from FM patients and age- and sexmatched normal controls. Initially the gene (promoters, exons, UTR) was sequenced in 14 FM patients and 24 controls. A single nucleotide polymorphism in the $3^{\prime}$ UTR (C2526T) was observed in 3/14 FM patients and no controls. For all subsequent genotyping assays a 241 bp region containing the SNP was amplified and sequenced for all patients and controls. Patient samples and controls were obtained from two FM clinics and an increased frequency in the C2526T SNP was observed in the FM patients as compared to controls (Table below). To determine whether the C2526T SNP was in linkage equilibrium with other SNPs in the $\mathrm{A}_{1}$ gene the frequency of 3 known SNPs upstream and 3 SNPs downstream of the C2526T SNP was determined in patients and controls by Taqman Assay and no differences were observed (data not shown). The overall frequency of the C2526T polymorphism in the FM patients was $17.3 \%$ as compared to $6.8 \%$ in the controls $(\mathrm{p}=0.019)$ 


\begin{tabular}{llll}
\hline & $\mathrm{C} / \mathrm{C}$ & $\mathrm{C} / \mathrm{T}$ & Frequency of C/T \\
\hline Fibromyalgia & & & 7 \\
Michigan & 31 & 7 & $18.4 \%$ \\
NYU & 11 & 14 & $21.4 \%$ \\
Florida & 73 & 24 & $16.1 \%$ \\
Total & 115 & & $17.3 \% *$ \\
Control & & 3 & $7.9 \%$ \\
Michigan & 35 & 5 & $6.3 \%$ \\
NYU & 75 & 8 & $6.8 \% *$ \\
Total & 110 & & \\
\hline
\end{tabular}

$* \mathrm{p}=0.019$

Previous reports have suggested that subgroups of patients with FM have SNPs in the serotonin receptor gene (5HT2AR) and COMT, an enzyme involved in metabolism of adrenergic agents but we observed no difference between controls and FM patients with regard to the frequency of these SNPs (Data not shown)

Conclusion: A SNP in the A1AR (C2526T) is associated with FM suggesting that in some patients genetically determined changes in adenosine receptor expression or function lead to fibromyalgia.

\title{
A Truncated P2X $\mathbf{X}_{7}$ Receptor Variant $\left(\mathbf{P}_{2} \mathrm{X}_{7-\mathrm{j}}\right)$ Endogenously Expressed in Cervical Cancer Cells Antagonizes the Full-Length P2X $_{7}$ Receptor through Hetero-Oligomerization
}

\author{
Ying-Hong Feng MD $\mathrm{PhD}^{1}$, Xin Li MD $\mathrm{PhD}^{2}$, Liqin Wang $\mathrm{MD}^{1}$, Lingying Zhou $\mathrm{MD}^{2}$, George \\ Gorodeski MD $\mathrm{PhD}^{2,3}$ \\ Department of Pharmacology ${ }^{1}$, Uniformed Services University of the Health Sciences, Bethesda, Maryland; \\ Reproductive Biology ${ }^{2}$, Physiology and Biophysics ${ }^{3}$, and Oncology ${ }^{3}$, CASE (Case Western Reserve) University, \\ Cleveland, Ohio, USA \\ gig@cwru.edu
}

In human cervical epithelial cells the $\mathrm{P}_{2} \mathrm{X}_{7}$ receptor mediates ATP-induced apoptosis by a mechanism that involves pore formation, augmented calcium influx, and calcium-dependent activation of the apoptotic mitochondrial pathway. However, the degree of ATP- and BzATP-induced apoptosis is smaller in cancer than in normal cervical cells [1]. A PCR product of a smaller size than the expected full-length $\mathrm{P}_{2} \mathrm{X}_{7}$ was identified in experiments trying to amplify the full-length $\mathrm{P}_{2} \mathrm{X}_{7}$ gene. DNA sequencing and gene analysis of the smaller PCR product revealed an identical $\mathrm{P}_{2} \mathrm{X}_{7}$ gene that lacks exon 8 except the $\mathrm{A}_{882}$, with a shift of coding frame to a new variant. The predicted truncated naturally occurring variant $\left(\mathrm{P} 2 \mathrm{X}_{7-\mathrm{j}}\right)($ Genebank Accession Number DQ399293) is a polypeptide of 258 amino acids that lacks the entire intracellular carboxy terminus, the second transmembrane domain, and the distal third of the extracellular loop of the full-length $\mathrm{P}_{2} \mathrm{X}_{7}$ receptor. The $\mathrm{P} 2 \mathrm{X}_{7-\mathrm{j}}$ cDNA was subcloned into various expression vectors and introduced into the host cells MDCK and HEK293 by transfection, and translation of a specific product (a protein cluster of $45-42 \mathrm{KDa}$ ) was confirmed. Expression of the $\mathrm{P}_{2} \mathrm{X}_{7-\mathrm{j}}$ as a naturally occurring protein was also confirmed in the human cancer cervical cell lines CaSki, HT3, SiHa, and Hela by Western blot with anti-P2 $\mathrm{X}_{7}$ antibody. The $\mathrm{P} 2 \mathrm{X}_{7-\mathrm{j}}$ was expressed mainly in the plasma membrane but compared to the full length receptor some of the $\mathrm{P}_{2} \mathrm{X}_{7-\mathrm{j}}$ also localized in nuclear/perinuclear regions. In host cells, inducible expression of full length $\mathrm{P} 2 \mathrm{X}_{7}$ alone, but not $\mathrm{P} 2 \mathrm{X}_{7-\mathrm{j}}$ alone resulted in significant decrease in cell number and increased apoptosis. In contrast, $\mathrm{P} 2 \mathrm{X}_{7-\mathrm{j}}$ expressing cells showed an increase in cell number and diminished baseline and BzATP-induced apoptosis. Similarly $\mathrm{P} 2 \mathrm{X}_{7-\mathrm{j}}$ expression showed diminished ligand-binding and channel function capacities and failed to form pores in response to treatment with BzATP. Co-expression of the P2X $\mathrm{X}_{7 \mathrm{j}}$ did not significantly affect expression of the full length $\mathrm{P} 2 \mathrm{X}_{7-\mathrm{j}}$, but it inhibited / blocked BzATP-induced P2 $\mathrm{X}_{7}$ channel activation, pore formation and apoptosis. The present results support the hypothesis that full length $\mathrm{P} 2 \mathrm{X}_{7} \mathrm{~mol}^{-}$ ecules homo-oligomerize as oligo-trimers. Interestingly, the $\mathrm{P} 2 \mathrm{X}_{7-\mathrm{j}}$ interacted with the $\mathrm{P} 2 \mathrm{X}_{7-\mathrm{j}}$ in a manner suggesting $\mathrm{P} 2 \mathrm{X}_{7-\mathrm{j}} / \mathrm{P} 2 \mathrm{X}_{7-\mathrm{j}}$ homo-oligomerization, and with the full-length $\mathrm{P} 2 \mathrm{X}_{7}$ suggesting $\mathrm{P} 2 \mathrm{X}_{7-\mathrm{j}} / \mathrm{P}_{2} \mathrm{X}_{7}$ heterooligomerization. $\mathrm{P} 2 \mathrm{X}_{7}$ immunoreactivities in HEK293 cells co-expressing the $\mathrm{P}_{2} \mathrm{X}_{7}$ plus the $\mathrm{P} 2 \mathrm{X}_{7-\mathrm{j}}$ suggested formation of inactive complexes of the $\mathrm{P} 2 \mathrm{X}_{7}$ receptor in the order of $\left[\mathrm{P} 2 \mathrm{X}_{7-\mathrm{j}}\right]_{3} \ggg\left[\mathrm{P} 2 \mathrm{X}_{7-\mathrm{j}}\right]_{2} /\left[\mathrm{P} 2 \mathrm{X}_{7}\right]>\left[\mathrm{P} 2 \mathrm{X}_{7}\right]_{2} /$ 
$\left[\mathrm{P} 2 \mathrm{X}_{7-\mathrm{j}}\right]$. These results identify a novel $\mathrm{P} 2 \mathrm{X}_{7}$ variant with apoptosis-inhibitory actions, and demonstrate a distinct regulatory property for a truncated variant to antagonize its full-length counterpart through hetero-oligomerization. This may represent a general paradigm for regulation of a protein function by its variant. In cancer cells, hetero-oligomerization of the $\mathrm{P} 2 \mathrm{X}_{7-\mathrm{j}}$ with the $\mathrm{P} 2 \mathrm{X}_{7}$ could result in inactive receptor, and abrogated $\mathrm{P} 2 \mathrm{X}_{7}$-mediated apoptosis could play a role in cervix neogenesis and enhance the growth of the cancer cells.

Support: AHA-SDG-0030019N, NHLBI-HL65492 (YHF); NIH HD29924, AG15955 (GIG).

1. Wang et al. Am J Physiol 287:C1349, 2004

\title{
A 1 Adenosine Receptor Antagonist, L-97-1, Increases Survival in Rats with Gram-Negative Septicemia
}

\author{
Masakatsu Goto ${ }^{1}$, Constance O. Vance ${ }^{2}$, and Constance N. Wilson ${ }^{2}$ \\ ${ }^{1}$ Department of Surgery, Loyola University of Chicago, Stritch School of Medicine, Maywood IL, ${ }^{2}$ Endacea, Inc., \\ Research Triangle Park, NC. \\ cwilson@endacea.nctda.org
}

Septicemia is a medical syndrome characterized by an overwhelming systemic response to infection that can rapidly lead to shock, organ failure and death. Following its release from Gram-negative bacteria, elevated levels of endotoxin in the plasma (endotoxemia) causes acute organ damage, including acute lung injury (ALI) and death. Previously, it was reported that $\mathrm{A}_{1}$ adenosine receptor (AR) antagonists block endotoxin-induced ALI and that endotoxin binds to and activates $\mathrm{A}_{1} \mathrm{ARs}$ on human pulmonary artery endothelial cells to induce the release of IL-6 and TXA 2 [1, 2]. L-97-1 is a water-soluble, small molecule A1 AR antagonist with high affinity and high selectivity for the human $A_{1}$ AR [3]. In a rat cecal ligation perforation (CLP) model of Gram-negative septicemia and endotoxemia, the effect of L-97-1 on mortality at 7 days (with and without antibiotics) following CLP was investigated. Administration of L-97-1 as an intravenous (i.v.) therapy post-CLP improved 7 day mortality in a dose-dependent manner (30-40\% survival) as compared to untreated CLP control (17\% survival) or antibiotics alone (23\% survival). In combination with antibiotics (gentamicin plus ampicillin), L-97-1 increased survival to $50-70 \%$ in a dose-dependent manner. In this animal model of Gram-negative septicemia and endotoxemia, L-97-1 as an adjuvant therapy to antibiotics is more effective than antibiotics alone to improve 7 day mortality. Despite the introduction of an FDA approved adjuvant therapy, Xigris ${ }^{\mathrm{TM}}$ (drotrecogin alfa), the mortality rate remains high for sepsis. As an adjuvant therapy to antibiotics, L-97-1 may improve the outcome for patients with Gramnegative septicemia.

1. Neely, CF et al. Am J Physiol 272:L353-L361, 1997.

2. Wilson, CN and Batra, VK. J. Endotoxin Res 8:263-271, 2002.

3. Obiefuna, PC et al. J Pharmacol Exp Ther 315:329-336, 2005.

Supported by STTR Phase I Grant \# AI056603

\section{A1 Adenosine Receptor Antagonist versus Montelukast Sodium on Airway Reactivity and Inflammation}

\author{
Ahmed Nadeem ${ }^{1}$, Peter C.M. Obiefuna ${ }^{1}$, Constance N. Wilson ${ }^{2}$ and S. Jamal Mustafa ${ }^{1}$ \\ ${ }^{1}$ Department of Pharmacology, East Carolina University, Greenville, NC; ${ }^{2}$ Endacea Inc, Research Triangle \\ Park, NC, USA \\ cwilson@endacea.nctda.org
}

Adenosine, an important signaling molecule in asthma, produces bronchoconstriction in allergic rabbits, primates, and humans by activating $A_{1}$ adenosine receptors (ARs) [1-3]. The effects of activation of $A_{1}$ ARs on airway inflammation in asthma are unknown. The effects of L-97-1, a water-soluble, small molecule $\mathrm{A}_{1}$ AR antagonist, are compared to montelukast sodium (MK), a cysteinyl leukotriene-1 $\left(\mathrm{CysLT}_{1}\right)$ receptor antagonist on early and late phase allergic responses (EAR and LAR), bronchial hyper-responsiveness (BHR) to histamine, and airway inflammation following House Dust Mite (HDM) administration in a hyper-responsive rabbit model of allergic 
asthma. Rabbits were made allergic by intraperitoneal injections of HDM extract within $24 \mathrm{~h}$ of birth followed by booster HDM injections. Hyper-responsive rabbits were subjected to aerosolized HDM (2500 AU), $1 \mathrm{~h}$ after intragastric administration of L-97-1 (1 mg/kg) or MK $(0.15 \mathrm{mg} / \mathrm{kg})$ and, lung dynamic compliance (Cdyn) and airway inflammation was assessed. Cdyn was significantly higher following treatment with L-97-1 at all time points and with MK at 60-300 min following HDM ( $\mathrm{p}<0.05)$; thus, L-97-1 blocks both EAR and LAR, whereas MK blocks only LAR. Both L-97-1 and MK significantly blocked BHR at $24 \mathrm{~h}(\mathrm{p}<0.05)$. Both L-97-1 and MK significantly reduced bronchoalverolar lavage (BAL) eosinophils at $6 \mathrm{~h}$ and neutrophils at 6 and $24 \mathrm{~h}(\mathrm{p}<0.05)$. As opposed to MK, L-97-1 significantly reduced BAL lymphocytes at 6 and $24 \mathrm{~h}$, while MK significantly reduced BAL macrophages at 6 and $24 \mathrm{~h}(\mathrm{p}<0.05)$. By blocking both bronchoconstriction and airway inflammation, L-97-1 may be an effective oral anti-asthma treatment.

1. Ali S et al. J Pharmacol Exp Ther 1994; 268: 1328-1334.

2. Nyce JW, Metzger WJ. Nature 1997; 385: 721-725.

3. Bjorck T et al. Am Rev Respir Dis 1992; 145: 1087-1091.

Supported by North Carolina Biotechnology Center Kenan Award CFA \#2000 CFG 8002 and STTR Phase I Grant \# HL070458.

\title{
A 2A Adenosine Receptor Down Regulation Leads to Increased Pro-Inflammatory Gene Expression in $\mathbf{A}_{2 \mathrm{~A}}$ Knockout Allergic Mice
}

\author{
S. Jamal Mustafa ${ }^{1}$, C. Ledent ${ }^{2}$, R. R. Morrison ${ }^{3}$ and A. Nadeem ${ }^{1}$ \\ ${ }^{T}$ Department of Physiology and Pharmacology and Center for Interdisciplinary Research in Cardiovascular \\ Sciences, West Virginia University, Morgantown, West Virginia, USA. ${ }^{2}$ University of Brussels, Belgium; ${ }^{3}$ Division \\ of Critical Care Medicine, St. Jude Children's Research Hospital, Memphis, Tennessee, USA.
}

Four types of adenosine receptors have been implicated in adenosine-mediated physiological and pharmacological responses in the lung. $\mathrm{A}_{2 \mathrm{~A}}$ receptor activation has been attributed to potent anti-inflammatory properties and its deficiency may lead to activation of pro-inflammatory transcription factor NF-kappa B. The present study was undertaken to investigate the role of adenosine $\mathrm{A}_{2 \mathrm{~A}}$ receptor on iNOS, Rel A (p65 subunit of NF-kappa $\mathrm{B}$ ) and $\mathrm{A}_{2 \mathrm{~A}}$ adenosine receptor gene expression along with the levels of pro-inflammatory cytokines in the lung in an allergic asthma model [1-4] in adenosine $\mathrm{A}_{2 \mathrm{~A}}$ receptor knockout (KO) mice. $\mathrm{A}_{2 \mathrm{~A}} \mathrm{KO}$ and wild-type (WT) mice were sensitized with two i.p. injections of ragweed $(200 \mu \mathrm{g})$ on days 1 and 6 . Aerosolization challenge was performed with $0.5 \%$ ragweed for 20 minutes both in the morning and afternoon on days 11,12 and 13 . Twenty-four hrs after the last challenge, bronchoalveolar lavage fluid (BALF) and lungs were collected from the mice for cytokine and gene expression assays, respectively. Allergen challenge in sensitized mice increased gene expression of both Rel A and i-NOS of WT and KO as compared to their respective controls $(p<0.01)$, with KO having greater expression of both of these genes than WT. $\mathrm{A}_{2 \mathrm{~A}}$ receptor expression was down-regulated by allergen challenge in WT sensitized mice as compared to WT controls whereas no transcripts of this receptor were detectable in KO mice. On the other hand, levels of pro-inflammatory cytokines (IL-2 and IL-4) were found to be higher in KO allergen challenged mice as compared to their respective WT sensitized and challenged mice $(p<-$ 0.01). Moreover, total nitrates and nitrites as a measure of nitric oxide production in the lung were also found to be higher in WT and $\mathrm{KO}$ allergen challenged mice as compared to their respective controls $(p<0.01)$ with $\mathrm{KO}$ sensitized and challenged mice having greater NO production than respective WT mice $(\mathrm{p}<0.05)$. These data showed that $\mathrm{A}_{2 \mathrm{~A}}$ receptor down-regulation resulted in increased gene expression of Rel, $\mathrm{A}$ which is believed to be involved in transcription of pro-inflammatory genes. The corresponding increased levels of pro-inflammatory cytokines and expression of i-NOS along with increased NO generation in the lung may be a result of this Rel A activation. These data suggest that $\mathrm{A}_{2 \mathrm{~A}}$ receptor deficiency might result in increased expression of pro-inflammatory transcription factor Rel A leading to downstream expression of pro-inflammatory genes in this model of asthma. (Supported by HL-027339).

1. Fan M, Mustafa SJ. Int Immunopharmacol 2006; 6:36-45.

2. Oldenburg PJ, Mustafa SJ. J Pharmacol Exp Ther 2005; 313:319-24.

3. Fan M, Qin W, Mustafa SJ. Am J Physiol Lung Cell Mol Physiol 2003; 284:L1012-9.

4. Fan M, Mustafa SJ. Pulm Pharmacol Ther 2000; 15:147-55. 


\title{
A Ad Adenosine Receptor Antagonists: Synthesis, Biological Activities and Molecular Modelling Analysis of Polysubstituted Adenines
}

\author{
$\underline{\text { Diego Dal Ben }},{ }^{1}$ Gloria Cristalli, ${ }^{1}$ Catia Lambertucci, ${ }^{1}$ Sauro Vittori, ${ }^{1}$ Karl-Norbert Klotz, ${ }^{2}$ Rosaria \\ Volpini ${ }^{1}$ \\ ${ }^{1}$ Dipartimento di Scienze Chimiche, Università di Camerino, via S. Agostino, 1, 62032 Camerino, Italy \\ ${ }^{2}$ Department. of Pharmacology, University of Wuerzburg, Wuerzburg, Germany \\ diego.dalben@unicam.it
}

The research on adenosine receptors has resulted in considerable progress in identifying selective agonists and antagonists and an increased understanding of the particular roles adenosine receptor subtypes play in physiological processes [1]. In the last years, $\mathrm{A}_{2 \mathrm{~A}}$ adenosine receptor antagonists have been proposed as an attractive pharmacological tool for the treatment of several diseases, such as motor dysfunctions, because of the interaction between $A_{2 A}$ and $D_{2}$ dopamine receptors in the basal ganglia, and $A_{2 A}$ antagonists could improve motor dysfunction in a variety of models relevant to Parkinson's disease. In addition, $\mathrm{A}_{2 \mathrm{~A}}$ adenosine receptor antagonists or the absence of $\mathrm{A}_{2 \mathrm{~A}}$ receptors is associated to neuroprotection. In this work our attention has been directed toward $\mathrm{A}_{2 \mathrm{~A}}$ adenosine receptor antagonists with adenine structure. In previous studies it was demonstrated that the introduction in 2 position of 9-ethyladenine of alkylamine or alkoxy chains leads to compounds endowed with affinity for the human adenosine receptors in the $\mu \mathrm{M}$ range [2,3]. In particular 9-ethyl-2-phenylethoxyadenine (2) and 9-ethyl-2-phenylethylaminoadenine (3) showing $\mathrm{K}_{\mathrm{i}} \mathrm{A}_{2 \mathrm{~A}}=0.12 \mu \mathrm{M}$ and $0.15 \mu \mathrm{M}$ respectively, were slightly $\mathrm{A}_{2 \mathrm{~A}}$ selective. In addition, the introduction of a bromine atom in 8 position of 9-ethyladenine (1) led to 8-bromo9-ethyladenine (4) endowed with improved affinity for all receptor subtypes. Starting from these observations and in the search for potent and selective adenosine receptor antagonists, a new series of 9-ethyladenine derivatives bearing different chains in 2 and 8 position was undertaken. Binding studies at human adenosine receptors showed that the new compounds are endowed with high affinity for the $\mathrm{A}_{2 \mathrm{~A}}$ receptor and are slightly selective for the same subtype. In particular, 8-bromo-9-ethyl-2-phenylethoxyadenine $\left(\mathbf{5} ; \mathrm{K}_{\mathrm{i}} \mathrm{A}_{2 \mathrm{~A}}=0.002 \mu \mathrm{M}\right)$ and 9-ethyl-8-furyl2-phenylethoxyadenine $\left(6 ; \mathrm{K}_{\mathrm{i}} \mathrm{A}_{2 \mathrm{~A}}=0.002 \mu \mathrm{M}\right)$ resulted to be two of the most potent $\mathrm{A}_{2 \mathrm{~A}}$ antagonists with adenine structure reported so far. Molecular modelling studies have been performed by docking analysis of the compounds in a rhodopsin-based model of the human $\mathrm{A}_{2 \mathrm{~A}}$ receptor, and the results have been compared with the binding data.

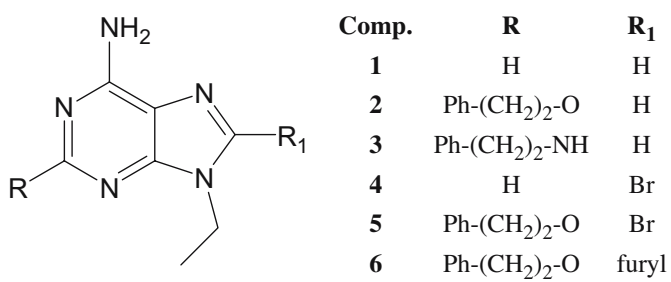

1. Cristalli, G. and Volpini, R (2003) Adenosine Receptors: Chemistry and Pharmacology. (eds.) Curr. Top. Med. Chem. 3, 355-469.

2. Camaioni, E., Costanzi, S., Vittori, S., Volpini, R., Klotz, K.N., Cristalli G. (1998) New substituted 9-alkylpurines as adenosine receptor ligands, Biorg. Med. Chem. 6, 523-533.

3. Klotz, K.N., Kachler, S., Lambertucci, C., Vittori, S., Volpini, R., Cristalli, G. (2003) 9-Ethyladenine derivatives as adendosine receptor antagonists: 2- and 8-substitution result in distinct selectivities, Naunyn-Schmiedeberg's Arch. Pharmacol. 367, 629-34.

\section{Absence of NTPDase1 Unmasks Vasoconstrictor Effects of Extracellular Uracil Nucleotides in Mouse Aortic Rings}

\author{
G. Kauffenstein $^{1}$, S.C. Robson ${ }^{2}$, J. Sévigny ${ }^{1}$. \\ ${ }^{T}$ Centre de Recherche en Rhumatologie et Immunologie, Université Laval, Québec, Canada. \\ ${ }^{2}$ Beth Israel Deaconess Medical Center, Harvard Medical School, Boston, MA, USA. \\ Gilles.kauffenstein@crchul.ulaval.ca
}


Extracellular nucleotides regulate vascular tone in different ways and can promote both vasodilation and vasoconstriction. Activation of certain endothelial $\mathrm{P} 2$ receptors induces the release of endothelium derived vasodilating agents (NO, $\left.\mathrm{PGI}_{2}, \mathrm{EDHF}\right)$ leading to a vasorelaxation. In contrast, the direct effect of nucleotides on vascular smooth muscle cells (SMC) P2 receptors induces the phosphorylation of myosin light chain, its association with actin leading to SMC contraction and a vasoconstriction. Nucleoside triphosphate diphosphohydrolase-1 (NTPDase1) is expressed on both vascular endothelium and smooth muscle. As NTPDase1 is the major ectonucleotidase regulating nucleotide concentrations and platelet activation in the environment of blood vessels, we propose that this enzyme may also regulate vasomotor tone. Despite normal adventitial expression of NTPDase2 in Entpd1 -/- mice vessels, the absence of NTPDase1 leads to a dramatic deficit in global nucleotidase activity (hydrolysis of ATP, ADP, UTP and UDP). Using ex vivo isometric contraction experiments we compared the vascular reactivity of aortic ring of Entpd1 -/- and $+/+$ mice. We found that the effect of exogenous nucleotides is enhanced in the absence of NTPDase1. While UDP and UTP $(100 \mu \mathrm{M})$ induced a strong contraction $(1.7$ fold of Phenylephrine-induced contraction) in Entpd1 -/- mice aorta rings, only a weak contraction could be measured in Entpd1+/+ mice aorta rings (0.1fold of Phenylephrine-induced contraction). The pharmacological profile of the response suggests that a $\mathrm{P}_{2} \mathrm{Y}_{6}$ like receptor is involved in the uracil nucleotide-induced vasoconstriction. Our data show that NTPDase1 regulates extracellular nucleotide signalling at the level of medial SMC.

\title{
Absence/Reduced Level of Diadenosine Triphosphatase (Fhit protein) is a Characteristic Feature of Several Neural Tumoural Cell Lines
}

\author{
$\underline{\text { P. Rotllán }}{ }^{1}$, C.R. Rodríguez-Ferrer ${ }^{1}$, S. Oaknin ${ }^{1}$, D. Lorenzo-Villegas ${ }^{2}$ and E. Castro $^{2}$ \\ Departments of Biochemistry and Molecular Biology. Universities of ${ }^{1}$ La Laguna and ${ }^{2}$ Las Palmas de Gran \\ Canaria. Canary Islands, Spain. \\ protllan@ull.es
}

Mammalian cells contain several intracellular diadenosine polyphosphates, $\mathrm{Ap}_{\mathrm{n}} \mathrm{A}$, a family of dinucleotides with intriguing biological functions. Among them, $\mathrm{Ap}_{3} \mathrm{~A}$ and $\mathrm{Ap}_{4} \mathrm{~A}$ receive most attention and appear to play antagonistic roles as intracellular signals in the control of cellular status. Cell proliferation and differentiation are associated with increases in $\mathrm{Ap}_{3} \mathrm{~A} / \mathrm{Ap}_{4} \mathrm{~A}$ ratio but apoptosis with decreases. Increased intracellular $\mathrm{Ap}_{4} \mathrm{~A}$ concentration may be involved in tumour cell growth suppression by inducing apoptosis. Diadenosine triphosphatase $\left(\mathrm{Ap}_{3} \mathrm{Aase}\right)$ and asymmetrical diadenosine tetraphosphatase $\left(\mathrm{Ap}_{4} \mathrm{Aase}\right)$ are the most important regulators of intracellular $\mathrm{Ap}_{3} \mathrm{~A}$ and $\mathrm{Ap}_{4} \mathrm{~A}$ levels. It has been reported that the novel tumour suppressor Fhit (fragil histidine triad) protein displays dinucleotide hydrolase in vitro and that the "old" enzyme $\mathrm{Ap}_{3}$ Aase and Fhit are identical proteins. Re-expression of Fhit protein in Fhit deficient cancer cells results in growth inhibition and restoration of apoptosis.

We have determined the activities and expression of Fhit-Ap $\mathrm{A}_{3}$ Aase and $\mathrm{Ap}_{4}$ Aase in soluble protein extracts from the following tumoural cell lines: C6 (rat glioma) Daoy (human medulloblastoma) and PC12 (rat pheochromocytoma). Soluble protein extracts from brain areas (hypothalamus, hippocampus, temporal cortex, frontal cortex and striatum), primary astrocytes from rat cerebellum and $\mathrm{HCN}-1 \mathrm{~A}$ cells were used as controls. The fluorogenic substrates $\varepsilon-\left(A p_{3} A\right)$ and $\varepsilon-\left(A p_{4} A\right)$ were used for measurement of $A p_{3} A$ ase and $A p_{4} A$ ase activities and polyclonal Fhit antibodies to detect Fhit- $\mathrm{Ap}_{3}$ Aase expression by western blotting. Both Fhit-Ap ${ }_{3}$ Aase and $\mathrm{Ap}_{4} \mathrm{Ase}$ were clearly detected in all brain areas with activity levels around 4 and $22 \mathrm{mU} / \mathrm{mg}$ respectively and $\mathrm{Ap}_{4} A$ Aase $/ \mathrm{Ap}_{3}$ Aase activity ratios ranging between 4 and 6 . Similar values were obtained for whole brain extracts. These ratios seem to be organ specific and limited between 4 and 10. Table I summarizes results obtained for cell cultures and whole brain.

Table 1. Fhit-Ap $\mathrm{p}_{3}$ Aase and $\mathrm{Ap}_{4}$ Aase activity (mU/mg protein) in extracts of whole brain and cell cultures. Values are means $\pm \mathrm{SD}$. $\mathrm{R}$ means $\mathrm{Ap}_{4} \mathrm{Aase} / \mathrm{Ap}_{3}$ Aase ratio.

\begin{tabular}{|c|c|c|c|c|c|c|c|}
\hline & $\mathrm{Ap}_{3}$ Aase & $\mathrm{Ap}_{4}$ Aase & $\mathrm{R}$ & & $\mathrm{Ap}_{3}$ Aase & $\mathrm{Ap}_{4}$ Aase & $\mathrm{R}$ \\
\hline Brain & $4.22 \pm 0.43$ & $22.10 \pm 2.21$ & $5.23 \pm 0.43$ & PC12 & $0.10 \pm 0.03$ & $5.46 \pm 0.72$ & $53.63 \pm 8.19$ \\
\hline Astrocytes & $2.25 \pm 1.09$ & $21.33 \pm 5.42$ & $10.05 \pm 2.47$ & C6 & $0.33 \pm 0.53$ & $15.98 \pm 2.32$ & $140.7 \pm 163.8$ \\
\hline $\mathrm{HCN}-1 \mathrm{~A}$ & $0.40 \pm 0.20$ & $9.97 \pm 3.88$ & $39.64 \pm 36.75$ & Daoy & 0 & $14.76 \pm 3.76$ & $\infty$ \\
\hline
\end{tabular}


Fhit- $\mathrm{Ap}_{3}$ Aase activity was extremely low in PC12 and C6 cells and practically undetectable in Daoy cells. These tumoural cell lines presented aberrant high $\mathrm{Ap}_{4} \mathrm{Aase} / \mathrm{Ap}_{3}$ Aase ratios at expense of low $\mathrm{Ap}_{3}$ Aase activity. Lack of Fhit- $\mathrm{Ap}_{3}$ Aase activity correlated with lack of Fhit protein expression as detected by western-blotting. Results suggest that alterations in metabolism of $\mathrm{Ap}_{\mathrm{n}} \mathrm{A}$ and their intracellular antitumour signalling pathways are involved in genesis/progression of neural tumours.

Acknowledgments: Work funded by grants TR5-02 and TR2003/06 (Gobierno de Canarias with participation of Hospiten SA, NovoNordisk Ibérica SA, Aventis Behring SA) and C03/06 (Red CIEN, FIS).

\section{Activation of ecto-5' ${ }^{\prime}$-nucleotidase by phospholipids}

A. Pexa, S. Schudeja, A. Deussen

Department of Physiologie, Medical Faculty Carl Gustav Carus, TU Dresden, Dresden, Germany

annette.pexa@tu-dresden.de

Backround: Ecto-5'-nucleotidase is the major enzyme controlling extracellular adenosine production in endothelial cells. Adenosine is a signal molecule involved in various hemodynamic and inflammatory processes. Activation of ecto-5'-nucleotidase increases adenosine concentration on the cell surface, and therefore may provide a tool to influence inflammatory processes. Furthermore, phospholipids are known modulators of various hemodynamic and inflammatory processes. Therefore adenosine might act as part of a signal transduction pathway in phospholipid signalling.

Methods: As experimental model we used human umbilical vein endothelial cells (HUVEC), pre-incubated with 5-10 $\mu \mathrm{M}$ of various phospholipids including $\gamma$-acyl- $\beta$-lyso- $\alpha$-phosphatidylcholine, $\beta$-arachidonyl- $\gamma$-palmityl- $\alpha$-phosphatidylcholine, $\beta, \gamma$-dipalmityl- $\alpha$-phosphatidyl-choline, $\beta, \gamma$-dipalmityl- $\alpha$-phosphatidylethanolamine, $\beta, \gamma$-dipalmityl- $\alpha$-phosphatidylserine, $\gamma$-acyl- $\beta$-lyso- $\alpha$-phosphatidylethanolamine, $\gamma$-acyl- $\beta$-lyso- $\alpha$-phosphatidylic acid, sphingosine-1-phosphate and sphingosylphosphorylcholine and $\beta$-acetyl- $\gamma$-O-hexadecyl- $\alpha$-phosphatidylcholine (known as platelet activating factor). In the cell supernatant the extracellular dephosphorylation rate of the fluorescent AMP-analogue $1, \mathrm{~N}^{6}$-etheno-5 AMP to $1, \mathrm{~N}^{6}$-etheno-adenosine was measured by HPLC.

Results: Out of these ten structurally related phospholipids only lysophosphatidylcholine (LPC), sphingosylphosphorylcholine (SPC) and platelet activating factor (PAF) increased the breakdown rate of AMP in a dose dependent manner. Interestingly, the activation can only be observed in a narrow concentration range between 5 and $20 \mu \mathrm{M}$. At higher phospholipid-concentrations the effect is blunted. Pharmacological blocking experiments with AOPCP, a specific inhibitor of ecto-5'-nucleotidase, showed that this effect was due to an activation of ecto-5'nucleotidase.

Although protein kinases $\mathrm{A}$ and $\mathrm{C}$ are known to activate ecto-5'-nucleotidase, application of blockers of both kinases did not diminish the effects of LPC, SPC and PAF. Furthermore, the effect does not seem to be mediated by G-protein-coupled receptors, as suramine and pertussis toxin had no effect.

Conclusions: Using information on the known molecular structures of tested phospholipids, a phosphatidylcholine residue in $\alpha$-position and a short chain length fatty acid esterified in $\beta$-position seem essential for activation of ecto-5'-nucleotidase by phospholipids. The signal transduction/activation mechanism of ecto-5'-nucleotidase remains unclear at present.

Lit.: Pexa A, Deussen A (2005) Modulation of ecto-5'-nucleotidase by phospholipids in human umbilical vein endothelial cells (HUVEC). Naunyn Schmiedebergs Arch Pharmacol 372:131-138

\section{Activation of $\mathrm{P} \mathrm{Y}_{1}$ and $\mathrm{P}_{2} \mathrm{X}_{7}$ receptors induce calcium/calmodulin-dependent protein kinase II phosphorylation in cerebellar granule neurons.}

${\underline{\mathbf{M}^{\mathrm{a}} \text { Teresa Miras-Portugal }}}^{(1)}$, David León ${ }^{(1)}$, Patricia Marín Garcia ${ }^{(1)}$, Felipe Ortega ${ }^{(1)}$, Jesús SánchezNogueiro $^{(1)}$, Cristina Hervás ${ }^{(1)}$, Nyrup M ${ }^{(2)}$

(1) Department of Biochemistry, Veterinary Faculty, Universidad Complutense de Madrid. 
(2) Department of Pharmacology and Pharmacotherapy. The Danish University of Pharmaceutical Science. mtmiras@vet.ucm.es

The activation of nucleotide receptors -both ionotropic, P2X, and most of metabotropic, P2Y- increases intracellular calcium concentration, resulting in calcium-calmodulin-dependent protein kinase II (CaMKII) activation. Stimulation of cerebellar granule neurons in culture -with different P2X and P2Y agonists and their effect on CaMKII phosphorylation- was studied using immunocytochemical and microfluorimetrical techniques. P2X agonist: 2'-3'-o-(4-benzoylbenzoyl)-adenosine 5'-triphosphate (BzATP), $\alpha, \beta$-methylene adenosine 5'-triphosphate $\left(\alpha, \beta\right.$-meATP) and diadenosine pentaphosphate $\left(\mathrm{Ap}_{5} \mathrm{~A}\right)$, and P2Y agonists: 2-(methylthyo)-adenosine diphosphate (2MeSADP) and uridine 5'-bisphosphate (UDP), tested induced a CaMKII phosphorylation but with a different immunostaining pattern in each group. Stimulation with $2 \mathrm{MeSADP}$ induced a $\mathrm{Ca}^{2+}$ release from intracellular stores and a significant CaMKII phosphorylation in both cell somas and fibres. This agrees with the subcellular distribution of $\mathrm{P}_{2} \mathrm{Y}_{1}$. MRS 2179, a specific P2Y $\mathrm{Y}_{1}$ inhibitor, antagonized the 2MeSADP effect. On the other hand, cerebellar granule neuron stimulation with BzATP, in $\mathrm{Mg}^{2+}$ free conditions, produced extracellular calcium entrance and, as a result, a significant increase in CaMKII phosphorylation mostly in fibers, which correspond with $\mathrm{P}_{2} \mathrm{X}_{7}$ subdistribution. Immunocytochemical and microfluorimetrical experiments, using $\mathrm{Zn}^{2+}$ and Brilliant Blue $\mathrm{G}$ $(\mathrm{BBG})$, as an specific $\mathrm{P} 2 \mathrm{X}_{7}$ antagonist, confirmed that BzATP was acting through the $\mathrm{P} 2 \mathrm{X}_{7}$ receptor. These results, indicate that $\mathrm{P}_{2} \mathrm{Y}_{1}$ and $\mathrm{P} 2 \mathrm{X}_{7}$, produce a significant increase in CaMKII phosphorylation, but show important differences in subcellular distribution and in effect duration. The abundant presence of $\mathrm{P} 2 \mathrm{X}_{7}$ at the synaptic structures suggest the relevant role played by this receptor in synaptic plasticity.

\title{
Activation of the adenosine A2A receptor protects mice deficient in CD8+ T-lymphocytes from liver ischemia-reperfusion injury.
}

\author{
McGreevy KS, Linden J. \\ Department of Medicine, University of Virginia, Charlottesville, VA 22908 USA \\ ksm3@hscmail.mcc.virginia.edu
}

BACKGROUND: The actions of T-lymphocyte subsets and their regulation through adenosine is of interest in many disease models, including ischemia-reperfusion injury. This study explores the effect of the selective adenosine $\mathrm{A}_{2 \mathrm{~A}}$ receptor agonist ATL-313 in animals depleted of $\mathrm{CD}^{+}$or $\mathrm{CD}^{+}$T-lymphocytes in a model of liver ischemia-reperfusion. METHODS: Immune-competent B6 mice were depleted of $\mathrm{CD}^{+}{ }^{+}$or $\mathrm{CD} 8^{+} \mathrm{T}$-lymphocytes in vivo by the introduction of an antibody to the respective cell type into the circulation. Rat monoclonal antibodies were prepared by an intraperitoneal injection of the hybridoma cell lines GK1.5 or 2.43 into SCID mice. Ascites fluid was collected and purified, and the final antibody concentration was adjusted to $1 \mathrm{mg} / \mathrm{ml}$ for storage. Experimental animals were tested for appropriate depletion of $\mathrm{CD}^{+}$or $\mathrm{CD}^{+}$T-lymphocytes through FACS analysis of the remaining T-lymphocyte population, labeled with commercially available antibodies. The antibodies were titered to determine the optimal dose of each for producing effective and selective depletion, found to be $0.05 \mathrm{mg}$ per mouse delivered on two consecutive days. On the fourth day after the second injection animals were subjected to liver ischemia/reperfusion, which was performed by application of a microaneurysm clamp to the hepatic triad for seventy-five minutes. Drug-treated animals received a bolus injection of $3 \mu \mathrm{g} / \mathrm{kg}$ ATL-313 fifteen minutes prior to reperfusion, and an Alzet pump delivering $1 \mathrm{ng} / \mathrm{kg} / \mathrm{min}$ ATL-313 at reperfusion, while control animals received vehicle. Blood was drawn 24 hours after the onset of ischemia, and a serum alanine aminotransferase (ALT) assay was performed to assess the degree of liver injury. RESULTS: In vehicle treated animals, the extent of injury in $\mathrm{CD}^{+}$depleted mice $(1846.2 \pm 440.8 \mathrm{IU} / \mathrm{L})$ was significantly less than that of wildtype $(5747.9 \pm 413.6 \mathrm{IU} / \mathrm{L})$ or $\mathrm{CD}^{+}$depleted mice $(5809.4 \pm 1097.1 \mathrm{IU} / \mathrm{L})$. ATL-313 provided significant protection in wild-type $(3472.0 \pm 666.0 \mathrm{IU} / \mathrm{L})$ and $\mathrm{CD}^{+}$depleted animals $(3475.5 \pm 424.9 \mathrm{IU} / \mathrm{L})$ but not in $\mathrm{CD} 4^{+}$ depleted animals $(1768.9 \pm 735.2 \mathrm{IU} / \mathrm{L})$. CONCLUSION: The loss of CD4 ${ }^{+} \mathrm{T}$-lymphocytes provides protection from liver ischemia-reperfusion injury, while the loss of $\mathrm{CD} 8^{+}$cells does not. A protective effect of the adenosine $\mathrm{A}_{2 \mathrm{~A}}$ receptor agonist ATL-313 is seen in wild-type and $\mathrm{CD} 8^{+}$depleted animals, but no further protection is conferred upon $\mathrm{CD}^{+}$depleted animals, suggesting that ATL-313 protects through an interaction with $\mathrm{CD} 4^{+}$cells. 
References:

1. Zwacka RM, Zhang Y, Halldorson J, Schlossberg H, Dudus L, Engelhardt JF. 1997. CD4+ T-Lymphocytes mediate ischemia/reperfusion-induced inflammatory responses in mouse liver. J. Clin. Invest. 100:279-289.

2. Day YJ, Li Y, Rieger JM, Ramos SI, Okusa MD, Linden J. 2005. A2A adenosine receptors on bone marrow-derived cells protect liver from ischemia-reperfusion injury. J. Immunol. 174:5040-6.

\title{
Activation-dependent Trafficking of NTPDase2 in Chinese Hamster Ovary Cells
}

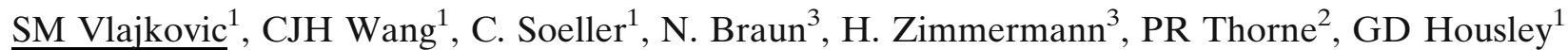

${ }^{T}$ Department of Physiology and ${ }^{2}$ Discipline of Audiology, Faculty of Medical and Health Sciences, The University of Auckland, New Zealand; ${ }^{3}$ Biozentrum der J.W. Goethe-Universität, AK Neurochemie, Frankfurt am Main, Germany

s.vlajkovic@auckland.ac.nz

Membrane-bound NTPDase2 is a member of the ecto-nucleoside triphosphate diphosphohydrolase (E-NTPDase) enzyme family involved in the regulation of P2 receptor signalling. NTPDase2 has broad substrate specificity for extracellular nucleotides, but hydrolyses nucleoside 5'-triphosphates with high preference over nucleoside $5^{\prime}$ diphosphates. In this study we have sought to determine how enzyme substrates acting on P2 receptors affect intracellular NTPDase2 trafficking. To achieve this, Chinese hamster ovary (CHO) cells expressing endogenous $\mathrm{P}_{2} \mathrm{X}_{7}$ and $\mathrm{P} 2 \mathrm{Y}_{2}$ receptor subunits were transiently transfected with rat-specific NTPDase2 cDNA (GenBank accession no. NM_172030) tagged with a green fluorescent protein (GFP) to allow direct visualisation of subcellular localisation and trafficking of NTPDase2.

Methods: Cells were transfected with plasmid containing recombinant GFP-NTPDase2 cDNA using Lipofectamine $^{\mathrm{TM}} 2000$ reagent. Transiently transfected $\mathrm{CHO}$ cells were imaged using an inverted confocal microscope (Zeiss LSM410) with 488-nm excitation. Cells were perfused $(1 \mathrm{ml} / \mathrm{min}$ ) with NTPDase2 substrates (ATP, UTP) and non-hydrolyzable ATP and ADP analogues ATP $\gamma$ S and ADP $\beta S(0.5 \mathrm{mM})$ in phosphate-free saline solution at room temperature. Z-stack image series were taken at $0,5,10,20$ and 30 minutes. P2 receptor inhibitors (suramin, PPADS, brilliant blue) were pre-incubated with $\mathrm{CHO}$ cells for 60 minutes before adding extracellular nucleotides. NTPDase2 incorporation into the cell membrane was determined at time intervals by comparative analysis of pixel intensity of fluorescence in the cytosolic and membrane compartments. To delineate the plasma membrane, CHO cells were stained with a membrane dye Di 4 ANNEPS (Molecular Probes). For calcium imaging, cells were loaded with $4 \mu \mathrm{M}$ Fluo-4 (Molecular Probes) in cell culture medium for 60 minutes before stimulation with ATP, UTP and ADP. This study confirmed the functional expression of endogenous ATP-preferring P2X receptors and ADP and UTP-preferring P2Y receptors. NTPDase 2 activity was measured in transiently transfected GFP-NTPDase2 CHO cells. Cells were perfused with NTPDase2 substrates ATP and UTP $(0.5 \mathrm{mM})$ individually to assess the hydrolysis rate. Perfusion was paused for 1 minute at time intervals used for cell imaging and a sample aliquot was removed for HPLC analysis.

Results and conclusion: (1) The present study shows that NTPDase2 tagged with GFP is functional. (2) ATP and its non-hydrolysable analogue $\mathrm{ATP} \gamma \mathrm{S}$ induce rapid membrane incorporation of NTPDase 2 from putative intracellular stores in CHO cells transiently transfected with GFP-NTPDase2, whilst UTP and ADP $\beta$ S are ineffective. (3) NTPDase2 trafficking requires extracellular $\mathrm{Ca}^{2+}$. (4) Pharmacological studies show that activation of P2X receptors involving $\mathrm{Ca}^{2+}$ entry, rather than $\mathrm{P} 2 \mathrm{Y}$ receptors releasing stored $\mathrm{Ca}^{2+}$, may be linked to trafficking of NTPDase2 to the cell membrane. (5) Initial hydrolysis rate for ATP correlates with increased NTPDase2 expression on the plasma membrane, whilst the initial velocity of UTP hydrolysis remains steady. (6) Increased trafficking of NTPDase 2 to the cell membrane may reflect a regulatory mechanism to limit excessive stimulation and desensitisation of $\mathrm{P} 2$ receptors.

Supported by the Health Research Council of New Zealand and Auckland Medical Research Foundation. 


\title{
Acute elevation of free fatty acids induces vasodilation which is not mediated by adenosine receptor stimulation
}

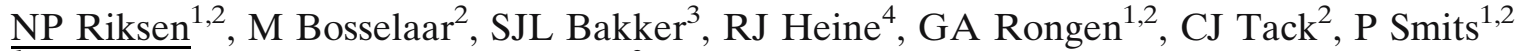 \\ ${ }^{1}$ Dept of Pharmacology-Toxicology, and ${ }^{2}$ Internal Medicine, Radboud University Nijmegen Medical Centre, ${ }^{3}$ De- \\ partment of Internal Medicine, University Medical Centre Groningen, ${ }^{4}$ Diabetes Centre, VU University Medical \\ Center, the Netherlands. \\ N.Riksen@aig.umcn.nl
}

Background Characteristics of the metabolic syndrome are associated with a hyperdynamic circulation. In these patients, a hyperdynamic circulation predicts future development of type 2 diabetes mellitus. Free fatty acids (FFA) have been implicated as mediators of this hyperdynamic circulation in patients with the metabolic syndrome. Previous in vitro studies have shown that FFA can inhibit the mitochondrial adenine nucleotide transporter, thus increasing cytosolic adenosine concentration. We hypothesized that FFA-induced vasodilation is mediated by adenosine receptor stimulation ${ }^{1}$.

Methods Nine healthy subjects participated in a randomised cross-over trial. Intralipid was administered intravenously for 2 hours with heparin to activate lipoprotein lipase to elevate plasma FFA. Glycerol/heparin was given on the other occasion as Control treatment. Forearm blood flow (FBF) was measured by venous occlusion plethysmography. During the last 15 minutes of infusion, the adenosine receptor antagonist caffeine was administered into the brachial artery of the non-dominant arm. In a validation study, adenosine was infused into the brachial artery $(5 \mu \mathrm{g} / \mathrm{min}$ per $\mathrm{dl}$ of forearm tissue) and subsequently caffeine was added $(90 \mu \mathrm{g} / \mathrm{min} / \mathrm{dl})$ to confirm effective adenosine receptor antagonism $(\mathrm{n}=6)$.

Results Administration of Intralipid increased plasma FFA from $334 \pm 108 \mu \mathrm{mol} / \mathrm{l}$ at baseline to $1269 \pm 234$, whereas Control infusion increased FFA from $338 \pm 114 \mu \mathrm{mol} / \mathrm{l}$ to only $432 \pm 175$. Intralipid infusion induced a hyperdynamic circulation: systolic blood pressure increased from $115 \pm 8$ to $122 \pm 8$ and from $116 \pm 10$ to $120 \pm 9 \mathrm{mmHg}$, pulse pressure increased from $53 \pm 6$ to $59 \pm 6$ and from $53 \pm 5$ to $55 \pm 9 \mathrm{mmHg}$ and FBF increased from $1.8 \pm 0.7$ to $2.7 \pm 1.8$ and from $2.3 \pm 0.7$ to $2.7 \pm 1.8 \mathrm{ml} / \mathrm{dl} / \mathrm{min}$ for Intralipid versus Control infusion, respectively. $(\mathrm{P}=0.05, \mathrm{P}<0.05$, and $\mathrm{P}$ $<0.05)$. In the validation study, adenosine-induced vasodilation was effectively reduced by caffeine $(\mathrm{P}<0.01)$. However, the FFA-induced vasodilation could not be reduced by administration of caffeine $(\mathrm{P}=\mathrm{NS})$.

Conclusion Acute elevation of plasma FFA levels induces an increased skeletal muscle blood flow, systolic blood pressure, and pulse pressure (hyperdynamic circulation). The vasodilator response is not mediated by adenosine receptor stimulation. Therefore, it is unlikely that adenosine mediates the association of a hyperdynamic circulation and the metabolic syndrome.

1. Bakker, S. J.; Gans, R. O.; ter Maaten, J. C.; Teerlink, T.; Westerhoff, H. V.; Heine, R. J. The potential role of adenosine in the pathophysiology of the insulin resistance syndrome. Atherosclerosis 2001, 155, 283-290.

\section{Acyl derivatives of coenzyme $A$ inhibit ADP-induced platelet function via antagonism at $\mathrm{P}_{2} \mathrm{Y}_{1}$ and $\mathrm{P}_{2} \mathrm{Y}_{12}$ receptors.}

Stan Heptinstall, Panagiotis Manolopoulos, Jacqueline R Glenn, Susan C Fox, Jane A May, Natalia Dovlatova, Siau-Wei Tang, Nadine Bauer, Martin Hesse, Neil R Thomas ${ }^{1}$ and Vera Ralevic. Centre for Integrated Systems Biology and Medicine and ${ }^{1}$ Centre for Biomolecular Sciences, University of Nottingham, Nottingham, UK.

s.heptinstall@nottingham.ac.uk

ADP plays a central role in platelet aggregation and is important in haemostasis and thrombosis. Individuals whose platelets respond poorly to ADP often present with easy bruising or bleeding. Also a pharmaceutical agent that inhibits ADP-induced platelet aggregation, clopidogrel, is in widespread use as an antithrombotic agent. ADP induces platelet aggregation acting via $\mathrm{P}_{2} \mathrm{Y}_{1}$ and $\mathrm{P}_{2} \mathrm{Y}_{12}$ receptors and clopidogrel acts as an antagonist at $\mathrm{P}_{2} \mathrm{Y}_{12}$ receptors. Interaction with $\mathrm{P}_{2} \mathrm{Y}_{1}$ induces rapid $\mathrm{Ca}^{2+}$ mobilisation leading to a change in the shape of platelets from smooth discs to spiny spheres and a transient aggregation response. Interaction with $\mathrm{P}_{2} \mathrm{Y}_{12}$ inhibits 
adenylate cyclase and activates PI-3-kinase which sustains the aggregation response. Both receptor subtypes are required for full aggregation induced by ADP. Antagonists acting at either P2 $\mathrm{Y}_{1}$ or P2 $\mathrm{Y}_{12}$ receptors (or both) could be usefully employed as anti-thrombotic agents. Following an observation that derivatives of coenzyme A (CoA) act as antagonists at $\mathrm{P}_{2} \mathrm{Y}_{1}$ receptors transfected into oocytes (1), we tested the effects of various acyl derivatives of $\mathrm{CoA}$ on platelet aggregation. Measurements were performed in both whole blood and platelet-rich plasma. CoAs containing a range of saturated and unsaturated acyl groups inhibited ADP-induced platelet aggregation. Those with saturated acyl groups containing 16-18 carbons were most effective and introduction of unsaturation into the fatty acid chains reduced efficacy. The effects of palmitoyl-CoA (16:0) were then tested on a variety of aspects of platelet function. Specificity for effects on platelet aggregation induced by ADP was demonstrated by showing that palmitoyl-CoA inhibited ADP-induced aggregation but not that induced by platelet activating factor, thrombin receptor activating peptide (TRAP) or serotonin. ADP-induced aggregation and also $P$-selectin expression (a marker of $\alpha$-granule release) were inhibited in a competitive way. Measurements of shape change were performed using the Biola laser aggregometer and also flow cytometry. Complete inhibition of ADPinduced shape change by palmitoyl-CoA indicated antagonism via $\mathrm{P}_{2} \mathrm{Y}_{1}$ receptors. Again there was no inhibition when other agonists were used, including agonists that act at $\mathrm{P} 2 \mathrm{X}_{1}$ receptors. $\mathrm{Ca}^{2+}$ mobilisation was measured using fluo 3-labelled platelets and flow cytometry. These studies also revealed complete inhibition by palmitoyl CoA of the increase in $\left[\mathrm{Ca}^{2+}\right]_{\mathrm{i}}$ that occurred in response to ADP, again indicative of antagonism at $\mathrm{P} 2 \mathrm{Y}_{1}$ receptors. Experiments were also performed to look for possible antagonistic effects of palmitoyl-CoA at $\mathrm{P}_{2} \mathrm{Y}_{12}$ receptors on platelets. This was done by examining platelet aggregation and/or $\mathrm{Ca}^{2+}$ mobilisation in response to collagen and to pairs of agonists: ADP and serotonin, and ADP and TRAP. Measurements of ADP-induced changes in cAMP and vasodilator-stimulated phosphoprotein phosphorylation were also performed. Results obtained with palmitoyl-CoA were compared with those for MRS 2179 (a P2Y 1 antagonist), AR-C69931 (a P2Y 12 antagonist) and combinations of these. The results were all consistent with palmitoyl-CoA inhibiting platelet function via antagonism at $\mathrm{P}_{2} \mathrm{Y}_{12}$ receptors in addition to $\mathrm{P}_{2} \mathrm{Y}_{1}$ receptors. Acyl derivatives of CoA such as those used in this investigation are natural agents that could be released into blood following cell and tissue activation and/or damage and thereby be involved in endogenous control of platelet function. They might also form the basis for a new class of antithrombotic drugs.

1. Coddou C et al. FEBS Lett. 2003;536:145-50

\title{
Adenine and adenosine salvage pathways in erythrocytes and the role of S-adenosylhomocysteine hydrolase. A theoretical study using elementary flux modes
}

\author{
Dimitar Kenanov and Stefan Schuster \\ Department of Bioinformatics, Friedrich Schiller University, Ernst-Abbe-Platz 2, 07743 Jena, Germany \\ Phone +49-3641-949580, Fax +49-3641-946452 \\ kenanov@minet.uni-jena.de
}

This talk is devoted to the study of redundancy and yield of salvage pathways in human erythrocytes. These cells are well known not to be able to synthesize ATP de novo. However, the salvage (recycling) of certain nucleosides or bases to give nucleotide triphosphates is operative. As the salvage pathways use enzymes consuming ATP as well as enzymes producing ATP, it is not easy to observe the net synthesis of ATP. As for pathways using adenosine, a straightforward assumption is that these pathways start with adenosine kinase. However, a pathway bypassing this enzyme and using S-adenosylhomocysteine hydrolase instead was reported. So far, this route has not been analysed in detail.

For the analysis we use the concept of elementary flux modes which can be described as a minimal set of enzymes able to operate at steady state with all irreversible reactions running in the right direction. This method opens up the possibility to study the various modes of behaviour of the biochemical system and it also allows detection of possible bypasses otherwise not easily understood by just observing the metabolic pathway. This tool enables one to simulate the interaction between several subsystems utilising substrates of interest or systems with enzyme deficiencies which can be used in investigating diseases caused be these deficiencies.

Using the concept of elementary flux modes, in the present study we investigate theoretically which salvage pathways exist in erythrocytes, which enzymes belong to each of these and what relative fluxes these enzymes 
carry. Here, we compute the net overall stoichiometry of ATP build-up from the recycled substrates and show that the network has considerable redundancy. For example, four different pathways of adenine salvage and 12 different pathways of adenosine salvage are obtained. They give different ATP/glucose yields, the highest being 3 : 10 for adenine salvage and 2: 3 for adenosine salvage provided that adenosine is not used as an energy source. Implications for deficiencies of the enzymes ADA and PNPase are also discussed.

\title{
Reference:
}

S. Schuster and D. Kenanov. Adenine and adenosine salvage pathways in erythrocytes and the role of S-adenosylhomocysteine hydrolase. A theoretical study using elementary flux modes. FEBS J. (2005) 272: 5278-90.

\section{Adenosine 5' -triphosphate (ATP) in exhaled breath condensate of patients with hypoxia}

\author{
Zs Lázár ${ }^{1}$, É Huszár ${ }^{1}$, M Valyon ${ }^{2}$, I Horváth ${ }^{1}$ \\ ${ }^{T}$ Department of Pathophysiology, ${ }^{2}$ Department of Clinical Diagnostics, National Korányi Institute of TB and Pu- \\ lmonology, Budapest, Hungary \\ zsofia.lazar@koranyi.hu
}

\begin{abstract}
ATP is released from many different cells in response to hypoxia activating purine receptors expressed by the same or neighboring cells leading to bronchoconstriction in the airways. We hypothesized that in hypoxia ATP concentration in the lungs is elevated compared to normal oxygenation. In our study we compared ATP concentrations in exhaled breath condensate (EBC) from patients with respiratory diseases causing hypoxia and healthy subjects, we studied whether clinical improvement of patients resulted in a decrease in ATP concentrations and investigated the correlation between ATP levels and partial arterial oxygen pressure. EBC was collected for 15 minutes from 15 patients with hypoxia and 8 healthy subjects. Simultaneously, blood gas of all participants was analyzed. In 9 subjects, EBC collection and blood gas analysis were repeated following clinical improvement. ATP was measured by a bioluminescence assay (ENLITEN ATP Assay System, Promega). Statistical analysis was performed non-parametrically. Data are expressed as median [range]. Patients exhibited profound hypoxia compared to healthy subjects $(7.51$ [4.67-8.62] kPa vs. 10.23 [8.32-13.47] $\mathrm{kPa}, \mathrm{p}<0.01)$ but there was no significant difference in EBC ATP concentration between the two groups (40 [22-473] pM vs. 33 [26-257] pM). Although clinical variables and $\mathrm{pO}_{2}$ demonstrate significant improvement during hospitalization $\left(\mathrm{n}=9, \mathrm{pO}_{2}\right.$ pretreated: 7.84 [5.07-8.62] kPa vs. $\mathrm{pO}_{2}$ at discharge: 8.85 [5.08-9.75] $\left.\mathrm{kPa}, \mathrm{p}<0.01\right)$, ATP values did not change significantly $(\mathrm{n}=$ 9, ATP pretreated: 26 [22-473] pM vs. ATP at discharge: 47 [18-77] pM) and there was no correlation between changes in $\mathrm{pO}_{2}$ and ATP concentration in EBC. In summary, ATP is detectable in EBC; however, our results suggest that hypoxia per se is not a major contributing factor to its level.
\end{abstract}

This study was supported by the Hungarian National Scientific Research Foundation (OTKA T-43396).

\section{Adenosine $\mathbf{A}_{1}$ and $\mathbf{A}_{2 \mathrm{~A}}$ Receptors Regulate Response to Infection with Schis- tosomiasis (S.Mansoni)}

Tuere F. Wilder ${ }^{1}$, Mazen Makarem ${ }^{2}$, Stephen J. Davies ${ }^{2}$ and Bruce N. Cronstein ${ }^{1}$

${ }^{T}$ Division of Clinical Pharmacology, Department of Medicine, NYU School of Medicine, New York, NY USA. ${ }^{2}$ Department of Microbiology and Immunology, USUHS, Bethesda, MD USA

Tuere.wilder@med.nyu.edu

Background: Schistosomiasis is a parasitic disease affecting more than 200 million people worldwide in which the pathology, including hepatic fibrosis and portal hypertension, results from host reactions to parasite. We have documented that adenosine released endogenously ligates adenosine $\mathrm{A}_{2 \mathrm{~A}}$ receptors to promote fibrosis but inhibit inflammation. It also has been documented that $\mathrm{A}_{1}$ receptor activation may serve to regulate pulmonary and renal inflammation in mice during chronic diseases. We therefore determined whether adenosine $\mathrm{A}_{1}$ and $\mathrm{A}_{2 \mathrm{~A}}$ receptors contribute to the pathophysiology of schistosomal infection. 
Methods: 3-10 month old female $\mathrm{A}_{1}$ and $\mathrm{A}_{2 \mathrm{~A}} \mathrm{KO}$ and WT littermate controls were infected with S. mansoni and sacrificed 4 or 8 weeks post-infection (p.i.). Worms were perfused from the portal vein of infected mice and measured. Fibrosis was digitally evaluated in 6 picrosirius red-stained sections/mouse. mRNA for IFN- $\gamma$, IL-4, and IL-10 from whole livers was analyzed by real time RT-PCR. Supernates of cultured mesenteric lymph node (MLN) cells stimulated with soluble egg antigen were assayed for IFN- $\gamma$, IL-4, IL-10 and IL-5 by ELISA.

Results: The worms from the $\mathrm{A}_{1} \mathrm{KO}$ mice were larger than their WT littermates 4 and 8 weeks p.i. There was also an increase in the percentage of females in pairs in the $\mathrm{A}_{1} \mathrm{KO}$ in comparison to the WT $(76 \pm 6 \mathrm{~N}=8$ vs. $36 \pm 19 \mathrm{~N}$ $=3, \mathrm{p}=0.02) 4$ weeks p.i. In the $\mathrm{A}_{2 \mathrm{~A}} \mathrm{KO}$ mice there was a 5 and 20 -fold increase in IFN- $\gamma$ and IL-4 message, respectively, 4 weeks p.i. This increase was accompanied by a striking 2-fold increase in hepatic fibrosis in the $\mathrm{A}_{2 \mathrm{~A}} \mathrm{KO}$ compared to the WT mice $(19 \pm 2 \mathrm{~N}=8$ vs. $8 \pm 1 \mathrm{~N}=7, \mathrm{p}<0.0001)$. In addition, the levels of all cytokines tested in supernates from cultured MLN cells showed at least a 2-fold increase in the $\mathrm{A}_{1} \mathrm{KO}$ and $\mathrm{A}_{2 \mathrm{~A}} \mathrm{KO}$ mice as compared to their WT controls. There were even more marked increases in IFN- $\gamma$ and IL-4 levels in the KO mice (Table I).

Table I

\begin{tabular}{llllllrr}
\hline $\begin{array}{l}\text { A1WT } \\
(\mathrm{ng} / \mathrm{ml})\end{array}$ & $\begin{array}{l}\text { A1KO } \\
(\mathrm{ng} / \mathrm{ml})\end{array}$ & P value & $\begin{array}{l}\text { Increase } \\
\text { between } \\
\text { groups }\end{array}$ & $\begin{array}{l}\text { A2AWT } \\
(\mathrm{ng} / \mathrm{ml})\end{array}$ & $\begin{array}{l}\text { A2AKO } \\
(\mathrm{ng} / \mathrm{ml})\end{array}$ & $\begin{array}{c}\text { P value } \\
\text { Increase } \\
\text { between } \\
\text { groups }\end{array}$ \\
\hline $\begin{array}{l}\text { IFN- } \gamma \\
\text { IL-4 }\end{array}$ & $170 \pm 7 \mathrm{~N}=3$ & $877 \pm 9 \mathrm{~N}=3$ & $<0.0001$ & 5 - fold & $\begin{array}{r}152 \pm 7 \mathrm{~N}=3 \\
31 \pm 1 \mathrm{~N}=3\end{array}$ & $\begin{array}{c}1409 \pm 17 \mathrm{~N}=3 \\
140 \pm 3 \mathrm{~N}=3\end{array}$ & $\begin{array}{c}0.0002 \\
<0.0001\end{array}$ \\
\hline
\end{tabular}

Conclusion: Endogenous adenosine modulates immune responses to schistosomal infection via interaction with its receptors. Increased hepatic fibrosis in infected $\mathrm{A}_{2 \mathrm{~A}} \mathrm{KO}$ mice most likely reflects enhanced immune reactions to parasites observed in these animals. On the other hand, although $\mathrm{A}_{1}$ receptors modulate the immune response they play no role in the development of hepatic fibrosis in infected mice but appear to play a permissive role in worm fecundity.

\section{Adenosine $A_{1}$ and $A_{2 A}$ Receptors, CD39/CD73KO Influence Granulomatous Responses to Mycobacterium Bovis BCG}

$\underline{\text { Firas M. Kara }}^{1}$, M. Carmen Montesinos ${ }^{2}$, Adam Mor $^{1}$, Simon Robson $^{3}$, Linda Thompson ${ }^{4}$ and Bruce N. Cronstein $^{1}$

${ }^{1}$ Division of Clinical Pharmacology, Department of Medicine, NYU School of Medicine, New York, NY USA, ${ }^{2}$ University of Valencia, Valencia, Span, ${ }^{3}$ Harvard School of Medicine, Boston, MA. ${ }^{4}$ Oklahoma Medical Research Foundation, Oklahoma City, OK

Firas.kara@med.nyu.edu

Background: Granuloma formation around infected macrophage is a defining cellular response to mycobacterium infections. Granulomas eliminate bacteria and also protect surrounding host tissue from destructive inflammatory responses. Without granuloma formation, mycobacterial infections can become widely disseminated and frequently lethal, as occurs in human AIDS-associated tuberculosis. Granuloma formation is characterized by a nodular aggregation of mononuclear cells and multinucleated giant cells. Adenosine, formed extracellularly from adenine nucleotides by nucleoside triphosphate dephosphorylase (CD39) and ecto-5'nucleotidase (CD73), modulates macrophage function by engaging cell surface adenosine receptors: A1 adenosine receptor activation promotes in vitro giant cell formation and enhances phagocytosis, whereas $\mathrm{A}_{2 \mathrm{~A}}$ receptor activation inhibits in vitro giant cell formation and phagocytosis. Therefore, we sought to study the formation of hepatic granulomas induced by $\mathrm{BCG}$ in receptor $\left(A_{1}\right.$ and $\left.A_{2 A}\right)$ and adenosine-producing enzyme (CD39), (CD73) and (CD39/CD73) knockout mice. Methods: 6 week old CD39KO, CD73KO and CD39/CD73KO, $\mathrm{A}_{2 \mathrm{~A}} \mathrm{KO}$ and $\mathrm{A}_{1} \mathrm{KO}$ and appropriate WT female mice were injected (IP) with BCG $\left(10^{7} \mathrm{CFU}\right)$. After 4 weeks mice were sacrificed and hepatic granuloma number, morphology and size analyzed digitally and expressed as granulomas $/ 10 \times$ field and pixels/granuloma, respectively. Results: Granuloma size did not differ among CD39KO, CD73KO and WT mice (3445 $\pm 796, \mathrm{~N}=3,2818 \pm 610$, N $=2$ vs. $2767 \pm 205, \mathrm{~N}=10)$ but were larger in CD39/73KO mice $(8295 \pm 2681, \mathrm{~N}=5, \mathrm{P}<0.0001$ vs. WT mice $)$. 
There were similar numbers of granulomas in the CD73KO and CD39/73KO mice and WT mice $(47 \pm 14 \mathrm{~N}=2$ and $35 \pm 12 \mathrm{~N}=5$ vs. $53 \pm 5 \mathrm{~N}=10$, respectively). CD39KO mice showed increased number of significantly granulomas than wild type mice $(63 \pm 26 \mathrm{~N}=3$ vs. $53 \pm 5 \mathrm{~N}=10, \mathrm{P}=0.02)$.

$\mathrm{A}_{1} \mathrm{KO}$ mice formed more granulomas than WT mice (22 \pm 9 vs. $13 \pm 6, \mathrm{n}=5$ for both, $\left.\mathrm{p}=0.004\right)$ although granulomas were smaller in $\mathrm{A}_{1} \mathrm{KO}$ mice $(1135 \pm 102$ vs. $1611 \pm 136, \mathrm{n}=5$ for both, $\mathrm{p}=0.004)$. In contrast, there were fewer granulomas in the $A_{2 A} \mathrm{KO}$ mice formed fewer granulomas $(4 \pm 2$ vs. $12 \pm 6, n=5$ for both, $p=0.00005)$ of the same size as WTs (Data not shown).

Conclusion: Adenosine, produced extracellularly from adenine nucleotides, alters the response to mycobacterial infections via interaction with $\mathrm{A}_{1}$ and $\mathrm{A}_{2 \mathrm{~A}}$ receptors on macrophages.

\section{Adenosine $\mathbf{A}_{1}$ receptor occupancy and sleep regulation: a positron emission tomography study}

Geissler $\mathrm{E}^{1,2}$, Ametamey $\mathrm{SM}^{1}$, Wyss $\mathrm{M}^{3}$, Treyer $\mathrm{V}^{3}$, Rétey $\mathrm{JV}^{2}$, Adam $\mathrm{M}^{2}$, Achermann $\mathrm{P}^{2}$, Schubiger $\mathrm{PA}^{1}$, Landolt $\mathrm{HP}^{2}$

${ }^{1}$ Center for Radiopharmaceutical Science of ETH, PSI and USZ, ETH Zürich, Switzerland

${ }^{2}$ Institute of Pharmacology and Toxicology, University of Zürich, Switzerland

${ }^{3}$ Division of Nuclear Medicine, University Hospital Zürich, Switzerland

landolt@pharma.unizh.ch

Recent findings support the notion that adenosine and adenosine receptors play an important role in human sleep regulation ${ }^{1,2}$. Post-mortem studies demonstrated a high density of adenosine $A_{1}$ receptors $\left(A_{1} A R\right)$ in brain regions involved in sleep regulation, such as thalamus, hippocampus, striatum, basal ganglia and cerebral cortex. Moreover, the non selective adenosine $\mathrm{A}_{1}$ and $\mathrm{A}_{2 \mathrm{~A}}$ receptor antagonist, caffeine, stimulates alertness and attenuates changes in the waking and sleep EEG, which are typically found after sleep deprivation. Nevertheless, the distinct roles of $A_{1}$ and $A_{2 A}$ receptors for sleep regulation are still controversial. The recent development of the potent and selective $\mathrm{A}_{1} \mathrm{AR}$ antagonist, 8-cyclopentyl-3-(3- ${ }^{18} \mathrm{~F}$-fluoropropyl)-1-propyl-xanthine $\left({ }^{18} \mathrm{~F}\right.$-CPFPX), offers the opportunity to directly visualize and quantify $\mathrm{A}_{1} \mathrm{AR}$ binding in the living human brain.

We established the radiosynthesis of ${ }^{18}$ F-CPFPX according to a recently reported two step reaction sequence ${ }^{3}$. We obtained the target compound ${ }^{18} \mathrm{~F}$-CPFPX in a radiochemical yield of about $10 \%$ and a radiochemical purity of $>99 \%$. Specific activity exceeded $100 \mathrm{GBq} / \mu \mathrm{mol}$.

In a still ongoing study, we combine quantitative EEG recordings and positron emission tomography (PET) brain imaging to determine whether $\mathrm{A}_{1} \mathrm{AR}$ occupancy is enhanced after 32 hours of prolonged wakefulness when compared to a baseline recording (after 8 hours of wakefulness). Moreover, we examine whether the sleep-deprivation induced changes in subjective vigilance, neurobehavioral performance and the EEG are reduced after $300 \mathrm{mg}$ slowrelease caffeine intake when compared to placebo. To date, two groups of 5 healthy young men completed the study. The first results support a high ${ }^{18} \mathrm{~F}$-CPFPX accumulation in sleep regulatory areas such as thalamus and cortex. In contrast, low ligand binding is found in cerebellum and brain stem.

We conclude that PET brain imaging with ${ }^{18} \mathrm{~F}$-CPFPX is a promising new tool to gain new insights into adenosinergic mechanisms of sleep regulation in humans. The effects of sleep deprivation and caffeine on cortical and subcortical A1AR occupancy remain to be determined.

Research supported by a Center for Neuroscience Zürich PhD Grant and the Swiss National Science Foundation.

(1) Landolt, H. P.; Rétey, J. V.; Tönz, K.; Gottselig, J. M.; Khatami, R.; Buckelmüller, J.; Achermann, P. Neuropsychopharmacol 2004, 29, 1933-1939.

(2) Rétey, J. V.; Adam, M.; Honegger, E.; Khatami, R.; Luhmann, U. F.; Jung, H. H.; Berger, W.; Landolt, H. P. Proc Natl Acad Sci USA 2005, 102, 15676-15681.

(3) Holschbach, M. H.; Olsson, R. A.; Bier, D.; Wutz, W.; Shiver, W.; Schuller, M.; Palm, B.; Coenen, H. H. J Med Chem 2002, 45, 5150-5156. 


\section{Adenosine A1 receptors are increased and sensitized in pick disease frontal cortex}

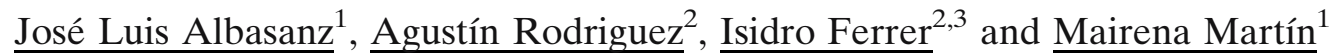

${ }^{T}$ Departamento de Química Inorgánica, Orgánica y Bioquímica, Facultad de Químicas, Centro Regional de Investigaciones Biomédicas, Universidad de Castilla-La Mancha, Ciudad Real.

${ }^{2}$ Departamento de Biología Celular y Anatomía Patológica, Facultad de Medicina, Universidad de Barcelona, campus de Bellvitge, Hospitalet de Llobregat.

${ }^{3}$ Instituto de Neuropatología, Servicio de Anatomía Patológica, IDIBELL-Hospital Universitario de Bellvitge, Hospitalet de Llobregat. SPAIN

jose.albasanz@uclm.es

Adenosine is a nucleoside widely distributed in nervous system where acts as a neuromodulator and neuroprotector through specific receptors. Adenosine receptors have been classified into four types: $A_{1}, A_{2 A}, A_{2 B}$ and $A_{3}$ receptors. $A_{1}$ and $A_{3}$ receptors inhibit adenylyl cyclase activity through Gi/o proteins, while $A_{2 A}$ and $A_{2 B}$ receptors stimulate adenylyl cyclase through Gs proteins. Adenosine acting through $\mathrm{A}_{1}$ receptors inhibits excitatory neurotransmitter release, therefore acting as a neuroprotector. Pick's disease $(\mathrm{PiD})$ is a fronto-temporal dementia characterized by severe atrophy of the frontal and temporal lobes that spares the pre-central gyrus and the posterior two-thirds of the superior temporal gyrus. This is accompanied by marked neuron loss, mainly in the upper cortical layers, and the appearance of typical phospho-tau-immunoreactive intraneuronal inclusions named Pick bodies, principally in the dentate gyrus of the hippocampus, CA1 region of the hippocampus, amygdala, septal nuclei, and upper layers of the entorhinal cortex and isocortex, together with phospho-tau-immunoreactive thorn-shaped and ramified astrocytes, and tau-positive bodies in oligodendroglia. The aim of the present work was to study $\mathrm{A}_{1}$ receptors/adenylyl cyclase (AC) pathway in post-mortem human cortex from Pick's disease (PiD) as compared with age matched non-demented controls. Adenosine $\mathrm{A}_{1}$ receptors, determined by radioligand binding and Western-blotting assays were significantly increased in PiD samples, suggesting the up-regulation of this receptor. Real time PCR analysis revealed an increase in mRNA coding $\mathrm{A}_{1}$ receptor in PiD cases. Adenylyl cyclase activity was determined in basal and stimulated conditions via stimulatory guanine nucleotide binding proteins (Gs) using GTP, or directly with forskolin. Basal AC activity was reduced in brains from PiD cases. This agrees with the decrease in AC I level detected by Western-blotting. No significant differences in GTP- or forskolin-stimulated adenylyl cyclase activity were observed between PiD and control cases. However, inhibition of forskolin-stimulated AC activity by a selective $\mathrm{A}_{1} \mathrm{R}$ agonist was significantly increased in brains from $\mathrm{PiD}$. These results show that adenosine $\mathrm{A}_{1}$ receptors/adenylyl cyclase transduction pathway is up-regulated and sensitized in cortex brain from Pick's disease.

\section{Adenosine $\mathbf{A}_{1}$ receptors in the phrenic motoneurons of adult rats: $\mathbf{A}$ biochemical study}

Saharan SR, Kizy T, and Nantwi, KD.

Department of Anatomy and Cell Biology, Wayne State University, School of Medicine, 540 E. Canfield, Detroit, MI 48202

knantwi@med.wayne.edu

Previous studies from our laboratory have demonstrated that in an animal model of spinal cord injury (SCI), a latent respiratory motor pathway can be activated to restore respiratory activity to a hemidiaphragm paralyzed by an upper cervical (C2) spinal cord hemisection. Activation of the motor pathway occurs during a reflex known as the "crossed phrenic phenomenon", by asphyxia or systemically administered theophylline. Theophylline acts via blockade of adenosine $\mathrm{A}_{1}$ receptors. Adenosine receptors are located in the phrenic motor neurons (PMN). The objective of the present study is to characterize adenosine receptors in the PMN of adult rats by radioligand binding. Binding Assays: The specific binding of the adenosine $\mathrm{A}_{1}$ - receptor selective ligand $\left[{ }^{3} \mathrm{H}\right]$-DPCPX to spinal cord membranes was determined using a rapid filtration assay. Spinal cord membranes were first incubated with adenosine deaminase (ADA, $5 \mathrm{U} / \mathrm{mg}$ protein) in $50 \mathrm{mM}$ Tris buffer, $2 \mathrm{mM} \mathrm{MgCl}_{2}$ at $\mathrm{pH} 7.4$ to remove endogenous adenosine. Membrane suspension (at $0.1 \mathrm{mg} / \mathrm{ml}$ protein in a final volume of $500 \mu \mathrm{l}$ ) was incubated for $1 \mathrm{~h}$ at $4^{\circ} \mathrm{C}$ with $\left[{ }^{3} \mathrm{H}\right]$-DPCPX in the 
absence of a competing compound and equilibrium binding reactions were terminated by vacuum filtration (Brandel harvester GF/B filters $0.5 \%$ PEI). Each filter was washed four times with $4 \mathrm{ml}$ of cold $50 \mathrm{mM}$ Tris-HCl, pH7.4. Filter discs were punched into vials using a Deposit/Dispenser. Three milliliter aliquots of 'Ultima Gold' Scintillation Cocktail were added into each vial prior to scintillation counting. Filter-bound radioactivity was determined by liquid scintillation counting using a Tricarb-2800-TR Liquid Scintillation counter. [ $\left.{ }^{3} \mathrm{H}\right]$-DPCPX was used (at 8-12 concentrations) in saturation binding. Competition studies used theophylline as the competing ligand at ten concentrations $(20 \mathrm{mM}-20 \mathrm{pM})$; against a standard concentration of $\left[{ }^{3} \mathrm{H}\right]$-DPCPX $(1 \mathrm{nM})$. Binding data were analyzed with (Graphpad software,). Results: In naïve animals, a single receptor binding site with $\mathrm{B}_{\max }$ and $\mathrm{K}_{\mathrm{d}}$ values of $256.00 \pm 32.13(\mathrm{fmol} / \mathrm{mg}$ protein) and $2.89 \pm 0.45(\mathrm{nM})$, respectively, was detected. Theophylline induced detection of a second binding site with a Bmax of $492.6 \pm 3.15(\mathrm{fmol} / \mathrm{mg})$ and $\mathrm{Kd} 14.09 \pm 2.06(\mathrm{nM})$ respectively. Hemisection induced an up-regulation of $A_{1}$ receptors; a Bmax $316.6 \pm 25.10 \mathrm{fmol} / \mathrm{mg}$ protein without apparent change in affinity $(\mathrm{Kd} 2.72 \pm 0.72 \mathrm{nM})$. It is concluded that $\mathrm{A}_{1}$ receptors in the PMN of adult rats are altered significantly by theophylline administration and C2 hemisection. The changes may underlie respiratory effects of theophylline. Furthermore, the up-regulation of the receptors after hemisection may be involved in theophylline-induced functional recovery.

\title{
Adenosine $\mathbf{A}_{1}$ Receptors Play a Critical Role in Osteoclast Formation
}

\author{
Firas M. Kara $^{1}$, Bertil Fredholm ${ }^{2}$ and Bruce N. Cronstein ${ }^{1}$ \\ ${ }^{1}$ Division of Clinical Pharmacology, Department of Medicine, NYU School of Medicine, New York, NY USA. \\ ${ }^{2}$ Karolinska Institutet, Stockholm, Sweden \\ Firas.kara@med.nyu.edu
}

Background: Osteoclasts are bone-resorbing, multinucleated giant cells that are essential for bone remodeling that are formed through the fusion of mononuclear precursor cells. Osteoclasts differentiate from hemopoietic precursors of the monocyte/macrophage lineage in the presence of M-CSF and receptor activator of NF- $\mathrm{kB}$ ligand (RANKL). Deficiency of osteoclasts leads to osteopetrosis, a condition characterized by increased bone density. Nonetheless, most bone diseases are due to increased bone resorption by osteoclasts and inhibition of osteoclastmediated bone resorption is a primary therapeutic objective. Indeed, most current therapies for osteoporosis are directed at inhibition of osteoclast function. Because we have previously reported that adenosine $\mathrm{A}_{1}$ receptor occupancy is required for fusion of stimulated human monocytes to form giant cells in vitro we determined whether there was a similar requirement for $\mathrm{A}_{1}$ receptor occupancy in osteoclast formation.

Methods: Spleens were harvested from female mice and, following isolation, splenocytes were resuspended in aMEM containing 10\% FBS. The cells were cultured in the presence of M-CSF (30 ng/ml) and RANKL (30 ng/ $\mathrm{ml}$ ) with or without various concentrations of the $\mathrm{A}_{1}$ receptor antagonist DPCPX or the $\mathrm{A}_{1}$ receptor agonist N6CPA. The culture was fed every 3 days by replacing half of the media containing the M-SCF, RANKL and adenosine receptor agonist/antagonist. After incubation for 7 days, cells were stained for tartrate-resistant acid phosphatase (TRAP). Osteoclasts were identified as TRAP-positive cells with 3 or more nuclei; the number of TRAP-positive multinucleated cells/well was then enumerated.

Results: We found that adenosine $A_{1}$ receptor occupancy is critical for the formation of osteoclasts by murine splenocytes incubated with M-CSF and RANKL in vitro. DPCPX inhibited osteoclast formation in a dose-dependent fashion ( $\mathrm{P}=0.0033)$; DPCPX inhibits osteoclastogenesis by directly acting on osteoclast precursors on day $0(95.00 \pm 2.309$ vs. $24.00 \pm 4.509 \mathrm{~N}=3, \mathrm{P}=0.0002)$ compared to day 3 and $6(95.00 \pm 2.309$ vs. $63.33 \pm 14.53 \mathrm{~N}=3$, $\mathrm{P}=0.0977$ ) since removal of DPCPX by adding N6CPA after 3 and 6 days after the start of incubation did not reverse the inhibition of osteoclastogenesis $(24.00 \pm 4.509 \mathrm{~N}=3$ vs. $29.33 \pm 2.333 \mathrm{~N}=3, \mathrm{P}=0.3528)$. In contrast to their wild type controls splenocytes from $\mathrm{A}_{1}$ receptor $\mathrm{KO}$ mice formed almost no osteoclasts in response to $\mathrm{M}$ CSF and RANKL $(120.4 \pm 23.93 \mathrm{~N}=5$ vs. $21.40 \pm 2.839 \mathrm{~N}=5, \mathrm{P}=0.0034)$. The osteoclast number and function in the $\mathrm{A}_{1} \mathrm{KO}$ mice did not appear to be normal as, in contrast to WT controls, osteoclasts in the $\mathrm{A}_{1} \mathrm{KO}$ mice are not attached to bone and there is little bone resorption associated with these osteoclasts.

Conclusion: These results indicate that endogenously released adenosine, acting at adenosine $\mathrm{A}_{1}$ receptors, plays a critical role in the formation of osteoclasts and bone remodeling. Study presented above will probably lead to new therapeutic approaches in several diseases that are characterized by excessive bone resorption. These results suggest that adenosine $\mathrm{A}_{1}$ receptor antagonists may be useful in the treatment of such conditions as osteoporosis, prosthetic joint loosening and other conditions in which osteoclasts play a pathogenic role (eg Paget's disease). 


\title{
ADENOSINE A ANTAGONISM IS PROTECTIVE IN A MODEL OF FOCAL CEREBRAL ISCHEMIA IN THE RAT
}

\author{
Pedata F., Gianfriddo M., Vannucchi M.G. ${ }^{1}$, Cipriani S., Giovannini M.G. and Melani A. \\ Department of Preclinical and Clinical Pharmacology, University of Florence, 50139 Florence, Italy; ${ }^{1}$ Department \\ of Histology, University of Florence, 50139 Florence, Italy. \\ felicita.pedata@unifi.it
}

Previous results indicate that the adenosine $\mathrm{A}_{2 \mathrm{~A}}$ antagonist SCH 58261 administered acutely immediately after focal ischemia reduces glutamate outflow and acute motor disturbance in the first hours after ischemia ${ }^{1}$.

The effect of a subchronic treatment of the $\mathrm{A}_{2 \mathrm{~A}}$ receptor antagonist, SCH58261, was studied in the same model of focal cerebral ischemia. Focal ischemia was induced by middle cerebral artery occlusion (MCAo) ${ }^{2}$. SCH58261 $(0.01 \mathrm{mg} / \mathrm{kg}$, i.p.) was administered $5 \mathrm{~min}, 6$ hours and 15 hours after MCAo. Soon after ischemia, contralateral turning behavior was evaluated as the number of rotations per hour between 3 and $4 \mathrm{~h}$ after MCAo. In SCH58261-treated rats $(n=14)$, the number of rotations per hour was significantly reduced with respect to vehicletreated rats $(\mathrm{n}=13)$ (mean \pm S.E.: $116.9 \pm 34.6$ vs $795.4 \pm 170.6, \mathrm{p}<0.0001)$. Twenty-four hour after MCAo, neurological deficit and ischemic brain damage were evaluated. SCH58261-treated rats $(n=14)$ showed significant improvement of the neurological score (mean \pm S.E: $10.8 \pm 0.4$ vs $8.8 \pm 0.5, p<0.001$ ) and reduction in the extent of the ischemic damage by $26 \%$ in the cortex $\left(41.1 \pm 2.8 \mathrm{~mm}^{3}\right.$ vs $\left.55.6 \pm 3.9 \mathrm{~mm}^{3}, \mathrm{p}<0.02\right)$ and by $45 \%$ in the striatum $\left(12.8 \pm 1.9 \mathrm{~mm}^{3}\right.$ vs $\left.23.2 \pm 2.7 \mathrm{~mm}^{3}, \mathrm{p}<0.01\right)$ with respect to vehicle-treated rats. The rats were then sacrificed for evaluation of phospho-p38 MAPK levels in the ischemic hemisphere. The phospho-p38 MAPK levels in the ischemic striatum of vehicle-treated rats $(n=5)$ were increased by $500 \%$ compared to the contralateral non ischemic striatum. In SCH58261-treated rats $(n=6)$, the phospho-p38 MAPK levels were significantly reduced by $70 \%$ in the ischemic striatum $(\mathrm{p}<0.01)$ with respect to vehicle-treated rats. In the striatum and cortex the phospho-p38 MAPK immunopositive cells showed swollen and hypertrophyc cell bodies, as well as processes, and exhibited morphological features of activated microglia. Twenty-four hour after MCAo, astrocytes were found only in the corpus callosum. Activated microglia immunostained by OX-42 or isolectin B4 was present in the same cortical and striatal areas where phospho-p38 MAPK immunopositive cells were detected. SCH 58261 reduced phospho-p38 MAPK immunoreactivity in the striatum and cortex without changing the microglial cell morphology.

Results indicate that the protective effect of the adenosine antagonist SCH 5826124 hours after ischemia is not due to reduced microglial activation but may involve inhibition of phospho-p38 MAPK and indicate that treatment with the $\mathrm{A}_{2 \mathrm{~A}}$ antagonist from the first to several hours after ischemia may be a useful therapeutic approach in cerebral ischemia. (Grant from University of Florence and Ente Cassa di Risparmio Firenze, Italy).

(1) Melani, A.; Pantoni, L.; Bordoni, F.; Gianfriddo, M.; Bianchi, L.; Vannucchi, M.G.; Bertorelli, R.; Monopoli, A.; Pedata, F. Brain Res. 2003, 959, 243-250.

(2) Melani, A.; Pantoni, L.; Corsi, C.; Bianchi, L.; Monopoli, A.; Bertorelli, R.; Pepeu, G.; Pedata, F. Stroke 1999, 30, $2448-2455$

\section{Adenosine $A_{2 A}$ receptor $\left(A_{2 A} R\right)$ antagonism prevents dermal fibrosis}

$\underline{\text { Patricia Fernandez }}^{1}$, Sean Trzaska ${ }^{1}$, Carmen Montesinos ${ }^{2}$, Michael H Pillinger $^{1}$, Allison B Reiss ${ }^{3}$, Bruce $\mathrm{N}_{\text {Cronstein }}^{1} \&$ Edwin SL Chan ${ }^{1}$

${ }^{1}$ New York University School of Medicine, New York, NY; ${ }^{2}$ University of Valencia, Valencia, Spain; ${ }^{3}$ Winthrop University Hospital, Mineola, NY

Patricia.Fernandez@med.nyu.edu

Background: Adenosine is a potent endogenous regulator of inflammation and tissue repair. Since the adenosine $A_{2 A}$ receptor $\left(\mathrm{A}_{2 \mathrm{~A}} \mathrm{R}\right)$ promotes dermal wound closure and increases dermal matrix deposition, we determined whether adenosine may also play a role in fibrosis in pathological conditions such as scleroderma. We therefore investigated the effect of $\mathrm{A}_{2 \mathrm{~A}} \mathrm{R}$ activation on fibroblast collagen production and examined the role of $\mathrm{A}_{2 \mathrm{~A}} \mathrm{R}$ in bleomycininduced dermal fibrosis, a model of scleroderma. 
Methods: Primary human dermal fibroblasts (DFs) were incubated with the $\mathrm{A}_{2 \mathrm{~A}} \mathrm{R}$ agonist CGS-21680 $(1 \mathrm{nM}-10 \mu \mathrm{M}$, $24 \mathrm{hrs})$ in the presence or absence of $\mathrm{A}_{2 \mathrm{~A}} \mathrm{R}$ antagonist ZM241385 $(1 \mu \mathrm{M})$. Collagen content in whole cell lysates and supernates was analyzed by Western blot and Sircol assay respectively. Collagen I and collagen III mRNA (10 hrs) were quantitated by real-time RT-PCR. Dermal fibrosis was induced with bleomycin ( $0.1 \mathrm{U}$ sc qod $\times 18$ days) in $\mathrm{A}_{2 \mathrm{~A}} \mathrm{R}$-deficient $\left(\mathrm{A}_{2 \mathrm{~A}} \mathrm{KO}\right)$ versus wild-type (WT) littermate mice and in C57BL/6 mice treated with or without ZM241385 (50 mg/kg/day ip) and compared to PBS-treated controls. Dermal morphometric measurements and hydroxyproline content (reflecting collagen) were analyzed at sacrifice.

Results: CGS-21680 treatment increased type I collagen in dermal fibroblast lysates by up to $151 \pm 21 \%$ in a dosedependent manner and this increase was reduced by coincubation with ZM241385 ( $1 \mu \mathrm{M}, \mathrm{p}<0.04)$. Total collagen content was increased in supernates of CGS-21680 stimulated DFs to $176 \pm 14 \%$ of control $(\mathrm{n}=3, \mathrm{p}<0.01)$, and this increase was completely abrogated by ZM241385 $(10 \mu \mathrm{M}, \mathrm{n}=3, \mathrm{p}=\mathrm{NS}$ vs. control). CGS-21680 increased collagen I mRNA $(146 \pm 15 \%$ control, $\mathrm{n}=4, \mathrm{p}<0.05)$ as much as TGF- $\beta(10 \mathrm{ng} / \mathrm{ml})$, with a lesser increase in collagen III mRNA (124 $\pm 11 \%$ control, $\mathrm{n}=4, \mathrm{p}=\mathrm{NS})$. Following bleomycin treatment, WT mice had greater punch biopsy thickness, skin-fold thickness and higher dermal tensile strength ( $\mathrm{p}<0.05$ for all, data not shown) than $\mathrm{A}_{2 \mathrm{~A}} \mathrm{KO}$ mice. Furthermore, dermal hydroxyproline content was higher in WT than $\mathrm{A}_{2 \mathrm{~A}} \mathrm{KO}$ mice $(27.4 \pm 2.3$ vs. $19.5 \pm 0.7 \mu \mathrm{g} / \mathrm{mg}$ tissue, WT vs. $\mathrm{A}_{2 \mathrm{~A}} \mathrm{KO}$, respectively, $\left.\mathrm{n}=5, \mathrm{p}<0.005\right)$. Compared to vehicle-treated mice, ZM241385-treated mice were also protected from bleomycin-induced increases in morphometric measures as well as in dermal hydroxyproline content $(26.2 \pm 1.0$ vs. $20.9 \pm 1.0 \mu \mathrm{g} / \mathrm{mg}$ tissue, control vs. $\mathrm{ZM}, \mathrm{n}=5, \mathrm{p}<0.005)$.

Conclusion: Adenosine, released in response to hypoxia or cell injury, promotes, collagen production by human dermal fibroblasts via $A_{2 A} R$ occupancy. More importantly, deletion or blockade of adenosine $A_{2 A} R$ protects mice from bleomycin-induced dermal fibrosis, a model of such fibrosing conditions as scleroderma, and suggests a novel therapeutic use for $\mathrm{A}_{2 \mathrm{~A}} \mathrm{R}$ antagonists for the prevention of fibrosis in the skin.

\title{
Adenosine $A_{2 A}$ receptor activation and IgG- $E$. coli-immune complexes synergistically up-regulate IL-10 production in mouse macrophages
}

\author{
Balázs Csóka, Zoltán H. Németh, György Haskó \\ Department of Surgery, UMDNJ-New Jersey Medical School, Newark, NJ 07103 \\ csokaba@umdnj.edu
}

IL-10 is produced by monocytes/macrophages, and it has important anti-inflammatory and immunomodulatory effects. This function of IL-10 is important for protecting the host from harmful effects of prolonged inflammatory responses in the context of microbial infection. Adenosine, an endogenous purine nucleoside, is formed at sites of metabolic stress associated with inflammation. Adenosine mediates its effects through four adenosine receptors $\left(\mathrm{A}_{1}\right.$, $\mathrm{A}_{2 \mathrm{~A}}, \mathrm{~A}_{2 \mathrm{~B}}, \mathrm{~A}_{3}$ ), all of which are expressed on macrophages. Stimulation of adenosine receptors results in increased IL-10 production ( 2-fold) in macrophages activated by endotoxin. However, the effect of adenosine on IL-10 production in IgG-E.coli immune complex-treated macrophages is not known. In this study we found that IgG-E. coli-immune complexes increased IL-10 production approximately two-fold in peritoneal macrophages. Adenosine dramatically ( 8-10-fold) enhanced IgG-E. coli immune complex-activated IL-10 production, indicating a synergistic effect. The $\mathrm{A}_{1}$ receptor agonist CCPA and the $\mathrm{A}_{3}$ adenosine agonist IB-MECA failed to mimic the stimulatory effect of adenosine on immune complex-induced IL-10 production. However, both the selective $\mathrm{A}_{2 \mathrm{~A}}$ receptor agonist CGS-21680 and the non-selective agonist NECA increased IL-10 production, and CGS-21680 was the most potent. To further confirm the role of $\mathrm{A}_{2 \mathrm{~A}}$ receptors in promoting immune complex-primed IL-10 production, the abilities of selective adenosine receptor antagonists were examined in preventing the stimulatory effect of adenosine. We found that only the selective $\mathrm{A}_{2 \mathrm{~A}}$ antagonist $\mathrm{ZM} 241385$ reversed the effect of adenosine on immune complex-induced IL-10 production. In addition, we found that adenosine had no effect on immune complex-primed IL-10 production in $\mathrm{A}_{2 \mathrm{~A}} \mathrm{KO}$ mouse macrophages, which also emphasizes the importance of $\mathrm{A}_{2 \mathrm{~A}}$ receptor ligation in IL-10 up-regulation. To investigate the intracellular mechanisms responsible for the augmented production of IL-10 by adenosine, we determined IL-10 mRNA levels using real-time PCR. We found that adenosine increased IL-10 mRNA levels in IgG-E. coli-immune complex-treated cells. We also examined the effect of adenosine on immune complex-induced IL-10 promoter activity by transfecting RAW264.7 cells with a construct in which luciferase expression is driven by the IL-10 promoter. We found that adenosine enhanced immune complex-induced IL-10 promoter activity, and this effect was even more enhanced when RAW264.7 cells were co-transfected with an $\mathrm{A}_{2 \mathrm{~A}}$ 
receptor protein overexpressing construct. Collectively, these results suggest that adenosine up-regulates IgG-E. coli immune complex-induced IL-10 production. This effect is mediated by $\mathrm{A}_{2 \mathrm{~A}}$ adenosine receptor activation, and is associated with increased IL-10 mRNA accumulation and enhanced IL-10 promoter activation. Moreover the synergistic effect of adenosine was more robust on IgG- $E$. coli-activated IL-10 production than on LPS-induced IL-10 production.

\title{
Adenosine $\mathbf{A}_{2 \mathrm{~A}}$ receptor activation reduces lung injury in trauma/hemorrhagic shock
}

\author{
György Haskó, Balázs Csóka, DaZhong Xu, Qi Lu, Zoltán H. Németh, Edwin A. Deitch \\ Department of Surgery, UMDNJ-New Jersey Medical School, Newark, New Jersey, USA \\ haskoge@umdnj.edu
}

Hemorrhagic shock and resuscitation trigger a global ischemia/reperfusion phenomenon, in which various inflammatory processes critically contribute to the ensuing tissue damage. Adenosine is an endogenous nucleoside that is released during shock. Activation of adenosine $\mathrm{A}_{2 \mathrm{~A}}$ receptors can broadly inactivate inflammatory cascades. The current study was designed to evaluate the effect of $\mathrm{A}_{2 \mathrm{~A}}$ receptor activation on organ injury and inflammation in the setting of global ischemia/reperfusion elicited by trauma/hemorrhagic shock and resuscitation. Adult male Sprague-Dawley rats were subjected to a laparotomy (trauma) and 90 minutes of hemorrhagic shock or trauma/sham shock. The selective $\mathrm{A}_{2 \mathrm{~A}}$ receptor agonist CGS-21680 (2-p-(2-carboxyethyl) phenethylamino-5'- $N$ ethyl-carboxamidoadenosine; $0.5 \mathrm{mg} / \mathrm{kg}$ ) or its vehicle was injected 30 min before shock or immediately after resuscitation. At 3 hours following resuscitation, animals were killed and tissue harvested for analysis. Lung permeability and pulmonary myeloperoxidase levels were used to quantitate lung injury. Intestinal injury was determined by histologic analysis of terminal ileum. Red blood cell (RBC) deformability was measured by a laserassisted ektacytometer. Pretreatment with CGS-21680 protected the lung but not the gut against shock-induced injury and prevented the shock-induced decrease in RBC deformability. Post-treatment with CGS-21680 ameliorated shock-induced lung injury but failed to prevent gut injury and preserve $\mathrm{RBC}$ deformability. $\mathrm{A}_{2 \mathrm{~A}}$ receptor agonists may represent a novel therapeutic approach in preventing organ injury following trauma/hemorrhagic shock.

\section{Adenosine $\mathbf{A}_{2 \mathrm{~A}}$ Receptor Agonist Protects Mice from Compression-Induced Spinal Cord Injury}

Yuesheng Li and Joel Linden

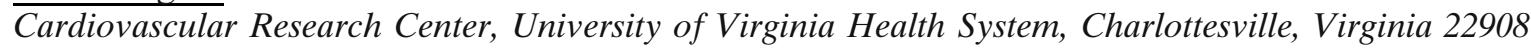
yjl4n@virginia.edu

The adenosine $A_{2 A}$ receptor $\left(A_{2 A} R\right)$ has been suggested as a predominant anti-inflammatory adenosine receptor1. In our previous studies, we have shown that activation of the $\mathrm{A}_{2 \mathrm{~A}} \mathrm{R}$ inhibits the activity of various inflammatory cells in vitro ${ }^{2-4}$ and in $v i v o^{5,6}$. Although inflammation clearly exacerbates ischemia-reperfusion injury (IRI) in heart, liver and kidney, it is not clear if this is also the case in spinal cord. We have developed a new system for scoring hindlimb locomotor dysfunction in mice, the mBBB scoring system. This system has improved our ability to evaluate compression-induced locomotor dysfunction following spinal cord injury (SCI) in mice ${ }^{7}$. We show that the $\mathrm{A}_{2 \mathrm{~A}} \mathrm{R}$ selective agonist, ATL313, transiently applied during or after spinal cord IRI persistently reduces mouse SCI based on the new mBBB locomotor score and a previously validated scoring system, $\mathrm{tBBB}^{8}$. Female mice were subjected to compression-induced SCI after laminectomy and periodically evaluated over 6 weeks. A $1 \times 2 \mathrm{~mm}$ region of spinal cord was compressed for 5 min with a $15 \mathrm{~g}$ weight applied to the dorsal surface of the cord at T12. ATL313 was administered IP for 5 minutes either before or just after injury, on the evening after surgery, and twice daily for the next 3 days. Compared to vehicle controls ATL313-treatment produced significant locomotor improvement that reached a plateau within 7 days and was sustained until the end of the experiment at 42 days. ATL313 did not influence SCI in $\mathrm{A}_{2 \mathrm{~A}} \mathrm{R}$ knockout mice. Ongoing experiments with 
bone marrow chimera mice created by irradiation and bone marrow transplantation indicate that spinal cord protection by ATL313 is due to receptors on bone marrow-derived cells and not due to receptors on neurons or the vasculature. The results suggest that inflammation does exacerbate SCI following spinal cord IRI, and that $\mathrm{A}_{2 \mathrm{~A}}$ agonists reduce injury by reducing inflammation. $\mathrm{A}_{2 \mathrm{~A}}$ agonist therapy may be useful for treating patients subjected to spinal cord ischemia during the repair vascular tears or aneurisms.

\section{References}

1. Linden, J. Adenosine in tissue protection and tissue regeneration. Molecular Pharmacology 2005, 67 (5), 13851387.

2. Lappas, C. M.; Rieger, J. M.; Linden, J. A(2A) adenosine receptor induction inhibits IFN-gamma production in murine CD4(+) T cells. Journal of Immunology 2005, 174 (2), 1073-1080.

3. Murphree, L. J.; Sullivan, G. W.; Marshall, M. A.; Linden, J. Lipopolysaccharide rapidly modifies adenosine receptor transcripts in murine and human macrophages: role of NF-kappa B in A(2A) adenosine receptor induction. Biochemical Journal 2005, 391, 575-580.

4. Sullivan, G. W.; Rieger, J. M.; Scheld, W. M.; Macdonald, T. L.; Linden, J. Cyclic AMP-dependent inhibition of human neutrophil oxidative activity by substituted 2-propynylcyclohexyl adenosine A(2A) receptor agonists. British Journal of Pharmacology 2001, 132 (5), 1017-1026.

5. Day, Y. J.; Huang, L. P.; McDuffie, M. J.; Rosin, D. L.; Ye, H.; Chen, J. F.; Schwarzchild, M. A.; Fink, J. S.; Linden, J.; Okusa, M. D. Renal protection from ischemia mediated by A(2A) adenosine receptors on bone marrow-derived cells. Journal of Clinical Investigation 2003, 112 (6), 883-891.

6. Day, Y. J.; Li, Y.; Rieger, J. M.; Ramos, S. I.; Okusa, M. D.; Linden, J. A2A adenosine receptors on bone marrow-derived cells protect liver from ischemia-reperfusion injury. Journal of Immunology 2005, 174 (8), 50405046.

7. Li, Y.; Oskouian, R. J.; Day, Y. J.; Kern, J. A.; Linden, J. Optimization of a Mouse Locomotor Rating System to Evaluate Compression-Induced Spinal Cord Injury: correlation of locomotor and morphological injury indices. Journal of Neurosurgery: Spine 2005, 4 (2), 165-173.

8. Ferguson, A. R.; Hook, M. A.; Garcia, G.; Bresnahan, J. C.; Beattie, M. S.; Grau, J. W. A simple post hoc transformation that improves the metric properties of the BBB scale for rats with moderate to severe spinal cord injury. Journal of Neurotrauma 2004, 21 (11), 1601-1613.

\section{Adenosine $\mathbf{A}_{2 \mathrm{~A}}$ receptor inactivation increases survival in polymicrobial sepsis}

György Haskó $^{1}$, Balázs Csóka ${ }^{1}$, Jeanette Wilmanski ${ }^{1}$, DaZhong Xu ${ }^{1}$, Qi Lu ${ }^{1}$, Catherine Ledent ${ }^{2}$, Edwin A. Deitch ${ }^{1}$, Pál Pacher ${ }^{3}$, Zoltán Spolarics ${ }^{1}$, and Zoltán H. Németh ${ }^{1}$

${ }^{1}$ Department of Surgery, UMDNJ-New Jersey Medical School, Newark, NJ 07103, USA; ${ }^{2}$ Institut de Recherche Interdisciplinaire en Biologie Humaine et Nucléaire, Université Libre de Bruxelles, B1070, Brussels, Belgium; ${ }^{3}$ National Institute on Alcohol Abuse and Alcoholism, National Institutes of Health, Bethesda, MD 20892-8115 haskoge@umdnj.edu

The mechanisms governing the impairment of bacterial clearance and immune function in sepsis are not known. Adenosine levels are elevated during tissue hypoxia and damage associated with sepsis. Adenosine has strong immunosuppressive effects, many of which are mediated by $\mathrm{A}_{2 \mathrm{~A}}$ receptors expressed on immune cells. We examined whether $\mathrm{A}_{2 \mathrm{~A}}$ receptors are involved in the regulation of immune function in cecal ligation and punctureinduced murine polymicrobial sepsis by genetically or pharmacologically inactivating $\mathrm{A}_{2 \mathrm{~A}}$ receptors. $\mathrm{A}_{2 \mathrm{~A}}$ receptor $\mathrm{KO}$ mice were protected from the lethal effect of sepsis and had improved bacterial clearance compared to WT animals. cDNA microarray analysis and flow cytometry revealed increased MHC II expression in $\mathrm{A}_{2 \mathrm{~A}}$-inactivated mice, suggesting improved antigen presentation as a mechanism of protection. Apoptosis was attenuated in the spleen of $\mathrm{A}_{2 \mathrm{~A}} \mathrm{KO}$ mice indicating preserved lymphocyte function. Levels of the immunosuppressive cytokines IL10 and IL-6 were markedly lower following $A_{2 A}$ receptor blockade. Similar to observations with $A_{2 A}$ receptor KO mice, an $\mathrm{A}_{2 \mathrm{~A}}$ receptor antagonist increased survival even when administered in a delayed fashion. These studies demonstrate that $\mathrm{A}_{2 \mathrm{~A}}$ receptor blockade may be useful in the treatment of infection and sepsis. 


\title{
ADENOSINE A2 $2_{A}$ RECEPTOR LEVELS IN DIFFERENT PERIPHERAL CELLS OF ALZHEIMER'S DISEASE PATIENTS
}

\author{
Lorenza Galimberti $^{1}$, Beatrice Arosio ${ }^{1}$, Carmen Calabresi ${ }^{1}$, Silvia Scurati ${ }^{1}$, Giorgio Annoni ${ }^{2}$ and Carlo \\ Vergani $^{1}$ \\ ${ }^{1}$ Department of Internal Medicine, Chair of Gerontology and Geriatrics, IRCCS Foundation, Ospedale Maggiore \\ Policlinico, Mangiagalli e Regina Elena, University of Milan, Milan, Italy; \\ ${ }^{2}$ Department of Clinical Medicine, Prevention and Medical Biotechnology, University of Milano-Bicocca, Milan, \\ Italy \\ lorenza.galimberti@unimi.it
}

Adenosine, released by cells and tissues, is an important endogenous purine neuromodulator which potently regulates inflammation. Its physiological effects are transduced through four different receptor subtypes (A1, $\mathrm{A} 2 \mathrm{~A}_{\mathrm{A}}, \mathrm{A} 2_{\mathrm{B}}$ and $\mathrm{A} 3$ ) that are variably expressed on immune and inflammatory cells. All adenosine receptors are Gprotein coupled receptors (GPCR) of the type 1 variety which act by modulating adenylyl cyclase ${ }^{(1)}$. In particular the $\mathrm{A} 2{ }_{\mathrm{A}}$ receptor subtype seems to be implicated in neuroprotective and anti-inflammatory mechanisms ${ }^{(2)}$; indeed the accumulation of extracellular adenosine in inflammatory and damaged tissues and the immunosuppressive properties of cAMP-elevating receptors indicate that signaling by $\mathrm{A} 2 \mathrm{~A}$ could be a possible natural mechanism of inhibition and/or termination of inflammation ${ }^{(3,4)}$. The aim of this study is to investigate if an altered expression and/or function of $\mathrm{A} 2 \mathrm{~A}$ receptors, could contribute to the inflammatory/neurodegenerative mechanisms involved in Alzheimer's disease (AD). We analysed the expression of this receptor in platelets and Peripheral Blood Mononuclear Cells (PBMCs) from AD patients and sex- and age-matched healthy controls (HC); furthermore we analysed a T/C single nucleotide polymorphism (SNP) at position 1083 of exon 2 of the $\mathrm{A} 2 \mathrm{~A}$ gene that seems to be correlated with other neurodegenerative disorders. We found no statistical significative differences in the distribution of this SNP between $\mathrm{AD}$ and $\mathrm{HC}$. By contrast the $\mathrm{A} 2_{\mathrm{A}}$ mRNA levels in platelets were $25 \%$ higher in $\mathrm{HC}$ than AD (1.09 and 0.82 respectively; $\mathrm{p}=0.000)$. To confirm these data we are analysing in a wider group of AD and $\mathrm{HC}$ the mRNA and protein expression of A2a in PBMCs. The preliminary results match with those obtained from platelets, suggesting a lower ability of $\mathrm{AD}$ subjects to terminate the inflammatory response and indicating a possible role of the $\mathrm{A} 2 \mathrm{~A}$ receptor in the pathogenesis of $\mathrm{AD}$.

1. Olah M.E., Stilles G.L. Adenosine receptor subtypes: characterization and therapeutic regulation. Annu. Rev. Pharmacol. Toxicol. 35, 1995; 581-606.

2. Ohta A., Sitkovsky M. Role of G-protein-coupled adenosine receptors in downregulation of inflammation and protection from tissue damage. Nature 414, 2001; 916-919.

3. Cronstein B.N. Adenosine, an endogenous anti-inflammatory agent. J.Appl. Physiol. 76, 1994;5-13.

4. Huang S., Koshiba M., Apasov S., Sitkovsky M. Role of $\mathrm{A} 2 \mathrm{~A}$ adenosine receptor-mediated signaling in inhibition of T cell activation and expansion. Blood 90, 1997; 1600-1610.

\section{Adenosine $\mathbf{A}_{2 \mathrm{~A}}$ receptors modulate psychomotor activity and brain injury by distinct cellular mechanisms}

Liqun Yuํㄹ Q Qing-yuan Huang ${ }^{1}$, Nelson Rebola ${ }^{4}$, Hai-Ying Shen ${ }^{1}$, Eric Kirsten Rapp ${ }^{1}$, Yuan-Ji Day ${ }^{3}$, Jarrod Ferrara ${ }^{1}$, Joana E. Coelho ${ }^{1}$, Paula M. Canas ${ }^{4}$, Zhi-Hong Huang ${ }^{2}$, Darcie Taylor ${ }^{1}$, Michael Moskowitz ${ }^{2}$, Michael Schwarzschild ${ }^{2}$, Joel Linden ${ }^{3}$, Rodrigo A. Cunha ${ }^{4}$ and Jiang-Fan Chen ${ }^{1 \#}$

${ }^{1}$ Department of Neurology, Boston University School of Medicine, Boston, MA; USA

${ }^{2}$ Neuroscience Center and Department of Neurology, Mass. General Hospital, Boston, MA; USA

${ }^{3}$ Department of Internal Medicine, University of Virginia, Charlottesville, VA; USA

${ }^{4}$ Center for Neuroscience, Institute of Biochemistry, University of Coimbra, Portugal

The adenosine $A_{2 A}$ receptor $\left(A_{2 A} R\right)$ has recently emerged as a leading non-dopaminergic therapeutic target for Parkinson's disease for its ability to regulate motor activity. Furthermore, $\mathrm{A}_{2 \mathrm{~A}} \mathrm{Rs}$ influence brain injury outcome 
in variety of neurological disease models, presumably through modulation of glutamate release. Using forebrain neuronal-specific $A_{2 A} R$ knockout $(K O)$ mice, we here provide the first direct evidence that $A_{2 A} R$-mediated control of motor function and neuroprotection involve distinct cellular mechanisms. By crossing the floxed $A_{2 A} R$ mice with the CaMKII-Cre transgenic line, we selectively depleted $\mathrm{A}_{2 \mathrm{~A}} \mathrm{R}$ mRNA and protein in forebrain neurons to the background level of the global $\mathrm{A}_{2 \mathrm{~A}} \mathrm{R} \mathrm{KO}$ mice, as demonstrated by in situ hybridization, immunochemistry and receptor binding assays. This genetic deletion of $\mathrm{A}_{2 \mathrm{~A}} \mathrm{Rs}$ in forebrain neurons abolished the psychomotor effect of the $\mathrm{A}_{2 \mathrm{~A}} \mathrm{R}$ selective agonist CGS21680 and antagonist KW-6002 and of the non-selective antagonist caffeine, and largely attenuated the pyschostimulant effect of cocaine. This demonstrates the key role of forebrain neuronal $\mathrm{A}_{2 \mathrm{~A}} \mathrm{Rs}$ in the modulation of psychomotor activity. In contrast, genetic deletion of the $\mathrm{A}_{2 \mathrm{~A}} \mathrm{R}$ in forebrain neurons did not confer protection against ischemic brain injury by middle cerebral arterial occlusion or against MPTP-induced dopaminergic neurotoxicity, despite abolishing CGS21680-mediated presynaptic facilitation of glutamate release in forebrain $\mathrm{A}_{2 \mathrm{~A}} \mathrm{R} \mathrm{KO}$ mice. Furthermore, intracerebral ventricular administration of KW-6002 into forebrain $A_{2 A} \mathrm{R} K O$ mice reinstated neuroprotection against MPTP neurotoxicity. These results provide the clearest data yet that $\mathrm{A}_{2 \mathrm{~A}} \mathrm{R}$ activity in forebrain neurons is critical to control psychomotor activity, but not for neuroprotection against brain injury, indicating that $\mathrm{A}_{2 \mathrm{~A}}$ Rs modulate motor activity and brain damage by distinct cellular mechanisms. This opens up the new possibility of selectively manipulating $\mathrm{A}_{2 \mathrm{~A}} \mathrm{R}$ 's motor and neuroprotective effects by targeting different cellular elements.

\title{
Adenosine $A_{2 A}$ Receptors Stimulate Collagen Production in LX-2 Cells via PKA, SRC and MAPK Cascade Signaling Pathway
}

\author{
Jiantu Che and Bruce N. Cronstein \\ Divisions of Clinical Pharmacology and Rheumatology, Department of Medicine, New York University School of \\ Medicine, New York, NY 10016
}

Background: We have previously reported that adenosine $\mathrm{A}_{2 \mathrm{~A}}$ receptor deletion protects mice from toxin-induced hepatic fibrosis and that adenosine $\mathrm{A}_{2 \mathrm{~A}}$ receptor occupancy promotes collagen production by hepatic stellate cells. We therefore determined the signaling pathways involved in adenosine receptor-mediated stimulation of a hepatic stellate cell line, LX-2 cells.

Methods: Soluble collagen was detected by Sircol assay according to the manufacturers directions. Type I and type III collagen mRNA expression was measured by real-time PCR. In addition, collagen I and III levels were detected semi-quantitatively by densitometric measurement of Western Blots. Phospho-Erk1/2 was detected by Western Blot.

Results: Soluble collagen was stimulated both in supernatant and the cells by CGS21680, an adenosine $\mathrm{A}_{2 \mathrm{~A}}$ receptor agonist, at concentrations ranging from $0.01-10 \mu \mathrm{M}(\mathrm{P}<0.005$ or $\mathrm{P}<0.001)$. Starting at 6 hours after treatment and reaching a peak at 24 hours. CGS21680 stimulated an increase in both mRNA and protein of collagens I and III (P $<$ 0.001). ZM241385, an adenosine $A_{2 A}$ receptor antagonist, completely blocked CGS21680-stimulated increases in soluble collagen (by $100 \%$ at 24 hours after treatment, $\mathrm{P}<0.001$ ) as well as collagen I (by $100 \%$ at 24 hours after treatment, $\mathrm{P}<0.001$ ) and III (by $100 \%$ at 24 hours after treatment, $\mathrm{P}<0.001$ ) with a relative potency profile consistent with blockade of an $\mathrm{A}_{2 \mathrm{~A}}$ receptor (maximal effect at $\mathbf{1} \mu \mathrm{M}$ ). Furthermore, the effect of CGS21680 on stimulation of soluble collagen was attenuated by the addition of Peptide Inhibitor of protein kinase A (PKAI, by $30 \%, 1 \mu \mathrm{M}, \mathrm{P}<0.005$ ), Src inhibitor II (SRCI, by 28\% P < 0.005), MEK-MAPK inhibitor U0126 (by $24 \%$, P < 0.005), the ERK MAPK inhibitor PD 98059 (by 37\%, P < 0.001), and the p38 MAPK inhibitor SB202190 (by $35 \%, P$ $<0.001$ ). Real-time PCR and Western blot analysis demonstrated that PKAI, SRCI, U0126 and PD98059 inhibited CGS21680-stimulated increases in both mRNA and protein of collagen I (by $50 \%, 48 \%, 44 \%$ and $42 \%$ respectively, $\mathrm{P}<0.001$ ), whereas SB202190 did not. In contrast, SB202190 inhibited CGS21680-stimulated type III collagen production (by $32 \%, \mathrm{P}<0.005$ ), which was independent of PKA, Src and ERK MAPK signaling pathway. The effect of PKAI, SRCI, U0123 and PD98059 on CGS21680-stimulated collagen production in LX-2 cells was associated with significantly decreased ERK1/2 phosphorylation.

Conclusions: These results further indicate that adenosine $\mathrm{A}_{2 \mathrm{~A}}$ receptors increase collagen production in LX-2 cells via a PKA-Src-MEK-ERK MAPK cascade or p38 MAPK signaling pathway. Moreover, these results provide further evidence that adenosine $\mathrm{A}_{2 \mathrm{~A}}$ receptor antagonists may be used to prevent hepatic fibrosis. Finally, prior studies indicate that coffee drinking prevents hepatic cirrhosis and our studies suggest that the mechanism by which coffee drinking prevents hepatic cirrhosis is via blockade of adenosine $\mathrm{A}_{2 \mathrm{~A}}$ receptors in the liver. 


\title{
Adenosine A2b receptor agonist mimics postconditioning: characterization of a selective adenosine A2b receptor agonist in a rabbit infarct model.
}

\author{
Thomas Krahn $^{1}$, Barbara Albrecht ${ }^{1}$, Ulrich Rosentreter ${ }^{2}$, Michael V. Cohen ${ }^{3}$, Natalia Solenkova ${ }^{3}$, \\ James M. Downey ${ }^{3}$ \\ From Depts. of Pharmacology ${ }^{1}$ and MedicinalChemistry ${ }^{2}$, Pharma Research Center, Bayer Health \\ Care, Wuppertal, Germany; Dept. of Physiology ${ }^{3}$, University of South Alabama, Mobile, AL USA \\ Th.Krahn:Thomas.Krahn@bayerhealthcare.com
}

There is a continuing clinical need for an agent given after the onset of ischemia that will protect the heart. Herein we present evidence that a specific agonist for the adenosine A2b receptor is cardioprotective when administered just prior to termination of $30 \mathrm{~min}$ of regional ischemia in rabbit hearts. The role of A2b receptors has been largely unexplored in adenosine-mediated cardioprotection because of the lack of specific A2b receptor agonists. Bayer Healthcare was the first to synthesize specific non-adenosine like A2b receptor agonists. ${ }^{1}$ These A2b specific agonists were characterized in $\mathrm{CHO}$ cells expressing human A1, A2a or A2b receptors. Here we report on BAY $\mathrm{A} 2 \mathrm{~b}$, a specific $\mathrm{A} 2 \mathrm{~b}$ agonist; $\mathrm{EC}_{50}$ values for receptor activation were $>10.000 \mathrm{nM}$ for $\mathrm{A} 1,>10.000 \mathrm{nM}$ for $\mathrm{A} 2 \mathrm{a}$ and 3 $\mathrm{nM}$ for A2b receptors. Major aspects concerning synthesis and selectivity of non-adenosine like A2b receptor agonists were also reproduced by other groups. ${ }^{2}$

BAY A2b was given to open-chest rabbits experiencing $30 \mathrm{~min}$ of regional ischemia and $3 \mathrm{~h}$ of reperfusion. Rabbits were anesthetized with sodium pentobarbital $(30 \mathrm{mg} / \mathrm{kg})$ and mechanically ventilated with $100 \%$ oxygen. The heart was exposed through a left thoracotomy and a ligature was passed under a coronary branch to create ischemia. Drug was given intravenously $10 \mathrm{ug} / \mathrm{kg}$ over $1 \mathrm{~min}$ starting $5 \mathrm{~min}$ prior to reperfusion and again $15 \mathrm{~min}$ after reperfusion. The heart was removed after $3 \mathrm{~h}$ of reperfusion. The risk zone was stained with fluorescent microspheres and the heart was cut into $2 \mathrm{~mm}$ slices and infarct size was determined by tetrazolium staining. ${ }^{3}$

No adverse hemodynamic effects were seen with the agent. A better than $50 \%$ reduction of infarct size was seen and was comparable to that in a third group receiving postconditioning ${ }^{4}$, an established cardioprotective intervention where the occluded artery is intermittently opened and closed for four 30-second cycles at the end of the ischemic insult. The A2b agonist BAY A2b is equivalent to postconditioning in its potency. In conclusion we have synthesized specific $\mathrm{A} 2 \mathrm{~b}$ receptor agonists and have shown that an $\mathrm{A} 2 \mathrm{~b}$ receptor agonist can reduce infarct size by application after the onset of ischemia.

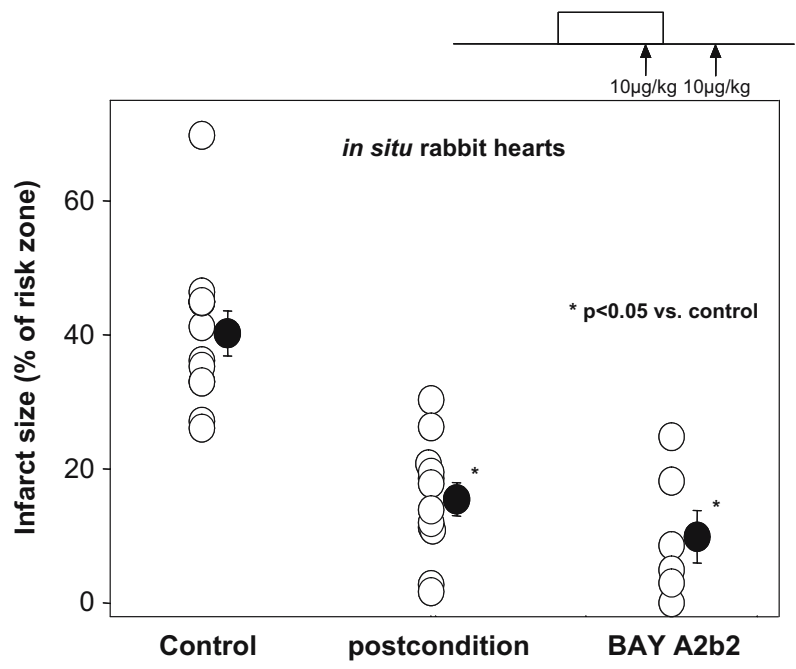

(1) Bayer HealthCare AG. WO0125210, 2001; WO03008384, 2003

(2) Beukers, M. W.; Chang, L. C. W.; von Frijtag Drabbe Künzel, J. K.; Mulder-Krieger, T.; Spanjersberg, R. F.; Brussee, J.; Ijzerman A. P. J. Med. Chem. 2004, 47, 3707

(3) Ytrehus, K.; Liu, Y.; Tsuchida, A.; Miura, T.; Liu, G. S.; Yang, X. M.; Herbert, D.; Cohen, M.V.; Downey, J. M. Am. J. Physiol. 1994, 267, H2383

(4) Zhao, Z.Q.; Corvera, J.S.; Halkos, M.E.; Kerendi, F.:Wang, N.P.; Guyton, R.A.; Vinten-Johansen, J. Am. J. Physiol. 2003, 285, H579-H588 


\title{
Adenosine and heme oxygenase 1 anti-inflammatory mechanisms are intimately interwoven in a regulatory loop involving the adenosine A2a receptor and carbon monoxide
}

\author{
A. Haschemi $^{1,2}$, L.E. Otterbein ${ }^{2}$, R. Marculescu ${ }^{1}$, S.C. Robson ${ }^{3}$, F.H. Bach ${ }^{2}$ and O. Wagner ${ }^{1}$ \\ ${ }^{T}$ Department of Laboratory Medicine, Medical University of Vienna, Austria. ${ }^{2}$ Department of Surgery and \\ ${ }^{3}$ Department of Medicine, Beth Israel Deaconess Medical Center, Harvard Medical School, Boston, USA. \\ arvand@gmx.net
}

Background: Adenosine and heme oxygenase 1 (HO-1) are crucial regulatory molecules that participate in inflammation and immunity. HO-1 is a protective, inducible enzyme responsible for intracellular heme degradation that exerts a wide range of anti-oxidative, anti-inflammatory and immunomodulatory actions. These are chiefly mediated by the respective heme degradation products viz. carbon monoxide $(\mathrm{CO})$, biliverdin/bilirubin and iron with apo-ferritin induction. In a similar manner, CD39 and other ecto-nucleotidases catalyze the phosphohydrolysis of extracellular nucleotides to generate the respective nucleosides. Adenosine is an endogenous nucleoside that is usually present at low concentrations in the extracellular space. However, with stress or in several patho-physiologic conditions, e.g. acute inflammatory responses, there may be dramatic increases in extracellular levels of all purinergic mediators. Adenosine modulates the kinetics and magnitude of immune responses via four different $\mathrm{G}$ protein-coupled receptors (A1, A2a, A2b and A3) that are differentially expressed on a variety of cells. In spite of their apparent lack of connection to one other, there are striking similarities in the anti-inflammatory profiles of adenosine and HO-1 and its products (as detailed above).

Aim: To elucidate possible cross talk between these two highly conserved molecules and/or catalytic products in the regulation of inflammatory and immune responses.

Results and Discussion: Adenosine exposure induces high level HO-1 expression in the macrophage cell line RAW264.7. This effect, appears to be mediated via the A2A receptor. In a reciprocal manner, high HO-1 expression remarkably sensitizes the macrophages to adenosine and potentiates the anti-inflammatory effects of adenosine and its analog $5^{\prime}$ - $N$-ethylcarboxamidoadenosine (NECA) in an in vitro model of LPS activation. Adenosine mediated inhibition of LPS-induced TNF- $\alpha$ expression, is in part, HO-1 dependent as blockade of HO1 via siRNA abrogates the effects of adenosine on TNF- $\alpha$. We demonstrate that HO- 1 boosts the expression of the anti-inflammatory A2a receptor and that $\mathrm{CO}$ can mimic and substitute for HO-1 in this response in RAW264.7 cells and mouse bone marrow derived macrophages which was not observed with biliverdin, one of its other products. Moreover, $\mathrm{CO}$ exposure prevents NECA induced down regulation of A2A receptors supporting the concept that HO-1 and adenosine are interrelated.

Conclusion: We demonstrate intimate cross talk between adenosine and HO-1/products. Based on these observations we can envision feedback loops, which may play a key role in the orchestration and resolution of inflammation.

\section{Adenosine deaminase deficiency promotes dermal fibrosis}

Patricia Fernandez ${ }^{1}$, Sean Trzaska ${ }^{1}$, Janci L Chunn ${ }^{2}$, Michael R Blackburn ${ }^{2}$, Bruce N Cronstein ${ }^{1} \&$ Edwin SL Chan ${ }^{1}$

${ }^{1}$ Department of Medicine, New York University School of Medicine, New York, NY;

${ }^{2}$ Department of Biochemistry and Molecular Biology, University of Texas-Houston Medical School, Houston, TX Patricia.Fernandez@med.nyu.edu

Background: Adenosine deaminase (ADA) catalyzes the deamination of adenosine to inosine. Recent studies indicate that chronic adenosine elevations lead to pulmonary fibrosis in ADA-deficient mice, suggesting a profibrotic role for adenosine in the lung [1]. We have also found that in a model of bleomycin-induced dermal fibrosis that mice lacking adenosine $\mathrm{A}_{2 \mathrm{~A}}$ receptors were protected from developing skin fibrosis. We therefore tested the hypothesis that chronic elevations of adenosine concentrations, due to either injury (as in the bleomycin-induced model of dermal fibrosis) or ADA deficiency, leads to dermal fibrosis.

Methods: Male ADA deficient mice (ADA KO) were supplemented with PEG-ADA enzyme therapy for the first three weeks after birth. After the last enzyme injection, animals were mantained without PEG-ADA for two 
weeks and then sacrificed. Dermal morphometric measurements were assessed on freshly excised skin. In some experiments mice were treated with the adenosine $A_{2 A}$ receptor antagonist ZM241385 $(50 \mathrm{mg} / \mathrm{kg} / \mathrm{day}$ IP) for the last 8 days prior to sacrifice when the mice were no longer treated with PEG-ADA.

Results: ADA KO mice showed significant increases in dermal thickness $(128.7 \pm 2.0 \%, \mathrm{n}=5, \mathrm{p}<0.001)$, skin-fold thickness $(140.6 \pm 2.8 \%, \mathrm{n}=5, \mathrm{p}<0.001)$ and breaking tension $(136.8 \pm 5.3 \%, \mathrm{n}=5, \mathrm{p}<0.001)$ compared with their wild type (WT) littermates. Dermal hydroxyproline content, a measure of collagen content, was also increased by $157.8 \pm 2.8 \%(\mathrm{n}=5, \mathrm{p}<0.001)$ in ADA KO compared to the WT mice. To determine whether the adenosine $\mathrm{A}_{2 \mathrm{~A}}$ receptor was involved in the dermal fibrosis observed in the ADA KO mice, we treated ADA KO mice with the $\mathrm{A}_{2 \mathrm{~A}}$ receptor antagonist ZM241385. After ZM241385 treatment, the skin of treated mice was not as thick $(27.2 \pm 8.3 \%$ decrease, $\mathrm{n}=4, \mathrm{p}<0.05)$, skin-fold thickness was $21.7 \pm 7.4 \%$ less $(\mathrm{n}=4, \mathrm{p}<0.05)$, and dermal tensile strength was reduced by $48.9 \pm 14.1 \%(\mathrm{n}=4, \mathrm{p}<0.05)$ compared with ADA KO mice treated with vehicle. Furthermore, dermal hydroxyproline content was decreased $(61.4 \pm 13.5 \%, \mathrm{n}=4, \mathrm{p}<0.001)$ in ZM treated ADA KO mice compared with vehicle-treated ADA KO mice.

Conclusion: The marked increase in adenosine levels seen in other tissues in adenosine deaminase deficiency may act similarly on skin to promote fibrogenesis. Pharmacological protection of ADA deficient mice with $\mathrm{A}_{2 \mathrm{~A}}$ receptor antagonist administration suggests a role for $\mathrm{A}_{2 \mathrm{~A}}$ receptors in the modulation of dermal fibrogenesis.

[1] Chunn JL, Molina JG, Mi T, Xia Y, Kellems RE, Blackburn MR. Adenosine-dependent pulmonary fibrosis in adenosine deaminase-deficient mice. J Immunol. 2005; 175:1937-46.

\title{
Adenosine inhibits the release of arachidonic acid and its metabolites (AAM) in activated human peripheral mononuclear cells
}

\author{
$\underline{\text { Sándor Sipka }}^{1}$, Ildikó Kovács ${ }^{1}$, Sándor Szántó ${ }^{1}$, Gyula Szegedi $^{2}$, László Brugós ${ }^{3}$, Géza Bruckner ${ }^{5}$, A. \\ József Szentmiklósi ${ }^{4}$ \\ ${ }^{1} 3$ rd Department of Internal Medicine, University of Debrecen, Hungary \\ ${ }^{2}$ Research Group of Autoimmune Diseases, Hungarian Accademy of Sciences, Debrecen, Hungary \\ ${ }^{3}$ Department of Pulmonology, University of Debrecen, Hungary \\ ${ }^{4}$ Department of Pharmacology and Pharmacotherapy, University of Debrecen, Hungary \\ ${ }^{5}$ Division of Clinical Nutrition, University of Kentucky, USA \\ sipka@iiibel.dote.hu
}

The effects of adenosine (Ado) and subtype-specific activators of adenosine receptors $\left(\mathrm{A}_{1}, \mathrm{~A}_{2 \mathrm{~A}}, \mathrm{~A}_{2 \mathrm{~B}}\right.$ and $\left.\mathrm{A}_{3}\right)$ were studied on the release of arachidonic acid and its metabolites (AAM) from human peripheral mononuclear cells (monocytes). In the cells activated by protein kinase C- specific phorbol ester (phorbol 12-myristate 13acetate) and $\mathrm{Ca}^{2+}$-ionophore (A-23187), adenosine and two subtype specific receptor agonists, CPA $\left(\mathrm{A}_{1}\right)$ and CGS-21680 $\left(\mathrm{A}_{2 \mathrm{~A}}\right)$ induced concentration dependent inhibitions in the release of AAM, whereas the stimulation of $\mathrm{A}_{2 \mathrm{~B}}$ and $\mathrm{A}_{3}$ receptors were ineffective. The rank order of potency in the inhibition of AAM release was as follows: CGS-21680 $=\mathrm{CPA}>$ adenosine $>$ NECA (in the presence of ZM-24185 and DPCPX as $\mathrm{A}_{2 \mathrm{~A}}$ and $\mathrm{A}_{1}$ adenosine receptor antagonists) $=$ IB-MECA. Adenosine inhibited the release of AAM only at and over the concentration of $10^{-4} \mathrm{M}$, whereas the inhibitory effect of $\mathrm{A}_{1}$ and $\mathrm{A}_{2 \mathrm{~A}}$ receptor specific agonists appeared at the range of $10^{-7} \mathrm{M}$. It can be concluded that adenosine physiologically may not have a significant effect on the AAM release of circulating monocytes, but in pathological conditions, where the local Ado concentrations increases, this nucleoside by the activation of $\mathrm{A}_{2 \mathrm{~A}}$ and $\mathrm{A}_{1}$ receptors can exert its antiinflammatory action via the decrease in the proinflammatory AAM production.

\section{Adenosine is induced during peritonitis and downregulates cytokine production and leukocyte recruitment}

$\underline{\text { Sigal Nakav }}^{1}$, Nadav Y. Ziv ${ }^{1}$, Boris Rogachev ${ }^{2}$, Julia Mazar ${ }^{1}$, Cidio Chaimovitz ${ }^{2}$, Moshe Zlotnik ${ }^{2}$ and Amos Douvdevani ${ }^{1,2}$. 
${ }^{1}$ Department of Clinical Biochemistry and ${ }^{2}$ Department of Nephrology, Soroka Medical Center, Ben-Gurion University of the Negev, Beer-Sheva, Israel.

sigs@bgu.ac.il

Adenosine is an endogenous immunomodulator that has been shown to exhibit anti-inflammatory and immunosuppressive effects. These anti-inflammatory effects depend mainly on ligation with its cell-surface receptors subtypes: $A_{2 A}$ receptor $\left(A_{2 A} R\right), A_{2 B}$ receptor $\left(A_{2 B} R\right), A_{1}$ receptor $\left(A_{1} R\right)$ and $A_{3}$ receptor $\left(A_{3} R\right)$ all of which are G-protein coupled. The generation of extracellular adenosine involves phosphohydrolysis of adenine nucleotide intermediates and is regulated by two enzymes, nucleotidase triphosphate dephosphorylase (CD39) which converts ATP to AMP, and 5' ectonucleotidase (CD73) which converts AMP to adenosine. Peritoneal mesothelial cells (PMC) form a monolayer that covers the peritoneal membrane. Their location between the peritoneal cavity and peritoneal blood vessels gives them a key role in intraperitoneal immune defense. Following stimulation with inflammatory cytokines and bacterial products, mesothelial cells express adhesion molecules and produce various cytokines and other pro-inflammatory mediators.

The aim of the present study was to elucidate the regulatory role of adenosine during peritonitis and to assess the regulation of CD39, CD73 on PMC during the inflammatory processes.

In a mouse model of $E$. coli-induced peritonitis we found a gradual increase of adenosine levels which peaked at 24 hours then gradually declined up to 72 hours from inoculation. The intra-peritoneal influx of leukocytes after inoculation was blocked by the $\mathrm{A}_{2 \mathrm{~A}} \mathrm{R}$ agonist CGS-21680. In inoculated mice, the $\mathrm{A}_{2 \mathrm{~A}} \mathrm{R}$ agonist also caused a significant decrease in sera and peritoneal levels of TNF- $\alpha$ and IL-6 as compared to untreated mice. Analysis of PMC mRNA and protein levels showed that both CD39 and CD73 levels increased $\sim 3$ fold higher than normal at the initial phase of inflammation and decreased at the resolution phase.

These data suggest that adenosine is a potent regulator of peritoneal inflammation and the upregulation of both CD73 and CD39 in the initial phase of peritonitis is responsible for the increase of peritoneal adenosine levels.

\title{
Adenosine modulates the release of catecholamines from rat carotid body chemoreceptor cells through an interaction between $\mathrm{D2}$ dopamine receptors and $\mathbf{A}_{2 B}$ adenosine receptors
}

\author{
Conde SV, Obeso A and Gonzalez C \\ Department of Biochemistry, Molecular Biology and Physiology, IBGM, Faculty of Medicine, University of \\ Valladolid, CSIC, Valladolid, Spain. \\ svconde.farm@fcm.unl.pt
}

Carotid bodies (CB) are major peripheral chemoreceptor organs sensing changes in blood $\mathrm{O}_{2}$ responding by generating action potentials at the carotid sinus nerve (CSN), which are integrated in the brainstem to induce a hyperventilatory compensatory response. Hypoxia, the physiological CB stimulus increases the release of dopamine ${ }^{7}$ and adenosine ${ }^{1,2}$ from rat CB. Adenosine is an excitatory neurotransmitter at the $\mathrm{CB}$, increasing CSN electrical activity and promoting hyperventilation through the activation of $\mathrm{A}_{2}$ adenosine receptors ${ }^{4,5,6}$. Recently, it has been described that caffeine inhibits the basal and evoked release of catecholamines (CA) from rat carotid body chemoreceptor cells (CBCC) through an action on $\mathrm{A}_{2 \mathrm{~B}}$ adenosine receptors ${ }^{3}$. The present work was performed in order to investigate possible interactions between $\mathrm{D} 2$ dopamine and $\mathrm{A}_{2 \mathrm{~B}}$ adenosine receptors responsible for the modulation of CA release in rat CBCC. Experiments were performed in CB removed from 3 months old Wistar rats. The effect of adenosine $\mathrm{A}_{2 \mathrm{~B}}$ and dopamine $\mathrm{D} 2$ receptor agonists and antagonists applied alone or conjunctly were studied on the basal and evoked release $\left(10 \% \mathrm{O}_{2}\right)$ of CA from CBCC. Dose response curves for these $A_{2 B}$ and $D 2$ receptor agonists and antagonists were performed. NECA, an $A_{2}$ receptor agonist (0.1-100 $\mu \mathrm{M})$, increase the basal and $10 \% \mathrm{O}_{2}$-evoked release of CA from rat $\mathrm{CB}$ in a dose-dependent manner. Haloperidol $(0.01-10 \mu \mathrm{M})$ and sulpiride $(0.1-10 \mu \mathrm{M})$, D2 dopamine antagonists, increase in a dose-dependent manner the basal and/or evoked release CA from CBCC. Propylnorapomorphine, D2 dopamine agonist (0.2-200 nM), induced a dose-dependent decrease in the basal and evoked release of CA from rat CBCC. NECA $(10 \mu \mathrm{M})$ when applied in association with haloperidol $(0.01-10 \mu \mathrm{M})$ potentiates the effect of this D2 antagonist, moving its doseresponse curve to the left. Sulpiride $(1 \mu \mathrm{M})$, reversed the inhibitory effect of caffeine on basal and stimulus induced release of CA from rat $\mathrm{CBCC}$. Therefore, our results suggest that an interaction between $\mathrm{A}_{2 \mathrm{~B}}$ adenosine 
and D2 dopamine receptors could exist in $\mathrm{CBCC}$, contrarily to the described in the CNS between $\mathrm{A}_{2 \mathrm{~A}}$ and $\mathrm{D} 2$ receptors, modulating the release of CA.

Supported by MEC (Spain) Project BFU2004-06394/BFI, Red Respira (Iciii/RTIC C03/011) and by JCyL grant VA 106A05. SV Conde is funded by a PhD grant from FCT (Portugal).

(1) Conde, S. V.; Monteiro, E. C. J. Neurochem. 2004, 89, 1148-1156.

(2) Conde S. V.; Monteiro, E. C. Adv. Exp. Med. Biol. 2006, 580, (in press).

(3) Gonzalez, C.; Conde, S. V.; Obeso, A. Rev. Neurol. 2005, 41 supl 2, 4.

(4) Monteiro, E. C.; Ribeiro, J. A. Naunyn-Schmiedeberg's Arch Pharmacol. 1987, 335, 143-148.

(5) Monteiro, E. C.; Ribeiro, J. A. Naunyn-Schmiedeberg's Arch Pharmacol. 1989, 340, 230-238.

(6) Runold, M.; Cherniak, N. S.; Prabhakar, N. R. Neurosci. Lett. 1990, 113, 111-114.

(7) Vicario, I.; Rigual, R.; Obeso, A.; Gonzalez, C. Am. J. Physiol Cell Physiol. 2000, 278, C490-C499.

\section{Adenosine produced via the CD73/ecto-5-nucleotidase pathway has no impact on erythropoietin production but is associated with reduced kidney weight}

Burcin Özüyaman ${ }^{1}$, Ulrich K.M. Decking ${ }^{1}$, Zhaoping Ding ${ }^{1}$, Anja Buchheiser ${ }^{1}$, Patrjcya Koszalka ${ }^{1}$, Axel Gödecke ${ }^{1}$, Norbert Braun ${ }^{2}$, Herbert Zimmermann ${ }^{2}$, Jürgen Schrader ${ }^{1}$

1 Institut für Herz- und Kreislaufphysiologie, Heinrich-Heine-Universität Düsseldorf, Germany

2 Biozentrum der J.W. Goethe-Universität, Institut für Zellbiologie und Neurowissenschaft, Frankfurt am Main, Germany

decking@uni-duesseldorf.de

CD73/ecto-5-nucleotidase which catalyzes the conversion of AMP to adenosine has been implicated in vascular homeostasis. In kidneys, exogenous adenosine is known to dose-dependently act as a vasodilator and constrictor and has been shown to enhance erythropoietin production. We therefore hypothesized that CD73-derived adenosine promotes erythropoietin production and determines basal kidney perfusion.

CD73 knockout mutants recently generated in our laboratory were compared to wild type controls. CD73 local expression was assessed by enzyme histochemistry and immunocytochemistry, CD73 activity determined by a phosphate assay, adenosine determined by HPLC, erythropoietin expression by real-time quantitative PCR, erythropoietin plasma concentration by an immunoassay, and renal plasma flow and glomerular filtration rate by $\mathrm{PAH}$ and FITC-inulin, respectively.

Of all organs investigated, kidneys showed the most prominent CD73 activity that was preferentially located in peritubular fibroblasts of the renal cortex and the glomerular mesangium. In the absence of CD73, alkaline phosphatase remained unchanged but tissue adenosine was reduced both under control conditions (by $76 \%$ ) and during normobaric hypoxia (by 72\%). Despite the loss of CD73 activity and substantial reduction in adenosine, EPO mRNA and plasma protein concentrations did not differ between WT and CD73 ${ }^{-1-}$ under basal conditions and following normobaric hypoxia $\left(8 \% \mathrm{O}_{2}\right)$ and carbon monoxide $(0.1 \% \mathrm{CO})$ inhalation (both for $\left.4 \mathrm{~h}\right)$. Although there was no difference in blood pressure and urine flow volume, the average weight of the two kidneys was reduced by $21 \%$ in the knockout (WT: $7.17 \pm 1.18 \mathrm{mg} / \mathrm{g}$ body wt; $\mathrm{CD} 73^{-1-}: 5.70 \pm 1.91 \mathrm{mg} / \mathrm{g}$ body wt). Measurement of renal plasma flow (RPF) and glomerular filtration (GFR) revealed no significant difference when related to the respective kidney weights.

Conclusion: The extracellular CD73 pathway is a major source of adenosine in the kidney. CD73 derived adenosine thas no impact on erythropoietin production under basal conditions and after hypoxic challenge but may influence kidney growth.

\section{Adenosine receptor activation protects against the development of diabetes in mouse models of type 1 diabetes}

Zoltán H. Németh $^{1}$, Balázs Csóka ${ }^{1}$, David Bleich ${ }^{2}$, Csaba Szabó ${ }^{1}$, Edwin A. Deitch $^{1}$, György Haskó $^{1}$

${ }^{7}$ Department of Surgery, ${ }^{2}$ Department of Medicine, UMDNJ-New Jersey Medical School, Newark, NJ 07103, USA nemethzo@umdnj.edu 
Type 1 diabetes or insulin-dependent diabetes mellitus (IDDM) is a disease affecting 1.2 million patients in the United States with 12,000 new cases diagnosed each year. The disease is characterized by the specific destruction of insulin-producing $\beta$-cells in the pancreatic islets of Langerhans by the immune system. The islets are invaded by immune cells, particularly by macrophages and $\mathrm{T}$ cells, and these cells are cytotoxic to islet $\beta$-cells, in part by generating cytokines and free radicals. Currently there is no available treatment to prevent or cure type 1 diabetes. Based on evidence that adenosine receptor occupancy suppresses inflammatory cytokine production, we hypothesized that selective stimulation of adenosine receptors using the potent and stable agonist $5^{\prime}$-N-ethylcarboxamidoadenosine (NECA) would attenuate the course of diabetes in multiple-low-dose streptozotocin (MLDS) treated and in non-obese-diabetic (NOD) mice. In the MLDS model, male CD-1 mice were treated with $40 \mathrm{mg} / \mathrm{kg}$ streptozotocin (STZ) for 5 consecutive days simultaneously with NECA or its vehicle, and after the cessation of STZ treatment NECA or vehicle treatment was continued for 21 additional days. We found that $0.03 \mathrm{mg} / \mathrm{kg}$ NECA treatment prevented the MLDS-induced hyperglycemia (blood sugar levels on day $21 \mathrm{in} \mathrm{mg} / \mathrm{dl}$ : control: $410.3 \pm 60$, NECA: $222.8 \pm 50.9$, mean \pm SEM, $\mathrm{p}<0.05$ ). The decrease in blood sugar levels was associated with preservation of pancreatic insulin content. To assess the extent of inflammation in the pancreas, we measured the pancreatic levels of tumor necrosis factor-alpha (TNF- $\alpha$ ), macrophage inflammatory protein 1-alpha (MIP-1 $\alpha)$, interleukin-12 (IL-12), and interferon-gamma (IFN- $\gamma$ ). Pancreatic contents of all four cytokines were significantly lower in mice treated with NECA than in vehicle-treated mice as shown in the table below.

\begin{tabular}{lll}
\hline Cytokine Level (pg/mg prot.) & Vehicle & NECA $(0.03 \mathrm{mg} / \mathrm{kg})$ \\
\hline TNF- $\alpha$ & $1.45 \pm 0.33$ & $0.62 \pm 0.03^{* * *}$ \\
MIP-1 $\alpha$ & $2.87 \pm 0.91$ & $0.47 \pm 0.02^{* *}$ \\
IL-12 & $20.19 \pm 6.88$ & $5.27 \pm 0.05^{*}$ \\
IFN- $\gamma$ & $0.37 \pm 0.13$ & $0.09 \pm 0.02^{*}$ \\
\hline
\end{tabular}

Results are means \pm SEM $(n=10) .{ }^{*} p<0.05, * * p<0.01$ versus vehicle-treated mice.

We next examined the effect of NECA in NOD mice, a genetic model of type 1 diabetes that has more in common with human IDDM than the chemically induced MLDS model. NOD mice were given a single dose of cyclophosphamide $(200 \mathrm{mg} / \mathrm{kg})$ and then daily injections of NECA $(0.01 \mathrm{mg} / \mathrm{kg})$. Even this low dose of NECA significantly decreased the blood glucose level in NOD mice (vehicle: $318.5 \pm 188 \mathrm{mg} / \mathrm{dl}$, NECA: $155.8 \pm 76 \mathrm{mg} / \mathrm{dl}$, mean \pm SEM, $\mathrm{p}<0.05)$. Taken together adenosine receptor stimulation might have great therapeutic potential in the treatment of humans suffering from IDDM.

\section{Adenosine receptors in colon carcinoma tissues and colon tumoral cell lines: focus on the $\mathbf{A}_{3}$ adenosine receptors}

${ }^{1}$ Stefania Gessi, ${ }^{1}$ Stefania Merighi, ${ }^{1}$ Katia Varani, ${ }^{1}$ Elena Cattabriga, ${ }^{1}$ Annalisa Benini, ${ }^{2}$ Carlo Feo, ${ }^{3}$ Edward Leung, ${ }^{3}$ Stephen MacLennan, and ${ }^{1}$ Pier Andrea Borea.

${ }^{1}$ Department of Clinical and Experimental Medicine, Pharmacology Unit and Interdisciplinary Center for the Study of Inflammation, Italy; ${ }^{2}$ Department of Surgery, Anesthesiology and Radiology and ${ }^{3}$ King Pharmaceutical, Cary, North Carolina.

gss@unife.it

The present study was undertaken to investigate the presence of adenosine receptors on human colon cancer and to evaluate the functional effect of these receptors on colon cancer cell biology. Therefore the expression of the $\mathrm{A}_{1}, \mathrm{~A}_{2 \mathrm{~A}}, \mathrm{~A}_{2 \mathrm{~B}}$ and $\mathrm{A}_{3}$ subtypes by means of quantitative real time RT-PCR and binding studies in both resected colon cancer tissues from patients undergoing surgery and HT29, DLD1 and Caco2 colon carcinoma cell lines have been analysed. Our results show that in human colon cancer tissues the density of $\mathrm{A}_{1}$ and $\mathrm{A}_{2 \mathrm{~A}}$ subtypes was quite low in comparison with that of the $\mathrm{A}_{3}$ receptor. Analogous results were found in carcinoma cell lines. $\left[{ }^{3} \mathrm{H}\right]$ MRE 3008F20 reveals an affinity and binding capacity very similar in all the cell lines investigated. Moreover the effect of adenosine and of selective adenosine antagonists for $A_{1}, A_{2 A}, A_{2 B}$ and $A_{3}$ subtypes on cell growth have been investigated. The results of this work suggest that adenosine modulated cell proliferation through the involvement of the $\mathrm{A}_{3}$ subtype. 


\title{
Adenosine receptors in exocrine pancreas
}

Ivana Novak, Susanne E. Hede and Mette R. Hansen

August Krogh Building, Institute of Molecular Biology and Physiology, University of Copenhagen Copenhagen, Denmark.

inovak@aki.ku.dk

Pancreatic acini secrete ATP in response to cholinergic stimulation. Recent experiments show that pancreatic juice also contains nucleotidases, CD39 and CD73. Thus apart from P2 receptors, also adenosine receptors could be potential regulators of exocrine secretion. The aim of the present study was to determine whether pancreatic ducts, which normally secrete $\mathrm{HCO}_{3}{ }^{-}$rich fluid, possess functional adenosine receptors.

Pancreatic ducts were obtained from collagense digests of rat pancreas. Electrical activity in single isolated ducts was monitored using whole-cell nystatin patch-clamp method (1). The membrane voltage $\left(V_{m}\right)$ was continuously monitored during experiments in zero current-clamp mode. Periodically, voltage was clamped and the whole-cell current and the total conductance $\left(G_{t}\right)$ were measured. In another set of experiments, the Fura-2 method was used to estimate $\left[\mathrm{Ca}^{2+}\right]_{i}$, in pancreatic ducts. In addition, RT-PCR analysis was carried out on isolated ducts and whole pancreas.

In about half of the ducts studied, adenosine $(1-100 \mu \mathrm{M})$ had no effect on resting $V_{\mathrm{m}}$ of about $-55 \mathrm{mV}$. However, in the other half of the ducts adenosine markedly and reversibly depolarized $V_{\mathrm{m}}$ by $10-40 \mathrm{mV}$, and also increased whole cell membrane conductances $\left(G_{\mathrm{m}}\right)$ by about $30 \%$. In such ducts lowering of the extracellular Cl- concentrations led to a further depolarization of $V_{\mathrm{m}}$, indicating activation of a Cl- conductance. In another set of experiments, the effect of adenosine on intracellular $\mathrm{Ca}^{2+}$ of isolated single ducts was monitored. However, adenosine had no significant effects in the Fura-2 ratio, indicating that intracellular signals were not mediated by $\mathrm{Ca}^{2+}$ signalling. RT-PCR analysis showed that the isolated ducts have transcripts for the following adenosine receptors: $\mathrm{A}_{1}, \mathrm{~A}_{2 \mathrm{~A}}, \mathrm{~A}_{2 \mathrm{~B}}$ and $\mathrm{A}_{3}$.

In summary, the present study shows that some pancreatic ducts express functional adenosine receptors that lead to opening of $\mathrm{Cl}$ - channels and possibly to the production of $\mathrm{HCO}_{3}^{-}$rich fluid. Since $\mathrm{Ca}^{2+}$ signals were not observed, it is likely that $\mathrm{A}_{2 \mathrm{~A}}$ or $\mathrm{A}_{2 \mathrm{~B}}$ receptors regulate CFTR Cl- channels in some pancreatic ducts.

The projects were supported by the Danish Medical and Science Research Councils, the Augustinus Foundation and the Lundbeck Foundation.

1. Novak, I. and M. J. Hug. Cell.Physiol.Biochem. 1995; 5: 344-352.

\section{Adenosine role in the formation of hepatic fibrosis}

\author{
Zhongsheng Peng ${ }^{1}$, Simon Robson ${ }^{2}$, Linda Thompson ${ }^{3}$ and Bruce N. Cronstein ${ }^{1}$ \\ ${ }^{1}$ NYU School of Medicine, New York, NY; ${ }^{2}$ Beth Israel-Deaconess Medical Center, Boston, MA; ${ }^{3}$ Oklahoma \\ Medical Research Foundation
}

Background: Adenosine is a potent endogenous regulator of tissue repair released from injured cells and tissues. Hepatic fibrosis results from chronic and acute hepatic injury and we have previously reported that adenosine, acting at $\mathrm{A}_{2 \mathrm{~A}}$ receptors, plays a role in hepatic fibrosis. The enzymes involved in formation of adenosine following hepatic injury have not been characterized.

Methods: Mice were treated with PBS, $\mathrm{CCl}_{4}$, thioacetamide or ethanol, sacrificed and their livers harvested. Liver slices were incubated in medium for $24 \mathrm{hrs}$ before adenosine concentration in the supernatant was measured by HPLC. Hepatic fibrosis was induced with thioacetamide in CD39 (nucleoside triphosphate phosphohydrolase) knockout mice, CD73 (ecto-5'nucleotidase) knockout mice, CD39/73 double knockout mice and C57BL/6 control mice and was quantified by digital analysis of picrosirius red stained slides and hydroxyproline content.

Results: Thioacetamide, carbon tetrachloride and ethanol treatment led to a marked increase in adenosine in supernates of hepatic slices $(419.4 \pm 22.8 \mathrm{nM}, 492.1 \pm 68.2 \mathrm{nM}, 694.9 \pm 35.8 \mathrm{nM}$, respectively, vs $274.0 \pm 14.7 \mathrm{nM}$ in PBS-treated mice, $\mathrm{n}=5$ and $P<0.05$ for all). Hepatic hydroxyproline content was significant decreased in CD39/ 73 knockout mice compared with C57BL/6 mice $(0.31 \pm 0.06 \mu \mathrm{g} / \mathrm{ml}$ versus $0.40 \pm 0.08 \mu \mathrm{g} / \mathrm{ml}, P<0.05)$. Hepatic 
fibrosis (\%fibrotic area/hepatic slice area) was decreased in CD39KO $(0.26 \pm 0.12 \%, \mathrm{p}<0.05)$, CD73KO $(0.31 \pm$ $0.21 \%, \mathrm{p}=\mathrm{NS})$ and CD39/CD73KO mice $(0.29 \pm 0.09 \%, \mathrm{p}<0.05)$ as compared to C57BL/6 $(0.42 \pm 0.17 \%)$.

Conclusion: These results are consistent with the hypothesis that following hepatic injury adenosine is produced extracellularly from adenine nucleotides and that the adenosine released promotes hepatic fibrosis. We conclude that inhibition of adenosine production or interaction with its receptor may help prevent hepatic fibrosis.

\title{
Adenosinergic Mechanisms Contribute to Individual Differences in the Effects of Sleep Deprivation on Psychomotor Performance and the EEG
}

\author{
Landolt $\mathrm{HP}^{1,2}$, Rétey $\mathrm{JV}^{1}$, Adam $\mathrm{M}^{1}$, Gottselig $\mathrm{JM}^{1}$, Khatami $\mathrm{R}^{1}$, Dürr $\mathrm{R}^{1}$, Achermann $\mathrm{P}^{1,2}$ \\ ${ }^{T}$ Institute of Pharmacology \& Toxicology, University of Zürich, Zürich, Switzerland \\ ${ }^{2}$ Center for Integrative Human Physiology, University of Zürich, Zürich, Switzerland \\ landolt@pharma.unizh.ch
}

Sleep deprivation impairs performance on neurobehavioral tasks and modulates the electro-encephalogram (EEG) in wakefulness and sleep. The changes in performance and the EEG are assumed to reflect a sleep-wake dependent, homeostatic process of sleep regulation ${ }^{1}$. Our observation that the adenosine receptor antagonist, caffeine, attenuates the EEG correlates of sleep homeostasis in wakefulness and sleep is consistent with the hypothesis that the adenosinergic system is prominently involved in sleep-wake regulation ${ }^{2}$. Recent research highlights the importance of studying individual differences in wakefulness and sleep as a novel approach to gain insights into physiological mechanisms underlying human sleep-wake regulation ${ }^{3}$. We recently found that genetic variability in the adenosinergic system contributes to inter-individual differences in the waking and sleep EEG ${ }^{4}$. Here, we hypothesized that adenosinergic mechanisms also play a role for individual differences in neurobehavioral performance decline associated with prolonged wakefulness. We investigated psychomotor vigilance task (PVT) performance and antero-posterior EEG power gradients during wakefulness and sleep following one night of sleep deprivation. The PVT is a visual reaction time task that is sensitive to sleep-deprivation induced impairments in sustained vigilant attention. Subjectively caffeine sensitive $(\mathrm{n}=12)$ and insensitive $(\mathrm{n}=10)$ young men (age range: 20-30 years) underwent two 40-hour waking periods. After 11 and 23 hours waking they received caffeine (200 mg per dose) and placebo in double-blind, cross-over fashion. The PVT and the EEG were assessed in 14 sessions at 3-hour intervals. To quantify the effect of prolonged waking, the data from three test sessions that occurred at analogous time of the day (at 11:00, 14:00 and 17:00 h) before and after the night without sleep were averaged. The data were analyzed with repeated-measures analyses of variance (rANOVA) and Spearman's rank correlation analyses. In the placebo condition, optimal PVT performance (fastest 10th percentile of speed) was more impaired by sleep deprivation in caffeine sensitive men than in caffeine insensitive men $(\mathrm{p}<0.04)$. This difference between the groups was not significant in the caffeine condition $(\mathrm{p}>0.05)$. In the placebo condition, sleep deprivation enhanced a fronto-occipital EEG power gradient (fronto-central vs. parieto-occipital bipolar EEG derivations) during waking $(6.5-8.5 \mathrm{~Hz}$ range) and non-rapid-eye-movement (nonREM) sleep $(<1 \mathrm{~Hz}$ range) in caffeine sensitive subjects when compared to caffeine insensitive subjects ( $<0.01$ and $\mathrm{p}<0.001)$. After caffeine these differences in the regional EEG power distribution between the groups were not significant $(\mathrm{p}>$ 0.05). The sleep-deprivation and caffeine-induced changes in PVT performance, as well as in the EEG during wakefulness and nonREM sleep were significantly correlated on an individual basis. These results indicate that adenosinergic mecha-nisms contribute to individual differences in the vulnerability to sleep-deprivation induced changes in psychomotor function and in the EEG. Elucidation of these mechanisms will help in the identification of persons at risk for impaired performance following sleep deprivation and in the develop-ment of agents to reduce the detrimental effects of prolonged waking on cognition and behavior. Research supported by the Swiss National Science Foundation (Grant \# 3100-067060.01).

(1) Finelli, L. A.; Baumann, H.; Borbély, A. A.; Achermann, P. Neurosci 2000, 101, 523-529.

(2) Landolt, H. P.; Rétey, J. V.; Tönz, K.; Gottselig, J. M.; Khatami, R.; Buckelmüller, J.; Achermann, P. Neuropsychopharmacol 2004, 29, 1933-1939.

(3) Van Dongen, H. P. A.; Vitellaro, K. M.; Dinges, D. F. Sleep 2005, 28, 479-496.

(4) Rétey, J. V.; Adam, M.; Honegger, E.; Khatami, R.; Luhmann, U. F.; Jung, H. H.; Berger, W.; Landolt, H. P. Proc Natl Acad Sci U S A 2005, 102, 15676-15681. 


\title{
Agonist-induced trafficking of the $\mathrm{P}_{1} \mathrm{Y}_{1}$ receptor quantified with the novel radioligand, $\left[{ }^{32} \mathbf{P}\right]$ MRS2500.
}

\author{
D. Houston, D.M. Bourdon, A.D. Qi, R.A. Nicholas, T. K. Harden. \\ Dept. Pharmacology, University of North Carolina School of Medicine, Chapel Hill, NC, USA. \\ dhouston@med.unc.edu
}

G-protein coupled receptors are responsible for transducing signals from a variety of extracellular hormones, nucleotides and neurotransmitters to the intracellular machinery. Subsequent to agonist activation, GPCR signaling can be terminated by desensitization followed by internalization into intracellular compartments. The occurrence and mechanisms of agonist-induced internalization of the P2Y family of GPCRs are poorly understood, primarily due to a lack of tools available for studying natively expressed receptors. Recently, we synthesized a high affinity, high specific radioactivity radioligand, $\left[{ }^{32} \mathrm{P}\right] \mathrm{MRS} 2500$, for the $\mathrm{P}_{2} \mathrm{Y}_{1}$ receptor $\left(\mathrm{P} 2 \mathrm{Y}_{1}-\mathrm{R}\right)$ and established its utility for detecting and quantifying $\mathrm{P}_{2} \mathrm{Y}_{1}-\mathrm{R}$ in a variety of mammalian tissues. An intact cell binding assay was established using this ligand and applied to measure agonist-induced loss of $\left[{ }^{32} \mathrm{P}\right] \mathrm{MRS} 2500$ binding sites in mammalian cell lines and intact platelets. Treatment of canine kidney (MDCK) cells with the P2 $\mathrm{Y}_{1}-\mathrm{R}$ agonist $2 \mathrm{MeSADP}$ resulted in a $50 \%$ decrease in the number of surface $\left.{ }^{32} \mathrm{P}\right] \mathrm{MRS} 2500$ binding sites. This decrease occurred with a t 1/2 of approximately 5-10 minutes and reached a steady-state within 30 minutes that was maintained for up to one hour. The human $\mathrm{P}_{2} \mathrm{Y}_{1}$ receptor stably expressed in MDCK cells (P2Y $\mathrm{Y}_{1}$-MDCK) exhibited similar kinetics for agonist-induced internalization. The extent of agonist-promoted loss of surface $\mathrm{P} 2 \mathrm{Y}_{1}-\mathrm{R}$ in $\mathrm{P}_{2} \mathrm{Y}_{1}$-MDCK cells was dependent on concentration of agonist, and internalization was inhibited almost completely by pretreatment of cells with $450 \mathrm{mM}$ sucrose, an inhibitor of clathrin-mediated endocytosis. The initial loss in surface $\mathrm{P}_{2} \mathrm{Y}_{1}-\mathrm{R}$ binding sites in $\mathrm{P}_{2} \mathrm{Y}_{1}$-MDCK cells was completely reversed within 15 minutes after removal of agonist from the medium. In human platelets, agonist-induced internalization occurred with much faster kinetics, with the maximal extent of internalization occurring within five minutes. Taken together, these data indicate that the $\mathrm{P}_{2} \mathrm{Y}_{1}-\mathrm{R}$ undergoes clathrin-mediated endocytosis in response to agonist stimulation.

\section{Agonists of the Adenosine $\mathbf{A}_{2 a}$ Receptor Reduce Leukocyte Adhesion and Platelet Aggregation in Sickle Cell Mice}

Robert Figler PhD ${ }^{1,2}$, Michael Steele ${ }^{2}$, Marek Marcinkiewicz MD, PhD ${ }^{2}$, Renata PolanowskaGrabowska $\mathrm{PhD}^{2}$, Kori Wallace ${ }^{2}$, Susi Srinivasan $\mathrm{PhD}^{2}$, Lynn Hedrick $\mathrm{PhD}^{2}$, Klaus Ley $\mathrm{MD}^{2}$, and Joel Linden $\mathrm{PhD}^{2}$

${ }^{1}$ Adenosine Therapeutics, LLC, P.O. Box 4632, Charlottesville VA 22905

${ }^{2}$ Cardiovascular Research Center, University of Virginia, Charlottesville VA 22908

raf2z@virginia.edu

It has become increasingly appreciated that chronic inflammation plays an important role in the etiology of vasoocclusive crisis (VOC) and the long-term pathologic manifestations of sickle cell disease (SCD). Central to this concept is evidence that VOC is not merely a result of the passive obstruction of the vasculature by deformed erythrocytes, but an active process involving heterotypic interaction of erythrocytes, leukocytes, platelets and the endothelium. In studies using a transgenic murine model of SCD (NY1DD) we have demonstrated the following: 1) increased adhesion of a monocytic cell line to primary aortic endothelial cells cultured from NY1DD mice; 2 ) increased aggregation of platelets isolated from NY1DD mice; 3 ) increased formation of circulating leukocyte/ platelet aggregates in the blood of NY1DD mice; and 4) increased adhesion of leukocytes to the endothelium of NY1DD mice in vivo.

This evidence of inflammation provides a rationale for the use of anti-inflammatory agents in the treatment of $\mathrm{SCD}$ and vaso-occlusive crisis. Adenosine has been demonstrated to act through the adenosine $\mathrm{A}_{2 \mathrm{~A}}$ receptor $\left(A_{2 A} R\right)$ found on bone marrow-derived cells to inhibit inflammation. ATL146e is an $A_{2 A}$-selective agonist that has already been shown to be safe in man and is currently in Phase III clinical trials. We investigated the effects of ATL146e on indices of platelet and leukocyte activation in SCD. 
We used an exteriorized cremaster muscle preparation to study the microcirculation in vivo as a means of assaying the effect of ATL146e on leukocyte interactions with the endothelium. At baseline, NY1DD mice have more than three times the number of adherent leukocytes/field when compared to congenic C57BL/6 mice. Treatment with ATL146e caused a pronounced reduction in leukocyte adhesion to wild type baseline levels when administered by either infusion (Alzet mini-pump, $10 \mathrm{ng} / \mathrm{kg} / \mathrm{min}$, overnight) or bolus injection (intraperitoneal, $5 \mu \mathrm{g} / \mathrm{kg}, 30$ minutes prior to cremaster exteriorization).

Single particle counting was used to study platelet aggregation in vitro. At baseline, platelets isolated from NY1DD mice and subjected to sheer stress exhibit significantly increased aggregation as compared to control platelets. When NY1DD mice were pretreated in vivo with ATL146e (Alzet mini-pump, $10 \mathrm{ng} / \mathrm{kg} / \mathrm{min}, \mathrm{O} / \mathrm{N}$ ) platelet activation in platelet rich plasma prepared from these animals and exposed to ADP added in vitro was reduced to below control levels. Further, flow cytometric analysis of whole blood indicates that NY1DD mice exhibit an increased number of circulating leukocyte/platelet aggregates and that this number can be significantly reduced by pretreatment with ATL146e.

These data support the hypothesis that anti-inflammatory therapy will reduce the severity of vaso-occlusive crises and indicate that adenosine $\mathrm{A}_{2 \mathrm{~A}}$ agonists may be clinically useful for the treatment of vaso-occlusive crisis in SCD.

\title{
Allosteric enhancers of A1 Adenosine Receptors Catalyze Oxidation of Thiols
}

\author{
Mahendra D. Chordia, ${ }^{a}$ Molly Zigler Karlinsey, ${ }^{\mathrm{a}}$ Heidi Figler, ${ }^{\mathrm{b}}$ Ray A. Olsson ${ }^{\mathrm{c}}$ and Joel Linden ${ }^{\mathrm{b}}$ \\ a.Department of Chemistry and b. Cardiovascular Research Center, University of Virginia, Charlottesville, VA \\ 22901 \\ c. Suncoast Cardiovascular Laboratory, University of South Florida, Tampa, FL 33612 \\ mdc3x@virginia.edu
}

Adenosine an endogenous ligand for four subtypes of $\mathrm{G}$ protein coupled adenosine receptors. Important physiological functions of Adenosine- $\mathrm{A}_{1}$ receptor activation have been described in many tissues including kidney, heart, adipose tissue and brain. Adenosine or synthetic adenosine analogs that occupy the orthosteric $\mathrm{A}_{1}$ receptor binding site have limited therapeutic potential due to cardiac and renal side effects and poor penetration into the brain. Allosteric enhancers (AE) of adenosine receptors have been described. ${ }^{1}$ These compounds decrease the dissociation kinetics of agonist radioligands from $\mathrm{A}_{1}$ receptors and facilitate adenosine signaling. Due to their lipophilic properties AEs may have clinical potential for producing analgesia, anti-seizure activity and sedation. Allosteric agents have little activity in the absence of adenosine and may selectively amplify adenosine signaling in hypoxic, ischemic or inflamed tissues that produce high levels of adenosine. Aminothiophenes, including PD 81,723 were the first compounds reported to have $\mathrm{AE} .^{2}$ We recently found that certain 2-aminothiazoles also have AE activity. ${ }^{3}$

We now report evidence suggesting that AEs act as thiol oxidants.. Reducing agents including DTT, N-acetylcysteine, glutathione and TCEP all block the activity of AE's to influence agonist radioligand binding. All compounds that are active as AEs can catalyze the oxidization of thiols such as glutathione to disulfides such as GSSH. The oxidizing power of AEs is well correlated with the receptor activity. In addition we discovered that AE's render $\mathrm{A}_{1}$ receptors resistant to solubilization in digitonin; certain disulfides have $\mathrm{AE}$ activity and $\mathrm{H}_{2} \mathrm{O}_{2}$ a strong oxidant has AE-like activity. Based on these observations we hypothesize that AEs catalyze oxidation of one or more Cys residues on the extracellular surface of A1 receptors to promote either intramolecular or intermolecular disulfide bonds. Agonist binding to the $\mathrm{A}_{1}$ receptor may be stabilized by and facilitate this oxidation. We hypothesize that formation of disulfides is responsible for stabilization of the ternary complex between ligand, receptors and G-proteins. This paper will discuss the detailed progress made towards unraveling mechanistic aspect of allosteric enhancer action on A1 adenosine receptors.

1. Gao, Z.; Kim, S.; IJzerman, A. P.; Jacobson, K. A. Mini-Rev.Med. Chem. 2005, 5, 545-553.

2. Bruns, R..F; Fergus, J.H.; Coughenour, L.L.; Courtland, G.G.; Pugsley, T.A.; Dodd, J.H.; Tinney, F.J. Mol. Pharm., 1990, 38, 950-958.

3. Chordia, M. D.; Zigler, M.; Murphree, L. J.; Figler, H.; MacDonald, T. L.; Olsson, R. A.; Linden, J. J. Med. Chem., 2005, 48, 5131-5139 and reference cited in there. 


\title{
Alteration of adenosine receptors in patients with chronic obstructive pulmonary disease
}

Katia Varani,${ }^{1}{ }^{*}$ Gaetano Caramori, ${ }^{2 *}$ Fabrizio Vincenzi, ${ }^{1}$ Ian Adcock, ${ }^{4}$ Paolo Casolari, ${ }^{2}$ Edward Leung, ${ }^{3}$ Stephen MacLennan, ${ }^{3}$ Stefania Gessi, ${ }^{1}$ Silvana Morello, ${ }^{4}$ Peter J Barnes ${ }^{4}$, Kazuhiro Ito, ${ }^{4}$ Kian Fan Chung, ${ }^{4}$ Giorgio Cavallesco, ${ }^{5}$ Gianfranco Azzena, ${ }^{5}$ Alberto Papi, ${ }^{2} *$ Pier Andrea Borea ${ }^{1 *}$

${ }^{1}$ Department of Clinical and Experimental Medicine, Pharmacology Section, and ${ }^{2}$ Department of Clinical and Experimental Medicine, Respiratory Disease Section, and ${ }^{5}$ Department of Surgical Sciences, Thoracic Surgery Section, University of Ferrara; Ferrara, Italy; ${ }^{3}$ King Pharmaceuticals Research \& Development, Cary, North Carolina, USA $;{ }^{4}$ Airway Disease Section, National Heart \& Lung Institute, Imperial College London, UK. $\left({ }^{*}\right.$ these authors contributed equally to this work).

Chronic obstructive pulmonary disease (COPD) is the fourth leading cause of mortality worldwide. Adenosine acts through four distinct receptors to mediate pro- and anti-inflammatory effects. The primary aim of this study is to investigate the expression in peripheral lung parenchyma, the major site of airflow obstruction in COPD, using immunohistochemistry, radioligand binding and real time quantitative polymerase chain reaction. Adenosine receptors were analyzed in age-matched smokers with COPD $(\mathrm{n}=14)$ and smokers with normal lung function (control group; $\mathrm{n}=20$ ). $\mathrm{A}_{1}, \mathrm{~A}_{2 \mathrm{~A}}, \mathrm{~A}_{2 \mathrm{~B}}$ and $\mathrm{A}_{3}$ receptors were differentially expressed in peripheral lung parenchyma. The affinity of $\mathrm{A}_{1}, \mathrm{~A}_{2 \mathrm{~A}}$ and $\mathrm{A}_{3}$ receptors was significantly decreased in COPD patients compared with control group $\left[\mathrm{K}_{\mathrm{D}}\left(\mathrm{A}_{1}\right)=3.15 \pm 0.19 *\right.$ versus $1.70 \pm 0.14 \mathrm{nM} ; \mathrm{K}_{\mathrm{D}}\left(\mathrm{A}_{2 \mathrm{~A}}\right)=7.88 \pm 0.68 *$ versus $1.87 \pm 0.09 \mathrm{nM} ; \mathrm{K}_{\mathrm{D}}\left(\mathrm{A}_{3}\right)$ $=9.34 \pm 0.27 *$ versus $4.41 \pm 0.25 \mathrm{nM} ; * \mathrm{p}<0.01]$ whereas their density was increased $\left[\operatorname{Bmax}\left(\mathrm{A}_{1}\right)=53 \pm 4 *\right.$ versus 32 $\pm 3 \mathrm{fmol} / \mathrm{mg}$ protein; $\operatorname{Bmax}\left(\mathrm{A}_{2 \mathrm{~A}}\right)=852 \pm 50 *$ versus $302 \pm 12 \mathrm{fmol} / \mathrm{mg}$ protein; $\operatorname{Bmax}\left(\mathrm{A}_{3}\right)=2078 \pm 108 *$ versus 770 $\pm 34 \mathrm{fmol} / \mathrm{mg}$ protein; $* \mathrm{p}<0.01$ ]. The affinity of $\mathrm{A}_{2 \mathrm{~B}}$ receptors was not altered but the density was significantly decreased in COPD patients compared with the control group $\left(\mathrm{Bmax}=66 \pm 5^{*}\right.$ versus $189 \pm 16 \mathrm{fmol} / \mathrm{mg}$ protein; $* \mathrm{p}<0.01)$. A significant correlation was found between the affinity and density of the adenosine receptors and forced expiratory volume in one second $\left(\mathrm{FEV}_{1}\right)$ /forced vital capacity $(\mathrm{FVC})$ ratio, an established index of airflow obstruction. In conclusion, this is the first report showing the presence of adenosine receptors differentially expressed in lung parenchyma in COPD compared with control smokers. These novel findings strengthen the hypothesis of a potential role played by adenosine receptors in the pathogenesis of COPD.

\section{ALTERED DISTRIBUTION, SIGNALLING AND FUNCTION OF A AND $A_{2 a}$ ADENOSINE RECEPTORS IN THE BRAIN OF WAG/RIJ RATS WITH GENETIC ABSENCE EPILEPSY BEFORE AND AFTER THE DISEASE APPEARANCE}

\author{
${ }^{1}$ D'Auro M., ${ }^{1}$ D'Alimonte I., ${ }^{2}$ Citraro R., ${ }^{1}$ Di Iorio P., ${ }^{1}$ Giuliani P., ${ }^{1}$ Caciagli F., ${ }^{2}$ De Sarro G., \\ ${ }^{1}$ Ciccarelli R. \\ ${ }^{1}$ Department of Biomedical Sciences, University of Chieti-Pescara. Chieti. Italy. ${ }^{2}$ Department of Experimental and \\ Clinical Medicine, "Magna Graecia” University. Catanzaro. Italy. \\ mimmidauro@yahoo.com
}

Adenosine shows anticonvulsant properties in different types of epilepsy, but its role in absence seizures is still to be defined. Here, we investigated the distribution as well as the signalling pathways and function of $\mathrm{A}_{1}$ and $\mathrm{A}_{2 \mathrm{~A}}$ adenosine receptors in genetically absence epileptic Wistar Albino Glaxo/Rijswijk (WAG/Rij) rats compared with August Copenhagen Irish (ACI) rats, not prone to develop this kind of epilepsy. In WAG/Rij rats, the disease onset occurs after 2 months of age, due to generation of abnormal oscillatory rhythms within the thalamocortical circuitry, including the reticular (nRT), ventroposterolateral and ventroposteromedial thalamic nuclei as well as the frontoparietal somatosensory cortex. Our study, carried out in young (1,5 months) and adult (6 months) animals, i.e. before and after seizure occurrence, focussed on cerebral areas above mentioned in comparison with hippocampus, not involved in seizure triggering. Immunohistochemistry and Western Blot analyses showed a 
lower density of $A_{1}$ receptors in thalamic and cortical areas from young WAG/Rij rats, as well as in the only nRt in adult epileptic animals as compared with ACI rats of the same age. In contrast, the distribution of $\mathrm{A}_{2 \mathrm{~A}}$ receptors was uniformly decreased and increased in all brain areas of young and adult WAG/Rij rats, respectively, as compared with matched control. Accordingly, the stimulation of $\mathrm{A}_{1}$ and $\mathrm{A}_{2 \mathrm{~A}}$ receptors was ineffective on the modulation of cAMP formation and $50 \mathrm{mM} \mathrm{K}^{+}$-evoked $\left[\mathrm{C}^{14}\right]$-glutamate release in brain slices from young epileptic in comparison with control. Moreover, the $\mathrm{A}_{2 \mathrm{~A}}$ receptor stimulation with 2-(4-(-2-carboxyethyl)-phenylamino)-5'-N-ethylcarboxamido- adenosine (CGS21680) increased either cAMP intracellular levels or $\mathrm{K}^{+}$-evoked labelled glutamate release to a greater extent in brain slices from adult WAG/Rij than ACI rats. On the contrary, the $\mathrm{A}_{1}$ receptor activation with 2-chloro- $\mathrm{N}^{6}$-cyclopentyladenosine (CCPA) inhibited forskolin-induced cAMP formation, but not $\mathrm{K}^{+}$-evoked labelled glutamate release, more remarkably in brain slices from adult WAG/Rij rat brains than from matched control. We also examined the modulation of the mitogen-activated protein kinase (MAPK) system, constituted by ERK1/2, p38 and JNK, because mostly p38 and JNK, when activated, are regarded as promoters of tissue stress and injury. Either CGS21680 or CCPA activated the entire MAPK system substantially in adult animals, more remarkably in brain slices from adult epileptic rats than from control. Thus, the reduced number of $\mathrm{A}_{1}$ and $\mathrm{A}_{2 \mathrm{~A}}$ receptors in WAG/Rij rat brain during development as well as the greater distribution of excitatory $\mathrm{A}_{2 \mathrm{~A}}$ receptors in adult epileptic rats, coupled to a significant alteration in signalling pathways and function linked to the activation of both receptors might deeply influence the pathogenesis of absence seizures, confirming some findings on the epileptogenic role of adenosine in such a disease $\mathrm{e}^{1,2}$.

1. Ilbay, G.; Sahin, D.; Karson, A.; Ates, N. Clin. Exp. Pharmacol. Physiol. 2001, 28, 643-646.

2. Ates, N; Sahin, D.; Ilbany, G. Epilepsy and Behaviour 2004, 5, 645-648.

\title{
AMP Deaminase and AMP-Regulated Protein Kinase Interaction in Heart Cells
}

\author{
Ryszard T Smolenski ${ }^{1,2)}$, Ada HY Yuen ${ }^{2)}$, Iwona Rybakowska ${ }^{1)}$, Tomasz Borkowski ${ }^{1)}$, Ewa M \\ Slominska ${ }^{1)}$, Krystian Kaletha ${ }^{1)}$, Magdi H Yacoub ${ }^{2)}$ \\ 1) Department of Biochemistry, Medical University of Gdansk, Poland and \\ 2) Heart Science Centre, Imperial College London, U.K. \\ r.smolenski@ic.ac.uk
}

The C34T mutation of the AMP deaminase 1 gene (AMPD1) induces protective effect demonstrated clinically in patients with dilated cardiomyopathy, ischemic heart disease and in donors of hearts for transplantation. However, the mechanism of this effect has not been established. The product of the gene, AMP deaminase (AMPDA) 1 is responsible for catabolism of AMP and decrease of this activity will lead to elevation of AMP concentration. This in turn may activate dephosphorylation pathway of AMP degradation leading to formation of adenosine. Alternatively, AMP could activate AMP regulated protein kinase (AMPK) and related signalling cascade that is known to induce cytoprotective mechanisms. We have shown earlier that this mutation leads to about $50 \%$ decrease in cardiac AMPDA activity and that increase in adenosine production is relatively small. The aim of this study was to evaluate whether AMPDA could modulate the activity of AMPK.

AMPDA was isolated from human skeletal muscle using two step column chromatography on phosphocellulose. A new assay system has been developed for measurement of AMPK activity using HPLC with UV and mass detection that allows simultaneous monitoring of the substrate peptide (AMARA) phosphorylation and adenine nucleotide concentration. This assay was used to study the effect of isolated AMPDA added to in vitro assay of AMPK activity in human heart homogenates performed in the presence or absence of AMP.

Addition of AMPDA at $1 \mu \mathrm{g}$ of protein reduced the activity of AMPK from $1.16 \pm 0.06$ to $0.43 \pm 0.10 \mathrm{nmol} / \mathrm{min} /$ $\mathrm{mg}$ prot $(\mathrm{n}=7, \mathrm{p}<0.001)$. Surprisingly, AMPDA reduced the activity of AMPK even in the absence of AMP from $0.72 \pm 0.10$ to $0.33 \pm 0.09 \mathrm{nmol} / \mathrm{min} / \mathrm{mg}$ prot $(\mathrm{p}<0.05)$. This effect was dependent on the amoun of the AMPDA added.

We conclude that AMPDA activity may affect the activity AMPK in the cell. This interaction has been observed even without AMP added to the assay, possibly through competing with or deamination of AMP already bound to AMPK. Interaction of AMP deaminase with AMP-regulated protein kinase may have important implications for 
the regulation of cell function and in particular may be responsible for beneficial effect of AMP deaminase inhibition in the heart.

\title{
An intracellular redox and mercury-sensitive site in a purinergic P2X receptor.
}

\author{
C. Coddou, J.G. Lillo, P. Bull and J.P. Huidobro-Toro.
}

Centro de Regulación Celular y Patología, J.V. Luco, Instituto MIFAB, Departamento de Fisiología, Facultad de Ciencias Biológicas, Pontificia Universidad Católica, Santiago, Chile.

ccoddou@puc.cl

P2Xs are ionotropic receptors differentially modulated by transition trace metals that bind to specific allostericsites. Extracellular histidines are essential for copper and zinc modulation in either $\mathrm{P}_{2} \mathrm{X}_{4}$ and $\mathrm{P} 2 \mathrm{X}_{2}$ receptors, however these residues are not relevant for mercury-induced modulation in either receptor. Mercury has oppositte effects on the $\mathrm{P} 2 \mathrm{X}_{2}$ and $\mathrm{P} 2 \mathrm{X}_{4}$ receptors, it potentiates the former but inhibits the latter. To identify the mercury binding-site we used chimeric receptors containing the $\mathrm{P}_{2} \mathrm{X}_{4}$ ectodomain in the backbone of the $\mathrm{P} 2 \mathrm{X}_{2}$ receptor. Chimeric $\mathrm{P} 2 \mathrm{X}_{4 / 2}$ and native receptors were expressed in X.laevis oocytes, the ATP-induced currents were analized by two electrode voltage-clamp. In the $\mathrm{P}_{2} \mathrm{X}_{4 / 2 \mathrm{a}}$ chimera, $10 \mu \mathrm{M} \mathrm{Hg}^{2+}$ increased $3.7 \pm 0.8$ fold the ATP-responses and displaced leftward the ATP concentration-response curve lowering the $\mathrm{EC}_{50}$ from $4.8 \pm 0.6$ $\mu \mathrm{M}$ to $2.4 \pm 0.8 \mu \mathrm{M}$. $(\mathrm{n}=8, \mathrm{p}<0.05)$. In the $\mathrm{P} 2 \mathrm{X}_{4 / 2 \mathrm{~b}}$ chimera (a splice variant of the $\mathrm{P}_{2} \mathrm{X}_{2}$ receptor, lacking a 68 amino acid sequence in the C-terminal region), mercury was inactive. Copper inhibited and zinc potentiated the ATP-responses much alike the $\mathrm{P}_{2} \mathrm{X}_{4}$ receptor, confirming the extracellular nature of the copper and zinc allosteric sites. We mutated Cys-430 by alanine, one of the 68 amino acid sequence of the $\mathrm{P} 2 \mathrm{X}_{2}$ receptor. In the C430A mutant the mercury-induced potentiation was significantly less than in the native $\mathrm{P} 2 \mathrm{X}_{2}$ receptor, although a smaller potentiation remained. The $\mathrm{C} 430 \mathrm{~A}$ mutant, of the $\mathrm{P} 2 \mathrm{X}_{4 / 2 \mathrm{a}}$ chimera was completely mercury-insensitive, suggesting that Cys-430 is a target for mercury. We test the potential role of Cys-430 as a redox sensitive residue with hydrogen peroxide $\left(\mathrm{H}_{2} \mathrm{O}_{2}\right)$. As mercury $\mathrm{H}_{2} \mathrm{O}_{2}$ potentiated the activity of the $\mathrm{P} 2 \mathrm{X}_{2}$ receptor by $2.8 \pm 0.3$-fold with $0.3 \mathrm{mM} \mathrm{H}_{2} \mathrm{O}_{2}(\mathrm{n}=5)$; the $\mathrm{P}_{2} \mathrm{X}_{4 / 2 \mathrm{a}}$ chimera was also potentiated by $\mathrm{H}_{2} \mathrm{O}_{2}(1 \mathrm{mM}, 1.9 \pm 0.2$-fold $)$. No potentiation was observed in the $\mathrm{P} 2 \mathrm{X}_{2} \mathrm{C} 430$ mutant nor in the $\mathrm{P} 2 \mathrm{X}_{4 / 2 \mathrm{~b}}$ chimera. Chemical alkylation of the $\mathrm{P} 2 \mathrm{X}_{2}$ receptor with the membrane-soluble methyl methanethiosulphonate MMTS also abolished the $\mathrm{H}_{2} \mathrm{O}_{2}$-potentiation. The $\mathrm{P}_{2} \mathrm{X}_{4}$ receptor was inhibited by $1 \mathrm{mM} \mathrm{H}_{2} \mathrm{O}_{2}$ by $50 \%$, and this effect was prevented with the addition of 1 $\mathrm{mM}$ DTT, a reducing agent. In conclusion we identified intracellular Cys-430 as a key amino acid for the potentiation induced by mercury and hydrogen peroxide on the activity of the $\mathrm{P} 2 \mathrm{X}_{2}$ receptor. This is the first report identifying an intracellular redox site in $\mathrm{P} 2 \mathrm{X}$ receptor channels highlighting an additional role of $\mathrm{P} 2 \mathrm{X}$ receptors as sensors of the cellular redox potential. Funded by FONDAP 13980001 grant. The Millenium Institute MIFAB contributed to fund the CRCP Center.

\section{Analysis of the role of extracellular cysteine residues in the P2Y12-receptor}

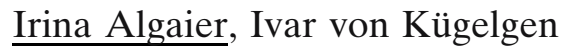

\author{
Department of Pharmacology, Universität of Bonn, Reuterstrasse 2b, 53113 Bonn, Germany \\ ialgaier@uni-bonn.de
}

ADP activates human platelets through two G-protein coupled receptors, the $\mathrm{P}_{2} \mathrm{Y}_{1}$ - as well as the $\mathrm{P}_{2} \mathrm{Y}_{12}$-receptor. Four extracellular cysteine residues of the $\mathrm{P}_{2} \mathrm{Y}_{12}$-receptor protein have been proposed to form disulfide bridges between the extracellular receptor domains and to represent the sites of action of the active metabolites of clopidogrel [1,2]. An important role of cysteine residues for receptor function has previously also been shown for the human $\mathrm{P}_{2} \mathrm{Y}_{1}$-receptor [3]. In order to study the role of the extracellular cysteine residues of the human $\mathrm{P} 2 \mathrm{Y}_{12^{-}}$ receptor we replaced cysteine residues by alanine residues by site-directed mutagenesis of the respective DNA sequence. Wild type $\mathrm{P}_{2} \mathrm{Y}_{12}$-receptor and mutant receptors were stably expressed in 1321N1-astrocytoma cells. 
Immunofluorescence staining was used to demonstrate the expression of the mutant receptors. Changes in receptor function were assessed by measuring 2-methylthio-ADP $(0.01 \mathrm{pM}$ to $1 \mu \mathrm{M})$ induced inhibition of cellular cAMP levels. The addition of isoprenaline $10 \mathrm{nM}$ elicited an increase in cellular cAMP levels (average increase by $55 \pm 4$ pM cAMP per well). In cells expressing wild type receptors, 2-methylthio-ADP caused a concentrationdependent inhibition of the cAMP production with an $\mathrm{EC}_{50}$ concentration of $0.9 \mathrm{nM}$ and a maximal inhibition by $47 \%$. In cells expressing recombinant receptors in which both the residue Cys17 in the N-terminus as well as the residue Cys270 in the third extracellular loop were replaced by alanine residues (Cys17Ala/Cys270Ala), 2-methylthio-ADP caused an inhibition in adenylate cyclase activity with a reduced maximal response (maximal inhibition by about $10 \%$ ). The same was true for cells expressing mutant receptors in which Cys97 (located near the exofacial end of transmembrane region 3, TM3) as well as Cys175 (in the extracellular loop 2) were replaced by alanine residues (Cys97Ala/Cys175Ala; maximal inhibition by about 10\%). In contrast, in cells expressing Cys17Ala/Cys97Ala-, Cys17Ala/Cys175Ala-, Cys97Ala/Cys270Ala- or Cys175Ala/Cys270Ala-mutant receptors any inhibitory action of 2-methylthio-ADP was lost. Additional experiments were performed to study the action of thiol agents at the $\mathrm{P} 2 \mathrm{Y}_{12}$-receptor. These compounds have also been proposed to interact with extracellular cysteine residues. p-Chloromercuribenzene sulfonic acid (pCMBS; 0.1 to $10 \mu \mathrm{M}$ ) and N-ethylmaleimide (NEM; 0.3 to $10 \mu \mathrm{M})$ abolished or markedly reduced the responses to receptor stimulation by 2-methylthio-ADP with $\mathrm{EC}_{50}$ concentrations of $0.9 \mu \mathrm{M}$ (pCMBS) and $10 \mu \mathrm{M}$ (NEM), respectively. Furthermore, in experiments performed under reducing conditions (presence of ascorbic acid $40 \mu \mathrm{M}$ ) receptor-mediated inhibition of adenylate cyclase activity was almost lost. In conclusion, our present results demonstrate that Cys17 (N-terminus) and Cys270 (third extracellular loop) form one disulfide bridge and that Cys97 (exofacial end of transmembrane region3, TM3) and Cys175 (second extracellular loop) the second bridge. Furthermore, the data indicate that the presence of only one intact disulfide bridges reduces the maximal responses to activation by agonists and that thiol reagents block the receptor. Hence, our findings are compatible with the view that the active metabolites of clopidogrel or prasugrel interact with the extracellular cysteine residues of $\mathrm{P}_{2} \mathrm{Y}_{12}$-receptor.

[1] Ding, Z., et al., Blood 2003, 101, 3908-3914.

[2] Savi, P., et al., Biochem. Biophys. Res. Comm. 2001, 283, 379-383.

[3] Hoffmann, C., et al., J.Biol.Chem. 1999, 274, 14639-14647.

\title{
Application of adenosine $5^{\prime}$-triphosphate (ATP) infusions in palliative home care: a multiccentre randomized clinical trial
}

\author{
S. Beijer, P.C. Dagnelie, N.E.G. Wijckmans \\ Department of Epidemiology, Maastricht University, P.O. Box 616, 6200 MD Maastricht, The Netherlands. \\ epid.unimaas.nl
}

Introduction: Palliative care in terminal cancer aims at alleviating suffering of patients. Complaints like progressive fatigue, deteriorating performance status, weight loss and decreasing functional abilities have a substantial impact on the quality of life of the patient and his/her environment. So far, few if any therapies are available to combat these devastating complaints. A randomized clinical trial in patients with advanced lung cancer showed favourable effects of regular ATP infusions on body weight, fat and fat-free mass, muscle strength, fatigue and quality of life, relative to untreated controls ${ }^{[1]}$. However, so far, no evidence is available about the effectiveness of ATP in other types of cancer, nor for the application of ATP infusions in a non-clinical setting. Based on this, we have initiated a study 1 . To reproduce the results, which were previously observed in lung cancer patients, in patients with other types of advanced cancer, and 2. To test the feasibility and safety of regular ATP infusions at the patient's home.

Subjects and methods: The study is targeted on cancer patients with a life expectancy of less than 6 months, who are (partly) mobile and suffer from at least one of the following three complaints: fatigue, weight loss, or anorexia (lack of appetite). Only patients are included with a life expectancy of $<6$ months, and for whom no systemic treatment is available: medical treatment options are restricted to palliative/supportive care. Patients are recruited through oncology departments of five participating hospitals, and family doctors. After eligible patients have signed informed consent, patients are stratified for tumour type and presence of weight loss (yes/no) and then 
randomly assigned to ATP or no ATP. The first ATP infusion is given at the day care centre of the participating hospitals, subsequent infusions are given at the patients' home by an experienced and trained nurse of the infusion team of regional community care organizations. Outcome parameters are assessed every two weeks, until 8 weeks.

Preliminary results: Preliminary results will be presented at the meeting. So far, we have enrolled and randomised 80 patients of a wide variety of tumour types. One of the problems encountered is a high rate of drop-out due to rapid deterioration of patients'condition, which is difficult to foresee at the time of inclusion: approximately $75 \%$ of patients complete 4 weeks of follow-up, and $50 \%$ complete the full 8 week study period.

\title{
Reference
}

1. Agteresch HJ, Dagnelie PC, Gaast A van der, Stijnen T, Wilson JHP. Randomized clinical trial of adenosine 5'triphosphate in patients with advanced non-small-cell lung cancer. Journal of the National Cancer Institute 2000; 92(4):321-328.

\section{ATP, adenosine, and P1/P2 receptors in the control of neutrophil chemotaxis.}

\author{
Yu Chen ${ }^{1}$, Ross Corriden ${ }^{1,2}$, Linda Yip ${ }^{1}$, Yoshiaki Inoue ${ }^{1}$, Cindy Cheung ${ }^{1}$, Paul A. Insel ${ }^{2,3}$, and Wolfgang G. \\ Junger $^{1}$

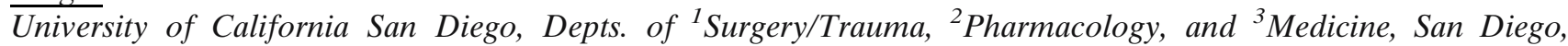 \\ California 92103.
}

As a key cell of the innate immune system, polymorphonuclear neutrophils (PMN) play an important role in inflammation and host defense. It is well known that PMN accumulate at sites of infection and inflammation where they kill bacteria, but these cells can also damage host tissues by releasing cytotoxic mediators. PMN possess complex mechanisms that allow them to travel to the source of chemotactic mediators released from bacteria or inflamed tissues. Although many aspects of PMN chemotaxis have been identified in recent years, it remains unclear how PMN amplify subtle differences in the concentration of chemoattractants within a gradient field.

Recent data from our laboratory indicate that chemoattractants such as the bacterial formyl peptide fMLP induce the release of cellular ATP from PMN at the leading edge that is closest to the source of fMLP. HPLC analysis revealed that PMN rapidly hydrolyze released ATP to adenosine. Using real-time RT-PCR analysis of all known P1 adenosine and P2 nucleotide receptor subtypes, we found that PMN and PMN-like HL-60 cells predominantly express the P2Y2 nucleotide and A2a and A3 adenosine receptor subtypes. Elimination of extracellular ATP or adenosine with apyrase or adenosine deaminase (ADA), respectively, dose-dependently blocked PMN activation and chemotaxis toward fMLP. Inhibition of P2 and A3 receptors significantly reduced chemotaxis, while agonists of these receptors, but not of A2a receptors, increased chemotaxis. Using fluorescent antibodies and HL-60 cells expressing P2Y2- and A3-EGFP fusion proteins, we found that cells migrating in a chemotactic gradient redistribute stimulatory A3 receptors to the leading edge (Fig.), while P2Y2 and A2a receptors remain uniformly distributed on the cell membrane. PMN of A3 knockout mice showed significantly reduced PMN migration in vitro and in a peritonitis model of inflammation.

Together these data imply that ATP release and positive feedback through P2Y2 receptors are essential steps needed for the amplification of initial chemotactic signals, while the accumulation of A3 receptors at the leading edge and positive feedback through adenosine predominantly generated at the leading edge maintains the locomotion of cells in a chemotactic gradient field. Based on these findings and conclusions, we propose a "pull-push" model of PMN chemotaxis whereby ATP release and adenosine formation together with stimulatory A3 receptors that are enriched at the leading edge increase cell migration, while suppressive A2a receptors at the receding edge facilitate membrane retraction.

This study was supported in part by grants from the National Institutes of Health (GM-51477, 60475, \& 66232). 


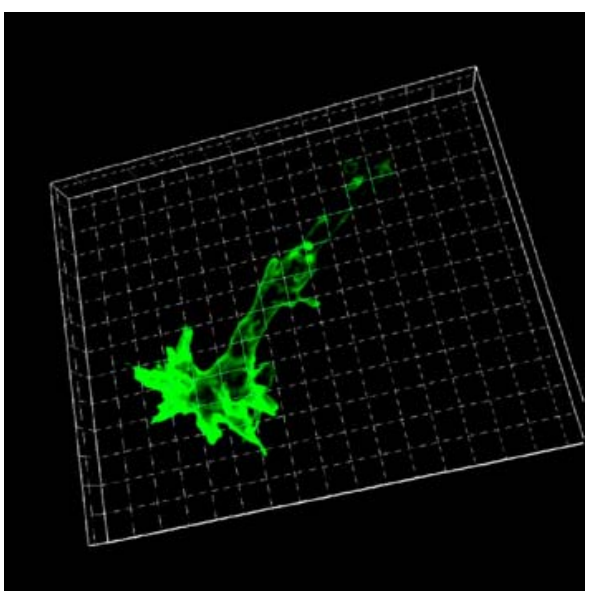

Fig. Confocal image of HL-60 cell expressing A3-EGFP receptor at the leading edge. The cell is migrating from the top right to the bottom left corner of the image shown.

\section{ATP as a neurotrasmitter of pain in migraine: a functional role for P2Y receptors in primary cultures from mouse trigeminal sensory ganglia.}

Fumagalli M. ${ }^{1 \ddagger}$, Ceruti S. ${ }^{1 \star}$, Verderio C. ${ }^{2 \ddagger}$, Abbracchio M.P. ${ }^{1 \ddagger}$

${ }^{1}$ Laboratory of Molecular and Cellular Pharmacology of Purinergic Trasmission, Department of Pharmacological Sciences, University of Milan and ${ }^{2}$ CNR Institute of Neuroscience, Cellular and Molecular Pharmacology, Department of Medical Pharmacology, Milan.

marta.fumagalli@unimi.it

${ }^{\ddagger}$ All authors equally contributed to this work

Migraine is a common episodic headache disorder affecting more than $10 \%$ of the population (1). Despite the availability of adjuvant drugs, treatment of migraine remains unsuccessful in a significant number of patients, suggesting that yet-unidentified causative agents remain to be discovered. In analogy to its role in other chronic pain states, ATP could represent a potential algogenic factor in trigeminal sensory terminals during migrainous attacks (2). On this basis, the present study was undertaken to characterize, in primary cultured cells from mouse trigeminal ganglia, the presence and function of ATP/P2 receptors, with special focus on $\mathrm{P} 2 \mathrm{Y}$ receptors. As a first step, we have characterized the percentage of the various cell types in our culture (i.e. neurons, astrocytes, and oligodendrocytes) by means of flow cytometry. Data showed that about $50 \%$ of cells were positive for oligodendrocyte markers, $20 \%$ for the astroglial marker GFAP, and $10 \%$ were neurons. Results were also confirmed by immunocytochemistry. Contaminating fibroblasts probably represents the remaining population. As a second step, we have characterized by RT-PCR the expression of all P2Y receptors cloned from rodent tissue (P2$\mathrm{Y}_{1,2,4,6,12,13,14}$ ) and of some "P2Y like" orphan G-protein-coupled receptors (oGPCRs). Results showed the presence of all P2Y receptors, together with four oGPCRs. Single-cell calcium imaging of Fura-2 loaded cells was then used to dissect the functionality of specific receptor subtypes by measuring their coupling to intracellular calcium $\left(\left[\mathrm{Ca}^{2+}\right]_{\mathrm{i}}\right)$ in the presence of subtype-selective P2 receptor agonist and antagonist ligands. We found that, despite the presence, at the mRNA level, of all P2Y receptors, only some of them are functionally coupled to increases of $\left[\mathrm{Ca}^{2+}\right]_{\mathrm{i}}$. In particular, application of ADP or $2 \mathrm{MeSADP}$, agonists at $\mathrm{P}_{2} \mathrm{Y}_{1}, \mathrm{P}_{2} \mathrm{Y}_{12}, \mathrm{P}_{2} \mathrm{Y}_{13}$, induced a $\left[\mathrm{Ca}^{2+}\right]_{\mathrm{i}}$ increases in approximately $20 \%$ of a sub-population of small and medium-size neurons and in almost all satellite-like glial cells. Treatment with the $\mathrm{P}_{2} \mathrm{Y}_{1}$ selective antagonist MRS2179 potently reduced $\left[\mathrm{Ca}^{2+}\right]_{\mathrm{i}}$ responses induced by ATP in both types of cells. Furthermore, in satellite-like glial cells, the ADP-induced $\left[\mathrm{Ca}^{2+}\right]_{\mathrm{i}}$ responses were significantly reduced by AR-C69931MX, an antagonist selective for $\mathrm{P}_{2} \mathrm{Y}_{12}$ and $\mathrm{P}_{2} \mathrm{Y}_{13}$ receptors, suggesting that a component of the $\left[\mathrm{Ca}^{2+}\right]_{\mathrm{i}}$ responses evoked by ADP derives from activation of $\mathrm{P} 2 \mathrm{Y}_{12}$ and $\mathrm{P} 2 \mathrm{Y}_{13}$. We also observed $\left[\mathrm{Ca}^{2+}\right]_{\mathrm{i}}$ increases in neurons and satellite-like glial cells stimulated with UTP, known to activate $\mathrm{P}_{2} \mathrm{Y}_{2}$ and $\mathrm{P}_{2} \mathrm{Y}_{4}$ receptors, and with UDP, which activates $\mathrm{P} 2 \mathrm{Y}_{6}$ receptors. Thus, in mouse trigeminal ganglia, 
$\mathrm{P}_{2} \mathrm{Y}_{1}$, and, to a lesser extent, $\mathrm{P}_{2} \mathrm{Y}_{2,4,6,12,13}$ contribute to ATP-induced calcium-dependent signalling. The elucidation of the interaction of these receptors with ionophoric $\mathrm{P} 2 \mathrm{X}$ receptors (in particular, $\mathrm{P} 2 \mathrm{X}_{3}$ ) after sensitization of trigeminal neurons with algogenic stimuli (e.g., NGF, BDNF or bradykinin), may help identifying new potential targets for the development of novel antimigraine drugs.

Sponsored by Comitato Telethon \#GGP04037 to MPA. AR-C69931MX was a kind gift of The Medicines Company, Waltham, MA, USA.

(1) Pietrobon, D. Neuroscientist, 2005 11:373-386.

(2) Burnstock, G. The Lancet, 1996 347:1604-1605.

\title{
ATP inhibits the inflammatory response in stimulated whole blood, even under conditions of severe oxidative stress
}

\author{
Els L.R. Swennen $^{1,2}{ }^{\text {, Aalt Bast }}{ }^{2}$, Pieter C. Dagnelie ${ }^{1}$ \\ ${ }^{T}$ Department of Epidemiology, NUTRIM, Maastricht University, P.O. Box 616, 6200 MD Maastricht, The \\ Netherlands. \\ ${ }^{2}$ Department of Pharmacology and Toxicology, NUTRIM, Maastricht University, P.O. Box 616, 6200 MD \\ Maastricht, The Netherlands. \\ e.swennen@farmaco.unimaas.nl
}

Over the past decades, evidence has accumulated which indicates that extracellular nucleotides and nucleosides may be important regulators of inflammatory and immune responses. Many diseases in which inflammatory reactions are involved are associated with oxidative stress.

The purpose of the present study was to determine the effects of ATP on TNF- $\alpha$, IL-10 and IL- 6 release in stimulated whole blood in the absence and presence of oxidative stress. Blood samples were drawn from healthy volunteers and incubated with ATP and lipopolysaccharide + phytohemagglutinin for $24 \mathrm{~h}$, without and with $\mathrm{H}_{2} \mathrm{O}_{2}$ as an inductor of oxidative stress.

In the absence of $\mathrm{H}_{2} \mathrm{O}_{2}$, ATP at $100 \mu \mathrm{M}$ and $300 \mu \mathrm{M}$ induced a reduction in TNF- $\alpha$ secretion by $32 \pm 8 \%$ (mean \pm SEM) and $65 \pm 4 \%$, respectively. Furthermore, these ATP concentrations induced an increase in IL-10 secretion by $93 \pm 5 \%$ and $166 \pm 7 \%{ }^{1}$. ATP- $\gamma-$ S and ADP also inhibited TNF- $\alpha$ release, but only ADP showed a stimulatory effect on IL-10 release. Co-treatment with adenosine deaminase did not reverse the ATP effect on TNF- $\alpha$ and IL10. In the presence of 1,5 and $10 \mathrm{mM} \mathrm{H}_{2} \mathrm{O}_{2}$, ATP at concentrations of $100 \mu \mathrm{M}$ and $300 \mu \mathrm{M}$ still inhibited TNF- $\alpha$ release and stimulated IL-10 release in stimulated blood ${ }^{2}$.

Our results demonstrate that even under circumstances of severe oxidative stress, ATP has marked anti-inflammatory properties in stimulated whole blood.

References

1. Swennen ELR, Bast A, and Dagnelie PC. Immunoregulatory effects of adenosine 5'-triphosphate on cytokine release from stimulated whole blood. Eur J Immunol. 2005; 35(3): 852-858

2. Swennen ELR, Dagnelie PC and Bast A. ATP inhibits hydroxyl radical formation and the inflammatory response of stimulated whole blood even under circumstances of severe oxidative stress. Free Radical research. 2006; 40(1): 53-58

\section{ATP metabolism in human blood}

\author{
Coolen JCM ${ }^{1,2}$, Swennen ELR ${ }^{1,2}$, Bast $\mathrm{A}^{2}$, Dagnelie PC ${ }^{1}$ \\ ${ }^{1}$ Department of Epidemiology, NUTRIM, Maastricht University, Maastricht, The Netherlands \\ ${ }^{2}$ Department of Pharmacology and Toxicology, NUTRIM, Maastricht University, Maastricht, The Netherlands \\ erik.coolen@epid.unimaas.nl
}

Background: Cells release ATP into the extracellular environment in order to retain homeostasis and in response to mechanical and pathophysiological stimuli (e.g. ischemia, hypoxia). Plasma ATP measurement is complicated 
by 1) degradation of ATP by circulating and cell-based ectonucleotidases and by 2) ATP release from erythrocytes and platelets. The aim of this study was to investigate the degradation profile of ATP in plasma after addition of ATP to blood in the absence or presence of LPS/PHA stimulation, because our group showed earlier that ATP inhibits the inflammatory response induced by LPS and PHA in blood.

Methods: Fresh blood was aliquoted into 24 -well plates and incubated with $300 \mu \mathrm{M}$ ATP for 30 min at $37^{\circ} \mathrm{C}$ and $5 \% \mathrm{CO}_{2}{ }^{1}$. Samples obtained $0,0.25,0.5,1,2,4,8$ and 24 hours after incubation with LPS/PHA or medium (control) were disposed of the cellular fraction and proteins by centrifugation. In a single run, ATP and metabolites were separated by methanol-gradient elution over a reversed-phase HPLC column, quantified by peak area through UV-absorption and identified by comparison of peak retention times with standards.

Results: Results indicated continuous ATP degradation to ADP and AMP, which was complete by 4 hours. The level of ADP initially remained constant, but started to decline after $30 \mathrm{~min}$. AMP increased during the first two hours, but slowly declined between 2 and 8 hours. Relatively high levels of hypoxanthine were observed after 24 hours. By that time, ATP, ADP and AMP were totally depleted. Similar results were obtained after incubation in the presence of LPS/PHA or medium.

Discussion: The simultaneous decline of ATP and ADP after 30 min possibly contributed to the strong increase of AMP and hypoxanthine. The decline of AMP after 2 hours, which was probably caused by ongoing depletion of its precursors ATP and ADP, did not lead to an increase in inosine or adenosine levels. This may have been caused by uptake by erythrocytes and intracellular conversion to inosine and hypoxanthine, followed by secretion from the erythrocytes ${ }^{2}$. Finally, the increase in plasma hypoxanthine levels after 2 hours was not matched by its degradation products xanthine and uric acid, which would suggest conversion to other products.

Conclusion: Our results so far indicate a slower ATP breakdown in blood than anticipated, which is accompanied by a rise in hypoxanthine after 24 hours.

1. Swennen, E. L.; Bast, A.; Dagnelie, P. C., Immunoregulatory effects of adenosine 5'-triphosphate on cytokine release from stimulated whole blood. Eur J Immunol 2005, 35, (3), 852-8.

2. Griffith, D. A.; Jarvis, S. M., Nucleoside and nucleobase transport systems of mammalian cells. Biochim Biophys Acta 1996, 1286, (3), 153-81.

\title{
$\mathrm{ATP} / \mathrm{P2} \mathrm{Y}_{2}$ receptor-mediated intimate interaction between astrocytes and pericytes
}

\author{
$\underline{\text { Schuichi Koizumi }}^{1}$, Kayoko Fujishita ${ }^{1}$, Koji Sueishi ${ }^{1,2}$, Yasufumi Kataoka ${ }^{2}$, Satoko Ohkubo ${ }^{1}$ and \\ Kazuhide Inoue $^{3}$ \\ ${ }^{1}$ Division of Pharmacology, National Institute of Health Sciences, 1-18-1 Kamiyoga, Setagaya, Tokyo 158-8501, \\ Japan. \\ ${ }^{2}$ Department of Pharmaceutical Care and Health Sciences, Faculty of Pharmaceutical Sciences, Fukuoka \\ University, 8-19-1 Nanakuma, Jonan, Fukuoka 814-0180, Japan. \\ ${ }^{3}$ Graduate School of Pharmaceutical Sciences, Kyusyu University, 3-1-1 Maidashi, Higashi, Fukuoka 812-8582, \\ Japan. \\ skoizumi@nihs.go.jp
}

Brain pericytes are cells that are located in ablumenal site of small blood vessels or capillaries with patchy structure. Since capillaries lack smooth muscles and pericytes express $\alpha$-smooth muscle actins, it is suggested that pericytes may control microcirculation by regulating tonus of capillaries. However, contraction or relaxation of brain pericytes is largely unknown. We previously reported that astrocytes release ATP that mediates glia-toneuron communications as well as glia-to-glia communications. In addition to this, astrocytes form endfeet to surround the vascular wall, suggesting that astrocytic ATP may also control vascular functions. Exogenously applied ATP and its analogues produced rises in $\left[\mathrm{Ca}^{2+}\right] \mathrm{i}$ in cultured pericytes obtained from rat forebrain with pharmacological profile of $\mathrm{P}_{2} \mathrm{Y}_{2}$ and $\mathrm{P}_{2} \mathrm{Y}_{1}$ receptors. Interestingly, when stimulated, pericytes showed contractile responses, which was inhibited by the P2 receptor antagonists. In addition, pericytes released ATP in response to mechanical stimulation or even spontaneously, and produced elevations in $\left[\mathrm{Ca}^{2+}\right] \mathrm{i}$, suggesting that pericytes could control function of capillaries by releasing endogenous ATP. We also demonstrate astrocytic control of pericytes or vascular tonus, which is also mediated by ATP/P2 receptors. 


\section{ATP reduces lactate dehydrogenase (LDH) and triacylglycerol levels in lung cancer patients- a potential explanation of ATP's favourable clinical effects?}

P.C. Dagnelie, T. Brouwer

Department of Epidemiology, Maastricht University, P.O. Box 616, 6200 MD Maastricht, The Netherlands

Dagnelie@epid.unimaas.nl

Background: Cancer-associated cachexia is a clinical syndrome of progressive weight loss associated with profound changes in host metabolism, including elevated lipolysis, proteolysis, and liver gluconeogenesis, and causing elevated plasma levels of lactate dehydrogenase (LDH) and triacylglycerols (TAG). At the same time, elevated LDH levels are a well-known marker of tumour progression and reduced survival. We previously reported that ATP infusions had marked beneficial effects on weight and muscle mass in patients with advanced lung cancer, ${ }^{1}$ as well as on survival in a subgroup of cachectic patients. ${ }^{2}$

Aim: To assess the effect of ATP infusion on plasma LDH and TAG levels in patients with advanced lung cancer. Methods: Blood values from our previously published clinical trial in patients with non-small-cell lung cancer (NSCLC, $\mathrm{N}=58$, stage IIIB/IV) were analysed. Patients were randomly assigned to either supportive care (control), or ATP infusions (total of 10 courses of $30 \mathrm{~h}$, given at 2- to 4-week intervals over 24 weeks). Differences over time were appraised by repeated-measures analysis.

Results: At baseline, ATP and control groups were similar with regard to prognostic variables, including weight, tumour stage, and plasma values of LDH and TAG. Over the 24-wk study period, LDH and TAG increased in the control group, indicating progression of tumour growth and deterioration of the cancer cachexia syndrome. In contrast, LDH and TAG remained stable in the ATP group (between-group difference: $\mathrm{P}<0.05$ for both LDH and TAG).

Discussion: The favourable effects of ATP on LDH and TAG levels in patients with advanced NSCLC may be due to anti-inflammatory effects of ATP. Swennen et al. ${ }^{3}$ recently showed that ATP inhibits the LPS-PHA inflammatory response in blood through inhibition of TNF-alpha release. TNF-alpha (a pro-inflammatory cytokine) has been associated with 1. elevated glucose-lactate cycling and 2. decreased lipoprotein lipase activity. Suppression of TNF by ATP could result in down-regulation of LDH production and increased clearance of plasma TAG, explaining the observed metabolic effects of ATP.

Conclusion: Our study demonstrates that ATP infusion has favourable effects on both serum LDH and TAG concentrations. These effects may partly explain the beneficial effects of ATP on body weight and survival in patients with NSCLC. Further investigations are needed for better understanding of the underlying mechanisms of these effects.

$\underline{\text { References: }}$

1. Dagnelie PC, Agteresch HJ. Drug Devel Res 2003; 59: 146-151 (7th Adenosine Meeting, Gold Coast, Australia).

2. Agteresch HJ, Burgers SA, van der Gaast A, Wilson JHP, Dagnelie PC. Anti-Cancer Drugs 2003; 14: 639-644. 3. Swennen ELR, Bast A, Dagnelie PC. Eur J Immunol 2005; 35: 852-858.

\section{ATP RELEASE THROUGH CONNEXINS HEMICHANNELS IN XENOPUS LAEVIS OOCYTES}

Laia Bahima $^{1}$, Xènia Grandes ${ }^{1}$, Jordi Aleu ${ }^{1}$, Mireia Martín-Satué ${ }^{1}$, Joan Blasi ${ }^{1}$, Jordi Marsal ${ }^{1}$, Luis C. Barrio $^{2}$, Bulat Ziganhin ${ }^{3}$ and Carles Solsona ${ }^{1}$

${ }^{1}$ Laboratory of Molecular and Cellular Neurobiology, Department of Pathology and Experimental Therapeutics, IDIBELL-Medical School, University of Barcelona, Bellvitge Campus, Feixa Llarga s/n, E-08907 L'Hospitalet de Llobregat, Spain.

${ }^{2}$ Unit of Experimental Neurology, Research Department, "Ramón y Cajal” Hospital, Carretera de Colmenar Viejo km 9, 28034 Madrid, Spain. 
${ }^{3}$ Department of Pharmacology, Pharmacognozy and Botany, Kazan State Medical University, 49 Butlerov Street, Kazan 420012, Russia

solsona@ub.edu

Usually, the controlled secretion of ATP occurs through the exocytosis of granules and vesicles. However, in some cells, and under certain circumstances, other mechanisms control ATP release. In gap junctions, connexins $(\mathrm{Cx})$ link the cytoplasm of two adjacent cells by establishing an intercellular channel and control the passage of ions and molecules up to $1 \mathrm{kDa}$. The channel is formed by two moieties called hemichannels or connexons. It has been suggested that connexons may be an alternative pathway for ATP release. Connexins, like Connexin 38 (Xenopus endogenous connexin) and Connexin 32 (Cx32) can, under certain circumstances, permeate for ATP (Bahima et al., 2006). We have investigated ATP release through hemichannels formed by Cx32. This protein is expressed in most of human organs, but, particulary, Cx32 mutations present in Schwann cells produce X-linked Charcot Marie Tooth disease (CMTX). Expressing Cx32 in experimental model of Xenopus oocytes, we have combined the use of two electrode voltage clamp to record the ionic current generated by the hemichannels and the bioluminscent reaction of luciferin-luciferase. The hemichannels formed by Cx32 were estimulated by depolarizing pulses resulting in an outward current. After this estimulation, during the repolarization period, there was an inward tail current coincident with the release of ATP from the oocyte. The area of this tail current correlated with the amount of ATP released. Moreover, when we treated the Cx32 injected oocytes with $5 \mu \mathrm{g} / \mathrm{ml}$ Brefeldin A, a vesicular assembly blocker, there were no significant changes in ATP release, indicating that there was no exocytotic process implicated on it. On the other hand, in oocytes coexpressing Cx32 and P2X1 receptor, we found a larger tail current because the autoactivation of the oocyte, due to the opening of P2X1 receptor by the ATP released. Altogether these results strongly relate the release of ATP to the activation of $\mathrm{Cx} 32$ hemichannels in Xenopus oocytes.

Acknowledgements: This work is supported by grants from the Spanish Government, MCYT (SAF2005-00736/ BFU2005-02202).

Bahima et al. 2006. Endogenous hemichannels play a role in the release of ATP from Xenopus oocytes. J. Cell Physiol. Jan;206(1):95-102

\section{ATP Stimulates Reactive Oxygen Species Generation by NADPH Oxidase via P2X7 Receptors}

Hewinson, J., Ward, S.G., \& MacKenzie, A.

Department of Pharmacy and Pharmacology, University of Bath, Bath, UK, BA2 7AY. prsjh@bath.ac.uk

Reactive oxygen species (ROS) play diverse roles in host defence and inflammation. They have important functions in pathogen destruction, as highlighted in diseases such as chronic granulomatous disease, and have an emerging role in the inflammatory process, especially in the regulation of cell adhesion molecule expression. A few publications have shown that ROS generation in immune cells may be stimulated by $\mathrm{ATP}^{1,2}$. However the receptors and pathways involved have not be defined in any detail, but are important in understanding the role of ATP in inflammation and disease. In this work ATP-induced ROS generation in the human macrophage was investigated.

All studies were performed using the human monocyte cell line THP-1 differentiated with phorbol 12-myristate 13-acetate and lipopolysaccahride, and real-time ROS generation was measured in cells pre-loaded with the ROSsensitive fluorescent compound dichlorofluorescin diacetate. In these cells ATP induced ROS generation was dose-dependent with a peak occurring at 3-5 mM $\left(\mathrm{EC}_{50} 269 \mu \mathrm{M}\right)$. ROS generation was not completely inhibited using 1-[N,O-bis-(5-Isoquinolinesulfonyl)-N-methyl-L-tyrosyl]-4-phenylpiperazine (KN-62; $10 \mu \mathrm{M}$ gave $52 \%$ inhibition with $3 \mathrm{mM}$ ATP) which, taken with the dose response data, suggests P2 receptors other than P2X7 may also signal ROS production. The detection of ATP $(3 \mathrm{mM})$-induced ROS generation was inhibited using $\mathrm{N}$ acetylcysteine (an antioxidant, concentrations $\geq 1 \mathrm{mM}$ gave complete inhibition) and diphenyleneiodonium (concentrations $\geq 30 \mu \mathrm{M}$ gave complete inhibition) but not with allopurinol $(\leq 30 \mu \mathrm{M})$ or $N \omega$-Nitro-L-arginine methyl 
ester $(\leq 100 \mu \mathrm{M})$. Therefore NADPH oxidase and not xanthine oxidoreductase (XOR) or nitric oxide synthase is responsible for the generation of ROS, even though the cell lysates were shown to contain active XOR. None of the effective compounds inhibited ATP-induced calcium influx into Fluo-4 loaded cells therefore discounting the possibility that direct inhibition of $\mathrm{P} 2$ receptors was occurring. In calcium-free medium ATP-induced ROS gen-

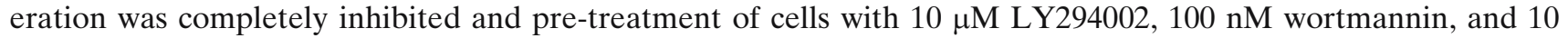
$\mu \mathrm{M}$ PD98059 resulted in partial inhibition of this pathway $(48,29$, and $29 \%$ inhibition, respectively), whereas treatment with $10 \mu \mathrm{M}$ Y-27632 (a ROCK inhibitor) and 0.3\% butan-1-ol (a phosphatidic acid formation inhibitor) had no effect. Therefore these results suggest the involvement of phosphatidyl inositol-3 kinase (PI3K) and extracellular-signal regulated kinase (ERK) in the ATP-induced ROS generation signalling pathway. All effective compounds were tested for cytotoxicity using a lactate dehydrogenase assay. At the concentrations tested none of the compounds were cytotoxic.

These results suggest that ATP can signal the upregulation of ROS production in human macrophages. The generation of ROS is ATP dose-dependent, occurs by NADPH activation, and involves a calcium-dependent pathway that may rely on PI3K and constitutes a pathway that potentially has a role in host-defence and inflammation.

(1) Ferrari, D.; Idzko, M; Dichmann, S; Purlis, D.; Virchow, C.; Norgauer, J.; Chiozzi, P.; Di Virgilio, F.; Luttmann, W. FEBS Letters 2000, 486, 217-224.

(2) Suh, B-C.; Kim, J-S.; Namgung, U.; Ha, H.; Kim, K-T. J Immunol 2001, 166, 6754-6763.

\title{
ATP-consuming and ATP-generating enzymes secreted by pancreas
}

\author{
Gennady Yegutkin ${ }^{1}$, Sirpa Jalkanen ${ }^{1}$ and Ivana Novak ${ }^{2}$ \\ ${ }^{T}$ MediCity Research Laboratory and Department of Medical Microbiology, Turku University and National Public \\ Health Institute, Turku, Finland; ${ }^{2}$ August Krogh Building, Institute of Molecular Biology and Physiology, \\ University of Copenhagen, Copenhagen, Denmark. \\ gennady.yegutkin@utu.fi
}

In exocrine glands, ATP and other nucleotides/sides are thought to be important regulators of salt and fluid transport. In rat pancreas ATP is released from acini into the series of excurrent ducts that possess P2 receptors and also adenosine receptors, as shown by the recent study (1). Close to acini the ATP concentrations are in the high micromolar range. However, in final pancreatic juice collected from the main duct of stimulated pancreas we detected very low concentrations of ATP and high nucleotidase activity (2). Thus, in order to understand acinoductal paracrine regulation, it is important to determine which ecto-enzymes are present in pancreatic secretion. For this purpose, in vivo experiments on rat pancreas were performed and the major purine-converting exchange enzyme activities were determined in pancreatic juice.

Animals were stimulated with cholecystokinin (CCK-8) infusion ( $5.6 \mathrm{pmol} / \mathrm{min} / \mathrm{per} 200 \mathrm{~g}$ animal) and pancreatic juice was collected from the common pancreatic bile duct. Purine-converting activities and transfer of $\gamma$-phosphate by pancreatic juice were determined by incubation of samples with $\left[{ }^{14} \mathrm{C}\right],\left[{ }^{3} \mathrm{H}\right]$ or $\left[\gamma^{-32} \mathrm{P}\right]$ labelled nucleotides, with/without cold nucleotides as second substrates, followed by subsequent separation by TLC and autoradiography (3). Pancreatic juice for enzymatic analysis was collected after $60 \mathrm{~min}$ of continuous CCK-8 stimulation when secretion was about $3 \mu \mathrm{l} / \mathrm{min}$. The time-courses of $\left[{ }^{14} \mathrm{C}\right] \mathrm{ATP}$ and $\left[{ }^{3} \mathrm{H}\right] \mathrm{ADP}$ hydrolyses show that pancreatic juice contains enzymes that convert ATP to ADP, which is further rapidly converted to AMP. Data indicate that the juice contain NTPDase that can hydrolyze both ATP and ADP about equally well, i.e. CD39. Reverse-phase HPLC analysis additionally shows that this enzyme has broad substrate specificity towards other nucleotides, UTP and ITP. In addition, secretion contains ecto-5'nucleotidase, i.e. CD73, converting AMP to adenosine. Activities of non-specific phosphatases, ecto-nucleotide pyrophosphatase/phosphodiesterases and adenosine deaminase were negligible. In addition to hydrolytic enzymes, there were also counteracting ATP-generating enzymes in pancreatic juice, adenylate kinase and NDP kinase, capable of sequentially phosphorylating AMP via ADP to ATP.

Taken together, following CCK-8 stimulation of pancreas, pancreatic juice contains significant activities of enzymes consistent with CD39 and CD73. There are also enzymes with lower activities that are able to regenerate ATP from AMP and ADP, where other NTP can be used as donors of $\gamma$-phosphoryl groups. This newly discov- 
ered richness of secreted enzymes underscores the importance of purinergic receptors in signalling along pancreatic ducts lumen, and regulated release of ATP-hydrolyzing and ATP-generating enzymes.

The projects were supported by the Danish Medical and Science Research Councils

1. Novak, I., S.E. Hede, and M.R. Hansen (this volume)

2. Sørensen, C. E., J. Amstrup, H. N. Rasmussen, I. Ankorina-Stark, and I. Novak. J Physiol 2003; 551(3): 881-892.

3. Yegutkin, G. G., S. S. Samburski, and S. Jalkanen. FASEB J 2003; 17: 1328-1330.

\title{
Attenuation of Reperfusion Lung Injury and Apoptosis by $\mathbf{A}_{2 \mathrm{~A}}$ Adenosine Receptor Activation is Associated with Modulation of Bcl-2 and Bax Expression and Activation of Extracellular Signal-Regulated Kinases
}

\author{
Julia Rivo, ${ }^{1}$ Evelyne Zeira, ${ }^{2}$ Eithan Galun, ${ }^{3}$ Sharon Einav, ${ }^{4}$ Joel Linden, ${ }^{5}$ Idit Matot ${ }^{6}$ \\ ${ }^{1,4,6}$ Department of Anesthesiology \& Critical Care Medicine, ${ }^{2,3}$ Goldyne Savad Institute of Gene Therapy, \\ Hadassah University Medical Center, The Hebrew University of Jerusalem, Jerusalem, Israel, ${ }^{5}$ Departments of \\ Medicine and Pharmacology, University of Virginia, Charlottesville, Virginia, USA. \\ iditm@hadssah.org.il
}

Background: Adenosine receptors (AR) and extracellular signal-regulated kinases (ERK) have been implicated in tissue protection and apoptosis regulation during ischemia-reperfusion (IR) injury. This study tests the hypothesis that reduction of reperfusion lung injury following $\mathrm{A}_{2 \mathrm{~A}} \mathrm{AR}$ activation is associated with attenuation of apoptosis, modulation of ERK activation, and alterations in anti- and pro-apoptotic protein expression (Bcl-2 and Bax, respectively).

Methods: The arterial branch of the left lower lung lobe in intact-chest, spontaneously breathing cats was occluded for $2 \mathrm{hr}$ and reperfused for $3 \mathrm{hr}$ (IR group). Animals were treated with the selective $\mathrm{A}_{2 \mathrm{~A}} \mathrm{AR}$ agonist ATL313 given 5 min before reperfusion alone, or in combination with the selective $\mathrm{A}_{2 \mathrm{~A}} \mathrm{AR}$ antagonist ZM241385.

Results: Western blot analysis showed significant reduction in expression of Bcl-2 and increase in expression of Bax after reperfusion compared with control lungs. Phosphorylated ERK1/2 levels were also increased following reperfusion. Apoptosis was preferentially increased in alveolar epithelial cells. Compared to the IR group, ATL313 markedly $(\mathrm{P}<0.01)$ attenuated indices of injury and apoptosis including the percentage of injured alveoli, wet/dry weight ratio, myeloperoxidase activity, in situ terminal deoxynucleotidyl transferase-mediated deoxyuridine triphosphate nick end-labeling (TUNEL) positive cells, and caspase 3 activity and expression. Furthermore, compared with reperfused lungs, in ATL313-pretreated lungs, Western blot analysis demonstrated substantial ERK1/2 activation, increased expression of Bcl-2, and attenuated expression of Bax. The protective effects of ATL313 were blocked by pretreatment with ZM241385.

Conclusions: In vivo activation of $\mathrm{A}_{2 \mathrm{~A}} \mathrm{AR}$ confers protection against reperfusion lung injury. This protection is associated with decreased apoptosis and involves ERK1/2 activation and alterations in anti-apoptotic Bcl-2 and pro-apoptotic Bax proteins. 
$\mathbf{a}$

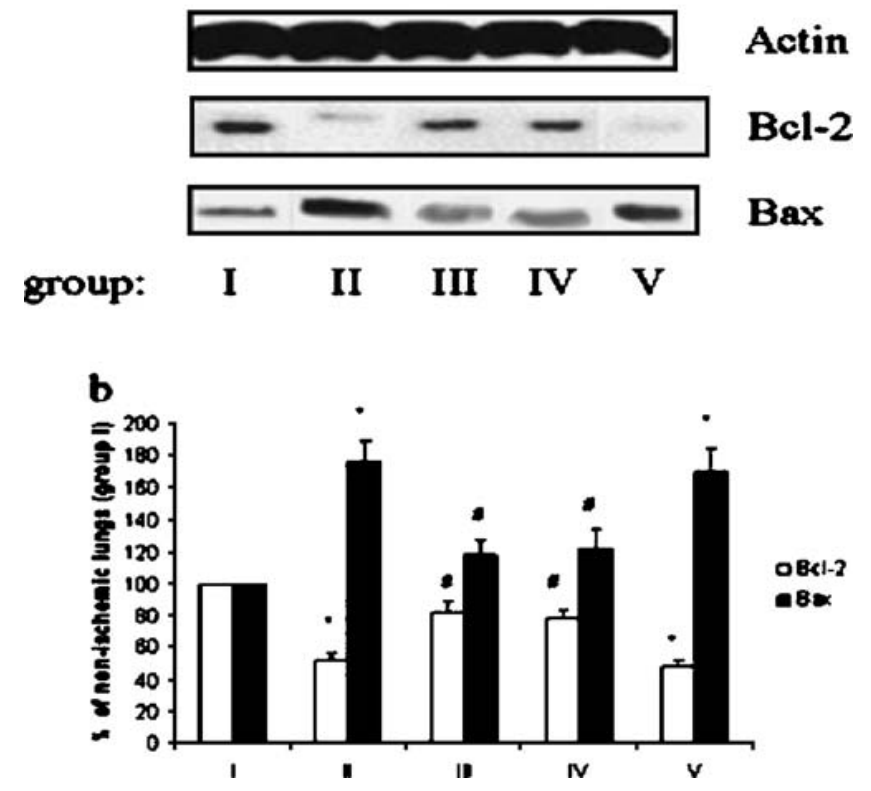

Western blot analysis of Bcl-2 and Bax protein levels in control, nonsichemic group (I), ischemia reperfusion (IR) \{II\}. ATL313 continuous infusion of $1 \mathrm{ng} \cdot \mathrm{kg} 1 \cdot \min 1$ administered before IR (III), ATL313 continuous infusion of $10 \mathrm{ng} \cdot \mathrm{kg} 1 \cdot \min 1$ administered before IR (IV). ZM241385 (V). (a) Representative immunoblots (b) Densitometric scan of Bcl-2 and Bax in lung tissue from the different groups. The intensities of bands in control treated tissue samples (no ischemia or reperfusion, group I) were expressed as $100 \%$. Data (mean \pm SEM) were obtained from Western blot assays performed at the end of reperfusion. Immunodetection of actin was performed as an internal control. $* \mathrm{p}<0.05$ compared with groups I, III, IV. ** $\mathrm{p}<0.01$ compared with groups II, V.

\section{Augmentation of cutaneous hypersensitivity by perceived acute stress is mediated by the adenosine $\mathrm{A} 1$ receptor.}

Sneha Mathew, Tuere Wilder, Bruce N Cronstein, Stephen J Oliver

Department of Medicine, Division of Clinical Pharmacology, New York University School of Medicine, New York, $N Y, U S A$.

olives03@med.nyu.edu

Adenosine is utilized by the body's tissues for modulation of a diverse range of activities that include sleep, cognitive function, immunity and pain perception. Acting through its specific receptors, adenosine modulates neurotransmitter function and release, behavior, systemic immunity and cell metabolism. Adenosine is generated extracellularly by degradation of released adenine nucleotides (ATP, ADP, AMP) and is constitutively present at low levels in all tissues. However, extracellular adenosine levels can markedly increase under metabolically stressful conditions such as inflammation or hypoxia. Biological systems have evolved to utilize extracellular adenosine levels to activate protective responses against physiological stressors such as ischemia and infection. Acting through the CNS, perceived psychological stress also affects biological functions within the body. In particular, differential effects of acute and chronic stress on skin immunity have been demonstrated in the well-established rodent model of non-painful restraint stress; with acute stress enhancing and chronic stress suppressing cutaneous delayed-type hypersensitivity (DTH), an antigen-specific immune response known to be mediated by CD8+ T cells $^{1,2}$. In this study, we utilize the restraint stress model and the hapten dinitrofluorobezene (DNFB, $1 \%$ in $4: 1$ acetone/olive oil) to induce delayed type hypersensitivity in the skin. In WT mice undergoing restraint stress DTH was augmented at $48 \mathrm{hrs}$ (mean change in pinna thickness, non-stressed [NS] vs acute stressed [AS]: $0.08 \mathrm{~mm}$ vs $0.31 \mathrm{~mm}$, respectively, $\mathrm{p}=0.008$ ). In contrast, stress does not enhance DTH responses in $\mathrm{A}_{1} \mathrm{KO}$ mice (mean change NS vs AS, $0.18 \mathrm{~mm}$ vs $0.19 \mathrm{~mm}$, respectively, $\mathrm{p}=0.73$ ). Further studies have failed to show any differences in stress-augmented plasma corticosterone and urinary norepinephrine levels between A1 receptor knockout 
(KO) and WT mice. Also, both A1 receptor KO and WT mice respond similarly to acute stress with rapid corticosterone-mediated deployment of monocytes and lymphocytes out of the blood vasculature into the surrounding tissues. However, acute stress associated increases in circulating blood neutrophils, known to be mediated by catecholamine release, were not seen in A1 KO mice (mean cell count, NS vs AS: $1.54 \times 10^{3}$ cells $/ \mu$ l vs $1.62 \times$ $10^{3}$ cells $/ \mu \mathrm{l}, \mathrm{p}=1.000$ ), in contrast to their WT littermates (mean cell count, NS vs AS: $1.18 \times 10^{3}$ cells $/ \mu \mathrm{l}$ vs $4.18 \times$ $10^{3}$ cells $\left./ \mu \mathrm{l}, \mathrm{p}=0.009\right)$. Others have suggested that the increased antigen specific $\mathrm{T}$ cell responses occurring with acute stress are due to norepinephrine-driven dermal dendritic cell (DC) migration to draining lymph nodes ${ }^{2}$. Using FITC (1\% in 1:1 acetone/dibutylphthalate) topically applied to shaved ventral skin, we show that the enhanced rates of MHC class II $^{\text {high }} \mathrm{CD} 11 \mathrm{c}+$ DC migration to draining lymph nodes observed in acutely stressed WT mice (DCs containing FITC, NS vs AS, at $6 \mathrm{hrs}, 17 \%$ vs $26.8 \%$; at $24 \mathrm{hrs}, 29.1 \%$ vs $39.8 \%$ ) do not occur in the adenosine A1 receptor KO mice under similar conditions (NS vs AS, at $6 \mathrm{hrs}, 27.4 \%$ vs $28.5 \%$; at $24 \mathrm{hrs}, 42.9 \%$ vs $38.1 \%$ ). Thus, our results suggest that acute stress enhances cutaneous immunity and that adenosine receptor A1 signaling plays a critical role in acute stress-altered cutaneous DTH immunity, at least in part through its modulating effects on norepinephrine release and dermal DC migration rates.

1. Dhabhar, F. S.; McEwen, B. S., Acute stress enhances while chronic stress suppresses immune function in vivo: a potential role for leukocyte trafficking. Brain Behav Immun 1997, 11, 286-306.

2. Saint-Mezard, P.; Chavagnac, C.; Bosset, S.; Ionescu, M.; Peyron, E.; Kaiserlian, D.; Nicolas, J.-F.; Berard, F., Psychological stress exerts an adjuvant effect on skin dendritic cell functions in vivo. Journal of Immunology 2003, 171, 4073-4080.

\section{Autocorrelation of Molecular Electrostatic Potential Surface Properties combined with Partial Least Squares Analysis as New Strategy for Predicting the Activity of Human $A_{3}$ Adenosine Receptor Antagonists tool to generate ligand-based 3D-QSARs.}

Magdalena Bacilieri $^{1}$, Stefano Moro ${ }^{1}$, Barbara Cacciari $^{2}$, Giampiero Spalluto $^{3}$,

${ }^{T}$ Molecular Modeling Section, Dipartimento di Scienze Farmaceutiche, Università di Padova, Via Marzolo 5, I-35131 Padova, Italy; ${ }^{2}$ Dipartimento di Scienze Farmaceutiche, Università degli Studi di Ferrara, Via Fossato di Mortara 17-19, I-44100 Ferrara, Italy; ${ }^{3}$ Dipartimento di Scienze Farmaceutiche, Università degli Studi di Trieste, Piazzale Europa 1, I-34127 Trieste, Italy

magdalena.bacilierri@unipd.it

Ligand based 3D-structure-activity relationship (3D-QSAR) is an ensemble of strategies to study new compounds starting from molecule structures and their corresponding chemical properties. Throughout the different approaches, the combination of autocorrelation vectors ${ }^{1}$ of molecular electrostatic potential ${ }^{2,3}$ (MEP) surface properties with the conventional partial least squares (PLS) analysis has been used for the prediction of the human $\mathrm{A}_{3}$ receptor antagonist activities. The autocorrelation allows in fact to compare molecules (and their properties) with different chemical structures and with different spatial orientation without any previous alignment (which is often not even possible).

A dataset of 358 structurally diverse human A3 receptor antagonists has been used to derive a novel ligand-based 3D-QSAR model. The molecular collection includes the most important chemical classes of human $\mathrm{A}_{3}$ antagonist structure-activity relationship. A remarkable quantitative model was generated, with a cross-validated correlation coefficient of 0.81 and a test set correlation coefficient of $0.82 .{ }^{4}$

The aim of this analysis was to employ a rigorously validated QSAR model for a possible application of virtual screening on available chemical databases to find out new compounds with high predicted activity. 


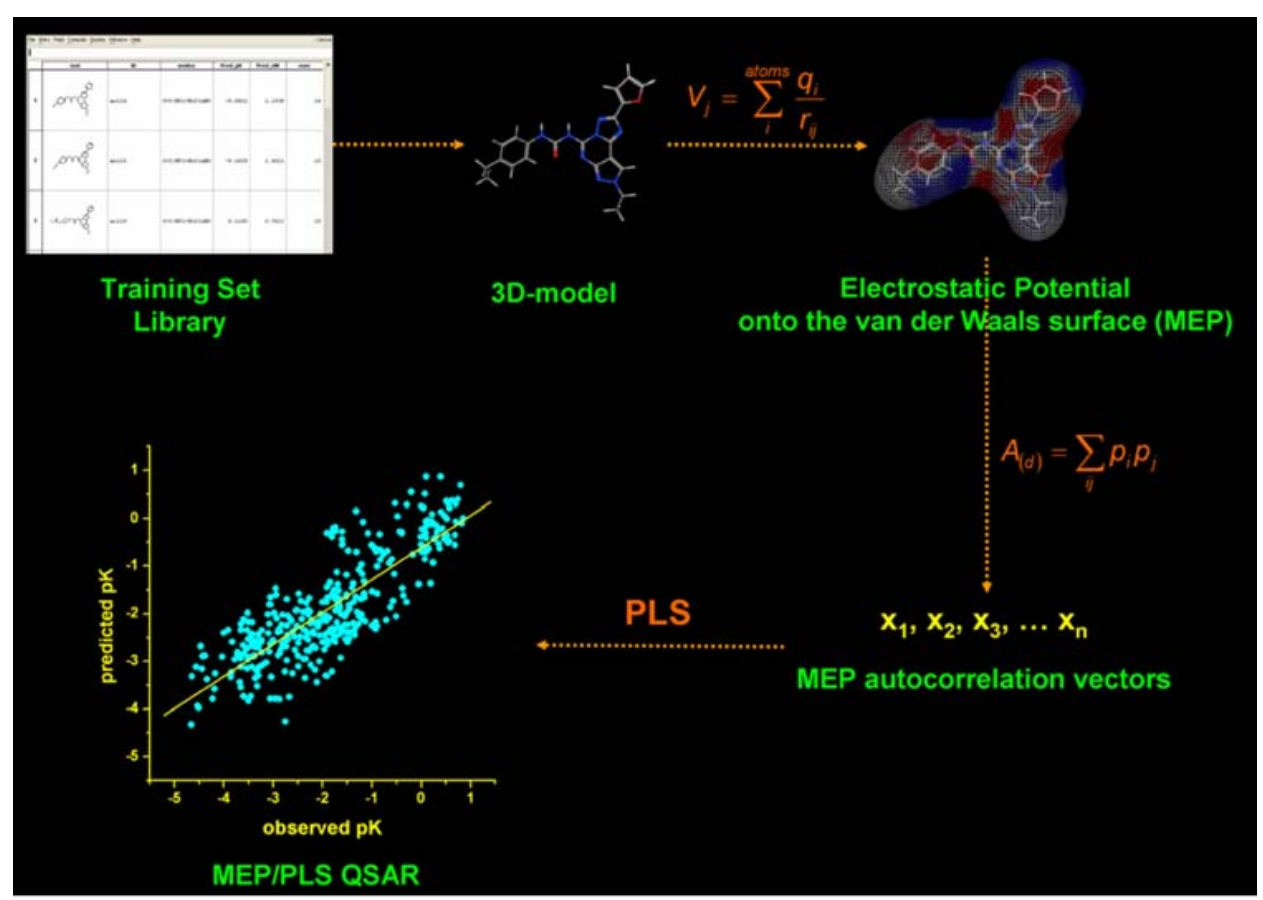

Flowchart of 3D-QSAR (autoMEP/PLS) approach

1. Wagener, M.; Sadowski, J.; Gasteiger, J. J. Am. Chem. Soc. 1995, 117, 7769-7776.

2. Gasteiger, J.; Li, X.; Rudolph, C.; Sadowski, J.; Zupan, J. J. Am. Chem. Soc. 1994, 116, 4608-4620.

3. Bauknecht, H.; Zell, A.; Bayer, H.; Levi, P.; Wagener, M.; Sadowski, J.; Gasteiger, J. J. Chem. Inf. Comput. Sci. 1996, 36, 1205-1213.

4. Moro, S.; Bacilieri, M.; Cacciari, B.; Spalluto, G. J. Med. Chem. 2005, 48, 5698-5704.

\section{BDNF-induced modulation of synaptic transmission requires functional adenosine $\mathbf{A}_{2 \mathrm{~A}}$ receptors in the mouse hippocampus.}

Martire A, Domenici MR, Potenza RL, Pepponi R, Felici F, Tebano MT, Popoli P. Istituto Superiore di Sanità, Rome, Italy.

patrizia@popoli@iss.it

Brain-derived neurotrophic factor (BDNF), a member of neurotrophin family, is able to enhance synaptic transmission and to induce long-term potentiation (LTP) either in vitro or in vivo. BDNF and its tyrosine kinase receptor $(\mathrm{TrkB})$ are highly expressed in the hippocampus where an interaction with adenosine $\mathrm{A}_{2 \mathrm{~A}}$ receptors $\left(\mathrm{A}_{2 \mathrm{~A}} \mathrm{Rs}\right)$ has been recently demonstrated (Diogenes et al, 2004). The aim of the present work was to evaluate BDNF effects in the hippocampus of $\mathrm{A}_{2 \mathrm{~A}} \mathrm{R}$ wild type (WT) and knock out (KO) mice (6 months of age). In electrophysiological experiments in hippocampal slices (450 um) from WT mice, application of BDNF (10-20 ng/ $\mathrm{ml}, 30 \mathrm{~min}$ ) increased the slope of excitatory post-synaptic field potentials (fEPSPs) recorded in the stratum pyramidale of $\mathrm{CA} 1$ region $(113 \pm 6.7 \%, \mathrm{P}<0.05$ vs basal, $\mathrm{N}=11)$. The co-application of the selective $\mathrm{A}_{2 \mathrm{~A}}$ antagonist ZM $241385(50 \mathrm{nM})$ abolished the BDNF-induced potentiation of fEPSP slope $(99.43 \pm 1.05 \%, \mathrm{~N}=6)$. Conversely, in slices from $\mathrm{A}_{2 \mathrm{~A}} \mathrm{R} \mathrm{KO}$ mice BDNF was no longer able to increase the fEPSP slope and it even reduced it $(88.83 \pm 3.29 \mathrm{P}<0.05 v s$ basal and $v s \mathrm{WT}, \mathrm{N}=12)$. Finally, enzyme immunoassay studies showed a tendency to a reduction in hippocampal BDNF levels in $A_{2 A} R$ KO vs WT mice. These results suggest that in the hippocampus the presence and the tonic activation of $\mathrm{A}_{2 \mathrm{~A}}$ Rs are necessary to allow BDNF-induced potentiation of synaptic transmission. Given the key role exerted by hippocampal BDNF on both synaptic plasticity and 
neuroprotection, it is of paramount importance to understand how the tone existing at $\mathrm{A}_{2 \mathrm{~A}}$ Rs may influence the BDNF effects under both physiological and pathological conditions.

Diogenes M. J.; Fernandes C. C.; Sebastiao A. M.; Ribeiro J. A. J. Neurosci. 2004; 24(12): 2905-2913.

\title{
Benzo $[d]$ isothiazol-3-yl-benzamidines: a new class of protective agents on culture of human chondrocytes stimulated by IL-1 $\beta$
}

\author{
AM. Panico $^{1}$, P. Vicini ${ }^{2}$, M. Incerti ${ }^{2}$, F. Garufi $^{1}$, S. Ronsisvalle ${ }^{1}$. \\ ${ }^{1}$ Dept. of Pharmaceutical Sciences, University of Catania, V.le Doria 6, 95125 Catania (Italy) \\ ${ }^{2}$ Pharmaceutical Dept., University of Parma, Parco Area delle Scienze 27/A, 43100 Parma (Italy) \\ panico@unict.it
}

Several articular pathologies evolve from a local inflammatory disease to a chronic process with distinct inflammatory and destructive components. The progressive damage of the articular cartilaginous tissue represents a central moment of the pathogenetic course, in which depletion of aggrecan, disregulated synthesis of matrix components like proteoglycans and collagen play a fundamental role [1]. Moreover, the functional alteration of cartilage results also from the interaction of different mediators, such as cytokines, e.g. interleukin-1 $1 \beta$ ?IL-1 $\beta$ ), that induces high levels of nitric oxide (NO), inhibits collagen and proteoglycans synthesis, increases susceptibility to injury by other oxidants (e.g. superoxide anion and hydroxyl radicals, peroxides, and reactive oxygen species, ROS).

The culture of human chondrocytes stimulate by IL-1 $\beta$ has been established, as a profit experimental model, for reproducing the mechanisms involved in the pathophysiology of arthritic diseases.

In the present study we determined the in vitro effects on human articular chondrocytes for a series of $N$ benzo[ $d]$ isothiazol-3-yl-benzamidines hydrochlorides designed to improve the effectiveness of $N$-benzo $[d]$ isothiazol-3-yl-amidines previously described by us as promising chondroprotective agents [2].
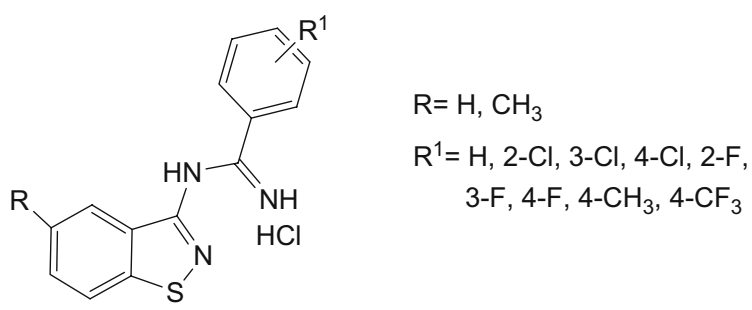

We investigated the influence of the new amidines on the production of NO, MMP-3, ROS, GAGs, key molecules involved in matrix destruction.

Our results clearly demonstrate that $N$-benzo[ $d]$ isothiazol-3-yl-benzamidines properly substituted can block multiple cartilage destruction during the arthritic inflammatory process, as simulated in our experimental model.

[1] Flugge, L.A., Miller-Deist L.A., Petillo P.A., 1999. Towards a molecular understanding of arthritis. Chemistry \& Biology 6, 157-166.

[2] Panico, AM; Vicini, P; Massimo, G; Cardile, V; Gentile, B; Avondo, S; Vittorio, F; Ronsisvalle, G; 2004. Protective effects of benzisothiazolylamidines on IL-1 $\beta$ induced alterations in human articular chondrocyte metabolism. Inflammation; 28 (4): 231-235. 


\title{
Beyond Purinergic Receptors: Drugging the Rest of the Purine-Binding Proteome
}

\author{
S. Hall, A. Barabasz, T. Barta, J. Daw, L. Dubois, J. Eaves, P. Fadden, B. Foley, T. Freed, L. Geng, G.
} Hanson, K. Hardemann, L. Hinkley, M. Jenks, M. Hu, K. Huang, M. Lewis, L. Liu, W. Ma, J. Otto, J. Partridge, B. Pronk, J. Rice, A. Scott, M. Silinski, P. Steed, J. Veal, K. Verleysen, R. Ware, T. Wadkins Serenex, Inc. 323 Foster Street, Durham, NC, 27701 USA

shall@serenex.com

Purinergic receptors represent an attractive class of druggable targets within the purine-binding proteome, yet there are many other enzyme families of interest as therapeutic targets within this large superfamily. We estimate that the purine-binding proteome contains at least 2000 members, with kinases composing the largest fractio$\mathrm{n}(25 \%)$ of this group. We report here a novel approach to screen hundreds of these targets in parallel, thus providing an efficient way to probe compound libraries as ligands to a broad variety of enzyme classes. We constructed and screened a targeted library of 8000 compounds using a novel affinity displacement assay (referred to as proteome mining). Multiple tissues were used as the source of native purine-binding proteins to profile this library. Hits were obtained for nearly 100 targets, including well validated targets such as dihydrofolate reductase and multiple kinases (e.g. CDK2, Her2, PDK1, AurA), and less well-validated targets such as the kinases Fer and Nek9. Importantly, the method also provided affinity information on potential toxicity targets (e.g. phosphorylase kinase, pyruvate carboxylase, glucose-6-phosphate dehydrogenase). Thus, this approach provides primary target affinity information as well as selectivity data across the entire purine-binding proteome. In addition, affinities of compounds for their protein targets could be determined directly from the same assay and these measurements were sufficiently quantitative and reproducible to drive the development of structure-activity relationships. Unexpectedly, selectivity within a gene family was not predictive of selectivity between gene families across the purine-binding proteome. The application of this screening approach to four diverse targets, dihydrofolate reductase, Hsp90, quinone reductase 2, and PI3kinase will be described. In each case, the use of the affinity displacement assay enabled the identification of novel, selective, orally active compounds. Taken together, these results support the use of proteome mining as both an alternative to single target biochemical screens and as a tool to drive SAR development.

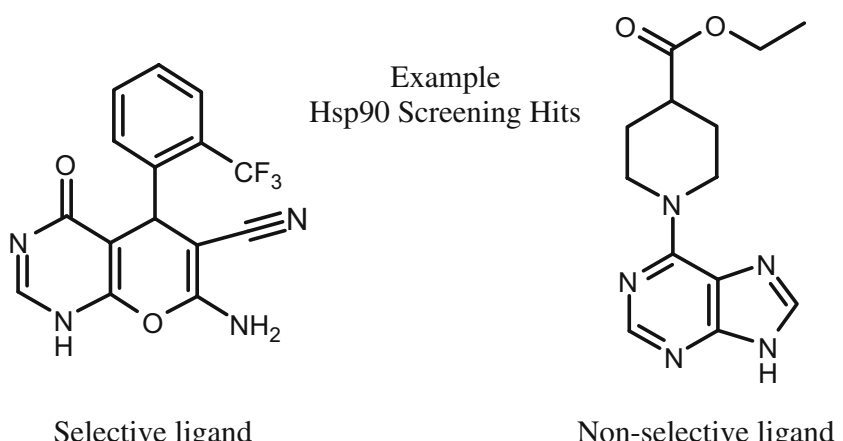

\section{Bioinformatic analysis of the $\mathrm{P} 2 X$ receptor family}

\author{
Digby $\mathrm{HR}^{1}$ Sutcliffe $\mathrm{MJ}^{2}$ \& Evans $\mathrm{RJ}^{1}$ \\ ${ }^{\mathrm{T}}$ Department Cell Physiology and Pharmacology, University of Leicester \\ ${ }^{2}$ School of Chemical Engineering and Analytical Science, University of Manchester \\ hrd1@le.ac.uk
}

P2X receptors for ATP are a family of ligand-gated ion channels consisting of seven receptor subunits that associate most likely as homo- and heterotrimeric channels. They are expressed throughout the body and have a 
variety of physiological roles which means the development of P2X receptor subtype selective drugs could have wide ranging therapeutic benefits. For example, $\mathrm{P} 2 \mathrm{X}_{1}$ receptors have been implicated in the regulation of thrombosis and $\mathrm{P} 2 \mathrm{X}_{3}$ receptor antagonists may act as analgesics and regulate bladder function. There are no crystal structures available for the P2X receptor family, they have no obvious similarity to other ion channels or ATP binding proteins and motifs such as the Walker motif are not present as in other ATP-sensitive proteins. For this reason, mutagenesis studies have been relied upon to identify important residues involved in ATP action at the receptors. However, the need for a structural model is imperative to functional studies aiding rational drug design and the development of pharmacophores. A portion of the extracellular loop of P2X receptor proteins shares a degree of homology with the catalytic domains of class II aminoacyl-tRNA synthetases and a homology model of the rat $\mathrm{P} 2 \mathrm{X}_{4}$ receptor has been published based on this similarity. However, existing models do not consider the entire protein sequence of the receptor and the likely trimeric structure. Ihave produced possible 3D models for the structure of the human $\mathrm{P} 2 \mathrm{X}_{1}$ receptor using ATP-binding proteins with known structures and similar ATP binding environments to the $\mathrm{P}_{2} \mathrm{X}_{1}$ receptor as predicted by mutagenesis studies. The structural models attempt to represent the extracellular ligand-binding loop. The models are currently being tested by site-directed mutagenesis. Supported by the Wellcome Trust

\title{
Biological Significance of the Endogenously Expressed Truncated $\mathbf{P 2 X}_{\mathbf{7}}$ Receptor Variant $\left(\mathbf{P 2 X}_{7-\mathrm{j}}\right)$ in Human Uterine and Skin Cancers
}

\author{
Xin Li MD PhD ${ }^{\mathrm{a}}$, Lingying Zhou MD ${ }^{\mathrm{a}}$, Ying-Hong Feng MD $\mathrm{PhD}^{\mathrm{b}}$, George Gorodeski MD PhD ${ }^{\mathrm{a}, \mathrm{c}}$ \\ Departments of Reproductive Biology ${ }^{\mathrm{a}}$, Physiology and Biophysics, and Oncology ${ }^{\mathrm{c}}$, CASE (Case Western \\ Reserve) University, Cleveland, Ohio, USA (gig@cwru.edu); and Pharmacology ${ }^{\mathrm{b}}$, Uniformed Services University \\ of the Health Sciences, Bethesda, Maryland.
}

The P2X7 system plays an important role in spontaneously occurring apoptosis and cell-number regulation in the human cervix (1). Recent studies from our lab revealed the presence of naturally occurring truncated variant of the human receptor $\mathrm{P} 2 \mathrm{X}_{7}$ in human cancer cervical epithelial cells. The novel protein $\left(\mathrm{P} 2 \mathrm{X}_{7-\mathrm{j}}\right)$, a polypeptide of 258 amino acids, lacks the entire intracellular carboxy terminus, the second transmembrane domain, and the distal third of the extracellular loop of the full-length $\mathrm{P}_{2} \mathrm{X}_{7}$ receptor. Expression experiments in host cells that lack endogenously the $\mathrm{P} 2 \mathrm{X}_{7}$ receptor revealed diminished ligand-binding and channel function capacities, and failure to form pores and mediate apoptosis in response to treatment with the $\mathrm{P}_{2} \mathrm{X}_{7}$ receptor agonist BzATP. The present study extended those preliminary experiments to other epithelial tissues, including the ectocervix (EctoCx), endometrium (EndoM), and skin. In the first experiment primary-secondary cultures of normal and cancer epithelial cells were generated on filters (2), and the degree of apoptosis was determined by the DNA solubilization assay (3) following $9 \mathrm{hrs}$ of treatment with $100 \mu \mathrm{M}$ BzATP. The results in Fig. 1 show diminished apoptosis in all three types of cancer cells compared to the corresponding normal cells. The second experiment determined mRNA (Fig. 2) and protein levels (Fig. 3) of the $\mathrm{P} 2 \mathrm{X}_{7-\mathrm{j}}$ and Full-Length $\mathrm{P} 2 \mathrm{X}_{7}$ receptor in normal and cancer ectocervix, endometrium, and skin tissues. mRNA levels were determined by Real-Time RT-PCR and normalized to GPDH; protein levels were determined by densitometry of the $\mathrm{P}_{2} \mathrm{X}_{7-\mathrm{j}}$ - specific $45-42 \mathrm{KDa}$ bands off Western blots using Alomone-M0 anti $\mathrm{P} 2 \mathrm{X}_{7} \mathrm{pAb}$ (which recognizes the proximal extracellular loop of the $\mathrm{P} 2 \mathrm{X}_{7}$ receptor) and normalized to tubulin. The data show that in the three types of tissues the ratios of $P 2 X_{7-j} / P 2 X_{7}$ mRNA (Fig. 2) and protein (Fig. 3) were greater in cancer than in normal cells. These results suggest a general pattern of upregulation of the $P 2 X_{7-j}$ or downregulation of the Full-Length $P 2 X_{7}$ in epithelial cancers. Because the $P 2 X_{7-j}$ variant antagonizes the Full-Length $\mathrm{P} 2 \mathrm{X}_{7}$ receptor (manuscript submitted), the present results suggest a novel pathophysiological mechanism of apoptosis inhibition through regulation of $\mathrm{P} 2 \mathrm{X}_{7}$ protein function by its variant the $\mathrm{P} 2 \mathrm{X}_{7-\mathrm{j}}$. Dysregulated apoptosis could play a role in carcinogenesis. 


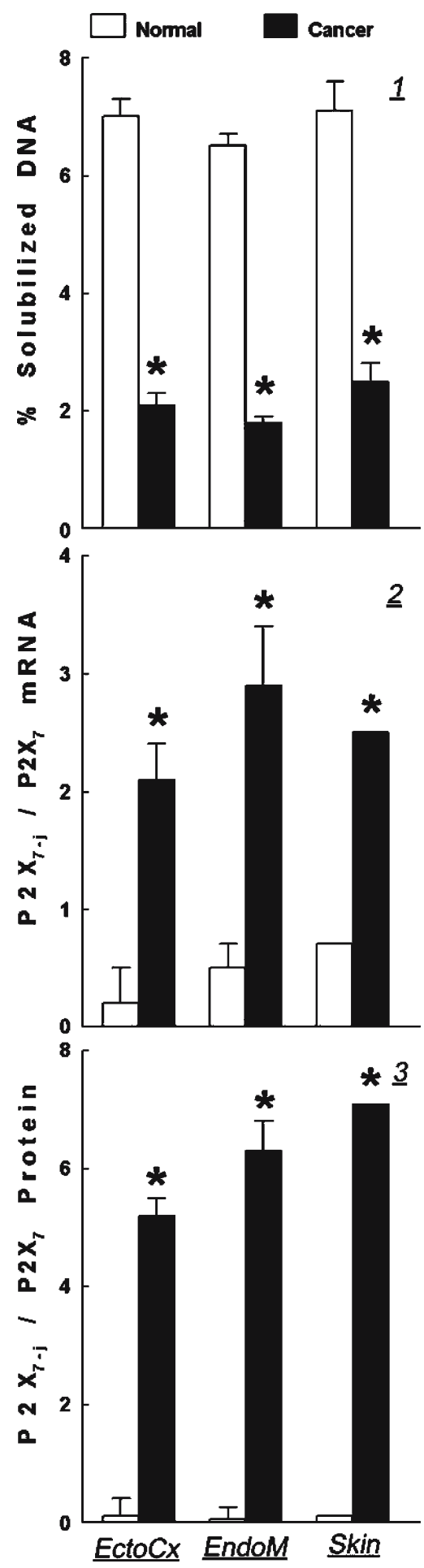

Support: AHA-SDG-0030019N, NHLBI-HL65492 (YHF); NIH HD29924, AG15955, and an unrestricted grant by CytoCore Inc (GIG). Patent pending.

1. Wang et al, Am J Physiol 287:C1349, 2004.

2. Gorodeski et al, Differentiation 56:107, 1994.

3. Wang et al, Endocrinology 145:5568, 2004. 


\title{
Bivalent adenosine antagonist-dopamine agonits for the treatment of Parkinson's disease
}

\author{
Aroa Soriano, Ruben Ventura, Vicent Casadó, Fernando Albericio*, Carmen Lluis, Miriam Royo*, \\ Rafael Franco. \\ Molecular Neurobiology Unit. Institut d'Investigacions Biomèdiques August Pi i Sunyer (IDIBAPS). University of \\ Barcelona. Diagonal 645. 08028 Barcelona and * Scientific Park PCB. University of Barcelona. Josep Samitier s/n. \\ 08028 Barcelona. \\ rfranco@ub.edu
}

G-protein-coupled (heptaspanning) membrane receptors are target for a wide range variety of diseases. Recent evidence shows that these receptors occur on the cell surface as homo and heterodimers. Therefore dimers but not monomers are the actual targets for therapeutical useful drugs (already marketed or under development). The heteromerization is one of the molecular basis involved in the negative cross-talk between adenosine and dopamine. The anti-parkinsonian actions of $\mathrm{A}_{2 \mathrm{~A}} \mathrm{R}$ antagonists in humans (already in phase 3 clinical trials) are to a substantital degree caused by blocking the action of endogenous adenosine on $A_{2 A} R$ of the $A_{2 A} R / D_{2} R$ heteromer. We have targeted $A_{2 A} R / D_{2} R$ heterodimers by the development of novel compounds that could potentially interact simultaneously with dopamine $\mathrm{D}_{2}$ and $\mathrm{A}_{2 \mathrm{~A}}$ receptors. Dopamine-adenosine bivalent ligands have mixed $A_{2 A}$ receptor antagonist and $D_{2}$ receptor agonist pharmacophores coupled by connecting spacers of variable lengths. These spacers are polyamide-poly (ethylene) glycol type oligomers composed by a precise number of monomer units $[(\mathrm{NH}-\mathrm{CH} 2 \mathrm{CH} 2 \mathrm{O}-\mathrm{CH} 2 \mathrm{CH} 2 \mathrm{O}-\mathrm{CH} 2 \mathrm{CH} 2-\mathrm{NH}-\mathrm{CO}-\mathrm{CH} 2 \mathrm{CH} 2-\mathrm{CO})]$ and were synthesized by a solidphase protocol. The potential of these bivalent ligands has already been confirmed in cell models and subsequently patented. Bivalent liagnds of given lengths are more potent than monovalent compounds and can simultaneously bind to both $\mathrm{D}_{2}$ and $\mathrm{A}_{2 \mathrm{~A}}$ receptors in the heterodimer. Their efficacy has been tested in cAMP level determination assays in transfected cells. A lead compound has been selected that has $\mathrm{D}_{2}$ receptor agonist activity and $\mathrm{A} 2 \mathrm{~A}$ receptor antagonists activity and higher relative efficacy than monovalent counterparts. These bivalent ligands prove that $A_{2 A} R / D_{2} R$ heteromers occur in striatum and they constitute novel therapeutical tools for PD. It is expected that these compounds are more efficacious than combined therapies using L-DOPA and adenosine antagonists and have less side effects.

\section{Blockade of adenosine $A_{2 A}$ receptors differently alters convulsive behaviour and prevents hippocampal damage in two models of temporal lobe epilepsy}

\author{
Lisiane O. Porciúncula, Paula M. Canas, Catarina R. Oliveira, Rodrigo A. Cunha \\ Ctr. Neuroscience Coimbra, Fac.Medicine, Univ.Coimbra, Portugal. \\ (loporciuncula@yahoo.com)
}

Endogenous adenosine in the brain is thought to prevent the development and spread of seizures via tonic anticonvulsant $\mathrm{A}_{1}$ receptor-mediated effects (Fredholm et al., 2005). However, adenosine modulation results from a balanced activation of inhibitory $A_{1}$ and facilitatory $A_{2 A}$ receptors $\left(A_{2 A} R s\right)$ and blockade of these $A_{2 A} R s$ affords neuroprotection against different brain insults (Cunha, 2005). Thus, we tested the role of $\mathrm{A}_{2 \mathrm{~A}} \mathrm{Rs}$ on the development of behavioural seizures and hippocampal damage in the two models of epilepsy: kainic acid (KA) ip administration and amygdala kindling.

Male Wistar rats injected ip with KA (10 mg/kg, ip) displayed rapid (within $30 \mathrm{~min}$ ) and severe convulsions (stage 4-5 of Racine's scale) and a pattern of neuronal damage (cresyl violet and FluoroJade staining) together with astrogliosis (GFAP immunoreactivity) and microgliosis (tomato lectin or CD11b immunoreactivity) in the hippocampus at $24 \mathrm{~h}$ or 7 days after KA injection. SCH58261 $(0.05 \mathrm{mg} / \mathrm{kg}$, ip, $30 \mathrm{~min}$ before KA) caused an inconsistent effect on convulsions (prevention, attenuation and no effect, 3 rats each) but abolished neuronal damage, astrogliosis and microgliosis in all 9 rats.

To confirm the role of $\mathrm{A}_{2 \mathrm{~A}} \mathrm{Rs}$ in the development of hippocampal damage resulting from behavioural seizures, we compared the effect of KA in wild type and $\mathrm{A}_{2 \mathrm{~A}} \mathrm{R}$ knockout male C57B16 mice. As occurred in rats, KA (35 mg/ $\mathrm{kg}, \mathrm{sc}$ ) injection in wild type mice triggered convulsions (stage 5-6 of modified Racine's scale) as well as hippo- 
campal damage typified by neuronal damage, astrogliosis and microgliosis observed $24 \mathrm{~h}$ after KA injection. However, in $\mathrm{A}_{2 \mathrm{~A}} \mathrm{R}$ knockout mice, $\mathrm{KA}$ injection caused a similar severity of convulsions but did not produce any hippocampal damage (no FluoroJade staining and no modification of GFAP- or CD11b-immunoreactivities compared to saline controls).

Finally, we investigated if $\mathrm{A}_{2 \mathrm{~A}} \mathrm{R}$ blockade would interfere with epileptogenesis using a model of epilepsy (amygdala kinding) that causes an onset of seizure activity slower (in the range of days) compared to kainate injection (within $30 \mathrm{~min}$ ). In vehicle injected control rats, tonic/clonic seizures were observed 8-10 days after starting the kindling protocol (sub-maximal stimulation $500 \mu \mathrm{A}, 1 \mathrm{msec}$ pulses at $50 \mathrm{~Hz}$ for $1 \mathrm{sec}$, twice a day, $10 \mathrm{AM}$ and 4 PM). In contrast, in $90 \%$ of kindled rats, the administration of SCH58261 $(0.05 \mathrm{mg} / \mathrm{kg}$, ip, $30 \mathrm{~min}$ before each stimulation period) delayed and attenuated the severity of convulsions. Likewise, astrogliosis and microgliosis observed after 15 days were prevented by SCH58261.

These results provide the first evidence that endogenous adenosine participates in the development of epilepticinduced damage through $A_{2 A} R$ activation. These $A_{2 A} R s$ might also aggravate epileptogenesis but this only evident in a chronic (kindling) rather than acute (KA) model of epilepsy. This questions the role of adenosine as an anti-epileptic agent and suggests that $\mathrm{A}_{2 \mathrm{~A}} \mathrm{R}$ antagonists may provide a novel strategy to control neuronal damage occurring in temporal lobe epileptic conditions. (Supported by FCT)

Cunha, R.A. Purinergic Signal. 2005 1, 111-134.

Fredholm B.B. et al. Int. Rev. Neurobiol. 2005 63, 191-270.

\title{
Blockade of adenosine $A_{2 A}$ receptors prevents $\beta$-amyloid $\left(A \beta_{1-42}\right)$-induced synaptotoxicity and memory impairment in rodents
}

\author{
Geanne M.A.Cunha ${ }^{1,2}$, Paula M.Canas ${ }^{1}$, Jiang-Fan Chen $^{3}$, Catarina R. Oliveira ${ }^{1}$, R.A. Cunha ${ }^{1}$ \\ ${ }^{1}$ Ctr. Neuroscience Coimbra, Fac. Medicine, Univ. Coimbra, Portugal, ${ }^{2}$ Dept. Physiol. Pharmacol., Federal Univ. \\ Ceara, Fortaleza, Brazil, ${ }^{3}$ Dept. Neurology, Boston Univ. School Medicine, MA, USA \\ (canas.paula@gmail.com)
}

Alzheimer's disease (AD) is the most common chronic neurodegenerative disease characterized clinically by an atrophy of hippocampal regions and a progressive cognitive impairment (Hardy and Selkoe, 2002). The parameters that correlate better with memory dysfunction in $\mathrm{AD}$ are the levels of soluble $\mathrm{A} \beta$, mainly $\mathrm{A} \beta_{1-42}$, and a decreased density of nerve terminals in cortical areas (Hardy and Selkoe, 2002). Thus, a major lead for the development of novel therapeutic strategies for $\mathrm{AD}$ might be to explore mechanisms able to prevent this early synaptotoxicity caused by $A \beta_{1-42}$.

Adenosine plays a prominent role in controlling brain neurodegeneration. In particular adenosine $\mathrm{A}_{2 \mathrm{~A}}$ receptor $\left(A_{2 A} R\right)$ blockade confers a robust neuroprotection in chronic noxious brain conditions (Cunha, 2005). Accordingly, we showed that caffeine and selective $A_{2 A} R$ antagonists prevent $A \beta$-induced toxicity in cultured neurons (Dall'Igna et al., 2003). Thus, we tested if the $A_{2 A} R$ antagonist SCH58261 (0.05 mg/kg, ip daily for 15 days) prevented the synaptotoxicity and memory impairment observed in rats two weeks after administration of $A \beta_{1-42}$ ( $2 \mathrm{nmol}, \mathrm{icv}$ ). The rats displayed a mnemonic deficit (Y maze) and a decrease of synaptophysin immunoreactivity (evaluated by immunohistochemistry and Western blot analysis), but neither cell death (evaluated by cresyl violet staining), nor microglia recruitment (evaluated by CD11b immunohistochemistry) was observed. In contrast, in SCH58261-treated rats, $A \beta_{1-42}$ failed to modify cognitive performance and there was no synaptotoxicity, i.e. reduction of synaptophysin immunoreactivity compared to control.

We attempted to confirm this key role of $A_{2 A}$ Rs in the development of the $A \beta_{1-42}$-induced synaptotoxicity and memory impairment using a genetic deletion rather than a pharmacological blockade of $\mathrm{A}_{2 \mathrm{~A}} \mathrm{Rs}$, i.e. using $\mathrm{A}_{2 \mathrm{~A}} \mathrm{R}$ knockout mice. As occurred in rats, there was a mnemonic deficit and a decrease of synaptophysin immunoreactivity, but neither cell death nor microglia recruitment in wild type C57B16 mice two weeks after administration of $A \beta_{1-42}(2 \mathrm{nmol}$, icv). In contrast, in preliminary experiments, none of these modifications were found two weeks after administration of $A \beta_{1-42}\left(2 \mathrm{nmol}\right.$, icv) in $\mathrm{A}_{2 \mathrm{~A}} \mathrm{R}$ knockout mice.

These results show that the blockade of $\mathrm{A}_{2 \mathrm{~A}} \mathrm{Rs}$, which are enriched in hippocampal synapses (Rebola et al., 2005), prevents $A \beta$-induced synaptotoxicity and consequent amnesia in rodents. This key role of $\mathrm{A}_{2 \mathrm{~A}} \mathrm{Rs}$ in the develo- 
pment of synaptic loss and memory impairment in an animal model of AD provides a rationale for a previous suggestion that caffeine consumption (an adenosine receptor antagonist) might attenuate the incidence of AD (Maia and de Mendonça, 2002). (Supported by CNPq Brazil, FCT, Pfizer award from SPN)

Dall'Igna, O.P. et al. Br. J. Pharmacol. 2003 138, 1207-1209.

Cunha, R.A. Purinergic Signal. 2005 1, 111-134.

Hardy, J.; Selkoe, D.J. (2002) Science 2002 297, 353-356.

Maia, L.; de Mendonça, A. Eur. J. Neurol. 2002 9: 377-382.

Rebola, N.; Canas, P.M.; Oliveira, C.R.; Cunha, R.A. Neuroscience 2005 132, 893-903.

\section{Blockade of $\mathrm{P}_{2} \mathrm{Y}_{1}$ receptors prevents the synaptotoxicity and memory impairment caused by $\mathbf{A} \beta_{1-42}$ administration.}

$\underline{\text { Ricardo J. Rodrigues }}^{1}$, Lisiane O. Porciúncula ${ }^{1}$, Paula M. Canas ${ }^{1}$, Christian Gachet ${ }^{2}$, Catarina R. Oliveira $^{1}$, Rodrigo A. Cunha.

${ }^{1}$ Center for Neuroscience and Cell Biology of Coimbra, Inst. Biochemistry, Fac. Medicine, Univ. of Coimbra, Coimbra, Portugal.

${ }^{2}$ INSERM U.311, Etablissement Français du Sang-Alsace, 10 rue Spielmann, B.P.N³6, 67065, Strasbourg Cedex

France

rodriguesrj@clix.pt

Amyloid beta-peptides $(\mathrm{A} \beta)$ are accepted to be a major cause of neuronal death in Alzheimer's disease (AD) (Hardy and Selkoe, 2002) which is predated by a synaptic loss (Selkoe, 2002). Thus, a major lead for the development of novel therapeutic strategies for AD might be to explore mechanisms able to prevent this early synaptotoxicity caused by $\mathrm{A} \beta_{1-42}$. Upon cell damage there is an increase of the extracellular levels of ATP, which can activate $\mathrm{P} 2$ receptors (P2Rs). Brain possesses both ionotropic P2XR and metabotropic P2YR which can control neuronal damage (Volonte et al., 2003). Since we showed that $\mathrm{P} 2 \mathrm{X}_{1-3} \mathrm{Rs}$ and $\mathrm{P} 2 \mathrm{Y}_{1,2,4} \mathrm{Rs}$ are located in nerve terminals (Rodrigues et al., 2005), we now investigated if $\mathrm{P} 2$ receptors could control damage in cultured hippocampal neurons and, in particular, the synaptotoxicity induced by the exposure to $A \beta_{1-42}$.

$\mathrm{A} \beta_{1-42}(500 \mathrm{nM}, 48 \mathrm{~h})$ led to the death of $22 \pm 3 \%$ of neurons, which displayed apoptotic features (nuclear condensation, cytochrome $\mathrm{c}$ release, caspase 3 activation). Blockade of P2Rs with $10 \mu \mathrm{M}$ PPADS prevented A $\beta_{1-}$ 42-induced neurotoxicity, which was mimicked by the blockade of P2YRs with $10 \mu \mathrm{M}$ reactive blue 2, but not P2X $\mathrm{X}_{1-3}$ Rs using $10 \mu \mathrm{M}$ NF023. The P2 $\mathrm{Y}_{1} \mathrm{R}$ antagonist MRS2179 $(10 \mu \mathrm{M})$ was also neuroprotective. This neurotoxicity was preceded (at $12 \mathrm{~h}$ ) by a synaptotoxicity and dendritic atrophy (with no neuronal death yet), which was abrogated upon blockade of P2Y1Rs in accordance with the synaptic localization of P2Y ${ }_{1}$ Rs. Interestingly we found an increased density of $\mathrm{P}_{2} \mathrm{Y}_{1} \mathrm{Rs}$ in hippocampal terminals of both rats (Wistar) and mice (C57-BL/6) two weeks after administration of $\mathrm{A} \beta_{1-42}(2 \mathrm{nmol}, \mathrm{icv})$ at a time where they displayed a mnemonic deficit (Y maze) and synaptotoxicity (reduced levels of immunoreactivity for synaptic markers such as SNAP-25 and synaptophysin) but no neuronal death (Fluoro-Jade C staining). Finally, KO P2 ${ }_{1} \mathrm{R}$ mice (C57-BL/6) did not display mnemonic deficit or synaptotoxicity two weeks after administration of $\mathrm{A} \beta_{1-42}$.

Altogether, these results show that pharmacological blockade or genetic inactivation of $\mathrm{P}_{2} \mathrm{Y}_{1}$ receptors is neuroprotective against synaptotoxicity/neurotoxicity and mnemonic impairment caused by $A \beta_{1-42}$. This indicates that extracelular ATP may be involved in the development of neurotoxicity caused by $\mathrm{A} \beta_{1-42}$, and prompts considering $\mathrm{P}_{2} \mathrm{Y}_{1} \mathrm{R}$ antagonists as potential candidates to interfere with the early events in AD. (Supported by FCT)

- Hardy J.; Selkoe D. J. Science 2002 297, 353-356.

- Rodrigues, R. J.; Almeida, T.; Richardson, P. J.; Oliveira, C. R.; Cunha, R. A. J. Neurosci. 2005 25, $6286-6295$.

- Selkoe, J. Science 2002 298, 789-791.

- Volonte, C.; Amadio, S.; Cavaliere, F.; D'Ambrosi, N.; Vacca, F.; Bernardi, G. Curr. Drug Targets CNS Neurol. Disord. 2003 2, 403-412. 


\title{
Blood leucocytes are mobile modulators of adenine nucleotide metabolism and play an important role in determining the effects of adenine nucleotides on platelet function.
}

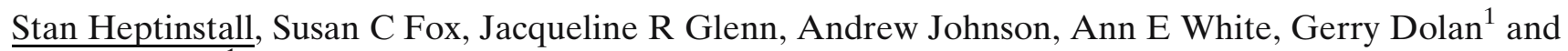 \\ Bethan Myers ${ }^{1}$. \\ Centre for Integrated Systems Biology and Medicine, University of Nottingham, and ${ }^{1}$ Department of Haematology, \\ Queen's Medical Centre, Nottingham, UK. \\ s.heptinstall@nottingham.ac.uk
}

Endothelial cells display the ecto-enzyme NTPDase (CD39) which converts ATP into ADP and ADP into AMP and it is believed that that these vascular cells play an important role in removing adenine nucleotides from blood and preventing ADP causing widespread platelet activation with potential pro-thrombotic consequences. Recently we have made several observations on the importance of leucocytes in adenine nucleotide metabolism and their effects on platelet function. First we found that ATP added to blood induces platelet aggregation although no such aggregation occurs in platelet-rich plasma (PRP). By adding autologous leucocytes back to the PRP we showed that leucocytes are involved in the aggregation response and through the use of selective antagonists showed that ADP is also involved (1). We then found that leucocytes (all neutrophils, all monocytes and a subset of lymphocytes) test positive for CD39. In addition we found modified platelet aggregation responses to both ATP and ADP in association with high leucocyte counts. Aggregation induced by ATP was more rapid while aggregation induced by ADP was followed by rapid disaggregation. This was the case in blood from patients with leucocytosis and also when leucocytosis was created experimentally in normal blood by adding autologous leucocytes (2). A systematic analysis of the role of blood cells and plasma enzymes in adenine nucleotide metabolism by HPLC was then performed. Studies in normal blood confirmed that leucocytes are the principle means through which ATP and ADP added to blood are broken down to ADP and AMP respectively, and that platelets and erythrocytes play virtually no part (3). Studies in leucocytosis demonstrated enhanced rates of conversion of ATP to ADP and of ADP to AMP and explained the effects of leucocytosis on platelet aggregation to ATP and ADP that we had demonstrated earlier (4). Recently we looked further at the role of CD39 on leucocytes in these processes. Blood was obtained from patients with leucocytosis and normal controls, and analyses were performed of platelet aggregation (measured in blood and PRP) and adenine nucleotide metabolism (measured by HPLC). Levels of CD39 on leucocytes were determined by flow cytometry. In all cases abnormalities in platelet aggregation in association with leucocytosis could be explained by enhanced adenine nucleotide metabolism brought about by increased amounts of CD39 on either myeloid or lymphoid cells. In one case in which leucocyte count returned to normal following successful chemotherapy, adenine nucleotide metabolism and CD39 also returned to normal. In summary, leucocytes provide a means of metabolising adenine nucleotides that is additional to that provided by vascular endothelial cells. Their mobility may enable modulation of platelet function throughout the entire vasculature as well as at its periphery.

1. Stafford NP et al. Arterioscler Thromb Vasc Biol. 2003;23:1928-33. 2. Glenn JR et al. Platelets 2005;16:159-70. 3. Heptinstall S et al. J Thromb Haemost. 2005;3:2331-9. 4 Heptinstall S et al. Arterioscler Thromb Vasc Biol. in press.

\section{Boranophosphate Isosteres of Dinucleoside Polyphosphates are Highly Potent and Selective P2Y -receptor Agonists $^{1}$}

\author{
$\underline{\text { Bilha Fischer }}^{\mathrm{a}}$, Victoria Nahum ${ }^{a}$, Mohan Tulapurkar ${ }^{b}$, Georg Reiser $^{b}$, Sébastien A. Lévesque ${ }^{c}$, and \\ Jean Sévigny $^{c}$ \\ bfischer@mail.biu.ac.il \\ a. Department of Chemistry, Bar-Ilan University, Ramat-Gan 52900, Israel \\ b. Institute for Neurobiochemistry, Faculty of Medicine, Otto von Guericke University, Leipziger Str. 44 D-39120 \\ Magdeburg, Germany c. Centre de recherche en Rhumatologie et Immunologie, Université Laval, Sainte-Foy, \\ Québec, Canada.
}


Dinucleoside polyphosphates, $\mathrm{Np}_{\mathrm{n}} \mathrm{N}^{\prime}$, exerting their physiological effects via P2-receptors, are attractive drug targets as they offer better stability and specificity compared to nucleotides. To further improve the properties of $\mathrm{Np}_{\mathrm{n}} \mathrm{N}^{\prime}$, which are still pharmacologically unsatisfactory, we developed novel boranophosphate isosters of dinucleoside polyphosphates, denoted as $\mathrm{Np}_{\mathrm{n}}(\mathrm{B}) \mathrm{N}$. These analogues were obtained in a facile and efficient synthesis as the exclusive products in a concerted reaction of two nucleoside phosphorimidazolides and inorganic boranophosphate. This unusual reaction is due to the pre-organization of three reactant molecules by the $\mathrm{Mg}^{2+}$ ion. We found that $\mathrm{Ap}_{3 / 5}(\beta \gamma-\mathrm{B}) \mathrm{A}$ analogues were potent $\mathrm{P} 2 \mathrm{Y}_{1}-\mathrm{R}$ agonists. $\mathrm{Ap}_{5}(\gamma-\mathrm{B}) \mathrm{A}$, was equipotent to 2-MeS-ADP $\left(\mathrm{EC}_{50} 6.3 \times 10^{-8} \mathrm{M}\right)$, thus making it one of the most potent $\mathrm{P} 2 \mathrm{Y}_{1}-\mathrm{R}$ agonists currently known. Moreover, $\mathrm{Ap}_{5}(\gamma-$ B)A did not activate $\mathrm{P} 2 \mathrm{Y}_{2}-\mathrm{R}$. In contrast, $\mathrm{Up}_{3 / 5}(\beta \gamma-\mathrm{B}) \mathrm{U}$ analogues were extremely poor agonists of both $\mathrm{P} 2 \mathrm{Y}_{1}-\mathrm{R}$ and $\mathrm{P}_{2} \mathrm{Y}_{2}-\mathrm{R} . \mathrm{Np}_{\mathrm{n}}(\mathrm{B}) \mathrm{N}$ analogues exhibited remarkable chemical stability under physiological conditions. Under conditions mimicking gastric juice, $\mathrm{Np}_{3}(\beta-\mathrm{B}) \mathrm{N}$ analogues exhibited half-life $\left(\mathrm{t}_{1 / 2}\right)$ of $1.3 \mathrm{~h}$, whereas $\mathrm{Np}_{5}(\gamma-\mathrm{B}) \mathrm{N}$ degraded at a much faster rate $\left(t_{1 / 2} 18 \mathrm{~min}\right)$. The hydrolysis of $A p_{3}(\beta-B) A$ by human nucleotide pyrophosphatase phosphodiesterases (NPP1 and NPP3) was slowed by $40 \%$ and $59 \%$, respectively, as compared to Ap ${ }_{3} \mathrm{~A}$. However, this effect of the boranophosphate was position-dependent, as $\mathrm{Np}_{5}(\gamma-\mathrm{B}) \mathrm{N}$ was degraded at a comparable rate to that of $\mathrm{Np}_{5} \mathrm{~N}$. In summary, $\mathrm{Ap}_{5}(\gamma-\mathrm{B}) \mathrm{A}$ appears to be a highly potent and selective $\mathrm{P} 2 \mathrm{Y}_{1}-\mathrm{R}$ agonist, as compared to the parent compound.

${ }^{1}$ A US patent application field on June 15, 2005.

\title{
C34T polymorphism in AMPD1 gene potentiates forearm reactive hyperemia in young healthy subjects.
}

\author{
NP Riksen ${ }^{1}$, Barbara Franke ${ }^{2}$, P Smits ${ }^{1}$, GA Rongen ${ }^{1}$ \\ ${ }^{1}$ Dept of Pharmacology-Toxicology, and ${ }^{2}$ Human Genetics, Radboud University Nijmegen Medical Center, the \\ Netherlands. \\ N.Riksen@aig.umcn.nl
}

Background The adenosine mono-phosphate deaminase (AMPD) enzyme converts AMP into IMP in muscle cells. The common variant C34T encodes a severely truncated protein and is associated with improved cardiovascular outcome in patients with coronary artery disease and heart failure, possibly due to increased levels of interstitial adenosine during ischemia. We hypothesize that in subjects with this polymorphism increased adenosine receptor stimulation during ischemia and reperfusion potentiates reactive hyperemia.

Methods In 100 healthy subjects the AMPD1 genotype was determined, as well as nucleoside uptake and adenosine kinase activity in erythrocytes. In 10 individuals heterozygous for the variant allele $(\mathrm{C} / \mathrm{T})$ and 10 matched controls $(\mathrm{C} / \mathrm{C})$ forearm blood flow $(\mathrm{FBF})$ was assessed with venous occlusion plethysmography during 3,5 and 5 minutes after 2, 5 and 13 minutes of forearm ischemia, with and without concomitant infusion of the nucleoside uptake inhibitor dipyridamole into the brachial artery $(7.4 \mathrm{nmol} / \mathrm{min}$ per $\mathrm{dl}$ of forearm tissue). Maximum FBF and FBF averaged per consecutive minute were analysed. After washout, vasodilation induced by intrabrachial infusion of sodium nitroprusside (SNP) and acetylcholine (ACh) was determined.

Results Both groups were similar in age, sex, weight, blood pressure, heart rate, baseline caffeine concentration, and $\mathrm{V}_{\max }$ and $\mathrm{K}_{\mathrm{m}}$ values for the nucleoside transporter and adenosine kinase. Maximal FBF after 2, 5 and 13 minutes of ischemia was $25.4 \pm 2.5,32.7 \pm 2.2$, and $38.6 \pm 2.6 \mathrm{ml} / \mathrm{min}$ per $\mathrm{dl}$ of forearm tissue in the $\mathrm{C} / \mathrm{T}$ group and $21.9 \pm 2.2,28.5 \pm 2.4$, and $41.0 \pm 3.3 \mathrm{ml} / \mathrm{min} / \mathrm{dl}$ in the $\mathrm{C} / \mathrm{C}$ group (mean $\pm \mathrm{SE}, P<0.05$, repeated measures ANOVA). One-minute averaged FBF was also higher in the $\mathrm{C} / \mathrm{T}$ group after 2 minutes of ischemia $(\mathrm{P}<0.05)$, but not after 5 minutes $(P=0.09)$ and 13 minutes $(P=0.6)$. One-minute averaged FBF was potentiated by dipyridamole in both groups for all periods of ischemia $(P<0.05)$. However, the effect of dipyridamole on maximum FBF after 2,5 and 13 minutes of ischemia was more pronounced in the $\mathrm{C} / \mathrm{C}$ group than in the $\mathrm{C} / \mathrm{T}$ group $(\mathrm{P}<0.05)$. Vasodilation induced by SNP and ACh did not differ between both groups $(P=0.9$ and $P=0.7$, respectively).

Conclusions Dipyridamole potentiates reactive hyperemia, suggesting predominant extracellular formation of adenosine during ischemia and early reperfusion. Heterozygous deficiency in AMPD1 augments reactive hyperemia but reduces the potentiating effect of dipyridamole during maximum vasodilation. These observations support the concept that the cellular uptake of adenosine is reduced in AMPD1 deficient subjects during ischemia and early reperfusion, resulting in increased adenosine receptor stimulation. This mechanism could well contri- 
bute to the observed improved cardiovascular outcome in patients with the C34T polymorphism of the AMPD1 gene.

\title{
$\mathrm{Ca}^{2+}$-dependent release of adenine and uridine nucleotides from A549 cells
}

\author{
Sabina Tatur ${ }^{1}$, Silvia Kreda ${ }^{2}$, Eduardo Lazarowski ${ }^{2}$ and Ryszard Grygorczyk ${ }^{1}$ \\ ${ }^{1}$ Research Centre, Centre hospitalier de l'Université de Montréal (CHUM) - Hôtel-Dieu, and Department of \\ Medicine, Université de Montréal, Montréal, Québec, Canada \\ ${ }^{2}$ Cystic Fibrosis Center, University of North Carolina School of Medicine, \\ Chapel Hill, NC 27599-7248, U.S.A. \\ ryszard.grygorczyk@umontreal.ca
}

Extracellular nucleotides play an important role in airway defense by controlling epithelial ion and fluid transport as well as ciliary beating. Nucleotide levels on airway surfaces, measured in vitro and ex vivo, show dynamic changes due to the combination of basal and stimulated release and their rapid metabolism. The mechanism of nucleotide release from epithelial cells is not well understood, and their hydrolysis at the airway surface makes it difficult to assess the magnitude and relative abundance of different nucleotide species released. In this study, to minimize cell surface hydrolysis, we used a low-volume $(300-\mu \mathrm{L})$ flow-through chamber $(1.3 \mathrm{ml} / \mathrm{min}$ perfusion rate), and examined adenine and uridine nucleotide content in perfusates of human A549 cells prior to and during $50 \%$ hypotonic shock. Aliquots of the perfusates were collected at 15- to 60-s intervals. ATP, ADP, AMP, and Ado were quantified by HPLC analysis of fluorescent etheno derivatives, and UTP and UDP were measured using HPLC-coupled radioenzymatic assays. After the onset of hypotonic shock, ATP, ADP, UTP, and UDP content in the perfusates increased markedly and peaked at approximately $2 \mathrm{~min}$, followed by a gradual decay during the next 15-20 min; changes in Ado and AMP at peak were relatively minor. The peak concentrations and fold increment (in brackets) were: $52 \mathrm{nM}$ ATP [5], $16 \mathrm{nM}$ ADP [5], $4 \mathrm{nM}$ AMP [2], $27 \mathrm{nM}$ Ado [1.3], $21 \mathrm{nM}$ UTP [>7], and $11 \mathrm{nM}$ UDP [12]. Nucleotide release was almost completely abolished from cells loaded with the calcium chelator BAPTA. Under isotonic conditions, elevation of intracellular calcium with the calcium ionophore ionomycin $(5 \mu \mathrm{M}, 3 \mathrm{~min})$ also released nucleotides with kinetics and relative abundance as above, although less robustly. ATP:ADP (3:1) and UTP:UDP (2:1) ratios in the perfusates from stimulated cells were markedly smaller than the cytosolic ratios of these species, suggesting that an NDP-rich compartment, e.g., the secretory pathway, contributed to nucleotide release. Laser confocal microscopy experiments illustrated increased uptake of FM1-43 into the plasma membrane upon hypotonic shock or ionomycin treatment, consistent with enhanced vesicle exocytosis under these conditions. In summary, our results strongly indicate that calcium-dependent exocytosis is responsible, at least in part, for adenine and uridine nucleotide release from A549 cells.

Supported by the Canadian Institutes of Health Research (to R.G.) and the National Institutes of Health USA (to E.L.).

\section{Caffeine intake induces an alteration of human neutrophil $A_{2 A}$ adenosine receptors}

Katia Varani ${ }^{1}$, Francesco Portaluppi ${ }^{1}$, Stefania Gessi $^{1}$, Stefania Merighi ${ }^{1}$, Fabrizio Vincenzi ${ }^{1}$, Annalisa Benini $^{1}$, Elena Cattabriga ${ }^{1}$, Alessandro Dalpiaz ${ }^{1}$, Fabrizio Bortolotti $^{1}$, Luiz Belardinelli ${ }^{2}$, Pier Andrea Borea $^{1}$

${ }^{1}$ Department of Clinical and Experimental Medicine and the Department of Pharmaceutical Sciences, University of Ferrara, Italy and the ${ }^{2}$ Department of Medicine, CV Therapeutics Inc, Palo Alto, CA.

Caffeine is the most widely used drug in the world and acts mainly through antagonism of the effects mediated by the adenosine receptor subtypes $\mathrm{A}_{1}, \mathrm{~A}_{2 \mathrm{~A}}, \mathrm{~A}_{2 \mathrm{~B}}$ and $\mathrm{A}_{3}$. Controversial results have been reported on the link between caffeine and inflammation. Recently, caffeine has been found to have anti-inflammatory activity in different human substrates. From this background the present study was designed to evaluate the effect of repeated caffeine administration at different doses and for different periods of time (400 or $600 \mathrm{mg} / \mathrm{d}$ for 1 week and 400 
$\mathrm{mg} / \mathrm{d}$ for 2 weeks) in human neutrophils. Blood and plasma levels of methylxanthines revealed no significant concentrations after caffeine abstinence. Neutrophils were obtained from peripheral venous blood of 33 healthy human volunteers at the end of 2 weeks of caffeine abstinence and at 1, 12, 24, 36, 48, 60 hours after the last dose of caffeine. Saturation binding assays showed an increase of affinity $\left(\mathrm{K}_{\mathrm{D}}\right)$ and density $(\mathrm{Bmax})$ of $\mathrm{A}_{2 \mathrm{~A}}$ adenosine receptors after caffeine intake at the doses of $400 \mathrm{mg} / \mathrm{d}$ for 14 days or $600 \mathrm{mg} / \mathrm{d}$ for 7 days. Interestingly, the increase in density of $\mathrm{A}_{2 \mathrm{~A}}$ receptors found at 1 hour after caffeine withdrawal was similar to that observed at 12 , 24 and 36 hour after the last dose of caffeine. On the other hand at 48 and 60 hour after the last dose of caffeine the binding parameters were not different from control condition or from the control group. The upregulation of $\mathrm{A}_{2 \mathrm{~A}}$ receptors does not appear to be ascribable to the synthesis of new receptors because were no changes in mRNA levels at different times after the last dose of caffeine. The $\mathrm{A}_{2 \mathrm{~A}}$ receptor alteration was accompanied by increases in cAMP accumulation and decreases in superoxide anion production after $\mathrm{A}_{2 \mathrm{~A}}$ stimulation by NECA. Binding and functional changes of $\mathrm{A}_{2 \mathrm{~A}}$ receptors gradually return to baseline after 48 hours of caffeine withdrawal. In summary, caffeine intake and subsequent withdrawal mediates a temporary upregulation of $\mathrm{A}_{2 \mathrm{~A}}$ receptors and as a consequence might represent the mechanism responsible for the observed anti-inflammatory effects.

\title{
Caffeine modulates P50 auditory sensory gating in healthy subjects.
}

$\underline{\text { Diogo R. Lara }}{ }^{1}$, Eduardo S. Ghisolfi, ${ }^{1}{ }^{2}$, Alice Schuch $^{2}$, Ivo M. Strimitzer Jr. ${ }^{2}$, Gustavo Luersen ${ }^{1}$, Fabíola F. Martins $^{1}$, Fernanda L. P. Ramos ${ }^{1}$, Jefferson Becker ${ }^{1}$

${ }^{1}$ Departamento de Ciências Fisiológicas, Faculdade de Biociências, PUCRS, Porto Alegre, Brazil; ${ }^{2}$ Departamento de Bioquímica, ICBS, UFRGS, Porto Alegre, Brazil.

drlara@pucrs.br

The P50 suppression paradigm is an index of sensory gating assumed to reflect an inhibitory process. Adenosine is a neuromodulator with mostly inhibitory activity that is released by physiological stimuli and can be blocked by non-selective adenosine receptor antagonists such as theophylline and caffeine. A previous study showed that a single dose of theophylline decreased P50 suppression in healthy volunteers. Here we investigated the effect of caffeine (0, 100, 200 and $400 \mathrm{mg}$ p.o.) on P50 sensory gating in 24 healthy volunteers (15 habitual caffeine highusers and 9 low-users). The $200 \mathrm{mg}$ and $400 \mathrm{mg}$ doses reduced P50 sensory gating (increase P50 ratio), whereas $100 \mathrm{mg}$ produced a non-significant effect. The effect of caffeine on P50 ration was independent of gender and habitual caffeine intake. High caffeine users also showed baseline differences, with lower $\mathrm{S}_{2}$ amplitudes compared to low-users. These results reinforce the participation of adenosine in the modulation of P50 sensory gating and suggest that both chronic and acute caffeine ingestion should be controlled for in studies using the P50 sensory gating paradigm.

\section{Caffeine recognizes differently adenosine $\mathbf{A}_{2 \mathrm{~A}}$ receptors in homo or heterodimers: A mechanism for caffeine tolerance.}

\author{
Gemma Navarro, Francisco Ciruela, Sergi Ferré, Carmen Lluis, Rafael Franco Vicent Casadó. \\ Molecular Neurobiology Unit. Institut d'Investigacions Biomèdiques August Pi i Sunyer (IDIBAPS). University of \\ Barcelona. Diagonal 645. 08028 Barcelona. \\ rfranco@ub.edu
}

Caffeine is the most world-wide consumed psychoactive drug and its only known molecular targets at non-pathological doses are adenosine $A_{1}$ and $A_{2 A}$ receptors. Of the four known adenosine receptors $\left(A_{1}, A_{2 A}, A_{2 B}\right.$ and $\left.A_{3}\right)$, adenosine $A_{1}\left(A_{1} R s\right)$ and $A_{2 A}\left(A_{2 A} R s\right)$ receptors are primarily responsible for the central effects of adenosine. The molecular mechanisms underlying the effects of caffeine are not fully elucidated. As indicated in another session of this Conference (Franco R) and in Ciruela et al., (2006), $\mathrm{A}_{1}$ adenosine receptors form heteromers with adenosine $A_{2 A}$ receptors. Functional studies showed that $A_{1} R-A_{2 A} R$ heteromers are responsible for a strong $\mathrm{A}_{1} \mathrm{R}-\mathrm{A}_{2 \mathrm{~A}} \mathrm{R}$ antagonistic cross-talk. The strength of this cross-talk would depend on the concentration of extracellular adenosine. To illustrate this, it can be expected that at adenosine concentrations high enough to 
activate $A_{2 A} R$, the signalling via $A_{1} R$ is severely impaired. In experiments of displacement of radioligand binding in transfected cells or in samples from striatum, it was found that $A_{1} R$ showed the same $K_{D}$ for caffeine in $A_{1} R$ $\mathrm{A}_{2 \mathrm{~A}} \mathrm{R}$ co-transfected than in $\mathrm{A}_{1} \mathrm{R}$-transfected cells. Also, as previously shown using other transfected cell lines, $A_{2 A}$ Rs displayed higher affinity (lower $K_{D}$ value) for caffeine than $A_{1} R s$ in single-transfected HEK cells. In contrast, the affinity of caffeine for $A_{2 A} R s$ in $A_{1} R-A_{2 A} R$ co-transfected cells was 12-times higher $(p<0.001)$ than in $\mathrm{A}_{2 \mathrm{~A}} \mathrm{R}$-transfected cells. The affinity of the $\mathrm{A}_{2 \mathrm{~A}} \mathrm{R}$ for caffeine was not altered when co-transfected with the dopamine $D_{2}$ receptor $\left(D_{2} R\right)$, even though they form $A_{2 A} R-D_{2} R$ heterodimers. These results demonstrate the existence of a selective reduction in the affinity of $A_{2 A} R$ for caffeine in the $A_{1} R-A_{2 A} R$ heteromer, i.e. the binding of caffeine to $A_{2 A} R s$ in the $A_{1} R-A_{2 A} R$ heteromer is qualitatively different from the binding to the non-heteromeric $A_{2 A} R$ or other heteromeric $A_{2 A}$ Rs.

An unresolved issue about caffeine is the strong tolerance for many of its behavioural and biochemical effects that develops after chronic treatment. It is generally assumed that tolerance to the behavioral effects of caffeine is mostly related to pharmacodynamic factors involving adenosine receptors, but there is no consensus about which are the significant changes in either $\mathrm{A}_{1} \mathrm{R}$ or $\mathrm{A}_{2 \mathrm{~A}} \mathrm{R}$ function after chronic caffeine treatment. The most commonly reported effect is the up-regulation of $\mathrm{A}_{1}$ Rs. Nevertheless, there are reports indicating a lack of significance in these changes in receptor density and there are even studies showing down-regulation of either $A_{1} R$ or $A_{2 A} R$ function. To test the role of $\mathrm{A}_{1} \mathrm{R}-\mathrm{A}_{2 \mathrm{~A}} \mathrm{R}$ heteromers in this phenomenon, competitive-inhibition experiments of $\left[{ }^{3} \mathrm{H}\right] \mathrm{R}$-PIA binding using CGS21680 were performed in striatal membrane preparations of caffeine-treated rats. The displacement curve of $\left[{ }^{3} \mathrm{H}\right] \mathrm{R}$-PIA binding by CGS21680 in striatal membranes from caffeine-treated rats was significantly $(\mathrm{p}<0.001)$ better fitted by a two-site model than by a single-site model. The $\mathrm{IC}_{50}$ value corresponding to the binding of CGS21680 to $\mathrm{A}_{1} \mathrm{R}(1.7 \pm 0.5 \mu \mathrm{M})$ was similar to that obtained in naïve rats $(1.8 \pm 0.5 \mu \mathrm{M})$. However, the inhibition of the $\left[{ }^{3} \mathrm{H}\right] \mathrm{R}$-PIA binding to $\mathrm{A}_{1} \mathrm{R}$ when CGS21680 binds to the $\mathrm{A}_{2 \mathrm{~A}} \mathrm{R}$ displays an EC50 value $(8 \pm 3 \mathrm{nM})$ about three fold lower than the value obtained in naïve rats $(22 \pm 6 \mathrm{nM})$. Interestingly, these results indicate that caffeine pre-treatment alters the function of the $A_{1} R-A_{2 A} R$ heteromers, increasing significantly $(p<0.02)$ the sensitivity of $A_{2 A} R$ to modulate (negatively) $A_{1} R s$.

Apart from the affinity of the $A_{1} R, A_{2 A} R$ and $A_{1} R-A_{2 A} R$ for adenosine and caffeine and from the strength of the $\mathrm{A}_{1} \mathrm{R}-\mathrm{A} 2 \mathrm{AR}$ intramembrane interaction, one more variable plays a substantial role when analyzing the effects taking place after chronic caffeine administration. In fact chronic treatment with the methylxanthine leads to a significant increase in the plasma and extracellular levels of adenosine. Since at relatively low adenosine levels there is little occupancy of $\mathrm{A}_{2 \mathrm{~A}}$ Rs most of the behavioral and biochemical effects following an acute administration of caffeine are due to $\mathrm{A}_{1} \mathrm{R}$ blockade. Under chronic caffeine treatment (or other conditions leading to increased adenosine levels), adenosine also binds and activate $\mathrm{A}_{2 \mathrm{~A}} \mathrm{R}$, which, in addition, has a reduced affinity for caffeine. This likely scenario would lead to a situation where the increased levels of adenosine acting on $\mathrm{A}_{2 \mathrm{~A}} \mathrm{Rs}$ to inhibit $A_{1} R$ function by means of the potentiated $A_{1} R-A_{2 A} R$ intramembrane interaction. Under these conditions, caffeine would have little effect on $A_{1} R s$, which would be already inhibited as a consequence of the $A_{1} R-A_{2 A} R$ intra-membrane inter-molecular interaction.

Ciruela et al (2006) J Neuroscience In the Press

\title{
Can Acadesine increase adenosine level in rat cardiomyocytes?
}

\author{
Jerzy Barankiewicz and Piotr Chomczynski \\ Molecular Research Center, Inc, Cincinnati, OH 45212 \\ jbarankiewcz@mrcgene.com
}

Acadesine (AICA-riboside) is thought to have a protective effect during cardiac ischemia via two mechanisms. Acadesine can protect energy metabolism by elevating adenine nucleotides concentration, or it can elevates adenosine (Ado) concentration, which acts via Ado receptors. In this study, we evaluated both of these mechanisms in isolated, spontaneously beating rat cardiomyocytes and neonatal rat heart slices under normoxic and various stress conditions.

In isolated rat cardiomyocytes and heart slices under normoxic conditions, $500 \mu \mathrm{M}$ radiolabeled acadesine was efficiently metabolized to the purine nucleotides ATP and GTP, although Hyp was the predominant product. However, as acadesine rose to surpass $1 \mathrm{mM}$ concnetration, radiolabeled ZMP accumulation increased and exceeded the ATP concentration. 
After 2 hrs incubation under anaerobic conditions in an argon atmosphere, ATP levels decreased three-fold in isolated rat cardiomyocytes. In the same cells transferred to aerobic conditions in the presence of $500 \mu \mathrm{M}$ acadesine, the ATP level appropriate for normoxic conditions was reconstituted after 2-3 hours incubation. Further incubation in this condition caused a continuing increase in ATP levels.

While no Ado accumulation was observed under the conditions described above, we also evaluated Ado accumulation in the presence of acadesine while chemically accelerating the cascade of ATP degradation. When antimycin-A or iodoacetate chemically stressed cardiomyocytes or heart slices, Hyp and inosine increased severalfold but not Ado accumulation was observed. However in the presence of 2'-deoxycoformycin - inhibitor of ADA activity, Ado accumulated but acadesine only slightly increased Ado concentration.

As opposed to Ado release and accumulation, we examined the effect of acadesine on Ado uptake in rat cardiomyocytes. Acadesine inhibited Ado uptake in these cells in a dose-dependent fashion under both normoxic and anaerobic conditions. This effect required however a relatively high concentration of acadesine.

In conclusion, these data indicate that acadesine treatment effectively supports reconstitution of ATP levels ischemic stress but it has no effect on Ado release and accumulation in rat cardiomyocytes. However, acadesine may affect Ado uptake in the same cells.

\title{
Cardiac expression of NTPDase1 and caveolins are altered in human disease
}

\author{
András Bodnár ${ }^{1}$, Péter Pócza ${ }^{1}$, Ida Matkó ${ }^{1}$, Beáta Sperlágh $^{2}$, Anna L. Kiss $^{1}$, Ágnes Kittel ${ }^{2}$ \\ ${ }^{1}$ Medical School, Semmelweis University, Budapest, Hungary, ${ }^{2}$ Institute of Experimental Medicine, Hungarian \\ Academy of Sciences, Budapest, Hungary \\ kittel@koki.hu
}

Pathological circumstances like inflammation or ischemic insult facilitate the release of adenine nucleotides among ATP, from several types of cells. Concentration of extracellular ATP is regulated by ectonucleotidases. Endothelial and smooth muscle cells possess high ecto-ATPase activity and it has been demonstrated that this enzyme activity is altered after LPS stimulus, ischemia, inflammation, viral infection and in multidrug resistance. Upregulation of increased ecto-ATPase activity is associated with the appearance of higher number of caveolae, specialized membrane invaginations, not only in endothelial and smooth muscle cells but, also in pericytes, astrocytes and multidrug resistant cancer cells. Several molecules involved in signalling (e.g. eNOS, G-proteins, receptors) are targeted to these membrane compartments; thus caveolae appear to integrate cellular activation events. The ecto-ATPase enzyme within these signal-transducing microdomains has been identified as NTPDase1/ CD391. NTPDase1/CD39 can co-associate with scaffolding domain of caveolin1 or 3, or of RanBPM, and other structural proteins of caveolae. The question has been arisen whether there is a link between higher NTPDase expression and expression of caveolins during disease. We demonstrated the ecto-ATPase activity of healthy and diseased human cardiac tissue by enzyme histochemistry, measured the concentration of the extracellular ATP and metabolites by HPLC and identified NTPDase1/CD39 by immunohistochemistry and Western blotting2. The presence of all the three types of caveolins were demonstrated in parallel ultracryo sections. We demonstrated the presence of caveolin1 in human cardiac muscle cells. Double immunostaining confirmed the co-localization of NTPDase1/CD39 and caveolin1 in endothelial cells, whereas the CD39 signal was weak in the cell membrane of cardiac muscle cells. Pathological samples from patients with ischemic heart disease exhibited significant increases in ecto-ATPase activity, as measured by HPLC and RT-PCR. Western blotting confirmed higher CD39 expression in diseased as compared with control tissues. This higher activity and expression level of NTPDase1 underline a putative protective roles in the cardiovascular system and also support the administration of soluble NTPDase1/CD39 in the treatment of ischemic complications. Caveolin3 expression was altered in pathological samples. The lower expression level of caveolin3 may indicate lability in contrast to caveolin1.The high level presence of caveolin1 in diseased cardiac muscle raises the possibility, that this caveolin isoform, at least in part, may substitute for caveolin3.

Research was supported by Hungarian grants ETT 480/2003, OTKA M036314.

\section{References}

(1) Koziak, K.; Kaczmarek, E.; Kittel, A.; Sevigny, J.; Blusztajn, J. K; Schulte Am Esch, J. 2nd; Imai, M.; Guckelberger, O.; Goepfert, C.; Qawi, I.; Robson, S. C. Palmitoylation targets CD39/endothelial ATP diphosphohydrolase to caveolae. 2000, J Biol Chem 275, 2057-62. 
(2) Kittel, A., Kiss, A.L., Mullner, N., Matko, I. \& Sperlagh, B. Expression of NTPDase1 and caveolins in human cardiovascular disease. Histochem Cell Biol 2005,124, 51-9.

\title{
CD73-deficient mice show increased lymphocyte migration through high endothelial venules (HEV) in draining lymph nodes during inflammation
}

\author{
Masahide Takedachi ${ }^{1}$, Yukihiko Ebisuno ${ }^{2}$, Stephanie McGee ${ }^{1}$ and Linda F. Thompson ${ }^{1}$ \\ ${ }^{T}$ Immunobiology and Cancer Program, ${ }^{2}$ Cardiovascular Biology Program, Oklahoma Medical Research \\ Foundation, Oklahoma City, Oklahoma, USA. \\ Masahide-Takedachi@omrf.ouhsc.edu
}

CD73 is a GPI-anchored cell surface protein with ecto-5'-nucleotidase enzyme activity which catalyzes the dephosphorylation of $5^{\prime}$-adenosine monophosphate to adenosine. As this molecule catalyzes the last step in the generation of adenosine from extracellular adenine nucleotides, it has the capacity to regulate the activation of adenosine receptors. For example, adenosine generated by CD73 can decrease endothelial cell permeability in vitro ${ }^{1)}$. Moreover, Thompson et al. ${ }^{2)}$ demonstrated that $c d 73$-deficient mice have defects in endothelial barrier function leading to neutrophil accumulation in tissues during hypoxia, a condition in which CD73 expression is up regulated. In this study, we asked whether CD73 might also have a role in regulating lymphocyte migration into lymph nodes during inflammation. Lymphocytes home from the blood stream to lymph nodes (LN) through a process of tethering, rolling, adhesion and transmigration through the vasculature. High endothelial venules $(\mathrm{H}-$ EV) are a key regulator of this process. The interaction of L-selectin on lymphocytes with peripheral-node addressins on HEV initiates tethering and rolling. Then, activation of $\beta 2$ integrin (LFA-1) on lymphocytes by chemokines induces firm adhesion with HEV. However little is known about the transmigration process and the factors that control HEV permeability. Here we show preliminary evidence for a role of CD73 in regulating the entrance of lymphocytes into draining LN under inflammatory conditions induced by LPS and poly (I:C). The high expression of CD73 on HEV has been already reported ${ }^{3)}$. The size of secondary lymphoid organs is normal in $c d 73$-deficient mice, making it likely that lymphocyte homing to peripheral LN is also normal under steady state conditions. To examine the role of CD73 in lymphocyte migration during an inflammatory response, $c d 73$-deficient mice and wild type controls were subjected to subcutaneous injection of LPS or poly(I:C) into the left hind footpad, and lymphocyte migration into the draining popliteal lymph nodes was examined. PBS was injected into contralateral site as a control. As expected, 24 hours after injection, the left draining lymph nodes were dramatically enlarged in both strains of mice $(\mathrm{p}<0.02)$; however, they were approximately twice as large in the $c d 73$ deficient mice as in the controls $(\mathrm{p}<0.04)$. To more directly assess lymphocyte migration, fluorescently labeled wild type splenocytes were injected i.v. 24 hours after LPS or poly (I:C) injection and the accumulation of labeled lymphocytes in the popliteal lymph nodes was measured after 1 hour. Although no difference was observed on the control side, the number of lymphocytes that migrated into the draining LN of $c d 73$-deficient mice was 2.5 -fold increased compared with that of wild type mice $(\mathrm{p}<0.01)$. These results suggest that CD73 on HEV modulates lymphocyte migration into peripheral draining lymph nodes during inflammation. Experiments are in progress to identify the specific adenosine receptors involved.

\section{References:}

1) Lennon PF et al: Neutrophil-derived 5'-adenosine monophosphate promotes endothelial barrier function via CD73-mediated conversion to adenosine and endothelial $\mathrm{A}_{2 \mathrm{~B}}$ receptor activation. J Exp Med 1998, 188:14331443.

2) Thompson LF et al: Crucial Role for Ecto-5'-Nucleotidase (CD73) in Vascular Leakage during Hypoxia. J Exp Med 2004, 200:1395-1405.

3) Yamashita Y et al: CD73 expression and fyn-dependent signaling on murine lymphocytes. Eur J Immunol 1998, 28:2981-90. 


\title{
Cells and drugs and rac and rho: A mechanistic look at chemotaxis mediated by the $\mathrm{P}_{2} \mathrm{Y}_{2}$ receptor
}

\author{
Laurie Erb, Zhongji Liao, Sriparna Bagchi, Cheikh I. Seye \& Gary A. Weisman \\ Department of Biochemistry, University of Missouri, Columbia, MO 65211 USA \\ erbl@missouri.edu
}

Chemotaxis, or directed cell migration, is a fundamental feature of eukaryotic cells and is important for many physiological events, including embryonic development, inflammation and wound healing. The ability of a cell to undergo chemotaxis requires the cell to assume a polarized morphology that is controlled by cell surface receptors that activate the Rho family of small GTPases, including Cdc42, Rac and Rho [1,2]. Upon activation of a chemoattractant receptor, $\mathrm{Cdc} 42$ and Rac localize at the leading edge of a cell and control directional cell movement and lamellipodium formation, respectively [1]. Rho localizes at the rear and sides of a cell and controls the formation of contractile actin-myosin stress fibers [3]. Together, these GTPases promote cell migration towards a chemoattractant by mediating extension of the actin cytoskeleton at the front edge of the cell and retraction of the cytoskeleton at the rear. Recent studies have shown that $G$ protein-coupled receptors activate Rac and Racdependent lamellipodia formation through $\mathrm{G}_{\mathrm{i} / \mathrm{o}}$, whereas the activity of Rho and Rho-dependent stress fiber formation is controlled by $\mathrm{G}_{12 / 13}$ [3]. Previously, we reported that the $G$ protein-coupled $P 2 \mathrm{Y}_{2}$ receptor $\left(\mathrm{P} 2 \mathrm{Y}_{2} \mathrm{R}\right)$ contains a consensus Arg-Gly-Asp (RGD) integrin-binding sequence that interacts with $\alpha_{V} \beta_{3}$ and $\alpha_{V} \beta_{5}$ integrins [4]. Furthermore, we found that the RGD domain in the $P 2 Y_{2} R$ is required for $G_{o^{-}}$but not $G_{q}$-mediated calcium signaling [4], leading us to speculate that $\alpha_{\mathrm{V}}$ integrin interaction with the $\mathrm{P} 2 \mathrm{Y}_{2} \mathrm{R}$ is important for nucleotideinduced chemotaxis. In this study, we show that mutation of the RGD sequence to RGE in the human $\mathrm{P} 2 \mathrm{Y}_{2} \mathrm{R}$ expressed in $1321 \mathrm{~N} 1$ astrocytoma cells completely prevents UTP-induced chemotaxis and stress fiber formation as well as activation of $G_{o}, G_{12}$, Rac, Rho and Vav2, a guanine nucleotide exchange factor for both Rac and Rho. In cells expressing the wild-type but not the RGE mutant $\mathrm{P} 2 \mathrm{Y}_{2} \mathrm{R}$, UTP also increases expression of vitronectin, an extracellular matrix protein that is a ligand for $\alpha_{\mathrm{v}} \beta_{3 / 5}$ integrins. $\mathrm{P} 2 \mathrm{Y}_{2} \mathrm{R}$-mediated chemotaxis, Rac and Vav2 activation, and vitronectin upregulation were inhibited by pretreatment of the cells with anti- $\alpha_{v} \beta_{5}$ integrin antibodies, $\alpha_{\mathrm{v}}$ antisense oligonucleotides or the $\mathrm{G}_{\mathrm{i} / \mathrm{o}}$ inhibitor, pertussis toxin. P2 $\mathrm{Y}_{2} \mathrm{R}$-mediated stress fiber formation and Rho activation were inhibited by anti- $\alpha_{\mathrm{v}} \beta_{5}$ integrin antibodies, $\alpha_{\mathrm{v}}$ antisense oligonucleotides or a dominant negative $G \alpha_{12}$ construct. Collectively, these results suggest that the $P 2 Y_{2} R$ requires interaction with $\alpha_{\mathrm{V}}$ integrins for coupling to $G_{o}$ and $G_{12}$ proteins involved in chemotaxis.

This study was supported by the National Institutes of Health Grants 1 P01-AG-018357 and 1 R01-DE-07389.

1. Burridge, K. And Wennerber, K. (2004) Cell 116: 167-179

2. Etienne-Manneville, S. and Hall A. (2002) Nature 420:629-635

3. Xu, J. et al., (2003) Cell 114: 201-214

4. Erb, L. et al., (2001) J. Cell Biol. 153: 491-501

\section{Cerebral Arteriolar Response to Neuronal Activation is Attenuated Following Subarachnoid Hemorrhage}

\author{
Joseph R. Meno ${ }^{1}$, Ik-Seong Park ${ }^{1}$, Taylor J. Abel ${ }^{1}$, Abhineet Chowdhary ${ }^{1}$, Thien-son K. Nguyen ${ }^{1}$, \\ H. Richard Winn ${ }^{2}$, Al C. Ngai ${ }^{1}$ \& Gavin W. Britz ${ }^{1}$ \\ ${ }^{1}$ Department of Neurological Surgery, University of Washington, Seattle, Washington 98104 \\ ${ }^{2}$ Department of Neurosurgery, Mount Sinai School of Medicine, New York, New York 10029 \\ richard.winn@mountsinai.org
}

Small diameter arterioles play a significant role in the maintenance of cerebral blood flow (CBF). However, the status of cerebral arteriolar reactivity and neurovascular coupling following SAH is unclear. The present study tests the hypothesis that SAH results in alterations in cerebral arteriolar response to neuronal activation. An endovascular filament model was used to induce SAH in halothane-anesthetized male Sprague-Dawley rats. Then, at 24, 48, 72, 96, and 120 hours post-SAH, pial arteriolar responses to contralateral sciatic nerve stimulation 
(SNS) were evaluated utilizing a closed cranial window technique. In addition, somatosensory evoked potentials (SEPs), $\mathrm{CO}_{2}$ reactivity, as well as dose-responses to topical application of adenosine (ADO) and sodium nitroprusside (SNP) were assessed. In sham-operated rats, SNS evoked a $23.6 \pm 1.8 \%$ increase in arteriolar diameter. In rats subjected to SAH, pial arteriolar response to SNS was significantly attenuated to $13.7 \pm 0.9 \%, 11.9 \pm 1.3 \%$ and $15.2 \pm 1.2 \%$ at 24,48 and 72 hours post-SAH, respectively $(p<0.05 ; \mathrm{n} \geq 7)$. By 96 and 120 hours post-SAH, SNSinduced dilations recovered and were similar to sham responses. In contrast, SEPs were unaffected, suggesting a direct effect of SAH on cerebral arterioles that cannot be attributed to alterations in evoked neuronal or metabolic activity. The effect of SAH on arteriolar reactivity to ADO or SNP paralleled our observations during SNS. Pial vasodilatation to ADO $(10 \mu \mathrm{M})$ and SNP $(1 \mu \mathrm{M})$ were significantly attenuated by $47 \%$ and $41 \%$, respectively, at 48 hours post-SAH $(p<0.05 ; \mathrm{n}=7)$, whereas responses at 96 - and 120 hours post-SAH were similar to sham. $\mathrm{SAH}$ also had no effect on $\mathrm{CO}_{2}$ reactivity suggesting that the attenuation of SNS-induced vasodilatation following $\mathrm{SAH}$ is not the result of non-specific cerebral arteriolar paralysis. The present study demonstrates that SAH significantly attenuates cerebral arteriolar response to neuronal activation; the effects of SAH peak at 48 hours and return to normal by 96 hours post insult. Moreover, vasodilatation to ADO and SNP (NO), purported mediators of CBF regulation during increased neuronal activity, were similarly attenuated by SAH. The results of the present study provide the first evidence for alterations in arteriolar reactivity during increased neuronal activity and underscore the importance of future investigations aimed at evaluating the status of cerebral arteriolar reactivity following SAH.

Acknowledgements: This study was supported by grants from the Research Foundation of the American Association of Neurological Surgeons (GWB), the American Heart Association (ACN) and the NIH (HRW, NS-21076)

\title{
Challenges and Oppurtunities in the Identification of Insulin-Like Growth Factor-I Receptor Kinase Inhibitors
}

\author{
Carlos Garcia-Echeverria \\ Novartis Institutes for BioMedical Research - Oncology Research, CH-4002 Basel, Switzerland \\ carlos.garcia-echeverria@novartis.com
}

The Insulin-like Growth Factor-I Receptor (IGF-IR) is a member of the insulin receptor family of tyrosine kinases. A broad range of experimental studies have revealed that IGF-IR function is implicated in most of the hallmarks of cancer, but it is probably the anti-apoptotic activity of this receptor that makes its kinase activity an attractive therapeutic target in anti-cancer drug discovery. In this context, the identification of specific low-molecular mass inhibitors of IGF-IR has proven to be a major challenge for medicinal chemistry due to the high sequence identity at the kinase domains of IGF-IR and InsR (around 84\%) and, in particular, at the ATP-binding pocket $(100 \%$ sequence identity). This presentation will cover the identification and characterization of a new series of IGF-IR kinase inhibitors. The selectivity achieved at the cellular level with these compounds suggest conformational differences between the native forms of IGF-IR and InsR from the unactivated to the fully activated form, that can effectively be exploited for drug discovery.

\section{Changes in levels of $3^{\prime}$-AMP, an intracellular P-site inhibitor of adenylate cyclase, and its forming enzyme activity in diabetic mice treated with insulinomimetic zinc (II) complex}

Akihiro Miyamoto $^{1}$, Hiroyuki Fujimori ${ }^{1}$, Takahiro Horiuchi ${ }^{1}$, Hiromu Sakurai ${ }^{2}$ \& Hidemitsu Pan-Hou ${ }^{1}$ ${ }^{T}$ Department of Analytical Chemistry in Hygiene, Faculty of Pharmaceutical Sciences, Setsunan University;

${ }^{2}$ Department of Analytical and Bioinorganic Chemistry, Kyoto Pharmaceutical University

05d101ma@edu.setsunan.ac.jp

Adenylate cyclase (AC) is regulated by a number of extracellular and intracellular signals. Intracellular adenosine $3^{\prime}$-monophosphate (3'-AMP), one of the degradation products of RNAs, is pharmacologically classified as a P-site 
inhibitor of AC. The 3'-AMP forming enzyme, one of the RNase, has been shown to exist in various organs of rat and mice [1,2]. The forming enzyme activity is shown to be inhibited by divalent metal ions, especially zinc ion, and activated by EDTA. However, the biological roles of 3'-AMP and its forming enzyme still remains unclear. Recently, Fujimori and Pan-Hou [3] reported that 2,5-dideoxyadenosine, a P-site inhibitor of AC, enhanced cellular ATP levels in PC 12 cells. As cAMP released by AC modulates glycolysis and insulin secretion, $3^{\prime}$-AMP and its forming enzyme might be involved in glucose metabolism in various organs including pancreas and liver. Streptozotocin (STZ) induces experimental diabetes in animals. Bis(picolinate)zinc (II) $\left[\mathrm{Zn}(\mathrm{pa})_{2}\right]$ has been known to possess potent insulinomimetic action of lowering blood glucose levels [4]. Zinc ion is not only an essential trace element but also has inhibitory effect on $3^{\prime}$-AMP forming enzyme activity. Therefore, it is of interest to know whether $3^{\prime}$-AMP levels and its forming enzyme activity in STZ-induced diabetic mice might be affected by the treatment of $\mathrm{Zn}(\mathrm{pa})_{2}$. The objective of our reserch was to examine the effect of $\mathrm{Zn}(\mathrm{pa})_{2}$ on $3^{\prime}$-AMP levels and its forming enzyme activity in STZ-induced diabetic mice.

Male ICR mouse were treated with intraperitoneal injections of STZ (40 mg/kg body weight) during five consecutive days, and then $\mathrm{Zn}(\mathrm{pa})_{2}(10 \mathrm{mg} / \mathrm{kg}$ body weight $\times 2$ days and $5 \mathrm{mg} / \mathrm{kg}$ body weight $\times 9$ days $)$ was intraperitoneally injected [4]. Determination of $3^{\prime}$-AMP and its forming enzyme activity by HPLC were performed according to the method described by Fujimori and Pan-Hou [1].

$\mathrm{Zn}(\mathrm{pa})_{2}$ effectively lowered the STZ-induced enhancement of blood glucose levels. Pancreatic $3^{\prime}$-AMP levels in the STZ-, Zn(pa) $2_{2}$ and STZ- Zn(pa $)_{2}$ groups decreased, and hepatic levels in the Zn(pa $)_{2}$ and STZ- Zn(pa $)_{2}$ groups increased. Pancreatic 3'-AMP forming enzyme activities in the $\mathrm{Zn}(\mathrm{pa})_{2}$ and STZ- $\mathrm{Zn}(\mathrm{pa})_{2}$ groups and hepatic the activities in the $\mathrm{Zn}(\mathrm{pa})_{2}$ group increased. These results suggested that $3^{\prime}$-AMP metabolism in pancreas and liver in the STZ-induced diabetes at early stage might be affected by the treatment of $\mathrm{Zn}(\mathrm{pa})_{2}$.

1) H. Fujimori, R. Sato, M. Yasuda and H. Pan-Hou, Biol. Pharm. Bull., 21, 1348 (1998).

2) H. Fujimori, T. Fujita, and H. Pan-Hou, J. Health Sci., 43, 314 (2001).

3) H. Fujimori and H. Pan-Hou, Biol. Pharm. Bull., 28, 358 (2005).

4) H. Sakurai, Y. Kojima, Y. Yoshikawa, K. Kawabe and H. Yasui, Coord. Chem. Rev., 226, 187 (2002).

\title{
Channel activity using the consensus segment of the $M 2$ transmembrane domain from $\mathrm{P} 2 \mathrm{X}_{7}$ receptor
}

\author{
Cristina Alves Magalhães de Souza ${ }^{1,2}$, Pedro Teixeira ${ }^{3}$, Robson Xavier Faria ${ }^{1}$, Oxana Krylova ${ }^{2}$, Peter $^{2}$ \\ Pohl $^{2} \&$ Luiz Anastacio Alves ${ }^{1}$ \\ ${ }^{1}$ Oswaldo Cruz Foundation - Dept. Immunology, Institute Oswaldo Cruz-Av. Brazil 4365-21045-900 RJ, Brazil. \\ ${ }^{2}$ Research Institute for Molecular Pharmacology - Robert Rössler-Strasse 10, 13125 Berlin, Germany. ${ }^{3}$ Research \\ Center - Monkida Foundation - Grajau, RJ, Brazil \\ souzacam@ioc.fiocruz.br
}

Subject: $\mathrm{P} 2 \mathrm{X}_{7}$ receptor modulates a spectrum of cellular events in a variety of cells of the immune system. Although the pharmacology and channel properties of the $\mathrm{P} 2 \mathrm{X}_{7}$ receptors have been studied intensively, the formation of the pore associated with the $\mathrm{P} 2 \mathrm{X}_{7}$ remains an open question. Therefore, in the present study, we have investigated the eletrophysiological characteristics of the M2 transmembrane domain portion from the P2X7 receptor. Methods and Results: Most of bioinformatics studies have shown that M2 transmembrane domain (TM2) from the P2X7 receptor is conformed as an alpha helix. Using new algorithms of bioinformatics and molecular dynamics, we verified that the consensus segment of TM2 might be conformed as a beta sheet configuration. To test this hypothesis, we verified experimentally in subsequent analyses whether this segment would be able to form a channel in a planar lipid bilayer. The segment was synthetized with high degree of purity and its activity onto the planar lipid bilayer was measured using voltage-clamp condition. We observed single-channel currents which had conductance of $10 \mathrm{pS}$ in symmetric conditions with $\mathrm{KCl}$, being cation selectivity. This peptide forms also channels in a mammalian cell line (HEK) when analyzed by patch clamp in cell attach configuration. Conclusion: The consensus peptide segment from the TM2 forms a channel that has characteristics similar to P2X7 channel but different from P2X7 associated pore. In addition, this study raises the possibility that some regions of TM2 are not in alpha helix configuration.

Financial Support: IOC, FAPERJ and CNPq 


\title{
Characterisation of functionally important residues in mouse $\mathrm{P}_{2} \mathrm{X}_{\mathbf{7}}$ receptor
}

\author{
Pablo Pelegrin, Mark Young \& Annmarie Surprenant \\ University of Sheffield, Department of Biomedical Science, Florey Building, Western Bank, Sheffield, UK, S10 2TN \\ p.pelegrin@sheffield.ac.uk
}

The ATP-gated $\mathrm{P}_{2} \mathrm{X}_{7}$ receptor is an unusual ion channel that couples to multiple downstream signalling cascades. Mouse $\mathrm{P}_{2} \mathrm{X}_{7}$, cloned from NTW8 microglial cells and heterologously expressed in HEK293 cells, is much less sensitive to ATP than the human or rat homologs, although in vivo experiments indicate that the endogenous mouse receptor is highly sensitive to ATP. We found single nucleotide variations in mouse cDNA sequences cloned from NTW8 microglial cells [T33,G661,T848] and C57BL/6 mice [G33,A661,C848]. These nucleotide variations result in three amino acid differences; $\left[\mathrm{Phe}^{11}, \mathrm{Ala}^{221}, \mathrm{Met}^{283}\right]$ from NTW8 microglia and $\left[\mathrm{Leu}^{11}, \mathrm{Thr}^{221}, \mathrm{~T}\right.$ $\mathrm{hr}^{283}$ ] from C57BL/6 mice. We expressed these receptors in HEK293 cells and compared membrane currents, ethidium uptake and surface membrane expression. We found that the presence of $\mathrm{Met}^{283}$ in the sequence derived from NTW8 cells caused massive impairment of the ATP response. Maximum current densities at mouse P2X $\mathrm{Met}^{283}$ were $<5 \%$ of those at mouse $\mathrm{P} 2 \mathrm{X}_{7} \mathrm{Thr}^{283}$, without change in the agonist concentration-response curve or receptor trafficking to the plasma membrane. Moreover, no ethidium uptake was observed for mouse $\mathrm{P}_{2} \mathrm{X}_{7}$ $\mathrm{Met}^{283}$. The corresponding mutation in rat $\mathrm{P} 2 \mathrm{X}_{7}\left(\mathrm{Thr}^{283}\right.$-Met) also yielded currents that were $<5 \%$ of wildtype and no ethidium uptake was observed. Furthermore, characterization of the mouse $\mathrm{P}_{2} \mathrm{X}_{7}$ cytoplasmic domain polymorphism $\mathrm{Pro}^{451} / \mathrm{Leu}^{451}$ surprisingly revealed similar maximum current densities and agonist $\mathrm{EC}_{50}$ values for both alleles. However, both ethidium uptake and agonist-induced rise in intracellular calcium concentration were significantly reduced at the $\left[\mathrm{Thr}^{283}, \mathrm{Leu}^{451}\right] \mathrm{P} 2 \mathrm{X}_{7}$ receptor. These results show that $\mathrm{Thr}^{283}$ in the ectodomain is critical for $\mathrm{P}_{2} \mathrm{X}_{7}$ receptor function and suggest that the intracellular residue at position 451 may affect downstream signalling independently of ion channel activity.

\section{Characterisation of $\mathbf{P} 2 X$ receptor expression and inflammatory function in human endothelial cells}

\author{
$\underline{\text { Heather L. Wilson }}^{1}$, Richard W. Varcoe ${ }^{2}$, Leanne Stokes ${ }^{2}$, Sheila E. Francis ${ }^{1}$, Steven K. Dower ${ }^{1}$, David \\ C. Crossman ${ }^{1} \&$ Annmarie Surprenant ${ }^{2}$ \\ ${ }^{1}$ School of Medicine and Biomedical Science, ${ }^{2}$ Department of Biomedical Science, University of Sheffield, S10 2JF. \\ U.K. \\ H.L.Wilson@sheffield.ac.uk
}

Interleukin-1 (IL-1) is the prototypical pro-inflammatory cytokine, functioning at the apex of a cascade effecting and co-ordinating a wide variety of inflammatory actions. Evidence from pathological specimens, animal models, genetic and clinical studies implicates IL-1 $\beta$, particularly of endothelial cell origin, in the pathogenesis of the atherosclerotic plaque and the arterial healing response to injury. The net effect of IL-1 signalling depends primarily on the balance between IL- $1 \beta$ and its naturally occurring receptor antagonist (IL-1ra) acting on type I IL-1 receptors (IL-1R1) at the target cell surface. IL-1 $\beta$ is a so-called leaderless secretory protein in that it lacks a signal peptide sequence directing it to the endoplasmic reticulum and Golgi apparatus for classical exocytotic release. In monocytes, release of leaderless IL-1 $\beta$ requires activation of the $\mathrm{P}_{2} \mathrm{X}_{7}$ receptor [1]. More recently, release of the leaderless intracellular isoform of IL-1ra (icIL-1ra), the only isoform produced in endothelial cells, was shown to occur via a P2X dependent process [2]. We therefore determined whether a similar mechanism exists for the release of IL-1 $\beta$ from human endothelial cells.

Expression studies using RT-PCR and qRT-PCR showed that human umbilical vein endothelial cells (HUVECs) predominantly express the $\mathrm{P}_{2} \mathrm{X}_{4}$ and $\mathrm{P} 2 \mathrm{X}_{7}$ purinergic receptor subtypes and that their expression is inducible under inflammatory conditions with IFN $\gamma$ containing combinations having the most pronounced effect. Treatment for $48 \mathrm{hr}$ with IFN $\gamma$ and TNF $\alpha$ resulted in a 100-fold and 65-fold increase in $\mathrm{P}_{2} \mathrm{X}_{4}$ and $\mathrm{P} 2 \mathrm{X}_{7} \mathrm{mRNA}$ levels respectively, relative to an untreated control. These changes in mRNA expression were confirmed at the protein level using western blotting, immunoprecipitation and FACS. 
Low level functional $\mathrm{P} 2 \mathrm{X}_{4}$ and $\mathrm{P} 2 \mathrm{X}_{7}$ responses were measured in HUVECs by whole cell patch-clamp recording with an increase in the mean current density recorded from IFN $\gamma / \mathrm{TNF} \alpha$ treated cells $(1.05 \pm 0.28 \mathrm{pA}$ in control and $2.63 \pm 0.39 \mathrm{pA}$ in treated HUVECs).

Intracellular pro-IL-1 $\beta$ synthesis is negligible in untreated HUVECs, whereas significant amounts are produced in response to inflammatory stimulation (up to $4000 \mathrm{pg} / \mathrm{ml}$ following treatment with IFN $\gamma / \mathrm{TNF} \alpha$, LPS and BzATP). In contrast, caspase- 1 is constitutively expressed in HUVECs but is similarly induced in response to inflammatory stimulation. Low-level release of biologically active $17 \mathrm{kDa}$ IL-1 $\beta$ was measured upon BzATP stimulation of HUVECs by ELISA and Western blots and was in the absence of cell death as shown by an LDH release assay. However, icIL-1ra was released under the same conditions, and at higher concentrations, so the net biological effect is generally anti-inflammatory, when measured using an IL-1 sensitive bioassay.

We conclude therefore, that P2X receptors play an important role in regulating secretion of leaderless pro- and anti-inflammatory cytokines in the endothelium; their expression and activation may determine the balance of IL$1 \beta$ and icIL-1ra released from endothelial cells, providing a target for altering the inflammatory state of the arterial vessel wall.

(1) MacKenzie, A.; Wilson, H. L.; Kiss-Toth, E.; Dower, S. K.; North, R. A.; Surprenant, A. Immunity 2001, 15, (5), 825-35.

(2) Wilson, H. L.; Francis, S. E.; Dower, S. K.; Crossman, D. C. J Immunol 2004, 173, (2), 1202-8.

\title{
Characterisation of a recombinant soluble apyrase from the blood-feeding sand fly Phlebotomus papatasi and anti-thrombotic activity in a rat model of acute thromboembolism
}

\author{
Thomas M. Smith ${ }^{1}$, Qi Zheng ${ }^{2}$ \& Min $\mathrm{Lu}^{2}$ \\ ${ }^{1}$ Department of Cardiovascular and Metabolic Diseases, Wyeth Research, Cambridge, MA, USA \\ ${ }^{2}$ Department of Biochemistry, Weill Medical College of Cornell University, New York, New York, USA
}

Adenosine nucleotides such as ADP found in the blood as a consequence of vascular injury and granule release from activated platelets are one of the most important physiological agonists of platelet recruitment, aggregation and thrombus formation. As such, the blockade of platelet $\mathrm{P}_{2} \mathrm{Y}_{12}$ ADP receptors by thienopyridine derivatives (e.g. clopidogrel) and the metabolic removal of ADP from the vascular milieu by ADP-hydrolyzing enzymes (apyrases) offer great advantages for the prevention and treatment of cardio- and cerebrovascular vascular events characterized by activated platelets.

We have previously reported the purification and crystal structure of a novel soluble human apyrase (see Dai et al., 2005. Cell. 116(5): 649-59). Based upon sequence homology to the apyrases identified in blood-feeding arthropods, the human enzyme was "re-engineered" using site-directed mutagenesis to alter the calcium binding and nucleotide substrate active site. As a result, the ADP hydrolyzing activity was enhanced more than 100 fold and this engineered human apyrase was shown to be an effective inhibitor of ADP and collagen induced platelet aggregation.

In spite of the enhanced ADPase activity of the engineered human apyrase however, the protein had lower $\mathrm{K}_{\text {cat }}$ and anti-platelet activity compared to the soluble salivary apyrases of blood-feeding arthropods. As a result, we expressed and purified the recombinant soluble apyrase from the blood-feeding sand fly Phlebotomus papatasi. The expressed Phlebotomus enzyme was a calcium-dependent ADP and ATPase, with a pH optimum of $\sim 7$, a low $\mathrm{K}_{\mathrm{M}}$ for ADP and ATP (61 uM and $65 \mathrm{uM}$, respectively), and a very high substrate turnover rate $\left(3.0 \times 10^{4} \mathrm{~min}^{-1}\right.$ and $2.9 \times 10^{4} \mathrm{~min}^{-1}$ for ADP and ATP, respectively).

In in vitro platelet aggregometry assays of human platelet rich plasma and PFA-100® analyses of human whole blood under high shear conditions, the Phlebotomus apyrase was a potent inhibitor of ADP induced platelet aggregation. The pharmacokinetics of the apyrase, administered intravenously at a single dose of $12.5 \mathrm{mg} / \mathrm{kg}$, was also investigated in rats. In vivo, the enzyme was distributed and eliminated rapidly, with a half-life of approximately 15 minutes. To examine the efficacy of the apyrase as an anti-thrombotic agent, a rat vascular electrolytic injury model was utilized. Intravenous administration of apyrase at a dose of $12.5 \mathrm{mg} / \mathrm{kg}$ showed a statistically significant $\sim 35 \%$ inhibition in occlusive thrombus formation compared to control animals. A clear dose-response relationship was observed, with approximately $22 \%$ and $15 \%$ inhibition seen at 1.0 and $0.3 \mathrm{mg} / \mathrm{kg}$ doses, respe- 
ctively. No inhibition was achieved at the $0.1 \mathrm{mg} / \mathrm{kg}$ dose. The platelet $\mathrm{P} 2 \mathrm{Y}_{12}$ ADP receptor antagonist clopidogrel at $10 \mathrm{mg} / \mathrm{kg}$ in this model exhibited $\sim 41 \%$ inhibition of thrombus formation.

Soluble apyrases act through the enzymatic "deletion" of the platelet pro-aggregant ADP, inhibiting platelet aggregation and thrombus formation. Thus, the unique anti-platelet actions of the apyrase enzymes make them particularly attractive in the context of therapeutic agents for the inhibition of platelet-mediated thrombotic disorders.

\title{
Characterization of Adenosine Metabolism and Coronary Flow Control by Adenosine in Mouse Heart
}

\author{
J. Weichsel, A. Pexa \& A. Deussen
}

Department of Physiology, Med. Fakultät Carl Gustav Carus, TU Dresden, Germany

johnwei@gmx.de

The murine heart preparation has been increasingly used in recent years, because of the opportunity to employ knockout or overexpression models. A further aspect relates to the use of expensive pharmacological tools, which is more economical in this species due to the small organ size. Aims of the present study were the characterization of adenosine metabolism and purine related coronary flow regulation of mouse heart.

Methods. C57B16 mice weighing 19-25 g were anaesthetized and heparinized (urethane $2 \mathrm{mg} / \mathrm{g}$ body mass, Liquemin ${ }^{\circledR} 500$ I.U.). Hearts were isolated and retrogradely perfused via the ascending aorta according to the Langendorff-method providing $37^{\circ} \mathrm{C}$ warm Krebs-Henseleit-buffer equilibrated with carbogen gas $\left(\mathrm{pO}_{2} 680 \mathrm{~mm} \mathrm{Hg}\right.$, $\mathrm{pCO}_{2} 35 \mathrm{~mm} \mathrm{Hg}$ ) at a perfusion pressure of $85 \pm 3 \mathrm{mmHg}$. Heart rate was kept constant by pacing (505 $\left.\pm 5 \mathrm{bpm}\right)$ and coronary flow was measured using an Ultrasonic $\AA$ flow probe. A PVC-balloon was inserted into the left ventricle via the left atrium to allow measurement of ventricular pressure development and $\mathrm{dP} / \mathrm{dt}$. A catheter advanced into the right atrium was used for collecting venous effluent perfusate for analysis of adenosine concentration (HPLC technique) and gas partial pressures.

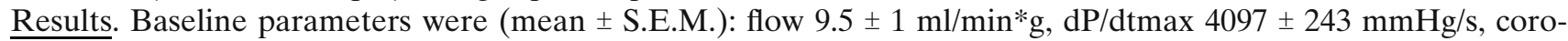
nary venous $\mathrm{pO}_{2} 160 \pm 7 \mathrm{mmHg}$, and adenosine concentration $18 \pm 3 \mathrm{nmol} / \mathrm{l}$. The reactive flow overshoot following a 20 s flow stop was $280 \pm 9 \%(n=10)$ of baseline flow. Elevation of the coronary arterial adenosine concentration by 80 and $200 \mathrm{nM}$ resulted in marked increases of coronary flow (2.8-, 3.4-fold), an increased contractility (1.5-, 1.3-fold) and elevated venous $\mathrm{pO}_{2}(2.3-, 2.7$-fold), respectively. Block of adenosine deamination and phosphorylation by EHNA $(5 \mu \mathrm{M})$ and ITU $(10 \mu \mathrm{M})$, respectively, augmented adenosine release 8.9-fold. In parallel, coronary flow rose 3.2-fold. In presence of EHNA and ITU, NBTI $(10 \mu \mathrm{M})$, a specific blocker of equilibrative nucleoside transport, only moderately lowered adenosine release by $18 \%$. Specific block of $\mathrm{A}_{1}$, (DPCPX $100 \mathrm{nM}$ ), $\mathrm{A}_{2 \mathrm{~A}}\left(\mathrm{ZM}-241385100 \mathrm{nM}, \mathrm{SCH}-58261100 \mathrm{nM}\right.$ ) and $\mathrm{A}_{2 \mathrm{~B}}$ (Alloxazine $10 \mu \mathrm{M}$, MRS-1754 $200 \mathrm{nM}$ ) adenosine receptors did neither change adenosine release nor baseline coronary flow significantly. However, the effect of exogenous adenosine $(80 \mathrm{nM}, 200 \mathrm{nM})$ on coronary flow was almost completely blocked by $\mathrm{A}_{2 \mathrm{~A}}$ antagonism, partly by $\mathrm{A}_{2 \mathrm{~B}}$ antagonism but not by blocking $\mathrm{A}_{1}$ adenosine receptors. During hypoxic perfusion, $\mathrm{A}_{2 \mathrm{~A}}$ antagonists lowered coronary flow by approximately $10 \%$.

Conclusions. 1) The coronary flow control of mouse heart exhibits a high sensitivity for adenosine. 2) Exogenous adenosine acts mainly via activation of $\mathrm{A}_{2 \mathrm{~A}}$-receptors. 3) Selective blockade of $\mathrm{A}_{2 \mathrm{~A}}$-receptors does not largely affect baseline coronary flow and adenosine release. 4) During hypoxic perfusion endogenous adenosine contributes to flow control. 5) Block of membrane transport of adenosine is rather insensitive to NBTI (NBMPR) as compared to other species.

\section{Characterization of the platelet $\mathrm{P}_{12} \mathrm{Y}_{12}$ receptor with the new, selective radiogland $\left[{ }^{3}\right.$ H]PSB-0413}

K.J. Griessmeier, A. El-Tayeb \& C.E. Müller

Pharmaceutical Sciences Bonn (PSB), Pharmaceutical Chemistry, Institute of Pharmacy, University of Bonn, Kreuzbergweg 26, 53115 Bonn, Germany

kerstin.griessmeier@uni-bonn.de 
The $\mathrm{P} 2 \mathrm{Y}_{12}$ ADP receptor is one of the major regulators of hemostasis and thrombosis and the target of antithrombotic thienopyridines and nucleotide analogs. It is a $\mathrm{G}$ protein-coupled receptor which upon activation inhibits adenylate cyclase activity $[1,2]$. The platelet $\mathrm{P}_{2} \mathrm{Y}_{12}$ receptor has been extensively characterized in functional assays $[1,2]$. However, characterization on the protein level has been hampered by the lacking of a selective radioligand.

In the present study, we characterized the $\mathrm{P}_{2} \mathrm{Y}_{12}$ receptor in human platelet membrane preparations using the newly synthesized enzymatically stable and $\mathrm{P} 2 \mathrm{Y}_{12}$-selective radioligand $\left[{ }^{3} \mathrm{H}\right] \mathrm{PSB}-0413$ (2-propylthioadenosine-5'adenylic acid (1,1-dichloro-1-phosphonomethyl-1-phosphonyl) anhydride, AR-C67085MX) [3]. [ $\left.{ }^{3} \mathrm{H}\right] \mathrm{PSB}-0413 \mathrm{~s}-$ howed a high affinity for $\mathrm{P}_{2} \mathrm{Y}_{12}$ receptors natively expressed in human platelets $\left(\mathrm{K}_{\mathrm{D}}=4.57 \pm 0.51 \mathrm{nM}\right)$ as determined in a saturation binding assay. The kinetically derived $\mathrm{K}_{\mathrm{D}}$ value was similar $(3.69 \pm 1.58 \mathrm{nM}$ at r.t. and 4.87 $\pm 2.81 \mathrm{nM}$ at $4^{\circ} \mathrm{C}$, respectively). A membrane preparation of human platelets showed a high expression level of $\mathrm{P}_{2} \mathrm{Y}_{12}$ receptors $\left(\mathrm{B}_{\max }=7.66 \pm 0.69 \mathrm{pmol} / \mathrm{mg}\right.$ of protein $)$. It was much higher than than the density of $\mathrm{P} 2 \mathrm{Y}_{1}$ receptors $(170 \mathrm{fmol} / \mathrm{mg}$ protein). A competition assay for the screening of ligands was established and a series of standard agonists and antagonists was evaluated. The rank order of potency for the inhibition of $\left[{ }^{3} \mathrm{H}\right] \mathrm{PSB}-0413$ binding was $\left(\mathrm{K}_{\mathrm{i}}\right.$ values): 2-methylthio-ADP $(4.88 \mathrm{nM})>\operatorname{ADP} \beta S(4.11 \mu \mathrm{M})>\operatorname{ATP} \gamma \mathrm{S}(13.2 \mu \mathrm{M})=\operatorname{ATP}(15.7 \mu \mathrm{M})$ $=\operatorname{ADP}(17.6 \mu \mathrm{M})$. GTP shift experiments confirmed that 2-methylthio-ADP and ADP were agonists and that ATP and the precursor of $\left[{ }^{3} \mathrm{H}\right] \mathrm{PSB}-0413$, PSB-0412 bearing a 2-propargyl instead of a 2-propyl substituent $\left(\mathrm{K}_{\mathrm{i}}\right.$ value $=2.41 \pm 0.25 \mathrm{nM}$ ), were antagonists at human $\mathrm{P}_{2} \mathrm{Y}_{12}$ receptors of platelets. Radioligand binding was not inhibited by $100 \mu \mathrm{M}$ of the following P2 receptor ligands: pyridoxalphosphate-6-azophenyl-2',4'-disulphonic acid (PPADS), $\beta, \gamma$-methylene-ATP, $\alpha, \beta$-methylene-ATP, UDP-glucose, AMP, IDP, UMP, UDP or UTP. The radioligand is selective versus the closely related $\mathrm{P} 2 \mathrm{Y}$ receptor subtypes $\mathrm{P} 2 \mathrm{Y}_{11}\left(\mathrm{EC}_{50}=1.5-8.9 \mu \mathrm{M}\right)$ and $\mathrm{P} 2 \mathrm{Y}_{13}\left(\mathrm{IC}_{50}=\right.$ 213-630 nM). These results indicate that $\left[{ }^{3} \mathrm{H}\right] \mathrm{PSB}-0413$ specifically labels the $\mathrm{P} 2 \mathrm{Y}_{12}$ receptor. In conclusion, $\left[{ }^{3} \mathrm{H}\right] \mathrm{PSB}-0413$ is the first selective high-affinity antagonist radioligand for the $\mathrm{P} 2 \mathrm{Y}_{12}$ receptor.

[1] Zhang, F.L.; Luo, L.; Gustafson, E.; Lachowicz, J.; Smith, M.; Qiao, X.; Liu, Y.; Chen, G.; Pramanik, B.; Laz, T.M.; Palmer, K.; Bayne, M.; Monsma, F.J. J. Biol. Chem., 2001, 276, 8608-8615.

[2] Hollopeter, G.; Jantzen, H.-M.; Vincent, D.; Li, G.; England, L.; Ramakrishan, V.; Yang, R.-B.; Nurden, P.; Nurden, A.; Julius, D.; Conley, P. B. Nature, 2001, 409, 202-207.

[3] El-Tayeb, A.; Griessmeier, K.J., Müller, C.E. Bioorg. Med. Chem. Lett., 2005, 15, 5450-5452.

Supported by the Deutsche Forschungsgemeinschaft (GRK-677)

\title{
Characterization of the receptor(s) that mediate BzATP responses in rat cerebellar astrocytes
}

\author{
Esmerilda G. Delicado, Luz María G. Carrasquero and Ma. Teresa Miras-Portugal \\ Departamento de Bioquímica, Facultad de Veterinaria, Universidad Complutense de Madrid, Spain \\ esmeril@vet.ucm.es
}

Cerebellar astrocyte cultures is one of the experimental models used to study nucleotide signaling in cerebellum. In previous works, we showed that cerebellar astrocytes possess a great diversity of functional P2Y receptors, ADP sensitive receptors, the $\mathrm{P}_{2} \mathrm{Y}_{1}$ and/or the $\mathrm{P}_{2} \mathrm{Y}_{13}$-like receptors, (see Poster of Carrasquero et al.) and ATP/ UTP sensitive receptors, the $\mathrm{P}_{2} \mathrm{Y}_{2} / \mathrm{P}_{2} \mathrm{Y}_{4}$ receptors, mainly coupled to PLC activation. These results contrast to those reported for astrocytes from other brain areas, such as cortex or hippocampus, which also express some ionotropic $\mathrm{P} 2 \mathrm{X}$ receptors, including the $\mathrm{P} 2 \mathrm{X}_{7}$ subtype. The $\mathrm{P} 2 \mathrm{X}_{7}$ receptor is an ionotropic $\mathrm{P} 2 \mathrm{X}$ receptor, only activated at millimolar ATP concentrations in vivo, which exhibits very peculiar characteristics. It was thought to be exclusive for cells of haematopoietic lineage, where its role in cellular toxicity and inflammatory process is well established. Recently, it has been also detected at the nervous system, where has resulted to be an "intriguing" receptor and far from induce cellular lysis or apoptosis, could exert other actions, such as differentiation, glutamate release, etc. Basing on these findings, we decided to explore their presence in cerebellar astrocytes using BzATP, described as the most specific agonist for this receptor subtype. We have found that cerebellar astrocytes displayed several BzATP responses, which are quite different to those elicited by other nucleotides, which would be mediated by $\mathrm{P} \mathrm{X}_{7}$-like receptors: i) BzATP-induced calcium responses were sustained, and not transient, as those obtained with the metabotropic agonists 2MeSADP and UTP, ii) Among the nucleotides BzATP produced the maximal ERK activation, iii) BzATP also produced long-term effects, inducing morphological changes, that 
leads to differentiation. Moreover, the presence of mRNA codifying of $\mathrm{P} 2 \mathrm{X}_{7}$ receptor and the protein was also confirmed. Although $\mathrm{P}_{2} \mathrm{X}_{4}$ subunits have been also detected, their functionality remains unclear.

The characterization of BzATP calcium responses was made by calcium imaging using fura-2. Most of tested cells $(80 \%)$ were sensitive to BzATP stimulations eliciting calcium responses, which are biphasic. They exhibited initial transients with an $\mathrm{EC}_{50}$ value of $12.2+1.6 \mu \mathrm{M}$, followed by a sustained responses, that were not desensitized. But, surprisingly, when BzATP challenges were applied in the absence of extracellular calcium, the initial transients were maintained, whereas as expected the sustained responses were completely abolished, which clearly indicated that the transients resulted from intracellular calcium mobilization. The same occurred by preincubation with 1 $\mu \mathrm{M}$ Brilliant Blue $\mathrm{G}$, concentration at which this antagonist selectively acts on $\mathrm{P} 2 \mathrm{X}_{7}$ receptors and stabilize the inactive form of the receptor, the sustained responses were abolished, but the transient was also observed. BzATP calcium responses were not affected by cross-desensitization with UTP or 2MeSADP, when were consecutively applied, though each agonist was able to desensitize itself, indicating that they are acting on different receptors. These data suggest that BzATP possess specific receptors, P2X7-like receptor type, in cerebellar astrocytes, which are also coupled to PLC activation.

\section{Chronic cyclosporine treatment increases total homocysteine levels and decreases adenine-nucleotides hydrolysis in rat blood serum}

Ana Elisa Böhmer ${ }^{1}$, Liz Marina B. P. Brum ${ }^{2}$, Carolina G. Souza ${ }^{1}$, Jean P. Oses ${ }^{1}$, Mariana Streit $^{1}$, Ricardo S. Bruch $^{2}$, João J.F. Sarkis ${ }^{1}$, Luis V. Portela ${ }^{1}$, Diogo O. Souza ${ }^{1}$

${ }^{1}$ Departamento de Bioquímica, Instituto de Ciências Básicas da Saúde, Universidade Federal do Rio Grande do Sul, Rua, Porto Alegre, RS, Brazil. ${ }^{2}$ Instituto de Cardiologia do Rio Grande do Sul, FUC, Porto Alegre, RS, Brazil. anaelisab@uol.com.br

Cyclosporine (CsA) is a potent immunosuppressant agent that has been extensively used in transplanted patients. However, CsA administration is accompanied by a variety of adverse effects being allograft vasculopathy the major cause of morbidity and mortality among renal and cardiac transplant recipients. The mechanisms involved in the vascular injury and the putative participation of CsA are poorly understood. CsA administration might affect homocysteine (Hcy) serum concentrations, a sulfur containing amino acid recognized as an independent risk factor for atherosclerosis and venous thromboembolism. Excessive production of free radicals, increase on platelet adhesiveness and decrease on tissue and plasmatic adenosine levels contribute to the vascular damage caused by Hcy. Extracellular adenosine has important benefic effects on the vascular system, avoiding thrombus formation and circulatory problems through the regulation of platelet aggregation, vasodilatation, coronary blood flow and inflammation. Thus, control of the ratio of adenine nucleotide/adenosine in the extracellular space by ecto-nucleotidases activities is one of the important steps for the maintenance of vascular homeostasis.

In this work, we examined the effects of chronic CsA administration on total serum Hcy levels, on the adeninenucleotides hydrolysis (ATP, ADP, AMP) through serum ecto-nucleotidases activities and its putative association with vascular disturbance in adult male Wistar rats. Animals were daily administered with CsA $5 \mathrm{mg} / \mathrm{kg}, 15 \mathrm{mg} / \mathrm{kg}$ or vehicle via gastric gavage during 8 weeks. Total Hcy concentrations in serum was measured using a commercial MEIA kit (Abbott, USA) and ATP, ADP and AMP hydrolysis were evaluated through phosphate release, using the method described by Oses et al. [1]. CsA induced a significant increase on total Hcy concentration and a decrease in ATP, ADP and AMP hydrolysis. The inhibition of nucleotides hydrolysis was negatively correlated with total Hcy levels and positively with uric acid levels. Increased platelets number and fibrinogen levels, involved in coagulation pathway, were also observed.

Altogether, these results indicate that chronic CsA treatment affects the homeostasis of vascular system by increasing platelets, fibrinogen and serum levels of total Hcy. Moreover, we postulated that by inhibiting adenine nucleotides hydrolysis, CsA might disrupt the equilibrium between adenine nucleotides/adenosine levels. Finally, these alterations could be implicated in the vascular complications reported in patients under CsA therapy.

(Supported by CNPq Brazil, FAPERGS).

Oses et al. Life Sciences 2004;74(26):3275-3284. 


\title{
Cloning and identification of the liver canalicular ECTO-ATPase as NTPDase8
}

\author{
M. Fausther $^{1}$, J. Lecka ${ }^{1}$, F. Kukulski ${ }^{1}$, J. Pelletier ${ }^{1}$, S.A. Lévesque ${ }^{1}$, H. Zimmermann ${ }^{2}$, J.A. Dranoff ${ }^{3}$ \\ and J. Sévigny 1 \\ ${ }^{1}$ Centre de Recherche en Rhumatologie et Immunologie, Université Laval, Québec, Canada \\ ${ }^{2}$ Biocenter, J.W. Goethe-University, AK Neurochemistry, Frankfurt am Main, Germany \\ ${ }^{3}$ Section of Digestive Diseases, Yale University School of Medicine, New Haven, CT, USA \\ michel.fausther@crchul.ulaval.ca
}

Background. Liver cells express various ecto-nucleotidases including CD39/ecto-nucleoside triphosphate diphosphohydrolase-1 (NTPDase1), CD39L1/NTPDase2 and CD73/ecto-5'-nucleotidase to regulate nucleotide and nucleoside levels at the cell surface. Since the these molecules control a number of critical cellular functions like cell volume autoregulation and ionic secretion, the biological activity of ecto-nucleotidases appears essential for liver homeostasis maintenance. Interestingly, the liver has one of the highest ecto-nucleotidase activity among tissues. In the liver, NTPDase1 is expressed by Kupffer cells and vascular endothelial cells whereas NTPDase2 is produced by portal fibroblasts and activated hepatic stellate cells. However, their combined activity appears much lower than the total ecto-nucleotidase activity observed in liver tissue. Histochemical studies showed that the high liver ecto-nucleotidase activity is mainly associated with the canalicular domain of hepatocytes and has the general characteristics of an NTPDase activity. We have recently reported, in mouse, the cloning of a novel ectonucleotidase, NTPDase 8 whose cDNA is highly expressed in liver. The Aim of this study was to identify the main ecto-nucleotidase(s) in the liver, particularly the one(s) expressed in the canalicular domain of hepatocytes. Methods. NTPDase8 cDNA was cloned from human and rat liver tissues and inserted in pcDNA3 expression vector. Constructs were used for transfection assays in COS-7 cell line. NTPDase8 was purified from rat liver by a 3-step chromatography. The biochemical characterization was performed with protein extracts from NTPDase8transfected cells or the purified protein. Antibodies against rat NTPDase8 and CD73 were generated and used to examine the expression pattern of these proteins, by Western blot and immunofluorescence. Results. Activity assays on protein extracts (from COS-7 cells expressing rat or human NTPDase8) and purified rat NTPDase8 regarding substrate specificity, ion requirement and sodium azide inhibition showed that the enzyme displayed similar biochemical properties as previously described for the purified porcine canalicular ecto-ATPase. The analyses of the primary structures showed high resemblance between rat and human NTPDase 8 when compared to porcine ecto-ATPase. The specificity of polyclonal antibodies against rat NTPDase8 and CD73 was confirmed by immunoblot and immunocytochemistry, using transfected cells. By western blot, the anti-rat NTPDase8 detected a protein with a molecular weight of $75 \mathrm{kDa}$ with the highest expression in liver and also some expression in kidney and jejunum. In liver sections, NTPDase 8 was expressed in the canalicular membrane domain of hepatocytes and co-localized with the canalicular marker MRP-2. Immunostaining for CD73 showed that this enzyme was also present in the bile canaliculi. Conclusions. In this work, we report the cloning of NTPDase8 in human and rat species, its identification as the canalicular ecto-ATPase and demonstrate that it represents the major ecto-nucleotidase activity of the liver. In addition, the canalicular localization of both NTPDase8 and CD73 suggest their potential involvement in biliary function.

\section{Cloning, expression and characterization of the Torpedo marmorata E-NTPDase1}

Mireia Martín-Satué $^{1}$, Benjamín Torrejón-Escribano ${ }^{2}$, Inma Gómez de Aranda ${ }^{1}$, Antonio Felipe ${ }^{3}$, Marc Elías ${ }^{1}$, Jordi Marsal $^{1}$, Joan Blasi ${ }^{1}$, and Carles Solsona ${ }^{1}$

${ }^{1}$ Cellular and Molecular Neurobiology laboratory, Dep. of Therapeuthics and Experimental Pathology, Medicine School, Bellvitge Campus, University of Barcelona, Spain

${ }^{2}$ Scientific and Technical Services, Bellvitge Campus, University of Barcelona, Spain

${ }^{3}$ Department of Biochemistry and Molecular Biology, University of Barcelona, Spain

martinsatue@ub.edu

Ecto-nucleoside triphosphate diphosphohydrolases (E-NTPDases) constitute a family of enzymes that hydrolyse both ATP and ADP to AMP, differing in their substrate preferences and tissue distribution. They are broadly 
expressed enzymes, mainly membrane bound, playing a relevant role in several physiological processes. An ENTPDase activity in electric organ membranes of Torpedo fishes was previously identified (1) and we have been using these membranes as a source of E-NTPDase activity to study the effect of new possible inhibitors $(2,3,4)$. Enzymology studies pointed to the classification of this enzyme into the class-1 E-NTPDases, since it shows nearly equal preference for ATP and ADP (5). We report here the cloning of its full-length cDNA and its characterization.

The clone, obtained by means of RACE (Rapid Amplification of $c$ DNA Ends)-PCR technique, contains an open reading frame of $1506 \mathrm{bp}$ and codes for a 502 aminoacids-protein that exhibits high homology with other ENTPDases1 previously identified, including those of zebrafish or Xenopus but also human, rat and mouse. Topology analyses revealed the existence of two transmembrane regions, two short cytoplasmic tails and a long extracellular domain containing the five highly conserved apyrase regions. Gene expression studies revealed that this gene is expressed in all the Torpedo tissues analysed. We have also characterized the expression of the recombinant protein expressed in COS and HeLa cells.

1. Grondal, EJM \& Zimmermann, H; J. Neurochem., 47 : 871-881, 1986.

2. Martí, E. et al.; Br. J. Pharmacol., 118: 1232-1236, 1996.

3. Martí, E. et al.; Biochim. et Biophys. Acta, 1282: 17-24, 1996.

4. Escalada, A. et al.; Neurochem. Res., 29: 1711-1714, 2004.

5. Sarkis, JJ \& Salto, C.; Brain Res. Bull., 26: 871-6, 1991.

This work was supported by grants of Ministerio de Educación y Ciencia from the Spanish Governement (C.Solsona, project no. SAF2005-00736) and Comisió de Recerca del Campus de Bellvitge (M. Martín-Satué, project no. ACESB/13) from the University of Barcelone, Spain.

\section{Co-localization and functional cross-talk between $A_{1}$ and $P_{2} Y_{1}$ purine receptors in the brain}

I. Tonazzini $^{1}$, M.L. Trincavelli ${ }^{1}$, L.H. Bergersen ${ }^{2}$, J. Storm-Mathisen ${ }^{2}$, M.P. Abbracchio ${ }^{3}$, C. Martini ${ }^{1}$

${ }^{T}$ Dept. of Psychiatry Neurobiology Pharmacology and Biotechnology, University of Pisa, Italy. ${ }^{2}$ Dept. of Anatomy and Centre for Molecular Biology and Neuroscience CMBN, University of Oslo, Norway. ${ }^{3}$ Dept. Pharmacological Science, Unviversity of Milan, Italy

tonazzini@farm.unipi.it

Adenosine and ATP are released from glia and neuron cells and are involved in a wide variety of both physiological and pathological processes by their interaction with specific receptors classified as P1 and P2, respectively (Abbracchio and Burnstock, 1998). Whereas the neuroprotective role of adenosine analogues active at the adenosine $A_{1}$ receptor subtype $\left(A_{1} A R\right)$ have been known for several years (Wardas, 2002), the role of purinergic $\mathrm{P}_{2} \mathrm{Y}_{1}$ receptor $\left(\mathrm{P}_{2} \mathrm{Y}_{1} \mathrm{R}\right)$, enough if not fully characterized, indicate important pathological functions/involvements: purine-mediated induction and maintenance of astrogliosis can be considered as a first response to limit the loss of neuronal tissue after insults (Franke and Illes, 2006). Recent data suggest the existence of a heteromeric complex between $\mathrm{A}_{1} \mathrm{ARs}$ and $\mathrm{P}_{2} \mathrm{Y}_{1}$ purinergic receptors with novel pharmacological and functional properties (Yoshioka and Nakata., 2004). As evidenced by G-protein coupled receptors heterodimerization studies, an important role in the control of receptor signalling and regulation processes has been demonstrated (Maggio et al., 2005).

In the present work we investigated the co-localization of $\mathrm{A}_{1}$ and $\mathrm{P}_{2} \mathrm{Y}_{1}$ receptors on rat brain tissues and their heterodimerization and functional cross-talk in astroglial cells (ADF).

Immunogold-electron microscopy quantification was used to study the cellular localization of $\mathrm{A}_{1}$ and $\mathrm{P}_{2} \mathrm{Y}_{1}$ receptors on rat hippocampus which is considered as a damage sensitive brain area. The results demonstrated a significantly high expression of both receptors on synaptic and astroglia membranes. Since glial elements represented the cell population in which the higher $\mathrm{A}_{1} / \mathrm{P}_{2} \mathrm{Y}_{1}$ co-localization occurred, an astroglial cell line was used to investigate the receptor functional cross-talk. $A_{1} A R$ and $P_{2} Y_{1} R$ expression in ADF cells was demonstrated by immunoblotting experiments; moreover, in co-immunoprecipitation assay we showed that these receptors constituted an heteromeric complex in basal conditions. 
$\mathrm{A}_{1} \mathrm{AR} / \mathrm{P}_{2} \mathrm{Y}_{1} \mathrm{R}$ functional interaction was investigated evaluating the heterologous regulation of $\mathrm{A}_{1} \mathrm{AR}$-cAMP pathway induced by the $\mathrm{P}_{2} \mathrm{Y}_{1}$-agonist, 2-MeSADP.

In control ADF cells, $\mathrm{A}_{1} \mathrm{AR}$ agonist, $\mathrm{CHA}$, was able to inhibit, in concentration dependent manner, the production of cAMP $\left(\mathrm{EC}_{50}=0,9 \mathrm{nM}\right)$. ADF cell exposure to 2-MeSADP inhibited $\mathrm{A}_{1} \mathrm{AR}$ functional response in time dependent manner with a maximal effect after 30 minutes. Moreover, a significant right shift of CHA doseresponse curve occurred. These data demonstrated $\mathrm{P}_{2} \mathrm{Y}_{1} \mathrm{R}$ induced heterologus $\mathrm{A}_{1} \mathrm{AR}$ desensitisation in ADF cells.

To clarify the cellular co-localization, the interaction and the function of heterodimerization of $\mathrm{A}_{1}$ and $\mathrm{P}_{2} \mathrm{Y}_{1}$ receptors and the possible alterations of $\mathrm{P}_{1}-\mathrm{P}_{2}$ receptor regulation processes during pathological conditions may help to clarify the patho-physiological functions of ATP and adenosine in CNS.

Abbracchio MP and Burnstock G, 1998. Jpn J Pharmacol, 78: 113-145.

Franke H and Illes P, 2006. Pharmacol Ther, 109 (3): 297-324.

Maggio R, Novi F, Scarselli M, Corsini GU, 2005. FEBS J, 272 (12): 2939-2946.

Wardas J, 2002. Pol J Pharmacol, 54: 313-326.

Yoshioda K and Nakata H, 2004. J Pharmacol Sci, 94: 88-94.

\title{
Comparison of NTPDase 1, 2 and 3 after refolding from bacterial inclusion bodies
}

\author{
Matthias Krause \& Norbert Sträter \\ From the Biotechnologisch-Biomedizinisches Zentrum, Fakultät für Chemie und Mineralogie der Universität \\ Leipzig, Deutscher Platz 5, 04103 Leipzig, Germany \\ matthias.krause@bbz.uni-leipzig.de
}

The ecto-Nucleoside triphosphate diphosphohydrolases or NTPDases are the enzymes responsible for signal termination and conversion in purinergic signalling. NTPDase1, 2 and 3 are localized on the cell surface, anchored to the membrane by two transmembrane helices. They catalyze the sequential removal of $\gamma$ - and $\beta$ - phosphate from ATP, ADP and other nucleotides.

Due to their involvement in physiological processes like blood clotting and pain perception, but also in certain types of cancer, they are now considered as potential new drug targets or drugs themselves. Soluble forms from mammalian cell culture have been shown to inhibit platelet aggregation in vivo. However, mammalian production of large amounts of therapeutic proteins is expensive and bears the risk of viral contamination. Therefore and for structural characterization we established an E. coli expression system for insoluble production of the extracellular domains of three NTPDases and optimized the in vitro refolding. The refolded proteins have been characterized in several aspects: substrate specificity, $\mathrm{K}_{\mathrm{M}}$ values for ATP and ADP, $\mathrm{pH}$ dependance and metal cofactor activation. Similarities and differences in the refolding behaviour and in enzymatic properties among the recombinant enzymes and in comparison to their wildtype counterparts will be discussed.

Our expression system allows for large scale production of active protein for further characterization and clinical application. X-ray structure determination of the refolded proteins will provide valuable insights in the rational design of medically relevant inhibitors.

\section{COMPLEX KINETICS OF P2X7-DEPENDENT SINGLE CHANNEL CURRENTS}

\author{
T Riedel $^{1}$, I Lozinsky ${ }^{1}$, G. Schmalzing ${ }^{2}$ and F Markwardt ${ }^{1}$

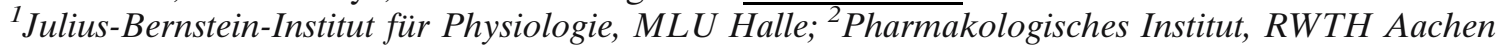 \\ fritz.markwardt@medizin.uni-halle.de
}

$\mathrm{P} 2 \mathrm{X}$ receptors are ATP-gated ion channels permeable to small inorganic cations. The $\mathrm{P} 2 \mathrm{X} 7$ receptor has several peculiar characteristics compared to the other members of the $\mathrm{P} 2 \mathrm{X}$ receptor family. The time course of activation 
and deactivation is dependent on species, number and duration of ATP applications. The initial part of activation is supposed to be so fast that it could not be investigated by conventional whole cell voltage clamp methods. Therefore, we expressed human P2X7 receptors heterologously in Xenopus oocytes and investigated the kinetics of activation and deactivation of P2X7-dependent single channel currents by means of the patch clamp method in the outside-out configuration. A combination of the U-tube technique and a piezo-driven liquid filament switch technique enabled the application and withdrawal of different concentrations of the agonist ATP $^{4-}$ within less then $1 \mathrm{~ms}$. Two different types of kinetics were observed and approximated by single exponential functions. A low-noise patch current component activated and deactivated with time constants of about $300 \mathrm{~ms}$. Its amplitude was dependent on $\left[\mathrm{ATP}^{4-}\right]$ and the seal resistance but independent of the expression of human $\mathrm{P} 2 \mathrm{X} 7$ receptors. It was attributed to non-specific effects of ATP on the seal resistance. Extracellular application of ATP evoked slightly inward rectifying single-channel currents with a conductance of about $10 \mathrm{pS}$ and mean open times of about $5 \mathrm{~ms}$ at negative membrane potentials. The mean closed times but not the mean open times were dependent on the activating ATP concentration. High ATP concentrations increase the probability of the additional opening of channels with a conductance of about $14 \mathrm{pS}$ and mean open times of about $20 \mathrm{~ms}$. At positive membrane voltages, the mean open time was increased to about $15 \mathrm{~ms}$. The channels activated with activation and deactivation time constants of about $15 \mathrm{~ms}$. The channel activation was only little dependent on the agonist concentration. Activation and deactivation were not continuously dependent on the membrane voltage. We conclude that binding of $\mathrm{ATP}^{4-}$ is not the only rate limiting step in the activation of $\mathrm{P} 2 \mathrm{X} 7$ channels and that the $\mathrm{ATP}^{4-}$ binding site is not located in the electrical field of the membrane.

This work was supported by the Deutsche Forschungsgemeinschaft (Ma 1581/12-1) and the Roux-programme of the ML university (FKZ 13/07).

\title{
Conduction of purine-induced responses in intracrebral arterioles: relative roles of endothelium and smooth muscle cells
}

\author{
Al C. Ngai ${ }^{1}$, Thien-Son K. Nguyen ${ }^{1}$, $\underline{\text { H. Richard Winn }}{ }^{2}$ \\ ${ }^{1}$ Department of Neurological Surgery, University of Washington School of Medicine, Seattle; ${ }^{2}$ Department of \\ Neurological Surgery, Mount Sinai School of Medicine, New York \\ richard.winn@mountsinai.org
}

Purines such as adenosine (Ado) and ATP elicit conducted dilation and/or constriction in cerebral arterioles. In this study, we evaluated the roles of the endothelium and smooth muscle in the conduction of vasomotor responses induced by purines along intracerebral arterioles. Penetrating intracerebral arterioles were isolated from Sprague Dawley rats, and cannulated with a concentric micropipette system. Upon pressurization to $60 \mathrm{~mm} \mathrm{Hg}$, the vessels developed spontaneous tone, contracting to $67 \pm 1 \%$ of passive diameter $(77 \pm 4 \mu \mathrm{m}, \mathrm{n}=9)$. The experimental protocol consisted of pulse-application of vasoactive agents (Ado or ATP) via micropipettes, onto a short segment of the arteriole. This induced both a direct local response, and a secondary conducted response that spread longitudinally along the vessel. Ado is an endothelium-independent dilating agent, whereas ATP-induced dilation is endothelium dependent. Pressure-pulse ejection of Ado (10 $\mathrm{mM}$ in pipette) elicited a local $14 \%$ dilation, and a $4 \%$ dilation at a site $500 \mu \mathrm{m}$ from the ejection site, whereas ATP $(10 \mathrm{mM})$ microapplication evoked biphasic local (18\% constriction, 9\% dilation) and conducted (11\% constriction, $8 \%$ dilation) responses. Endothelial cells within an $100 \mu \mathrm{m}$ segment (positioned between the application and observation sites) were injured by a luminal light-dye (L-D) technique using luminal Na Fluorescein. Alternatively, vascular smooth muscle (VSM) cells were selectively damaged by adventitial L-D treatment. Endothelial injury did not significantly affect the conducted dilation responses to Ado, but virtually eliminated the conducted responses (both dilation and constriction) to ATP. VSM injury abolished conducted dilation responses to Ado, but did not affect ATP-dilation. However, VSM injury attenuated the remote constriction responses to ATP microapplication. Our data suggest that the endothelium is the predominant pathway for the conduction of ATP-induced dilation, whereas the smooth muscle layer primarily mediates the conduction of Ado-induced dilation. Both the endothelium and smooth muscle appear to be involved in the propagation of ATP-induced constriction.

Funded by NIH Grant NS-21076 and AHA Grant 0255703N. 


\title{
CONSTITUTIVELY ACTIVE MUTANTS OF THE HUMAN ADENOSINE A 2 RECEPTOR REVEAL INVERSE AGONISM
}

\author{
Qilan $\mathrm{Li}^{1}$, Kai Ye ${ }^{1}$, Clara C. Blad ${ }^{1}$, Hans den Dulk ${ }^{2}$, Jaap Brouwer ${ }^{2}$, Ad P. IJzerman ${ }^{1}$, \\ Margot Beukers ${ }^{1}$ \\ ${ }^{1}$ Medicinal Chemistry (LACDR) and ${ }^{2}$ Molecular Genetics (LIC), Gorlaeus Laboratories, Einsteinweg 55, 2300 RA, \\ Leiden, the Netherlands. \\ Q.li@chem.leidenuniv.nl
}

The human adenosine $A_{2 B}$ receptor belongs to class A G-protein-coupled receptors. In our previous work, constitutively active mutant (CAM) human adenosine $A_{2 B}$ receptors were identified from a random mutation bank based on their ability to grow in histidine-deficient medium [1]. In the current study, three known $\mathrm{A}_{2 \mathrm{~B}}$ receptor antagonists, MRS1706, ZM241385 and DPCPX were tested on wild-type and 9 CAM A 2 B receptors with different levels of constitutive activity in a yeast growth assay.

The compounds' ability to antagonize the agonist NECA on the wild-type receptor was assessed first. The $\mathrm{pA}_{2}$ values of MRS1706, ZM241385 and DPCPX were derived from a Schild analysis, and found to be in good agreement with available literature.

Since the wild-type receptor lacks constitutive activity, we employed CAM receptors to investigate whether these antagonists could be reclassified as inverse agonists. All three compounds turned out to be inverse agonists for the adenosine $\mathrm{A}_{2 \mathrm{~B}}$ receptor as they were able to fully reverse the basal activity of the 4 low-level CAM $\mathrm{A}_{2 \mathrm{~B}}$ receptors: T42A, F84S, F84L and F84L/S95G. The basal activity of 3 medium-level CAM A 2 B receptors, N36S/T42A, T42A/ V54A and N36S/T42A/T66A, was partially inhibited.

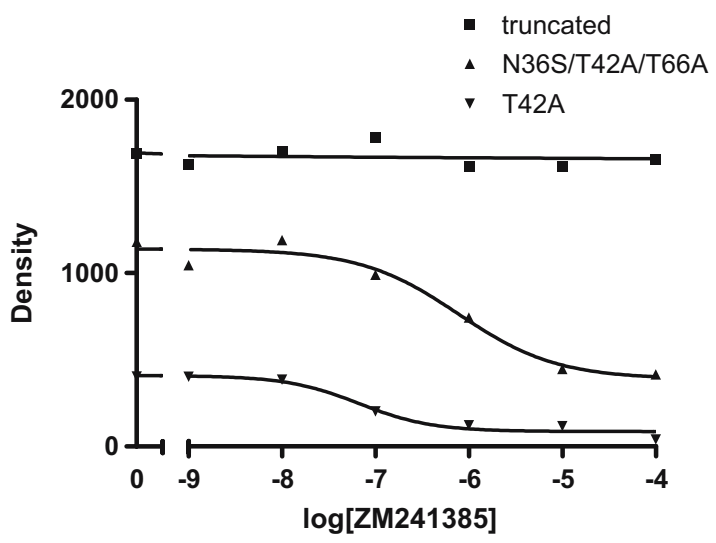

Figure 1. Inverse agonism of ZM241385 on CAM receptors with varying levels of constitutive activity.

We also discovered 2 highly constitutively active or locked mutants, A18T/A23V/C83Y/A106V/R112S and Q214L/I230N/V240M/V250M/N254Y/T257S/K269stop (a truncated receptor). Their basal activity could not be reversed by any of the three compounds. The inverse agonistic properties of ZM241385 on the T42A, N36S/T42A/ T66A and the truncated receptor are shown in figure 1 as a typical example.

The rank order of potency of the compounds matched the rank order obtained in the antagonist assay on the wildtype receptor, that is ZM241385 = MRS1706 > DPCPX. However, ZM241385 was the strongest inverse agonist as demonstrated by the rank order of intrinsic activities ZM241385 $>$ MRS1706 $=$ DPCPX.

In conclusion, this study is the first to describe inverse agonism on the human adenosine $A_{2 B}$ receptor. Moreover, the use of receptor mutants with varying levels of constitutive activity enabled us to appreciate differences in intrinsic activity of the inverse agonists.

${ }^{1}$ : Beukers et al. Mol Pharmacol (2004) 65: 702. 


\title{
Contractile Effects of Adenosine, Coronary Flow and Perfusion Pressure in Murine Myocardium
}

\author{
Laura Willems and John P. Headrick \\ Heart Foundation Research Centre, Griffith University Southport, QLD 4217, Australia \\ j.headrick@griffith.edu.au
}

There is mixed evidence adenosine receptors (ARs) may directly enhance myocardial contractility, though this remains contentious. Identifying such effects is important in clarifying physiological and protective functions of adenosine. We assessed inotropic actions of adenosine $(50 \mu \mathrm{M})$ and selective AR activation with $100 \mathrm{nM} \mathrm{N}^{6}$ cyclohexyladenosine (CHA; $\mathrm{A}_{1} \mathrm{AR}$ agonist), $25 \mathrm{nM}$ 2-[p-(2-carboxyethyl) phenethylamino]-5'-N-ethylcarboxamidoadenosine (CGS-21680; $\mathrm{A}_{2 \mathrm{~A}} \mathrm{AR}$ agonist), and $100 \mathrm{nM}$ 2-chloro- $\mathrm{N}^{6}$-(3-iodobenzyl)-adenosine-5'-N-methyluronamide (Cl-IB-MECA; $\mathrm{A}_{3} \mathrm{AR}$ agonist) in $\mathrm{C} 57 / \mathrm{BL} / 6 \mathrm{~J}$ mouse hearts paced at 420 beats $/ \mathrm{min}$, and perfused at constant-pressure, constant-flow, or under conditions of stable flow and pressure (achieved with nitroprussidemediated vasodilatation in constant-flow hearts, prior to AR activation). Adenosine and CGS-21680 induced significant (albeit modest) positive inotropy in constant-pressure perfused hearts (up to $10 \mathrm{mmHg}$ elevations in systolic force). Inotropic effects of adenosine and CGS-21680 paralleled coronary vasodilatation (with up to $10 \mathrm{ml} /$ $\mathrm{min} / \mathrm{g}$ elevations in flow). Neither CHA or Cl-IB-MECA altered force or flow. Under conditions of constant flow, adenosine and CGS-21680 reduced systolic pressure in parallel with coronary perfusion pressure. When changes in coronary flow and perfusion pressure were prevented (eliminating potential Gregg-related effects), CGS-21680 no longer modified contractile force. However, adenosine itself still significantly enhanced systolic pressure by up to $10 \mathrm{vmmHg}$, independently of changes in coronary flow, perfusion pressure, and heart rate. Relations between flow, perfusion pressure, and ventricular performance evidence significant Gregg-related effects in murine myocardium - ventricular systolic pressure increases transiently by $\sim 1 \mathrm{mmHg}$ per $\mathrm{ml} / \mathrm{min} / \mathrm{g}$ change in flow during the initial 1-2 min of hyperemia, and in a sustained manner by $\sim 1 \mathrm{mmHg}$ per $\mathrm{mmHg}$ change in coronary perfusion pressure. These effects contribute to inotropic effects of AR agonists when coronary flow or pressure are uncontrolled. In summary, we find no evidence of direct $A_{1}$ or $A_{3} A R$-mediated changes in contractility in intact myocardium. Inotropic actions of $\mathrm{A}_{2 \mathrm{~A}} \mathrm{AR}$ agonism are indirect, involving Gregg-related effects. Despite no $\mathrm{A}_{1}$, $\mathrm{A}_{2 \mathrm{~A}}$ or $\mathrm{A}_{3} \mathrm{AR}$-mediated effects, the endogenous agonist adenosine exerts a modest inotropic action independently of flow and perfusion pressure. The mechanistic basis of this response remains to be identified.

\section{Contribution of PKC to the desensitisation of relaxation to luminally-perfused purinoceptor agonists in rat small mesenteric arteries}

\author{
Polly Winter \& Kim. A. Dora \\ Department of Pharmacy \& Pharmacology, University of Bath, Bath BA2 7AY, UK \\ pr9pw@bath.ac.uk
}

Protein kinase $\mathrm{C}(\mathrm{PKC})$ contributes to the desensitisation of the $\mathrm{P}_{2} \mathrm{Y}_{1}$ purinoceptor in response to prolonged treatment with the non-hydrolyzable analogue of ADP, adenosine $5^{\prime}-[\beta$-thio]diphosphate (ADP $\beta S$ ) in isolated blood platelets [1]. The aim of this study was to ascertain the extent of PKC modulation of the vasodilatation of small rat mesenteric arteries in response to luminal perfusion of ATP, the non-hydrolysable analogue of ATP, adenosine-5'-(3-thiotriphosphate) ATP $\gamma \mathrm{S}$, and ADP $\beta$ S.

Segments of third order mesenteric arteries were isolated from male Wistar rats (200-250 g), killed by schedule 1 methods of the Animals Scientific Procedures Act 1986 (UK). Arteries were cannulated, mounted in a pressure myograph (Danish Myotechnology), pressurized to $50 \mathrm{mmHg}$ and perfused with MOPS at $37^{\circ} \mathrm{C}$. Following precontraction with phenylephrine a multi-channel syringe pump was used to perfuse agonists through the lumen at $90 \mu \mathrm{l} \cdot \mathrm{min}^{-1}$. Once the peak relaxation response was established the time course of desensitisation of the relaxation response was assessed over a 2 min period. Values are means \pm s.e.mean of the percentage relaxation to maximum diameter.

Luminal perfusion of ATP $(3 \mu \mathrm{M})$ and ATP $\gamma \mathrm{S}(3 \mu \mathrm{M})$ resulted in peak vasodilatation responses of $91.3 \pm 1.5 \%$ and $80.5 \pm 4.0 \%$ respectively. These levels of relaxation were maintained throughout the 2 min post-peak period 
(3 $\mu \mathrm{M}$ ATP, $96.4 \pm 0.8 \% ; 3 \mu \mathrm{M}$ ATP $\gamma \mathrm{S}, 78.4 \pm 12.1 \%)$ and were unchanged following luminal incubation (15 min) with the selective inhibitor of PKC, bisindolylmaleimide 1 (BIS $1 \mu \mathrm{M})(3 \mu \mathrm{M}$ ATP, $94.6 \pm 1.4 \% ; 3 \mu \mathrm{M}$ ATP $\gamma \mathrm{S}, 88.8$ $\pm 1.5 \%)$. In contrast, luminal perfusion of ADP $\beta S(1 \mu \mathrm{M})$ and ATP $(1 \mu \mathrm{M})$ resulted in relaxation responses of 70.0 $\pm 6.6 \%$ and $40.5 \pm 8.2 \%$ respectively that decayed over the 2 min post-peak period $(1 \mu \mathrm{M}$ ADP $\beta S, 41.5 \pm 6.1 \% ; 1$ $\mu \mathrm{M}$ ATP, $28.9 \pm 9.3 \%)$. Prior luminal incubation with BIS increased the peak relaxation response to ADP $\beta S$ (1 $\mu \mathrm{M})(94.8 \pm 1.0 \%, P<0.05$; students paired t-test $)$ and ATP $(1 \mu \mathrm{M})(70.4 \pm 14.5 \%)$. The decay of the responses observed in response to these two agonists was also significantly reversed $(1 \mu \mathrm{M}$ ADP $\beta S, 89.1 \pm 6.6 \%$; $1 \mu \mathrm{M}$ ATP, $67.9 \pm 13.8 \%, P<0.01$, students paired t-test).

PKC has been found to contribute to the desensitisation of the relaxation response to ADP $\beta S(1 \mu \mathrm{M})$ and ATP $(1$ $\mu \mathrm{M})$ supporting a role for $\mathrm{PKC}$ in the desensitisation of $\mathrm{P}_{2} \mathrm{Y}_{1}$ receptors within the endothelium of isolated rat mesenteric arteries. Activation of $\mathrm{P}_{2} \mathrm{Y}_{2}$ or $\mathrm{P}_{2} \mathrm{Y}_{4}$ receptors with $\mathrm{ATP} \gamma \mathrm{S}(3 \mu \mathrm{M})$ did not evoke a response that decayed over time. Variation in the downstream pathways beyond each P2Y purinoceptor subtype may therefore exist.

This work was kindly supported by the British Heart Foundation

1) Hardy, A.R. et al., 2005. Blood., 105 (9); 3552-3560

\section{Contributions of P2Y and P2X receptors to nucleotide-induced signaling in macrophages revealed by single-cell $\mathrm{Ca}^{2+}$ measurements and knockout mice}

Adriana del Rey ${ }^{1}$, Vijay Renigunta ${ }^{1}$, Alexander H. Dalpke ${ }^{2}$, Jens Leipziger ${ }^{3}$, Joana E. Matos ${ }^{3}$, Bernard Robaye $^{4}$, Marylou Zuzarte ${ }^{1}$, Annemieke Kavelaars ${ }^{5}$ and Peter J. Hanley ${ }^{1}$

${ }^{1}$ Institute of Physiology, Marburg University, Germany; ${ }^{2}$ Department of Hygiene \& Medical Microbiology, Heidelberg University, Germany; ${ }^{3}$ Institute of Physiology \& Biophysics, Aarhus University, Denmark; ${ }^{4}$ Institute of Interdisciplinary Research, Université Libre de Bruxelles, Belgium; and ${ }^{5}$ Psychoneuroimmunology, University Medical Center Utrecht, The Netherlands

hanley@mailer.uni-marburg.de

Antigen-presenting cells express two families of nucleotide receptors: ATP-gated cation channels (P2X receptors) and $\mathrm{P} 2 \mathrm{Y}$ receptors, a subset of the $\mathrm{G}$ protein-coupled receptor superfamily. To identify the receptors mediating the $\mathrm{Ca}^{2+}$ responses of resident peritoneal macrophages to extracellular UTP, ATP and UDP, we performed $\mathrm{Ca}^{2+}$ measurements on single cells isolated from various knockout mice. To obviate the confounding effects of extracellular $\mathrm{Ca}^{2+}$ sources, experiments were performed in $\mathrm{Ca}^{2+}$-free buffer. Note that when ATP is applied to a macrophage in the presence of $\mathrm{Ca}^{2+}$, the observed increase in cytosolic $\left[\mathrm{Ca}^{2+}\right]\left(\left[\mathrm{Ca}^{2+}\right]_{\mathrm{i}}\right)$ can be attributed to endoplasmic reticular (ER) $\mathrm{Ca}^{2+}$ release, $\mathrm{Ca}^{2+}$ influx through $\mathrm{Ca}^{2+}$ release-activated $\mathrm{Ca}^{2+}$ (CRAC) channels or $\mathrm{Ca}^{2+}$ influx via P2X receptors (Figure 1). We found that application of UTP (or ATP), under $\mathrm{Ca}^{2+}$-free conditions, to macrophages isolated from wild-type mice induced a transient (lasting $1 \mathrm{~min}$ ) increase in $\left[\mathrm{Ca}^{2+}\right]_{\mathrm{i}}$ with halfmaximal response at concentrations $<1 \mu \mathrm{M}$. However, macrophages isolated from $\mathrm{P}_{2} \mathrm{Y}_{2} / \mathrm{P}_{2} \mathrm{Y}_{4}$ double knockout mice did not respond to either UTP or ATP. Furthermore, the nucleotide-induced $\mathrm{Ca}^{2+}$ response of macrophages isolated from $\mathrm{P}_{2} \mathrm{Y}_{4}-/-$ mice was normal whereas cells from $\mathrm{P}_{2} \mathrm{Y}_{2}-/-$ mice were completely unresponsive. Thus, P2 $Y_{2}$ is the sole receptor which couples extracellular UTP or ATP to phospholipase C- $\beta$ (PLC- $\beta$ ) activity and ER $\mathrm{Ca}^{2+}$ release. In contrast to UTP, UDP (purified with hexokinase) was an essentially ineffective agonist in both $\mathrm{P}_{2} \mathrm{Y}_{2}$-deficient and wild-type macrophages, suggesting that the $\mathrm{P} 2 \mathrm{Y}_{6}$ receptor is weakly expressed. In $\mathrm{P} 2 \mathrm{Y}_{2}-1-$ macrophages, though, the $\mathrm{Ca}^{2+}$ responses to 'pure' $\mathrm{P} 2 \mathrm{X}$ receptor activation could be studied by applying ATP in the presence of extracellular $\mathrm{Ca}^{2+}$. 


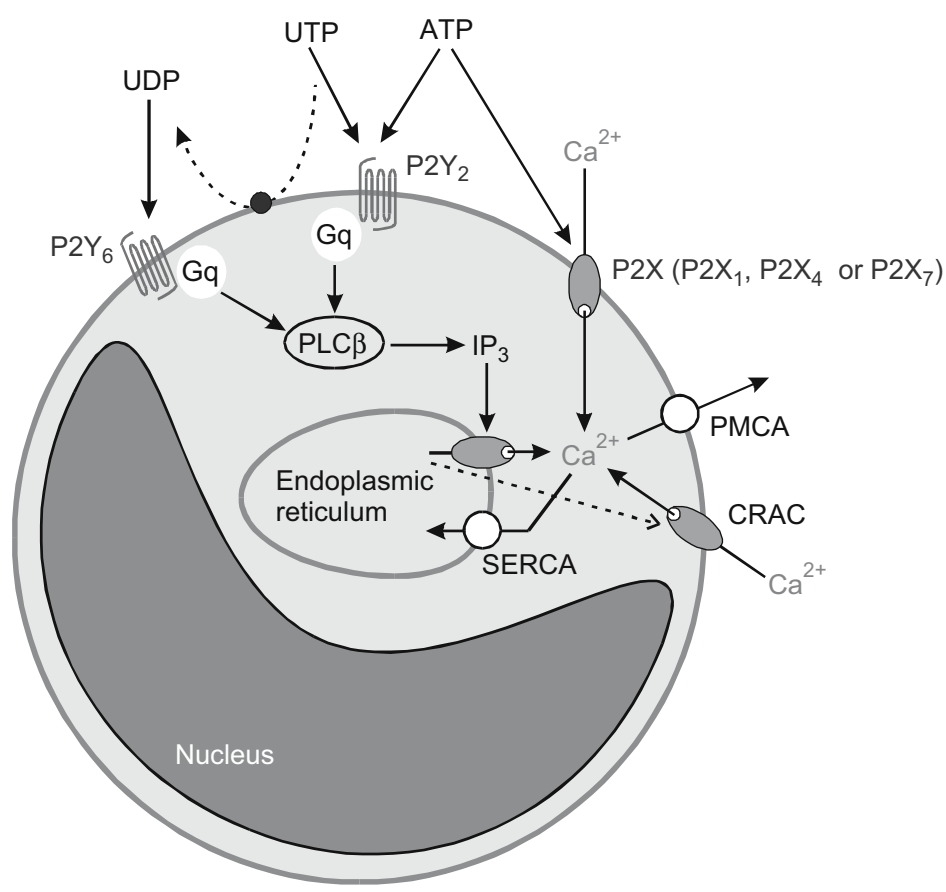

Figure 1. Schematic diagram of $\mathrm{Ca}^{2+}$ signaling in a mouse macrophage induced by extracellular nucleotides.

\title{
Conventional protein kinase $\mathrm{C}$ isoforms mediate human sperm capacitation induced by $A_{1}$ adenosine receptor agonist
}

\author{
Lavinia Liguori, Rossana Palazzo, Ilaria Bellezza \& Alba Minelli \\ Dipartimento di Medicina Sperimentale Scienze Biochimiche, Sezione Biochimica Cellulare, Università di Perugia, \\ via del Giochetto, 06123, Perugia, Italia. \\ albaminelli@virgilio.it
}

Mammalian ejaculated spermatozoa are infertile and the acquisition of the fertilizing capacity is obtained via a sequential two-steps activation process, named capacitation and acrosome reaction. Capacitation, regarded as a signal transduction receptor-mediated phenomenon, is an emerging concept $(1,2)$ and our data, showing that the stimulation of $\mathrm{A}_{1} \mathrm{AR}$ triggers a cascade of signalling events that leads to capacitation, support this concept. The constant presence of $\mathrm{A}_{1} \mathrm{AR}$ on spermatozoa suggested a functional role (3), later identified either in human or in murine species as capacitative $(4,5)$. Murine spermatozoa, lacking the $\mathrm{A}_{1} \mathrm{AR}$, showed a delayed capacitation indicating that the $\mathrm{A}_{1} \mathrm{AR}$ modulates the velocity of the process without affecting the number of capacitated cells (4). We have already shown that the signalling initiated by $A_{1} A R$ proceeds via PLC and IP3 release (5). The comparative analysis of the effects of CCPA and FCSu during a $3.5 \mathrm{~h}$ incubation shows that CCPA is as effective as FCSu, a known inducer of capacitation, although it does not stimulate $\mathrm{O}_{2}{ }^{-}$- production and does not cause an increase of the phosphorylation either of the tyrosine residues or of the Thr-Glu-Tyr-motif suggesting that when the $A_{1} A R$ is activated, its signalling proceeds via kinase-induced events that target proteins different from those obseved in the presence of FCSu. Treatment of human spermatozoa with different protein kinases inhibitors shows that receptor type PTK, PKA, and PI3K are not involved in the CCPA-mediated response whereas PKC is responsible for the capacitative effect. All the PKC inhibitors tested, acting with different mechanism and on different PKC isoforms, caused a significant decrease of CCPA-induced capacitation, indicating that all these PKCs are activated via $\mathrm{A}_{1} \mathrm{AR}$. This result was not unexpected after the reported involvement of PLC in the $\mathrm{A}_{1} \mathrm{AR}$ signalling (5). Kinexus analysis of the phosphorylation levels of PKC isoforms after CCPA stimulation shows the activation of the alpha isoform and disactivation of the gamma. PKC alpha isoform has been related to alteration of cell permeability whereas PKCgamma isoform has been linked to G $\beta$ phosphorylation thereby en- 
hancing the potency of $\mathrm{G} \beta \gamma$ to stimulate adenylyl cyclase activity. Our results are consistent with the increased permeability occurring during the capacitative event and with the shift to Gai inhibitory signalling induced by $\mathrm{A}_{1} \mathrm{AR}$ activation.

1 Mededovic, S., and Fraser, L.R. (2005) Mechanisms of action of angiotensin II on mammalian sperm function. Reproduction, 129, 211-218.

2 Rossato, M., Ion Popa, F., Ferigo, M., Clari, G., and Foresta, C. (2005) Human sperm express cannabinoid receptor $\mathrm{Cb} 1$, the activation of which inhibits motility, acrosome reaction, and mitochondrial function. $J$ Clin Endocrinol Metab, 90, 984-991.

3 Minelli, A., Allegrucci, C., Piomboni, P., Mannucci, R., Lluis, C., and Franco, R. (2000) Immunolocalization of A1 adenosine receptors in mammalian spermatozoa. J. Histochem. Cytochem, 48, 1163-1171.

4 Minelli, A., Liguori, L., Bellezza. I., Mannucci, R., Jhoansson, B., and Fredholm, BB. (2004) Involvement of A1 adenosine receptors in the acquisition of fertilizing capacity. J. Androl, 25, 286-292.

5 Allegrucci, C., Liguori, L., and Minelli A. (2001) Stimulation by $\mathrm{N}^{6}$-cyclopentyladenosine of A1 adenosine receptors, coupled to $\mathrm{G} \alpha$ i2 protein subunit, has a capacitative effect on human spermatozoa. Biol. Reprod, 64, 1653-1659.

\title{
Conversion of Nucleoside-Based $A_{3}$ Adenosine Receptor Agonists into Potent and Selective Antagonists: Overcoming the Problem of Species Differences
}

\author{
$\underline{\text { Kenneth A. Jacobson }}^{1}$, Bhalchandra V. Joshi ${ }^{1}$, Athena Klutz ${ }^{1}$, Soo-Kyung Kim ${ }^{1}$, Hyuk Woo Lee ${ }^{2}$, Hea
} $\mathrm{Ok} \mathrm{Kim}^{2}$, Lak Shin Jeong ${ }^{2}$ and Zhan-Guo Gao ${ }^{1}$

${ }^{1}$ Molecular Recognition Section, Laboratory of Bioorganic Chemistry, National Institute of Diabetes and Digestive and Kidney Diseases, National Institutes of Health, Bethesda, MD 20892, USA.

${ }^{2}$ Laboratory of Medicinal Chemistry, College of Pharmacy, Ewha Womans University, Seoul 120-750, Korea kajacobs@helix.nih.gov

We have systematically probed the effects of base and ribose substitution of adenosine on both the affinity and intrinsic efficacy at the $\mathrm{A}_{3}$ adenosine receptor (AR). The abilities of a nucleoside to bind to and to activate the receptor are structurally distinct. Based on this observation we designed new nucleoside antagonists of the $\mathrm{A}_{3} \mathrm{AR}$, which bind potently and selectively, but do not activate the receptor. The goal is to convert agonists that are $\mathrm{A}_{3}$ AR-selective in both human and rat into species independent $\mathrm{A}_{3} \mathrm{AR}$ antagonists. Previously, the spirolactam nucleoside derivative MRS1292 was designed as a cross-species $\mathrm{A}_{3} \mathrm{AR}$ antagonist, based on chemical constraint of the ribose moiety [1]. This derivative was shown to lower intraocular pressure in the mouse, supporting the use of $\mathrm{A}_{3}$ antagonists in the treatment of glaucoma [2]. The highly selective agonists of the $\mathrm{A}_{3} \mathrm{AR}, \mathrm{Cl}$-IB-MECA (2chloro- $N^{6}$-(3-iodobenzyl)-5'-N-methylcarboxamidoadenosine) and its $4^{\prime}$-thio analogue, were successfully converted into selective antagonists simply by appending a second $N$-methyl group on the $5^{\prime}$-uronamide position [3]. The 2-chloro-5'-( $N, N$-dimethyl)uronamido analogues bound to, but did not activate the human $\mathrm{A}_{3} \mathrm{AR}$, with $\mathrm{K}_{\mathrm{i}}$ values of $29 \mathrm{nM}\left(4^{\prime}-\mathrm{O}, \mathrm{MRS} 3771\right)$ and $15 \mathrm{nM}\left(4^{\prime}-\mathrm{S}\right.$, LJ1256), showing $>100$-fold selectivity over $\mathrm{A}_{1}, \mathrm{~A}_{2 \mathrm{~A}}$, and $\mathrm{A}_{2 \mathrm{~B}} \mathrm{ARs}$. Competitive antagonism was demonstrated by Schild analysis. We used rhodopsin-based, dynamic molecular modeling of the $\mathrm{A}_{3} \mathrm{AR}$ and ligand docking to characterize the putative ligand-binding region and identified distinct amino acid residues related to binding of ligands, such as agonist Cl-IB-MECA and antagonist MRS3771, and activation. In general, removing H-bonding ability or introducing steric rigidity in the region of the ribose $5^{\prime}$ uronamide lowers efficacy at the $\mathrm{A}_{3} \mathrm{AR}$, consistent with predictions derived from modeling. 

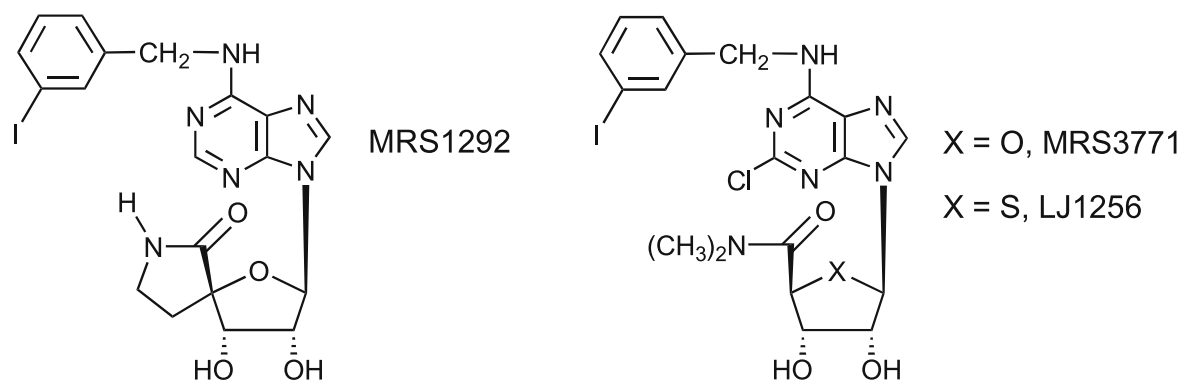

1. Gao, Z.G., et al. J. Med. Chem. 2002, 45, 4471-4484.

2. Yang, H., et al. Current Eye Res. 2005, 30, 747-754.

3. Gao, Z.G., et al. Bioorg. Med. Chem. Lett. 2006, 16, 596-601.

\title{
Cooperation between Gq and Gs-linked signaling pathways in regulation of IL-4 by adenosine in mast cells
}

\author{
Sergey Ryzhov, Anna E. Goldstein, Italo Biaggioni and Igor Feoktistov \\ Vanderbilt University, Nashville, TN 37232, USA. \\ igor.feoktistov@vanderbilt.edu
}

Adenosine provokes bronchoconstriction in asthmatics through acute activation of mast cells and plays an important role in chronic inflammation by upregulating Th2 cytokines in mast cells, thus promoting IgE synthesis by B-lymphocytes. This effect is mediated by $\mathrm{A}_{2 \mathrm{~B}}$ receptors because neither the selective $\mathrm{A}_{2 \mathrm{~A}}$ agonist CGS21680 nor the selective $A_{3}$ agonist IB-MECA reproduced it, and the selective $A_{2 B}$ antagonist IPDX prevented it. To get insight into molecular mechanisms underlying this unique role of $\mathrm{A}_{2 \mathrm{~B}}$ adenosine receptors in promoting Th2 inflammation, we studied intracellular pathways linking this receptor to upregulation of IL-4 in HMC-1 mast cells. $\mathrm{A}_{2 \mathrm{~B}}$ receptors stimulate adenylate cyclase via coupling to Gs proteins, and stimulate phospholipase $\mathrm{C} \beta$ through coupling to Gq proteins. Inhibition of phospholipase C $\beta$ with U73122 completely blocked adenosine-induced IL-4 secretion. The protein kinase inhibitor Ro-32-0432 had no effect on $\mathrm{A}_{2 \mathrm{~B}}$-mediated, but inhibited PMA-stimulated IL-4 secretion. In contrast, chelation of intracellular $\mathrm{Ca}^{2+}$ inhibited both adenosine and ionomycin-induced IL-4 secretion. This $\mathrm{Ca}^{2+}$-sensitive pathway most likely includes calcineurin and NFAT, because adenosine-induced IL4 secretion was blocked with cyclosporine A or 11R-VIVIT peptide. Gs-linked pathways also play a role in the $\mathrm{A}_{2 \mathrm{~B}}$-dependent stimulation of IL-4 secretion; inhibition of adenylate cyclase with $2^{\prime}, 5^{\prime}$-dideoxyadenosine, or protein kinase A with Rp-cAMP or H-89, attenuated adenosine-dependent IL-4 secretion. Although stimulation of adenylate cyclase with forskolin did not increase IL-4 secretion on its own, it potentiated the effect of Pasteurella multocida toxin by 2 -fold and ionomycin by 3 -fold. Both forskolin and adenosine upregulated NFATc1 expression. We conclude that $\mathrm{A}_{2 \mathrm{~B}}$ receptors upregulate IL-4 through Gq signaling that is potentiated via crosstalk with Gs-coupled pathways. Thus, our data explain the necessity and underscore the importance of dual coupling of $A_{2 B}$ receptors to $\mathrm{Gs} / \mathrm{Gq}$ proteins with concurrent stimulation of diverse intracellular pathways for adenosine-dependent regulation of IL-4 production in human mast cells.

\section{Cysteine scanning mutagenesis of the extracellular loop region 286-329 of the Human P2X 1 receptor.}

Jonathan Roberts* and Richard J. Evans

Department of Cell Physiology \& Pharmacology, University of Leicester, Leicester, LE1 9HN, UK; *jar20@le.ac.uk

As P2X receptors show little homology to other ATP binding proteins that have been crystallized models of agonist binding to $\mathrm{P} 2 \mathrm{X}$ receptors have been developed from analysis of site-directed mutagenesis studies. Alanine 
mutagenesis of conserved amino acids has highlighted key residue K309, proposed to co-ordinate ATP phosphate group binding, and the motif N290F291R292 co-ordinating binding of the adenine ring at $\mathrm{P}_{2} \mathrm{X}_{1}$ receptors. To further develop the P2X receptor model and investigate the role of N290F291R292 and K309, adjacent nonconserved amino acids and the contribution of the 20 amino acid linker to the second transmembrane domain, we have undertaken substituted cysteine scanning mutagenesis (SCAM) of the region S286-I329 of the human P2X receptor. Analysis of the effects of these mutants on ATP potency, regulation by methanethiosulphonate compounds and determination of the accessibility of residues in a MTSEA-biotinylation assay has allowed us to identify amino acids involved in ATP binding, channel properties and information on the secondary structure of this section of the extracellular domain.

Cysteine substitution was well tolerated and functional responses were recorded from all of the mutant channels. R295 and R305 however produced small currents of 16 and 94 nA respectively (10mM ATP). Substitution by cysteine reduced ATP potency at N290C, F291C, R292C and K309C receptors $(\sim 125,135,20$ and 240 fold respectively) supporting previous alanine mutagenesis. At the majority of residues (27/44) MTSEA (+ve charge) had no affect on ATP activation but caused a large inhibition of ATP responses for mutants N290C, R292C, G321C and $\mathrm{I} 328 \mathrm{C}$ (4\%, 4\%, 41\% and 41\% relative to control respectively). In contrast at mutants F291C and K309C addition of MTSEA potentiated ATP evoked responses by $164 \%$ and $168 \%$ relative to control respectively. MTSES (-ve charge) had no effect on ATP response at the majority of residues (34/44) but significantly inhibited ATP response at N290C, R292C, K309C, D316C and G321C (41\%, 10\%, 6\%, 69\% and 44\% relative to control respectively). F291C and A323C were both potentiated by $129 \%$ and $138 \%$ relative to control respectively. At N290, F291, R292 and K309 modulation of ATP responses by MTS reagents were coupled to a change in ATP potency indicating that these residues are involved in ATP binding. In contrast for mutants D316C, G321C, A323C and I328C, located in the linker region near to the channel pore, MTS reagents had no effect on ATP potency indicating involvement with gating or ionic flow. MTSEA biotin labels accessible cysteine residues and indicates possible local environment secondary structure around N290F291R292, K309 and the 20 amino acid linker region. Western blotting with $\mathrm{P}_{2} \mathrm{X}_{1} \mathrm{Ab}$ on MTSEA biotin treated samples immunoprecipitated with streptavidin agarose showed three areas of accessibility, firstly 286-294 which contains the ATP binding region (N290F291R292), secondly 300-303 which contains a glycosylation motif (NGT) and thirdly the linker region close to the channel pore (D327-I329C). Interestingly K309 was not accessible to MTSEA biotin possibly due to the conformation of the binding pocket. These results provide a strong body of evidence supporting ATP binding at

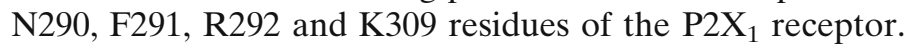

Supported by the Wellcome Trust

\section{Co-release of ATP, NPY and NA from peripheral sympathetic nerves, modulation by CGRP and bradykinin.}

J.P. Huidobro-Toro and M.V. Donoso.

Centro Regulación Celular y Patología, Instituto MIFAB, Departamento Fisiología, Facultad Ciencias Biológicas, P. Universidad Católica de Chile, Casilla 114-D, Santiago, Chile.

jphuid@bio.puc.cl

To ascertain the extracellular role of purines in synaptic transmission and particularly adenosine $5^{\prime}$ triphosphate (ATP) as a sympathetic co-transmitter, we investigated whether ATP is co-released together with noradrenaline (NA) and neuropeptide Y (NPY) from sympathetic nerve terminals. To examine the role of peptides as modulators of sympathetic co-transmission, we investigated whether calcitonin gene related peptide (CGRP) or bradykinin (BK) modulated the co-release of the sympathetic co-transmitters ATP, NA, and NPY. We used the prostatic half of the rat vas deferens from adult Sprague Dawley rats, a preparation enriched in sympathetic nerve endings. The tissues were mounted in superfusion baths and maintained with Tyrode buffer $\left(37^{\circ} \mathrm{C}\right)$ supplemented with $1 \mathrm{mM}$ desipramine, gassed with $95 \% \mathrm{O} 2 / 5 \% \mathrm{CO} 2$ and superfused at a flow of $2 \mathrm{ml} / \mathrm{min}$. Neurotransmitter release was elicited by electrical field stimulation $(16 \mathrm{~Hz}, 60 \mathrm{~V}, 1 \mathrm{~ms}$ during $1 \mathrm{~min})$. All 3 sympathetic co-transmittters were assayed in a same sample aliquot from the collecting fluid. The overflow of ATP, NA and NPY were analytically quantified from a same aliquot sample of the perfusion buffer. As controls, non-stimulated buffer samples were also analyzed. Neurochemicals were determined by HPLC procedures: electrochemical detection quantified NA, while ATP and metabolites were detected as fluorescent derivatives. RIA quantified NPY. Ele- 
ctrical nerve ending depolarization elicited the co-release of ATP and NA plus NPY. The time course of ATP and NA overflow was different; the release of NA was considerably delayed as compared to ATP. These differences in the time course of overflow might indicate differential rates of diffusion from the tissues. The net outflow of ATP was 4-10 times larger than the outflow of NA. The application of 10 nM CGRP, a concentration that reduced the twitching of the electrically evoked muscle contraction, significantly reduced the overflow of ATP, NA and irNPY released by electrical stimulation. $30 \mathrm{nM}$ BK, which increased 3-fold the electrically evoked smooth muscle twitching, halved the overflow of ATP and the outflow of adenosine from $3421 \pm 526$ to $1403 \pm 131 \mathrm{pmol}(\mathrm{p}<0.05$, $\mathrm{n}=4)$. The total NA and ir-NPY overflow was also reduced by BK, as shown in the following Table:

\begin{tabular}{cccc}
\hline Control & BK & CGRP & \\
ATP (pmol) & $95.79 \pm 23.54(7)$ & $45.56 \pm 35.36(3)$ & $37.74 \pm 10.65(3)$ \\
NA (pmol) & $46.92 \pm 9.22(7)$ & $19.24 \pm 8.40^{*}(3)$ & $11.75 \pm 4.98^{*}(3)$ \\
Ir-NPY (fmol) & $32.05 \pm 11.20(4)$ & $15.56 \pm 4.43(2)$ & $12.08 \pm 0.91(2)$ \\
\hline
\end{tabular}

In conclusion, electrical nerve ending depolarization elicited the overflow of extracellular ATP and NA consonant with their proposed co-transmitter role in sympathetic neuroeffector junctions. The much larger release of ATP might be related to its affinity for excitatory postjunctional $\mathrm{P} 2 \mathrm{X}$ receptors, highlighting its role in sympathetic cotransmission. CGRP is a presynaptic sympathetic modulator, while BK has a dual action on sympathetic neuroeffector junctions highlighting the modulator role of neuropeptides in sympathetic co-transmission. The intracellular signaling mechanisms triggered by CGRP and BK to control sympathetic co-transmission is under investigation. Funded by FONDAP 13980001 and MIFAB.

\section{CP-532,903 provides cardioprotection in two different mouse models of ischemia/reperfusion injury via an $A_{3}$ adenosine receptor-mediated mechanism}

Tina C. Wan, Zhi-Dong Ge, John A. Auchampach.

Department of Pharmacology and Toxicology, Medical College of Wisconsin, WI, USA

twan@mcw.edu

CP-532,903 is a $3^{\prime}$-aminoadenosine- $5^{\prime}$-uronamide derivative shown previously to be a highly selective agonist for the human and rabbit $\mathrm{A}_{3}$ adenosine receptor (AR). The goal of this study was to determine the selectivity of CP$532,903(\mathrm{CP})$ for the mouse $\mathrm{A}_{3} \mathrm{AR}$ and to characterize its cardioprotective profile in an in vivo mouse model of infarction and an isolated mouse heart model of global ischemia/reperfusion injury. In vitro binding assays conducted with HEK 293 cells expressing recombinant mouse ARs and [ $\left.{ }^{125} \mathrm{I}\right] \mathrm{I}-\mathrm{AB}-\mathrm{MECA}$ determined that CP binds to the mouse $\mathrm{A}_{3} \mathrm{AR}$ with high affinity $(9.6 \pm 2.5 \mathrm{nM})$ and $\sim 100$-fold selectivity versus the $\mathrm{A}_{1} \mathrm{AR}$. In cAMP assays, $\mathrm{CP}$ did not stimulate cAMP production in HEK 293 cells expressing either the recombinant mouse $\mathrm{A}_{2 \mathrm{~A}} \mathrm{AR}$ or the $\mathrm{A}_{2 \mathrm{~B}} \mathrm{AR}$ at a concentration of $10 \mu \mathrm{M}$, demonstrating poor potency of the compound for mouse $\mathrm{A}_{2} \mathrm{ARs}$. Infarct size in control mice subjected to 30 min of coronary occlusion and $24 \mathrm{~h}$ of reperfusion was $57 \pm 2 \%$ of the area at risk. In mice pretreated with 30 or $100 \mu \mathrm{g} / \mathrm{kg}$ of $\mathrm{CP}$, infarct size was significantly reduced to $43 \%$ and $39 \%$ of the area at risk, respectively. Administration of $\mathrm{CP}$ produced a short-lived reduction in mean arterial blood pressure and significantly increased plasma histamine levels, responses likely due to $\mathrm{A}_{3} \mathrm{AR}$-mediated degranulation of mast cells. In the isolated mouse heart model, coronary infusion of 10,30 or $100 \mathrm{nM} \mathrm{CP}$ for 10 minutes prior to $20 \mathrm{~min}$ of global ischemia and $45 \mathrm{~min}$ of reperfusion produced a concentration-dependent improvement in recovery of left ventricular developed pressure and $+/-\mathrm{dP} / \mathrm{dt}(\sim 30 \%$ at 30 and $100 \mathrm{nM})$ compared to vehicle-treated hearts. In contrast to the in vivo studies, infusion of $\mathrm{CP}$ in isolated hearts produced no changes in hemodynamic parameters. The selectivity of $\mathrm{CP}$ in the two mouse models for the $\mathrm{A}_{3} \mathrm{AR}$ was confirmed by repeating the studies using $\mathrm{A}_{3} \mathrm{AR}$ gene 'knock-out' mice. We conclude that the $\mathrm{A}_{3} \mathrm{AR}$ agonist $\mathrm{CP}$ provides protection against ischemia/reperfusion injury in mouse hearts by activating the $\mathrm{A}_{3} \mathrm{AR}$. 


\section{Critical Role of 5' - Ectonucleotidase (CD73) in Cardiac Ischemic Preconditioning}

Tobias Eckle $^{1}$, Almut Grenz ${ }^{2}$, Marion Faigle ${ }^{1}$, Thomas Weissmüller ${ }^{1}$, Linda Thompson ${ }^{3}$, Sean P. Colgan $^{4}$, Hartmut Oßwald ${ }^{2}$ and Holger K. Eltzschig ${ }^{1,4}$

${ }^{1}$ Clinic of Anesthesiology and Intensive Care Medicine, and ${ }^{2}$ Institute of Pharmacology, Tübingen University Hospital, ${ }^{3}$ Department of Microbiology and Immunology, University of Oklahoma Health Sciences Center, and ${ }^{4}$ Center for Experimental Therapeutics and Reperfusion Injury, Brigham and Women's Hospital, Harvard Medical School, Boston, MA 02115, USA

${ }^{1}$ t.eckle@arcor.de

Acute myocardial infarction (AMI) is among the leading causes of morbidity and mortality in the western countries. Cardiac ischemic preconditioning (IP) has been described as the strongest form of in vivo protection during AMI. Some evidence suggests that increased nucleotide phosphohydrolysis via CD73 [conversion of adenosine monophosphate (AMP) to adenosine] and nucleoside signaling via adenosine receptors ${ }^{1}$ contributes to the infarct size limiting effects of $\mathrm{IP}^{2}$. However, previous studies are limited to pharmacological approaches or indirect lines of evidence. Therefore, we established a murine model of cardiac IP and studied cardioprotective effects using a genetic approach.

Following cardiac IP, CD73 mRNA was induced in preconditioned myocardium $(18,4 \pm 5,7$ - fold, $n=6, p<0,05)$. Immunhistochemistry and enzyme activity measurements confirmed robust induction of CD73 protein and function by IP in preconditioned hearts. Using our murine model of cardiac IP, infarct sizes were reduced following IP by $69 \%$, from $42.1 \%$ to $13,5 \%$ in wildtype mice. Alpha-beta-methylene-ADP (APCP, a highly selective CD73 inhibitor) treatment completely abolished the infarct size limiting effects of cardiac IP. Cd73 $3^{-/-}$mice had significantly bigger infarct sizes at baseline. Moreover, the infarct size limiting effects of IP were completely abolished in these animals. Adenosine treatment of $\mathrm{cd}^{-1-}$ mice resulted in a partial reconstitution of the cardioprotective effects of IP (IP-Ado in $\mathrm{cd}^{-/-}: 54 \% \pm 3,0$ vs. IP+Ado in $\mathrm{cd}^{-/-} 39,3 \% \pm 3,3$ infarct size; $\mathrm{p}<0,05$ ).

Taken together, our data reveal for the first time direct in vivo evidence that CD73 is critical for the observed cardioprotective effects of IP. Further studies will need to define the contribution of individual adenosine receptors to cardioprotection by IP with the goal to identify novel therapeutic targets in the treatment of medical conditions associated with myocardial ischemia.

References:

(1) Eltzschig HK, Ibla JC, Furuta GT, Leonard MO, Jacobson KA, Enjyoji K, Robson SC, Colgan SP. J Exp Med. 2003, 198, 783-796.

(2) Colgan, S.P., H.K. Eltzschig, T. Eckle, and L.F. Thompson. Purinergic Signalling. 2006, in press.

\section{Cross regulation of adenosine and uridine nucleotides salvage synthesis in rat and human brain cytosol}

Piero L. Ipata ${ }^{1}$, Lodovico Lutzenberger ${ }^{2}$ and Catia Barsotti ${ }^{1}$. ipata@dfb.unipi.it

${ }^{1}$ Department of Biology, Section of Biochemistry; ${ }^{2}$ Department of Neuroscience, Section of Neurosurgery; University of Pisa, Pisa, Italy.

The de novo pathways for purine and pyrimidine biosynthesis are cross-regulated, i.e., accumulation of a purine nucleotide (ATP) activates pyrimidine synthesis and inhibits purine synthesis, and vice versa. In bacteria the molecular mechanism relies on the modulation of a single enzyme, aspartate transcarbamylase (1). This enzyme is activated by elevated ATP levels, which can be viewed as a signal of purine sufficiency, and inhibited by CTP and UTP, a signal of pyrimidine sufficiency. In mammals the modulated enzyme is carbamoyl phosphate synthetase (2). However, it is well established that several tissues and organs, including brain, rely more heavily on the salvage synthesis of nucleotides from preformed bases and nucleosides, rather than on de novo synthesis from simple precursors (3). This raises the following question: how do these districts maintain the right balance between their purine and pyrimidine nucleotide pools? 
The rationale of our experimental procedure is the following. Since the purine and pyrimidine salvage processes occur at the base and nucleoside level, respectively $(4,5)$, rat brain cytosol is incubated in the presence of either radioactive adenine and cold uridine, to follow the rate of purine salvage, or with cold adenine and radioactive uridine, to follow the rate of pyrimidine salvage. We emphasize that this experimental procedure allows to measure the rate of purine salvage when also pyrimidine salvage is operative, and vice versa. The time course of radioactive purine and pyrimidine nucleotides formation is followed, in the presence of different concentrations of effectors.

Our results show that in rat and human brain cytosol the two processes of purine and pyrimidine nucleotide salvage respond in an opposite manner to the fluctuation of intracellular [purine nucleoside triphosphates]/[pyrimidine nucleoside triphosphates] ratio (PUR/PYR ratio). The metabolic sensor is a two enzyme system which maintains uridine homeostasis, composed of uridine phosphorylase (UPase) and uridine kinase (Uk). When the activity of Uk is inhibited by relatively high UTP and CTP (6) concentration (low PUR/PYR ratio), uridine accumulates and the equilibrium of the UPase reaction is shifted towards uridine phosphorolysis. The ribose-1-P produced forms PRPP, an obligate substrate of adenine salvage (7). At a high ratio these effects are reversed. Uridine kinase becomes fully active, and pyrimidine salvage is favoured over purine salvage.

1. Allewell, N.M. (1989) Escherichia coli aspartate transcarbamoylase: structure, energetics, and catalytic and regulatory mechanisms, Annu. Rev. Biophys. Biophys. Chem. 18: 71-92.

2. Jones, M. E. (1980) Pyrimidine nucleotide biosynthesis in animals: genes, enzymes, and regulation of UMP biosynthesis, Annu. Rev. Biochem. 49: 253-279.

3. Stone, T.W., and Simmonds, H.A. (1991) Purine: Basic and Clinical Aspects, Kluwer, Dordrecht.

4. Kim, Y. A., King, M. T., Teague Jr, W.E., Rufo Jr, G. A., Veech, R. L., and Passonneau, J.V. (1992) Regulation of the purine salvage pathway in rat liver, Am. J. Physiol. 26: E344-E352.

5. Löffler, M., Fairbanks, L., D. Zameitat, E., Marinaki, A. M., and Simmonds, H. A (2005) Pyrimidine pathways in health and disease, Trends Mol. Med. 11: 430-437.

6. Orengo, A. (1969) Regulation of enzymic activity by metabolites. I. Uridine-cytidine kinase of Novikoff ascites rat tumor, J. Biol. Chem. 244: 2204-2209.

7. Barsotti, C., Tozzi, M.G., Ipata, P.L. (2002) Purine and pyrimidine salvage in whole rat brain. Utilization of ATP-derived ribose-1-phosphate and 5-phosphoribosyl-1-pyrophosphate generated in experiments with dialyzed cell-free extracts, J. Biol. Chem. 277: 9865-9869.

\title{
Cross-talk between human cysteinyl-leukotriene and extracellular nucleotide receptors: implication in inflammation
}

\author{
Valérie Capra ${ }^{1}$, Maria Rosa Accomazzo ${ }^{1}$, Simona Citro ${ }^{1}$, Liaman K. Mamedova ${ }^{3}$, Kenneth A.
} Jacobson $^{3}$, Maria Pia Abbracchio ${ }^{2}$ and G. Enrico Rovati ${ }^{1}$

${ }^{1}$ Laboratory of Molecular Pharmacology, Section of Eicosanoids Pharmacology and ${ }^{2}$ Laboratory of Molecular and Cellular Pharmacology of Purinergic Transmission, Department of Pharmacological Sciences, University of Milan, Via Balzaretti, 9, 20133 Milan, Italy

${ }^{3}$ Molecular Recognition Section, Laboratory of Bioorganic Chemistry, National Institute of Diabetes and Digestive and Kidney Diseases, National Institutes of Health, Bethesda, Maryland 20892, USA.

GEnrico.Rovati@unimi.it

Cysteinyl-leukotrienes (cysteinyl-LTs) and extracellular nucleotides mediate inflammatory responses via specific G protein-coupled receptors (GPCRs), the CysLTR and the P2YR, respectively. Evidence has begun to accumulate for the existence of a multifaceted cross-talk between the cysteinyl-LT and the nucleotide systems, considering that these mediators accumulate at sites of inflammation and inflammatory cells express both classes of receptors. For example, in rat microglia, activation of P2Y1R and CysLTR mediate co-release of both nucleotides and cysteinyl-LTs. Furthermore, we recently demonstrated that two $\mathrm{CysLT}_{1} \mathrm{R}$ selective antagonists marketed for asthma therapy functionally interact with P2YR signaling pathways by inhibiting nucleotide-induced calcium mobilization. Herein, we investigated the molecular basis of desensitization and trafficking of the human CysL$\mathrm{T}_{1} \mathrm{R}$ in response to $\mathrm{LTD}_{4}$ or nucleotides. Exposure of the CysLT $\mathrm{R}_{1} \mathrm{R}$ endogenously expressed in monocyte-macrophage like U937 cells to agonist induced a rapid homologous desensitization followed by receptor internalization. Activation of P2YR with ATP or UDP induced $\mathrm{CysLT}_{1} \mathrm{R}$ heterologous desensitization. Conversely, $\mathrm{LTD}_{4}$-induced CysLT $\mathrm{R}$ activation had no effect on P2YR responses. Furthermore, ATP/UDP-induced 
CysLT $_{1} \mathrm{R}$ desensitization was unable to cause receptor internalization and was dependent upon PKC, at variance with homologous desensitization. Hence, $\mathrm{CysLT}_{1} \mathrm{R}$ desensitization and trafficking are differentially regulated by its cognate ligand or by extracellular nucleotides. This cross-talk may have a profound physiological implication in the regulation of responses at the sites of inflammation, and may represent just an example of a feed-back mechanism utilized by the cells to fine tune their responses. Furthermore, it is also possible that other inflammatory stimuli heterologously desensitize the $\mathrm{CysLT}_{1} \mathrm{R}$ in a similar way, as is the case for the chemoattractant receptor system.

Partially supported by FIRB2001 to G.E.R. and by the Italian CARIPLO Foundation (2004 Projects "Promuovere la ricerca scientifica e tecnologica in tema di salute e scienze della vita" to G.E.R. and M.P.A., Project coordinated by prof. Francesco Clementi, University of Milan, Italy).

\title{
CVT-3619, a novel partial $A_{1}$ adenosine receptor agonist, lowers plasma free fatty acids, triglycerides and improves insulin sensitivity without cardiovascular effects.
}

\author{
Arvinder K. Dhalla, Mei-Yee Wong, Melissa Santikul, Michelle Smith, John C. Shryock and Luiz \\ Belardinelli. \\ Department of Pharmacological Sciences, CV Therapeutics Inc. Palo Alto, CA. USA 94304. \\ email:arvinder.dhalla@cvt.com
}

Elevated plasma free fatty acid (FFA) levels have been linked to the onset of insulin resistance. $\mathrm{A}_{1}$ adenosine receptor $\left(\mathrm{A}_{1} \mathrm{R}\right)$ agonists are potent anti-lipolytic agents which inhibit adipose tissue lipolysis and lower circulating FFA levels. Many $A_{1} R$ agonists have been developed in the past for the purpose of lowering FFA; however, their concurrent effects on the cardiovascular system are major limitations for their therapeutic utility. In the present study we report that CVT-3619, a novel partial $\mathrm{A}_{1} \mathrm{R}$ agonist, causes reduction in circulating FFA levels and improves insulin sensitivity without any significant effect on heart rate and blood pressure. Male Sprague Dawley rats (250-300 gms) implanted with indwelling arterial and venous cannulas for blood sampling and drug administration were used in the study. CVT-3619 $(1-10 \mathrm{mg} / \mathrm{kg}$, PO dose) decreased both FFA and triglyceride (TG) levels $(15-57 \%)$ in a dose-dependent manner. CVT-3619 did not have any significant effect on heart rate and blood pressure up to $10 \mathrm{mg} / \mathrm{kg}$, PO. At 25 and $50 \mathrm{mg} / \mathrm{kg}$ dose of CVT-3619 there was a small $(10 \%)$ but significant decrease in heart rate. The FFA lowering effects of three consecutive single doses CVT-3619 were not significantly different suggesting that there was no tachyphylaxis of the effect of CVT-3619. In addition, the anti-lipolytic effect of CVT-3619 was not associated with a rebound increase in FFA levels as seen with Nicotinic acid. TG secretion $(\mathrm{mg} / \mathrm{dl} / \mathrm{min})$ by the liver was significantly $(\mathrm{p}<0.001)$ reduced with CVT-3619 treatment (slope for control $5.5 \pm 0.12$; CVT-3619 $3.7 \pm 0.17$ ). CVT-3619 also lowered FFA and TG levels in rats with impaired insulin sensitivity caused by 2 weeks of high fat diet feeding. An oral glucose tolerance test (OGTT) in high fat diet fed rats treated with CVT-3619 for 2 weeks showed improved insulin sensitivity as compared to untreated control rats as indicated by the area under the curve for the time-course of the changes in glucose and insulin plasma levels (AUCg x AUCi). In conclusion, CVT-3619 is a novel, partial, orally bioavailable $\mathrm{A}_{1} \mathrm{R}$ agonist that lowers circulating FFA and TG levels resulting in improved insulin sensitivity in a diet-induced model of insulin resistance with minimal cardiovascular effects.

\section{Design and Synthesis of purine and pyrimidine nucleosides carrying phosphate mimics groups at the 5 '-position as potential $\mathbf{P 2}$ receptor ligands ${ }^{1}$}

Maria-Cruz Bonache, Lisa Buzzoni, Nunzia Ciliberti and Stefano Manfredini

Department of Pharmaceutical Sciences, University of Ferrara,

Via Fossato di Mortara 17-19, 44100 Ferrara, Italia

Extracellular nucleotides regulate a wide variety of functional responses in many cell types by stimulation of both G-protein coupled receptors (P2Y) and ATP-gated ion channel receptors (P2X). Selective ligands for purinergic 
and pyrimidinergic P2 receptors are urgently needed in order to investigate their physiological role and the pharmacological potential of such compounds ${ }^{2}$. A useful approach to the discovery of novel P2 receptor ligands may consists in the design of purine and pyrimidine nucleosides carrying phosphate mimics groups at the $5^{\prime}$ position. These compounds should be featured with structural changes compatible for the recognicition by the receptors but stable to the enzymatic hydrolisys. Consequently, we have recently explored the possibility to obtain a new class of stable nucleotide analogs endowed with isosteric substitution of the diphosphate group. The substitution of the diphosphate moiety of natural substrates with phosphonoacetic acid ester and amide moieties should give a stronger interaction with the active site, thereby resulting in potent ligands. On these premises, on the hand, we have prepared new nucleosides analogs designed in order to obtain bioisosters at the diphosphate moiety. Taking into account our previous results have explored 5'-phosphono acetic acid, amide and ester analogs of inosine, adenosine, uridine, cytidine and ribofuranosiltimine. In this communication, the synthesis of these compounds will be reported.

${ }^{1}$ This work was supported by FIRB, grant number RBNE03YA3L_008

${ }^{2}$ Ralevic and Bursntock G. Pharmacol. Reviews, 1998,50, 413-492

\title{
Design and synthesis of selective $\mathbf{A}_{2 \mathrm{~B}}$ adenosine receptor antagonists: New mono-N-1 alkyl 8-(pyrazol-4-yl) xanthines
}

\author{
$\underline{\text { Rao Kalla }},{ }^{\mathrm{a}}$ Elfatih Elzein, ${ }^{\mathrm{a}}$ Thao Perry, ${ }^{\mathrm{a}}$ Xiaofen Li, ${ }^{\mathrm{a}}$ Tennig Maa, ${ }^{\mathrm{b}}$ Arthur Gimbel, ${ }^{\mathrm{b}}$ Dewan Zeng, ${ }^{\mathrm{b}}$ \\ and Jeff Zablocki ${ }^{\mathrm{a}}$ \\ ${ }^{a}$ Department of Bioorganic Chemistry, ${ }^{b}$ Department of Drug Research and Pharmacological Sciences, CV The- \\ rapeutics Inc., 3172 Porter Drive, Palo Alto, CA 94304, USA \\ rao.kalla@cvt.com
}

Adenosine is an endogenous ligand that binds to four adenosine receptor subtypes $-A_{1}, A_{2 A}, A_{2 B}$, and $A_{3}$. Activation of the $\mathrm{A}_{2 \mathrm{~B}}$ adenosine receprot (AdoR) on mast cells may play a putative role in asthma through mast cell degranulation leading to the release of inflammatory cytokines (e.g. interleukin-8). Furthermore, activation of $\mathrm{A}_{2 \mathrm{~B}}$ AdoRs on bronchial smooth muscle cells (BSMC) has been demonstrated to lead to the production of interleukin- 6 and monocytic chemotactic peptide- 1 , a process that can be blocked by selective $A_{2 B}$ AdoR antagonists. Therefore, potent and selective $\mathrm{A}_{2 \mathrm{~B}}$ adenosine receptor (AdoR) antagonists may play a beneficial role in understanding the role of $\mathrm{A}_{2 \mathrm{~B}}$ AdoRs in physiological and pathological conditions and as a potential treatment for asthma. In our efforts to identify a slective high affinity $\mathrm{A}_{2 \mathrm{~B}}$ AdoR antagonist, we explored 8-pyrazol-4-yl xanthine derivatives that represents a new class adenosine receptor antagonists. ${ }^{1}$ Previously, we reported the SAR of 1,3-disubstituted 8-pyrazolyl xanthine derivatives that displayed high affinity for the $\mathrm{A}_{2 \mathrm{~B}}$ AdoR and good selectivity. ${ }^{2,3}$ We have shown that substitution of the pyrazole nitrogen with a meta-substituted benzyl group (1) or with oxadiazoles (2) and isoxazoles increased the $\mathrm{A}_{2 \mathrm{~B}}$ AdoR selectivity. Herein, we describe our efforts to increase the $\mathrm{A}_{2 \mathrm{~B}}$ AdoR selectivity of 8-pyrazolyl xanthines by exploring the effects of mono-substitution at the N-1 and N-3 positions of the xanthine. The N-1 substituted 8-pyrazolyl xanthines $\mathbf{3}$ and $\mathbf{4}$ have displayed higher selectivity compared to their corresponding disubstituted derivatives $\mathbf{1}$ and $\mathbf{2}$. The synthesis of mono substituted 8pyrazolyl xanthines and their SAR will be discussed in detail.<smiles>[R]n1c(=O)n(CCC)c(=O)c2[nH]c(-c3cnn(Cc4cccc(C(F)(F)F)c4)c3)nc21</smiles>

1. $\mathrm{R}_{1}=\mathrm{n}-$ propyl; $\mathrm{h}\left(\mathrm{A}_{2 \mathrm{~B}}\right)-14 \mathrm{nM} ; \mathrm{h}\left(\mathrm{A}_{1}\right)-170 \mathrm{nM}$ $\mathrm{h}\left(\mathrm{A}_{2 \mathrm{~A}}\right)-400 \mathrm{nM} ; \mathrm{h}\left(\mathrm{A}_{3}\right)-56 \mathrm{nM}$

3. $\mathrm{R}_{1}=\mathrm{H} ; \mathrm{h}\left(\mathrm{A}_{2 \mathrm{~B}}\right)-8 \mathrm{nM} ; \mathrm{h}\left(\mathrm{A}_{1}\right)->6000 \mathrm{nM}$ $\mathrm{h}\left(\mathrm{A}_{2 \mathrm{~A}}\right)->5000 \mathrm{nM} ; \mathrm{h}\left(\mathrm{A}_{3}\right)-700 \mathrm{nM}$

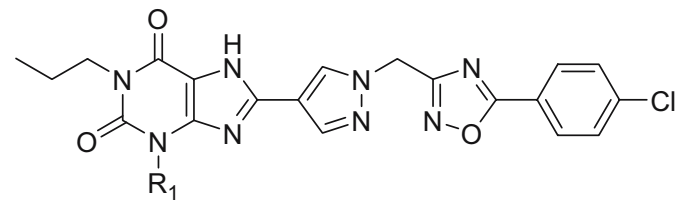

2. $\mathrm{R}_{1}=\mathrm{n}$-propyl; $\mathrm{h}\left(\mathrm{A}_{2 \mathrm{~B}}\right)-39 \mathrm{nM} ; \mathrm{h}\left(\mathrm{A}_{1}\right)-2900 \mathrm{nM}$ $\mathrm{h}\left(\mathrm{A}_{2 \mathrm{~A}}\right)$ - > $5000 \mathrm{nM} ; \mathrm{h}\left(\mathrm{A}_{3}\right)->6000 \mathrm{nM}$

4. $\mathrm{R}_{1}=\mathrm{H} ; \mathrm{h}\left(\mathrm{A}_{2 \mathrm{~B}}\right)-14 \mathrm{nM} ; \mathrm{h}\left(\mathrm{A}_{1}\right)->6000 \mathrm{nM}$ $\mathrm{h}\left(\mathrm{A}_{2 \mathrm{~A}}\right)$ - > $5000 \mathrm{nM} ; \mathrm{h}\left(\mathrm{A}_{3}\right)->6000 \mathrm{nM}$ 
(1) Kalla, R.; Perry, T.; Elzein, E.; Varkhedkar, V.; Li, X.; Ibrahim, P.; Palle, V.; Xiao, D.; Zablocki, J. A 2 Adenosine Receptor Antagonists. Patent US 6825349 (2004); WO 0342214 (2003). (2) Kalla, R.; Perry, T.; Elzein, E.; Palle, V.; Li, X.; Varkhedkar, V.; Maa, T.; Nguyen, M.; Wu, Y.; Maydanik, V.; Lustig, D.; Leung, K.; Zeng, D.; Zablocki, J. 227th ACS National Meeting, Anaheim, CA, United States, March 28-April 1, 2004, MEDI-253. (3) Elzein, E.; Kalla, R.; Li, X.; Perry, T.; Parkhill, E.; Palle, V.; Varkhedkar, V.; Gimbel, A.; Zeng, D.; Lustig, D.; Leung, K.; Zablocki, J. Bioorg. Med. Chem. Lett. 2006, 16, 302-306.

\title{
DETERMINANTS FOR THE CAMP BINDING SITE AT THE S-ADENOSYLHOMOCYSTEINE-(SAH)-HYDROLASE
}

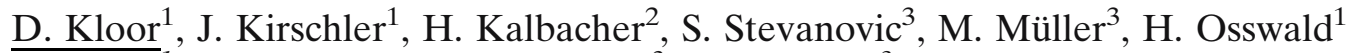 \\ Depts. of ${ }^{1}$ Pharmacology and Toxicology, ${ }^{2}$ Biochemistry, ${ }^{3}$ Immunology, Eberhard-Karls Universität, \\ 72074 Tübingen, Germany \\ doris.kloor@uni-tuebingen.de
}

SAH-hydrolase catalyzes the reversible hydrolysis of SAH to adenosine and homocysteine. One mol of the enzyme contains $4 \mathrm{~mol}$ of $\mathrm{NAD}^{+}$which is the essential cofactor of the enzyme ${ }^{1}$. As recently shown purified SAHhydrolase exhibits two adenosine binding sites one with a high and one with a low affinity. These adenosine binding sites are controlled by the enzyme-bound $\mathrm{NAD}^{+} / \mathrm{NADH}$ ratio. The existance of the two sepatrate binding sites were identified after covalent labeling with 8 -azido-adenosine ${ }^{2}$. Since adenosine competes with cAMP at the high affinity binding site of the enzyme ${ }^{3}$, we examined in the present study the effect of cAMP on the enzyme activity and its binding characteristics to SAH-hydrolase in its native, $\mathrm{NAD}^{+}$and $\mathrm{NADH}$ form.

Methods: SAH-hydrolase was purified to homogeneity from bovine kidney with chromatographical methods. The activity of SAH-hydrolase was assayed in the direction of hydrolysis. With the spectrophotometric titration assay we determined the ability of adenosine and cAMP to reduce the enzyme bound $\mathrm{NAD}^{+}$at $327 \mathrm{~nm}$. To gain further insight into the role of $\mathrm{NAD}^{+}$in cAMP binding we removed $\mathrm{NAD}^{+}$of the enzyme by dialysis and the apo-enzyme was reconstituted either with $100 \% \mathrm{NAD}^{+}$or with $100 \% \mathrm{NADH}$. For saturation binding experiments the enzyme $(10 \mu \mathrm{g} / \mathrm{ml})$ was incubated with ${ }^{3} \mathrm{H}-\mathrm{cAMP}(0.01-5 \mu \mathrm{mol} / \mathrm{l})$ two hours at room temperature in Tris/Hepes $\mathrm{pH}$ 7.4. To identify the cAMP binding site, SAH-hydrolase was incubated with $\left[2-{ }^{3} \mathrm{H}\right]-8$-azido-cAMP. After irradiation the reaction mixture was digested by Asp-N or trypsin and the peptides purified by HLPC were sequenced.

Results: cAMP enhanced the hydrolytic activity of SAH-hydrolase by $30 \%$ at $5 \mu \mathrm{mol} / \mathrm{l}$. In contrast to adenosine, cAMP did not reduces the tightly bound $\mathrm{NAD}^{+}$in the titration assay. In the presence of cAMP the conversion of $\mathrm{NAD}^{+}$to NADH by adenosine is decreased in a concentration dependent manner. Saturation experiments performed by increasing concentrations of ${ }^{3} \mathrm{H}-\mathrm{cAMP}$ and with the three differnt forms of SAH-hydrolase, native, $\mathrm{NAD}^{+}$and $\mathrm{NADH}$, indicated only one binding site with a high affinity. This binding site was identified after irradiation of the enzyme with $\left[2-{ }^{3} \mathrm{H}\right]-8$-azido-cAMP. One photolabeled peptide was isolated as $\operatorname{Trp}^{310}-\mathrm{Val}^{325}$ from the native SAH-hydrolase and one peptide as $\mathrm{Asn}^{314}-\mathrm{Gln}^{324}$ from both, $\mathrm{NAD}^{+}$and NADH forms.

Conclusion: Our data show that the cAMP binding site of the SAH-hydrolase is independent of the $\mathrm{NAD}^{+}$/ NADH ratio of the enzyme. We conclude that the cAMP binding site is identical with the high affinity binding site of adenosine. This implies that the inhibitory effect of adenosine on the SAH-hydrolase can be antagonized by cAMP. Whether this cAMP action may have physiological implications remains to be determined.

References

1. Palmer, J.L.; Abeles, R.H.; J. Biol. Chem. 1979, 254:1217-1226.

2. Kloor, D.; Lüdtke, A.; Stoeva, S.; Osswald, H.; Biochem. Pharmacol. 2003, 66:2117-2123.

3. Kloor, D.; Danielyan, L.; Osswald, H.; Biochem. Pharmacol, 2002, 64:1201-1206. 


\title{
DETERMINATION OF GLIAL CELL ACTIVATION AND C-FOS IMMUNOREACTIVITY IN THE BRAIN OF HUNTINGTON TRANSGENIC MICE
}

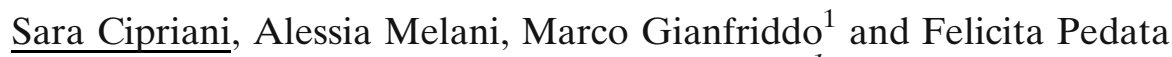 \\ Dept. Of Pharmacology, University of Florece, Italy; ${ }^{1}$ Sienabiotech S.p.A, Siena, Italy. \\ scipriani@unifi.it
}

Huntington disease (HD) is an autosomal, dominantly inherited neurodegenerative disorder characterized by progressive motor and cognitive disturbances caused by an expansion in CAG repeats in the IT15 gene which encodes the huntingtin protein. A pathogenetic role for excitotoxic cell death mediated by increased glutamatergic excitoxicity in the striatum has been proposed. Drugs able to modulate striatal levels of glutamate are thus candidates to protect striatal neurons from neurodegeneration. We have recently demonstrated that the extracellular concentration of adenosine increases in the striatum of HD transgenic (R6/2) mice and that the selective antagonist of adenosine $\mathrm{A}_{2 \mathrm{~A}}$ receptors, $\mathrm{SCH}$ 58261, directly administered in the striatum, significantly reduces glutamate outflow ${ }^{1}$. Furthermore we demonstrated that the p38 mitogen-activated protein kinase (MAPK) pathway is activated in the neurons of the striatum of HD transgenic mice ${ }^{1}$. The p38 MAPK is known to be a factor activated and inducing the expression of pro- and inflammatory mediators and involved in the early gene c-Fos phosphorylation. Products of Fos family are players in inducing inflammatory gene expression in glial cells. In HD patient brain microglial activation in the striatum and in the cortex ${ }^{2}$ and reactive astrogliosis in the striatum during the late stage of pathology ${ }^{3}$ were reported.

A first aim was therefore to investigate glial cells and c-Fos activation in heterozygous transgenic R6/2 male and wild-type mice at different ages, 10-11 (presymptomatic phase) and 14 week old (symptomatic phase), and to evaluate the effect of selective adenosine $\mathrm{A}_{2 \mathrm{~A}}$ receptor antagonist $\mathrm{SCH} 58261$. SCH 58261 was subchronically administered $(0.01 \mathrm{mg} / \mathrm{kg}$ i.p.) at time: $-20,-17$ and $-2 \mathrm{~h}$ from sacrificed. Mice were transcardically perfused with paraformaldheyde and brains were cut by cryostat into $30 \mu \mathrm{m}$ thick slices. Microglial cells stained by isolectine-B4 and astrocytes immunostained by GFAP were not activated in the striatum of 10-11 $(n=5)$ and 14 week old R6/2 mice $(n=3)$. Astroglial cells were detected only in the cingolate cortex of 14 week old mice $(n=3)$. In the brain of 10-11 week old R6/2 treated mice $(n=5)$ specific c-Fos immunostaining was not changed in comparison to wildtype mice $(n=6)$. On the other hand, specific c-Fos immunostaining was increased in the piriform cortex, but not in the striatum, of 14 week old mice $(n=3)$ in comparison to wild-type mice $(n=6)$. The selective antagonist of adenosine $A_{2 A}$ receptors decreased c-Fos immunoreactivity in the piriform cortex of 14 week old mice $(n=3)$.

The results demonstrate that in transgenic R6/2 mice in the terminal phase, there is a modest activation of glial cells and activation of c-Fos early gene in the cortex, and that adenosine $\mathrm{A}_{2 \mathrm{~A}}$ antagonism reduces c-Fos activation in the cortex. (Grant by Italian Ministry of Health and Fondazione Monte dei Paschi di Siena, Italy).

(1) Gianfriddo, M., Melani, A., Turchi, D., Giovannini, M.G., Pedata, F.. Neurobiol. Dis. 2004, 17, 77-88.

(2) Sapp, E., Kegel, K.B., Aronin, N., Hashikawa, T., Uchiyama, Y., Tohyama, K., Bhide, P.G., Vonsattel, J.P., DiFiglia, M. J Neuropathol. Exp. Neurol. 2001, 60, 161-172.

(3) Vonsattel, J.P., Myers, R.H., Stevens, T.J., Ferrante, R.J., Bird, E.D., Richardson, E.P., Jr. J. Neuropathol. Exp. Neurol. 1985, 44, 559-577.

\section{Developing an assay to probe the activity of the $\mathbf{P} 2 \mathrm{X}_{1}$ receptor with radio-labelled 2-azido ATP}

*Kelvin C. Agboh, Jonathan Roberts, Andrew Powell ${ }^{1}$ \& Richard J. Evans

Department of Cell Physiology \& Pharmacology, University of Leicester, Leicester, LE1 9HN, UK;

${ }^{1}$ Gene Expression \& Protein Biochemistry, GlaxoSmithKline, Stevenage, SG1 2NY, UK

"kca2@le.ac.uk

Mutagenesis of conserved residues at the $\mathrm{P}_{2} \mathrm{X}_{1}$ receptor has revealed reductions in the potency of ATP which could be attributed to either binding or gating effects. To discriminate between them, we developed an assay to 
probe binding at the $\mathrm{P} 2 \mathrm{X}_{1}$ receptor using 2-azido ATP. Whole cell patch clamp recordings show that 2-azido ATP is a potent agonist at the $\mathrm{P}_{2} \mathrm{X}_{1}$ receptor. The ATP analogue contains a photo-active azido $\left(-\mathrm{N}_{3}\right)$ group that is chemically inert until activation by UV light. Upon irradiation, a covalent linkage between the compound and the active site of the receptor is formed. Cross-linking should cause permanent $\mathrm{P}_{2} \mathrm{X}_{1}$ receptor occupation, therefore subsequent ATP applications should produce responses that are greatly reduced from the control.

HEK293 cells stably expressing the $\mathrm{P}_{2} \mathrm{X}_{1}$ receptor were incubated in $30 \mu \mathrm{M}$ 2-azido ATP and UV irradiated for 3 min, with the UV source positioned $7.5 \mathrm{~cm}$ above the cells. Under these conditions and following a 10 min wash, $\mathrm{UV}$ or ATP and UV had no effect on the amplitude of the $\mathrm{P}_{2} \mathrm{X}_{1}$ receptor response. However, UV irradiation with 2 -azido ATP caused an $86 \%$ reduction in currents elicited by $1 \mu \mathrm{M}$ ATP application $\left(\mathrm{EC}_{50}\right.$ concentration). Control responses were reduced from $3.47 \pm 0.28 \mathrm{nA}(n=15)$ to $0.49 \pm 0.09 \mathrm{nA}(n=30)$. This reduction was maintained throughout the recorded $45 \mathrm{~min}$ time period. The effects of cross-linking were prevented by pre-treatment with $300 \mu \mathrm{M}$ ATP or $100 \mu \mathrm{M}$ suramin, this provided $29 \%$ and $73 \%$ receptor protection respectively.

To detect the binding of 2-azido ATP to the receptor, we used a ${ }^{32} \mathrm{P}$ labelled version of the compound. HEK293 cells stably expressing the $\mathrm{P}_{2} \mathrm{X}_{1}$ receptor were treated with radio-labelled 2-azido ATP. To determine the level of non-specific binding, non-transfected HEK293 cells were treated similarly. After lysis, the cross-linked P2X receptor was isolated by immunoprecipitation with the $\mathrm{P} 2 \mathrm{X}_{1}$ antibody (Alomone) and the samples were run on a $10 \%$ SDS gel. Exposure of the dried gel to autoradiography film directly provided evidence of 2-azido ATP binding at the $\mathrm{P}_{2} \mathrm{X}_{1}$ receptor, no binding to non-transfected HEK293 cells and the reduced binding which occurred at cells protected by excess ATP. Previously shown mutations of the NFR region (290-292) and the positive lysine at the 309 position to either an alanine or cysteine residue revealed a significantly reduced ATP potency when compared to the wildtype. When expressed in Xenopus oocytes, binding of the radio-labelled 2-azido ATP was significantly decreased in these mutants when compared to the wildtype. This decrease is indicative of a reduction in direct binding to the $\mathrm{P}_{2} \mathrm{X}_{1}$ receptor and provides further evidence that the NFR region and the lysine residue at 309 contribute to the binding site of the $\mathrm{P} 2 \mathrm{X}_{1}$ receptor.

\title{
Development and structure-activity relationships of ectonucleotidase inhibitors
}

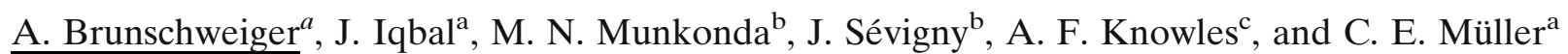 \\ ${ }^{a}$ Pharmaceutical Sciences Bonn (PSB), Pharmaceutical Chemistry, Institute of Pharmacy, University of Bonn, \\ Kreuzbergweg 26, Bonn, Germany \\ ${ }^{b}$ Centre de Recherche en Rhumatologie et Immunologie, Sainte-Foy, Québec, Canada \\ ${ }^{c}$ Department of Chemistry, San Diego State University, San Diego, CA 92182-1030, USA \\ andreas.brunschweiger@uni-bonn.de
}

Full abstract can only be sent late

\section{Development of a novel, automated method for the characterization and screening of NTPDase inhibitors by in-capillary enzymatic microreaction}

\author{
Jamshed Iqbal $^{1}$, Herbert Zimmermann ${ }^{2}$ and Christa E. Müller ${ }^{1}$ \\ ${ }^{T}$ Pharmazeutisches Institut Poppelsdorf, Universität Bonn, Kreuzbergweg 26, D-53115 Bonn; \\ ${ }^{2}$ AK Neurochemie, Biozentrum der J.W. Goethe-Universität, Marie-Curie-Str. 9, D-60439 Frankfurt am Main, \\ Germany. \\ jamshed.iqbal@uni-bonn.de
}

The ecto-nucleoside triphosphate diphosphohydrolases (EC 3.6.1.5) represent a major and ubiquitous family of ecto-nucleotidases. They catalyze the sequential hydrolysis of the $\gamma$ - and $\beta$-phosphate residues of nucleoside triand diphosphates, producing the corresponding nucleoside monophosphate derivatives.1 The activation of P2 receptors is controlled by ecto-nucleotidases capable of hydrolyzing nucleoside tri- and diphosphates.1 Inhibition of ecto-nucleotidases can thus result in a potentiation of purinergic signaling, supporting the notion that endogenous ecto-nucleotidases reduce the effective concentration of the released nucleotide. 2 Inhibitors of ecto-nucl- 
eotidases could thus represent valuable tools for amplifying the biological effects induced by extracellularly released nucleotides.

Several methods have been used for the determination of Michaelis-Menten constants $\left(\mathrm{K}_{\mathrm{m}}\right.$ values), and inhibition constants $\left(\mathrm{K}_{\mathrm{i}}\right.$ values for enzyme inhibitors) of NTPDases, including radioisotopic, HPLC and spectrophotometric assays. All of these methods are time-consuming and suffer from further serious drawbacks. Capillary electrophoresis (CE) has recently emerged as a versatile technique for the quantitative analysis of nucleotides 3 and for the monitoring enzymatic reactions. ${ }^{4} \mathrm{CE}$ systems have been successfully applied for assaying enzyme activity, including the determination of Michaelis-Menten constants $\left(\mathrm{K}_{\mathrm{m}}\right.$ values), and inhibition constants $\left(\mathrm{K}_{\mathrm{i}}\right.$ values for enzyme inhibitors), exhibiting a number of advantages over conventional methods. These include rapid separation of substrate and product, ultra-low sample volume requirements, and high throughput by automation. CE is particularly useful for investigating enzymatic reactions involving charged substrates or products, e.g. for the monitoring of phosphorylation or dephosphorylation reactions.

In the present study we have developed an easy, rapid, nonradioactive at-capillary-inlet CE method for the screening and characterization of NTPDases inhibitors. ${ }^{5}$ The enzymes used in this study were expressed in $\mathrm{CHO}$ cells. Different standard compounds were tested as NTPDase inhibitors by this new method and results were in close agreement with data obtained from standard methods. The newly developed CE-based NTPDases inhibition and characterization assay is suitable for automation using 96-well plates and can be used for the high throughput screening of novel potential NTPDase inhibitors and substrates.

(1) Zimmermann, H. Naunyn Schmiedebergs Arch.Pharmacol. 2000, 362, 299-309.

(2) Bültmann, R.; Driessen, B.; Goncalves, J.; Starke, K. Naunyn Schmiedebergs Arch.Pharmacol. 1995, 351, 555560.

(3) Qurishi, R.; Kaulich, M.; Müller, C. E. J.Chromatogr.A 2002, 952, 275-281.

(4) Kaulich, M.; Qurishi, R.; Müller, C. E. Cell Mol.Neurobiol. 2003, 23, 349-364.

(5) Iqbal, J.; Vollmayer, P.; Braun, N.; Zimmermann, H.; Müller, C.E. Purinergic Signalling 2005, 1, 349-358.

\title{
Diadenosine polyphosphate analogue controls postsynaptic excitation in CA3- CA1 synapses via a nitric oxide (NO)-dependent mechanism
}

\author{
Sergei Melnik, ${ }^{1}$ Michael Wright, ${ }^{2}$ Julian A. Tanner, ${ }^{2}$ Timur Tsintsadze, ${ }^{1}$ Vera Tsintsadze, ${ }^{1}$ Andrew D. \\ Miller, ${ }^{2}$ and Natalia Lozovayaa ${ }^{1}$ \\ ${ }^{T}$ Department of Cellular Membranology, Bogomoletz Institute of Physiology, Ukraine; ${ }^{2}$ Imperial College Imperial \\ College Genetic Therapies Centre, Imperial College London, UK. \\ a.miller@imperial.ac.uk
}

Previously, we have described the modulatory effect of diadenosine polyphosphates $A p_{4} A$ and $A p_{5} A$ on synaptic transmission in the rat hippocampal slices, mediated by presynaptic receptors (Klishin et al., 1994). We have reexamined this phenomenon probing with a non-hydrolysable $\mathrm{Ap}_{4} \mathrm{~A}$ analogue diadenosine $-5^{\prime}, 5^{\prime}-\mathrm{P}^{1}, \mathrm{P}^{4}-\left[\beta, \beta^{\prime}\right.$-methylene]tetraphosphate $\left(\mathrm{AppCH} \mathrm{CH}_{2} \mathrm{ppA}\right)$ of high purity and low salt content. In contrast to our previous report we now describe how $\mathrm{AppCH}_{2} \mathrm{ppA}$ at low micromolar concentrations exerts a pure postsynaptic effect giving strong non-desensitizing inhibition of orthodromically evoked field potentials (OFPs), without affecting the amplitude of excitatory postsynaptic currents (EPSCs) and antidromically evoked field potentials (AFPs) recorded in the CA1 zone of hippocampus. The effects of $\mathrm{AppCH}_{2} \mathrm{ppA}$ on OFPs are eliminated by a $\mathrm{P} 2$ receptor antagonist PPADS but not mimicked by purinoceptor agonists, $\alpha, \beta$-methylene-ATP and ATP- $\gamma$-S, indicating that a P2-like receptor is involved but not one belonging to the conventional $\mathrm{P} 2 \mathrm{X} / \mathrm{P} 2 \mathrm{Y}$ receptor classes. In contrast to $\mathrm{AppCH}_{2} \mathrm{ppA}$, ATP inhibits the EPSCs. Diadenosine polyphosphate receptor (P4) antagonist, $\mathrm{Ip}_{4} \mathrm{I}$, was unable to modulate AppC$\mathrm{H}_{2} \mathrm{ppA}$ effects. Thus, the PPADS-sensitive P2-like receptor for $\mathrm{AppCH}_{2} \mathrm{ppA}$ appears to selectively control dendritic excitation of the CA1 neurons. The specific nitric oxide (NO)-scavenger 2-phenyl-4,4,5,5-tetramethylimidazoline-1-oxyl-3-oxide (PTIO) is shown to attenuate significantly $\mathrm{AppCH}_{2}$ ppA mediated inhibitory effects, indicating that $\mathrm{NO}$ is involved in the cascade of events initiated by the $\mathrm{AppCH}_{2} \mathrm{ppA}$. Further downstream mediation by adenosine A1 receptors is also demonstrated. Such spatially selective postsynaptic dendritic inhibition may influence dendritic electrogenesis in pyramidal neurons and consequently mediate control of neuronal network activity. 
Klishin A, Lozovaya N, Pintor J, Miras-Portugal MT, and Krishtal O (1994) Possible functional role of diadenosine polyphosphates: negative feedback for excitation in hippocampus. Neuroscience 58:235-236.

\title{
Diadenosine Polyphosphates Are Selective Vasoconstrictors In Human Coronary Artery Bypass Grafts.
}

\author{
AR Conant ${ }^{1}$, WC Dihmis ${ }^{1}$, AWM Simpson ${ }^{2}$ \\ ${ }^{1}$ The Cardiothoracic Centre, Liverpool NHS Trust, Thomas Drive, Liverpool, L14 3PE, UK ${ }^{2}$ Dept Human \\ Anatomy and Cell Biology, University of Liverpool, L69 3GE UK \\ awms@liv.ac.uk
}

Nucleotides released by degranulating platelets are potential causative agents of post-operative contraction in coronary artery bypass grafts. Diadenosine polyphosphates $\left(\mathrm{Ap}_{\mathrm{n}} \mathrm{A}\right)$ are released alongside mono-nucleotides and have activity at several P2 receptors. Diadenosine polyphosphates are relatively long-lived vasoactive molecules, as their stability is enhanced by the preferential metabolism of mono-nucleotides by ecto-nucleotidases. Therefore diadenosine polyphosphates may be the cause of local nucleotide-mediated vasoconstriction at sites of tissue injury and inflammation in coronary artery bypass grafts.

Following ethical committee approval, sections of human radial and internal mammary artery and saphenous vein were obtained surplus to surgery with informed patient consent. Changes in tension were recorded upon application of diadenosine polyphosphates or the $\mathrm{P}_{2} \mathrm{X}_{1}$ receptor agonist $\alpha \beta$ methyleneATP.

Radial artery and saphenous vein demonstrated robust concentration-dependent vasoconstriction to $\mathrm{Ap}_{4} \mathrm{~A}, \mathrm{Ap}_{5} \mathrm{~A}$ and $\mathrm{Ap}_{6} \mathrm{~A}$ and $\alpha \beta$ methyleneATP. Cross-desensitisation revealed that diadenosine-mediated vasoconstriction apparently occurred via the $\mathrm{P}_{2} \mathrm{X}_{1}$ receptor. In contrast, in the internal mammary artery $\alpha \beta$ methyleneATP-mediated vasoconstriction was weak or absent in the majority of samples. Consistent with activity at the $\mathrm{P} 2 \mathrm{X}_{1}$ receptor diadenosine polyphosphate-mediated vasoconstriction was also absent. However in radial artery and saphenous vein, responses to $\mathrm{Ap}_{5} \mathrm{~A}$ could not be inhibited by PPADS but were inhibited by suramin or NF279 at high concentrations. Indeed in the presence of PPADS, $\mathrm{Ap}_{5} \mathrm{~A}$-mediated vasoconstriction was enhanced suggesting that ectonucleotidase activity was reducing the availability of $\mathrm{Ap}_{5} \mathrm{~A}$ at the smooth muscle. Consistent with these findings enhanced $\mathrm{Ap}_{5} \mathrm{~A}$-mediated vasoconstriction occurred when $\mathrm{Ap}_{5} \mathrm{~A}$-responses were repeated in the presence of excess ATP.

Our data would implicate diadenosine polyphosphates as a potential cause of post-operative vasoconstriction in coronary artery bypass grafts. However this activity is limited to saphenous vein and radial artery grafts since in comparison the internal mammary artery is relatively insensitive to the diadenosine polyphosphates.

\section{Diastereoselectivity of the $\mathbf{P 2} Y_{11}$ receptor: molecular insights}

\author{
Ecke, D. ${ }^{1}$, Nahum,V. ${ }^{2}$, Tulapurkar, M.E. ${ }^{1}$, Fischer, B. ${ }^{2}$, Reiser, G. ${ }^{1}$ \\ ${ }^{T}$ Institut für Neurobiochemie, Medizinische Fakultät, Otto-von-Guericke-Universität Magdeburg, Magdeburg, \\ Germany \\ ${ }^{2}$ Department of Chemistry, Bar-Ilan University, Ramat-Gan 52900, Israel \\ denise.ecke@medizin.uni-magdeburg.de
}

The $\mathrm{P}_{2} \mathrm{Y}_{1}$ and $\mathrm{P} 2 \mathrm{Y}_{11}$ receptors are closest homologues among the family of G-protein-coupled $\mathrm{P} 2$ receptors and are activated by adenine nucleotides exclusively 1,2 . We stably expressed the $\mathrm{P}_{2} \mathrm{Y}_{11}$ receptor in $1321 \mathrm{~N} 1$ cells and monitored the cells for the change in fluorescence intensity of the calcium indicator Fura-2 after agonist stimulation. By the use of this method we found a clear difference in potencies of several borano/sulfur-modified diastereoisomers of ATP. The $\mathrm{P}_{2} \mathrm{Y}_{11}$ receptor clearly prefers the (B) isomers (ATP- $\alpha-\mathrm{B}$ Sp isomers and ATP- $\alpha$-S $\mathrm{Rp}$ isomers) of the derivatives tested. The (B) isomers of all derivatives tested were more potent at the $\mathrm{P} 2 \mathrm{Y}_{11}$ receptor than the corresponding (A) isomers (ATP- $\alpha-\mathrm{B}$ Rp isomers and ATP- $\alpha-\mathrm{S}$ Sp isomers) and the parent compounds. In former studies we have shown that ATP- $\alpha-\mathrm{S}$ and ATP- $\alpha-\mathrm{B}$ diastereoisomers display a stereoselective activity at the $\mathrm{P}_{2} \mathrm{Y}_{1}$ receptor 3, 4. Here we report that the $\mathrm{P}_{2} \mathrm{Y}_{11}$ receptor exhibits exactly the opposite chiral discrimination. Moreover, the (A) isomer of 2-MeS-ATP- $\alpha-\mathrm{B}$ was found to be most selective at the P2 $\mathrm{Y}_{1}$ receptor being 3000-fold more potent at this subtype than at the $\mathrm{P}_{2} \mathrm{Y}_{11}$ receptor. The 2-MeS-ATP- $\alpha$-B diastere- 
oisomers are ineffective at the $\mathrm{P}_{2} \mathrm{Y}_{2}$ receptor5. Thus, the 2-MeS-ATP- $\alpha-\mathrm{B}(\mathrm{A})$ isomer can be used for subtypeselective activation of the $\mathrm{P}_{2} \mathrm{Y}_{1}$ receptor in tissues expressing also the $\mathrm{P} 2 \mathrm{Y}_{2}$ and $\mathrm{P} 2 \mathrm{Y}_{11}$ receptors. The distinct opposite diastereoselective activity of ATP derivatives at the $\mathrm{P}_{2} \mathrm{Y}_{11}$ and the $\mathrm{P} 2 \mathrm{Y}_{1}$ receptor will allow deciphering structural differences of the ligand recognition sites between these receptor subtypes. Presently, we are working on the molecular basis of the diastereoselectivity. By means of mutagenesis and rhodopsin-based homology $\mathrm{m}-$ odelling we aim to characterize the putative stereoselective nucleotide recognition site of the $\mathrm{P}_{2} \mathrm{Y}_{11}$ receptor. This will aid in the development of subtype-selective agonists.

1. Communi, D.; Govaerts, C.; Parmentier, M.; Boeynaems, J. M., Cloning of a human purinergic P2Y receptor coupled to phospholipase C and adenylyl cyclase. J Biol Chem 1997, 272, (51), 31969-73.

2. Burnstock, G., Purinergic signalling. Br J Pharmacol 2006, 147 Suppl 1, S172-81.

3. Nahum, V.; Zundorf, G.; Levesque, S. A.; Beaudoin, A. R.; Reiser, G.; Fischer, B., Adenosine 5'-O-(1-boranotriphosphate) derivatives as novel P2Y(1) receptor agonists. J Med Chem 2002, 45, (24), 5384-96.

4. Major, D. T.; Nahum, V.; Wang, Y.; Reiser, G.; Fischer, B., Molecular recognition in purinergic receptors. 2. Diastereoselectivity of the h-P2Y1-receptor. J Med Chem 2004, 47, (18), 4405-16.

5. Tulapurkar, M. E.; Laubinger, W.; Nahum, V.; Fischer, B.; Reiser, G., Subtype specific internalization of P2Y1 and P2Y2 receptors induced by novel adenosine 5'-O-(1-boranotriphosphate) derivatives. Br J Pharmacol 2004, $142,(5), 869-78$.

\title{
DIFFERENT BEHAVIOUR OF P2X 7 RECEPTORS IN CORTICAL vS. HIPPOCAMPAL ASTROCYTES
}

\author{
Bianco F., Colombo A, Mele R, Matteoli M. and Verderio C. \\ CNR-Institute of Neuroscience, Univ. of Milano Dept. of Medical Pharmacology, \\ Center of Excellence on Neurodegenerative Diseases, Milano Italy. \\ f.bianco@in.cnr.it
}

$\mathrm{P}_{2} \mathrm{X}_{7}$ is an ionotropic purinergic receptor with the characteristic to undergo transition from a selective channel to an aspecific pore, permeable to molecules up to $900 \mathrm{kD}$. Prolonged activation of the $\mathrm{P}_{2} \mathrm{X}_{7}$ receptor and pore transition can cause cell permeabilization and lysis.

The mechanisms leading to pore formation are just starting to be unravelled. One hypothesis suggests that pore formation derives from dilatation of the channel through recruitment of additional receptor subunits (Wiley et al, 1998; Virginio et al, 1999; Smart et al, 2002) while another hypothesis suggests that activation of $\mathrm{P}_{2} \mathrm{X}_{7}$ determines synthesis of a second messenger, which is responsible for pore opening. In this view, pore opening might occur through a membrane protein distinct from the purinergic receptor (Persechini et al., 1998; Gu et al, 2001).

In line with the recent observation that MAPK may be involved in pore formation. (Faria et al, 2005), here we show that the capability of $\mathrm{P}_{2} \mathrm{X}_{7}$ receptor to form an aspecific pore requires p38 MAPK activation, as pretreatment with the MAPK inhibitor SB, strongly prevented pore transition, as assayed by Yo-Pro uptake. We also provide evidence for different behaviour of the $\mathrm{P}_{2} \mathrm{X}_{7}$ receptor in primary astrocytes from two rat brain regions, cortex and hippocampus. While $\mathrm{P} 2 \mathrm{X}_{7}$ receptor in cortical astrocytes undergoes transition from selective ionotropic channel to aspecific pore, $\mathrm{P} 2 \mathrm{X}_{7}$ receptor in hippocampal astrocytes does not form a pore. The different ability to undergo pore transition affects cytokine release. Following pore formation in cortical astrocytes exposed to LPS, caspase-1 is activated, and the enzyme is able to process the pro-inflammatory cytokine IL1-beta which is released in the extracellular medium in its active form. $\mathrm{P} 2 \mathrm{X}_{7}$ activation in hippocampal astrocytes does not result in IL1-beta secretion, although the pro-cytokine is present in the cytosol of astrocytes activated with LPS. The different mechanisms involved in $\mathrm{P}_{2} \mathrm{X}_{7}$ receptor activation, by affecting the ability of cortical and hippocampal astrocytes to release proinflammatory cytokines, might influence the onset on neuroinflammation during neurodegenerative processes in different brain areas.

- Faria RX, DeFarias FP, and Alves LA. Are second messengers crucial for opening the pore associated with P2X7 receptor? Am J Physiol Cell Physiol 288: C194-C205, 2005. 
- Gu BJ, Zhang WY, Worthington RA, Sluyter R, Dao-Ung P, Petrou S, Barden JA, and Wiley JS. A Glu-496 to Ala polymorphism leads to loss of function of the human P2X7 receptor. J Biol Chem 276: 11135-11142, 2001.

- Persechini PM, Bisaggio RC, Alves-Neto JL, and Coutinho-Silva R. Extracellular ATP in the lymphohematopoietic system: P2Z purinoceptors off membrane permeabilization. Braz J Med Biol Res 31: 25-34, 1998

- Smart ML, Panchal RG, Bowser DN, Williams DA, and Petrou S. Pore formation is not associated with macroscopic redistribution of P2X7 receptors. Am J Physiol Cell Physiol 283: C77-C84, 2002.

- Virginio C, MacKenzie A, Rassendren FA, North RA, and Surprenant A. Pore dilation of neuronal P2X receptor channels. Nat Neurosci 2: 315-321, 1999.

- Wiley JS, Gargett CE, Zhang W, Snook MB, and Jamieson GP. Partial agonists and antagonists reveal a second permeability state of human lymphocyte P2Z/P2X7 channel. Am J Physiol Cell Physiol 275: C1224-C1231, 1998.

\title{
Differential Effect of Cyclic Stress and Constant Stress on ATP Release in Human Airway Epithelial Cells
}

\author{
Brian Button and Richard C. Boucher \\ Cystic Fibrosis Research and Treatment Center, University of North Carolina, Chapel Hill, North Carolina, USA. \\ Brian_Button@med.unc.edu
}

The airway surface liquid (ASL) layer lining the superficial cells of the airways is crucial for regulating mucociliary clearance and removal of noxious materials from the lungs. As a result of ion channel and fluid regulation, the height of the ASL is maintained at $\sim 7 \mu \mathrm{m}$, where cilia are fully erect, capable of proper cilia beating. Our overarching hypothesis is that the concentration of extracellular ATP and adenosine (ADO), via the activation of $\mathrm{P}_{2} \mathrm{Y}_{2}$ and $\mathrm{A}_{2 \mathrm{~b}}$ purinoceptors located in the luminal membrane, modulate the activity of ion channels involved in fluid secretion and absorption, to maintain the ASL at this level. The significance of ATP/ADO on ASL regulation in airway epithelia is underscored by experiments that demonstrate: 1) addition ATP/ADO induces ASL fluid secretion and 2) Removal of extracellular ATP/ADO induces unregulated ASL fluid absorption. We have previously demonstrated that mechanical stimulation of airway epithelial cultures, using cyclic stress to emulate the stresses experienced in the lung during normal tidal breathing, elicits ATP release, to levels that are sufficient to stimulate ASL secretion. In addition to cyclic stresses in the lung, constant (static) stress, such as those associated with bronchoconstriction of the airways, can also be observed. However, the ability of such constant stress to stimulate ATP release and fluid secretion, like cyclic stress, is unknown. The goal of this study was to directly compare ATP release and its subsequent effect on fluid secretion, on airway cultures under physiological levels of cyclic or static stress. In these studies, well-differentiated human airway cultures were exposed to 30 minutes of: 1) constant transepithelial pressure of $20 \mathrm{cmH}_{2} \mathrm{O}$, 2) cyclic pressure, oscillating between atmospheric pressure and 20 $\mathrm{cmH}_{2} \mathrm{O}$ at 20 cycles/ min, or 3) atmospheric pressure only. To determine the rate of ATP release, a cocktail of ATPase inhibitors (ebselen, $\beta \gamma$ MeATP and AP5A) were added to the luminal solution in order to prevent ATP hydrolysis. The cumulative ATP present in the ASL at 30 minutes was assessed by microsampling the ASL and quantified using a conventional luciferin-luciferase assay. In control cultures, the ATP release rate was $378 \pm 69$ $\mathrm{fmol} / \mathrm{cm}^{2} / \mathrm{min}$, similar to previously reported values. In cultures undergoing cyclic stress, ATP release was significantly stimulated, to $2514 \pm 823 \mathrm{fmol} / \mathrm{cm}^{2} / \mathrm{min}$. In contrast, the rate of ATP release during constant stress was 422 $\pm 126 \mathrm{fmol} / \mathrm{cm}^{2} / \mathrm{min}$, significantly less than cyclic stress at the same pressure (stress) magnitude. Further, exposures of airway cultures to static pressure $\left(20 \mathrm{cmH}_{2} \mathrm{O}\right.$ for $\left.24 \mathrm{~h}\right)$ failed to induce a significant change in steady-state ASL height, as measured by confocal microscopy ( $7.5 \pm 1.6$ versus $7.8 \pm 0.9 \mu \mathrm{m}$ for control). However, cyclic stress $(20$ $\mathrm{cmH}_{2} \mathrm{O} / 20 \mathrm{cycles} / \mathrm{min}$ for $24 \mathrm{~h}$ ) resulted in a doubling of ASL height (to $14.2 \pm 1.7 \mu \mathrm{m}$ ). The cyclic stress-induced secretion was completely inhibited by the pre-treatment with apyrase/ADA to inhibit accumulation of ATP/ADO (to $4.4 \pm 0.2 \mu \mathrm{m}$ ), demonstrating that ASL secretion requires the stimulation of ATP release, which was not observed in cultures under constant stress. These results provide the first evidence of differential effects of cyclic and static stress on ATP release from airway epithelial cells. While the mechanism by which airway epithelial cells differentiate between cyclic and constant stress remains to be elucidated, this model system is useful in delivering the stimuli required to investigate this phenomenon. Supported by the Cystic Fibrosis Foundation. 


\section{Differential effects of $\mathbf{A}_{2 \mathrm{~A}}$ adenosine receptor gene transfer and cyclic $\mathrm{AMP}$ elevation on the induction of distinct NF- $\mathrm{B}$ target genes in vascular endothelial cells}

Elaine W. Strong and Timothy M. Palmer

Division of Biochemistry \& Molecular Biology, IBLS, University of Glasgow, Scotland, U.K. 0311557s@student.gla.ac.uk

The $\mathrm{A}_{2 \mathrm{~A}}$ adenosine receptor $\left(\mathrm{A}_{2 \mathrm{~A}} \mathrm{AR}\right)$ is a critical non-redundant suppressor of inflammatory responses in vivo. One important mechanism by which this seems to occur is suppression of the NF- $\mathrm{B}$ pathway, which controls the induction of multiple genes encoding proteins that initiate and sustain pro-inflammatory responses. We have previously demonstrated that $\mathrm{A}_{2 \mathrm{~A}} \mathrm{AR}$ gene transfer in human umbilical vein endothelial cells (HUVECs) suppresses NF-кB activation in response to stimuli such as TNF $\alpha$ and LPS by reducing the nuclear accumulation of p50/p65 heterodimers. To assess the functional sequelae of this phenomenon, we have compared the effects of $\mathrm{A}_{2 \mathrm{~A}} \mathrm{AR}$ gene transfer in HUVECs on the accumulation of three important gene products known to be controlled at least in part by NF-кB; cyclooxygenase-2 (COX-2), E-selectin and vascular cell adhesion molecule-1 (VCAM1). Characterisation of COX-2 induction in response to TNF $\alpha$ revealed that while sustained induction (24 hr) was solely dependent on activation of p38 MAP kinase, earlier time-points at which induction was first detectable (8 hr) were also sensitive to inhibition of the NF- $\mathrm{B}$ pathway. At this time-point, $\mathrm{A}_{2 \mathrm{~A}} \mathrm{AR}$ gene transfer alone was sufficient to reduce $\mathrm{TNF} \alpha$-stimulated $\mathrm{COX}-2$ induction compared with controls, while no effect of the $\mathrm{A}_{2 \mathrm{~A}} \mathrm{AR}$ was detectable at $24 \mathrm{hr}$. Surprisingly, addition of the $\mathrm{A}_{2 \mathrm{~A}} \mathrm{AR}$-selective agonist CGS21680 to $\mathrm{A}_{2 \mathrm{~A}} \mathrm{AR}$-expressing HUVECs actually reversed the effect seen with receptor alone. Time-course experiments in control cells using elevated concentrations of the adenylyl cyclase activator forskolin demonstrated that cAMP elevation was sufficient to promote the transient induction of COX-2 even in the absence of TNF $\alpha$. However, in the presence of $\mathrm{TNF} \alpha$, exposure to different concentrations of forskolin exerted a biphasic effect, suppressing COX-2 induction at low concentrations while enhancing it at higher concentrations. These data suggest that the effects of $\mathrm{A}_{2 \mathrm{~A}} \mathrm{AR}$ expression with or without agonist on TNF $\alpha$-mediated COX-2 induction can be accounted for completely by corresponding changes in cAMP. Comparative analysis with E-selectin revealed that induction of this gene was more straightforward, being sensitive to NF- $\mathrm{BB}$ inhibition alone with no contribution of any other signalling pathway being detectable. Moreover, while induction was suppressed by $\mathrm{A}_{2 \mathrm{~A}} \mathrm{AR}$ gene transfer, the suppression was more pronounced in the presence of CGS21680 and correlated with an ability of cAMP-elevating stimuli to block TNF $\alpha$-mediated E-selectin induction in control cells. Interestingly, TNF $\alpha$-mediated induction of VCAM-1 was unaffected by either $\mathrm{A}_{2 \mathrm{~A}} \mathrm{AR}$ gene transfer or pre-treatment of control cells with cAMP-elevating agents. Taken as whole, these data argue that the ability of the $\mathrm{A}_{2 \mathrm{~A}} \mathrm{AR}$ to modulate the expression patterns of defined $\kappa \mathrm{B}$-sensitive target genes in HUVECs can be explained completely by the ability of the receptor to couple positively to adenylyl cyclase and elevate intracellular cAMP levels. They also suggest that the net effects of $\mathrm{A}_{2 \mathrm{~A}} \mathrm{AR}$ gene transfer on specific $\mathrm{\kappa B}$-regulated target genes will vary depending on the nature of their control by cAMP elevation.

\section{DIFFERENTIAL EXPRESSION OF E-NTPDASES IN GLIOMA CELL LINES IN RELATION TO ASTROCYTES}

M. R. Wink ${ }^{1}$, E. Braganhol ${ }^{1}$, A. S. K. Tamajusuku ${ }^{1}$, E.A. Casali ${ }^{1}$, G. Lenz ${ }^{2}$, L. F. Zerbini ${ }^{3}$, T. A. Libermann $^{3}$, J. Sévigny ${ }^{4}$, A. M. O. Battastini ${ }^{1}$ and S. C. Robson ${ }^{5}$.

${ }^{1}$ Departamento de Bioquímica, ICBS, UFRGS, Rua Ramiro Barcelos, 2600-anexo, CEP 90035-003, Porto Alegre, RS, Brazil, ${ }^{2}$ Departamento de Biofísica, IB, UFRGS, Porto Alegre, RS, Brazil; ${ }^{3}$ BIDMC Genomics Center and New England Baptist Bone and Joint Institute, Beth Israel Deaconess Medical Center, Harvard Medical School, Boston, MA 02215, USA ${ }^{4}$ Centre de Recherche en Rhumatologie et Immunologie, Centre Hospitalier Universitaire de Québec, Université Laval, Québec, Canada and ${ }^{5}$ Department of Medicine, Beth Israel Deaconess Medical Center, Harvard Medical School, 99 Brookline Avenue, Boston, MA 02215,USA

abattastini@terra.com.br 
Extracellular nucleotides and nucleosides modulate glial cell growth and are important factors involved in inflammation, stroke and progression of tumor cells. It is known that ATP and adenosine induce proliferation in human astrocytoma cells and that ectonucleotidases play a key role in purinergic signaling regulation. We undertook a comparative study of the degradation of extracellular nucleotides by primary astrocytes cultures and glioma cell lines. We have previously observed that extracellular ATP and ADP degradation by glioma cell is lower than by astrocyte cultures. In the present work, we have analyzed the pattern of phosphohydrolysis of extracellular ATP by HPLC and have characterized the nature of E-NTPDases expression by RT-PCR, quantitative real-time PCR, immunocytochemistry as well as Western blotting analysis in astrocytes and glioma cell cultures. We noted that astrocytes generate AMP as 50\% of the degradation product at $3 \mathrm{~h}$, whereas this nucleotide did not represent more than 5\% of extracellular product in the glioma cell lines. Among the nuclosides and the non-phosphorylated degradation products, normal astrocyte cultures generate twice as much inosine and hypoxanthine (14 and 15\%) when compared to adenosine (7\%). Glioma cell lines had almost undetectable levels of adenosine, generating inosine as the major non-phosphorylated degradation product. By quantitative real-time PCR, we identified Entpd2 (CD39L1) as the dominant Entpd gene expressed by rat hippocampal, cortical and cerebelar astrocytes, while C6 glioma cells exhibit low expression of all NTPDases investigated. The substantial decreases in ATP and ADP hydrolysis observed in glioma cultures were substantiated by the absence of NTPDase2. ATP is recognized as a mitogenic factor and potentiates the proliferation in human glioma cells. Therefore, we suggest that reduction of the ATP hydrolysis in glioma cell lines may be part of a process to grant high progression rates of these tumor cells. We propose that alterations in the ecto-nucleotidase expression may represent an important mechanism associated with malignant transformation of glioma cells.

Support: NIH grants HL-63972 (Dr. S.C. Robson) and CNPq, FAPERGS (Dr. A.M.O. Battastini)

\title{
Differential regulation of NTPdase1 and NTPdase2 by cholesterol.
}

\author{
$\underline{\text { A.Papanikolaou }}^{1}$, A.Papafotika ${ }^{1,2}$, A.Xilouri $^{3}$, J.Mitsios $^{4}$, A.Tselepis ${ }^{4}$, and S.Christoforidis ${ }^{1,2}$ \\ Laboratory of Biological Chemistry, Medical School, University of Ioannina \\ Biomedical Research Institute/Foundation for Research and Technology, Ioannina \\ Department of Obstetrics and Gynecology, University Hospital of Ioannina, Ioannina \\ Laboratory of Biochemistry, Department of Chemistry, University of Ioannina, Greece. \\ me00897@cc.uoi.gr
}

Nucleoside triphosphate diphosphohydrolases (NTPDases) are a family of ectonucleotidases that differentially hydrolyze $\gamma$ and $\beta$ phosphate groups of extracellular nucleotides. NTPDase 1 is the major ecto-nucleotidase at the luminal surface of blood vessels and hydrolyzes both triphospho- and diphosphonucleosides thereby terminating platelet aggregation in response to ADP. In contrast, NTPDase2 is a preferential nucleoside triphosphatase in the adventitia of blood vessels. By converting ATP to ADP, NTPDase2 activates platelet aggregation in the case of vessel damage.

The enzymatic activity of NTPDase1 depends on cholesterol-rich domains of the membrane which points to a NTPdase1-dependent protective role of cholesterol in thrombosis. If this is true, given the platelet-activating action of NTPDase2, cholesterol should exert an opposite effect on this NTPDase. To address this question we increased the cholesterol content of isolated heart membranes using cholesterol complexed with methyl- $\beta$-cyclodextrin and measured its effect on ecto-ATPase enzymatic activity. Interestingly, treatment of membranes with increasing concentrations of cholesterol resulted in a corresponding inhibition of the enzymatic activity of NTPdase2.

To obtain further insights into this inhibition, we measured the endogenous cholesterol levels in the heart membranes. We were surprised by the finding that the cholesterol content in heart membranes (where NTPdase 2 is enriched) is 10 times lower than the cholesterol amount in placental membranes (where NTPdase1 is enriched). It is therefore likely that NTPDase2 is localized in cholesterol-poor domains for maintaining a high enzymatic activity.

A key function of NTPDase2 is to promote platelet plug formation in the case of vessel injury. To examine the role of cholesterol on NTPDase2-dependent activation of platelet aggregation, we performed platelet aggregation assays in the presence of heart membranes incubated with cholesterol. High levels of platelet aggregation were obtained when heart membranes were added to platelets and ATP, which were significantly reversed when heart membranes had been incubated with cholesterol. 
The above data raise the possibility that in pathological cases associated with high cholesterol levels, such as hypercholesterolemia and atherosclerosis, a cholesterol-dependent protective effect against thrombosis is of crucial importance. Consistent with this view, we found that, similarly to cholesterol, oxysterols and oxidized LDL exert an inhibitory effect on NTPdase2. Therefore, a concomitant differential control of NTPdase1 and NTPdase 2 activities by cholesterol may result in a fine tuning of extracellular nucleotide levels, providing a tight control of purinergic receptor signaling. Such a regulatory mechanism is expected to apply not only in thromboregulation, but also in other functions regulated by purinergic receptors where the fine balance of ATP/ADP ratio is of major importance.

\title{
Discovery of new potent, non-nucleotide ligands at human P2Y 11-receptors results in NF546, the first non-nucleotide P2Y receptor agonist, and NF340, the so far most potent and selective $\mathrm{P}_{2} \mathrm{Y}_{11}$ antagonist
}

\author{
Sabine Meis, Darunee Hongwiset, and Matthias U. Kassack \\ Institute of Pharmacy, University of Bonn, An der Immenburg 4, D-53121 Bonn (Germany) \\ sabine.meis@uni-bonn.de; kassack@uni-bonn.de
}

$\mathrm{P}_{2} \mathrm{Y}_{11}$ receptors are $\mathrm{G}$ protein-coupled receptors coupled to both, the phosphoinositide and the cAMP pathway 1. Up to now, only suramin as a non-selective and low potency compound $\left(\mathrm{pK}_{\mathrm{i}}=6.52\right)$ and NF157 recently introduced by our group as a more selective and potent ligand $\left(\mathrm{pK}_{\mathrm{i}}=7.35\right)$ have been described as $\mathrm{P}^{2} \mathrm{Y}_{11}$ antagonists 2 . Non-nucleotide agonists at P2Y receptors are not yet available. The aim of this study was to further improve the potency and selectivity of NF157 and to search for non-nucleotide $\mathrm{P}_{2} \mathrm{Y}_{11}$ agonists. A large number of sulfonic and phosphonic acid derivatives were screened at $\mathrm{P}_{2} \mathrm{Y}_{11}$ receptors recombinantly expressed in 1321N1 astrocytoma cells. Compounds were first studied with a previously described microplate-reader based $\mathrm{Ca}^{2+}$-assay 3. Compounds active in the $\mathrm{Ca}^{2+}$-assay were then also examined in a cAMP luciferase reporter-gene assay at $\mathrm{P}_{2} \mathrm{Y}_{11}$ receptors and further tested for selectivity at other P2Y-receptors.

The tetrasulfonic acid derivative NF340 was identified as a competitive antagonist with nanomolar potency $\left(\mathrm{pK}_{\mathrm{i}}=\right.$ 7.77) which is 2.6fold more potent than NF157. Concentrations up to $100 \mu \mathrm{M}$ showed no effects at other P2Y receptors $\left(\mathrm{P}_{2} \mathrm{Y}_{1}, \mathrm{P}_{2} \mathrm{Y}_{2}\right.$, and $\mathrm{P}_{2} \mathrm{Y}_{6}$, recombinantly expressed in $1321 \mathrm{~N} 1$ astrocytoma cells). Schild analysis with $\mathrm{ATP} \gamma \mathrm{S}$ as agonist gave a slope not significantly different from unity. The $\mathrm{pA}_{2}$ value was estimated as 8.07. The screening also identified the first non-nucleotide agonist at $\mathrm{P} 2 \mathrm{Y}$ receptors. The tetraphosphonic acid derivative NF546 showed almost the same intrinsic activity $(95 \%)$ at $\mathrm{P}_{2} \mathrm{Y}_{11}$ receptors as the standard agonist ATP $\gamma$ S in both assays, $\mathrm{Ca}^{2+}$ and cAMP. An $\mathrm{EC}_{50}$ of $542 \mathrm{nM}$ was obtained in the $\mathrm{Ca}^{2+}$-assay. Schild analyses with NF546 as agonist and NF157 or NF340 as antagonists were performed. The slopes of both Schild plots were not significantly different from unity, and the $\mathrm{pA}_{2}$ values of NF157 and NF340 using NF546 as agonist showed no difference from the results using ATP $\gamma$ S as agonist: NF157 / NF546: $\mathrm{pA}_{2}=7.66 ;$ NF157 / ATP $\gamma$ S: $\mathrm{pA}_{2}=7.77 ;$ NF340 / NF546: $\mathrm{pA}_{2}$ $=8.00 ; \mathrm{NF} 340 / \mathrm{ATP} \gamma \mathrm{S}: \mathrm{pA}_{2}=8.06$. NF546 showed no effect at other P2Y receptors up to $10 \mu \mathrm{M}$.

In conclusion, NF340 was identified as a selective, non-nucleotide antagonist at $\mathrm{P}_{2} \mathrm{Y}_{11}$ receptors with improved potency over the recently described NF157. NF546 is the first non-nucleotide P2Y agonist and selective for P2Y 11 receptors. Both compounds may serve as useful tools in studying the physiological role of $\mathrm{P}_{2} \mathrm{Y}_{11}$ receptors.

(1) Communi, D.; Govaerts, C.; Parmentier, M.; Boeynaems, J. M. J.Biol.Chem. 1997, 272, 31969-31973.

(2) Ullmann, H.; Meis, S.; Hongwiset, D.; Marzian, C.; Wiese, M.; Nickel, P.; Communi, D.; Boeynaems, J. M.; Wolf, C.; Hausmann, R.; Schmalzing, G.; Kassack, M. U. J.Med.Chem. 2005, 48, 7040-7048.

(3) Kassack, M. U.; Hofgen, B.; Lehmann, J.; Eckstein, N.; Quillan, J. M.; Sadee, W. J.Biomol.Screening. 2002, 7, 233- 246.

This project was supported by Bischoefliche Studienfoerderung Cusanuswerk, Deutsche Forschungsgemeinschaft (GRK677), and Deutscher Akademischer Austauschdienst.

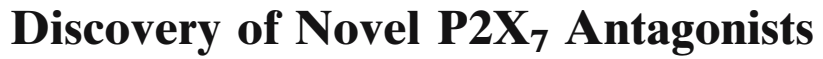

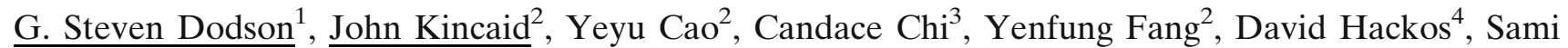
Hussain $^{4}$ Carl Kaub $^{2}$, Luna Lui ${ }^{3}$, Eileen Rose ${ }^{1}$, Jennifer Shumilla ${ }^{5}$ Qingling Zhang ${ }^{3}$, Michael G. Kelly. 
${ }^{1}$ Department of Discovery Biology, ${ }^{2}$ Department of Medicinal Chemistry, ${ }^{3}$ Department of Drug Metabolism and Pharmacokinetics, ${ }^{4}$ Department of in vitro Pharmacology, ${ }^{5}$ Department of in vivo Pharmacology

Renovis, Inc.

dodson@renovis.com,kincaid@renovis.com

The $\mathrm{P} 2 \mathrm{X}_{7}$ receptor is a ligand-gated ion channel that mediates a non-selective cation conductance in response to extracellular ATP. Extended exposure to agonist leads to the formation of a pore permeable to large molecular weight dyes. $\mathrm{P} 2 \mathrm{X}_{7}$ is expressed in a fairly limited set of cell types, primarily the hematopoietic lineages and glia, where it is thought to play a role in inflammatory process important in human disease states. In monocytes, macrophages and microglia primed with an inflammatory signal $\mathrm{P} 2 \mathrm{X}_{7}$ functions to initiate the post translational processing and release of leaderless cytokines such as IL-1 $\beta$. Published work in $\mathrm{P}_{2} \mathrm{X}_{7}$ knockout mice shows that the absence of $\mathrm{P} 2 \mathrm{X}_{7}$ activity in vivo can modulate an inflammatory response and attenuate the severity of $\mathrm{mAb}$ induced arthritis. In addition, absence of $\mathrm{P} 2 \mathrm{X}_{7}$ attenuates mechanical and thermal hyperalgesia in the Seltzer model of neuropathic pain and inflammatory hypersensitivity in FCA induced inflammation.

Our continued interest in the discovery and development of novel analgesic and anti-inflammatory therapeutics has led to the identification of multiple series of novel, selective and bioavailable $\mathrm{P}_{2} \mathrm{X}_{7}$ receptor antagonists.

Herein, we will disclose the in vitro and in vivo characterization of $\mathrm{RN}-6189$, an early biological lead that inhibits human $\mathrm{P}_{2} \mathrm{X}_{7}$ current in transfected HEK293 cells with an $\mathrm{IC}_{50}$ of $34 \pm 1.6 \mathrm{nM}$. In addition, $\mathrm{RN}-0276189$ inhibits both 2',3'-O-(4-benzoylbenzoyl)-adenosine 5'-triphosphate (BzATP) dependent pore formation and IL-1 $\beta$ release in THP-1 cells in the same concentration range. Further property based drug design has led us to identify novel compounds with improved potency and pharmacokinetic properties that are currently in preclinical evaluation.

\title{
Distinct effects of ticlopidine and clopidogrel on vascular ecto-nucleotidases and impact on haemostasis.
}

\author{
Lecka J. ${ }^{1}$, Rana M.S. ${ }^{1}$, Zimmermann H. ${ }^{2}$ and Sévigny J. ${ }^{1}$ \\ ${ }^{T}$ Centre de recherche en Rhumatologie et Immunologie, Université Laval, Ste-Foy, Québec, Canada. \\ ${ }^{2}$ Biocenter, J.W. Goethe-University, AK Neurochemistry, Frankfurt am Main, Germany. \\ e-mail: joanna.lecka@crchul.ulaval.ca
}

Ticlopidine (Tyklid) and clopidogrel (Plavix) are antiaggregatory drugs that irreversibly inhibit P2Y 12 receptor ${ }^{1}$. The real active metabolites that reduce platelet activation are produced by the liver after daily administration of the drugs ${ }^{2}$. $\mathrm{P}_{12} \mathrm{Y}_{12}$ receptor is activated by extracellular ADP which leads to platelet aggregation. Adenosine, the product of ADP dephosphorylation, inhibits platelet aggregation via activation of $\mathrm{A} 22_{\mathrm{a}}$ receptors ${ }^{3}$. Blood level of both purine ligands is regulated by ectonucleotidases, mainly NTPDase 1 and ecto-5'-nucleotidase ${ }^{4}$. As both of these enzymes have an important implication in haemostasis, we examined in vitro whether ticlopidine and clopidogrel could affect their biochemical activities. Concentrations of $30 \mu \mathrm{M}$ ticlopidine and $10 \mu \mathrm{M}$ clopidogrel have been reported in the plasma of treated patients. At this concentration, ticlopidine reduced ATPase and ADPase activity by $30 \%$ while $100 \mu \mathrm{M}$ of both drugs inhibited $60 \%$ of ADP hydrolysis; they had no effect at $10 \mu \mathrm{M}$. Since NTPDase1 is an important inhibitor of platelet aggregation, inhibiting this enzyme would be expected to generate undesired effects in treated patients. Indeed, the antiagreggatory effect of human NTPDase1 was significantly weaker in the presence of ticlopidine, as seen in platelet aggregation assays. Additionally, ticlopidine (but not clopidogrel) inhibited rat ecto-5'-nucleotidase by $11 \%$ at $100 \mu \mathrm{M}$ and $50 \%$ at $1 \mathrm{mM}$ level. Interestingly, the human ortholog of ecto-5'-nucleotidase was insensitive to both drugs. We observed that the inhibition of the vascular ectonucleotidases by ticlopidine is direct and did not require preincubation. Our data suggest that the action of ticlopidine in haemostasis is complex and not exclusively antiaggregatory while its analog clopidogrel may be more suitable for therapeutic use.

1. Gachet C. The platelet P2 receptors as molecular targets for old and new antiplatelet drugs.Pharmacol Ther. 2005 Nov;108(2):180-92. Epub 2005 Jun 13. Review.

2. Kam P.C., Nethery C.M. The thienopyridine derivatives (platelet adenosine diphosphate receptor antagonists), pharmacology and clinical developments.Anaesthesia. 2003 Jan;58(1):28-35. Review. 
3. Hourani S.M. Purinoceptors and platelet aggregation. J Auton Pharmacol. 1996 Dec;16(6):349-52. Review. 4. Lecka J., Komoszynski M., The role of ecto-purines (e-purines) in regulation of blood pressure, haemostasis and etiology of atherosclerosis. Progress in Medical Research (2003). Review.

\section{Distribution and Regulation of the nucleoside transporter, ENT1}

Kay Barnes $^{1}$, Halina Dobrzynski ${ }^{2}$, Sophie Foppolo ${ }^{1}$, Paul R. Beal ${ }^{3}$, James Tellez ${ }^{2}$, William C. Claycomb ${ }^{7}$, Carol E. Cass ${ }^{4,5,6}$, James D. Young ${ }^{4}$, Rudi Billeter-Clark ${ }^{1}$, Mark R. Boyett ${ }^{2}$ and Stephen A. Baldwin ${ }^{1}$.

From the ${ }^{1}$ Institute of Membrane and Systems Biology, University of Leeds, Leeds, LS2 9JT, United Kingdom, the ${ }^{2}$ Division of Cardiovascular and Endocrine Sciences, University of Manchester, Manchester M13 9XX, United Kingdom, the ${ }^{3}$ Department of Biology, University of York, United Kingdom, the ${ }^{4}$ Membrane Protein Research Group, Departments of Physiology and ${ }^{5}$ Onconlogy, University of Alberta and the ${ }^{6}$ Cross Cancer Institute, Edmonton, Alberta T6G 2H7, Canada, ${ }^{7}$ Department of Biochemistry and Molecular Biology, Louisiana State, University Health Sciences Center, New Orleans, Louisiana 70112, U.S.A.

k.barnes@leeds.ac.uk

ENT1 is a well-characterized member of the equilibrative (SLC29) nucleoside transporter family that has an important role in regulation of adenosine concentrations in the vicinity of extracellular purinergic receptors. ENT1 has been designated as belonging to the es (equilibrative inhibitor-sensitive) subtype of nucleoside transporters as it is potently inhibited by NBMPR (nitrobenzylmercaptopurine riboside) in the nanomolar range. It has 11 transmembrane helices with a large intracellular loop joining transmembrane domains 6 and 7, a cytoplasmic $\mathrm{N}$-terminus and and extracellular C-terminus.

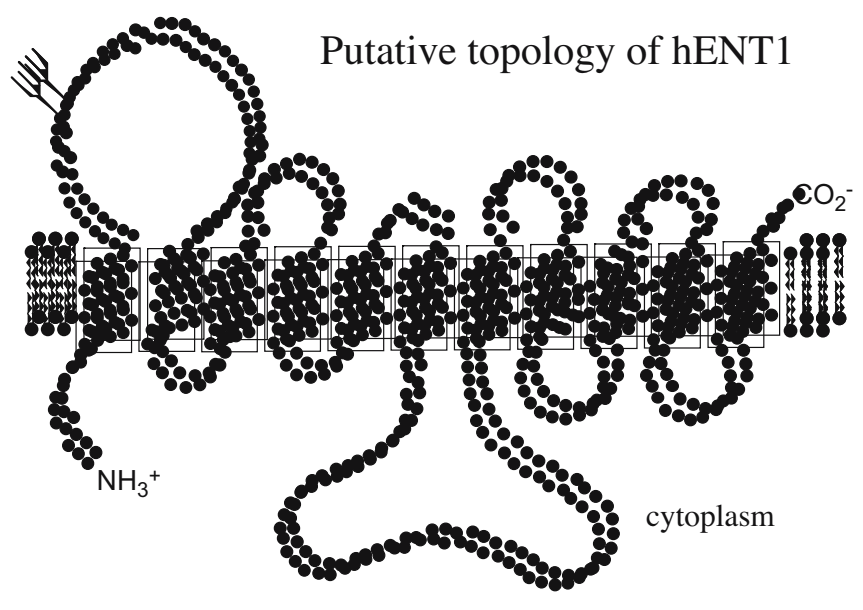

In this study we have used qualitative and quantitative immunological approaches to examine the relative distribution of rENT1 in the ventricles, atria and SA node of the rat heart. In parallel experiments using RT PCR and tissue samples prepared from the same rats hENT1mRNA transcripts showed a similar distribution to the transporter protein. Although little is known about the mechanism of nucleoside transporter regulation, activity of the transporter can be modulated by activators and inhibitors of protein kinase $\mathrm{C}$ and protein kinase $\mathrm{A}$ and by the serine/threonine protein kinase, casein kinase II. Using a bioinformatic approach we have identified potential phosphorylation sites in hENT1 that may be important in transporter regulation and expression constructs encoding hENT1 mutants lacking the potential phosphorylation sites have been prepared. 


\title{
Sequential activation of adenosine $A_{1}$ receptors terminate inhibition of acetylcholine release caused by ADP-sensitive P2Y $_{1}$ receptors on myenteric motoneurons
}

\author{
Margarida Duarte-Araújo, M. Alexandrina Timóteo \& Paulo Correia-de-Sá \\ Laboratório de Farmacologia, Unidade Multidisciplinar de Investigação Biomédica (UMIB), Instituto de Ciências \\ Biomédicas de Abel Salazar (ICBAS) - Universidade do Porto, Portugal. \\ mdcma@icbas.up.pt
}

Purine nucleotides and nucleosides are responsible for modulating gastrointestinal function. Besides the direct effect of ATP on neuronal P2X receptors, the nucleotide may indirectly control nerve excitability due to the formation of metabolites. In the myenteric plexus, ATP actions are rapidly terminated by intense ecto-NTPDase 1 (ecto-diphosphohydrolase) activity generating AMP, which is then converted into adenosine by ecto-5'-nucleotidase. Alternative formation of ADP by ecto-ATPase might be physiologically relevant to restrain acetylcholine $(\mathrm{ACh})$ release from myenteric motoneurons via $\mathrm{P}_{2} \mathrm{Y}_{1}$ purinoceptors activation. The co-existence of inhibitory $\mathrm{P}_{2} \mathrm{Y}_{1}$ and adenosine $\mathrm{A}_{1}$ receptors in the rat myenteric plexus lead us to investigate their role to control $\left[{ }^{3} \mathrm{H}\right]-\mathrm{ACh}$ release from stimulated (5 Hz, 200 pulses) cholinergic motoneurons.

The experiments were performed at $37^{\circ} \mathrm{C}$ on longitudinal muscle-myenteric plexus of rat ileum, superfused with gased $\left(95 \% \mathrm{O}_{2}+5 \% \mathrm{CO}_{2}\right)$ Tyrode's solution. The release of $\left[{ }^{3} \mathrm{H}\right]$-ACh from myenteric motoneurons loaded with $\left[{ }^{3} \mathrm{H}\right]$-choline $(2.5 \mu \mathrm{Ci} / \mathrm{mL})$, was induced by electrical field stimulation $(5 \mathrm{~Hz}, 200$ pulses, $0.5 \mathrm{~ms}, 60 \mathrm{~V})\left(\mathrm{S}_{1}\right.$ and $\left.\mathrm{S}_{2}\right)$. Test drugs were added 15 minutes before $S_{2}$, and their effects in transmitter release were expressed by the $S_{2} / S_{1}$ ratios. When we evaluated changes in the effect of tested drugs induced by a modifier, the latter compound was applied 15 min before starting sample collection and hence it was present during $S_{1}$ and $S_{2}$.

Selective blockade of $\mathrm{A}_{1}$ - and $\mathrm{P}_{2} \mathrm{Y}_{1}$-receptors respectively with 1,3-dipropyl-8-cyclopentyl xanthine (DPCPX, 10 $\mathrm{nM})$ and MRS2179 $(300 \mathrm{nM})$ increased $\left[{ }^{3} \mathrm{H}\right]$-ACh release by $43 \pm 12 \%(\mathrm{n}=3)$ and $27 \pm 3 \%(\mathrm{n}=4)$, respectively. A reduction of tritium outflow was observed by activating adenosine $\mathrm{A}_{1}$ receptors, with $\mathrm{R}-\mathrm{N}^{6}$-phenylisopropyl adenosine (R-PIA, $300 \mathrm{nM},-36 \pm 4 \%, \mathrm{n}=4)$, or P2Y purinoceptors, with ADP $\beta S(30 \mu \mathrm{M},-27 \pm 3 \%, \mathrm{n}=4)$. The inhibitory action of ADP $\beta S(30 \mu \mathrm{M})$ was blocked by MRS2179 $(300 \mathrm{nM})$, but the P2Y ${ }_{1}$-receptor antagonist was devoid of effect on R-PIA (300 nM) inhibition. On the contrary, DPCPX (10 $\mathrm{nM})$ attenuated inhibition by R-PIA (300 $\mathrm{nM}$ ), while significantly enhancing (to $42 \pm 5 \%, \mathrm{n}=4)$ ADP $\beta S(30 \mu \mathrm{M})$-induced depression. While ADP $\beta S$ (30 $\mu \mathrm{M})$ failed to modify the action of R-PIA $(300 \mathrm{nM})$, activation of adenosine $\mathrm{A}_{1}$ receptors occluded the inhibitory effect of ADP $\beta S(30 \mu \mathrm{M})$.

We provided evidence showing that adenosine exerts a fine-tuning control of tonic ACh release inhibition mediated by released adenine nucleotides at the myenteric synapse. The results indicate that over stimulation of inhibitory $\mathrm{P}_{2} \mathrm{Y}_{1}$ purinoceptors is cut-short by sequential activation of inhibitory $\mathrm{A}_{1}$ receptors by endogenously generated adenosine.

Work supported by FCT (POCTI/45549/FCB/2002, participation of FEDER funding).

\section{EARLY AND TRANSIENT ALTERATION OF ADENOSINE A RECEPTOR SIGNALLING IN A MOUSE MODEL OF HUNTINGTON DISEASE}

\author{
Alessia Tarditi, Alessandra Camurri, Katia Varani ${ }^{1}$, Pier Andrea Borea ${ }^{1}$, Ben Woodman ${ }^{2}$, Gillian Bates ${ }^{2}$, \\ Elena Cattaneo and Maria P. Abbracchio \\ Department of Pharmacological Sciences, University of Milan, Italy, and ${ }^{1}$ Department of Clinical and Experimental \\ Medicine, Pharmacology Unit, University of Ferrara, Italy ${ }^{2}$ Department of Medical and Molecular Genetics, GKT \\ School of Medicine, King's College London, UK
}

Huntington Disease (HD) is a dominantly-inherited neurodegenerative disorder featuring progressive worsening chorea, psychiatric disturbances and cognitive impairment due to brain cell loss. $\mathrm{A}_{2 \mathrm{~A}}$ receptors expressed on 
GABAergic striatal neurons have been suggested to play a pathogenetic role. Previous data demonstrated the presence of an aberrant alteration of $\mathrm{A}_{2 \mathrm{~A}}$ receptor-dependent adenylyl cyclase in an in vitro model of the disease (striatal cells expressing mutant huntingtin) and in peripheral circulating cells of HD patients, suggesting that the aberrant $\mathrm{A}_{2 \mathrm{~A}}$ receptor phenotype may represent a novel potential biomarker of $\mathrm{HD}$. Despite evidence suggesting a facilitatory role of $\mathrm{A}_{2 \mathrm{~A}}$ receptors in neuronal death, a direct correlation between the peripheral $\mathrm{A}_{2 \mathrm{~A}}$ receptor change in HD subjects and the status of this receptor in HD brain is still lacking. One of the best characterized animal models of HD is represented by the R6/2 mice. We thus investigated in this model the presence, expression and functionality of striatal $\mathrm{A}_{2 \mathrm{~A}}$ receptors at different developmental stages in comparison with age-matched wild type animals. A transient increase in $\mathrm{A}_{2 \mathrm{~A}}$ receptor density $(\mathrm{Bmax})$ with no changes in affinity $\left(\mathrm{K}_{\mathrm{D}}\right)$ and $\mathrm{A}_{2 \mathrm{~A}}$ receptor-dependent cAMP production at early presymptomatic ages (7-14 postnatal days) was found. The increase of $\mathrm{A}_{2 \mathrm{~A}}$ receptor-mediated adenylyl cyclase responsiveness in R6/2 mice was even more marked, as demonstrated by an $\mathrm{EC}_{50}$ value of the $\mathrm{A}_{2 \mathrm{~A}}$ receptor agonist CGS 21680 ten fold lower with respect to corresponding $\mathrm{EC}_{50}$ value in wild type mice $(175 \pm 23 \mathrm{nM}$, with respect to $1922 \pm 197 \mathrm{nM})$. Both alterations normalized to control values starting from postnatal day 21. In contrast, $\mathrm{A}_{2 \mathrm{~A}}$ receptor mRNA, as detected by real time PCR, dramatically decreased starting from PND21 until late symptomatic stages (12 weeks of age). The discrepancy between $\mathrm{A}_{2 \mathrm{~A}}$ receptor expression and density suggests compensatory mechanisms. These data, reproducing ex vivo the previous observations in vitro, support the hypothesis that an alteration of $\mathrm{A}_{2 \mathrm{~A}}$ receptor signalling is present in HD and might represent an interesting target for neuroprotective therapies.

Sponsored by the Italian Ministry of Health (progetto "Ruolo del recettore $\mathrm{A}_{2 \mathrm{~A}}$ dell'adenosina e metabotropici del glutammato nelle demenze da neurodegenerazione striatale", Alz4) and by the Hereditary Disease Foundation.

\title{
Ecto-5' nucleotidase (CD73) activity and expression in the rat ileum
}

\author{
$\underline{\text { Anna Bin }}^{1}$, Maria Cecilia Giron ${ }^{1}$, Sabrina Etteri $^{1}$, Francesca Bongiovanni ${ }^{1}$, Chiara Florio $^{2}$, \\ Rosa Maria Gaion ${ }^{1}$ \\ ${ }^{1}$ Department of Pharmacology and Anesthesiology, University of Padova; \\ ${ }^{2}$ Department of Biomedical Sciences, University of Trieste \\ anna.bin@unipd.it
}

INTRODUCTION: Ecto-5'nucleotidase (CD73) is a purine salvage enzyme located on the external surface of plasma membrane in many cell types. CD73 is responsible for adenosine formation from AMP and has a regulatory role in neurotransmitter release, functions to control blood flow and may play a role in antiinflammatory responses ${ }^{1-3}$. Concerning the intestinal tract, expression of CD73 has been demonstrated in human duodenal mucosa, intestinal epithelial cells and in the longitudinal muscle strips of guinea pig ileum ${ }^{1,4}$.

AIMS: The purpose of the present study was to evaluate the metabolism of exogenous AMP by rat ileum strips (ISs) and the role of CD73 in the production of extracellular adenosine (ADO) in the intestine. Expression of CD73 in ileum smooth muscle cells (ISMCs) was also examined.

METHODS: Rat ISs were incubated in vitro in $2 \mathrm{ml}$ of Tyrode solution with exogenous AMP $(50 \mu \mathrm{M})$ for 5 or 60 minutes and $1 \mathrm{ml}$ of the medium was used for the determination of AMP, ADO, inosine and IMP by HPLC. ISMCs were prepared according to the method of $\mathrm{Khan}^{5}$ and the presence of CD73 was detected by confocal fluorescent microscopy using anti-CD73 antibody.

RESULTS: AMP added to the incubation medium was metabolised by $82 \%$ and $100 \%$ within 5 and 60 min, respectively. Treatment with $\alpha, \beta$ methylene-ADP (AOPCP, $200 \mu \mathrm{M}$ ), a CD73 inhibitor, allowed the recovery of endogenous AMP and IMP (at $5 \min 0.2 \pm 0.08$ and $0.8 \pm 0.2 \mu \mathrm{M}$, respectively, and at $60 \mathrm{~min} 0.5 \pm 0.1$ and $2.2 \pm 0.3 \mu \mathrm{M}$, respectively). AOPCP also reduced the clearance of exogenous AMP, whose concentrations at 5 and 60 min rose to $18.7 \pm 1.8$ and $4.4 \pm 0.9 \mu \mathrm{M}$, respectively. The addition of AMP caused a marked increase in ADO concentration in the medium. This increase was inhibited by AOPCP, indicating that the nucleoside is rapidly formed from AMP. Inosine showed the same trend as ADO, in accordance with the high activity of intestinal ecto- $\mathrm{ADA}^{6}$. Addition of AMP in AOPCP-pretreated samples did not significantly modify IMP levels, showing that the contribution of AMP deaminase to exogenous AMP metabolism is irrelevant. Immunocytochemical analysis demonstrated the high expression of CD73 on the surface of ISMCs. 
CONCLUSIONS: These results suggest that CD73 is a critical enzyme responsible for the generation of adenosine. Its expression on ISMCs, shown here for the first time, points out the possible key role in the control of intestinal motility. This is the first step in the development of further studies aimed to understand how CD73 expression can be affected by drugs and by pathological conditions.

\title{
REFERENCES
}

1. Strohmeier, G.R.; Lencer, W.I.; Patapoff T.W.; Thompson L.F.; Carlson, S.L.; Moe, S.J.; Carnes, D.K.; Mrsny, R.J.; Madara, J.L. J. Clin. Invest. 1997, 99, 2588-2601.

2. Narravula, S.; Lennon, P.F.; Mueller, B.U.; Colgan S.P. J. Immunol. 2000, 165, 5262-5268.

3. Hunsucker, S.A.; Mitchell, B.S.; Spychala J. Pharmacol. Ther. 2005, 107, 1-30.

4. Nitahara, K.; Kittel, A.; Liang, S.D.; Vizi, E.S. Neuroscience 1995, 67, 159-168.

5. Khan, I.; Blennerhassett, M.G.; Kataeva G.V.; Collins, S.M. Gastroenterology 1995, 108, 1720-1728.

6. Xu, P.A.; Kellens, R.E. Biochem. Biophys. Res. Commun. 2000, 269, 749-757.

\section{Ecto-5' ${ }^{\prime}$-nucleotidase (CD73) and Mucosal Inflammation}

\author{
Sean P. Colgan $^{1}$, Linda F. Thompson ${ }^{2}$, Andreas Robinson ${ }^{1}$ and Nancy A. Louis ${ }^{1,3}$ \\ ${ }^{T}$ Center for Experimental Therapeutics, Brigham and Women's Hospital and Harvard Medical School, Boston, MA \\ USA; ${ }^{2}$ Immunobiology and Cancer Research Program Oklahoma Medical Research Foundation, Oklahoma City, \\ OK; ${ }^{3}$ Division of Newborn Medicine, Brigham and Women's Hospital and Harvard Medical School, Boston, MA \\ USA. \\ colgan@zeus.bwh.harvard.edu
}

Sites of inflammation are characterized by significant shifts in metabolic activity1. Shifts in energy supply and demand can result in diminished delivery and/or availability of oxygen, leading to inflammation-associated tissue hypoxia and metabolic acidosis. These shifts in tissue metabolism, as indicated by previous studies, are frequently associated with vasculitis and profound recruitment of inflammatory cell types, particularly myeloid cells such as neutrophils (PMN) and monocytes. Under such conditions, we and others have observed activation of the global hypoxia regulatory transcription factor hypoxia-inducible factor (HIF). A significant HIF target gene during mucosal inflammatory disease (e.g. inflammatory bowel disease, colitis) is CD732. Previous studies have determined that CD73 is regulated by HIF at the gene promoter level3. Since tissue profiling studies revealed highest expression of CD73 in mucosal tissues, particularly the intestine4, we have addressed the role of CD73 in model inflammatory disease in vitro and in vivo. Studies using intestinal epithelial cells have revealed that high expression of CD73 is protective for a number of epithelial properties (e.g. barrier function, ion transport), likely through generation of adenosine at the epithelial apical surface. Inflammatory studies in mice conditionally lacking the alpha subunit of HIF-1 in intestinal epithelial cells have shown that the loss of functional HIF-1 correlates with decreased expression of CD73 and enhanced inflammatory disease in these mice. More recent studies have addressed the role of CD73 colitic disease progression. These ongoing studies have revealed that the induction of colitis increases epithelial expression of CD73 mRNA and activity during the acute phase colitic disease. Studies utilizing Cd73-null mice4 have revealed that relative that the loss of CD73 correlated with more severe clinical symptoms of colitis (weight loss, colon length), and increased levels of inflammatory markers (neutrophil numbers). Mechanisms of increased susceptibility with decreased CD73 will be discussed. Taken together, these studies provide unique insight into tissue microenvironmental changes during model inflammatory disease and identify CD73 as a critical control point during mucosal insult.

\section{References:}

1. Karhausen J, Haase VH, Colgan SP. Inflammatory Hypoxia: Role of Hypoxia-Inducible Factor. Cell Cycle 2005;4:256-258.

2. Karhausen JO, Furuta GT, Tomaszewski JE, Johnson RS, Colgan SP, Haase VH. Epithelial hypoxia-inducible factor-1 is protective in murine experimental colitis. J Clin Invest 2004;114:1098-1106.

3. Synnestvedt K, Furuta GT, Comerford KM, Louis N, Karhausen J, Eltzschig HK, Hansen KR, Thompson LF, Colgan SP. Ecto-5'-nucleotidase (CD73) regulation by hypoxia-inducible factor-1 (HIF-1) mediates permeability changes in intestinal epithelia. J. Clin. Invest. 2002;110:993-1002. 
4. Thompson LF, Eltzschig HK, Ibla JC, Van De Wiele CJ, Resta R, Morote-Garcia JC, Colgan SP. Crucial role for ecto-5'-nucleotidase (CD73) in vascular leak during hypoxia. J. Exp. Med. 2004;200:1395-1405.

\title{
Ecto-ATP as a danger signal: Overexpression of P2RX7 on $\mathrm{CD4}^{+} \mathrm{CD25}^{+}$ regulatory $\mathrm{T}$ cells correlates with a higher susceptibility to ATP induced cell death
}

\author{
Sahil Adriouch, Sandra Hubert, Friedrich Haag, Olivier Boyer and Michel Seman \\ EA1556 University Denis Diderot-Paris7 and INSERM U519, Faculty of Medicine and Pharmacy, 22 \\ Boulevard Gambetta, 76000, Rouen, France \\ michel.seman@univ-rouen.fr
}

The P2X7 receptor is expressed on most cells of the immune system including $\mathrm{T}$ lymphocytes. On these cells, $\mathrm{P} 2 \mathrm{X} 7$ expression can be modulated depending on cell activation. Allelic polymorphism of P2X7 such as the P451L natural mutation in the mouse also affects its expression on the surface of naive $\mathrm{T}$ cells. In both wild type and $451 \mathrm{~L}$ mutant ${ }^{1}$, a higher density of $\mathrm{P} 2 \mathrm{RX} 7$ is found on the subset of $\mathrm{CD} 4{ }^{+} \mathrm{CD} 25^{+} \mathrm{CD} 62 \mathrm{~L}^{\text {high }}$ Foxp $3^{+}$regulatory $\mathrm{T}$ cells compared to naïve CD25 T cells. Overexpression of P2RX7 may thus represent another marker of Tregs in both mouse and man. Consistently, Tregs are more prone to apoptosis induced by exposure to ATP or the P2RX7 agonist bzATP. In the mouse, P2RX7 activation can also be induced by ART2 mediated ADP-ribosylation ${ }^{2}$. In C57Bl/6 mice which harbour the 451L mutant but have a high density of ART2 on their surface, ART2 substrate, $\mathrm{NAD}^{+}$, induces the death of $\mathrm{CD} 4^{+} \mathrm{CD} 25^{+} \mathrm{T}$ cells but not that of $\mathrm{CD} 4^{+} \mathrm{CD} 25^{-}$cells. By contrast, Tregs from $\mathrm{BALB} / \mathrm{c}$ mice, which harbour the 451P wild type P2RX7 but a very low density of ART2, are resistant to NAD induced cell death but not to ATP induced cell death. Results suggest that ATP also plays a new role as a danger signal in the immune system. ATP released during inflammation and infection may contribute to the induction of the adaptative immune response by inducing the death of suppressive regulatory $\mathrm{T}$ cells.

Supported by grants from the Ministère de la Recherche, Ligue Nationale contre le cancer, Association pour la recherche sur le cancer.

1. Adriouch, S., et al., Cutting edge: a natural P451L mutation in the cytoplasmic domain impairs the function of the mouse P2X7 receptor. J Immunol, 2002. 169(8): p. 4108-12.

2. Seman, M., et al., NAD-induced T cell death: ADP-ribosylation of cell surface proteins by ART2 activates the cytolytic P2X7 purinoceptor. Immunity, 2003. 19(4): p. 571-82.

\section{Ecto-nucleotide pyrophosphatase/phosphodiesterase as part of a multiple system for nucleotide hydrolysis by platelets from rats: Kinetic characterization and biochemical properties}

\author{
Cristina Ribas Fürstenau ${ }^{1}$, Danielle da Silva Trentin ${ }^{1}$, Maria Luiza, Morais Barreto-Chaves ${ }^{2}$ \\ and João José Freitas Sarkis ${ }^{1}$ \\ ${ }^{1}$ Departamento de Bioquímica, Universidade Federal do Rio Grande do Sul, Porto Alegre, RS, Brazil \\ ${ }^{2}$ Departamento de Anatomia, Universidade de São Paulo, São Paulo, SP, Brazil \\ jjsarkis@plugin-com.br
}

Platelets are known to play a major role in the maintenance of endothelial integrity and hemostasis ${ }^{1}$. ADP acts to potentiate platelet aggregation ${ }^{2}$. ATP has been considered as a competitive inhibitor or ADP-induced platelet aggregation $^{3}$. The action of nucleotides is terminated by a surface-located enzyme cascade that sequentially degrades nucleoside $5^{\prime}$-di and $5^{\prime}$-triphosphates to their respective nucleosides ${ }^{4}$. Since nucleotides exert different responses in diverse tissues, it is norteworthy that cells can co-express two or more enzymes for nucleotide hydrolysis. As earlier described ${ }^{5}$, two enzymes are present on the ecto-surface of intact platelets: ecto-nucleoside 
triphosphate diphosphohydrolase (E-NTPDase) and ecto-5'-nucleotidase. In this study, we describe an ecto-nucleotide pyrophosphatase/phosphodiesterase (E-NPP) activity in rat platelets. Using p-nitrophenyl 5'-thymidine monophosphate ( $p$-Nph-5'-TMP) as a substrate, we demonstrate an enzyme activity that shares the major biochemical properties described for E-NPPs: alkaline $\mathrm{pH}$ and divalent cation dependence, and blockade of activity by metal ion chelator. $\mathrm{K}_{\mathrm{M}}$ and $\mathrm{V}_{\max }$ values for $p$-Nph-5'-TMP hydrolysis were found to be $106 \pm 18 \mathrm{mM}$ and $3.44 \pm$ $0.18 \mathrm{nmol} \mathrm{p}$-nitrophenol/min/mg (mean $\pm \mathrm{SD}, \mathrm{n}=5)$. We hypothesize that an E-NPP is co-localized with an ENTPDase and an ecto- $5^{\prime}$-nucleotidase on the platelet surface. Thus, $0.25 \mathrm{mM}$ suramin inhibited $p$-Nph-5'-TMP, ATP and ADP hydrolysis, while $0.5 \mathrm{mM}$ AMP decreased only $p$-Nph-5'-TMP hydrolysis. Besides, 5.0, 10 and $20 \mathrm{mM}$ sodium azide just inhibited ATP and ADP hydrolysis. Angiotensin II (5.0 and 10nM) affected only ADP hydrolysis. Gadolinium chloride $(0.2$ and $0.5 \mathrm{mM})$ strongly inhibited the ATP and ADP hydrolysis. The set of results presented here reinforces our hypothesis of the possible co-localization of E-NTPDase and E-NPP activities that, together with and ecto-5'-nucleotidase could constitute a multienzymatic complex for the complete nucleotide hydrolysis in the ecto-surface of intact rat platelets. The enormous tissue distribution of the members of the E-NTPDase and E-NPP families make it difficult to assign specific functions to these enzymes in individual tissues ${ }^{4}$. However, since these enzymes present different kinetic properties, they can act under distinct physiological conditions and can be differently regulated. By converting ADP, released from aggregated platelets to AMP, E-NTPDase may play an important role in the prevention of microthrombus formation ${ }^{5}$. In addition, phosphodiesterases may act as "guard dogs" to prevent subversion of the cell by destroying incoming DNA or RNA $^{6}$. The E-NPP described here represents a novel insight into the control of platelet purinergic signaling.

1. Birk, A.V.; Bubman, D.; Broekman, M.J.; Robertson, H.D., Drosopoulos, J.H.; Marcus, A.J.; Szeto, H.H. J Lab. Clin. Med. 2002,139, 116.

2. Qawi, I.; Robson, S.C. Curr. Drug Targets, 2000, 1, 285.

3. Coade, S.B.; Pearson, J.D. Circ. Res. 1989, 65, 531.

4. Zimmermann, H. Trends Pharmacol. Sci. 1999, 20, 231.

5. Frassetto, S.S.; Dias, R.D.; Sarkis, J.J.F. Mol. Cell. Biochem. 1993, 129, 47.

6. Goding, J.W.; Terkeltaub, R.; Maurice, M.; Deterre, P.; Sali, A.; Belli, S.I. Immunol. Rev. 1998, 161, 11.

\title{
Effect of Diadenosine Polyphosphates in Achondroplasic Chondrocytes: Inhibitory Effect of Ap4A on FGF9 induced MAPK cascade
}

\author{
Ana Isabel Guzmán, Marta Irazu and Jesús Pintor \\ Departamento de Bioquímica y Biología Molecular IV, E.U. Óptica, Universidad Complutense de Madrid, c/Arcos \\ de Jalón s/n 28037 Madrid, Spain. \\ aguzman@opt.ucm.es
}

Achondroplasia (dwarfism) is a genetic pathology due to a mutation in the gene that encodes for the fibroblast growth factor type 3 receptor in cartilage cells, the chondrocytes. Studies carried out in different populations have demonstrated that the most common mutation in achondroplasia is Gly380Arg position in the receptor transmembrane domain. This mutation produces a sustained activation of the receptor altering the normal equilibrium between proliferation and maturation therefore inhibiting normal growth of the bone (L'Hote et Knowles., 2005). Rat chondrocytes which have been transfected with the wild type form of human FGFR3 receptor and with the mutated type form, were used to analyse in depth the intracellular signal transduction pathways triggered by overactivated FGFR3. In this sense we have confirmed the induction of mitogen-activated protein kinases (MAP kinases) cascade. This pathway seems to be involved in alteration of chondrocyte differentiation process. Additionally this pathway elicits a lower synthesis of the extracellular matrix inhibiting bone growth (Yasoda et al., 2004).

Therefore a good aproache for the treatment of achondroplasia could be focused to limit the activity of the MAP kinase cascade, which is overstimulated by mutated FGFR3.

In order to modify the MAP kinase activity and taking into account that previously we have observed the presence of $\mathrm{P} 2$ receptors in achondroplasic chondrocytes, we have examined the effect of different dinucleotides and nucleotides on MAP kinase activation.

Diadenosine triphosphate, $\mathrm{Ap}_{3} \mathrm{~A}$ and diadenosine pentaphosphate $\mathrm{Ap}_{5} \mathrm{~A}$ did not modify phosphorylation of MAP kinase in achondroplasic chondrocytes. In contrast, diadenosine tetraphosphate, $\mathrm{Ap}_{4} \mathrm{~A}$ reduced phosphorylation 
of MAP kinase. Other nucleotides assayed, both naturally occurring such as ATP, ADP, UTP and UDP or synthetic, increased or did not modify the levels of phosphorylated MAP kinase. This fact opens a new perspective inviting to consider $\mathrm{Ap}_{4} \mathrm{~A}$ as a new therapeutic target for the treatment of achondroplasia.

L'Hote CG, Knowles MA. Cell responses to FGFR3 signalling: growth, differentiation and apoptosis. Exp Cell Res 2005; 304(2):417-31.

Yasoda A, Komatsu Y, Chusho H, Miyazawa T, Ozasa A, Miura M, et al. Overexpression of CNP in chondrocytes rescues achondroplasia through a MAPK-dependent pathway. Nat Med 2004; 10(1):80-6.

This work has been supported by a research grant from la Comunidad de Madrid CAM GR/SAL/057372004, Fundación López Hidalgo and Fundación ICO.

\section{EFFECT OF THE NEUROLEPTIC Chlorpromazine ON THE NUCLEOTIDE AND PHOSPHOLIPID METABOLISM IN HUMAN LYMPHOCYTES.}

Gergely Keszler, Rita Sümeg, Tanjana Spasokoukotskaja, Lynette D. Fairbanks,+ Annamaria Stenger*, H. Anne Simmonds+ and Maria Staub**

Semmelweis University, Medical Faculty, Department of Medical Chemistry, Molecular Biology and Pathobiochemistry 1444-Budapest, POB 260, Hungary

* Health Service, Pediatric Department, District II, Budapest, Hungary

+Purine Research Laboratory, United Medical and Dental Schools, Guy's Hospital, London, SE1 9RT, United Kingdom.

Chlorpromazine (CPZ) the amphiphilic amine, widely used as neuroleptic drug, decreased the ATP concentration by $30 \%$ in human lymphocytes, at relatively low concentration $(30 \mu \mathrm{M})$ and short incubation time $(60 \mathrm{~min})$ without influencing the GTP, UTP and CTP concentrations. CPZ had the most pronounced effect on the nucleotide containing phospholipid precursors of the cells. At the same conditions, CDP-ethanolamine (CDP-EA) decreased from 87 to $75 \mathrm{pmol} / \mathrm{million}$ cells, while CDP-choline (CDP-Ch) from 12 to $0 \mathrm{pmol} / \mathrm{million}$ cells during incubation with $30 \mu \mathrm{M} \mathrm{CPZ}$ for 60 minutes. The effect of the anti-leukemic drug, arabinosyl-cytosine (araC) was also measured on the phospholipid precursor concentration, as it has been shown, deoxycytidine (dCyt) and its analogue araC can be also metabolized into the the phospholipids. AraC $(50 \mathrm{nM})$ only slightly decreased the CDP-EA (from 87 to $85 \mathrm{pmol}$ ) concentration, while it doubled the CDP-Ch concentration (12 to $25 \mathrm{pmol}$ ) of the cells during 60 min incubation. The incorporation of labeled dCyt into dCDP-EA and dCDP-Ch were both inhibited by CPZ, while araC increased the labeling of dCDP-EA and slightly enhanced it in dCDP-Ch.

The inositol-phospholipid pathway can only be labelled from extracellular dCyt, if its turnover was inhibited by CPZ. Labelled dCyt appeared in dCDP-DAG only in the presence of CPZ, while its incorporation into DNA decreased.

Similar effect of CPZ on the phospholipid biosynthesis were found in Y79 neuroblastoma cell lines to that, measured in lymphocytes. Thus low concentrations of CPZ inhibited at many places the membrane phospholipid synthesis, which might be its primary effect on neural functions.

\section{Effect of adenosine receptor activation on lipopolysaccharide (LPS)-evoked cytokine production in BV-2 microglia cells}

Balázs Koscsó, Zsolt Selmeczy, E. Sylvester Vizi, György Haskó

Department of Pharmacology, Institute of Experimental Medicine, Hungarian Academy of Sciences, Budapest, Hungary

koscso@koki.hu

Microglia, the intrinsic macrophages of the central nervous system, are normally quiescent, but rapidly respond to various kinds of noxious stimuli and these responses are relatively uniform irrespective of the noxius agent. One 
of these noxious agents is bacterial LPS, which induces elaboration of a wide array of proinflammatory mediators including cytokines and free radicals, as well as modulating the expression of cell surface markers. Although the sequence of intracellular events during microglial activation in response to LPS has been well defined, we have relatively little knowledge about the extracellular signals which affect the inflammatory processes of microglia. Adenosine is an endogenous purine nucleoside interacting with specific $G$ protein-coupled receptors $\left(A_{1}, A_{2 A}\right.$, $\mathrm{A}_{2 \mathrm{~B}}$, and $\mathrm{A}_{3}$ ), and is well recognized as an effective modulator of the immune system. In this study, we investigated whether adenosine receptors regulate production of the proinflammatory cytokine TNF- $\alpha$ in the BV-2 murine microglial cell line. BV-2 cells were treated with increasing concentrations of different adenosine receptor agonists ( $\mathrm{N}^{6}$-benzyl 5'-N-ethylcarboxamidoadenosine (NECA), a non-selective adenosine receptor agonist; 2-chloro-N6-cyclopentyl-adenosine (CCPA), an $\mathrm{A}_{1}$ receptor agonist; CGS-21680, an $\mathrm{A}_{2 \mathrm{~A}}$ receptor agonist; and $\mathrm{N}^{6}-(3-$ iodobenzyl)-adenosine-5'-N-methyluronamide (IB-MECA), an $\mathrm{A}_{3}$ receptor agonist) 30 minutes before addition of $10 \mu \mathrm{g} / \mathrm{ml}$ LPS. $24 \mathrm{~h}$ later, the supernatants were collected and analyzed for TNF- $\alpha$ content using ELISA. Our results showed that pretreatment with adenosine receptor agonists decreased LPS-induced TNF- $\alpha$ production in $\mathrm{BV}-2$ cells in the following order of potency: IB-MECA $\geq$ CCPA $>$ NECA $>$ CGS-21680, indicating a primary role for $\mathrm{A}_{3}$ receptors. Next, the cells were pretreated with various selective adenosine receptor antagonists (8-cyclopentyl-1,3-dipropylxanthine (DPCPX), $\mathrm{A}_{1}$ antagonist; 4-(2-[7-amino-2-(2-furyl)[1,2,4] triazolo [2,3-a] [1,3,5] triazin-5-ylamino]ethyl)phenol (ZM241385), an $\mathrm{A}_{2 \mathrm{~A}}$ antagonist; benzo[g]pteridine-2,4(1H,3H)-dione isoalloxazine (alloxazine), an $\mathrm{A}_{2 \mathrm{~B}}$ receptor antagonist; and 5-propyl-2-ethyl-4-propyl-3-(ethylsulfanylcarbonyl)-6-phenylpyridine-5-carboxylate (MRS-1523), an $\mathrm{A}_{3}$ receptor antagonist) before treatment with CCPA or IB-MECA. MRS1523, but not other antagonists, reversed the suppressive effects of both IB-MECA and CCPA on LPS-induced TNF- $\alpha$ production. These results demonstrate that $\mathrm{A}_{3}$ receptor stimulation suppresses TNF- $\alpha$ production by BV-2 microglia. Thus, adenosine may be an important endogenous anti-inflammatory mediator in the brain.

\title{
Effect of low frequency electromagnetic fields on $A_{2 A}$ and $A_{3}$ adenosine receptors in human neutrophils
}

\author{
$\underline{\text { Katia Varani }^{1}}{ }^{\text {, Stefania Gessi }}{ }^{1}$, Stefania Merighi ${ }^{1}$, Fabrizio Vincenzi $^{1}$, Elena Cattabriga ${ }^{1}$, \\ Annalisa Benini $^{1}$, Ruggero Cadossi ${ }^{2}$, Pier Andrea Borea ${ }^{1}$ \\ ${ }^{1}$ Department of Clinical and Experimental Medicine, Pharmacology Unit, University of Ferrara, Ferrara, Italy, \\ ${ }^{2}$ Laboratory of Biophysics, IGEA, Carpi, Italy
}

The present study was designed to evaluate the binding and functional characterization of $A_{2 A}$ and $A_{3}$ adenosine receptors in human neutrophils exposed to low frequency, low energy, pulsing electromagnetic fields (PEMFs). Saturation binding experiments on $\mathrm{A}_{2 \mathrm{~A}}$ and $\mathrm{A}_{3}$ adenosine receptors revealed a single class of binding sites with similar affinity in control and in PEMF-treated human neutrophils $\left(\mathrm{K}_{\mathrm{D}}=1.05 \pm 0.10\right.$ and $1.08 \pm 0.12 \mathrm{nM}$ for $\mathrm{A}_{2 \mathrm{~A}}$ receptors and $K_{D}=2.36 \pm 0.16$ and $2.45 \pm 0.15 \mathrm{nM}$ for $A_{3}$ receptors, respectively). PEMFs treatment revealed that the $A_{2 A}$ and $A_{3}$ receptor density was statistically increased (Bmax $=126 \pm 10$ and $215 \pm 15 * \mathrm{fmol} / \mathrm{mg}$ protein and Bma$\mathrm{x}=451 \pm 18$ and $736 \pm 25^{*} \mathrm{fmol} / \mathrm{mg}$ protein, respectively, $\left.{ }^{*}, \mathrm{P}<0.01\right)$. In the adenylyl cyclase assays the $\mathrm{A}_{2 \mathrm{~A}}$ agonists, HE-NECA and NECA increased cAMP accumulation in untreated human neutrophils with an $\mathrm{EC}_{50}$ value of 43 (40-47) and 255 (228-284) nM, respectively. The capability of the same compounds to stimulate cAMP levels in PEMF treated human neutrophils was increased with an $\mathrm{EC}_{50}$ value of 10 (8-13)* and 61 (52-71)* nM, respectively, ${ }^{*}, \mathrm{P}<0.01$. In the superoxide anion production assays examined agonists inhibited the generation of $\mathrm{O}_{2}{ }^{-}$in untreated human neutrophils with an $\mathrm{EC}_{50}$ value of 3.6 (3.1-4.2) and of 23 (20-27) nM, respectively. In PEMF treated human neutrophils, the same compounds show an $\mathrm{EC}_{50}$ value of $1.6(1.2-2.1)^{*}$ and of $6.0(4.7-7.5)^{*} \mathrm{nM}$, respectively, ${ }^{*}, \mathrm{P}<0.01$. Similarly, typical $\mathrm{A}_{3}$ agonists such as $\mathrm{Cl}$-IB-MECA and IB-MECA were able to inhibit cyclic AMP accumulation and superoxide anion production. The potencies of Cl-IB-MECA and IB-MECA were statistically increased after exposure to PEMFs. These data indicate in human neutrophils treated with PEMFs the presence of significant alterations in the $\mathrm{A}_{2 \mathrm{~A}}$ and $\mathrm{A}_{3}$ adenosine receptor density and functionality. In summary, these results should serve as an impetus for further investigation of the pharmacological changes in the adenosine receptors and PEMF treatment. From a clinical point of view a clarification of the potential effects of PEMFs facilitate the development of alternative treatments or the elaboration of novel promising therapeutic tools. 


\title{
Effect of $\mathrm{P2}$ receptor antagonists on human ecto-nucleotidases.
}

\author{
M.N. Munkonda, S.A. Lévesque, F. Kukulski, C. Legendre, J. Lecka and J. Sévigny. \\ Centre de recherche en Rhumatologie-Immunologie du CHUL, Département de médecine, Université Laval, \\ Québec, Canada. \\ sebastien.levesque@crchul.ulaval.ca
}

Background: Through the activation of P2-receptors, extracellular nucleotides regulate a variety of biological functions. Concentrations of extracellular nucleotides are modulated by ectonucleotidases such as by members of the ecto-nucleoside triphosphate diphosphohydrolase (E-NTPDase) and ecto-nucleotide pyrophosphatases/phosphodiesterases (E-NPPs) families. These enzymes hydrolyze the terminal phosphate residue of tri- and/or diphosphonucleosides, and, in concert with ecto-5'-nucleotidase, facilitates the formation of adenosine ${ }^{1}$. Four different NTPDases, bound to plasma membrane, appears important in the control of nucleotide concentration, namely NTPDase1 (CD39), NTPDase2, 3 and 8. ${ }^{2}$ Also, NPP1 and NPP3 could be involved in the control of extracellular nucleotides concentrations. ${ }^{1}$ The lack of specific inhibitors for these enzymes impedes the research on their functions. It has already been reported that several P2 receptor antagonists such as Reactive Blue-2 (RB2) and suramin also inhibit ecto-nucleotidases. ${ }^{3}$ In this study, we investigated the effect of several P2-receptor antagonists (suramin, NF279, NF449, RB-2 and MRS2179) on the activity of human NTPDases 1, 2, 3 and 8 and human NPP1 and NPP3. Methods: Recombinant human NTPDases and NPPs were produced by transfecting COS-7 or HEK 293T cells with an expression vector encoding each of these enzymes. The inhibition of NTPDase activity (ATP and ADP hydrolysis) was measured by the quantification of released phosphate $\left(\mathrm{P}_{\mathrm{i}}\right)$. The inhibition NPPs activity (pnp-TMP hydrolysis) was measured by the quantification of para-nitrophenol produce using a colorimetric assay. Results: Most NTPDases were inhibited by the P2-receptor antagonists tested. Broad range antagonists RB-2 $(100 \mu \mathrm{M})$ blocked all NTPDases activity by over $87 \%$ while suramin $(100 \mu \mathrm{M})$ inhibited mainly NTPDases 2 and 3 to $51 \pm 2 \%$ and $66 \pm 5 \%$ respectively. RB- 2 completely inhibited human NPP1 and decreased activity of human NPP3 by about $40 \%$ while suramin inhibited these enzymes by $70 \pm 2 \%$ and $8 \pm 3 \%$, respectively. More specific antagonists like suramin derivative NF279 (P2X $\left.\mathrm{X}_{1}, \mathrm{P} 2 \mathrm{X}_{7}\right)$, NF449 (P2X $\left.\mathrm{X}_{1}\right)$ blocked NTPDases 1 to 3 by over $70 \%$ at concentration of $100 \mu \mathrm{M}$ while MRS2179 (P2Y $)_{1}$ only affected human NTPDase3 by about $50 \%$. Interestingly, NTPDase 3 could be blocked by NF279 and NF449 at lower concentrations with EC50 of 0,1 and 5 $\mu \mathrm{M}$ respectively. All these specific antagonists blocked human NPP1 activity by over $75 \%$ while they had minimal effect on human NPP3 (<15\%). These data on P2-receptor antagonists are relevant for the interpretation of pharmacological experiments with these molecules. Some of these P2 receptor antagonists may also be used as inhibitors of ecto-nucleotidases and even discriminate between NTPDase3 activity and the other ecto-nucleotidases.

(1) Zimmermann, H. Naunyn Schmiedebergs Arch. Pharmacol., 2000, 362, 299-309.

(2) Kukulski, F.; Lévesque S.A.; Lavoie E.G.; Lecka, J.; Bigonnesse F.; Knowles, A.F.; Robson, S.C.; Kirley, T.L.; Sévigny, J. Purinergic Signalling, 2005, 1, 193-204.

(3) Gendron, F-P; Benrezzak, O.; Krugh, B.W.; Kong Q.; Weisman, G.A.;Beaudoin, A.R. Current Drug Target, 2005, 3, 229-245.

\section{Effect of Sulphate-Reducing Bacteria Infection on ATP-induced Apoptosis of Human Intestinal Epithelial Cells.}

Carolina de Oliveira Souza ${ }^{1,2}$, Robson Coutinho-Silva ${ }^{1}$, Maurício Magalhães de Paiva ${ }^{2}$, and Cláudia Mara Lara Melo Coutinho ${ }^{2,3}$

${ }^{1}$ Instituto de Biofísica Carlos Chagas Filho, Universidade Federal do Rio de Janeiro, Brasil. ${ }^{2}$ Instituto Oswaldo Cruz, FIOCRUZ, Rio de Janeiro, RJ, Brasil. ${ }^{3}$ Instituto de Biologia, Universidade Federal Fluminense, Niterói, RJ, Brasil.

ccoutinho@ioc.fiocruz.br

Sulphate-reducing bacteria (SRB) are a group of anaerobic organisms involved in the reduction of sulphate to sulphide and are present in the majority of natural environments, plants, animals and humans. SRB were found 
colonizing the human gut and have been associated with the development of ulcerative colitis, a kind of chronic inflammatory bowel disease (IBD). Recently, it was shown that nucleoside-nucleotide free diet protects rat colonic mucosa from damage in a model of IBD. It was also shown that P2X and P2Y subtype receptors can be positively modulated in smooth muscle and immune cells, respectively, on human IBD. Here, we evaluated if the interaction of SRB with human intestinal epithelial cells can interfere with ATP-induced apoptosis on these cells. Material and Methods: a subconfluent culture of HCT8 human intestinal epithelial cells $\left(10^{6}\right.$ total cells) was infected or not with pure SRB strain Desulfovibrio indonensiensis (final concentration of $10^{6} \mathrm{cells}^{-\mathrm{ml}^{-1}}$ ) and, after $24 \mathrm{~h}$ of infection, the cells were washed and treated with $2 \mathrm{mM}$ of ATP for 12 to $48 \mathrm{~h}$. The attached cells were then released from the wells with PBS/EDTA and recovered in Falcon tubes, washed and centrifuged for $7 \mathrm{~min}$ at $250 \mathrm{~g}$. The cells were finally resuspended in apoptosis buffer containing $0.1 \%$ sodium citrate, $0.1 \%$ Triton X-100 and $5 \mathrm{~g} /$ $\mathrm{ml}$ of propidium iodate. The DNA content of the acquired cells (10.000 cells per tube) was analyzed using a FACScan Becton Dickinson flow cytometry. SRB-infected and control epithelial cells were processed for scanning electron microscopy analysis.

Results: we observed that both ATP and SRB induced specific apoptosis on $11 \pm 2 \%$ and $6 \pm 1 \%$ of HCT 8 cells, respectively. When SRB were led to infect the cells followed by ATP treatment, we observed stimulatory effect on ATP-induced apoptosis growing up to $19 \pm 2 \%$ of cells. In addition, scanning electron microscopy analyses showed that SBR were found attached to the surface of the epithelial cells. It was also observed significant differences on the morphological pattern of the infected epithelial cells, especially increased number of microvilla projecting from their plasma membranes.

Conclusion: Our data suggest that SRB attach and cause apoptosis to intestinal epithelial cells and such bacterial infection can interfere with $\mathrm{P} 2$ receptor signaling of these epithelial cells. We hypothesize that SRB together with $\mathrm{P} 2$ receptor can have a role on the establishment of IBD.

Acknowledgments: CNPq, FAPERJ and PETROBRAS.

\title{
Effector-coupling of the $\mathbf{A}_{2 \mathrm{~A}}$ adenosine receptor is contingent on the action of retinoic acid in neuroblastoma cells
}

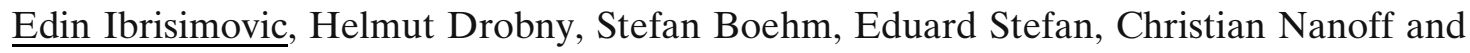 \\ Oliver Kudlacek \\ Institute of Pharmacology, Center for Biomolecular Medicine and Pharmacology, Medical University of Vienna, \\ Austria \\ edin.ibrisimovic@meduniwien.ac.at
}

The $\mathrm{A}_{2 \mathrm{~A}}$-adenosine receptor has been implicated in the pathogenesis of movement disorders, as a drug target it holds therapeutic potential. We have investigated if SH-SY5Y neuroblastoma cells (SH-cells) which endogenously express an $\mathrm{A}_{2 \mathrm{~A}}$-receptor and can be differentiated into a neuron-like phenotype may serve as a model for the regulation of $\mathrm{A}_{2 \mathrm{~A}}$-receptor signalling in nerve cells. We find that receptor activation facilitated neurotransmitter release and that the receptor molecule was targeted to cell extensions reflecting previous evidence obtained on brain slices. An additional finding in SH-cells was that coupling of the receptor to its prototypical effector adenylyl cyclase is contingent on pre-treatment of cells with all-trans retinoic acid (RA). We have investigated if changes in the signal transduction complement accounted for the enhanced coupling. Productive receptor-effector coupling correlated with an increase in catalyst activity but the treatment with RA had a stronger effect on receptor- ( 20-fold) than on forskolin-mediated cAMP formation ( 4-fold). The maximum effect occurred one day after pre-treatment of cells with RA. Both the time course and the regulation pattern consistent with the presence of type I and V isoforms were suggestive of transcriptional regulation via the RA-receptor. However, we did not detect alterations in the mRNA level specific for these and other adenylyl cyclase isoform transcripts in SH-cells (type I, III, V, VII, IX) nor in the amount of membrane bound adenylyl cyclase type I and type V protein. In G-protein levels we found a $\sim 20 \%$ increase in $\mathrm{G} \alpha_{\mathrm{s}}$ with no changes in $\mathrm{G} \alpha_{\mathrm{i} / \mathrm{o}}$ or $\mathrm{G} \beta \gamma$. Nevertheless, the increase could hardly explain the increment in activity nor the altered regulatory pattern. The $\mathrm{A}_{2 \mathrm{~A}}$-receptor level was constantly low $(\sim 100 \mathrm{fmol} / \mathrm{mg})$ even upon the phenotypic changes induced by treatment with RA. Our current hypothesis is that RA fortifies coupling by an indirect effect via an autocrine regulatory mechanism. This is based on the following evidence. RA is known to induce the BDNF receptor trkB in SH-cells and we detected 
BDNF transcript in SH-cells treated with RA. Conversely, addition of a trkB inhibitor (K252a) during the RAdependent maturation interval impaired the effect, i.e. coupling of adenylyl cyclase to the $\mathrm{A}_{2 \mathrm{~A}}$-receptor. This raises the issue if $\mathrm{RA}$ might affect $\mathrm{A}_{2 \mathrm{~A}}$-signalling in the adult brain.

\title{
Effects of adenosine receptor agonists and antagonists in WAG/Rij rats, a genetic model of generalized absence epilepsy.
}

\author{
${ }^{1}$ Citraro R., ${ }^{1}$ Ferreri G., ${ }^{1}$ Russo E., ${ }^{2} D^{\prime}$ Auro M., ${ }^{2}$ Ciccarelli R., ${ }^{2}$ De Sarro G. \\ ${ }^{1}$ Chair of Pharmacology, ${ }^{2}$ Department of Experimental and Clinical Medicine, "Magna Graecia" University. \\ Catanzaro. Italy. ${ }^{2}$ Department of Biomedical Sciences, University of Chieti-Pescara. Chieti. Italy. \\ r.ciccarelli@dsb.unich.it
}

Adenosine exerts anticonvulsant effects in convulsant epilepsies, mainly via inhibitory $\mathrm{A}_{1}$ receptors, whereas the pharmacological blockade or genetic inactivation of the facilitatory $\mathrm{A}_{2 \mathrm{~A}}$ subtypes seems to attenuate convulsions ${ }^{1}$. However, the effects of $\mathrm{A}_{1}$ and $\mathrm{A}_{2 \mathrm{~A}}$ receptor activation were not yet evaluated in a particular type of epilepsy such as absence seizures, characterised by brief interruption of consciousness associated with generalised, synchronous spike-wave discharges (SWDs) in the EEG. Thus, we wanted to test whether the focal bilateral microinjection of $\mathrm{A}_{1}$ and $\mathrm{A}_{2 \mathrm{~A}}$ selective adenosine receptor agonists and antagonists into thalamic nuclei (nucleus reticularis thalami, NRT; ventroposterolateral, VPL; ventroposteromedial, VPM) and the peri-oral region of the somatosensory cortex (S1po), brain areas mainly involved in absence seizure triggering, modified the number and/ or duration of SWDs in a genetic animal model of absence epilepsy, the WAG/Rij rat. In animals equipped with fronto-parietal cortical electrodes for EEGraphic recordings and two additional guide cannulae for bilateral focal microinjection, drugs were focally administered in a volume of $0.5 \mu \mathrm{l} / \mathrm{side}^{2}$. We evaluated the effects of drugs selectively active on $\mathrm{A}_{1}$ (agonist $=\mathrm{CCPA}$; antagonist $=\mathrm{DPCPX}$ ) and $\mathrm{A}_{2 \mathrm{~A}}$ receptors (agonists= CGS21680 and 2HE-NECA; antagonist= SCH58261). Independently of the site of administration, CCPA dose-dependently reduced the number of SWDs, while affected their duration differently, i.e. CCPA increased or decreased SWD duration when microinjected in VPM or VPL, respectively, whereas duration was not significantly modified in the other areas examined. The $\mathrm{A}_{2 \mathrm{~A}}$ agonists had no effects in the VPL, reduced the number and increased SWD duration in the VPM and S1po, while, in the NRT, they increased SWD number without affecting the duration. The $\mathrm{A}_{1}$ antagonist dose-dependently reduced the SWD number in all the examined areas, without affecting the duration, except in NRT, where the duration was significantly increased. Similarly, the $A_{2 A}$ antagonist significantly reduced SWD number in all injected areas, modifying their duration only in NRT (increase) and VPL (reduction). In conclusion, these results are mostly in agreement with a few previous findings indicating either a protective activity by non selective adenosine receptor blockade with theophylline ${ }^{3}$ or a pro-convulsive adenosine $^{4}$ effect in the same animal model of absence epilepsy. Further studies need to better evaluate whether, in this kind of epilepsy, is prevailing the pro-convulsant $\mathrm{A}_{1}$-mediated effect, probably related to an increased inhibitory function in the brain, which plays a significant role in the absence seizure pathogenesis, or the pro-convulsant activity of the excitatory $\mathrm{A}_{2 \mathrm{~A}}$ receptors.

1. Ribeiro, J.A., Sebastiao, A.M., de Mendonca, A. Prog. Neurobiol. 2002, 68: 377-392.

2. Russo, E., Constanti, A., Ferreri, G., Citraro, R., De Sarro, G. Neuropharmacology 2004, 4: 865-78.

3. Ates, N, Sahin, D., Ilbany, G. Epilepsy \& Behaviour 2004, 5: 645-648.

4. Ilbany, G., Sahin, D., Karson, A., Ates, N. Clin. Exp. Pharmacol. Physiol. 2001, 28: 643-646.

\section{EFFECTS OF ADENOSINE RECEPTOR ANTAGONISTS ON AMITRIPTYLINE-INDUCED QRS PROLONGATION IN ISOLATED RAT HEARTS}

\author{
Akgun A, Kalkan S, Hocaoglu N, Gidener S, Tuncok Y. \\ Dokuz Eylul University, School of Medicine, Department of Pharmacology, Izmir, Turkey. \\ aylin.akgun@deu.edu.tr
}


Objective: In our previous study, we had demonstrated that adenosine $\mathrm{A}_{1}$ and $\mathrm{A}_{2 \mathrm{a}}$ antagonists prevented hypotension and QRS prolongation in an in-vivo model of rat amitriptyline toxicity ${ }^{1}$. The aim of the study was to investigate the effects of adenosine receptor antagonists on amitriptyline-induced cardiotoxicity in isolated rat hearts. Methods: A randomized controlled experimental study was performed with hearts of adult male Wistar rats mounted on a cannula of the Langendorff isolated perfused heart apparatus with constant flow. Left Ventricular Developed Pressure (LVDP), dp/dt $\mathrm{dmax}_{\text {, }}$ QRS duration and Heart Rate (HR) were measured. Two experimental protocols were performed. The amitriptyline concentration that prolongated the QRS duration more than $150 \%\left(10^{-4} \mathrm{M}\right)$ was accepted as the control group for the first protocol and the concentration that prolongated the QRS duration 50-75\% $\left(10^{-4.25} \mathrm{M}\right)$ was accepted as the control group for the second protocol. In the first protocol, $10^{-4} \mathrm{M}$ amitriptyline was infused for 60 minutes following pretreatment with a selective adenosine $\mathrm{A}_{1}$ receptor antagonist, DPCPX (8-cyclopentyl-1,3-Dipropylxanthine, $10^{-4}$ to $10^{-6} \mathrm{M}$ ) or a selective adenosine $\mathrm{A}_{2 \mathrm{a}}$ receptor antagonist, $\mathrm{CSC}$ (8-3-chlorostyryl -caffeine, $10^{-4}$ to $\left.10^{-6} \mathrm{M}\right)$. At the second protocol, $10^{-4.25} \mathrm{M}$ amitriptyline was infused for 60 minutes following pretreatment with DPCPX $\left(10^{-4} \mathrm{M}\right)$ or CSC $\left(10^{-5} \mathrm{M}\right)$. Statistical analyses were performed by Student's t test for paired data and ANOVA followed by Tukey-Kramer for multiple comparison tests. Results: At the first protocol, $10^{-4} \mathrm{M}$ amitriptyline infusion following pretreatment with $10^{-4} \mathrm{M}$ DPCPX shortened QRS duration at 50 min. significantly when compared to control group (146.7 $\pm 13.3 \%$ for DPCPX pretreatment, $275.0 \pm 40.3 \%$ for control, $\mathrm{p}<0.05)$. Amitriptyline infusion following pretreatment with $10^{-4}$ to $10^{-6} \mathrm{M}$ DPCPX or $10^{-5}$ or $10^{-6} \mathrm{M}$ CSC did not change LVDP, $\mathrm{dp} / \mathrm{dt}_{\max }$ and HR when compared to control ( $\mathrm{p}>0.05$ ). At the second protocol, pretreatment with $10^{-4} \mathrm{M}$ DPCPX shortened the QRS duration $(122.2 \pm 7.0 \%$ at 40 min., $\mathrm{p}<0.05 ; 120.8 \pm 6.7 \%$ at 50 min., $\mathrm{p}<0.01 ; 120.8 \pm 6.7 \%$ at 60 min., $\mathrm{p}<0.05)$. Pretreatment with $10^{-5} \mathrm{M}$ CSC prolongated QRS duration (185.4 $\pm 20.8 \%$ at $20 \mathrm{~min}$., $\mathrm{p}<0.05 ; 187.5 \pm 12.5 \%$ at $30 \mathrm{~min}$., $\mathrm{p}<0.05 ; 218.8 \pm 14.6 \%$ at 60 min., $\mathrm{p}<0.05)$. Amitriptyline infusion $\left(10^{-4.25} \mathrm{M}\right)$ following pretreatment with $10^{-4} \mathrm{M}$ DPCPX or $10^{-5} \mathrm{M}$ CSC did not change LVDP, dp/dt $\mathrm{dax}_{\max }$ and HR when compared to control $(\mathrm{p}>0.05)$. Conclusion: While $10^{-4} \mathrm{M}$ DPCPX shortened QRS prolongation, $10^{-5} \mathrm{M}$ CSC prolongated QRS duration in the isolated rat hearts with prolonged QRS by 50-75 \% induced by $10^{-4.25} \mathrm{M}$ amitriptyline. However, $10^{-4} \mathrm{M}$ DPCPX was not sufficient to shorten QRS prolongation induced by the higher concentration of amitriptyline $\left(10^{-4} \mathrm{M}\right)$. According to our results, adenosine $\mathrm{A}_{1}$ receptors might have a role in amitriptyline-induced QRS prolongation. Further studies with electrophysiological effects of adenosine antagonists on action potential for amitriptyline cardiotoxicity is needed to explain the exact mechanism.

(1) Kalkan, S.; Aygoren, O.; Akgun, A.; Gidener, S.; Guven, H.; Tuncok, Y. J Toxicol Clin Toxicol 2004, 42:945954.

\title{
Effects of adenosine-angiotensin II interactions on renal function
}

\author{
M. Morato ${ }^{1,2}$, J. Marques-Lopes ${ }^{1}$, T. Sousa ${ }^{1}$, D. Pinho ${ }^{1}$, M. Couto ${ }^{1}$, S. Fontes ${ }^{1}$, A. Albino-Teixeira ${ }^{1}$ \\ ${ }^{1}$ Institute of Pharmacology and Therapeutics, Faculty of Medicine of Porto and IBMC; ${ }^{2}$ Department of Pharma- \\ cology, Faculty of Pharmacy of Porto; University of Porto, Portugal; \\ mmorato@ff.up.pt
}

Selective antagonists of adenosine $A_{1}$ but not $A_{2}$ receptors produce diuresis and natriuresis $(1,2)$. Non-selective antagonism of adenosine receptors with 1,3-dipropyl-8-sulfophenylxanthine (DPSPX) induces hypertension (3) and activation of the renin-angiotensin system (4,5). Angiotensin II induces tubular sodium reabsorption (6). This study aimed at assessing renal function in DPSPX-hypertensive rats.

DPSPX $(90 \mu \mathrm{g} / \mathrm{kg} / \mathrm{h}$; i.p.) was continuously infused from day 0 to day 7 to male Wistar rats $(240-270 \mathrm{~g})$. Shamoperated $(S ; n=6)$ and DPSPX (PX; $n=5)$-treated rats were housed in metabolic cages from day -2 to day 14 with free access to water and rat chow. Intakes were monitored every day. Twenty-four hours urine was daily collected. Plasma was collected on day 14. Sodium and potassium levels were quantified with ion-sensitive electrodes. Urea was measured by an enzymatic test, creatinine by the Jaffé method and total proteins by the biuret reaction. Systolic blood pressure (SBP) was determined by tail-cuff. Statistical analysis was performed using Student's $t$ test. DPSPX increased SBP $(S=123.20 \pm 1.13 \mathrm{mmHg} ; \mathrm{PX}=147.57 \pm 5.83 \mathrm{mmHg} ; \mathrm{p}<0.05)$. Infusion of DPSPX decreased food and water intake until day 7 and urinary excretion rate throughout the study (Table). Body weight gain was similar between S and PX groups. DPSPX-induced hypertension was associated with reduced urinary sodium, potassium and urea excretion rates (Table). No difference was found in urinary creatinine or protein excretion 


\begin{tabular}{|c|c|c|c|c|}
\hline & Day 7 & & Day 13 & \\
\hline & SHAM & DPSPX & SHAM & DPSPX \\
\hline Food intake $(\mathrm{g})$ & $24.33 \pm 0.67$ & $21.8 \pm 0.37 *$ & $24.0 \pm 1.10$ & $20.8 \pm 2.75$ \\
\hline Water intake $(\mathrm{mL})$ & $30.67 \pm 1.17$ & $26.6 \pm 0.68^{*}$ & $29.3 \pm 3.39$ & $29.6 \pm 1.36$ \\
\hline Uexcretion rate $(\mathrm{mL} / \mathrm{min})$ & $10.8 \pm 0.77$ & $6.8 \pm 0.52 *$ & $13.0 \pm 1.11$ & $7.0 \pm 0.65^{*}$ \\
\hline $\mathrm{UNa}^{+}\left(\mathrm{mg} / \mathrm{d} /{ }^{\prime} \mathrm{kg}\right)$ & $5.8 \pm 0.14$ & $4.7 \pm 0.15^{*}$ & $6.1 \pm .022$ & $3.9 \pm 0.70^{*}$ \\
\hline $\mathrm{UK}^{+}(\mathrm{mg} / \mathrm{d} / \mathrm{kg})$ & $4.1 \pm 0.24$ & $2.9 \pm 0.15^{*}$ & $5.8 \pm 0.34$ & $3.3 \pm 0.30 *$ \\
\hline Uurea $(\mathrm{g} / \mathrm{d} / \mathrm{kg})$ & $1.91 \pm 0.04$ & $1.52 \pm 0.04 *$ & $2.10 \pm 0.06$ & $1.47 \pm 0.12 *$ \\
\hline
\end{tabular}

between S and PX groups. On day 14, there was no difference in plasma sodium, potassium, urea, creatinine or protein levels between S and PX groups. Creatinine clearance was similar between S and PX groups. Fraccional excretion of potassium was lower in DPSPX-hypertensive rats $(\mathrm{S}=0.6 \pm 0.06 \%$; $\mathrm{PX}=0.4 \pm 0.02 \% ; \mathrm{p}<0.05)$ while fraccional excretion of sodium was similar to that of sham-operated rats.

We concluded that DPSPX-induced hypertension is associated with decreased excretion of fluid, electrolyte and urea. Future studies will clarify the net result of the concomitant antagonism of adenosine receptors with increased plasma angiotensin II levels on renal function.

(1) Collis, M.G.; Shaw, G.; Keddie, J.R. J. Pharm. Pharmacol. 1991, 43, 138-139.

(2) Suzuki, F.; Shimada, J.; Mizumoto, H.; Karasawa, A.; Kubo, K.; Nonaka, H.; Ishii, A.; Kawakita, T. J. Med. Chem. 1992, 35, 3066-3075.

(3) Albino-Teixeira, A.; Matias, A.; Polonia, J.; Azevedo, I. J. Hypertens. 1991, 9, S196-S197.

(4) Sousa, T., Morato, M., Albino-Teixeira, A. Eur. J. Pharmacol. 2002, 441, 99-104.

(5) Morato, M., Sousa, T., Guimarães, S., Moura, D., Albino-Teixeira, A. Eur. J. Pharmacol. 2002, 455, 135-141.

(6) Van der Mark, J.; Kline, R.L. Am. J. Physiol. 1994, 266, R739-R748.

Supported by POCTI/NSE/45409/2002 and POCTI/NSE/38952/2001 of FCT and FEDER

\title{
Effects of exogenous cAMP on rat intestinal motility
}

Maria Cecilia Giron, Anna Bin, Elisabetta Ciervo, Chiara Zoppellaro, Federica Bianchi,

\author{
Rosa Maria Gaion \\ Department of Pharmacology and Anesthesiology, University of Padova \\ cecilia.giron@unipd.it
}

INTRODUCTION: In these last few decades it has been proposed that cyclic AMP (cAMP) could be an extracellular mediator, supported by the following facts: in several cell types cAMP was shown to be extruded into interstitial fluids and to bind to specific plasma membrane sites. Moreover extracellular cAMP can affect specific functions in a variety of cells and tissues ${ }^{1,2}$. AIMS: The purpose of the present investigation was to evaluate the action of exogenous cAMP on the isolated rat ileum and to determine whether any such action is a) affected by inhibition of tissue phosphodiesterase or ecto-phosphodiesterase or ecto-5'nucleotidase activity, b) mediated by P1 receptors and/or release of endogenous acetylcholine, c) possibly governed by the adrenergic activity.

METHODS: To measure the muscle tension, ileum segments (approximately $2 \mathrm{~cm}$ in length, $0.2 \pm 0.03 \mathrm{~g}$ wet tissue weight $)$ from Wistar rats $(250 \pm 50 \mathrm{~g}$ b. w.) were mounted vertically in double-jacketed organ baths containing 10 $\mathrm{mL}$ of Tyrode solution $\left(\mathrm{NaCl} 136 \mathrm{mM}, \mathrm{KCl} 2.7 \mathrm{mM}, \mathrm{CaCl}_{2} 1.4 \mathrm{mM}, \mathrm{MgCl}_{2} 0.46 \mathrm{mM}, \mathrm{NaH}_{2} \mathrm{PO}_{4} 0.32 \mathrm{mM}\right.$, $\mathrm{NaHCO}_{3} 12 \mathrm{mM}$, glucose $5 \mathrm{mM}$ ), maintained at $37^{\circ} \mathrm{C}$ and continuously aerated with a mixture of $95 \% \mathrm{O}_{2}$ and $5 \%$ $\mathrm{CO}_{2}$. Changes in tension were recorded by isometric transducers coupled to a pen recorder, and they were expressed as a percentage of the maximal response to carbachol $(1 \mu \mathrm{M})$ measured at the beginning of each experiment.

RESULTS: Addition of cAMP $(0.1 \mu \mathrm{M}$ to $1 \mathrm{mM})$ evoked a slight, transient relaxation which, in most cases $(90 \%)$, was followed by a marked, concentration-dependent contraction. Contractions began within $30 \mathrm{~s}$ after application to the bath and the peak response was reached within $60 \mathrm{~s}$. cAMP-induced contraction was sustained (over 15 $\mathrm{min}$ ) and reversible with washing. Repeated administration of $0.5 \mathrm{mM}$ cAMP, followed by washing at $15 \mathrm{~min}$ intervals, did not induce tachyphylaxis. Pretreatments with 3-isobutyl-1-methylxanthine (IBMX, $10 \mu \mathrm{M})$, a PDEinhibitor, 1,3-dipropyl-8-p-sulfophenylxanthine (DPSPX, $0.1 \mathrm{mM}$ ), an ecto-PDE-inhibitor, and $\alpha, \beta$ methylene- 
ADP (AOPCP, $0.1 \mathrm{mM}$ ), an ecto-5'NT-inhibitor, partially attenuated cAMP-induced contractions by $29 \%$, 37\% and $50 \%$, respectively. Pretreatment of the preparations with 8-phenyltheophylline (8-PT, $10 \mu \mathrm{M})$, a P1 antagonist, or with atropine $(1 \mu \mathrm{M})$, a muscarinic antagonist, did not significantly influence cAMP effect on ileum tension. Concentration-dependent contractions induced by cAMP were significantly reduced by in vivo treatment of rats with reserpine, to deplete norepinephrine (NE) stores in nerve terminals.

CONCLUSIONS: These experimental observations suggest that extracellular cAMP may act directly on intestinal smooth muscle by binding to specific cell membrane sites ${ }^{1,3}$, but degradation products of the cyclic nucleotide are also involved in its effects on rat ileum, that are controlled by the adrenergic system.

REFERENCES

1. Bankir, L.; Ahloulay, M.; Devreotes, P.N.; Parent, C.A. Am. J. Physiol. Renal Physiol. 2002, 282, F376-392.

2. Bär, H.P. Adv. Cyclic Nucleotide Res. 1974, 4, 195-237.

3. Sorbera, L.A.; Morad, M. Science 1991, 253, 1286-1289.

\section{Effects of genetic deletion of adenosine deaminase and $\mathrm{A} 1$ receptors in normoxic and ischemic hearts}

Melissa Reichelt*1, Laura Willems*1, Jose G. Molina2, Chun-Xiao Sun2, Janci C. Noble2, Kevin J. Ashton1, Jurgen Schnermann3, Michael R. Blackburn2, and John P. Headrick1†

*Equal first authorship

1 Heart Foundation Research Center, Griffith University Southport, QLD 4217, Australia,

2Department of Biochemistry and Molecular Biology, University of Texas Health Science Center

at Houston, Medical School, Houston, TX 77030, USA, and

3National Institute of Diabetes and Digestive and Kidney Diseases, National Institutes of Health,

Bethesda, MD 20892, USA

m.reichelt@griffith.edu.au

Adenosine deaminase (ADA) may be multifunctional,regulating adenosine levels and receptor (AR) agonism and functionality. We assessed effects of ADA, A1AR, and dualADA/A1AR knockout (KO) on AR-mediated responses and ischemic tolerance (20-25 min ischemia 45 min reperfusion) in murine hearts. Neither ADA or A1AR KO modified basal contractility, though ADA KO reduced resting heart rate (an effect abrogated by A1AR KO). AR mediated bradycardia and dilation with 2-chloroadenosine was unaltered by ADA KO, and A1AR KO eliminated bradycardia. Adenosine efflux was increased 10- to 20-fold by ADA KO (at the expense of inosine). Deletion of ADA improved outcome from $25 \mathrm{~min}$ ischemia, reducing diastolic pressure ( $21 \pm 4$ vs. $38 \pm 3$ $\mathrm{mmHg})$ and $\mathrm{LDH}$ efflux $(0.12 \pm 0.01 \mathrm{vs} .0 .21 \pm 0.02 \mathrm{U} / \mathrm{g} / \mathrm{min}$ ischemia), and enhancing pressure development $(89 \pm 6$ vs. $66 \pm 5 \mathrm{mmHg}$ ). Protection was also evident after $20 \mathrm{~min}$ ischemia, and mimicked by the ADA inhibitor EHNA $(5 \mu \mathrm{M})$. ADA KO enhanced tolerance in A1AR KO hearts, though effects on diastolic function were eliminated. Absence of ADA does not alter functional sensitivities of cardiovascular A1 or A2ARs, despite enhanced adenosine levels, but enhances ischemic tolerance. Conversely, $\mathrm{A}_{1} \mathrm{AR} \mathrm{KO}$ impairs ischemic tolerance. Effects of ADA KO on diastolic dysfunction are A1AR-specific while other ARs contribute to changes in contractile recovery and cell death.

\section{EFFECTS OF THE ADENOSINE RECEPTOR ANTAGONISTS ON AMITRIPTYLINE-INDUCED VASODILATION IN RAT ISOLATED AORTA}

Kalkan S, Hocaoglu N, Akgun A, Gidener S, Tuncok Y. Dokuz Eylul University, School of Medicine, Department of Pharmacology, Izmir, Turkey. sule.kalkan@deu.edu.tr

Background: Although, we had demonstrated that adenosine receptor antagonists prevented hypotension in an invivo rat model of amitriptyline toxicity ${ }^{1}$, it was not clear that whether adenosine receptors in heart or in vascul- 
ature were dominant. The aim of the study was to investigate the role of adenosine $\mathrm{A}_{2 \mathrm{a}}$ receptors on amitriptylineinduced vasodilation in rat isolated aorta. Methods: After determining $\mathrm{EC}_{80}$ of noradrenalin (the concentration of noradrenalin that produces $80 \%$ of maximal contractile response) as $10^{-5} \mathrm{M}, \mathrm{IC}_{50}$ value of amitriptyline was calculated as the drug concentration causing a half-maximal inhibition of contractile responses to noradrenalin (NA) in rat isolated aorta. In the experimental groups, tissues were first contracted with $\mathrm{EC}_{80}$ of NA, then, DPCPX (8cyclopentyl-1,3-Dipropylxantine, a selective adenosine $\mathrm{A}_{1}$ antagonist, $\left.10^{-9}-10^{-5} \mathrm{M}\right)$, CSC (8-(3-chlorostyryl), a selective $\mathrm{A}_{2 \mathrm{a}}$ antagonist, $10^{-9}-10^{-5} \mathrm{M}$ ) or DMSO (dimethyl sulfoxide, a solvent for adenosine antagonists) were incubated. Following the amitriptyline incubation, NA was administered again. $\mathrm{IC}_{50}$ of amitriptyline was compared in the presence of the DPCPX, CSC or DMSO. Statistical analysis was done by Student's t test. Results: Amitriptyline inhibited $49.9 \pm 3.7 \%$ contractile response to NA on aorta segments at $1.8 \times 10^{-5} \mathrm{M}\left(\mathrm{IC}_{50}\right)$. DPCPX increased amitriptyline-induced inhibition on contractile response to NA dose dependently. CSC decreased amitriptyline-induced inhibition on contractile response to NA at $10^{-5} \mathrm{M}$. DMSO did not change amitriptyline- induced inhibition on contractile response to NA, significantly. Conclusion: Adenosine $A_{2 a}$ receptor stimulation seems to be responsible for amitriptyline-induced vasodilation and hypotension since adenosine $\mathrm{A}_{1}$ antagonist, DPCPX, increased amitriptyline-induced.

(1) Kalkan, S.; Aygoren, O.; Akgun, A.; Gidener, S.; Guven, H.; Tuncok, Y. J Toxicol Clin Toxicol 2004, 42:945-954.

\title{
Effects of the membrane lipid environment on adenosine $A_{1}$ and $A_{2 A}$ receptors in rat brain striatum
}

\author{
A. Themann, A. C. Schiedel and C. E. Müller \\ Pharmaceutical Sciences Bonn (PSB), Pharmaceutical Chemistry, Institute of Pharmacy, University of Bonn, \\ Kreuzbergweg 26, 53115 Bonn, Germany \\ andrea.themann@uni-bonn.de
}

Lipid rafts are plasma membrane microdomains rich in cholesterol and sphingolipids, which provide a particularly ordered lipid environment. They play a central role in many cellular processes, including membrane sorting and trafficking, cell polarization, and signal transduction processes. Certain G protein-coupled receptors (GPCRs) and associated molecules were shown to be enriched in cholesterol-rich microdomains which may act as signalling platforms [1]. The family of adenosine receptors, which are GPCRs, consists of four subtypes designated $\mathrm{A}_{1}, \mathrm{~A}_{2 \mathrm{~A}}$, $\mathrm{A}_{2 \mathrm{~B}}$ and $\mathrm{A}_{3}$. Adenosine $\mathrm{A}_{1}$ and $\mathrm{A}_{2 \mathrm{~A}}$ receptors are expressed in high density in the striatum (caudate-putamen). Striatal $\mathrm{A}_{2 \mathrm{~A}}$ receptors have been identified as novel targets for the treatment of Parkinson's disease and related motoric disorders $\left(\mathrm{A}_{2 \mathrm{~A}}\right.$ antagonists) and for schizophrenia ( $\mathrm{A}_{2 \mathrm{~A}}$ agonists) [2,3]. Lipid rafts were described as detergent-insoluble membranes (DRMs) that can be isolated by sucrose-gradient centrifugation after treatment of membranes with the detergent Triton X-100 at $4{ }^{\circ} \mathrm{C}$ [4]. When striatal membranes were subjected to $0.4 \%$ Triton X-100 followed by sucrose-gradient centrifugation, we found that $\mathrm{A}_{1}$ and $\mathrm{A}_{2 \mathrm{~A}}$ receptors were highly enriched in DRMs as shown by radioligand binding studies using the $A_{1}$ agonist radioligand $\left[{ }^{3} \mathrm{H}\right] \mathrm{CCPA}$, and the $\mathrm{A}_{2 \mathrm{~A}}$ agonist radioligand $\left[{ }^{3} \mathrm{H}\right] \mathrm{CGS} 21680$, respectively. However, we could also demonstrate that DRMs are detergent-induced artefacts, which are not representative of the structures in an intact cell membrane.

To examine the modulatory role of cholesterol on ligand binding and G-protein coupling of adenosine $\mathrm{A}_{1}$ and $\mathrm{A}_{2 \mathrm{~A}}$ receptors, cholesterol was depleted from rat brain striatal membranes using methyl- $\beta$-cyclodextrin $(\mathrm{M} \beta C D)$ [5]. Saturation binding experiments with the $A_{1}$ agonist $\left[{ }^{3} \mathrm{H}\right] \mathrm{CCPA}$, the $\mathrm{A}_{1}$ antagonist $\left[{ }^{3} \mathrm{H}\right] \mathrm{DPCPX}$, the $\mathrm{A}_{2 \mathrm{~A}}$ agonist $\left[{ }^{3} \mathrm{H}\right] \mathrm{CGS} 21680$ and the $\mathrm{A}_{2 \mathrm{~A}}$ antagonist $\left[{ }^{3} \mathrm{H}\right] \mathrm{ZM} 241385$ were performed at native and cholesterol-depleted membranes. Cholesterol depletion significantly altered the affinity of $\mathrm{A}_{1}$ (increase) and $\mathrm{A}_{2 \mathrm{~A}}$ agonists (decrease) without affecting antagonist affinities. In addition, it reduced the number of receptors $\left(B_{\max }\right.$ values) recognized by agonists and antagonists. Furthermore, $\left[{ }^{35} \mathrm{~S}\right] \mathrm{GTP} \gamma \mathrm{S}$ binding assays were performed to assess receptor function. Our data provide evidence that the cholesterol content influences the interaction of ligands with the highly expressed adenosine $A_{1}$ and $A_{2 A}$ receptors in rat brain striatum. These results may have relevance for statintreated patients, and in Alzheimer's disease where enhanced cholesterol levels have been observed.

[1] Ostrom, R.S.; Insel, P.A. Br. J. Pharmacol. 2004, 143, 235-245.

[2] Müller, C.E. Drugs of the Future 2000, 25, 1043-1052.

[3] Yan, L.; Burbiel J.C.; Maass, A.; Müller, C.E. Expert Opin. Emerg. Drugs 2003, 8, 537-576. 


\section{Electrophysiological characterization of aminoglycoside antibiotics effects on the $\mathrm{P}_{2} \mathrm{X}_{2}$ receptor and a $\mathbf{P 2 X} \mathrm{X}_{2} / \mathrm{P} 2 \mathrm{X}_{1}$ receptor chimera after heterologous expression in Xenopus oocytes}

Eva-Verena Bongartz and Jürgen Rettinger

Max-Planck-Institute of Biophysics, Frankfurt am Main, Germany

Eva.Bongartz@mpibp-frankfurt.mpg.de

$\mathrm{P} 2 \mathrm{X}$ receptors are cation-selective ligand-gated ion channels that open upon extracellular binding of ATP. Aminoglycoside antibiotics work by binding to the $30 \mathrm{~S}$ ribosomal subunit of Gram-negative bacteria, causing inhibition of protein synthesis. Their clinical use is limited by toxic side effects that include cochlear and vestibular toxicity as well as nephrotoxicity. Micromolar concentrations of the aminoglycosides streptomycin or gentamycin are commonly used in the culture medium of Xenopus laevis oocytes. It has previously been described that neomycin causes voltage dependent inhibition of ATP-induced whole cell current of Guinea-pig outer hair cells (OHC) (1) which have later been shown to express $\mathrm{P}_{2} \mathrm{X}_{2}$ receptors. To further characterize aminoglycoside inhibition at defined $\mathrm{P} 2 \mathrm{X}$ receptors and determine their mode of action we investigated the inhibitory potency of five different aminoglycoside antibiotics at homomeric rat $\mathrm{P} 2 \mathrm{X}_{2}$ receptors and at a non-desensitizing $\mathrm{P} 2 \mathrm{X}_{2} / \mathrm{P} 2 \mathrm{X}_{1}$ receptor chimera which exhibits the ligand-binding properties of the $\mathrm{P} 2 \mathrm{X}_{1}$ receptor (2). The respective subunits were expressed in Xenopus laevis oocytes and the ATP induced membrane currents were analysed using the twoelectrode voltage-clamp technique. At $-60 \mathrm{mV}$, all five aminoglycosides inhibited ATP induced inward currents with varying potencies. The inhibition was dose dependent and displayed fast on-set and off-set. $\mathrm{P}_{2} \mathrm{X}_{2}$ receptor currents were most potently blocked by streptomycin with an $\mathrm{IC}_{50}$ value of $35 \mu \mathrm{M}$, the weakest inhibition was induced by paromomycin with half-maximum inhibition at $1.5 \mathrm{mM}$. The rank order of potency at $\mathrm{P}_{2} \mathrm{X}_{2}$ receptors was streptomycin $>$ neomycin $>$ gentamycin $>$ kanamycin $>$ paromomycin. This rank order differs from the results obtained from native P2X receptors on OHCs where neomycin was significantly more effective than streptomycin and gentamycin. Similar to what has been found on OHCs, the inhibition was voltage-dependent and greatly reduced at more positive membrane potentials. Likewise, the ATP-current could not be reversed by increasing the ATP concentration, indicating that the effect was non-competitive. To further analyze the blocking mechanism we tested the aminoglycosides on a non-desensitizing $\mathrm{P} 2 \mathrm{X}_{2} / \mathrm{P} 2 \mathrm{X}_{1}$ receptor chimera in which the $\mathrm{N}$-terminal and first transmembrane portion of the $\mathrm{P} 2 \mathrm{X}_{1}$ were replaced by the corresponding regions of the $\mathrm{P}_{2} \mathrm{X}_{2}$ receptor subunit and compared it to the properties of known $\mathrm{P} 2 \mathrm{X}$ receptor antagonists. The onset rates of inhibition of PPNDS, NF449 or TNP-ATP at this chimera were limited by the slow dissociation rate of ATP $(\tau=63 \mathrm{~s})$. In contrast, all tested aminoglycosides displayed a fast onset of inhibition at the chimera thus clearly indicating an inhibitory action independent of ATP dissociation. Taken together, we show that aminoglycosides block the rat $\mathrm{P} 2 \mathrm{X}_{2}$ receptor with a rank order of potency different from that at native $\mathrm{P} 2 \mathrm{X}$ receptors on guinea-pig outer hair cells, and that the $\mathrm{P} 2 \mathrm{X}_{2} / \mathrm{P} 2 \mathrm{X}_{1}$ chimera provides a fast screening model to distinguish between competitive and noncompetitive antagonism.

(1) Lin X.; Hume RI.; Nuttall AL. J. Neurophysiol. 1993, 70(4), 1593-1605.

(2) Rettinger J.; Schmalzing G. J. Biol. Chem. 2004 279(8), 6426-6433

\section{Endothelial Catabolism of Extracellular Adenosine during Hypoxia: Role of Surface Adenosine Deaminase and CD26}

Holger K. Eltzschig, ${ }^{1}$ Marion Faigle, ${ }^{1}$ Simone Knapp, ${ }^{1}$ Jorn Karhausen, ${ }^{1}$ Juan Ibla, ${ }^{2,} 4$

Kirsten C. Odegard, ${ }^{2}$ Peter C. Laussen, ${ }^{2}$ Linda F. Thompson, ${ }^{3}$ and Sean P. Colgan ${ }^{4}$

${ }^{1}$ Department of Anesthesiology and Intensive Care Medicine, Tübingen University Hospital, D-72076, Tübingen, Germany and ${ }^{2}$ Department of Anesthesiology, Perioperative and Pain Medicine, Children's Hospital, Harvard Medical School, Boston, Massachusetts, USA, and ${ }^{3}$ Immunobiology and Cancer Program, Oklahoma Medical 
Research Foundation (OMRF), Oklahoma City, OK 73104 and ${ }^{4}$ Center for Experimental Therapeutics and Reperfusion Injury, Brigham and Women's Hospital, Harvard Medical School, Boston, MA 02115, USA

heltzschig@partners.org

Extracellular levels of adenosine significantly increase during conditions of limited oxygen availability (hypoxia) (1-3). Therefore, we reasoned that adenosine clearance mechanisms must exist to counter potentially deleterious influences of adenosine (4). As guided by microarray results revealing a nearly fifty-fold induction of endothelial adenosine deaminase (ADA) mRNA in hypoxia, we pursued the hypothesis that hypoxia coordinates adaptive catabolism of adenosine. Utilizing in vitro and in vivo models of adenosine signaling, we confirmed induction of ADA at the protein and functional levels. Further studies revealed that the ADA complexing protein CD26 is coordinately induced by hypoxia, effectively localizing ADA activity at the endothelial cell surface. Functional studies in murine hypoxia models revealed that inhibition of ADA with coformycin enhances protective physiologic responses mediated by adenosine (e.g. vascular leak, neutrophil accumulation, pulmonary edema). Analysis of plasma ADA activity in pediatric patients with chronic hypoxia (mean oxygen saturation of $81.9 \pm 6 \%$ ) undergoing cardiac surgery (Bi-directional Glenn procedure) revealed a $4.1 \pm 0.6$-fold increase in plasma ADA activity compared to controls. Taken together, these results demonstrate induction of ADA as an innate metabolic adaptation to elevated extracellular adenosine levels during hypoxia, and identify ADA inhibition as potential therapeutic strategy for vascular leakage and excessive inflammation associated with acute hypoxia.

1. Eltzschig, H.K., P. Abdulla, E. Hoffman, K.E. Hamilton, D. Daniels, C. Schonfeld, M. Loffler, G. Reyes, M. Duszenko, J. Karhausen, A. Robinson, K.A. Westerman, I.R. Coe, and S.P. Colgan. 2005. HIF-1-dependent repression of equilibrative nucleoside transporter (ENT) in hypoxia. J. Exp. Med. 202:1493-1505.

2. Eltzschig, H.K., L.F. Thompson, J. Karhausen, R.J. Cotta, J.C. Ibla, S.C. Robson, and S.P. Colgan. 2004. Endogenous adenosine produced during hypoxia attenuates neutrophil accumulation: coordination by extracellular nucleotide metabolism. Blood 104:3986-3992.

3. Eltzschig, H.K., J.C. Ibla, G.T. Furuta, M.O. Leonard, K.A. Jacobson, K. Enjyoji, S.C. Robson, and S.P. Colgan. 2003. Coordinated adenine nucleotide phosphohydrolysis and nucleoside signaling in posthypoxic endothelium: role of ectonucleotidases and adenosine A2B receptors. J Exp Med 198:783-796.

4. Blackburn, M.R. 2003. Too much of a good thing: adenosine overload in adenosine-deaminase-deficient mice. Trends Pharmacol Sci 24:66-70.

\title{
Endothelium-dependent adenosine $\mathbf{A}_{2 \mathrm{~B}}$-receptor-mediated relaxation of corpora cavernosa is impaired in men with vasculogenic impotence
}

\author{
M. Faria $^{1}$, M.T. Magalhães-Cardoso ${ }^{1}$, J.-M. Lafuente-de-Carvalho ${ }^{2} \&$ P. Correia-de-Sá ${ }^{1}$ \\ ${ }^{T}$ Laboratório de Farmacologia, Unidade Multidisciplinar de Investigação Biomédica (UMIB), and \\ ${ }^{2}$ Serviço de Urologia, Hospital Geral de Santo António (HGSA), Instituto de Ciências Biomédicas de Abel Salazar \\ (ICBAS) - Universidade do Porto, Portugal. \\ (migfaria@icbas.up.pt)
}

Purinergic transmission is important for initiation and maintenance of penile erection. Smooth muscle relaxation to ATP may be mediated directly (via P2-purinoceptors) or indirectly, via adenosine generated by ectonucleotidases (Mantelli et al., 1995, J. Androl., 16, 312-317). Due to its short half-life, adenosine has been used as an agent for the diagnosis of vasculogenic impotence (Kilic et al., 1994, Int. J. Impot. Res., 6, 191-198). Therefore, we aimed at characterizing the adenosine receptors regulating human corpus cavernosum (HCC) smooth muscle tone. We also evaluated the pattern of extracellular catabolism of ATP in order to probe its contribution for adenosine generation in HCC. HCC specimens were collected from organ donors (control subjects) and from patients with vasculogenic erectile dysfunction (ED) according to the rules internationally accepted and approved by the Ethics Committees of HGSA and ICBAS of the University of Porto. Longitudinal strips of corpus cavernosum tissue were mounted in a 12-ml organ bath and superfused with oxygenated $\left(95 \% \mathrm{O}_{2}+5 \% \mathrm{CO}_{2}\right)$ Tyrode's solution at $37^{\circ} \mathrm{C}$ and connected to an isometric force transducer. To test tissue relaxant responsiveness, cumulative concentration responses to adenosine and its stable analogues were evaluated in pre-contracted strips with 1 $\mu \mathrm{M}$ phenylephrine (PE). For the kinetic experiments of $30 \mu \mathrm{M}$ ATP, AMP and adenosine catabolism in HCC strips, samples $(75 \mu \mathrm{l})$ were collected from the bath at different times up to $45 \mathrm{~min}$ for HPLC analysis of the 
variation of substrate disappearance and product formation (Cunha \& Sebastião, 1991, Eur. J. Pharmacol., 197, 83-92). We showed that HCC possesses a high ecto-NTPDase 1 activity converting ATP directly into AMP, which is then dephosphorylated into adenosine by ecto-5'-nucleotidase. Contrasting with most tissues, HCC strips slowly inactivates adenosine. Adenosine and 5'-N-ethyl-carboxamide adenosine (NECA) fully relaxed HCC (IC50 values of $30 \mu \mathrm{M}$ and $3 \mu \mathrm{M}$, respectively) in control subjects. The selective $\mathrm{A}_{2 \mathrm{~A}}$ receptor agonist, CGS21680C, produced only a partial relaxation $(30-50 \%)$ of HCC $($ IC50 0.1 $\mu \mathrm{M})$. MRS1706 $\left(10 \mathrm{nM}\right.$, a selective $\mathrm{A}_{2 \mathrm{~B}}$ receptor antagonist) attenuated NECA-induced relaxation without affecting the effect of CGS 21680C. The relaxing effects of both agonists were attenuated by ZM241385 $\left(50 \mathrm{nM}\right.$, a selective $\mathrm{A}_{2 \mathrm{~A}}$ receptor antagonist). In contrast to CGS21680C, NECA-induced relaxation was partially attenuated by NO synthase inhibition with $\mathrm{N}^{\mathrm{G}}$-nitro-Larginine (L-NOARG, $100 \mu \mathrm{M})$ and by the cyclooxygenase inhibitor, indomethacin $(10 \mu \mathrm{M})$. Patients with endothelial cell dysfunction (insensitivity to ACh) were partially resistant to NECA, but kept relaxation of HCC strips to CGS 21680C. Data indicate that extracellular adenosine causes HCC relaxation via the activation of both CGS21680C-sensitive $\left(\mathrm{A}_{2 \mathrm{~A}}\right)$ and -insensitive $\left(\mathrm{A}_{2 \mathrm{~B}}\right)$ receptors. Penile vessels from impotent men with severe vascular diseases are partially resistant to relaxation by adenosine, probably due to endothelial $\mathrm{A}_{2 \mathrm{~B}}$ receptor dysfunction.

Work supported by FCT (POCTI/45549/FCB/2002, participation of FEDER funding) and UP/Fundação Ilídio Pinho.

\title{
EPOXYEICOSATRIENOIC ACIDS (EETS) MEDIATE ADENOSINE- INDUCED RENAL VASODILATION VIA A 2A $_{\text {RECEPTORS }}$
}

\author{
Mairéad A. Carroll, Anabel B. Doumad, Jing Li, Elvira L. Liclican and John C. McGiff \\ Department of Pharmacology, New York Medical College, Valhalla, New York 10562, USA \\ Corresponding author: mairead_carroll@nymc.edu
}

Autocrine, endocrine and paracrine signals act on preglomerular microvessels (PGMV), where they generate a variety of mediators/modulators of the renal circulation. Activation of renal adenosine ${ }_{1}$ receptors $\left(A_{1} R\right)$ and $A_{2} R$ participate in the regulation of vascular tone and tubular function. $A_{1} R$ activation inhibits adenylyl cyclase (AC) via $\mathrm{Gi}_{\alpha}$, resulting in constriction of PGMV, whereas $A_{2 A} R$ activation stimulates $A C$ via $G_{\alpha}$, increasing renal blood flow. 1 The PGMV are endowed with high levels of cytochrome P450 (P450) which metabolize arachidonic acid to EETs and 20-hydroxyeicosatetraenoic acid (HETE). A dynamic and antagonistic interaction between EETs and 20-HETE is evident in their independent and opposing actions on autoregulation of blood flow; 2 20HETE being a constrictor of PGMV and, in contrast, EETs dilate PGMV by activating calcium-dependent potassium $\left(\mathrm{K}^{+} \mathrm{Ca2+}\right)$ channels of smooth muscle cells. 20-HETE has been shown to contribute to the ATP-induced constriction of afferent arterioles via $\mathrm{P}_{2 X} \mathrm{R}$. Our study on vascular mechanisms served by EETs has uncovered a close relationship between stimulation of the $\mathrm{A}_{2 \mathrm{~A}} \mathrm{R}$ and increasing P450 epoxygenase activity; 11,12-EET was identified as the likely candidate mediator of PGMV dilatation on activation of the $A_{2 A} R$. 3 EET levels in PGMVs increased from 1.06 to 3.86 and $7.57 \mathrm{ng} / \mathrm{mg}$ protein/15 min in response to 10 and $100 \mu \mathrm{M}$ CGS 21680, a selective $A_{2 A} R$ agonist, whereas, an $A_{1} R$ agonist failed to stimulate EET formation. Both $A_{2 A} R$ antagonism and inhibition of epoxygenase activity prevented elevation of EET levels produced by CGS 21680.

We have investigated the commonality of the signal transduction pathway - sequential inhibition of $\mathrm{Gs}_{\alpha}$, AC, protein kinase $\mathrm{A}$ (PKA) and $\mathrm{K}^{+} \mathrm{Ca} 2+$ channel activity - to the vasoactive responses to $\mathrm{A}_{2 \mathrm{~A}} \mathrm{R}$ activation by CGS 21680, and 11,12-EET. Rat microdissected arcuate arteries $(c a 120 \mu \mathrm{m})$ were cannulated and pressurized to $80 \mathrm{mmHg}$. Vessels were superfused with Krebs' solution and preconstricted with phenylephrine. 3-aminobenzamide $(10 \mu \mathrm{M})$, an inhibitor of mono-ADP ribosyltranferases, reduced responses to 11,12-EET (3 nM) and CGS $21680(10 \mu \mathrm{M})$ by $70 \%(\mathrm{p}<0.05)$, without affecting the response to sodium nitroprusside $(10 \mu \mathrm{M})$. Like cholera toxin $(100 \mathrm{ng} / \mathrm{ml}), 11,12$-EET $(100 \mathrm{nM})$ stimulated ADP ribose formation in homogenates of arcuate arteries. Incubation of 11,12-EET ( $3 \mathrm{nM}$ to $3 \mu \mathrm{M})$ with PGMV resulted in an increased cAMP levels $(\mathrm{p}<0.05)$, suggesting that $\mathrm{A}_{2 \mathrm{~A}} \mathrm{R}$ coupled EET release is upstream of AC. An inhibitor of AC activity, SQ22536 $(10 \mu \mathrm{M})$, decreased responses to CGS 21680 and 11,12-EET, reducing the internal diameter from $22 \pm 2 \mu \mathrm{m}$ to $1 \pm 1 \mu \mathrm{m}$ and from $24 \pm 5$ $\mu \mathrm{m}$ to $9 \pm 5 \mu \mathrm{m}(\mathrm{p}<0.05)$, respectively. Myristolated PKI $(5 \mu \mathrm{M})$, an inhibitor of PKA, diminished the dilator responses to CGS 21680 and 11, 12-EET by $88 \%$ and $95 \%$, respectively. The responses to both 11,12-EET and CGS 21680 were significantly reduced by superfusion of iberiotoxin $(100 \mathrm{nM})$, an inhibitor of $\mathrm{K}^{+} \mathrm{Ca} 2+$ channel activity. 
Thus, in rat PGMV, EETs mediate $\mathrm{A}_{2 \mathrm{~A}} \mathrm{R}$-induced dilation. EETs stimulate mono-ADP ribosyltransferase resulting in $\mathrm{Gs}_{\alpha}$ activation and sequential activation of $\mathrm{AC}$, PKA and $\mathrm{K}^{+} \mathrm{Ca2}+$ channel activity. We submit that EET production by PGMVs mediate the renal protective effects of adenosine. In addition, activation of the $\mathrm{A}_{2 \mathrm{~A}} \mathrm{R}$ epoxygenase pathway in response to salt loading, greatly enlarges the scope of this pathway; namely, the assignment of an antihypertensive function to adenosine via release of EETs. [4]

1. Fredholm BB, Arslan G, Halldner L, et al. Structure and function of adenosine receptors and their genes. Naunyn Schmiedebergs Arch Pharmacol 2000;362:364-374.

2. Imig JD, Falck JR.Inscho EW. Contribution of cytochrome P450 epoxygenase and hydroxylase pathways to afferent arteriolar autoregulatory responsiveness. Br J Pharmacol 1999;127:1399-1405.

3. Cheng MK, Doumad AB, Jiang H, et al. Epoxyeicosatrienoic acids mediate adenosine-induced vasodilation in rat preglomerular microvessels (PGMV) via A2A receptors. Br J Pharmacol 2004;141:441-448.

4. Liclican EL, McGiff JC, Pedraza PL, et al. Exaggerated response to adenosine in kidneys from high salt-fed rats: role of epoxyeicosatrienoic acids. Am J Physiol Renal Physiol 2005;289:F386-F392.

\section{Evaluation of adenosine $A_{2 A}$ receptor loss in a preclinical model of Huntington disease using $\left[{ }^{11} \mathrm{C}\right] \mathrm{SCH} 442416$ as a radioligand.}

Moresco $\mathrm{RM}^{1,2,3}$, Belloli $\mathrm{S}^{1,2,3}$, Todde $\mathrm{S}^{1,2,3}$, Matarrese $\mathrm{M}^{1,2,3}$, Carpinelli $\mathrm{A}^{1,2,3}$, Turolla $\mathrm{E}^{1,2,3}$, Popoli $\mathrm{P}^{5}$, Pèzzola $\mathrm{A}^{5}$, Simonelli $\mathrm{P}^{1,2,3}$, Lecchi $\mathrm{M}^{4}$ and Fazio $\mathrm{F}^{1,2,3}$. IBFM-CNR ${ }^{1}$, Università degli Studi Milano Bicocca ${ }^{2}$ e Istituto Scientifico San Raffaele ${ }^{3}$, Milano, Università degli Studi di Milano Statale4 Milano, Istituto Superiore di Sanità ${ }^{5}$, Roma, Italy. sara.belloli@hsr.it

Huntington's disease (HD) is a neurological disorder characterized by progressive degeneration of striatal neurons resulting in abnormal involuntary movements, psychiatric and cognitive abnormalities, and death. Adenosine $A_{2 A}$ receptor are mainly expressed in the striatum where they are functionally linked with $D_{2}$ dopamine receptors. Adenosine $\mathrm{A}_{2 \mathrm{~A}}$ receptor antagonists have been indicated as potential neuroprotective drugs, although the mechanisms and efficacy of these compounds remain to be elucidated. A loss of $\mathrm{A}_{2 \mathrm{~A}}$ receptors has been reported at 8 days after the injection of quinolinic acid (QA) a neurotoxin that produces several neurochemical modification that approximate Huntington's disease $(1,2)$. Behavioral studies in rodents indicate that a single injection of QA induces behavioral modifications that progress over time (3). However only few studies investigated the neurochemical modifications, including the $\mathrm{A}_{2 \mathrm{~A}}$ receptors loss, present at later times after $\mathrm{QA}$ administration. Aim of this study was to evaluate whether the loss of $\mathrm{A}_{2 \mathrm{~A}}$ receptors observed at 8 days progresses over time and if this loss is correlated with other neurochemical modifications including dopaminergic $\mathrm{D}_{2}$ receptors loss and activation of microglial cells. For this purpose we measured ex-vivo the expression of $\mathrm{A}_{2 \mathrm{~A}}$ receptors until 60 days after the monolateral intrastriatal injection of QA using $\left[{ }^{11} \mathrm{C}\right] \mathrm{SCH} 442416$ as a radioligand. Adenosine $\mathrm{A}_{2 \mathrm{~A}}$ receptor expression was correlated with that of $\mathrm{D}_{2}$ dopamine receptors and with the presence of activated microglial cells using respectively $\left[{ }^{11} \mathrm{C}\right]$ Raclopride and $\left[{ }^{11} \mathrm{C}\right] \mathrm{PK} 11195$ as radioligands associated with ex-vivo or in vivo techniques. Results of the study showed a progressive decrease of $\left[{ }^{11} \mathrm{C}\right] \mathrm{SCH} 442416$ binding in the lesioned striatum that reach a maximum reduction of approximately $58 \%$ of non lesioned striatum binding $(\mathrm{p}<0.05)$. A progressive loss of $\mathrm{D}_{2}$ receptors was simultaneously observed by ex-vivo and in vivo techniques (YAP-(S)PET; ISE, Italy); such a loss was maximal at 60 days after injection $(60 \% ; \mathrm{p}<0.05)$. The different rate of reduction between adenosine and dopamine receptors produced a progressive reduction in $\mathrm{D}_{2}$ over $\mathrm{A}_{2 \mathrm{~A}}$ ratios (approximately 2.10 at 8 days until 0.8 at 60 days). Interestingly the decrease of $A_{2 A}$ and $D_{2}$ receptors was paralleled by a four times increase of $\left[{ }^{11} \mathrm{C}\right]$ PK11195 binding that was maximum at 8 days after injection $(\mathrm{p}<0.01)$. The results of our study indicate a progressive degeneration of intrastriatal neurons as indicated by the simultaneous loss of adenosine and dopamine receptors. Moreover, the different rate of loss of $\mathrm{A}_{2 \mathrm{~A}}$ and $\mathrm{D}_{2}$ receptors and the consequent modifications in their binding ratios may indicate a different response of the two neurochemical system to the neurotoxic insult. Interestingly, neuronal degeneration is followed by a constant activation of microglial cells as indicated by the increase of $\left[{ }^{11}\right.$ C]PK11195 binding that was maximum at 8 days and then remained stable. Finally, the results of our study confirm the feasibility of the use of molecular imaging techniques to follow disease progression in selected preclinical models of neurodegeneration. 


\title{
Evidence for a patho-physiological and pharmacological role of guanine-based purines as a new extracellular signalling system.
}

\author{
$\underline{\text { M.P. Rathbone }}{ }^{1}$ and F. Caciagli ${ }^{2}$. \\ Departments of Medicine ${ }^{1}$, McMaster University, Hamilton, Ontario, Canada, and Biomedical Sciences ${ }^{2}$, \\ "G. d'Annunzio" University of Chieti-Pescara. Chieti. Italy. \\ Email:mrathbon@mcmaster.ca
}

In addition to the adenine-based purinergic intercellular signaling systems, involving adenine, adenosine and adenosine phosphates, over the last 15 years analogous guanine-based systems have been discovered. Most of these are involved in "trophic" effects, affecting the growth, differentiation and survival of various cells. Indeed, guanine-based purines act synergistically with certain growth factors such as NGF, and also stimulate the production and release from cells of several growth factors and cytokines. Guanine-based purinergic signaling has been particularly investigated in cells of the nervous system and muscle. However, in addition other tissues, including skin, respond to these compounds, indicating that, like adenine based purinergic signaling, guanine based signaling may be widespread throughout many cell types and organs.

Guanine-based purines are released from cells, and when cells are damaged the release increases substantially. Indeed, under conditions simulating ischemia, cells release more guanine-based purines than adenine based purines. Moreover, the extracellular concentration of the guanine-based purines is higher than that of the adeninebased counterparts.

There is evidence that in some cases guanosine produces its effects through entering cells and interacting with an NGF-inducible protein kinase. But there is also evidence that guanosine may interact with unique receptors on the surface of cells. Similarly, there is evidence that GTP may also have cell-surface receptors that mediate some of its effects. Moreover, guanosine is metabolized to guanine by the enzyme purine nucleoside phosphorylase (PNP). Experimental studies have indicated that exogenous extracellular guanosine is relatively persistent compared to adenosine, but a large proportion of guanosine is metabolized to guanine. Emerging evidence indicates that guanine may also have its own extracellular signaling system that is distinct from guanosine. Certainly, this would be of particular interest, since the enzyme that metabolizes guanine, guanine deaminase, shows 50 fold regional variations in brain. This degree of regional variation is characteristically associated with enzymes that degrade neurotransmitters.

It appears that the concept of intercellular signaling by guanine-based purines is now well substantiated. Since GTP, guanosine and guanine have different biological effects, different receptive mechanisms and likely different signal transduction mechanisms, it could be suggested the intriguing possibility that the extracellular interconversion of these guanine derivatives provides an extra layer of signal regulation by cells.

\section{Evidence for Extracellualr ATP in Plants: Localization and Functional Significance in Root Hair Growth and Signaling}

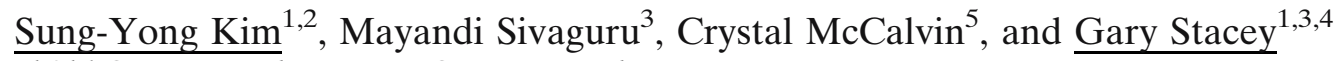 \\ sk2kb@mizzou.edu, staceyg@missouri.edu \\ ${ }^{1}$ Division of Plant Sciences, University of Missouri-Columbia. \\ ${ }^{2}$ National Center for Soybean Biotechnology. \\ ${ }^{3}$ Division of Biological Sciences and Molecular Cytology Core, University of Missouri-Columbia. \\ ${ }^{4}$ Division of Biochemistry, Department of Microbiogoy and Immunology. University of Missouri-Columbia. \\ ${ }^{5}$ Department of Microbiology, University of Tennessee-Knoxville.
}


Transgenic Lotus japonicus plants expressing the soybean ectoapyrase (GS52), with a predicted extracellular catalytic domain, showed increased root infection when inoculated with the nitrogen-fixing symbiotic bacterium, Mesorhizobium loti. These data suggest the presence of extracellular ATP (eATP) in plants and a role for this molecule in symbiotic infection. A variety of recent publications have shown that exogenous addition of ATP can affect plant processes. Indeed, a recent publication points to an essential role for eATP in plant cell viability. What is missing from these investigations is clear demonstration of the presence of eATP under normal plant growth conditions. We constructed a novel reporter protein where the cellulose binding domain was fused with the ATP-requiring enzyme, luciferase. This construct, in the presence of the substrate luciferin, is expected to produce luminescence only when eATP is present. Indeed, when added to legume plant roots, luminescence was seen and localized to the interstitial spaces between cells of the root and the tips of growing root hair cells. The secretion of eATP was dependent on $\mathrm{Ca}_{2}{ }^{+}$, as indicated by reduced luminescence upon addition of $\mathrm{GdCl}, \mathrm{LaCl}$ (calcium channel blockers) or BAPTA (calcium chelator) and an increase in the intensity upon addition of $\mathrm{CaCl}_{2}$. Treatment of roots with brefeldin A, an inhibitor of vesicular trafficking, also blocked eATP release. In addition, reactive oxygen species (ROS) production is also dependent on cytosolic calcium and its gradients are also closely associated with root hair tip growth. Interestingly, addition of exogenous ATP significantly increased the production of ROS, but addition of adenosine, AMP, ADP, or non-hydrolyzable ATP ( $\beta \gamma$ meATP) did not stimulate ROS production. These data suggest that eATP is released from the root hair tip during vesicle exocytosis, concomitant with polar growth. We postulate that eATP may act on polar growth through the production of ROS. Taken together, our current work strongly suggests that eATP is present in plants and likely is an additional signal involved in the earliest events of root hair infection by rhizobia.

\section{Evidence for the involvement of basic amino acid residues in transmembrane regions 6 and 7 of the human platelet P2Y $_{12}$-receptor in ligand recognition}

Kristina Hoffmann, Irina Algaier, Ivar von Kügelgen

Department of Pharmacology, University of Bonn, Reuterstrasse 2b, 53113 Bonn, Germany

Kristina.hoffmann@uni-bonn.de

The $\mathrm{P}_{2} \mathrm{Y}_{12}$ receptor plays a crucial role in ADP-induced platelet aggregation. The ligand binding site of this receptor has yet not been fully characterized. A patient with a congenital bleeding disorder has been shown to carry a polymorphism with a change of R256 to glutamine in the upper third of transmembrane region 6 (TM6) [1]. When expressed in cells, the R256Q mutant receptor had a defective receptor function. For the P2 $\mathrm{Y}_{1}$-receptor, several amino acid residues in TM6 and TM7 are involved in ligand recognition [2]. We now studied whether the corresponding amino acid residues are involved in ligand recognition of the P2Y12-receptor. Mutations were introduced in the encoding DNA sequence by site directed mutagenesis. Wild type $\mathrm{P}_{2} \mathrm{Y}_{12}$-receptors and mutant receptors were stably expressed in human 1321N1-astrocytoma cells. Immunofluorescence staining revealed that the expression levels of the mutant receptors were at least as high as those of wild type receptors. Receptor function was then determined by measuring the 2-methylthio-ADP-induced inhibition of cellular cAMP levels. For this purpose, cellular cAMP accumulation was increased by addition of isoprenaline $10 \mathrm{nM}$ (increase of $52 \pm 2$ pmol cAMP per well). In cells expressing wild type receptors, 2-methylthio-ADP caused a concentration-dependent inhibition of the cAMP production with an $\mathrm{EC}_{50}$ concentration of $0.9 \mathrm{nM}$ and a maximal effect of $55 \%$ inhibition. In cells transfected with the S101A mutant receptor (TM3), the maximal response to stimulation by 2methylthio-ADP was decreased (maximal inhibition by 29\%). Next we studied mutants with a change in TM6. 2Methylthio-ADP inhibited cellular cAMP production in cells expressing the R256K mutant receptor (change of a basic residue to another basic residue) by a similar degree as observed for the wild type receptor ( $\mathrm{EC}_{50}$ concentration of about $0.2 \mathrm{nM}$, inhibition by $39 \%$ ). When R256 was replaced by alanine (R256A), there was a clear decrease in agonist potency with an $\mathrm{EC}_{50}$ concentration of about $10 \mathrm{nM}$ (maximal inhibition by $23 \%$ ). In cells expressing the R256D mutant (replacement of a basic residue by an acidic residue), the maximal effect was decreased even more (only 18\% inhibition left). Several mutations caused a total loss of receptor function. In cells expressing Y259D mutant receptors, 2-methylthio-ADP $(0.01 \mathrm{nM}$ to $1 \mu \mathrm{M})$ failed to elicit any inhibitory effect. Similarly, 2-methylthio-ADP caused no effect in cells with the H253A/R256A double mutant receptor (TM 6). In cells expressing the K280A mutant receptor (TM7), there was also no inhibition due to stimulation by 2-methylthio-ADP. AR-C69931MX ( $\mathrm{N}^{6}$-(2-methylthioethyl)-2-(3,3,3-trifluoropropylthio)- $\beta, \gamma$-dichloromethylene-ATP) a- 
cts as a potent antagonist at the wild type $\mathrm{P}_{2} \mathrm{Y}_{12}$ receptors when used in nanomolar concentrations [3]. At the R256A mutant receptor, AR-C69931MX $30 \mathrm{nM}$ shifted the concentration-response curve to the right, excluding a major change in the affinity of the antagonist at this mutant receptor. In summary, our data indicate the involvement of the residues H253, R256, Y259 (TM 6) and K280 (TM 7) in ligand recognition of the human P2Y 12 receptor. S101 (TM3) may play an intermediate role.

[1] Cattaneo et al, 2003, PNAS, 100, 1978-1983

[2] Jacobson et al, 2002, J Med. Chem., 45, 4057-4093

[3] Takasaki et al, 2001, Mol. Pharmacol., 60, 432-439

\title{
Evidence for trophic effects of guanosine in SH-SY5Y neuroblastoma cells but not in EA.hy926 and HUVEC endothelial cells
}

\author{
C. Florio ${ }^{1}$, S. Pacor ${ }^{1}$, U. Traversa $^{1,2}$ \\ ${ }^{1}$ Department of Biomedical Sciences, ${ }^{2}$ BRAIN Centre for Neuroscience, University of Trieste, Italy. \\ traversa@units.it
}

It is known that after brain injury there is early proliferation of microglia, followed by astrogliosis and then, at a later stage, proliferation of new capillary endothelial cells. The trophic effects of guanosine (GUO), which is released at high levels by hypoxia, included: i) the stimulation of the synthesis and release of neurotrophic factors which stimulate the proliferation of microglia and astrocytes and protect neurons against excitotoxic death, ii) the stimulation of PI3-K and MAPK pathways, which protect astrocytes against apoptosis. Both actions appeared to be mediated by putative GUO receptors. We questioned whether guanosine could also exert trophic effects both in endothelial and neuronal cells.

The proliferation experiments with EA.hy926 human cell line were performed in: i) different FBS conditions, starvation ( $0 \%)$, stand-by (1\%) and proliferating (10\%); ii) different exposure times (24-48 hrs) to different GUO concentrations $(12.5-300 \mu \mathrm{M})$; iii) different times of starvation (24-48-72 hrs). GUO did not significantly increase the proliferation in any conditions tested. Similarly, it was unable to modify proliferation of HUVEC cells. Studies to identify putative GUO binding sites were unsatisfactory. On the other hand, RT-PCR analysis to evaluate the expression of a orphan GPCR (G3), hypothesised to be the putative receptor for GUO since RNAsi abolished the GUO-induced phosphorylation of AKT or ERK1/2 in astrocytes, gave negative results in both cell lines. Moreover, GUO did not caused an increase in cAMP levels in the absence or presence of forskolin $(3 \mu \mathrm{M})$.

In SH-SY5Y human neuroblastoma cells a treatment for $24 \mathrm{~h}$ with GUO (12.5-300 $\mu \mathrm{M})$ induced a concentrationdependent proliferative effect which was evident when the cultures were maintained in starvation ( $0 \%$ FBS), whereas it was not detected when the cells were cultured in $2 \%$ serum. The proliferative effect was also maintained in more severe conditions, prolonging the starvation up to $72 \mathrm{hrs}$. In this condition, when the $0 \%$ FBS medium was replaced with $2 \%$ FBS medium the cells restarted to growth and once again the addition of GUO was without effect. The cytofluorimetric analysis showed that GUO induced a concentration-dependent shift of cell cycle from $\mathrm{G}_{0}-\mathrm{G}_{1}$ phase towards $\mathrm{S}$ phase. The proliferative effects of GUO were not counteracted by the P1 receptor antagonists, DPCPX or DMPX, ruling out a role of endogenous adenosine in mediating GUO action. Suramine, a non specific $\mathrm{P} 2$ receptor antagonist, per se induced an increase in cell growth that was not modified by GUO. The proliferative effects of GUO were increased by the presence of $1 \mathrm{mM}$ propentophylline, a non specific inhibitor of purine transporters, and was significantly reduced by the pre-treatment of SH-SY5Y cells with $10 \mu \mathrm{M}$ PD098,059 and/or 30 $\mu$ M LY-294,002 inhibitors of MAPKs and PI-3K pathways, respectively. GUO produced a weak, but significant, increase in cAMP levels and potentiated the forskolin action which were not changed by cell treatments with ADA, DPCPX or DMPX. Preliminary experiments showed the presence of high affinity binding sites for GUO in SH-SY5Y cells that also express the G3 protein. In conclusion, these results show that guanosine exert trophic effects also on neurons with mechanisms that could be similar to those involved in its trophic action on glia, and reinforce the hypothesis of the existence of extracellular recognition binding sites for guanosine. 


\title{
EXAGGERATED RENAL RESPONSE TO ADENOSINE IN HIGH SALT- FED RATS: ROLE OF EPOXYEICOSATRIENOIC ACIDS (EETs)
}

\author{
Elvira L. Liclican, John C. McGiff and Mairéad A. Carroll \\ Department of Pharmacology, New York Medical College, Valhalla, New York 10595, USA \\ Corresponding author: Elvira_liclican@nymc.edu
}

Adenosine plays a critical role in the regulation of renal vascular tone and tubular function, thus modulating renal blood flow and glomerular filtration rate. In the renal microcirculation, activation of the adenosine ${ }_{1}$ receptor $\left(A_{1} R\right)$ and $A_{2 A} R$ subtypes has opposing effects: vasoconstriction in response to $A_{1} R$ activation enhances proximal tubular $\mathrm{NaCl}$ reabsorption, whereas endothelium-dependent relaxation via $\mathrm{A}_{2 \mathrm{~A}} \mathrm{R}$ promotes natriuresis1. Adenosine stimulation of $\mathrm{A}_{2 \mathrm{~A}} \mathrm{R}$ in rat preglomerular microvessels is linked to production of EETs, cytochrome P-450 (CYP) epoxygenase metabolites of arachidonic acid2. EETs are important modulators of cardiovascular function and their contribution to blood pressure regulation has been established in several different animal models. EETs are thought to be natriuretic by virtue of their ability to dilate the renal vasculature as well as regulate $\mathrm{Na}^{+}$ transport in proximal and distal tubules. An increase in natriuretic EETs is one of the significant components of the kidney's adaptive response to prevent elevation of blood pressure in response to high salt (HS) intake. It is also documented that renal ARs are affected by $\mathrm{Na}^{+}$intake3, and mice lacking $\mathrm{A}_{2 \mathrm{~A}} \mathrm{R}$ exhibit elevated blood pressure4. As adenosine levels are increased by dietary salt intake, we propose that adenosine is the stimulus for increased renal epoxygenase activity in response to salt loading. More specifically, we hypothesize that HS intake increases the renal response to adenosine, resulting in increased epoxygenase activity and EET levels.

Male Sprague-Dawley rats were fed HS $(4.0 \% \mathrm{NaCl})$ or normal salt $(\mathrm{NS} ; 0.4 \% \mathrm{NaCl})$ diet. On day 8 , isolated kidneys were perfused with Krebs' buffer and preconstricted to $c a 150 \mathrm{~mm} \mathrm{Hg}$ with phenylephrine $\left(10^{-7} \mathrm{M}\right)$. Renal effluent eicosanoids were analyzed by GC/MS. Bolus injections of the stable adenosine analog 2-chloroadenosine (2-CA; 0.1-10 $\mu$ g) resulted in dose-dependent dilation; at $10 \mu \mathrm{g}$, perfusion pressure (PP) was lowered to a greater extent in the kidneys of HS rats compared to NS rats $(-60 \pm 4$ vs. $-31 \pm 8 \mathrm{~mm} \mathrm{Hg} ; \mathrm{p}<0.05)$ and the area of response was increased ( $27 \pm 6$ vs. $\left.9 \pm 4 \mathrm{~mm}^{2} ; \mathrm{p}<0.05\right)$, as was EET release (132 \pm 23 vs. $\left.38 \pm 18 \mathrm{ng} ; \mathrm{p}<0.05\right)$. HS treatment increased $\mathrm{A}_{2 \mathrm{~A}} \mathrm{R}$ and $\mathrm{CYP} 2 \mathrm{C} 23$ epoxygenase protein expression. A selective epoxygenase inhibitor, $N$-methylsulfonyl-6-(2-propargyloxyphenyl) hexanamide (MS-PPOH; $12 \mu \mathrm{M}$ ), significantly reduced the response to 2-CA in HS rats; PP, area of response, and EET release decreased by $40 \%, 70 \%$ and $81 \%$, respectively, whereas lesser changes were evident in NS kidneys. In addition to the increase in EET levels with HS intake, a ca $97 \%$ increase of total purines (as an index of flux through the adenosine pathway) was measured in renal effluents obtained from HS-fed rats compared to NS-fed rats (2357 \pm 268 vs. $1197 \pm 127 \mathrm{nmol} / \mathrm{L} ; \mathrm{p}<0.05)$. In vivo, infusion of MS$\mathrm{PPOH}\left(5 \mathrm{mg} / \mathrm{kg}\right.$ daily for 3 days) via osmotic pumps increased blood pressure and decreased $\mathrm{U}_{\mathrm{Na}} \mathrm{V}$ in HS- compared to NS-fed rats.

Thus, the greater vasodilator response to 2-CA seen in kidneys of HS-fed rats was mediated by increased EET release, presumably via upregulation of $\mathrm{A}_{2 \mathrm{~A}} \mathrm{R}$ and epoxygenase expression. As EETs have renal vasodilator and natriuretic properties, $\mathrm{A}_{2 \mathrm{~A}} \mathrm{R}$ activation may be a key link in a renal mechanism that contributes to the regulation of blood pressure.

1. Jackson EK,Dubey RK. Role of the extracellular cAMP-adenosine pathway in renal physiology. Am J Physiol Renal Physiol 2001;281:F597-F612.

2. Cheng MK, Doumad AB, Jiang H, et al. Epoxyeicosatrienoic acids mediate adenosine-induced vasodilation in rat preglomerular microvessels (PGMV) via A2A receptors. Br J Pharmacol 2004;141:441-448.

3. Zou AP, Wu F, Li PL, et al. Effect of chronic salt loading on adenosine metabolism and receptor expression in renal cortex and medulla in rats. Hypertension 1999;33:511-516.

4. Ledent C, Vaugeois JM, Schiffmann SN, et al. Aggressiveness, hypoalgesia and high blood pressure in mice lacking the adenosine A2a receptor. Nature 1997;388:674-678. 


\title{
"Exchange Protein Activated by cAMP" (Epac)-mediated "Suppressor of Cytokine Signalling-3" (SOCS-3) induction: a novel $A_{2 A}$ adenosine receptor- triggered anti-inflammatory signalling pathway
}

\author{
William A. Sands, Hayley Woolson, Gillian R. Milne and Timothy M. Palmer \\ Division of Biochemistry \& Molecular Biology, IBLS, University of Glasgow, Scotland, U.K. \\ Presenting author e-mail: W.Sands@bio.gla.ac.uk
}

While the anti-inflammatory effects of the $A_{2 A}$ adenosine receptor $\left(A_{2 A} A R\right)$ are well-established, the molecular mechanisms responsible for these effects remain unclear. Here we demonstrate that selective activation of endogenous $\mathrm{A}_{2 \mathrm{~A}}$ ARs by CGS21680 induces the expression of "suppressor of cytokine signalling-3" (SOCS-3) but not the related protein SOCS-1 in human umbilical vein endothelial cells (HUVECs). The effect could be recapitulated by elevation of intracellular cyclic AMP (cAMP) but was resistant to inhibition of PKA and was instead mimicked by either selective activation of the cAMP-activated Rap GEF "Epac" or transient expression of constitutively active Val12Rap1a. Importantly, SOCS-3 induction was associated with a PKA-independent ability of cAMP to inhibit IL-6-mediated STAT1 and STAT3 activation by an interleukin-6 (IL-6) trans-signalling complex comprising IL-6 and soluble IL-6 receptor- $\alpha$ (hereafter termed IL-6/sIL-6R $\alpha$ ) via gp130, a well-characterised target for inhibition by SOCS-3. Attenuation of SOCS-3 induction using either morpholino antisensemediated knockdown or employing SOCS-3-null murine embryonic fibroblasts (MEFs) abolished the inhibitory effect of cAMP on IL-6/sIL-6R $\alpha$-activated STAT phosphorylation whereas inhibition of SHP-2, another key negative regulator of gp130, was without effect. These data suggest that SOCS-3 is the dominant mediator of cAMP's effects. Interestingly, cAMP elevation activated the ERK pathway in HUVECs, and this was required to observe SOCS-3 induction. Subsequent in silico analysis of the human SOCS-3 promoter identified several potential sites for ERK regulation, including consensus motifs for binding of the CAAT enhancer binding protein (CEBP) transcription factor family. Chromatin immunoprecipitation assays confirmed that either elevation of cAMP or selective activation of Epac stimulated the rapid accumulation of CEBP $\beta$ on the SOCS-3 promoter in HUVECs. Together, these data argue for the existence of a novel cAMP/Epac/CEBP $\beta / S O C S-3$ pathway for limiting pro-inflammatory signalling from Class I cytokine receptors in endothelial cells, and illuminate a new mechanism by which the $\mathrm{A}_{2 \mathrm{~A}} \mathrm{AR}$ may mediate its potent anti-inflammatory effects.

\section{Exposure to $\mathrm{Hg}^{2+}$ and $\mathrm{Pb}^{2+}$ alters NTPDase and ecto-5' - -nucleotidase activities in central nervous system of zebrafish (Danio rerio)}

Carla Denise Bonan ${ }^{1}$, Mario Roberto Senger ${ }^{1,2}$, Eduardo Pacheco Rico ${ }^{1,2}$, Marcelo de Bem Arizi ${ }^{1}$, Ana Paula Guedes Frazzon ${ }^{3}$, Renato Dutra Dias ${ }^{1}$, Maurício Reis Bogo ${ }^{1}$.

${ }^{1}$ Faculdade de Biociências, Pontifícia Universidade Católica do Rio Grande do Sul. Porto Alegre, RS, Brazil ${ }^{2}$ Departamento de Bioquímica, UFRGS, Porto Alegre, RS, Brazil. ${ }^{3}$ Departamento de Farmacologia e Toxicologia, FFFCMPA, Porto Alegre, RS, Brazil.

cbonan@pucrs.br

Neurotransmission can be affected by exposure to heavy metals, such as mercury and lead. ATP is a signaling molecule that can be inactivated by ecto-nucleotidases. Ecto-nucleotidases are ubiquitous enzymes with a broad phylogenetic distribution, occurring in many vertebrate tissues. Zebrafish is a consolidated model system in neuroscience and toxicological studies. The zebrafish genome project has demonstrated regions of syntenic relationship with human genome. Purinoceptors were already identified in this teleost and, recently, we characterized the presence of a NTPDase and an ecto-5'-nucleotidase activities in brain membranes of zebrafish. Considering that mercury and lead are important environmental contaminants and the presence of purinergic receptors and enzyme activities involved in extracellular catabolism of nucleotides in zebrafish brain, the aim of present study was to investigate the effect of mercury chloride $\left(\mathrm{HgCl}_{2}\right)$ and lead acetate $\left[\mathrm{Pb}\left(\mathrm{CH}_{3} \mathrm{COO}\right)_{2}\right]$ on NTPDase and ecto-5'-nucleotidase activities and expression in central nervous system of zebrafish. In vitro exposure to $\mathrm{HgCl}_{2}$ decreased ATP and ADP hydrolysis in an uncompetitive mechanism and AMP hydrolysis in a non-competitive 
manner in brain membranes of zebrafish. $\mathrm{Pb}\left(\mathrm{CH}_{3} \mathrm{COO}\right)_{2}$ inhibited ATP hydrolysis in an uncompetitive manner, but not $\mathrm{ADP}$ and $\mathrm{AMP}$ hydrolysis. In vivo exposure to $\mathrm{HgCl}_{2}$ or $\mathrm{Pb}(\mathrm{CH} 3 \mathrm{COO})_{2}(20 \mu \mathrm{g} / \mathrm{L}$, during $24 \mathrm{~h}$, $96 \mathrm{~h}$ and 30 days) caused differential effects on ecto-nucleotidase activities. Exposure to $\mathrm{HgCl}_{2}$ during $96 \mathrm{~h}$ caused a significant inhibition of ATP (37\%), ADP (42\%) and AMP (39\%) hydrolysis Interestingly, after 30 days of exposure to $\mathrm{HgCl}_{2}$, ATP hydrolysis return to the control level and ADP hydrolysis was strongly increased $(118 \%)$ when compared to the control values. AMP hydrolysis at this time of exposure remaining inhibited (32\%). However, after $96 \mathrm{~h}$ of exposure to $\mathrm{Pb}\left(\mathrm{CH}_{3} \mathrm{COO}\right)_{2}$, it is possible to observe a significant decrease on ATP hydrolysis $(17 \%)$, but not on ADP and AMP hydrolysis. The chronic exposure to $\mathrm{Pb}\left(\mathrm{CH}_{3} \mathrm{COO}\right)_{2}$ during 30 days also promoted a significant decrease of ATP (33\%), ADP (37\%) and AMP (40\%) hydrolysis in brain membranes of zebrafish. To verify if the chronic exposure to mercury or lead during 30 days was able to modify ecto-nucleotidases expression, RT-PCR experiments were performed. There were no changes in the expression of NTPDase1 and 5'-nucleotidase, following 30 days of exposure to both metals. The results indicate that the significant alterations observed probably are related to post-translational changes of the enzymes. Therefore, this study demonstrated that ectonucleotidases can be a potential target related to neurotoxicity induced by mercury and lead and a possible indicator of the biological impact of exposure to heavy metal contaminants.

Supported by: FAPERGS, CNPq, CAPES, TWAS.

\title{
Expression and function of $\mathbf{A}_{2 \mathrm{~A}}$ and $\mathbf{A}_{3}$ adenosine receptors in mouse neutrophils
}

\author{
Dharini van der Hoeven, John A. Auchampach \\ Department of Pharmacology and Toxicology, Medical College of Wisconsin, WI, USA \\ jauchamp@mcw.edu
}

Adenosine is a potent anti-inflammatory agent, which partially explains its beneficial effects when administered in models of tissue injury and inflammation including ischemia/reperfusion injury. The aims of this study were to comprehensively characterize the expression profile of the four AR subtypes in mouse neutrophils and to determine their roles in regulating neutrophil functions. Neutrophils were isolated from bone marrow by Percoll density gradient centrifugation and immunomagnetic selection with an anti-Gr-1 antibody resulting in isolates that were $99 \%$ pure based on cytospin images and FACS analysis. Real-time PCR analysis of purified neutrophil populations revealed that mRNA expression levels of the $\mathrm{A}_{2 \mathrm{~A}}$ and $\mathrm{A}_{3} \mathrm{AR}$ were highest (3809.32 \pm 391.38 and $3524.19 \pm 685.17$ copies of mRNA/50 ng of total RNA, respectively) followed by the $\mathrm{A}_{2 \mathrm{~B}} \mathrm{AR}(162.89 \pm 32.87$ copies of mRNA/ $50 \mathrm{ng}$ of total RNA). mRNA expression of the $\mathrm{A}_{1} \mathrm{AR}$ was negligible. Radioligand binding analysis using the $A_{2 A} A R$ antagonist ${ }^{125}$ I-ZM-241385 and the $A_{1} / A_{3} A R$ agonist $\left[{ }^{125}\right.$ I]I-AB-MECA also suggested that $\mathrm{A}_{2 \mathrm{~A}}$ and $\mathrm{A}_{3} \mathrm{AR}$ proteins are abundantly expressed in mouse neutrophils, confirming the results of the quantitative PCR studies. We subsequently examined the role of $A_{2 A}$ and $A_{3} A R s$ in regulating neutrophil superoxide $\left(\mathrm{O}_{2}^{-}\right)$production and chemotaxis. In $\mathrm{O}_{2}^{--}$assays, Percoll gradient purified neutrophils were treated with AR agonists for 30 minutes prior to stimulation with activating agents and $\mathrm{O}_{2}{ }^{--}$produced was measured using the chemiluminescent probe 2-methyl-6-(p-methoxyphenyl)-3,7-dihydroimidazo [1,2-alpha]pyrazin-3-one (MCLA, 0.5 $\mu \mathrm{M})$. The non-specific AR agonist NECA $(300 \mathrm{nM})$, the $\mathrm{A}_{2 \mathrm{~A}} \mathrm{AR}$ agonist CGS-21680 $(100 \mathrm{nM})$ and the $\mathrm{A}_{3} \mathrm{AR}$ agonist CP-532,903 (100 nM) inhibited $\mathrm{O}_{2}^{-}$production by wild-type (WT) neutrophils activated with $\mathrm{N}$-formyl peptide fMLP $(1 \mu \mathrm{M})$, complement C5a $(3 \mathrm{nM})$ and platelet activating factor (PAF, $100 \mathrm{nM})$, but not with phorbol myristate acetate (PMA, $800 \mathrm{nM})$. Subsequent concentration-response studies revealed that both CGS21680 and CP-532,903 inhibited $\mathrm{O}_{2}{ }^{--}$production by neutrophils with similar potencies $\left(\mathrm{EC}_{50}\right.$ values of $16.43 \pm 2.07$ $\mathrm{nM}$ and $38.10 \pm 1.60 \mathrm{nM}$ respectively) and efficacies ( $45 \%$ and $\sim 55 \%$ inhibition, respectively). In chemotaxis assays, Percoll gradient purified WT neutrophils were treated with CGS-21680 or CP-532,903 (100 nM) for 30 minutes and directed migration towards fMLP $(30 \mathrm{nM}-10 \mu \mathrm{M})$ or C5a $(300 \mathrm{pM}$ to $100 \mathrm{nM})$ was measured using a standard 48-well chemotaxis chamber with polycarbonate membranes $\left(5 \mu \mathrm{m}\right.$ pore size). Activation of the $\mathrm{A}_{3} \mathrm{AR}$ with $100 \mathrm{nM}$ CP-532,903 (but not the $\mathrm{A}_{2 \mathrm{~A}} \mathrm{AR}$ with CGS 21680) inhibited fMLP-induced chemotaxis of mouse neutrophils producing 25\% inhibition elicited by 1 and $3 \mu \mathrm{M}$ fMLP. Interestingly, CP-532,903 did not inhibit C5a-induced neutrophil chemotaxis. In both the assays for $\mathrm{O}_{2}^{--}$production and chemotaxis, the specificity of the agonists for $\mathrm{A}_{2 \mathrm{~A}}$ and $\mathrm{A}_{3} \mathrm{ARs}$ was confirmed by repeating the experiments with neutrophils obtained from $\mathrm{A}_{2 \mathrm{~A}} \mathrm{AR}$ or $A_{3} A R$ gene 'knock-out' mice. In conclusion, while activation of the $A_{2 A}$ or $A_{3} A R$ inhibits neutrophil $\mathrm{O}_{2}{ }^{-}$ production, activation of the $\mathrm{A}_{3} \mathrm{AR}$ may also lead to stimulant-specific inhibition of neutrophil chemotaxis. 


\title{
EXPRESSION AND FUNCTION OF PURINERGIC RECEPTORS IN HUMAN TYPE-B SYNOVIOCYTES. A PRELIMINARY STUDY.
}

\author{
${ }^{1}$ F. Caporali, ${ }^{2}$ A. Gamberucci, ${ }^{1}$ G. Pompella, ${ }^{1}$ P.L. Capecchi, and ${ }^{1}$ F. Laghi Pasini. \\ ${ }^{T}$ Department of Clinical Medicine and Immunological Science, Division of Clinical Immunology, and \\ ${ }^{2}$ Department of Pathophysiology, Experimental Medicine, and Public Health, University of Siena, Siena, Italy. \\ caporali7@unisi.it
}

Type-B synoviocytes are actively involved in joint injury during chronic and acute rheumatic diseases producing cytokines and inflammatory mediators.

The aim of this study was to investigate $\mathrm{P} 2$ receptors $(\mathrm{P} 2 \mathrm{R})$ expression and role in human B-type synoviocytes obtained from articular biopsies.

mRNA levels of P2R were analysed by RT-PCR. Human primary synoviocytes were found to express mRNA for the following P2X and P2Y receptors: $\mathrm{P} 2 \mathrm{X}_{1}, \mathrm{P} 2 \mathrm{X}_{2}, \mathrm{P} 2 \mathrm{X}_{4}, \mathrm{P} 2 \mathrm{X}_{5}, \mathrm{P} 2 \mathrm{X}_{6}, \mathrm{P} 2 \mathrm{X}_{7}$, and $\mathrm{P} 2 \mathrm{Y}_{1}, \mathrm{PY}_{4}, \mathrm{P}_{2} \mathrm{Y}_{11}, \mathrm{P} 2 \mathrm{Y}_{12}, \mathrm{P}_{2} \mathrm{Y}_{13}$ and $\mathrm{P}_{2} \mathrm{Y}_{14}$. Moreover we observed $\mathrm{P} 2 \mathrm{X}_{7}$ expression by Western Blotting.

We analyzed whether synovial cells have the ability to mobilize calcium when challenged with agonists that interact with the purinergic receptors family.

ATP $(1 \mathrm{mM})$ induced an increase in intracellular free calcium concentration $\left(\left[\mathrm{Ca}^{2+}\right]_{\mathrm{i}}\right)$ in FURA-2 loaded synoviocytes in presence and in absence of extracellular calcium, suggesting that calcium rise depends either on influx across the plasma membrane or on release from intracellular stores and so both P2X and P2Y receptors appear to be involved in calcium mobilization in synoviocytes.

To determine the specific role of $\mathrm{P} 2 \mathrm{X}$ receptors we stimulated synoviocytes with a $\mathrm{P} 2 \mathrm{X}_{7}$ receptor agonist, BzATP $(500 \mu \mathrm{M})$, which was able to induce a rise in $\left[\mathrm{Ca}^{2+}\right]_{\mathrm{i}}$ in the presence of extracellular calcium; conversely, no changes in $\left[\mathrm{Ca}^{2+}\right]_{\mathrm{i}}$ were observed when BzATP was tested in the absence of extracellular calcium.

Since BzATP was reported to activate also $\mathrm{P}_{2} \mathrm{X}_{1}$ and $\mathrm{P} 2 \mathrm{X}_{3}$ receptors, we investigated the calcium mobilizing activity of $\alpha, \beta$ MeATP, a specific agonist of these receptors. Indeed after $\alpha, \beta$ MeATP $(100 \mu \mathrm{M})$ stimulation no significant $\left[\mathrm{Ca}^{2+}\right]_{\mathrm{i}}$ rise was observed, indicating the possible $\mathrm{P} 2 \mathrm{X}_{7}$ receptor activation.

On the basis of the previous considerations we investigated the involvement of $\mathrm{P} 2 \mathrm{X}_{7}$ receptor in the IL-6 release in ELISA experiments through BzATP stimulation.

Synoviocytes spontaneously released low levels of the proinflammatory cytokine interleukin-6 (IL-6) as measured in the supernatant by ELISA. Stimulation with IL-1 $\beta$ increased such secretion after 3-6-24h incubation. We observed a time-dependency in the secretion of IL-6 after BzATP $(100-500 \mu \mathrm{M})$ treatment. In agrement with the hypothesis about the $\mathrm{P}_{2} \mathrm{X}_{7}$ receptor involvement we used a $\mathrm{P} 2 \mathrm{X}_{7}$-selective antagonist, oxidized ATP and we observed that the increase in the cytokine release was inhibited.

This very preliminary study suggests that: i) human synoviocytes express mRNA for several P2X and P2Y receptors; ii) $\mathrm{P}_{2} \mathrm{X}_{7}$ activation is probably associated with cytokine release; iii) $\mathrm{P} 2 \mathrm{R}$ antagonism may represent a therapeutic target for the pharmacological manipulation of the proinflammatory activity of synoviocytes.

\section{Expression and role of the adenosine A2a receptor in the dorsal horn of rat spinal cord.}

\author{
H. Dumont ${ }^{1,2}$, E. Guntz ${ }^{1,2}$, D. Gall ${ }^{2}$, A. de Kerchove d'Exaerde ${ }^{2}$, S.N. Schiffmann ${ }^{2}$, M. Sosnowski ${ }^{1}$. \\ ${ }^{1}$ CHU St Pierre, Brussels, Belgium \\ ${ }^{2}$ Lab. Neurophysiology, ULB, Brussels, Belgium \\ maurice_sosnowski@stpierre-bru.be
}

Background and goal of the study: Adenosine is an endogenous neuromodulator that acts on 4 different subtypes of G-coupled protein receptors (A1, A2a, A2b, A3). The presence of A2a receptor is well established in the central nervous system, predominantly in the striatum where A2a receptor activation was described as a modulator of the NMDA receptor $(1,2)$. In contrast, the distribution and the role of A2a receptor in the spinal cord 
remain unclear (3). The goal of this study is to explore the presence and the role of A2a receptor in the spinal cord pain pathways.

Material and Methods: Total RT-PCR was first performed to assess the expression of the adenosine A2a receptor gene in the lumbar enlargement of the rat spinal cord. Second, single-cell RT-PCR was performed on acute slices in order to identify cells expressing A2a receptor mRNA and to quantify the proportion of lumbar dorsal horn neurons that express it. Third, we investigated whether the activation of A2a receptor has any modulatory effect on the activity of NMDA receptor by using the whole cell patch clamp technique on projection neurons from lamina II.

Results and Discussion: RT-PCR performed on the entire lumbar spinal cord revealed the presence of the adenosine A2a receptor transcript. RT-PCR performed on single cell identified as projection neurons revealed the presence of the adenosine A2a receptor transcript in 5 out of 32 cells. Electrophysiological recordings did not show a significant difference between the current induced in presence of $10 \mu \mathrm{M}$ NMDA and the one induced in presence of $10 \mu \mathrm{M}$ NMDA plus $0.1 \mu \mathrm{M}$ CGS 21680, the A2a receptor agonist $(-107.32 \pm 16.93 \mathrm{pA}$ vs $-83.43 \pm 9.31$ $\mathrm{pA}, \mathrm{n}=7$, student's $t$-test, $\mathrm{p}>0.05$ ).

Conclusion: Adenosine A2a receptor is present in the dorsal horn of the lumbar spinal cord. The receptor is expressed on $15 \%$ of the lamina II projection neurons. A2a receptor agonists seem not able to modulate the NMDA receptor activity.

References: 1) Schiffmann SN, Neurosci Lett. 1991; 130(2):177-81. 2) Wirkner K, Br J Pharmacol. 2000;130(2):259-69. 3) Brooke R.E., J Neurosci. 2004; 24(1):127-37.

\title{
Expression of ecto-alkaline phosphatase in the murine neurogenic subventricular zone
}

\author{
D. Langer $^{1}$, Y. Ikehara ${ }^{2}$, H. Takebayashi ${ }^{3}$ and H. Zimmermann ${ }^{1}$ \\ ${ }^{T}$ Institute of Cell Biology and Neuroscience, Biocenter, J.W. Goethe-University, 60439 Frankfurt, Germany, \\ ${ }^{2}$ Department of Cell Biology, Fukuoka University School of Medicine, Fukuoka 814-0180, Japan, \\ ${ }^{3}$ Division of Molecular Neurobiology and Bioinformatics, National Institute for Physiological Sciences, \\ Okazaki 444-8787, Japan \\ D.Langer@zoology.uni-frankfurt.de
}

In the adult murine brain, neurogenesis persists in two brain regions, the subgranular layer of the hippocampal dentate gyrus and the subventricular zone (SVZ) of the lateral ventricles. In the SVZ, multipotential and selfrenewing astrocyte-like stem cells (type B cells) are the source of newly generated neuroblasts (type A cells). The highly proliferating type $\mathrm{C}$ cells are thought to represent intermediates between type A and type B cells. The neuroblasts migrate along the ventricular surface and the rostral migratory stream (RMS) towards the olfactory bulb (OB). Forming a network of tightly associated cells, the migrating cells pass through tunnels formed by processes of type $\mathrm{B}$ cells. In the $\mathrm{OB}$, the type A cells move radially and differentiate into granular or periglomerular interneurons. We have previously shown that the ecto-nucleotidase NTPDase2 is selectively associated with type $\mathrm{B}$ cells of the $\mathrm{SVZ}^{1}$ and neural progenitors in the hippocampal dentate gyrus ${ }^{2}$ and that neurosphere cells cultured from SVZ stem cells express functional $\mathrm{P} 2 \mathrm{Y}_{1}, \mathrm{P}_{2} \mathrm{Y}_{2}$ and $\mathrm{P} 1$ receptors whose activation synergistically supports growth factor-mediated cell proliferation. ${ }^{3}$

Using enzyme histochemistry and immunocytochemistry we demonstrate that an additional ecto-nucleotidase is expressed by cells of the adult murine SVZ and RMS, the tissue non specific form of alkaline phosphatase (TNAP). TNAP is capable of hydrolyzing nucleoside tri-, di- and monophosphates with an alkaline $\mathrm{pH}$ optimum. In contrast to NTPDase2, TNAP can generate extracellular adenosine. Interestingly, TNAP is not expressed by adult hippocampal progenitors. Using double immunohistochemistry and various cell markers, we demonstrate that TNAP does not colocalize with the NTPDase2-expressing type B cells. It rather is associated with their descendants, the Olig2-expressing type C cells and the doublecortin or PSA-NCAM-expressing type A cells. At physilogical $\mathrm{pH}$, the ATP-hydrolyzing activity of TNAP in the neurogenic zone is lower than that of NTPDase2. During brain ontogeny, TNAP is already expressed within the mouse embryonic neurogenic zones at day 14 (E14), several days before NTPDase2. Taken together these results further support the notion that signaling via extracellular nucleotides and presumably also nucleosides supports both embryonic and adult mammalian neurogenesis. Ecto-nucleotidases would have the potential to control the availability of agonists at P2 and P1 receptors whose cellular expression pattern in the neurogenic zones requires further investigation. 
(1) Braun, N., Sevigny, J., Mishra, SK., Robson, SC., Gerstberger, R., Hammer, K., Zimmermann, H., J. Neurosci. 2003, 17, 1355-1364

(2) Shukla, V., Zimmermann, H., Wang, L., Kettenmann, H., Raab, S., Hammer, K., Sevigny, J., Robson, SC., Braun, N., J. Neurosci. Res. 2005, 80, 600-610,

(3) Mishra, SK., Braun, N., Shukla, V., Füllgrabe, M., Schomerus, C., Korf, HW., Gachet, C., Ikehara, Y., Sevigny, J., Robson, SC., Zimmermann, H., Development 2006, 133, 675-684

\section{EXPRESSION OF HUMAN ECTO 5'-NUCLEOTIDASE IN PIG ENDOTHELIUM AND ITS EFFECTS ON ADENOSINE PRODUCTION, NK CELL-MEDIATED LYSIS AND PLATELET FUNCTION}

Foy N Osborne ${ }^{1)}$, Kameljit K Kalsi ${ }^{1)}$, Charlotte Lawson ${ }^{1)}$, Marialuisa Lavitrano ${ }^{2)}$, Magdi H Yacoub 1), Marlene L Rose ${ }^{1)}$, Ryszard T Smolenski ${ }^{1)}$

1) Heart Science Centre, Imperial College at Harefield Hospital, Harefield, U.K.

2) Department Medicina Sperimentale Ambientale e Biotecnologie Mediche, University of Milano-Bicocca, Via Cadore, 48, 20052 Monza, Milano, Italy

r.smolenski@ic.ac.uk

The use of xenogeneic cells, tissues and organs is one possible solution to circumvent the shortage of human organs for allotransplantation. Pigs are considered as optimal candidates and major obstacle after transplantation of pig organs into primates, hyperacute rejection has been partially resolved in transgenic pigs expressing human complement regulatory proteins such as human decay accelerating factor (hDAF) or by knocking-out $\alpha 1,3$-galactosyltransferase. However, delayed xenograft rejection or acute vascular rejection that occurs within days to weeks after pig-to-primate organ transplantation remains unresolved. This process is mediated by immune and haemostatic mechanisms involving antibodies, natural killer (NK) cells and monocytes. Ecto-5'-nucleotidase (E$\left.5^{\prime} \mathrm{N}\right)$ is an endothelial surface enzyme that controls conversion of extracellular nucleotides that triggers thrombosis and immune response into into immunosuppressive and antithrombotic adenosine. We evaluated whether expression of human $5^{\prime} \mathrm{N}$ on pig endothelial cells (EC) attenuates human NK cell mediated cytotoxicity and inhibit platelet aggreggation and adhesion.

A pig EC line was stably transfected with human E5 ${ }^{\prime} \mathrm{N}$ and human NK cell adhesion and cytotoxicity towards pig EC cultures was measured by flow cytometry and intracellular enzyme release. E5 ${ }^{\prime} \mathrm{N}$ activity in pig EC lysates increased from $0.68 \pm 0.07$ to $1013 \pm 293 \mathrm{nmol} / \mathrm{min} / \mathrm{mg}$ protein, whilst the rate of AMP to adenosine metabolism by intact cells increased from $0.37 \pm 0.05$ to $>300 \mathrm{nmol} / \mathrm{min} / \mathrm{mg}$ protein in non-transfected and transfected cells, respectively. The rate of adenosine production in transfected cells increased also with ATP as the extracellular substrate. Cytotoxicity of human NK cells was reduced from $10.7 \pm 0.4 \%$ and $11.1 \pm 1.1 \%$ with non-transfected pig EC to $5.2 \pm 0.2 \%$ and $5.0 \pm 0.2 \%$ in transfected cells with $50 \mu \mathrm{M}$ and $250 \mu \mathrm{M}$ AMP respectively. Reduction of cytotoxicity in $\mathrm{E}^{\prime} \mathrm{N}$-transfected $\mathrm{EC}$ was abolished by the E5 ${ }^{\prime} \mathrm{N}$ inhibitor and was mimicked in non-transfected EC by the addition of adenosine, demonstrating the key role of adenosine produced by $\mathrm{E}^{\prime} \mathrm{N}$ in inhibiting $\mathrm{NK}$ cell cytotoxicity. Platelet aggregation measured using aggregometer was markedly reduced by supernatants of cells transfected with $5^{\prime} \mathrm{N}$ added to platelet reach plasma. Adhesion of fluorescently labelled platelets to E5 $5^{\prime} \mathrm{N}$ transfected pig endothelial cells was reduced to $39 \pm 9 \%$ of the adhesion observed in non-transfected cells. Both effects on platelet function were abolished by the $\mathrm{E} 5^{\prime} \mathrm{N}$ inhibitor. We suggest that over-expression of $\mathrm{E} 5^{\prime} \mathrm{N}$ in $\mathrm{EC}$ of transgenic pigs is a possible strategy to ameliorate rejection after xenotransplantation.

\section{Extracellular ATP: a critical modulator of hypoxia-induced pulmonary artery adventitial fibroblast proliferation}

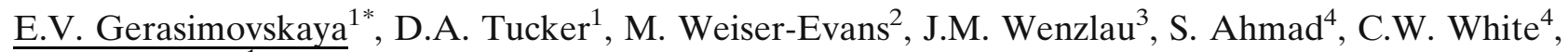
K.R. Stenmark ${ }^{1}$,

${ }^{1}$ Department of Pediatrics and ${ }^{2}$ Department of Medicine University of Colorado at Denver and Health Sciences Center, Barbara Davis Center for Childhood Diabetes and JMRC ${ }^{3}$, Denver, CO, USA;

*E-mail: Evgenia.Gerasimovskaya@UCHSC.edu 
Nucleotides such as ATP and UTP are emerging as a ubiquitous family of extracellular signaling molecules. They have been shown to play a key role in transducing mitogenic, contractile, metabolic, and secretory signals in a variety of cells through release and subsequent binding to the $\mathrm{P} 2\left(\mathrm{P} 2 \mathrm{X}\right.$ and $\mathrm{P} 2 \mathrm{Y}$ ) family of purinergic receptors ${ }^{1,2}$. However, little is known regarding an autocrine role for ATP in hypoxia-induced vascular cell responses. In a neonatal model of hypoxic pulmonary hypertension, we showed that Pulmonary Artery (PA) adventitial fibroblasts proliferate both in vivo and in vitro in response to hypoxic conditions ${ }^{3,4}$. We therefore examined the hypothesis that hypoxia-induced adventitial fibroblast proliferation would be mediated by hypoxia-induced changes in ATP release and/or its extracellular degradation. We found that acute hypoxia $\left(3 \% \mathrm{O}_{2} 10-60 \mathrm{~min}\right)$ increased extracellular ATP concentrations in adventitial fibroblasts and that chronic hypoxia $\left(3 \% \mathrm{O}_{2}, 14-30\right.$ days $)$ markedly attenuated the rate of extracellular ATP hydrolysis by ectonucleotidase(s) suggesting that at least two different cellular mechanisms may contribute to elevated extracellular ATP levels. Exogenous ATP $(100 \mu \mathrm{M})$ stimulated thymidine incorporation and increased the phosphorylation of Akt, Erk1/2, mTOR, and p70S6K in fibroblasts as did UTP, UDP, ADP, ADP $\beta$ S, MeSATP, $\alpha \beta$ MeATP, BzATP and some other agonists, indicating that both $\mathrm{P} 2 \mathrm{Y}$ and $\mathrm{P} 2 \mathrm{X}$ purinoceptors mediate mitogenic responses. PCR analysis revealed that adventitial fibroblasts express P2Y1,2,6 as well as P2X2,4,6,7 receptor subtypes. The rank order of potency of various agonists to activate each individual kinase pathway (ERK1/2, PI3K/Akt or mTOR/p70S6K) indicates that in these cells, purinergic receptors are coupled to the proliferative responses in a pathway-specific manner. Importantly, in this ATP-activated signaling network, a translational pathway, involving mTOR, p70S6K and S6 ribosomal protein, plays a central role in integrating Erk1/2 and PI3K/Akt pathways. We also found that ATP $(100 \mu \mathrm{M})$ and hypoxia $\left(3 \% \mathrm{O}_{2}\right)$, induced expression and activation of the Egr-1 transcription factor, and both stimuli acted, in part, through Gai-initiated ERK1/2 signaling pathway. Apyrase $(2.5 \mathrm{U} / \mathrm{ml})$, as well as the non-selective P2 receptor antagonists, suramin, cibacron blue 3GA, and PPADS (all used at $100 \mu \mathrm{M}$ ) attenuated hypoxia- and ATP-induced DNA synthesis, indicating an activation and a functional role of $\mathrm{P} 2 \mathrm{Y} / \mathrm{P} 2 \mathrm{X}$ purinoceptors in hypoxia-induced proliferative responses. In addition, suramin, cibacron blue 3GA and apyrase, markedly attenuated hypoxiainduced ERK1/2 activation and Egr-1 expression. Collectively, our findings demonstrate that PA adventitial fibroblasts can be considered as endogenous source and a target of extracellular nucleotides within the vascular wall and that a hypoxia-induced autocrine loop of ATP signaling plays a critical role in the regulation of fibroblast proliferation under hypoxic conditions.

1. Ralevic V, Burnstock G. Pharmacol Rev. 1998, 50:413-92.

2. Di Virgilio F, Solini A. Br J Pharmacol. 2002, 135(4):831-842.

3. Stenmark KR, Mecham RP. Annu Rev Physiol. 1997, 59:89-144.

4. Stenmark KR, Gerasimovskaya EV, Nemenoff RA, Das M 2002, Chest, 122:326-334.

\title{
Extracellular ATP: a potential regulator of vasa vasorum neovascularization in hypoxia-induced pulmonary vascular remodeling
}

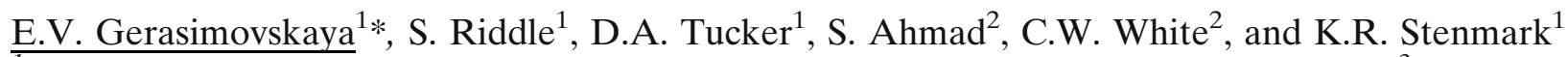 \\ ${ }^{T}$ Department of Pediatrics University of Colorado at Denver and Health Sciences Center; and ${ }^{2}$ National Jewish \\ Medical and Research Center, Denver, $C O$ \\ *E-mail: Evgenia.Gerasimovskaya@UCHSC.edu
}

Pathological vascular remodeling is a key component and frequently life-threatening consequence, of vascular diseases in both the systemic and pulmonary circulation ${ }^{1,2}$. In a neonatal model of hypoxic pulmonary hypertension, we have demonstrated that hypoxia-induced pulmonary artery (PA) remodeling is associated with marked increases in adventitial thickening and the expansion of vasa vasorum network ${ }^{2}$. However, the precise cellular and molecular mechanisms contributing to these changes remain unclear. Extracellular adenine nucleotides are increasingly recognized as important regulators of vascular function ${ }^{3,4}$. Since hypoxia has been shown to stimulate ATP release in endothelial cells and fibroblasts ${ }^{5,6}$, we hypothesized that the endothelium of newly forming adventitial vasa vasorum may represent another, as yet unidentified, source of extracellular ATP and that ATP may mediate or modulate angiogenic responses in vasa vasorum endothelial cells (VVEC). To test this hypothesis, VVEC were isolated from PA adventitia of chronically hypoxic calves and characterized for the expression of endothelial markers. We found that acute hypoxic exposure $\left(1 \%\right.$ and $\left.3 \% \mathrm{O}_{2}, 10-60 \mathrm{~min}\right)$ induced an increase in 
extracellular ATP in VVEC conditioned media from 0.02 to 2.12 nmol. Quinacrine staining revealed ATP containing vesicles in the cytosol and around the nuclei. We showed that hypoxia-induced ATP release could be attenuated by the PI3K inhibitors LY294002 $(20 \mu \mathrm{M})$ and Wortmannin $(1 \mu \mathrm{M})$; the Rho-kinase inhibitor Y27632 $(10 \mu \mathrm{M})$; and by the inhibitor of exocytosis NEM $(4 \mu \mathrm{M})$ suggesting that regulated exocytosis and PI3K/Rho kinase pathways are involved in hypoxia-stimulated ATP release. We also found that exogenous ATP and hypoxia stimulated growth and tube formation in VVEC and induced activation of PI3K/Akt, ERK1/2, and mTOR/ p70S6K, all of which are known to be associated with mitogenic and angiogenic signaling in endothelial cells. Notably, the magnitude of ATP-induced mitogenic responses in VVEC reached 20 fold, suggesting that these cells represent physiologically relevant sources and the targets of adenine nucleotides. Finally, we demonstrated that hypoxia-induced mitogenic responses in VVEC were significantly attenuated by the P2 receptor antagonists, suramin $(100 \mu \mathrm{M})$ and cibacron blue $3 \mathrm{GA}(100 \mu \mathrm{M})$. In summary, our data support the idea that ATP released by VVEC into the local adventitial environment may serve as a potent autocrine/paracrine factor contributing to vasa vasorum neovascularization in hypoxic pulmonary hypertension. The characterization of purinergic receptors and coupled signaling pathways in VVEC will provide unique targets for therapeutic strategies aimed at inhibiting pathologic angiogenesis in the blood vessel wall.

1.Rupnick MA, Panigrahy D, Zhang CY, et al. Proc Natl Acad Sci U S A. 2002; 99:10730-10735.

2.Davie NJ, Crossno JT Jr, Frid MG, et al. Am. J. Physiol. Lung Cell Mol. Physiol. 2004; 286:L668-678.

3.Burnstock G. Arterioscler Thromb Vasc Biol. 2002; 22:364-373.

4. Di Virgilio F, Solini A. Br J Pharmacol. 2002, 135(4):831-842.

5.Gerasimovskaya EV, Davie NJ, Ahmad S, Tucker DA, White CW, Stenmark KR, Chest. 2005, 128:608S-610S. 6.Gerasimovskaya EV, Ahmad S, White CW, Jones PL, Carpenter TC, Stenmark KR. J Biol Chem. 2002, 277(47): 44638-44650.

\title{
Extracellular ATP acting on murine RAW 246.7 macrophages enhances thrombin generation in the absence of cell death.
}

\author{
Moore, S.F. \& MacKenzie, A.B. \\ Department of Pharmacy and Pharmacology, University of Bath, Bath. BA2 7AY. U.K. \\ sfm22@bath.ac.uk
}

Extracellular adenosine 5'triphosphate (ATP) acting at the $\mathrm{P} 2 \mathrm{X}_{7}$ receptor $\left(\mathrm{P} 2 \mathrm{X}_{7} \mathrm{R}\right)$ has been shown to be important for macrophage inflammatory responses including the release of pro-inflammatory cytokines and formation of reactive oxygen species. Host inflammatory responses are closely linked to coagulation where macrophages can participate in events leading to the formation of thrombin in atherosclerosis and inflammation ${ }^{1}$. Cloned $\mathrm{P} 2 \mathrm{X}_{7} \mathrm{Rs}$ couple to the rapid externalization of phosphatidylserine (PS), a cofactor for the assembly of the prothrombinase complex, in the absence of cell death ${ }^{2}$. The objective of the present study was to characterize the pharmacological properties of endogenous murine P2X receptors expressed by a RAW264.7 macrophage cell line and the potential role in prothrominase activity.

Fluorescent measurements of intracellular $\mathrm{Ca}^{2+}$ concentrations in Fluo-4-AM loaded cells or Ethidium Bromide (EtBr, MW $390 \mathrm{Da}$ ) influx have been used to evaluate functional P2X receptor expression. Unless stated, all experiments were performed in saline containing $147 \mathrm{mM} \mathrm{NaCl}, 2 \mathrm{mM} \mathrm{KCl}, 2 \mathrm{mM} \mathrm{CaCl}, 1 \mathrm{mM} \mathrm{MgCl}, 12 \mathrm{mM}$ glucose and 10mM HEPES $(\mathrm{pH} 7.4)$ at $37^{\circ} \mathrm{C}$. ATP $(0.1 \mu \mathrm{m}-3 \mathrm{mM})$ elicited a dose dependent increase in intracellular $\mathrm{Ca}^{2+}$ with a peak response at $3 \mathrm{mM}$. ATP-stimulated a dose-dependent increase in EtBr uptake with an $\mathrm{EC}_{50} 944 \pm 7.4 \mu \mathrm{M}(n=3)$ providing evidence for the expression of the $\mathrm{P} 2 \mathrm{X}_{7} \mathrm{R}$. Using $1 \mathrm{mM}$ ATP, EtBr uptake was observed to increase 2-fold with the removal of extracellular divalent cations $(n=3)$ but was unaffected by replacement of extracellular $\mathrm{Na}^{+}$with $154 \mathrm{mM} \mathrm{N}$-methyl D-glucamine $(n=3)$ or isotonic $\mathrm{KCl}(n=3)$. Pre-application of $3 \mu \mathrm{M}$ Ivermectin did not alter ATP induced EtBr uptake $(n=3)$. EtBr uptake evoked by $3 \mathrm{mM}$ ATP was blocked by $\mathrm{KN}-62$ with an $\mathrm{IC}_{50} 1.25 \pm 0.001 \mu \mathrm{M}(n=3)$.

Brief activation of $\mathrm{P}^{2} \mathrm{X}_{7} \mathrm{Rs}\left(<10\right.$ mins) is associated with PS externalization ${ }^{3}$ and reversible membrane blebbing. Exposure of PS on the outer leaflet of the plasma membrane is associated with a number of macrophage functions such as the adhesion to endothelium ${ }^{4}$ and the assembly of the procoagulant enzyme complexes ${ }^{1}$. Prothrombin is activated to thrombin by the enzyme complex termed "prothrombinase" consisting the enzyme factor Xa, cofac- 
tor/factor $\mathrm{Va}, \mathrm{Ca}^{2+}$ and negatively changed phospholipids most commonly PS. Assays performed in the presence of factors $\mathrm{Va}$ and Xa demonstrate that exposure to $3 \mathrm{mM}$ ATP ( $>2$ mins) enhanced the prothrombinase activity (3 fold) on macrophages. By contrast, only prolonged stimulation with $3 \mathrm{mM}$ ATP ( $>30$ mins) triggered the release of lactate dehydrogenase which was blocked by $\mathrm{KN}-62\left(\mathrm{IC}_{50}=0.283 \pm 0.04 \mu \mathrm{M}, n=3\right)$. This data indicates that extracellular ATP can rapidly trigger an increase in prothrombinase activity in the absence of cell death.

(1) Ananyeva, N.M.; Kouiavskaia, D.V.; Shima, M. \& Saenko, E.L. Blood. 2002. 99, 4475-4485

(2) MacKenzie, A.B.; Young, M.T.; Adinolfi, E. \& Surprenant, A. J. Biol. Chem. 2005. 280, 33968-33976

(3) Wilson, H.L.; Francis, S.E.; Dower, S.K. J. Immunol. 2004. 173, 1202-1208

(4) Lesauskaite, V.; Ivanviene, L.; Valanciute, A. Medicina. 2003. (Kaunas). 39, 529-534

\section{Extracellular ATP and adenosine - two important immunomodulators regulating leukocyte-endothelial adhesion}

Gennady G. Yegutkin*11, Tiina Henttinen ${ }^{2}$, Andrey Mikhailov ${ }^{2}$, Sergei Samburski ${ }^{1}$, Sirpa Jalkanen ${ }^{1}$

${ }_{1}$ MediCity Laboratory and Department of Medical Microbiology, Turku University and National Public Health Institute, FIN-20520 Turku, Finland

${ }^{2}$ Turku Centre for Biotechnology, University of Turku/Åbo Akademi University, POB 123, 20521 Turku, Finland

*E-mail: gennady.yegutkin@utu.fi

Leukocyte-endothelial interactions involve both cell adhesion through specific receptor-ligand pairs and bidirectional cell signaling, and are affected by soluble mediators, which modulate adhesive molecules and signaling events in both cell types. Extracellular ATP and ADP are important signaling molecules mediating leukocytosis, thrombosis and several inflammatory responses in the vasculature, whereas adenosine has a non-redundant counteracting role in the attenuation of inflammation and tissue damage in vivo. The duration and magnitude of purinergic signaling is governed by a network of ectoenzymes and endothelial and lymphoid cells are generally characterized by counteracting, ATP-inactivating and ATP-regenerating/adenosine-eliminating, phenotypes, respectively. Here we have identified a link between the adhesion cascade and extracellular purine turnover. Upon adhesion, lymphocytes prevent adenosine formation in the endothelial microenvironment that, as a consequence, impairs the vascular barrier function and facilitates subsequent step of leukocyte transmigration into the tissue. Together, these leukocyte adhesion-mediated shifts in the local nucleotide and nucleoside concentrations may represent a previously unrecognised paracrine mechanism affecting the functional state of the targeted endothelium and co-ordinately regulating lymphocyte trafficking between the blood and tissues.

\section{Extracellular ATP inhibits neutrophil apoptosis by increasing intracellular cAMP, but independently of increases in intracellular calcium levels, via P2-purinoceptors}

Kathryn R. Vaughan ${ }^{1}$, Leanne Stokes ${ }^{2}$, Annmarie Surprenant ${ }^{2}$, Moira K. Whyte ${ }^{1}$.

${ }^{T}$ Academic Unit of Respiratory Medicine, School of Medicine and ${ }^{2}$ Biomedical Sciences, University of Sheffield, $U K$

K.R.Vaughan@sheffield.ac.uk

At sites of inflammation ATP is released from activated platelets and endothelial cells, and delays neutrophil apoptosis in a concentration- and time-dependent manner, with significant effects occurring within 10 minutes of ATP exposure (\% apoptosis at 5 hours: $23.0 \pm 4.9$ (mean \pm SEM) control $v s 9.9 \pm 1.210 \mu \mathrm{M}$ ATP, $p<0.05$ ). Typical P2X-receptor agonists BzATP and $\beta \gamma$ MeATP, the stable ATP analogue ATP- $\gamma$-S, and the typical P2Y-receptor agonist 2MeSATP, delayed neutrophil apoptosis to levels comparable to ATP, whereas the P2Y-agonist UTP was without effect. We found highly-pure (i.e. PBMC depleted) populations of human neutrophils express P2X-1, P2X-5, P2Y-1, P2Y-2, P2Y-4, P2Y-6 and P2Y-11 receptor mRNA. Increases in intracellular calcium $\left[\mathrm{Ca}^{2+}\right]_{\mathrm{i}}$ were demonstrated by $\mathrm{Ca}^{2+}$ fluorimetry to be induced by UTP and thus independent of the delay of neutrophil apoptosis. We then investigated other possible mechanisms by which ATP inhibits apoptosis. Inhibition of cAMP- 
dependent protein kinases completely abrogated ATP-mediated neutrophil survival (\% apoptosis at 16 hours: $46.2 \pm 5.0$ control $v s 27.5 \pm 3.9100 \mu \mathrm{M}$ ATP, $\mathrm{p}<0.05$, vs $56.4 \pm 6.30 .7 \mathrm{mM} R p$-8-Br-cAMPS with $100 \mu \mathrm{M}$ ATP, $\mathrm{p}<0.01)$, whilst ATP increased intracellular cAMP $[\mathrm{cAMP}]_{\mathrm{i}}$ levels. These data suggest that ATP-mediated delays in neutrophil apoptosis are likely P2Y-11 receptor mediated via increases in [cAMP $]_{i}$, and that these effects are not associated with increases in $\left[\mathrm{Ca}^{2+}\right]_{\mathrm{i}}$.

\title{
Extracellular ATP signaling affects contractility of skeletal muscle
}

\author{
D. Sandonà ${ }^{1}$, D. Danieli-Betto ${ }^{2}$, E. Germinario ${ }^{2}$, S. Gastaldello ${ }^{1}, \underline{\text { R. Betto }}^{3}$ \\ ${ }^{1}$ Department of Biomedical and Experimental Sciences, University of Padova, ${ }^{2}$ Department of Human Anatomy \\ and Physiology, University of Padova, and ${ }^{3}$ Laboratory of Muscle Biology and Physiopathology, C.N.R. Institute \\ of Neuroscience, Italy. \\ (romeo.betto@bio.unipd.it)
}

Muscle contraction consumes more than $50 \%$ of the ATP produced by the striated cells, moreover, the working muscle also releases some of its precious nucleotide into the extracellular milieu. However, growing evidence shows that this release is not a pure waste. It appears in fact that skeletal muscle cells utilize extracellular ATP to stimulate relevant autocrine/paracrine signaling pathways, related, for example, to the differentiative program. Present work was aimed at investigating the purinergic signaling of adult skeletal muscle. First, we confirmed, at the single fiber level, the release of ATP during contractile activity. The electrically stimulation (five 30-mV pulses of $2 \mathrm{~ms}$ duration with $500 \mathrm{~ms}$ interval) of cultured rat flexor digitorum brevis muscle fibers, isolated by the collagenase method, caused a substantial, rapid (seconds) release of ATP into the extracellular fluid, whose level returned to baseline with an estimated half-decay time of about 20 min. The decrease of the ATP concentration in the extracellular milieu represents an indirect observation of the presence of an ATP-hydrolyzing activity at muscle fiber surface, that we measured as high as $41 \pm 4$ nmol Pi liberated per mg protein $(\mathrm{n}=3)$. Consistently, RT-PCR and WB analyses show the presence of NTPDase- 1 and NTPDase-2, NPP1, and $\alpha$-sarcoglycan in muscle fibers. Different purinergic receptors have been reported to be expressed in striated cells, a finding that we confirmed in adult muscle fibers. Moreover, we demonstrated that $\mathrm{P}_{2} \mathrm{X}_{4}$ is localized mainly in the T-tubule membranes, i.e., the critical site of excitation-contraction coupling of skeletal muscle.

Because all the elements of extracellular ATP signaling are present in skeletal muscle, we speculated that both the enhanced $\mathrm{Ca}^{2+}$ entry as well as the subsequent activation of $\mathrm{Ca}^{2+}$-dependent intracellular processes could modulate muscle contraction, especially during sustained contractile activity. Therefore, we examined different in vitro stimulation protocols on a typical slow-twitch muscle (soleus) in order to mimic such prolonged activity. We found that stimulation of soleus muscle at low frequencies (between 0.016 and 0.05 pulses/s) produced a slow progressive rise of twitch tension (potentiation) which, after 40 twitches, was about $20 \%$ higher than the initial value. We then applied different protocols devoted either to prevent or stimulate any possible effect of extracellular ATP in tension potentiation. The removal of extracellular ATP by specific enzymes (hexokinase/apyrase), the inhibition of P2X receptors by a cocktail of P2 blockers (PPADS, suramin, and RB-2), or $\mathrm{Ca}^{2+}{ }^{-f r e e}$ conditions, all abolished tension potentiation. The addition of $\mathrm{Zn}^{+}$or ivermectin, at levels known to stimulate $\mathrm{P} 2 \mathrm{X}_{4}$, were without effects, suggesting that the receptor is already fully activated. On the contrary, elevated doses of $\mathrm{Zn}^{+}$, ivermectin or 2 meSATP reduced twitch tension potentiation.

Taking together, these data reveal that ATP-mediated $\mathrm{Ca}^{2+}$ entry plays, by still unknown mechanism/s, an important role in modulating the contractile activity of skeletal muscle and add new hints regarding the extracellular ATP signaling of this tissue.

The work was funded by MIUR (PRIN03), Telethon Italy and CNR.

\section{Extracellular interconversion of nucleotides indicates the presence of an ecto- adenylate kinase activity in the rat hippocampus}

\author{
Beáta Sperlágh and E. Sylvester Vizi \\ Department of Pharmacology, Institute of Experimental Medicine, Hungarian Academy of Sciences, Budapest, \\ Hungary \\ sperlagh@koki.hu
}


Whereas the enzymatic mechanisms, responsible for the inactivation of extracellular ATP and giving rise to the formation of adenosine are well delineated in the nervous system, less is known about the reverse process i.e. the rephosporylation of nucleotides and/or nucleosides to ATP. Although an early study indicated that there is a catalytic activity in the extracellular space to convert 2 ADP to ATP and AMP in rat brain synaptosomes with $\mathrm{K}_{\mathrm{m}}$ in the millimolar range (Nagy et al., 1989) and similar ecto-adenylate kinase (EC 2.7.4.3) activity has been reported in non-neuronal cells (Yegutkin et al., 2002), the reaction products of such an enzymatic conversion has not been directly demonstrated in intact brain preparations. To fulfill this objective, rat hippocampal slices and synaptosomes were exposed to increasing concentrations of various nucleotides and nucleosides and their extracellular interconversion were analysed by the HPLC-UV technique. If the slices were exposed to ATP (20-100$500 \mu \mathrm{M}$ ), it converted to ADP, AMP, adenosine, inosine and hypoxanthine according to the enzymatic process catalysed by the known families of ectonucleotidases. On the other hand, when the slices were challenged with ADP, in addition to the generation of adenosine, inosine and hypoxanthine, a parallel formation of ATP and AMP was also observed, which was strictly dependent on the initial concentration of ADP. A similar process was also observed in purified synaptosomes; however AMP formation in this case prevailed, and ATP generation could be only observed at higher initial ADP concentrations (100-500 $\mu \mathrm{M})$. In the presence of the specific adenylate kinase inhibitor diadenosine pentaphosphate (Ap5A, $200 \mu \mathrm{M})$, the decomposition of ADP and the parallel formation of ATP in the slices was significantly slowed down, although some additional ATP generation was also detected, which was probably due to the breakdown of Ap5A to ATP, or to the release of ATP by P2 receptor activation (Ballerini et al., 1996), homo- or heteroexchange (Sperlágh et al., 2003) or by other mechanism. Nevertheless, no substantial change in the interconversion of ADP to ATP and AMP was found in the presence of the nucleoside transport inhibitor dypiridamole $(3 \mu \mathrm{M})$, the P2 receptor antagonist PPADS $(30 \mu \mathrm{M})$ and the connexin hemichannel inhibitor flufenamic acid $(50 \mu \mathrm{M})$. When slices were exposed to AMP or adenosine, ectokinase activity was not detected, i.e. neither ATP nor ADP formation was observed.

In summary, here we demonstrate the parallel formation of ATP and AMP from ADP in the rat hippocampus, indicating that there is an ecto adenylate kinase activity in this brain area. This activity, which functions in the micromolar range, and is only partly associated to nerve terminals might represent the missing link of the nucleotide utilizing enzyme chain present in the brain tissue.

\title{
References
}

Nagy, A.K., Shuster, T.A. and Delgado-Escueta, A.V. (1989) Rat brain synaptosomal ATP:AMP-phosphotransferase activity. J Neurochem 53, 1166-1172.

Yegutkin, G. G., Henttinen, T., Samburski, S. S., Spychala, J. and Jalkanen, S. (2002) The evidence for two opposite, ATP-generating and ATP-consuming, extracellular pathways on endothelial and lymphoid cells. Biochem J 367, 121-128.

Sperlagh, B., Szabo, G., Erdelyi, F., Baranyi, M. and Vizi, E. S. (2003) Homo- and heteroexchange of adenine nucleotides and nucleosides in rat hippocampal slices by the nucleoside transport system. Br J Pharmacol 139, 623-33.

Ballerini, P., Rathbone, M.P., Di Iorio, P., Renzetti, A., et al. (1996) Rat astroglial P2Z (P2X7) receptors regulate intracellular calcium and purine release. Neuroreport, 7, 2533-7.

\section{Extracellular $\mathrm{NAD}^{+}$activates the $\mathrm{P} \mathrm{Y}_{11}$ purinergic receptor}

\author{
${ }^{1}$ Moreschi, I., ${ }^{1}$ Bruzzone, S., ${ }^{2}$ Nicholas, R.A., ${ }^{1}$ Zocchi, E. and ${ }^{1}$ De Flora, A. \\ ${ }^{1}$ Department of Experimental Medicine, Section of Biochemistry, and Center of Excellence for Biomedical \\ Research, University of Genova, Viale Benedetto XV/1, 16132 Genova, Italy; ${ }^{2}$ Department of Pharmacology, \\ University of North Carolina, Chapel Hill, NC, USA. \\ email: iliana.moreschi@unige.it
}

Extracellular $\beta-\mathrm{NAD}^{+}\left(\mathrm{NAD}^{+}{ }_{\mathrm{e}}\right)$ has been implicated in the regulation of intracellular calcium concentration $\left(\left[\mathrm{Ca}^{2+}\right]_{\mathrm{i}}\right)$ in many cell types and by means of different mechanisms (1-6). In human granulocytes, micromolar $\mathrm{NAD}_{\mathrm{e}}^{+}$activates cell-specific functional responses, i.e. superoxide and NO generation and chemotaxis, by triggering the following signalling cascade: i) activation of adenylate cyclase (AC) and accumulation of cyclic AMP 
(cAMP), ii) activation of protein kinase A, iii) stimulation of ADP-ribosyl cyclase activity with subsequent overproduction of cyclic ADP-ribose (cADPR), a universal $\mathrm{Ca}^{2+}$ mobilizer through activation of ryanodine receptors, and iv) influx of extracellular $\mathrm{Ca}^{2+}(7)$. Here we demonstrate that the addition of millimolar $\mathrm{NAD}^{+}$to human granulocytes promotes the intracellular production of both Inositol-1,4,5-trisphosphate $\left(\mathrm{IP}_{3}\right)$ and cAMP, leading to a two-step $\left[\mathrm{Ca}^{2+}\right]_{\mathrm{i}}$ elevation: an initial $\mathrm{IP}_{3}$-mediated $\mathrm{Ca}^{2+}$ release from intracellular stores, followed by a second cADPR-mediated sustained influx of extracellular $\mathrm{Ca}^{2+}$. The use of suramin, a broad inhibitor of the P2Y purinoceptors sub-family, abrogated the $\mathrm{NAD}^{+}$-induced increases of $\left[\mathrm{IP}_{3}\right]_{\mathrm{i}},[\mathrm{cAMP}]_{\mathrm{i}},[\mathrm{cADPR}]_{\mathrm{i}}$ and $\left[\mathrm{Ca}^{2+}\right]_{\mathrm{i}}$, thus indicating a role for a phospholipase C (PLC)- and AC-coupled P2Y receptor in initiating the signalling pathway triggered by $\mathrm{NAD}_{\mathrm{e}}^{+}$. The $\mathrm{P} 2 \mathrm{Y}_{11}$ receptor is the only known member of the $\mathrm{P} 2 \mathrm{Y}$ receptor sub-family coupled to both PLC and AC (8). Therefore, we investigated $\mathrm{Ca}^{2+}$ responses to $\mathrm{NAD}^{+}$in an $\mathrm{hP}^{2} \mathrm{Y}_{11}$-transfected $1321 \mathrm{~N} 1$ astrocytoma cells (9), compared to native $\mathrm{P}_{2} \mathrm{Y}_{11}$-negative cells. Micromolar $\mathrm{NAD}^{+}$induced a two-step elevation of $\left[\mathrm{Ca}^{2+}\right]_{\mathrm{i}}$ in transfected cells, but not in the control cells, due to the enhanced intracellular production of $\mathrm{IP}_{3}$, cAMP and cADPR. Thus, our results strongly suggest that $\beta-\mathrm{NAD}_{\mathrm{e}}^{+}$is an endogenous agonist of the $\mathrm{P}_{2} \mathrm{Y}_{11}$ purinoceptor.

\title{
References
}

1. De Flora, A., Zocchi, E., Guida, L., Franco, L., and Bruzzone, S. (2004) Ann. N.Y. Acad. Sci. 1028, 176-191

2. Verderio, C., Bruzzone, S., Zocchi, E., Fedele, E., Schenk, U., De Flora, A., and Matteoli, M. (2001) J. Neurochem. $\mathbf{7 8}, 1-13$

3. Sun, L., Adebanjo, O.A., Moonga, B.S., Corisdeo, S., Anandatheerthavarada, H.K., Biswas, G., Arakawa, T., Hakeda, Y., Koval, A., Sodam, B., et al. (1999) J. Cell Biol. 146, 1161-1172

4. Romanello, M., Bicego, M., Pirulli, D., Crovella, S., Moro, L., and D'Andrea, P. (2002) Biochem. Biophys. Res. Commun. 299, 424-431

5. Seman, M., Adriouch, S., Scheuplein, F., Krebs, C., Freese, D., Glowacki, G., Deterre, P., Haag, F., and KochNolte, F. (2003) Immunity 19, 571-582

6. Gerth, A., Nieber, K., Oppenheimer, N.J., and Hauschildt, S. (2004) Biochem. J. 382, 849-856

7. Bruzzone, S., Moreschi, I., Guida, L., Usai, C., Zocchi, E., and De Flora, A. (2006) Biochem J. 393, 697-704

8. Communi, D., Robaye, B., and Boeynaems, J-M. (1999) Br. J. Pharmacol. 128, 1199-1206

9. Qi, A-D., Kennedy, C., Harden, T.K., and Nicholas, R. (2001) Br. J. Pharmacol. 132, 318-326.

\section{EXTRACELLULAR NUCLEOTIDES ENHANCE EGF-INDUCED C-FOS EXPRESSION IN TWO BREAST CANCER CELL LINES, BY DRIVING THE EGF RECEPTOR INTO A DETERGENT-INSOLUBLE MEMBRANE FRACTION.}

\author{
A. Gartland $^{1}$, R.A. Hipskind ${ }^{2}$, and J.A. Gallagher ${ }^{3}$. \\ ${ }^{T}$ Academic Unit of Bone Biology, The University of Sheffield, Sheffield, S10 2RX, UK. ${ }^{2}$ IGMM, CNRS, 1919 Route \\ de Mende. 34293 Montpellier. Cedex 5, France. ${ }^{3}$ Human Bone Cell Research Group, Department of Human \\ Anatomy and Cell Biology, The University of Liverpool, Liverpool, L69 3GE U.K. \\ a.gartland@sheffield.ac.uk
}

There is increasing evidence that extracellular nucleotides play a pivotal role in cancer growth, metastasis, and pain. We have previously shown that extracellular nucleotides synergise with growth factors to induce expression of the proto-oncogene $c$-fos in various cell types. In this study, we have examined the effect of extracellular nucleotides on growth factor-induced signalling in two breast cancer cell lines, HS578T and T47D. Using RT-PCR we found expression of various P2Y and P2X receptor subtypes in both cell lines. Co-stimulation of these cells with extracellular nucleotides and EGF lead to induction of endogenous $c$-fos above that observed with either treatment alone. This effect was recapitulated using HS578T and T47D $c$-fos reporter cells and was more pronounced with EN that predominantly act via P2Y receptors. EGF-induced phosphorylation of p44/p42 MAPK was unaffected in either cell type following co-stimulation, although phosphorylation of AKT and ERK5 was effected. EGFR phosphorylation appeared diminished upon co-stimulation, however the levels of total EGFR were also diminished in cold $1 \%$ Triton lysates in both cell types. When lysates were prepared in denaturing hot SDS buffer no reduction in EGFR levels was observed. This suggests that extracellular nucleotides may lead to inclusion of 
the EGFR into a Triton-insoluble membrane fraction, possibly lipid rafts, which may explain facilitated signalling to the $c$-fos promoter. Synergy between extracellular nucleotides and growth factors may represent an important mechanism promoting the growth and spread of tumours. This may be particularly relevant for breast cancer metastasis to bone as bone cells constitutively release ATP, and in response to mechanical strain. This could play a key role in creating an environment propitious for breast cancer cell growth after metastasis.

\title{
Extracellular nucleotides mediate LPS-induced IL-8 release from monocytes and neutrophil migration.
}

\author{
Filip Kukulski, Fethia Ben Yebdri, Julie Lefebvre, Jean Sévigny. \\ Centre de recherche en Rhumatologie et Immunologie, Université Laval, Ste-Foy, Québec, Canada. \\ filip.kukulski@crchul.ulaval.ca
}

Increasing evidence suggests that extracellular nucleotides may mediate some of the proinflammatory effects of gram negative bacteria lipopolysaccharides (LPS). For instance, $\mathrm{P}_{2} \mathrm{Y}_{6}$ receptor activation is required for the LPSinduced secretion of interleukin-8 (IL-8) by THP-1 cells. Knowing that peripheral blood leukocytes (monocytes, lymphocytes and neutrophils) and HUVEC also express P2Y ${ }_{6}$ mRNA and that they respond to LPS by the secretion of IL-8, we have tested whether extracellular nucleotides mediate IL-8 release by these cells. As expected, all cells analyzed released IL-8 in response to LPS. However, this release was nucleotide-dependent only in monocytes, as it was significantly inhibited by a nucleotide scavenger (apyrase) and $\mathrm{P}_{2} \mathrm{Y}_{6}$ receptor antagonists (MRS 2578 and reactive blue 2). Importantly, the former inhibition by apyrase was not due to the formation of adenosine. In further support for a role of $\mathrm{P}_{2} \mathrm{Y}_{6}$ in IL-8 release by monocytes, these cells secreted important amounts of IL-8 when stimulated with UDP.

IL-8 is a major human chemokine, so we next tested the chemotactic activity of conditioned media from treated monocytes on neutrophil migration. Using modified Boyden chambers with an endothelial monolayer, we observed that the media of LPS-stimulated monocytes recruited significantly more neutrophils than the corresponding media to which LPS were added simultaneously with apyrase. Conditioned media of UDP-stimulated monocytes were as effective to induce neutrophil migration as those of the cells treated with LPS. In addition, IL-8 neutralizing antibodies added to the media of LPS- or UDP-stimulated monocytes substantially decreased their chemotactic activity, demonstrating that IL-8 was the main chemokine responsible for cellular migration in these assays.

In conclusion, extracellular nucleotides have the ability to regulate neutrophil recruitment to inflamed tissues by mediating IL-8 release from human monocytes/macrophages.

\section{Feed forward cycle of hypotonic stress-induced ATP release, purinergic receptor activation and growth stimulation of prostate cancer cells}

\author{
$\underline{\text { Rajender Nandigama }}^{\#}$, Manju Padmasekar ${ }^{\#}$, Maria Wartenberg ${ }^{\S}$, and Heinrich Sauer ${ }^{\#}$ \\ From the Department of Physiology, Justus-Liebig-University Gießen", Germany and the Department of Cell \\ Biology, GKSS Research Center, Teltow, Germany ${ }^{\S}$ \\ E-mail: n_rajenderreddy@yahoo.com
}

ATP is released in many cell types upon mechanical strain, the physiological function of extracellular ATP is largely unknown, however. Here we report that ATP released upon hypotonic stress stimulated prostate cancer cell proliferation, activated purinergic receptors, increased intracellular $\left[\mathrm{Ca}^{2+}\right]_{i}$ and initiated downstream signalling cascades that involved mitogen activated protein kinases (MAPKs) ERK1/2 and p38 as well as PI3-kinase. MAPK activation, the calcium response as well as induction of cell proliferation upon hypotonic stress were inhibited by preincubation with the ATP scavenger apyrase, indicating that hypotonic stress-induced signalling pathways are elicited by released ATP. Hypotonic stress increased prostaglandin $\mathrm{E}_{2}\left(\mathrm{PGE}_{2}\right)$ synthesis. Consequently, ATP release was inhibited by antagonists of PI3-kinase (LY294002 and wortmannin), phospholipase $\mathrm{A}_{2}$ 
(MAFP), cyclooxygenase-2 (COX-2) (indomethacin, etodolac, NS398) and 5,8,11,14-eicosatetraynoic acid (ETYA), which are involved in arachidonic acid metabolism. Furthermore, ATP release was abolished in the presence of the adenylate cyclase (AC) inhibitor MDL-12,330A, indicating regulation of ATP-release by cAMP. The hypotonic stress-induced ATP release was significantly blunted when the ATP-mediated signal transduction cascade was inhibited on different levels, i.e. purinergic receptors were blocked by suramin and PPADS, the $\mathrm{Ca}^{2+}$ response was inhibited upon chelation of intracellular $\mathrm{Ca}^{2+}$ by BAPTA, and ERK1,2 as well as p38 were inhibited by UO126 and SB203580, respectively. In summary our data demonstrate that hypotonic stress initiates a feed forward cycle of ATP release and purinergic receptor signalling resulting in proliferation of prostate cancer cells.

\section{References-:}

1. Burnstock, G. (1999) J.Anat. $194 \pm$ Pt 3), 335-342

2. Insel, P. A., Ostrom, R. S., Zambon, A. C., Hughes, R. J., Balboa, M. A., Shehnaz, D., Gregorian, C., Torres, B., Firestein, B. L., Xing, M., and Post, S. R. (2001) Clin.Exp.Pharmacol.Physiol 28, 351-354

3. Schwiebert, E. M. and Zsembery, A. (2003) Biochim.Biophys.Acta 1615, 7-32

4. Post, S. R., Jacobson, J. P., and Insel, P. A. (1996) J.Biol.Chem. 271, 2029-2032

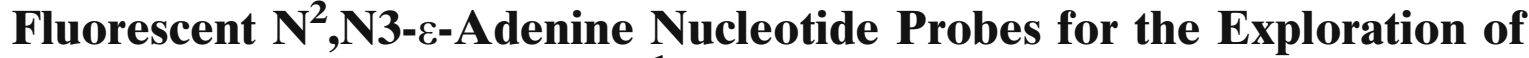 P2Y-receptors and NTPDases ${ }^{1}$}

$\underline{\text { Bilha Fischer }}^{a}$, Einat Sharon ${ }^{a}$, Sebastien Levesque $^{b}$, Mercedes N. Munkonda $^{b}$, Jean Sévigny $^{b}$, Denise Ecke ${ }^{c}$, and Georg Reiser ${ }^{c}$

a. Department of Chemistry, Bar-Ilan University, Ramat-Gan 52900, Israel

b. Centre de recherche en Rhumatologie et Immunologie, Université Laval, Sainte-Foy, Québec, Canada. c. Institute for Neurobiochemistry, Faculty of Medicine, Otto von Guericke University, Leipziger Str. 44 D-39120 Magdeburg, Germany

e-mail: bfischer@mail.biu.ac.il

The fields of P2Y-receptors and NTPDases lack analytical tools for the exploration of these proteins. Therefore, we designed a novel probe: $\mathrm{N}^{2}, \mathrm{~N} 3$-etheno-adenosine-5'-triphosphate, $\left(\mathrm{N}^{2}, \mathrm{~N} 3-\varepsilon-\mathrm{ATP}\right)$ for improving the adenine fluorescence characteristics ( and $\lambda_{\max }$ ) while preserving its H-bonding pattern, required for molecular recognition. Here, we describe four novel syntheses of the target $\varepsilon$-nucelotide, and related analogues. These methods are short (3-4 step syntheses), facile, and provide the product regiospecifically and in a reasonable yield. In addition, we report on the spectral properties of $\mathrm{N}^{2}, \mathrm{~N} 3-\varepsilon-\mathrm{ATP}$, including absorption and emission spectra, and dependence of the spectral features on $\mathrm{pH}$ and polarity of the medium. Specifically, maximum emission of $\mathrm{N}^{2}, \mathrm{~N} 3-\varepsilon-\mathrm{ATP}$ in water is observed at $420 \mathrm{~nm}(0.03$, excitation at $290 \mathrm{~nm})$. The biochemical properties of $\mathrm{N}^{2}, \mathrm{~N} 3-\varepsilon-\mathrm{ATP}$ were evaluated with respect to P2 $\mathrm{Y}_{1}$-receptor and NTPDase1 and 2. $\mathrm{N}^{2}, \mathrm{~N} 3-\varepsilon$-ATP was found equipotent to ATP at the $\mathrm{P}_{2} \mathrm{Y}_{1}$-receptor, and was hydrolysed by NTPDase1 and 2 at about the same rate as ATP. However, substitution at the exocyclic amine of $\mathrm{N}^{2}, \mathrm{~N} 3-\varepsilon$-ATP significantly reduced affinity to $\mathrm{P}_{2} \mathrm{Y}_{1}-\mathrm{R}$ and NTPDase1. These observations emphasize the importance of free $\mathrm{N} 1$ and $\mathrm{N}^{6}$ positions for the recognition of ATP-based probes by target proteins. Furthermore, $\mathrm{N}^{2}, \mathrm{~N} 3-\varepsilon$-ATP was fluorescent also in the protein-bound state. Based on the unique fluorescence and full recognition by target proteins, we propose $\mathrm{N}^{2}, \mathrm{~N} 3-\varepsilon$-ATP and related nucleotides as useful probes for studies of these proteins.

${ }^{1}$ A US provisional patent application filed on 24 January, 2005.

\section{Functional analysis of the role of single amino acid residues in the human $\mathrm{P}_{2} \mathrm{Y}_{2}$ receptor}

Petra Hillmann $^{1}$, Ivar von Kügelgen ${ }^{2}$, Geun-Yung $\mathrm{Ko}^{3}$, Hans-Dieter Höltje ${ }^{3}$ and Christa E. Müller ${ }^{1}$

${ }^{T}$ Pharmaceutical Sciences Bonn, Pharmaceutical Institute, University of Bonn, Kreuzbergweg 26, 53115 Bonn, Germany 
${ }^{2}$ Department of Pharmacology, University of Bonn, Reuterstrasse 2b, 53113 Bonn, Germany

${ }^{3}$ Pharmaceutical Institute, University of Düsseldorf, Building 26.23, Universitätsstrasse 1, 40225 Düsseldorf, Germany

hillmann@uni-bonn.de

$\mathrm{P}_{2} \mathrm{Y}_{2}$ receptors belong to the family of $\mathrm{G}$ protein-coupled nucleotide (P2) receptors. They are activated by the physiological nucleotides UTP and ATP. There is a lack of potent and selective P2 $\mathrm{Y}_{2}$ receptor ligands [1] which are required as pharmacological tools. In addition, such compounds have potential as novel therapeutics, e.g. as antiinflammatory agents, for the treatment of coronary vasospastic disorders, as analgesics, or as neuroprotective drugs [1,2]. In order to design potent and specific agonists and antagonists for the $\mathrm{P}_{2} \mathrm{Y}_{2}$ receptor, information about the structure, constitution, and conformation of the orthosteric binding site and of potential allosteric binding sites will be highly useful.

The present study focuses on investigating the importance of single amino acids for ligand binding and activation of the $\mathrm{P}_{2} \mathrm{Y}_{2}$ receptor. The amino acid residues $\mathrm{His}^{262}, \mathrm{Arg}^{265}$ and $\mathrm{Arg}^{292}$ were previously shown to be part of the nucleotide binding site [3]. Four amino acids in the first extracellular loop ( $\mathrm{Arg}^{95}$, Gly ${ }^{96}$, Asp ${ }^{97}$, and Leu ${ }^{108}$ ) were found to be essential for apical targeting of the $\mathrm{P}_{2} \mathrm{Y}_{2}$ receptor but do not interfere with receptor activation [4]. We have designed several $\mathrm{P}_{2} \mathrm{Y}_{2}$ receptor mutants, including R177_180A, R194H and R272A. A retroviral transfection system was used to overexpress the wild-type and the mutated receptors in 1321N1 astrocytoma cells. Characterization of the mutants was performed using a fluorimetric calcium assay. A series of physiological nucleotides, including the agonists ATP and UTP, as well as dinucleotide derivatives, have been tested at the receptor mutants. Several non-competitive $\mathrm{P}_{2} \mathrm{Y}_{2}$ receptor antagonists previously developed in our group, including flavonoids [5], biflavonoids, and anthraquinone derivatives were also used to study the mutated $\mathrm{P}_{2} \mathrm{Y}_{2}$ receptors.

Furthermore, the role of the disulfide bonds was investigated. Under disulfide reducing conditions [6] (1-10 mM dithiothreitol) activation of the $\mathrm{P}_{2} \mathrm{Y}_{2}$ receptor by UTP or ATP was strongly affected. Calcium release from the endoplasmatic reticulum was reduced by up to $73 \%$ while the muscarinic M3 receptor, endogenously expressed on the cells, showed a very moderate decrease in activation of only $19 \%$. These results are consistent with a computer-generated model of the $\mathrm{P}_{2} \mathrm{Y}_{2}$ receptor and indicate the existence of a disulfide bond in the extracellular loops $\left(\mathrm{Cys}^{25}-\mathrm{Cys}^{278}\right)$, which is essential for receptor activation.

[1] Brunschweiger; Müller Curr. Med. Chem. 2006, 12, 763-771

[2] Moriyama et al., J. Neuroscience 2003, 23, 6058-6062

[3] Erb et al., J. Biol. Chem. 1995, 270, 4185-4188

[4] Qi et al., J. Biol. Chem. 2005, 280, 29169-29175

[5] Kaulich et al., Drug Dev. Res. 2003, 59, 72-81

[6] Heerding et al., Biochemistry 2001, 40, 8369-8377

Supported by the Deutsche Forschungsgemeinschaft (GRK 804)

\title{
Functional P2Y Receptors in Achondroplasic Chondrocytes
}

\author{
Ana Isabel Guzmán, Marta Irazu and Jesús Pintor \\ Departamento de Bioquímica y Biología Molecular IV, E.U. Optica, Universidad Complutense de Madrid, c/Arcos \\ de Jalón s/n, E-28040 Madrid, Spain \\ jpintor@vet.ucm.es
}

Chondrocytes are important cells in the development and growth of bones in mammals. Several molecules, hormones, cytokines and growth factors drive the chondrocytes to a proper maturation and development. Among all of them, transforming growth factors (TGF- $\beta \mathrm{s}$ ), bone morphogenetic proteins (BMP), insulin-like growth factors, (IGFs), fibroblast growth factors (FGFs), interleukins (ILs) and others, interplay an organised sequence of events that permit the normal development of bones. Alteration in any of these substances, or more commonly in any of their receptors, will produce changes in ossification that will lead to a group of pathologies termed as bone displasias. One of these bone syndromes is achondroplasia, the most common type of dwarfism. Achondroplasia (dwarfism) is a genetic pathology due to a mutation in the gene that encodes for the fibroblast growth factor type 
3 receptor (FGFR3, G380A). This mutation severely affects the chondrocytes, the consequence of such failure being an acute reduction in the bone length.

We have investigated the presence of $\mathrm{P} 2 \mathrm{Y}$ receptors in achondroplasic chondrocytes by means of immunocytochemical techniques and intracellular $\mathrm{Ca}^{2+}$ measurements. Also, we have explored the ability of these cells to degrade extracellular nucleotides and dinucleotides by means of the HPLC technique.

Rat achondroplasic chondrocytes present $\mathrm{P} 2 \mathrm{Y}_{1}, \mathrm{P} 2 \mathrm{Y}_{2}, \mathrm{P}_{2} \mathrm{Y}_{6}$ and $\mathrm{P} 2 \mathrm{Y}_{11}$ receptors as observed after immunocytochemical analysis. At least some of the P2Y receptors were active since the perfusion of cells with various nucleotides and dinucleotides produced significant changes in the intracellular $\mathrm{Ca}^{2+}$ concentration. Among them, diadenosine polyphosphates $\left(\mathrm{Ap}_{3} \mathrm{~A}, \mathrm{Ap}_{4} \mathrm{~A}\right.$ and $\left.\mathrm{Ap} \mathrm{p}_{5} \mathrm{~A}\right)$, ATP and UTP were the most effective compounds. At a wide range of concentrations $\left(10^{-9} \mathrm{M}\right.$ to $\left.10^{-3} \mathrm{M}\right)$, ATP provided the highest increment of intracellular calcium, followed by UTP, $\mathrm{Ap}_{4} \mathrm{~A}, \mathrm{Ap}_{5} \mathrm{~A}$ and $\mathrm{Ap}_{3} \mathrm{~A}$. When analysed the $\mathrm{pD}_{2}$ values obtained from the concentration response curves, it was possible to obtain the following potency order: $A_{3} A=A p_{4} A=A p_{5} A=A T P=U T P$. Four different antagonists were used to investigate P2Y receptors: MRS2179, suramin, PPADS and reactive blue 2 (RB-2). Among them the most effective on the $\mathrm{Ca}^{2+}$ transient elicited by $\mathrm{Ap}_{4} \mathrm{~A}$ was PPADS.

Diadenosine polyphosphates were weakly hydrolysed by extracellular ecto-nucleotidases, indicating that the effects observed were due to the dinucleotides rather than to their cleavage products.

This work has been supported by a research grant from la Comunidad de Madrid CAM GR/SAL/057372004, Fundacion López Hidalgo and Fundación ICO.

\title{
G PROTEIN-COUPLED RECEPTORS TOPOLOGY: LIGAND-BASED HOMOLOGY MODELING AS NEW APPROACH TO SIMULATE THE REORGANIZATION INDUCED BY THE ANTAGONIST BINDING.
}

\author{
Erika Morizzo $^{1}$, Francesca Deflorian ${ }^{1}$, Magdalena Bacilieri ${ }^{1}$, Giampiero Spalluto ${ }^{2}$, Stefano Moro $^{1}$
}

${ }^{T}$ Molecular Modeling Section, Dipartimento di Scienze Farmaceutiche, Università di Padova, Via Marzolo 5, I-35131 Padova, Italy; ${ }^{2}$ Dipartimento di Scienze Farmaceutiche, Università degli Studi di Trieste, Piazzale Europa 1, I-34127 Trieste, Italy

erika.morizzo@unipd.it

G protein-coupled receptors (GPCRs) constitute a very large family of heptahelical, integral membrane proteins that mediate a wide variety of physiological processes, ranging from the transmission of the light and odorant signals to the mediation of neurotransmission and hormonal actions. GPCRs are dysfunctional or dysregulated in several human diseases and are estimated to be the targets of $>40 \%$ of the drugs used in clinical medicine today ${ }^{1}$. The crystal structure of rhodopsin provides the first information on the three-dimensional structure of GPCRs, which now supports homology modeling studies and structure-based drug-design approaches ${ }^{2}$.

In this work we review our recent work ${ }^{3}$ on adenosine receptor, a family of GPCRs and, in particular, on Adenosine $A_{3}$ receptor subtype antagonists. We will focus on a different approach to computationally inspect the reorganisation of the human A3 receptor induced by the antagonist-binding. Ligand-based homology modeling is a new strategy to simulate the antagonist-like conformational states of the receptor induced by the ligand binding. The success of this computational tool is due to the synergic interaction between theory and experiment. 


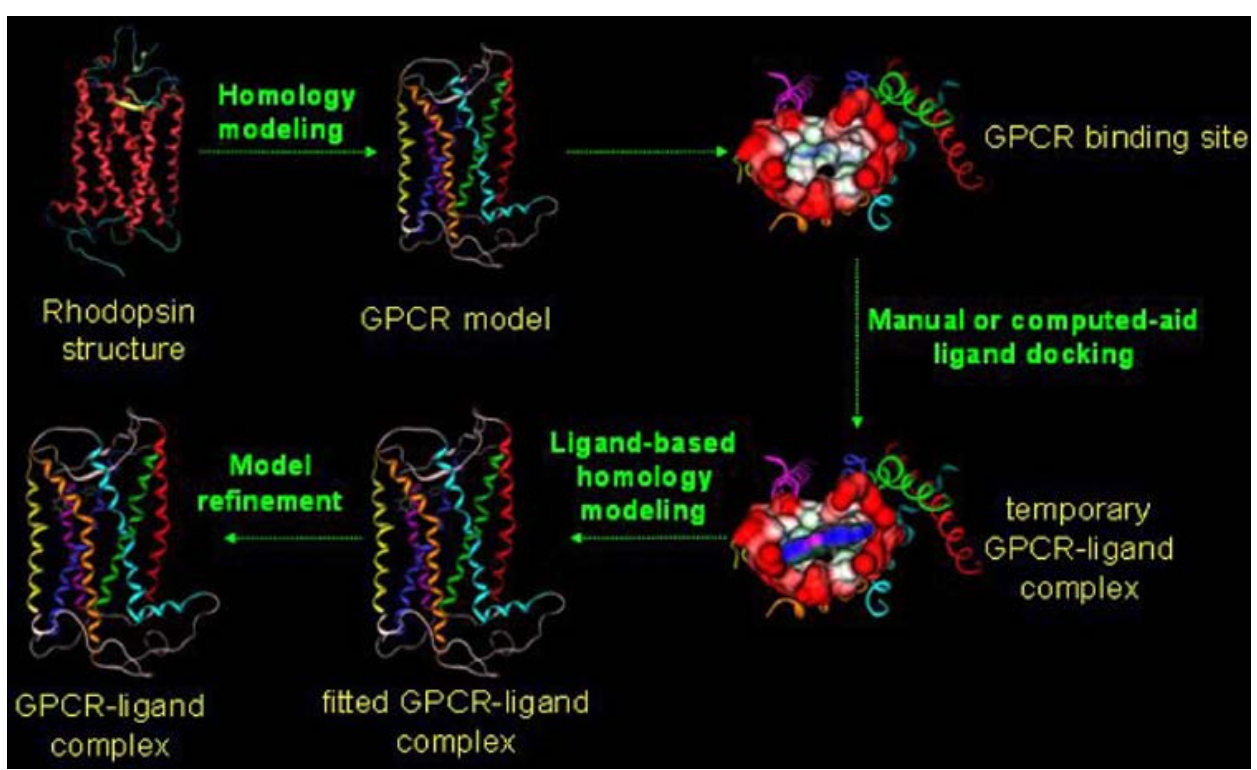

Flow chart of the ligand-based homology modeling technique considering an evolution of a conventional homology modeling algorithm implemented by Molecular Operating Environment modeling software.

${ }^{1}$ Moro, S.; Bacilieri, M.; Deflorian, F.; Spalluto, G. New J. Chem. 2006, 30, 1-8.

${ }^{2}$ Moro, S.; Deflorian, F.; Bacilieri, M.; Spalluto, G. Curr. Med. Chem. 2006, 13, 763-771.

${ }^{3}$ Moro, S.; Deflorian, F.; Bacilieri, M.; Spalluto, G. Curr. Pharm. Des. 2006, 12, in press.

\title{
Gene expression profiling for the identification of G-protein coupled receptors in human platelets
}

\author{
Oscar Ö Braun ${ }^{1}$, Stefan Amisten ${ }^{1}$, Anders Bengtsson ${ }^{2}$ \& David Erlinge ${ }^{1}$ \\ Department of Cardiology ${ }^{1}$ and Rheumatology ${ }^{2}$, Lund University Hospital, Lund, Sweden \\ oscar.braun@med.lu.se
}

G protein coupled receptors (GPCRs) play an important role in regulating platelet activation and thrombus formation. Aberrant platelet activation may lead to thrombus formation and occlusion of blood vessels that may result in myocardial infarction and stroke. It is of great clinical importance to further understand the mechanisms influencing platelet activation. The aim of this study was to generate an expression profile for G-protein coupled receptors in circulating human platelets. Several studies have explored platelet gene expression based on large amounts of blood, time consuming platelet purification methods and samples of varying grade of contamination by other cell types. In this study, a rapid platelet purification process was used in combination with a very sensitive leukocyte contamination detection system to generate a platelet gene expression profile using the Affymetrix Human HG-U133 Plus 2.0 GeneChip ${ }^{\circledR}$. The generated gene expression profile was used in combination with quantitative real-time PCR (Q-RT-PCR) to characterize the G-protein coupled receptor gene expression in circulating platelets. A platelet gene expression profile with low levels of leukocyte contamination revealed expression of 3,408 genes in circulating platelets. Gene expression of $24 \mathrm{G}$-protein coupled receptors was validated by Q-RT-PCR. The thrombin receptor $(1865 \pm 178 \%)$ was the most abundant GPCR expressed on platelets, followed by the ADP receptor $\mathrm{P}_{2} \mathrm{Y}_{12}(459 \pm 88 \%)$, succinate receptor $1(257 \pm 48 \%)$, $\mathrm{P}_{2} \mathrm{Y}_{1}(100 \%)$, the orphan receptor $\mathrm{P}_{2 \mathrm{RY}}(68.2 \pm 3.3 \%)$, the LPA receptor GPR23 $(48.2 \pm 11 \%)$, the orphan receptor GPR92 $(26.1 \pm$ $3.3 \%)$, the alpha 2 adrenergic receptor $(18.4 \pm 4.4 \%)$, the orphan receptor EBI2 $(6.31 \pm 0.42)$, two adenosine receptors $\mathrm{A}_{2 \mathrm{~A}}(2.94 \pm 0.24 \%)$ and $\mathrm{A}_{2 \mathrm{~B}}(2.88 \pm 0.16 \%)$ and the LPA receptor EDG2 $(2.59 \pm 0.39 \%)$. In conclusion we show for the first time that the $A_{2 B}$ receptor is expressed, in equal amount as the $A_{2 A}$ receptor, in human platelets. Interestingly enough we also conclude that eight among the 24 most abundant GPCRs expressed in human platelets are or were thought to be purinergic receptors. 


\title{
Generation of antibodies that recognize ENTPDs 1,2,5, and 6 and human ENPP-2 (Autotaxin) in native conformation
}

\author{
Caroline Jung, Gudrun Dubberke, Friedrich Koch-Nolte, and Friedrich Haag \\ Institute of Immunology, University Hospital, Martinistr. 52, Hamburg, 20246 Germany \\ haag@uke.uni-hamburg.de
}

Signalling through purinoreceptors is effectively controlled by nucleotide-metabolising enzymes, which regulate the availability of extracellular nucleotides. Prominent roles are played by members of the ecto-nucleoside triphosphate diphosphohydrolase (ENTPD, CD39-like) and ecto-nucleotide pyrophosphatase/phosphodiesterase (ENPP) families. Despite their importance for the control of purinergic signalling, investigation of these enzyme families has been hampered by the lack of available antibodies, especially such ones that recognize the enzymes in their native conformation. This is in part due to the structural complexity of these enzymes, which makes it difficult to produce sufficient quantities of recombinant protein for immunization purposes.

Genetic immunization with cDNA expression vectors poses an attractive alternative to conventional immunization strategies, since the protein of interest is produced in native conformation by cells of the immunized host (1). We used this approach to raise antibodies against mouse ENTPDs 1, 2, 5, and 6, human ENTPDs 2 and 6, as well as human ENPP-2 (Autotaxin). All antisera recognized their target antigens in immunofluorescence analysis of HEK cells transfected with the respective cDNAs, either on the surface of living cells or, in the case of the secreted proteins ENTPD5 and ENPP2, in fixed and permeablized cells. These studies show that genetic immunization is a feasible method for raising antisera against ecto-enzymes in their native conformation, even in cases where recombinant production/purification of the antigen is difficult.

supported by DFG grant No310/6-1 to FH and FKN

(1) Adriouch, S.; Dubberke, G.; Diessenbacher, P.; Rassendren, F.; Seman, M.; Haag, F.; Koch-Nolte, F., Probing the expression and function of the P2X7 purinoceptor with antibodies raised by genetic immunization. Cell Immunol 2005, 236, (1-2), 72-77.

\section{Generation of antibodies that recognize P2X1, P2X4, and P2X7 in native conformation}

\author{
Sina Möller, Sahil Adriouch, Friedrich Haag, and Friedrich Koch-Nolte \\ Institute of Immunology, University Hospital, Martinistr. 52, Hamburg, 20246 Germany \\ nolte@uke.uni-hamburg.de
}

P2X purinoceptors contain cytosolic N- and C- termini, two transmembrane domains and an extracellular ligandbinding domain with five conserved intra-chain disulfide bridges. Peptide immunizations have generated antisera that are useful for detecting P2X family members by immunoblot analyses. In general however, these antipeptide antibodies do not recognize the native purinoceptors on the cell surface. Antibodies against native purinoceptors are devoutly to be desired for monitoring purinoceptor expression on the cell surface. Production of soluble recombinant purinoceptors in sufficient quantities for immunizations is frought with inherent difficulties due to the presence of cysteine residues and glycosylation sites. Genetic immunization with full length cDNA expression vectors poses an attractive alternative immunization strategy since the protein of interest is produced in native conformation by cells of the immunized host (1). Usind cDNA immunization technology we have successfully raised antibodies that recognize human and mouse P2X1, P2X4, and P2X7 on the surface of living cells and on paraformaldehyde-fixed cells. We illustrate the utility of these antibodies for monitoring cell surface expression of wild type and mutant P2X receptors by immunofluorescence microscopy and flow cytometry of transiently transfected HEK cells.

supported by DFG grant No310/6-1 to FH and FKN 
(1) Adriouch, S.; Dubberke, G.; Diessenbacher, P.; Rassendren, F.; Seman, M.; Haag, F.; Koch-Nolte, F., Probing the expression and function of the P2X7 purinoceptor with antibodies raised by genetic immunization. Cell Immunol 2005, 236, (1-2), 72-77.

\title{
Glycolysis Dependent And Independent Adenosinergic Protection In Ischaemic-Reperfused Mouse Heart
}

\author{
Benjamin D. Hack ${ }^{1}$, Melissa E. Reichelt ${ }^{1}$, Jason Peart ${ }^{1}$, G. Paul Matherne ${ }^{2}$, and John P. Headrick ${ }^{1}$ \\ ${ }^{1}$ Heart Foundation Research Center, Griffith University Southport, QLD, AUSTRALIA, \\ ${ }^{2}$ Department of Pediatrics, University of Virginia Health Sciences Center, Charlottesville, VA, USA \\ j.Headrick@griffith.edu.au
}

Glycolysis is important to ischaemic tolerance, and may be crucial to cardioprotection via adenosine and its receptors. We assessed effects of glycolytic inhibition on functional tolerance to $25 \mathrm{~min}$ ischaemia and $60 \mathrm{~min}$ reperfusion, and protection mediated by adenosine or $\mathrm{A}_{1}$ adenosine receptor $\left(\mathrm{A}_{1} \mathrm{AR}\right)$ overexpression in mouse hearts. Control hearts $(11 \mathrm{mM}$ glucose as substrate) rapidly developed ischaemic contracture (time to onset or $\mathrm{TOC}=272 \pm 22 \mathrm{~s}$; peak contracture or $\mathrm{PC}=80 \pm 6 \mathrm{mmHg})$, and displayed significant diastolic dysfunction $(27 \pm 1$ $\mathrm{mmHg}$ ) with incomplete recovery of left ventricular developed pressure (LVDP) to only $58 \pm 3 \mathrm{mmHg}(46 \pm 4 \%$ of pre-ischaemia) after reperfusion. Glycolytic inhibition with $10 \mathrm{mM}$ pyruvate+iodoacetic acid (IAA; 150 followed by $50 \mu \mathrm{M}$ ), which reduced GAPDH activity by $\sim 98 \%$, markedly delayed ischaemic contracture (TO$\mathrm{C}=779 \pm 72 \mathrm{~s} ; \mathrm{PC}=63 \pm 4 \mathrm{mmHg}$ ). This effect was duplicated by pyruvate alone, and by treatment with $5 \mathrm{mM} 2,3$ butanedione monoxime (uncoupling contractile function from $\left[\mathrm{Ca}^{2+}\right]$ ) or $10 \mu \mathrm{M}$ BIIB-513 (inhibiting $\mathrm{Na}^{+}-\mathrm{H}^{+}$ exchange). Despite delayed contracture, glycolytic inhibition worsened functional recovery (LVDP=26 $\pm 3 \mathrm{~mm}$ $\mathrm{Hg} ; 22 \pm 3 \%)$, due to exaggerated diastolic dysfunction $(47 \pm 3 \mathrm{mmHg})$. In glucose-perfused hearts, adenosine $(25$ $\mu \mathrm{M})$ and cardiac $\mathrm{A}_{1} \mathrm{AR}$ overexpression delayed ischaemic contracture and improved post-ischaemic recovery (diastolic pressures $=12-16 \mathrm{mmHg}, \mathrm{LVDP}=75-80 \mathrm{mmHg}$ ), as did inhibition of adenosine kinase with $5 \mu \mathrm{M}$ iodotubercidin to enhance endogenous [adenosine]. None of these strategies modified ischaemic contracture when glycolysis was inhibited, whereas adenosinergic improvements in post-ischaemic outcome were still evident. These data collectively reveal that: i) glycolysis improves post-ischaemic functional recovery in murine myocardium despite accelerating contracture; ii) ischaemic contracture involves $\mathrm{Ca}^{2+}$ - or ion-dependent processes; iii) adenosine/ $\mathrm{A}_{1} \mathrm{AR}$-mediated inhibition of ischaemic contracture is glycolysis-dependent; whereas iv) adenosinergic enhancement of post-ischaemic outcome involves glycolysis-independent mechanisms.

\section{GSK-3 is involved in 2MeSADP signalling in cerebellar granule neurons}

Raquel Pérez-Sen, Felipe Ortega, and MªTeresa Miras-Portugal. Departmento de Bioquímica. Facultad de Veterinaria. Universidad Complutense de Madrid. Email: rpsen@vet.ucm.es

Cerebellar granule neurons express a great variety of nucleotidic receptors, both of ionotropic $\left(\mathrm{P}_{2} \mathrm{X}_{1,2,3,4,7}\right)$ and metabotropic type $\left(\mathrm{P}_{2} \mathrm{Y}_{1,4,12}\right)$. These receptors are heterogeneously distributed along the granule cell cytoarchitecture between cell bodies and prolongations. Functional responses to nucleotides, measured as increases in the intracellular calcium concentration, were dependent on the differentiation state of the culture, becoming significant in mature cultures (1). The intracellular nucleotide signalling beyond the calcium signal was investigated beginning with MAPK kinases and GSK-3, proteins which play a key role in multiple processes of survival and differentiation in granule neurons $(2,3)$.

We first analyzed the effect of metabotropic nucleotidic agonists, among them, the highest levels of phosphorylation of ERK-1,2 and GSK-3, were found for the ADP receptor agonist, 2MeSADP. The responses found for the pyrimidine agonists UTP and UDP were much lower. These results agree well with the expression levels of metabotropic nucleotidic receptors in granule neurons, as the major population of nucleotide responding cells was sensitive to stimulation with 2MeSATP and 2MeSADP. Although the effect of 2MeSADP was clearly metabotropic, it seemed to be mediated by other $\mathrm{P} 2 \mathrm{Y}$ receptor different from $\mathrm{P}_{2} \mathrm{Y}_{1}$ and $\mathrm{P} 2 \mathrm{Y}_{12}$, as it remained in the 
presence of the specific $\mathrm{P}_{2} \mathrm{Y}_{1}$ and $\mathrm{P} 2 \mathrm{Y}_{12}$ antagonists MRS-2179 and 2MeSAMP, respectively. In addition, the 2MeSADP-mediated increases in ERK-1,2 and GSK-3 phosphorylation were both sensitive to treatment with Pertussis toxin and wortmanin, suggesting the existence of a $\mathrm{G}_{\mathrm{i}}$ coupled ADP receptor mediating these effects in cultured granule neurons, and that PI3-kinase is a critical intermediate step. These data are relevant considering that the PI3-kinase-Akt-GSK-3 cascade is an important survival signalling pathway in this cellular model $(3,4)$. Taking into account that GSK-3 phosphorylation at $\operatorname{Ser}^{9,21}$ involves the inactivation of this enzyme, one of the main substrates of GSK-3 activity was examined, that is $\beta$-catenin. In the absence of GSK-3 activity, degradation of $\beta$-catenin is abolished and this protein accumulates in the cytosol, and can reach the nucleus where it acts as a transcriptional regulator (5). 2MeSADP was able to induce translocation of $\beta$-catenin to the nucleus of cerebellar granule neurons, implying effects of this nucleotidic agonist at the transcriptional level.

1. Hervás et al., 2003. J. Neurosci. Res. 73:384-399.

2. Subramaniam et al., 2005. J. Neurosci. 25:2838-2852.

3. Chin et al., 2005. Mol. Brain Res. 137:193-2001.

4. Miller et al., J. Biol.. Chem. 272:9847-9853.

5. Nelson and Nusse 2004. Science 303:1483-1487.

\title{
Guanosine protects SY5Y neuroblastoma cells against MPP ${ }^{+}$-induced apoptosis
}

\author{
K.M. Pettifer, C. Bau, S. Jiang, E.S. Werstiuk, M.P.Rathbone. \\ Department of Medicine, McMaster University, Hamilton, Ontario, Canada. \\ Email: pettifkm@mcmaster.ca
}

Guanosine, an non-adenine based purine nucleoside exerts trophic and neuroprotective effects in the central nervous system. Apoptosis (a morphological form of programmed cell death) has been inmplicated in the pathophysiology of Parkinson's disease (PD), and thought to contribute to cell death of the dopaminergic neurons in the substantia nigra and striatum. 1-Methyl-4-pheny-1,2,3,6- tetrahydropyridinium iodide $\left(\mathrm{MPP}^{+}\right)$is a dopaminergic neurotoxin that mimics the intracellular characteristics of PD, such as apoptosis. We have shown recently, that guanosine protects different types of cells from apoptosis induced by various stimuli $(1,2)$. We therefore evaluated the effect of guanosine on $\mathrm{MPP}^{+}$-induced apoptosis in human SH-SY5Y neuroblastoma cells. We added guanosine to neuroblastoma cells prior to, or at the same time, or $24 \mathrm{~h}$ after the adminsitration of $\mathrm{MPP}^{+}$and determined the extent of apoptosis by oligonucleosomal ELISA. We also measured caspase-3-activity by a spectorphotometric assay, and $\alpha$-synuclein protein expression by an ELISA.

Exposure of neuroblastoma cells to $\mathrm{MPP}^{+}(10 \mu \mathrm{M}-5 \mathrm{mM}$ for $24-72 \mathrm{~h})$ induced apoptosis in a concentration-, and time-dependent manner $\left(p<0.01 ; 500 \mu \mathrm{M} \mathrm{MPP}^{+}\right.$vs. vehicle at $\left.48 \mathrm{~h}\right)$. Administration of guanosine $(100 \mu \mathrm{M})$ prior to, at the same time, or after the addition of $\mathrm{MPP}^{+}$abolished $\mathrm{MPP}^{+}$-induced apoptosis $\left(p<0.05\right.$; vs. $\mathrm{MPP}^{+}$at 48h). Neither $\mathrm{MPP}^{+}$nor guanosine had any significant effect on $\alpha$-synuclein protein expression. Treatment of $\mathrm{SH}-$ SY5Y cells with $\mathrm{MPP}^{+}(500 \mu \mathrm{M})$ significantly increased caspase- 3 activity $(p<0.05$ at $72 \mathrm{~h}$ vs. control at 0 time), and this was abolished by pre-treatment, or co-treatment with guanosine. In contrast, administration of guanosine 24 , or $48 \mathrm{~h}$ after $\mathrm{MPP}^{+}$addition had no significant effect on the activity of this enzyme. Our data show that pretreatment, or co-treatment of SH-SY5Y neuroblastoma cells with guanosine prevents $\mathrm{MPP}^{+}$-induced apoptosis, and this is mediated by inhibiting caspase-3 activation. More importantly, guanosine can also reverse the proapoptotic effect of $\mathrm{MPP}^{+}$when it is added after DNA fragmentation is already in progress. These findings reveal a unique neuroprotective effect of guanosine, with a potential for effective pharmacological intervention in PD.

\section{References:}

1. DiIorio P, Ballerini P, Traversa U, Nicoletti F, D’Alimonte I, Kleywegt S, Werstiuk ES, Rathbone MP, Cacaigli F, Ciccarelli R. 2004. The anti-apoptotic effect of guanoisne is mediated by the activation of the PI3-kiase/AKT/ PKB pathway in cultured rat astrocytes. Glia, 46: 356-368.

2. Pettifer KM, Kleywegt S, Bau C, Ramsbottom JD, Vertes E, Ciccarelli R, Caciagli F, Werstiuk ES, Rathbone MP. 2004. Guanosine protects SH-SY5Y cells against $\beta$-amyloid-induced apoptosis. NeurReport, 15: 833-836. 


\title{
$\mathrm{H}_{2} \mathrm{O}_{2}$ ACTIVATES PURINERGIC SIGNALLING IN OSTEOBLASTS
}

$\underline{\text { Paola D}^{\prime}} \underline{\text { Andrea }}^{1}$, Milena Romanello ${ }^{1}$, Massimiliano Bicego ${ }^{1}$, Thomas H. Steinberg ${ }^{2}$ and Gianluca Tell ${ }^{3}$.

${ }^{1}$ Dipartimento di Biochimica, Biofisica e Chimica delle Macromolecole, Università degli Studi di Trieste, via Giorgieri 1, 34127 Trieste - Italy. ${ }^{2}$ Department of Internal Medicine, Washington University School of Medicine, 660 South Euclid Avenue, St. Louis, MO 63110, USA. ${ }^{3}$ Dipartimento di Scienze e Tecnologie Biomediche, Università degli Studi di Udine, P.le Kolbe 1, 33100 Udine - Italy.

dandrea@bbcm.units.it

Osteoblasts are largely exposed to toxic and non-toxic doses of ROS due, for instance, to bone resorption processes by osteoclasts. High doses of ROS play an important role in controlling osteoblastic differentiation, by acting at the transcriptional level through NF-kB and Egr-1 transcription factors. In this study we examined the consequences of $\mathrm{H}_{2} \mathrm{O}_{2}$ administration on osteoblast calcium signalling. In the highly differentiated HOBIT cell line, characterized by an elevated sensitivity to nanomolar extracellular ADP and UTP, addition of $1 \mathrm{mM}_{2} \mathrm{O}_{2}$ induced oscillatory increases the cytosolic calcium concentration that were followed by a steady and sustained calcium increase. Long lasting rhythmic calcium activity was induced by lower $\mathrm{H}_{2} \mathrm{O}_{2}$ doses $(10-50 \mu \mathrm{M})$. These calcium increases, due to both release from intracellular stores and influx from the extracellular milieu, were totally prevented by incubating the cells with suramin or apyrase. Similar results were obtained with the osteosarcoma cell line MG-63. At difference with HOBIT, these cells are totally unrensponsive to ADP and respond to micromolar concentrations of UTP. In SAOS and in ROS17/2.8 osteosarcoma cells, $1 \mathrm{mM} \mathrm{H}_{2} \mathrm{O}_{2}$ induced a slow, moderated and sustained increase of calcium which was dependent on influx from the extracellular medium and could not be prevented by suramin and apyrase. SAOS cells responded to micromolar ADP but were largely unresponsive to UTP, while ROS17/2.8 were totally insensitive to ATP, ADP and UTP in keeping with the evidence that these cells lack functional purinergic receptors. In transfected ROS 17/2.8, permanently over-expressing the $\mathrm{P} 2 \mathrm{Y} 2$ receptor (ROS/P2Y2), spontaneous calcium oscillations were observed in $60 \%$ of the population and nanomolar concentration of extracellular ATP or UTP activated calcium oscillations in silent cells. Spontaneous activity was inhibited by suramin and apyrase. $\mathrm{H}_{2} \mathrm{O}_{2}(10-50 \mu \mathrm{M})$ mimicked ATP administration, inducing oscillatory calcium activity that was blocked by suramin and apyrase. These results suggested that mild oxidation activated purinergic signalling in osteoblast expressing P2Y2 receptors. The possibility that oxidative stress could induce ATP release was evaluated by measuring the concentration of extracellular ATP with the highly sensitive luciferin-luciferase luminescence method. Dose- and time-course experiments, however, failed to reveal an increase of extracellular ATP following $\mathrm{H} 2 \mathrm{O} 2$ administration, indicating that the oxidative condition did not promote ATP release.

The possibility that mild oxidation of P2Y2 receptor could lead its sensitization was explored in ROS/P2Y2 cells. The sensitivity of these cells to nanomolar UTP concentrations was decreased by three orders of magnitude in the presence of DTT, which was effective also in inhibiting spontaneous calcium oscillations. On the other hand, the membrane-impermeant oxidant 5,5'-dithiobis (2-nitrobenzoic acid) (DTNB) induced calcium oscillations that were inhibited by incubating the cells with suramin or apyrase.

Taken together, these results indicate that mild oxidative stress could activate purinergic signalling in osteoblasts through the sensitization of $\mathrm{P} 2 \mathrm{Y} 2$ receptor.

\section{Hepatic Adenosine Regulates Blood flow and Sensory Reflex Control of Renal Function}

\author{
W. Wayne Lautt, Zhi Ming, and Dallas J. Legare \\ Department of Pharmacology \& Therapeutics, Faculty of Medicine, University of Manitoba, Winnipeg, Manitoba, \\ Canada \\ Email:wlautt@cc.umanitoba.ca
}

This presentation represents an overview of studies that demonstrate a unique role for adenosine in regulation of hepatic arterial blood flow and as a sensory neurotransmitter that monitors hepatic regional blood flow and results in reflex control of renal function including accounting for renal fluid retention in liver cirrhosis. 
The hepatic arterial buffer response (HABR) is the inverse response of the hepatic artery to changes in portal blood flow. This material has been reviewed (1). Adenosine is produced, in an oxygen-independent manner, at a constant rate and is secreted into the small extravascular space of Mall that surrounds the terminal branches of the portal vein and hepatic artery. The concentration of adenosine is regulated by washout into the portal blood. Reduction in portal blood flow leads to accumulation of adenosine and dilation of the hepatic artery. This mechanism is sufficiently powerful to regulate the hepatic artery from full constriction to full dilation.

We tested the hypothesis that the renal dysfunction seen in chronic liver disease was not related to a baroreceptor reflex detecting portal hypertension but rather to a reflex dependent upon detection of reduced intrahepatic blood flow. By this hypothesis, reduction in portal flow leads to accumulation of adenosine in the space of Mall where sensory nerves exist. The sensory nerves are activated to produce a sympathetic reflex inhibition of renal urine output thus tending to elevate circulating blood volume, increase cardiac output, and correct the reduction in portal flow. This mechanism is demonstrated in normal animals showing that intraportal adenosine inhibits urinary output and the effect can be blocked by denervation of the liver, the kidney, or application of an adenosine receptor antagonist directly to the liver (2). The same mechanism is shown to function in a chronic cirrhotic rat model (3). The adenosine receptor subtype is shown to be $A_{1}$ through the use of selective $A_{1}$ and $A_{2}$ antagonists (unpublished). Intraportal caffeine, previously shown by us to have very weak in vivo $\mathrm{A}_{2}$ antagonistic actions was also effective in blocking this hepatorenal reflex and suggests a potential therapeutic intervention through the use of slow release caffeine to treat renal dysfunction at both an early and late stage of chronic liver disease.

1. Lautt WW. The 1995 Ciba-Geigy Award Lecture. Intrinsic regulation of hepatic blood flow. Can J Physiol Pharmacol 1996; 74: 223-33.

2. Ming Z, Smyth DD, Lautt WW. 2002. Decreases in hepatic portal flow trigger a hepatorenal reflex to inhibit renal sodium and water excretion in rats: role of adenosine. Hepatology 2002; 35: 167-75.

3. Ming Z, Lautt WW. Reflex regulation by portal blood flow on renal function in healthy and cirrhotic rats: role of adenosine - a conceptual review. Proc West Pharmacol Soc 2004; 47: $33-4$.

\title{
Heteromeric assembly of $\mathbf{P 2} \mathrm{X}_{5}$ subunits at the plasma membrane
}

\author{
Chaumont S. ${ }^{[1]}$, Compan V..$^{[2]}$, Rassendren F. ${ }^{[2]}$ \\ [1] Division of Neurobiology MRC Laboratory of Molecular Biology, Cambridge, UK. \\ [2] Institut de Génomique Fonctionnelle, CNRS UMR5302, Montpellier, France. \\ Email:vincent.compan@igh.cnrs.fr
}

Purinergic P2X receptors are cationic channels activated by extra-cellular ATP. They are expressed throughout the body and mediate a multitude of functions, including muscle contraction or neuronal excitability. To date, seven subunits have been cloned, showing two transmembrane domains and a large extra-cellular loop. These receptors assemble as trimeric association of homo- or heteromeric subunits. Based on co-immunoprecipitation several potential heteromeric P2X receptors have been proposed (Torres et al., 1999), but these might represent heteromeric assemblies that are not expressed at the plasma membrane. I addition, only few heteromeric P2X receptors have been functionally characterized. We have previously characterized a stabilization motif (YXXXK) located in the proximal C-terminal region of all P2X subunits (Chaumont et al., 2004). Mutation of this motif induces a reduction of the surface expression of homomeric-mutated subunits due to their lack of stabilization at the plasma membrane. However, co-expression of a mutated subunit with wild type homo- or heteromeric subunits, rescues normal trafficking of mutated subunits.

In the present study, we used this property to systematically investigate all possible heteromeric P2X receptors expressed at the cell surface. Our results show that only a subset of heteromeric receptors are expressed at the plasma membrane; this includes 4 new combinations: $\mathrm{P} 2 \mathrm{X}_{2 / 5}, 4 / 3,4 / 5$ and $4 / 7$, suggesting a specificity of interaction between subunits.

Interestingly, in the case of the $\mathrm{P} 2 \mathrm{X}_{5}$ subunit, we observed that wild type homomeric receptors are mostly intracellularly expressed but that co-expression with either $\mathrm{P}_{2} \mathrm{X}_{2}$ or $\mathrm{P}_{2} \mathrm{X}_{4}$ subunits strongly enhance their surface expression. This was further confirmed by immuno-staining of tagged subunits and co-immunoprecipitation of the subunits after surface protein biotynilation. Using brain extracts $\mathrm{P} 2 \mathrm{X}_{2}$ and $\mathrm{P} 2 \mathrm{X}_{5}$ were co-immunoprecipitated, demonstrating that in vivo $\mathrm{P} 2 \mathrm{X}_{5}$ is likely to be associated to other $\mathrm{P} 2 \mathrm{X}$ subunits. 
Co-expression of $\mathrm{P} 2 \mathrm{X}_{5}$ and a trafficking-deficient $\mathrm{P} 2 \mathrm{X}_{2}$ subunit $\left(\mathrm{P}_{2} \mathrm{X}_{2}[\mathrm{~K} 366 \mathrm{~A}]\right)$ also resulted in a normal $\mathrm{P} 2 \mathrm{X}_{2}$ expression at the cell surface. Homomeric $\mathrm{P}_{2} \mathrm{X}_{2}[\mathrm{~K} 366 \mathrm{~A}]$ subunits are not functional, but have quasi-normal function when co-expressed with $\mathrm{P} 2 \mathrm{X}_{3}$, we therefore used this mutant to investigate the functional properties of heteromeric $\mathrm{P}_{2} \mathrm{X}_{2 / 5}$ receptors. However, no rescue of $\mathrm{P} 2 \mathrm{X}_{2}[\mathrm{~K} 366 \mathrm{~A}]$ currents by WT $\mathrm{P} 2 \mathrm{X}_{5}$ could be obtained, suggesting that $\mathrm{P} 2 \mathrm{X}_{2 / 5}$ receptors are composed by two $\mathrm{P} 2 \mathrm{X}_{2}$ subunits and a single $\mathrm{P} 2 \mathrm{X}_{5}$, and that two functional subunits are necessary for a receptor to be fully active.

Taken together, our results show that $\mathrm{P} 2 \mathrm{X}_{5}$ subunit is expressed at the cell surface only in hetreomeric receptors composed of either $\mathrm{P} 2 \mathrm{X}_{1},{ }_{2}$ or ${ }_{4}$ subunits. In addition, it is also suggested that only one $\mathrm{P}_{2} \mathrm{X}_{5}$ subunit is present in a heterotrimeric receptor. The precise function of $\mathrm{P} 2 \mathrm{X}_{5}$ subunit in heteromeric $\mathrm{P} 2 \mathrm{X}$ receptors is currently being investigated.

Torres GE, Egan TM, Voigt MM J. Biol. Chem. 274:6653-6659, 1999

Chaumont S, Jiang LH, Penna A, North RA, Rassendren F. J. Biol. Chem. 279:29628-29638, 2004

\title{
High glucose disturbs purinergic signalling system in rat retinal cell cultures
}

\author{
Tiago Pereira ${ }^{1}$, Denisa Gouveia ${ }^{1}$, António Ambrósio ${ }^{3}$ and Paulo Santos ${ }^{1,2}$ \\ ${ }^{1}$ Center for Neuroscience and Cell Biology, ${ }^{2}$ Dept. of Zoology and ${ }^{3}$ Fac. of Medicine, University of Coimbra, \\ Portugal \\ E-mail of presenting author: tosper@sapo.pt
}

Diabetic retinopathy (DR), the leading cause of blindness within the working-age group in western countries, has been traditionally regarded as a vascular disorder. However, growing evidence indicates that this is also a neurodegenerative and inflammatory process. The underlying mechanisms are largely unknown and several intercellular messengers, such as glutamate, VEGF, and insulin, have been suggested to play a role in this pathogenesis. We propose, nonetheless, another extracellular signalling molecule as a possible relevant player in DR, ATP. In the retina, ATP can be released by exocytosis, and can activate several P2 receptors, which have been shown to be expressed in this tissue. ATP has been associated with several cellular functions, such as microglia activation, modulation of glutamate release and cell death, which seem to be altered in DR. We also found, in previous work, that the incubation of primary retinal cell cultures with a high concentration of glucose (HG) is associated with a deregulation in ATP release and degradation. Therefore, ATP is a compelling candidate for a role in the pathogenesis of DR.

We have investigated the expression of several P2X receptors (1, 2, 3, 4 and 7), by immunoblotting and immunocytochemistry, and found that all these receptors are expressed in our cell cultures. P2X4 is particularly expressed in microglia and its total expression is decreased when the retinal cells are incubated in HG.

We have also studied the effect of $\mathrm{HG}$ incubation on intracellular $\mathrm{Ca}^{2+}\left(\left[\mathrm{Ca}^{2+}\right]_{\text {in }}\right)$ changes induced by $\mathrm{P} 2$ receptors' agonists and antagonists in these retinal cultures. We have found that there are clearly two distinct populations of retinal cells that are responsive to ATP and BzATP: neuronal and microglial. In both populations, ATP and BzATP induce an increase in $\left[\mathrm{Ca}^{2+}\right]_{\text {in }}$, but the latter only does so in the presence of extracellular $\mathrm{Ca}^{2+}$. Furthermore, these $\left[\mathrm{Ca}^{2+}\right]_{\text {in }}$ changes induced by BzATP are dependent on extracellular $\mathrm{Na}^{+}$in neurons, whereas in microglia, they are not. Therefore, BzATP activates P2X receptors in both retinal neurons and microglia, and this activation results in $\mathrm{Ca}^{2+}$ influx through P2X channels in microglia, and probably through VSCC in neurons. We also found that basal $\left[\mathrm{Ca}^{2+}\right]_{\text {in }}$ levels in neuronal cells incubated with $\mathrm{HG}$ are higher than in control conditions, but $\left[\mathrm{Ca}^{2+}\right]_{\text {in }}$ changes induced by BzATP are not significantly different between control and HG cells. This implies that $\mathrm{HG}$ incubated neurons are subjected to higher $\left[\mathrm{Ca}^{2+}\right]_{\text {in }}$ both in basal and stimulated (BzATP) conditions.

We have also found that HG neurons are more susceptible to inhibition by PPADS. This suggests that: either there is a modification of the P2X expression profile in HG neurons; a phenotypically selective neuronal death associated with $\mathrm{HG}$; or a post-translational modification that changes P2X functional characteristics. The immunoblotting results are in agreement with the first two hypotheses.

In conclusion, the incubation of primary retinal cell cultures with a high concentration of glucose induces changes that indicate that the purinergic signalling system is altered in these cells, particularly in neurons. The extent and implications of such changes are yet unclear, but if proven relevant in the context of DR, it would mean that extracellular ATP dynamics and receptors could be aimed as new targets for the therapy or prevention of DR. 


\title{
HUMAN P $P_{2 Y}$ RECEPTORS EXHIBIT INDIVIDUAL INTERACTION PATTERNS WITH $\beta$-ARRESTIN-1 AND -2
}

\author{
C. Hoffmann, N. Mohr, M.J. Lohse and C. Krasel* \\ Institut für Pharmakologie und Toxikologie, Universität Würzburg, Versbacher Strasse 9, 97078 Würzburg. \\ * Present address: University of Reading, School of Pharmacy, Whiteknights, P.O. Box 228 Reading, Berkshire, \\ RG6 6AJ, United Kingdom \\ C.Hoffmann@toxi.uni-wuerzburg.de
}

Interaction of G protein-coupled receptors (GPCRs) with arrestins is an important step in receptor desensitization. GPCRs have been subdivided into class A and B receptors according to their interaction pattern with $\beta$ arrestin-1, and -2. By means of confocal microscopy, we have investigated receptor internalization and interaction of the human $\mathrm{P}_{2 \mathrm{Y}}$ receptors $1,2,4,6,11$, and 12 with different arrestins.

Human P2Y receptors were C-terminally fused with YFP and transiently expressed in HEK-293 cells. Upon agonist stimulation receptor internalization was observed for $\mathrm{P}_{2 \mathrm{Y} 1}-\mathrm{YFP}, \mathrm{P}_{2 \mathrm{Y} 2}-\mathrm{YFP}$, and $\mathrm{P}_{2 \mathrm{Y} 4}-\mathrm{YFP}$ receptor constructs, whereas no internalization was detectable for $\mathrm{P}_{2 \mathrm{Y} 6}-\mathrm{YFP}, \mathrm{P}_{2 \mathrm{Y} 11}$-YFP, and $\mathrm{P}_{2 \mathrm{Y} 12}$-YFP receptor constructs. Next we measured $\beta$-arrestin translocation upon $P_{2 Y}$ receptor stimulation. HEK-293 cells transfected only with cDNA coding for $\beta$-arrestin-1-GFP, or $\beta$-arrestin-2-YFP showed no arrestin translocation upon stimulation with ADP, ATP, UDP, or UTP. However, upon co-transfection of each individual wild-type $P_{2 Y}$ receptor with $\beta$ arrestin-1-GFP or $\beta$-arrestin-2-YFP and stimulation with the corresponding agonist, a differential translocation pattern for individual $\mathrm{P}_{2 \mathrm{Y}}$-receptors was found. The $\mathrm{P}_{2 \mathrm{Y} 1}$-receptor stimulated with ADP strongly translocated $\beta$ arrestin-2-YFP, while only a slight translocation was observed for $\beta$-arrestin-1-GFP, classifying this receptor as class $\mathrm{A}$ receptor. The $\mathrm{P}_{2 \mathrm{Y} 4}$-recptor showed a strong translocation that was comparable for $\beta$-arrestin-1-GFP and $\beta$ arrestin-2-YFP when stimulated with UTP, classifying this receptor as class B receptor. No arrestin translocation was observed for the $\mathrm{P}_{2 \mathrm{Y} 11^{-}}$and $\mathrm{P}_{2 \mathrm{Y} 12^{2}}$-receptor, when stimulated with ATP and ADP respectively. The $\mathrm{P}_{2 \mathrm{Y}^{-}}$ receptor showed slight translocation for $\beta$-arrestin-2-YFP when stimulated with UDP, but only when GRK-2 was transfected as well. The translocation was too weak to be quantified as a loss of cytoplasmic fluorescence, but nevertheless clearly visible at the plasma membrane. The most interesting observation was made in the case of the $\mathrm{P}_{2 \mathrm{Y} 2}$-receptor. The arrestin translocation pattern was dependent of the ligand used for stimulation. UTP translocated $\beta$-arrestin-1-GFP or $\beta$-arrestin-2-YFP equally strong, whereas ATP translocated $\beta$-arrestin-1-GFP to a much lower extent than $\beta$-arrestin-2-YFP. Therefore the $\mathrm{P}_{2 \mathrm{Y} 2}$ receptor can be classified as a class $\mathrm{A}$ receptor when stimulated with ATP, or as a class B receptor when stimulated with UTP.

\section{Hydrolytic Ectoenzymatic Activity on Diadenosine Polyphosphates in Synaptic and Plasmatic Membranes Isolated from Rat Brain and Cerebellum}

\author{
$\underline{\text { P. Rotllán }}^{1}$, A.C. Asensio ${ }^{1}$, C.R. Rodríguez-Ferrer ${ }^{1}$, A Castañeyra $^{2}$, S. Oaknin ${ }^{1}$ \\ Departments of ${ }^{1}$ Biochemistry and Molecular Biology and ${ }^{2}$ Anatomy. University of La Laguna, Tenerife, Canary \\ Islands, Spain. \\ (protllan@ull.es)
}

Several diadenosine polyphosphates, mainly $\mathrm{Ap}_{4} \mathrm{~A}$ and $\mathrm{Ap}_{5} \mathrm{~A}$, have been identified as releasable components of storage granules in specialized neurosecretory cells and function as extracellular signals in nervous system. Accordingly, they bind to plasma membrane purinergic receptors (specific dinucleotide, P1 and P2), displaying physiological actions as neurotransmitters/ neuromodulators. The inactivation of $\mathrm{Ap}_{\mathrm{n}} \mathrm{A}$ by hydrolytic ectoenzymes at the cell surface provides a mechanism to regulate the receptor-mediated actions of $\mathrm{Ap}_{\mathrm{n}} \mathrm{A}$. To date, a systematic investigation of the ectoenzymatic system operating in brain and cerebellum for inactivation of exocytotically released $\mathrm{Ap}_{\mathrm{n}} \mathrm{A}$ has been lacking.

We describe here the main biochemical characteristics of ectoenzymatic activity responsible for hydrolysis of extracellular $\mathrm{Ap}_{\mathrm{n}} \mathrm{A}$ in rat brain and cerebellum. Highly purified plasma and plasma synaptic membranes and synaptosomes obtained by sucrose gradient ultracentrifugation from several brain regions (hypothalamus, hippocampus, temporal cortex, frontal cortex and striatum) and cerebellum were used as ectoenzyme source, while the 
diethenoadenosine polyphosphates $\varepsilon-\left(\mathrm{Ap}_{\mathrm{n}} \mathrm{A}\right)$ were used as fluorogenic substrates. All $\varepsilon-\left(A p_{n} \mathrm{~A}\right), \mathrm{n}=2-5$, were broken down to $\varepsilon$-adenosine as end-product by all membrane preparations. Partially purified ectoenzymatic activity by sequential ion-exchange and gel filtration chromatography cleaved all substrates into mononucleotide moieties: $\varepsilon$-AMP and the corresponding $\varepsilon$-adenosine $(n-1) 5$ phosphate. Ectoenzymatic hydrolysis reached maximal activity at pH 9.0 (range 6.5-9.0) and was activated by $\mathrm{Ca}^{2+}$ and $\mathrm{Mg}^{2+}$ ions with maximal effects around 2.0 $\mathrm{mM}$ cation. EDTA drastically reduced activity and $\mathrm{Zn}^{2+}$ was required for enzyme reactivation. Hydrolysis of substrates by membranes and synaptosomes followed hyperbolic kinetics with $\mathrm{K}_{\mathrm{m}}$ values in the 3-10 $\mu \mathrm{M}$ range and similar $\mathrm{V}_{\max }$ values. $\mathrm{Ap}_{\mathrm{n}} \mathrm{A}(\mathrm{n}=2-5)$ and heparin behaved as competitive inhibitors. AMP, ATP, $\alpha, \beta$-methylene ADP, ADP $\beta S, A T P \gamma S, \beta, \gamma$-methylene ATP and suramin were also inhibitors. Synaptic membranes from cerebellum, hypothalamus and hippocampus presented the highest activity and no significant changes were observed between young and aged animals. Plasma membranes showed a more homogeneous distribution but a general increase in activity was detected in aged animals, especially in hypothalamus and cerebellum, about $150 \%$.

This ectoenzymatic activity shared characteristics typical of members of the E-NPP (ecto-nucleotide pyrophosphatase phosphodiesterase) family, which contains at least three isoenzymes: NPP1, NPP2 and NPP3, with very similar catalytic properties. These results, together with previous data by others on cloning and expression of NPP ectoenzymes, suggest that NPP2 could play a significant role in extracellular inactivation of $\mathrm{Ap}_{\mathrm{n}} \mathrm{A}$ in brain and cerebellum. Increased activity in plasma membranes of aged animals could play a deleterious role in aged brain by limiting neuroprotective actions reported for extracellular $\mathrm{Ap}_{4} \mathrm{~A}$.

Acknowledgments: Work funded by grants TR5-02 and TR2003/06 (Gobierno de Canarias with participation of Hospiten SA, NovoNordisk Ibérica SA, Aventis Behring SA) and C03/06 (Red CIEN, FIS).

\title{
Hypoxia-induced pulmonary damage in transgenic sickle cell mice is reduced after administration of adenosine $\mathbf{A}_{2 \mathrm{~A}}$ receptor agonists
}

\author{
Kori L. Wallace, Robert A. Figler, Melissa A. Marshall, David K. Glover, Joel Linden \\ Cardiovascular Research Center; University of Virginia; Box 801394; Charlottesville, VA 22908; \\ klw5g@virginia.edu
}

Sickle cell disease (SCD) is an inherited disorder arising from at least one mutant sickle hemoglobin (HbS) allele. $\mathrm{HbS}$ is poorly soluble when deoxygenated and in hypoxic conditions polymerizes reversibly to form a gelatinous network of fibrous polymers. Although polymerization of deoxygenated $\mathrm{HbS}$ is the primary event in the molecular pathogenesis of SCD, recent findings have revealed that chronic inflammation plays an important role in the etiology of vaso-occlusive crisis (VOC) and the long-term pathologic manifestations of SCD.1 SCD patients and transgenic mice (NY1DD) expressing human $\mathrm{B}^{\mathrm{S}}$-globin exhibit increased plasma levels of pro-inflammatory cytokines, increased numbers of circulating endothelial cells, leukocytosis, and elevated expression of cell-surface activation markers on leukocytes and endothelial cells. ${ }^{2}$

Adenosine acts through the adensoine $A_{2 A}$ receptor $\left(A_{2 A} A R\right)$ found on bone marrow derived cells to inhibit inflammation. We used hypoxia/reoxygenation to induce VOC in NY1DD mice and assessed plasma cytokine levels, lung inflammation, and pulmonary function following the prophylactic administration of the $\mathrm{A}_{2 \mathrm{~A}} \mathrm{AR}$ agonist ATL146e $(10 \mathrm{ng} / \mathrm{kg} / \mathrm{min})$ or the acute administration of ATL313 $(5 \mathrm{ug} / \mathrm{kg}$ bolus, $1 \mathrm{ug} / \mathrm{kg}$ booster $)$ given immediately at the start of reoxygenation. Compared to vehicle treated NY1DD mice, $\mathrm{A}_{2 \mathrm{~A}} \mathrm{AR}$ agonist treated mice displayed: 1) decreased plasma pro-inflammatory cytokine levels; 2) Improved pulmonary function as measured by whole-body plethysmography. Pulmonary minute volume was markedly increased in NY1DD mice during and after hypoxia, and this was improved to near normal levels by treatment with $\mathrm{A}_{2 \mathrm{~A}} \mathrm{R}$ agonists; 3) Decreased pulmonary vascular congestion as evidenced by histology; and 4) Reduced pulmonary hypoxia based on 2-nitroimidazole deposistion in hypoxic cells in lung tissue. Pulmonary hypoxia also was non-invasivley assessed in living mice with whole body $\gamma$-immaging following injection of animals with BRU59-21, a 99mTc-labeled 2nitroimidazole. The agent accumulated in the lungs of NY1DD mice after exposure to hypoxia and this was reduced by $\mathrm{A}_{2 \mathrm{~A}} \mathrm{R}$ agonsits treatment. These data suggest that $\mathrm{A}_{2 \mathrm{~A}} \mathrm{AR}$ agonists may be clinically useful for the treatment of VOC in SCD. NIH grant \#PO1 HL073361. 
1. Platt, O. S. Sickle cell anemia as an inflammatory disease. J. Clin. Invest 2000, 106 (3), 337-338.

2. Belcher, J. D.; Bryant, C. J.; Nguyen, J.; Bowlin, P. R.; Kielbik, M. C.; Bischof, J. C.; Hebbel, R. P.; Vercellotti, G. M. Transgenic sickle mice have vascular inflammation. Blood 2003, 101 (10), 3953-3959.

\title{
IB-MECA, an $\mathbf{A}_{3}$ Adenosine Receptor Agonist Prevents Bone Resorption In Rats with Adjuvant Induced Arthritis
}

\author{
Lea Rath-Wolfson², Sara Bar- Yehuda ${ }^{1,3}$, Lea Madi', Pnina Fishman ${ }^{1,3}$ \\ ${ }^{1}$ Can-Fite BioPharma Ltd., Kiryat-Matalon, Petah-Tikva, 49170, Israel. ${ }^{2}$ Department of Pathology, and \\ ${ }^{3}$ Felsenstein Medical Research Center, ${ }^{2,3}$ Rabin Medical Center, Sackler Faculty of Medicine Tel-Aviv University, \\ Petach-Tikva 49100, Israel.
}

Objectives: The anti-inflammatory effect of adenosine is partially mediated via the $\mathrm{A}_{3}$ adenosine receptor $\left(\mathrm{A}_{3-}\right.$ $\mathrm{AR}$ ), a Gi protein associated cell surface receptor. The highly selective $\mathrm{A}_{3} \mathrm{AR}$ agonist, IB-MECA was earlier shown to prevent the clinical and pathological manifestations of arthritis in experimental animal models of collagen and adjuvant induced arthritis (AIA). In this study we tested the effect of IB-MECA on the prevention of bone resorption in AIA rats and looked at the molecular mechanism of action.

Methods: rats with AIA were treated orally twice daily with IB-MECA starting upon onset of disease and clinical score was evaluated every other day. At study termination the foot, knee and hip region of both vehicle and IBMECA treated animals were subjected to histomorphometric analysis. Western blot analysis was carried out on paw protein extracts.

Results: IB-MECA ameliorated the clinical manifestations of the disease and reduced pannus and fibrosis formation, attenuated cartilage and bone destruction, decreased the number of osteoclasts and reduced bone formation. In cell protein extracts derived from paw of AIA rats, $\mathrm{A}_{3} \mathrm{AR}$ was highly expressed in comparison to naïve animals. In paw extracts derived from IB-MECA treated AIA rats, down-regulation of the $\mathrm{A}_{3} \mathrm{AR}$ protein expression level was noted. PI3K, PKB/Akt, IKK, NF- $\mathrm{B}$, TNF- $\alpha$ and RANKL were down-regulated whereas caspase 3 was up-regulated.

Conclusions: IB-MECA via $\mathrm{A}_{3} \mathrm{AR}$ activation, induces modulation of proteins which control survival and apoptosis resulting in the amelioration of the inflammatory process and the preservation of bone mass in AIA rats.

\section{Identification and characterization of a functional $\mathbf{P 2} \mathrm{X}_{7}$ splice variant with an alternative transmembrane domain}

\author{
Annette Nicke $^{1}$, Marianela Masin ${ }^{2}$, Benjamin Marquez-Klaka ${ }^{1}$, Jürgen Rettinger $^{3}$, Florentina Soto ${ }^{2}$ \\ ${ }^{T}$ MPI for Brain Research, Frankfurt am Main, Germany \\ ${ }^{2}$ MPI for Experimental Medicine, Göttingen, Germany \\ ${ }^{3}$ MPI of Biophysics, Frankfurt am Main, Germany \\ anicke@gwdg.de
}

The $\mathrm{P}_{2} \mathrm{X}_{7}$ receptor is found on different immune cells where it has been shown to be involved in interleukin release, cytoskeletal rearrangements, L-selectin shedding and activation of phospho-lipase D and p38MAP kinase. Its presence in neuronal cells is still under debate. It is distinguished from other P2X receptors by its very long intracellular C-terminus, a low ATP sensitivity, and its ability to induce "cell permeabilization", meaning that upon prolonged ATP application the opening of a permeation pathway for large cations is induced. Although the C-terminus has been shown to be involved in this process, the mechanism of this "pore dilation" is still poorly understood. Also, it is not clarified to which extend this is an intrinsic property of the $\mathrm{P} 2 \mathrm{X}_{7}$ protein or requires the interaction with other proteins.

Here we describe the biochemical and functional properties of a $\mathrm{P} 2 \mathrm{X}_{7}$ splice variant $\left(\mathrm{P} 2 \mathrm{X}_{7(\mathrm{i})}\right)$ which was cloned from a rat lung cDNA library and compare it with the originally cloned $\mathrm{P} 2 \mathrm{X}_{7}$ receptor. In the splice variant, the N-terminus and $80 \%$ of the proposed first transmembrane domain are replaced by an alternative sequence derived from a yet unidentified exon in the $\mathrm{P}_{2} \mathrm{X}_{7}$ gene, which is highly lipophilic.

Upon heterologous expression in Xenopus oocytes, both the $\mathrm{P} 2 \mathrm{X}_{7(\mathrm{i})}$ and the $\mathrm{P} 2 \mathrm{X}_{7 \text { (a) }}$ subunit are expressed as complex glycosylated proteins of identical size. Analysis of the digitonin-solubilized receptor complexes by BN- 
PAGE analysis and subsequent western blotting revealed identical mobilities of both receptors and $\mathrm{P} 2 \mathrm{X}_{7}$ complexes solubilized from various native tissues. Partial dissociation by SDS revealed two additional bands of higher mobility demonstrating that both $\mathrm{P} 2 \mathrm{X}_{7}$ isoforms assembled as trimers.

Two-electrode voltage-clamp analysis showed that the $\mathrm{P} 2 \mathrm{X}_{7(\mathrm{i})}$ receptor was functional and had a similar ATP sensitivity as the $\mathrm{P}^{2} \mathrm{X}_{7 \text { (a) }}$ receptor. Current recordings and analysis of reversal potentials in N-methyl-D-glucamine (NMDG) solution revealed a gradual permeability increase for NMDG upon prolonged activation by ATP. However, the permeability shift was markedly less than the one observed for the $\mathrm{P}_{2} \mathrm{X}_{7 \text { (a) }}$ receptor. Unlike the $\mathrm{P}_{2} \mathrm{X}_{7 \text { (a) }}$ receptor, the splice variant exhibited already a high permeability ratio $\left(\mathrm{P}_{\mathrm{NMDG}} / \mathrm{P}_{\mathrm{Na}}\right)$ at the begining of the ATP application suggesting that the channel is constitutively dilated upon opening.

Duplex RT-PCR with primer pairs selective for the $\mathrm{P} 2 \mathrm{X}_{7(\mathrm{a})}$ and $\mathrm{P} 2 \mathrm{X}_{7(\mathrm{i})}$ isoforms confirmed the presence of the $\mathrm{P} 2 \mathrm{X}_{7(\mathrm{i})}$ transcript in lung and showed a dominant expression in the spleen.

Taken together, we describe a novel $\mathrm{P} 2 \mathrm{X}_{7}$ isoform with an alternative TM1 domain and a high propensity to form NMDG permeable pores indicating that residues critically involved in pore dilation are located within the TM1 domain. This splice variant will help to elucidate the process of pore dilation and might provide an explanation for the distinct properties described for $\mathrm{P}_{2} \mathrm{X}_{7}$ receptors in different tissues.

\section{IDENTIFICATION OF A NOVEL NATURALLY OCCURING NUCLEO-

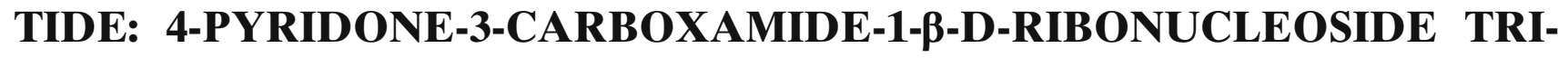 PHOSPHATE IN HUMAN ERYTHROCYTES AND ITS RELATION WITH ATP CONCENTRATION}

Ewa M. Slominska ${ }^{1,2)}$, Elizabeth A. Carrey ${ }^{2,3)}$, Henryk Foks ${ }^{4)}$, Czeslawa Orlewska ${ }^{4)}$, Ewa Wieczerzak ${ }^{5)}$, Pawel Sowinski ${ }^{6)}$, Magdi H. Yacoub ${ }^{7)}$, Anthony M. Marinaki ${ }^{2)}$, H. Anne Simmonds ${ }^{2)}$, Ryszard T. Smolenski ${ }^{1,7)}$

${ }^{1)}$ Department of Biochemistry, Medical University of Gdansk, Poland,

${ }^{2)}$ Purine Research Unit, Guy's Hospital, London, U.K.

${ }^{3)}$ University College London Institute of Child Health, London, U.K.,

${ }^{4)}$ Department of Organic Chemistry, Medical University of Gdansk, Poland,

${ }^{5)}$ Department of Organic Chemistry, University of Gdansk, Poland,

${ }^{6)}$ Laboratory of NMR Spectroscopy, Chemical Faculty, Gdansk University of Technology, Poland,

${ }^{7)}$ Heart Science Centre, Imperial College London, Harefield, U.K.

e-mail: eslom@amg.gda.pl.

We report the identification of a hitherto unknown nucleotide that is present in micromolar concentrations in the erythrocytes of healthy humans and massively accumulates in the erythrocytes of patients with renal failure. The unknown nucleotide was isolated from the triphosphate fraction of erythrocyte extracts obtained from patients with renal failure using anion-exchange high performance liquid chromatography (HPLC). Next, the isolated nucleotide was degraded using alkaline phosphatase to obtain nucleoside and further processed by acid hydrolysis to obtain the free base. The purified nucleotide, nucleoside and base were then characterized using HPLC with UV and tandem mass detection, nuclear magnetic resonance (NMR), and infrared (IR) spectroscopy. This analysis revealed the molecular weight of the nucleotide $(\mathrm{m}=510)$, nucleoside $(\mathrm{m}=270)$ and base $(\mathrm{m}=138)$, the structure of the heterocyclic ring of the base as 4-pyridone-3-carboxamide (4PY) and hence the nucleoside as 4pyridone-3-carboxamide-1- $\beta$-D-ribonucleoside (4PYR). This base and the nucleoside were then chemically synthesized and their identity with natural compounds confirmed. The unknown nucleotide has therefore been identified as 4-pyridone-3-carboxamide-1- $\beta$-D-ribonucleoside triphosphate (4PYTP). An erythrocyte concentreation established in the normal human erythrocytes was established at 13.0 $\pm 4.7 \mu \mathrm{M} / \mathrm{L}$ erythrocytes (range: 6-18) and its concentration in the advanced chronic renal failure was $162 \pm 189 \mu \mathrm{M} / \mathrm{L}$ erythrocytes (range: 45-670). Subsequently, we demonstrated formation of the unknown nucleotide established here as 4PYTP in the intact human erythrocytes during incubation with 4PYR accompanied by progressive depletion of cellular ATP and accumulation of the IMP. Formation of 4PYTP and the effects on ATP concentration was abolished by inhibition of adenosine kinase with 5'-iodotubercidin. No 4PYTP formation was noted with 4PY as the substrate. The base constituent structure of this new nucleotide is related to oxidized nicotinamide nucleotides and this metabolite could be part 
of the pathway for excretion of nicotinamide catabolites. 4PYTP may be important for or interfere with any ATP related mechanism inside or outside the cell and its structural similarity with UTP, an agosnist for P2 receptors is worth noting. However, physiological role of 4PYTP and the consequences of accumulation in renal failure remain to be established.

\title{
Identification of an inter-subunit cross-link between substituted cysteine residues located in the putative ATP binding site
}

\author{
Benjamin Marquez-Klaka ${ }^{1}$, Yogesh Bhargava ${ }^{2}$, Jürgen Rettinger ${ }^{2}$, Annette Nicke $^{1}$ \\ ${ }^{T}$ MPI for Brain Research, Frankfurt am Main, Germany \\ ${ }^{2}$ MPI for Biophysics, Frankfurt am Main, Germany \\ bmk@mpih-frankfurt.mpg.de
}

P2X receptors are ATP-gated ion channels and are assembled as homo- or heterotrimers of homo-logous subunits (1). Each subunit contains two transmembrane domains linked by a large extra-cellular loop containing the ATP binding site. Although single residues that directly contribute to ATP binding have been identified, the location of the binding site remains unclear. Mutagenesis data based on an ATP binding site model deduced from secondary structure predictions, suggest an intra-subunit location (2). However, this model does not take into account three conserved lysine residues $\left(\mathrm{K} 68, \mathrm{~K} 70\right.$, and $\mathrm{K} 309$ in $\left.\mathrm{P} 2 \mathrm{X}_{1}\right)$ which are critical for high-affinity ATP binding $(3,4)$. The location of these residues on opposite ends of the extracellular loop opens the possibility of an inter-subunit ATP-binding site, similar as in the nicotinic acetylcholine receptor.

Here we used a disulfide cross-linking approach to identify pairs of residues that are in close proximity within the ATP binding site to determine whether this is formed within one or between two neighbouring subunits. Hexahistidyl-tagged $\mathrm{P} 2 \mathrm{X}_{1}$ mutants in which single residues supposed to contribute to ATP binding were substituted by cysteine residues were expressed in Xenopus laevis oocytes. Functional expression of these binding site mutants was confirmed by two-electrode-voltage clamp analysis of the respective mutations in a non-desensitzing $\mathrm{P} 2 \mathrm{X}_{2 / 1}$ chimera. This chimera contains the $\mathrm{N}$-terminus and the first transmembrane domain of the $\mathrm{P} 2 \mathrm{X}_{2}$ subunit and the high-affine ligand-binding domain of the fast desensitizing $\mathrm{P} 2 \mathrm{X}_{1}$ receptor and has been shown to provide a valid model for the ATP-binding site of the $\mathrm{P}_{2} \mathrm{X}_{1}$ receptor (5). Upon metabolic labelling, $\mathrm{P} 2 \mathrm{X}$ receptor complexes were purified under non-denaturing conditions. Blue native gel electrophoresis (BN-PAGE) analysis of these mutants showed exclusively trimeric complexes thus excluding artificial disulfide formation due to aggregation of receptor complexes. Non-reducing SDS-PAGE analysis of pairwise expressed mutants revealed a spontaneous and specific dimer formation between the K68C and F291C mutants, suggesting an inter-subunit disulfide crosslink. In support of this finding, the use of short cysteine reactive cross-linkers pointed to a close approximation of residues R292C and K70C across the interface of two neighbouring subunits. An almost complete spontaneous cross-link into trimers was achieved with the K68C/F291C double mutant. Deglycosylation analysis and BNPAGE analysis of the selectively labelled plasma membrane protein confirmed that this double mutant was correctly folded and inserted into the plasma membrane.

In summary, we provide first evidence that loops from neighbouring $\mathrm{P} 2 \mathrm{X}$ subunits contribute to the formation of the ATP-binding site in $\mathrm{P} 2 \mathrm{X}$ receptors.

(1) Nicke, A.; Bäumert, H. G.; Rettinger, J.; Eichele, A.; Lambrecht, G.; Mutschler, E.; Schmalzing, G. EMBO J. 1998, 17, 3016-28.

(2) Yan, Z.; Liang, Z.; Tomic, M.; Obsil, T.; Stojilkovic, S. S. Mol. Pharmacol. 2005, 67, 1078-88.

(3) Jiang, L. H.; Rassendren, F.; Surprenant, A.; North, R. A. J. Biol. Chem. 2000, 275, 34190-6.

(4) Ennion, S.; Hagan, S.; Evans, R. J.; J. Biol. Chem. 2000, 275, 29361-7.

(5) Rettinger, J.; Schmalzing, G. J. Biol. Chem. 2004, 279, 6426-33. 


\title{
Identification of Molecular Anti-inflammatory Mechanisms of Adenosine: Cullin-1 Deneddylation during Hypoxic Preconditioning
}

\author{
Joseph Khoury $^{1,2}$, Juan C. Ibla ${ }^{1,2}$ and Sean P.Colgan ${ }^{2}$ \\ From the Departments of Anesthesia Perioperative and Pain Medicine, Children's Hospital ${ }^{1}$ and Center for \\ Experimental Therapeutics and Reperfusion Injury, Brigham and Women's Hospital ${ }^{2}$ and Harvard Medical School, \\ Boston, MA 02115, USA. \\ jkhoury@Zeus.BWH.Harvard.edu
}

Hypoxic preconditioning (HPC) is a unique phenomenon of endogenous protection that renders cells tolerant to more severe challenges of hypoxia. The molecular mechanism by which HPC confers such protection is currently an area of intense investigation. Here, we sought to define the specific anti-inflammatory properties of HPC. Using protocols of HPC, mice and cultured epithelial cells were profiled for known pathways common to pro and anti-inflammatory responses. Guided by cDNA microarray analysis of lung tissue from preconditioned mice, we identified significant repression of NFKB regulated genes elicited by HPC. HPC evoked a $95 \pm 3 \%$ repression of the $\mathrm{NF} \kappa \mathrm{B}-$ dependent gene IL-6, as was the case for other NFкB regulated genes. We confirmed the functional downregulation of $\mathrm{NF}_{\kappa} \mathrm{B}$ activation using a NFKB luciferase reporter assay. Transfected cells were exposed to an HPC protocol $\left(2 \% \mathrm{O}_{2}\right.$ for 45 minutes followed by re-oxygenation $21 \% \mathrm{O}_{2}$ for 20 minutes for 3 cycles) followed by 24 hours of anoxia $\left(0 \% \mathrm{O}_{2}\right)$, and under such conditions, HPC cells showed a significant down-regulation of NFkB activity compared to control $(p=0.01)$. Subsequent experiments revealed that the anti-inflammatory properties of HPC could be conferred in soluble supernatants from HPC cells $(p=0.05)$. An HPLC profiling of soluble supernatants identified an active nucleotide fraction which carried significant anti-inflammatory bioactivity. This active fraction was identified as adenosine. Led by studies demonstrating the inhibition of NFkB activation occurs by

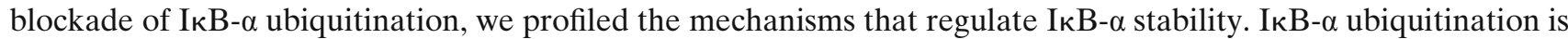
mediated by the E3 ubiquitin ligase complex, which in turn is regulated by the modification of the Cullin-1 subunit by the ubiquititin-like protein NEDD-81. The state of Cullin-1 neddylation has been implicated in NFKB suppression by bacteria2. Using the general adenosine receptor agonist NECA, we probed the state of neddylation of Cullin-1. We found a dose dependent dennedylation of Cullin-1 in response to adenosine receptor stimulation both in vitro and in vivo. Taken together these results demonstrate that HPC results in the extra-cellular accumulation of adenosine responsible for the deneddylation of Cullin-1 and the subsequent suppression of $\mathrm{NF}_{\kappa} \mathrm{B}$ mediated pathways. These results define a novel molecular regulatory pathway by which adenosine provides potent anti-inflammatory conditions.

\section{References:}

1. Maniatis T. A ubiquitin ligase complex essential for the NF-kappaB, Wnt/Wingless, and Hedgehog signaling pathways. Genes Dev 1999;13:505-10.

2. Collier-Hyams LS, Sloane V, Batten BC, Neish AS. Cutting edge: bacterial modulation of epithelial signaling via changes in neddylation of cullin-1. J Immunol 2005;175:4194-8.

\section{Identification of the Ecto Nucleoside Triphosphate Diphosphohydrolases Regulating Bacterial Clearance in Human Airways}

\author{
Maryse Picher, Lauranell H. Burch ${ }^{2}$, Mercedes N. Munkonda ${ }^{3}$, Brian Button ${ }^{1}$, Julie Pelletier ${ }^{3}$ and \\ Jean Sevigny ${ }^{3}$ \\ ${ }^{1}$ Cystic Fibrosis Center, University of North Carolina, NC, USA \\ ${ }^{2}$ Laboratory of Respiratory Biology, NIEHS, Durham, NC, USA \\ ${ }^{3}$ Centre de Recherche en Rhumatologie et Immunologie, CHUL, Ste-Foy, QC, CA \\ pichm@med.unc.edu
}

In human airways, extracellular nucleotides are among the most potent mediators of mucociliary clearance (M$\mathrm{CC}$ ). Their interaction with $\mathrm{P}_{2} \mathrm{Y}_{2}$ receptors on epithelial surfaces stimulates mucus secretion, cilia beating activity and maintains airway hydration. These mechanisms are impaired in chronic lung diseases including cystic fibrosis 
(CF). Clinical trials revealed that aerosolized nucleotides only provide transient improvement of MCC due to their rapid metabolism. In the present study, we demonstrate that P2 receptor-mediated MCC is regulated by ecto nucleoside triphosphate diphosphohydrolases (E-NTPDases; ATP $\rightarrow$ ADP $\rightarrow$ AMP). All experiments were performed on polarized primary cultures of human nasal or bronchial epithelial cells maintained at air-liquid interface. Cyclic compressive stress (CCS) mimicking normal breathing $\left(20 \mathrm{cmH}_{2} \mathrm{O} ; 0.5 \mathrm{~Hz}\right)$ restored $\mathrm{MCC}$ in $\mathrm{CF}$ cultures by a mechanism involving ATP release, P2 receptor activation and a $75 \%$ reduction in ectoATPase activities. The E-NTPDase inhibitor, azide $(20 \mathrm{mM})$, prevented CCS-mediated reduction in ATP metabolism. The biochemical properties of airway E-NTPDases were investigated on static cultures. Azide reduced the metabolism of $1 \mathrm{mM}$ UTP and UDP by $45 \%$ and $55 \%$ on the mucosal surface compared to $<10 \%$ on the serosal surface. Nucleotide specificity generated in the presence of azide was consistent with those of NTPDase 1 and 3 (ATP = UTP $<$ GTP $<$ CTP), but not azide-insensitive NTPDase 2 or 8 . While the azide-sensitive activity ratio for UTP/ UDP (6.2) obtained with $1 \mathrm{mM}$ nucleotides was consistent with NTPDase 3, assays conducted with $0.01 \mathrm{mM}$ substrates generated a ratio (1.3) supporting NTPDase 1 expression. The co-expression of NTPDase 1 and NTPDase 3 in human airway epithelia was confirmed by RT-PCR and RNAse protection assays. These two azidesensitive E-NTPDase activities were distinguished using ebselen (2-phenyl-1,2-benziso selenazol-3(2H)-one) and Pseudomonas aeruginosa lipopolysaccharide (LPS). Biochemical assays performed on protein extracts from transfected COS-7 cells indicated that acute exposures $(15 \mathrm{~min})$ to $30 \mu \mathrm{M}$ ebselen significantly inhibited NTPDase 3 (40\%) while LPS inhibited NTPDase $1(50 \%)$. Based on these compounds, both activities were detected on the mucosal surface of nasal and bronchial cultures, while low $(<15 \%)$ NTPDase 1 activity was also detected on serosal surfaces. On the mucosal surface, whereas NTPDase 3 was unaffected by cell type, NTPDase 1 activity was 2-fold higher on bronchial than nasal epithelia, results were confirmed at the mRNA level. Collectively, these experiments demonstrate the functional co-expression of NTPDase 1 and NTPDase 3 on airway mucosa. Furthermore, the fact that ebselen mimicked the effects of CCS on E-NTPDase activities suggest that NTPDase 3 plays a major role in the regulation of nucleotide-mediated MCC.

Work supported by the Cystic Fibrosis Foundation PICHER 05G0

\title{
Identifying Neuronal Release Modulation of Noradrenaline and its Cotransmitter ATP using Isolated Perfused Kidneys of Alpha2A-Adrenoceptor Knockout Mice
}

\author{
Oliver Vonend, Sina Habbel, Johannes Stegbauer, Lars Christian Rump \\ Experimental Nephrology, Ruhr-University Bochum, Germany \\ Email: Oliver.Vonend@ruhr-uni-bochum.de
}

Sympathetic overactivity and increased neurotransmitter spillover are closely associated with progressive renal failure. The present study investigates whether the alpha2A-adrenoceptor-subtype represents the presynaptic autoinhibitory adrenoceptor that regulates noradrenaline (NA) and ATP release in mouse kidney. Moreover we evaluated the renal phenotype of alpha2A adrenoceptor $\mathrm{KO}$ mice. This is of particular interest, since sympahtetic neurotransmitters are able to mediate maladaptive changes within the kidney (Vonend 2004).

Wildtype and alpha2A-adrenoceptor-KO mice (C57/B16) were anaesthetized, renal arteries were cannulated and kidneys isolated perfused with Krebs-Henseleit solution at a constant rate $(7.25 \mathrm{ml} / \mathrm{min} \mathrm{g})$. Electrodes were placed around the renal arteries to stimulate the renal nerves. The perfusion solution was collected and endogenously released NA was measured by HPLC. The perfusion pressor was monitored continuously. To evaluate renal phenotype blood pressure, puls, serum-creatinine, serum-BUN, urine-sodium and albumine excretion were measured.

Renal nerve stimulation (RNS) induced a frequency $(1,2,5,7.5,10,15 \mathrm{~Hz})$ dependant release of NA (1156+/ $-223,2509+/-332,6728+/-584,11542+/-1167,19094+/-1241,23846+/-2388 \mathrm{pg}$ NA / g kidney $+/-\mathrm{SE})$. In KO mice RNS at $5 \mathrm{~Hz}$ increased NA release 2.7 fold. In wildtype animals the non selective alpha-adrenoceptor blocker phentolamine increased RNS induced NA-release in a concentration dependent manner up to $350 \%$ of control. No facilitation by phentolamine was observed in KO mice. Phentolamine decreased the RNS induced pressor responses in WT mice at higher frequencies. NA does not seem to be responsible for pressor responses at lower frequencies. Pressor reponses to $1 \mathrm{~Hz}$ and $2 \mathrm{~Hz}$ cannot be blocked by prazosin. Inhibition of presynaptic alpha-receptors increased non-adrenergic (purinergic) pressor responses up to $300 \%$ in WT but not in KO mice. Apart from a higher pulsrate in $\mathrm{KO}$ mice no significant differences were detectable between both strains. 
We established an in vitro mouse model to analyse real sympathetic neurotransmitter release. Experiments with knockout mice demonstrated that the alpha2A-adrenoceptor subtype is responsible for modulating NA and ATP release in mouse kidney. ATP seems to be the predominant transmitter at physiological stimulation frequencies. Even though the alpha2A-adrenoceptor $\mathrm{KO}$ mice is characterised by sympathetic overactivity no renal phenotype was apparent. Compensatory mechanisms might play a role here. Another explanation could be the genetic background. C57/B16 mice seem to be not susceptible for the development of glomerulosclerosis (Ma 2003).

Vonend, O., Turner, C. M., Chan, C. M., Loesch, A., et al., Kidney Int 2004, 66, 157-166. Ma, L. J. and Fogo, A. B., Kidney Int 2003, 64, 350-355.

\title{
Imbalance Between Airway Adenosine Production and Elimination in Cystic Fibrosis
}

\author{
Maryse Picher ${ }^{1}$, Andrew Hirsh ${ }^{1}$ and Lauranell H. Burch ${ }^{2}$ \\ ${ }^{T}$ Cystic Fibrosis Center, University of North Carolina, NC, USA \\ ${ }^{2}$ Laboratory of Respiratory Biology, National Institute of Environmental Health Sciences, Durham, NC, USA \\ pichm@med.unc.edu
}

Adenosine is a multi-faceted signaling molecule mediating key aspects of airway defenses, including bacterial clearance. However, chronically-elevated lung adenosine initiates inflammatory responses in animal models. And bronchoalveolar lavage from patients with chronic lung diseases exhibits high adenosine concentrations. The source of this excess adenosine has not been identified. We recently showed that airway adenosine is generated by sequential dephosphorylation of locally-released ATP on epithelial surfaces. The cell surface enzyme (ectonucleotidase) responsible for the conversion of AMP to adenosine was identified as ecto 5'-nucleotidase (ecto 5'NT). In the present study, we identified the proteins regulating the elimination of airway adenosine. Experiments conducted on polarized primary cultures of human nasal epithelial cells showed that $10 \mu \mathrm{M}$ adenosine is eliminated from mucosal surfaces by deamination $(60 \%)$ and cellular uptake $(40 \%)$. The cell surface conversion of adenosine to inosine was completely inhibited by $10 \mu \mathrm{M}$ erythro9-(2-hydroxy-3-nonyl)adenine (EHNA), selective inhibitor of adenosine deaminase 1 (ADA1). This enzyme displayed $K_{m}$ and $V_{\max }$ values of $24 \mu \mathrm{M}$ and 0.14 nmole. $\min ^{-1} \cdot \mathrm{cm}^{-2}$. Adenosine transport was $\mathrm{Na}^{+}$-dependent and inhibited by $2 \mathrm{mM}$ phloridzin, inhibitor of the concentrative nucleoside transporters (CNTs). Apparent $\mathrm{K}_{\mathrm{m}}$ and $\mathrm{V}_{\max }$ values for adenosine uptake were $17 \mu \mathrm{M}$ and 7.2 nmoles.min ${ }^{-1} \cdot \mathrm{cm}^{-2}$. The permeability coefficient ratio of adenosine/mannitol (18/1) indicated a minor contribution of paracellular absorption. Competitive studies showed that adenosine uptake is not affected by hypoxanthine but broadly inhibited by purines and pyrimidines $($ inosine $=$ uridine $>$ guanosine $=$ cytidine $>$ thymidine). Messenger RNA for ecto 5'-NT, ADA1 and CNT3 was detected throughout airway epithelia, while the expression of CNT2 was restricted to nasal epithelia. Cultured nasal epithelial cells from cystic fibrosis patients exhibited a 5-fold increased rate in adenosine accumulation from exogenous $10 \mu \mathrm{M}$ ATP. This finding was correlated with a 4-fold increase in adenosine production by ecto 5'-NT and a 3-fold decrease in adenosine elimination by ADA1. This study suggests that the excess airway adenosine detected in chronic lung diseases may arise from an imbalance between the rates of adenosine production and elimination on airway epithelial surfaces. The identification of the proteins regulating airway adenosine, as well as pharmacological tools that regulate their activities, may lead to a better understanding of the roles of chronically-elevated adenosine in lung diseases.

This study was supported by the Cystic Fibrosis Foundation (Picher 05G0)

\section{Improved Syntheses of 2-(Aryl)alkoxyadenosine - Adenosine $\mathbf{A}_{2 \mathrm{~A}}$ Agonists for the Treatment of Chronic Wounds}

\author{
Allan R. Moorman, ${ }^{1}$ Eckard Wolfe, ${ }^{2}$ David Dime, ${ }^{2}$ Michael P. Scannell, \\ ${ }^{3}$ Thiagarajan Balasubramanian, ${ }^{3}$ Russell J. Outcalt, ${ }^{3}$ and Pier Giovanni Baraldi ${ }^{4}$
}


${ }^{1}$ King Pharmaceuticals Research and Development, Inc., 4000 CentreGreen Way, Suite 300, Cary, 27513 North Carolina; USA, 2Toronto Research Chemical, 2 Brisbane Road, North York, Ontario, M3J 2J8, Canada, 3Scynexis, Inc., 3501C Tricenter Boulevard, Durham, North Carolina 27713, 4 Dipartimento di Scienza Farmaceutiche, Università di Ferrara, 44100 Ferrara, Italy.

E-mail: allan.moorman@kingpharm.com

The development of chronic wounds, such as neuropathic foot ulcerations, is a major complication of diabetes. Evidence suggests that a selective adenosine $\mathrm{A}_{2 \mathrm{~A}}$ agonist, when applied topically, can significantly enhance the rate of healing of these wounds. The mechanism of this enhanced capacity is thought to involve both an antiinflammatory component as well the down-regulation of the anti-angiogenic matrix protein thrombospondin 1. Moreover, this enhanced capacity toward wound closure occurs in both normal animals, as well as those with an impaired healing capacity, such as occurs in diabetes.

One class of adenosine $\mathrm{A}_{2 \mathrm{~A}}$ receptor agonists that has drawn attention, the 2-aralkoxy- and 2-alkoxyadenosines, was described by Olsson and coworkers. Although these compounds are both potent and selective adenosine $\mathrm{A}_{2 \mathrm{~A}}$ agonists with demonstrated in vivo efficacy in models of wound healing, their development has been hampered by the lack of a robust and scaleable synthesis. Previous reports indicate that protection of the 2'- and 3'-hydroxyls with an ethoxymethylidene or isopropylidene moiety is important to prevent formation of a $2 \rightarrow 2$ ' polymeric product and that the lability of the glycosidic bond to the acidic conditions required to remove these protecting groups contributes to the low yields observed.

Here we describe two approaches to the synthesis of this class of molecules, in particular 2-[2-(4-chloropheny1)ethoxy]adenosine (MRE-0094, 1), a compound currently in clinical development for the treatment of diabetic foot ulcers. In the first approach, 2-amino-6-chloro-purine riboside triacetate (2) is hydrolytically diazotized and then coupled with 2-(4-chlorophenyl)ethyl bromide. Concurrent displacement of the 6-chloro moiety and ammonolysis of the acetyl-protecting groups affords the desired MRE-0094. The second approach utilizes the $\operatorname{tris}(T$ ERT-butyldimethylsilyl)-protected 2-chloroadenosine (3) to condense with 2-(4-chlorophenyl)ethanol, followed fluoride ion deprotection to afford the target molecule. These approaches permit the preparation of kilogram quantities of the clinical candidate, as well as numerous analogues, in improved overall yields.

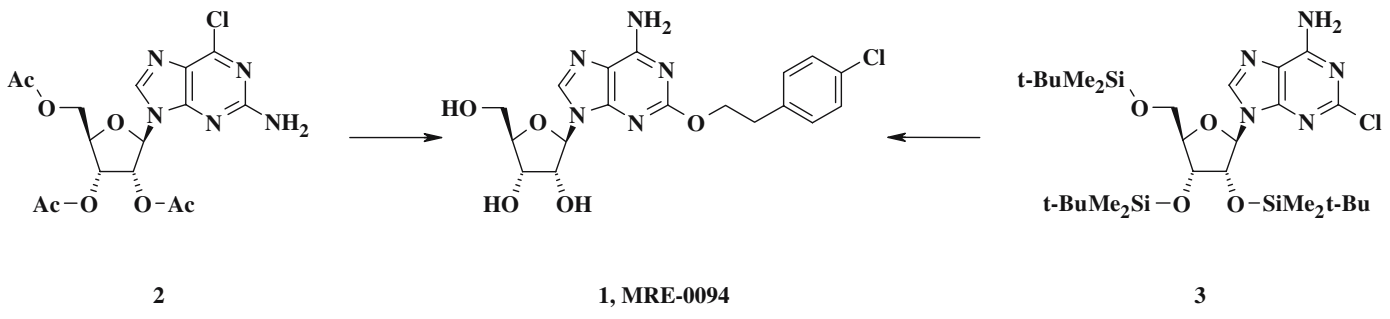

\title{
In rat hepatocytes, different adenosine receptor subtypes use different secondary messengers to increase the rate of ureagenesis.
}

\author{
Guinzberg $\mathrm{PR}^{1}$, Uribe $\mathrm{CS}^{2}$, Díaz-Cruz $\mathrm{A}^{3}$ and Piña $\mathrm{E}^{1 *}$. \\ ${ }^{1}$ Department of Biochemistry, School of Medicine, ${ }^{2}$ Institute for Cellular Physiology and ${ }^{3}$ Department of Nutrition, \\ School of Veterinary Medicine. UNAM.* \\ epgarza@servidor.unam.mx
}

In rat hepatocytes, the role of cAMP and $\mathrm{Ca}^{2+}$ as secondary messengers in the ureagenic response to stimulation of specific adenosine receptor (AR) subtypes was explored. Analyzed receptor subtypes were: $A_{1}, A_{2 A}, A_{2 B}$ and $A_{3}$. Each receptor subtype was stimulated with a specific agonist while blocking all other receptor subtypes with a battery of specific antagonists. For the $A_{1}$ and $A_{3} A R$ subtypes, the secondary messenger was the cytoplasmic $\mathrm{Ca}^{2+}$ concentration $\left(\left[\mathrm{Ca}^{2+}\right]_{\text {cyt }}\right)$. Accordingly, the $\mathrm{A}_{1}$ or $\mathrm{A}_{3}$-mediated increase in $\left[\mathrm{Ca}^{2+}\right]_{\text {cyt }}$ and in ureagenic activity were both inhibited by chelating $\mathrm{Ca}^{2+}$ with either EGTA or BAPTA-AM. Also, $\mathrm{Gd}^{3+}$ blocked both the increase in $\left[\mathrm{Ca}^{2+}\right]_{\mathrm{cyt}}$ and ureagenesis, suggesting that a $\mathrm{Ca}^{2+}$ channel may be involved in the response to both $\mathrm{A}_{1}$ and $\mathrm{A}_{3}$. $\mathrm{A}$ partial effect was observed with the sarcoplasmic reticulum $\mathrm{Ca}^{2+}$-ATPase inhibitor thapsigargin. The concentra- 
tion of cyclic AMP ([cAMP]) increased in response to stimulation of either the $A_{2 A}$ or the $A_{2 B} A R$ subtypes, while it decreased slightly in response to stimulation of either $A_{1}$ or $A_{3}$. The stimulation of either the $A_{2 A}$ or $A_{2 B}$ AR subtypes resulted in an increase in [cAMP] and an ureagenic response which were not sensitive to EGTA, BAPTA-AM, $\mathrm{Gd}^{3+}$ or to thapsigargin. In addition, the adenylyl cyclase inhibitor MDL12,330A blocked the ureagenic response to $A_{2 A}$ and $A_{2 B}$, but not the response to either $A_{1}$ or $A_{3}$. Our results indicate that in the ureagenic liver response to adenosine, the secondary messenger for both, the $A_{1}$ and $A_{3}$ adenosine receptor subtypes is $\left[\mathrm{Ca}^{2+}\right]_{\text {cyt }}$, while the message from the $\mathrm{A}_{2 \mathrm{~A}}$ and $\mathrm{A}_{2 \mathrm{~B}} \mathrm{AR}$ subtypes is relayed by [cAMP]. Grants IN211502-2, IN210401 from DGAPA, UNAM.

\section{In Vitro Evaluation of Adenosine 5'-monophosphate as a Tumor Metabolic Imaging Agent}

Steve Y. Cho, MD; Edward H. Abraham ${ }^{2}$, MD; Josh Polster ${ }^{1}$, MD; James M. Engles ${ }^{1}$, BS; Richard L. $\mathrm{Wahl}^{1} \mathrm{MD}$

${ }^{1}$ Division of Nuclear Medicine, Russel H. Morgan Department of Radiology and Radiological Sciences, Johns Hopkins Medical Institutions, Baltimore, MD; ${ }^{2}$ Department of Radiation Oncology, Tufts-New England Medical Center

email: eabraham@tufts-nemc.org

Key words:[2- $\left.{ }^{3} \mathrm{H}\right]$ Adenosine $5^{\prime}$-monophosphate, in vitro, cancer cells, nucleoside transporter

Mini-abstract: In vitro evaluation of the mechanism of intracellular uptake of adenosine generated from adenosine monophosphate in various human tumor cell lines, and its potential as a metabolic tumor PET radiotracer Abstract: Adenosine appears to play an important role in tumor growth and metastasis. Synthesis of $\left[{ }^{11} \mathrm{C}\right]$ Adenosine $5^{\prime}$-monophosphate $\left(\left[{ }^{11} \mathrm{C}\right] \mathrm{AMP}\right)$ serves as a precursor for $\left[{ }^{11} \mathrm{C}\right]$ Adenosine and has been recently reported as a potential tumor imaging radiotracer. Methods: A variety of human tumor cell lines (SKOV-3, SCC-15, U251, U87, Raji, and Daudi) were incubated with $3.7 \mathrm{KBq}(0.1 \mu \mathrm{Ci})$ of $\left[2-{ }^{3} \mathrm{H}\right]$ Adenosine $5^{\prime}$-monophosphate $\left(\left[{ }^{3} \mathrm{H}\right] \mathrm{AMP}\right)$, $\left[5,6-{ }^{3} \mathrm{H}\right]$ 2-Fluoro-2-deoxy-D-glucose $\left(\left[{ }^{3} \mathrm{H}\right] \mathrm{FDG}\right)$, or $\left[2-{ }^{3} \mathrm{H}\right]$ Adenosine $\left(\left[{ }^{3} \mathrm{H}\right]\right.$ Adenosine $)$ in low physiologic glucose serum free media. Selected cells were exposed to caffeine, dipyridamole, adenosine $5^{\prime}$-( $\alpha, \beta$-methylene)diphosphate (APCP), or cold adenosine prior to exposure to the radiotracer. R-Phycoerytherin conjugated mouse antihuman monoclonal antibody to human CD73 was used for immunophenotyping. Results: There was significant intracellular uptake of $\left[{ }^{3} \mathrm{H}\right] \mathrm{AMP}$ on the order of 10 to 100 times the uptake of $\left[{ }^{3} \mathrm{H}\right] \mathrm{FDG}$ in corresponding cells, depending on the particular tumor cell line. Pre-exposure of SKOV-3 cells to caffeine, a competitive inhibitor of adenosine receptors, did not affect cellular uptake of $\left[{ }^{3} \mathrm{H}\right]$ AMP. However, pre-exposure of SKOV-3 cells to dipyridamole, an equilibrative nucleoside transporter inhibitor (ENT), APCP, a CD73 (ecto-5' ${ }^{\prime}$-nucleotidase) inhibitor, or cold adenosine significantly inhibited cellular uptake of $\left[{ }^{3} \mathrm{H}\right] \mathrm{AMP}$. SKOV-3 uptake of $\left[{ }^{3} \mathrm{H}\right]$ Adenosine was inhibited by dipyridamole but not APCP. U251 uptake of $\left[{ }^{3} \mathrm{H}\right]$ AMP was significantly inhibited by dipyridamole and APCP. U87 uptake of $\left[{ }^{3} \mathrm{H}\right]$ AMP was only partially inhibited by dipyridamole and APCP. However, Raji and Daudi cells had significantly lower uptake of $\left[{ }^{3} \mathrm{H}\right]$ AMP compared to $\left[{ }^{3} \mathrm{H}\right] \mathrm{FDG}$, but had significantly increased uptake of $\left[{ }^{3} \mathrm{H}\right]$ Adenosine which was inhibited by dipyridamole. Raji and Daudi cells were negative, but the SKOV-3 cells were positive for CD73 cell surface expression. Conclusions: Cancer cell lines evaluated in vitro had significantly elevated uptake of radiolabeled AMP generated adenosine, on the order of 10-100 fold, in comparison to radiolabeled FDG. The mechanism of intracellular uptake is predominantly dependent on ENTs after conversion of AMP to adenosine by CD73 in SKOV-3, SCC-15, and U251 cells. Raji and Daudi cells have low uptake of radiolabeled AMP due to a lack of CD73 expression. This in vitro evaluation using $\left[{ }^{3} \mathrm{H}\right] \mathrm{AMP}$ with tumor cell lines supports the potential for the use of $\left[{ }^{11} \mathrm{C}\right]$ AMP to target the nucleoside transport pathway in PET tumor imaging.

\section{In Vivo Imaging of $A_{1}$ Adenosine Receptors in the Human Brain with PET}

$\underline{\text { Andreas Bauer }}^{1}$, David Elmenhorst ${ }^{1}$, Philipp Meyer ${ }^{1}$, Andreas Matusch ${ }^{1}$, Markus Dehnhardt ${ }^{1}$, Dirk Bier $^{2}$, Ray A. Olsson ${ }^{2}$, Marcus H. Holschbach ${ }^{2}$ 
The $A_{1}$ adenosine receptor $\left(A_{1} A R\right)$ is widely distributed in the body and particularly prevalent in the central nervous system. Activation of neuronal $\mathrm{A}_{1} \mathrm{ARs}$ results in decreased adenylate cyclase activity through activation of pertussis toxin-sensitive $\mathrm{G}_{\mathrm{i}}$ proteins and $\mathrm{K}^{+}$channels, respectively, as well as $\mathrm{K}_{\mathrm{ATP}}$ channels. $\mathrm{A}_{1}$ ARs are involved in the regulaion of sleep and arousal, cognition and memory, neuronal damage and degeneration. Therapeutic implications for epilepsy and multiple sclerosis as well as neurodegenerative diseases such as Parkinson's and Alzheimer's disease are currently under investigation. To investigate alterations of the $\mathrm{A}_{1} \mathrm{AR}$ in normal and diseased states of the human brain we have recently developed a positron emission tomography (PET) method to image and quantify the $\mathrm{A}_{1} \mathrm{AR}$ in the living human brain (Holschbach et al. 2002, Bauer et al. 2003, Meyer et al. 2005).

Methods: Quantitative dynamic $\left[{ }^{18} \mathrm{~F}\right] \mathrm{CPFPX}$ receptor PET was performed in healthy subjects and patients suffering from brain tumor (glioma) or temporal lobe epilepsy. Regional cerebral ligand distribution was evaluated by volume of interest and kinetic analyses, respectively, after co-registration of PET and individual magnetic resonance data sets (Bauer et al. 2003, Meyer et al. 2004).

Results: $\left[{ }^{18} \mathrm{~F}\right] \mathrm{CPFPX}$ PET displayed a high ligand accumulation throughout the brain with a region-specific binding pattern. Ligand distribution was in accordance with in vitro autoradiographic studies on the regional binding pattern of $\mathrm{A}_{1}$ ARs. Highest values of total distribution volume were found in temporal and occipital cortex, striatum and thalamus, lowest values were detected in pons and cerebellum. The data point to significant species differences of the distribution of cerebral $\mathrm{A}_{1} \mathrm{ARs}$ in rodents and man. In first clinical applications we observed a belt of increased $A_{1} A R$ binding in the immediate tumor vicinity in patients suffering from high grade glioma (Bauer et al. 2005). In vitro inspection of tumor specimens pointed to a primarily microglial and astrocytic source of increased $\mathrm{A}_{1} \mathrm{AR}$ expression. In patients with unilateral temporal lobe epilepsy we found a reduction of $\mathrm{A}_{1} \mathrm{AR}$ binding in the hippocampus and mesial parts of the temporal lobe which are in accordance with a reduced density of $\mathrm{A}_{1} \mathrm{ARs}$ as described in in vitro studies.

Conclusion: $\left[{ }^{18} \mathrm{~F}\right] \mathrm{CPFPX}$ PET is a promising tool for the investigation of the in vivo distribution of the cerebral $\mathrm{A}_{1} \mathrm{AR}$. It offers a unique opportunity to investigate the in vivo behaviour of the cerebral $\mathrm{A}_{1} \mathrm{AR}$ and permits the investigation of human pathologies in the course of disease.

Bauer, A.; Holschbach, M.H.; Meyer, P.T.; Boy, C; Herzog, H.; Olsson, R.A.; Coenen, H.H.; Zilles, K. Neuroimage. 2003, 19, 1760.

Bauer, A.; Langen, K.J.; Holschbach, M.H.; Olsson, R.A.; Cremer, M.; Weber, S.; Coenen, H.H.; Zilles, K. J. Nucl. Med. 2005, 46, 450.

Holschbach, M.H.; Olsson, R.A.; Bier, D.; Wutz, W.; Sihver, W.; Schuller, M.; Palm, B.; Coenen, H.H. J. Med. Chem. 2002, 45, 5150.

Meyer, P.T.; Bier, D.; Holschbach, M.H.; Boy, C.; Olsson, R.A.; Coenen, H.H.; Zilles, K.; Bauer, A. J. Cereb. Blood Flow Metab. 2004, 24, 323.

Meyer, P.T.; Elmenhorst, D.; Bier, D.; Holschbach, M.H.; Matusch, A.; Coenen, H.H.; Zilles, K.; Bauer, A. Neuroimage. 2005, 24, 1192.

\title{
Increased risk of acute myocardial infarction and elevated levels of C-reactive protein in carriers of the Thr87 variant of the ATP receptor P2Y 11
}

\author{
Stefan Amisten $^{1}$, Olle Melander ${ }^{2}$, Anna-Karin Wihlborg${ }^{1}$, Göran Berglund ${ }^{2}$ and David Erlinge ${ }^{1}$ \\ ${ }^{T}$ Department of Cardiology, ${ }^{2}$ Diabetes and Endocrinology, Lund University Hospital, Lund, Sweden \\ stefan.amisten@med.lu.se
}

Extracellular ATP acting on the membrane receptor $\mathrm{P}^{2} \mathrm{Y}_{11}$ regulates inflammatory cells and has inotropic effects on cardiac myocytes. We hypothesized that polymorphisms in the receptor could influence the risk of acute myocardial infarction (AMI).

In a case-control study with 1244 AMI cases and 2488 paired controls, the common dimorphism Ala-87-Thr of the $\mathrm{P}_{2} \mathrm{Y}_{11}$ gene $(19.2 \%$ Thr-87 allele distribution in controls) was associated with acute myocardial infarction (odds ratio $1.21,95 \%$ C.I. $1.02-1.44, \mathrm{P}=0.03)$. Even stronger associations were found in the AMI subgroups containing 
family history AMI cases $(1.32,1.02-1.69, \mathrm{P}=0.03)$, as well as early onset AMI $(1.43,1.13-1.82, \mathrm{P}=0.003)$. The strongest association was seen in a third subgroup consisting of individuals with early onset AMI in combination with a family history of AMI (1.56 95\% C.I. $1.11-2.18, \mathrm{P}=0.01)$, supporting a genetic mechanism.

In the case-control study, as well as in the early onset AMI subgroup, homozygous carriers of the Thr-87 variant of P2RY $\mathrm{R}_{11}$ showed a stronger association with AMI than heterozygous carriers: Thr-87/Thr-87 (odds ratio 1.93, 1.02 - 3.66) vs. Thr-87/Ala-87 $(1.18,0.98-1.41, \mathrm{P}=0.08)$; early onset AMI subgroup: Thr-87/Thr-87 (2.48, $1.02-$ $6.05, \mathrm{P}=0.046)$ vs. Thr-87/Ala-87 $(1.39,1.08-1.78, \mathrm{P}=0.009)$.

To determine a possible mechanism for the increased prevalence of AMI, we examined a cardiovascular group population $(\mathrm{n}=6101)$, with data of several cardiovascular risk factors. Interestingly, the inflammatory marker Creactive protein was significantly elevated in carriers of the Thr-87 variant of $\mathrm{P} 2 \mathrm{RY} \mathrm{Y}_{11}\left(\mathrm{CRP}_{\mathrm{Thr}-87} 0.16(0.07-0.30)\right.$ $\left.\mathrm{mg} / \mathrm{ml}, \mathrm{CRP}_{\text {Ala- } 87} 0.13(0.07-0.28) \mathrm{mg} / \mathrm{ml}, \mathrm{P}=0.001\right)$. No difference was seen for blood pressure, lipids, body-mass index or diabetes mellitus.

In conclusion, the common Ala-87-Thr polymorphism of the $\mathrm{P}_{2} \mathrm{Y}_{11}$ receptor is strongly associated with AMI probably by stimulating inflammation. Our findings make the $\mathrm{P} 2 \mathrm{Y}_{11}$ receptor a promising novel drug target in the prevention of myocardial infarction and cardiovascular inflammatory disease.

\section{Inflammatory role of adenosine in human lung cells}

Dewan Zeng, Hongyan Zhong and Luiz Belardinelli

Department of Pharmacological Sciences, CV Therapeutics, Inc., Palo Alto, California 94304.

dewan.zeng@cvt.com

It has been suggested that adenosine might have both pro- and anti-inflammatory actions depending on the pathophysiology of the diseases. In the lungs of asthmatics, inhaled adenosine or adenosine monophosphate (AMP) increases the release of histamine, tryptase and leukotrienes, suggesting a pro-inflammatory role of adenosine presumably due to the activation of adenosine receptors present in mast cells. The objective of our study was to determine the potential role of adenosine and its receptors in the human lung cells such as bronchial epithelial cells, airway smooth muscle cells and lung fibroblasts. Among the four subtypes of adenosine receptors $\left(A_{1}, A_{2 A}, A_{2 B}\right.$ and $\left.A_{3}\right)$, the $A_{2 B}$ receptor is expressed at the highest level in all three types of lung cells. In airway smooth muscle cells, activation of $A_{2 B}$ receptors leads to the release of IL-6 and MCP-1. Similarly, in lung fibroblasts, activation of $A_{2 B}$ receptors increases the release of IL-6, which in turn promotes the differentiations of fibroblasts into myofibroblasts. In bronchial epithelial cells, activation of $A_{2 B}$ receptors increases the release of IL19 , which leads to the release of TNF- $\alpha$ from a monocytic cell line. Altogether, these results suggest that activation of $\mathrm{A}_{2 \mathrm{~B}}$ receptors in the human lung cells increases the release of several pro-inflammatory cytokines and chemokines, which could lead to deleterious inflammatory responses in the lung.

\section{Influence of $A_{1}$ and $P 2$ receptor-activation on angiotensin II mediated facilitation of noradrenaline release in the rat mesenteric vessels}

Talaia C., Hodan J., Morato M., Gonçalves J., Queiroz G.

Laboratory of Pharmacology, CEQOFFUP, Faculty of Pharmacy, University of Porto, Rua Aníbal Cunha, 164, 4050-047 Porto, Portugal

Interactions between prejunctional inhibitory $\alpha_{2}$-autoreceptors and facilitatory angiotensin $\mathrm{AT}_{1}$ receptors have been described in several sympathetic innervated tissues (1). However, the influence of other inhibitory receptors, namely the purinoceptors, on the angiotensin II (ANG II) receptor-mediated facilitation of noradrenaline (NA) release was never investigated in tissues where ATP is a sympathetic cotransmitter. Therefore, the influence of P2 and adenosine $A_{1}$ receptors on modulation of NA release mediated by ANG II was studied in the rat mesenteric artery and vein, tissues where ATP is an important cotransmitter (2) and where adenosine and ATP are neuromodulators (3).

Mesenteric arteries and veins from male Wistar rats $(250-350 \mathrm{~g})$ were isolated, incubated with $1 \mu \mathrm{M}\left[{ }^{3} \mathrm{H}\right]-\mathrm{NA}$ and superfused with a physiological solution containing $0.4 \mu \mathrm{M}$ desipramine. Tissues were stimulated with trains of 100 pulses at $2 \mathrm{~Hz}$ or 20 pulses at $50 \mathrm{~Hz}(50 \mathrm{~mA}, 1 \mathrm{~ms})$. The effects of drugs on evoked NA release (estimated as 
tritium overflow) were calculated as \% of change from the corresponding controls, expressed as mean \pm S.E.M, and tested for significance by one-way ANOVA followed by Dunnett's test.

Stimulation with trains of 20 pulses at $50 \mathrm{~Hz}$, elicited an overflow of tritium that was not changed by $\alpha_{2}$-adrenoceptor-antagonist yohimbine $(1 \mu \mathrm{M})$. However, tritium overflow elicited by trains of 100 pulses delivered at $2 \mathrm{~Hz}$ was enhanced by yohimbine $(1 \mu \mathrm{M})$ in the mesenteric artery and vein up to $489 \pm 31 \%(\mathrm{n}=4, P<0.05)$. The adenosine $\mathrm{A}_{1}$ receptor antagonist 8-cyclopentyl-1,3-dipropylxanthine (DPCPX, $0.1 \mu \mathrm{M}$ ) and the P2 receptor antagonist reactive blue $2(\mathrm{RB} 2,30 \mu \mathrm{M})$ did not change tritium overflow evoked by stimulation at $2 \mathrm{~Hz}$. When $\alpha_{2^{-}}$ adrenoceptors were blocked with yohimbine $(1 \mu \mathrm{M})$, DPCPX $(0.1 \mu \mathrm{M})$, but not RB2 $(30 \mu \mathrm{M})$, enhanced tritium overflow elicited at $2 \mathrm{~Hz}$ (artery: $15 \pm 5 \%$; $=6, P<0.05$; vein: $28 \pm 4 \% ; \mathrm{n}=5, P<0.05$ ). When tissues were stimulated under marked ongoing $\alpha_{2}$-autoinhibition (100 pulses at $\left.2 \mathrm{~Hz}\right)$, ANG II (0.01-100 nM) enhanced tritium overflow up to $89 \pm 7 \%(\mathrm{n}=6, P<0.05)$ and $69 \pm 7 \%(\mathrm{n}=6, P<0.05)$ in the artery and vein, respectively. This facilitatory effect was attenuated in the presence of yohimbine $(1 \mu \mathrm{M})$ to $32 \pm 3 \%(\mathrm{n}=5, P<0.05)$ and $24 \pm 6 \%$ (n $=6, P<0.05)$ in the artery and vein, respectively, and abolished when tissues were stimulated with trains of 20 pulses at $50 \mathrm{~Hz}$. In the presence of yohimbine, the enhancement of tritium overflow caused by ANG II (10 nM) was not changed by DPCPX $(0.1 \mu \mathrm{M})$ in the artery but was attenuated in the vein from $24 \pm 6 \%(\mathrm{n}=4)$ to $14 \pm 3 \%$ $(\mathrm{n}=8, P<0.05)$.

In conclusion, in the mesenteric artery and vein facilitation of NA release by ANG II is amplified by tonic activation of inhibitory receptors: the $\alpha_{2}$-autoreceptors in mesenteric artery, the $\alpha_{2}$-autoreceptors and adenosine $\mathrm{A}_{1}$ receptores in the vein.

(1) Cox et al. (2000) Br J Pharmacol 129, 1095 -1102.

(2) Bobalova et al. (2001) Clin Exp Pharm Physiol 28, 397-401.

(3) Shinozuka et al. (2001) Jpn J Pharmacol 85, 41-46.

Supported by FCT Projects (POCTI/SAU-FCB/60714/2004 and CEQOFFUP - I\&D 226/94)

\title{
Inhibition of Ischemic Contracture: A 'Supraphysiological' Response to Exogenous But Not Endogenous Adenosine
}

\author{
Melissa E. Reichelt ${ }^{1}$, Laura Willems ${ }^{1}$, Jason Peart ${ }^{1}$, G. Paul Matherne ${ }^{2}$, Michael R. Blackburn ${ }^{3}$, and \\ John P. Headrick $^{* 1}$ \\ ${ }^{T}$ Heart Foundation Research Center, Griffith University, QLD, Australia, \\ ${ }^{2}$ Department of Pediatrics, University of Virginia Health Sciences Center, Charlottesville, VA, USA, and \\ ${ }^{3}$ Department of Biochemistry and Molecular Biology, University of Texas Health Science Center at Houston, \\ Medical School, Houston, TX 77030, USA \\ j.headrick@griffith.edu.au
}

Inhibition of ischemic contracture development was one of the first documented cardioprotective actions of adenosine. However, whether this represents a normal protective function of endogenous adenosine is not known. Moreover, the relevance of delayed contracture to adenosinergic improvements in post-ischemic outcome is unclear. We assessed effects of exogenous and endogenous adenosine on ischemic contracture, and relationships between contracture and post-ischemic outcomes, in hearts from C57/B16 mice. Untreated wild-type hearts developed a peak ischemic contracture (PC) of $75 \pm 5 \mathrm{mmHg}$ at $8.5 \pm 0.8 \mathrm{~min}$, with a time to reach $20 \mathrm{mmHg}$ (termed time to onset of contracture; TOC) of $4.4 \pm 0.3 \mathrm{~min}$. Adenosine $(50 \mu \mathrm{M})$ significantly delayed TOC to $6.7 \pm 0.5$. This protection was mimicked by pre-treatment with the stable analogue 2-chloroadenosine $(3 \mu \mathrm{M})$ and an $\mathrm{A}_{1}$ adenosine receptor (AR) agonist (50 nM CPA), but not by $\mathrm{A}_{3} \mathrm{AR}$ (150 nM Cl-IB-MECA) or $\mathrm{A}_{2 \mathrm{~A}} \mathrm{AR}$ agonism (20 nM CGS-21680). A selective role for $\mathrm{A}_{1}$ ARs was supported by abrogation of AR-mediated effects in $\mathrm{A}_{1} \mathrm{AR}$ gene knockout (KO) hearts, and contracture inhibition with cardiac $\mathrm{A}_{1} \mathrm{AR}$ overexpression. Importantly, adenosinemediated inhibition of contracture was evident with prolonged (10 to $15 \mathrm{~min}$ ) but not brief ( $3 \mathrm{~min}$ ) pre-treatment. Endogenously generated adenosine was also ineffective - $\mathrm{A}_{1} \mathrm{AR} \mathrm{KO}$ and non-selective $(50 \mu \mathrm{M}$ 8-sulfophenyltheophylline) and $\mathrm{A}_{1} \mathrm{AR}$ selective (150 nM DPCPX) antagonism all failed to alter intrinsic contracture development in the absence of AR agonists. Although inhibition of contracture does not, therefore, play any role in cardioprotection via endogenous adenosine, delayed contracture with exogenous $\mathrm{AR}$ agonism and $\mathrm{A}_{1} \mathrm{AR}$ overexpression was associated with improved coronary reflow (reflecting reduced vascular compression) and enhanced functional recovery. In summary, our data confirm that adenosinergic inhibition of ischemic contracture is solely 
$\mathrm{A}_{1} \mathrm{AR}-\mathrm{mediated}$, and represents a 'supra-physiological' response that is only evident with significant pre-ischaemic AR agonism or enhanced $\mathrm{A}_{1} \mathrm{AR}$ density. Endogenous adenosine does not normally limit ischemic contracture, potentially due to rapidity of contracture development $v s$. the time required for expression of AR-mediated protection.

\title{
Interactions between Subunits in Agonist Activayion of Heteromeric P2X $_{2 / 3}$ Receptors.
}

\author{
William J. Wilkinson $^{1}$, Lin-Hua Jiang ${ }^{2}$, R. Alan North ${ }^{1}$ \\ ${ }^{T}$ Faculty of Life Sciences, University of Manchester, Manchester, M13 9PT, UK \\ ${ }^{2}$ School of Biomedical Sciences, University of Leeds, Leeds, LS2 9JT, UK \\ william.wilkinson@postgrad.manchester.ac.uk
}

Seven genes encode P2X subunits, which can form homo- and heteromeric ATP-gated cation channels. P2X homomeric and $\mathrm{P} 2 \mathrm{X}_{2 / 3}$ heteromeric channels are predominantly found in a subset of primary sensory neurons. Pharmacological inhibition of $\mathrm{P}_{2} \mathrm{X}_{3}$-containing receptors and studies of $\mathrm{P} 2 \mathrm{X}_{3}$-knockout mice have highlighted the importance of this receptor in animal models of chronic inflammatory and neuropathic pain.

Previous work has identified two conserved lysine residues in the P2X receptor ectodomain that may be involved in ATP binding ${ }^{1,2}$. In this study we have further investigated these residues (Lys ${ }^{69}$, Lys $^{308}$; rat P2 $\mathrm{X}_{2}$ numbering), using heterologous expression of P2X receptor mutants in HEK293 cells. Currents evoked by ATP were then measured using whole-cell patch clamp recording.

Both rat $\mathrm{P} 2 \mathrm{X}_{2}$ and rat $\mathrm{P} 2 \mathrm{X}_{3}$ receptors containing the single point mutations K69A or K308A (K63A or K299A in $\mathrm{P} 2 \mathrm{X}_{3}$ ) gave no current in response to up to $1 \mathrm{mM}$ ATP. Co-expression of either of the $\mathrm{P} 2 \mathrm{X}_{2}$ point mutants with $\mathrm{P} 2 \mathrm{X}_{3}$ wild type subunits produced currents with a distinct $\mathrm{P} 2 \mathrm{X}_{2 / 3}$ phenotype (i.e. sustained currents to the ATP analogue $\alpha \beta$-metylene ATP) $\mathrm{EC}_{50}$ values were $27 \pm 3.3 \mu \mathrm{M}\left(\mathrm{P}_{2} \mathrm{X}_{2 / 3}\right.$ wild type $\left.(n=5)\right) 22 \pm 2.6 \mu \mathrm{M}\left(\mathrm{P}_{2} \mathrm{X}_{2}[\mathrm{~K} 69 \mathrm{~A}]+\right.$ $\left.\mathrm{P}_{2} \mathrm{X}_{3}(n=5)\right)$ and $44 \pm 2.6 \mu \mathrm{M}\left(\mathrm{P}_{2} \mathrm{X}_{2}[\mathrm{~K} 308 \mathrm{~A}]+\mathrm{P} 2 \mathrm{X}_{3}(n=8)\right)$.

However, conversely co-expression of $\mathrm{P} 2 \mathrm{X}_{3}$ subunits carrying equivalent point mutations with $\mathrm{P} 2 \mathrm{X}_{2}$ wild type subunits did not produce a functional heteromeric phenotype. Surprisingly the doubly mutated $\mathrm{P} 2 \mathrm{X}_{2}$ receptor (K69A,K308A) could not be "rescued" into a functional heteromer by co-expression with wild type $\mathrm{P}_{2} \mathrm{X}_{3}$ subunits. The $\mathrm{P} 2 \mathrm{X}_{2 / 3}$ heteromeric channel activated by $\alpha \beta$-meATP has been shown to contain a single $\mathrm{P} 2 \mathrm{X}_{2}$ subunit and two $\mathrm{P}_{2} \mathrm{X}_{3}$ subunits ${ }^{3}$. Therefore, the simplest interpretation of the one-way rescue of "dead" $\mathrm{P} 2 \mathrm{X}_{2}$ subunits by wild type $\mathrm{P} 2 \mathrm{X}_{3}$ subunits is that only two $\left(\mathrm{P} 2 \mathrm{X}_{3}\right)$ subunits are required to bind the agonist $(\alpha \beta$-meATP) in order for the channel to open. The finding that the doubly mutated $\mathrm{P}_{2} \mathrm{X}_{2}[\mathrm{~K} 69 \mathrm{~A}, \mathrm{~K} 308 \mathrm{~A}]$ receptor cannot be rescued by $\mathrm{P} 2 \mathrm{X}_{3}$ suggests that the agonist binding site is contributed to by lysines on two adjacent subunits. Therefore in this case, mutating both lysines to alanine in the single $\mathrm{P}_{2} \mathrm{X}_{2}$ subunit would leave the heteromeric receptor with only one binding site (i.e. carrying two lysines) located between the $\mathrm{P} 2 \mathrm{X}_{3}$ subunits, which would insufficient for the channel to function.

This work was funded by the Medical Research Council and the Wellcome Trust.

1. Jinag L-H, Rassendren F, Surprenant A, North RA, J. Biol. Chem. 2000 275, 34190-6

2. Ennion S, Hagan S, Evans RJ, J Bio Chem 2000 275, 29361-67

3. Jiang L-H, Kim M, Spelta V, Bo X, Surprenant A, North RA, J. Neurosci. 2003 23, 8903-10

\section{Interactions between stress-induced diadenosine polyphosphates and heat shock proteins; how and why?}

Michael Wright and Andrew D. Miller Imperial College Genetic Therapies Centre, Imperial College London. a.miller@imperial.ac.uk 
Diadenosine polyphosphates $\left(\mathrm{Ap}_{\mathrm{n}} \mathrm{As}\right)$ were first discovered in cells in the $1960 \mathrm{~s}$ and in spite of the research of many groups, their intracellular functions have remained elusive. Roles have been identified that are both positive and negative to cell survival leading McLennan to ask the question famously, "Diadenosine polyphosphates; friend of foe?" However, the reason for this lack of clear-cut progress in understanding has been the curiously high metabolic lability of $\mathrm{Ap}_{\mathrm{n}} \mathrm{As}$ in the face of batteries of "nudix" hydrolase enzymes that are found in different cells of different organisms. Also, there have been few reliable chemical methodologies for the preparative synthesis of $\mathrm{Ap}_{\mathrm{n}} \mathrm{As}$ and useful $\mathrm{Ap}_{\mathrm{n}} \mathrm{A}$ analogues. Fortunately, reproducible preparative synthesis of $\mathrm{Ap}_{\mathrm{n}} \mathrm{As}$, analogues, fluorescent-labelled analogues and affinity labels is now possible using a combination of Escherichia coli ( $E$. coli) stress-induced enzyme (LysU) catalyzed phosphate-phosphate bond formation, synthesis and powerful anion-exchange chromatography.

The mechanism of LysU induced catalysis will be described in detail including the effect of phosphorylation. In particular, we report that active LysU has a dual catalytic activity, initially producing diadenosine- $5^{\prime}, 5^{\prime}-P^{1}, P^{4}$ triphosphate $\left(\mathrm{Ap}_{4} \mathrm{~A}\right)$ from ATP, before converting the tetraphosphate to a triphosphate. Therefore, LysU is also an efficient diadenosine- $5^{\prime}, 5^{\prime}-P^{1}, P^{3}$-triphosphate $\left(\mathrm{Ap}_{3} \mathrm{~A}\right)$ synthase. Mechanistic investigations reveal that $\mathrm{Ap}_{3} \mathrm{~A}$ formation requires reversal of $\mathrm{Ap}_{4} \mathrm{~A}$ synthesis once complete, to regenerate ATP and a lysyl-adenylate intermediate, of which the latter is attacked by inorganic phosphate so generating ADP in situ. This in situ ADP can then attack further regenerated lysyl-adenylate giving rise to $\mathrm{Ap}_{3} \mathrm{~A}$. LysU may be a key control enzyme for recovery from cellular stress responses that has the capacity to produce an accomodatory "spike" of $\left[\mathrm{Ap}_{4} \mathrm{~A}\right]$ several mins post stress-induction followed by a prolonged restoration of modulatory $\left[\mathrm{Ap}_{3} \mathrm{~A}\right]$ levels with time leading to [A$\left.\mathrm{p}_{3} \mathrm{~A}\right] /\left[\mathrm{Ap}_{4} \mathrm{~A}\right]$ ratio 1.

The nature and properties of putative $\mathrm{Ap}_{4} \mathrm{~A}$ interactions with E. coli molecular chaperone GroEL and cAMP receptor protein (CRP) are also investigated. We show that GroEL is an $A_{4} A$ binding protein that binds $A p_{4} A$ at a set of binding sites (one per monomer) distinct from the GroEL ATP/ADP sites. Ap $\mathrm{p}_{4} \mathrm{~A}$ binding appears to enhance ATPase rates at higher temperatures, encourages the release of bound ADP and may promote substrate protein release through differential destabilization of the substrate protein-GroEL complex. We suggest that such effects should result in enhanced GroEL/GroES chaperoning activities that could be a primary reason for the improved yields of refolded substrate protein observed during GroEL/GroES-assisted folding/refolding at $30^{\circ} \mathrm{C}$ and above in the presence of $\mathrm{Ap}_{4} \mathrm{~A}$. We propose that $\mathrm{Ap}_{4} \mathrm{~A}$ has a modulator or "repressor" function reducing the tendency of GroEL to convert to its storage conformation from an active molecular chaperone conformational state. $\mathrm{Ap}_{4} \mathrm{~A}$ could contribute significantly to E. coli ability to sustain a "skeleton" physiology and metabolism during stress and aid the restoration to normality immediately post stress. In contrast, we are unable to obtain any data to support a direct role for $\mathrm{Ap}_{4} \mathrm{~A}$ interactions with CRP.

\title{
Interventions on neonatal adenosinergic system: evaluation of neurochemical and nociceptive aspects.
}

\author{
Da Silva, R. S ${ }^{1}$., Tonial, E. M. ${ }^{1}$, Silveira, V. G. da ${ }^{2}$, Torres, I. L. S. ${ }^{3}$, Lara, D. R. ${ }^{1}$, Bonan, C.D. ${ }^{1}$ \\ ${ }^{1}$ Laboratório de Pesquisa Bioquímica, PUCRS, ${ }^{2}$ Laboratório de Enzimologia, UFRGS, \\ ${ }^{3}$ Centro Universitário,UNIVATES. Rio Grande do Sul - Brazil \\ rosane.silva@pucrs.br
}

The activation of adenosinergic system exerts a modulator role since early phases of development. The expression of adenosinergic receptors reaches the pattern of adult brain before the birth (1). Adenosine receptor activation in embryonic and neonatal phases has been demonstrated to produce impairment of global animal development, ventriculomegaly and loss of white brain matter $(2 ; 3)$. This information raises the question about the susceptibility of immature brain towards some drugs, such as caffeine. Caffeine is a metylxanthine that exhibits stimulant effects, which are based on unspecific blockade of adenosine receptors. In a work of our laboratory we verify that chronic administration of caffeine $(1 \mathrm{~g} / \mathrm{L})$ in the drink water of rat dams during gestational and lactational phases altered the pattern of MK-801-induced hyperlocomotion, in 21 days-old litters (4). These effects are persistent to the deprivation of caffeine seven days before the experiment. In the present study, it has been evaluated the effects of caffeine administration $(1 \mathrm{~g} / \mathrm{L})$ during gestational and lactational period on the pain threshold and enzymes activities from cerebral preparation of the litters. Additionally, we verified the stress induced analgesia in litters at 50 days of age. Young (14 days old) rats submitted to caffeine treatment have an enhancement of pain 
threshold, which is lost in adult life, probably by a reestablishment of the balance of adenosine receptor activation. Young rats which were deprivated of caffeine 7 days before the experiment did not differ of control group. Stress induced-analgesia is not altered by caffeine treatment, which may be related to an independent action between the analgesia induced by adenosine or opioids. Caffeine treatment of dams promoted distinct alterations in activities of both nucleotidases and acetylcholinesterease activities from hippocampus preparations of neonates at 7 , 14 and 21 days of age. Maternal caffeine intake altered nucleotidase and AChE activities from hippocampus of neonate rats in a time- and treatment- dependent manner. However, the alterations occurs in different ways, since that cholinesterase activity increased and nucleotidase decreased after caffeine treatment. Such alterations in the immature brain could promote alterations in adult life, which must be further evaluated. This set of results indicate that intervention on adenosinergic system during gestational and neonatal phases is able to promote adaptations, probably related to up-regulation of adenosine receptors. These adaptations can reestablish the normal neuromodulatory control of adenosine, considering the relevance of the adenosinergic control on the systems evaluated to the organism maintenance.

(1). Adén, U, Herlenius, E, Tang, L-Q, Fredholm, B B. Pediatr. Res. 2000, 48 (2), 177-183.

(2). Zimmerberg, B, Carr, K L, Scott, A, Lee, H H, Weider, J M. Pharmacol. Biochem. Behav. 1991, 39 (4), $883-$ 888.

(3). Turner C P, Yan H, Schwartz M, Othman T, Rivkees S A. NeuroReport. 2002, 13,1199-1204.

(4). Da Silva, R S, Hoffman, A, De Souza, D O, Lara, D R, Bonan, C D. Eur. J. Pharmacol. 2005; 509 (2-3): 155-159.

\title{
Intracellular signaling underlying ATP-induced chemotaxis of microglia
}

\author{
Ohsawa K. ${ }^{1}$, Irino Y. ${ }^{1}$, Nakamura $\mathrm{Y}^{1}$., Akazawa C. ${ }^{1}$, Inoue K. ${ }^{2}$ and Kohsaka S. ${ }^{1}$ \\ 1) Dept. Neurochem., Natl. Inst. Neurosci., Tokyo, Japan. ${ }^{2)}$ Dept. Pharmcol., Grad. Sch. Pharm. Sci., Kyushu \\ Univ., Fukuoka, Japan. \\ kohsaka@ncnp.go.jp
}

Resident microglia exhibit ramified shapes in the normal brain, however, in response to pathological stimuli, they transform rapidly themselves into more motile amoeboid form called activated microglia and migrate toward the lesioned sites, where the accumulating microglia secret a variety of substances ${ }^{1)}$ to repair the tissues. Thus, microglial cell migration is an important initial step of amelioration of the damaged nervous system.

Extracellular ATP is known to regulate physiological functions of microglia ${ }^{2)}$. Microglia possesses both ionotropic P2X receptors (P2X4R and P2X7R) and G-protein-coupled P2Y receptors (P2Y2R, P2Y6R and P2Y12R). We previously showed that ATP induced membrane ruffling and chemotaxis of microglia and suggested that the membrane ruffling is mediated by Gi/o-protein-coupled P2Y12R ${ }^{3,4)}$. We showed here that the ATP-induced chemotaxis of microglia is also regulated by the ionotropic receptor, P2X4R in addition to the P2Y12R.

Stimulation of G-protein-coupled receptor lead to activation of phospholipase C (PLC) and phosphoinositide 3kinase (PI3K). We examined the effect of PLC and PI3K inhibitors on the formation of membrane ruffling and the chemotaxis of microglia following the stimulation by ATP. A PLC inhibitor inhibited both membrane ruffling and chemotaxis, while PI3K inhibitors suppressed only chemotaxis without inhibiting the membrane ruffling. These observations indicate that PLC activation is essential for both of the membrane ruffling and the chemotaxis, while the activation of PI3K is necessary only for the chemotaxis. Phosphorylation of Akt, which is known to be a downstream target of PI3K pathway, was enhanced by ATP stimulation. The increase in Akt phosphorylation was suppressed by chelating extracellular calcium. These results indicate that activation of PI3K pathway is modulated by the extracellular calcium influx suggesting a possibility that ionotropic P2XRs are involved in the PI3K activation. Therefore, we examined the effect of various P2XRs antagonists on the ATP-induced chemotaxis of microglia. TNP-ATP significantly inhibited the chemotaxis, but neither PPADS nor BBG were effective. Furthermore, we constructed the lentivirus vector expressing short hairpin RNAi against P2X4R and introduced the vector into microglia and showed that suppression of P2X4R reduced the ATP-induced chemotaxis of the cells. These results clearly indicate that P2X4R in addition to P2Y12R are involved in the ATP-induced chemotaxis of microglia. 
1) Kreutzberg, G.W. Trends. Neurosci., 1996, 19, 312-318.

2) Inoue, K. Glia, 2002, 40, 156-163.

3) Honda, S.; Sasaki, Y.; Ohsawa, K.; Imai, Y.; Nakamura, Y.; Inoue, K.; Kohsaka, S. J. Neurosci., 2001, 21, 19751982.

4) Sasaki, Y.; Hoshi, M.; Akazawa, C.; Nakamura, Y.; Tsuzuki, H.; Inoue, K.; Kohsaka, S. Glia, 2003, 44, $242-250$.

\title{
Involvement of adenosine $\mathbf{A}_{1}$ and $\mathbf{A}_{2 \mathrm{~A}}$ receptors in sleep-wake regulation
}

\author{
Yoshihiro Urade ${ }^{1}$ Wei-Min Qu, ${ }^{1}$ Zhi-Li Huang, ${ }^{1,2}$ Naomi Eguchi, ${ }^{1,3}$ Osamu Hayaishi ${ }^{1}$
}

${ }^{T}$ Department of Molecular Behavioral Biology, Osaka Bioscience Institute, Osaka 565-0874, Japan. ${ }^{1}$ National Key Laboratory of Medical Neurology, Shanghai Medical College of Fudan University, Shanghai 200032, P.R.China.

${ }^{3}$ Waseda-Olympus Bioscience Institute, Helios \#05-01/02, 11 Biopolis Way, Singapore 138667.

uradey@obi.or.jp

We summarize recent progress in the research of adenosine involved in sleep-wake regulation. Adenosine is recognized as a major humoral sleep-inducing factor, accumulated in the brain during the prolongation of wakefulness. Because adenosine is rapidly metabolized in vivo; converted to AMP by adenosine kinase in the brain parenchyma and to inosine by adenosine deaminase dominantly expressed in the leptomeninges (1), we first used stable analogs of adenosine to identify the adenosine receptor subtype involved in sleep regulation. The i.c.v. infusion into wild-type (WT) mice of cyclopentyl adenosine, an agonist of $A_{1}$ receptors $\left(A_{1} R\right)$, did not change the sleep-wake cycle; whereas CGS21680, an agonist of $A_{2 A}$ receptors $\left(A_{2 A} R\right)$, induced potent non-REM sleep $(2,3)$. The infusion of $\mathrm{A}_{2 \mathrm{~A}} \mathrm{R}$-agonist increased the expression of fos protein in the ventrolateral preoptic area (VLPO), one of the sleep centers, in a non-REM sleep-dependent manner. The activation of VLPO neurons was associated with a decrease in fos expression in the histaminergic tuberomammillary nucleus (TMN), one of the arousal centers. The GABAergic inhibition of TMN was found to be involved in non-REM sleep induction by adenosine $\mathrm{A}_{2 \mathrm{~A}} \mathrm{R}$-agonist (3). The neural network between VLPO and TMN is considered to play a key role in the regulation of vigilance states. We then examined the sleep-wake cycles of WT, $A_{1} R$-gene knockout $(\mathrm{KO})$, and $\mathrm{A}_{2 \mathrm{~A}} \mathrm{R}-\mathrm{KO}$ mice. Compared with WT mice, $\mathrm{A}_{1} \mathrm{R}-\mathrm{KO}$ mice did not show any changes in non-REM and REM sleep regulation under basal conditions and exhibited the same amount of rebound sleep after 6-hr sleep deprivation during the light period. On the other hand, $\mathrm{A}_{2 \mathrm{~A}} \mathrm{R}-\mathrm{KO}$ mice showed a slight increase in non-REM and REM sleep during the dark period under the basal conditions and exhibited almost no rebound of non-REM sleep after the 6-hr sleep deprivation (4). Moreover, caffeine inhibited non-REM and REM sleep in WT and $A_{1} R-K O$ mice, but not at all in $A_{2 A} R-K O$ mice (5). These results, taken together, indicate that $A_{2 A} R$, but not $A_{1} R$, is important for sleep regulation and suggest that the genetic depletion of $\mathrm{A}_{2 \mathrm{~A}} \mathrm{R}$ is compensated by other system(s) but is associated with altered sleep-wake regulation1.

(1) Okada, T., Mochizuki, T., Huang, Z.-L., Eguchi, N., Sugita,Y., Urade, Y., Hayaishi, O. Biochem. Biophys. Res. Commun. 2003, 312, 29-34.

(2) Urade,Y., Eguchi, N., Qu,W.-M., Sakata, M., Huang, Z.-L., Chen, J.-F., Schwarschild, M. A., Fink, J. S., Hayaishi, O. Neurology 2003, 61:S94-S96.

(3) Hong, Z.-Y., Huang, Z.-L., Qu, W.-M., Eguchi, N., Urade, Y., Hayaishi, O. J. Neurochem. 2005, 92, 1542-1549.

(4) Hayaishi, O., Urade, Y., Eguchi, N., Huang, Z.-L. Arch. Itali. Biol. 2004, 142, 533-539.

(5) Huang, Z.-L., Qu, W.-M., Eguchi, N., Chen, J.-F., Schwarschild, M. A., Fredholm, B. B., Urade, Y., Hayaishi, O. Nat. Neurosci. 2005, 8, 858-859.

\section{Involvement of extracellular ATP on the growth of gliomas in central nervous system}

Morrone $\mathrm{FB}^{1,2}$, Oliveira $\mathrm{DL}^{2}$, Gamermann $\mathrm{PW}^{2}$, Stella $\mathrm{J}^{2}$, Wofchuck $\mathrm{S}^{2}$, Wink $\mathrm{MR}^{3}$, Meurer $\mathrm{L}^{4}$, $\overline{\text { Edelwieis MI }}^{4}$, Lenz $\mathrm{G}^{3}$, AMO Battastini ${ }^{3}$ 
${ }^{1}$ Faculdade de Farmácia, PUCRS, ${ }^{2}$ Departamento de Bioquímica, ICBS, ${ }^{3}$ Departamento de Biofísica IB, ${ }^{4}$ Departamento de Patologia, HCPA, UFRGS, Porto Alegre, RS, Brazil

ATP is an important signaling molecule on the peripheral and central nervous system (CNS). Nucleotides and nucleosides induce proliferation in glioma cell lines and are hydrolyzed very slowly by gliomas when compared with astrocytes. Gliomas growth can be involved with ATP liberation mechanism that occurs by the injury caused by tumor resection. Glioma cells present a clear resistance to death induced by cytotoxic concentrations of ATP when compared with normal brain tissue. To deplete the extracellular ATP, the enzyme apyrase was tested on the treatment of gliomas implanted in the rats CNS. One million cells of gliomas in $3 \mu$ of DMEM were injected in the right striata of rats male Wistar, 250-270g. After 20 days, the rats were decapitated and the brain sectioning and stained with hematoxylin and eosine (H\&E). We performed immunohistochemical experiments with Ki67, CD31 and VEGF (vascular endothelial growth factor). Total RNA was isolated from cultured glioma C6 cells and the cDNA was analyzed by Real Time-PCR with primers for the NTPDase family. Our results showed that C6 cells effectively have a low expression of all NTPDases investigated, in comparison with normal astrocytes. The rats with implanted glioma co-injected with apyrase had a significant reduction in the tumor size $(\mathrm{p}<0.05)$ in comparison with the rats injected only with gliomas or with gliomas plus inactivated apyrase (apyrase control). According to the pathological analysis, the malignant gliomas induced by C6 injection and co-injected with apyrase presented a significant reduction in the mitotic index and other histological characteristics that indicate a less invasive/proliferative tumor. Reduction of proliferation induced by apyrase co-injection was confirmed by counting the percentage of Ki67 positive glioma cell nuclei. According to counts with CD31, vessel density and neoformation was higher in the C6 group 20 days after implantation, in comparison with the group treated with apyrase. Confirming this observation, rats treated with apyrase presented less VEGF staining in comparison to the control group (glioma alone). These results indicate the participation of ATP and the ecto-nucleotidases may be associated with the development of this type of brain tumor in an in vivo glioma model.

Key words: Extracelular ATP, tumor growth, glioma, apyrase

Correspondence to: Fernanda B Morrone

fmorrone@portoweb.com.br

\section{Involvement of $\mathrm{P2Y}_{6 \text { (like) }}$ receptor in UTP-evoked relaxation of the mouse aorta}

$\underline{\text { Pieter-Jan D. F. Guns }}^{\text {a }}$, Tim Van Assche ${ }^{\mathrm{a}}$, Fransen Paul ${ }^{\mathrm{a}}$, Bernard Robaye ${ }^{\mathrm{b}}$, Jean-Marie Boeynaems ${ }^{\mathrm{c}}$, Hidde Bult $^{\text {a }}$

${ }^{a}$ Division of Pharmacology, University of Antwerp, 2610 Wilrijk, Belgium

${ }^{b}$ Institute of Interdisciplinary Research, Institute of Biology and Molecular Medicine, Free University of Brussels, 6041 Gosselies, Belgium

${ }^{c}$ Institute of Interdisciplinary Research, School of Medicine, Free University of Brussels, 1070 Brussels, Belgium pieter-jan.guns@ua.ac.be

Objectives: Previously, we documented the presence of functional $\mathrm{P} 2 \mathrm{Y}_{1}, \mathrm{P}_{2} \mathrm{Y}_{2}$ and $\mathrm{P} 2 \mathrm{Y}_{6}$ receptors in murine aorta endothelial cells. In general, endothelium-dependent relaxation evoked by ATP and UTP is mainly attributed to $\mathrm{P}_{2} \mathrm{Y}_{2}$ receptors. In the mouse aorta, however, the antagonist suramin showed different apparent $\mathrm{pKb}$-values for ATP $(4.53 \pm 0.07)$ and UTP $(5.19 \pm 0.03)$. Particularly the pKb for UTP was different from values reported in rat aorta, suggesting that other P2Y receptors might be involved in the action of UTP. Therefore this study was aimed to identify the receptors for UTP.

Methods: In view of the lack of selective purinergic agonists and antagonists, we used $\mathrm{P} 2 \mathrm{Y}_{2}-$ and $\mathrm{P} 2 \mathrm{Y}_{4}$-knockout mice. Isometric force development of thoracic aorta segments of wild type (WT), P2 $\mathrm{Y}_{2}$ - and P2 $\mathrm{Y}_{4}$-deficient (CD1/ 129SV) mice was measured in organ baths. Rings were contracted with phenylephrine and then exposed to nucleotides.

Results: ATP and UTP evoked complete relaxation in $\mathrm{P}_{2} \mathrm{Y}_{4}$-deficient aortas that did not differ in any aspect from that of WT mice. Moreover, $\mathrm{P}_{2} \mathrm{Y}_{4}$ mRNA was not detected in whole aorta homogenates of WT mice, whereas $\mathrm{P}_{2} \mathrm{Y}_{1}, \mathrm{P}_{2} \mathrm{Y}_{2}$ and $\mathrm{P}_{2} \mathrm{Y}_{6}$ mRNA was present. $\mathrm{P}_{2} \mathrm{Y}_{2}$-deficient mice, however, showed impaired ATP- and ATP $\gamma \mathrm{S}$ evoked relaxation, indicating that ATP and ATP $\gamma \mathrm{S}$ activate predominantly $\mathrm{P}_{2} \mathrm{Y}_{2}$-receptors. In contrast, UTPevoked relaxation in $\mathrm{P}_{2} \mathrm{Y}_{2}$-knockout mice was indistinguishable from that of WT mice. Further, MRS2179, a $\mathrm{P}_{2} \mathrm{Y}_{1}$-selective antagonist did not inhibit UTP-responses. 
Conclusion: The functional experiments with $\mathrm{P}_{2} \mathrm{Y}_{4}-\mathrm{knockout}_{\text {mice }}$ and mRNA data clearly demonstrate that $\mathrm{P}_{2} \mathrm{Y}_{4}$ receptors are not involved in UTP-induced relaxation of the murine aorta. Further, the results of the $\mathrm{P} 2 \mathrm{Y}_{2^{-}}$ knockout mice indicate that the $\mathrm{P}_{2} \mathrm{Y}_{2}$-subtype is not the main UTP-receptor. And MRS2179 excluded the involvement of the $\mathrm{P}_{2} \mathrm{Y}_{1}$ subtype. Therefore, UTP most likely acts on a $\mathrm{P}_{2} \mathrm{Y}_{6 \text { (like) }}$ receptor subtype. Furthermore, this $\mathrm{P} \mathrm{Y}_{6 \text { (like) }}$ hypothesis is supported by identical pKb-values of suramin for UTP $(5.19 \pm 0.03)$ and UDP(5.26 \pm 0.06$)$ and by indistinguishable dose response-curves of UTP and UDP. This was unexpected since UTP mainly activates $\mathrm{P}_{2} \mathrm{Y}_{2}$ in human and rat arteries.

\title{
Is in vitro vasorelaxation by ticlopidine related to P2Y12 receptors?
}

\author{
G. Froldi, M. Montopoli, E. Ragazzi, RM Gaion, L. Caparrotta and P. Dorigo \\ Department of Pharmacology and Anesthesiology -Pharmacology Division-Largo E. Meneghetti 2, University of \\ Padova (Italy). \\ g.froldi@unipd.it
}

Ticlopidine is an antiplatelet agent also known as ADP receptor antagonist in vivo. The compound does not inhibit ADP-induced platelet aggregation in vitro; hepatic biotrasformation into an active metabolite is required for its action. The mechanism of antiplatelet action appears to be the irreversible alteration of the platelet surface P2Y12 receptor, resulting in a reduction of ADP-induced platelet aggregation. Recently, P2Y12 receptor has been identified in human blood vessels (1). The aim of our research was to study in vitro direct effects of ticlopidine on resistance vessel. Rat-tail arterial rings were placed in a tissue bath containing Krebs-Ringer solution and their viability was assessed by $10 \mu \mathrm{M}$ phenylephrine and $10 \mu \mathrm{M}$ acethylcholine. Ticlopidine from $0.01 \mu \mathrm{M}$ to $1 \mu \mathrm{M}$ per se did not significantly change resting tension of arterial rings; only at $10 \mu \mathrm{M}$ it slightly decreased the basal tone of tissues. Ticlopidine per se evoked relaxation of arteries precontracted by $80 \mathrm{mM} \mathrm{KCl}, 0.5 \mu \mathrm{M}$ phenylephrine (Phe) and $0.5 \mu \mathrm{M}$ 5-hydroxytryptamine (5-HT), as shown in the figure. The potency order was 5-HT $\geq \mathrm{Phe}>\mathrm{KCl}$.

2-MethioADP (10 nM to $100 \mu \mathrm{M})$, a selective agonist of P2Y1 and P2Y12 receptors, induced a fast and transient vasoconstriction. Preincubation with $1 \mu \mathrm{M}$ ticlopidine did not change vasoconstriction by $10 \mu \mathrm{M}$ 2-MethioADP, whereas $300 \mu \mathrm{M}$ suramine inhibited it. Also we studied the influence of suramine on concentration-related vasorelaxation by ticlopidine in tissues precontracted with 5-HT. Vasorelaxation induced by ticlopidine was never inhibited, indicating that the effect is not related to $\mathrm{P} 2$ receptors.

Our experimental observations suggest that ticlopidine can directly induce vasorelaxation of rat resistance arteries. Moreover, experimental data indicate that its effect is not linked to P2Y receptors and that other mechanisms must be involved.

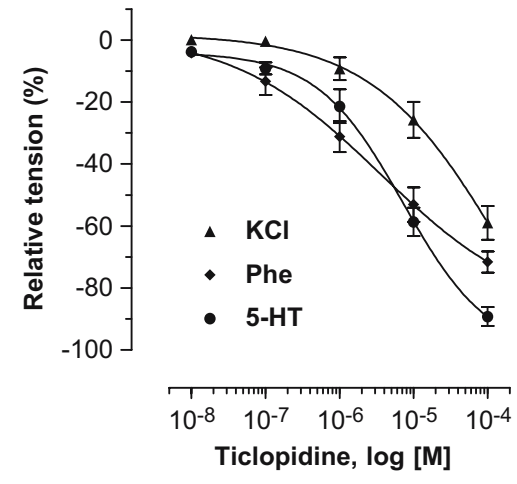

\section{Reference}

1) Wihlborg AK, Wang L, Braun OO, Eyjolfsson A, Gustafsson R, Gudbjartsson T, Erlinge D.

ADP receptor P2Y12 is expressed in vascular smooth muscle cells and stimulates contraction in human blood vessels. Arterioscler Thromb Vasc Biol. 2004, 24(10): 1810-5. 


\title{
Kinetic characterization of an NTPDase and $5^{\prime}$-nucleotidase by a crude synaptosomal fraction isolated from rat heart.
}

\author{
Bárbara Rücker, Manoela Enger Almeida, Maria Luiza Moraes de Barreto Chaves and João José \\ Freitas Sarkis. \\ Departamento de Bioquímica, Universidade Federal do Rio Grande do Sul, Porto Alegre, RS, Brazil \\ barucker@ig.com.br
}

The first suggestions that purines acted in a cardioprotective role came with the demonstration that adenosine mediated vasodilatation during hypoxia to increased blood flow and thus maintain oxygen delivery to the heart ${ }^{1,2}$. Besides, adenosine is an important extracellular signalling molecule with aintithrombotic and antiinflamatory properties. In heart, ATP is involved in positive ionotropic effects, may induce various forms of arrhythmia besides hypertrophy and apoptosis ${ }^{2,3}$. Traditionally, the inactivation of adenine nucleotides signaling is attributed to its breakdown by cell-membrane bound enzymes, classified as ATPases, ecto-apyrases and ecto-5'-nucleotidases $^{4,5}$. The objective of the present study is to characterize the enzymes involved in ATP, ADP e AMP hydrolysis in cardiac synaptosomes of adult male rats. The crude synaptosomal-mitochondrial fraction was prepared as previously described by Aloyo et al, $1991^{6}$. The reaction was initiated by the addition of nucleotides $(1.0 \mathrm{mM}$ ATP, ADP or AMP) after 10 minutes of pre-incubation at $37^{\circ} \mathrm{C}$. The nucleotide hydrolysis was determined by the Malachite Green method previously described by Chan et al, $1986^{7}$. The protein concentration and the incubation time were chosen in order to ensure the linearity of the reaction. Then, about $6 \mu \mathrm{g} /$ tube of protein and 6 minutes of incubation were used in enzymatic assays for ATP/ADP. The mitochondria ATPase inhibitors (olygomycin $2 \mu \mathrm{g} / \mathrm{mL}$ and sodium azide $100 \mu \mathrm{M}$ ) were used in all enzymatic ATP assays. For the study of AMP hydrolysis, 10 $\mu \mathrm{g} /$ tube of protein and $10 \mathrm{~min}$ were used. Both enzymes were cation-dependent: NTPDase showed a preference for ion $\mathrm{Ca}^{2+}$, while $5^{\prime}$-nucleotidase showed a preference for ion $\mathrm{Mg}^{2+}$. In addition, both activities were blockade by metal ion chelator. $\mathrm{K}_{\mathrm{M}}$ and $\mathrm{V}_{\max }$ values for ATP, ADP and AMP hydrolysis were found to be $143 \pm 16 \mathrm{mM}$ and $564 \pm 89 \mathrm{nmol} \mathrm{Pi} / \mathrm{min} / \mathrm{mg}$ protein $(\mathrm{n}=3), 68 \pm 17 \mathrm{mM}$ and $216 \pm 37 \mathrm{nmol} \mathrm{Pi} / \mathrm{min} / \mathrm{mg}$ protein $(\mathrm{n}=4), 65 \pm 9 \mathrm{mM}$ and $68 \pm 6 \mathrm{nmol} \mathrm{nmol} \mathrm{Pi} / \mathrm{min} / \mathrm{mg}$ protein $(\mathrm{n}=3)$, respectively. Extracellular nucleotides are known to regulate several physiological responses, including vascular tone, cardiac funtion and haemostasis. There is evidence that purines contribute to a number of processes involved in normal cardiovascular function, and that disturbances in purinergic signaling are involved in some cardiovascular diseases ${ }^{2}$. These results present first evidences about the kinetic characterization for nucleotide hydrolysis by crude synaptosomal-mitochondrial fraction obtained from adult rats. Then these results may represent a new insight about the purines participation on the cardiovascular system.

1. Berne, R.M. Am. J. Physiol. 1963, 204, 317.

2. Ralevic, V. and Burnstock, G. Drug News Perspect. 2003, 16, 133.

3. Vassort, G. Physiol. Rev. 2001, 81, 767.

4. Zimmermann, H. Biochem. J. 1992, 285, 345.

5. Plesner, L. Int. Rev. Cytol. 1995, 158, 141.

6. Aloyo, V.J.; McIlvain, H.B.; Bhavsar, V.H. and Roberts, J. Life Sci. 1991, 48, 1317.

7. Chan, K.; Delfert, D.; Junges, K.D. Anal. Biochem. 1986, 157, 375.

\section{Lead optimization of a human $A_{3}$ adenosine receptor antagonist: the application of the 3D-QSAR (auto-MEP/PLS) approach as an efficient pharmacodynamic- driven filtering method for small-size virtual library.}

Magdalena Bacilieri ${ }^{1}$, Stefano Moro ${ }^{1}$, Barbara Cacciari $^{2}$, Karl-Norbert Klotz ${ }^{3}$, Giampiero Spalluto ${ }^{4}$,

$T$ Molecular Modeling Section, Dipartimento di Scienze Farmaceutiche, Università di Padova, Via Marzolo 5, I-35131 Padova, Italy; ${ }^{2}$ Dipartimento di Scienze Farmaceutiche, Università degli Studi di Ferrara, Via Fossato di Mortara 17-19, I-44100 Ferrara, Italy; ${ }^{3}$ Institut für Pharmakologie und Toxikologie, Universität Würzburg, 
Versbacher Str. 9, D-97078 Würzburg, Germany; ${ }^{4}$ Dipartimento di Scienze Farmaceutiche, Università degli Studi di Trieste, Piazzale Europa 1, I-34127 Trieste, Italy

magdalena.bacilieri@unipd.it

In recent work ${ }^{1,2}$, we have reported that the combination of particular descriptors such as molecular electrostatic potential (MEP) surface properties (autocorrelation vectors) with the conventional partial least squares (PLS) analysis can be used to produce a robust ligand-based 3D structure-activity relationship (autoMEP/PLS) for the prediction of the human $\mathrm{A}_{3}$ receptor antagonist activities. Following step of this project is the application of the 3D-QSAR (autoMEP/PLS) approach as efficient and alternative pharmacodynamic filtering method for small-size virtual library. We have derived therefore a small sized combinatorial library (729 compounds) on one of the scaffold known as potent human $\mathrm{A}_{3}$ antagonist, the pyrazolo-triazolo-pyrimidines ${ }^{3}$. Once predictions have been computed, the most interesting analogues were further prioritized for synthesis and pharmacological characterization. Remarkably, we found that all the new synthetized compounds are correctly predicted as potent human $\mathrm{A}_{3}$ antagonists ${ }^{4}$.

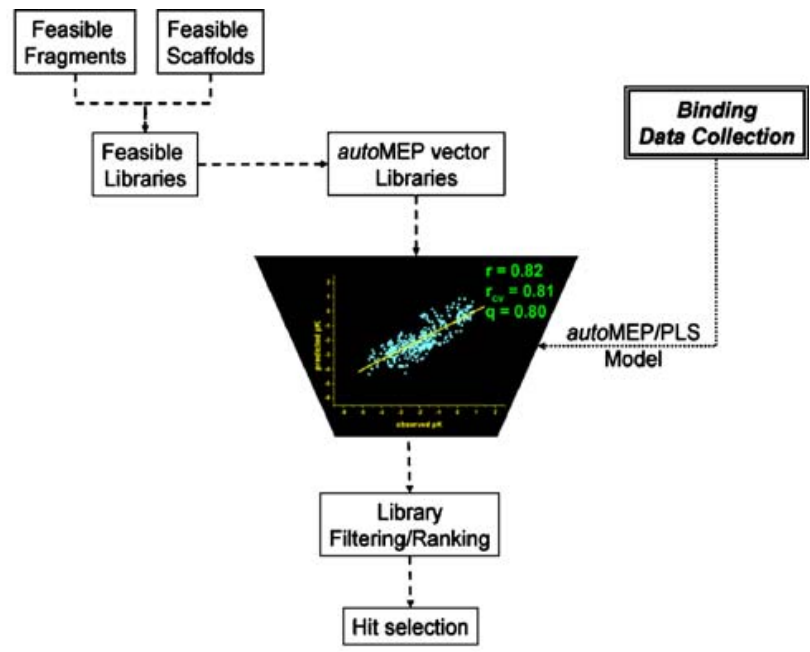

Flowchart of (autoMEP/PLS) model application as pharmacodynamic-driven filthering method.

1. Moro, S.; Bacilieri, M.; Ferrari, C.; Spalluto, G. Curr. Drug. Disc. Techn. 2005, 2, 13-21.

2. Moro, S.; Bacilieri, M.; Cacciari, B.; Spalluto, G. J. Med. Chem. 2005, 48, 5698-5704.

3. Moro, S.; Braiuca, P.; Deflorian, F.; Ferrari, C.; Pastorin, G.; Cacciari, B.; Baraldi, P.G.; Varani, K.; Borea, P.A.; Spalluto, G. J. Med. Chem. 2005, 48, 152-156.

4. Moro, S.; Bacilieri, M.; Cacciari, B.; Klotz, K.N.; Spalluto, G. Biorg. Med. Chem. submitted.

\title{
Local effect of adenosine 5 '-triphosphate on NSAID-induced permeability changes in the human small intestine
}

\author{
M.J.L. Bours ${ }^{1}$, R.J.M. Brummer ${ }^{2}$, F.J. Troost $^{2}$, A. Bast ${ }^{3}$ and P.C. Dagnelie ${ }^{1}$ \\ ${ }^{T}$ Dept of Epidemiology, Maastricht University, Maastricht, The Netherlands \\ ${ }^{2}$ Dept. of Clinical Dietetics/Gastroenterology, Internal Medicine, University Hospital Maastricht, Maastricht, \\ The Netherlands \\ ${ }^{3}$ Dept. of Pharmacology and Toxicology, Maastricht University, Maastricht, The Netherlands \\ m.bours@epid.unimaas.nl
}

Introduction: Non-steroidal anti-inflammatory drugs (NSAIDs) are among the most prescribed anti-inflammatory and analgesic drugs worldwide. However, use of NSAIDs is associated with an elevated risk of mucosal damage in 
the gastrointestinal tract. Adenosine 5'-triphosphate (ATP) signalling has been implicated in the control of epithelial function and may play a protective role in the small intestine.

Objective: To determine the local effect of ATP on small intestinal permeability changes induced by short-term challenge of the NSAID indomethacin in healthy humans.

Subjects and methods: Mucosal permeability of the small intestine was assessed by the lactulose/rhamnose (L/R) permeability test, i.e. ingestion of a test drink containing $5 \mathrm{~g}$ lactulose and $0.5 \mathrm{~g}$ L-rhamnose followed by total urine collection for five hours. Urinary excretion of lactulose and rhamnose was determined by fluorescent detection high-pressure liquid chromatography (HPLC). As a control condition, basal permeability of the small intestine was assessed. As a model of increased small intestinal permeability two doses of indomethacin were ingested prior to ingestion of the test drink ( $75 \mathrm{mg}$ and $50 \mathrm{mg}$ at $-9 \mathrm{hrs}$ and $-1 \mathrm{hr}$, respectively). Placebo or 30 $\mathrm{mg} / \mathrm{kg}$ ATP was administered concomitantly with indomethacin through a naso-intestinal tube.

Results: Median urinary $\mathrm{L} / \mathrm{R}$ ratio $(\mathrm{g} / \mathrm{g})$ observed in the control condition was 0.030 (range: 0.015-0.051). In comparison with the control condition, urinary $\mathrm{L} / \mathrm{R}$ ratio after ingestion of indomethacin and administration of placebo was significantly increased $(0.038(0.016-0.131)$; $\mathrm{P}=0.04)$. Urinary $\mathrm{L} / \mathrm{R}$ ratio after ingestion of indomethacin and administration of ATP $(0.027(0.015-0.067))$ was significantly lower than the L/R ratio observed in the placebo condition $(\mathrm{P}=0.02)$.

Conclusions: Administration of ATP directly into the small intestine during short-term challenge of the NSAID indomethacin completely prevents the NSAID-induced increase in small intestinal permeability in healthy humans. These findings would suggest that ATP could also be beneficial in the treatment of intestinal disorders in which intestinal permeability changes are involved such as Crohn's disease.

\title{
Localization of nucleoside triphosphate diphosphohydrolyse-3 in mouse digestive associated glands
}

\author{
Elise G. Lavoie and Jean Sévigny \\ Centre de recherche en Rhumatologie et Immunologie, CHUQ research center, Université Laval, Québec, Canada \\ Elise.lavoie@crchul.ulaval.ca
}

Introduction: In the digestive system, extracellular nucleotide signalling, via the activation of P2 receptors, participates in the regulation of various functions including electrolyte and glandular secretion, smooth muscle contraction and the control of blood flow. More specifically, in the associated organs, $\mathrm{P} 2$ receptors seem implicated in the Sjögren's syndrome $\left(\mathrm{P}_{2} \mathrm{Y}_{2}\right)$ in the salivary glands [1], and, in pancreas, in insulin secretion $\left(\mathrm{P}_{2} \mathrm{Y}_{1}\right)[2]$. By the hydrolysis of $\gamma$ and $\beta$ phosphates of extracellular nucleotides, nucleoside triphosphate diphosphohydrolyses (NTPDases) may regulate some of these pathways. NTPDases 1-3 and 8 are the most important member of ENTPDase family that regulate nucleotides levels at the cell surface. In the digestive associated glands (pancreas and salivary glands), a general localisation has firstly demonstrated the presence of NTPDases in the plasma membrane of acinar cells, duct epithelial cells and blood vessels of pig pancreas and parotid glands. The parotid myoepithelial cells were also stained. In mouse, NTPDase1 was located in the pancreatic acinar cells and NTPDase2 in the duct epithelial cells of pancreas and in myoepithelial cells of sub-mandibular glands.

Aim: In this work, we determined the tissular localisation of the third member of the E-NTPDase family (NTPDase3) in the mouse salivary glands (parotid, sublingual and sub-mandibular glands) as well as in pancreas. We also localised NTPDases 1 and 2 in parotid and sublingual glands to complete the previous studies.

Results: NTPDases were localised by immunological techniques with new antibodies and by enzyme histochemistry. In the pancreas, a strong level of NTPDase3 expression was detected in Langerhans islet cells. In parotid and submandibular glands, NTPDase 3 immunostaining was on the apical side of serous acinar cells. A weak signal was detected in the sublingual glands. As expected, staining for NTPDases 1 and 2 was seen in blood vessels in sublingual and parotid glands. NTPDase2 was principally localised on the cells surrounding the ducts. These results suggest that NTPDase 3 may play a role in the regulation of endocrine secretion in pancreas and in the serous secretion of salivary glands by controlling the activation of nearly expressed $\mathrm{P} 2$ receptors.

1. Schrader, A.M., J.M. Camden, and G.A. Weisman, P2Y2 nucleotide receptor up-regulation in submandibular gland cells from the NOD.B10 mouse model of Sjogren's syndrome. Arch Oral Biol, 2005. 50(6): p. 533-40. 
2. Léon, C., et al., The P2Y1 receptor is involved in the maintenance of glucose homeostasis and in insulin secretion in mice. Purinergic Signalling, 2005. 1: p. 145-151.

\title{
Looking for the $P 2$ receptor subtypes involved in the presynaptic inhibition induced by ATP at the mouse neuromuscular junction
}

\author{
Silvana De Lorenzo, Mariela I. Veggetti, Salomón Muchnik, Adriana Losavio \\ Laboratorio de Neurofisiología, Instituto de Investigaciones Médicas Alfredo Lanari, Facultad de Medicina, \\ Universidad de Buenos Aires, Buenos Aires, Argentina. \\ idimneurofisio@gmail.com
}

At the neuromuscular junction, ATP is co-released with the neurotransmitter ACh and once in the synaptic space, it is degraded to the presynaptically active metabolite adenosine. However, it was demonstrated that ATP is able to modulate $\mathrm{ACh}$ release through a mechanism independent of the action of adenosine via its own $\mathrm{P} 2$ purinoceptors. At mice diaphragms, we found that $100 \mu \mathrm{M}$ ATP and $30 \mu \mathrm{M}$ of the non hydrolysable ATP analogue $\beta \gamma$ imido ATP reduced MEPP frequency by $45.3 \%$ and $55.9 \%$ respectively, through the modulation of the voltagedependent $\mathrm{Ca}^{2+}$ channels related to spontaneous secretion ( $\mathrm{L}$ and $\mathrm{N}$-type $\mathrm{Ca}^{2+}$ channels). $\beta \gamma$-imido ATP-induced modulation on MEPP frequency was antagonized by the non-specific P2 receptor antagonist suramine (suramine $97.7 \pm 4.0 \%$ of control values; suramine + ATP $103.9 \pm 1.7 \%, \mathrm{n}=4$ ). To investigate the type of P2 receptor involved in the inhibition induced by ATP, the effect of $\beta \gamma$-imido ATP on MEPP frequency was evaluated on diaphragm muscles of CF1 mice in the presence of different P2 antagonists. We found that $10 \mu \mathrm{M}$ PPADS (antagonist of P2X receptors, although it was also described its action on the $\mathrm{P}_{2} \mathrm{Y}_{1}, \mathrm{P}_{2} \mathrm{Y}_{4}, \mathrm{P}_{2} \mathrm{Y}_{6}$ and $\mathrm{P} 2 \mathrm{Y}_{13}$ receptors) did not modify the effect of $\beta \gamma$-imido ATP (PPADS $95.9 \pm 2.7 \%$ of control values; PPADS + $\beta \gamma$-imido ATP $50.7 \pm 2.4 \%, \mathrm{n}=4)$, while the $\mathrm{P} 2 \mathrm{Y}_{4,6,11,12,13}$ receptor antagonist reactive blue $2(5 \mu \mathrm{M})$, prevented the effect of the ATP analog (RB $98.8 \pm 2.8 \%$ of control values, RB $+\beta \gamma$-imido ATP $100.3 \pm 4.7 \%, \mathrm{n}=8$ ), suggesting that the effect was mediated by P2Y receptors. On the other hand, pertussis toxin $\left(2 \mu \mathrm{M} \mathrm{ml}^{-1}\right.$ for $\left.12-14 \mathrm{~h}\right)$ and NEM $(10 \mu \mathrm{M})$, which are blockers of $\mathrm{G}_{\mathrm{i} / \mathrm{o}}$ proteins, abolished the action of the nucleotide (PTX $+\beta \gamma$-imido ATP $102.8 \pm$ $1.6 \%, \mathrm{n}=4 ; \mathrm{NEM}+\beta \gamma$-imido ATP $122.9 \pm 5.6 \%, \mathrm{n}=4)$, indicating that the P2Y receptors involved are coupled to $\mathrm{G}_{\mathrm{i} / \mathrm{o}}$ proteins. Amongst the P2Y receptors, $\mathrm{P} 2 \mathrm{Y}_{12}$ and ${ }_{13}$ are linked to $\mathrm{G}_{\mathrm{i} / \mathrm{o}}$ proteins; thus, we studied the effect of $\beta \gamma$-imido ATP in the presence of the P2 $\mathrm{Y}_{12}$ and 13 antagonists 2MeSAMP $(30 \mu \mathrm{M})$ and AR-C69931MX $(1 \mu \mathrm{M})$. Both antagonists prevented the effect of $\beta \gamma$-imido ATP (2-MeSAMP $89.5 \pm 0.4 \%$ of control values, 2 -MeSAMP + $\beta \gamma$-imido ATP $88.5 \pm 0.6 \%, \mathrm{n}=4$; AR-C69931MX 97.0 $\pm 4.9 \%$, AR-C69931MX $+\beta \gamma$-imido ATP $94.0 \pm 3.6 \%, \mathrm{n}=$ 4). Moreover, AR-C69931MX also antagonized the presynaptic modulation exerted by $150 \mathrm{nM} 2 \mathrm{MeSADP}$ (ARC69931MX 93.7 $\pm 2.1 \%$ of control values, AR-C69931MX + 2MeSADP $94.6 \pm 2.0 \%, n=4$ ), which is the preferential agonist for these subtypes of receptors (2MeSADP $53.9 \pm 1.6 \%, \mathrm{n}=4$ ).

The present results suggest that $\mathrm{P}_{2} \mathrm{Y}_{12}$ or 13 receptors mediate the inhibition of spontaneous ACh secretion induced by adenine nucleotides at mammalian neuromuscular junctions.

\section{Lowered activity of adenosine kinase leads to adenosine release and decrease of diabetic $\mathbf{T}$ lymphocytes proliferation potential}

\author{
Tadeusz Pawelczyk, Monika Sakowicz-Burkiewicz, Katarzyna Kocbuch, Marzena Grden \\ Department of Molecular Medicine, Medical University of Gdansk, 80-211 Gdansk, Poland \\ tkpaw@amg.gda.pl
}

Several aspects of immunity are altered in diabetic patients, which are generally more susceptible to some specific infection and ultimate complications. The proliferative response of CD4+ T cells derived from type- 1 diabetic patients to the primary protein antigens was reported to be significantly reduced [1], but the mechanisms responsible for impaired lymphocyte proliferation are largely unclear. Diabetic lymphocytes among functional alterations display several metabolic changes including decreased rate of glucose and glutamine oxidation [2]. Our 
previous studies documented decreased activity of adenosine kinase (AK) [3] and altered nucleoside transporters in diabetic lymphocytes $[4,5]$. These changes may lead to impairment in energy provision especially under conditions of increased energy consumption and elevation of nucleotides metabolites including adenosine. The present study was undertaken to investigate the effect of insulin and changes in glucose concentration on adenosine metabolism, transport and receptor-mediated action in rat $\mathrm{T}$ lymphocytes. Experiments were conducted on isolated rat $\mathrm{T}$ lymphocytes cultured in medium containing various glucose and insulin concentrations. Proliferation of T cells was induced with either the Con A or anti-CD3 plus anti CD28 mAbs.

Performed experiments showed that insulin and glucose differentially affected the activities of adenosine metabolizing enzymes in resting and proliferating $\mathrm{T}$ cells. However, the vulnerability of $\mathrm{T}$ cells to metabolic stress induced by 2-deoxyglucose was determined by insulin but not by glucose concentration. Irrespective of incubation conditions the proliferation of $\mathrm{T}$ cells was not depended on expression level and functional state of nucleoside transporters ENT1, ENT2, and CNT2. Inhibition of AK activity with 5-iodotubercidin lowered the proliferation potential of $\mathrm{T}$ cells to the level observed in insulin-deprived cells. Moreover, insulin-deprived cells but not cells cultured in the presence of insulin released significant quantities of adenosine. Under resting conditions the level of cAMP was $\sim 6$-fold higher in cells cultured at $20 \mathrm{mM}$ glucose and the absence of insulin comparing to cells grown at low glucose and the presence of insulin. T lymphocytes cultured at high glucose $(20 \mathrm{mM})$ and the absence of insulin displayed increased level of $A_{2 A}$ adenosine receptor mRNA and decreased level of $A_{3}$ receptor mRNA. The expression level of $\mathrm{A}_{1}$ and $\mathrm{A}_{2 \mathrm{~B}}$ receptor was not affected by changes in insulin and glucose concentrations. Experiments with specific adenosine receptors agonists and antagonists showed that adenosine-induced suppression of proliferation of $\mathrm{T}$ cells cultured at high glucose and the absence of insulin was mediated by $\mathrm{A}_{2 \mathrm{~A}}$ adenosine receptor but not by $\mathrm{A}_{2 \mathrm{~B}}$ receptor. Treatment of $\mathrm{T}$ cells grown at high glucose and the absence of insulin with $\mathrm{H}-89(10 \mu \mathrm{M})$, a specific protein kinase A (PKA) inhibitor restored suppressed T cell proliferation.

Concluding, imparied proliferation of insulin-deprived T lymphocytes is evoked by decreased expression of AK what in turn leads to outflow of adenosine from the cell. Released adenosine by acting on $\mathrm{A}_{2 \mathrm{~A}}$ receptor induces, cAMP production leading to activation of PKA and suppression of T cell proliferation.

1. Eibl, N., Spatz, M., Fischer, G.F., Mayr, W.R., Samstag, A., Wolf, H.M., Schernthaner, G., \& Eibl, M.M. (2002) Clin. Immunol. 103, 249-259

2. Otton, R., Mendonca, J.R., \& Curi, R. (2002) J. Endocrinol. 174, 55-61

3. Pawelczyk, T., Sakowicz, M., \& Podgorska M. (2003) Exp. Cell Res. 286, 152-163

4. Sakowicz, M., Szutowicz, A., \& Pawelczyk, T. (2004) Biochem. Pharmacol. 68, 1309-1320

5. Sakowicz, M., Szutowicz, A., \& Pawelczyk, T. (2005) Int. Immunol. 17, 145-154

\section{Lung shows a great content in inactive ecto-5' ${ }^{\prime}$-nucleotidase (CD73)}

Morote-García, J.C., Campoy, F.J., Vidal, C.J., Muñoz-Delgado, E. Department of Biochemistry and Molecular Biology-A, University of Murcia, Spain. jcmorote@um.es

Ecto-5'-nucleotidase (eNT or CD73) converts extracellular AMP into adenosine. In lung, most of the eNT activity is located in the epithelial surface covering the lumen of the respiratory ways ${ }^{1}$. The importance of the eNTgenerated adenosine in this organ ${ }^{1,2}$, and the finding of non-catalytic (inactive) eNT variants in muscle ${ }^{3}$ and liver $^{4}$ of normal and dystrophic (merosin-negative) mice, prompted us to explore the presence of inactive eNT in lung, as well as the effects of the merosin-deficient muscular dystrophy on pulmonary eNT.

During the multi-step extraction protocol of eNT from lung, the addition of Triton X-100 + sodium deoxycholate produces a 5-fold increase in eNT activity ${ }^{5}$. Probably, the activity latency can be explained by the influence of the lipidic environment upon the nucleotidase activity ${ }^{6}$, and by the preferential location of the protein in caveolae and lipid rafts $^{7}$, which are crucial for keeping the pulmonary air-blood barrier in optimal conditions ${ }^{8}$.

Inactive eNT variants can be easily detected by comparing samples with the same units of activity in a Western $b l o t^{3}$. Therefore, samples from tissues where inactive eNT has already been identified, such as muscle, liver and kidney $^{3,4}$, were subjected to Western blotting together with samples from lung, all with the same units of eNT activity. The result showed:1) a 4.4-fold increase in the intensity of lung-eNT bands respect to that of the other organs, meaning that this tissue contains a high amount of inactive $\mathrm{eNT}^{5}$; and 2) a smaller size of the eNT subunit in lung (66 
$\mathrm{kDa})$ compared to the one of muscle, liver and kidney $(72 \mathrm{kDa})^{5}$. The great content of inactive eNT in lung might represent a stock of enzyme susceptible of activation for facing pathological situations in which a high amount of adenosine is required, as it happens in hypoxic episodes ${ }^{9}$. On the other hand, digestion with peptide- $N$-glycosidase F shows that the size difference of the lung-eNT subunit is due to its lower state of glycosylation. No differences between normal and dystrophic lung exists in eNT activity, the immunolabeling intensity or size of the subunit. At the transcriptional level, a single PCR product was identified, revealing that active and inactive eNT variants differ in post-translational processing ${ }^{5}$. Again, no differences between normal and dystrophic lung were detected ${ }^{5}$. Research supported by MCYT of Spain (SAF2001/0279) and the Seneca Foundation (PI83/00840/FS01).

(1) Picher, M., Burch, L. H., Hirsh, A. J., Spychala, J., Boucher, R. C. J. Biol. Chem. 2003, 278, 13468-13479.

(2) Thompson, L. F., Eltzschig, H. K., Ibla, J. C., Van De Wiele, C. J., Resta, R., Morote-García, J. C., Colgan, S. P. J. Exp. Med. 2004, 200, 1395-1405.

(3) García-Ayllón, M. S., Campoy, F. J., Vidal, C. J., Muñoz-Delgado, E. J. Neurosci. Res. 2001, 66, 656-665.

(4) Morote-García J.C., García-Ayllón M.S., Campoy F.J., Vidal C.J., Muñoz-Delgado E. Int. J. Biochem. Cell Biol. 2004, 36(3), 422-433.

(5) Morote-Garcia J.C., Sanchez Del Campo L.F., Campoy F.J., Vidal C.J., Munoz-Delgado E. Int. J. Biochem. Cell Biol. (in press)

(6) Lehto, M. T., Sharom, F. J. Biochem. J. 1998, 332, 101-109.

(7) Strohmeier, G. R., Lencer, W. I., Patapoff, T. W., Thompson, L. F., Carlson, S. L., Moe, S. J., Carnes, D. K., Mrsny, R. J. y Madara, J. L. J. Clin. Invest. 1997, 99, 2588-2601.

(8) Palestini, P., Calvi, C., Conforti, E., Daffara, R., Botto, L. y Miserocchi, G. J. Appl. Physiol. 2003, 95, 1446-1452.

(9) Eltzschig, H. K., Ibla, J. C., Furuta, G. T., Leonard, M. O., Jacobson, K. A., Enjyoji, K., Robson, S. C. y Colgan, S. P. J. Exp. Med. 2003, 198, 783-796.

\section{Mast cells secrete IL-15 by microvesicles shedding upon P2X $_{7}$ receptor stimulation}

Elena Bulanova, Vadim Budagian, Zane Orinska and Silvia Bulfone-Paus

Department of Immunology and Cell Biology, Research Center Borstel, D-23845 Borstel, Germany

ebulanova@fz-borstel.de

Mast cells are recognized as the key cells of allergic inflammatory reactions. They express and secrete a number of pro-inflamatory cytokines and chemokines. Interleukin-15 (IL-15) is a potent anti-apoptotic cytokine and a regulator of T, B and NK cells differentiation and proliferation. Bone marrow-derived mast cells (BMMCs) constitutively express IL-15 mRNA and this expression is further upregulated by LPS stimulation. However, there is no evidence for IL-15 cytokine secretion from activated mast cells. Recently, we have reported that ATP induces apoptosis of BMMCs, as well as triggers pro-inflamatory cytokine secretion, presumably in the time period between commitment to apoptosis and actual cell death. ${ }^{1}$ Here we show that BMMCs release annexin-V-positive microvesicles upon Bz-ATP stimulation. These vesicles contain biologically active IL-15, which later appears in the vesicle-free supernatant and stimulates the proliferation of the IL-15-dependent CTLL cell line. The IL-15containing microvesicles were also found in the supernatants from THP-1 monocytic cell line upon agonistic P2X7 stimulation. Thus, the microvesicle shedding mechanism constitutes one of secretory pathways for a release of IL15 in mast cells and monocytes, which might play an important role in regulation by IL-15 of diverse physiological processes or their pathological deviations.

${ }^{1}$ E. Bulanova, V. Budagian, Z. Orinska, M. Hein, F. Petersen, L. Thon, D. Adam, and S. Bulfone-Paus. Extracellular ATP induces cytokine expression and apoptosis through P2X7 receptor in murine mast cells. J. Immunol. 2005, 174, 3880-3890. 


\title{
Mechanism of 2-chloroadenosine toxicity to PC3 cell line.
}

Ilaria Bellezza ${ }^{1}$, Massimliano Agostini ${ }^{2}$, Zoran Culig ${ }^{3}$, Alba Minelli ${ }^{1}$.

${ }^{T}$ Dipartimento di Medicina Sperimentale Scienze Biochimiche, Sezione Biochimica Cellulare, ${ }^{2}$ Dipartimento di Medicina Clinica e Sperimentale, Sezione di Farmacologia, Università di Perugia, via del Giochetto, 06123, Perugia, Italia. $^{3}$ Department of Urology, Innsbruck Medical University, Anichstrasse 35, A-6020 Innsbruck, Austria. ilibelle@email.it

Adenosine, a ubiquitous nucleoside, serves as a building block of nucleic acids and energy storage molecules, as a substrate for multiple enzymes, and as an extracellular modulator of cellular activity. Extracellular adenosine can be taken up by the cell and undergo a series of metabolic transformations or can bind to specific G-proteincoupled receptors (1). Adenosine has been linked to a variety of physiological processes where its effects depend either on extracellular adenosine concentration or on the expression of different adenosine receptor subtypes and the signal transduction mechanism initiated by the binding of specific agonists. 2-chloroadenosine (2-CADO), a non-selective adenosine receptors agonist as well as an hydrolysis- and deaminase-resistant adenosine analogue, induces apoptosis in several cells with mechanisms still to be resolved (2). Prostate cancer (PCa), the second leading cause of cancer deaths in men, is a complex disease whose etiology involves genetic changes, activated oncogenes and growth factors, androgenic hormones, and dietary factors (3). In this study we have used the PC3 cell line that reflects molecular pathways of clinical androgen-independent PCa (4), to investigate the effects and the mechanism of action of 2-CADO on cellular proliferation, cell cycle kinetic, and DNA synthesis. We have shown that analogue uptake and its phosphorylations are fundamental pre-requisites for cellular response and that the strong cytotoxic effect exerted by 2-CADO on PC3 cells derives from irreversible cytotoxic effect due to permanent aberrant situations. 2-CADO elicits toxic actions by distributing cells into S-phase causing DNA strand breaks, and interfering with DNA synthesis through enzymes that lead to a nucleotide pool imbalance. The observed disappearance of all deoxy-ribonucleoside triphosphate suggests a strong inhibition of ribonucleotide reductase, one of the rate-limiting enzyme in DNA biosynthesis whereas all ribonucleoside triphosphates, after a marked initial reduction, showed a small re-synthesis, probably due to enzymes involved in de novo synthetic purine and pyrimidine nucleotide pathways whose activities have not been inhibited by 2-CADO treatment. The use of inhibitors of different key enzymes of nucleotides metabolism and DNA biosynthesis has shown that these enzymes are also inhibited by 2-CADO. Only 5-azacytidine was capable of potentiating the cytotoxic effect of 2CADO suggesting that the two compounds target different enzymes and that 2-CADO does not inhibit DNA methylation reactions.

1. Ralevic, V., and Burnstock, G. (1998) Receptors for purines and pyrimidines. Pharmacol Rev, 50, 413-92.

2. Bellezza, I., Agostini, M., Liguori, L., Minelli, A. (2005) Cytostatic effect of the nucleoside analogue 2-Chloroadenosine on human prostate cancer cell line. Current Pharmaceutical Analysis, 1, 265-272

3. Routh, J.C., Leibovich, B.C. (2005) Adenocarcinoma of the prostate: epidemiological trends, screening, diagnosis, and surgical management of localized disease. Mayo Clin Proc, 80, 899-907.

4. Culig, Z., Steiner, H., Bartsch, G., Hobisch, A. (2005) Mechanisms of endocrine therapy-responsive and unresponsive prostate tumours. Endocr Relat Cancer, 12, 229-244.

\section{Mechanism of extracellular nucleotide-induced eNOS activation in human endothelial cells.}

\author{
Cleide Gonçalves da Silva, Anke Specht, Elzbieta Kaczmarek \\ Beth Israel Deaconess Medical Center, Harvard Medical School, Boston, MA, USA. \\ cdasilva@bidmc.harvard.edu
}

Nitric oxide (NO) that is generated by endothelial NO synthase (eNOS), regulates vascular tone and leukocyte adhesion to the endothelium, inhibits vascular smooth muscle cell proliferation and platelet aggregation, and modulates cell apoptosis and angiogenesis. These diverse functions give endothelial-derived NO a significant influence on vascular function and integrity. The pathological effects of altered NO production and bioavailability 
have been linked to hypertension, hypercholesterolaemia, endothelial cell dysfunction, atherosclerosis, diabetes, heart failure, and poor wound healing. In endothelial cells (EC), eNOS can be activated by various stimuli, including vascular endothelial growth factor, bradykinin, tumor necrosis factor alpha, histamine, thrombin, endothelin-1, and angiotensin II. Multiple protein kinases, including phosphoinositide-3- kinase (PI3K), cAMP-dependent protein kinase (PKA), extracellular signal-regulated kinase (ERK), protein kinase C (PKC), $\mathrm{Ca}^{2+} /$ calmodulin-dependent kinase (CaMK) and AMP-activated protein kinase (AMPK) have been show to be involved in the activation of eNOS by phosphorylation of Ser-1177. ATP, which extracellular concentration can rapidly increase in response to several stress events, also can induce generation of NO. However, a precise mechanism of this observation has not been identified.

In this study we demonstrate that extracellular nucleotides, ATP, ADP and UTP (but not UDP or BzATP) mediate eNOS phosphorylation on Ser-1177 in human umbilical vein EC (HUVEC). We conclude that P2Y1, $\mathrm{P} 2 \mathrm{Y} 2$ and/or P2Y4 receptors, while not $\mathrm{P} 2 \mathrm{Y} 6, \mathrm{P} 2 \mathrm{Y} 11$ and $\mathrm{P} 2 \mathrm{X}$ receptors, are involved in eNOS activation in HUVEC. We have also investigated a role of various kinases in extracellular nucleotide-initiated pathways of eNOS activation. We excluded PI3K, ERK and CaMK II, since an inhibition of these kinases had no effect on nucleotide-induced phosphorylation of Ser-1177 in eNOS. Recently, we revealed that in EC, extracellular nucleotides induce AMPK activation in the $\mathrm{Ca}^{2+}$ - and CaMK kinase-dependent manner. In addition, our data indicate that nucleotide-induced phosphorylation of eNOS depends on an increase in the concentration of intracellular $\mathrm{Ca}^{2+}$ since BAPTA-AM (a chelator of $\mathrm{Ca}^{2+}$ ) totally inhibits eNOS activation. We presume that CaMK and AMPK might be upstream components of purinergic activation of eNOS in EC. We hypothesize that P2 receptors could be a target for the pharmacologic regulation of eNOS activity and its effects on EC function.

\section{Methanol changes ectonucleotidase and acetylcholinesterase activities from central nervous system of zebrafish (Danio rerio)}

$\underline{\text { Maurício Reis Bogo }}^{1}$, Eduardo Pacheco Rico ${ }^{1,2}$, Denis Broock Rosemberg ${ }^{1}$, Mario Roberto Senger ${ }^{1,2}$, Marcelo de Bem Arizi ${ }^{1}$, Giovana Farinon Bernardi ${ }^{1}$, Renato Dutra Dias ${ }^{1}$, Carla Denise Bonan ${ }^{1}$

${ }^{1}$ Faculdade de Biociências, Pontifícia Universidade Católica do Rio Grande do Sul. Porto Alegre, RS, Brazil;

${ }^{2}$ Departamento de Bioquímica, UFRGS, Porto Alegre, RS, Brazil.

mbogo@pucrs.br

Methanol has been described as an environment contaminant that affects the aquatic biota. Furthermore, it is also recognized as a neurotoxin capable of producing visual impairment or blindness, affecting optic nerve andretina. Studies have suggested that ATP and acetylcholine are co-released together in a $\mathrm{Ca}^{+2}$-dependent manner. After being released, the neurotransmitter acetylcholine is metabolized into choline and acetate by acetylcholinesterase (AChE), whereas ectonucleotidases controls the ATP and its metabolites levels. The zebrafish genome has demonstrated regions of syntenic relationship with human genes. In addition, this species has being useful in biochemistry and toxicological studies, due to the presence of similar physiological response to apomorphic animals when exposed to different drugs. Considering (i) the NTPDase and ecto-5'-nucleotidase activities in brain membranes of zebrafish recently characterized; (ii) the co-release of ATP and acetylcholine at nerve endings; (iii) the use of methanol in zebrafish embryo cryoconservation protocols, the aim of this study is to test the in vivo (acute) and in vitro effects of methanol on ectonucleotidase and acetylcholinesterase activities in zebrafish brain.

The in vitro experiments demonstrated that methanol promoted a significant inhibition of ATP hydrolysis (19\% and $34 \%$ ) at 1.5 and $3.0 \%$, respectively, but ADP hydrolysis decreased only at $3.0 \%$ (29\%). However, ecto-5'nucleotidase was not affected by methanol treatment. After 1 hour of in vivo exposure at varying methanol concentrations $(0.25,0.5$ and $1.0 \%)$, there were significant decrease of ATP $(26.5 \%$ and $45.1 \%)$ and ADP $(26.3 \%$ and $30.0 \%$ ) hydrolysis at 0.5 and $1.0 \%$. The acute treatment with methanol was not able to promote any significant change on ecto-5'-nucleotidase activity in zebrafish brain membranes in all concentrations tested. The significant inhibition on NTPDase activity in zebrafish brain membranes observed after in vivo methanol treatment could be attributed to an indirect effect of this compound, because both methanol concentrations $(0.5$ and $1 \%)$ did not promote any significant change on ATP and ADP hydrolysis when added directly to the reaction medium. The in vitro exposure of methanol did not present significant effect on AChE activity when compared to control 
group. Nevertheless, zebrafish exposed to methanol had a significant inhibition of AChE activity in all concentrations tested (30-38\%). Based on these findings, it is possible to suggest that methanol metabolites, such as formaldehyde or formate could act on zebrafish brain NTPDases and AChE, modulating ATP, ADP and ACh hydrolysis.

Supported by: FAPERGS, CNPq, CAPES, TWAS

\section{Microvesicle shedding as a mechanism of release of proinflammatory cytokines in dendritic cells and macrophages}

Cinzia Pizzirani, Sara Gulinelli, Davide Ferrari, Paola Chiozzi, and Francesco Di Virgilio

Department of Experimental and Diagnostic Medicine, Section of General Pathology, University of Ferrara, Via Borsari 46, 44100, Ferrara, Italy

cinziapizzirani@libero.it

The activation by extracelluar ATP of purinergic P2 receptors (P2R), in particular of $\mathrm{P} 2 \mathrm{X}_{7} \mathrm{R}$, has been shown to be a potent stimulus for maturation and release of proinflammatory cytokines by immune cells ${ }^{1,2}$. How cytokines are externalized is still under investigation, even if in the last years there is evidence in human monocytes ${ }^{3}$ and microglia ${ }^{4}$ of a mechanism of IL-1 $\beta$ release mediated by shedding of small microvesicles.

Here we demonstrate that monocyte-derived dendritic cells and macrophages release, respectively, IL-1 $\beta$ and IL18 by the same mechanism. Extracellular $\mathrm{Ca}^{2+}$ chelation highly reduced the phenomenon, suggesting the release of microvesicles to be a $\mathrm{Ca}^{2+}$-dependent mechanism. The release of microvesicles is triggered by activation of $\mathrm{P}_{2} \mathrm{X}_{7}$ receptor with millimolar concentrations of ATP and its other agonist BzATP. Moreover, pretreatment of dendritic cells and macrophages with oxidized ATP, a covalent blocker of $\mathrm{P} 2 \mathrm{X}_{7} \mathrm{R}$, inhibits microvesicle shedding. The microvesicles are clearly of plasma membrane origin as confirmed from the presence of a series of proteins that characterize antigen presenting cells such as MHC-II molecules, together with P2 receptors. The cytokines stored in the microvesicles are released in the medium in the presence of cytotoxic extracellular ATP concentrations. This suggests a dual role for the nucleotide: as a danger signal for the immune cells, triggering the externalization of IL-1 $\beta$ and IL-18 and, after microvesicle shedding, as a stimulus able to induce microvesicles lysis and cytokine delivery.

1. Ferrari D, Chiozzi P, Falzoni S, Dal Susino M, Melchiorri L, Baricordi OR, Di Virgilio F. Extracellular ATP triggers IL-1 beta release by activating the purinergic P2Z receptor of human macrophages. J Immunol. 159: 1451-8, 1997.

2. Labasi JM, Petrushova N, Donovan C, McCurdy S, Lira P, Payette MM, Brissette W, Wicks JR, Audoly L, Gabel CA. Absence of the P2X7 receptor alters leukocyte function and attenuates an inflammatory response. J Immunol 168: 6436-45, 2002

3. MacKenzie A, Wilson HL, Kiss-Toth E, Dower SK, North RA, Surprenant A. Rapid secretion of interleukin1beta by microvesicle shedding. Immunity 15: 825-35, 2001.

4. Bianco F, Pravettoni E, Colombo A, Schenk U, Moller T, Matteoli M, Verderio C. Astrocyte-derived ATP induces vesicle shedding and IL-1 beta release from microglia. J Immunol 174: 7268-77, 2005.

\section{Mitochondrial dysfunction induced by a cytotoxic adenine dinucleotide produced by ADP-ribosyl cyclases from cADPR}

\footnotetext{
${ }^{1}$ Bruzzone, S., ${ }^{1}$ Basile, G., ${ }^{2}$ Dodoni, G., ${ }^{2}$ Kaludercic, N., ${ }^{1}$ Millo, E., ${ }^{2}$ Di Lisa, F., ${ }^{1}$ De Flora, A., and ${ }^{1}$ Zocchi, E.

${ }^{1}$ Dept. of Experimental Medicine, Section of Biochemistry, and Center of Excellence for Biomedical Research, University of Genova, Viale Benedetto XV/1, Genova, Italy;

${ }^{2}$ Dept. of Biological Chemistry, University of Padova, Viale G. Colombo 3, Padova, Italy. santina.bruzzone@unige.it
} 
ADP-ribosyl cyclases (ADPRCs) are a family of multifunctional enzymes, present from protists to mammals and higher plants, that generate a number of products, i.e. cyclic ADP-ribose (cADPR), ADP-ribose, nicotinic acid adenine dinucleotide and the ADP-ribose dimer, affecting the intracellular free calcium concentration $\left(\left[\mathrm{Ca}^{2+}\right]_{\mathrm{i}}\right)$. Recently, ADPRCs from Porifera, molluscs and mammals have been shown to catalyse an additional reaction on cADPR, introducing adenine into the $\mathrm{C1}^{\prime}-\mathrm{N} 1$ bond of the cyclic nucleotide and generating three new adenine homodinucleotides, called P18, P24 and P31 from their HPLC retention times (1). P31 proved to be diadenosine 5',5-P1P2-diphosphate (Ap2A). P18 and P24 are two isomers of Ap2A, each containing an unusual N-glycosidic bond between the newly introduced adenine and one ribose: C1'-N1 in P18 and C1'-N3 in P24 (1). They are the first dinucleotides featuring a non-canonical N-glycosidic bond demonstrated in $\mathrm{ADPRC}^{+}$animal cells. P18 and P24 both show a potent cytotoxic effect on cell lines and an even more severe growth-inhibitory effect on HP colony growth (with $\mathrm{IC}_{50}$ values of 1.0 and $0.18 \mu \mathrm{M}$, respectively) (1).

These results prompted us to investigate the mechanisms underlying the cytotoxic effect of P18 and of P24, also in view of their potential use in clinical hematology as antileukemic agents. Results obtained identify mitochondria as a major target of P24 toxicity, with micromolar concentrations of the dinucleotide, i) dissipating the mitochondrial proton gradient in intact cells, ii) inhibiting the respiratory chain in isolated mitochondria, acting on complex I, and, iii) inducing opening of the permeability transition pore (PTP). Conversely, P18 does not show significant effects on any of these functions, indicating the absolute requirement for a $\mathrm{C}^{\prime}-\mathrm{N} 3$ bond in the adenine dinucleotide to exert its mitochondrial effects.

These results identify P24 as a novel endogenous regulator of mitochondrial function in mammalian cells, thus extending the physiological functions of the ADPRC product family to include control of cell respiration. Interestingly, cADPR and Ap2A partially antagonise P24-induced cytotoxicity and proton gradient dissipation, suggesting that the relative intracellular concentrations of these ADPRC products may decide upon cell life and death.

\title{
References
}

1. Basile, G., Taglialatela-Scafati, O., Damonte, G., Armirotti, A., Bruzzone, S., Guida, L., Franco, L., Usai, C., Fattorusso, E., De Flora, A., and Zocchi, E. (2005) Proc. Natl. Acad. Sci. USA 102, 14509-14.

\section{Modification by kainate-induced convulsions of the density of presynaptic P2X receptors in the rat hippocampus}

\author{
$\underline{\text { Jean Pierre Oses }}^{1,2}$, Ana Patrícia Simões ${ }^{1}$, Paula Canas ${ }^{1}$, Ricardo J. Rodrigues ${ }^{1}$, João J. F. Sarkis ${ }^{2}$, \\ Catarina R. Oliveira ${ }^{1}$, Rodrigo A. Cunha ${ }^{1}$ \\ ${ }^{1}$ Ctr. Neuroscience Coimbra, Inst. Biochemistry, Fac.Medicine, Univ.Coimbra, Portugal, ${ }^{2}$ Dept. Of Biochemisty, \\ ICBS, UFRGS, Brazil.
}

(jean.Pierre.oses@gmail.com)

Epilepsy is a common neurological condition, affecting $0.4-1 \%$ of the world's population. Temporal lobe epilepsy is a chronic neurologic disorder characterized by recurrent seizures that have a behavioral expression in the form of convulsions accompanied by a modification of limbic circuits and the frequent presence of neurodegenerative features. Seizure activity results from a broken excitatory-inhibitory balance in the brain circuits and there is evidence for a role of excitatory amino acids, mainly glutamate, in the neurotoxic effects of seizures, mostly through activation of ionotropic glutamate receptors. Previous studies have shown that ATP is released upon stimulation of hippocampal glutamatergic afferent (Wieraszko et al., 1989), in particular upon stimulation at higher frequencies (Cunha et al., 1996) that are characteristic of seizure activity. Furthermore, we have recently shown that ionotropic receptors for ATP $\left(\mathrm{P} 2 \mathrm{X}_{1-3}\right)$ are presynaptically located and control the release of glutamate from hippocampal nerve terminals (Rodrigues et al., 2005). Thus, as a starting point to explore the potential role of these P2X receptors in the control of seizure activity and consequent neurodegeneration, we now explored if the density of these receptors was modified by a convulsive period triggered by the administration of kainate, a validated animal model of temporal lobe epilepsy (Ben-Ari, 1985).

Male Wistar rats were divided into 2 groups, i.e. control (saline-injected) and kainate $(10 \mathrm{mg} / \mathrm{kg}$, intra-peritoneally). All kainate-injected rats displayed a rapid (within $30 \mathrm{~min}$ ) period of severe convulsions, which reached stage $4-5$ of the Racine's scale. The animals were sacrificed 24 hours after kainate administration. Total membranes and 
nerve terminal membranes were prepared from the hippocampi of both kainate and saline-injected rats for Western blot analysis of $\mathrm{P} 2 \mathrm{X}_{1-3}$ receptor density, using previously validated antibodies (Rodrigues et al., 2005).

Our preliminary results indicate that the density of $\mathrm{P} 2 \mathrm{X}_{1}$ receptors was not modified in total membranes, but was selectively decreased in nerve terminal membranes of kainate-injected rats compared to saline controls. In contrast, the density of $\mathrm{P} 2 \mathrm{X}_{2}$ receptors was decreased both in total membranes and in nerve terminal membranes, whereas the density of $\mathrm{P}_{2} \mathrm{X}_{3}$ receptors was not modified in either total or nerve terminal membranes of kainate compared to saline control rats.

These results suggest that there is a global tendency for a decrease of presynaptic P2X receptors in the hippocampus of rats that have suffered a convulsive period. However, the discrete decrease of particular P2X receptor subtypes is consistent with the hypothesis that these receptors may contribute for the development of seizures and/or of neurodegeneration during epilepsy, an issue that is currently being explored. (Supported by CNPq Brazil and FCT).

Ben-Ari,Y. Neuroscience 1985 14, 375-403.

Cunha, R.A.; Vizi, E.S.; Ribeiro, J.A.; Sebastião, A.M.; J. Neurochem. 1996 67, 2180-218.

Rodrigues, R.J. et al. J. Neurosci. 2005 27, 6286-6295.

Wieraszko A.; Goldsmith, G.; Seyfried, T.N. Brain Res. 1989 485, 244-250.

\title{
Modification of adenosine modulation of acetylcholine release in the hippocampus of aged rats
}

\author{
Ricardo J Rodrigues ${ }^{1}$, Luísa V Lopes ${ }^{2}$, Paula M Canas ${ }^{1}$, Rodrigo A Cunha ${ }^{1}$ \\ ${ }^{1}$ Ctr. Neuroscience Coimbra, Fac.Medicine, Univ.Coimbra, ${ }^{2}$ Inst. Pharmacol. Neurosciences, Inst. Mol. Medicine, \\ Univ. Lisbon, Portugal. \\ (lvlopes@fm.ul.pt)
}

Adenosine is a neuromodulator that acts through activation of inhibitory $\mathrm{A}_{1}$ receptors $\left(\mathrm{A}_{1} \mathrm{Rs}\right)$ and facilitatory $\mathrm{A}_{2 \mathrm{~A}} \mathrm{Rs}$, which are mainly located in synapses (Fredholm et al., 2005). Upon ageing, adenosine modulation is modified with increased levels of adenosine, increased density and effects of $\mathrm{A}_{2 \mathrm{~A}} \mathrm{Rs}$ and a decrease of $\mathrm{A}_{1} \mathrm{Rs}$ (Lopes et al., 1999; Cunha et al., 2001). Notably, $\mathrm{A}_{2 \mathrm{~A}} \mathrm{R}$ antagonists recover memory deficits in aged animals (Predinger et al., 2005). Since age-related memory deficits are associated with a decreased a cholinergic function, we now investigated how aging affects the density of adenosine receptors in cholinergic terminals and the tonic adenosine modulation of acetylcholine (ACh) release in the rat hippocampus.

In young adult rats $(2$ months old), $64.4 \pm 3.1 \%$ of cholinergic terminals (immuno-positive for vesicular ACh transporters) were endowed with $\mathrm{A}_{1} \mathrm{Rs}(\mathrm{n}=5)$ and $35.9 \pm 3.4 \%$ possessed $\mathrm{A}_{2 \mathrm{~A}} \mathrm{Rs}(\mathrm{n}=5)$. In aged rats $(24$ months old), the percentage of cholinergic terminals with $\mathrm{A}_{1} \mathrm{Rs}$ was preserved $(53.3 \pm 5.2 \%, \mathrm{n}=7, \mathrm{P}>0.05)$, whereas that of $\mathrm{A}_{2 \mathrm{~A}}$ Rs was larger $(49.0 \pm 3.4 \%, \mathrm{n}=7, \mathrm{P}<0.05)$ and $\mathrm{A}_{1}$ Rs and $\mathrm{A}_{2 \mathrm{~A}}$ Rs were co-located in $36.4 \pm 4.4 \%(\mathrm{n}=7)$ of cholinergic terminals.

Electrical stimulation $(40 \mathrm{~V}, 3 \mathrm{msec}, 2 \mathrm{~Hz}$ for $2 \mathrm{~min})$ of hippocampal slices triggered ACh release that was larger in young than aged rats (Lopes et al., 1999). $\mathrm{A}_{1} \mathrm{R}$ blockade (50 $\mathrm{nM}$ DPCPX) enhanced $(17.2 \pm 3.8 \%, \mathrm{n}=5) \mathrm{ACh}$ release in young rats and this tonic $\mathrm{A}_{1} \mathrm{R}$-mediated inhibition was greater in aged rats $(35.2 \pm 4.0 \%, \mathrm{n}=6, \mathrm{P}<0.05)$. There was also a more pronounced role of adenosine tonically facilitating ACh release in aged rats since the blockade of $\mathrm{A}_{2 \mathrm{~A}}$ Rs with ZM241385 $(20 \mathrm{nM})$ decreased $\mathrm{ACh}$ release by $11.5 \pm 1.3 \%(\mathrm{n}=7)$ in aged rats and was devoid of effects $(-4.0 \pm 1.4 \%, \mathrm{n}=6)$ in young adult rats. This indicates that lower levels of adenosine in young adults are only tonically inhibiting $\mathrm{ACh}$ release through $\mathrm{A}_{1} \mathrm{Rs}$, whereas the greater levels of adenosine in aged rats (Cunha et al., 2001) cause a greater $A_{1} R$-mediated inhibition and a simultaneous $A_{2 A} R$-mediated facilitation of ACh release. Accordingly, removing endogenous extracellular adenosine with $2 \mathrm{U} / \mathrm{ml}$ adenosine deaminase (converts adenosine into its centrally inactive metabolite, inosine) caused a similar facilitation of ACh release in young adult $(15.3 \pm 2.8 \%, \mathrm{n}=6)$ and aged rats $(19.3 \pm 1.1 \%, \mathrm{n}=7, \mathrm{P}>0.05)$.

These results indicate that there is an enhanced $A_{2 A} R$ density and facilitation of ACh release to compensate for the enhanced $A_{1} R$ density and presumably greater tonic $A_{1} R$ modulation of $A C h$ release, preserving the global adenosine modulation of $\mathrm{ACh}$ release in aged rats. Furthermore, since $\mathrm{A}_{2 \mathrm{~A}} \mathrm{R}$ antagonists inhibit $\mathrm{ACh}$ release, it is 
unlikely that the beneficial effects of $\mathrm{A}_{2 \mathrm{~A}} \mathrm{R}$ antagonists on memory performance in aged rats result from modulation of ACh release. (Supported by Portuguese Society of Neuroscience, Pfizer and FCT).

Cunha, R.A.; Almeida, T.; Ribeiro, J.A. J. Neurochem. 2001 76, 372-382.

Fredholm B.B. et al. Int. Rev. Neurobiol. 2005 63, 191-270.

Lopes, L.V.; Cunha, R.A.; Ribeiro, J.A. J. Neurochem. 1999 73, 1733-1738

Prediger, R.D.; Batista, L.C.; Takahashi, R.N. Neurobiol. Aging 2005 26, 957-964

\section{Modulation by LL-37 of the purinergic responses of mouse peritoneal Macrophages}

Stéphanie Pochet $^{1}$, Elie Kabré ${ }^{2}$, Manuela De Lorenzi ${ }^{1}$, Do Duc Khanh $\operatorname{Tran}^{1}$ and Jean-Paul Dehaye ${ }^{1}$

${ }^{1}$ Biochemistry and Cellular Biology, Pharmacy, Université libre de Bruxelles, Belgium

${ }^{2}$ Biochemistry and Immunology, UFR-SDS, Université Ouagadougou, Burkina-Faso

spochet@ulb.ac.be

LL-37 is a cationic peptide composed of 37 amino acids residues first described as antimicrobial agent but which possesses more potent immunomodulatory properties in physiological conditions. It is formed after the cleavage of its inactive pro-form, the human cathelicidin hCAP-18 by proteinase-3. LL-37 is produced by neutrophils, monocytes, epithelial cells,...Its structure in $\alpha$-helix accounts for its destabilizing effect on microbial membranes but LL-37 is also involved in apoptosis, chemokine production, angiogenesis, wound healing, anti-endotoxin activity and chemotaxis (Bowdish et al., 2005). LL-37 was shown to induce interleukin-1 $\beta$ processing and release in human LPS-primed monocytes after $\mathrm{P}_{2} \mathrm{X}_{7}$ receptor activation (Elssner et al, 2004).

The aim of the study was to examine the role of $\mathrm{P} 2 \mathrm{X}_{7}$ receptor in LL-37 responses observed in mouse peritoneal macrophages and to study its possible modulatory effects on purinergic responses. The experiments were conducted in peritoneal macrophages from normal mice and $\mathrm{P}_{2} \mathrm{X}_{7}$ receptor $\mathrm{KO}$ mice (Dr Gabel, Pfizer, Groton, $\mathrm{Ct}$ ). $3 \mu \mathrm{M}$ LL-37 increased the intracellular concentration of calcium $\left(\left[\mathrm{Ca}^{2+}\right]_{\mathrm{i}}\right)$ in normal and KO mice. Similarly, LL37, $1 \mu \mathrm{M}$, stimulated a phospholipase $\mathrm{A}_{2}\left(\mathrm{PLA}_{2}\right)$ activity in both mice. This shows that, in mouse peritoneal macrophages, the effects of LL-37 are not mediated by $\mathrm{P} 2 \mathrm{X}_{7}$ receptors. We then tested if this peptide could modulate the cellular responses to purinergic agonists. The calcium response of macrophages to $1 \mathrm{mM}$ ATP was modified after a preincubation of the cells with $1 \mu \mathrm{M}$ LL-37. Indeed, the $\mathrm{P}_{2} \mathrm{X}_{7}$ part of the response was completely lost and the remaining ATP response was similar to that obtained in KO mice. This inhibitory effect was reversible, elicited by low LL-37 concentrations $(0.3 \mu \mathrm{M})$, also observed on the Bz-ATP response and reproduced by polymyxin $\mathrm{B}$, another cationic antibiotic. We then examined if this inhibitory effect was present on other $\mathrm{P}_{2} \mathrm{X}_{7^{-}}$ mediated responses. Pore formation and phospholipase D activation induced by $1 \mathrm{mM}$ ATP were not abolished by $1 \mu \mathrm{M}$ LL-37 while PLA 2 activation was potentiated by the peptide.

In conclusion, LL-37 does not stimulate $\mathrm{P} 2 \mathrm{X}_{7}$ receptors in mice peritoneal macrophages but modulates their responses. The involved mechanisms will be discussed.

Bowdish DM, Davidson DJ, Lau YE, Lee K, Scott MG, Hancock RE. (2005) Impact of LL-37 on anti-infective immunity. J Leukoc Biol. 77,451-459.

Elssner A, Duncan M, Gavrilin M, Wewers MD. (2004) A novel P2X7 receptor activator, the human cathelicidinderived peptide LL37, induces IL-1 beta processing and release. J Immunol 172, 4987-4994.

S. Pochet is a postdoctoral researcher of the Fonds National de la Recherche Scientifique of Belgium 


\title{
Modulation of cardiac sarcoplasmic reticulum calcium channel by adenosine: a protein kinase $\mathrm{C}$ - dependent pathway
}

\author{
Sandra Ghelardoni, Sabina Frascarelli, Vittoria Carnicelli, Simonetta Ronca-Testoni, Riccardo Zucchi \\ Dipartimento di Scienze dell'Uomo e dell'Ambiente, University of Pisa, Pisa, Italy \\ s.ghelardoni@med.unipi.it
}

Adenosine is known to increase myocardial resistance to ischemia and reperfusion ${ }^{1,2}$ but the mechanisms responsible for cardioprotection are not entirely understood. In rat heart, $\mathrm{A}_{3}$ adenosine receptor stimulation was found to reduce $\left[{ }^{3} \mathrm{H}\right]$-ryanodine binding and sarcoplasmic reticulum (SR) $\mathrm{Ca}^{2+}$ release, potentially leading to a depletion of the SR $\mathrm{Ca}^{2+}$ pool, and to the delay of the development of cytosolic $\mathrm{Ca}^{2+}$ overload during ischemia ${ }^{3}$. In the present work we investigated the effect of the transduction pathway responsible for the anti-ischemic effect in an isolated rat heart model.

Hearts were perfused for 20 min with control buffer or with $100 \mathrm{nM}$ IB-MECA ( $\mathrm{N}^{6}$-(iodobenzyl)-adenosine-5' $-\mathrm{N}$ methyluronamide), an $\mathrm{A}_{3}$ receptor agonist, $10 \mu \mathrm{M}$ U-73122, a phospholipase $\mathrm{C}$ inhibitor, or $2 \mu \mathrm{M}$ chelerythrine, a protein kinase $\mathrm{C}$ inhibitor. At the end of each perfusion, the hearts were homogenized and used for one of the following procedures: $\left[{ }^{3} \mathrm{H}\right]$-ryanodine binding assay, RT-PCR or western blotting experiments.

Perfusion with IB-MECA produced a significant increase in coronary flow while in the presence of chelerythrine no significant change was observed in hemodynamic variables. U-73122 determined a remarkable negative inotropic action ( $75 \%$ reduction in cardiac output, $\mathrm{P}<0.01 ; 35 \%$ reduction in systolic aortic pressure, $\mathrm{P}<0.05)$. IBMECA perfusion caused a significant decrease in ryanodine binding (Bmax: $379 \pm 15$ vs. $434 \pm 15 \mathrm{fmol} / \mathrm{mg}$ of protein, $\mathrm{P}<0.05)$, which was abolished by chelerythrine $(\mathrm{Bmax}=430 \pm 17 \mathrm{fmol} / \mathrm{mg}$ of protein, $\mathrm{P}=\mathrm{NS}$ vs control), but not by U-73122 (Bmax $=362 \pm 17 \mathrm{fmol} / \mathrm{mg}$ of protein, $\mathrm{P}<0.05$ vs. control). RT-PCR experiments showed that ryanodine receptor gene expression was not affected by IB-MECA. In Western blot experiments, ryanodine receptor phosphorylation on serine 2809 was not modified after perfusion with IB-MECA. In conclusion, the modulation of SR $\mathrm{Ca}^{2+}$ release by IB-MECA can be dependent on protein kinase $\mathrm{C}$ activation which, in our model, is not due to phospholipase $\mathrm{C}$ activation. Changes in ryanodine receptor gene expression or direct phosphorylation of the ryanodine receptor on serine 2809 residue do not appear to be involved in the molecular mechanisms of adenosine cardioprotection.

1. Berne, R.M., Cardiovasc. Res., 1993, 27(1), p. 2.

2. Ely, S.W. and. Berne, R.M, Circulation, 1992, 85(3), p. 893-904.

3. Zucchi R, Yu G, Ghelardoni S, Ronca F, Ronca-Testoni S. Cardiovasc. Res., 2001, 50, 56-64,

\section{Modulation of Endothelial ATP-Signaling by Hypoxia: Functional Consequences of HIF-1 dependent P2Y 2 Induction}

Thomas Weissmüller $^{1}$, Stefanie Kube ${ }^{1}$,Tobias Eckle ${ }^{1}$, Marion Faigle ${ }^{1}$, Andreas Robinson ${ }^{3}$, Volkhard A. J. Kempf $^{2}$, Sean P. Colgan ${ }^{3}$, and Holger K. Eltzschig ${ }^{1,3}$

${ }^{1}$ Department of Anesthesiology and Intensive Care Medicine, and ${ }^{2}$ Institut für Medizinische Mikrobiologie und Hygiene, Tübingen University Hospital, and ${ }^{3}$ Center for Experimental Therapeutics and Reperfusion Injury, Brigham and Women's Hospital, Harvard Medical School, Boston, MA 02115, USA.

Thomas.Weissmueller@freenet.de

Extracellular levels of adenine nucleotides (i.e. ATP, ADP, AMP) are elevated by various mechanisms during hypoxia $(1,2)$. Since vascular endothelial cells express multiple ATP receptors $(2)$, we considered the possibility that hypoxia might transcriptionally regulate the profile of endothelial ATP receptors. An mRNA screen of endothelial P2-receptors revealed that the $\mathrm{P}_{2} \mathrm{Y}_{2}$ isoform of ATP receptors was selectively upregulated by hypoxia. Using an siRNA approach, functional examination of endothelia exposed to hypoxia showed robust increases in $\mathrm{P}_{2} \mathrm{Y}_{2}$-dependent induction of vascular cell adhesion molecule-1 (VCAM-1) expression. Furthermore, using in vitro models of ATP-signaling, we showed increased endothelial permeability following $\mathrm{P}_{2} \mathrm{Y}_{2}-$ stimulation in 
post-hypoxic endothelia, and such alterations of permeability were absent following siRNA repression of the $\mathrm{P}_{2} \mathrm{Y}_{2}$ receptor. Moreover, loss and gain of function studies in cell lines with genetically modified activity of the transcription factor HIF-1 revealed that $\mathrm{P}_{2} \mathrm{Y}_{2}$ induction by hypoxia maps, at least in part, to HIF-1 dependent gene regulation. Taken together, these results identify the selective induction of $\mathrm{P} 2 \mathrm{Y}_{2}$ receptor by hypoxia. Such results define a potential pro-inflammatory signaling pathway mediated by extracellular ATP during hypoxia.

1. Eltzschig, H.K., L.F. Thompson, J. Karhausen, R.J. Cotta, J.C. Ibla, S.C. Robson, and S.P. Colgan. Blood 2004.

2. Weissmuller, T., H.K. Eltzschig, and S.P. Colgan. Purinergic Signalling 2005.

This work was supported by a Fortune grant F1211250.1 to TW and HKE.

\section{Modulation of myenteric motoneurons by endogenous adenosine: On the role of secreted adenosine deaminase}

Paulo Correia-de-Sá, Sara Adães, M. Alexandrina Timóteo, Cátia Vieira, Teresa Magalhães-Cardoso, Carlos Nascimento, Margarida Duarte-Araújo

Laboratório de Farmacologia/UMIB, Instituto de Ciências Biomédicas de Abel Salazar (ICBAS), Universidade do Porto, L. Prof. Abel Salazar, 2, 4099-003 Porto, Portugal

farmacol@icbas.up.pt

Adenosine is a ubiquitous component of cells that act as a homeostatic regulator in the nervous system (Cunha, 2001). Its main role in the nervous system is to regulate neuronal activity modulating neurotransmitter release, the postsynaptic component, and the nonsynaptic components. Besides the well-characterized inhibitory effect of adenosine in the gastrointestinal tract operated by neuronal $\mathrm{A}_{1}$ receptors (e.g. Nitahara et al., 1995), the involvement of $\mathrm{A}_{2}$ receptors mediating excitation of myenteric neurons was reported with conflicting results (Christofi et al., 1994). Recently, we demonstrated that endogenous adenosine plays a predominant facilitatory action on $\left[{ }^{3} \mathrm{H}\right]$ acetylcholine $\left(\left[{ }^{3} \mathrm{H}\right] \mathrm{Ach}\right)$ release from myenteric neurons of the rat ileum, via the activation of prejunctional facilitatory $\mathrm{A}_{2 \mathrm{~A}}$ receptors (Duarte-Araújo et al., 2004). The co-existence of both receptor subtypes on cholinergic neurons prompted the question of how does adenosine discriminate between these receptors to regulate synaptic transmission in the longitudinal muscle-myenteric plexus (LM-MP) of the rat ileum. Electrical stimulation of the LM-MP increased the outflow of adenosine, inosine and hypoxanthine. Myenteric neurons seem to be the main source of endogenous adenosine, since blockade of action potentials with tetrodotoxin $(1 \mu \mathrm{M})$ or omission of $\mathrm{Ca}^{2+}$ (plus EGTA, $1 \mathrm{mM}$ ) in the buffer essentially abolished nucleosides release, while adenosine outflow remained unchanged when smooth muscle contractions were prevented by nifedipine $(1 \mu \mathrm{M})$. Inhibition of ecto-5'-nucleotidase by concanavalin $\mathrm{A}\left(0.1 \mathrm{mg} \mathrm{ml}^{-1}\right)$ produced only a moderate decrease $(\sim 25 \%)$ on adenosine accumulation in the LM-MP, indicating that the extracellular catabolism of released ATP might not be a major source of the nucleoside. Data using the acetylcholinesterase inhibitor, physiostigmine $(10 \mu \mathrm{M})$, and several subtype-specific muscarinic receptor antagonists, 4-DAMP (100 nM), AF-DX $116(10 \mu \mathrm{M})$ and muscarinic toxin-7 $(1 \mathrm{nM})$, suggest that cholinergic motoneurons are endowed with muscarinic $\mathrm{M}_{3}$ autoreceptors facilitating the outflow of adenosine. Surprisingly, bath samples collected after stimulating the LM-MP exhibited a relatively high adenosine deaminase (ADA) activity $\left(0.60 \pm 0.07 \mathrm{U} \mathrm{ml}^{-1}\right)$, which increased in parallel with the accumulation of adenosine and its deamination products. Our findings are in keeping with the hypothesis that ADA secretion, along with a lessefficient dipyridamole-sensitive nucleoside transport system, may restrict endogenous adenosine actions to the synaptic region channelling to facilitatory $\mathrm{A}_{2 \mathrm{~A}}$ receptors activation. Such a local environment may also limit diffusion of exogenously added adenosine towards the active zones, as we showed that this constrain may be overcome by inhibiting ADA activity with erythro-9(2-hydroxy-3-nonyl) adenine $(50 \mu \mathrm{M})$.

Cunha, R.A., 2001. Neurochem. Int. 38, 107-125.

Nitahara, K., Kittel, A., Liang, S.D., Vizi, E.S., 1995. Neuroscience 67, 159-168.

Christofi, F.L., Baidan, L.V., Fertel, R.H., Wood, J.D., 1994. Neurogastroenterol. Mot. 6, 67-78.

Duarte-Araújo, M., Nascimento, C., Timóteo, M.A., Magalhães-Cardoso, M.T., Correia-de-Sá, P., 2004. Br. J. Pharmacol. 141, 925-934.

Research supported by FCT (POCTI/FCB/45549/2002, participation of FEDER funding). Determination of ADA activity was performed by Ms. M.J. Carvalho and M.I. Garrido (Lab. Química Clínica - HGSA-SA, Porto). 


\title{
Modulation of neurotransmitter release by $P 2 X$ and $P 2 Y$ receptors in the rat spinal cord
}

\author{
Attila Heinrich, Lilla Papp, Cecilia Csölle, E. Sylvester Vizi and Beáta Sperlágh \\ Laboratory of Molecular Pharmacology, Institute of Experimental Medicine, Hungarian Academy of Sciences, \\ Budapest, Hungary, \\ heinrich@koki.hu
}

Under normal conditions, pain is associated with electrical activity in small-diameter fibers of dorsal root ganglion (DRG) of the spinal cord. In addition, numerous studies have shown that the descending noradrenergic pathway from the locus coeruleus plays a crucial role in the modulation of sensory transmission in the spinal cord and thereby attenuates pain sensation. Electrophysiological studies suggest that glutamatergic transmission in the spinal cord is under the control of P2X receptors (Nakatsuka and Gu, 2001) and previous data (e.g. Sperlágh et al., 2002, Papp et al., 2004) indicate that the release of both glutamate and noradrenaline are subject to modulation by presynaptic facilitatory P2X and inhibitory P2Y receptors in the CNS, although there is a considerable regional heterogeneity in the underlying receptors subtypes involved in these actions. In this study rat spinal cord slices were stimulated electrically and the effect of different purinergic agonists and antagonists on $\left[{ }^{3} \mathrm{H}\right]$ noradrenaline and $\left[{ }^{3} \mathrm{H}\right]$ glutamate release were examined.

Among agonists, ATP, ADP and 2-methylthioadenosine 5'-disphosphate (2MeSADP) decreased concentrationdependently the electrical stimulation-evoked tritiated NA efflux from superfused rat spinal cord slices with the following rank order of agonist potency: 2MeSADP > ADP > ATP. The inhibitory effect of ATP could be counteracted by reactive blue $2(\mathrm{RB} 230 \mu \mathrm{M})$ and by the P2Y12/13 receptor antagonist 2-methylthioadenosine $5^{\prime}$ monophosphate (2-MeSAMP, $10 \mu \mathrm{M})$, and partly by the P2Y1 receptor antagonist MRS $2179(10 \mu \mathrm{M})$, but not by suramin $(300 \mu \mathrm{M})$ and PPADS $(30 \mu \mathrm{M})$. RB2 $(30 \mu \mathrm{M})$ and 2MeSAMP $(10 \mu \mathrm{M})$, per se, had no significant effect. On the other hand, 2-methylthioadenosine-5'-triphosphate (2-MeSATP, 10-300 $\mu \mathrm{M}$ ), and ADP at a lower concentration range $(100-600 \mu \mathrm{M})$ increased electrically evoked tritium overflow. The facilitatory effect of 2-MeSATP was antagonized by PPADS $(30 \mu \mathrm{M})$ and by the P2X1 receptor selective antagonist 4,4',4,4'-[Carbonylbis[imino5,1,3-benzenetriyl bis (carbonyl-imino)]] tetrakis (benzene-1,3-disulfonic acid) octasodium salt (NF449, $100 \mathrm{nM}$ ), but not by MRS $2179(10 \mu \mathrm{M})$.

When the release of $\left[{ }^{3} \mathrm{H}\right]$ glutamate measured, ATP, 2-MeSATP, and 2-MeSADP all decreased electrically evoked tritium overflow, with the following rank order of agonist potency: 2MeSADP > ATP > 2-MeSATP. The effect of ATP was fully antagonised by suramin $(300 \mu \mathrm{M})$ and by 2-MeSAMP $(10 \mu \mathrm{M})$, and partly by MRS $2179(10 \mu \mathrm{M})$, and PPADS $(30 \mu \mathrm{M})$.

In conclusion nucleotides exert dual and opposite modulation on the release of noradrenaline in the spinal cord of the rat. Whereas the inhibitory modulation is most likely mediated by P2Y12 and/or P2Y1 receptors, the facilitatory modulation is mediated by P2X1 receptors. In addition the release of glutamate is also subject to similar inhibitory modulation; however, the identity of underlying P2 receptor subtype awaits further investigation.

\section{References}

Nakatsuka, T. and Gu, J. G. (2001) ATP P2X receptor-mediated enhancement of glutamate release and evoked EPSCs in dorsal horn neurons of the rat spinal cord. J Neurosci 21, 6522-31.

Papp, L., Balazsa, T., Kofalvi, A., Erdelyi, F., Szabo, G., Vizi, E. S. and Sperlagh, B. (2004a) P2X receptor activation elicits transporter-mediated noradrenaline release from rat hippocampal slices. J Pharmacol Exp Ther 310, 973-80.

Sperlagh, B., Kofalvi, A., Deuchars, J., Atkinson, L., Milligan, C. J., Buckley, N. J. and Vizi, E. S. (2002) Involvement of $\mathrm{P} 2 \mathrm{X} 7$ receptors in the regulation of neurotransmitter release in the rat hippocampus. J Neurochem 81 , $1196-211$. 


\title{
Modulation of $\mathrm{P}_{2} \mathrm{Y}_{2}$ receptor trafficking and $\mathrm{P}_{2} \mathrm{Y}_{2}$ receptor-mediated proliferation
}

\author{
Tulapurkar, M.E., Schäfer, R. and Reiser G. \\ Institut für Neurobiochemie, Medizinische Fakultät, Otto-von-Guericke Universität, Leipziger Str. 44, 39120 \\ Magdeburg, Germany. \\ georg.reiser@medizin.uni-magdeburg.de
}

Nucleotides in the extracellular fluids serve as important signaling molecules which act via the P2 receptor family. The $\mathrm{P}_{2} \mathrm{Y}_{2}$ receptor is a unique member of the P2Y receptor family which responds equipotently to ATP and UTP. We have generated a stably transfected HEK 293 cell line overexpressing rat P2 $\mathrm{Y}_{2}$ receptor with a GFP tag on the C-terminus. We investigated the role of different proteins and factors that could be involved in the endocytosis of the receptor. The localization of the expressed receptor was seen exclusively on the plasma membrane. This is in accordance to the endogenous distribution of the $\mathrm{P}_{2} \mathrm{Y}_{2}$ receptor. To confirm the functional expression of the transfected receptor we stimulated the cells with ATP or UTP $(100 \mu \mathrm{M})$ and monitoring the rise in $\left[\mathrm{Ca}^{2+}\right]_{\mathrm{i}}$ using Fura-2. We could show that the endocytosis of the receptor proceeds via clathrin-dependent pathway (Tulapurkar et. al., 2005). We further investigated the role of kinases (Tulapurkar et. al., 2006) and other proteins that could modulate receptor trafficking. The cells were pre-incubated with $0.75 \% \mathrm{v} / \mathrm{v}$ 1-butanol, an inhibitor of phospholipase-D2 $\left(\mathrm{PLD}_{2}\right)$, and then stimulated with $100 \mu \mathrm{M}$ UTP. It was observed that pre-incubation delayed the endocytosis of the receptor and it delayed the reappearance of the receptor. To confirm the role of $\mathrm{PLD}_{2}$ in endocytosis of the receptor the cells were pre-treated with $0.75 \% \mathrm{v} / \mathrm{V}$ 2-butanol, before stimulation with UTP. Then the kinetics of endocytosis was not affected. These results confirmed that $\mathrm{PLD}_{2}$ modulates the endocytosis of the $\mathrm{P}_{2} \mathrm{Y}_{2}$ receptor. Kinases are also known to modulate endocytosis of G-protein coupled receptors (GPCRs) and the proliferation mediated via them. Pre-incubation of the cells with U0126, an inhibitor of MEK did not affect the endocytotic kinetics of the receptor, but delayed the reappearance of the receptor. ATP and UTP, equipotent agonists at the $\mathrm{P}_{2} \mathrm{Y}_{2}$ receptor in terms of the $\mathrm{Ca}^{2+}$ responses, exhibited, however, an interesting difference in terms of modulating reappearance of the endocytosed $\mathrm{P}_{2} \mathrm{Y}_{2}$ receptor. Endocytosis of the receptor proceeded normally on stimulation of the cells with $100 \mu \mathrm{M}$ of ATP or UTP for either 30 or $60 \mathrm{~min}$. In case of cells that were stimulated for $30 \mathrm{~min}$ with either agonist, the endocytosed receptor reappeared on the plasma membrane 1 hour after withdrawal of the agonist. After $60 \mathrm{~min}$ of exposure of the cells to agonist and $60 \mathrm{~min}$ of withdrawal for ATP treated cells the receptor completely reappeared on the plasma membrane, whereas in case of UTP-treated cells there was a partial reappearance of the receptor on the plasma membrane. This indicates that although ATP and UTP are equipotent at the $\mathrm{P}_{2} \mathrm{Y}_{2}$ receptor in terms of $\mathrm{Ca}^{2+}$ response but not in terms of reappearance of the receptor. The nucleotides specifically stimulated and modulated the proliferation of the HEK 293 cells. This stimulation in proliferation was not observed, when cells were treated with agonist for proteaseactivated receptor or agonists of different growth factor receptors. This indicates that nucleotides could be used to modulate specifically the growth of cells and trafficking of the receptor is a finely modulated process.

Tulapurkar ME, Schafer R, Hanck T, Flores RV, Weisman GA, Gonzalez FA, Reiser G. Cell Mol Life Sci. 2005 62, 1388-99.

Tulapurkar ME, Zündorf G, Reiser G J Neurochem.2006 96, 624-34.

\section{MODULATION OF SYNAPTIC TRANSMISSION BY ADENOSINE A AND NPY RECEPTORS ON RAT CORTICAL NEURONS}

Sichardt, K.; Beck-Sickinger, A.G.; Nieber, K.

Institute of Pharmacy, Dept. Pharmacology, University of Leipzig, D-04103 Leipzig, Germany, sichardt@uni-leipzig.de

G-protein coupled receptors (GPCRs) have been extensively characterised with respect to both, ligand binding and activation of various signalling pathways. There are considerable evidences that signalling via GPCRs can be 
regulated by inputs from GPCRs coupled to other pathways. Such an interaction may have important implications for the pathophysiological consequences of receptor activation.

In the central nervous system (CNS) neuroprotection during hypoxia is based on the inhibition of glutamate release from presynaptic terminals followed by inhibition of postsynaptic potentials. Two GPCR subtypes are involved in this process on cortical neurons: the adenosine $A_{1}$ receptor $\left(A_{1} R\right)$ and the neuropeptide $Y Y_{1}$ receptor $\left(Y_{1} R\right)$. Both are located presynaptical and inhibit the glutamate release. Therefore the aim of the present study was to evaluate the effect of the two GPCRs on the synaptic transmission on pyramidal cells of the rat cingulate cortex and to demonstrate a possible interaction between the two GPCRs. Intracellular recordings with glasmicroelectrodes were made on pyramidal cells in layer $\mathrm{V}$ of the cingulate cortex in rat brain slices. Postsynaptic potentials (PSPs) were evoked by electrical stimulation with a concentric bipolar electrode in layer I. Previously we found that activation of $\mathrm{A}_{1}$ Rs by endogenous released adenosine or by the selective $\mathrm{A}_{1} \mathrm{R}$ agonist N6-cyclopentyladenosine (CPA, $1 \mathrm{nM}-10 \mu \mathrm{M})$ depressed the PSPs [1,2]. Approximately $60 \%$ of the neurons responded to NPY and the selective $Y_{1}$ receptor agonist [F7, P34]pNPY suggesting a moderate distribution of $Y_{1} R$ in the cingulate cortex. NPY and [F7, P34]pNPY inhibited the PSPs in a concentration dependent manner after a superfusion about 5 minutes. The inhibition of NPY (1nM) and [F7, P34]pNPY (1nM) was nearly 40\%. The effect was antagonised by the $Y_{1}$ selective antagonist BIBP3226 (50nM). In further experiments an interaction between $\mathrm{A}_{1} \mathrm{R}$ and $\mathrm{Y}_{1} \mathrm{R}$ was tested. Two different types of experiments were conducted. Firstly, NPY and CPA were superfused successively without washout, each in a concentration which induced maximum inhibition in individual experiments. NPY $(100 \mathrm{nM})$ and CPA $(10 \mu \mathrm{M})$ depressed the PSPs in the same range as in individual experiments. Secondly the selective $\mathrm{A}_{1} \mathrm{R}$ antagonist 1,3-dipropyl-8-cyclopentylxanthine (DPCPX) was superfused 5 minutes before application of NPY. DPCPX $(0.1 \mu \mathrm{M})$ decreased the NPY induced inhibition compared to the inhibition of NPY alone. The current results suggest that the two GPCRs induce the inhibition of the PSPs independently from each other. So far, an interaction cannot be excluded and it must be investigated whether a desensitization by NPY exists.

[1] Brand, A, Vissiennon, Z, Eschke, D, Nieber, K: Adenosine $A_{1}$ and $A_{3}$ receptors mediate inhibition of synaptic transmission in rat cortical neurons. Neuropharmacology 2001; 40: 85-95.

[2] Hentschel, S, Lewerenz, A, Nieber, K: Activation of A3 receptors by endogenous adenosine inhibits synaptic transmission during hypoxia in rat cortical neurons. Rest. Neurol. Neurosci. 2003; 21: 55-63.

\title{
Modulation of $\mathbf{T}$ cell function by $\mathrm{ATP}$, adenosine, and P1/P2 receptors.
}

\author{
Linda Yip $^{1}$, Cindy Cheung ${ }^{1}$, Yu Chen ${ }^{1}$, Ross Corriden ${ }^{1,2}$, Naoyuki Hashiguchi ${ }^{1}$, Paul A. Insel ${ }^{2,3}$, and \\ Wolfgang G. Junger ${ }^{1}$ \\ University of California San Diego, Depts. of ${ }^{1}$ Surgery/Trauma, ${ }^{2}$ Pharmacology, and ${ }^{3}$ Medicine, San Diego, \\ California 92103. \\ lyip@ucsd.edu
}

$\mathrm{T}$ cell activation helps control the immune system. Here we examined the role of ATP, adenosine, $\mathrm{P} 1 / \mathrm{P} 2$ receptors, and ecto-enzymes in regulating $\mathrm{T}$ cell function. Using Real-Time RT-PCR, we found that human CD ${ }^{+} \mathrm{T}$ cells and Jurkat $\mathrm{T}$ cells express similar patterns of multiple $\mathrm{P} 1$ and $\mathrm{P} 2$ receptor subtypes: $\mathrm{T}$ cells express high levels of $\mathrm{A}_{2 \mathrm{~A}}$ adenosine receptors and the $\mathrm{P}_{2} \mathrm{X}_{4}$ and $\mathrm{P} 2 \mathrm{X}_{5}$ nucleotide receptor subtypes, and moderate to low levels of $\mathrm{A}_{1}$ (Jurkat only), $\mathrm{A}_{2 \mathrm{~B}}$, and $\mathrm{A}_{3}$ adenosine receptors and $\mathrm{P} 2 \mathrm{X}_{1}, \mathrm{P} 2 \mathrm{X}_{7}, \mathrm{P}_{2} \mathrm{Y}_{1}, \mathrm{P} 2 \mathrm{Y}_{4}, \mathrm{P}_{2} \mathrm{Y}_{6}\left(\mathrm{CD} 4{ }^{+}\right.$only), $\mathrm{P} 2 \mathrm{Y}_{11}, \mathrm{P} 2 \mathrm{Y}_{12}$ (Jurkat only), and $\mathrm{P} 2 \mathrm{Y}_{14}$ nucleotide receptors. Cell stimulation with phytohemagglutinin (PHA; $50 \mathrm{ng} / \mathrm{ml}$ ) and phorbol ester (PMA; $5 \mathrm{ng} / \mathrm{ml}$ ) altered these expression patterns in Jurkat cells. In particular, $\mathrm{P}_{2} \mathrm{X}_{1}$ and $\mathrm{P}_{2} \mathrm{X}_{5}$ receptor mRNA and protein expression increased in response to cell stimulation (see Fig.), suggesting a role for these receptors in $\mathrm{T}$ cell activation.

$\mathrm{T}$ cell activation elicited a rapid release of $\sim 0.1 \%$ of the total cellular ATP content ( $\sim 36 \mathrm{pmol} / \mathrm{cell})$ from Jurkat cells. HPLC studies demonstrated that ATP released in response to PHA/PMA stimulation was rapidly hydrolyzed to ADP, AMP, adenosine, and inosine, most likely by ecto-enzymes. Resting Jurkat cells hydrolyze ATP, reducing exogenously added ATP $(5 \mu \mathrm{M})$ by $50 \%$ in $<1 \mathrm{~h}$ to form ADP, AMP, and inosine; but little adenosine was observed, suggesting a short half-life. Indeed, we found that Jurkat cells rapidly converted exogenously added adenosine to inosine. Complete adenosine deamination occured in $<1 \mathrm{~min}$. Using the adenosine deaminase 
(ADA) inhibitor EHNA and the nuceloside transport inhibitor dipyridamole, we found that both reuptake by nucleoside transporters and rapid hydrolysis by ADA localized outside the cell likely account for low levels of extracellular adenosine.

By measuring interleukin-2 (IL-2) mRNA induction and IL-2 expression with Real-Time RT-PCR and ELISA, respectively, we found that ATP and adenosine elict opposing actions on T cell activation. Agonists of P2 receptors (ATP and ATP $\gamma \mathrm{S}$ ) enhanced IL-2 expression in Jurkat cells, while the P1 agonist adenosine and $\mathrm{A}_{2 \mathrm{~A}}$ receptor-selective agonist (CGS 21680) suppressed IL-2 expression. Selective agonists for other P1 receptor subtypes had little effect.

Together, these results suggest that $\mathrm{T}$ cell function is tightly controlled by the coordinated activation of components involved in release of and response to ATP and adenosine. T cell stimulation may be supported by positive feedback through ATP release and P2 receptor activation, while adenosine, formed from released ATP, may terminate the $\mathrm{T}$ cell activation process through a negative feedback loop that involves $\mathrm{A}_{2 \mathrm{~A}}$ receptor activation. Fine-tuning of the $\mathrm{T}$ cell response may occur through changes in the activity of ecto-enzymes that regulate extracellular ATP and adenosine concentrations, and through changes in the expression patterns of specific P1 and $\mathrm{P} 2$ receptors.

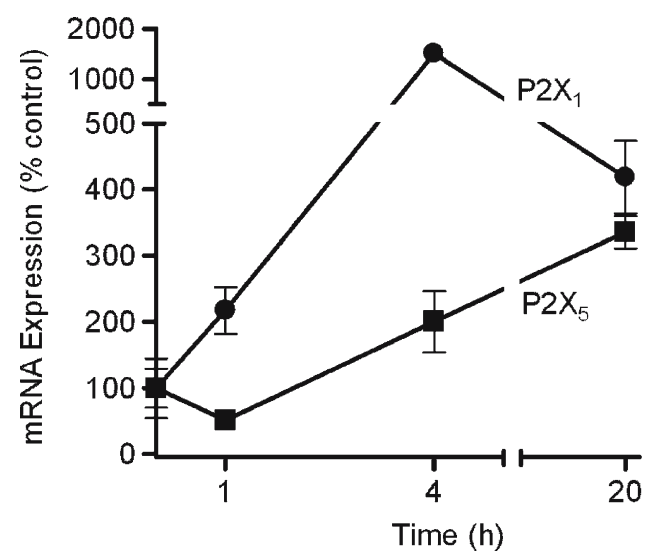

Fig. Changes in $\mathrm{P} 2 \mathrm{X}_{1}$ and $\mathrm{P} 2 \mathrm{X}_{2}$ mRNA expression after stimulation with PHA and PMA for indicated times.

This study was funded in part by grants from the National Institutes of Health (GM-51477 \& 60475).

\section{Modulation of the antigen receptor-induced $\mathrm{Ca}^{2+}$ response in human $\mathrm{B}$ lymphocytes by activation of the $\mathrm{P} 2 \mathrm{X} 7$ receptor}

M Klapperstück and F Markwardt

Julius-Bernstein-Institute for Physiology, Martin-Luther-University Halle, Magdeburger Straße 6, D-06097 Halle/ Saale, Germany

manuela.klapperstueck@medizin.uni-halle.de

Cells release ATP during inflammatory processes or tissue damage. In our study we investigated the interaction of the P2X7-receptor on the calcium signalling of B lymphocytes induced by B cell receptor (BCR) ligation. In B cells ligation of $\mathrm{BCR}$ induces a biphasic $\mathrm{Ca}^{2+}$ response. Activation of the $\mathrm{P} 2 \mathrm{X} 7$ receptor raises a cationic ion current which carries sodium, calcium and potassium. The extent of the accompanying increase in the global intracellular calcium concentration is dependent on the degree of the depolarisation of the cell membrane, which in turn is dependent on the activation and expression level of the P2X7 receptor. B lymphocytes were isolated from human from tonsils and purified by means of the nylon wool method. The cells were loaded with $\mathrm{Ca}^{2+}$ sensitive and membrane potential-sensitive dyes. The fluorescence signals were measured by flow cytometry. The cells were stimulated by the ligation of the $\mathrm{B} B C R$ by human $\mathrm{F}\left(a b^{\prime}\right) 2$ anti- $\mu$ antibodies in combination with rising concentrations of the P2X7 receptor agonist BzBzATP. We found a concentration dependent effect of BzBzATP on the BCR-Ca ${ }^{2+}$-response in P2X7-receptor expressing cells which was inhibiting at low concentrations $(1 \mu \mathrm{M})$ and additive at higher concentrations $(50 \mu \mathrm{M})$ of BzBzATP. The interaction of these two receptors might be significant for the modulation of the innate immune response. 


\title{
Modulatory role of adenosine $A_{2 A}$ receptors as basis for the use of $A_{2 A}$ antagonists in the treatment of Parkinson's disease.
}

\author{
Morelli M. ${ }^{(1,2)}$, Borsini F. ${ }^{(3)}$, Pontis S. ${ }^{(1)}$, Tronci E. ${ }^{(1)}$, Simola N. ${ }^{(1)}$, Carta AR. ${ }^{(1,2)}$, Schintu N. ${ }^{(1)}$, \\ Pinna A. ${ }^{(1,4)}$
}

(1) Department of Toxicology, University of Cagliari, Italy; (2) Center of Excellence for Neurobiology of Drug of Abuse, Italy; (3) Dept. of Pharmacology, Sigma-tau, Rome, Italy; (4) CNR Institute for Neuroscience, Cagliari, Italy.

morelli@unica.it

The search of therapies alternative to L-DOPA or dopamine receptor agonists for the treatment of Parkinson's disease $(\mathrm{PD})$ is very active and adenosine $\mathrm{A}_{2 \mathrm{~A}}$ receptors, for their interaction with the dopamine $\mathrm{D}_{2}$ receptor, have became particularly attractive. Recent evidences obtained in rodent and primate models of PD and preliminary clinical trials, indicate that adenosine $\mathrm{A}_{2 \mathrm{~A}}$ receptor antagonists might represent a new valuable therapeutic tool for the treatment of PD.

In this study we evaluated the effect of the $\mathrm{A}_{2 \mathrm{~A}}$ receptor antagonists $\mathrm{SCH} 58261$ and ST 1535 in the unilateral 6hydroxydopamine (6-OHDA) rat model of PD.

In 6-OHDA lesioned rats, acute administration of SCH 58261 and ST 1535 counteracted the impairments in the initiation of stepping movements and in the adjusting step induced by the lesion and increased the turning behavior induced by L-DOPA. In chronic studies, SCH 58261 or ST 1535 + L-DOPA induce lower diskinetic movements than L-DOPA alone and did not induce long-term increase in GAD67 (the synthesizing enzyme of GABA) and dynorphin mRNA in striatum.

The data indicate that $\mathrm{A}_{2 \mathrm{~A}}$ receptor antagonists ameliorate the motor impairment which characterize PD. Furthermore the neuronal modifications observed in rat striatum after chronic treatment with SCH 58261 or ST $1535+$ L-DOPA, suggest that such treatments might not produce detrimental long-term changes as L-DOPA alone.

\section{Molecular and functional evidence of a $\mathbf{P 2 X} \mathbf{X}_{7}$-like receptor in $\mathbf{P 2 X}_{7}$ knockout mouse brain.}

Patricia Marín García, Jesús Sánchez-Nogueiro, David León, Miriam León Otegui, María Diez Zaera, Miguel Díaz Hernández, María Teresa Miras-Portugal.

Departamento de Bioquímica y Biología Molecular, Facultad de Veterinaria, Universidad Complutense de Madrid.Spain.

patricia8149@bio.ucm.es

$\mathrm{P} 2 \mathrm{X}_{7}$ expression in mammalian central and peripheral nervous system is a matter of controversy because there are not really specific agents for this receptor subtype. In this research, $\mathrm{P} 2 \mathrm{X}_{7}$ expression and functionality was studied in C57Bl6J wild type (WT) and P2X 7 knockout (KO) mice from Dr. Gabel (Pfizer, Solle et al., 2001).

RT-PCR experiments using specific pairs of primers to amplify the disrupted region in $\mathrm{P}_{2} \mathrm{X}_{7} \mathrm{KO}$ mice showed that this DNA fragment is only present in WT mice. However, when the specific primers that codify for a region before to the disrupted one or back to it were used, DNA fragments were amplified in WT and KO animals. In western blotting and immunocytochemical experiments in brain, no differences from WT versus $\mathrm{P}_{2} \mathrm{X}_{7} \mathrm{KO}$ were found. However, western blotting assays in macrophages showed a $\mathrm{P} 2 \mathrm{X}_{7}$ protein expression only in WT mouse. Calcium imaging experiments were made to assess the $\mathrm{P}_{2} \mathrm{X}_{7}$ functionality. The physiological agonist, as ATP, and pharmacological agonist as Benzoyl-ATP were tested in medium without magnesium; and so, inhibitors as zinc or Brilliant Blue (BBG). A similar functional response in WT and $\mathrm{KO}$ mice was observed.

YO-PRO assays in a micro plate reader showed that no pore was formed in cerebellum granule neurons from WT and $\mathrm{KO}$.

These results indicate that in $\mathrm{P} 2 \mathrm{X}_{7} \mathrm{KO}$ mice brain may exist another protein very similar to $\mathrm{P} 2 \mathrm{X}_{7}$ receptor, which could assume its role in $\mathrm{KO}$ mice brain. 


\title{
Molecular mechanisms involved in mediating the anti-inflammatory effect of IB-MECA in adjuvant induced arthritis
}

\author{
Avivit Ochaion, Sara Bar-Yehuda, Shira Cohen and Pnina Fishman ${ }^{1}$ \\ Can-Fite BioPharma Ltd., Kiryat-Matalon, Petah-Tikva, 49170, Israel.
}

Activation of the $A_{3}$ adenosine receptor $\left(A_{3} A R\right)$ was found to mediate anti-inflammatory effects via the natural ligand adenosine or synthetic $\mathrm{A}_{3} \mathrm{AR}$ agonists such as IB-MECA or Cl-IB-MECA. In this study we demonstrate that the NF-kB signal transduction pathway is responsible for the ability of IB-MECA to prevent the clinical and pathological manifestations of arthritis in an experimental animal model of adjuvant induced arthritis (AIA). $\mathrm{A}_{3} \mathrm{AR}$ protein expression level was highly expressed in the paw, synovia, drain lymph nodes (DLN) and peripheral blood mononuclear cells (PBMNC) of rats with AIA in comparison to naïve animals. Upon treatment with IB-MECA, down-regulation of $\mathrm{A}_{3} \mathrm{AR}$ expression was noted, both in the inflammatory tissues as well as in the PBMNC. Analysis of synovia and DLN protein extracts revealed a decreased expression level of PI3K, PKB/Akt, IKK, NF-kB and TNF- $\alpha$, known to affect survival and apoptosis of inflammatory cells. Additionally, the level of caspase-3, was up-regulated, demonstrating that apoptosis of inflammatory cells took place.

As a result of above events, AIA rats responded to IB-MECA treatment by a decrease in the clinical and pathological score of the disease. The response to IB-MECA was neutralized by pre-treatment of the animals with the antagonist MRS1220, confirming that the efficacy of the synthetic agonist was A3AR mediated. Taken together, IB-MECA an orally bioavailable molecule activates $A_{3} A R$, generating down-stream signaling pathway which results in an anti-inflammatory effect. The finding that $\mathrm{A}_{3} \mathrm{AR}$ expression level in the PBMNC reflects receptor status in the remote inflammatory sites suggests $\mathrm{A}_{3} \mathrm{AR}$ as a follow up bio-marker.

Taken together, high $\mathrm{A}_{3} \mathrm{AR}$ expression is found in the synovia and the immune cells in the DLN and PBMNC. IB-MECA, an orally bioavailable molecule, activates $\mathrm{A}_{3} \mathrm{AR}$ inducing receptor down-regulation and the initiation of a molecular mechanism which involves de-regulation of the PI3K - NF- $\kappa \mathrm{B}$ signaling pathway. As a result, a potent anti-inflammatory effect, manifested in the improvement of disease clinical and pathological score, takes place. The finding that $\mathrm{A}_{3} \mathrm{AR}$ expression level in the PBMNC and DLN reflects receptor status in the remote inflammatory site suggests $\mathrm{A}_{3} \mathrm{AR}$ as a follow up bio-marker.

\section{MOLECULAR MODELLING OF A A $_{2 \mathrm{~B}}$ ADENOSINE RECEPTOR}

\author{
Vladimir A. Palyulin ${ }^{1}$, Andrei A. Ivanov ${ }^{1}$, Pier Giovanni Baraldi ${ }^{2}$, Nikolai S. Zefirov ${ }^{1}$ \\ ${ }^{1}$ Department of Chemistry, Moscow State University, Moscow 119992 Russia \\ ${ }^{2}$ Universitá di Ferrara, Dipartimento di Scienze Farmaceutiche, Via Fossato di Mortara 17/19 44100 Ferrara, Italy \\ vap@org.chem.msu.su
}

The molecular model of $\mathrm{A}_{2 \mathrm{~B}}$ subtype of human adenosine receptor was built in homology with bovine rhodopsin. The model includes a transmembrane domain, all extracellular and intracellular hydrophobic loops and terminal domains. The molecular docking of adenosine as well as some known agonists and antagonists had been made and their binding modes had been studied. The model of $\mathrm{A}_{2 \mathrm{~B}}$ receptor subtype had been compared with the models obtained for other subtypes of adenosine receptors $\left(\mathrm{A}_{1}, \mathrm{~A}_{2 \mathrm{~A}}, \mathrm{~A}_{3}\right)$ and possible reasons of differences in ligand activities with respect to different receptor subtypes were discussed. 


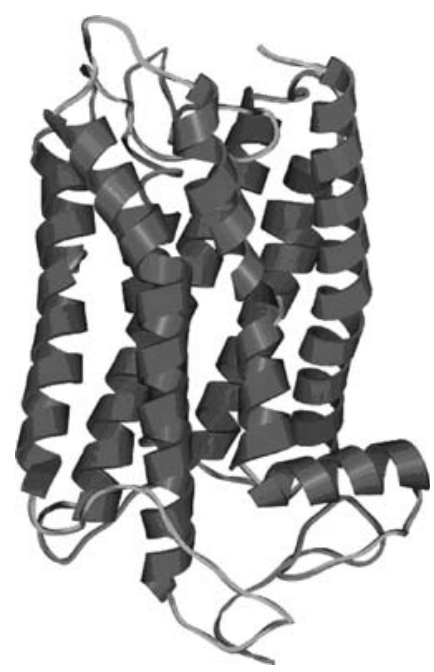

The molecular dynamics simulations of ligand-receptor complexes inserted into the phospholipid bilayer were carried out. The conformational changes of the $\mathrm{A}_{2 \mathrm{~B}}$ receptor occurring during molecular dynamics simulations were explored, and the stable binding modes of the studied ligands were analysed. According to the models presented in this work, the residues involved in ligand recognition were determined for the $A_{2 B}$ adenosine receptor. The binding modes of the $\mathrm{A}_{2 \mathrm{~B}}$ receptor ligands demonstrate good agreement with the site-directed mutagenesis data.

Ivanov, A.A.; Baskin, I.I.; Palyulin, V.A.; Piccagli, L.; Baraldi, P.G.; Zefirov N.S. Molecular modeling and molecular dynamics simulation of the human $\mathrm{A}_{2 \mathrm{~B}}$ adenosine receptor. The study of the possible binding modes of the $\mathrm{A}_{2 \mathrm{~B}}$ receptor antagonists. J. Med. Chem., 2005, 48, 6813-6820.

\title{
MOLECULAR SIGNALLING MEDIATING THE PROTECTIVE EFFECT OF A ADENOSINE AND mGLU3 METABOTROPIC GLUTAMATE RECEPTOR ACTIVATION AGAINST APOPTOSIS INDUCED BY OXYGEN/GLUCOSE DEPRIVATION IN CULTURED RAT BRAIN ASTROCYTES
}

\author{
${ }^{1}$ D'Alimonte I., ${ }^{1}$ Nargi E., ${ }^{1}$ Ballerini P., Masciulli A., ${ }^{2,3}$ Bruno V., ${ }^{1}$ Caciagli F., ${ }^{1}$ Ciccarelli R. \\ ${ }^{T}$ Department of Biomedical Sciences, University of Chieti-Pescara. Chieti. Italy. ${ }^{2}$ Department of Pharmacology and \\ Physiology, "La Sapienza" University. Rome. Italy. ${ }^{3}$ Istituto Neurologico Mediterraneo (Neuromed). Venafro. \\ Italy.i.dalimonte@dsb.unich.it
}

Astrocyte apoptotic death occurs in acute and chronic neurodegenerative disorders. Since astrocytes are essential for neuronal survival and synaptic function as well as for neurogenesis and neural repair, interventions aimed at blocking or preventing astrocyte death may contribute to neuroprotection. Here, we investigated whether the activation of $\mathrm{A}_{1}$ adenosine or mGlu3 metabotropic glutamate receptors was protective against apoptosis induced in cultured rat brain astrocytes by 3-hour exposure to combined oxygen-glucose deprivation (OGD). Addition of $\mathrm{N}^{6}$-chlorocyclopentyladenosine (CCPA, 2.5-75 nM) or (-)2-oxa-4-aminocyclo-[3.1.0] hexane-4,6-dicarboxylic acid (LY379268, 0.25-7.5 $\mu \mathrm{M}$ ), selective $\mathrm{A}_{1}$ and mGlu3 receptor agonists, respectively, to the astrocyte medium for 4 $\mathrm{h}$, starting from $1 \mathrm{~h}$ prior to cell exposure to OGD, reduced in dose-dependent fashion the apoptotic death rate (maximal reduction by about 50\%) caused by OGD. The combined addition of the two agents prior to OGD produced less than additive effects. Protection was abrogated by cell pre-treatment with either the respective $\mathrm{A}_{1}$ [8-cyclopentyl-1,3-dipropylxanthine, DPCPX, 100nM] or mGlu3 [(2S,1'S,2'S)-2(9-xanthylmethyl)-2-(2'-carboxycyclopropyl)glycine, LY341495, $1 \mu \mathrm{M}$ ] receptor antagonists or pertussis toxin $(200 \mathrm{ng} / \mathrm{ml})$, an inhibitor of metabotropic receptor-coupled Gi protein activity. CCPA and LY379268 anti-apoptotic effects were also nullified by the presence of U0126 and LY294002, inhibitors of extracellular signal-regulated kinase (ERK)1/2/mitogen-activated 
protein kinase (MAPK) and phosphoinositide-3-kinase (PI3K) pathways, respectively. These enzymatic cascades have been most implicated in maintaining cell survival. Immunoblot analysis of the molecular pathways involved in either apoptotic or protective events showed that 3-h OGD promoted, yet after $30 \mathrm{~min}$, the phosphorylation of p38 and c-Jun N-terminal kinase (JNK) in the MAPK system, which are considered as promoters of cell stress and injury. This event was abrogated by cell pretreatment with CCPA or LY379268. OGD also caused a transient and delayed (at $2 \mathrm{~h}$ ) phosphorylation of Akt, downstream target in the survival PI3K pathway, and of apoptosis signalregulating kinase 1 (ASK1) at Ser83, leading to its inactivation. However, this effect was obscured by cell pretreatment with CCPA or LY379268, which promoted Akt and ASK1 phosphorylation as early as 5 min and up to $3 \mathrm{~h}$. CCPA and LY379268 also induced ERK1/2 phosphorylation and the expression of the antiapoptotic protein Bcl- $\mathrm{X}_{\mathrm{L}}$, both effects being abrogated by LY294002. Finally, CCPA and LY379268 inactivated the apoptotic factor Bad causing its phosphorylation at Ser112 and Ser136, dependent on ERK1/2 and PI3K activity, respectively, as these effects were counteracted by U0126 and LY294002, inhibitors of those pathways. Thus, the activation of $\mathrm{A}_{1}$ adenosine or mGlu3 receptors, which share common signalling pathways, protects astrocytes against OGD-induced apoptosis via the activation of intracellular pathways classically related to cell survival.

\title{
Multiple P2X receptors are involved in the modulation of apoptosis in human mesangial cells
}

\author{
A.Solini $^{1}$, E.Santini ${ }^{1}$, D.Chimenti ${ }^{1}$, P.Chiozzi ${ }^{1}$, F.Pratesi ${ }^{1}$, S.Cuccato ${ }^{1}$, E.Ferrannini ${ }^{1}$, G.Pugliese ${ }^{2}$ and \\ F.Di Virgilio ${ }^{3}$ \\ ${ }^{1}$ Dept. of Internal Medicine, University of Pisa, ${ }^{2}$ Dept. of Clinical Sciences, La Sapienza University of Rome and \\ ${ }^{3}$ Section of General Pathology, University of Ferrara, Italy \\ a.solini@med.unipi.it
}

As in other tissues, cell loss through apoptosis (Apo) participates in maintaining renal tissue homeostasis; moreover, it has been considered as a major mechanism for either resolution of glomerular hypercellularity in glomerulonephritis or loss of cellularity and progression to glomerulosclerosis in chronic renal disease. Aims of this study were to investigate the role of extracellular ATP (eATP) in mediating Apo in human mesangial cells (HCM), and to identify the subtype(s) of P2 receptors (P2r) involved in this effect. HMC were probed with different concentrations of nucleotides in the presence or absence of the P2X antagonist oxidized-ATP (oATP) or the selective inhibitor of human $\mathrm{P}_{2} \mathrm{X}_{7}, \mathrm{KN} 62$. We assessed morphological changes by contrast-phase mycroscopy; Apo rate by Annexin V/propidium iodide based flow cytometry; activation (act) of caspase-3 by fluorimetric technique; plasma membrane potential alterations (PMDep) by bisoxonol uptake; calcium increase $\left[\left(\mathrm{Ca}^{2+}\right) \mathrm{i}\right]$ by Fura-2/AM uptake; P2Rs exp by RT-PCR, WB and immunofluorescence (IF). eATP caused dose-dependent shrinkage, emission of neurite-like protrusions, formation of small blebs in HMC; these varicosities were fully prevented by oATP. BzATP induced alterations only at high concentrations and to a lower extent than ATP; its effects were abolished by KN62. eATP $1 \mathrm{mM}$ caused Apo in 14.6\% of HMC (positive control:17.4\% with TNF $\alpha$ ); oATP fully prevented ATP- or BzATP-triggered Apo (back to 6.5\%). BzATP was less effective than ATP and KN62 not only did not prevent BzATP effect but even potentiated it (from $11.7 \%$ to $26.7 \%$ ). Caspase- 3 act was $1376 \pm 324$ AU with ATP and $1039 \pm 83$ AU with 1 mM BzATP. eATP-stimulated Apo was a late event: 3 or 6 hrs of incubation did not induce any cell death. To characterize either the mechanism of ATP-dependent Apo and the level of $\mathrm{P}_{2} \mathrm{X}_{7}$ activity, we monitored $\left(\mathrm{Ca}^{2+}\right) \mathrm{i}$ and PMDep. eATP triggered a fast, dose-dependent $\mathrm{Ca}^{2+}$ release from intracellular stores (from $75 \pm 12$ to $181 \pm 26 \mathrm{nM}$ with $1 \mathrm{mM}$ ATP), which was not followed by the delayed plateau, suggesting that $\mathrm{Ca}^{2+}$ influx from the extracellular space is negligible. eATP also induced a transmembrane ion flux as shown by PMDep $(46 \pm 13 \%)$. BzATP caused a smaller $\left(\mathrm{Ca}^{2+}\right) \mathrm{i}$ as compared with ATP (from $55 \pm 15$ to $90 \pm 18 \mathrm{nM}$ with $1 \mathrm{mM}$ ATP), and was uneffective in inducing PMDep at all concentrations tested. Irrespective of this relatively scarce functional activity, $\mathrm{P} 2 \mathrm{X}_{7}$ was expressed in $\mathrm{HMC}$, as shown by WB and IF, with the typical ring-like structures localized at the periphery of the cells. Overall, these data suggest that $\mathrm{P}_{2} \mathrm{X}_{7}$ have a negligible role in ATP-mediated Apo, and that $\mathrm{P} 2 \mathrm{Xr}(\mathrm{s})$ others than it could participate in this process. In addition to $\mathrm{P} 2 \mathrm{X}_{7}, \mathrm{HMC}$ express $\mathrm{P} 2 \mathrm{X}_{2}, \mathrm{P} 2 \mathrm{X}_{3}, \mathrm{P} 2 \mathrm{X}_{4}, \mathrm{P} 2 \mathrm{X}_{6}, \mathrm{P}_{2} \mathrm{Y}_{2}, \mathrm{P}_{2} \mathrm{Y}_{5}$ and $\mathrm{P}_{2} \mathrm{Y}_{6}$ subtypes; given the protective effect of oATP and the lack of effect of UTP, we focused our attention on others P2Xr, expecially $\mathrm{P}_{2} \mathrm{X}_{4}$, already, even seldom, involved in Apo phenomena induced by eATP. We then tested the effect of the P2X blocker TNP-ATP, which, when co-incubated with BzATP and KN62, reduced Apo by 30-40\%, thus confirming 
the role of $\mathrm{P}_{2} \mathrm{X}_{4}$ in HMC Apo. Moreover, $\mathrm{P} 2 \mathrm{X}_{4}$ was largely represented in $\mathrm{HMC}$, as shown by IF, and its exp was upregulated either by ATP and BzATP. In conclusion, P2R stimulation or dysfunction, particularly distinct changes in $\mathrm{P}_{2} \mathrm{X}_{4}$ exp, accompany Apo in $\mathrm{HMC}$, showing the relevance of the purinergic signaling in controlling the fate of these cells.

\title{
Mutagenesis of Conserved Residues in Human Ecto-ATPase (E-NTPDase 2)
}

\author{
Reem Javed, KyokoYarimizu, Nicole Pelletier, and Aileen F. Knowles \\ Department of Chemistry and Biochemistry, San Diego State University, San Diego, California, U.S.A. \\ aknowles@chemistry.sdsu.edu
}

The human ecto-ATPase (E-NTPDase 2) contains conserved motifs (five ACR and four conserved regions) and conserved lysine $(\mathrm{K})$ and arginine $(\mathrm{R})$ residues that are also present in other cell surface E-NTPDases. Some of these are presumably involved in substrate binding and catalysis. In addition, the protein contains six potential Nglycosylation sites. To determine the importance of these motifs and amino acid residues for the function and expression of human ecto-ATPase, we have carried out site-directed mutagenesis using its cDNA that is inserted in the expression vector pcDNA3. Activity of the mutants and protein expression are evaluated after transfection of the mutant cDNA in HEK293 cells.

Results obtained with seven lysine mutants (K39A, K57A, K62A, K62R, K182A, K182R, K428A) and six arginine mutants (R155A, R155K, R158A, R245A, R311A, R394A) indicate the following. (i) There is negligible protein expression upon mutation of K62, which is located in conserved region 1 (CR1) immediately following ACR1, and K182 which is downstream of ACR3. (ii) Protein expression is not restored in K62R but partial activity and protein expression are obtained in the K182R mutant. (iii) R155, which is located in CR3, suffers loss of protein expression and activity when mutated to alanine, but both are restored in the R155K mutant.

Conserved region 1 (CR1) of human ecto-ATPase with the sequence ${ }^{58}{ }^{8}$ PADKENDTGIV ${ }^{69}$ is a critical region for the function of the enzyme. It not only contains the invariable K62, but also contains the conserved Nglycosylation site $\left({ }^{64} \mathrm{NDT}\right)$ as well as ${ }^{65} \mathrm{DTG}^{67}$, that is similar to the phosphate-binding motifs (DXG) in ACR1 and 4, which have been shown to be important for E-NTPDase 3 activity [1]. The D65A and G67A mutants of human ecto-ATPase have only $10-30 \%$ activity of the wild type enzyme as well as a reduction in protein expression. Activity is partially restored in the D65E mutant indicating the importance of a negatively charged residue at that site. Mutagenesis of other residues in this conserved region is in progress.

Mutation of asparagine at the six N-glycosylation sites (N64, N88, N129, N294, N378 and N443) individually to glutamine indicated the importance of N64 (in CR1) and N443 (in ACR5), the two N-glycosylation sites that are conserved in all cell-surface E-NTPDases [2,3]. N64Q and N443Q mutants have $<20 \%$ ATPase activity of the wild-type enzyme. The N378Q mutant is fully active even though N378 is glycosylated in the WT protein. N88Q, N129Q, N294Q mutants suffer variable loss of activity. Double N (to Q) mutants other than N64 and N443 all have reduced protein expression and activity, with N88Q/N294Q being the least active.

Our results indicate that, in addition to the five ACR, CR1 and possibly the other three CR are also important for the function of cell surface E-NTPDases. (Supported by the California Metabolic Research Foundation. We also wish to acknowledge the generous gift of the anti-C-terminus antibody of the human ecto-ATPase by Dr. Terence L. Kirley.)

References:.

1. Smith, T. M. and Kirley, T. L. Biochemistry 1999, 38, 321-328

2. Mateo, J., Kreda, S., Henry, C. E., Harden, T. K. and Boyer, J. L. J. Biol. Chem. 2003, 278, 39960-39968

3. Wu, J. J., Choi, L. E., and Guidotti, G. Mol Biol. Cell 2005, 16, 1661-1672 


\title{
N6-benzyladenine induces increased expression of adenine phosphoribosyltransferase mRNA in mammalian leukaemia cells.
}

\author{
Ivo Frydrych and Petr Mlejnek \\ Department of Biology, Faculty of Medicine, Palacky University, Hnevotinska 3, Olomouc 77515, Czech Republic, \\ mlejnek_petr@volny.cz
}

Abstract Adenine phosphoribosyltransferase (APRT, EC 2.4.2.7) is a highly conserved purine salvage enzyme that catalyzes the conversion of adenine and 5-phosphoribosyl-1-pyrophosphate to AMP. In mammals, APRT is present in all tissues and provides the only known mechanism for the metabolic salvage of adenine (Simmonds et al. 1995). In addition, APRT plays a crucial role in the activation of purine antimetabolites such as 2,6-diaminopurine (Turker and Martin 1985). APRT appears to be responsible also for toxic effects of N6-substituted derivatives of adenine (cytokinins) in plants (Mlejnek et al. 2005). The role of APRT in the activation of N6substituted derivatives of adenine in mammalian cell is presumable but less clear as their toxicity is much lower than those in plants (Mlejnek and Dolezel 2005).

We observed that N6-benzyladenine induced elevated expression of APRT gene in human leukaemia cells at least at the mRNA level. Our results further supported the idea that the cytotoxic effects of N6-benzyladenine also required the APRT enzymatic activity for its cytotoxicity to develop. The properties of N6-benzyladenine could be of potential therapeutic value either for the treatment of APRT deficiency and/or for potentiation of cytotoxic effects of other purines activation of which depends on APRT activity.

Acknowledgement This work was supported by grant \#MSM 6198959216 (Ministry of Education, Youth and Sports) and in part by the Czech Grant Agency, grant GA301/04/1239.

\section{References}

1. Simmonds HA., Sahota A, and Van Acker KJ., APRT deficiency: 2,8-dihydroxyadenine lithiasis. In: The Metabolic and Molecular Bases of Inherited Disease (7th ed.), edited by Stanbury JB, Wyngaarden JB, and Fredrickson DG, New York: McGraw-Hill, 1995.

2. Turker MS and Martin GM., (1985) Induction of adenine salvage in mouse cell lines deficient in adenine phosphoribosyltransferase. Mol Cell Biol. 5(10): 2662 - 2668

3. Mlejnek P, Dolezel P, and Prochazka S., (2005) Intracellular conversion of cytokinin bases into corresponding mononucleotides is related to cell death induction in tobacco BY-2 cells. Plant Science 168 (2): 389-395

4. Mlejnek P, Dolezel P, (2005) Apoptosis induced by N6-substituted derivatives of adenosine is related to intracellular accumulation of corresponding mononucleotides in HL-60 cells Toxikology in Vitro 19 (7): 985-990.

\section{$\mathbf{N}^{6}$-Cycloalkyl-2-Substituted Adenosine Derivatives as High Affinity and Selective Adenosine $\mathbf{A}_{1}$ Receptor Agonists}

\author{
Elfatih Elzein, ${ }^{\text {a }}$ Rao Kalla, ${ }^{\text {a }}$ Xiaofen Li, ${ }^{\text {a }}$ Thao Perry, ${ }^{\text {a }}$ Tim Marquart, ${ }^{\text {a }}$ Mark Micklatcher, ${ }^{\text {a }}$ Li, Yuan, \\ Yuzhi Wu, ${ }^{\mathrm{b}}$ Dewan Zeng, ${ }^{\mathrm{b}}$ Jeff Zablocki ${ }^{\mathrm{a}}$ \\ ${ }^{a}$ Department of Bioorganic Chemistry, ${ }^{b}$ Department of Drug Research and Pharmacological Sciences, CV Thera- \\ peutics Inc., 3172 Porter Drive, Palo Alto, CA 94304, USA \\ elfatih.elzein@cvt.com
}

Adenosine is an endogenous purine nucleoside that modulates a variety of physiological functions as a result of its activation of specific $G$ protein-coupled receptors defined as $A_{1}, A_{2 A}, A_{2 B}$ and $A_{3}$ adenosine receptors (AdoRs). Physiological responses that are mediated by the $\mathrm{A}_{1}$-AdoR include cardiac (negative inotropic, negative chronotropic and negative dromotropic effects) and anti-lipolytic effects. Therefore, $\mathrm{A}_{1}$-AdoR agonists have received much attention as anti-arrhythmic and anti-lipolytic agents. In general, selective $\mathrm{A}_{1}$-AdoR agonists were obtained by monosubstitution of the $\mathrm{N}^{6}$-position of adenosine (e.g. CPA, CHA), whereas substitution at the C-2 position of adenosine yielded selective $A_{2 A}$-AdoR agonists (e.g. CVT-3146 (1), CGS21680). However, a wide range of $\mathrm{N}^{6}$ substituted adenosine derivatives have been reported to have high binding affinity for both $\mathrm{A}_{1}$ and $\mathrm{A}_{3}$-AdoRs and that represented a new challenge to discover selective $\mathrm{A}_{1}$-AdoR agonists. ${ }^{1}$ Even though CCPA is considered to be one of the most selective $\mathrm{A}_{1}$-AdoR agonists known to date, it displayed only 50 fold selectivity for the $\mathrm{A}_{1}$-AdoR 
over the $\mathrm{A}_{3}$-AdoR. Hence, more selective $\mathrm{A}_{1}$-AdoR agonists are needed. Simultaneous substitution at the $\mathrm{N}^{6}$ and C-2 positions of adenosine have resulted in compounds with different activity and selectivity profiles and some of these disubstituted compounds have high affinity and selectivity for the $\mathrm{A}_{1}$-AdoR. ${ }^{2,3}$ In previous communications we have shown that introducing a methyl group into the $\mathrm{N}^{6}$ position of compound $\mathbf{1}$ induces an increase in the affinity for the human $\mathrm{A}_{3}$-AdoR and simultaneously decreases the affinity for the $\mathrm{A}_{1}$ and $\mathrm{A}_{2 \mathrm{~A}}$-AdoRs, resulting in significant enhancement in $\mathrm{A}_{3}$-AdoR selectivity. ${ }^{4}$ During that study, we have also observed that increasing the size of the $\mathrm{N}^{6}$ substituent from methyl to ethyl and propyl resulted in a decrease in the $\mathrm{A}_{3}$-AdoR affinity and an increase in the $\mathrm{A}_{1}$ and $\mathrm{A}_{2 \mathrm{~A}}$-AdoRs affinity and selectivity. This prompted us to explore the effect of introducing substituents that are conducive to high $\mathrm{A}_{1}$-AdoR binding affinity (e.g. cycloalkyls) into the $\mathrm{N}^{6}$ position of our 2pyrazolyl adenosine derivatives with the idea of enhancing $\mathrm{A}_{1}$-AdoR binding affinity and selectivity. This resulted in analogs with very high affinity and selectivity for the $\mathrm{A}_{1}$-AdoR (e.g. 2a \& $\mathbf{2 b}$ ). The synthesis and biological activities of these new $\mathrm{N}^{6}$-C-2-disubstituted adenosine derivatives will be discussed.<smiles>[R6]c1cnn(-c2nc(N[B])c3ncn(C4OC(CO)[C@@H](O)[C@H]4O)c3n2)c1</smiles>

1. $\mathrm{R}_{1}=\mathrm{H}, \mathrm{R}_{2}=\mathrm{CONHCH}_{3}(\mathbf{C V T}-3146)$

2a. $\mathrm{R}_{1}=$ norbornyl, $\mathrm{R}_{2}=\mathrm{H}$

$\mathrm{K}_{\mathrm{i} \text { (low) }} \mathrm{hA}_{1}=0.4 \mathrm{nM}, \mathrm{K}_{\mathrm{i}} \mathrm{hA}_{2 \mathrm{~A}}=>5000 \mathrm{nM}, \mathrm{K}_{\mathrm{i}} \mathrm{hA}_{3}=1270 \mathrm{nM}$

2b. $\mathrm{R}_{1}=$ cyclopentyl, $\mathrm{R}_{2}=\mathrm{CONHCH}_{3}$

$\mathrm{K}_{\mathrm{i} \text { (low) }} \mathrm{hA}_{1}=1.7 \mathrm{nM}, \mathrm{K}_{\mathrm{i}} \mathrm{hA}_{2 \mathrm{~A}}=>5000 \mathrm{nM}, \mathrm{K}_{\mathrm{i}} \mathrm{hA}_{3}=1420 \mathrm{nM}$

(1) Gao et al., Biochem. Pharmacol., 2003, 65, 1675. (2) Beukers et al., J. Med. Chem., 2003, 46, 1492. (2) Jagtap et al., Bioorg. Med. Chem. Lett., 2004, 14, 1495. (4) Elzein et al., J. Med. Chem., 2004, 47, 4766.

\title{
$\mathbf{N}^{6}$-cyclopentyladenosine: selective brain targeting by nasal administration of microparticles
}

\author{
Alessandro Dalpiaz ${ }^{1}$, Paolo Giunchedi ${ }^{2}$, Elisabetta Gavini ${ }^{2}$, Gaia Colombo ${ }^{1}$, Fabrizio Bortolotti ${ }^{1}$, Luca \\ Ferraro $^{3}$, Sergio Tanganelli ${ }^{3}$, Angelo Scatturin ${ }^{1}$, Enea Menegatti ${ }^{1}$, Paolo Colombo ${ }^{4}$ \\ ${ }^{1}$ Department of Pharmaceutical Sciences, Ferrara University, Italy \\ ${ }^{2}$ Department of Drug Sciences, Sassari University, Italy \\ ${ }^{3}$ Department of Experimental and Clinical Medicine, Pharmacology Section, Ferrara University. \\ ${ }^{4}$ Department of Pharmacy, Parma University,Italy \\ E-mail: dla@unife.it
}

The adenosine derivative $\mathrm{N}^{6}$-cyclopentyladenosine (CPA) has been proposed as a potent antiischemic drug for the central nervous system (CNS). ${ }^{1}$ On the other hand, this compound has not yet entered in the clinical use being (i) quickly degraded in blood; (ii) unable to reach the brain by the systemic way; (iii) able to induce relevant side effects at other organs. ${ }^{1}$ Recently, the nasal route has been proposed as a promising way to directly deliver into the brain those drugs unable to overcome the physiologic barriers between blood and CNS. ${ }^{2,3}$ Here, we report a study regarding the preparation of powder formulations containing CPA for nasal delivery. The powders have been obtained by spray-drying in the presence either of mannitol and lecithin, or chitosan as carriers. The drug content was detected by UV-spectrophotometric analysis, morphology by scanning electronic spectroscopy and particle size by laser diffraction. In vitro drug release studies from microspheres were carried out according to USP 24. Ex vivo mucoadhesive tests were performed on sheep nasal mucosa blowing an air stream over the microspheres spread onto the mucosa and calculating the detection of drug content by HPLC, upon dissolution in water of microparticles still adhered onto the mucosa. The permeability of both free and microencapsulated drug was studied across sheep nasal mucosa using phosphate buffer ( $\mathrm{pH}$ 6.5) as receptor solution. Male Wistar rats received an intravenous infusion of drug. Nasal administration was also performed using insufflators Monopowder $P ®$ (Valois Dispray, France). After the administration, blood and liquor samples, as well as the olfactory bulb and ventricular sections of the brain were withdrawn and the respective drug amounts analysed by HPLC. According 
to our results, the drug influenced the particle size and morphology in comparison with blank microparticles. The drug dissolution rate increased or decreased after loading in mannitol or chitosan microparticles, respectively. About $60 \%$ of chitosan microspheres was recovered from mucoadhesive tests. The permeation rate of free drug was lower or higher than that of the encapsulated drug in mannitol or chitosan microspheres, respectively. The drug was not found in the central nervous system (CNS) after intravenous administration, which led to blood concentrations in the micromolar range. However, after nasal administration of the same dose, the drug was found in the CNS (up to micromolar range in the liquor, up to $0.2 \mathrm{ng} / \mathrm{mg}$ tissue in the brain sections). The CPA amounts detected in rat blood after nasal administration of the powders appeared negligible with respect to the amounts detected after intravenous administration.

We can conclude that nasal administration of microparticles prepared by spray-drying appears a promising strategy to obtain the selective CNS targeting of antiischemic adenosine derivatives.

(1) Dalpiaz A., Manfredini S. Curr. Med. Chem. 2002, 9, 1923-1937.

(2) Illum L. Eur. J. Pharm. Sci. 2000, 11, 1-18.

(3) Illum L. J. Pharm. Pharmacol. 2004, 56, 3-17.

\title{
N6-isopentenyladenosine arrests tumor cell proliferation by inhibiting farnesyl diphosphate synthase and protein prenylation.
}

\author{
C. Laezza*, M. Notarnicola $\uparrow$, M. G. Caruso $\dagger$, M. Macchiał, G.Portella, $\S$ S Pisanti^ ${ }^{\wedge}$ A Malfitano^${ }^{\wedge}$ C. \\ Grimaldi ^, A. Santoro ^, P. Gazzerro^ ${ }^{\wedge}$ and M. Bifulco^. \\ *Istituto di Endocrinologia e Oncologia Sperimentale. I.E.O.S., CNR, †I.R.C.C.S. "S. de Bellis" Castellana \\ G. (Bari), $\ddagger$ Dipartimento di Scienze Farmaceutiche, Universita di Pisa, §Dipartimento di Biologia e Patologia. \\ Cellulare e Molecolare "L.Califano," Universita" di Napoli "Federico II”, ^Dipartimento di Scienze \\ Farmaceutiche, Universita' di Salerno), Italy
}

The physiological effects of a variety of N6-substituted adenine and adenosine derivatives called cytokinins have been documented in plants, but information on their occurrence and function in other biological system is limited. Here we investigated the anti-proliferative effect of N6-isopentenyladenosine ( $\left.\mathrm{i}^{6} \mathrm{~A}\right)$, an adenosine and isoprenoid derivative, in a thyroid cell system, FRTL-5 wild-type, and K-ras transformed KiMol cells. Addition of $\mathrm{i}^{6} \mathrm{~A}$ to FRTL-5 cells caused a dose-dependent arrest of the G0-G1 cell phase transition associated with a reduction of cells in the $\mathrm{S}$ phase that was much more evident in KiMol cells. I6A arrested tumor cell proliferation by inhibiting farnesyl diphosphate synthase (FPPS) and protein prenylation. Indeed the addition of farnesol reversed these effects and i6A affected protein prenylation, in particular lamin B processing. $\mathrm{I}^{6} \mathrm{~A}$ effect was not mediated by the adenosine receptor but was due to a direct modulation of FPPS enzyme activity as a result of its uptake inside the cells. $\mathrm{I}^{6} \mathrm{~A}$ inhibited FPPS activity more efficaciously in KiMol cells than in normal FRTL-5. Moreover, the i6A anti-proliferative effect was evaluated in vivo in a nude mouse xenograft model, where KiMol cells were implanted subcutaneously. Mice treated with i6A showed a drastic reduction in tumor volume. Our findings indicate that this isoprenoid end product might be used for antineoplastic therapy, an application emulating that of the lovastatin and/or farnesyltransferase inhibitors.

chilaez@hotmail.com

\section{New $\mathbf{A}_{2 A}$ Adenosine Receptor Antagonists: Anti Parkinson's Activity and Metabolism Studies}

$\underline{\text { Ram Chandra Mishra }},{ }^{1}$ Catia Lambertucci, ${ }^{1}$ Rosaria Volpini, ${ }^{1}$ Sara Finaurini, ${ }^{2}$ Jan N. M. Commandeur, ${ }^{2}$ Micaela Morelli, ${ }^{3}$ Gloria Cristalli ${ }^{1}$

${ }^{1}$ Dipartimento di Scienze Chimiche, Università di Camerino, via S. Agostino, 1, 62032 Camerino, Italy

${ }^{2}$ LACDR-Division of Molecular Toxicology, Department of Pharmacochemistry, Vrije Universiteit, De Boelelaan 1083, NL-1081 HV Amsterdam, the Netherlands 
${ }^{3}$ Department of Toxicology, University of Cagliari, Via Ospedale, 72, 09124 Cagliari, Italy ramchandra.mishra@unicam.it

In search for new adenosine receptor antagonists our attention has been directed toward the synthesis of adenine derivatives, since 8-bromo-9-ethyladenine resulted to be a compound endowed with good affinity at human adenosine receptors and slight selectivity toward the $A_{2 A}$ subtype (8-bromo-9-ethyladenine, $K_{i} A_{1}=280 n M, K_{i} A_{2 A}$ $=52 \mathrm{nM}, \mathrm{K}_{\mathrm{i}} \mathrm{A}_{2 \mathrm{~B}}=840 \mathrm{nM}, \mathrm{K}_{\mathrm{i}} \mathrm{A}_{3}=28,000 \mathrm{nM}$ ). Hence, in the search of potent and selective adenosine receptor antagonists, the synthesis of a new series of 8-substituted 9-ethyladenines was undertaken.

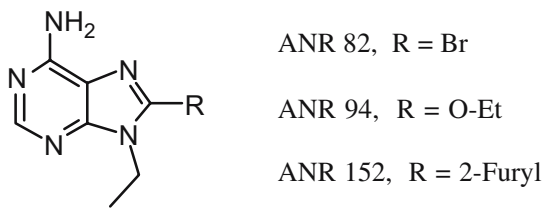

In particular, 9-ethyladenine substituted in 8-position with halogens, alkyl, alkoxy groups and heteroaromatic rings have been synthesized and tested in binding studies at adenosine receptors. ${ }^{1}$ Among them, the three most promising compounds in term of $\mathrm{A}_{2 \mathrm{~A}}$ binding affinity and selectivity (see figure), have been tested in two in vivo models of Parkinson's disease: 1) the haloperidol catalepsy reversal and 2) the 6-OHDA (6-hydroxydopamine) model of contralateral turning behviour. ${ }^{2}$ The adenine derivatives, ANR 82, ANR 94 and ANR 152, reversed the haloperidol induced catalepsy. In this model compounds ANR 82 and ANR 152 had maximum effect soon, after administration, which lasted for about 80 minutes while ANR 94 showed slower but longer lasting effect than the previous two. In the 2nd Parkinson model compounds ANR 94 and ANR 152 were able to potentiate L-DOPA (L-dihydroxyphenylalanine) induced contralateral turning in L-DOPA sensitized rats, while ANR 82 was found to be completely ineffective. Since these differences in efficacies of the ANR compounds in the two models could be explained in terms of different half-life, metabolic studies on rat liver microsomal cytochrome P450 (CYP) activity are in progress and results will be presented.

1. Klotz, K. N., Kachler, S., Lambertucci, C., Vittori, S., Volpini, R., Cristalli, G. (2003) 9-Ethyladenine derivatives as adenosine receptor antagonists: 2- and 8-substitution result in distinct selectivities, Naunyn-Schmiedeberg ${ }^{1}$ s Arch. Pharmacol. 367, 629-634.

2. Pinna, A., Volpini, R., Cristalli G., Morelli, M. (2005) New adenosine $A_{2 A}$ receptor antagonists: Actions on Parkinson's disease models, Eur. J. Pharm. 512, 157-164.

\title{
New messanger molecules of the adrenal glands
}

\author{
V. Jankowski, W. Zidek, M. van der Giet, J. Jankowski, \\ Vera.Jankowski@charite.de
}

\section{Charité - Campus Benjamin Franklin, Med. CliniC IV, Berlin Germany}

Dinucleoside polyphosphates have been characterised as extracellular mediators controlling numerous physiological functions like vascular tone or cell proliferation. Here we describe the isolation and identification of dinucleoside polyphosphates ApnA (with $n=2-3$ ), ApnG (with $n=2-6$ ) as well as GpnG (with $n=2-6$ ) from adrenal glands. These dinucleoside polyphosphates are localized in granules of the adrenal glands.

The dinucleoside polyphosphates diadenosine diphosphate $\left(\mathrm{Ap}_{2} \mathrm{~A}\right)$, diadenosine triphosphate $\left(\mathrm{Ap}_{3} \mathrm{~A}\right)$, the adenosine guanosine polyphosphates $\left(\mathrm{Ap}_{n} \mathrm{G}\right)$ and diguanosine polyphosphates $\left(\mathrm{Gp}_{\mathrm{n}} \mathrm{G}\right)$, both with phosphate group (p) numbers (n) ranging from 2 to 6 , were identified by fractionating them to homogeneity by preparative size-exclusion- and affinity-chromatography as well as analytical anion-exchange and reversed-phase-chromatography from deproteinized adrenal glands and by analysis of the homogenous dinucleoside polyphosphates containing fractions with post-source-decay (PSD) matrix-assisted laser desorption/ionisation mass spectrometry (MALDI-MS). 
The identity of the dinucleoside polyphosphates was confirmed by retention time comparison with authentic dinucleoside polyphosphates. Enzymatic analysis demonstrated an interconnection of the phosphate groups with the adenosines in the 5-positions of the riboses in all dinucleoside polyphosphates purified from adrenal glands. In conclusion, the identification of these dinucleoside polyphosphates in adrenal gland granules emphasizes that these dinucleoside polyphosphates can be released from the adrenal glands upon stimulation into the circulation.

\section{New Pyrrolo[2,1-f]purine-2,4-dione and Imidazo[2,1-f]purine-2,4-dione Derivatives as Potent and Selective Human $A_{3}$ Adenosine Receptor Antagonists}

Pier Giovanni Baraldi, ${ }^{\S}$ Delia Preti,${ }^{\S}$ Mojgan Aghazadeh Tabrizi,${ }^{\S}$ Francesca Fruttarolo, ${ }^{\S}$ Romeo Romagnoli, ${ }^{\S}$ Naser Abdel Zaid, 'Allan R. Moorman, ${ }^{\circ}$ Stefania Merighi, ${ }^{\#}$ Katia Varani, and Pier Andrea Borea \#

${ }^{\S}$ Dipartimento di Scienze Farmaceutiche, "Dipartimento di Medicina Clinica e Sperimentale-Sezione di Farmacologia, Università di Ferrara, 44100 Ferrara Italy, College of Pharmacy, An-Najah National University, Nablus, ${ }^{\circ}$ King Pharmaceutical Research and Development, Inc., 4000 CentreGreen Way, Suite 300, Cary, North Carolina 27513

$\mathrm{A}_{3}$ adenosine receptors and their ability to regulate cell survival represent a promising therapeutic target in diseases in which excessive cell death is either undesirable, such as neurodegeneration, or desirable, such as cancer and inflammation. ${ }^{1}$ The clarification of the role of adenosine and its receptors in cancer development may hold great promise for the chemotherapeutic treatment of patients affected by malignancies.

Different classes of compounds with non-xanthine structures have been reported to be $\mathrm{A}_{3}$ adenosine receptor antagonists. ${ }^{2}$ In a recent work, compounds presenting an additional fused ring on the xanthine nucleus have been reported to exhibit antagonistic activity with various levels of affinity and selectivity towards the four adenosine receptors subtypes $\mathrm{A}_{1}, \mathrm{~A}_{2 \mathrm{~A}}, \mathrm{~A}_{2 \mathrm{~B}}$ and $\mathrm{A}_{3} .{ }^{3}$ In particular, $1 \mathrm{H}, 3 \mathrm{H}$-pyrido[2,1-f]purine-2,4-diones ${ }^{4}$ have been claimed as potent $\mathrm{A}_{3}$ receptor antagonists. The report by Priego et al. ${ }^{4}$ about the mentioned $1 \mathrm{H}, 3 \mathrm{H}$-pyrido[2,1-f]purine2,4-diones, highlighted the importance of a benzyl and a propyl moieties at the 1 and 3 positions, respectively. We therefore evaluated the effect of the introduction of a benzyl and a propyl at the 1 and 3 positions respectively in a new series of fused xanthine derivatives. In particular, we performed the synthesis of 1-benzyl-3-propyl-7-aryl/ alkyl-1H,6H-pyrrolo[2,1-f]purine-2,4-dione (general structure 1) and 1-benzyl-3-propyl-7-aryl/alkyl-1 $\mathrm{H}, 8 \mathrm{H}$-imidazo[2,1-f]purine-2,4-dione (general structure 2) derivatives ${ }^{5}$ among which, very potent and selective $\mathrm{A}_{3}$ adenosine receptors antagonists have been identified. In particular, 1-benzyl-7-methyl-3-propyl-1 $\mathrm{H}, 8 \mathrm{H}$-imidazo[2,1-f]purine-2,4-dione shows a subnanomolar affinity towards the desired receptor target with a noteworthy selectivity versus the other adenosine receptors subtypes $\left(\mathrm{K}_{\mathrm{i}}(\mathrm{hA})=0.8 \mathrm{nM}, \mathrm{K}_{\mathrm{i}}\left(\mathrm{hA}_{1} / \mathrm{hA}_{3}\right)=3163, \mathrm{~K}_{\mathrm{i}}\left(\mathrm{hA} 2 \mathrm{~A} / \mathrm{hA}_{3}\right)\right.$ $\left.>6250, \mathrm{IC}_{50}\left(\mathrm{hA}_{2 \mathrm{~B}}\right) / \mathrm{K}_{\mathrm{i}}\left(\mathrm{hA}_{3}\right)=2570\right)$. Interestingly, a notable concordance between binding and functional experiments performed with $\mathrm{hA}_{3}$ receptor, has been revealed.

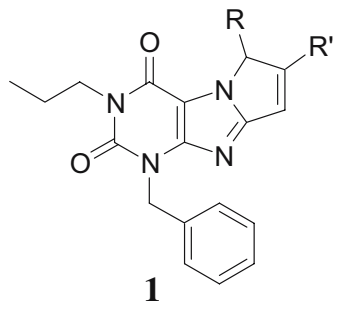<smiles></smiles>

1. Merighi et al. Pharmacol. Ther. 2003, 100(1), 31-48.

2. Baraldi et al. Drug. Dev. Res. 2003, 58, 315-329.

3. Drabczyńska et al. Eur. J. Med. Chem. 2003, 38, 397-402.

4. Priego et al. J. Med. Chem. 2002, 45, 3337-3344.

5. Baraldi et al. J. Med. Chem. 2005, 48, 4697-4701. 


\title{
New roles for extracellular ATP and UTP on keratinocytes : alteration of serum factors signaling pathways, lamellipodia dynamic and cell migration.
}

Salma Taboubi, Julie Milanini, Parat Fabrice, Jean-Claude Hubaud ${ }^{1}$ and Maxime Lehmann. FRE CNRS 2737, Faculté de Pharmacie, 27 Bd Jean Moulin, 13005 Marseille, France. ${ }^{1}$ DIPTA, 505 Rue Pierre BERTHIER, 13855 Aix en Provence.

salma.taboubi@gmail.com

Keratinocytes are the predominant cells of the skin. They express several purinergic receptors including P2Y2 (receptor for ATP and UTP). Purinergic receptor functions have been investigated and revealed their involvement in keratinocyte differentiation, proliferation and apoptosis. After wound healing, keratinocytes stimulated by numerous growth factors undergo profound morphological changes and migrate directionally to initiate reepithelialization. In the wound bed, ATP is released by damaged cells and platelets. The potential activity of extracellular nucleotides on keratinocyte dynamic was never studied before. The aim of our study was to determine whether ATP or UTP may regulate keratinocyte shape and migration.

By wound healing assays, we surprisingly discovered that ATP $(100 \mu \mathrm{M})$ inhibited by $50 \%$ the serum-induced migration of keratinocytes. Using video-microscopy, we observed that ATP and UTP destructed lamellipodia (migrating structures) after 10 min of treatment. Due to a desentization of the cells, this effect was transiant and lamellipodia started to regrow after $30 \mathrm{~min}$ of treatment. By kymography assays, we demonstrated that the dynamic of the newly synthesized lamellipodia was dramaticaly decreased. Finally, we observed that these ATPmediated effects were accompanied by a profound reorganization of the actin network. In keratinocytes, lamellipodia formation and cell migration are known to require activation of MAPK and PI3K/Akt pathways by growth factors. Here, we further report that ATP and UTP unexpectedly inhibited the phosphorylation of Akt and Erk1,2 induced by serum factors. As described above for lamellipodia, this inhibition was transiant and preceded lamellipodia destruction. ATP and UTP were equipotent to alter keratinocyte shapes and to inhibit Akt and Erk1,2 activity. This observation suggests that the Gaq-coupled P2Y2 receptor may be involved in these events. This work evidences new crosstalks between purinergic P2Y receptors and growth factors receptors and shows that ATP and UTP regulate keratinocyte migration and shape changes.

\section{NMDA GLUTAMATE RECEPTOR BINDING IN SPINAL CORDS OF MICE LACKING THE ADENOSINE A2A RECEPTOR}

\author{
M.J. Hussey, G.D. Clarke, I. Kitchen and S.M.O. Hourani. University of Surrey, Guildford, UK \\ m.hussey@surrey.ac.uk
}

Adenosine is a neuromodulator with complex effects on pain pathways. Mice lacking the adenosine $\mathrm{A}_{2 \mathrm{~A}}$ receptor are hypoalgesic (Ledent et al., 1997), and have altered analgesic responses to opioid agonists (Bailey et al., 2002). The adenosine $\mathrm{A}_{2 \mathrm{~A}}$ receptor is absent from the spinal cord despite mRNA transcripts in dorsal root ganglion, indicating transport of receptors to the peripheral terminal only (Kaelin-Lang et al., 1998), and the $\mathrm{A}_{2 \mathrm{~A}}$ agonist CGS21680 causes hyperalgesia in the paw pressure test (Khasar et al., 1995). These findings suggest a role for the $\mathrm{A}_{2 \mathrm{~A}}$ receptor in sensitizing afferent fibres projecting to the spinal cord. As glutamate is a primary nociceptive transmitter we have used spinal cord binding of $\left[{ }^{3} \mathrm{H}\right]-\mathrm{MK} 801$, an antagonist radioligand for the NMDA receptor, as an indirect measure of spinal nociceptive processing in $\mathrm{A}_{2 \mathrm{~A}}$ receptor knockout mice, to investigate whether peripheral $\mathrm{A}_{2 \mathrm{~A}}$ receptors influence pain transmission.

Adult male wildtype and $\mathrm{A}_{2 \mathrm{~A}}$ receptor knockout mice (CD1) were killed and the spinal cords removed. Sections $(20 \mu \mathrm{m})$ were cut from cervical, thoracic, lumbar and sacral regions. Total binding was determined by incubation with 70nM [ $\left.{ }^{3} \mathrm{H}\right]-\mathrm{MK} 801$ for 1 hour. Adjacent sections were incubated in the additional presence of $1 \mathrm{mM}$ unlabelled MK801 to determine non-specific binding. Sections were apposed to $\left[{ }^{3} \mathrm{H}\right]$ sensitive film (Hyperfilm, Amersham) with microscale standards and developed after 3 weeks. Quantitative analysis was carried out using an MCID imaging system. Statistical analysis of $\left[{ }^{3} \mathrm{H}\right]-\mathrm{MK} 801$ binding to NMDA glutamate receptors was carried out using 2-way ANOVA for the factors genotype and region.

There was a substantial reduction in binding of $\left[{ }^{3} \mathrm{H}\right]-\mathrm{MK} 801$ in all regions of the spinal cords of $\mathrm{A}_{2 \mathrm{~A}}$ knockout mice $(\mathrm{P}<0.001)$. The mean overall decrease was $61.3 \%$ throughout the spinal cord with the highest decreases seen 
in cervical and lumbar regions. We are also investigating time dependent changes in $\left[{ }^{3} \mathrm{H}\right]-\mathrm{MK} 801$ binding after inflammatory challenge with $\mathrm{PGE}_{2}$. Initial results show a decreased level of binding in $\mathrm{A}_{2 \mathrm{~A}}$ receptor knockout mice.

The decrease in NMDA glutamate receptor binding could reflect reduced peripheral sensory input to the spinal cord and be related to the hypoalgesia in this genotype. These results support a key role for the adenosine $\mathrm{A}_{2 \mathrm{~A}}$ receptor in peripheral pain pathways.

Bailey, A.; Ledent, C.; Kelly, M.; Hourani, S. M. O.; Kitchen, I., J Neurosci 2002, 22, (21), 9210-20. Khasar, S. G.; Wang, J. F.; Taiwo, Y. O.; Heller, P. H.; Green, P. G.; Levine, J. D., Neuroscience 1995, 67, (1), $189-95$. Kaelin-Lang, A.; Lauterburg, T.; Burgunder, J. M., Neurosci Lett 1998, 246, (1), 21-4.

Ledent, C.; Vaugeois, J. M.; Schiffmann, S. N.; Pedrazzini, T.; El Yacoubi, M.; Vanderhaeghen, J. J.; Costentin, J.; Heath, J. K.; Vassart, G.; Parmentier, M., Nature 1997, 388, (6643), 674-8.

This work was supported by a BBSRC CASE studentship in collaboration with GlaxoSmithKline.

\section{Non-phosphate analogues of adenine nucleotides}

Erki Enkvist, Gerda Raidaru, Asko Uri

Institute of Organic and Bioorganic Chemistry, University of Tartu, Jakobi 2, Tartu, Estonia

Erki.Enkvist@ut.ee

Extra-cellular adenine nucleotides regulate cellular processes via activation of purinoceptors. Many agonists and antagonists of nucleotide receptors are phosphate-containing analogues of native nucleotides with high negative charge in small volume close to adenine moiety. We have replaced phosphate groups of nucleotides with nonhydrolyzable carboxylate groups retaining high negative charge of the compounds. Flexible synthetic methods can be used for the preparation of compounds with different positioning of carboxylate groups. Fragments of molecules containing protected carboxylates were synthesised in solution or on solid-phase and connected to appropriate adenosine or adenine derivatives. Final deprotection and/or cleavage from the synthesis resin gave the desired compounds. Adenosine-oligoaspartate conjugates revealed moderate antagonistic activity towards $\mathrm{P}_{2} \mathrm{Y}_{1}$ receptor ${ }^{1}$. Analogues 2-thioalkyl derivatives inhibited $\mathrm{P}_{2} \mathrm{Y}_{12}$ receptors of rat brain and human platelets ${ }^{2}$. Direct connection of carboxylate-containing structures to the C9-position of adenine gave a class of compounds were the ribose moiety was missing. Some of these derivatives weakly inhibited ADP induced platelet aggregation ${ }^{3}$.
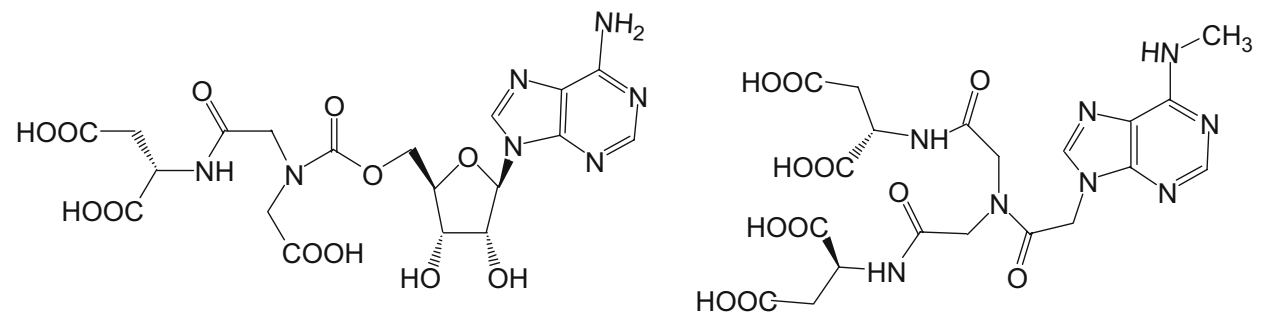

1. Sak, K., Uri, A., Enkvist, E., Raidaru, G., Subbi, J., Kelve, M and Järv, J. Biochem. Biophys. Res. Commun., 2000, 272, 327-331.

2. Vasiljeva, K. S.; Uri, A.; Laitinen, J. Neuropharmacology, 2003, 45, 145-154.

3. Enkvist, E., Raidaru, G., Uri, A., Patel, R., Redick, C., Boyer, J. L., Subbi, J., Tammiste, I. Nucleosides, Nucleotides Nucleic Acids, 2006, 25, 141-157. 


\section{Novel Potent and Selective Human Adenosine $\mathbf{A}_{3} d$}

Federico Da Settimo, ${ }^{\mathrm{c}}$ Giampaolo Primofiore, ${ }^{\complement}$ Concettina La Motta,${ }^{\complement}$ Laura Mugnaini, ${ }^{\complement}$ Sabrina Taliani, ${ }^{\complement}$ Francesca Simorini, ${ }^{\complement}$ Anna Maria Marini,,${ }^{\complement}$ E. Novellino, ${ }^{\S}$ G. Greco, ${ }^{\S}$ B. Cosimelli, ${ }^{\S}$ Maria Letizia Trincavelli, ${ }^{£}$ Claudia Martini ${ }^{£}$ ${ }^{c}$.Dipartimento di Scienze Farmaceutiche, Università di Pisa, Via Bonanno 6, 56126 Pisa, Italy. ${ }^{\S}$ Dipartimento di Chimica Farmaceutica e Tossicologica, Università di Napoli "Federico II", Via Domenico Montesano, 49, 80131 Napoli, Italy. ${ }^{£}$ Dipartimento di Psichiatria, Neurobiologia, Farmacologia e Biotecnologie, Università di Pisa, Via Bonanno 6, 56126 Pisa, Italy

lamotta@farm.unipi.it

Adenosine is an endogenous nucleoside which exerts its physiological functions through activation of specific cell membrane receptors, classified as $\mathrm{A}_{1}, \mathrm{~A}_{2 \mathrm{~A}}, \mathrm{~A}_{2 \mathrm{~B}}$ and $\mathrm{A}_{3}$ receptors. Adenosine $\mathrm{A}_{3}$ receptor, cloned in the early 1990s, plays a key role in both stimulation and inhibition of cell growth, in the release of inflammatory mediators, in the response to ischemia of the brain and heart and in glaucoma. Therefore, $\mathrm{A}_{3}$ selective antagonists represent an attractive therapeutic tool with potential cerebroprotective, anti-inflammatory, anti-asmathic and anti-glaucoma activity.

A number of different heterocyclic compounds have been identified as promising leads for $\mathrm{A}_{3}$ receptor antagonists, either xanthines or non-xanthines, showing varying degrees of potency and receptor subtype selectivity. As only few of them reach advanced clinical trials, the attention of the medicinal chemistry is still direct towards the discovery of more specific and effective antagonists.

In this study we report the synthesis and the biological evaluation of novel pyrazolo[3,4- $d]$ pyrimidines, substituted at positions 2, 4 and 6 of the heterocyclic core, which proved to be potent and selective antagonists at the human adenosine $\mathrm{A}_{3}$ receptor with binding affinity in the nanomolar range.

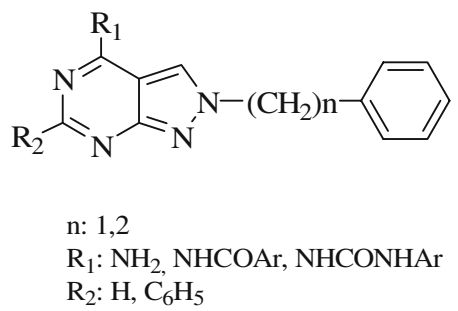

[1] P. G. Baraldi, B. Cacciari, R. Romagnoli, S. Merighi, K. Varani, P. A. Borea, G. Spalluto. Med. Res. Rev. 2000, 20,103-128.

[2] C. E. Muller. Curr. Top. Med. Chem. 2003, 3, 445-462.

\section{Novel Synthetic Stratergies Towards $A_{1}$ Adenosine Receptor Full and Partial Agonists}

$\underline{\text { Trent D. Ashton }}^{\mathrm{a}}$, Peter J. Scammells ${ }^{\mathrm{a}}$, Stephen P. Baker ${ }^{\mathrm{b}}$

${ }^{a}$ Department of Medicinal Chemistry, Victorian College of Pharmacy, Monash University, 381 Royal Parade, Parkville VIC 3052.

${ }^{\mathrm{b}}$ Department of Pharmacology \& Therapeutics, University of Florida College of Medicine, Box 100267, Gainesville FL 32610, USA.

trent.ashton@vcp.monash.edu.au

Partial agonists of the $\mathrm{A}_{1}$ adenosine receptor are envisaged to act as therapeutic agents in the treatment of arrhythmias. Their potential stems from less pronounced cardio vascular effects, less receptor down regulation and tissue selectivity. ${ }^{1-3}$ Our work has focused on the synthesis of a series of $N^{6}$-substituted-5'-modified adenosines of the general formula (2) through concise novel synthetic pathways ${ }^{4}$ and their assessment as partial or full 
agonists. Modification of this synthesis also allows divergent access to similar series with variation of the $N^{6}$ substituent.
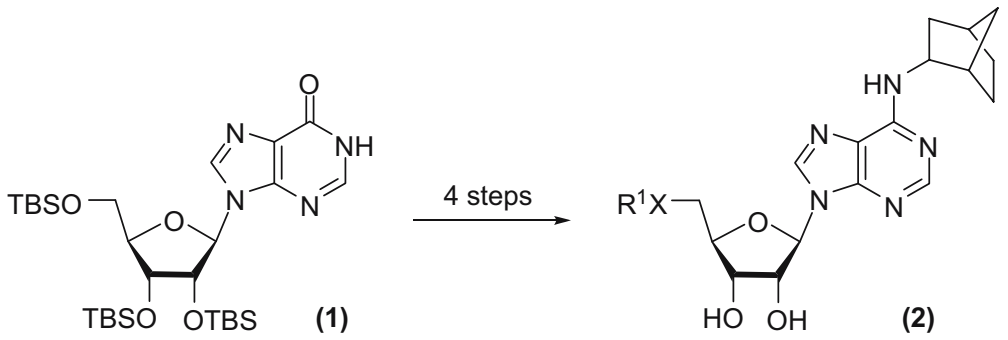

As the nature of the $N^{6}$-substituent has been shown to influence the potential for partial agonism,5 the development of novel $N^{6}$-substituents has also been investigated. Structures such as $\mathbf{3}$ and similar analogues have been the focus of this series.

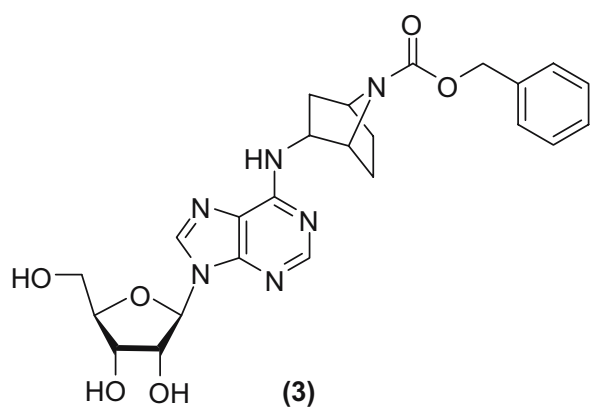

(1) Dhalla, A. K.; Shryock, J. C.; Shreeniwas, R.; Belardinelli, L. Pharmacology and Therapeutic Applications of A $_{1}$ Adenosine Receptor Ligands. Current Topics in Medicinal Chemistry 2003, 3, 369-385.

(2) Zablocki, J. A.; Wu, L.; Shryock, J.; Belardinelli, L. Partial A 1 Adenosine Receptor Agonists from a Molecular Perspective and Their Potential Use as Chronic Ventricular Rate Control Agents During Atrial Fibrillation. Current Topics in Medicinal Chemistry 2004, 4, 839-854.

(3) Hutchinson, S. A.; Scammells, P. J. A 1 Adenosine Receptor Agonists: Medicinal Chemistry and Therapeutic Potential. Current Pharma. Design 2004, 10, 2021-2039.

(4) Ashton, T. D.; Scammells, P. J. An Improved Synthesis of 5'-Deoxy-5'-fluoroadenosines. Bioorg. Med. Chem. Lett. 2005, 15, 3361-3363.

(5) Morrison, C. F.; Elzein, E.; Jiang, B.; Ibrahim, P. N.; Marquart, T. et al. Structure-Affinity Relationships of 5'-Aromatic Ethers and 5'-Aromatic Sulfides as Partial A1 Adenosine Agonists, Potential Supraventricular AntiArrhythmic Agents. Bioorg. Med. Chem. Lett. 2004, 14, 3793-3797.

\section{NUCLEOTIDE RELEASE ASSOCIATED WITH MUCIN SECRETION IN POLARIZED AIRWAY EPITHELIAL CELLS}

Silvia M Kreda, Seiko Okada, Catharina van Heusden, Wanda O’Neal, Richard C Boucher, and Eduardo R Lazarowski,

Cystic Fibrosis/Pulmonary Research and Treatment Center, The University of North Carolina at Chapel Hill,

Chapel Hill, NC, USA.

Silvia_kreda@med.unc.edu

Nucleotides and nucleotide-sugars within the airway surface liquid play important roles in innate lung defense but the mechanisms of airway epithelial nucleotide release are poorly understood. In this study, we report that polarized monolayers of CalU-3 cells, a human airway-derived epithelial cell line known to express high levels of the cystic fibrosis transmebrane regulator (CFTR) $\mathrm{Cl}^{-}$channel, produce and secrete mucins (e.g. MUC5AC) trough 
the apical surface. A fraction of cells (5-40\%) displayed apically localized secretory mucin granules that resembled those observed in native goblet cells, as revealed by electron microscopy and MUC5AC immunostaining/ confocal microscopy. Secreted MUC5AC was detected (by slot blotting) in the mucosal but not serosal bath of unstimulated CalU-3 cells. Adenine nucleotides and UDP-glucose were present also in the apical (but not in the basolateral) bath of resting cells. Elevation of intracellular $\mathrm{Ca}^{2+}(1-10 \mu \mathrm{M}$ ionomycin) promoted exocytosis trough the apical membrane, as judged by increased (i) FM 1-43 membrane fluorescence, (ii) secretion of MUC5AC into the apical bath (slot blotting), and (iii) massive discharge of MUC5AC granules (immunostaining). Similarly, intracellular granules that were pre-labeled with either FM1-43, quinacrine, or acridine orange were released upon increase in intracellular $\mathrm{Ca}^{2+}$. These exocytotic changes were paralleled by enhanced release of ATP and UDP-glucose to the apical but not to the basal bath. Removal of $\mathrm{Ca}^{2+}$ from bathing solutions or disruption of the cytoskeleton $(5 \mu \mathrm{M}$ cytochalasin D) cancelled ionomycin-promoted both exocytosis and nucleotide release. In contrast to $\mathrm{Ca}^{2+}$-mediated changes, elevation of cyclic AMP levels and activation of CFTR (10 $\mu$ M forskolin) failed to promote exocytosis/mucin secretion or nucleotides release from CalU-3 cells. These studies indicate that CalU-3 cultures can differentiate into cells that express mucin granules competent for regulated secretion. Secretion of mucins may be accompanied by nucleotide release from mucous cells, suggesting that regulated exocytosis of specialized glycoproteins may be an important mechanism to control the secretion of nucleotides and nucleotide sugars in complex airway epithelia.

\title{
Opposing Effects of Extracellular ATP and Adenosine on Osteoblast Differentiation in Human Valve Interstitial Cells
}

\author{
L Osman $^{1}$, RT Smolenski ${ }^{1}, \mathrm{M} \mathrm{Amrani}^{2}, \mathrm{MH}$ Yacoub ${ }^{1}$, AH Chester ${ }^{1}$ \\ ${ }^{T}$ Imperial College, Harefield Heart Science Centre, ${ }^{2}$ Department of Cardiothoracic Surgery, Royal Brompton and \\ Harefield NHS Trust, Harefield, U.K. \\ lana.osman@imperial.ac.uk
}

Calcific aortic valve disease is an active process ranging from aortic sclerosis to severe calcification with unknown cellular mechanisms. Extracellular ATP is known to be an important molecule in bone remodelling, however, its breakdown product, adenosine has been shown to exert anti-inflammatory properties. We hypothesise that both extracellular ATP and adenosine will exert opposite effects on osteoblast differentiation in human valve cells. We therefore, aim to asses the ability of extracellular ATP and adenosine to promote or inhibit the expression of osteoblast-cell markers in human valve interstitial cells (VICs).

Primary cultures of human aortic VICs were treated for 21 days with ATP $(100 \mu \mathrm{M})$, ATP- $\gamma$-S $(10 \mu \mathrm{M})$, 2-Methylthio-ADP $(10 \mu \mathrm{M})$; two stable agonists to the P2Y receptor. Alkaline phosphatase (ALP) (an osteoblast marker) activity and expression were measured using a colorimetric assay and immunocytochemistry staining. Furthermore, cultures of VICs were also grown in osteogenic media in the presence and absence of adenosine $(30 \mu \mathrm{M})$ for 21 days and ALP activity and expression were determined.

Extracellular ATP and the stable agonists to the P2Y receptor were able to mimic the effects of osteogenic media. Valve ICs treated with osteogenic media significantly increased ALP activity from $3.1 \pm 1.0 \mathrm{nmol} / \mathrm{min} / \mathrm{mg}$ protein in control cells to $14.4 \pm 1.4 \mathrm{nmol} / \mathrm{min} / \mathrm{mg}$ protein. In addition, both ATP and ATP- $\gamma-\mathrm{S}$ increased the ALP activity to $12.1 \pm 1.9 \mathrm{nmol} / \mathrm{min} / \mathrm{mg}$ and $11.1 \pm 1.5 \mathrm{nmol} / \mathrm{min} / \mathrm{mg}$ protein, respectively. This increase was reduced in valve ICs treated with 2-Methylthio-ADP to $5.9 \pm 1.2 \mathrm{nmol} / \mathrm{min} / \mathrm{mg}$ protein after 21 days of treatment $(\mathrm{n}=3)$. Adenosine treatment inhibited the effect of osteogenic media by significantly reducing the activity and expression of ALP from $10.4 \pm 0.8 \mathrm{nmol} / \mathrm{min} / \mathrm{mg}$ protein to $3.4 \pm 0.6 \mathrm{nmol} / \mathrm{min} / \mathrm{mg}$ protein in human VICs $(\mathrm{n}=3)$.

In conclusion, extracellular ATP and stable agonists to P2Y receptor induced the transformation of VICs into osteoblast-like cell phenotype, while adenosine was capable of inhibiting this transformation process. These opposite effects may have important implications in understanding the regulatory effect of extracellular nucleotides on valve calcification and helps in identifying new therapeutic targets. 


\title{
Opposite effects of the $A_{2 A}$ receptor agonist CGS 21680 in the striatum of Huntington Disease (HD) versus wild-type (WT) mice.
}

\author{
A. Martire $^{1}$, G. Calamandrei ${ }^{2}$, F. Felici ${ }^{1}$, M.L. Scattoni ${ }^{2}$, M.T. Tebano ${ }^{1}$, A. Pintor ${ }^{1}$, P. Popoli ${ }^{1}$ \\ (1)Department of Drug Research and Evaluation, (2) Department of Cell Biology and Neuroscience Istituto \\ Superiore di Sanità, Rome, Italy. \\ alberare@iss.it
}

Adenosine $\mathrm{A}_{2 \mathrm{~A}}$ receptors $\left(\mathrm{A}_{2 \mathrm{~A}} \mathrm{Rs}\right)$ have been proposed as possible targets to treat Huntington's disease (HD). However, the effects of $\mathrm{A}_{2 \mathrm{~A}} \mathrm{R}$ ligands may become unpredictable in presence of HD mutation, since mutant huntingtin was found to influence both the function and the expression of $\mathrm{A}_{2 \mathrm{~A}} \mathrm{R}$ in cellular models of HD. The aim of the present work was to investigate whether the HD mutation influenced $A_{2 A} R$-mediated effects on basal synaptic transmission and on NMDA-dependent toxicity. Transgenic R6/2 mice in a frankly symptomatic phase (12-13 weeks) and age-matched WT mice were used. The animals were decapitated, the brain removed and corticostriatal slices (300 $\mu \mathrm{m}$ thick) cut with a vibratome. Extracellular field potentials (FPs) were recorded in the striatum; the mean basal FP amplitude was calculated, and the effects of the drugs expressed as percentage variation with respect to basal values. Application of $75 \mu \mathrm{M}$ NMDA to WT slices induced a transient disappearance of the FP followed by a recovery of $67.9 \pm 8.2$ and $89.3 \pm 9 \%$ of basal after 30 and 50 min of washout, respectively $(\mathrm{N}=8)$. In slices from HD mice, the mean FP recovery was significantly reduced $(39.4 \pm 4$ and $47.8 \pm 5 \%$ of basal after 30 and 50 min of washout, respectively; $\mathrm{N}=9, \mathrm{P}<0.05 v s$ WT in both cases). The co-application of $100 \mathrm{nM}$ CGS $21680\left(\mathrm{~A}_{2 \mathrm{~A}} \mathrm{R}\right.$ agonist $)$ reduced the FP recovery in slices from WT mice $(35.2 \pm 10$ and $46.7 \pm 13.5 \%$ after 30 and $50 \mathrm{~min}$, respectively, $\mathrm{N}=6, \mathrm{P}<0.05$ vs NMDA alone). Interestingly, CGS 21680 exerted the opposite effect (i.e. a significant increase in FP recovery), in slices from R6/2 HD mice (59.7 $8 \%$ after $30 \mathrm{~min}, \mathrm{P}<0.05$, and $65.8 \pm 9, \mathrm{NS}, \mathrm{N}=7)$. The $\mathrm{A}_{2 \mathrm{~A}} \mathrm{R}$ antagonist $\mathrm{ZM} 241385$ did not influence the FP recovery in WT or HD slices. These results show that the pharmacology of $\mathrm{A}_{2 \mathrm{~A}} \mathrm{Rs}$ is altered by the HD mutation. In particular, $\mathrm{A}_{2 \mathrm{~A}} \mathrm{Rs}$ oppositely modulate NMDA-induced toxicity in the striatum of HD vs WT mice. These results may have important implications for the neuroprotective potential of $\mathrm{A}_{2 \mathrm{~A}} \mathrm{R}$ antagonists in $\mathrm{HD}$.

\section{Orthogonal Activation of the Reengineered $\mathbf{A}_{3}$ Adenosine Receptor (Neoceptor) Using Tailored Nucleoside Agonists}

Kenneth A. Jacobson ${ }^{\mathrm{a}}$, Heng T. Duong ${ }^{\mathrm{a}}$, Tatiana Sonin, ${ }^{\mathrm{b}}$ Soo-Kyung Kim ${ }^{\mathrm{c}}$, Philippe Van Rompaey ${ }^{\mathrm{c}}$, Serge Van Calenbergh $^{\mathrm{d}}$, Liaman Mamedova ${ }^{\mathrm{a}}$, Hea Ok Kim ${ }^{\mathrm{c}}$, Myong Jung Kim ${ }^{\mathrm{c}}$, Ae Yil Kim ${ }^{\mathrm{c}}$, Bruce T. Liang $^{\mathrm{b}}$, Lak Shin Jeong ${ }^{\mathrm{c}}$, and Zhan-Guo Gao ${ }^{\mathrm{a}}$

${ }^{a}$ Molecular Recognition Section, Lab. of Bioorganic Chemistry, National Inst. of Diabetes and Digestive and Kidney Diseases, NIH, Bethesda, MD 20892, USA.

${ }^{\mathrm{b}}$ Department of Cardiology, University of Connecticut Health Center, Farmington, CT 06030, USA.

${ }^{\mathrm{c}}$ Lab. of Medicinal Chemistry, College of Pharmacy, Ewha Womans Univ., Seoul 120-750, Korea.

${ }^{\mathrm{d}}$ Lab. for Medicinal Chemistry, Ghent University, Harelbekestraat 72, B-9000 Ghent, Belgium.

kajacobs@helix.nih.gov

An integrated approach to the study of drug-receptor interactions, based on probing the receptor structure through site-directed mutagenesis and molecular modeling, has been applied to adenosine receptors (ARs) and other seven transmembrane-spanning receptors. Selective AR agonists are sought for therapeutic application, ${ }^{1}$ however, because of the widespread distribution of native ARs, their activation is inherently nonselective. An alternative approach to overcome the lack of specificity of conventional agonist therapy can be the reengineering of the GPCRs and their agonists. A reengineered receptor (neoceptor) could be selectively activated by a modified agonist, but not by the endogenous agonist. ${ }^{2}$ Synthetic nucleoside agonists have been specifically tailored to serve as neoligands, i.e. to activate only receptors in which the putative binding sites have been modified. This orthogonal approach to receptor activation, intended for eventual gene therapy, has been explored for $\mathrm{A}_{3}$ and $\mathrm{A}_{2 \mathrm{~A}}$ ARs. $^{1-3}$ Assisted by rhodopsin-based molecular modeling, we pinpointed mutations of the $\mathrm{A}_{3} \mathrm{AR}$ for selective affinity enhancement following complementary modifications of adenosine. Ribose modifications examined incl- 
uded, at 3': amino, aminomethyl, azido, guanidino, ureido; and at 5': uronamido, azidodeoxy. $N^{6}$ variations included: 3-iodobenzyl, 5-chloro-2-methyloxybenzyl, and methyl. As predicted by the molecular modeling and ligand docking, certain adenosine derivatives modified in the $3^{\prime}$-position displayed selective affinity enhancement at a mutant (H272E), but not the wild-type, $\mathrm{A}_{3} \mathrm{AR}$. An $N^{6}$-(3-iodobenzyl)-3'-ureido adenosine derivative MRS3481 (LJ720) activated phospholipase $\mathrm{C}$ in COS-7 cells $\left(\mathrm{EC}_{50}=0.18 \mu \mathrm{M}\right)$ or phospholipase $\mathrm{D}$ in chick primary cardiomyocytes mediated by the H272E neoceptor, but not wild-type, $\mathrm{A}_{3} \mathrm{AR}$. The affinity enhancements for MRS3481 and the corresponding 3'-acetamidomethyl analogue MRS3174 were $>100$-fold and $>20$-fold, respectively. MRS3481 concentration-dependently protected cardiomyocytes transfected with the neoceptor against hypoxia. Unlike MRS3481, adenosine activated the wild-type $\mathrm{A}_{3} \mathrm{AR}\left(\mathrm{EC}_{50}\right.$ of $\left.1.0 \mu \mathrm{M}\right)$, but had no effect on the $\mathrm{H} 272 \mathrm{E}$ mutant $\mathrm{A}_{3} \mathrm{AR}(100 \mu \mathrm{M})$. This truly orthogonal pair comprising an engineered receptor and a modified agonist should be useful for elucidating signaling pathways and could be therapeutically applied to diseases following organ-targeted delivery of the neoceptor gene.

1. Jacobson, K.A., et al. Chem. Biol. 2005, 30, 237-247.

2. Jacobson, K.A., et al. J. Med. Chem. 2001, 44, 4125-4136.

3. Kim, S.K., et al. J. Med. Chem. 2003, 46, 4847-4859.

\title{
Osmotic stress enhances neutrophil degranulation by the release of ATP and the activation of $P 2$ and $A 3$ receptors
}

\author{
$\underline{\text { Yu Chen }}^{1}$, Linda Yip ${ }^{1}$, Ross Corriden ${ }^{1,2}$, Paul A. Insel ${ }^{2}$, and Wolfgang G. Junger ${ }^{1}$ \\ University of California San Diego, Depts. of ${ }^{1}$ Surgery/Trauma, and ${ }^{2}$ Pharmacology, San Diego, California 92103. \\ y20chen@ucsd.edu
}

Polymorphonuclear neutrophils (PMN) play a crucial role in the defense against invading bacteria, fungi, and protozoa. However, excessive activation of PMN is involved in the pathology of various diseases, including major post-traumatic complications. Hypertonic fluids can be used to resuscitate trauma patients who have suffered severe blood loss. Our previous work has shown that hypertonic resuscitation fluids can reduce the risk of posttraumatic complications by suppressing PMN function. We have found that hypertonic saline (HS) induces the release of cellular ATP from PMN and that ATP is quickly hydrolyzed to adenosine, which in turn activates A2a adenosine receptors expressed on the PMN cell surface. However, we and other researchers have found augmentation of the degranulation and enzyme release of PMN when the cells are activated before they are exposed to HS. Here we studied if this enhancement of PMN degranulation also involves feedback mechanisms related to ATP release and purinergic receptors.

We found that, like HS, exogenously added ATP and the non-hydrolyzable form ATP $\gamma$ S enhance enzyme (elastase) release and the expression of the degranulation marker CD11b of PMN previously stimulated by the peptide, fMLP. These effects were paralleled by corresponding increases in ERK and p38 MAPK activation patterns. Removal of extracellular ATP with apyrase or inhibition of P2 receptors with suramin abolished the enhancing effect of HS on elastase release. Agonists of A3, but not A2, adenosine receptors also enhanced elastase release and p38 MAPK activation of fMLP-stimulated PMN, while A1 receptor agonists had the opposite effect (Fig.). Studies with antagonists of these receptors showed that HS enhances degranulation via A3 receptors. 


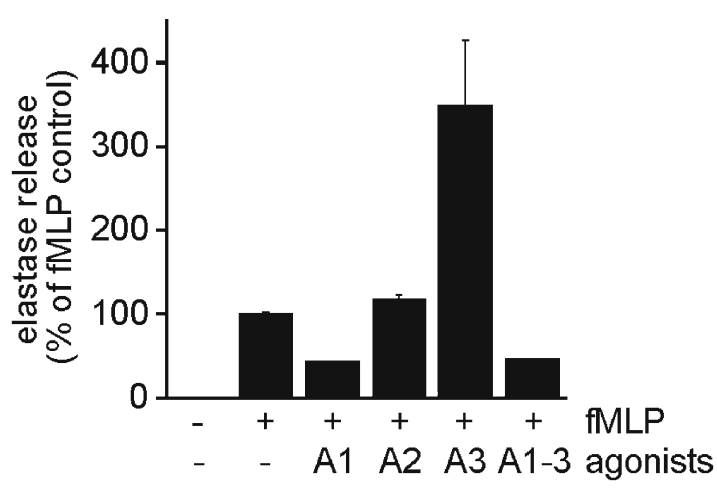

Fig. Agonist of A3 receptors (IB-MECA) enhanced elastase release of fMLP-stimulated PMN, while A1 receptor agonist $(C P A)$ decreased $P M N$ degranulation.

We conclude that pharmacological manipulation of the receptors that control the action of HS on PMN responses could be a useful therapeutic approach to adjust the efficacy of hypertonic fluid resuscitation by reducing the risk of exacerbated PMN activation and tissue damage in trauma patients.

This study was supported in part by grants from the U.S. National Institutes of Health (GM-51477 \& 60475).

\section{P2 RECEPTOR ANTAGONISM AND MAPK ACTIVATION IN A MODEL OF FOCAL CEREBRAL ISCHEMIA}

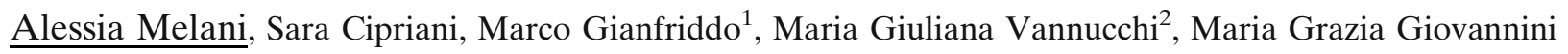
and Felicita Pedata

Dept. of Pharmacology, University of Florence, Italy; ${ }^{1}$ Sienabiotech S.p.A., Siena, Italy;

${ }^{2}$ Dept. of Istology, University of Florence, Italy. alessia.melani@unifi.it

Extracellular adenosine $5^{\prime}$-triphosphate (ATP) levels increase under ischemic conditions in rat striatum in vivo ${ }^{1}$. Data in the literature support the idea that ATP exerts an excitotoxic role on P2 receptors in the brain. We recently demonstrated that, in a model of focal cerebral ischemia, the non selective P2 receptor antagonist, Reactive Blue 2 (RB2), reduced both the striatal and the cortical damage and improved the neurological deficit, $24 \mathrm{~h}$ after ischemia ${ }^{2}$. The aim of the present study was to evaluate if neuroprotective effect of RB2 involves activation of mitogen-activated protein kinases (MAPKs) during cerebral ischemia.

Permanent focal cerebral ischemia was induced by right middle cerebral artery occlusion (MCAo) in the rat. RB2 $(100 \mathrm{mg} / \mathrm{kg}$, i.p.) was administered $5 \mathrm{~min}$ after MCAo. Between 3 and $4 \mathrm{~h}$ after MCAo an acute motor disturbance was evaluated as number of rotations per hour. Twenty-four hour after ischemia, neurological deficit was evaluated by a battery of sensory-motor tests. Rats were then sacrificed by transcardiac perfusion with $4 \%$ paraformaldehyde solution. Brains were cut in $30-\mu \mathrm{m}$-thick coronal slices to evaluate ischemic brain damage by acetate cresyl violet staining and activation of p38-, ERK1/2- and JNK-MAPK by specific antibodies. Neurons, microglia and oligodendrocytes were also stained by NeuN, OX-42 and MAG specific antibodies, respectively.

Vehicle-treated rats, soon after ischemia, showed a definite turning behaviour contralateral to ischemic hemisphere. RB2 did not modify this acute motor disturbance. Twenty-four hours after ischemia, vehicle-treated rats showed an extensive ischemic area in the vascular territories supplied by the MCA, the striatum and the sensorymotor cortex, and a clear neurological deficit. RB2 protected against ischemic striatal and cortical damage and against the neurological deficit. In vehicle-treated rats $24 \mathrm{~h}$ after MCAo, phospho-p38 MAPK, evaluated by Western Blot, increased by $500 \%$ in the ischemic striatum. Phospho-p38 MAPK and OX-42 immunoreactive cells were localized in the ventral striatum and frontoparietal cortex of the ischemic hemisphere. OX-42 and phosphop38 MAPK immunoreactive cells showed overlapping morphological features typical of reactive microglia. Phospho-ERK1/2 immunopositive cells were localized in the cortex and in the striatum of the ischemic hemisphere and showed morphological features of reactive microglia. Phospho-JNK immunopositive cells were present in the corpus callosum and in the white matter of the striatal nuclei of the ischemic hemisphere. RB2 did not modify activation of any of the MAPK investigated. 
In conclusion, our data show that the protective effect of the unselective P2 receptor antagonist RB2 does not involve modulation of MAPKs.

Grant by University of Florence and Ente Cassa di Risparmio di Firenze, Italy.

(1) Melani, A.; Turchi, D.; Vannucchi, M.G.; Cipriani, S.; Gianfriddo, M; Pedata, F. Neurochem. Int. 2005, 47, 442-448.

(2) Melani, A.; Amadio, S.; Gianfriddo, M.; Vannucchi, M.G.; Volontà, C.; Pedata, F.; Sancesario, G. J. Cereb. Blood Flow Metab. 2006 In Press.

\section{P2 receptors from heart to brain: emergence of complexity on a receptor family.}

Cinzia Volontè $^{1}$, Susanna Amadio ${ }^{1}$, Fabio Cavaliere ${ }^{1}$, Nadia D’Ambrosi ${ }^{1}$, Marcos Frizzo ${ }^{2}$, Juliana Karl Frizzo $^{1}$, Fabrizio Vacca ${ }^{1}$, Monica Colpi ${ }^{3}$, Geoffrey Burnstock ${ }^{4}$

${ }^{1}$ Santa Lucia Foundation/CNR, Via Del Fosso di Fiorano 64, 00143 Rome, ITALY

${ }^{2}$ Departamento de Ciências Morfológicas, ICBS, UFRGS, Porto Alegre, BRAZIL

${ }^{3}$ Dip. di Fisica G. Occhialini, Universita' di Milano-Bicocca, Milano, ITALY

${ }^{4}$ Autonomic Neuroscience Institute, Royal Free \&University College Medical School, London, UK cinzia.volonte@inmm.cnr.it

The present work seeks to offer a new perspective on a family of receptors, the P2 receptors, specific for nucleoside tri- and di-phosphates of both purines and pyrimidines. The prototype ligand is ATP, one of the most diffuse extracellular signaling molecules in many organs and tissues, including the central nervous system. We emphasize here that while decoding the inputs of various different, but closely related, extracellular ligands such as ATP, ADP, UTP, UDP, UDP-glucose, P2 receptors are a clear example of the emergence of increasing biological complexity. They are represented by ionotropic P2X and metabotropic P2Y proteins, by seven P2X and eight distinct P2Y subunits; they couple to various ligands, own very heterogeneous ligand binding characteristics, molecular properties, transduction mechanisms, cellular localization, and protein-protein interactions. While the reason for this sophistication is currently unknown, a few compelling issues emerge in looking at such a rich variety. We ask, for instance, why so many different receptor subtypes are necessary for triggering biological properties and functions, and if these receptors are more than the sum of their single entities. A very first possibility is that newly synthesized P2 proteins are casually located on the cell surface (stochastic hypothesis). Otherwise, distinct P2 subunits could be engaged on the different cellular phenotypes by a strict genetic control (genetic determinism) and/or selective recruitment under different physiopathological conditions and epigenetic stimuli (epigenetic determinism). Nevertheless, the most proper way to dissect the vast biological scenario and molecular diversities among P2 subunits and, at the same time, to integrate and upgrade this assortment into a final common outcome, is to regard these receptors as a "receptor web", that is, a dynamic architecture of P2 proteins legitimated by an energetic advantage. This condition is in turn sustained by a process of "fine tuning", a mechanism endorsing the dynamic nature of all biological reactions.

In the present analysis we would like to stimulate a scientific query on what contributes to the biological emergence of such a vast $\mathrm{P} 2$ receptor sophistication.

The present work was supported by grants from MIUR 2004 and FIRB 2001. J.K.F. is a recipient of a postdoctoral fellowship from the Brazilian funding agency CNPq.

\section{P2-Receptor mediated immune-responses: a link to asthmatic airway inflammation?}

Marco Idzko $^{1,2}$, Hamida Hammad ${ }^{2}$, Menno v Nimwegen ${ }^{2}$, Davide Ferrari ${ }^{3}$, Elisabeth Panther ${ }^{4}$, Mirjam Kool $^{2}$, Francesco Di Virigilio ${ }^{3}$, Bart N Lambrecht ${ }^{2}$

Dept. of Pneumology ${ }^{1}$ and Gastroenterology ${ }^{4}$, University of Freiburg, Germany, Dept. of Pneumology ${ }^{2}$, Erasmus MC, Rotterdam, Netherlands, Dept. of Experimental and Diagnostic Medicine ${ }^{3}$, University of Ferrara, Italy 
Extracellular nucleotides has been shown to modulate in vitro the function of various cell types, including eosinophils, lymphocytes, dendritc cells and airway epithelial cells, which are involved in the pathogenesis of asthmatic airway inflammation. Therefore, the aim of the present study was investigate the role of P2-Receptors mediated immune response in vivo an experimental asthma. Hereby we could show in a murine asthma model (Balb/c mice) that local application of P2X and P2Y receptor antagonist (suramin, PPADS and oATP) suppresses Th2-dependent eosinophilic airway inflammation and induction of bronchial hyperresponsiveness when given during the allergen challenge phase (Figure 1A and B). Furthermore, local suramine treatment inhibited the migration of lung DCs to the mediastinal lymph nodes and abolished the induction of a Th2 response to ovalbumin in these nodes. In contrast, intratracheal injection of ATP together with FITC-labelled OVA in naive Balb/c induce a significant recruitment of OVA-FITC ${ }^{+}$-lung DC to the mediastinal lymph node, compared mice receiving vehicle/FITC-labelled OVA. Finally, we could show here, that the levels of extracellular ATP are increased in the airway of sensitized ("asthmatic") mice compared to naive controls (Figure 2). These data demonstrate that endogenous release of ATP occurs during asthmatic airway inflammation, and that application of P2-receptor antagonists to the lung targets the key functions of airway DCs thus suppressing the cardinal features of asthma. Future studies in asthma patients will demonstrate if this is a feasible strategy.

Figure 1 A

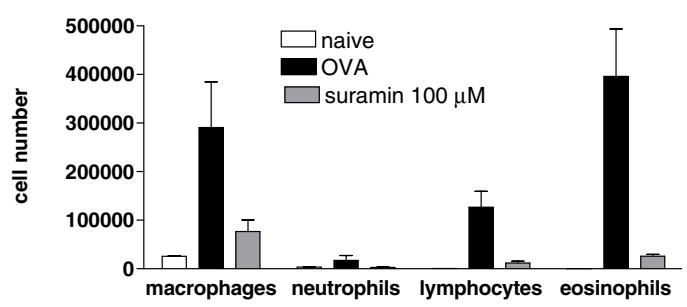

B

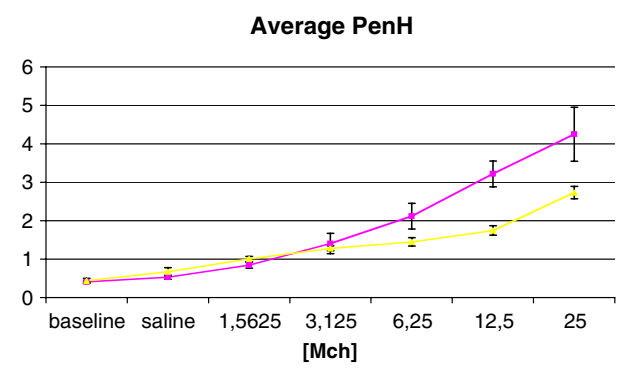

Figure 2

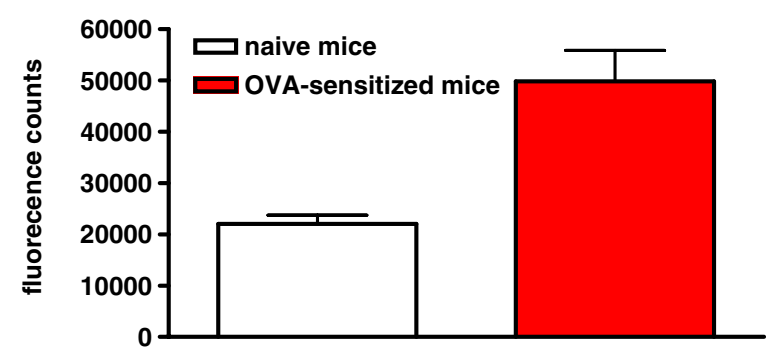

\section{$\mathbf{P 2 X}_{4}$ receptor function in rat macrophage}

\section{Leanne Stokes \& Annmarie Surprenant}

Department of Biomedical Science, University of Sheffield, Florey Building, Firth Court, Western Bank, Sheffield, S10 2TN. UK.

l.stokes@sheffield.ac.uk

Macrophages play a key role in inflammation due to the synthesis and release of pro-inflammatory cytokines, for example one of the known functional roles of the ATP-gated ion channel $\mathrm{P}_{2} \mathrm{X}_{7}$ in macrophages is the release of mature IL-1? following treatment with LPS and ATP. P2X family, is often co-expressed with $\mathrm{P} 2 \mathrm{X}_{7}$ in immune cells, but to date the physiological role of $\mathrm{P} 2 \mathrm{X}_{4}$ is unclear. Our group has shown functional expression of $\mathrm{P}_{2} \mathrm{X}_{4}$ but not $\mathrm{P} 2 \mathrm{X}_{7}$ in the NR8383 rat alveolar macrophage cell line (Bowler et al, 2003) making this cell line a useful model for the study of $\mathrm{P} 2 \mathrm{X}_{4}$ function in isolation from $\mathrm{P} 2 \mathrm{X}_{7}$. We are investigating whether activation of $\mathrm{P}_{2} \mathrm{X}_{4}$ can induce or influence cytokine secretion or affect key macrophage 
functions. Classical activation of NR8383 macrophages with combinations of IFN- $\gamma$, TNF- $\alpha$ and LPS has differing effects on the macrophage $\mathrm{P}_{2} \mathrm{X}_{4}$ response. This appears to be correlated to regulation of cell surface expression of $\mathrm{P}_{2} \mathrm{X}_{4}$ as determined by cell surface biotinylation experiments.

Bowler et al, 2003. $\mathrm{P} 2 \mathrm{X}_{4}, \mathrm{P}_{2} \mathrm{Y}_{1}$ and $\mathrm{P}_{2} \mathrm{Y}_{2}$ receptors on rat alveolar macrophages Br. J. Pharmacol. 140, 567-575.

\title{
$\mathrm{P2X}_{7}$ purinergic receptor is involved in the process of renal inflammation and fibrosis of unilateral ureteral obstruction in mice
}

\author{
Romulo G. Gonçalves ${ }^{1}$, Letícia Gabrich ${ }^{1}$, Cesonia A. Martinusso ${ }^{1}$, Pedro M. Persechini ${ }^{2}$, Robson \\ Coutinho-Silva ${ }^{2}$, Maurilo Leite $\mathrm{Jr}^{1}$. \\ ${ }^{1}$ Hospital Universitário Clementino Filho, Universidade Federal do Rio de Janeiro. \\ ${ }^{2}$ Instituto de Biofísica Carlos Chagas Filho, Universidade Federal do Rio de Janeiro. \\ mleitejr@hucff.ufrj.br
}

Purinergic receptors have been involved in several cellular mechanisms including those related to inflammation and immunological response. Receptors of the $\mathrm{P}_{2} \mathrm{X}_{7}$ type have been demonstrated in granulocytes, monocytes/ macrophages, $\mathrm{B}$ and $\mathrm{T}$ lymphocytes. The present study was designed to investigate the role of these receptors on the inflammatory and fibrogenic response of unilateral ureteral obstruction (UUO), by using $\mathrm{P}_{2} \mathrm{X}_{7}$ knockout mice. C57B16 mice were submitted to surgical left ureteral obstruction and sacrificed after 14 days. Histopathology using HE, PAS and Sirius red staining and immunohistochemistry with $\mathrm{f} 4 / 80$ for macrophages, $\alpha$-SMA for myofibroblasts and TGF- $\beta$ were identified. Protocols were as follows: 1) UUO non-treated; 2$)$ UUO P2X 7 (-/-) mice; 3) Control; 4) Sham-operated. Mice were sacrificed 14 days after the surgery. Sirius red was significantly higher in group $1(3917,1 \pm 2416,8)$, compared to groups $2(1416,8 \pm 1155,7 \mathrm{P}<0.001), 3(319,4 \pm 384,1 \mathrm{P}<0.001)$ and 4 (349, $0 \pm 340,0 \mathrm{P}<0.001)$. The pro-fibrotic cytokine TGF- $\beta$ was markedly high in group $1(14,6 \pm 8,9)$ compared to groups 2 $(7,1 \pm 5,7 \mathrm{P}<0,001), 3(2,0 \pm 2,4 \mathrm{P}<0,001)$ and $4(2,1 \pm 2,3 \mathrm{P}<0,001)$. The percentage of myofibroblasts cells was significantly higher in group $1(22,1 \pm 13,1)$ compared to groups $2(8,3 \pm 8,3 \mathrm{P}<0,001), 3(0,8 \pm 1,5 \mathrm{P}<0,001)$ and $4(1,3 \pm 2,5$ $\mathrm{P}<0,001)$. The number of positive inflammatory cells was significantly higher in group $1(68,0 \pm 20,7)$, compared to groups $2(43,2 \pm 19,1 \mathrm{P}<0.001), 3(1,1 \pm 1,2 \mathrm{P}<0,001)$ and $4(1,4 \pm 1,3 \mathrm{P}<0,001)$. Atrophic tubules measure by PAS was $7.8 \pm 3.6$ per field in group 1 , whereas in $\mathrm{UUO} \mathrm{P}_{2} \mathrm{X}_{7}(-/-)$ mice we observed a lower number of atrophic tubules comprising aproximately $44 \%$ less atrophy than the findings in kidney tissue from group 1 . Control and shamoperated animals did not exhibit tubular atrophy. There was no statistical difference between groups 3 and 4 in all analysis. In $\mathrm{P}_{2} \mathrm{X}_{7}$ knockout mice submitted to UUO, the inflammation and fibrosis were of lower magnitude than that observed in UUO wild type mice. These findings constitute the first evidence that $\mathrm{P}_{2} \mathrm{X}_{7}$ receptors are implicated in the mechanism of macrophage infiltration and collagen deposition in response to ureteral obstruction in mice.

\section{$\mathrm{P2X}_{7}$ receptor activation caused mitochondrial membrane depolarization through sodium influx in submandibular glands}

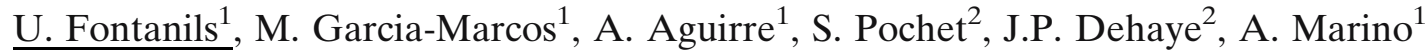 \\ gbbforou@lg.ehu.es
}

We investigated the effect of extracellular ATP on mitochondrial inner membrane depolarization in rat submandibular glands. High ATP concentrations caused a sustained depolarization of mitochondrial membrane. This response was blocked in the presence of magnesium or apyrase and reproduced by low concentrations of $2,3-O$ (4-benzoylbenzoyl) adenosine 5-triphosphate (BzATP), suggesting the implication of the $\mathrm{P}_{2} \mathrm{X}_{7}$ purinergic receptor. This fact was confirmed comparing the response to ATP of wild-type and $\mathrm{P} 2 \mathrm{X}_{7}$ knock-out $\left(\mathrm{P} 2 \mathrm{X}_{7} \mathrm{R}^{-l-}\right)$ mice. Blockade of calcium uptake by mitochondrial uncouplers (FCCP or rotenone) or a mitochondrial calcium uniporter inhibitor (ruthenium red) potentiated the cytosolic increase of calcium provoked by ATP, suggesting a 
mitochondrial uptake of calcium after $\mathrm{P} 2 \mathrm{X}_{7}$ activation in normal conditions. However, $\mathrm{P} 2 \mathrm{X}_{7}$ induced mitochondrial membrane depolarization was not affected by extracellular calcium removal. Moreover, neither ruthenium red nor ruthenium 360 (two blockers of the mitochondrial calcium uniporter) affected the mitochondrial membrane depolarization by ATP. On the other hand, $\mathrm{P}_{2} \mathrm{X}_{7}$ activation is known to increase the permeability to sodium. Extracellular sodium replacement with N-methyl-D-glucosamine almost completely blocked the mitochondrial depolarization. The effect of ATP was not reproduced by the sodium ionophore monensin but was partially suppressed by the mitochondrial $\mathrm{Na} / \mathrm{Ca}$ exchanger inhibitor 7-chloro-5-(2-chlorophenyl)-1,5-dihydro-4,1benzothiazepin-2(3H)-one (CGP-37157). Interestingly, cyclosporine A had no effect on ATP-induced mitochondrial depolarization, indicating that the mitochondrial permeability transition pore is not essential for this phenomenon.

It is concluded that $\mathrm{P} 2 \mathrm{X}_{7}$ receptor activation by ATP depolarizes the mitocondrial inner membrane. The uptake of extracellular sodium plays a major role in this process, at least in part through the mitochondrial $\mathrm{Na} / \mathrm{Ca}$ exchanger. Sodium uptake seems to be necessary but not sufficent, suggesting the implication of other $\mathrm{P}_{2} \mathrm{X}_{7}$ related responses in the mitochondrial membrane depolarization.

${ }^{1}$ Departamento de Bioquímica y Biología Molecular, Facultad de Ciencias, Universidad del País Vasco, Barrio Sarriena S/N Leioa, P.O. Box 644, 48080 Bilbao, Spain

${ }^{2}$ Laboratoire de Biochimie et de Biologie Cellulaire, Institut de Pharmacie C.P. 205/3, Université Libre de Bruxelles, B-1050 Brussels, Belgium

\title{
$\mathrm{P2X}_{7}$ receptor mediated proliferation: a mitochondrial-reticular explanation
}

\author{
Maria Giulia Callegari, Paolo Pinton, Rosario Rizzuto, Francesco Di Virgilio, Elena Adinolfi \\ ${ }^{1}$ Department of Experimental and Diagnostic Medicine Section of General Pathology, University of Ferrara, Via \\ Borsari 46, 44100, Ferrara, Italy \\ elena.adinolf@unife.it
}

The $\mathrm{P} 2 \mathrm{X}_{7}$ receptor is an ATP gated ion channel, that is endowed with a strong pro-apoptotic activity but also promotes cell survival in the absence of serum. While the pro-apoptotic and pro-necrotic activity of the receptor has been widely investigated, little is known about its growth promoting function. In the last years we focused our attention on the organellar network, fundamental for both cell survival and death, studying the behavior of mitochondria and endoplasmic reticulum (ER) in HEK cells expressing the $\mathrm{P}_{2} \mathrm{X}_{7}$ receptor. Thanks to targeted calcium sensing probes we were able to measure increased mitochondrial calcium levels and enhanced reticular calcium uptake in receptor transfected cells versus mock. Experiments with thapsigargin on cell populations allowed us to estimate a total content of reticular calcium significantly higher for $\mathrm{P} 2 \mathrm{X}_{7}$ transfectants. $\mathrm{P} 2 \mathrm{X}_{7}$ expressing clones also resulted more sensitive to carbachol stimulation that caused a burst in both reticular and mitochondrial calcium levels.

This organellar calcium increase correlated with longer survival in the absence of serum and enhanced ATP production. These data allow us to propose a model in which $\mathrm{P}_{2} \mathrm{X}_{7}$ expression will determine an increased cellular calcium influx that will be stored in both mitochondria ad ER causing on one hand an higher energy production and on the other stimulating the proliferative pathways involving a release of calcium from the endoplasmic reticulum.

\section{$\mathrm{P2X}_{7}$ Receptor-mediated Cell Death in Murine T Lymphocytes}

\author{
Hitoshi Harada $^{1}$, Mitsutoshi Tsukimoto ${ }^{1}$, Akira Ikari ${ }^{1}$, Kuniaki Takagi ${ }^{1}$ and Masakuni Degawa ${ }^{1,2}$ \\ ${ }^{T}$ School of Pharmaceutical Sciences, University of Shizuoka \\ ${ }^{2}$ The COE Program in the 21st Century, University of Shizuoka \\ 52-1 Yada Suruga, Shizuoka 422-8526, JAPAN \\ harada@u-shizuoka-ken.ac.jp
}

The ATP-gated $\mathrm{P} 2 \mathrm{X}_{7}$ receptor is a plasma membrane receptor belonging to the family of $\mathrm{P} 2 \mathrm{X}$ purinoceptors. Its activation leads to multiple downstream events including influx of ions, pore formation to allow the passage of 
larger molecular weight species and cell death by apoptosis and/or necrosis. However, the molecular mechanism of $\mathrm{P} 2 \mathrm{X}_{7}$ receptor-mediated cell death is still unknown. The apoptotic cell death is thought to be correlated with the pore formation, but does not directly result from the dilatation of pore [1]. The activities of intracellular $\mathrm{Ca}^{2+}$ mobilization and pore formation by $\mathrm{P} 2 \mathrm{Z} / \mathrm{P} 2 \mathrm{X}_{7}$ receptor are modulated during $\mathrm{T}$ cell differentiation in murine thymus and spleen [2]. On the other hand, ERK1/2 phosphorylation is involved in $\mathrm{P}_{2} \mathrm{X}_{7}$ receptor-mediated necrotic cell death in murine thymocytes [3]. Recently, we have reported the involvement of cell shrinkage following pore formation in $\mathrm{P}_{2} \mathrm{X}_{7}$ receptor-mediated apoptotic cell death and the essential role of extracellular $\mathrm{Cl}^{-}$in it using a clone of chicken DT40 lymphocytes stably transfected with the rat $\mathrm{P}_{2} \mathrm{X}_{7}$ receptor [4]. In this study, to assess the role of $\mathrm{P} 2 \mathrm{X}_{7}$ receptor in cells endogenously expressing $\mathrm{P} 2 \mathrm{X}_{7}$ receptors, we investigated $\mathrm{P} 2 \mathrm{X}_{7}$ receptormediated cell death during murine $\mathrm{T}$ cell differentiation and maturation.

$\mathrm{P}_{2} \mathrm{X}_{7}$ receptor expression and its activities (pore formation, ERK1/2 phosphorylation and cell shrinkage) were higher in splenocytes than thymocytes. T cells of different developmental stages differed in sensitivity to P2 $\mathrm{X}_{7}$ receptor-mediated cell shrinkage. Immature thymocytes (CD4-8- and CD4+8+), mature thymocytes (CD4+8- or CD4-8+) and peripheral T cells (splenic CD4+ and CD8+) showed increasing reactivity. Decrease of extracellular $\mathrm{Cl}^{-}$suppressed the cell shrinkage without inhibition of ATP-induced ERK1/2 phosphorylation, whereas treatment with U0126 (a MEK inhibitor) suppressed the ERK1/2 phosphorylation but not the cell shrinkage. Decrease of extracellular $\mathrm{Cl}^{-}$and treatment with U0126 suppressed ATP-induced cell death, respectively. Moreover, treatment with U0126 in a buffer containing low concentration of $\mathrm{Cl}^{-}$inhibited ATP-induced cell death additively. In conclusion, we demonstrate the presence of two independent pathways, one involving cell shrinkage and the other involving phosphorylation of ERK1/2, in $\mathrm{P}_{2} \mathrm{X}_{7}$ receptor-mediated $\mathrm{T}$ cell death and the increase in $\mathrm{P} 2 \mathrm{X}_{7}$ receptor activity during $\mathrm{T}$ cell maturation. Our findings could explain the so-called " $\mathrm{P} 2 \mathrm{X}_{7}$ receptor-mediated apoptotic and/or necrotic cell death"and suggest the particular importance of peripheral $\mathrm{T}$ cell death in $\mathrm{P} 2 \mathrm{X}_{7}$ receptor-regulated immune responses.

1. Virginio, C.; MacKenzie, A.; North, R. A.; Surprenant, A., Kinetics of cell lysis, dye uptake and permeability changes in cells expressing the rat $\mathrm{P} 2 \mathrm{X}_{7}$ receptor. J Physiol 1999, 519, 335-46.

2. Chused, T. M.; Apasov, S.; Sitkovsky, M., Murine T lymphocytes modulate activity of an ATP-activated P2Ztype purinoceptor during differentiation. J Immunol 1996, 157, 1371-80.

3. Auger, R.; Motta, I.; Benihoud, K.; Ojcius, D. M.; Kanellopoulos, J. M., A role for mitogen-activated protein kinase $\left(\right.$ Erk1/2) activation and non-selective pore formation in $\mathrm{P}_{2} \mathrm{X}_{7}$ receptor-mediated thymocyte death. $J$ Biol Chem 2005, 280, 28142-51.

4. Tsukimoto, M.; Harada, H.; Ikari, A.; Takagi, K., Involvement of chloride in apoptotic cell death induced by activation of ATP-sensitive $\mathrm{P}_{2} \mathrm{X}_{7}$ purinoceptor. J Biol Chem 2005, 280, 2653-8.

\title{
P2Y RECEPTOR ACTIVATION BY URIDINE 5'-TRIPHOSPHATE ENHANCED TOLERANCE TO ISCHEMIA-REPERFUSION IN MOUSE HEART
}

\author{
$\underline{\text { Shirley Wee }}^{1}$, John Headrick ${ }^{1}$ \\ ${ }_{1}^{1}$ Heart Foundation Research Centre, Griffith University, Australia. \\ s.wee@griffith.edu.au
}

\begin{abstract}
Effects of the P2Y receptor agonist, uridine 5'-triphosphate (UTP) were investigated in isolated hearts in terms of functional tolerance to ischemia-reperfusion. Langendorff perfused C57 mouse hearts were subjected to 20 min global ischemia, followed by $45 \mathrm{~min}$ of reperfusion. Three experimental groups were studied with $250 \mathrm{nM}$ or $1 \mu \mathrm{M}$ UTP: i) 5 minutes pre-treatment prior to ischemia (Pre-ISCH), ii) 5 minutes treatment during reperfusion (PostISCH), or iii) 5 minutes pre-ischemia and 5 minutes post-ischemia (Pre- \& Post-ISCH). Post-ischemic functional recoveries and tissue necrosis (LDH efflux) were assessed. Control (CTRL) hearts recovered $67 \pm 3 \mathrm{mmHg}$ left ventricular developed pressure (LVDP) ( $50 \%$ of pre-ischemia) and exhibited sustained diastolic contracture $(31 \pm 2 \mathrm{mmHg})$ at the end of $45 \mathrm{~min}$ reperfusion. Pre-ischemic UTP treatment $(250 \mathrm{nM}$ and $1 \mu \mathrm{M})$ did not modify LVDP recovery, but end-diastolic contracture was significantly reduced $(17 \pm 1$ and $21 \pm 4 \mathrm{mmHg}$ respectively, $\mathrm{p}<0.05)$. Pre-ischemic UTP treatment also resulted in a significantly higher ( $\sim 95 \%$ of pre-ischemia) coronary flow
\end{abstract}


recovery when compared to CTRL hearts. ( $75 \%$ of pre-ischemia, $\mathrm{p}<0.05)$. Functional recovery was not altered by post-ischemic UTP treatment at either concentration. Interestingly, Pre- \& Post-ISCH UTP at 250nM not only significantly reduced end-diastolic contracture $(17 \pm 3 \mathrm{mmHg})$ but also resulted in a higher recovery of left ventricular developed pressure $(89 \pm 6 \mathrm{mmHg})$. However, Pre- \& Post-ISCH UTP at the higher concentration $(1 \mu \mathrm{M})$ failed to change functional recovery. Whilst post-ischemic LDH efflux indicated a protective role of UTP in terms of reducing tissue necrosis in treated hearts, the higher $1 \mu \mathrm{M} \mathrm{UTP}$, although not significant, appeared to worsen tissue injury with Pre - \& Post-ISCH UTP (CTRL, $23 \pm 1 \mathrm{U} / \mathrm{g}$ vs $27 \pm 4 \mathrm{U} / \mathrm{g}$ ). In summary, the P2Y agonist, UTP when infused pre-ischemia enhances tolerance to ischemia, whilst post-ischemic treatment did not modify functional recovery. In addition, the cardioprotective role of UTP in reducing tissue necrosis is dose-dependent. A recent study has shown release of UTP during cardiac ischemia and indicates that UTP may have a role in cardiac regulation. ${ }^{1}$ Current results suggest P2Y activation by UTP may exert a myocardial protective role in ischemiareperfusion. Given apparent dose-dependent effects of UTP, it is possible a combination of exogenous and endogenous UTP may, under certain conditions, exert differing effects. High $(1 \mu \mathrm{M})$ UTP may, together with local UTP, enhance injury. Lower (250nM) UTP may act beneficially.

1. Erlinge, D., Harnek, J., Van Heusden, C., Olivecrona, G., Jern, S., Lazarowski, E. Uridine triphosphate (UTP) is released during cardiac ischemia. International Journal of Cardiology 2005; 100:427-433

\title{
P2Y receptor mediated calcium signalling in dissociated mouse spinal cord cultures.
}

\author{
A E Atterbury-Thomas ${ }^{1}$, Catherine Leon $^{2}$, ${\text { Christian } \text { Gachet }^{2} \text {, \& R J Evans }}^{1}$ \\ ${ }^{T}$ Cell Physiology and Pharmacology, University of Leicester, Leicester, UK \\ ${ }^{2}$ Institut National de la Sante et de la Recherche Medicale (INSERM) U.311, EFS-Alsace 10, Srasboug Cedex, \\ 67065, France.
}

Ionotropic P2X $\left(\mathrm{P} 2 \mathrm{X}_{1-7}\right)$ and metabotrophic P2Y(1,2,4,6,11,12,13) receptors are expressed throughout the nervous system, however their contribution to nucleotide signalling in spinal cord (SC) remains to be determined. RTPCR analysis indicated the presence of all P2 receptor transcripts in the SC, apart from $\mathrm{P}_{2} \mathrm{Y}_{4}$. In this study we have used fluorescent calcium imaging and western blotting to investigate the response of the SC to applied nucleotides. SC cells from neonatal mice were dissociated and cultured for 3-5 days. Propidium iodide staining and immunocytochemical analysis with glial fabrillary acidic protein (GFAP) indicated that the cultures consisted of $\sim 45 \%$ neurons, with the remainder glial in origin.

The contribution of defined $\mathrm{P} 2$ receptors was determined using wild-type (WT) and $\mathrm{P} 2 \mathrm{Y}_{1}$ receptor-deficient (KO) mice. In WT application of both ADP $(10 \mu \mathrm{M})$ a $\mathrm{P}_{2} \mathrm{Y}_{1}, \mathrm{P}_{2} \mathrm{Y}_{12}$, and $\mathrm{P}_{2} \mathrm{Y}_{13}$ receptor agonist and the pyrimidine nucleotide UTP $(100 \mu \mathrm{M})$ a $\mathrm{P}_{2} \mathrm{Y}_{2}$ and $\mathrm{P}_{2} \mathrm{Y}_{4}$ agonist evoked a reproducible sustained response in $\sim 100 \%$ and $\sim 70 \%$ of cells $\left(\mathrm{F}_{525}\right.$ ratio 2.4,1.74) respectively, which remained largely unchanged in the absence of extracellular calcium.

ADP $(10 \mu \mathrm{M})$ did not evoke a calcium response in P2Y $\mathrm{Y}_{1} \mathrm{KO}$ cells, whereas UTP $(100 \mu \mathrm{M})$ responses were unaffected. ADP $(100 \mu \mathrm{M})$ responses were abolished in the presence of pyridoxalphosphate-6-azophenyl-2'-5'-disulfonate (PPADS) and Suramin, both broad-spectrum P2 receptor antagonists. MRS 2179 a P2Y ${ }_{1}$ selective antagonist caused a concentration dependant inhibition to application of ADP.

Western Blot analysis on WT cells showed that application of ADP $(10 \mu \mathrm{M})$ activated ERK phosphoryation in a time dependant manner (10 mins optimal) and this was almost completely inhibited by the phospholipase C inhibitor U73122. Application of BAPTA, a calcium-specific chelator, did not inhibit ADP evoked ERK phosphorylation, however pre-incubation of cultured cells with PPADS or suramin substantially reduced ERK phosphorylation. In contrast to ADP evoked calcium respones that were abolished ADP evoked ERK phosphorylation was unaffected in $\mathrm{P}_{2} \mathrm{Y}_{1} \mathrm{KO}$ SC.

Our results suggest that a range of functional $\mathrm{P} 2$ receptor subtypes are expressed by the $\mathrm{SC}$ which are coupled to an increase in intracellular calcium demonstrating the presence of a functional ADP-sensitive $\mathrm{P}_{2} \mathrm{Y}_{1}$ receptors and additionally UTP-sensitive $\mathrm{P}_{2} \mathrm{Y}_{2}$ receptors in mouse SC. However western blot analysis reveals that $\mathrm{P}_{2} \mathrm{Y}_{1}$ coupled to PLC is not responsible for subsequent ERK activation seen in response to ADP and this activation is calcium independent. 


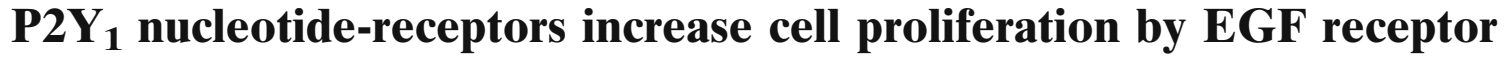 trans-activation
}

$\underline{\text { Sonja Buvinic }}^{1,2,3}$, Marcela Bravo-Zehnder ${ }^{1,2,4}$, Francisco Palma ${ }^{1,2,3}$, Alfonso González ${ }^{1,2,4}$ and J. Pablo Huidobro-Toro ${ }^{1,2,3}$

${ }^{1}$ Centro Regulación Celular y Patología (CRCP) and ${ }^{2}$ Instituto MIFAB, ${ }^{3}$ Departamento Fisiología, Facultad de Ciencias Biológicas, ${ }^{4}$ Departamento de Inmunología clínica y Reumatología, Facultad de Medicina, P. Universidad Católica de Chile, Santiago, Chile.

sbuvinic@bio.puc.cl

Metabotropic $\mathrm{P} 2 \mathrm{Y}_{1}$ receptors $\left(\mathrm{P} 2 \mathrm{Y}_{1} \mathrm{R}\right)$ are G-protein coupled receptors (GPCRs) activated by extracellular nucleotides that regulate a wide range of physiological events. Several GPCRs have been described as cell proliferation inductors, by trans-activation of the epidermal growth factor receptor (EGFR). Considering that nucleotides are continuously released from cells, we hypothesized that they might act as local trophic factors. We assessed whether P2Y $Y_{1}$, like other GPCRs, could modulate cell proliferation via the EGFR. Epithelial FRT cells and cancerous HeLa cells responded to the selective $\mathrm{P}_{2} \mathrm{Y}_{1} \mathrm{R}$ agonist 2-MeSADP by increasing $\left[\mathrm{H}^{3}\right]$-thymidine incorporation with $\mathrm{EC}_{50}$ values of $42 \pm 7 \mathrm{nM}$ and $76 \pm 3 \mathrm{nM}$, respectively, an effect totally abolished by the $\mathrm{P} 2 \mathrm{Y}_{1} \mathrm{R}$ antagonist MRS2179. 2-MeSADP also increased the tyrosine phosphorylation of EGFR, by 3-4 fold in FRT cells and more than 60 fold in HeLa cells, leading to activation of ERK1/2 proteins. Inhibitors of EGFR kinase (AG1478), PKC (Ro318220), src (PP2) and metalloproteases (Ilomastat), and a negative dominant of Gaq, all reduced the rise in cell proliferation evoked by 2-MeSADP. Apyrase, used to degrade extracellular nucleotides, reduced $15-25 \%$ the basal proliferation rate of FRT and HeLa cells, suggesting a trophic role of the nucleotides endogenously released by each cell type. Nucleotides quantitation by high performance liquid cromatography following derivatization of purines demonstrated that HeLa and FRT cells endogenously release near to 200nM ATP after a mechanical stimuli such as the gently change of culture media; basal values of 20-50 nM ATP were reached after 15-30 min. The released ATP was totally degaded to AMP using apyrase. On the other hand, epithelial MDCK cells overexpressing $\mathrm{P}_{2} \mathrm{Y}_{1} \mathrm{R}$ increased their proliferation rate through a mechanism that depended on EGFR activation, as judged by AG1478 inhibition. Cells overexpressing the $\mathrm{P}_{2} \mathrm{Y}_{1} \mathrm{R}$ showed increased levels of REGF expression and a basal activated form of ERK proteins, suggesting that regulation of REGF through nucleotide receptors could involve other mechanisms, different than those involved in trans-activation. These results revealed that $\mathrm{P} 2 \mathrm{Y}_{1} \mathrm{R}$ activation trans-activates the EGFR leading to increased proliferation of both epithelial and cancerous cells. While nucleotides permanently released to the media could be involved in the maintenance of the basal proliferation rate, endogenous nucleotides widely released after cell damage and tissue injury could also play a role in tissue repairing.

(Funded by FONDAP grant 13980001; Millennium MIDEPLAN project MIFAB also funded the CRCP Center)

\section{$\mathrm{P}_{2} \mathrm{Y}_{1}$ receptors mediates release of tissue plasminogen activator (t-PA) in response to reactive coronary hyperemia in a pig model}

\author{
Goran Olivecrona $^{1}$, Matthias Gotberg ${ }^{1}$, Jan Harnek $^{2}$, Kenneth A Jacobson ${ }^{3}$, Sverker Jern ${ }^{4}$, \\ David Erlinge ${ }^{1}$ \\ ${ }^{1}$ Department of Cardiology, Lund University, ${ }^{2}$ Department of Radiology, Lund University, Lund, Sweden, \\ ${ }^{3}$ Molecular Recognition Section, NIH, Bethesda, MD, ${ }^{4}$ Hjart-Karlinstitutionen, Kliniskt experimentella Forskning- \\ slaboratoriet, Sahlgrenska University Hospitalöstra, Gothenburg, Sweden \\ goran.olivecrona@med.lu.se
}

Background: The endothelial $\mathrm{P}_{2} \mathrm{Y}_{1}$ receptor is responsible for a large part of the reactive hyperemia following cardiac ischemia. Tissue plasminogen activator (t-PA) increases during reactive hyperemia. We postulated that 
the release of t-PA during reactive hyperemia could be mitigated through blocking the coronary endothelial $\mathrm{P}_{2} \mathrm{Y}_{1}$ receptor.

Methods: t-PA was measured in peripheral arterial blood and locally in the venous blood from the coronary sinus (CS) in a porcine model. 2-MeSADP $\left(10^{-5} \mathrm{M}\right)$, alone or as co-infusion with a selective $\mathrm{P}_{2} \mathrm{Y}_{1}$ receptor blocker, MRS $2179\left(10^{-3} \mathrm{M}\right)$ was locally delivered in the left anterior descending artery (LAD) through the tip of a coronary angioplasty balloon. In separate pigs the coronary artery was occluded with the balloon for ten min. During the first and tenth min of coronary ischemia, $2.5 \mathrm{ml}$ of MRS $2179\left(10^{-3} \mathrm{M}\right)$ was delivered distal to the occlusion in 8 pigs, 10 pigs were used as controls.

Results: 2-MeSADP increased levels of t-PA in the CS by $85 \%$ which could completely be inhibited by co-infusion with MRS 2179. During cardiac ischemia and reperfusion t-PA increased significantly, an effect that could be significantly inhibited by MRS 2179. Conclusion: Intra coronary administered MRS 2179, a selective P2Y $\mathrm{Y}_{1}$ receptor blocker, significantly reduces the increased levels of t-PA caused by both 2-MeSADP and cardiac ischemia + reperfusion in coronary arteries. Thus, ADP acting on the endothelial $\mathrm{P}_{2} \mathrm{Y}_{1}$ receptor may play a major role in the release of t-PA during post- ischemic hyperemia.

\section{Net t-PA during ischemia / reperfusion}

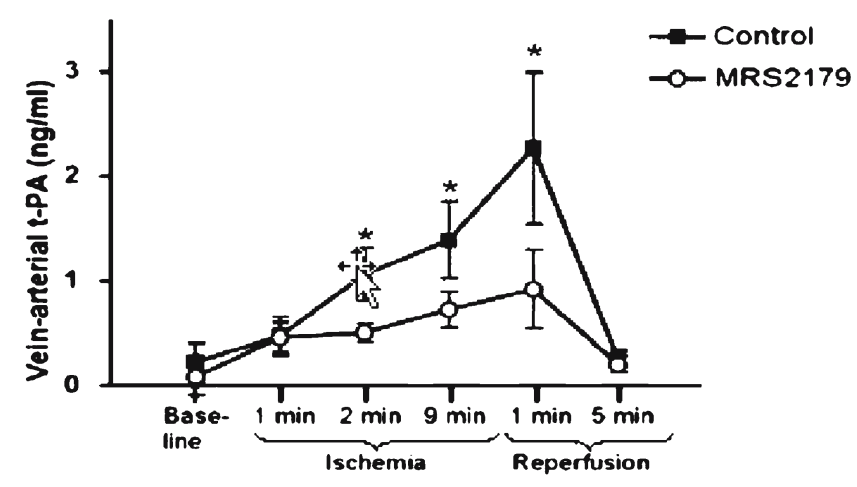

\section{$\mathrm{P}_{1} \mathrm{Y}_{1}$ signalling in human monocyte derived dendritic cells}

Blandine Maître, Béatrice Hechler, Catherine Léon, Jean-Pierre Cazenave, Christian Gachet. INSERM U311. Etablissement Français du Sang-Alsace, Strasbourg, France blandine.maitre@efs-alsace.fr

Adenosine triphosphate (ATP) and adenosine diphosphate (ADP) have been reported to regulate human monocyte derived dendritic cells (DCs) by inhibiting IL-12 production and by modulating expression of costimulatory molecules at their cell surface. ATP is thought to interact with DCs through the P2 $\mathrm{Y}_{11}$ receptor [1] whereas the receptors activated by ADP are not yet well characterized [2]. The aim of the present study was to investigate whether ADP could stimulate the $\mathrm{P}_{2} \mathrm{Y}_{1}$ receptor in DCs and, if yes, the possible role of this receptor in maturation of DCs and in regulation of the immune response. In human immature DCs, ADP $(50 \mu \mathrm{M})$ induced an increase in intracellular $\mathrm{Ca}^{2+}$ both in the presence and absence of external calcium, which was absent in mature dendritic cells. This intracellular $\mathrm{Ca}^{2+}$ rise was totally inhibited by MRS2179 $(250 \mu \mathrm{M})$, a potent and selective antagonist of $\mathrm{P}_{2} \mathrm{Y}_{1}$. Moreover, ADP induced an increase in intracellular $\mathrm{Ca}^{2+}$ in murine bone marrow derived dendritic cells (BMDC), that was absent in BMDC derived from $\mathrm{P}_{2} \mathrm{Y}_{1}$ deficient mice. To determine whether ADP could influence maturation of human monocyte derived DCs, stimulation with $100 \mathrm{ng} / \mathrm{mL}$ lipopolysaccharide (LPS) in the presence or the absence of $50 \mu \mathrm{M}$ ADP for 24 hours was performed. ADP had a synergistic effect with LPS on the increased cell surface expression of DCs maturation markers, CD83, HLA-DR, CD86 but not CD80 and this effect was partially reversed by MRS2179 suggesting that, not only the $\mathrm{P}_{2} \mathrm{Y}_{1}$ receptor, but other P2Y receptors are involved in this response. Finally, ADP inhibited Il-12p70 and Il-12p40 secretion by human monocyte derived DCs induced by LPS $(100 \mathrm{ng} / \mathrm{mL})$. Increase of CD86 at the cell surface of DCs and inhibition of IL-12 secretion suggest that ADP could potentiate a Th2 response. In conclusion, these results indicate that ADP 
interacts with immature DCs through different P2Y receptors, of which $\mathrm{P}_{2} \mathrm{Y}_{1}$ and that ADP, like ATP, could modulate the immune response by acting on immature DCs.

1. Wilkin, F., et al., The P2Y11 receptor mediates the ATP-induced maturation of human monocyte-derived dendritic cells. J Immunol, 2001. 166(12): p. 7172-7.

2. Marteau, F., et al., Involvement of multiple P2Y receptors and signaling pathways in the action of adenine nucleotides diphosphates on human monocyte-derived dendritic cells. J Leukoc Biol, 2004. 76(4): p. 796-803.

\title{
Permeation properties of single human purinergic $\mathbf{P 2 X} 7$ receptors
}

\author{
T. Riedel ${ }^{1}$, G. Schmalzing ${ }^{2}$ and F. Markwardt ${ }^{1}$
}

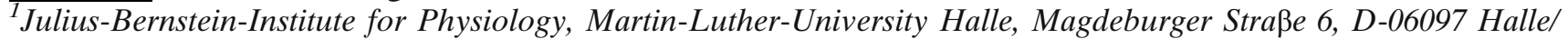
Saale, Germany, ${ }^{2}$ RWTH Aachen, Department for Molecular Pharmacology, Wendlingweg 2, D-52074 Aachen, Germany

thomas.riedel@medizin.uni-halle.de

Purinergic P2X7 receptors belong to the family of receptor-operated ion channels. They are mainly expressed in cells of the immune and inflammatory system and in epithelium. P2X7 receptors can induce a nonselective membrane permeability after prolonged or repeated stimulation by an unknown mechanism. To investigate this in more detail, single channels currents of human P2X7 receptors heterologously expressed in Xenopus oocytes were recorded using the patch clamp technique in the outside-out configuration using $\mathrm{Cs}^{+}$as intracellular cation. Substitution of extracellular $\mathrm{Na}^{+}$ions by inorganic cations of increasing size successively decreased the single channel conductance and shifted the reversal potential to more negative values. In extracellular solutions containing the large cations tris ${ }^{+}$or choline ${ }^{+}$, no single channel inward currents at negative membrane potentials up to $-160 \mathrm{mV}$ but outward currents probably carried by $\mathrm{Cs}^{+}$at positive potentials were detected. Substitution of $\mathrm{Na}^{+}$ by organic cations prolonged the mean open times and increased the open probability of single P2X7 channel currents. Additionally, the deactivation time course was slowed. These single channel permeation properties did not change during repeated or prolonged ATP applications. This was also found in single channel recordings in the cell attached configuration. We conclude that single P2X7 channels are only permeable to small cations independent of the duration of agonist application. Substitution of $\mathrm{Na}^{+}$by large inorganic cations not only decreases the single channel conductance but also has a pronounced influence of the hP2X7 channel kinetics.

This work was supported by the Deutsche Forschungsgemeinschaft (Ma 1581/12-1) and the Roux-programme of the ML university (FKZ 13/07).

\section{Pharmacological and safety evaluation of the platelet $\mathrm{P}_{2} \mathrm{Y}_{12}$ receptor antagonist INS50589 in healthy human volunteers.}

José L. Boyer, Fred L. Johnson, Philip T. Leese, Todd Durham, Christopher S. Crean, Ramesh Krishnamoorthy, Paul S. Watson and Donald J. Kellerman

Inspire Pharmaceuticals, Inc. 4222 Emperor Boulevard, Suite 200, Durham, North Carolina, 27703. USA JBoyer@inspirepharm.com

Platelets play a critical role in hemostasis and are involved in the pathogenesis of arterial thrombosis, where platelet-rich thrombi are the hallmark of the disease. Platelet-specific cell-surface receptors as well as intracellular signaling enzymes are the targets of current antiplatelet therapeutic agents. A number of antiplatelet drugs are used in the management of cardiovascular and cerebrovascular thrombotic conditions. A key extracellular signaling molecule involved in platelet activation is $\mathrm{ADP}$, which binds to $\mathrm{P} 2 \mathrm{Y}_{1}$ and $\mathrm{P} 2 \mathrm{Y}_{12}$ surface receptors. A direct and reversible antagonist of the platelet $\mathrm{P}_{2} \mathrm{Y}_{12}$ receptor, INS50589, is under development by Inspire for the acute treatment of cardiovascular conditions where a strict control of platelet function is desired.

A phase 1 study involving 36 healthy volunteers was conducted to evaluate the pharmacological, pharmacokinetic and safety properties of a continuous intravenous administration of INS50589 at doses of $0.1,0.3,1.0$ and $3.0 \mathrm{mg} / \mathrm{kg} / \mathrm{h}$ for four hours. Platelet function before, during and after the administration of the drug was assessed 
by impedance whole blood aggregometry, thromboelastography, and measurement of bleeding time. A dosedependent inhibition of ADP-induced platelet aggregation following the administration of INS50589 was observed as early as 15 minutes, and the level of inhibition was maintained during the four-hour infusion period, returning to predose levels following discontinuation of the infusion. The recovery rate of platelet function was also proportional to the administered dose of INS50589 and can be associated with the systemic elimination of the drug. At a dose rate of $0.1 \mathrm{mg} / \mathrm{kg} / \mathrm{h}$, the extent of inhibition of platelet aggregation was $60 \%$ of the predose level when stimulated with $5 \mu \mathrm{M}$ ADP, and complete inhibition was observed at doses of $0.3,1$ and $3 \mathrm{mg} / \mathrm{kg} / \mathrm{h}$. The plasma levels of the drug were proportional to the dose level and decreased rapidly upon discontinuation of the drug. All subjects that initiated treatment completed the study and no serious adverse events were reported. INS50589 was well tolerated and no clinically significant changes in physical examination, serum chemistry, hematology, and electrocardiogram were observed during or after treatment.

Intravenous administration of INS50589 produces a rapid and reversible modulation of platelet function. The results of this study support the advancement of this clinical candidate for acute use where a rapid and strict control of platelet function is required.

\section{Pharmacological characterization of novel adenosine ligands in recombinant and native human $\mathbf{A}_{2 B}$ receptors}

Katia Varani ${ }^{1}$, Stefania Gessi ${ }^{1}$, Stefania Merighi ${ }^{1}$, Fabrizio Vincenzi ${ }^{1}$, Elena Cattabriga ${ }^{1}$, Annalisa Benini $^{1}$, Karl-Norbert Klotz ${ }^{2}$, Pier Giovanni Baraldi ${ }^{3}$, Mojgan Aghazadeh Tabrizi ${ }^{3}$, Stephen Mac Lennan $^{4}$, Edward Leung ${ }^{4}$ and Pier Andrea Borea ${ }^{1}$

${ }^{1}$ Department of Clinical and Experimental Medicine, Pharmacology Unit, and ${ }^{3}$ Department of Pharmaceutical Sciences, University of Ferrara, Ferrara, Italy; ${ }^{2}$ Institut für Pharmakologie, Universitat Würzburg, Germany, ${ }^{4}$ King Pharmaceuticals Research \& Development, Cary, USA

The present study was designed to evaluate the effects of novel and recognised compounds at human recombinant $\mathrm{A}_{2 \mathrm{~B}}$ adenosine receptors expressed in Chinese hamster ovary $\left(\mathrm{hA}_{2 \mathrm{~B}} \mathrm{CHO}\right)$, in human embryonic kidney 293 $\left(\mathrm{h} \mathrm{A}_{2 \mathrm{~B}} \mathrm{HEK} 293\right)$ and at endogenous $\mathrm{A}_{2 \mathrm{~B}}$ receptors in human mast cells (HMC-1). Saturation binding experiments performed using the new high affinity $\mathrm{A}_{2 \mathrm{~B}}$ adenosine radioligand $\left[{ }^{3} \mathrm{H}\right]-\mathrm{N}$-benzo[1,3]dioxol-5-yl-2-[5-(2,6-dioxo1,3-dipropyl-2,3,6,7-tetra hydro- $1 H$-purin-8-yl)-1-methyl-1H-pyrazol-3-yloxy]-acetamide ([3 $\mathrm{H}]$-MRE2029 F20) revealed a single class of binding sites in $\mathrm{hA}_{2 \mathrm{~B}} \mathrm{CHO}, \mathrm{hA}_{2 \mathrm{~B}} \mathrm{HEK} 293$ and HMC-1 cells with $\mathrm{K}_{\mathrm{D}}(\mathrm{nM})$ of $1.65 \pm$ $0.18,2.83 \pm 0.34,2.62 \pm 0.27$ and $\mathrm{Bmax}$ (fmol/mg protein) of $36 \pm 4,475 \pm 50$ and $128 \pm 15$, respectively. The pharmacological profile of new compounds, determined in inhibition binding experiments in $\mathrm{hA}_{2 \mathrm{~B}} \mathrm{HEK} 293$ cells using $\left[{ }^{3} \mathrm{H}\right]$-MRE 2029F20, showed a rank order of potency typical of the $\mathrm{A}_{2 \mathrm{~B}}$ receptors with $\mathrm{Ki}$ values in the range 3.2-28 nM. In functional assays recognised and novel compounds were studied by evaluating their capability to modulate the cAMP production in $\mathrm{hA}_{2 \mathrm{~B}} \mathrm{CHO}$ and in HMC-1 cells. In particular, novel compounds showed an high potency and MRE 2029F20 was the best compound with a $K_{B}$ of $23 \pm 3 \mathrm{nM}$ and of $19 \pm 2 \mathrm{nM}$, respectively. New compounds were also able to inhibit cAMP levels in the absence of NECA and in the presence of forskolin stimulation in $\mathrm{hA}_{2 \mathrm{~B}} \mathrm{CHO}$ and in $\mathrm{HMC}-1$ cells. In each cell line examined all novel compounds at the $1 \mu \mathrm{M}$ concentration were able to decrease cAMP levels by 30-50\%. Interestingly, in HEK293 cells MRE 2029F20 reduced cAMP basal levels with an $\mathrm{IC}_{50}$ value of $2.9 \pm 0.3 \mathrm{nM}$. These results suggest that novel compounds are antagonists with an inverse agonist activity in recombinant and native human $\mathrm{A}_{2 \mathrm{~B}}$ receptors. As a consequence, the development of potent and selective $\mathrm{A}_{2 \mathrm{~B}}$ compounds appears promising to increase the knowledge of the potential role of $\mathrm{A}_{2 \mathrm{~B}}$ receptors in the pathogenesis of several disorders.

\section{Pharmacological profile of P2Y receptors involved in feed-back inhibition of noradrenaline release in sympathetic innervated tissues}

Queiroz G. ${ }^{1}$, Talaia C., Gonçalves J. 
1'gloria@ff.up.pt, Laboratory of Pharmacology, CEQOFFUP, Faculty of Pharmacy, University of Porto, Rua Aníbal Cunha, 164, 4050-047 Porto, Portugal

In the sympathetic nervous system, ATP is co released with noradrenaline (NA) from postganglionic nerve terminals and acts not only as a neurotransmitter but also as a neuromodulador (1). Adenine nucleotides, such as ATP and its analogues, have been shown to inhibit NA release, by direct activation of presynaptic inhibitory P2Yreceptors (2). However, the receptor subtype involved has not been identified. The aim of the present study was to identify the subtype of P2Y-receptor involved on modulation of NA release in two sympathetically innervated tissues, the epididymal portion of vas deferens and tail artery of rat, and if they are activated by the released ATP. Epididymal portions of vasa deferentia and tail arteries from male Wistar rats (250-350 g) were incubated with 0.1 $\mu \mathrm{M}\left[{ }^{3} \mathrm{H}\right]-\mathrm{NA}$ and superfused with a physiological solution containing $0.4 \mu \mathrm{M}$ desipramine and antagonists of adenosine $\mathrm{A}_{1}$ receptors (DPCPX, $0.1 \mu \mathrm{M}$ ) and $\alpha_{2}$-adrenoceptors (yohimbine, $1 \mu \mathrm{M}$ ). Tissues were stimulated with trains of 100 pulses at $8 \mathrm{~Hz}$ or $5 \mathrm{~Hz}(50 \mathrm{~mA}, 1 \mathrm{~ms})$. The effects of $\mathrm{P} 2$ agonists and antagonists on evoked NA release (estimated as tritium overflow) were expressed in \% of respective controls as mean \pm S.E.M, and were tested for significance by one-way ANOVA followed by Dunnett's test.

The P2-receptor agonists 2MeSATP $(1-100 \mu \mathrm{M})$, 2MeSADP $(1-100 \mu \mathrm{M})$, ADP $(1-300 \mu \mathrm{M})$ and ATP $(1-300 \mu \mathrm{M})$, decreased tritium overflow up to $53 \pm 7 \%(\mathrm{n}=6 ; P<0.01)$, in the epididymal portion and up to $61 \pm 6 \%(\mathrm{n}=6$; $P<0.01$ ), in tail artery. The order of potency was: 2 MeSADP $\geq 2$ MeSATP $>$ ADP $\geq$ ATP, in the epididymal portion of vas deferens and ADP $>2 \mathrm{MeSATP} \geq 2 \mathrm{MeSADP}$ in tail artery. The effects of 2MeSATP $(1-100 \mu \mathrm{M})$ were antagonized in both tissues by RB2 $(10 \mu \mathrm{M})$, PPADS $(10-30 \mu \mathrm{M})$ and by the selective P2Y1 antagonist MRS $2179(10 \mu \mathrm{M})$ but were not changed by the selective P2Y12/P2Y13 antagonists, 2MeSAMP $(30 \mu \mathrm{M})$ or MRS 2395 $(30 \mu \mathrm{M})$. The P2Y-receptor antagonists, RB2 $(1-30 \mu \mathrm{M})$, MRS $2179(1-100 \mu \mathrm{M}), 2 \mathrm{MeSAMP}(1-100 \mu \mathrm{M})$ and MRS $2395(30 \mu \mathrm{M})$ increased tritium overflow up to $142 \pm 5 \%(\mathrm{n}=4 ; P<0.01)$, in the epididymal portion of vas deferens. The order of potency was: RB2 $>$ MRS 2179 2MeSAMP $\geq$ MRS 2395. In the tail artery, they caused no change on tritium overflow evoked at $5 \mathrm{~Hz}$. In the epididymal portion of vas deferens, the effect of 2MeSADP $\left(30 \mu \mathrm{M} ; \mathrm{S}_{2} / \mathrm{S}_{1}=62 \pm 2 \% ; \mathrm{n}=8\right)$ was abolished when tissues were pre-incubated with pertussis toxin $\left(8 \mu \mathrm{g} / \mathrm{ml} ; \mathrm{S}_{2} / \mathrm{S}_{1}\right.$ $=96 \pm 6 \% ; \mathrm{n}=4, P<0.01)$ and was not changed by inhibition of phospholipase $\mathrm{C}$ or protein kinase $\mathrm{C}$ with U-73122 $\left(\mathrm{S}_{2} / \mathrm{S}_{1}=60 \pm 3 \% ; \mathrm{n}=4\right)$ and Ro 32-0432 $\left(\mathrm{S}_{2} / \mathrm{S}_{1}=58 \pm 3 \%, \mathrm{n}=4\right)$, respectively.

It is concluded that, in the epididymal portion of vas deferens and tail artery of the rat adenine nucleotides modulate NA release through activation of inhibitory P2 receptors coupled to $\mathrm{G}_{\mathrm{i} / \mathrm{o}}$-proteins with a pharmacological profile that resemble PY1 receptors.

(1) Burnstock G (2004) Curr Op Pharmacol 4:47-52.

(2) von Kügelgen I et al. (1999) Prog Brain Res 120:173-182.

Supported by FCT Projects (POCTI/SAU-FCB/60714/2004 and CEQOFFUP - I\&D 226/94)

\section{Pharmacology of AS-16; a novel and selective Adenosine A2B receptor antagonist.}

Stephen MacLennan, ${ }^{1}$ Allan R. Moorman, ${ }^{1}$ Katia Varani, ${ }^{2}$ Stefania Gessi, ${ }^{2}$ Stefania Merighi, Mojgan Aghazadeh Tabrizi, ${ }^{3}$ Pier Giovanni Baraldi, ${ }^{3}$ Pier Andrea Borea, ${ }^{2}$ William M. Abraham, ${ }^{4}$ Edward Leung ${ }^{1}$.

${ }^{1}$ King Pharmaceuticals Research \& Development, Cary, North Carolina, USA; ${ }^{2}$ Department of Clinical and Experimental Medicine, Pharmacology Section, ${ }^{3}$ Department of Pharmaceutical Sciences, University of Ferrara; Ferrara, Italy; Miller School of Medicine University of Miami, at ${ }^{4}$ Mount Sinai Medical Center, Florida, USA.

There is considerable evidence to suggest that adenosine, through activation of $A_{2 B}$ receptors on mast cells and smooth muscle cells, is involved in the pathophysiology of asthma (Holgate, 2005). Here we describe the pharmacology of a novel selective $A_{2 B}$ receptor antagonist AS-16 (2-(4-benzyloxy-phenyl)-N-[5-(2,6-dioxo-1,3-dipropyl-2,3,6,7-tetrahydro-1H-purin-8-yl)-1-methyl-1H-pyrazol-3-yl]-acetamide) which may have therapeutic utility in asthma and respiratory disease. In radioligand binding studies utilizing the selective $\mathrm{A}_{2 \mathrm{~B}}$ receptor ligand $\left[{ }^{3} \mathrm{H}\right]$ MRE-2029, AS-16 has an affinity of $22 \mathrm{nM}$ for the human recombinant $\mathrm{A}_{2 \mathrm{~B}}$ receptors expressed in HEK-293 cells. 
It is selective over the other adenosine receptor subtypes, and monoamine receptors in general, having an affinity greater than $1,000 \mathrm{nM}$ at these targets. In functional assays, using recombinant $\mathrm{A}_{2 \mathrm{~B}}$ receptors in $\mathrm{CHO}$ cells and endogenous $A_{2 B}$ receptors in the human mast cell line HMC-1, AS-16 demonstrates an affinity $\left(K_{B}\right)$ of $75 \mathrm{nM}$ and $87 \mathrm{nM}$ respectively.,.

The in vivo efficacy of AS-16 has been examined in sheep which are naturally sensitive to the allergen Ascaris suum. In these animals antigen-induced bronchospasm has two phases similar to what occurs with allergen challenge studies in patients, an immediate increase in airflow resistance (early airway response), followed several hours later by a second increase in airway resistance (late airway response). AS-16 $(2 \mathrm{mg} / \mathrm{kg}$ ) was dosed orally twice-daily for 2 days prior to antigen challenge. This dosing regime gave a consistent plasma concentration (70$100 \mathrm{ng} / \mathrm{ml}$ ) during the experimental phase of the studies. AS-16 reduced the early airway response by approximately $20 \%$ and almost completely abolished the late airway response. A further feature of the sheep model, which is also consistent with studies in patients, is that antigen challenge induces airway hyperresponsiveness (AHR). AS-16 (2 mg/kg p.o.) completely prevented the AHR to inhaled carbachol, assessed $24 \mathrm{~h}$ after allergen challenge. Finally, AS-16 (2 mg/kg p.o.) was able to completely block adenosine-induced bronchospasm, suggesting that in sheep adenosine effects are mediated via the $A_{2 B}$ receptor.

Thus, AS-16 is a novel, selective $\mathrm{A}_{2 \mathrm{~B}}$ receptor antagonist which will be a useful tool to further explore the pathophysiology of asthma and respiratory disease.

References

Holgate, S.T. (2005) Br. J. Pharmacol., 145, 1009-1015.

\title{
Polydeoxyribonucleotide improves angiogenesis and wound healing in experimental burn wounds.
}

\author{
$\underline{\text { Alessandra Bitto }}^{1}$, Letteria Minutoli ${ }^{1}$, Francesca Polito ${ }^{1}$, Domenica Altavilla ${ }^{1}$, Giulia Cattarini ${ }^{2}$ and \\ Francesco Squadrito ${ }^{1}$. \\ ${ }^{1}$ Department of Clinical and Experimental Medicine and Pharmacology - Section of Pharmacology - University \\ of Messina, Italy \\ ${ }^{2}$ Mastelli s.r.l., San Remo, Italy \\ abitto@unime.it
}

We investigated the effects of polydeoxyribonucleotide (PDRN) a compound holding a mixture of deoxyribonucleotide polymers of different lengths, in an experimental model of burn wounds. This compound stimulates "in vitro" fibroblast proliferation and collagen production, likely stimulating the $\mathrm{A}_{2}$ purinergic receptor. In our experiment C57BL/6 male mice of 25-30 g were immersed in 80 degrees $\mathrm{C}$ water for 10 seconds to achieve a partialthickness scald burn. Animals were treated daily for 14 days with PDRN ( $8 \mathrm{mg} / \mathrm{kg} / \mathrm{ip})$ or its vehicle $(100 \mu \mathrm{l})$. On day 14 , burn areas were used for measuring malondialdehyde content, histological damage, neo-angiogenesis by immunohistochemistry and expression (Western blot) of the specific endothelial marker CD31 as well as quantification of micro-vessel density, endothelial and inducible nitric oxide synthase (eNOS and iNOS) expression and wound nitrite content. PDRN decreased tissue oxidative stress index, increased epithelial proliferation, extracellular matrix maturation, and augmented neoangiogenesis as suggested by the marked increase in micro-vessel density and by the robust expression of the specific endothelial marker CD31. PDRN was also able to improve the recruitment and differentiation of endothelial progenitor cells as confirmed by the immuno-staining with VEGFR2 a specific surface marker of committed endothelial cells. Furthermore, PDRN caused a marked expression of eNOS (vehicle $8.1 \pm 1.5$ integrated intensity; PDRN $15.2 \pm 1.8$ integrated intensity) and iNOS (vehicle $7.1 \pm 1.8$ integrated intensity; PDRN $14.2 \pm 1.3$ integrated intensity) and increased wound nitrite content. Our data suggest that PDRN may be an effective therapeutic approach to improve clinical outcomes after thermal injury.

\section{References}

1: Altavilla D, Galeano M, Bitto A, Minutoli L, Squadrito G, Seminara P, Venuti FS, Torre V, Calo M, Colonna M, Lo Cascio P, Giugliano G, Scuderi N, Mioni C, Leone S, Squadrito F. Lipid peroxidation inhibition by raxofelast improves angiogenesis and wound healing in experimental burn wounds. Shock. 2005 Jul;24(1):85-91. 
2: Galeano M, Deodato B, Altavilla D, Squadrito G, Seminara P, Marini H, Stagno d'Alcontres F, Colonna M, Calo M, Lo Cascio P, Torre V, Giacca M, Venuti FS, Squadrito F. Effect of recombinant adeno-associated virus vector-mediated vascular endothelial growth factor gene transfer on wound healing after burn injury. Crit Care Med. 2003 Apr;31(4):1017-25.

3: Thellung S, Florio T, Maragliano A, Cattarini G, Schettini G. Polydeoxyribonucleotides enhance the proliferation of human skin fibroblasts: involvement of A2 purinergic receptor subtypes. Life Sci. 1999;64(18):1661-74.

\title{
Potential role for neural adenosine $\mathbf{A}_{2 \mathrm{~A}}$ receptors in the dilations caused by increases in tissue adenosine.
}

Richard J. Rivers, Naris Thengchaisri, Timothy M. Moore, and Larysa Voytenko

Johns Hopkins University, Department of Anesthesiology and Critical Care Medicine Baltimore, Maryland, USA email: (rrivers3@jhmi.edu)

We recently reported a distinct vascular network response initiated by elevated tissue concentrations of adenosine, but the role of specific adenosine receptor subtypes has not been elucidated. To determine this, remote arteriolar responses were tested in anesthetized hamster cheek pouch when adenosine (or its analogues) were applied ( $3 \mu \mathrm{m}$ tipped micropipette, 1 psi, 10 seconds) into the tissue $500 \mu \mathrm{m}$ away from the arteriole $(\mathrm{n}=67$, baseline diameter $22.4 \pm 0.6 \mu \mathrm{m}$ ). Dilations did not decay along the arteriole when measured $1000 \mu \mathrm{m}$ further away. Control dilations caused by tissue adenosine $(5 \pm 0.4 \mu \mathrm{m})$ were not altered by superfusion of the $\mathrm{A}_{1}$ receptor antagonist DPCPX $(1 \mu \mathrm{M} ; 4.6 \pm 0.3 \mu \mathrm{m}), \mathrm{A}_{2 \mathrm{~B}}$ receptor antagonist alloxazine $(1 \mu \mathrm{M} ; 6 \pm 0.8 \mu \mathrm{m}), \mathrm{A}_{3}$ receptor antagonist MRS1220 $(5 \mathrm{nM} ; 5.6 \pm 0.8 \mu \mathrm{m})$ or intracellular P-site antagonist NBTI $(1 \mu \mathrm{M})$, but were abolished by the selective $\mathrm{A}_{2 \mathrm{~A}}$ receptor antagonist ZM241385 $(0.1 \mu \mathrm{M} ; 0.8 \pm 0.2 \mu \mathrm{m})$, indicating that activation of $\mathrm{A}_{2 \mathrm{~A}}$ receptors mediates these network responses. Direct arteriolar application of ZM241385 (0.1 $\mu \mathrm{M} ; 4.9 \pm 0.4 \mu \mathrm{m})$ did not alter the dilation caused by tissue adenosine, on the other hand, local application of ZM241385 into the tissue inhibited adenosine induced network responses $(1.9 \pm 0.3 \mu \mathrm{m})$ similar to superfusion. Furthermore, application of $\mathrm{A}_{2 \mathrm{~A}}$ receptor agonist CGS21680 $(1 \mu \mathrm{M})$ but not $\mathrm{A}_{1}$ receptor agonist CPA $(100 \mu \mathrm{M})$ in the tissue mimicked adenosine network response.

The location of these tissue receptors is unknown. Using antibodies to the adenosine A2a subtype we found numerous cells in the tissue that labeled during immunohistochemical studies. This includes capillary endothelium and small neurons, with cell bodies, that co-labeled with calcitonin gene-related peptide (CGRP) and that innervated the arterioles. We tested for a role for endothelium damaging the endothelium in the dilating arterioles. Despite loss of response to bradykinin and persistent dilation to nitroprusside, the response to increases in tissue adenosine persisted. Thus, these data suggest a novel role of tissue $\mathrm{A}_{2 \mathrm{~A}}$ receptor in initiating the adenosine vascular network response and that the A2a receptors on the endothelium are unlikely to be playing a role during increases in tissue adenosine.

This research was supported by National Heart, Lung, and Blood Institute Grant R01 HL-072922 and American Heart Association Grant-in-Aid 0455730U.

\section{Priming of STAT1 and STAT3 for cytokine-triggered degradation by the prote- asome upon $A_{2 A}$ adenosine receptor $\left(A_{2 A} A R\right)$ expression}

\author{
Mohammed Safhi, William A Sands and Timothy M. Palmer \\ Division of Biochemistry \& Molecular Biology, IBLS, University of Glasgow, Scotland, U.K. \\ Presenting author e-mail: 0410422s@student.gla.ac.uk
}

While the $\mathrm{A}_{2 \mathrm{~A}}$ adenosine receptor $\left(\mathrm{A}_{2 \mathrm{~A}} \mathrm{AR}\right)$ is known to elevate cAMP, the molecular mechanisms underlying its anti-inflammatory effects remain obscure, particularly with respect to its effects on cytokines that utilise the JAKSTAT signalling pathway. Thus, since we had found that adenovirus-mediated $\mathrm{A}_{2 \mathrm{~A}} \mathrm{AR}$ gene transfer into human umbilical vein endothelial cells (HUVECs) profoundly suppresses NF- $\mathrm{B}$ activation, the effect of $\mathrm{A}_{2 \mathrm{~A}} \mathrm{AR}$ expression on cytokine activation of the JAK-STAT pathway was also tested in this system. Activation of the JAKSTAT pathway was achieved by exposure of HUVECs to an interleukin-6 (IL-6) trans-signalling complex com- 
prising IL-6 and soluble IL-6 receptor- $\alpha$ (hereafter termed IL-6/sIL-6R $\alpha$ ). In control cells, IL-6/sIL-6R $\alpha$ exposure produced a time-dependent and sustained phosphorylation of STAT3 on Tyr705 and STAT1 on Tyr701 that peaked between 15 and $30 \mathrm{~min}$ exposure and only began to diminish by $60 \mathrm{~min}$. Expression of the $\mathrm{A}_{2 \mathrm{~A}} \mathrm{AR}$ resulted in a more transient phosphorylation of STAT1 and STAT3 that peaked at 15 min before diminishing to basal levels by 30 and 60 min. Interestingly, detailed analysis of total STAT1 and STAT3 levels following IL-6/ sIL-6R $\alpha$ exposure revealed that both of these proteins were down-regulated in a cytokine-dependent manner in $\mathrm{A}_{2 \mathrm{~A}} \mathrm{AR}$-expressing but not control cells, with maximal down-regulation occurring after $2 \mathrm{hr}$. This reflected a cytokine-inducible degradation of STAT1 and STAT3, as down-regulation was abolished by pre-treatment with the proteasome inhibitor MG132. Further experiments under conditions in which proteasomal degradation was blocked revealed that the suppression of IL-6/sIL-6R $\alpha$-stimulated STAT phosphorylation observed in $\mathrm{A}_{2 \mathrm{~A}} \mathrm{AR}$ expressing cells could be completely accounted for by the down-regulation phenomenon. Moreover, the cytokine inducibility of the effect reflected a requirement for JAK-mediated phosphorylation of STAT proteins, since pretreatment with a concentration of JAK inhibitor sufficient to block IL-6/sIL-6R $\alpha$-stimulated STAT phosphorylation also abolished their down-regulation. Finally, the effect of $\mathrm{A}_{2 \mathrm{~A}} \mathrm{AR}$ expression on cytokine regulation of STAT degradation appeared not to be restricted to either IL-6 or vascular endothelial cells, since stable expression of the $\mathrm{A}_{2 \mathrm{~A}} \mathrm{AR}$ in rat $\mathrm{C} 6$ glioma cells suppressed the ability of interferon- $\gamma$ (IFN $\gamma$ ) to promote the tyrosine phosphorylation of STAT1, and this was accompanied by an IFN $\gamma$-induced time-dependent degradation of STAT1 protein. This was associated with an attenuation of the ability of IFN $\gamma$ in conjunction with NF- $\mathrm{B}$ mobilising stimuli to mediate the induction of iNOS. Taken together, these data argue that expression of the $\mathrm{A}_{2 \mathrm{~A}} \mathrm{AR}$ primes STAT proteins for phosphorylation-triggered degradation by the proteasome, and thus leading to reduced target gene transcription, by regulating the activity and/or expression of E3 ligases specific for JAK-phosphorylated STATs.

\section{PROMOTION OF RAT ALVEOLAR MACROPHAGE FUSION INTO MULTINUCLEATED GIANT CELLS (MGC) BY GMCSF INVOLVES THE PARTICIPATION OF THE P2X 7 RECEPTOR}

Irma Lemaire $^{1}$, Bin Zhang ${ }^{1}$, Natacha Leduc ${ }^{1}$, Simonetta Falzoni ${ }^{2}$ and Francesco Di Virgilio ${ }^{2}$ ${ }^{1}$ Department of Cellular \& Molecular Medicine, Faculty of Medicine, University of Ottawa, Ottawa, ON, Canada and ${ }^{2}$ Department of Experimental and Diagnostic Medicine, Section of General Pathology, University of Ferrara, Ferrara, Italy.

ilemaire@uottawa.ca

Multinucleated giant macrophages are a hallmark of many chronic inflammatory reactions but the mechanisms regulating MA fusion and MGC formation remain unclear. In previous work, we have demonstrated that granulocyte-macrophage colony stimulating factor (GMCSF), a cytokine produced during inflammation, promotes the fusion of rat alveolar macrophages (MA) into MGC, and such response was partly dependent on IL-6 production. The purinergic $\mathrm{P} 2 \mathrm{X}_{7}$ receptor has been implicated both in MGC formation and in the induction of the IL-1 $\rightarrow$ IL-6 cytokine cascade. In the present study, we investigated the potential role of the $\mathrm{P} 2 \mathrm{X}_{7}$ receptor in mediating GMCSF effects on MA function and fusion into MGC. To this aim, parallel experiments were performed using primary cultures of rat alveolar MA expressing the native receptor as well as HEK293 cells stably transfected with the rat $\mathrm{P} 2 \mathrm{X}_{7}$ receptor or a mock vector. Incubation of rat alveolar MA with GMCSF for 3 to 5 days increased in a dose-dependent fashion $\pm 2.5-10 \mathrm{ng} / \mathrm{ml}$ ) MA fusion into multinucleated MA by up to 3-fold. Interestingly, pretreatment of MA with oxidized ATP $(\mathrm{oATP})(100 \mu \mathrm{M})$, an irreversible blocker of $\mathrm{P}_{2} \mathrm{X}_{7}$, decreased significantly GMCSF-induced MGC formation (fusion index and MGC numbers) at all doses tested $\pm 51 \%$ of control at $10 \mathrm{ng} / \mathrm{ml}$ GMCSF), suggesting the participation of $\mathrm{P}_{2} \mathrm{X}_{7}$. In support of this, GMCSF altered $\mathrm{P} 2 \mathrm{X}_{7}$-dependent pore forming activity, a hallmark of $\mathrm{P}_{2} \mathrm{X}_{7}$ function. Rapid $(5 \mathrm{~min})$ stimulation of MA with either GMCSF $(2.5-10 \mathrm{ng} / \mathrm{ml})$ or ATP $(5 \mathrm{mM})$ was performed under similar conditions and pore forming activity was measured by ethidium bromide (EtBr) uptake. As observed with ATP, GMCSF increased the number of MA that became permeabilized to $\operatorname{EtBr}(17 \%)$, albeit at a lower level than ATP $(30 \%)$. Such effect was reduced by pre-treatment of MA with oATP \pm down to $58 \%$ of control). In addition, GMCSF up-regulated the expression of $\mathrm{P}_{2} \mathrm{X}_{7}$ receptor during MGC formation. Incubation with GMCSF $(10 \mathrm{ng} / \mathrm{ml})$ increased the percentage of $\mathrm{P}_{2} \mathrm{X}_{7}$ receptor immu- 
noreactive MA (55\% compared to $27 \%$ for unstimulated MA). Enhanced P2X $\mathrm{X}_{7}$ expression was seen at $24 \mathrm{~h}$ and up to $72 \mathrm{~h}$ at which time it correlated with increased MGC formation. In agreement with this, stable expression of rat $\mathrm{P}_{2} \mathrm{X}_{7}$ receptor in HEK293 transfected cells was associated with higher numbers of MGC compared to HEK293 cells transfected with a mock vector and that do not express $\mathrm{P}_{2} \mathrm{X}_{7}$. Quite interestingly, Polymyxin B (PMB) which increases selectively $\mathrm{P} 2 \mathrm{X}_{7}$ pore-forming activity in $\mathrm{P} 2 \mathrm{X}_{7}$ transfected cells but not in mock transfectants, also potentiated $\mathrm{P}_{2} \mathrm{X}_{7}$-dependent pore formation and IL-1 $\beta$ release in rat alveolar MA as well as GMCSF-induced MGC formation by rat MA (1.7-fold). All together, our data indicate that GMCSF promotion of MGC may result from its ability to stimulate $\mathrm{P} 2 \mathrm{X}_{7}$ function and expression and that its effect can be further increased by agents that potentiate $\mathrm{P} 2 \mathrm{X}_{7}$ receptor activity. Our study point to the $\mathrm{P} 2 \mathrm{X}_{7}$ receptor as an important effector pathway in the promotion of MGC formation by GMCSF, a cytokine produced during chronic inflammatory reactions. (Supported by CIHR, NSERC,AIRC, IMESR and CNR Italy).

\title{
Protection from Bleomycin-Induced Pulmonary Injury by Activating $\mathbf{A}_{2 \mathrm{~A}}$ or Inhibiting A $\mathbf{A}_{2 B}$ Adenosine Receptors
}

\author{
Elaine Cagnina and Joel Linden \\ Cardiovascular Research Center, University of Virginia, Charlottesville, VA 22901 \\ reb2w@virginia.edu
}

Pulmonary inflammation and fibrosis are significant side effects that complicate treatment with the anti-neoplastic drug, bleomycin (BLM). BLM is also used in mice to model idiopathic pulmonary fibrosis since it elicits mild transient inflammation followed by progressive fibrosis. Activation of the $\mathrm{A}_{2 \mathrm{~A}}$ adenosine receptor is known to inhibit pulmonary macrophage activation and neutrophil chemotaxis, and activation of the $A_{2 B}$ receptor stimulates activation of pulmonary fibroblasts. This study investigated the effects of the selective $\mathrm{A}_{2 \mathrm{~A}}$ receptor agonist, ATL313 (1.44 $\mu \mathrm{g} / \mathrm{kg} / \mathrm{day})$, or the selective $\mathrm{A}_{2 \mathrm{~B}}$ receptor antagonist, ATL851 (10 mg/kg/day), on BLM (0.075 U)induced inflammatory cell accumulation in the broncheoalveolar lavage (BAL) fluid or pulmonary fibrosis over a 2 week period. Drugs were administered continuously by subcutaneous Alzet minipumps implanted just prior to BLM exposure. ATL313 reduced BLM-induced neutrophil and lymphocyte recruitment into the BAL at 3 days post BLM treatment. ATL851 enhanced 2-week survival and reduced pulmonary collagen deposition and alveolar damage at 2 weeks. ATL851 also blocked release of the pro-fibrotic cytokine IL-6 from isolated murine pulmonary fibroblasts stimulated with the adenosine agonist $N$-ethylcarboxamidoadenosine. On the basis of these data we speculate that pulmonary fibrosis in response to BLM is triggered by an early inflammatory event that is attenuated by activation of $\mathrm{A}_{2 \mathrm{~A}}$ receptors and progresses in part due to adenosine-mediated activation of $\mathrm{A}_{2 \mathrm{~B}}$ receptors that stimulate mitogenic cytokine release from fibroblasts and possibly other pulmonary cells.

\section{Protective activity of guanosine in an in vitro model of Parkinson's disease.}

\author{
Buccella S., Romano S., D’Alimonte I., Nargi E., Ballerini P., Fischione G., Caciagli F., Di Iorio P. \\ Department of Biomedical Sciences. University of Chieti-Pescara. Chieti. Italy. \\ Email: silvana.buccella@unich.it
}

Parkinson's disease (PD) is a neurodegenerative disorder characterized by loss of dopaminergic neurons in the substantia nigra, where an inhibition of mitochondrial complex I activity has been reported [1]. 6-Hydroxydopamine (6-OHDA), which is widely used to generate in vitro and in vivo models of PD, induces both apoptotic and necrotic cell death by inhibiting mitochondrial metabolic function and by inducing endoplasmic reticulum stress [2].

This study aimed at verifying whether exogenous guanosine (eGUO), which exerts protective and anti-apoptotic effects in different in vitro models of neurodegenerative disorders [3,4], was able to protect against the toxic effect of 6-OHDA.

Exposure of SH-SY5Y human neuroblastoma cells or cultured astrocytes of rat brain to 6-OHDA (12.5-200 $\mu \mathrm{M})$ for $12 \mathrm{~h}$ caused a dose-dependent decrease of cell viability, evaluated by MTT assay. The maximal effect ( $75 \%$ 
of which $\sim 40 \%$ of apoptotic cells) was reached at $150 \mu \mathrm{M}$ with an apparent $\mathrm{EC}_{50}$ of $50 \mu \mathrm{M}$. The toxic effect of $50 \mu \mathrm{M} 6$-OHDA was evident after $3 \mathrm{~h}$ of cell treatment, whereas a longer time of exposure to lower concentrations of 6-OHDA needed to cause a significant effect. Within $3 \mathrm{~h}, 6-\mathrm{OHDA}(50 \mu \mathrm{M})$ induced phosphorylation of the stress-activated protein kinases (JNK and p38) and it also elicited a sustained ERK1/2 activation. These effects were associated with an activation of caspase cascade and GSK3beta factor. A modest and delayed increase of AKT phosphorylation was also found (2-3 h after cell exposure to 6-OHDA for $3 \mathrm{~h}$ ).

Pre-treatment of SH-SY5Y cells or astrocytes with 10-300 $\mu \mathrm{M}$ of eGUO, starting from $0.5 \mathrm{~h}$ before and carried on the time of cell exposure $(3 \mathrm{~h})$ to $50 \mu \mathrm{M}$ 6-OHDA, dose-dependently counteracted the toxin-induced cell death, with a maximal reduction of the apoptotic cells by about $70 \%$. A similar protective effect of eGUO was also observed when it was either co-administered $(3 \mathrm{~h})$ with $50 \mu \mathrm{M}$ 6-OHDA or administered (for $3 \mathrm{~h}$ at least) immediately after cell exposure to the toxin.

As we previously found [4], eGUO induced an early and PI-3 kinase-mediated increase of ERK1/2 and Akt phosphorylation. This effect was dose-dependent and reached the maximal degree after 10-15 min, lasting for the following 30-40 min at least. Downstream these factors, eGUO caused an inhibition of pro-apoptotic factors, such as GSK3beta, and a delayed activation (4 h) of anti-apoptotic proteins, such as Bcl-2.

Cell pre-treatment or co-treatment with eGUO reduced the 6-OHDA-induced phosphorylation of JNK and p38 as well as the activation of caspases and GSK3beta factor without affecting the increased levels of pERK1/2; these effects were reduced by inhibiting the PI-3 kinase pathway. The protective effect of eGUO administered after cell exposure to 6-OHDA seems to be linked to further molecular mechanisms, likely involving the Bcl-2 protein activation downstream signals.

1. Bharath, S.; Hsu, M.; Kaur, D.; Rajagopalan, S.; Andersen, J.K. Biochem. Pharmacol. 2002, 64, 1037-1048.

2. Chen, G.; Bower, K.A.; Ma, C.; Fang, S.; Thiele, C.J.; Luo, J. Faseb J. 2004, 8, 1162-1164.

3. Rathbone, M.P.; Middlemiss, P.J.; Gysberg, J.W.; Andrew, C.; Herman M.A.R.; Reed, J.K.; Ciccarelli, R.; Di Iorio, P.; Caciagli, F. Prog. Neurobiol. 1999, 59, 663-690.

4. Di Iorio, P.; Ballerini, P.; Traversa, U.; Nicoletti, F.; D’Alimonte, I.; Kleywegt, S.; Werstiuk, E.S.; Rathbone, M.P.; Caciagli, F.; Ciccarelli, R. Glia. 2004, 46, 356-368.

\title{
Purine and pyrimidine nucleosides preserve human astrocytoma cell adenylate energy charge in conditions of hypoxia
}

\author{
Francesco Balestri, Michela Giannecchini, Maria Grazia Tozzi, Marcella Camici \\ Dipartimento di Biologia, Unità di Biochimica, Via S. Zeno 51, 56100 Pisa \\ camici@dfb.unipi.it
}

The brain depends on both glycolysis and mitochondrial oxidative phosphorylation for maintainance of ATP pools. Astrocytes play an integral role in brain functions providing trophic supports and energy substrates for neurons. A number of papers have been published in which a neuroprotective effect of purine nucleosides in conditions of hypoxia has been reported. Whether nucleosides exert their protective action by interacting with specific receptors, or after their entry into the cell and metabolic conversion to energetic intermediates, is still matter of debate. While in some cases the action of adenosine is receptor-mediated $(1,2)$, to explain the effect of its deamination product, inosine, the contribution of hitherto unknown specific receptors has been invoked (3). On the other hand, several papers report a receptor-independent mechanism of nucleoside action, which ultimately involves the phosphorolytic cleavage with generation of phosphorylated sugar which is used as energy source (4-7). To address this question, we have used a human astrocytoma cell line (ADF) which has been subjected to metabolic stress conditions by exclusion of glucose and pre-incubation with oligomycin (an inhibitor of oxidative phosphorylation). This treatment brings about a depletion of the ATP pool, with a concomitant increase in the AMP levels, which results in a significant decrease of the adenylate energy charge (AEC) to a value of 0.7. A further incubation of the oligomycin pre-treated cells in DMEM medium devoid of glucose brings about a decrease in the AEC up to 0.4. The presence of glucose, inosine and guanosine in the DMEM medium preserves the AEC. Besides purine nucleosides, also pyrimidine nucleosides, such as uridine and, to a lesser extent, cytidine, are able to preserve the AEC. The determination of lactate in the incubation medium indicates that nucleosides can preserve the ATP pool through anaerobic glycolysis, thus pointing to a relevant role of the phosphorolytic clea- 
vage of the $\mathrm{N}$-glycosidic bond of nucleosides which generates, without energy expense, the phosphorylated pentose, which through the pentose phosphate pathway and glycolysis can be converted to energetic intermediates.

1. Abbracchio, M.P., and Burnostock, G. (1998) Purinergic signalling: pathophysiological roles. Jpn. J. Pharmacol. 78, 113-145.

2. Tomaselli, B., Podhraski, V., Heftberger, V., Böck, G., and Baier-Bitterlich, G. (2005) Purine nucleoside-mediated protection of chemical hypoxia-induced neuronal injuries involves p42/44 MAPK activation. Neurochem. Int. 46, $513-521$.

3. Haun, S.E., Segeleon, J.E., Trapp, V.L., Clotz, M.A., and Horrocks, L.A. (1996) Inosine mediates the protective effect of adenosine in rat astrocyte cultures subjected to combined glucose-oxygen deprivation. J. Neurochem. 67, 2051-2059.

4. Jurkowitz, M.S., Litsky, M.J., Browning, M.J., and Hohl, C.M. (1998) Adenosine, inosine and guanosine protect glial cells during glucose deprivation and mitochondrial inhibition: correlation between protection and ATP preservation. J. Neurochem. 71, 535-548.

5. Litsky, M.L., Hohl, C.M, Lucas, J.H., and Jurkowitz, M.S. (1999) Inosine and guanosine preserve neuronal and glial cell viability in mouse spinal cord cultures during chemical hypoxia. Brain Res. 821, 426-432.

6. Yoo, B-K., Choi, J.W., Yoon, S.Y., and Ko K.H. (2005) Protective effect of adenosine and purine nucleos(t)ides against the death by hydrogen peroxide and glucose deprivation in rat primary astrocyres. Neurosci. Res. 51, 39-44.

7. Giannecchini, M., Matteucci, M., Pesi, R., Sgarrella, F., Tozzi, M.G., and Camici, M. (2005) Uptake and utilization of nucleosides for energy repletion. Int. J. Biochem. Cell Biol. 37, 797-808.

\title{
Purinergic $\mathbf{P 2 X}_{2}$ receptor-channels as modulators of electrical excitability in resting and agonist-stimulated anterior pituitary gonadotrophs
}

\author{
Hana Zemkova $^{1,2}$, Ales Balik ${ }^{1}$, Yonghua Jiang ${ }^{2}$ and Stanko S. Stojilkovic ${ }^{2}$ \\ ${ }^{1}$ Department of Cellular and Molecular Neuroendocrinology, Institute of Physiology, ASCR, Prague, Czech \\ Republic, ${ }^{2}$ Section on Cellular Signaling, Endocrinology and Reproduction Research Branch, NICHD, NIH, \\ Bethesda, Maryland 20892-4510, zemkova@biomed.cas.cz.
}

The anterior pituitary is composed of the five major hormone-secreting cell types, corticotrophs, lactotrophs, thyrotrophs, somatotrophs and gonadotrophs, and their function is regulated by numerous hypothalamic and intrapituitary factors. ATP may also contribute to the control of pituitary functions, since these cells release ATP and express several subtypes of ion-conducting purinergic $\mathrm{P} 2$ receptor-channels (P2XRs), calcium-mobilizing P2Y receptors, and ecto-nucleotidase eNTPDase 1-3 (1). However, the specificity in the expression of P2 receptors among secretory cell types and their biophysical properties and roles in spontaneous and receptor-controlled electrical activity, calcium signaling, and hormone secretion have been incompletely characterized. Our RT-PCR analysis revealed that mRNA transcripts for $\mathrm{P}_{2} \mathrm{X}_{1}, \mathrm{P} 2 \mathrm{X}_{2 \mathrm{ab}}, \mathrm{P} 2 \mathrm{X}_{3}, \mathrm{P} 2 \mathrm{X}_{4}$ and $\mathrm{P} 2 \mathrm{X}_{7}$ were expressed in pituitary cells from embryonic, neonatal and adult rats. In further studies, we focused on functional identification of P2XR subtypes expressed in gonadotrophs from all three age groups. These cells fire action potentials spontaneously and respond to activation of gonadotropin-releasing hormone receptors with long-lasting membrane potential and calcium oscillations, which are dependent on periodic calcium release from $\operatorname{Ins}(1,4,5) \mathrm{P}_{3}$-sensitive store and associated voltage-gated calcium influx (2). Gonadotrophs from all three age groups also responded to ATP, but with non-oscillatory, depolarizing, slowly desensitizing, and rapidly deactivating current, indicating that these cells express P2XRs but not P2YRs. The amplitudes of P2X current responses and the rates of receptor desensitization, but not the rates of receptor deactivation, were dependent on ATP concentration. The kinetics of receptor activation, deactivation, desensitization, and resensitization in gonadotrophs were comparable with those observed in cells expressing recombinant $\mathrm{P} 2 \mathrm{X}_{2} \mathrm{R}$ and/or $\mathrm{P} 2 \mathrm{X}_{4} \mathrm{R}$. The ATP-induced current was blocked by pyridoxal 5-phosphate 6-azophenyl-2',4'-disulphonic acid, suramin, reactive blue-2 and elevated extracellular calcium concentrations, which is consistent with the expression of $\mathrm{P} 2 \mathrm{X}_{2} \mathrm{R}$ subtypes in gonadotrophs. Activation of these channels resulted in rapid plasma membrane depolarization, initiation of firing of action potentials in quiescent cells, increase in the frequency of spiking in spontaneously active cells and stimulation of gonadotropin release in perifused pituitary cells. Effects of ATP on firing of action potentials were also blocked with suramin and pyridoxal 5-phosphate 6-azophenyl-2',4'-disulphonic acid, confirming the relevance of $\mathrm{P} 2 \mathrm{X}_{2} \mathrm{R}$ inward current in depolarization of cells. ATP also influenced gonadotropin-releasing hormone-induced current and membrane potential oscillations and hormone release in an extracellular calcium-dependent manner. These oscillations were facilitated, slowed or stopped, depending of ATP concentration, the time of its application and the level of calcium content in intracellular stores. These results indicate that in gonadotrophs $\mathrm{P}_{2} \mathrm{X}_{2} \mathrm{Rs}$ function as pacema- 
king channels and modulators of gonadotropin-releasing hormone-controlled electrical activity and secretion.

1. Stojilkovic SS, Koshimizu T. 2001. Signaling by extracellular nucleotides in anterior pituitary cells. Trends Endocrinol Met 12:218-225.

2. Stojilkovic SS, Reinhart J, Catt KJ. 1994. Gonadotropin-releasing hormone receptors: structure and signal transduction pathways. Endocrine Rev 15:462-499.

\title{
Purinergic receptors are recruited in lipid microdomains at the plasma membrane of brain astrocytes
}

\author{
Elena Saba $^{1}$, Silvia Ferrario ${ }^{2}$, Stefania Ceruti ${ }^{2}$, Maria P. Abbracchio ${ }^{2}$, Patrizia Rosa ${ }^{1}$ \\ ${ }^{T}$ CNR-Institute of Neuroscience, Dep. Pharmacology, Via Vanvitelli 32, 20129 Milan \\ ${ }^{2}$ Laboratory of Molecular and Cellular Pharmacology of Purinergic Transmission, Department of Pharmacological \\ Sciences, Via Balzaretti 9, 20133, University of Milan, Italy \\ e-mail:e.saba@in.cnr.it
}

Purinergic (P2) receptors are expressed in many excitable and non-excitable cells where they mediate a great number of physiological actions, including smooth muscle contractility, neuroendocrine secretion and synaptic transmission. In addition, several data have provided evidence for a role of these receptors in physiopathological events, such as platelet aggregation, chloride secretion by epithelia, hematopoiesis, and immunological reaction. In the nervous system, P2 receptors mediate neurotransmission, release of proinflammatory cytokines, reactive astrogliosis. Extracellular nucleotides activate multiple P2 receptors in neurons and glial cells, including P2X receptors and G protein-coupled P2Y receptors (Abbracchio \& Verderio 2006). Recent studies have demonstrated the localization of some P2 receptors in lipid rafts. Given their ability to concentrate selected molecules locally, lipid rafts are known to function in protein sorting, cytoskeleton regulation, signal transduction and receptor/ion channel signaling or turnover. Therefore, we investigated whether P2Y receptors, known to be expressed in astrocytes, are recruited in lipid rafts. To this aim, primary astrocytic cultures from rat brain cortices were treated with non-ionic detergent at $4^{\circ} \mathrm{C}$ and the so-called detergent resistant membranes (DRMs, believed to represent lipid microdomains in intact cell membrane) were separated from the soluble proteins on sucrose gradients by floatation. Analysis of the gradient fraction showed that the lipid microdomain markers, GM1 and caveolin, were mainly localized in DRMs (Taverna et al. 2004) and floated in the low density region of the gradients. In line with previous results, significant amounts of the t-SNARE SNAP-23 and syntaxin3 were also present in the low density fractions. When the distribution in the gradients of $\mathrm{P}_{2} \mathrm{Y}_{1}$ and $\mathrm{P}_{2} \mathrm{Y}_{6}$ was analyzed, we found that a consistent amount of $\mathrm{P}_{2} \mathrm{Y}_{6}$ receptor localized in DRM containing fraction. Because several evidence suggested that a number of non-physiological rearrangements may occur upon the addition of Triton X-100 to membranes (Mukherjee and Maxfield, 2004), to further demonstrate the localization of $\mathrm{P}_{2} \mathrm{Y}_{6}$ receptor in lipid microdomains we used a morphological approach. To this aim "membrane sheets" were obtained from cultured astrocytes by sonication and were immunolabeled for t-SNARE proteins or P2 receptors. As expected, SNAP-23 or syntaxin 3 showed a punctate staining on the inner membrane leaflet. Interestingly, $\mathrm{P}_{2} \mathrm{Y}_{6}$ showed a similar clustered distribution. Irrelevant staining was observed when the membrane sheets were labeled with non-immune mouse or rabbit IgG. Altogether these results are in line with the biochemical data and confirm the presence of $\mathrm{P}_{2} \mathrm{Y}_{6}$ in lipid microdomains. Studies are in progress to investigate the molecular organization of these domains and their functional role in reactive astrocytes.

Abbracchio, M.P; Verderio, C. Novartis Found Symp 2006, in press. Mukherjee, S.; Maxfield, F.R. Annu. Rev. Cell Dev. Biol. 2004, 20, 839-866.

Taverna, E.; Saba, E.; Rowe, J.; Francolini, M.; Clementi, F.; Rosa, P., J. Biol. Chem. 2004, $279,5127-5134$.

Supported by The Italian Ministry of University and Research, COFIN-MIUR2004 to MPA 


\title{
Rapid ATP-induced Release of Matrix Metalloproteinase 9 Is Mediated by The $\mathrm{P2X}_{7}$ Receptor
}

\author{
Ben J. Gu \& James S. Wiley \\ The Department of Medicine, University of Sydney at Nepean Hospital, Penrith, New South Wales 2750, Australia. \\ Email: gub@med.usyd.edu.au
}

Matrix metalloproteinase-9 (MMP-9) activity is required for inflammatory response, leucocyte recruitment and tumor invasion. There is increasing evidence to suggest the $\mathrm{P} 2 \mathrm{X}_{7}$ receptor of mononuclear cells, which is activated by extracellular ATP, is involved in inflammatory responses. In this study, ATP caused a rapid release of MMP-9 and a moderate decrease in TIMP-1 release from human peripheral blood mononuclear cells (PBMC) over a 30 min time course. The release was time and dose-dependent, and dissociated from ATP-induced cell death. BzATP, which is the most potent agonist for the $\mathrm{P} 2 \mathrm{X}_{7}$ receptor, also caused a similar effect at a lower dosage. ATP-induced MMP-9 release was inhibited by the $\mathrm{P}_{2} \mathrm{X}_{7}$ receptor antagonists, periodate oxidized ATP and KN62 , or by calcium chelators, as well as by a loss-of-function polymorphism in the $\mathrm{P}_{2} \mathrm{X}_{7}$ receptor, but not by Brefeldin A, monensin or cycloheximide, or by anti-TNF-alpha or anti-IL-1beta monoclonal antibodies. Results from purified subsets of PBMC showed monocytes were the major source for MMP-9 and TIMP-1 release and ATP remained effective in purified monocyte and $\mathrm{T}$ cell populations. These observations suggest a novel role for $\mathrm{P}_{2} \mathrm{X}_{7}$ as a pro-inflammatory receptor involved in rapid MMP-9 release and leucocyte recruitment.

\section{Real-time detection of ATP release using a two enzyme assay system}

\author{
$\underline{\text { Ross Corriden }}^{1,2}$, Paul A. Insel ${ }^{2}$, and Wolfgang G. Junger ${ }^{1}$ \\ University of California San Diego, Depts. of ${ }^{1}$ Surgery/Trauma, and ${ }^{2}$ Pharmacology, San Diego, California 92103. \\ E-mail: rcorriden@ucsd.edu
}

Many different non-excitatory cell types release ATP in response to mechanical or biochemical stimulation. The mechanisms responsible for this release, however, are not well understood. This is partly due to the difficulty of studying the dynamics of ATP release immediately outside the cell membrane. Current methods for the measurement of extracellular ATP do not allow for convenient visualization of release using a microscope. Here we developed a method which allows us to both visualize and quantify localized ATP release. This method consists of a two enzyme system which uses ATP and NADP as substrates that result in production of NADPH, a fluorophore, which can be easily detected by fluorescence microscopy and hence serves as an indicator of extracellular ATP.

The two enzymes, hexokinase and glucose 6-phosphate dehydrogenase (G6PD), are added together with NADP to living cells (e.g., human neutrophils), and placed in a stage incubator of an inverted fluorescence microscope equipped with a $100 \mathrm{~W}$ mercury lamp. Images are acquired with an Orca II ER camera and analyzed with Openlab imaging software. In the presence of glucose, ATP released from the cells is converted by hexokinase to the product glucose 6-phosphate. G6PD in turn converts glucose 6-phosphate and NADP to the products 6-phosphogluconate and NADPH. We optimized a filter set that allows us to record in real-time the production of NADPH. This filter set consists of a $340 \mathrm{~nm}$ band pass exciter, a $400 \mathrm{~nm}$ dichroic mirror, and a $434 \mathrm{~nm}$ band pass emission filter. Figure 1 shows a sample image of a migrating human neutrophil. A fluorescence signal outside the moving cell in close proximity to the cell membrane indicates that migrating neutrophils release ATP in a polarized fashion with greatest ATP release observed at the leading edge. We calibrated fluorescence intensity values using ATP solutions of known concentrations. Our new method allows the detection of ATP concentrations as low as $1 \mu \mathrm{M}$, and it has a sufficiently wide dynamic range, being able to detected concentrations of up to $1 \mathrm{mM}$. This assay may provide a valuable tool for researchers who are interested in visualizing and quantifying the release of ATP in different cell systems. 

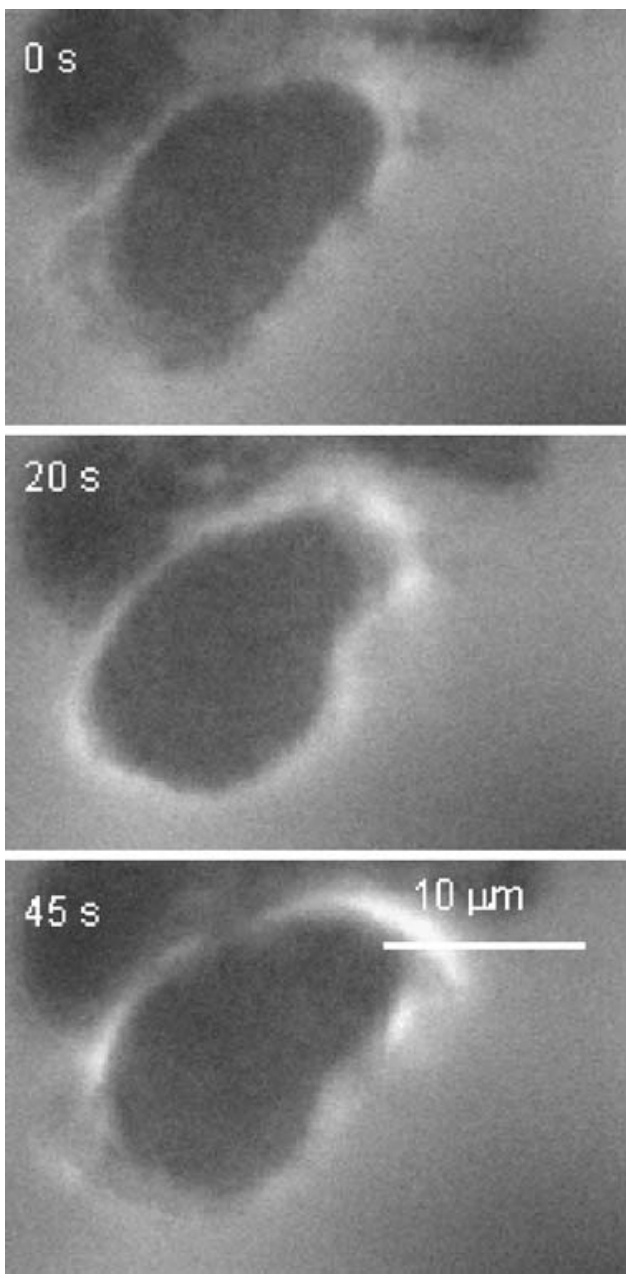

Fig. 1 Fluorescence images of a neutrophil extending a pseudopod towards the upper right hand corner. A series of images was taken at the indicated intervals $(0,20,45 \mathrm{~s})$.

This study was supported in part by grants from the U.S. National Institutes of Health (GM-51477, 60475, \& 66232).

\section{Real-time measurements of ATP and adenosine release during oxygen/glucose deprivation in area CA1 of rat hippocampal slices.}

Bruno G. Frenguelli ${ }^{1}$, Enrique Llaudet ${ }^{2,3}$ \& Nicholas Dale ${ }^{2,3},{ }^{1}$ Neurosciences Institute, Division of Pathology \& Neuroscience, University of Dundee, Ninewells Hospital, Dundee, UK, DD1 9SY, ${ }^{2}$ School of Biological Sciences, University of Warwick, Coventry, UK, CV4 7AL and ${ }^{3}$ Sarissa Biomedical Ltd, Barclays Venture Centre, Sir William Lyons Road, Coventry, UK, CV4 7EZ.

b.frenguelli@dundee.ac.uk

The purines ATP and adenosine are released into the extracellular space during insults to the mammalian brain such as head injury and cerebral ischemia. Under these conditions adenosine exerts a neuroprotective influence, primarily via the adenosine $\mathrm{A}_{1}$ receptor, whereas activation of ATP P2X or P2Y receptors may contribute to neuropathology. Although the release of ATP and adenosine is a pivotal event in the brain's response to ische- 
mia, the mechanisms underlying their release remain unclear. We have therefore used microelectrode biosensors for adenosine and ATP to measure in real-time their release from area CA1 during oxygen/glucose deprivation. $400 \mu \mathrm{m}$ thick hippocampal slices were prepared using standard techniques from 11-26 day old rat pups. Extracellular or whole-cell patch-clamp recordings $\left(33-34^{\circ} \mathrm{C}\right)$ were made from stratum radiatum and CA1 neurones, respectively. Microelectrode $(50 \mu \mathrm{m})$ biosensors for ATP and adenosine/inosine, along with a null (no-enzyme) sensor to check for the presence of electroactive interferences, were inserted into stratum radiatum. The biosensors possessed a selectivity screen that almost completely eliminated responses to potential electroactive interferences such as ascorbate, urate, dopamine and serotonin. The sensors were calibrated with exogenous adenosine or ATP and the integrity of the screen assessed by applying serotonin at the end of each experiment. Biosensors were purchased from Sarissa Biomedical Ltd www.sarissa-biomedical.com).

Omission of oxygen and glucose from the perfusion medium (ischemia; replaced by $\mathrm{N}_{2}$ and sucrose, respectively) caused the rapid release of adenosine and the depression of the fEPSP. In the early stages of ischemia, no ATP release was observed. However, during prolonged ischemia in older animals (22-26 days), ATP (1-2 $\mu \mathrm{M})$ was released around the time of the extra- or intracellularly-recorded anoxic depolarisation. Upon reoxygenation, a second phase of adenosine, and, to a lesser extent ATP $(2-3 \mu \mathrm{M})$, release was observed. Neither ATP nor adenosine release was prevented by glutamate receptor antagonism ( $5 \mathrm{mM}$ kynurenic acid), gap junction blockade (100 $\mu \mathrm{M}$ carbenoxelone) or inhibition of glial cell metabolism (100 $\mu \mathrm{M}$ fluorocitrate). ATP release was not a harbinger of widespread cell death or lysis as ATP release occurred in the presence of kynurenic acid, which, once washed from the slice, allowed recovery of the fEPSP. Omission of $\mathrm{Ca}^{2+}$ in the aCSF did not prevent ATP release and indeed increased adenosine release ( 3-fold increase after 5 mins ischemia). However, combined omission of extracellular $\mathrm{Ca}^{2+}$ together with chelation of residual extracellular $\mathrm{Ca}^{2+}$ with $1 \mathrm{mM}$ EGTA greatly reduced ATP, but not adenosine release. Inhibition of nucleoside transport with dipyrimadole $(10 \mu \mathrm{M})$ and NBTI $(5 \mu \mathrm{M})$, sufficient to depress the fEPSP by $\sim 50 \%$, did not prevent either ATP or adenosine release.

Our findings suggest complex and perhaps independent mechanisms underlying the release of adenosine and ATP during in vitro ischemia. A better understanding of the release mechanisms might provide novel therapeutic targets in acute human brain injury.

\title{
Reduced atherosclerotic lesions in $\mathrm{P}_{2} \mathrm{Y}_{1} / \mathrm{ApoE}$ double knockout mice
}

\author{
Béatrice Hechler, Catherine Léon, Monique Freund, Jean-Pierre Cazenave, Christian Gachet \\ INSERM U311, Etablissement Français du Sang-Alsace, Strasbourg, France \\ beatrice.hechler@efs-alsace.fr
}

Atherosclerosis is a multi-factorial disease of the arterial wall involving a complex interplay between circulating blood cells (leukocytes and platelets), endothelial and smooth muscle cells of the vasculature. The P2Y $\mathrm{Y}_{1}$ receptor is present on all those cell types, and plays a key role in platelet physiology. The aim of the present study was to evaluate the potential involvement of $\mathrm{P}_{2} \mathrm{Y}_{1}$ in the development of atherosclerotic lesions in mice. Therefore, we intercrossed atherosclerosis-prone apolipoprotein $\mathrm{E}$ deficient mice $\left(\mathrm{ApoE}^{-1-}\right)$ with mice lacking the $\mathrm{P} 2 \mathrm{Y}_{1}$ receptor $\left(\mathrm{P} 2 \mathrm{Y}_{1}^{-l-}\right)$ to generate $\mathrm{P} 2 \mathrm{Y}_{1} / \mathrm{ApoE}$ double knockout mice $\left(\mathrm{P}_{2} \mathrm{Y}_{1}^{-1-} / \mathrm{ApoE}^{-1-}\right)$. Mice were maintained on regular chow for 30 weeks before collection of the whole aortas and hearts for analysis of atherosclerotic lesion size and composition. Plasma cholesterol and triglyceride levels were unchanged between $\mathrm{ApoE}^{-/-}$and $\mathrm{P} 2 \mathrm{Y}_{1}^{-/-} /$ $\mathrm{ApoE}^{-l-}$ mice. In the entire aorta, atherosclerotic lesion areas, as quantified after staining with oil red $\mathrm{O}$, tended to be reduced in $\mathrm{P}_{2} \mathrm{Y}_{1}^{-1-} / \mathrm{ApoE}^{-1-}$ mice as compared to ApoE ${ }^{-1-}$ mice, although the difference did not reach statistical significance $(\mathrm{P}=0.0981$, Student's test $)$. In contrast, in the aortic roots, $\mathrm{P} 2 \mathrm{Y}_{1}^{-1-} / \mathrm{ApoE}^{-/-}$mice displayed a $24 \%$ reduction in atherosclerotic lesion areas as compared to $\mathrm{ApoE}^{-1-}$ mice $\left(0.41 \pm 0.03 \mathrm{~mm}^{2}\right.$ vs $0.54 \pm 0.03$ $\mathrm{mm}^{2}, \mathrm{n}=15, \mathrm{P}=0.003$, Student's test). This reduction in size was associated with a reduction in the percentage of total plaque area occupied by macrophages, identified with Mac-3 staining $(7.6 \pm 0.4 \%$ vs $9.6 \pm 0.8 \%, \mathrm{n}=15, \mathrm{P}=$ 0.0372) and a slight reduction in the smooth muscle cell content, while the content in collagen fibres remained similar between the two genotypes. These results suggest that the $\mathrm{P}_{2} \mathrm{Y}_{1}$ receptor contributes to atherosclerotic lesion development in $\mathrm{ApoE}^{-l-}$ mice. Whether this effect is entirely due to platelet inhibition or whether the endothelial and/or leukocyte $\mathrm{P}_{2} \mathrm{Y}_{1}$ receptor are involved requires further studies. 


\section{Reduced inflammatory and neuropathic pain in $\mathbf{P 2 X}_{4}$ receptor deficient mice}

Ulmann L. ${ }^{(1)}$, Hatcher J.P. ${ }^{(2)}$, Hughes J.P.(3), Mander P. ${ }^{(3)}$, Green P. ${ }^{(3)}$, A.J. Reeve ${ }^{(3)}$, Buell G. ${ }^{(4)}$, Chessell I. $^{(2)}$, Rassendren F.A ${ }^{(1)}$

(1)Laboratoire de Génomique Fonctionnelle, CNRS UPR2580, Montpellier, France. (2) Pain Research, N\&GI CEDD, (3) Neuro-Cell Sciences, GlaxoSmithKline, Harlow, UK., (4) Glaxo Institute of Molecular Biology, Geneva, Switzerland.

Corresponding author: lulmann@igh.cnrs.fr

Extracellular ATP is recognized as a mediator of acute and chronic pain through its binding to several types of $\mathrm{P} 2 \mathrm{X}$ receptors. It has been recently shown that in the spinal cord, antisense-mediated $\mathrm{P}_{2} \mathrm{X}_{4}$ receptor knockdown

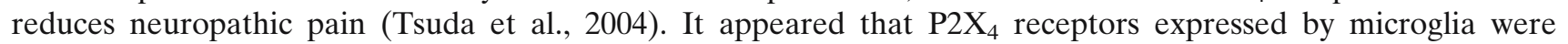
involved in mediating neuropathic pain. Similarly, disruption of $\mathrm{P}_{2} \mathrm{X}_{7}$ gene impairs both neuropathic and inflammatory pain, the later being associated with a reduced secretion of the pro-inflammatory cytokine IL- $1_{\beta}$ (Chessell et al., 2005).

In the present study, the involvement of $\mathrm{P}_{2} \mathrm{X}_{4}$ receptors in inflammatory and neuropathic pain was investigated using $\mathrm{P} 2 \mathrm{X}_{4}$ deficient mice. Inflammatory pain was induced by a FCA injection in the hind paw of mice. 24h later, $\mathrm{P}_{2} \mathrm{X}_{4}$ WT mice showed a significant hyperalgesia, compared to baseline responses. $\mathrm{P} 2 \mathrm{X}_{4} \mathrm{KO}$ mice showed no hyperalgesia. Cytometric analysis of cytokines of FCA injected paws demonstrated that the anti-inflammatory cytokine IL-10 content was up-regulated in $\mathrm{P} 2 \mathrm{X}_{4} \mathrm{KO}$ mice whereas IL- $1_{\beta}$ release was unaffected. Quantitative PCR from paw tissues, did not revealed major modification of cytokine gene expression following FCA challenge in KO animals compared to WT. Neither cytokine content nor expression was changed in spinal cord from $\mathrm{P}_{2} \mathrm{X}_{4}$ $\mathrm{KO}$ mice. Using LacZ staining, $\beta$-galactosidase and $\mathrm{P} 2 \mathrm{X}_{4}$ immunostaining, only a slight up-regulation of $\mathrm{P} 2 \mathrm{X}_{4}$ receptors in the spinal cord was observed up to 7 days after FCA injection. The absence of Cd11b staining, a specific marker of microglial cells support the view that P2X4 receptors expressed by microglia are not involved in short term FCA-induced inflammatory pain.

Following sciatic nerve ligation, $\mathrm{P} 2 \mathrm{X}_{4} \mathrm{KO}$ mice showed a significant decrease in mechanical hyperalgesia up to 25 days compared to WT. Strong LacZ staining, $\beta$-galactosidase labelling and $\mathrm{P} 2 \mathrm{X}_{4}$ expression was observed in the dorsal horn of the spinal cord. Double immunostaining showed co-localization of $\beta$-galactosidase and CD11b markers, indicating that up-regulation of $\mathrm{P}_{2} \mathrm{X}_{4}$ receptor is associated with microglial activation. In addition, in neuropathic animals lacZ staining was also observed in supra-spinal centres such as in thalamic nuclei or discrete cortical areas, suggesting an implication of $\mathrm{P}_{2} \mathrm{X}_{4}$ receptors along pain related pathways in the central nervous system. Finally, results from western blotting, YO-PRO up-take and immunocytochemistry on microglial culture showed that $\mathrm{P}_{2} \mathrm{X}_{7}$ receptors were still present and functional in $\mathrm{P} 2 \mathrm{X}_{4} \mathrm{KO}$ mice.

Our results indicate that $\mathrm{P}_{2} \mathrm{X}_{4}$ receptors are involved in both inflammatory and neuropathic pain through different mechanisms. In inflammatory pain, $\mathrm{P}_{2} \mathrm{X}_{4}$ receptors expressed by immune cells recruited at the injury site certainly mediate pain processing through peripherally secreted cytokines whereas in neuropathic pain, $\mathrm{P} 2 \mathrm{X}_{4}$ expressed by microglia are likely to be involved.

Tsuda M, Shigemoto-Mogami Y, Koizumi S, Mizokoshi A et al. Nature 424:778-783, 2004

Chessell IP, Hatcher JP, Bountra C, Michel AD Hughes JP et al. Pain 114:386-396, 2005

\section{Regulation of adenosine receptors on peritoneal mesothelial cells during peritonitis}

$\underline{\text { Sigal Nakav }}{ }^{1}$, Nadav Y. Ziv ${ }^{1}$, Boris Rogachev ${ }^{2}$, Julia Mazar ${ }^{1}$, Cidio Chaimovitz $^{2}$, Moshe Zlotnik ${ }^{2}$ and Amos Douvdevani ${ }^{1,2}$.

${ }^{1}$ Department of Clinical Biochemistry and ${ }^{2}$ Department of Nephrology, Soroka Medical Center and Ben-Gurion University of the Negev, Beer-Sheva, Israel. sigs@bgu.ac.il.

Adenosine is an endogenous immunomodulator that has been shown to exhibit anti-inflammatory and immunosuppressive effects. These anti-inflammatory effects depend mainly on the ligation with its cell-surface receptors 
subtypes: $A_{2 A}$ receptor $\left(A_{2 A} R\right), A_{2 B}$ receptor $\left(A_{2 B} R\right)$ which interact with Gs to stimulate adenylyl cyclase activity thereby elevating cAMP levels that have potent immunosuppressive effects. In contrast, the $A_{1}$ receptor $\left(A_{1} R\right)$ and $A_{3}$ receptor $\left(A_{3} R\right)$, through interaction with members of the Gi/Go family, reduce levels of cAMP. We have previously demonstrated that adenosine levels increase during peritonitis; furthermore, $\mathrm{A}_{2 \mathrm{~A}} \mathrm{R}$ agonist of the adenosine receptor strongly diminish leukocyte recruitment following E. coli inoculation.

Peritoneal mesothelial cells (PMC) form a monolayer that covers the peritoneal membrane. Their location between the peritoneal cavity and peritoneal blood vessels gives them a key role in intraperitoneal immune defense. Following stimulation with inflammatory cytokines and bacterial products, mesothelial cells express adhesion molecules and produce various cytokines and other pro-inflammatory mediators.

The aim of this study was to assess the regulation of adenosine receptors on PMC during inflammatory processes. Peritonitis was induced in CD1 mice by intraperitoneal injection of E. coli. Protein and mRNA were extracted from the peritoneum at various time points and adenosine receptor levels were analyzed by western blot and real time PCR analysis. Receptor mRNA and protein levels were evaluated in human PMC following inflammatory stimulation.

In a mouse model of peritonitis, $\mathrm{A}_{1} \mathrm{R}$ protein levels on PMC peaked at 12 hours after inoculation and then returned to baseline at 24 hours while the high affinity $\mathrm{A}_{2 \mathrm{~A}} \mathrm{R}$ protein level remained at its summit up to 24 hours at which point adenosine concentration reached its highest level in the peritoneal fluid. The low affinity $\mathrm{A}_{2 \mathrm{~B}}$ receptor maintained a slowly sustained elevation up to 48 hours. Treatment of isolated PMC with TNF $\alpha$, and IL-1 up regulated of $A_{2 A} R$ and $A_{2 B} R$ mRNA and protein while IFN- $\gamma$ decreased $A_{2 A} R$ levels; however promote the increase of $\mathrm{A}_{2 \mathrm{~B}} \mathrm{R}$ levels.

The sequential increase of $\mathrm{A}_{1}, \mathrm{~A}_{2 \mathrm{~A}}$ and $\mathrm{A}_{2 \mathrm{~B}}$ receptors, as observed in our study, suggests that the complex expression of adenosine receptors is probably part of the regulation of adenosine signaling during peritonitis. The early increase of the $A_{1} R$ stimulates leukocyte influx into the inflamed area, while the upregulation of the $A_{2 A}$ and $A_{2 B}$ receptors that appear at a later stage moderates the number of leukocytes in the exudate thereby reducing the potential damage to the peritoneal tissue caused by the cytotoxic molecules released by the leukocytes.

\title{
Regulation of adenosine receptors on peritoneal mesothelial cells during peritonitis
}

\author{
Sufaro Yuval ${ }^{1}$, Gad Shaked ${ }^{2}$, Reuven Gurfinkel ${ }^{3}$, Cidio Chaimovitz ${ }^{4}$, Amos Douvdevani ${ }^{1,4}$. \\ ${ }^{T}$ Department of Clinical Biochemistry, ${ }^{2}$ Department of Trauma care, ${ }^{3}$ Department of Plastic Surgery, and \\ ${ }^{4}$ Department of Nephrology, Soroka Medical Center and Ben-Gurion University of the Negev, Beer-Sheva, Israel. \\ sufaro@yahoo.com.
}

Among the vast array of regulatory functions attributed to adenosine it has been shown to mediate vasodilatation of blood vessels and to exert negative chronotrophic and ionotrophic upon the myocardium. These regulatory effects depend mainly on the ligation with its cell-surface receptors subtypes: $A_{2 A}$ receptor $\left(A_{2 A} R\right)$, and the $A_{1}$ receptor $\left(A_{1} R\right)$ (and possibly $A_{3}$ receptor $\left(A_{3} R\right)$ ) respectively.

Major trauma often represents a state of severe hemodynamic shock caracterized by fatal dysfunction of the cardiovascular system and impaired metabolism. It has been established that in such stressfull conditions, resulting in tissue ischemia, the concentration of adenosine in the extracellular fluid rises dramatically.

The aim of this study was to assess the role of adenosine in the acute phase of major trauma. To test our hypothesis we chose thermal injury as our model. In order to asses the presence of adenosine following thermal injury, blister fluids from burn patients were collected in the Emergency Room. Adenosine levels were determined using high performance liquid chromatography (HPLC). In a murine thermal injury model, full thickness $40 \%$ of total body surface area (TBSA) dorsal burn was inflicted upon female CD1 mice with (immediately postburn and every 12 hours for 3 days) or without $A_{1}$ and $A_{3}$ receptors antagonists. Survival, Blood glucose, and major organs architecture by histology were evaluated. All experiments were approved by the local institutional Helsinki committee.

We found adenosine concentration in blister fluids sampled during the first 12 hours post burn to be significantly elevated. In a mouse model of thermal injury, survival of burned mice treated with $A_{1} R$ antagonist and $A_{3} R$ antagonist simultaneously $(\mathrm{N}=12)$, improved dramatically demonstrating survival of $62 \%$ in comparison with 
$12 \%$ in the burned untreated group $(\mathrm{N}=12)$. There was no significant difference in survival of burned mice treated solely with either $A_{1} R$ antagonist or $A_{3} R$ antagonist. 24 hours post burn, blood glucose levels of burned mice treated with $A_{1} R$ antagonist and $A_{3} R$ antagonist simultaneously $(N=6)$ were not significantly different in comparison with untreated mice. Liver histology demonstrated central lobular ischemia of burned mice which was not present in mice treated with $A_{1} R$ antagonist and $A_{3} R$ antagonist simultaneously $(N=6)$.

Tissue adenosine is present in higher concentrations in burn patients. The increased survival of burned mice treated with $A_{1} R$ and $A_{3} R$ antagonists is suggestive for improved cardiovascular response to severe trauma. The unchanged survival of burned mice treated solely with either $A_{1} R$ or $A_{3} R$ antagonists implies of their synergistic affect in this process. The conserved liver tissue may imply of an additional positive effect by maintaining the blood flow to major organs during the acute phase of trauma. This data not only, strongly suggests of adenosine's involvement in the morbidity from major trauma but also opens a new path for the development of new efficient therapeutics.

\title{
Regulation of ATP release from naive and inflamed airway epithelia
}

\author{
Seiko F. Okada ${ }^{1}$, Carla M.P. Ribeiro ${ }^{1}$, Robert A. Nicholas ${ }^{2}$, Eduardo R. Lazarowski ${ }^{1}$ and Richard C. \\ Boucher $^{1}$. Cystic Fibrosis/Pulmonary Research and Treatment Center ${ }^{1}$ and Dept of Pharmacology ${ }^{2}$, the \\ University of North Carolina at Chapel Hill, NC, USA. seiko_okada@med.unc.edu
}

In airway epithelia, extracellular ATP and its metabolite adenosine (ADO) facilitate mucociliary clearance essential for pulmonary host defense. Thus, elucidating the ATP and ADO concentrations in the thin $(\sim 7 \mu \mathrm{m})$ film of airway surface liquid and linking these to regulation of epithelial function is important, but doing so has been difficult to achieve. Here we measured ATP concentrations ([ATP]s) at the apical surface of well-differentiated primary human bronchial epithelial (HBE) cells with luciferase fused to the IgG-binding domain of Staphylococcus protein A (SPA-luc) ${ }^{1}$. Real-time measurements of [ATP] by SPA-luc attached to the apical HBE culture surface via an antibody against endogenously expressed keratan sulfate were compared with those determined with soluble luciferase dissolved in bulk mucosal liquid. [ATP] on resting HBE surfaces were in the low nM range as measured by both methods. Inhibition of extracellular ATPases by the addition of $\beta, \gamma$-methylene-ATP, levamisole and ebselen resulted in ATP accumulation at a rate of $\sim 250 \mathrm{fmol} / \mathrm{cm}^{2} / \mathrm{min}$, which reflected basal ATP release. Following 33\% hypotonic challenge, HBE cells swelled and [ATP] transiently reached $\sim 1 \mu \mathrm{M}$ as measured by cell-attached SPA-luc, reflecting an increase in ATP release rate to $200-900 \mathrm{pmol} / \mathrm{cm}^{2} / \mathrm{min}$. The peak [ATP] in mucosal liquid following hypotonic challenge as measured by soluble luciferase was volume dependent, with measurements in a small volume $\left(25 \mu \mathrm{l} / \mathrm{cm}^{2}\right)$ approximating those of cell-attached SPA-luc.

These techniques were applied to address whether inflammation augments ATP release from airway epithelia, similar to what has been reported in other systems. A challenge with supernatant of mucopurulent material (SMM) collected from cystic fibrosis airways was utilized to induce inflammation in HBE cells ${ }^{2}$. Mucosal exposure to SMM for $48 \mathrm{~h}$ augmented hypotonicity-induced ATP release from HBE cultures (peak [ATP] = $1130 \pm 200$ and $2050 \pm 150 \mathrm{nM}$ in vehicle- and SMM-treated cultures, respectively), which paralleled the increased secretion of inflammatory markers such as IL-8. No difference was observed in resting state ATP concentrations and release rates between vehicle- and SMM-treated cultures. Because SMM treatment increased $\mathrm{Ca}^{2+}$ i stores and augmented agonist-induced $\mathrm{Ca}^{2+}{ }_{i}$ mobilization in HBE cells2, we examined the effect of $\mathrm{Ca}^{2+}{ }_{i}$ chelation on ATP release. BAPTA treatment did not significantly alter the peak [ATP] following hypotonic challenge in vehicle-treated cultures; however, it reduced peak [ATP] of SMM-treated cultures to the range of vehicle-treated (840 \pm 40 and $1330 \pm 250 \mathrm{nM}$ in vehicle- and SMM-treated cultures, respectively). Fourteen days after the cessation of SMM- or vehicle-exposure, both IL-8 secretion and ATP release from SMM-treated cultures returned to the range of those of vehicle-treated. These findings suggest that inflammation in HBE cells augmented hypotonicity-induced ATP release in a reversible fashion. In contrast to the hypotonicity-induced ATP release from naive HBE cells having observed to be largely $\mathrm{Ca}^{2+}{ }_{i}$-independent, the $\Delta[$ ATP $]$ gained by inflammation was mostly $\mathrm{Ca}^{2+}{ }_{\mathrm{i}}$-dependent, suggesting that inflammation conferred $\mathrm{Ca}^{2+}{ }_{\mathrm{i}}$-dependent pathways of ATP release to HBE cells, which were not major players in non-inflamed epithelia.

(Supported by National Institute of Health and Cystic Fibrosis Foundation.) 
1:Joseph, S. M.; Buchakjian, M. R.; Dubyak, G. R., Colocalization of ATP release sites and ecto-ATPase activity at the extracellular surface of human astrocytes. J Biol Chem 2003, 278, (26), 23331-42.

2: Ribeiro, C. M.; Paradiso, A. M.; Schwab, U.; Perez-Vilar, J.; Jones, L.; O'Neal, W.; Boucher, R. C., Chronic airway infection/ inflammation induces a $\mathrm{Ca} 2+\mathrm{i}$-dependent hyperinflammatory response in human cystic fibrosis airway epithelia. $J$ Biol $C h e m$ 2005, 280, (18), 17798-806.

\title{
Regulation of E-NTPDase Activities by the Transmembranous Domains
}

\author{
Wei.-Chieh Chang ${ }^{\mathrm{a}}$, Yonghee Lee ${ }^{\mathrm{a}}$, Takashi Mukasa ${ }^{\mathrm{a}}$, Cheryl $\mathrm{Li}^{\mathrm{a}}$, Jean Sévigny ${ }^{\mathrm{b}}$, and Aileen F. \\ Knowles $^{\text {a }}$ \\ ${ }^{a}$ Department of Chemistry and Biochemistry, San Diego State University, San Diego, California, U.S.A. ${ }^{b}$ Centre de \\ recherché en Rhumatologie et Immunologie, Universite Laval, Québec, Canada aknowles@chemistry.sdsu.edu
}

The cell surface E-NTPDases (E-NTPDase 1, 2, 3, 8) are anchored to the membrane by two transmembranous domains (TMD), one each at the N- and C-termini. Of the three E-NTPDases that we cloned, expressed and characterized, the chicken E-NTPDase 8 is unusual in that it remains active in the presence of high concentrations of certain detergents, e.g., NP-40 [1]. In contrast, human E-NTPDase 2 and 8 are inhibited by low concentrations of NP-40 which can be prevented by prior treatment of the enzymes by a cross-linking agent, glutaraldehyde $[2,3]$. Furthermore, the human E-NTPDase 2 is susceptible to substrate inactivation [2] whereas the chicken and human E-NTPDase 8 are not $[1,3]$.

The TMD of the chicken E-NTPDase 8 and human E-NTPDase 2 have different amino acid sequences. In order to elucidate the roles of the TMD in the two E-NTPDases in their different responses to detergents and substrate inactivation, we generated the following constructs: (i) chimeras (ck-hu ACR1, ACR5, and ACR1,5) in which the $\mathrm{N}$ - or C-terminal regions of chicken E-NTPDase 8 or both are exchanged for the corresponding regions of the human E-NTPDase2, (ii) chimeras (hu-ck ACR1, ACR5 and ACR1,5) in which the N-and C-terminal regions or both of the human E-NTPDase 2 are exchanged for the corresponding regions of the chicken E-NTPDase 8, and (iii) soluble extracellular domains (ECD) of the three E-NTPDases. Except for hu-ck ACR1, all are expressed in HEK293 cells upon transfection with the cDNA constructs.

Chimeras containing one or both of the N- or C-terminal TMD of the human E-NTPDase 2, regardless of the parent molecule, are all susceptible to inhibition by NP-40 and substrate inactivation. Interestingly, substrate inactivation is more pronounced in ck-hu ACR1 than in ck-hu ACR1,5. However, the chimera of human ENTPDase 2 with both chicken TMD (hu-ck ACR1,5) is now insensitive to NP-40 inhibition nor substrate inactivation. These results suggest that catalysis at the active site in the ECD is affected by the interhelical interaction of the respective pairs of TMD, which is affected differently by NP-40 in the two enzymes.

As expected, none of the soluble enzymes of the three E-NTPDases are affected by NP-40. However, their enzymatic characteristics are significantly altered from the membrane-bound enzymes. Both soluble human E-NTPDase 2 and 8, in contrast to the membrane-bound enzymes, show a preference for CaATP, and the soluble human E-NTPDase 2 is no longer inactivated by substrates. Unexpectedly, soluble chicken E-NTPDase 8 shows marked substrate inactivation in a temperature dependent manner. Thus the presence of TMD in the chicken E-NTPDase 8 abrogates substrate inactivation of its ECD, which is exactly the opposite of that observed with the soluble and membrane-bound human E-NTPDase 2. (Supported by the California Metabolic Research Foundation.)

References:

1. Mukasa, T., Lee, Y. and Knowles, A. F. Biochemistry 2005, 44, 11660-11170

2. Knowles, A. F. and Chiang, W-C. Arch. Biochem. Biophys. 2003, 418, 217-227

3. Knowles, A. F. and Li, C. (submitted) Biochemistry

\section{Regulation of epithelial $\mathrm{K}^{+}$channels by P2Y2 and P2Y4 receptors}

\author{
Susanne E. Hede $^{1}$, Jan Amstrup ${ }^{1}$, Dan A. Klaerke ${ }^{2}$ and Ivana Novak ${ }^{1}$ \\ ${ }^{T}$ August Krogh building, Institute of Molecular Biology and Physiology, University of Copenhagen, Denmark. \\ ${ }^{2}$ Section for Physiology and Biochemistry, The Royal Veterinary and Agricultural University, Denmark. \\ sehede@aki.ku.dk
}


Secretion in epithelia of airways, pancreas, small intestine and sweat glands is initiated by opening of Cl- channels (e.g. CFTR), and $\mathrm{K}^{+}$channels that keep the driving force for the secretory process. In our previous studies on native rat pancreatic ducts we have shown that activation of $\mathrm{P} 2 \mathrm{Y} 2$ and $\mathrm{P} 2 \mathrm{Y} 4$ receptors causes increased intracellular $\mathrm{Ca}^{2+}$, and surprisingly inhibition of $\mathrm{K}^{+}$channels that would decrease the secretion (1). However, the identity of the $\mathrm{K}^{+}$channels associated with the epithelia of pancreatic ducts was not known. Therefore the aim of the project was to determine the molecular identities of the $\mathrm{K}^{+}$channels expressed in the native pancreatic duct and to elucidate at a cellular level how the purinergic receptors P2Y2 and P2Y4 regulate epithelial $\mathrm{K}^{+}$channels. To resolve which $\mathrm{K}^{+}$channels are present in pancreatic ducts, we performed RT-PCR experiments and identified transcripts for BK (Slo-1), IK and KCNQ1 but not SK. We also tested whether the recently discovered BK subtypes Slo-2 and Slo-3 were expressed, but they could not be detected.

To elucidate at a cellular level how the pancreatic purinergic receptors P2Y2 and P2Y4 regulate the epithelial IK and BK and KCNQ1 channels, we co-expressed the channels with one of the receptors in Xenopus oocytes and measured currents by two-electrode voltage clamp. The purinergic receptors were stimulated by UTP $\left(10^{-4} \mathrm{M}\right)$. When human BK and IK were expressed in oocytes together with human P2Y2 and P2Y4 receptors, these channels could be activated or inhibited, depending on the combination of channel and receptor. The most notable finding was that P2Y2 receptors inhibited BK (20\%) similar to what we have found in native rat ducts. Co-expression of P2Y4 receptors stimulated the BK-channel (30\%). IK stimulated both P2Y2 (5 fold) and P2Y4 receptors (10 fold)(2). The KCNQ-1 channel was expressed with its $\beta$-subunits (KCNE1 or KCNE3), but none of these constellations were affected by stimulation of the purinergic receptors. Taken together, the combination of P2Y2 and BK channels in the oocytes give similar data as in the native pancreatic ducts and indicates that this constellation is dominant. Traditionally, the P2Y2 receptors have been assumed to couple to Gq and its downstream effectors, including PKC. We tested if PKC could be the link between activation of P2Y2 and inhibition of BK channels. In these experiments the PKC activator PMA $(n=4)$ failed to inhibit the BK currents in P2Y2 and BK expressing oocytes. Also pre-incubation of the oocyte with the PKC inhibitor staurosporine $(\mathrm{n}=4)$ could not prevent inhibition of BK. Based on these studies we expect that the P2Y2 induced inhibition of BK current is independent of PKC and other intracellular signalling partners have to be considered.

This work was supported by The Lundbeck Foundation, The Augustinus Foundation and the Danish Medical and Science Research Council.

1. Hede SE, Amstrup J, Christoffersen BC, Novak I (1999) J.Biol.Chem. 274:31784-31791

2. Hede SE, Amstrup J, Klaerke DA, Novak I (2005) Pflugers Arch. 450 (6):429-36.

\title{
Synthesis and structure-activity relationships of base-modified UDP and UTP derivatives and analogs at the human $\mathrm{P}_{2} \mathrm{Y}_{2}, \mathrm{P}_{4} \mathrm{Y}_{4}$ and $\mathrm{P}_{2} \mathrm{Y}_{6}$ receptors
}

\author{
Ali El-Tayeb $^{1}$, Aidong $\mathrm{Qi}^{2}$ and Christa E. Müller ${ }^{1}$ \\ ${ }^{T}$ Pharmaceutical Sciences Bonn (PSB), Pharmaceutical Chemistry, Institute of Pharmacy, University of Bonn, \\ Kreuzbergweg 26, Bonn, Germany \\ ${ }^{2}$ Department of Pharmacology, University of North Carolina School of Medicine, Chapel Hill, USA \\ E-mail address of presenting author a.el-tayeb@uni-bonn.de
}

The human P2Y receptor subtypes are activated by different physiological nucleotides, including the purines ATP and ADP, and the pyrimidines UTP, UDP and UDPglucose, depending on the receptor subtype. Four P2Y receptor subtypes are sensitive to uracil nucleotides, the P2Y 2 (UTP, also ATP), the P2Y 4 (UTP), the P2Y 6 (UDP), and the $\mathrm{P}_{2} \mathrm{Y}_{14}$ (UDPglucose). ${ }^{1}$ There is a lacking of potent, selective and enzymatically stable agonists and antagonists for uracil nucleotide-activated P2Y receptors. Such compounds are required as pharmacological tools and have considerable potential as novel drugs. ${ }^{1}$ Only limited information on structure-activity relationships of uracil nucleotide derivatives and analogs is available so far.

In the present study, we synthesized a series of base-modified UTP, UDP and UMP derivatives as well as UTP analogs in which the triphosphate group was replaced by an enzymatically more stable residue. Base-modified uracil nucleotides were obtained starting from the uracil derivatives, which were silylated and subsequently reacted with benzoyl- or acetyl-protected ribose in the presence of a Friedel-Crafts catalyst according to a modified Hilbert-Johnson method, to afford the protected nucleosides. After deprotection ${ }^{2}$ an N3-substituent could be introduced by alkylation with alkyl halogenides in the presence of $\mathrm{K}_{2} \mathrm{CO}_{3}$ in acetone/DMF. ${ }^{3}$ The obtained nuc- 
leosides were susceptible to phosphorylation in the $5^{\prime}$-position according to a procedure described by $\mathrm{Ludwig}^{4}$ to afford the corresponding mono-, di-, and/or triphosphates.
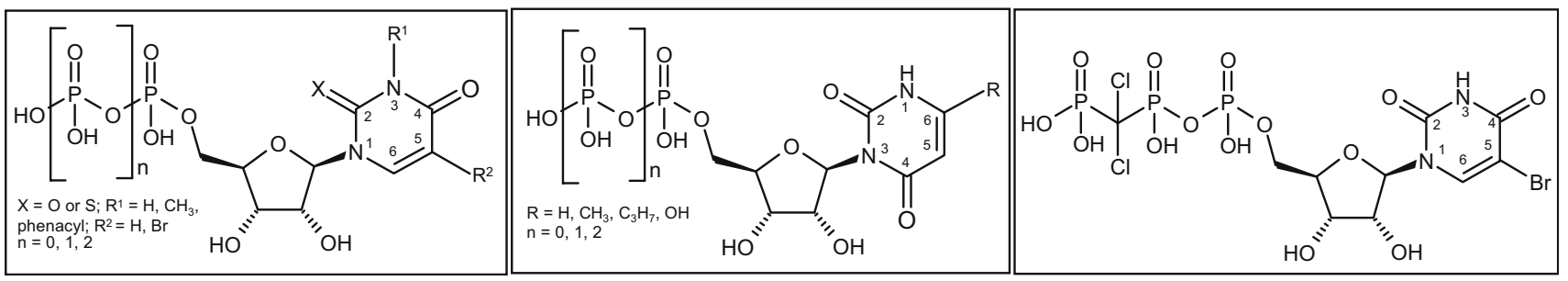

The synthesized nucleotides were investigated for their potency to increase $\mathrm{P}_{2} \mathrm{Y}_{2^{-}}, \mathrm{P}_{2} \mathrm{Y}_{4^{-}}$, and $\mathrm{P} \mathrm{Y}_{6}$-receptormediated inositol phosphate accumulation in recombinant astrocytoma $1321 \mathrm{~N}$ cells expressing the respective receptor subtype. Several of the synthesized nucleotides showed high activity at certain receptor subtypes. N3phenacyl-UDP was a potent and selective $\mathrm{P}_{2} \mathrm{Y}_{6}$ agonist $\left(\mathrm{EC}_{50}=70 \mathrm{nM}\right)$, and 2-thio-UTP was a potent P2 $\mathrm{Y}_{2}$ agonist $\left(\mathrm{EC}_{50}=50 \mathrm{nM}\right)$. A 5-Bromo-UTP analog stabilized by a $\mathrm{P} \beta \mathrm{P} \gamma$-dichloromethylene bridge to enhance the enzymatic stability was synthesized and showed relatively high activity at $\mathrm{P}_{2} \mathrm{Y}_{2}$ and $\mathrm{P} 2 \mathrm{Y}_{6}$ receptors with $\mathrm{EC}_{50}$ values of 354, and $120 \mathrm{nM}$, respectively.

1. Brunschweiger, A.; Müller, C. E. Curr. Med. Chem. 2006, 12, 763-771.

2. Hamamura, H.; Sato and K.; Moffatt, G. J. J. Med. Chem. 1972, 15, 1061-1065.

3. Yamamoto, I; Kimura, T.; Tateoka, Y.; Watanabe, K.; Ho, K. I. J. Med. Chem. 1987, 30, $2227-2231$.

4. Ludwig, L. Acta Biochim. Biophys. Acad. Sci. Hung. 1981, 16, 131-133.

\section{Synthesis of pyrazolo[3,4-b]pyridines selective antagonists of $\mathbf{A}_{1}$ adenosine receptors}

$\underline{\text { Schenone S. }}{ }^{1}$, Brullo C. ${ }^{1}$, Ranise A $^{1}$, Bondavalli F. ${ }^{1}$, Mosti L. ${ }^{1}$, Fossa P. ${ }^{1}$, Menozzi G. ${ }^{1}$, Trincavelli L. ${ }^{2}$, Martini $\mathrm{C}^{2}$, Manetti $\mathrm{F}^{3}$, Tintori $\mathrm{C}^{3}$.

${ }^{1}$ Dipartimento di Scienze Farmaceutiche, Università di Genova, Viale Benedetto XV, I-16132, Genova, Italy; ${ }^{2}$ Dipartimento di Psichiatria, Neurobiologia, Farmacologia e Biotecnologie, Università di Pisa, Via Bonanno 6, I-56126, Pisa, Italy; ${ }^{3}$ Dipartimento Farmaco Chimico Tecnologico, Università degli Studi di Siena, Via Alcide de Gasperi 2, I-53100, Siena, Italy.

schensil@unige.it

$\mathrm{A}_{1}$ adenosine receptors ( $\left.\mathrm{A}_{1} \mathrm{ARs}\right)$ are widely distributed, ubiquitously expressed in the central nervous system and in internal organs such as heart, kidneys, liver and bladder. Adenosine causes via $\mathrm{A}_{1} \mathrm{ARs}$ locomotor depression, anxiolysis and sedation in the CNS, while in the heart has negative chronotropic, dromotropic and inotropic effects. Moreover $\mathrm{A}_{1} \mathrm{ARs}$ stimulation provokes in the kidney vasoconstriction and a decrease in the glomerular filtration rate.

$\mathrm{A}_{1} \mathrm{AR}$ antagonists have therapeutic potential in the treatment of various forms of dementia, depression and as cognition enhancers in geriatric therapy. Moreover $\mathrm{A}_{1} \mathrm{AR}$ antagonists are currently studied as potassium saving diuretics and for the treatment of acute renal failure.

Among the non xanthine derivatives $\mathrm{A}_{1} \mathrm{AR}$ antagonists, only few examples of pyrazolo[3,4-b]pyridine derivatives are reported in literature, the most active of them showing affinity of $0.3 \mu \mathrm{M}$, but scarce selectivity.

A few years ago, in this context we have synthesized a series of 4-amino-1-(2-chloro-2-phenylethyl)-1H-pyrazolo[3,4-b]pyridine-5-carboxylic acid ethyl esters, possessing an interesting antagonistic profile, the most active compound showing a $50 \mathrm{nM}$ affinity toward $\mathrm{A}_{1} \mathrm{ARs}{ }^{1}$ Very recently, on the basis of our pseudoreceptor model, we have synthesized new compounds bearing different amino groups on $\mathrm{C} 4$ and different esters on $\mathrm{C} 5$, obtaining more active compounds with $\mathrm{K}_{\mathrm{i}}$ lower than $10 \mathrm{nM}^{2}$ 


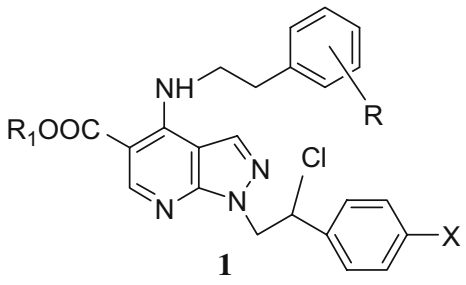

We reported here the synthesis and the affinity data of other pyrazolo[3,4-b]pyridine derivatives $\mathbf{1}$, following SAR studies on all the inhibitors previously reported, bearing differently substituted phenylethylamino groups on C4 and an activated phenyl ring on the $\mathrm{N} 1$ side chain.

(1.) Bondavalli, F.; Botta, M.; Bruno, O.; Ciacci, A.; Corelli, F. et al. Synthesis, molecular modeling studies, and pharmacological activity of selective $\mathrm{A}_{1}$ receptor antagonists. J. Med. Chem. 2002, 45, 4875-87.

(2.) Manetti, F.; Schenone, S.; Bondavalli, F.; Brullo, C. at al. Synthesis and 3D QSAR of new pyrazolo[3,4-b]pyridines: potent and selective inhibitors of $\mathrm{A}_{1}$ adenosine receptors. J. Med. Chem. 2005, 48, 7172-85.

\section{Synthesis, Characterization, and Quantitation of the Four Single-Inversion Epimers of 2-Chloroadenosine}

James J. Carey, ${ }^{1}$ Allan R. Moorman, ${ }^{1 *}$ Robert P. Rodebaugh, ${ }^{2}$ Darryl LeBlanc, ${ }^{2}$ Cliff F. Sargent, ${ }^{2}$ Michael P. Scannell, ${ }^{2}$ Jie Li, ${ }^{3}$ Bradley H. Wolfe, ${ }^{3}$ Marc W. Andersen, ${ }^{3}$ Richard Vinson, ${ }^{4}$ and Krzysztof Golebiowski $^{4}$

${ }^{1}$ King Pharmaceuticals Research and Development, Inc., 4000 CentreGreen Way, Suite 300, Cary, North Carolina 27513. ${ }^{2}$ Scynexis, Inc., 3501C TriCenter Boulevard, Durham, North Carolina 27713. ${ }^{3}$ Cardinal Health, 160 Magellan Lab Court, Morrisville, North Carolina 27560. ${ }^{4}$ Metrics Inc., 1240 Sugg Parkway, Greenville, North Carolina 27834. allan.moorman@kingpharm.com

The importance of chirality to the biological activity of drug molecules is well precedented. In certain cases, the stereochemical integrity of a raw material can be compromised during chemical processing steps used to manufacture starting material or drug substance. The development of pharmaceutical agents based upon adenosine introduces four stereogenic centers in the ribosyl-moiety that may be impacted. Because 2-chloroadenosine serves as an important starting material for a number of 2-modified adenosine analogues, we undertook the synthesis of the four single-inversion epimers $\left(\mathbf{1}, \mathbf{2}, \mathbf{3}\right.$, and $\mathbf{4}$, respectively) of this key starting material. The syntheses of the $1^{\prime}-$, $3^{\prime}$ - and $4^{\prime}$-epimers were accomplished by coupling an appropriately protected sugar molecule with 2,6-dichloroadenine, promoted by tin tetrachloride, followed by regioselective displacement of the 6-chloro group with ammonia, and deprotection. The $2^{\prime}$-epimer (2) was synthesized from commercially available 2-chloroadenosine by inversion of the hydroxyl group at the $2^{\prime}$-position. This poster will describe the synthesis and characterization of these epimers, as well as a chromatographic method for the quantitation of their relative content in 2-chloroadenosine. These epimeric materials also serve as starting materials for the synthesis of the corresponding epimers of adenosine $\mathrm{A}_{2 \mathrm{~A}}$ receptor agonists currently in clinical development.
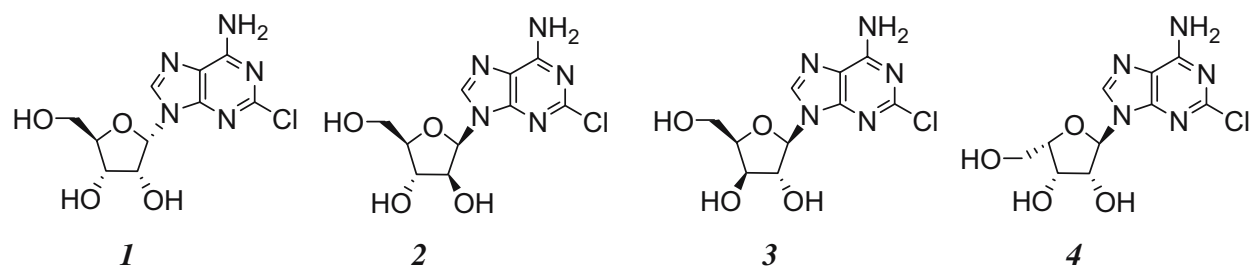


\title{
Involvement of multiple receptors in the regulation of murine dendritic cells by purines
}

\author{
Abduelhakem Ben Addi ${ }^{1}$, Pamela Conley ${ }^{2}$, Jean-Marie Boeynaems $>^{1,3}$ and Bernard Robaye ${ }^{1}$ \\ ${ }^{1}$ Institute of Interdisciplinary Research, IBMM, School of Medicine, Université Libre de Bruxelles, Gosselies, \\ Belgium \\ ${ }^{2}$ Portola Pharmaceuticals, South San Francisco, California \\ ${ }^{3}$ Department of medical chemistry, Erasme Hospital, Brussels, Belgium \\ jmboeyna@ulb.ac.be
}

There is now strong evidence that ATP induces a semi-maturation state of human monocyte-derived dendritic cells via the activation of the $\mathrm{P}_{2} \mathrm{Y}_{11}$ receptor. In the same cells, ADP exerts partially similar effects via the activation of $\mathrm{G}_{\mathrm{i}}$-coupled receptors, which have not been identified. Whereas the mouse is largely used for in vivo studies of immunity and inflammation, very few data are available on the modulation of murine dendritic cells by purines. We have shown that ADP $\beta$ S increases cytosolic $\mathrm{Ca}^{2+}$ in Flt-3 ligand-expanded splenic dendritic cells. That response was abolished by AR-C69931MX and AR-C67085MX. It was also abolished in $\mathrm{P}_{2} \mathrm{Y}_{12}{ }^{-/-}$but not in $\mathrm{P}_{2} \mathrm{Y}_{13}{ }^{-/-}$mice. ADP $\beta S$ reduced the production of IL-12p70 by splenic dendritic cells stimulated by cell-bound CD40 ligand. This inhibition was relieved by AR-C69931MX, which by itself increased the production of IL-12. However the reduction by ADP $\beta S$ was maintained in $\mathrm{P} 2 \mathrm{Y}_{12}{ }^{-/-}$and $\mathrm{P} 2 \mathrm{Y}_{13}{ }^{-/-}$mice. This might suggest that both $\mathrm{P}_{2} \mathrm{Y}_{12}\left(\right.$ via $\mathrm{Ca}^{2+}$ ) and $\mathrm{P}_{2} \mathrm{Y}_{13}$ (via another signal) decrease IL-12. In bone marrow derived dendritic cells, ADP $\beta S$ inhibited the production of IL-12 induced by lipopolysaccharide. However that inhibition was resistant to ARC69931MX and MRS 2179, but it was abolished by 8-(p-sulfophenyl)theophylline. These studies show that multiple receptors play a role in the regulation of dendritic cells by purines, depending on species and subpopulation:

- in human monocyte-derived dendritic cells, ATP induces a semi-maturation state via activation of the P2 $\mathrm{Y}_{11}$ receptor;

- in murine bone marrow derived dendritic cells, it is adenosine that plays a dominant role;

- in murine splenic dendritic cells, the $\mathrm{P}_{2} \mathrm{Y}_{12}$ receptor is functionally expressed, but another AR-C69931MXsensitive receptor, probably $\mathrm{P}_{2} \mathrm{Y}_{13}$, seems to be involved also in the action of ADP.

\section{Targeted knockdown of cytosolic $5^{\prime}$-nucleotidase (cN-II) with siRNA.}

\author{
Maria Giovanna Careddu*, Simone Allegrini*, Marcella Camici, Rossana Pesi and Maria Grazia Tozzi. \\ Dipartimento di Biologia, Università di Pisa. *Dipartimento di Scienze del Farmaco, Università di Sassari. \\ m.tozzi@dfb,unipi,it
}

Cytosolic 5'-nucleotidase (cN-II) belongs to a family of proteins involved in the hydrolysis of intracellular nucleotides. Members of this family differ widely in cellular location, substrate specificity, regulation and aminoacid sequence (1). cN-II has been described as a bifunctional enzyme since catalyzes both the hydrolysis of IMP and GMP and the transfer of phosphate from a mononucleotide donor to a nucleoside acceptor such as inosine, guanosine, deoxyinosine and several nucleoside analogs in use as antiviral or antineoplastic purine prodrugs $(2,3)$. Furthermore, cN-II seems to be responsible for the resistance to several purine derivative drugs. Therefore, it would seem that cN-II plays a fundamental role in the effectiveness of several purine drugs and its activity has been indicated as predictive of patient survival in acute myeloid leukaemia $(4,5)$. cN-II is ubiquitously present in cells and organs and its involvement in the intracellular production of adenosine from AMP has been hypothesized at least where cN-I (nucleotidase specific for AMP) is absent. We purified and characterized this enzyme from different sources and obtained the bovine recombinant enzyme which presents a $99 \%$ homology with human $\mathrm{cN}-\mathrm{II}$ (6). We demonstrated that the enzyme catalyze the hydrolase/phosphotransferase reaction through the formation of a covalent enzyme-phosphate intermediate and described its complex regulation depending on intracellular energy charge (7). Furthermore, on the bases of site directed mutagenesis we identified aminoacid residues involved in catalysis $(8,9)$. In order to study the metabolic pathways in which this enzyme plays a fundamental role and to unequivocally assess its involvement in adenosine production, we decided to utilize the siRNA technique to obtain the $\mathrm{cN}-\mathrm{II}$ knockdown. Here we report our more recent results on the silencing of $\mathrm{cN}-$ II both in mouse and in human cells with the small interfering RNA technique, utilizing different viral vectors. We 
obtained constitutive siRNA intracellular production causing a complete knockout of the enzyme. Since cN-II silencing proved to be lethal for cells we are now developing a viral vector harboring an inducible promotor.

1-Bianchi V., and Spychala J. (2003) Mammalian 5'-nucleotidase. J.B.C. 278, 46196-46198

2-Banditelli S., Baiocchi C., Pesi R., Allegroni S., Turriani M., Ipata P.L. Camici M., Tozzi M.G. The phosphotransferase activity of cytosolic $5^{\prime}$-nucleotidase: a purine analogs phosphorylating enzyme. (1996) Int,J,Biochem.Cell Biol., 28, 711-720

3-Johnson M.A. and Fridland A. (1969) Phosphorylation of 2'-3'-dideoxyinosine by cytosolic 5'-nucleotidase of human lymphoid cells. Med. Pharmacol. 36, 291-205

4-Galmarini C.M., Mackey J.R., and Dumontet C. (2001) Nucleoside analogues: mechanisms of drug resistence and reversdal strategies. Leukemia, 15, 875-890

5-Mackey J.R., Galmarini C., Graham K.A., Joy A.A., Delmer A., Dabbagh L., Giubrecht D., Jewel L.D., Lai R., Lang T., Hanson J., Young J.D., Merie-Beral H., Binet J., Cass C.E., and Dumontet C. (2005) Quantitative analysis of nucleoside trasporter and metabolism gene expression in chronic lymphocytic leukaemia (CLL) identification of fuldarabine sensitive populations. Blood, 105, 767-774

6- Pesi R, Turriani M., Allegrini S., Scolozzi C., Camici M., Ipata P.L. and Tozzi M.G. (1994) The bifunctional cytosolic 5'-nucleotidase:regulation of phosphotransferase and nucleotidase activities. Arch. Biochem. Biophys., 312, 75-80

7- Allegrini S., Pesi R., Tozzi M.G., Fiol C., Johnson B., and Eriksson S. (1997) Bovine cytosolic IMP/GMP specific 5'-nucleotidase: cloning and expression of active enzyme in Eescherichia coli. Biochem.J 328, 483-487

8 Allegrini S., Scaloni A. Careddu M.G., Cuccu G., D’Ambrosio C., Pesi R., Camici M., Ferrara L., and Tozzi M.G.. (2004)

Mechanistic studies on bovine cytosolic 5'-nucleotidase II Eur.J.Biochem 271, 4881-4691

\title{
The adenosine $A_{2 A}$ receptor agonist CGS-21680 fails to ameliorate the course of dextran sulphate-induced colitis in mice
}

\author{
Zsolt Selmeczy, E. Sylvester Vizi, György Haskó \\ Department of Pharmacology, Institute of Experimental Medicine, Hungarian Academy of Sciences, Budapest, \\ Hungary \\ e-mail:selmeczy@koki.hu
}

Crohn's disease and ulcerative colitis, collectively referred to as inflammatory bowel disease, are chronic spontaneously relapsing disorders of unknown cause. Several murine models of intestinal inflammation have an important role in the research of these disorders. One of the murine models of inflammatory bowel disease is colitis induced by oral administration of dextran sulphate sodium (DSS). Some of the symptoms and pathophysiologic features of DSS-induced colitis are similar to those found in human inflammatory bowel disease. Meanwhile, it is well recognised that certain naturally occurring purines, mainly adenosine and its analogues, are effective modulators of the immune system. Physiological actions of adenosine result from its occupancy of cell surface adenosine receptors $\left(\mathrm{A}_{1}, \mathrm{~A}_{2 \mathrm{~A}}, \mathrm{~A}_{2 \mathrm{~B}}, \mathrm{~A}_{3}\right)$, which are expressed on immune cells. As $\mathrm{A}_{2 \mathrm{~A}}$ receptors dominate in mediating the anti-inflammatory effects of adenosine, in this study we investigated the effect of CGS-21680 (2-p(2-Carboxyethyl)phenethylamino-5'-N-ethylcarboxamidoadenosine hydrochloride), an adenosine $\mathrm{A}_{2 \mathrm{~A}}$ receptor agonist, in a model of DSS-induced colitis in NMRI mice. Mice were fed $5(\mathrm{w} / \mathrm{v}) \%$ DSS, and were treated intraperitoneally with $0.5 \mathrm{mg} / \mathrm{kg}$ CGS-21680 or appropriate control (10 (v/v) \% DMSO) for 10 days. Parameters recorded in these experiments were body weight, colon length, and bleeding from rectum. Furthermore, levels of two chemokines, macrophage inflammatory protein (MIP)-1 $\alpha$ and MIP-2, as well as four proinflammatory cytokines, interferon gamma (IFN $\gamma$ ), interleukin (IL)-1 $\beta$, IL-12 and tumor necrosis factor-alpha (TNF- $\alpha$ ) were determined from homogenates of colon biopsies. DSS-induced colitis significantly decreased body weight (control: $110.9 \pm 10.3 \%$ vs. DSS: $81.3 \pm 10.2 \%, \mathrm{P}<0.001$ ) and colon length (control: $5.96 \pm 0.56 \mathrm{~cm} v s$. DSS: $3.62 \pm 0.51 \mathrm{~cm}, \mathrm{P}$ $<0.001$ ), and it increased the incidence of rectal bleeding compared to DSS-untreated animals. Treatment of DSSinduced animals with CGS-21680 failed to affect these parameters (bodyweight, DSS: $81.3 \pm 10.2 \%$ vs. CGS+DSS: $76.2 \pm 8.3 \%$; colon length, DSS: $3.62 \pm 0.51 \mathrm{~cm} v s$. CGS+DSS: $3.69 \pm 0.47 \mathrm{~cm}$ ). Among investigated chemokines and cytokines, levels of MIP-1 $\alpha$, MIP-2 and IL-1 $\beta$ were elevated during DSS-induced colitis by 25-, 65- and 15-fold compared with controls, respectively, while the concentrations of IFN $\gamma$, IL-12 and TNF- $\alpha$ did not change following CGS-21680 administration. CGS-21680 had no effect on the production of MIP-1 $\alpha$, MIP-2 and IL-1 $\beta$. According to our results, CGS-21680 is ineffective in ameliorating DSS-induced colitis in mice.

Research was supported by a grant from Hungarian National R\&D Programme 1A/036/2004. 


\title{
THE ADENOSINE A2a RECEPTOR ENHANCES T CELL IMMMUNO-SUPPRESSION FOLLOWING TRAUMA
}

Charles C. Caldwell, Andre Martignoni, Maria Reid, Holly Goetzmann and Lisa Choi

Department of Surgery, University of Cincinnati College of Medicine, Cincinnati, OH 45267; and Department of Research, Shriner's Hospital for Children, Cincinnati, Ohio 45229.

charles.caldwell@uc.edu

In ancient Greece, the treatment of trauma consisted of bandaging, immobilization of fractures, amputations, and removal of foreign objects. Between this time and the present, exponential advances in treatment and understanding the pathophysiology of trauma have resulted in historical increases in trauma patient outcome. However, the trauma patient subsequently committed into the ICU faces a significant possibility of multi-organ dysfunction (MODS). Further, following trauma, the patient is susceptible to infections that would normally be cleared. Hypoxic conditions are known to exist following trauma and during sepsis. Hypoxia leads to an increase of extracellular adenosine. In leukocytes, activation of the adenosine A2a receptor (A2aR) increases intracellular cAMP, which, in turn, inhibits inflammatory cytokine secretion and enhances anti-inflammatory cytokine release. In our studies, we have induced trauma injury by inflicting mice with an $18 \%$ total body surface area dorsal scald burn. Here, we show that this trauma results in a systemic inflammatory response that peaks at 12-24 hours as measured by a 200 -fold increase in serum IL-6. Following injury, splenic T cells show marked immunosuppression as determined by ex vivo stimulation of T cells and measurement of the T cell-secreted cytokines IFN-gamma and IL-4. As compared to T cells from sham animals, IFN-gamma secretion taken from traumatized animals decreased by as much as $75 \%$. In contrast, T cells taken from A2aR-deficient animals showed some decreased interferon-gamma production, but still 2-fold higher than T cells from sham animals. A common observation in the ICU is that there is lymphocyte depletion following trauma injury. This is considered significant in that it has been recently demonstrated that the adoptive transfer of apoptotic lymphocytes worsens survival during sepsis due to increased immunosuppression. Here, we show similar reduction of IFN-gamma production in T cells from mice one day after only being injected with apoptotic lymphocytes. Thus, we conclude that adenosine, acting through the A2aR, plays a role in increasing immunosuppression following trauma, possibly by exacerbating a bystander effect driven by apoptotic lymphocytes through an undetermined mechanism. We expect that pharmacological treatment with $\mathrm{A} 2 \mathrm{aR}$ antagonists will result in less immunosuppression coupled with less susceptibility to subsequent infections. Support: SHC Project 8560

\section{The adenosine A2a receptor is pro-apoptotic in lymphoyctes following trauma}

\author{
Andre Martignoni, Maria Reid, Holly Goetzmann, Lisa Choi and Charles C. Caldwell
} Department of Surgery, University of Cincinnati College of Medicine, Cincinnati, OH 45267; and Department of Research, Shriner's Hospital for Children, Cincinnati, Ohio 45229. E-mail: Andre.Martignoni@med.uni-muenchen.de

Trauma leads to a number of systemic physiological changes associated with alterations in the immune system. In injuries, the systemic inflammatory response syndrome (SIRS) is first observed, followed by the compensatory anti-inflammation response syndrome (CARS). These systemic effects include tissue damage, inflammation, lymphocyte apoptosis, and subsequently, immunosuppression. Of the leukocytes that are involved in SIRS and CARS, the macrophage and the lymphocyte play important roles. It is known that the adenosine A2a receptor $(\mathrm{A} 2 \mathrm{aR})$ is expressed on these cells. The A2aR mediates leukocyte functions by increasing the intracellular concentration of cAMP. It has been reported that corticosteroids and increased intracellular cAMP cooperate synergistically to cause the loss of lymphocyte viability ${ }^{1}$. Further, it has been shown that the pro-apoptotic molecule Bim was upregulated using a cAMP agonist in dexamethasone-treated lymphomas ${ }^{2}$. This is considered significant in that it has been recently demonstrated that the adoptive transfer of apoptotic lymphocytes worsens survival following trauma due to increased immunosuppression ${ }^{3}$. In our studies, we have induced trauma injury by inflicting mice with an $18 \%$ total body surface area dorsal scald burn. Here, we show that this trauma results in a $90 \%$ depletion of the total number of splenic naive $\mathrm{T}$ and follicular $\mathrm{B}$ lymphocytes within one day of the trauma. 
Analysis of TUNEL staining of the spleen 12 hours following the injury show a significant increase in DNA strands with exposed 3'-hydroxyl ends, a hallmark of late stage apoptosis. However, in this model, there was a significant reduction of lymphocyte depletion in mice either genetically or pharmacologically devoid of the A2aR. This is significant in that we and others have shown that lymphocytes are protective against a subsequent infection following injury. Thus, adenosine, acting through the A2aR, plays a role in increased lymphocyte depletion following trauma. Altogether, we expect that antagonism of the A2aR, either genetically or pharmacologically, will decrease the anti-inflammatory phenotype following thermal injury, at least partially by decreasing lymphocyte apoptosis. We expect that this antagonism will then enhance survival during the subsequent infection. Support: SHC Project 8560.

1. Gruol, D. J.; Altschmied, J., Synergistic induction of apoptosis with glucocorticoids and $3^{\prime}, 5^{\prime}$-cyclic adenosine monophosphate reveals agonist activity by RU 486. Mol Endocrinol 1993, 7, (1), 104-13.

2. Zhang, L.; Insel, P. A., The pro-apoptotic protein Bim is a convergence point for cAMP/protein kinase A- and glucocorticoid-promoted apoptosis of lymphoid cells. J Biol Chem 2004, 279, (20), 20858-65.

3. Hotchkiss, R. S.; Chang, K. C.; Grayson, M. H.; Tinsley, K. W.; Dunne, B. S.; Davis, C. G.; Osborne, D. F.; Karl, I. E., Adoptive transfer of apoptotic splenocytes worsens survival, whereas adoptive transfer of necrotic splenocytes improves survival in sepsis. Proc Natl Acad Sci U S A 2003, 100, (11), 6724-9.

\section{The carboxyl terminus of the $\mathbf{A}_{2 \mathrm{~A}}$-adenosine receptor is a docking site for several interaction partners}

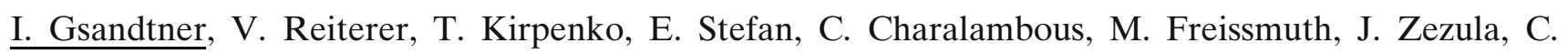
Nanoff, O. Kudlacek

Institute of Pharmacology, Center for Biomolecular Medicine and Pharmacology, Währinger Straße 13a; A-1090 Vienna, Austria

E-mail: ingrid.gsandtner@meduniwien.ac.at

The $A_{2 A}$ adenosine receptor is a prototypical $G_{s}$-coupled receptor and has an extended carboxyl terminus. Truncation of the C-terminus by 100 amino acids has only very modest effects on the signalling pathways controlled by the receptor (i.e. the $\mathrm{G}_{\mathrm{s}}$-induced cAMP accumulation and stimulation of mitogen-activated protein (MAP) kinase, which is independent of heterotrimeric $G$ proteins). In order to understand the biological function of the long C-terminus, we screened a human library for proteins that bind to the last 120 amino acids of the $\mathrm{A}_{2 \mathrm{~A}}$ receptor. Using the appropriate LexA-fusion protein, we identified several potential binding partners, including ARNO/cytohesin-2, (a nucleotide exchange factor for the small G protein Arf6), the deubiquitinating enzyme USP4 and SAP102, a protein that belongs to the MAGUK (membrane associated guanylat kinase-like domain) proteins. The interaction site was mapped by employing baits comprising C-termini of varying length: USP4 required the distal portion of the carboxyl terminus, because it failed to bind to truncated LexA fusion proteins which lacked the last 50 or 100 amino acids. In contrast, ARNO/cytohesin-2 bound to the proximal segment, i.e. the first 20 amino acids adjacent to the 7th transmembrane domain of the receptor. All interactions were confirmed by pull-down assays and by co-immunoprecipitation. The following functional effects were assigned to the interactors: USP4 greatly enhanced cell surface expression of the receptor by relaxing overprotective quality control in the ER; this was associated with an increased accumulation of cAMP after agonist stimulation. ARNO/ cytohesin-2 or its catalytic inactive mutant did not alter the level of $\mathrm{A}_{2 \mathrm{~A}}$ receptor expression, and did not affect $\mathrm{G}_{\mathrm{s}}$-dependent stimulation of adenylyl cyclase. This was also true for agonist induced receptor desensitisation. However, the presence of ARNO/cytohesin-2 was required for alternative (i.e. $\mathrm{G}_{\mathrm{s}}$-independent) signalling to mitogen activated kinase (MAP kinase). This conclusion was based on the following observations: (i) dominant negative ARNO and (ii) dominant negative Arf6 efficiently suppressed sustained phosphorylation of MAP kinase, while (iii) brefeldin A (an inhibitor of other sec7-containg ARF-GEFs) did not affect the time course of MAP kinase stimulation. SAP102 interacted with the $\mathrm{A}_{2 \mathrm{~A}}$-receptor via its (catalytically inactive) GUK domain and colocalized with $\mathrm{A}_{2 \mathrm{~A}}$-adenosine receptor in dendritic spines of hippocampal neurons.

These results demonstrate that the extended carboxyl terminus of the $\mathrm{A}_{2 \mathrm{~A}}$-adenosine receptor subserves a role that is irrelevant to $\mathrm{G}$ protein coupling. It is however a docking site of several proteins that are relevant to sorting, targeting and prolonged signalling. 


\title{
The Distribution and Functional Characterization of ENT4, a bifunctional nucleoside and organic cation transporter
}

Kay Barnes $^{1}$, Halina Dobrzynski ${ }^{2}$, Sophie Foppolo ${ }^{1}$, Paul R. Beal ${ }^{3}$, James Tellez $^{2}$, William C. Claycomb ${ }^{7}$, Carol E. Cass ${ }^{4,5,6}$, James D. Young ${ }^{4}$, Rudi Billeter-Clark ${ }^{1}$, Mark R. Boyett ${ }^{2}$ and Stephen A. Baldwin ${ }^{1}$. From the ${ }^{1}$ Institute of Membrane and Systems Biology, University of Leeds, Leeds, LS2 9JT, United Kingdom, the ${ }^{2}$ Division of Cardiovascular and Endocrine Sciences, University of Manchester, Manchester M13 9XX, United Kingdom, the ${ }^{3}$ Department of Biology, University of York, United Kingdom, the ${ }^{4}$ Membrane Protein Research Group, Departments of Physiology and ${ }^{5}$ Onconlogy, University of Alberta and the ${ }^{6}$ Cross Cancer Institute, Edmonton, Alberta T6G 2H7, Canada, ${ }^{7}$ Department of Biochemistry and Molecular Biology, Louisiana State, University Health Sciences Center, New Orleans, Louisiana 70112, U.S.A.

k.barnes@leeds.ac.uk

The nucleoside, adenosine, plays multiple roles in the efficient functioning of the heart by regulating coronary blood flow, cardiac pacemaking and the contractility of the heart. It is produced by the cardiac myocardium under stress and acts as a major local autocrine and paracrine regulator of tissue function in situations of reduced oxygen supply relative to demand. In the mammalian heart the release and uptake of nucleosides such as adenosine is mediated by members of the equilibrative nucleoside transporter (ENT) or SLC8 family. The results of probing a multiple tissue expression RNA array with an oligonucleotide corresponding to a portion of the hENT4cDNA demonstrated that ENT4, a recently identified member of the equilibrative nucleoside transporter family is widely distributed in human tissues, but is particularly abundant in the heart, brain and skeletal muscle. A quantitative survey of the distribution of rENT4 in distinct regions of the rat heart was conducted using densitometry of Western blots of whole tissue lysates probed with affinity purified anti-peptide antibodies raised against residues 301-319 of hENT4: the transporter was more abundant in the ventricular than the atrial tissue and virtually absent from the SA and AV nodes. Using the specific antibodies to hENT4 ${ }_{301-319}$ and immunofluorescence microscopy of endothelial and cardiac cells, it was revealed that in contrast to ENT3, but like ENT1 and ENT2, the human-, mouse- and rat- ENT4 proteins, hENT4, mENT4 and rENT4 respectively, all have a predominantly plasma membrane location. Characterization of the transporter activity in Xenopus oocytes and in mammalian cells revealed the protein to be a low affinity adenosine transporter. It was partially sensitive to the nucleoside transporter inhibitors dipyridamole and dilazep, insensitive to nitrobenzylthioinosine (nitrobenzylmercaptopurine riboside; NBMPR), and strongly dependent upon $\mathrm{pH}$, the optimum $\mathrm{pH}$ value of mENT4 and hENT4 activity being 5.5 and 6.0, respectively. The presence in the heart of a purine nucleoside transporter able to function optimally at acidic $\mathrm{pH}$ might aid restoration of normoxia following, for example, an ischaemic attack when the blood $\mathrm{pH}$ may fall as low as 6.6 or below. In contrast, ENT4-mediated serotonin influx was not proton-dependent, similar activity being recorded at both acidic and physiological $\mathrm{pH}$. We propose that ENT4 is a cell surface protein that in addition to its published role as an organic cation transporter is a genuine nucleoside transporter.

\section{THE EFFECT OF ANOXIA AND ADENINE NUCLEOTIDE POOL ON NUCLEOTIDE RELEASE FROM ERYTHROCYTES}

\author{
Ewa M Slominska ${ }^{1)}$, Magdi H Yacoub ${ }^{2)}$, Ryszard T Smolenski ${ }^{1,2)}$ \\ ${ }^{1)}$ Department of Biochemistry, Medical University of Gdansk, Poland. \\ 2) Heart Science Centre, Imperial College at Harefield Hospital, U.K. \\ eslom@amg.gda.pl.
}

ATP release from the erythrocytes has recently been found to be linked to haemoglobin oxygenation status and suggested as the mechanism that controls vascular tone and other $\mathrm{P} 2$ receptor dependent processes such as platelet aggregation or cell migration and proliferation. However, little is known about the metabolic factors that affect erythrocyte ATP release. One of the crucial elements could be adenine nucleotide pool in the erythrocytes. This is particularly relevant to pathological conditions such as chronic renal failure or erythrocyte AMP deaminase deficiency where elevation of erythrocyte ATP concentration has been observed or Tarui's disease and 5'nucleotidase superactivity where depletion of erythrocyte ATP was noted. We aimed therefore to evaluate wh- 
ether increase in erythrocyte adenine nucleotide pool will affect extracellular ATP concentration under normal and anoxic conditions.

Fresh washed human erythrocytes were first preincubated for $120 \mathrm{~min}$ in Hepes buffered, $2 \%$ albumin supplemented Krebs solution containing $5 \mathrm{mM}$ adenosine and $5 \mathrm{mM}$ ortophosphate to increase ATP pool (A) while 5 $\mathrm{mM}$ ortophosphate was used in controls $(\mathrm{C})$. At the end of preincubation erythrocytes were washed and both A and $\mathrm{C}$ cells were incubated for a further $90 \mathrm{~min}$ under air oxygen tension (OA, OC) or in $\mathrm{CO} 2$ supplemented anoxic environment induced with BD Gaspak system (DA, DC). At the end of incubation, erythrocytes and incubation medium were rapidly separated and analysed for ATP and its metabolites content by HPLC.

Preincubation with adenosine and orthophosphate in A resulted in elevation of the ATP concentration to $2370 \pm$ $40 \mu \mathrm{mol} / 1$ erythrocytes as compared to $1170 \pm 18 \mu \mathrm{mol} / 1$ erythrocytes in controls $(\mathrm{C})(\mathrm{n}=5, \pm \mathrm{SEM}, \mathrm{p}<0.001$. No differences in GTP or NAD concentrations were observed. This was accompanied by increased in extracellular ATP concentration from $0.50 \pm 0.05$ to $0.68 \pm 0.08 \mu \mathrm{mol} /$ erythrocytes $1(\mathrm{p}<0.05)$ under normoxic conditions and from $3.42 \pm 0.59$ to $7.10 \pm 1.90 \mu \mathrm{mol} / \mathrm{l}$ erythrocytes $(\mathrm{P}<0.05)$ during anoxia after $90 \mathrm{~min}$ incubation. No GTP, NAD, haemoglobin or lactate dehydrogenase release has been observed.

We confirmed that hypoxia induces ATP rerlease from the erythrocytes. Furthermore, we have demonstrated here that erythrocyte ATP pool is additional factor that controls the extracellular ATP concentration. Changes in the disease conditions that affect erythrocyte nucleotide pool such as renal failure could affect signaling mediated by erythrocyte ATP release. On the other hand the effect we demonstrated here provides opportunity to control ATP mediated signaling by regulation of erythrocyte nucleotide pool.

\title{
The Effects of Metabolic Stimulators and Inhibitors on Histamine-Induced ATP Release in HaCaT Cells.
}

\author{
H.E.Burrell, J.A. Gallagher \& A.W.M. Simpson. \\ Department of Human Anatomy \& Cell Biology, School of Biomedical Sciences, The Sherrington Buildings, \\ Ashton Street, Liverpool, L69 3GE, U.K. \\ Email: H.E.Burrell@liv.ac.uk
}

Extracellular nucleotides, acting via P2 receptors, are regulators of important cellular functions such as proliferation, differentiation and apoptosis. In the epidermis, activation of the $\mathrm{P}_{2} \mathrm{Y}_{2}$ receptor results in an increase in proliferation in the basal layer, while the $\mathrm{P}_{2} \mathrm{X}_{5}$ receptor is involved in differentiation in the granular layer, and the $\mathrm{P}_{2} \mathrm{X}_{7}$ receptor is confined to the apoptotic cornified layer. Extracellular nucleotides are also released from cells via a variety of postulated mechanisms. These principally fall into two groups: anion channels and exocytosis. While the production of ATP by mitochondria and its subsequent release into the cytoplasm has previously been studied [1], the effect of changes in cytosolic ATP on ATP release have not been investigated. In this study, we have investigated the effects of different metabolic stimulators and inhibitors on ATP release from the HaCaT keratinocyte cell line. HaCaT cells were seeded into 12 well plates at a density of $1 \times 10^{5}$ cells per well and allowed to adhere and grow overnight until confluence. Cells were then washed with warm PBS and serumstarved in $0.5 \mathrm{ml}$ HEPES-buffered saline for 1 hour prior to treatment. The medium bathing the cells was then sampled $(200 \mu \mathrm{l})$ in duplicate and the ATP content analysed using luciferin-luciferase luminometry in a Berthold tube luminometer [2]. We have previously reported that $\mathrm{HaCaT}$ cells release ATP under static, unstimulated conditions [2]. We now show that release can be concentration-dependently stimulated by addition of histamine (100-10,000 nM). The time-course of ATP release (0-5 minutes) also differs depending on the concentration of histamine. While low concentrations of histamine $(100 \mathrm{nM})$ induce a spike of ATP release lasting for a period of seconds, high histamine concentrations $(10,000 \mathrm{nM})$ induce a longer release of ATP, which lasts for a period of minutes. We postulate that these differences reflect previously published changes in $\left[\mathrm{Ca}^{2+}\right]_{i}$ signalling [1]. Histamine-induced ATP release is concentration-dependently stimulated by addition of glucose (5-15 mM, when added for 1 hour prior to addition of histamine) or $1 \mathrm{mM}$ sodium succinate (added for 2 minutes prior to histamine) in comparison with glucose or sodium succinate alone. Conversely, histamine-induced ATP release is inhibited by addition of $1 \mathrm{mM}$ extracellular calcium in the presence or absence of glucose (added for 1 hour prior to addition of histamine), indicating that ATP release from HaCaT cells is unlikely to occur via exocytosis and may occur through hemichannels [3]. Addition of oligomycin $(6 \mu \mathrm{M})$ for 5 minutes prior to addition of histamine 
$(10,000 \mathrm{nM})$ also inhibits the histamine-induced release of ATP. These studies show for the first time that stimulation or inhibition of mitochondrial ATP production directly affects ATP release by the cells.

1) Jouaville, L.S., Pinton, P., Bastianutto, C., Rutter, G.A. \& Rizzuto, R. (1999). Proc. Natl. Acad. Sci. USA 96: 13807-13812.

2) Burrell, H.E., Wlodarski, B., Foster, B.J., Buckley, K.A., G. R. Sharpe, Quayle, J.M., Simpson, A.W.M. \& Gallagher, J.A. (2005). J. Biol. Chem. 280: 29667-29676.

3) Bahima, L., Aleu, J., Elias, M., Martin-Satue, M., Muhaisen, A., Blasi, J., Marsal, J. \& Solsona, C. (2006). J. Cell Physiol. 206: $95-102$.

\title{
The Expression and Immunolocalisation of Soluble NTPDases in the Cochlea
}

\author{
$\underline{\text { MG O}}^{\prime} \underline{\text { Keeffe }}^{1,2}$, SM Vlajkovic ${ }^{1}$, GD Housley ${ }^{1}$, SC Robson $^{3}$, and PR Thorne ${ }^{2}$ \\ Department of Physiology ${ }^{1}$ and Discipline of Audiology ${ }^{2}$, Faculty of Medical and Health Sciences, University of \\ Auckland, New Zealand; Beth Israel Deaconess Medical Center ${ }^{3}$, Harvard Medical School, Boston, USA \\ Presenting author email: m.okeeffe@auckland.ac.nz
}

Ecto-nucleotidase triphosphate diphosphohydrolase (E-NTPDase) is a family of enzymes that catalyse the dephosphorylation of extracellular nucleotides and thus regulate the signalling function of P2 receptors (1). In the cochlea, P2X and P2Y receptors are associated with regulation of sound transduction, electrochemical homeostasis, blood flow and auditory neurotransmission (2). There is also evidence that changes in purinergic and pyrimidinergic signalling may be associated with cochlear pathology and hearing loss (3). The distribution of membrane-bound NTPDase1 and NTPDase2 in cochlear tissues has been established and is consistent with regulation of extracellular nucleotide concentrations and a putative otoprotective role (4). In contrast to other E-NTPDase family members that contain two transmembrane regions, NTPDase5 and NTPDase6 lack the C-terminus and can be cleaved to produce a soluble protein. Both enzymes are located intracellularly, but have intra- and extracellular functionality as they can be released from cells. NTPDase 5 and 6 have high preference for nucleoside 5'-diphosphates, such as UDP and GDP $(5,6)$.

Methods: This study investigates the expression of NTPDase5 and NTPDase6 mRNAs (RT-PCR) in the rat cochlea, and their distribution in cochlear tissues (Western blotting, immunohistochemistry). Enzyme localisation was determined using polyclonal antibodies raised in rabbits by the injection of the encoding cDNA or the enzyme-specific peptide.

Results and conclusion: Immunoperoxidase histochemistry and confocal immunofluorescence demonstrated NTPDase5-specific immunolabelling in the perikarya of the primary auditory neurones in the spiral ganglion and their central neural processes. NTPDase5 may be required for termination of extracellular UDP signalling via $\mathrm{P}_{2} \mathrm{Y}_{6}$, and possibly $\mathrm{P}_{2} \mathrm{Y}_{14}$ receptor subunits associated with auditory neurotransmission. In the organ of Corti, NTPDase5 is localised to the supporting Deiters' cells, inner border cells and inner phalangeal cells surrounding the sensory hair cells. These cells are known to express UTP/UDP-preferring P2Y receptor subunits, suggesting the involvement in regulation of UDP signalling associated with sensory transduction and cochlear amplification. By contrast, NTPDase6 expression is confined to the cytoplasm of the inner hair cells. Intracellular distribution of NTPDase6 resembles the localisation of the glycogen stores in the inner hair cells, suggesting a role for this soluble enzyme in the glycosylation of proteins and lipids that may be required for sensory transduction. Functional studies characterising the respective roles of NTPDase5 and NTPDase6 in cochlear function are underway. Supported by the Auckland Medical Research Foundation and Health Research Council of New Zealand.

1. Zimmermann, H. (2001) Drug Development Research, 52, 44-56.

2. Housley GD et al. (2002) Audiology \& Neuro-Otology, 7, 55-61.

3. Thorne PR et al (2002) Audiology \& Neuro-Otology, 7, 180-184.

4. Vlajkovic SM et al. (2004) Neuroscience, 126, 763-773.

5. Mulero, JJ et al. (1999) Journal of Biological Chemistry, 274 (29), 20064-20067.

6. Braun, N. et al. (2000) Biochemical Journal, 351, 639-647. 


\title{
The guanine-based purinergic system as a new target for neuroprotection against glutamatergic excitotoxicity.
}

\author{
Diogo O. Souza * \\ Department of Biochemistry ICBS, Institute of Basic Sciences of Health, "Rio Grande do Sul" Federal University. \\ Porto Alegre, RS. Brazil. \\ E-mail:diogo@ufrgs.br
}

Glutamate is the main excitatory neurotransmitter in mammalian CNS, essential for brain activities, as those involved in development, aging, memory, and adaptation to the environment. However, hyper-activation of the glutamatergic system may be potentially neurotoxic, involved in the pathogenesis of various acute and chronic brain injuries.

Our group has given strong evidence that the guanine-based purinergic system is effectively neuroprotective against glutamate toxicity, in acute and chronic animal models, both in vitro and in vivo studies. Although the administration of guanine derivatives (GD) exerts neuroprotection, our results strongly indicate that the active compound is the nucleoside guanosine (GUO).

In in vivo studies carried out in rat and mouse, GD protect against brain damage caused by hyper-activation of the glutamatergic system. Indeed: i) chronically, GMP administration in rat striatum protects cells against death caused by quinolinic acid (QA); ii) acutely, GMP or GUO i.c.v., i.p. or orally administered protect against seizures induced by QA (or $\alpha$-dendrotoxin). In in vitro studies carried out in brain slices, GUO protects cell against death caused by in vitro ischemia.

Searching for mechanisms implicated in this neuroprotection, we demonstrated that: i) GUO stimulates the astrocytic glutamate uptake (in astrocyte cultures and brain slices), the main process involved in endogenous neuronal protection; ii) QA induced-seizures decrease glutamate uptake by cortical brain slices and this decrease is reversed by GUO when it acts as anticonvulsant; iii) Brain ischemia decreases glutamate uptake by hippocampal slices and i.p. GUO administration prevents this decrease. Thus we propose that the stimulatory effect on glutamate uptake is involved in the neuroprotective actions of GUO.

These results encourage further studies aiming at the therapeutic use in humans of GUO in acute (hypoxia, ischemia, brain traumatism) and chronic (neurodegenerative diseases) brain injuries involving glutamate excitotoxicity.

\section{The immune-response modifier imiquimod is an adenosine receptor antagonist}

Karl-Norbert Klotz, ${ }^{1}$ Michael P. Schön, ${ }^{2}$ Margarete Schön ${ }^{2}$

University of Würzburg, Department of Pharmacology and Toxicology, ${ }_{1}^{1}$ and Rudolf-Virchow-Center, DFGResearch Center for Experimental Biomedicine, ${ }^{2}$ Versbacher Str. 9, D-97078 Würzburg, Germany

klotz@toxi.uni-wuerzburg.de

Imiquimod is an imidazoquinoline derivative which is in use for topical treatment of skin diseases. It acts as an immune-response modifier and has shown antiviral and antitumoral activity both in vitro and in clinical applications. A number of studies have shown that imiquimod and related compounds mediate their effects on immune responses through activation of toll-like receptors TLR7 and/or TLR8. Activation of the NF- $\mathrm{kB}$ pathway ultimately leads to an increased production of cytokines like TNF $\alpha$, IL-2, IL-6, IL-12, GM-CSF, TNF $\alpha$, and chemokines, e.g. IL-8, MIP-1 $\alpha$ and MIP-1 $\beta$.<smiles>CC(C)Cn1cnc2c(N)nc3ccccc3c21</smiles> 
The structure of imiquimod resembles the large family of adenosine receptor antagonists that is derived form the nonselective triazoloquinazoline compound CGS 15943. Therefore, we determined binding affinity of imiquimod at adenosine receptors in CHO cells stably transfected with the human subtypes. In addition, their effect on adenylyl cyclase activity was tested in order to establish their potential functional role in adenosine receptormediated signal transduction.

In competition binding experiments we found that imiquimod binds to $A_{1}$ receptors with a $K_{i}$ of $2.9 \mu \mathrm{M}$, to $A_{2 A}$ receptors with a $\mathrm{K}_{\mathrm{i}}$ of $2.2 \mu \mathrm{M}$, and to the $\mathrm{A}_{3}$ subtype with a $\mathrm{K}_{\mathrm{i}}$-value of $14.6 \mu \mathrm{M}$. A potential interaction with the $\mathrm{A}_{2 \mathrm{~B}}$ adenosine receptors was investigated in adenylyl cyclase studies. In concentrations up to $100 \mu \mathrm{M}$ no effect of imiquimod on cyclase activity was detected. However, $100 \mu \mathrm{M}$ imiquimod caused an about $30-50 \%$ inhibition of NECA-stimulated cyclase activity suggesting that it acts as a weak $\mathrm{A}_{2 \mathrm{~B}}$ antagonist. Likewise, we could not detect activation of adenylyl cyclase in $\mathrm{CHO}$ cells transfected with $\mathrm{A}_{2 \mathrm{~A}}$ adenosine receptors, again suggesting that imiquimod is a receptor antagonist. In $\mathrm{CHO}$ cells stably transfected with $\mathrm{A}_{1}$ or $\mathrm{A}_{3}$ adenosine receptors a small but reproducible inhibition of forskolin-stimulated cyclase activity was observed. Although this observation may suggest that imiquimod exhibits some partial agonistic activity at these subtypes, experimental evidence argues against such a notion: Imiquimod inhibits forskolin-stimulated cyclase activity in untransfected $\mathrm{CHO}$ cells or in $\mathrm{A}_{2 \mathrm{~A}}-\mathrm{CHO}$ cells to the same degree as in $\mathrm{A}_{1}-\mathrm{CHO}$ or $\mathrm{A}_{3}-\mathrm{CHO}$ cells. These results suggest that imiquimod is a nonselective adenosine receptor antagonist which, in addition to the adenosine receptor-mediated effects, shows an inhibitory effect on adenylyl cyclase activity downstream from the $G$ protein-coupled adenosine receptors. We have characterized the immune-response modifier imiquimod as a nonselective adenosine receptor antagonist with affinity to the receptor subtypes comparable to the prototypical xanthine antagonist theophylline. The blockade of $\mathrm{A}_{2 \mathrm{~A}}$ adenosine receptors may contribute to the pro-inflammatory response which is induced by imiquimod. The additonal receptor-independent effect on cAMP levels may add a further complementary mechanism resulting in the intense inflammatory response that is observed in clinical treatment with imiquimod.

\section{The mobility of the $A_{2 A}$ receptor is not restricted by the actin cytoskeleton C. Charalambous, L. Milan-Lobo, I. Gsandtner, O. Kudlacek, H. Farhan, J. Zezula, H.H. Sitte, M. Freissmuth}

Institute of Pharmacology, Center of Biomlecular Medicine \& Pharmacology. Medical University of Vienna Währinger Str. 13a; A-1090 Vienna, Austria

Christoforos.charalambous@meduniwien.ac.at

The $A_{2 A}$-adenosine receptor is a prototypical $G_{s}$-protein coupled receptor. The $A_{2 A}$-adenosine receptor has long been known to activate adenylyl cyclase in a manner inconsistent with collision coupling (1). In contrast, many other Gs-coupled receptors such as the $\beta_{2}$-adrenergic receptor activate cAMP formation with kinetics consistent with a random walk and collision coupling (2). We have investigated the basis of this phenomenon by determining the diffusion rate of the $\mathrm{A}_{2 \mathrm{~A}}$-receptor tagged with the yellow fluorescent protein (YFP) and heterologously expressed in HEK293 cells. The YFP-moiety was bleached and fluorescence recovery after photobleaching (FRAP) was measured by confocal laser microscopy. As a control, we employed the YFP-tagged CRF-receptor-2, which is similar in size as the $\mathrm{A}_{2 \mathrm{~A}}$-receptor. We also verified that both, the $\mathrm{A}_{2 \mathrm{~A}}$-receptor and the CRF-receptor-2 form homodimers (assessed by fluorescence resonance energy transfer, =FRET-microscopy, using YFP and CFPtagged versions of the receptors). Under basal conditions, i.e. in the absence of agonist, fluorescence recovered with comparable half-lives $(\mathrm{t} / 2=\sim 10 \mathrm{~s})$ for the two receptors. Upon agonist activation, the mobility of the CRFreceptor increased; this effect was however absent in the $\mathrm{A}_{2 \mathrm{~A}}$-receptor. It has been proposed that the $\mathrm{C}$-terminus of the $\mathrm{A}_{2 \mathrm{~A}}$-receptor is linked to the actin cytoskeleton via binding of $\alpha$-actinin (3). In addition, the C-terminus of the $\mathrm{A}_{2 \mathrm{~A}}$-receptor binds to $\mathrm{ARNO} /$ cytohesin-2, which is an exchange factor for Arf6 (which regulates the actin cytoskeleton, ref 4). We have therefore tested whether manipulations of the actin cytoskeleton affected the mobility of the receptor: (i) treatment of cells with latrunculin A to disrupt the cortical actin, (ii) expression of the receptor in the absence and presence of wild type and dominant negative ARNO to manipulate the ability of the receptor to signal to cortical actin, (iii) truncation of the C-terminus of the receptor to affect its ability to bind to $\alpha$-actinin. None of these manipulations had a detectable effect on the FRAP lifetime of the wild type receptor and 
its mutated versions. We therefore conclude that the restricted collision coupling mode is a property specified by the core of the receptor and unrelated to the actin cytoskeleton.

\title{
References:
}

1. Braun S, Levitzki A. (1979) Adenosine receptor permanently coupled to turkey erythrocyte adenylate cyclase. Biochemistry 18:2134-2138

2. Rimon G, Hanski E, Braun S, Levitzki A. (1978) Mode of coupling between hormone receptors and adenylate cyclase elucidated by modulation of membrane fluidity. Nature 276:394-396

3. Burgueno J, Blake DJ, Benson MA, Tinsley CL, Esapa CT, Canela EI, Penela P, Mallol J, Mayor F Jr, Lluis C, Franco R, Ciruela $\mathrm{F}(2003)$ The adenosine $\mathrm{A}_{2 \mathrm{~A}}$ receptor interacts with the actin-binding protein $\alpha$-actinin. $J$ Biol Chem 278:37545-37552 4. Gsandtner I, Charalambous C, Stefan E, Ogris E, Freissmuth M, Zezula J (2005) Heterotrimeric G protein-independent signaling of a G protein-coupled receptor. Direct binding of ARNO/cytohesin-2 to the carboxyl terminus of the A2A adenosine receptor is necessary for sustained activation of the ERK/MAP kinase pathway. J Biol Chem. 280:31898-31905

\section{The N-terminal fatty amino acid 6-methylheptanoic/octanoic-DAB is crucial for polymyxin B-mediated modulation of $\mathrm{P}^{2} \mathrm{X}_{7}$ receptor functions in immune and non immune cells}

Davide Ferrari, ${ }^{1 *}$ Cinzia Pizzirani, ${ }^{1 *}$ Sara Gulinelli, ${ }^{1}$ Giulia Callegari, ${ }^{1}$ Paola Chiozzi, ${ }^{1}$ Marco Idzko, ${ }^{2}$ Elisabeth Panther, ${ }^{2}$ and Francesco Di Virgilio ${ }^{1}$

${ }^{1}$ Department of Experimental and Diagnostic Medicine, Section of General Pathology, and Interdisciplinary Center for the Study of Inflammation (ICSI), University of Ferrara, I-44100 Ferrara, Italy. ${ }^{2}$ Department of Pneumology and Gastroenterology, University of Freiburg, Freiburg, Germany. "D.F. and C.P. contributed equally to this work. E-mail:dfr@unife.it.

We previously showed ${ }^{1}$ that the antibiotic polymyxin B (PMB), which binds to and neutralizes the toxic residue of bacterial lypopolysaccharide (LPS), greatly amplifies cellular responses mediated by the $\mathrm{P}_{2} \mathrm{X}_{7}$ receptor $\left(\mathrm{P} 2 \mathrm{X}_{7} \mathrm{R}\right)$. However, the molecular mechanism involved was not elucidated. In the present study we show that PMB effects depend on the presence of its N-terminal fatty amino acid 6-methylheptanoic/octanoic-diaminobenzoic residue as deletion of this residue abolished PMB-dependent modulation of ATP triggered responses in HEK293 stably expressing the $\mathrm{P}_{2} \mathrm{X}_{7}$ receptor $\left(\mathrm{HEK} 293-\mathrm{hP} 2 \mathrm{X}_{7}\right)$. In contrast to $\mathrm{PMB}$, the polymyxin $\mathrm{B}$ nonapeptide $(\mathrm{PMBN})$, which is the deacylated amino derivative of PMB lacking the N-terminal fatty amino acid 6-methylheptanoic/ octanoic-diaminobenzoic residue, was unable to potentiate a) the ATP-induced $\mathrm{Ca}^{2+}$ increase, b) pore formation and consequently ATP-mediated plasma membrane permeabilization, and c) ATP-dependent cytotoxicity also in natively expressing $\mathrm{P} 2 \mathrm{X}_{7}$ cells such as human macrophages. In addition, PMBN was unable to revert the effect of the $\mathrm{P}_{2} \mathrm{X}_{7}$ reversible blocker KN-62 and did not induce cell fusion. However, PMBN was partially active when the more potent $\mathrm{P} 2 \mathrm{X}_{7} \mathrm{R}$ agonist benzoylbenzoyl-ATP (BzATP) was used in place of ATP. In summary, our data show that interaction of $\mathrm{PMB}$ with $\mathrm{P} 2 \mathrm{X}_{7} \mathrm{R}$ depends on the presence of the highly hydrophobic N-terminal region of this antibiotic.

\section{THE ROLE OF PHOSPHORYLATED P38 MAPK IN THE PROTECTIVE MECHANISM OF ADENOSINE RECEPTOR ACTIVATION AGAINST HYPOXIC CONDITIONS}

\author{
Leshem $\mathrm{D}^{1}$, Hochhauser $\mathrm{E}^{2}$, Kaminski $\mathrm{O}^{2}$,Shneyvays $\mathrm{V}^{2}$, Cheporko $\mathrm{Y}^{2}$, Vidne BA ${ }^{2}$, Shainberg $\mathrm{A}^{1^{*}}$. \\ ${ }^{1}$ Bar-Ilan University, Ramat Gan, Israel. ${ }^{2}$ FMRC, Rabin Medical Center, Tel Aviv University, Tel Aviv, Israel \\ (*shaina@mail.biu.ac.il).
}


Activation of either $A_{1}$ adenosine receptor $\left(A_{1} R\right)$ or $A_{3}$ adenosine receptor $\left(A_{3} R\right)$ elicits protection against infarction, ischemia or hypoxia. The mechanism of this protection is not fully understood. Recently it was also shown that ischemic preconditioning attenuated ischemia/reperfusion (I/R)-induced cardiac dysfunction through modulation of p38 MAPK. The purpose of this study is to investigate the involvement of p38 MAPK in the mechanism of adenosine receptor activation in cardioprotection in cardiomyocyte cultures as well as in the whole heart.

Cultured cardiomyocytes were incubated with SB203580 (a specific inhibitor for phosphorylated p38) for 15 min, then treated with CCPA or Cl-IB-MECA (the agonists of $\mathrm{A}_{1}$ and $\mathrm{A}_{3}$ adenosine receptors, respectively) before being subjected to 90 min hypoxia. Levels of LDH released from the cells, and ATP content were measured. Phosphorylated p38 MAPK was examined using Western blot analysis on both cell culture and isolated rat hearts that were injected with CCPA or Cl-IB-MECA $(10 \mathrm{nM}) 24$ hours before I/R.

Results: Both $\mathrm{A}_{1} \mathrm{R}$ and $\mathrm{A}_{3} \mathrm{R}$ agonists reduced hypoxia-induced injury both in vivo and in-vitro. However, when SB203580 was given together with these agonists, the protection was prevented as revealed by LDH release, ATP content and mitochondrial membrane potential. It was also shown that phosphorylated p38 appeared only in heart pretreated with adenosine receptor agonists.

Conclusions: CCPA and Cl-IB-MECA protect both cell culture and isolated hearts against ischemia. This protection was partially related to the increased phosphorylation of p38 MAPK before and during ischemia. It is known that phosphorylation of p38 MAPK activates intracellular signaling which protects the cytoskeleton against degradation.

\title{
Ticlopidine and clopidogrel affect vascular smooth muscle cell proliferation in culture
}

\author{
M. Montopoli, E. Ragazzi, L. Caparrotta and G. Froldi \\ Department of Pharmacology and Anesthesiology - Pharmacology Division- Largo E. Meneghetti 2, University of \\ Padova. E-mail: monica.montopoli@unipd.it
}

Extracellular nucleotides have been shown to mediate the proliferation and migration of vascular smooth muscle cells (VSMC) involved in intimal lesions following vascular injury. Ticlopidine and clopidogrel are prodrugs that are converted in the liver into irreversible antagonists of P2Y12 receptors, having the highest expression among the $\mathrm{P} 2$ receptors in platelets. The aim of our research was to study direct effects of thienopyridines on VSMC proliferation. We carried out experiments in VSMC derived from rat aorta and cell vitality was measured by MTT test. $1 \mu \mathrm{M}$ Ticlopidine per se decreased cell proliferation, whereas at $100 \mu \mathrm{M}$ it significantly stimulated VSMC proliferation (Figure). Incubation of VSMC with ticlopidine (1-100 $\mu \mathrm{M})$ and $50 \mu \mathrm{M}$ ADP, a well known activator of VSMC proliferation, added up effects of single substances. 2-MethioADP $(0.1-1 \mu \mathrm{M})$, a stable analogue of ADP with high affinity to P2Y1 and P2Y12 receptors, only slightly increased VSMC proliferation. $0.1 \mu \mathrm{M}$ 2-MethioADP did not modify effects of ticlopidine $(1-100 \mu \mathrm{M})$ on VSMC proliferation. 1 and $10 \mu \mathrm{M}$ Clopidogrel slightly decreased SMC proliferation, whereas at $100 \mu \mathrm{M}$ an increased proliferation was evidenced $(+140 \% \pm 7)$. The effects induced by clopidogrel were not affected by the presence of $50 \mu \mathrm{M}$ ADP or $0.1 \mu \mathrm{M}$ 2-MethioADP; as for ticlopidine, no inhibition between adenine nucleotides and clopidogrel on the VSMC proliferation was evidenced. It has been shown that clopidogrel significantly inhibits intimal proliferation after arterial injury in rabbit by unknown mechanisms (1) and it has been suggested that ticlopidine enhances the interleukin $1 \beta$-stimulated NO release in cultured rat smooth muscle cells via cAMP- and pKA-dependent mechanism (2). Our experimental data indicate that thienopyridines, without hepatic biotrasformation, can directly influence vascular cell growth in culture, since both ticlopidine and clopidogrel at micromolar concentrations inhibit VSMC proliferation, whereas at higher concentrations stimulate it. Also our results indicate that thienopyridines action is independent from P2Y1 and P2Y12 receptors. Further researches on the proliferative effects of the two antiplatelet drugs are under evaluation. 


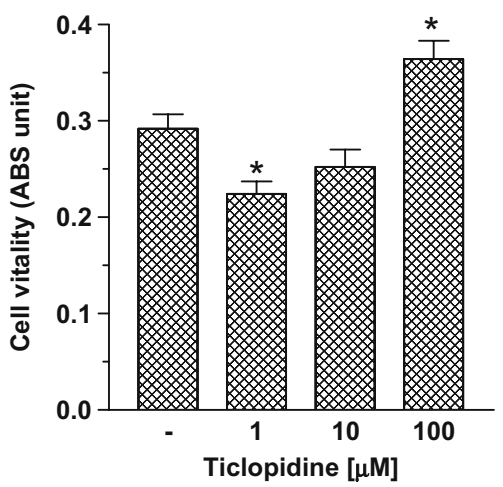

\section{References}

1) Herbert JM, Tissinier A, Defreyn G, Maffrand JP. Inhibitory effect of clopidogrel on platelet adhesion and intimal proliferation after arterial injury in rabbits. Arterioscler Thromb. 1993, 13:1171-9.

2) Inoue M, Kusano E, Ito C, Akimoto T, Iimura O, Nemoto J, Amemiya M, Muto S, Asano Y. The anti-platelet agent, ticlopidine, upregulates interleukin-1-Beta-stimulated nitric oxide production in cultured rat vascular smooth muscle cells. Exp Nephrol. 2002, 10:267-74.

\section{Toll-Like Receptors (TLRs) Synergistically Enhance Adenosine $\mathbf{A}_{2 \mathrm{~A}}$ Receptor $\left(A_{2 A} R\right)$-Mediated Induction of a Switch in Macrophages from an Inflammatory to an Anti-Inflammatory, Angiogenic Phenotype.}

S. Joseph Leibovich, Ph.D.*, Thomas W. Lysz, Genie Elson, Grace Pinhal-Enfield, Ph.D., Joseph Quispe, and Stan Grinberg

Department of Cell Biology \& Molecular Medicine \& The Cardiovascular Research Center, New Jersey Medical School, UMDNJ, 185 South Orange Avenue, Newark, NJ 07103, USA.

E-mail:leibovic@umdnj.edu

TLR2, 4, 7 and 9 agonists synergize with $\mathrm{A}_{2 \mathrm{~A}} \mathrm{R}$ agonists to induce a phenotypic switch of macrophages from an inflammatory to an anti-inflammatory, angiogenic phenotype ${ }^{1,2}$. This switch strongly up-regulates expression of VEGF, IL-10 and sphingosine kinase-1 (SK-1), while strongly down-regulating expression of TNF $\alpha$, IL-12, MIP1 $\alpha$ and matrix metalloproteinase-9 (MMP9). Up-regulation of VEGF expression is mediated at both the transcriptional and mRNA stability levels, and involves the induction of expression of Hypoxia-Inducible Factor-1 $\alpha$ (HIF1$\alpha)$ mRNA and stabilization of HIF1- $\alpha$ protein. The Hypoxia Response Element (HRE) in the promoter of the VEGF gene is critically required for the $A_{2 A} R$-depenent induction of VEGF transcription, while putative NF- $\kappa B$ sites in this promoter are not. In contrast, the down-regulation of $T N F \alpha$ expression by $\mathrm{A}_{2 \mathrm{~A}} \mathrm{R}$ agonists is mediated at the translational level rather than transcriptionally, and is also independent of NF- $\mathrm{B}$ activation. As reported by Murphree et $\mathrm{al}^{3}$, TLRs up-regulate expression of $\mathrm{A}_{2 \mathrm{~A}} \mathrm{Rs}$, as well as of $\mathrm{A}_{2 \mathrm{~B}}$ Rs on macrophages, and this upregulation may play an important role in the angiogenic switch. The TLR and $\mathrm{A}_{2 \mathrm{~A}} \mathrm{R}$-dependent induction of VEGF expression is not blocked by adenylyl cyclase inhibitors (SQ22536, 2',5'-dideoxyadenosine), by Protein Kinase-A (PK-A) inhibitors, or by MAP-kinase inhibitors, suggesting a cAMP/PK-A independent signaling pathway. Also, specific inhibitors of Gsa signaling such as NF449, do not block the up-regulation of VEGF or down-regulation of TNF $\alpha$ expression. In contrast, inhibition of myo-inositol specific phospholipase-C (PL-C) with selective inhibitors suggests that PL-C signaling is specifically involved in both the up-regulation of VEGF transcription and the down-regulation of TNF $\alpha$ expression. Specific ablation of PL-C $\beta$ isoforms using siRNA in LPS-treated macrophages suggests a key role for the PL-C $\beta 2$ isoform in this pathway. Ablation of PL-C $\beta 2$ strongly up-regulates expression of VEGF in LPS-treated macrophages, while simultaneously down-regulating $\mathrm{TNF} \alpha$-expression, thus mimicking the effects of $\mathrm{A}_{2 \mathrm{~A}} \mathrm{R}$ agonists. This suggests an important role for PL-C $\beta 2$ signaling in the regulation of the switch of macrophages from an inflammatory to an anti-inflammatory, angiogenic phenotype. 
1. Leibovich, S. J.; Chen, J. F.; Pinhal-Enfield, G.; Belem, P. C.; Elson, G.; Rosania, A.; Ramanathan, M.; Montesinos, C.; Jacobson, M.; Schwarzschild, M. A.; Fink, J. S.; Cronstein, B., Synergistic up-regulation of vascular endothelial growth factor expression in murine macrophages by adenosine $\mathrm{A}_{2 \mathrm{~A}}$ receptor agonists and endotoxin. Am. J. Pathol. 2002, 160, $2231-2244$. 2. Pinhal-Enfield, G.; Ramanathan, M.; Hasko, G.; Vogel, S. N.; Salzman, A. L.; Boons, G. J.; Leibovich, S. J., An angiogenic switch in macrophages involving synergy between Toll-like receptors 2, 4, 7, and 9 and adenosine A(2A) receptors. Am $J$ Pathol 2003, 163, (2), 711-21.

3. Murphree, L. J., Sullivan, G.W., Marshall, M.A., Linden, J., Lipopolysaccharide rapidly modifies adenosine receptor transcripts in murine and human macrophages: role of NF-кB in $\mathrm{A}_{2 \mathrm{~A}}$ adenosine receptor induction. Biochem. J. 2005, 391, 575-580.

* Supported in part by a grant from the US Public Health Service (RO1-GM068636)

\title{
Two new pathways of AMP-activated protein kinase (AMPK) activation in endothelial cells. Involvement of $\mathrm{P} 2$ receptors and adenosine transporters.
}

\author{
Cleide Gonçalves da Silva ${ }^{1}$, Robert Jarzyna ${ }^{1,2}$, Anke Specht ${ }^{1}$, Elzbieta Kaczmarek ${ }^{1}$ \\ ${ }^{1}$ Beth Israel Deaconess Medical Center, Harvard Medical School, Boston, MA, USA; \\ ${ }^{2}$ Warsaw University, Warsaw, Poland. \\ ekaczmar@bidmc.harvard.edu
}

\begin{abstract}
AMPK plays a key role in the regulation of energy homeostasis and is activated in response to cellular stress, including hypoxia/ischemia and hyperglycemia. AMPK is a heterotrimeric Ser/Thr kinase consisting of a catalytic alpha subunit and regulatory beta and gamma subunits. Depletion of ATP followed by an increase in the AMP level and AMP:ATP ratio lead to activation of AMPK. However, AMPK can also be phosphorylated and activated by the mechanism independent of changes in the AMP:ATP ratio. AMPK is activated allosterically by AMP and by phosphorylation of Thr-172 on the alpha subunit, which is catalyzed by upstream kinases, including LKB1 and $\mathrm{Ca}^{2+} /$ calmodulin-dependent kinase kinase (CaMKK). Activated AMPK turns on catabolic pathways that generate ATP and turns off pathways that consume ATP by phosphorylation of multiple targets. Since AMPK is essential in controlling the metabolism of glucose and fatty acids, its role in obesity and type 2 diabetes is of major importance. AMPK is expressed in skeletal muscle, brain, liver, adipocyte and pancreas. AMPK has been also localized in endothelial cells (EC), however pathways of its activation, as well as the functions of this kinase in the endothelium are still not well understood.

The stress events are accompanied by rapid release of extracellular nucleotides from damaged tissues or activated EC and platelets. We demonstrate that extracellular nucleotides (ATP, ADP and UTP, but not UDP) and adenosine, independently induce phosphorylation and activation of AMPK in human umbilical vein EC (HUVEC) by the mechanism that is not linked to changes in the AMP:ATP ratio. HUVEC express NTPDases, as well as 5'-nucleotidase, hence nucleotides can be metabolized to adenosine. However, inhibition of $5^{\prime}$-nucleotidase had no effect on ATP/ADP/UTPinduced phosphorylation of AMPK, indicating that AMPK activation occurred as a direct response to nucleotides. Pharmacological evaluation of nucleotide-evoked phosphorylation of AMPK in HUVEC led to the conclusion that AMPK activation was mediated by P2Y1, P2Y2 and/or P2Y4 receptors, while P2Y6, P2Y11 and P2X receptors were not involved. The nucleotide-induced phosphorylation of AMPK was affected by changes in the concentration of intracellular $\mathrm{Ca}^{2+}$ and by CaMKK, while most likely it was not dependent on LKB1 kinase. Adenosine-induced phosphorylation of AMPK was not mediated by P1 receptors but required adenosine uptake by equilibrative nucleoside transporters followed by its (intracellular) metabolism to AMP. Moreover, adenosine effect was $\mathrm{Ca}^{2+}$ - and CaMKK-independent while probably associated with upstream LKB1. We hypothesize that $\mathrm{P} 2$ receptors and adenosine transporters could be novel targets for the pharmacologic regulation of AMPK activity and its downstream effects on EC function.
\end{abstract}

\section{Typical neuroleptics regulated $A_{2 A}$ adenosine receptors in human platelets of patients affected by bipolar disorder.}

M. Letizia Trincavelli, M. Montali, S. Cuboni, E. Cerrai, A. Ciapparelli, A. Lucacchini, GB Cassano, L. Dell'Osso, C. Martini. 
Department of Psychiatry, Neurobiology, Pharmacology and Biotechnology. University of Pisa; Italy. email: ltrincavelli@farm.unipi.it

Neuroleptic drugs, potent dopamine receptor antagonists, are commonly used in the treatment of psychotic and affective illness. An antagonistic functional interactions between $A_{2 A}$ adenosine receptors (ARs) and $D_{2}$ dopamine receptors (DRs) has been demonstrated in the central nervous system suggesting that the adenosine system may be involved in the pathogenesis of basal ganglia disorders. In a previous work we have demonstrated that chronic treatment with typical antipsychotics induced a significant modulation on $\mathrm{A}_{2 \mathrm{~A}} \mathrm{AR}$ binding parameters and receptor responsiveness in platelets from psychotic patients with respect to healthy volunteers, pointing to $\mathrm{A}_{2 \mathrm{~A}}$ ARs as a possible target to test the efficacy of typical antipsychotic therapy.

In this work we evaluated the effect of typical neuroleptics on $\mathrm{A}_{2 \mathrm{~A}} \mathrm{AR}$ binding parameters in human platelets of patients affected by bipolar disorder (BD) under chronic treatment with these drugs, in particular evaluating the correlation between $D_{2}$ DR occupancy and the $A_{2 A} A R$ alterations. A cohort of 28 patients affected by $B D$ with or without psychotic symptoms were consecutively recruited from the Department of Psychiatry at the University of Pisa (Pisa, Italy). All patients were naturalistically treated with antipsychotic drugs for at least one month. Control group included 32 healthy volunteers with no history of mental disorder, alcoholism, or drug abuse and with no medical illness, as determined by clinical interview. The study was approved by the local Ethical Committee in accordance with the Declaration of Helsinki (1996) and with the Guidelines of the Good clinical Practice (1995).

$\mathrm{A}_{2 \mathrm{~A}} \mathrm{AR}$ equilibrium binding parameters were determined on platelet membranes obtained from healthy volunteers and from patients, by saturation binding studies using the selective $\mathrm{A}_{2 \mathrm{~A}} \mathrm{AR}$ antagonist, $\left[{ }^{3} \mathrm{H}\right] \mathrm{ZM}_{241385}$. For correlation studies, the mean dosage of drugs was reported as equivalent of chlorpromazine.

The obtained results demonstrated that in $\mathrm{BD}$ patients, typical neuroleptics induced a significant decrease in $\mathrm{A}_{2 \mathrm{~A}}$ AR ligand affinity values with respect to healthy volunteers demonstrating a significant alteration in receptor comphormational state. On the contrary, no significant alterations in maximum density of $A_{2 A} A R$ binding sites was detected. By the means of ANOVA statistical test a significative correlation between $A_{2 A} A R$ affinity values and the mean drug dosage was detected in relation to severity illness.

These results suggest $A_{2 A} A R$ are selectively regulated by $D_{2} D R$ antagonists in relation to dopamine receptor occupancy and to the individual responsiveness to the drugs.

Sundram et al. (2003) Current Mol. Med. 3: 393-407.

Martini C. et al. (2006) J. Psych. Res. 40: 81-88.

\section{Upregulation of P2Y2 receptors by retinoids in normal human epidermal keratinocytes}

Kayoko Fujishita $^{1}$, Kazuhide Inoue ${ }^{2}$ and Schuichi Koizumi ${ }^{1}$

${ }^{T}$ Division of Pharmacology, National Institute of Health Sciences, 1-18-1 Kamiyoga, Setagaya, Tokyo 158-8501, Japan.

${ }^{2}$ Graduate School of Pharmaceutical Sciences, Kyusyu University, 3-1-1 Maidashi, Higashi, Fukuoka 812-8582, Japan.

fujishit@nihs.go.jp

Retinoids, general term of vitamin A derivatives, play critical regulatory roles in growth and differentiation in epidermis. They are often used clinically to the cure of some skin troubles or disorders, such as pigmentation and wrinkles. So far, however, molecular mechanisms by which retinoids reveal their therapeutic effects have only limited attention, let alone their effects on P2 receptors, receptors that regulate various skin functions. In this study, we used normal human epidermal keratinocytes (NHEKs) and assessed the effect of retinoids on P2 receptors. DNA array analysis showed that among P2 receptors in NHEKs, mRNAs for P2Y2 receptors are selectively upregulated by the treatment with all-trans retinoic acid (ATRA), an agonist to RAR (retinoic acid receptor). ATRA increased the mRNA for the P2Y2 receptor in a concentration- (1 nM to $1 \mu \mathrm{M})$ and an exposure time- ( 2 to $24 \mathrm{hr}$ ) dependent manner. Am80, a synthesized agonist to RAR, showed a similar increment, whereas 9-cis retinoic acid (9-cis RA), an agonist to RXR (retinoid $\times$ receptor), induced a lesser enhancement of 
P2Y2 genes. These results indicate that retinoids upregulate P2Y2 mRNAs mainly via RAR. Moreover, fura-2 based $\mathrm{Ca}^{2+}$ imaging analysis revealed that ATRA also increased function of P2Y2 receptors in NHEKs. P2Y2 receptors are important for proliferation in basal layer of skin (ref 1,2), and our present results indicated that retinoids selectively upregulated $\mathrm{P} 2 \mathrm{Y} 2$ receptor-mediated responses in NHEKs. All these findings suggest that retinoids would, at least in part, achieve their growth effects via upregulation of P2Y2 receptors, thereby leading to therapeutic gain of retinoids against ailments and aging events in skin.

1. Greig AV et al., J Invest Dermatol 120: 1007-1015, 2003

2. Burrell HE et al., J Invest Dermatol 120: 440-447, 2003

\title{
Uracil nucleotides protect murine HL-1 cardiomyocytes from cell-cycle changes and apoptotic/necrotic death induced by adenine nucleotides and tumor necrosis factor-alpha
}

\author{
Alessia Mazzola*, Emanuela Amoruso*, Elena Tremoli, Maria P. Abbracchio \\ ${ }^{T}$ Laboratory of Molecular and Cellular Pharmacology of Purinergic Transmission, Department of Pharmacological \\ Sciences, University of Milan, and Monzino Cardiologic Center IRCCS, Milan, Italy \\ alessia.mazzola@unimi.it*These authors equally contributed to this work
}

Despite available therapies, chronic heart failure (CHF) remains a major cause of morbidity and mortality in western countries, suggesting that key pathogenic mechanisms still have to be uncovered. Pyrimidine and purine nucleotides are released from heart sympathetic terminals and hypoxic cardiomyocytes. In addition to their intracellular functions in signalling and genetic coding, nucleotides have an important role as extracellular signalling molecules. P2 receptors activated by nucleotides consist of two families: seven ligand-gated ion channels (the $\mathrm{P} 2 \mathrm{X}_{1-7}$ receptors) and eight G-protein-coupled receptors (the $\mathrm{P}_{2} \mathrm{Y}_{1,2,4,6,11,13,14}$ receptors) (1). At variance from $\mathrm{P} 2 \mathrm{X}$ receptors, some $\mathrm{P} 2 \mathrm{Y}$ receptors can also or exclusively respond to uracil nucleotides. We recently demonstrated the presence of four $\mathrm{P} 2 \mathrm{Y}$ receptors ( $\mathrm{P} 2 \mathrm{Y}_{2,4,6,13}$ receptors) in an in vitro model of murine cardiomyocytes (HL-1 cells) (see: Amoruso et al., accompanying abstract), the only cell line that continuously divide, spontaneously contract and maintain a differentiated adult cardiac phenotype in culture (2). In the present study, we have focused our attention on the differential role of adenine (ATP and ADP) and uracil nucleotides (UTP, UDP, UDP-glucose) in regulating the viability of HL-1 cells. Exposure of HL-1 cells to ATP or ADP (500 $\mu \mathrm{M})$ for 24 hours induced significant apoptosis and necrosis. The effect induced by ATP was concentration-dependent. In line with previous data (Banfi et al., 3, and Amoruso et al., accompanying abstract), these effects were increased by pretreatment of cells with the cytokine tumor necrosis factor alpha (TNF- $\alpha, 10 \mathrm{ng} / \mathrm{ml})$ for 16 hours. On the contrary, uracil nucleotides (UTP, UDP and UDP-glucose, $500 \mu \mathrm{M}$ ), utilized either alone or with TNF- $\alpha$, did not induce apoptosis or necrosis "per se", but significantly reduced cardiomyocyte death induced by adenine nucleotides. Flow-cytometry analysis showed that ATP and ADP-induced cell death was also associated to altered cellcycle progression; in particular, a reduction of the number of HL-1 cells in S and G2/M phase was observed. Uracil nucleotides also effectively prevented the alterations of cell-cycle progression induced by adenine nucleotides in the presence of the proinflammatory cytokine. Globally, these data suggest that, in cardiomyocytes, activation of specific P2Y receptor subtypes by uracil nucleotides can counteract induction of cell death by ATP and ADP. We are currently evaluating the role of mitochondria and the involvement of caspase cascades in our experimental model. The present results suggest an important role for some $\mathrm{P} 2 \mathrm{Y}$ receptor subtypes $\left(\mathrm{P} 2 \mathrm{Y}_{2}, \mathrm{P}_{2} \mathrm{Y}_{4}\right.$ and/or $\mathrm{P}_{2} \mathrm{Y}_{6}$ ) in cardiomyocyte survival and cardioprotection and may lead to the identification of new therapeutic strategies for heart disease.

The authors warmly thank Professor William Claycomb, LSU Health Sciences Center, New Orleans, LA, USA, for the kind gift to HL-1 cells

(1) Abbracchio, M.P.; Boeynaems, J.M.; Barnard, E.A.; Boyer, J.L.; Kennedy, C.; Mira-Portugal, M.T.; King, B.F.; Gachet, C.; Jacobson, K.A.; Weisman, G.A.; Burnstock, G. Trends Pharmacol Sci, 24, 52-55.

(2) White, S.M.; Costantin, P.E.; Claycomb, W.C. Am. J. Physiol. Heart Circ.Physiol. 2004, 286 (3), H823-829.

(3) Banfi, C; Ferrario, S; De Vincenti, O; Ceruti, S; Fumagalli, M; Mazzola, A; D' Ambrosi, N; Volonte, C; Fratto, P; Vitali, E; Burnstock, G; Beltrami, E; Parolari, A; Polvani, G,; Biglioli, P; Tremoli, E; Abbracchio, MP. J Mol Cell Cardiol. 2005 39:929-39. 


\title{
Uracil nucleotides are involved in cardiac Protection of the heart from ischemic stress
}

\author{
Asher Shainberg $^{1 *}$, Smadar Yitzhaki ${ }^{1}$, Vladimir Shneyvays ${ }^{1}$ and Edith Hochhauser ${ }^{2}$. \\ ${ }^{T}$ Faculty of Life Sciences, Bar-Ilan University, Ramat Gan, Israel. ${ }^{2}$ FMRC, Rabin Medical Center, Tel Aviv Uni- \\ versity, Tel Aviv, Israel (*shaina@mail.biu.ac.il).
}

Considerable effort has been devoted towards improving functional recovery and reducing the extent of infarction after ischemic episodes since coronary heart disease remains a major worldwide threat. It was found that the heart was significantly protected against ischemic injury, if it was first preconditioned (PC) by a brief ischemia. Accumulating evidence suggests that adenosine, adrenoceptors, bradykinin, opioid and ATP receptors contribute to PC.

Massive amounts of nucleotides are released during ischemia and hypoxia in the cardiovascular system. Whereas the effect of purine nucleotides (ATP) in myocardial infarction was intensively studied, the role of pyrimidine nucleotides (UTP) under hypoxic condition has not been demonstrated. The principal aim of our study is to elucidate the protective effects of UTP and pyrimidinergic receptor activation against detrimental factors of ischemia/hypoxia as well as to investigate the mechanism by which the relevant pyrimidine receptor is coupled to its respective intermediate effectors, such as $\mathrm{IP}_{3}$ receptors, and the downstream cascade, which exerts distinctive cardioprotective responses.

Results: We found that UTP significantly reduced cardiomyocyte death induced by hypoxia [1]. This effect of UTP was not observed with its derivative, UDP. Even incubation (1 hour) with UTP, 24 hours before exposing the cells to hypoxic conditions, protected the cells. The cardioprotective effect of UTP was reduced in the presence of the non-selective P2 antagonist - suramin. In addition, UTP caused a transient increase of $\left[\mathrm{Ca}^{2+}\right]_{\mathrm{i}}$ level in cardiomyocytes. PPADS or $\mathrm{RB}_{2}$, other antagonists of $\mathrm{P} 2$ receptors, abolished $\left[\mathrm{Ca}^{2+}\right]_{\mathrm{i}}$ elevation caused by UTP. Using various inhibitors of the $\mathrm{Ca}^{2+}$ signaling pathway, we have shown that UTP originating from intracellular sources, elevated the $\left[\mathrm{Ca}^{2+}\right]_{\mathrm{i}}$ level via PLC and the $\mathrm{IP}_{3}$ receptor. Interestingly, BAPTA-AM, a $\left[\mathrm{Ca}^{2+}\right]_{\mathrm{i}}$ chelator, and other inhibitors of the $\mathrm{Ca}^{2+}$ signaling pathway, did not prevent the protective effect caused by UTP. Preliminary studies in an in vivo model of myocardial infarction showed the same protective effects.

Conclusion: This study describes the cellular protective role of UTP nucleotide on cardiomyocytes against hypoxic damage, which is mediated via nucleotide receptor(s).

1. Yitzhaki, S., Shneyvays, V., Jacobson, K.A. and Shainberg, A. Involvement of uracil nucleotides in protection of cardiomyocytes from hypoxic stress. Biochem. Pharmacol. 69, 1215-1223, 2005.

\section{Vascular endothelial growth factor regulation by adenosine via hypoxia-inducible factor-1 in hypoxic human glioblastoma cells}

\author{
Stefania Merighi ${ }^{1}$, Annalisa Benini ${ }^{1}$, Prisco Mirandola ${ }^{2}$, Stefania Gessi ${ }^{1}$, Katia Varani $^{1}$, Edward Leung ${ }^{3}$, \\ Stephen Maclennan ${ }^{3}$, Pier Andrea Borea ${ }^{1,4}$ \\ ${ }^{1}$ Department of Clinical and Experimental Medicine, Pharmacology Unit; University of Ferrara, 44100, Ferrara, \\ Italy; ${ }^{2}$ Department of Anatomy, Pharmacology and Forensic Medicine, Human Anatomy Section, University of \\ Parma, 43100, Parma, Italy; ${ }^{3}$ King Pharmaceuticals Research \& Development, Cary, North Carolina 27513, U.S.A.; \\ ${ }^{4}$ Interdisciplinary Center for the Study of Inflammation; University of Ferrara, 44100, Ferrara, Italy. \\ mhs@unife.it
}

Hypoxia-inducible factor-1 (HIF-1) is a key regulator of genes crucial to many aspects of cancer biology. Here we show that in the human A172 and U87MG glioblastoma cell lines under hypoxic conditions $\left(1 \% \mathrm{O}_{2}\right)$ adenosine up-regulates HIF- $1 \alpha$ protein expression in a dose- and time- dependent manner, exclusively via the $\mathrm{A}_{3}$ receptor subtype. The response to adenosine was generated at the cell surface since the inhibition of $\mathrm{A}_{3}$ receptor expression, by using small interfering RNA, abolished the nucleoside effects. We investigated the effect of $\mathrm{A}_{3}$ receptor antagonists on HIF-1 and vascular endothelial growth factor (VEGF) expression. We found that $\mathrm{A}_{3}$ antagonists inhibit adenosine-induced HIF-1 $\alpha$ and VEGF protein accumulation in the hypoxic cells. Investigations 
of the molecular mechanism showed that $\mathrm{A}_{3}$ receptor stimulation activates $\mathrm{p} 44 / \mathrm{p} 42$ and p38 MAPKs that are required for $\mathrm{A}_{3}$-induced increase of HIF-1 $\alpha$ and VEGF. Further studies are required to demonstrate the in vivo relevance of these observations with regard to the proposed role for adenosine as a key element in situations of hypoxia and tumors.

\title{
Which is the receptor mediating ADP signaling in rat cerebellar astrocytes?
}

\author{
Luz María Gutiérrez Carrasquero, Esmerilda García Delicado and María Teresa Miras-Portugal. \\ Departamento de Bioquímica. Facultad de Veterinaria. Universidad Complutense de Madrid. Spain \\ luzmaria@vet.ucm.es
}

During the last years it has been demonstrated that nucleotides are important signaling molecules in the SNC, some of the actions previously attributed to ATP appear to be mediated by other nucleotides, such as ADP. ADP possesses specific metabotropic receptors, the $\mathrm{P} 2 \mathrm{Y}_{1}, \mathrm{P}_{2} \mathrm{Y}_{12}$ and $\mathrm{P} 2 \mathrm{Y}_{13}$ subtypes. The characterization was made in heterologous system expression, but going to native tissues is quite difficult, with the exception of platelets, which are an excellent model for ADP receptor signaling studies. Although the three receptors exhibit high affinity for ADP and 2MeSADP, they differ in the relative potency of the two agonists; P2 $\mathrm{Y}_{1}$ is coupled to PLC activation, whereas $\mathrm{P} 2 \mathrm{Y}_{12}$ and $\mathrm{P} 2_{13}$ receptors are negatively coupled to adenylate cyclase. With respect to antagonists, MRS2179 is selective for $\mathrm{P}_{2} \mathrm{Y}_{1}$, but, to date, there are no antagonists able to discriminate between $\mathrm{P} 2 \mathrm{Y}_{12}$ and $\mathrm{P} 2 \mathrm{Y}_{13}$ receptors. The unique selective antagonist for $\mathrm{P}_{2} \mathrm{Y}_{12}$ receptor is a metabolite originated from clopidogrel metabolism, which limits its use to animal models.

Several studies suggested the existence of ADP Gi-coupled receptors in rat cerebellum, by which we decided to investigate their presence in cultured cerebellar astrocytes. In previous studies we found that all cerebellar astrocytes responded to ADP and ATP stimulations with metabotropic calcium responses, which were mediated by $\mathrm{P} 2 \mathrm{Y}_{1}$ and $\mathrm{P}_{2} \mathrm{Y}_{2} / \mathrm{P}_{2} \mathrm{Y}_{4}$ receptors, respectively. However, additional studies carried out using the new available agonist 2MeSADP, have demonstrated that calcium responses and other intracellular cascades triggered by ADP and/or $2 \mathrm{MeSADP}$ could be mediated mainly by a $\mathrm{P}_{2} \mathrm{Y}_{13}$-like receptor. This was supported by the following data: 1.- ADP and $2 \mathrm{MeSADP}$ inhibited adenylate cyclase activation induced by isoproterenol, with similar $\mathrm{IC}_{50}$ values, which suggested the involvement of a $\mathrm{P}_{2} \mathrm{Y}_{13}$ receptor. These experiments were carried out in the presence of MRS-2179.

2.- Microfluorimetric experiments using fura-2 showed that 2 MeSADP induces intracellular calcium mobilization. These responses were comparable to those elicited by UTP. However, when the effect of MRS-2179 was checked, cells exhibited heterogeneous responses: there were cells in which 2MeSADP responses were abolished or diminished in different extents, and there was also a subpopulation, whose responses were not affected, indicating that another receptor different from $\mathrm{P}_{2} \mathrm{Y}_{1}$ is being activated.

3.-Finally, we studied the effect of 2MeSADP in ERK activation. We observed that the stimulation of the cells with this compound induced an increased in phosphorylated ERK levels. This effect was dosis-dependent with a $\mathrm{EC}_{50}$ of $4.85 \pm 1.5 \mathrm{nM}$ and insensitive to MRS-2179. ERK activation was sensitive to Pertussis Toxin, src protein inhibitors and long-term treatment with phorbol esters.

All these findings would indicate that $\mathrm{P}_{2} \mathrm{Y}_{13}$-like receptor present in cerebellar astrocytes triggered several intracellular cascades, that at first were supposed to be mediated by P2 $\mathrm{Y}_{1}$, such as PLC activation and the parallel MAP kinase cascade activation, and which have not been described for the cloned $\mathrm{P} 2 \mathrm{Y}_{13}$ receptor. The question that arises is what it is role of $\mathrm{P}_{2} \mathrm{Y}_{1}$ receptor?

\section{Xanthines foster up-regulation of export-deficient variants of the $\mathbf{A}_{\mathbf{1}}$-adenosine receptor}

Laura Málaga-Diéguez, Halyna Pankevych, Oliver Kudlacek and Christian Nanoff

Institute of Pharmacology. Center for Biomolecular Medicine and Pharmacology, Medical University of Vienna, Austria

laura.malaga-dieguez@meduniwien.ac.at 
Caffeine consumption fails to up-regulate the $\mathrm{A}_{1}$ adenosine receptor in the CNS in vivo. However, we find that the lipophilic xanthines 1,3-dipropyl-8-cyclopentylxanthine (DPCPX) and isobutyl-methylxanthine (IBMX) can act as efficient pharmacochaperones for the $\mathrm{A}_{1}$-receptor in cultivated cell lines. Using $\mathrm{A}_{1}$-receptor variants with wild-type sequence or with sequence mutations in the receptor carboxyl-(c-)terminus we have assessed the mechanism by which xanthines up-regulate the receptor. (i) Both the low- and high-affinity antagonist ligands DPCPX and IBMX caused an increase in receptor density; agonists, by contrast, had no effect. When an exportdeficient receptor was tested the pharmacochaperones raised the level of surface expressed receptors to the range of wild-type receptors. For the mutants the drug-induced increase was marked as assessed by radioligand binding ( 15-fold with $1 \mu \mathrm{M}$ DPCPX; 6-fold with $50 \mu \mathrm{M}$ IBMX). FACS (fluorescence activated cell sorting) using an antibody against an extracellular epitope indicated that the plasma membrane was the site where receptor density increased. On the wild-type receptor, however, the effect was more modest (1.6-fold). (ii) By contrast, the recombinant $\mathrm{A}_{1}$-receptor expressed in bacteria was completely refractory to pharmacochaperoning suggesting that the ligand does not act as a scaffold during co-translational membrane insertion and folding. Rather, we believe that the effect of the pharmacochaperone set in once the receptor was mature enough to pass the endoplasmic reticulum (ER) quality control since our previous findings had indicated that $\mathrm{A}_{1}$-receptor retained in the ER fails to bind ligand. (iii) Pharmacochaperoning was critically controlled by an ER-associated cytosolic heat-shock protein, DRiP78 (dopamine receptor interacting protein, member of the hsc40 family), that was found to directly bind to the $\mathrm{A}_{1}$-receptor c-terminus. Overexpression of DRiP78 reduced receptor surface expression and, in addition, blunted the pharmaco-chaperone effect. This inhibition was reverted by sequence specific RNA-interference. The findings point to the ER-exit gate as the bottle neck in the export pathway where DRiP78 acts as a guardian. (iv) The export deficient receptor variants had been created by mutating single amino acid residues present in the conserved $\mathrm{NPXXY}(\mathrm{X})_{6} \mathrm{~F}$ motif within the junction between helices 7 and 8 . Exchanging either $\mathrm{Y}$ or $\mathrm{F}$ to alanine resulted in receptors that gave very low surface expression levels. Nevertheless, the F/A mutant receptor was perfectly capable of inhibiting cAMP formation. As opposed to the wild-type receptor, however, the surface level of the F/A variant was not augmented by DRiP78 RNAi. Thus, antagonist ligands can up-regulate the $\mathrm{A}_{1^{-}}$ adenosine receptor provided that the receptor is functional but retained intracellularly; we speculate that pharmacochaperoning is effective unless retention is critically controlled by the components of the ER-quality control machinery. 